WATER-LEVEL DATA FOR

SELECTED WELLS ON OR NEAR

THE IDAHO NATIONAL ENGINEERING LABORATORY,

1949 THROUGH 1982

By Jack T. Barraclough, Jefferson C. Bagby, Luke J. White, and Rodger G. Jensen.

U.S. GEOLOGICAL SURVEY

Open-File Report 84-239

Prepared in cooperation with the

U.S. DEPARTMENT OF ENERGY

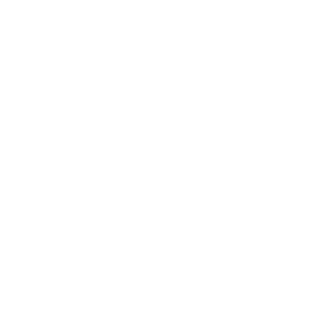

Id aho Falls, Id aho

July 1984 
UNITED STATES DEPARTMENT OF THE INTERIOR

WILLIAM P. CLARK, Secretary

GEOLOGICAL SURVEY

Dallas L. Peck, Director

For additional information write to :

Project Chief

U.S. Geological Survey-WRD

P.0. Box 2230

INEL, CF-690, Rm 164

Idaho Falls, Idaho 83401
Copies of this report can be purchased from:

Open-File Services Section Western Distribution Branch Box 25425, Federal Center Denver, Colorado 80225 
List of wells for which records are published ........... . iv

Conversion factors....................... . viii

Abstract . . . . . . . . . . . . . . . . . . . . . . . 1

Introduction . . . . . . . . . . . . . . . . . . . . . 1

Purpose and scope... . . . . . . . . . . . . . . 4

Acknowledgments . . . . . . . . . . . . . . . . . . . 4

Numbering systems used for wells . . . . . . . . . . . . . . . 11

USGS site identification numbers . . . . . . . . . . . . . 11

Idaho well-numbering system . . . . . . . . . . . . 11

Local well names .. . . . . . . . . . . . . . . 11

Regional hydrology . . . . . . . . . . . . . . . . 17

Collection of records. . . . . . . . . . . . . . . . . . 17

Water-level data collection methods........... . 17

Conclusions . . . . . . . . . . . . . . . . . . . . 18

Selected references . . . . . . . . . . . . . . . . 19

\section{ILLUSTRATIONS}

Figure 1. Relief map of Idaho showing the location of the INEL, Snake River Plain, and generalized ground-water flow lines of the Snake River Plain aquifer (from Barraclough, Lewis, and Jensen, 1981) ........ 2

2. Location of the INEL facilities... . . . . . . . . . 3

3. Site identification numbers and locations of selected wells near the INEL . . . . . . . . . . . . . . 5

4. Local names and locations of selected wells near the INEL . . . . . . . . . . . . . . . . 6

5. Site identification numbers and locations of selected wells on the INEL . . . . . . . . . . . . . . 7

6. Local names and locations of selected wells on the INEL - 8

7. Site identification numbers and locations of selected wells in the ICPP-TRA area . . . . . . . . . . . 9

8. Local names and locations of selected wells in the ICPPTRA area .. . . . . . . . . . . . . . . 10

9. Diagram showing Idaho well-numbering system . . . . . 12

\section{TABLES}

Table 1. Site identification numbers, local well names, and Idaho we11 numbers . . . . . . . . . . . . . . . . . 13

2. Key to abbreviations used in this report . . . . . . . 23

3. Water-level data for selected wells on or near the INEL . 24 
LIST OF WELLS FOR WHICH RECORDS ARE PUBLISHED

We11 name

$01 \mathrm{~S} 27 \mathrm{E} 14 \mathrm{dcc} 1$

$04 \mathrm{~N} 26 \mathrm{E} 32 \mathrm{cbb} 1$

ANP 3 disposal

ANP 5

ANP 6

ANP 7

ANP 9

ANP 10

ARA 2

Arbor test

Arco test

AREA II

Cerro Grande

CFA 2

Corehole 1

Corehole 2A

CPP 1

CPP 2

CPP 3 disposal

$D$ and $W$ Hansen

EBR I

EBR II 1

EBR II 2

EOCR

FET 3 disposal

Fire Station 2

Grazing 2

Highway 2

Highway 3

Highway 1-A

Highway 1-B

Highway 1-C

Id aho Fish and Game

IET 1

INEL 1

Inland Brick

Leo Roger's 1

Linderman and Bo

LOFT 1

LOFT 2

LPTF disposal

LPTF production

MTR test

No name

No own 24

NRF 1

NRF 2

NRF 3
Site ID number

Page

431946113161401 . . . . . . . . . . . 24

433748113234001 . . . . . . . . . . 25

435053112423201 . . . . . . . . . . 34

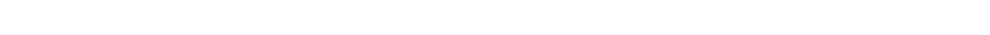

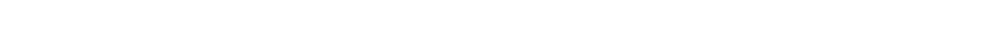

435522112444201 . . . . . . . . . . . . 37

434856112400001 . . . . . . . . . . 38

434909112400401 . . . . . . . . . . 33

433107112492201 . . . . . . . . . . . . 404

433509112384801 . . . . . . . . . . 41

434001113215201 . . . . . . . . . . 43

433223112470201 . . . . . . . . . . . 44

432618112555501 . . . . . . . . . . 45

433144112563501 . . . . . . . . . . 47

432927112410101 . . . . . . . . . . 50

434558112444801 . . . . . . . . . . 51

433433112560201 . . . . . . . . . . 52

433432112560801 . . . . . . . . . 54

433413112560401 . . . . . . . . . 56

435045113031701 . . . . . . . . . 57

433051113002601 . . . . . . . . . . . . 58

433546112391601 . . . . . . . . . . 59

433544112391301 . . . . . . . . . . 60

433120112535101 . . . . . . . . . 61

435124112433701 . . . . . . . . . . 62

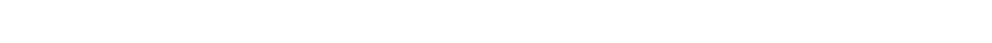

431551112493201 . . . . . . . . . . . 64

433307112300001 . . . . . . . . . . 65

433256113002501 . . . . . . . . . . 67

433218112191603 . . . . . . . . . . . 68

433218112191602 . . . . . . . . . . . 69

433218112191601 . . . . . . . . . . 70

435504112222301 . . . . . . . . . . . 73

435153112420501 . . . . . . . . . . 83

433717112563501 . . . . . . . . . . 84

433819113191601 . . . . . . . . . . 85

432533112504901 . . . . . . . . . . . 88

435028112202601 . . . . . . . . . . . . . . . . 90

435120112432101 . . . . . . . . . . . . . 91

435119112431801 . . . . . . . . . . . . 92

434946112412401 . . . . . . . . . . . 93

434952112411301.0 .0 .0 .0 .0 .094

433520112572601 . . . . . . . . . . 95

435038112453401 . . . . . . . . . . 108

435332112331801 . . . . . . . . . . 109

433859112545401 . . . . . . . . . . . . 110

433854112545401 . . . . . . . . . . . 111

433858112545501 . . . . . . . . . . 113 
LIST: OF WELLS FOR WHICH RECORDS ARE PUBLISHED (continued)

Wel1 name

Site ID number

Page

OMRE

433116112534701 . . . . . . . . . 114

$P$ and $W 1$

$P$ and $W 2$

$P$ and $W 3$

Park Bell

PSTF test

2nd Owsley

R. Archer

Simplot 1

Simplot 2

Site 1

Site 4

Site 6

Site 9

Site 14

Site 16

Site 17

Site 19

SPERT 1

Sweet Sage

T. Smith

TAN 1

TAN 2

TRA 1

TRA 3

USBLM 1

USGS 1

USGS 2

USGS $3 \mathrm{~A}$

USGS 4

USGS 5

USGS 6

USGS 7

USGS 8

USGS 9

USGS 11

USGS 12

USGS 13

USGS 14

USGS 15

USGS 16

USGS 17

USGS 18

USGS 19

USGS 20

USGS 21

USGS 22

USGS 23

435416112460401 . . . . . . . . . . 115

435419112453101 . . . . . . . . . . . 116

435443112435801 . . . . . . . . . . 117

440058112293605 . . . . . . . . . . 118

434941112454201 . . . . . . . . . . . . 119

434819112380501 . . . . . . . . . . 30

434857112185801 . . . . . . . . . . . 120

434751112571801 . . . . . . . . . . . 121

431929112421701 . . . . . . . . . . . 122

432854113201001 . . . . . . . . . . . 123

433617112542001 . . . . . . . . . . . 133

433826112510701 . . . . . . . . . . . 134

433123112530101 . . . . . . . . . . 135

434334112463101 . . . . . . . . . . 124

433546112391501 . . . . . . . . . 130

434027112575701 . . . . . . . . . 131

433522112582101 . . . . . . . . . 132

433252112520401 . . . . . . . . . . . 137

435033112593701 . . . . . . . . . . . 138

432021112404801 . . . . . . . . . . . 140

435056112420001 . . . . . . . . . . . 141

435100112420701 ............ 142

433521112573801 ............ 143

433522112573501 ............ . 144

435728112281101 . . . . . . . . . . 145

432700112470801 ............ 151

433320112432301 . . . . . . . . . . 207

433732112335401 ............ 252

434657112282201 . . . . . . . . . . . 268

433543112493801 . . . . . . . . . . . 291

434031112453501 . . . . . . . . . . . . 299

434915112443901 . . . . . . . . . . 304

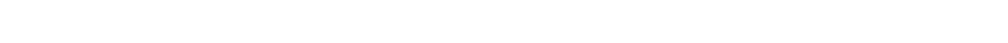

432740113044501 . . . . . . . . . . 330

432336113064201 . . . . . . . . . . . 169

434126112550701 . . . . . . . . . . . . 173

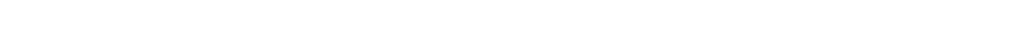

432019112563201 . . . . . . . . . . 183

434234112551701 . . . . . . . . . . 188

431333113001701 . . . . . . . . . . . . 189

433937112515401 . . . . . . . . . . 190

434540112440901 . . . . . . . . . 193

434426112575701 . . . . . . . . . . 196

433253112545901 . . . . . . . . . . . 211

434307112382601 . . . . . . . . . . . 215

433422113031701 . . . . . . . . . . . 225

434055112595901 . . . . . . . . . . 228 
LIST OF WELLS FOR WHICH RECORDS ARE PUBLISHED (cont inued)

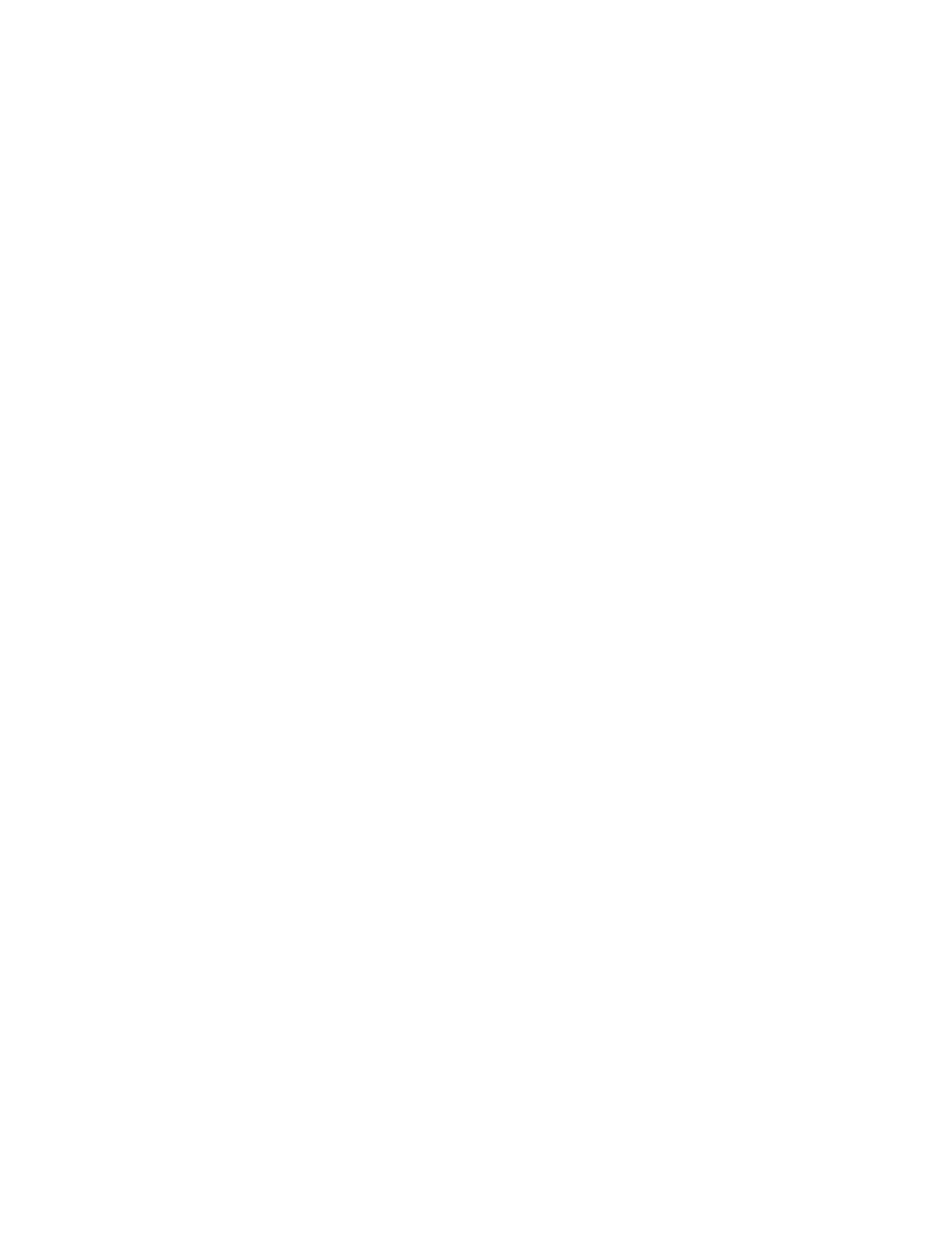


LIST OF WELLS FOR WHICH RECORDS ARE PUBLISHED (cont inued)

We11 name

USGS 97

USGS 98

USGS 99

USGS 100

USGS 101

USGS 103

USGS 104

USGS 105

USGS 106

USGS 107

USGS 108

USGS 109

USGS 110

Weaver and Lowe

Water table
Site ID number

Page

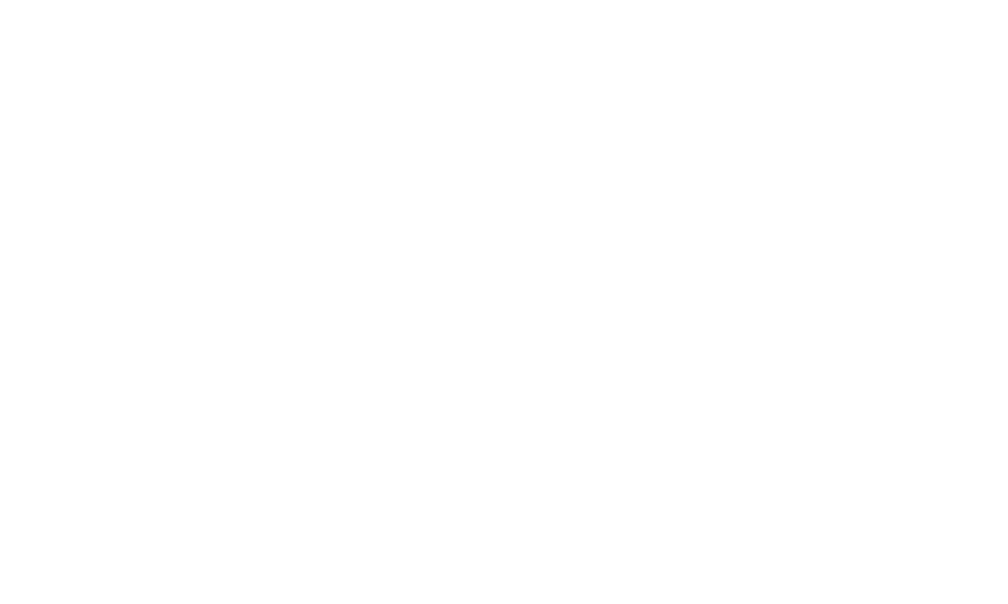




\section{CONVERSION FACTORS}

Conversion factors for terms used in this report are listed below for readers who prefer to use metric or other units.

\section{Multiply}

feet ( $f t$ )

square miles $\left(\mathrm{mi}^{2}\right)$

acre-feet (acre-ft)

gallons (gal)
By

0.3108

2.590

$1,233.0$

3.7850

\section{To obtain}

meters (m).

square kilometers $\left(\mathrm{km}^{2}\right)$ cubic meters $\left(\mathrm{m}^{3}\right)$

liters (1)

Temperature in degrees Celsius $\left({ }^{\circ} \mathrm{C}\right)$ can be converted to degrees

Fahrenheit $\left({ }^{\circ} \mathrm{F}\right)$ as follows: ${ }^{\circ} \mathrm{F}=1.8\left({ }^{\circ} \mathrm{C}+32\right)$

MVGD is equivalent to Mean Vertical Geodetic Datum, or Mean Sea Level, in publications before 1978 .

The use of trade names in this report is for identification purposes only and does not constitute endorsement by the U.S. Geological Survey. 


\title{
HATER-LEVEL DATA FOR SELECTED WELLS ON OR NEAR THE IDAHO NATIONAL ENGINEERING LABORATORY, 1949 THROUGH 1982
}

\author{
By Jack T. Barraclough, Jefferson C. Bagby, Luke J. White \\ and Rodger G. Jensen
}

\section{ABSTRACT}

Since 1949, the U.S. Geological Survey (USGS) has maintained a project office at the Idaho National Engineering Laboratory (INEL). During the past 30 years of INEL operations, water-level measurements have been regularly collected from the Geological Survey and Department of Energy (DOE) observation wells penetrating the Snake River Plain aquifer underlying the INEL, as well as private wells in the vicinity of the INEL. The water-level data from these wells have allowed the Geological Survey scientists to observe and describe hydrologic conditions in the Snake River Plain aquifer.

These data have been assembled on magnetic tape along with waterquality and source-term data and are available for the comparison and evaluation of various predictive digital ground-water solute-transport models.

This report is a tabulation of the water-level data available on magnetic tape. It includes water-level data for wells on or near the INEL which penetrate the Snake River Plain aquifer. The majority of the data reported are for 1949 through 19.82 , although some earlier data are included. Maps showing the locations of the wells measured are included, as well as a reference 1 ist of reports which interpret the data.

\section{INTRODUCTION}

The Idaho National Engineering Laboratory (INEL) was established as the National Reactor Testing Station in 1949, covering approximately 886 square miles of the eastern Snake River Plain (fig. 1), and used by the U.S. Department of Energy (DOE) for nuclear reactor experiments. The INEL contains fifty-two reactors, a nuclear fuel reprocessing plant, and a radioactive-waste burial ground. Sixteen of the reactors, the reprocessing plant, and the burial ground are still being used (fig. 2).

In 1949, the Atomic Energy Commission (now DOE) requested that the Geological Survey investigate and describe the water resources of the INEL and adjacent areas. The Geological Survey has since established a network of observation wells, used to monitor the fluctuations in water level and movement of radionuclide and chemical wastes in the Snake River Plain aquifer. Water levels measured. in these observation wells are used in solving various hydrologic problems, in addition to being used in waterresources development. 


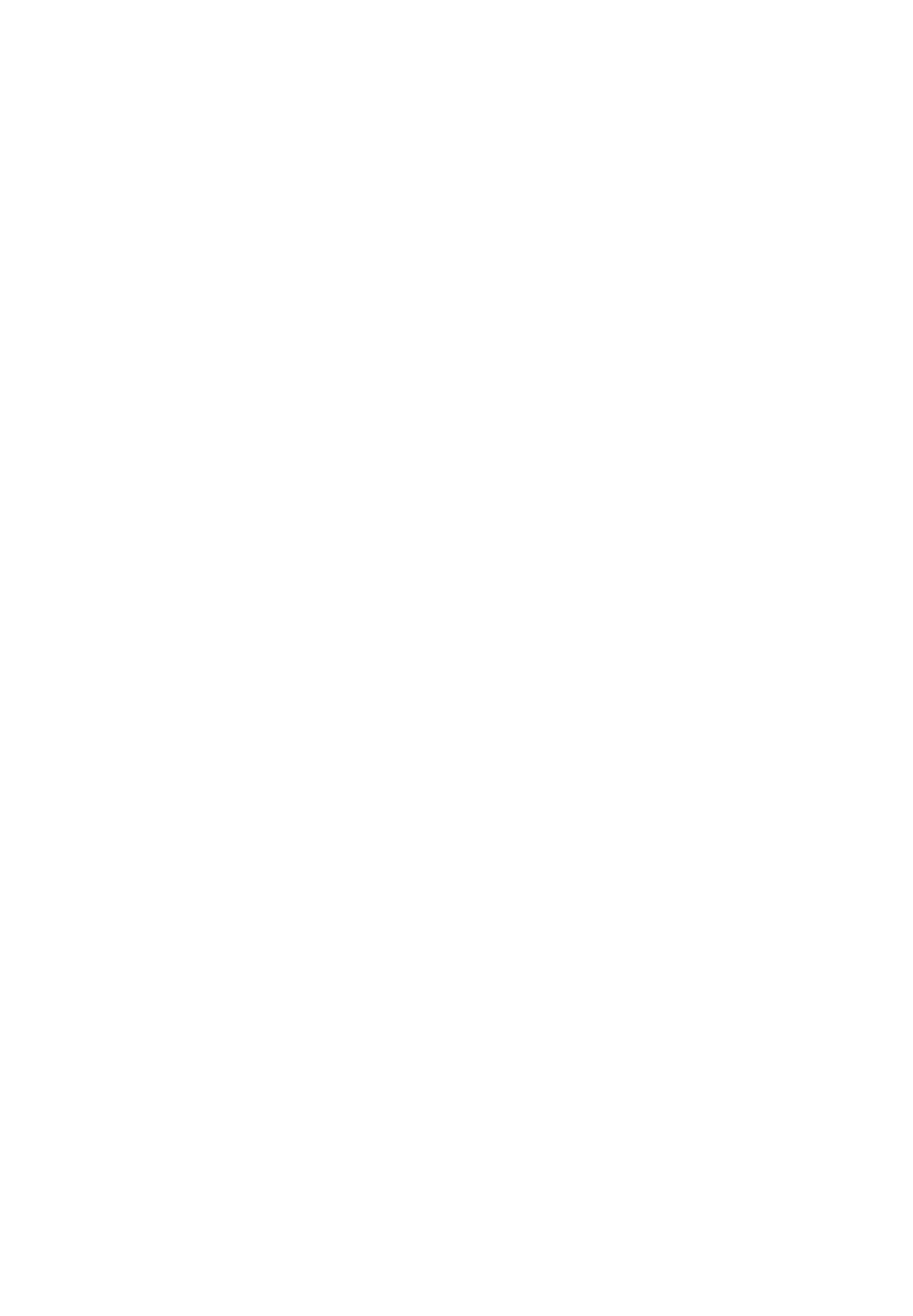

Figure 1.--Relief map of Idaho showing the location of the INEL, Snake River Plain, and generalized ground-water flow lines of the Snake River Plain aquifer (from Barraclough, Lewis, and Jensen, 1981). 


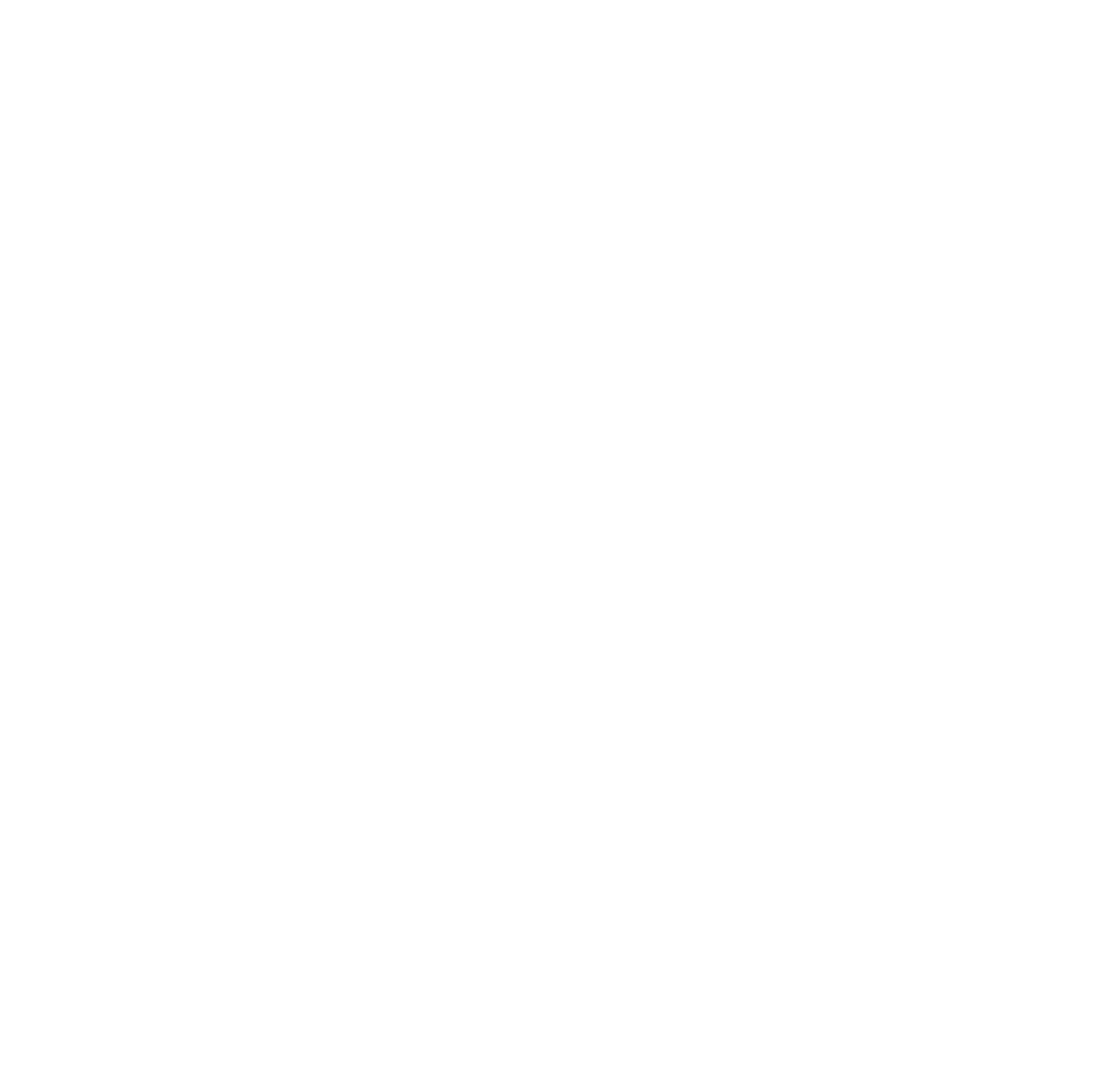

Figure 2.--Location of the INEL facilities. 
An IBM magnetic tape has been compiled in 1600 bit-per-inch ASCII format, containing the following information:

o A water-quality file from selected Geological Survey monitoring wells penetrating the Snake River Plain aquifer in the vicinity of the INEL. This file included both chemical and radionuclide data.

o A file of water-level data for these wells.

o A data file of aqueous wastes disposed of at the INEL.

A set of U.S. Geological Survey open-file reports has been prepared to provide written documentation of the data on the magnetic tape. This is one of those reports. A report is in preparation which will describe the format and use of the magnetic tape, and provide other information necessary to use this data set for modeling.

\section{Purpose and Scope}

The purpose of this report is to provide written documentation of the water-level data that have been recorded on the INEL ground-water data base magnetic tape. The data are for wells that penetrate the Snake River Plain aquifer and are located on or close to the INEL. The data are 1 isted in tabular form, and maps (figures 3 to 8 ) are provided to show the locations of wells from which data were collected. A brief introduction to the INEL and its hydrogeological characteristics has been included. A 1 ist of reports which interpret this data has been provided at the end of the text.

\section{Acknowledgments}

We thank the following Geological Survey personnel: Kirk Sheppard, Chris Ahlstrom, Tami Belnap, Amy Marlette, Lisa Gayheart, Isabel Anderson, and Jean Eagleton who spent hundreds of hours searching-for, keypunching, and editing the data. Credit is also given to Darwin Mecham (EG\&G Idaho, Inc.), computer-system manager; and to programmers Donald N. Hollister (EG\&G Idaho, Inc.), and Deven Browning (Geological Survey). Thanks are also extended to the people in the Geological Survey Idaho District office who helped retrieve and sort archived records. Assistance was also provided by Norman Hutchison, Dave Maddy, and Roger Booker of the Geological Survey WATSTORE program office in Reston, Virginia. Special thanks are extended to Thomas F. Gesel1, Chief, Dosimetry Branch, Radiological and Environmental Sciences Laboratory (RESL), DOE-ID; and to Fred L. Kalbeitzer; Research Physicist, Dosimetry Branch, RESL, DOE-ID who allowed us to use their VAX $11 / 750$ computer for the assembly of this set of documents and the magnetic tape. 


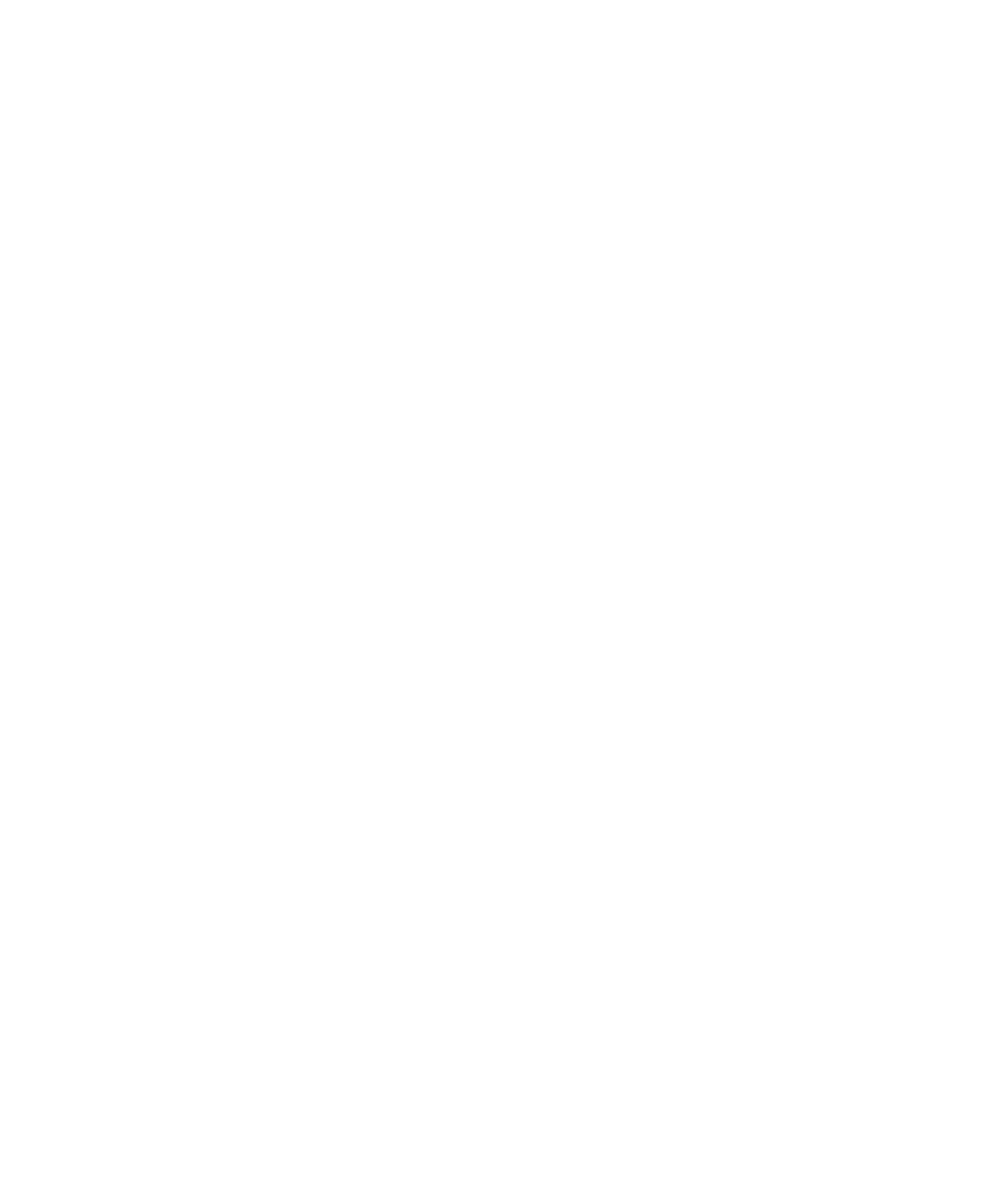

Figure 3.--Site identification numbers and locations of selected wells near the INEL. 


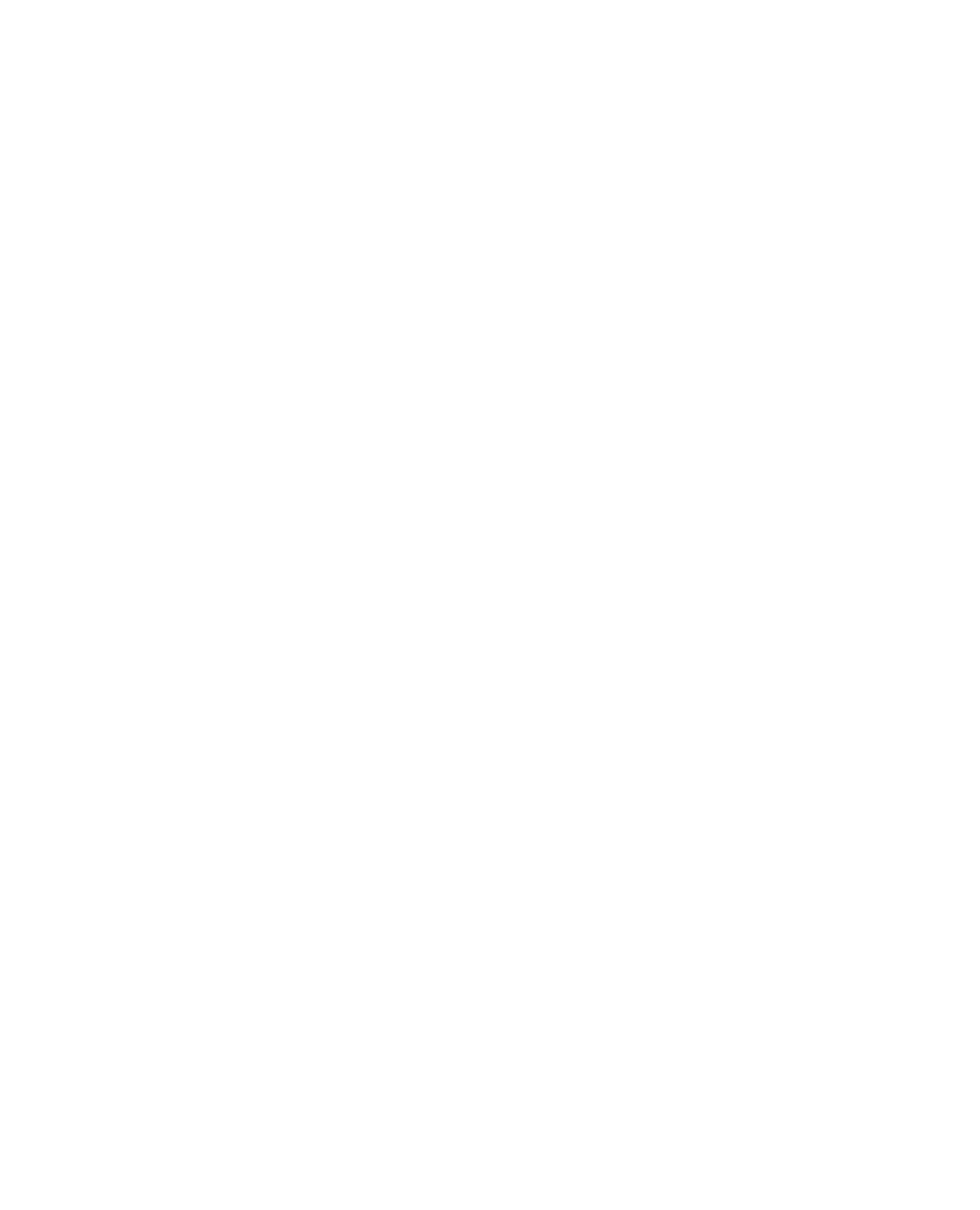

Figure 4.--Local names and locations of selected wells near the INEL. 


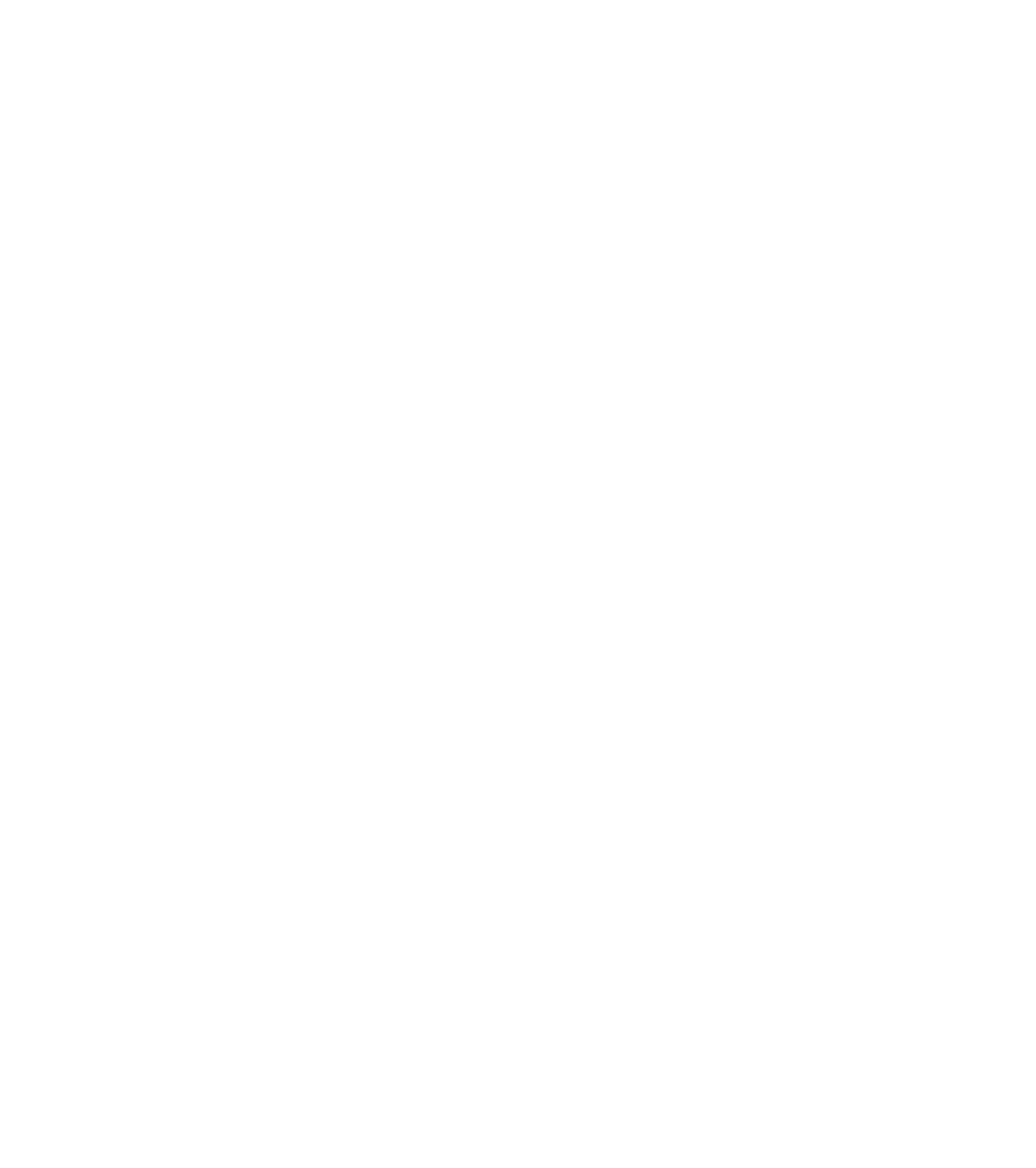

Figure 5.--Site identification numbers and locations of selected wells on the INEL. 


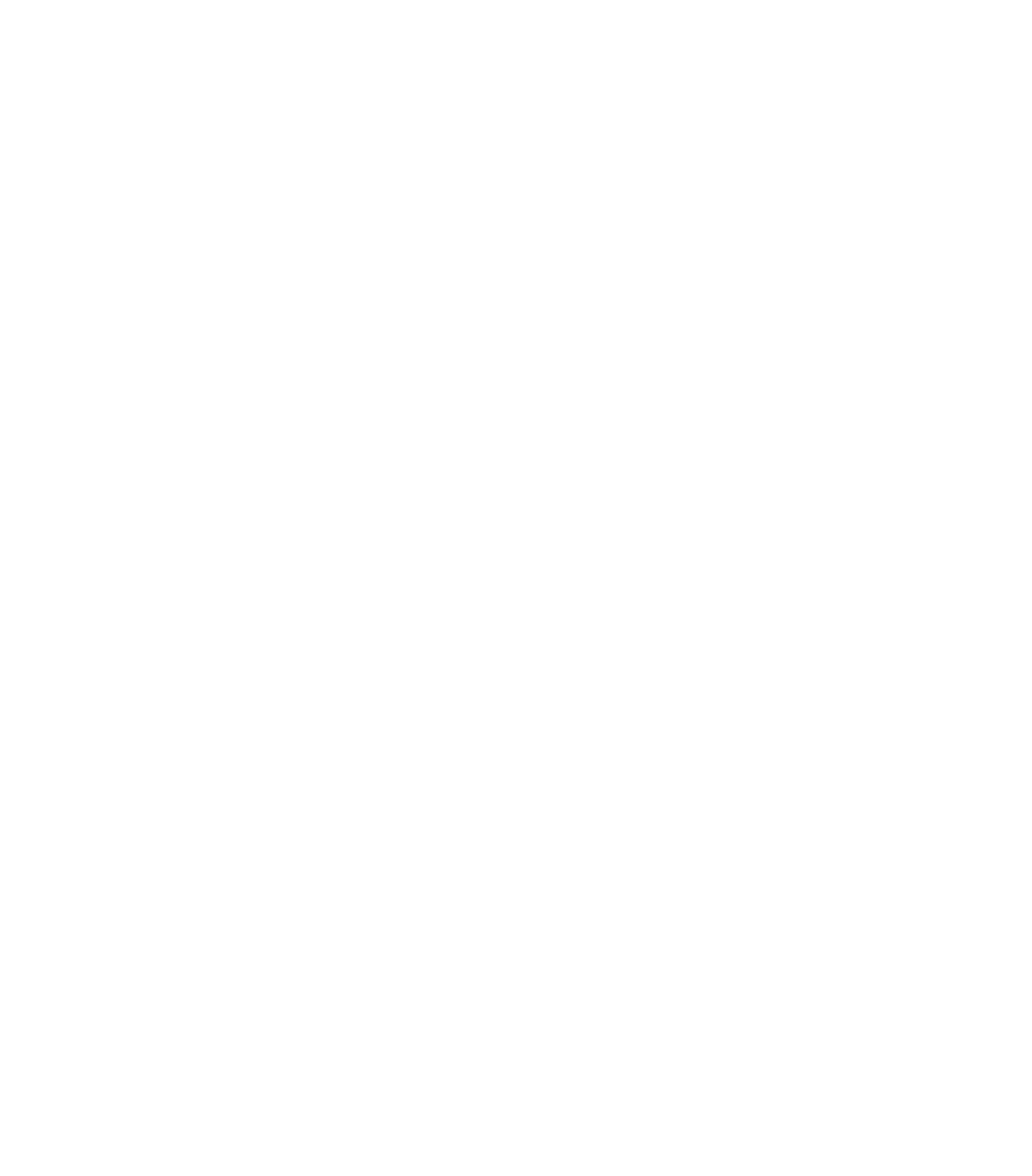

Figure 6.--Local names and locations of selected wells on the INEL. 
$113^{\circ} 57^{\prime 3} 30^{\circ}$

$112^{\circ} 55^{\circ} 00^{\prime \prime}$

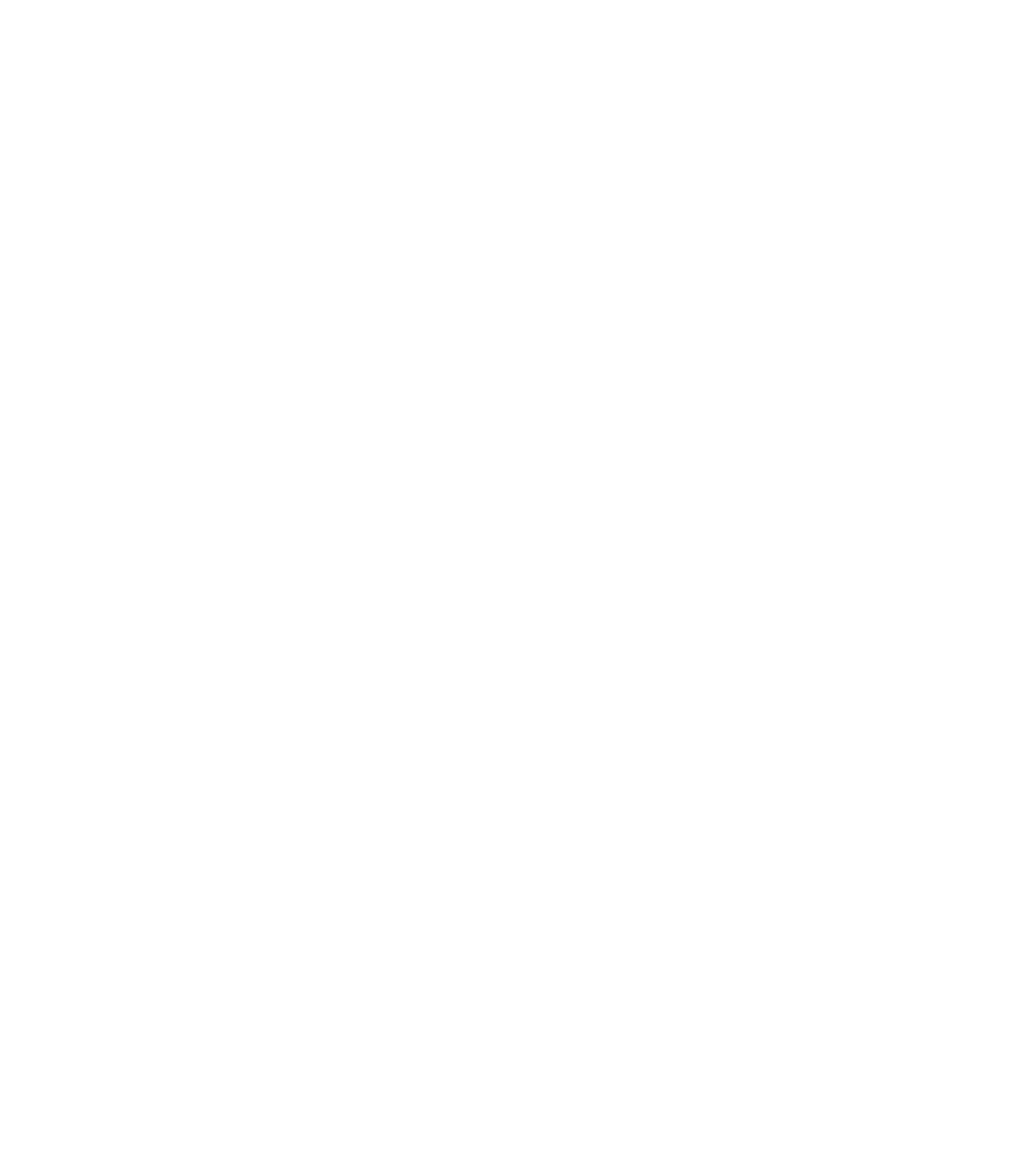

Figure 7.--Site identification numbers and locations of selected wells in the ICPP-TRA area. 


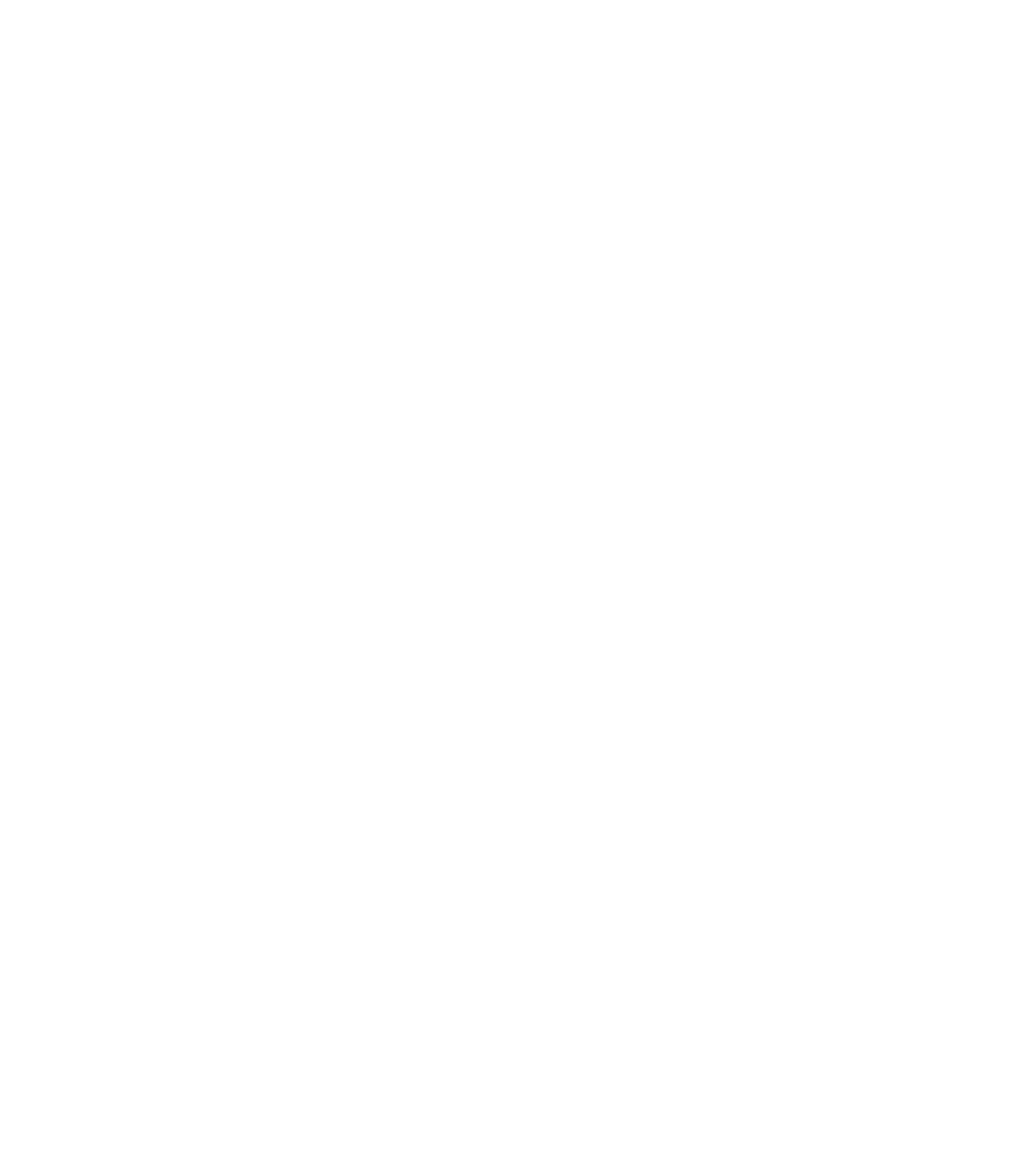

Figure 8.--Local names and locations of selected wells in the ICPPTRA area. 
In the following sections the various systems used for identifying well sites are discussed, so that the reader can better understand the maps and tables contained in this report.

USGS Site Ident ification Numbers

The latitude and longitude grid system is used as the well site numbering system of the Geological Survey. The system provides the geographic location of the well or miscellaneous site and a unique number for each well site. The number consists of 15 digits. The first 6 digits denote degrees, minutes, and seconds of 1 atitude; the next 7 digits denote degrees, minutes, and seconds of longitude; and the 1 ast 2 digits (assigned sequentially through time) identify the wells or other sites to within one second of latitude and longitude.

\section{Id aho We11-Numbering System}

The Idaho we11-numbering system locates wells within the official rectangular subdivisions of the public lands, with reference to the Boise base line and meridian. The first segment of a well-number indicates the township and position north, $\mathrm{N}$, or south, $\mathrm{S}$, of the Boise base 1 ine; the second the range and position east, $E$, or west, $W$, of the Boise meridian; and the third the number of the section in which the well is situated. The letters following the section number indicate the well location within the section: the first letter denotes the 160-acre tract, the second the 40-acre tract, and the third the 10-acre tract in which the wel1 occurs. The subdivisions of the section are designated $a, b, c$, and $d$ and are assigned in a counter clockwise direction beginning in the northeast quarter of each subdivision ( $\mathrm{fig} .9$ ). The last numeral is a serial number assigned when the we11 is inventoried. Thus, we $1107 \mathrm{~S}-17 \mathrm{E}-06$ acal is in the northeast quarter of the southwest quarter of the northeast quarter of section 6, township 7 south of the Boise base line, range 17 east of the meridian, and was the first well inventoried in that tract.

\section{Loca1 Names}

Local names are common names which have been assigned to each we 11. The local name for a particular well location may change with time, but the Geological Survey or Idaho identification numbers do not. The local names for the wells in this report are listed in two places. First they are listed in the front of the text as an index, sorted in alphabetical order. Also included in this index are the site identification number and the page number where the data for that particular well begins. Local names are 1 isted in table 1 , sorted by site identification number, and the local well name and Idaho well number. By using these two lists, it is possible to cross reference the water-level data table by either well name or site identification number. Additional information concerning abbreviations used in the data table is included in table 2, preceding the data table. 


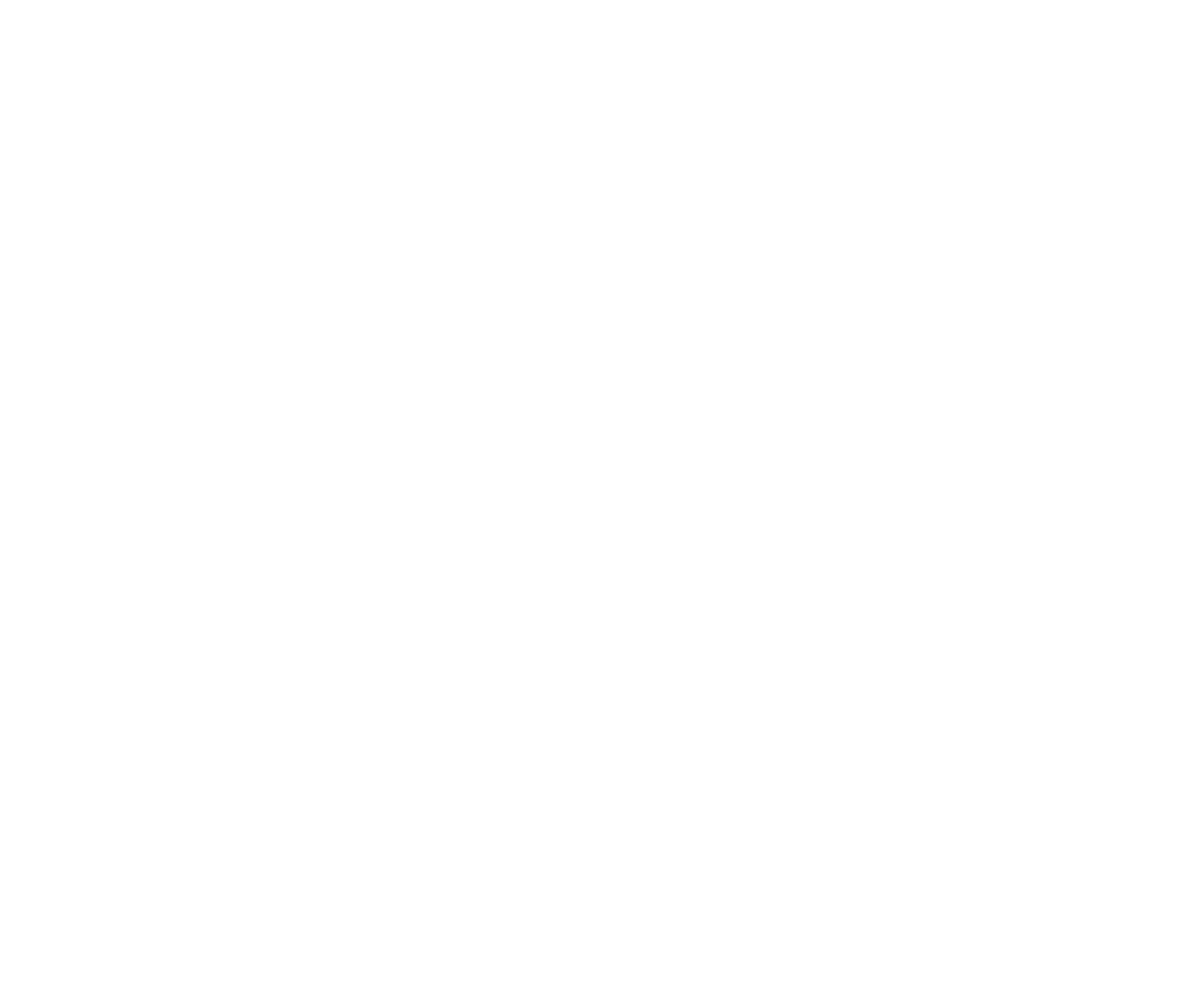

Figure 9.--Diagram showing Idaho well-numbering system. 
Table 1.--Site identification numbers, local well names, and Idaho well numbers (table sorted by site identification number).

\begin{tabular}{|c|c|c|c|c|}
\hline Site ID number & Local name & Id aho & we11 & 1 number \\
\hline 431333113001701 & USGS 16 & $02 \mathrm{~S}$ & $30 \mathrm{E} 3$ & $30 \mathrm{bbb} 1$ \\
\hline 431551112493201 & Grazing 2 & $02 S$ & $31 \mathrm{E}$ & $10 \mathrm{bdd} 1$ \\
\hline 431929112421701 & Simpl ot 2 & $01 \mathrm{~S}$ & $32 \mathrm{E} 2$ & $22 \mathrm{bdb} 1$ \\
\hline 431946113161401 & $01 \mathrm{~S} 27 \mathrm{E} 14 \mathrm{dcc} 1$ & $01 \mathrm{~s}$ & $27 \mathrm{E} 1$ & $14 \mathrm{dcc} 1$ \\
\hline 43201.9112563201 & USGS 14 & $01 \mathrm{~S}$ & $30 E_{1} 1$ & $15 \mathrm{bca} 1$ \\
\hline 432021112404801 & T. Smith & $01 \mathrm{~s}$ & $32 \mathrm{E} 1$ & $14 a b c 1$ \\
\hline 432336113064201 & USGS 11 & $01 \mathrm{~N}$ & $29 E^{3}$ & $30 \mathrm{bbd} 1$ \\
\hline 432533112504901 & Leo Roger's 1 & $01 \mathrm{~N}$ & $31 \mathrm{E} 0$ & $08 c d d 1$ \\
\hline 432618112555501 & Cerro Grande & $01 \mathrm{~N}$ & $30 \mathrm{E} 1$ & 10bbai \\
\hline 432659112582601 & USGS 108 & $02 \mathrm{~N}$ & $29 \mathrm{E} 3$ & $35 c c c 1$ \\
\hline 432700112470801 & USGS 1 & $02 \mathrm{~N}$ & $31 \mathrm{E} 3$ & $35 \mathrm{dcc} 1$ \\
\hline 432701113025601 & USGS 109 & $02 \mathrm{~N}$ & $29 \mathrm{E} 3$ & $3 \operatorname{lcdcl}$ \\
\hline 432703113001801 & USGS 105 & $02 \mathrm{~N}$ & $29 \mathrm{E} 3$ & $33 \mathrm{dccl}$ \\
\hline 432714112560701 & USGS 103 & $02 \mathrm{~N}$ & $30 \mathrm{E} 3$ & $31 \mathrm{cbc} 1$ \\
\hline 432717112501501 & USGS 110 & $02 \mathrm{~N}$ & $30 \mathrm{E} 3$ & $35 \mathrm{~d}$ ad 1 \\
\hline 432731113143901 & USGS 13 & $02 \mathrm{~N}$ & $27 \mathrm{E} 3$ & $33 a c c 2$ \\
\hline 432730113044501 & USGS 9 & $02 \mathrm{~N}$ & $28 \mathrm{E} 3$ & $35 \mathrm{accl}$ \\
\hline 432854113201001 & Site 1 & $02 \mathrm{~N}$ & $26 E 2$ & $22 \mathrm{ddal}$ \\
\hline 432854113201002 & Water table & $02 \mathrm{~N}$ & $26 \mathrm{E} 2$ & $22 \mathrm{dda} 2$ \\
\hline 432856112560801 & USGS 104 & $02 \mathrm{~N}$ & $29 \mathrm{E} 2$ & $24 \mathrm{~d}$ ad 1 \\
\hline 432927112410101 & Corehole 1 & $02 \mathrm{~N}$ & $32 E 2$ & $22 \mathrm{aba} 1$ \\
\hline 43293511.3080001 & USGS 86 & $02 \mathrm{~N}$ & $28 \mathrm{E} 2$ & $21 \mathrm{bbb} 1$ \\
\hline 432940113030201 & USGS 88 & $02 \mathrm{~N}$ & $29 E 1$ & $18 c c d 1$ \\
\hline 432942112532801 & USGS 107 & $02 \mathrm{~N}$ & $30 \mathrm{E} 1$ & $16 c c a l$ \\
\hline 432952113020501 & USGS 90 & $02 \mathrm{~N}$ & $29 \mathrm{E} 1$ & $17 \mathrm{cbc} 1$ \\
\hline 432959112593101 & USGS 106 & $02 \mathrm{~N}$ & $29 \mathrm{E} 1$ & $15 \mathrm{cbal}$ \\
\hline 433005113032801 & USGS 89 & $02 \mathrm{~N}$ & $28 \mathrm{E} \quad 1$ & $13 \operatorname{add} 1$ \\
\hline 433013113024201 & USGS 87 & $02 \mathrm{~N}$ & $29 E 1$ & $18 \mathrm{bdal}$ \\
\hline 433023112561501 & USGS 83 & $02 \mathrm{~N}$ & $29 \mathrm{E} 1$ & $13 a a a 1$ \\
\hline 433051113002601 & EBR I & $02 \mathrm{~N}$ & $29 \mathrm{E} 0$ & $09 \mathrm{c}$ a 1 \\
\hline 433107112492201 & ARA 2 & $02 \mathrm{~N}$ & $30 \mathrm{E} 1$ & $12 a b d 1$ \\
\hline 433116112534701 & OMRE & $02 \mathrm{~N}$ & $30 \mathrm{E} 0$ & $08 a a a 1$ \\
\hline 433120112535101 & EOCR & $02 \mathrm{~N}$. & $30 \mathrm{E} \quad 0$ & $05 d d d 1$ \\
\hline 433121113115801 & USGS 8 & $02 \mathrm{~N}$ & $27 \mathrm{E} \mathrm{C}$ & $02 d d c l$ \\
\hline 433123112530101 & Site 9 & $02 \mathrm{~N}$ & $30 \mathrm{E} \mathrm{C}$ & $04 d c c 1$ \\
\hline 433144112563501 & CFA 2 & $02 \mathrm{~N}$ & $29 \mathrm{E} \mathrm{C}$ & $01 \mathrm{dbb} 1$ \\
\hline 433218112191601 & Highway $1-\mathrm{C}$ & $02 \mathrm{~N}$ & $35 \mathrm{E} \mathrm{C}$ & $02 b b c 1$ \\
\hline 433218112191602 & Highway $1-B$ & $02 \mathrm{~N}$ & $35 \mathrm{E} \mathrm{C}$ & $02 b b c 2$ \\
\hline 433218112191603 & Highway $1-A$ & $02 \mathrm{~N}$ & $35 \mathrm{E}$ & $02 b b c 3$ \\
\hline 433223112470201 & AREA II & $03 \mathrm{~N}$ & $31 E 3$ & $35 \mathrm{dcal}$ \\
\hline 433246112571201 & USGS 85 & $03 \mathrm{~N}$ & $29 \mathrm{E} 3$ & $36 \mathrm{bcbl}$ \\
\hline 433252112520401 & Spert 1 & $03 \mathrm{~N}$ & $30 \mathrm{E} 3$ & $34 \mathrm{bad} 1$ \\
\hline 433253112545901 & USGS 20 & $03 \mathrm{~N}$ & $30 \mathrm{E} 3$ & 31 aad 1 \\
\hline 433255112381801 & USGS 101 & $03 \mathrm{~N}$ & $32 \mathrm{E} 3$ & 36 add 1 \\
\hline
\end{tabular}


Table 1.--Site identification numbers, local well names, and Idaho well numbers (table sorted by site identification number). (cont inued)

\begin{tabular}{|c|c|c|c|}
\hline Site ID number & Local name & Id aho & well number \\
\hline 433256113002501 & Highway 3 & $03 \mathrm{~N}$ & $29 \mathrm{E} 33 \mathrm{bad} 1$ \\
\hline 433307112300001 & Highway 2 & $03 N$ & $31 \mathrm{E} \quad 32 \mathrm{bbc} 1$ \\
\hline 433315112560301 & USGS 77 & $03 N$ & $30 \mathrm{E} 30 \mathrm{ccb} 1$ \\
\hline 433320112432301 & USGS 2 & $03 \mathrm{~N}$ & $32 \mathrm{E} 29 \mathrm{ddcl}$ \\
\hline 433322112564301 & USGS 38 & $03 \mathrm{~N}$ & $29 \mathrm{E} 25 \mathrm{cad} 1$ \\
\hline 433326112564801 & USGS 37 & $03 \mathrm{~N}$ & $29 \mathrm{E} 25 \mathrm{caal}$ \\
\hline 433330112565201 & USGS 36 & $03 \mathrm{~N}$ & $29 \mathrm{E} 25 \mathrm{bddl}$ \\
\hline 433334112565501 & USGS 34 & $03 \mathrm{~N}$ & $29 \mathrm{E} 25 \mathrm{bdc} 1$ \\
\hline 433339112565801 & USGS 35 & $03 \mathrm{~N}$ & $29 \mathrm{E} 25 \mathrm{bdbl}$ \\
\hline 433343112470001 & USGS 39 & $03 \mathrm{~N}$ & $29 \mathrm{E} 25 \mathrm{bbd} 1$ \\
\hline 433344112554101 & USGS 67 & $03 N$ & $30 \mathrm{E} \quad 30 \mathrm{bad} 1$ \\
\hline 433344112561601 & USGS 57 & $03 N$ & $29 \mathrm{E} \quad 25 \mathrm{abd} 1$ \\
\hline 433351002560601 & USGS 51 & $03 N$ & $30 \mathrm{E} \quad 30 \mathrm{bbbl}$ \\
\hline 433354112554701 & USGS 59 & $03 N$ & $30 \mathrm{E} \quad 30 \mathrm{bab} 1$ \\
\hline 4333561125742.01 & USGS 84 & $03 N$ & $29 \mathrm{E} 23 \mathrm{dcd} 1$ \\
\hline 433401112551001 & USGS 82 & $03 N$ & $30 \mathrm{E} \quad 19 \mathrm{ddc} 2$ \\
\hline 433401112560301 & USGS 48 & $03 \mathrm{~N}$ & $30 \mathrm{E} \quad 19 \mathrm{ccc} 1$ \\
\hline 33402112561801 & USGS 45 & $03 \mathrm{~N}$ & $29 E 24 d d c 1$ \\
\hline 433403112555401 & USGS 49 & $03 \mathrm{~N}$ & $30 \mathrm{E} 19 \mathrm{ccal}$ \\
\hline 433404112561301 & USGS 42 & $03 N$ & $29 \mathrm{E} 24 \mathrm{dda} 2$ \\
\hline 433407112560301 & USGS 47 & $03 \mathrm{~N}$ & $30 \mathrm{E} 19 \mathrm{ccbl}$ \\
\hline 433407112561501 & USGS 46 & $03 \mathrm{~N}$ & 29E 24dda3 \\
\hline 433409112561301 & USGS 41 & $03 \mathrm{~N}$ & 29E 24ddal \\
\hline 433409112562101 & USGS 44 & $03 \mathrm{~N}$ & $29 E \quad 24 d d b 1$ \\
\hline 433411112561101 & USGS 40 & $03 \mathrm{~N}$ & $29 \mathrm{E} 24 \mathrm{~d}$ ad 1 \\
\hline 433413112560401 & CPP 3 disposal & $02 \mathrm{~N}$ & $30 \mathrm{E} \quad 19 \mathrm{cbc} 1$ \\
\hline 433414112554201 & USGS 52 & $03 \mathrm{~N}$ & $30 \mathrm{E} \quad 19 \mathrm{cac} 1$ \\
\hline 4333415112561501 & USGS 43 & $03 \mathrm{~N}$ & $29 \mathrm{E} 24 \mathrm{~d}$ ad 2 \\
\hline 433422113031701 & USGS 22 & $03 N$ & $29 \mathrm{E} \quad 19 \mathrm{cbbl}$ \\
\hline .433425112573201 & USGS 76 & $03 N$ & 29E 23adcl \\
\hline 433432112560801 & CPP 2 & $03 \mathrm{~N}$ & 29E 24adal \\
\hline 433433112560201 & CPP 1 & $03 \mathrm{~N}$ & $30 \mathrm{E} \quad 19 \mathrm{bcb} 1$ \\
\hline 433447112574501 & USGS 65 & $03 N$ & 29E 23abbl \\
\hline 433445113.202801 & Weaver and Lowe & $03 \mathrm{~N}$ & 26E 22abal \\
\hline 433500112572502 & USGS 58 & $03 \mathrm{~N}$ & $29 \mathrm{E} \quad 14 \mathrm{dda} 2$ \\
\hline 433503112400701 & USGS 100 & $03 \mathrm{~N}$ & $32 \mathrm{E} \quad 14 \mathrm{cdd} 1$ \\
\hline 433505112581901 & USGS 79 & $03 \mathrm{~N}$ & $29 \mathrm{E} 14 \mathrm{cbdl}$ \\
\hline 433509112384801 & Arbor test & $03 \mathrm{~N}$ & $32 \mathrm{E} 13 \mathrm{dca} 1$ \\
\hline 433520112572601 & MTR test & $03 \mathrm{~N}$ & $29 \mathrm{E} \quad 14 \mathrm{add} 1$ \\
\hline 433522112573501 & TRA 3 & $03 \mathrm{~N}$ & $29 \mathrm{E} \quad 14 \mathrm{adbl}$ \\
\hline 433522112582101 & Site 19 & $03 \mathrm{~N}$ & $29 \mathrm{E} \quad 14 \mathrm{bcbl}$ \\
\hline 433543112493801 & USGS 5 & $03 N$ & $30 \mathrm{E} \quad 12 \mathrm{cdd} 1$ \\
\hline 433544112391301 & EBR II 2 & $03 \mathrm{~N}$ & $32 \mathrm{E} \quad 13 \mathrm{bbd} 3$ \\
\hline 433546112391501 & Site 14 & $03 \mathrm{~N}$ & $32 \mathrm{E} \quad 13 \mathrm{bbd} 1$ \\
\hline
\end{tabular}


Table 1.--Site identification numbers, local well names, and Idaho well numbers (table sorted by site identification number).

(cont inued)

\begin{tabular}{|c|c|c|c|c|}
\hline Site ID number & Local name & Id aho & we 1 & 11 number \\
\hline 433546112391601 & EBR II 1 & $03 \mathrm{~N}$ & $32 \mathrm{E}$ & $13 b$ bd 2 \\
\hline 433548112562301 & Fire Station 2 & $03 N$ & $29 E$ & $12 \mathrm{ddb} 1$ \\
\hline 433617112542001 & Site 4 & $03 \mathrm{~N}$ & $30 \mathrm{E}$ & $08 \mathrm{bda} 1$ \\
\hline 433657112563601. & USGS 98 & $03 N$ & $29 E$ & $01 \mathrm{dbb} 1$ \\
\hline 433705112552101 & USGS 99 & $03 N$ & $30 \mathrm{E}$ & $06 a c d 1$ \\
\hline 433717112563501 & INEL 1 & $03 N$ & $29 \mathrm{E}$ & $01 a b d 1$ \\
\hline 433732112335401 & USGS $3 \mathrm{~A}$ & $03 \mathrm{~N}$ & $33 \mathrm{E}$ & $03 a b a 1$ \\
\hline 433748113234001 & $04 \mathrm{~N} 26 \mathrm{E} 32 \mathrm{cbb} 1$ & $04 \mathrm{~N}$ & $26 \mathrm{E}$ & $32 \mathrm{cbbl}$ \\
\hline 433807112551501 & USGS 97 & $04 \mathrm{~N}$ & $30 \mathrm{E}$ & $31 \mathrm{abd} 1$ \\
\hline 433826112510701 & Site 6 & $04 \mathrm{~N}$ & $30 \mathrm{E}$ & $26 \mathrm{ccal}$ \\
\hline 433854112545401 & NRF 2 & $04 N$ & $30 \mathrm{E}$ & $30 a d a 1$ \\
\hline 433858112545501 & NRF 3 & $04 \mathrm{~N}$ & $30 \mathrm{E}$ & $30 a \operatorname{ad} 2$ \\
\hline 433859112545401 & NRF 1 & $04 \mathrm{~N}$ & $30 \mathrm{E}$ & $30 a$ ad 1 \\
\hline 433937112515401 & USGS 17 & $04 \mathrm{~N}$ & $30 E$ & $22 \mathrm{bdd} 1$ \\
\hline 434001113215201 & Arco test & $04 \mathrm{~N}$ & $26 \mathrm{E}$ & $21 \mathrm{abb} 1$ \\
\hline 434027112575701 & Site 17 & $04 \mathrm{~N}$ & $29 E$ & $14 \mathrm{caal}$ \\
\hline 434031112453501 & USGS 6 & $04 \mathrm{~N}$ & $31 \mathrm{E}$ & $16 \mathrm{adcl}$ \\
\hline 434055112595901 & USGS 23 & $04 \mathrm{~N}$ & $29 \mathrm{E}$ & $09 \mathrm{dcd} 1$ \\
\hline 434126112550701 & USGS 12 & $04 N$ & $30 \mathrm{E}$ & $07 \mathrm{adb} 1$ \\
\hline 434234112551701 & USGS 15 & $04 N$ & $30 \mathrm{E}$ & $06 a b a 1$ \\
\hline 434307112382601 & USGS 21 & $05 N$ & $32 \mathrm{E}$ & $36 a \mathrm{dd} 1$ \\
\hline 4343141.12322901 & USGS 33 & $05 \mathrm{~N}$ & $33 \mathrm{E}$ & 35 daa 1 \\
\hline 434334112463101 & Site 14 & $05 \mathrm{~N}$ & $31 \mathrm{E}$ & $28 c c c 1$ \\
\hline 434407112285101 & USGS 29 & $05 \mathrm{~N}$ & $34 \mathrm{E}$ & 29daal \\
\hline 434426112575701 & USGS 19 & $05 \mathrm{~N}$ & $29 \mathrm{E}$ & $23 c d d 1$ \\
\hline 434444112322101 & USGS 32 & $05 N$ & $33 \mathrm{E}$ & $23 \mathrm{dda} 1$ \\
\hline 434540112440901 & USGS 18 & $05 \mathrm{~N}$ & $31 \mathrm{E}$ & $14 \mathrm{bccl}$ \\
\hline $4345581 \cdot 12444801$ & Corehole $2 \mathrm{~A}$ & $05 \mathrm{~N}$ & $31 \mathrm{E}$ & $15 \mathrm{~b}$ ad 1 \\
\hline 434600112360101 & USGS 28 & $05 N^{\circ}$ & $33 \mathrm{E}$ & 17 add 1 \\
\hline 434601112315401 & USGS $30 \mathrm{~A}$ & $05 \mathrm{~N}$ & $33 \mathrm{E}$ & $13 \mathrm{bdc} 1$ \\
\hline 434601112315402 & USGS $30 \mathrm{~B}$ & $05 \mathrm{~N}$ & $33 \mathrm{E}$ & $13 \mathrm{bdc} 2$ \\
\hline 434601112315403 & USGS $30 \mathrm{C}$ & $05 N$ & $33 \mathrm{E}$ & $13 \mathrm{bdc} 3$ \\
\hline 434625112342101 & USGS 31 & $05 \mathrm{~N}$ & $33 \mathrm{E}$ & $10 \mathrm{cdc} 1$ \\
\hline 434657112282201 & USGS 4 & $05 \mathrm{~N}$ & $34 \mathrm{E}$ & $09 \mathrm{bdal}$ \\
\hline 434751112571801 & Simplot 1 & $05 \mathrm{~N}$ & $29 \mathrm{E}$ & $01 \mathrm{bbb} 1$ \\
\hline 434819112380501 & 2nd Owsley & $06 \mathrm{~N}$ & $32 \mathrm{E}$ & 36 add 1 \\
\hline 434851112321801 & USGS 27 & $06 \mathrm{~N}$ & $33 \mathrm{E}$ & $26 \mathrm{ddb} 1$ \\
\hline 434856112400001 & ANP 9 & $06 \mathrm{~N}$ & $32 \mathrm{E}$ & $26 \mathrm{cdbl}$ \\
\hline 434857112185801 & R. Archer & $06 \mathrm{~N}$ & $35 \mathrm{E}$ & $27 \mathrm{dd}$ al \\
\hline 434909112400401 & ANP 10 & $06 \mathrm{~N}$ & $32 \mathrm{E}$ & $26 c a b l$ \\
\hline 434915112443901 & USGS 7 & $06 \mathrm{~N}$ & $31 \mathrm{E}$ & $27 \mathrm{bdd} 1$ \\
\hline 434941112454201 & PSTF test & $06 \mathrm{~N}$ & $31 \mathrm{E}$ & $21 \mathrm{dccl}$ \\
\hline 434946112412401 & LPTF disposal & $06 \mathrm{~N}$ & $32 \mathrm{E}$ & $22 \mathrm{ccal}$ \\
\hline 434952112411301 & LPTF production & $06 \mathrm{~N}$ & $32 \mathrm{E}$ & $22 \mathrm{cac} 1$ \\
\hline
\end{tabular}


Table 1.--Site identification numbers, local well names, and Idaho well numbers ( $t a b l e$ sorted by site identification number). (cont inued)

\begin{tabular}{|c|c|c|}
\hline Site ID number & Local name & Idaho we 11 number \\
\hline 435028112202601 & Linderman and Bo & $06 \mathrm{~N} \quad 35 \mathrm{E} \quad 21 \mathrm{aab} 1$ \\
\hline 435033112593701 & Sweet Sage & 06N 29E l6ddd 1 \\
\hline 435038112453401 & No name 1 & $06 \mathrm{~N} \quad 31 \mathrm{E} \quad 16 \mathrm{ccc} 1$ \\
\hline 435045113031701 & $D$ and $W$ Hansen & $06 \mathrm{~N} \quad 28 \mathrm{E} \quad 13 \mathrm{ddal}$ \\
\hline 435053112420801 & USGS 24 & $06 \mathrm{~N} 3 \mathrm{IE} 13 \mathrm{dbb} 1$ \\
\hline 435053112423201 & ANP 3 disposal & $06 \mathrm{~N} \quad 31 \mathrm{E} \quad 13 \mathrm{Cab} 1$ \\
\hline 435056112420001 & TAN 1 & $06 \mathrm{~N} 3 \mathrm{IE} 13 \mathrm{acd} 1$ \\
\hline 435100112420701 & TAN 2 & 06N 3lE 13accl \\
\hline 435119112431801 & LOFT 2 & $06 \mathrm{~N} \quad 31 \mathrm{E} \quad 14 \mathrm{abb} 2$ \\
\hline 435120112432101 & LOFT 1 & $06 \mathrm{~N} \quad 31 \mathrm{E} \quad 14 \mathrm{abbl}$ \\
\hline 435124112433701 & FET disposal 3 & $06 \mathrm{~N} \quad 31 \mathrm{E} \quad 11 \mathrm{cdc} 1$ \\
\hline 435152112443101 & ANP 6 & $06 \mathrm{~N} \quad 3 \mathrm{lE}$ 10accl \\
\hline 435153112420501 & IET 1 & $06 \mathrm{~N} \quad 31 \mathrm{E} \quad 12 \mathrm{acdl}$ \\
\hline 435212112394001 & USGS 26 & 06N 32E llabal \\
\hline 435308112454101 & ANP 5 & $07 \mathrm{~N} 31 \mathrm{E} 33 \mathrm{dcd} 1$ \\
\hline 435339112444601 & USGS 25 & 07N $31 \mathrm{E} 34 \mathrm{bddl}$ \\
\hline 435416112460401 & $\mathrm{P}$ and $\mathrm{W} 1$ & $07 \mathrm{~N} 31 \mathrm{E} 28 \mathrm{cacl}$ \\
\hline 435419112453101 & $\mathrm{P}$ and $\mathrm{W} 2$ & $07 \mathrm{~N} 31 \mathrm{E} 28 \mathrm{dab} 1$ \\
\hline 435443112435801 & $P$ and $W 3$ & $07 \mathrm{~N} \quad 31 \mathrm{E} 26 \mathrm{bbcl}$ \\
\hline 435504112222301 & Idaho Fish and Game & 07N $\quad 35 \mathrm{E} \quad 20 \mathrm{cbd} 1$ \\
\hline 435522112444201 & ANP 7 & $07 \mathrm{~N} \quad 31 \mathrm{E} \quad 22 \mathrm{bdd} 1$ \\
\hline 435728112281101 & USBLM 1 & $07 \mathrm{~N} \quad 34 \mathrm{E} \quad 04 \mathrm{cdcl}$ \\
\hline 440058112293605 & Park Bell & $08 \mathrm{~N} \quad 34 \mathrm{E} \quad 17 \mathrm{ccc} 7$ \\
\hline
\end{tabular}


The eastern Snake River Plain, a large downwarped structural basin, is approximately $12,000 \mathrm{mi}^{2}$ in area ( $\mathrm{fig} .1$ ). This basin has been filled to its' present level with approximately 2,000 to 10,000 feet of thin basaltic lava flows, rhyolite deposits, and interbedded sediments. Nearly all of the eastern Snake River Plain is underlain by the Snake River Plain aquifer, containing in excess of 1 billion acre-feet of water. Groundwater flow in this aquifer is generally south-southwest (fig. 1) at relatively high velocities of 5-20 feet per day (Robertson, Schoen, and Barraclough, 1974, p. 13). Aquifer transmissivity is also high, ranging from 1 million to 100 million gallons per day per foot (Robertson, Schoen, and Barraclough, 1974, p. 12).

The Snake River Group of Quaternary age includes basaltic volcanic rocks and interbedded sediments which comprise the aquifer. The basement is thought to be older volcanic and sedimentary rocks, in addition to any underlying crystalline rocks. Primary and secondary permeabilities of the basalt consist of intercrystalline and intergranular porespace, fractures, cavities, macroscopic interstitial voids, interflow zones, and lava tubes. The direction of ground-water movement is locally complicated throughout the aquifer by the variety and degree of interconnection of these openings.

Ground-water recharge to the aquifer in the vicinity of the INEL is principally by underflow from the northeastern part of the plain and from adjacent drainages to the west and north of the INEL. Most of the ground water underlying the INEL entered the ground in the uplands to the north, northeast, and northwest of the site. This ground water moves southwestward through the aquifer, and discharges at springs along the valley of the Snake River near Hagerman (fig. 1). Small amounts of recharge may be derived from local precipitation (about 8 inches annually) on the plain.

\section{Collection of Records}

The regular collection of water-level data in the INEL area began in 1949. Few records are available prior to that time. Since 1949, the Geological Survey has continually expanded the network of observation wells in the vicinity of the INEL. As a result, the number of water-level records per year increases with $t$ ime, with the bulk of the records being collected after 1960 .

\section{Water-Level Data Collection Methods}

Water-level measurements taken by the Geological Survey project office at INEL are normally collected using methods described in Garber and Koopman, 1968, using A 1,000-foot steel surveyor's tape. The tape is lowered from a standard measuring point to some estimated length (hold distance), reeled back to the surface, and the wetted tape length (the cut distance) is subtracted to arrive at the vertical distance between water 
measuring. point. Measuring point height above 1 and surface is then subtracted to adjust the measurement to land-surface datum (LSD). Finally, the adjusted distance is subtracted from land-surface datum elevation above the mean geodetic vertical datum (MGVD) to arrive at the elevation of the water table above MGVD. If difficulties in measurement are encountered, the water level is remeasured until two measurements agree within 0.01 feet. Accuracy of these measurements is generally considered to be \pm 0.01 foot.

In addition to steel-tape measurements, water levels are sometimes measured at the INEL using electric tapes, mechanical floats and recorders, or electronic pressure transducers. Electric tapes generally have a pair of electrodes at the end of the tape. Water acts as an electrolyte, when it is contacted in the well bore, the circuit between the two electrodes is closed allowing the flow of electricity from a battery on the surface and causes the deflection of an adjacent voltage meter. Distance from the measuring point is then read by a tape attached to the electrical cord and the water-table elevation is calculated using the same method as above.

Mechanical floats are installed in a few wells on the INEL. Waterlevel fluctuations through time in these wells are recorded on a Stevens A35 strip chart recorder. A few INEL observation wells have been equipped with pressure transducers and strip chart recorders. Both mechanical float and pressure transducer installations are calibrated periodically with a steel surveyor's tape.

\section{CONCLUSIONS}

This report is one of a series of reports in preparation which summarize the data collected over the last 34 years at the INEL. This report, in conjunction with the other reports encapsulating the data, is intended to provide a useful working data set for government agencies and private industry. In magnetic tape format, this data should become a useful tool for hydrologic modelers working with arid-climate problems. 
Bagby, J. C., Ghering, G. E., Jensen, R. G., and Barraclough, J. T., 1981, A wind-powered, ground water monitoring installation at a radioactive waste management site in Idaho: U.S. Geological Survey Water-Resources Investigations, Open-File Report 81-493, 28 p.

Barraclough, J. T., Lewis, B. D., and Jensen, R. G., 1981, Hydrologic conditions at the Idaho National Engineering Laboratory, Idaho, Emphasis: 1974-1978: U.S. Geological Survey Water Supply Paper $2191,52 \mathrm{p}$.

Barraclough, J. T., and Jensen, R. G., 1976, Hydrologic data for the Idaho National Engineering Laboratory site, Idaho, 1971 to 1973: U.S. Geological Survey Open-File Report 75-318 (IDO-22055), 52 p.

Barraclough, J. T., Robertson, J. B., and Janzer, V. J., 1976, Hydrology of the solid waste burial ground, as related to the potential migration of radionuclides, Idaho National Engineering Laboratory, with a section on Drilling and sample analyses, by L. G. Saindon: U.S. Geological Survey Open-File Report 76-471 (IDO-22056), 183 p.

Barraclough, J. T., Teasdale, W. E., and Jensen, R. G., 1967, Hydrology of the National Reactor Testing Station, Idaho, 1965: U.S. Geological Survey Open-File Report IDO-22047, 107 p.

Barraclough, J. T., Teasdale, W. E., Robertson, J. B., and Jensen, R. G., 1967, Hydrology of the National Reactor Testing Station, Idaho, 1966: U.S. Geological Survey Open-File Report IDO-22048, 95 p.

Bowman, C. R., and Bennett, Richard, Tracer studies at the Loss of Fluid Test (LOFT). Issued by Texas Instruments, Inc., Science Services Division, Dallas, Texas, 1968.

Carrigan, P. H., Jr., 1972, Probability of exceeding capacity of floodcontrol system at the National Reactor Testing Station, Idaho: U.S. Geological Survey Open-File Report IDO-22052, 102 p.

Crosthwaite, E. G., 1973, A progress report on results of test-drilling and ground-water investigations of the Snake River Plain aquifer, southeastern Idaho, part 1-Mud Lake region, 1969-70 and part 2observation wells south of Arco and west of Aberdeen: Idaho Department of Water Administration, Water Information Bulletin 32, $60 \mathrm{p}$.

Doherty, D. J., 1979, Drilling data from exploration wel1 1, NE 1/4, Sec. 22, T. 2N., R. 32E., Bingham County, Idaho: U.S. Geological Survey Open-File Report 79-1225, 1 sheet.

Doherty, D. J., McBroome, L. A., and Kuntz, M. A., 1979, Probable geological interpretation and 1 ithologic $\log$ of the exploratory geothermal test well (INEL-1), Idaho National Engineering Laboratory, eastern Snake River Plain, Idaho: U.S. Geological Survey Open-File Report 79-1248, 7 p. 
Druffel, Leroy, Stiltner, G. J., and Keefer, T. N., 1979, Probable hydrologic effects of a hypothetical failure of Mackay Dam on the Big Lost River valley from Mackay, Idaho, to the Idaho National Engineering Laboratory: U.S. Geological Survey Water-Resources Investigations 79-99 (IDO-22058), 47 p.

Garber, M. S., and Koopman, F. C., 1968, Methods of measuring water levels in deep wells: U.S. Geological Survey Techniques of Water-Resources Investigations, book 8 , chapter Al, $23 \mathrm{p}$.

Goldstein, F. J., and Weight, W. D., 1982, Subsurface information from eight welis drilled at the Idaho National Engineering Laboratory, southeastern Idaho: U.S. Geological Survey Open-File Report 82-644, $29 \mathrm{p}$.

Hardaway, J. E. et al, Gas injection experiment NRTS, Idaho--Measurement of Xenon-133 flux and surface-level air concentrations. Issued by Isotopes, a Teledyne Company, Westwood, New Jersey, 1968.

Hawkins, D. B., and Schmalz, B. L., Environmental tritium studies at the National Reactor Testing Station, IDO-12043 (August 1965).

Humphrey, T. G., and Tingey, F. H., 1978, The subsurface migration of radionuclides at the Radioactive Waste Management Complex, 1976-1977: U.S. Department of Energy, Idaho Operations office Publication, TREE-1171, 98 p.

Idaho Department of Health and Welfare, 1977, Idaho regulations for public drinking water systems; Idaho Department of Health and Welfare, Division of Environment Publication, Title 1, Chaptr 8, 44 p.

Jones, P. H. 1961, Hydrology of waste disposl, National Reactor Testing Station, Idaho, an interim report: U.S. Atomic Energy Commission, Idaho Operations office Publication, IDO-22042-USGS, 152 p.

Lamke, R. D., 1969 Stage-discharge relations on Big Lost River, within National Reactor Testing Station, Idaho: U.S. Geological Survey Open-File Report IDO-22052, 29 p.

Leenheer, J. A., and Bagby, J. C., 1982, Organic solutes in ground water at the Idaho National Engineering Laboratory: U.S. Geological Survey Water-Resources Investigations 82-15, $39 \mathrm{p}$.

Lewis, B. D., and Goldstein, F. J., 1982, Evaluation of a predictive ground-water solute-transport model at the Idaho National Engineering Laboratory, Idaho: U.S. Geological Survey Water-Resources Investigations $82-25,71 \mathrm{p}$.

Morris, D. A., Use of Chemical and Radioactive Tracers at the National Reactor Testing Station, Idaho, in Isotope Techniques in the Hydrologic Cycle. (Geophysical Monograph No. 11) Washington, D.C.: American Geophysical Union, 1967 p. 130-142. 
Morris, D. A., Barraclough, J. T., Chase, G. H., Teasdale, W. E., and Jensen, R. G., 1965, Hydrology of subsurface waste disposal, National Reactor Testing Station, Idaho, annual progress report, 1964: U.S. Atomic Energy Commission, Idaho Operations Office Publication, IDO22047-USGS, 186 p.

Morris D. A., Barraclough, J. T., Hogenson, G. M., Shuter, Eugene, Teasdale, W. E., Ralston, D. A., and Jensen, R. G., 1964, Hydrology of subsurface waste disposal, National Reactor Testing Station, Idaho, annual progress report, 1963: U.S. Atomic Energy Commission, Idaho Operations Office Publication, IDO-22046-USGS, 97 p.

Morris, D. A., Hogenson, G. M., Shuter, Eugene, and Teasdale, W. E.; 1963, Hydrology of waste disposal, National Reactor Testing Station, Idaho, annual progress report, 1962: U.S. Atomic Energy Commission, Idaho Operations Office Publication, IDO-22044-USGS, 99 p.

Mundorff, M. J., Broom, H. C., Kilburn, C., Reconnaissance of the hydrology of the Little Lost River Basin, Idaho, Washington, D.C.: U.S. Government Printing office, 1963. U.S. Geological Survey WaterSupply Paper 1539-Q.

Mundorff, M. J., Crosthwaite, E. G., and Kilburn, Chabot, 1964, Ground water for irrigation in the Snake River Basin in Idaho: U.S. Geological Survey Water-Supply Paper 1654, 224 p.

Nace, R. L., and Barraclough, J. T., Ground-water recharge from the Big Lost River below Arco, Idaho, IDO-22016 (January 1952).

Nace, R. L., Stewart, J. W., Walton, W. C., Barraclough, J. T., Peckham, A. E., Theis, C. V., Johnson, A. I., and McQueen, I. S., 1959, Geography, geology and water resources of the National Reactor Testing Station, Idaho, Part 3, Hydrology and water resources: U.S. Atomic Energy Commission, Idaho Operations office Publication, IDO-22034USGS, $253 \mathrm{p}$.

Norvitch, R. F., Thomas, C. A., and Madison, R. J., 1969, Artificial recharge to the Snake River Plain aquifer in Idaho; An evaluation of potential and effect: Idaho Department of Reclamation, Water Information Bullet in $12,59 \mathrm{p}$.

Olmsted, F. H., 1962, Chemical and physical character of ground water in the National Reactor Testing Station, Idaho: U.S. Atomic Energy Commission, Idaho Operations office Publications, IDO-22043-USGS, 142 p.

Polzer, W. L., Percival, D. R., and Barraclough, J. T., 1976, Special analyses for Plutonium and Americium in water from the Snake River Plain aquifer: U.S. Department of Energy, Idaho Operations office Publication, IDO-12081, 9 p.

Robertson, J. B., 1969, Behavior of Xenon 133 gas after injection underground molecular diffusion materials balance barometric pressure effects: U.S. Geological Survey Open-File Report IDO-22051, 37 p. 
1974, Digital modeling of radioactive and chemical waste transport in the Snake River Plain aquifer at the National Reactor Testing Station, Idaho: U.S. Geological Survey Open-File Report IDO-22054, 41 p.

1977, Numerical modeling of subsurface radioactive solute transport from waste-seepage ponds at the Idaho National Engineering Laboratory: U.S. Geological Survey Open-File Report 76-717 (IDO-22057), 68 p.

Robertson, J. B., and Barraclough, J. T., 1973, Radioactive- and chemicalwaste transport in ground water at National Reactor Testing Station, Idaho: 20-year case history, and digital model: Underground Waste Management and Artific ial Recharge, v. 1, p. 291-322.

Robertson, J. B., Schoen, Robert, and Barraclough, J. T., 1974, The influence of 1 iquid waste disposal on the geochemistry of water at the National Reactor Testing Station, Idaho, 1952-1970: U.S. Geological Survey Open-File Report IDO-22053, 231 p.

Schmalz, B. L., (comp.), Injection of gas into the lithosphere at the National Reactor Testing Station, IDO-12069 (June 1969).

U.S. Environmental Protection Agency, 1976, National interim primary drinking water regulations: U.S. Environmental Protection Agency, Office of Water Supply Publication, EPA-570/9-76-003, 159 p.

U.S. Public Health Service, Drinking water standards, Public Health Service Publication No. 956, Washington, D.C.: U.S. Government Printing Of $\mathrm{f}$ ice, $1962,61 \mathrm{p}$.

Walker, E. H., 1964, Subsurface geology of the National Reactor, Testing Station, Idaho: U.S. Geological Survey Bullet in 1133-E, 22 p.

Walton, W. C., Analysis of aquifer tests at the National Reactor Testing Station, Idaho, 1949-1957, Boise: U.S. Geological Survey, 1958, 38 p. 
Table 2.--Key to abbreviations used in this report

LSD: Land Surface Datum

MP: Measuring Point

MSL: Mean Sea Level as of 1929. Equivalent to National Geodetic Vertical Datum (NGVD) of 1929.

TOC: Top of Casing

MET: Method of Measurement

S: Steel tape

V: Calibrated electric tape

T: Electric tape

Z: Other

STA: Status of well

D: Dry

P: Pumping

$\mathrm{N}$ : Measurement discont inued

$\mathrm{R}$ : Recently pumping

0 : Obstruction

V: Foreign substance

E: Flowed recently

S: Nearby pumping

Idaho National Engineering Laboratory installation names and abbreviations used in this report

Name of installation

Abbreviation

Advanced Test Reactor

ATR

Aircraft Nuclear Propulsion

ANP

Argonne Fast Source Reactor

AFSR

Army Reactor Area

Auxiliary Reactor Equipment Area

ARA

Boiling Water Reactor

AREA

Central Facilities Area

BORAX

Engineering Test Reactor

CFA

Experimental Beryllium Oxide Reactor

ETR.

Experimental Breeder Reactor No. 1

Experimental Breeder Reactor No. 2

EBOR

EBR I

EBR II

Experimental Organic Cooled Reactor

EOCR

Field Engineering Test Facility

FET

Gas Cooled Reactor Experiment

GCRE

Idaho Chemical Processing Plant

ICPP.

Initial Engineering Test Facilty

IET

Loss of Fluid Test Facility

LOFT

Low Power Test Facility

LPTF

Materials Testing Reactor

MTR

Naval Reactors Facility

Organic Moderated Reactor Experiment

NRF

OMRE

Power Burst Facility

PBF

Propulsion System Test Facility

PSTF

RWMC

Radioactive Waste Management Complex

SPERT

Test Area North

TAN

Test Reactor Area

TRA

Transient Reactor Test Facility

TREAT

Zero Power Reactor No. 3

ZPR-III 


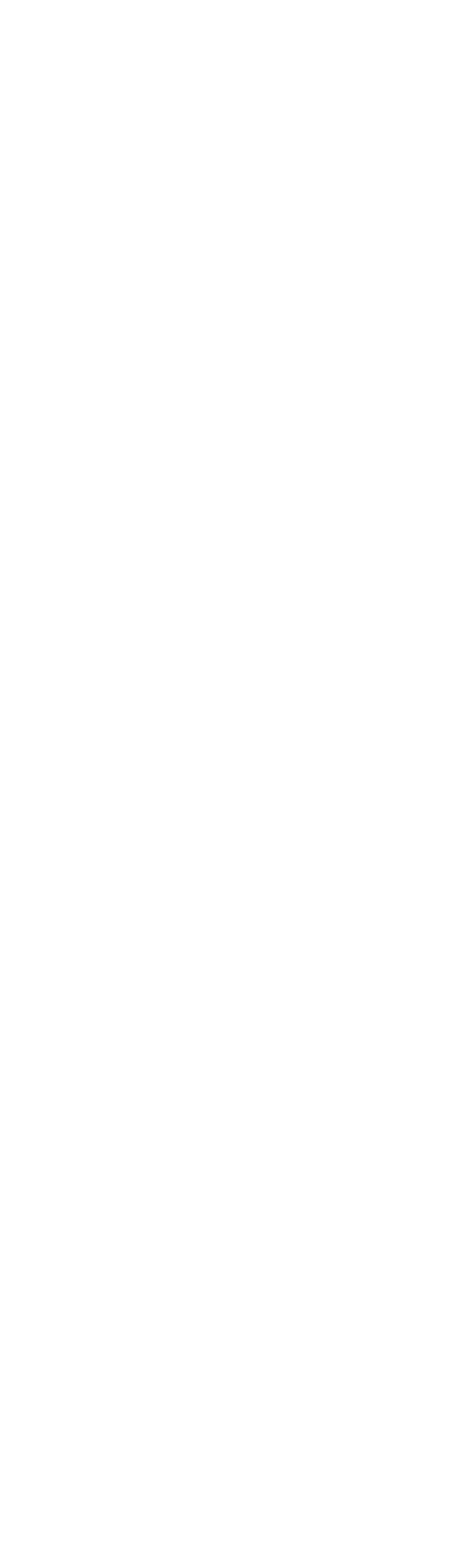

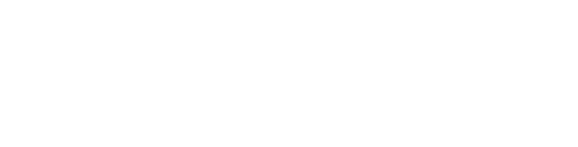

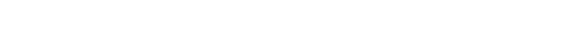

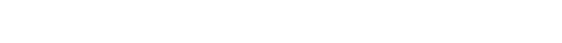

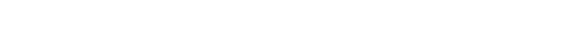

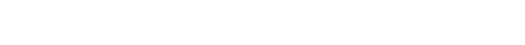

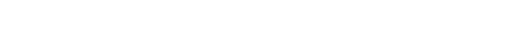

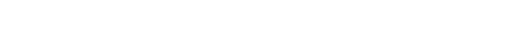

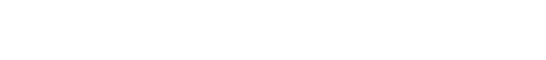

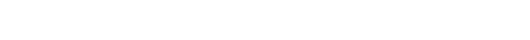

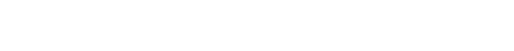

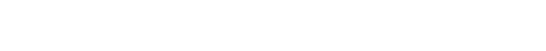

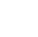

is

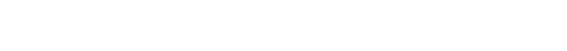

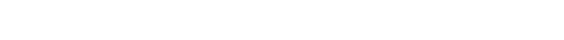

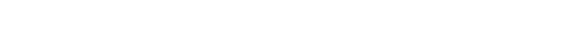
×ง

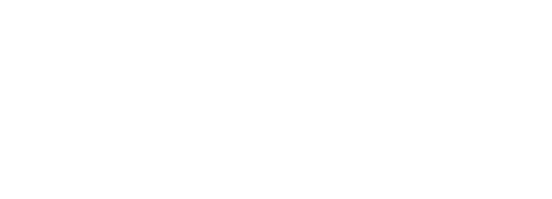

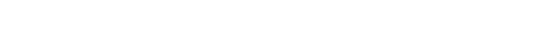
5

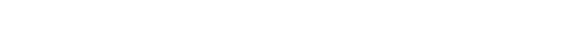

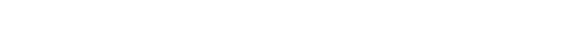

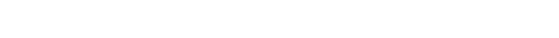

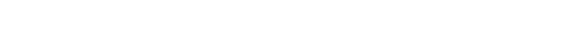

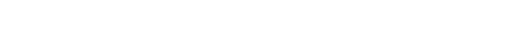

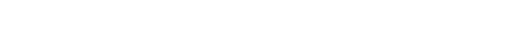

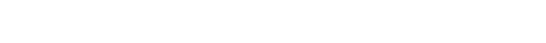

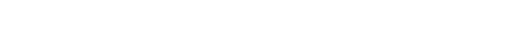

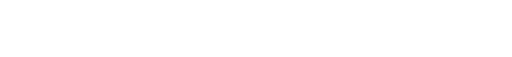

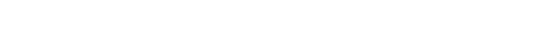
$\Sigma$ 5

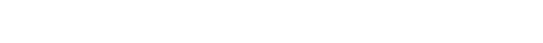
a

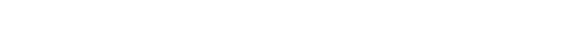

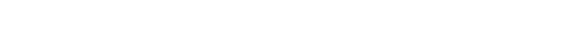

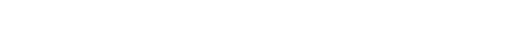

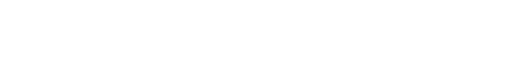

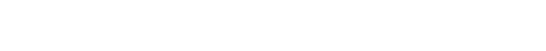

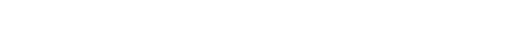

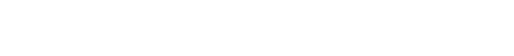




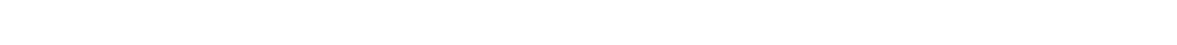
แ

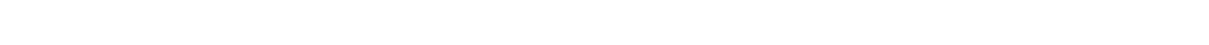

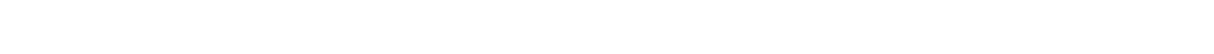

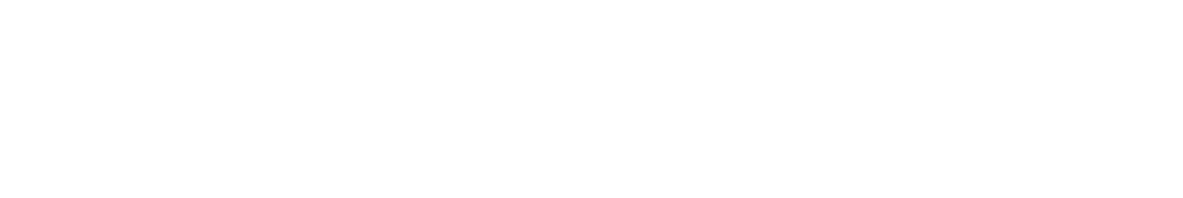
$\stackrel{w}{\Sigma}$

$\frac{a}{n}$

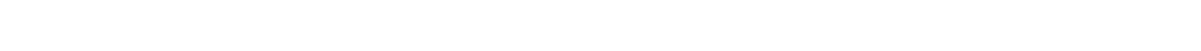
แ

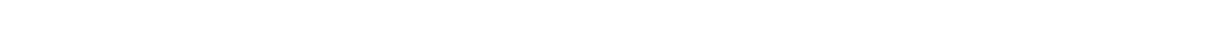

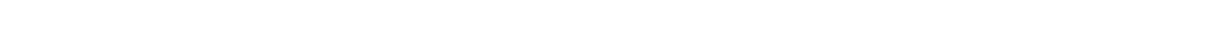

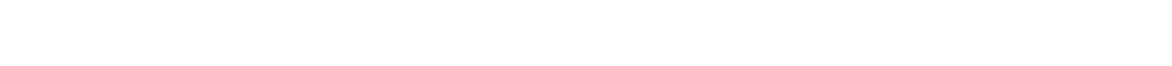

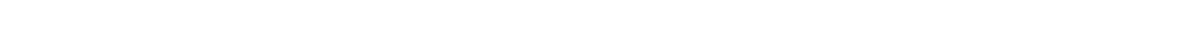

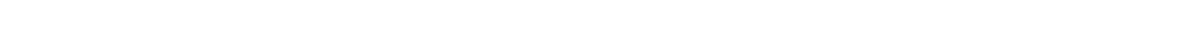

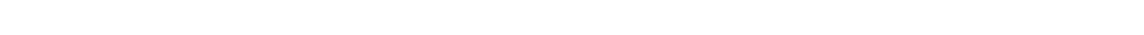

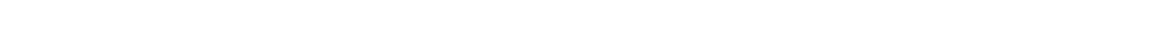

$$
\begin{aligned}
& \text { țescosescoses } \\
& \text { ह }
\end{aligned}
$$

" w दूo З

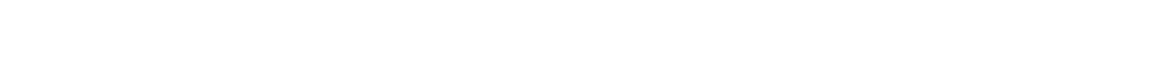

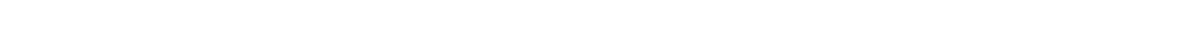

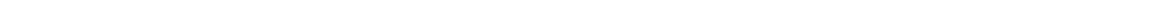

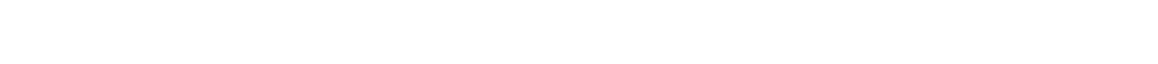

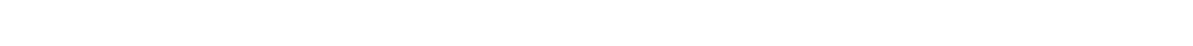
$\alpha$ is

mon

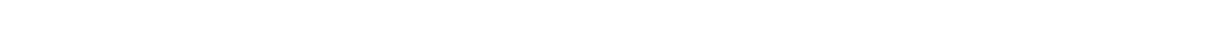

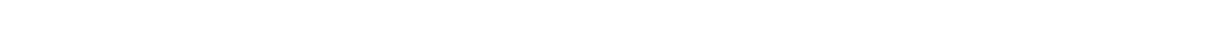

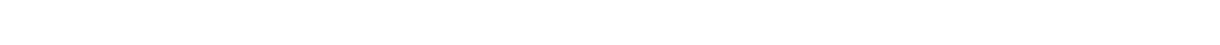

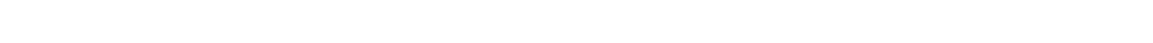

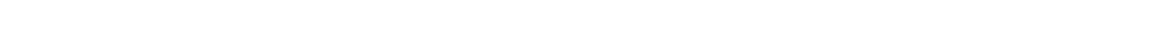

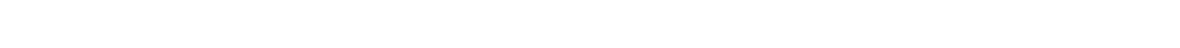

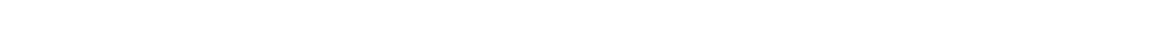

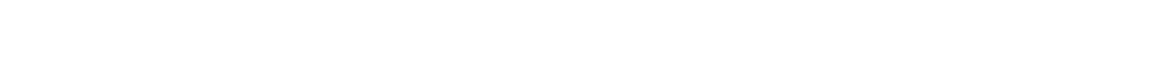


is

"

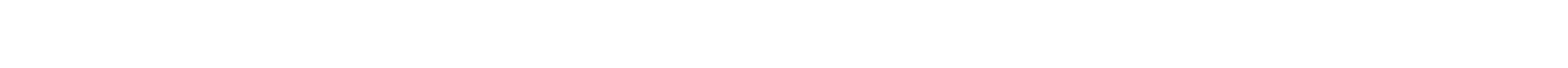
3.

w

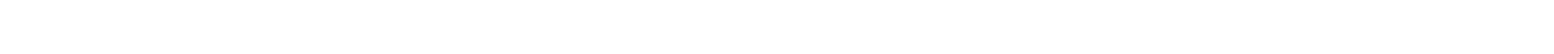

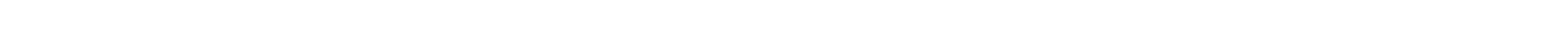

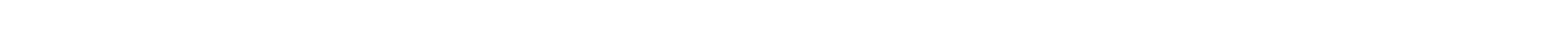

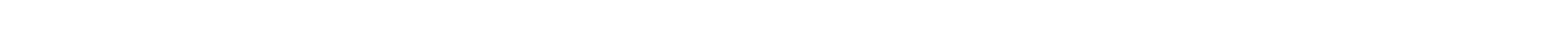

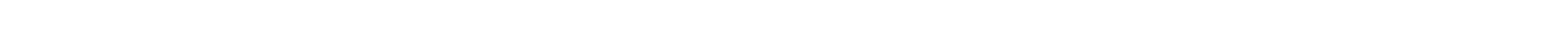

5
i
in

แ

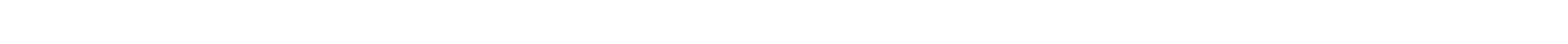

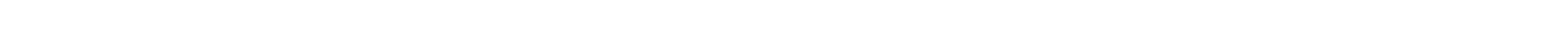

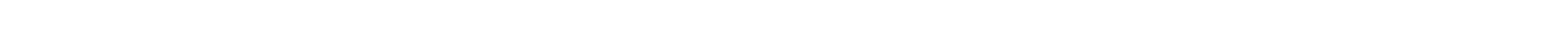

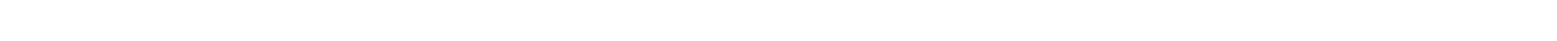

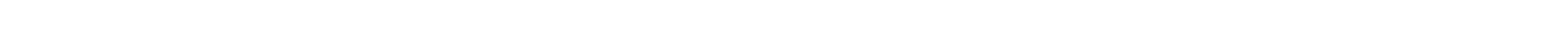

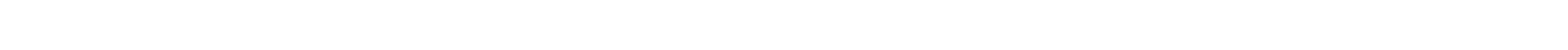

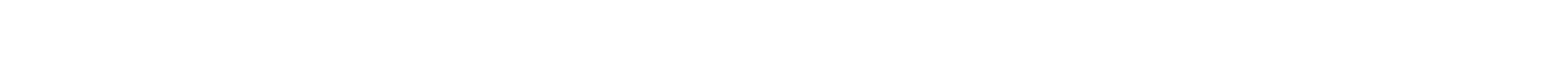

흘

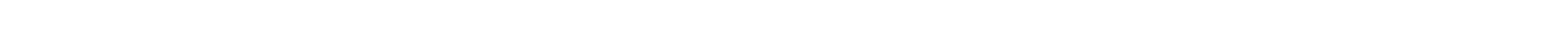

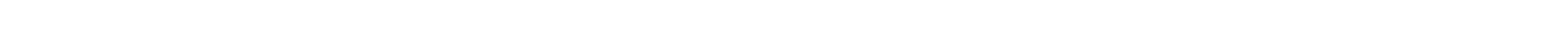

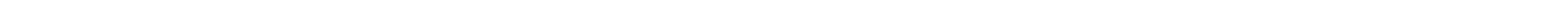

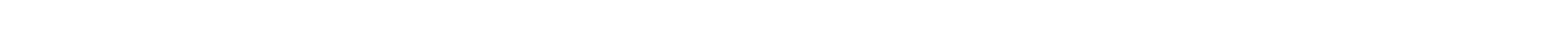

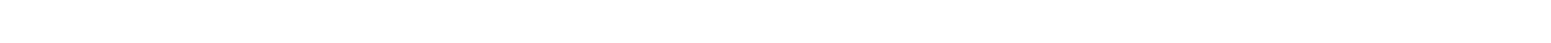

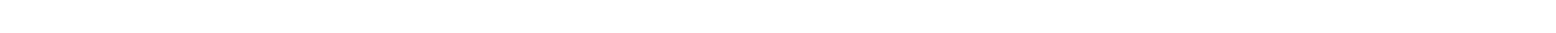

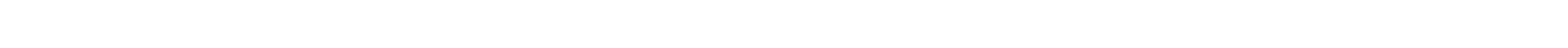
ONMOF

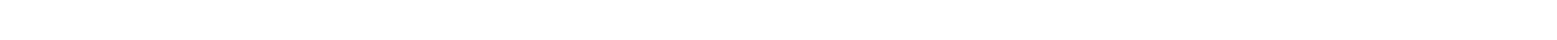

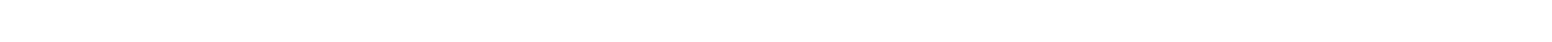

us
$\Sigma$
$\vdots$
5

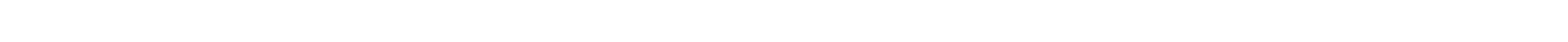

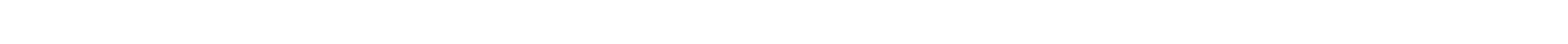

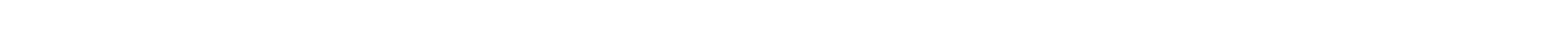

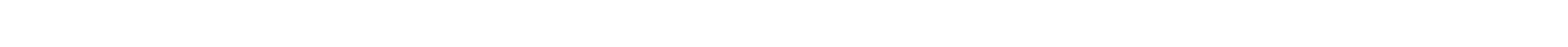

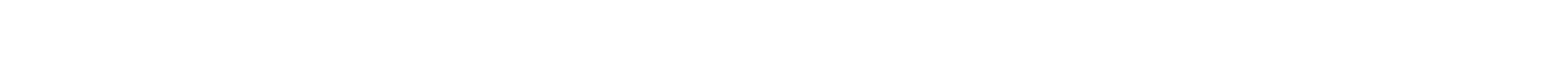

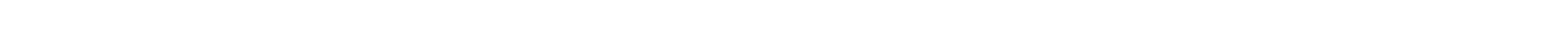

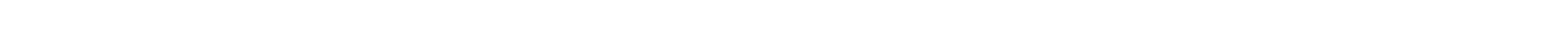

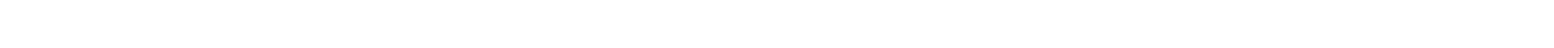


cosencosensosenesenes

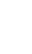
"

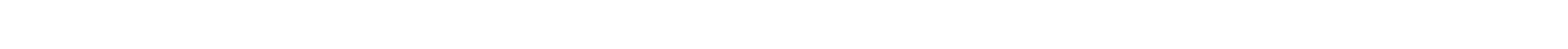
-

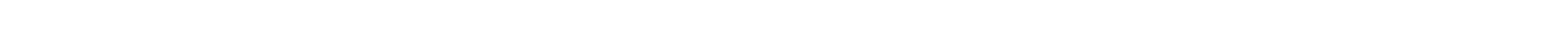

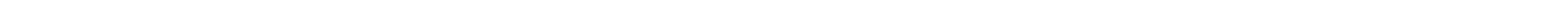

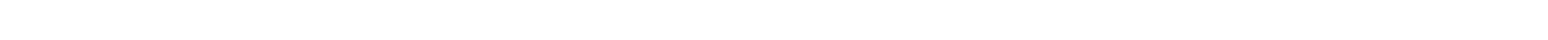

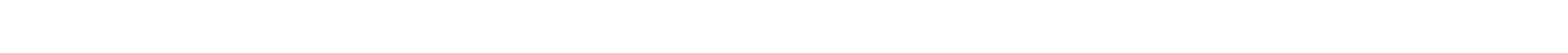
“

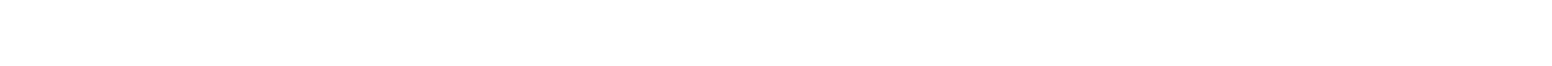

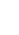

$\underline{\mathbf{z}}$

$\underset{1}{4}$

N a

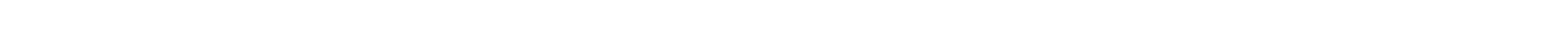

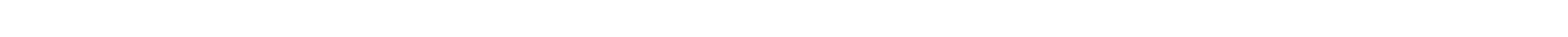

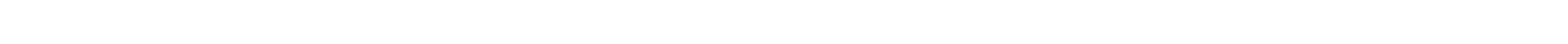

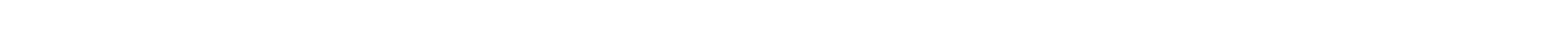
w LEF - F ‘र

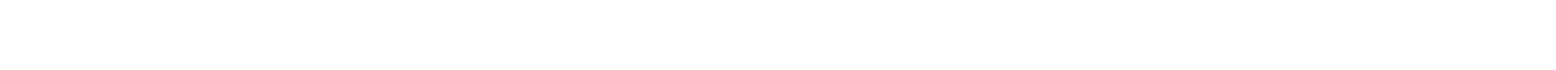

栏

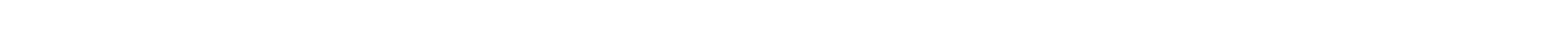

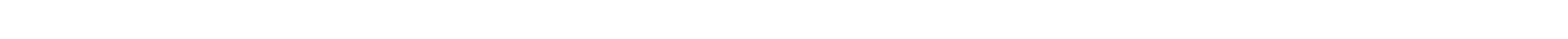

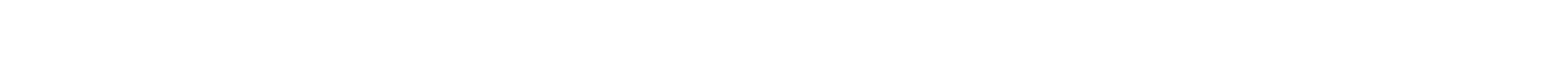

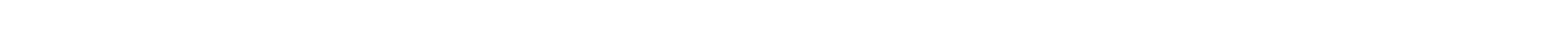

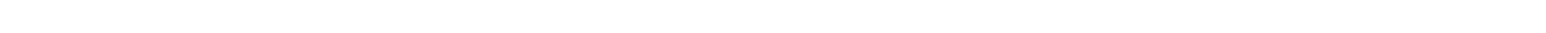
w 世

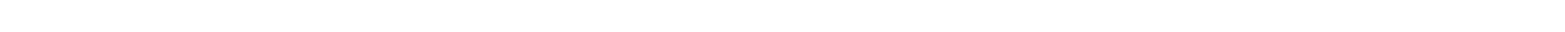

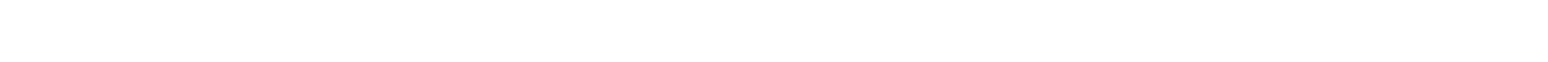

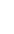

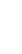

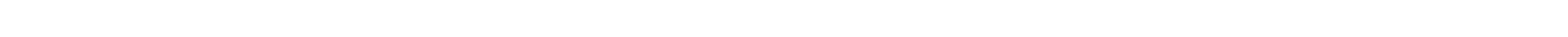
u w? :

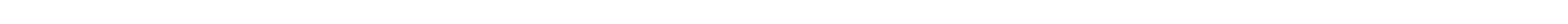

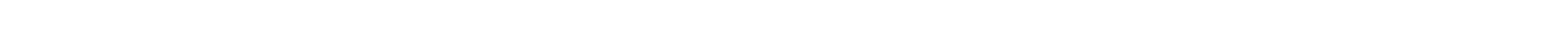

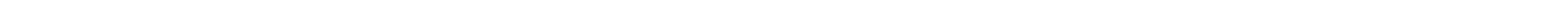

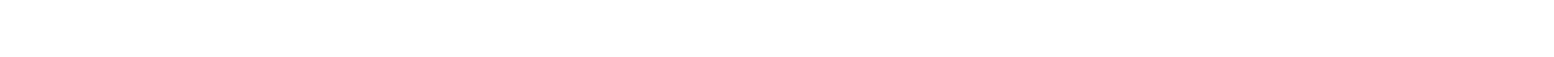

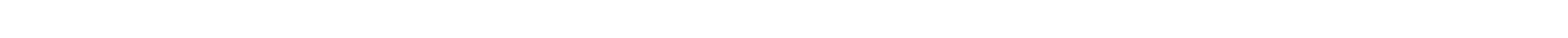
๔O

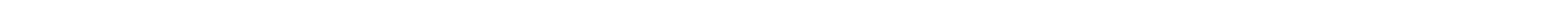

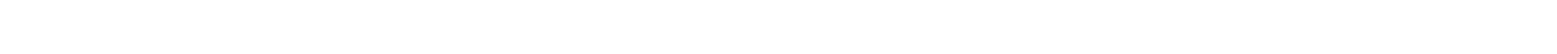




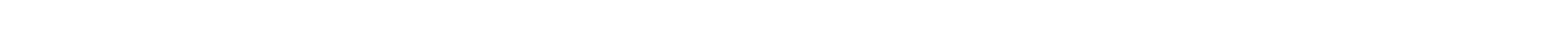

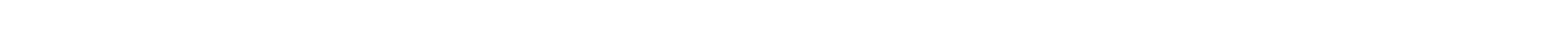

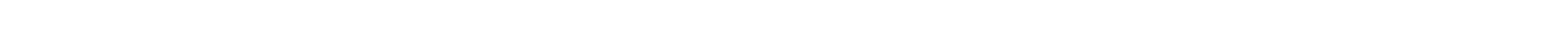

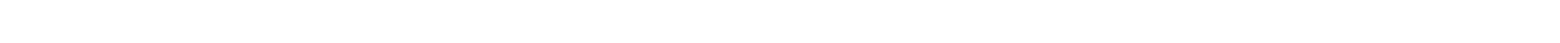

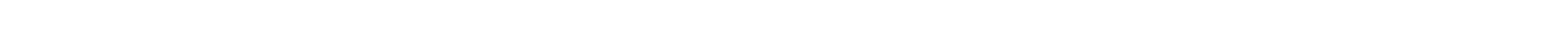

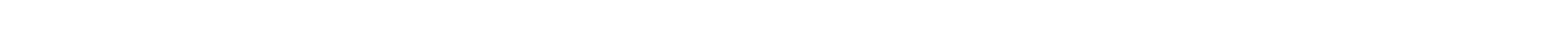
แ

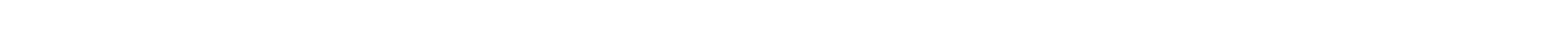

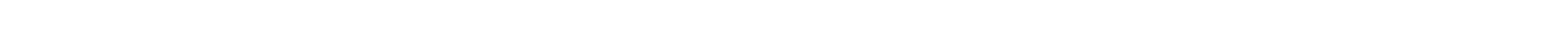

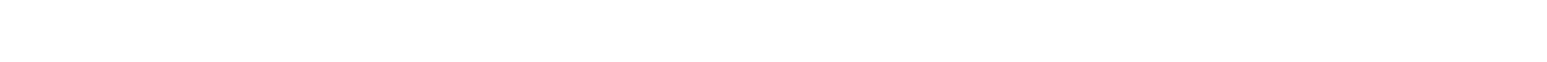

立

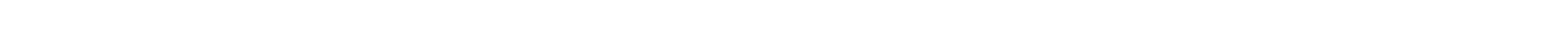

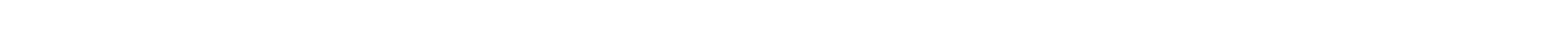

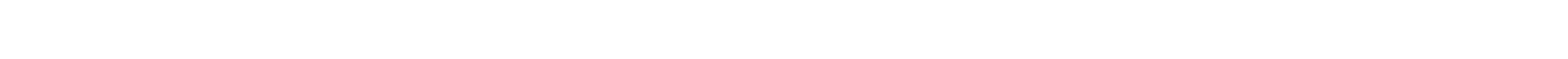

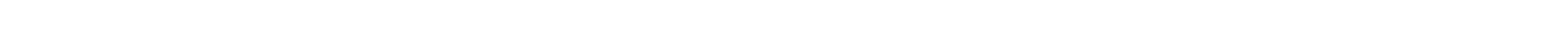

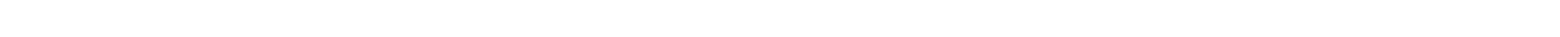

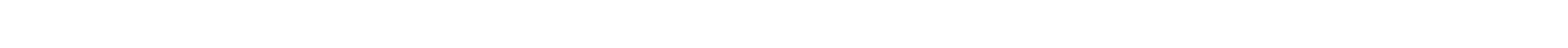

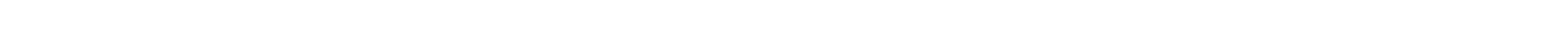
EะN MOE

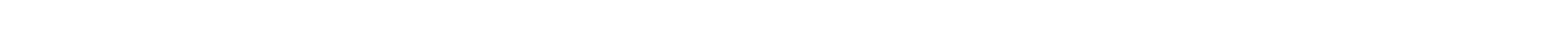
点
is

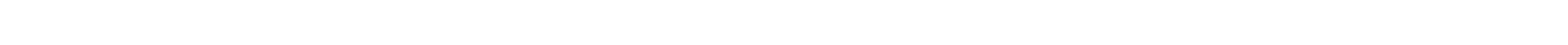

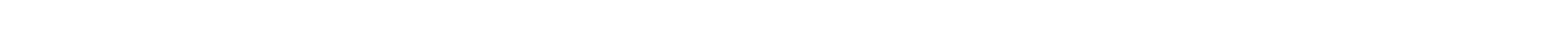

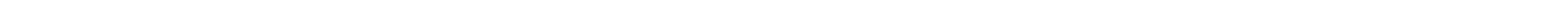
З

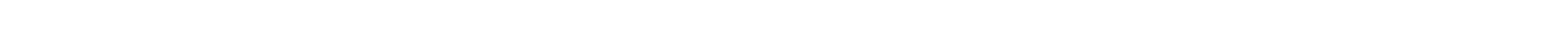

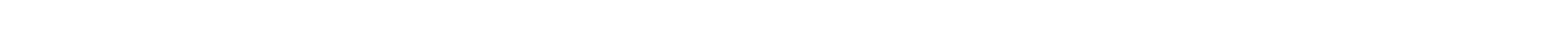

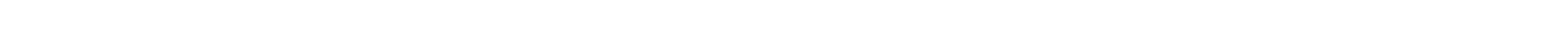

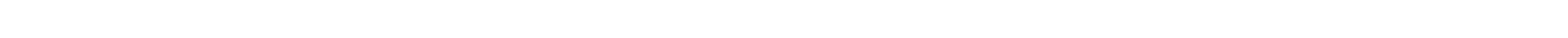

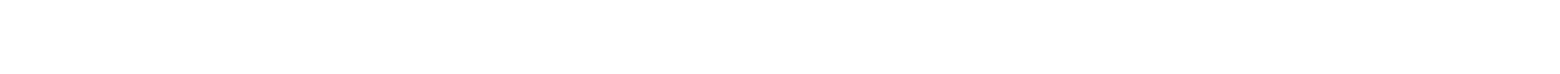

$\stackrel{\omega}{\Sigma}$ is

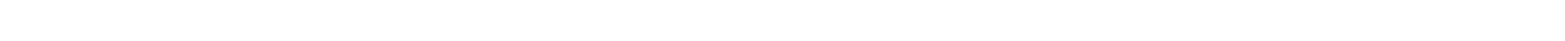

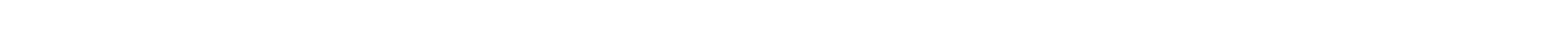
4 แล

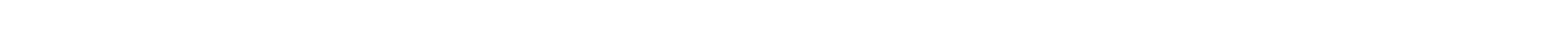

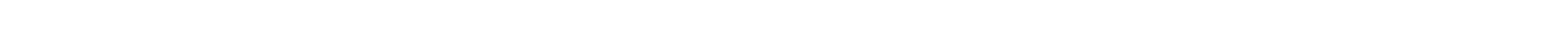

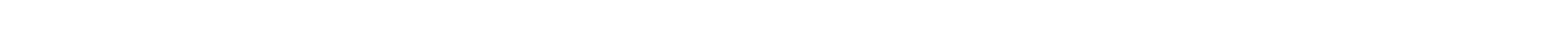

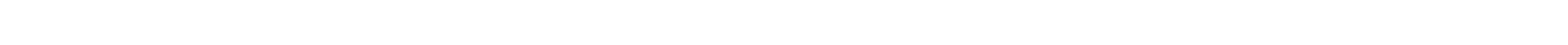

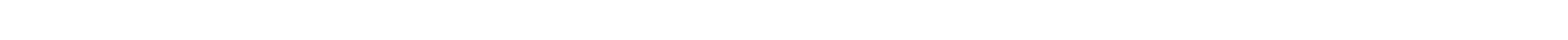

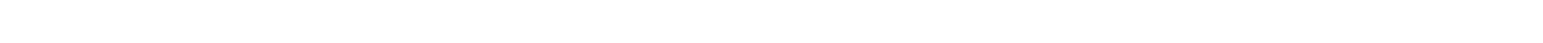



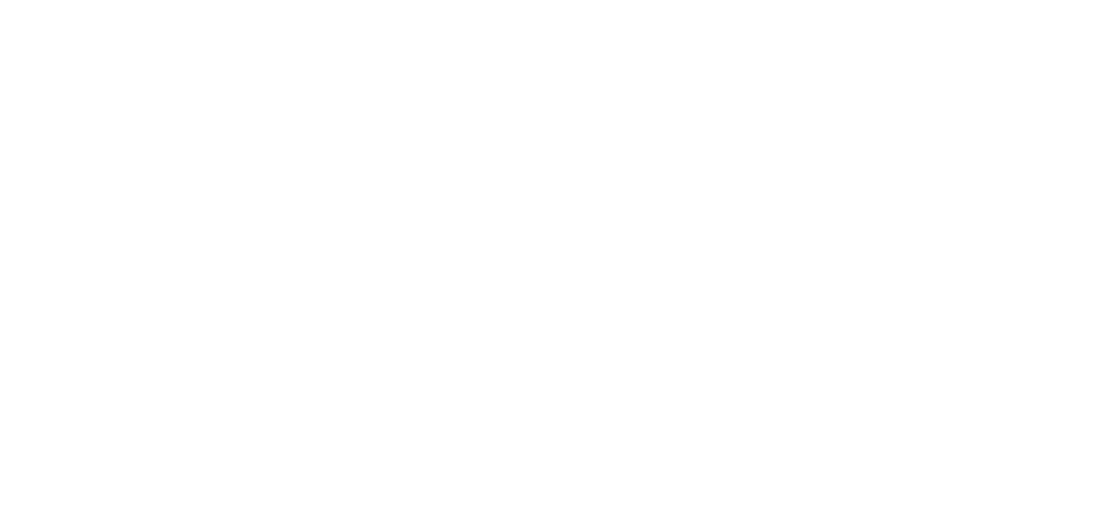

$\stackrel{5}{2}$

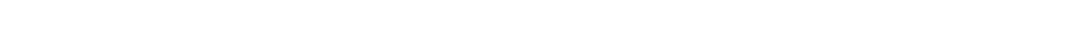

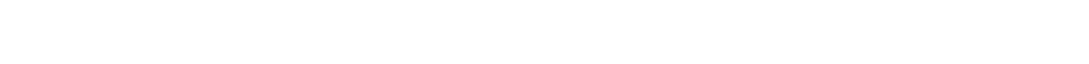

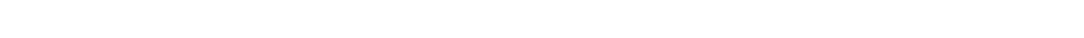

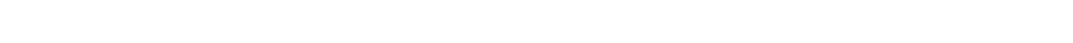

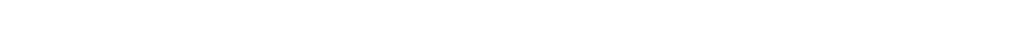

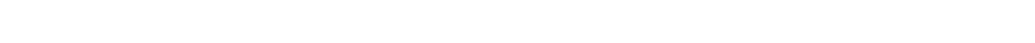

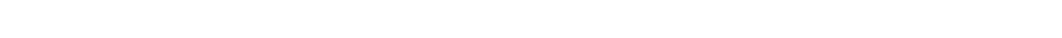
-

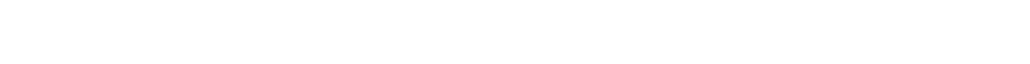

w in

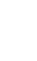

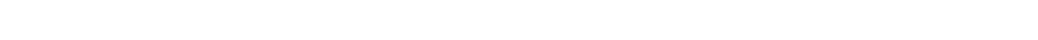

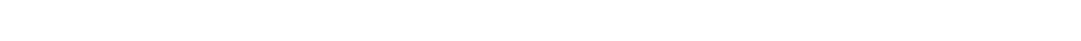
- Z ও

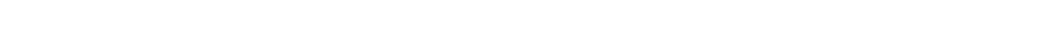

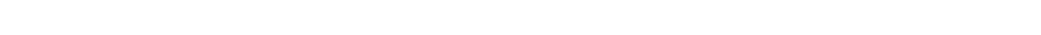

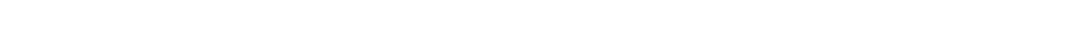
O-

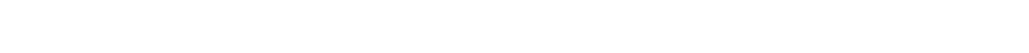$$
+
$$$$
\text { i }
$$$$
\Sigma
$$$$
\text { is }
$$

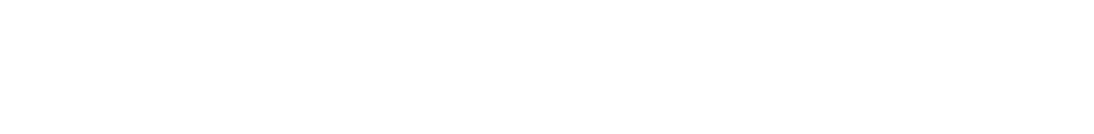

$000000000000000000000000000000000-$ 作 แ

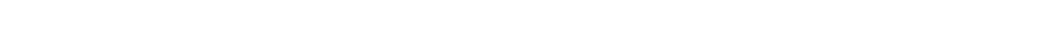

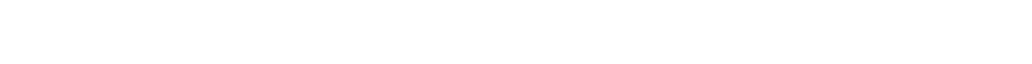




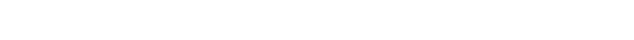

is

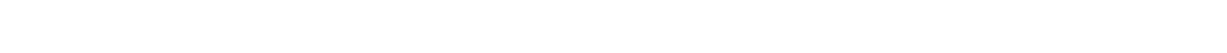
แ Z

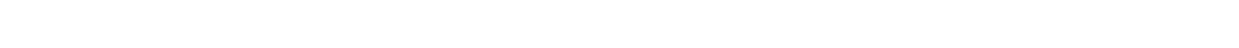

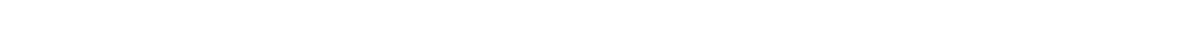

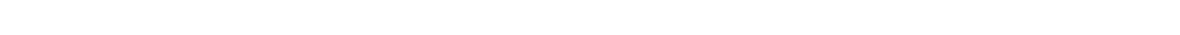

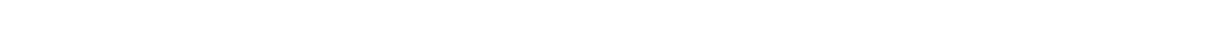

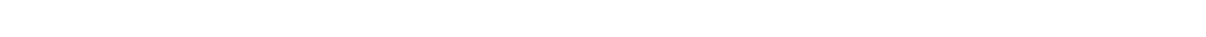

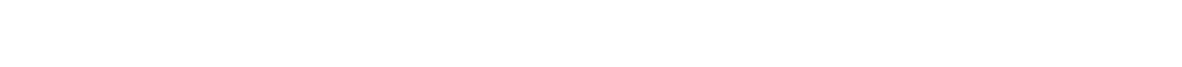

党

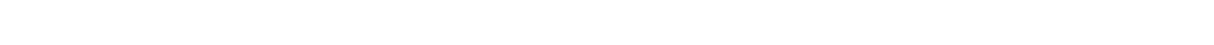
w w Е

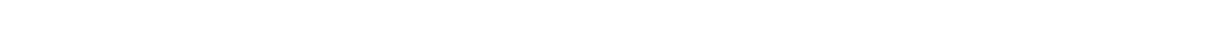

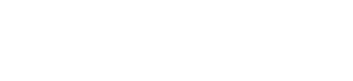
$z^{\infty} \geq x$ U $z$

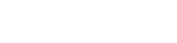

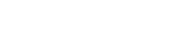
$>0 z$ 更 wis

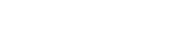

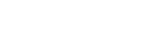
药

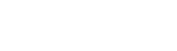

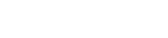

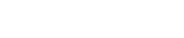
눙유 =ㅇㅇㅇ응

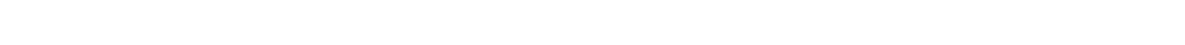

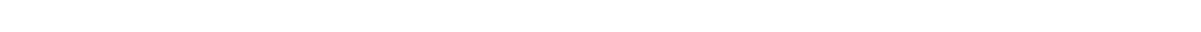

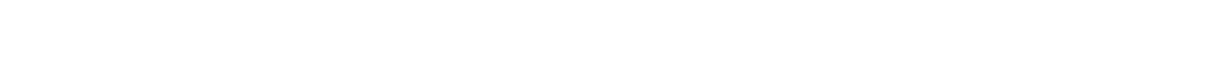

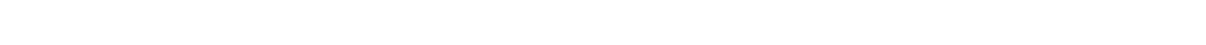

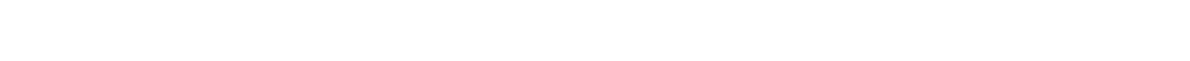

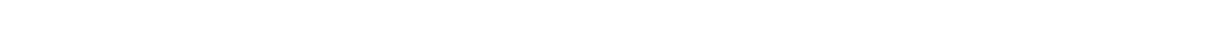
$\alpha$ is

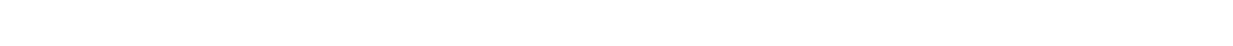
w

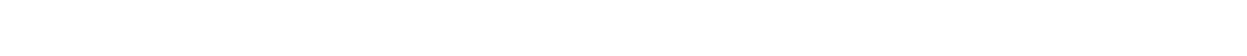

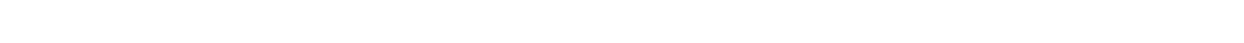

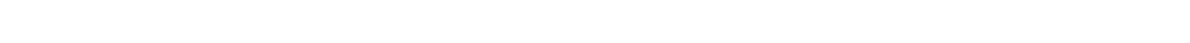

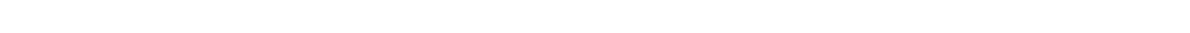

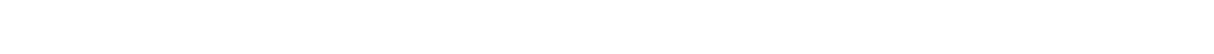

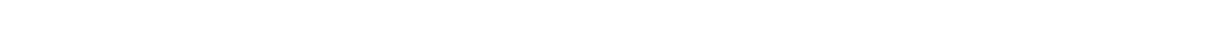

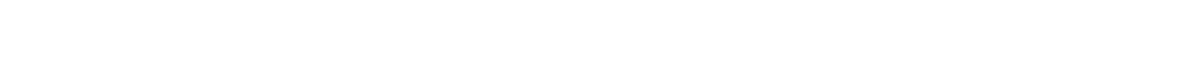

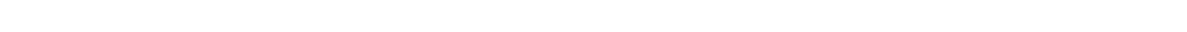

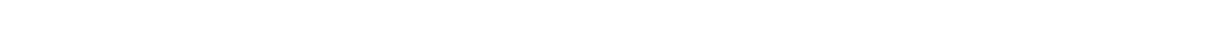
in

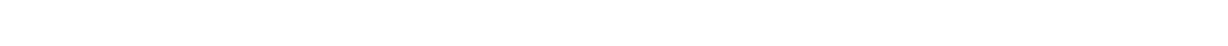

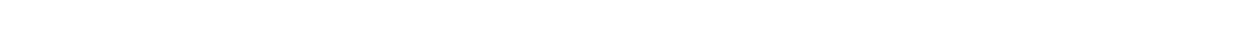
屯ำ

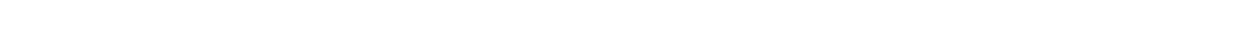

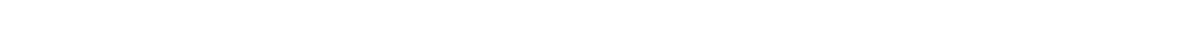

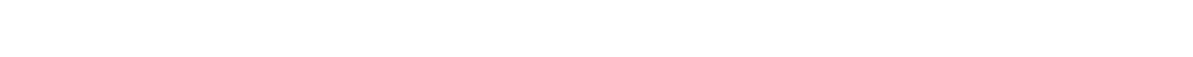

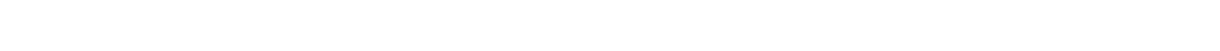

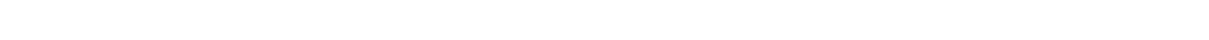

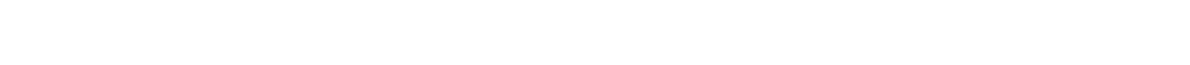




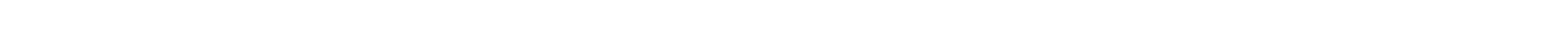

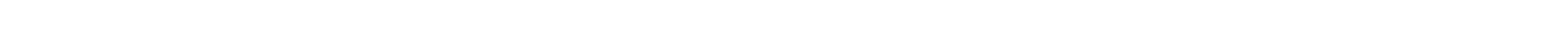

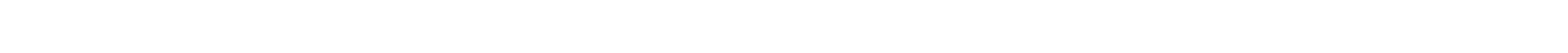

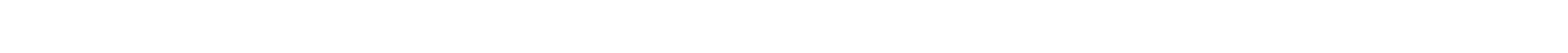

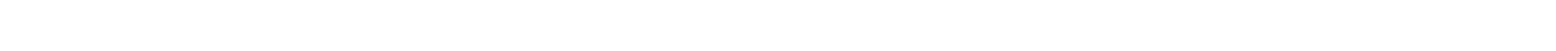

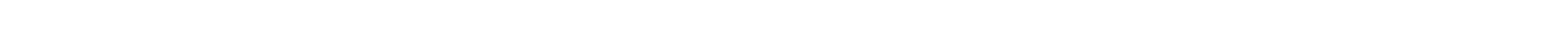

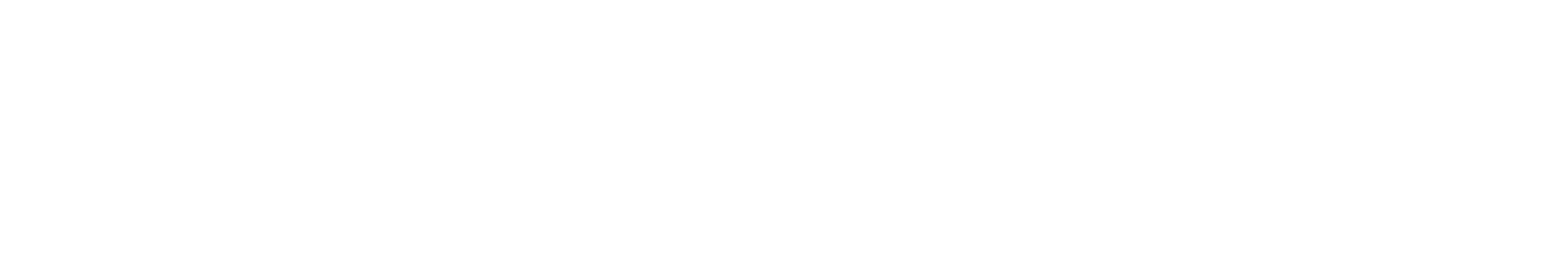

นกับกN

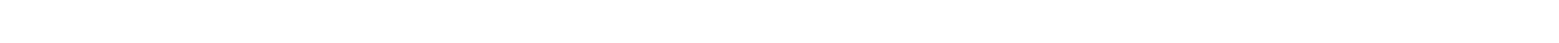

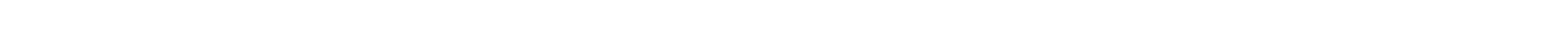

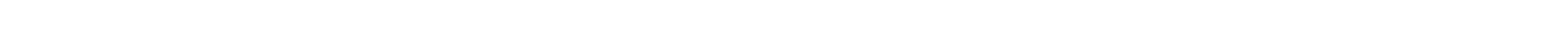

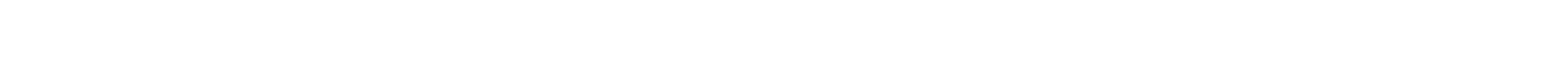
(1)

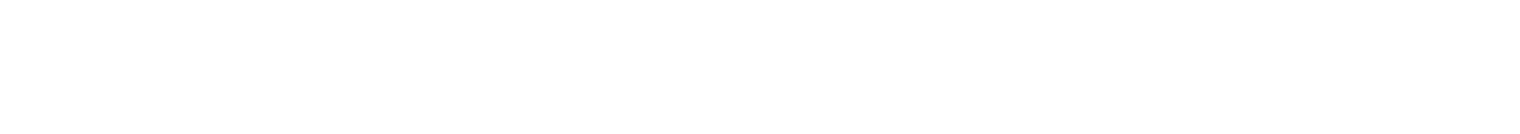

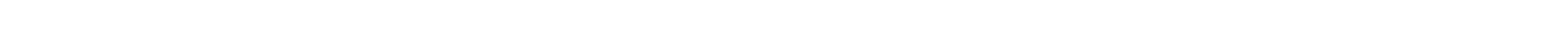
a w

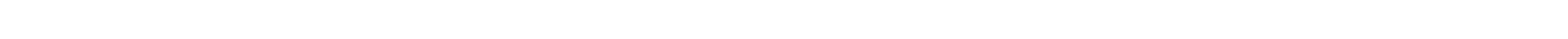

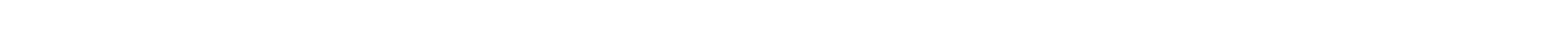

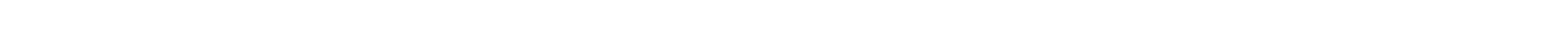

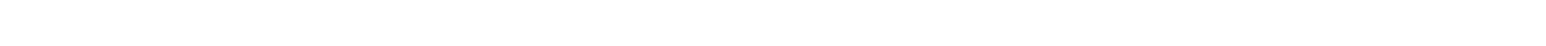

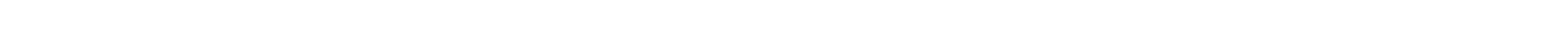

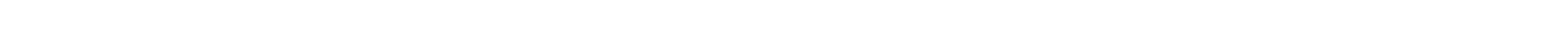
0 O-FNNMmOG

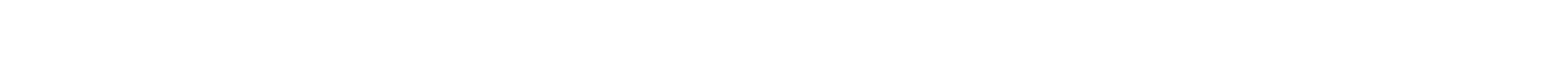

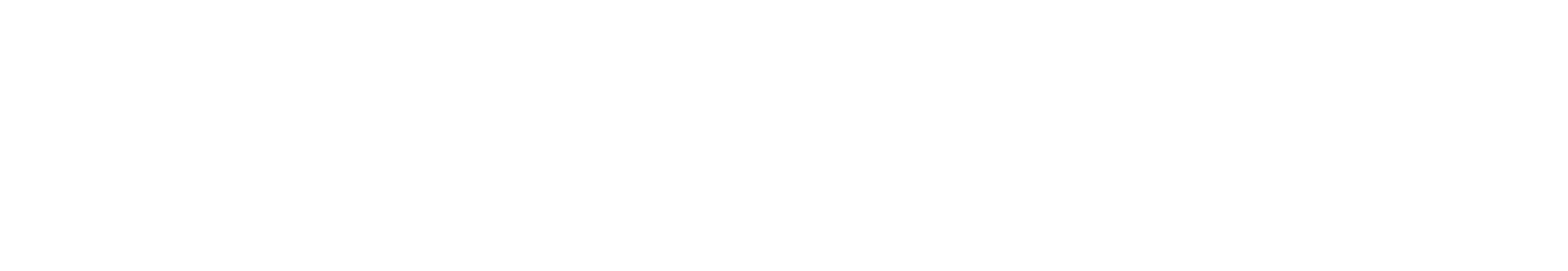

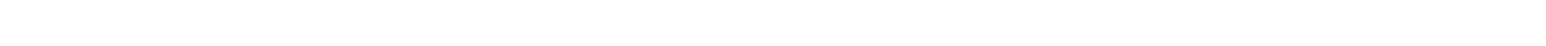

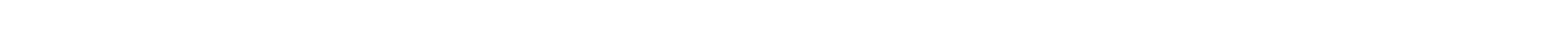

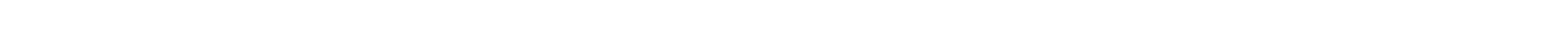

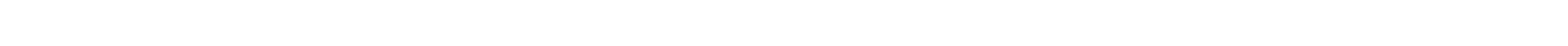

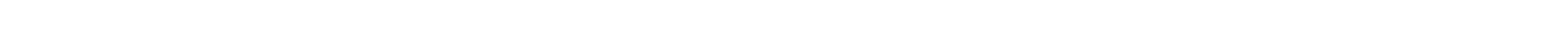

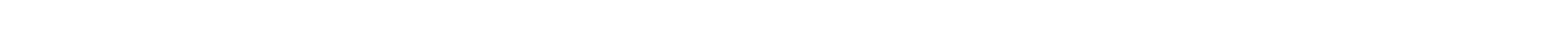




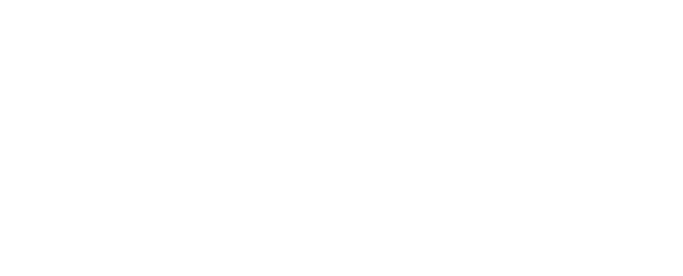

NON

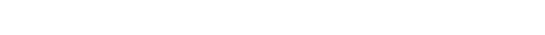

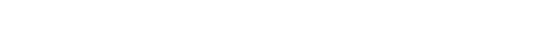

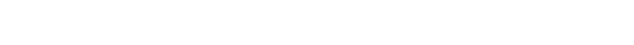

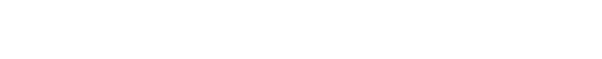

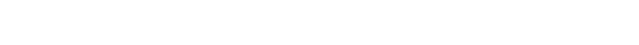

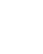

"⿻ำ

世山以 แ "

ang

2
0
0
0
0
0

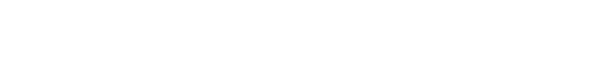

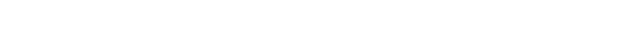

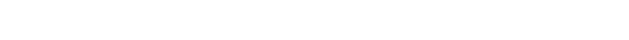

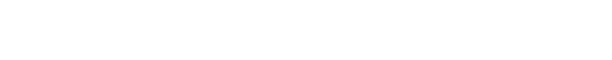

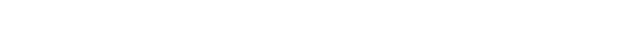

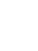

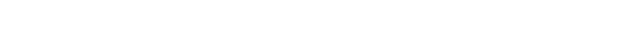

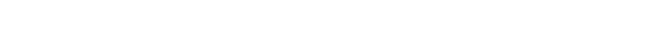

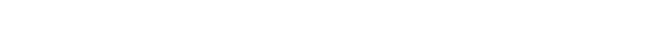

a o ommmmmmmmmgen

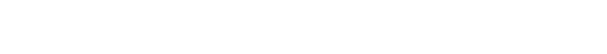

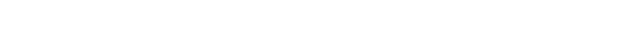

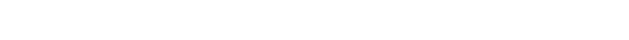

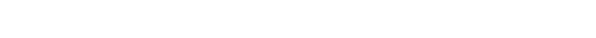

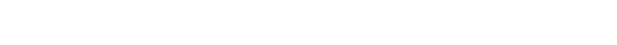

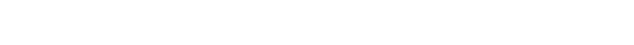
is

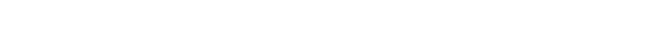

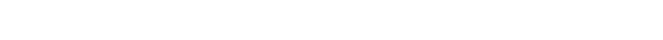

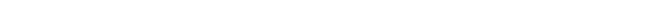

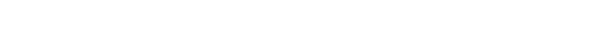

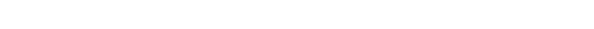

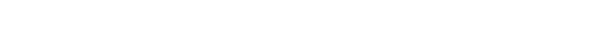

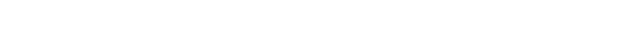

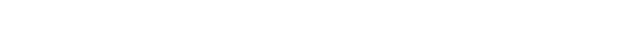

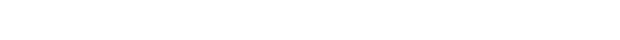

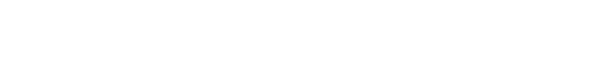




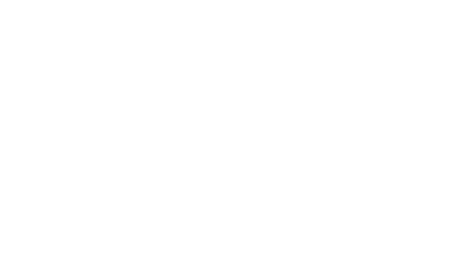

告

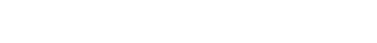

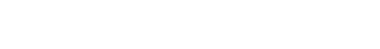

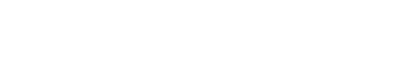

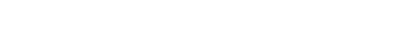

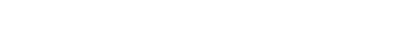

tusescosicsescosencososos

5

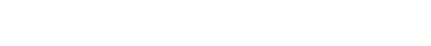

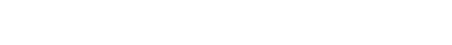
L MNMmMm\&MmMẼ

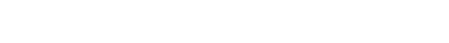

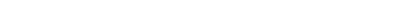

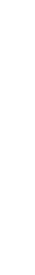

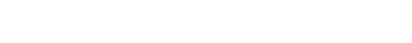

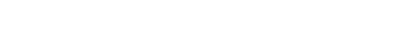

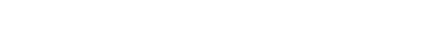

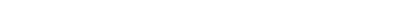

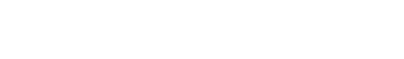

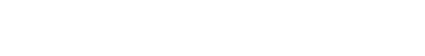
in

๔ - $\quad$ w - $\quad$ J

I $=\frac{1}{2}$

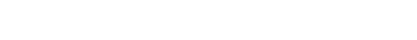

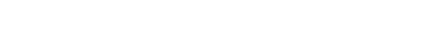
ON-TNN-FNNNN-F

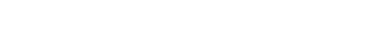

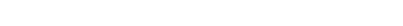

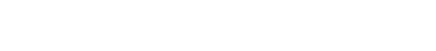
$\underset{1}{a}$ is

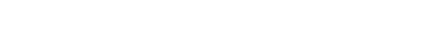

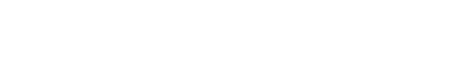

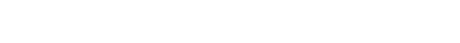

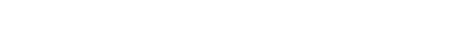

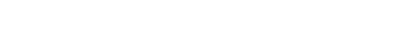

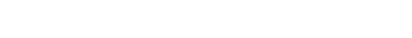

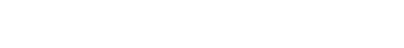

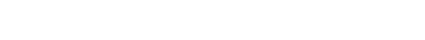

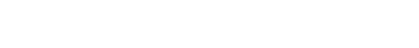

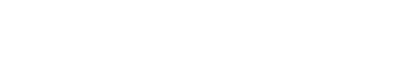



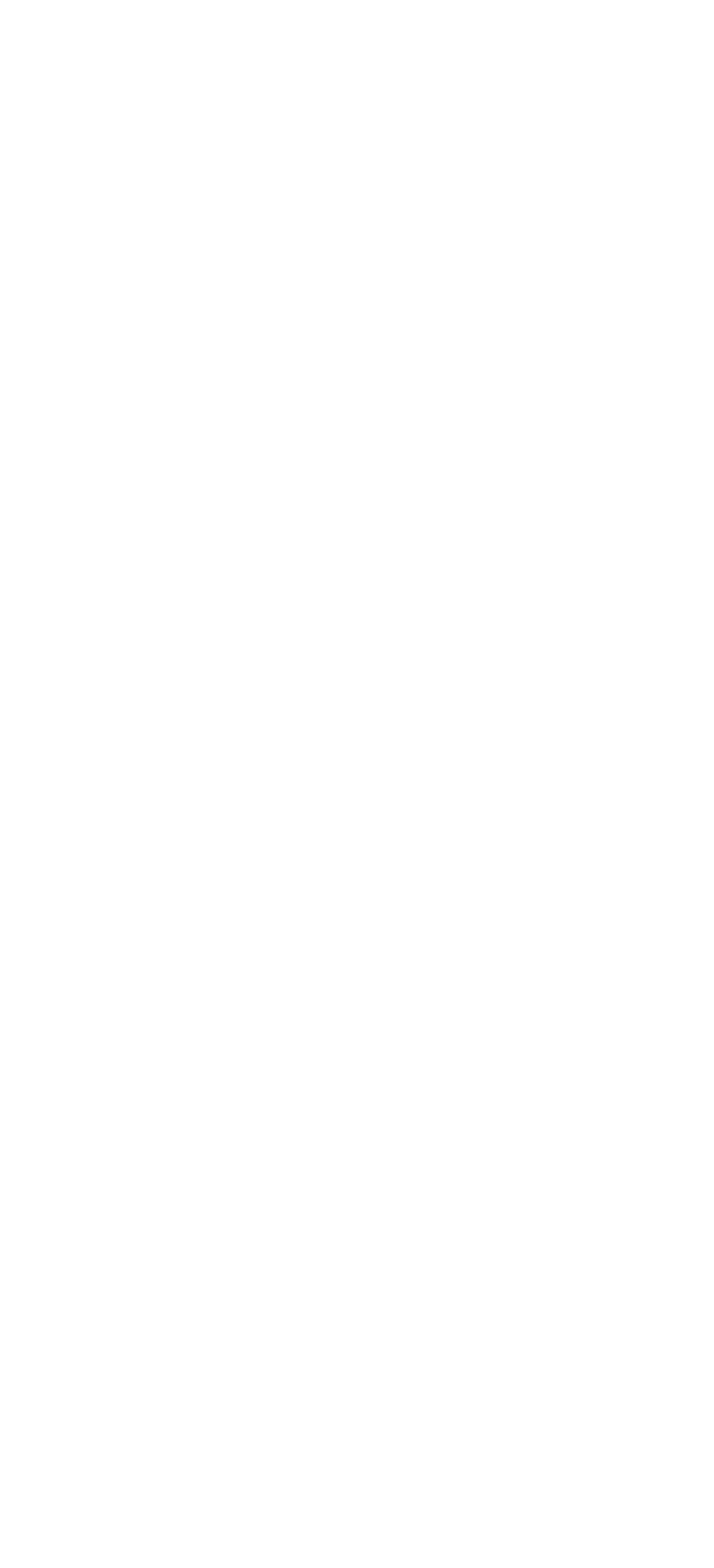

min om monor wa2a minamonana

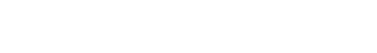

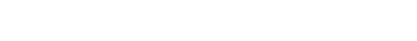
5

-

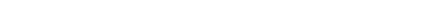

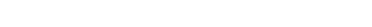

$\operatorname{con} 2 \pi=$

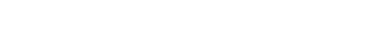

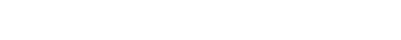

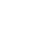

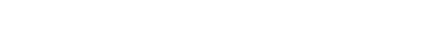
$>-1-0-n$

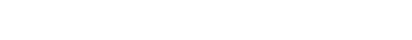

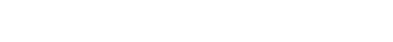
aaaaaaำ

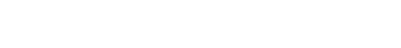

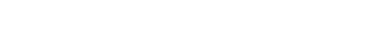



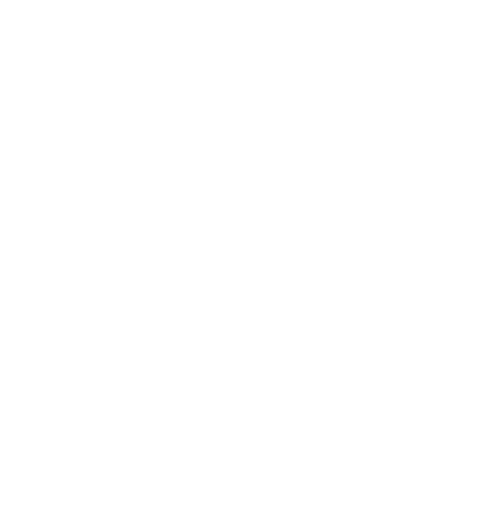

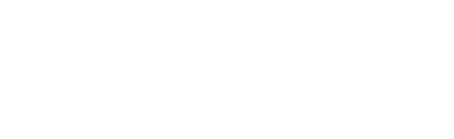

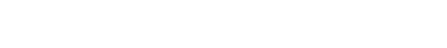

w ǘ

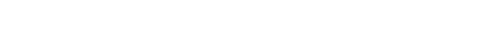
$x$ -

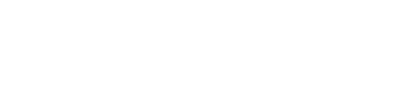
$\dot{\sim}^{n} \dot{\sim} \dot{\sim} \dot{\sim} \dot{\sim}$

$\ddot{\sim} \omega \ddot{\circ}$

a

$\ddot{\sim} \ddot{\Sigma} \ddot{\sim} \ddot{a} \ddot{\sim}$

○ा

$n \sum_{n=1}^{n} w$

$-\pi$

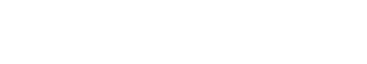

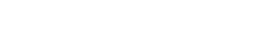

$=\dot{0}$

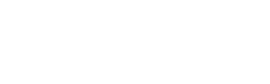

0

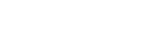

足芒

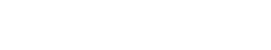

$\because \vec{a}$.

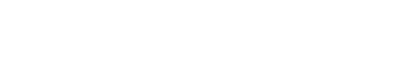

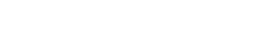

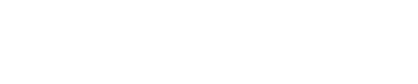

늠

a $z$ j

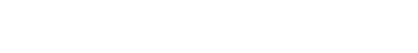

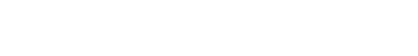

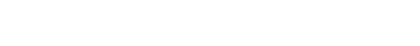

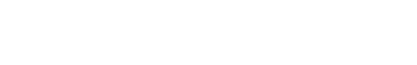

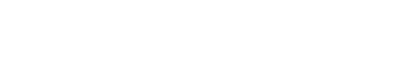

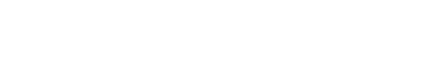
2

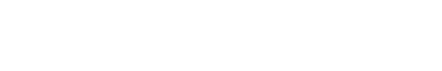

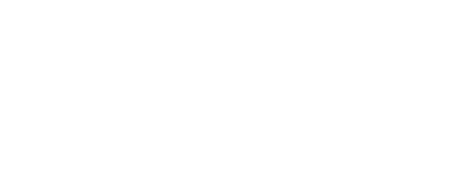

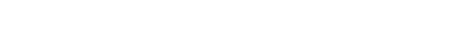

is

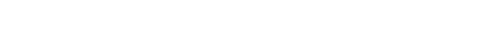

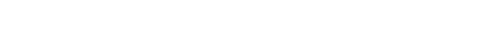

w

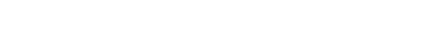

$\hat{n} \hat{n} \hat{n} \hat{n} \hat{n} \tilde{n} \hat{n} \hat{n} \tilde{n} \tilde{n}$

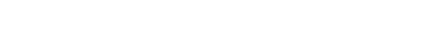

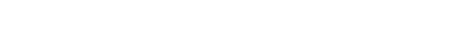

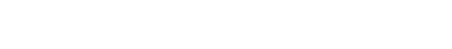

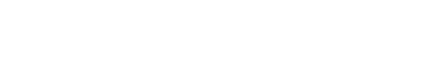

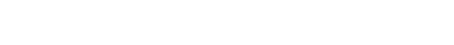
is

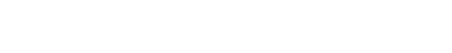
แน $\rightarrow-0 \dot{0} 0 \dot{0} 0 \dot{0}-\dot{0} 0$ स vinn num $z^{2} z^{2} z^{2} z^{2} z^{2} z$

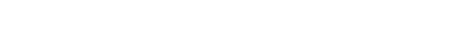

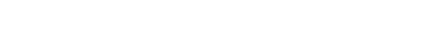

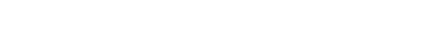

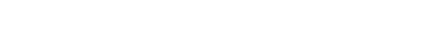

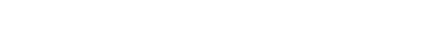

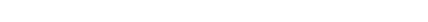

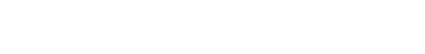




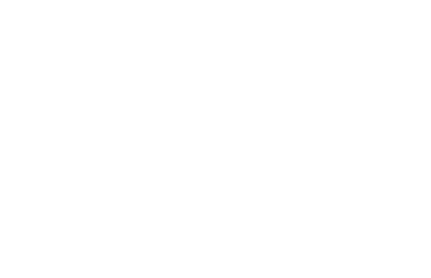

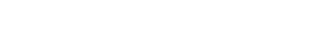

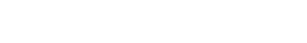

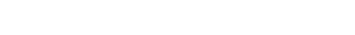

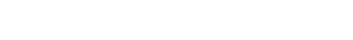

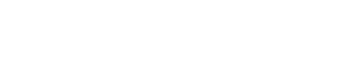

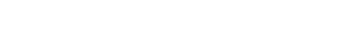
品

ก ㅇำ

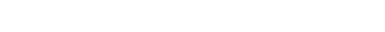
" w $m$ का का $x_{0}, t \rightarrow \infty$

$8_{0}^{0} 0$ o $-\frac{\pi}{\alpha} \sim$

西

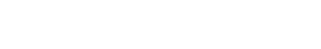

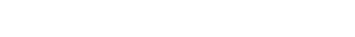

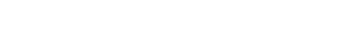

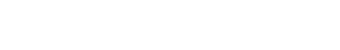

tuscosescrosenesesos $\sum$

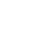

inum

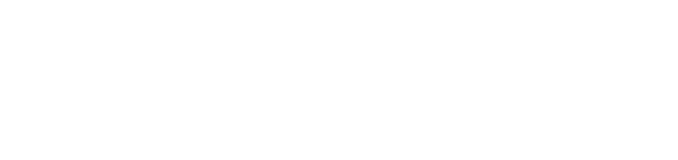

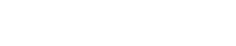

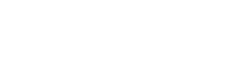

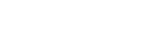

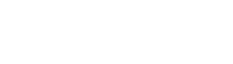
눈인 운 c. w w w w a a a 0 - 0 znzam

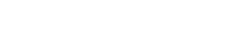
İ $=-\div$ ox-onono

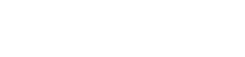

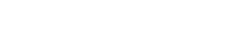
$\ddot{u} \stackrel{w}{\omega}-\ddot{w} \sim \ddot{m} m$

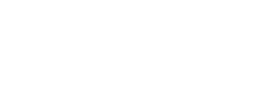

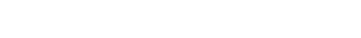

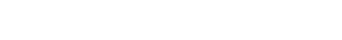

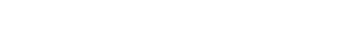
OEะOENNEN-NE

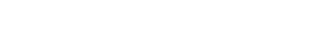

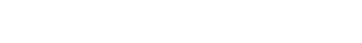
in

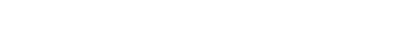

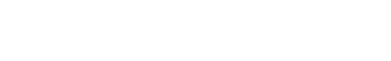

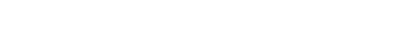

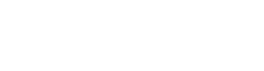

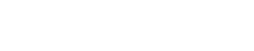

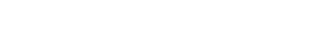

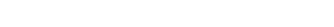

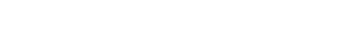

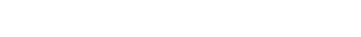

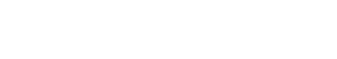



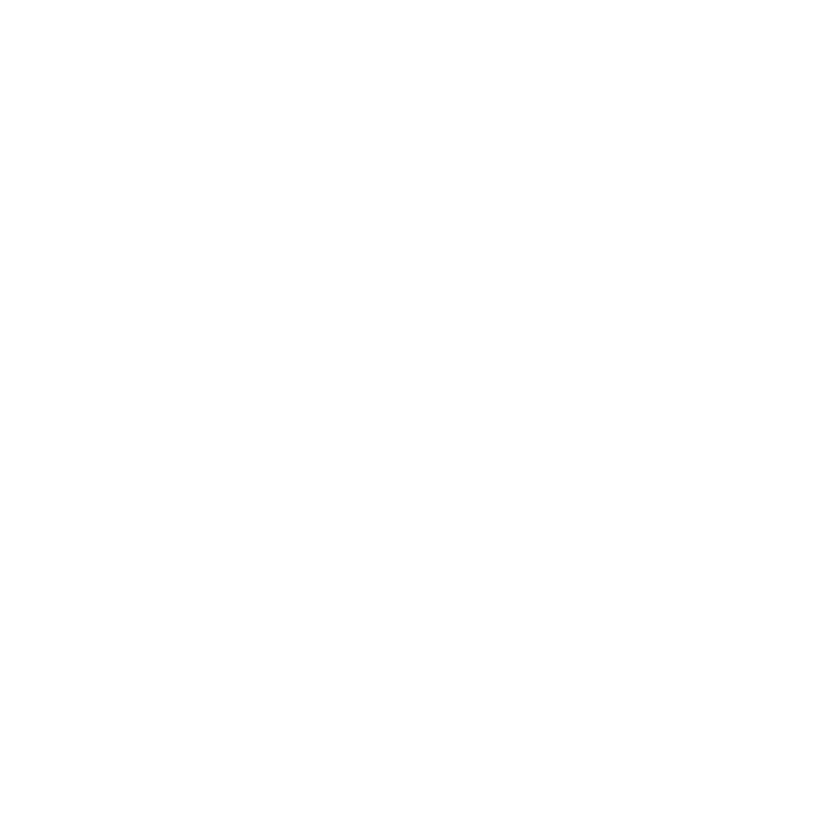

3

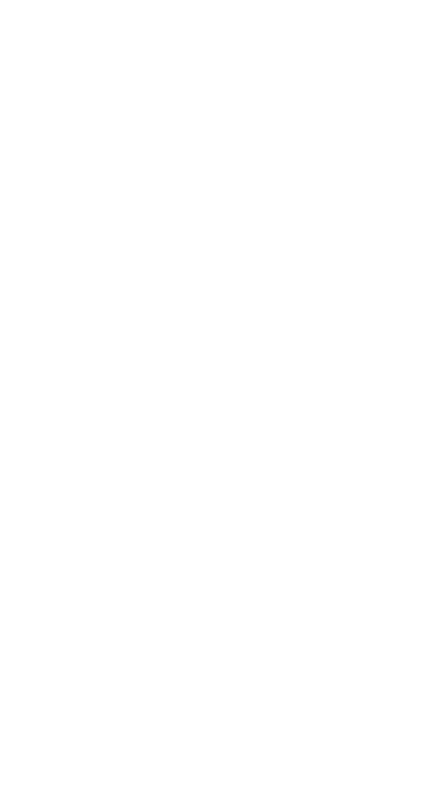

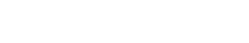

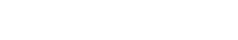

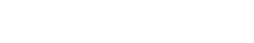

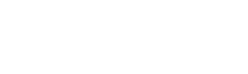

\&

w =

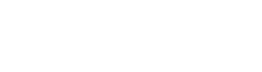

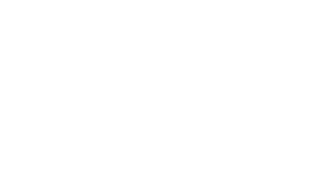

Eusencosencosen

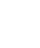

$m \infty n \infty \infty \infty 00$

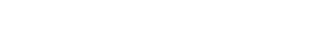

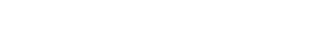
a d.uñ

$z^{z} z^{z} z^{z} z^{2}$

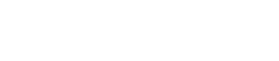

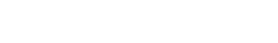

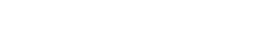

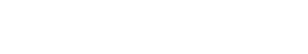
- OONENENNN

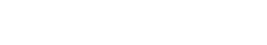

tosesenisunesen in

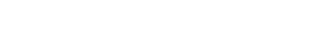
$m \sim \dot{m} \dot{\sim} \dot{\sim} \dot{\sim} \dot{\sim} \dot{\sim}$

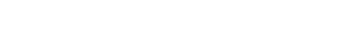
d ninn

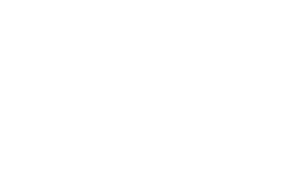




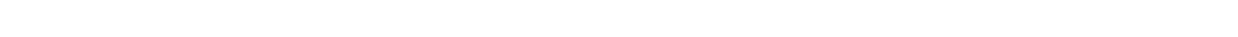

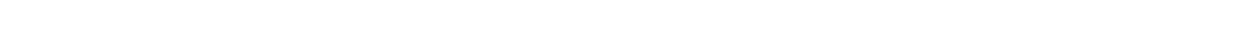
แ

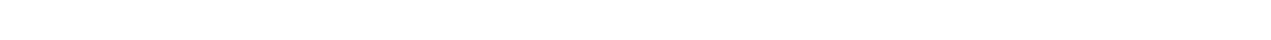

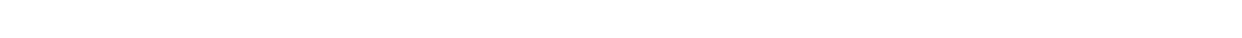

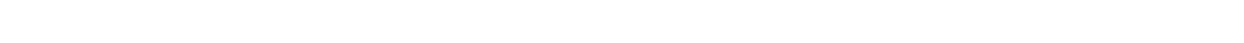
is is
n
is
is
$\sin \sin \theta$
in

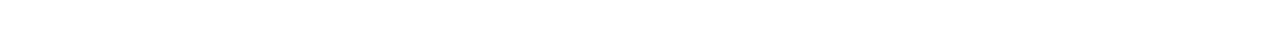

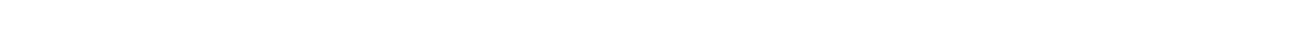

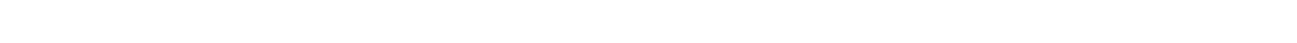

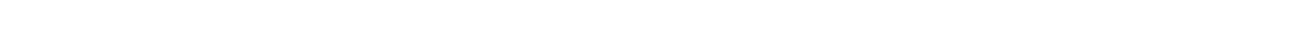

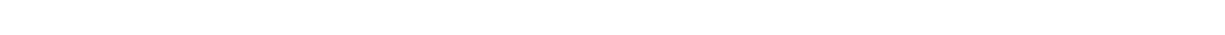

$\stackrel{0}{\circ}$

I

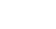
I is

is

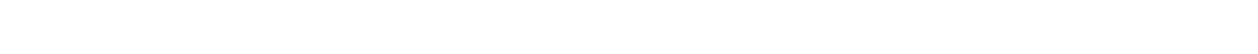

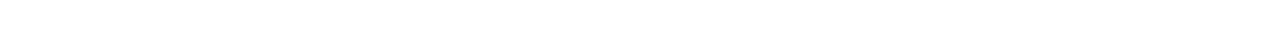

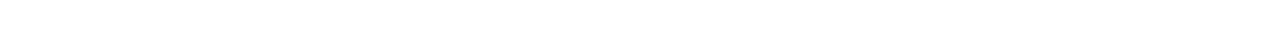

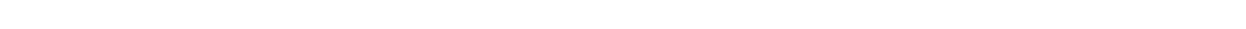

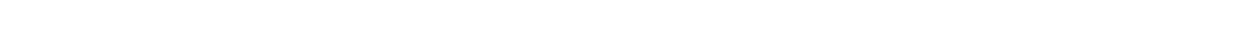

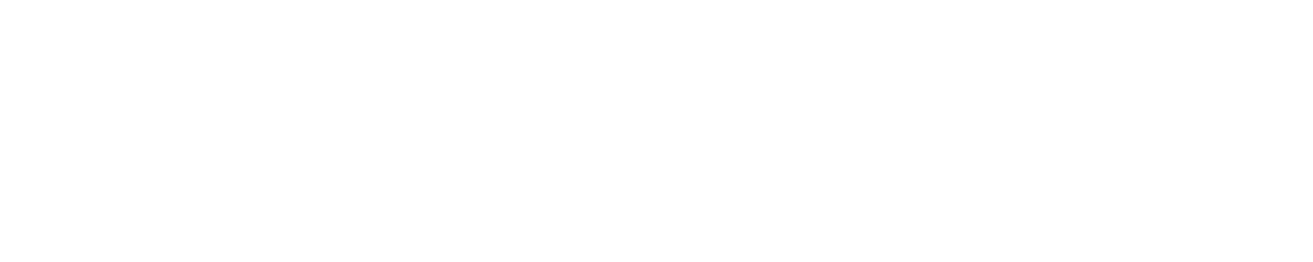

$$
5
$$$$
\text { twen isenes }
$$

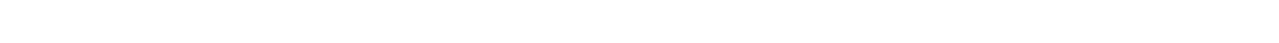

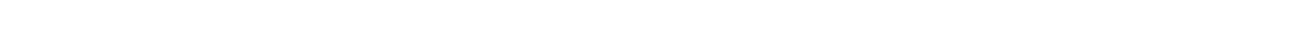
西

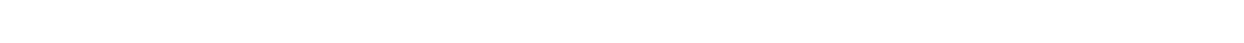

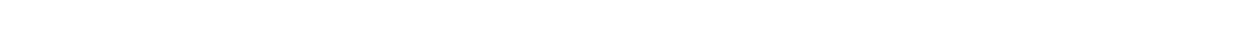

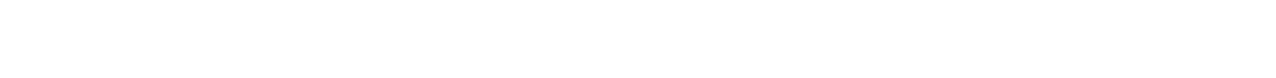

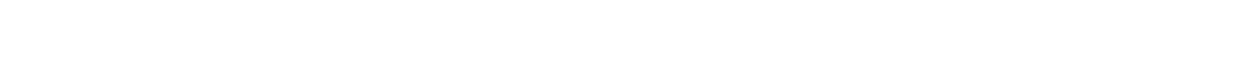

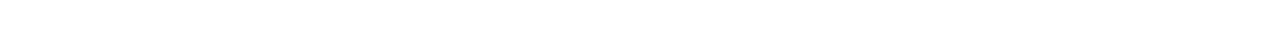

is

O a

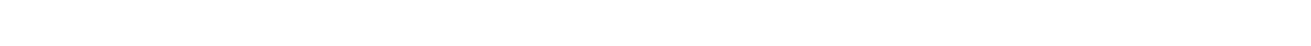
व

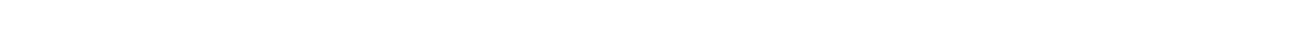

$\vdash \ddot{u}-$

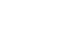

0

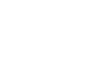

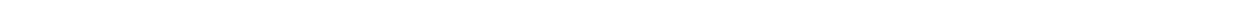

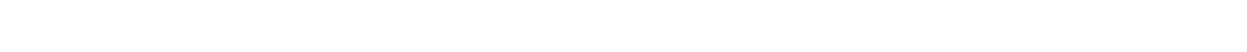

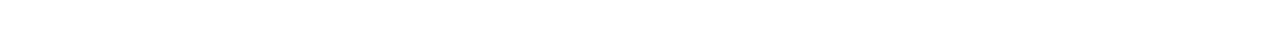

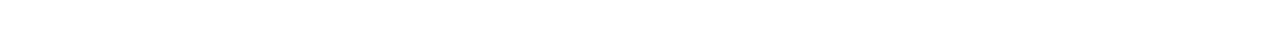

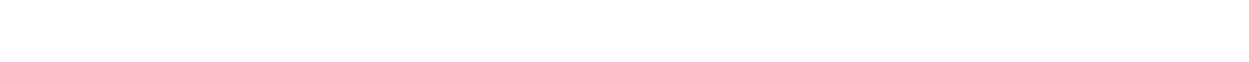




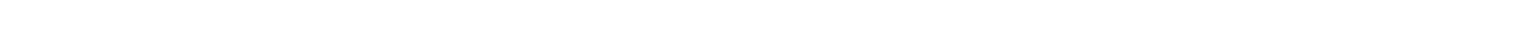

in

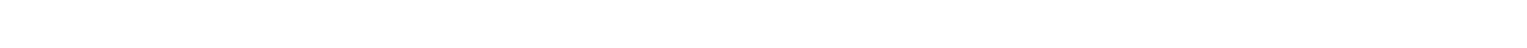

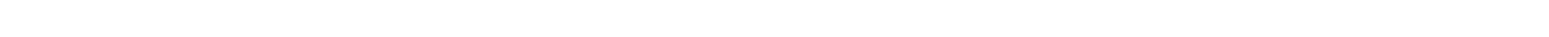

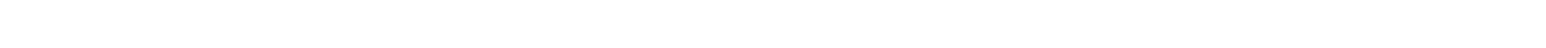

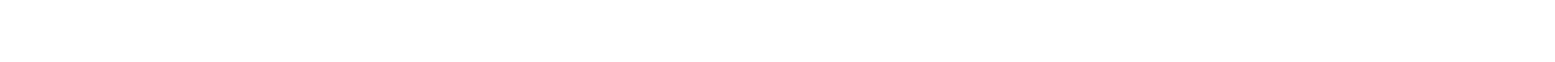

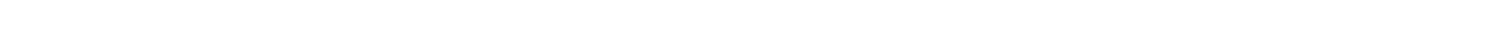

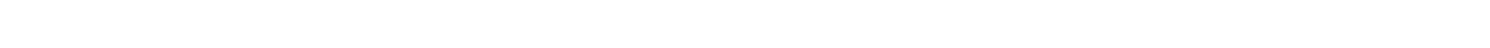

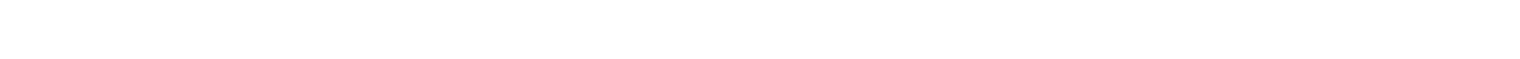

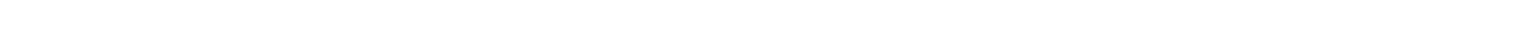

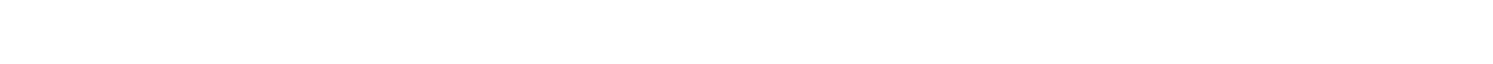

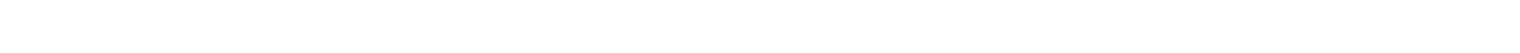

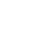

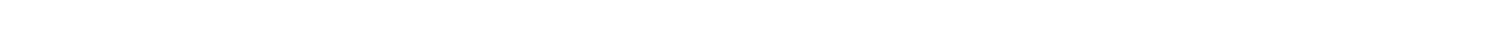

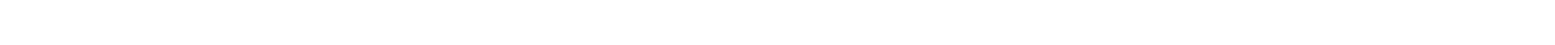

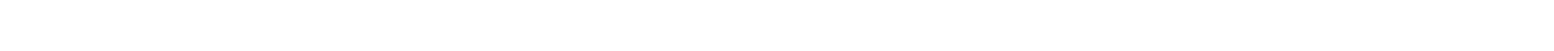

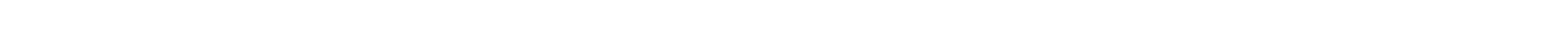

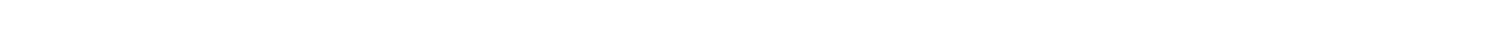

곤

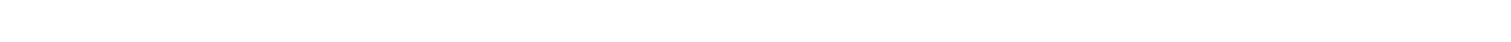
แ

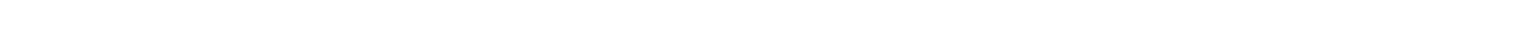

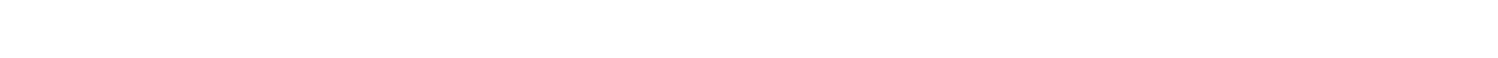

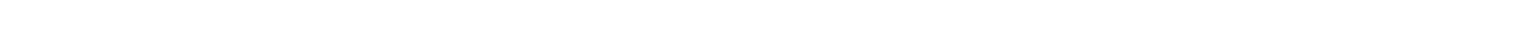

$+$

in

mo \%

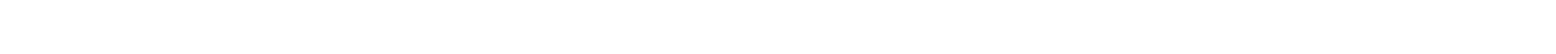

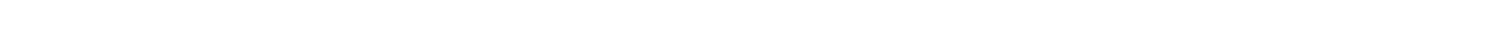

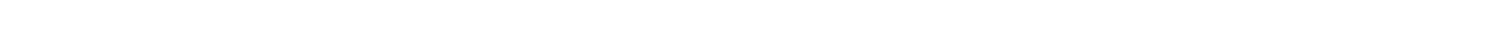

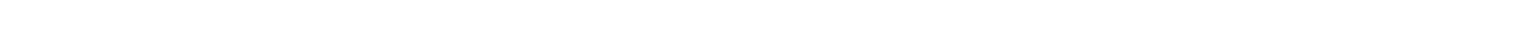
$\ln 00 \ln 20$

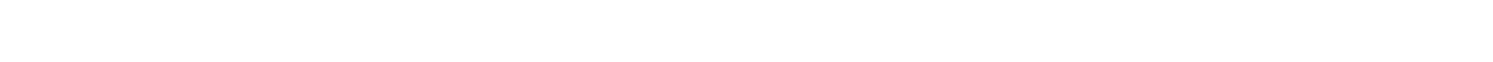

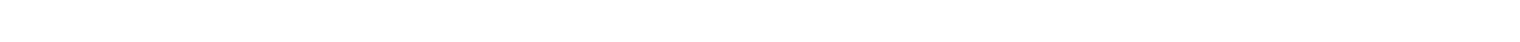
$\Sigma$

$\pm$

น ú

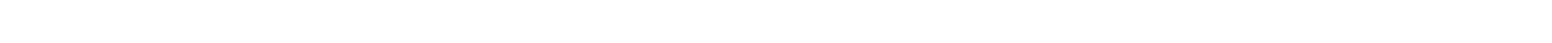
乐

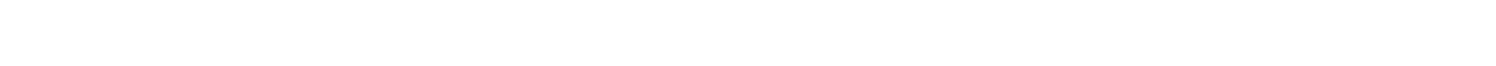

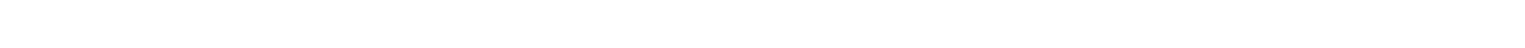

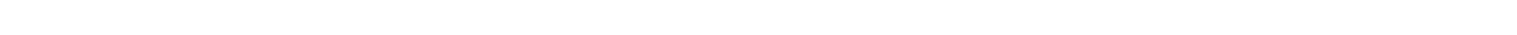

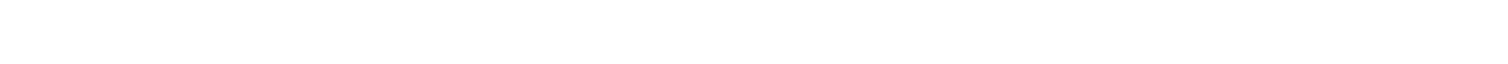




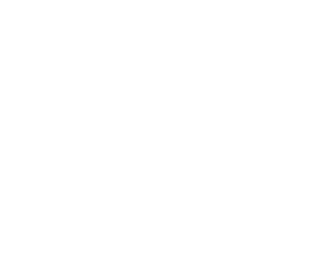

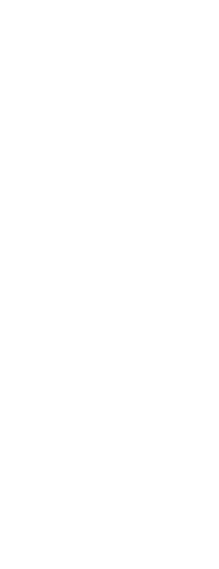

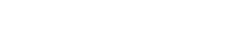

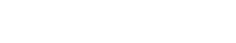
แララララニニニラ

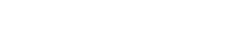
옹으능ํำ

toncosonosos

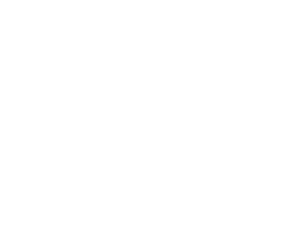

뜬

$-50$

5.

品。

aㄴ

马는

更

00

我立这

ziñ

$\propto \sim 0 \Omega$

$\sim \infty$

w

iั0.

$z$ 에

m

널

」过

$x 0 \leqslant n$

แล口乞

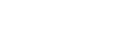

xm.s

< :

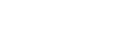

$5 \div \infty$ o

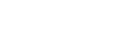

s.

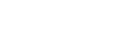

政品

isa?

ว언 w

员范

$\sum$ ut w w

a a

田年

Jowa a z

w $z^{2}$

$\alpha \underset{0}{\infty}$

今心

w

임

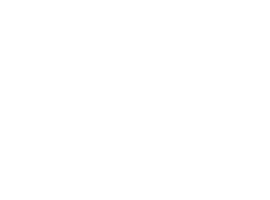

tosesencosenes

is

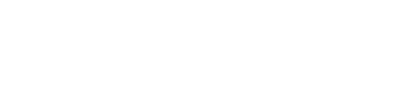

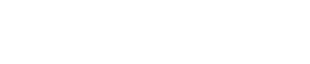

ii $\quad$ on

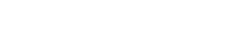

แேニニニニニニニ

0000nNnNN

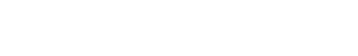

ก̃z

$=1$

กั้

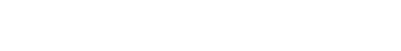

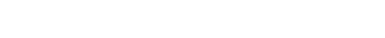

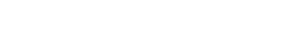

$\ddot{m}$

dimmmmmm

ก $\sim \sim \sim \operatorname{mon} \alpha$

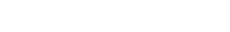

และ์

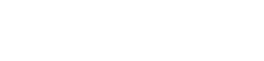

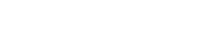


정

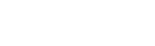

过出娄

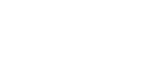
ㅇํ은 a 山oa 잉

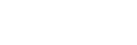
áá山吅 ะ0! 눙워 心 $z$ 一次告

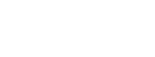

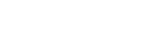
웅응 而议出

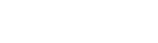
14a政 wo,

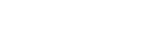
o 2 ? 은원 울 孞 5

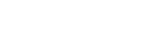
żaㄴ 준 $0^{\text {s. }} \div \leq$ 世n 0 : Jis

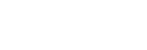

운

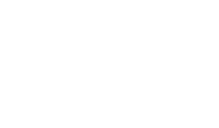

in 00

乞

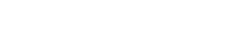

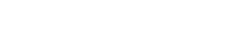

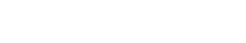

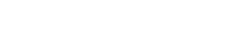

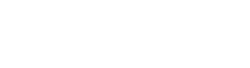

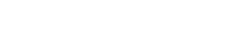

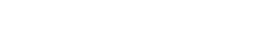
山出山出出 a mazozoz

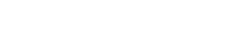
ーㅜ드눙

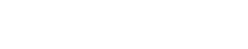
ta 0 a $\leftarrow \leftarrow$ $\ddot{\mu}-\ddot{w} \sim \ddot{w} m \ddot{\omega}+$ z七.上告。 a z z z z z z

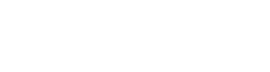
:

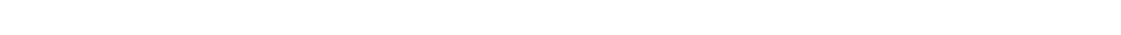
is

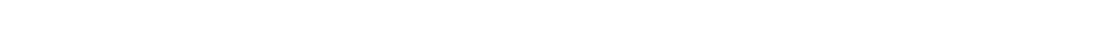

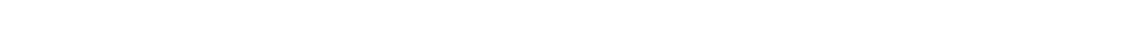
แóó

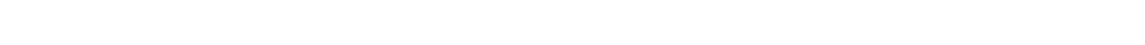

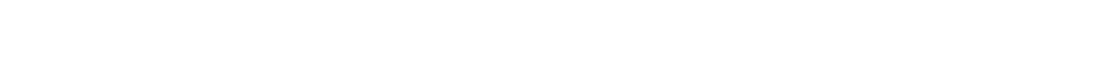

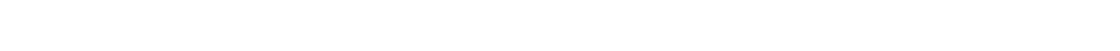

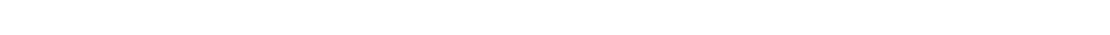

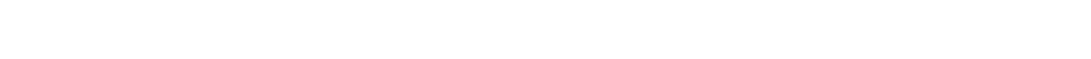

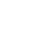

\&

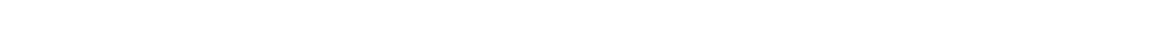

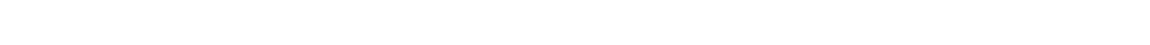

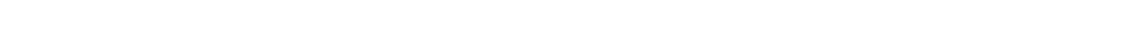

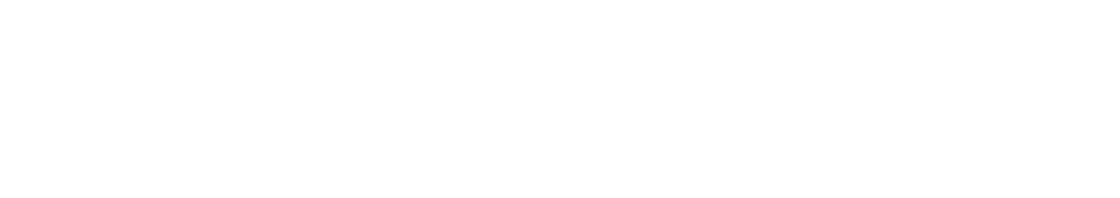

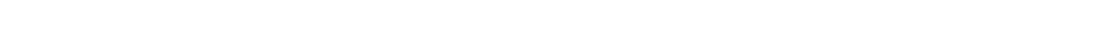

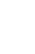

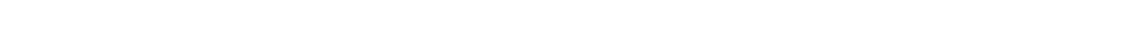

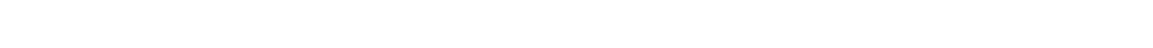

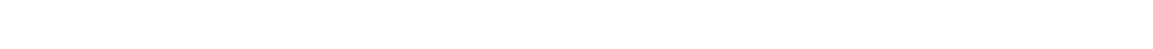

$0000005-5-5-55 \sim m$ tง

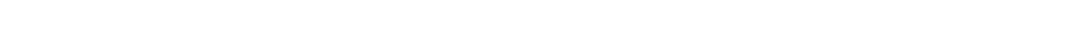

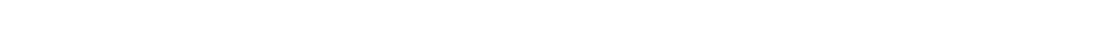

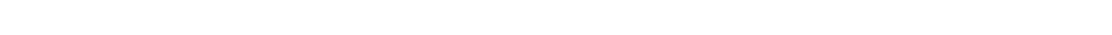
OQEニЕ

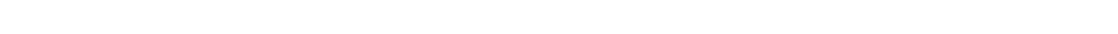
足

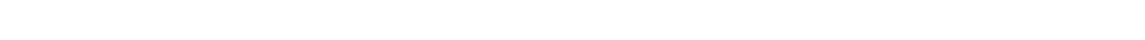
ш ú

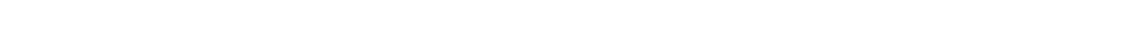

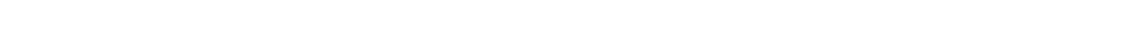

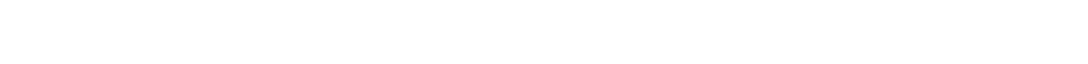

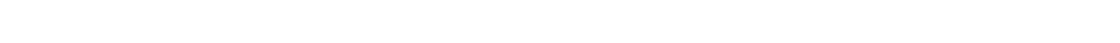

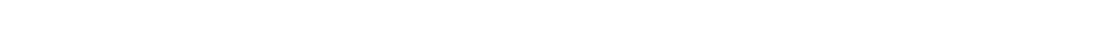

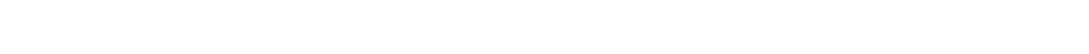




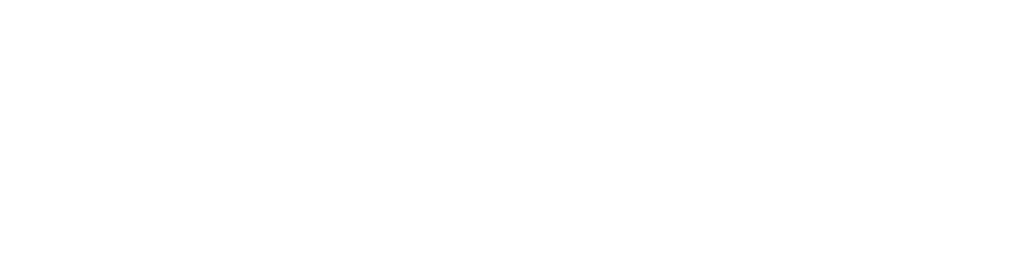

OD. or

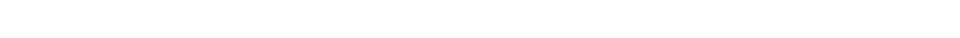

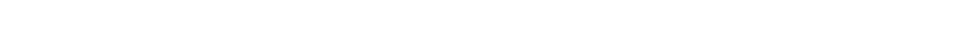

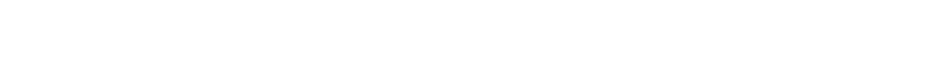

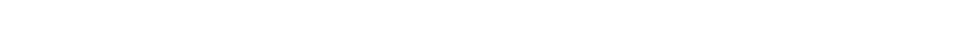

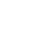

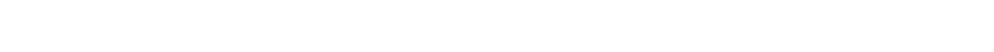

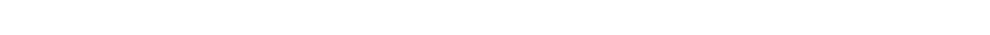

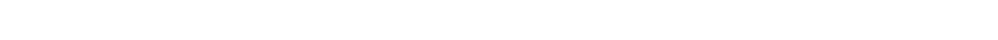

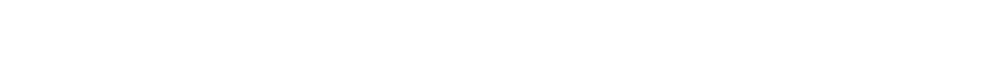

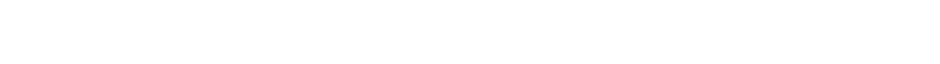

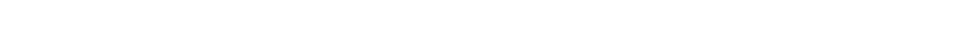

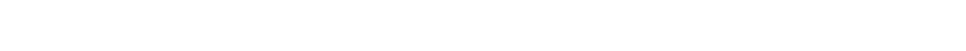

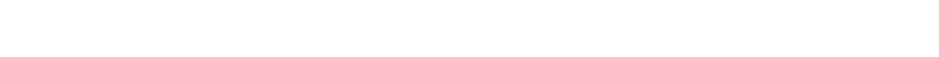

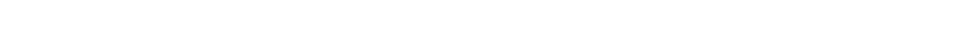

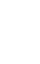

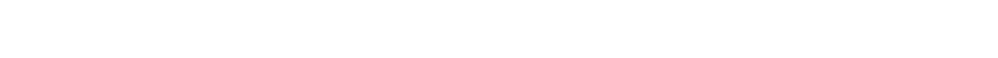
L

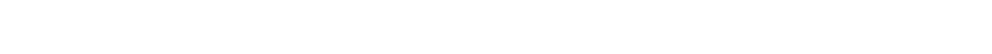

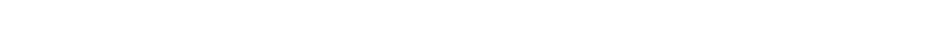

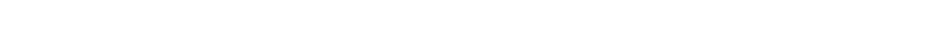

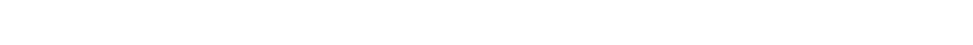
$\sin \pi \sim \sin$

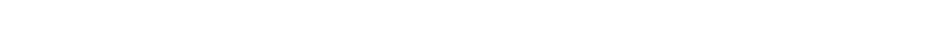

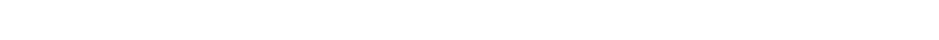

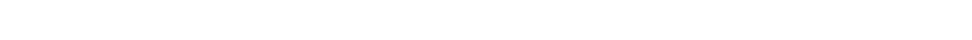

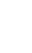

"mนnmm-

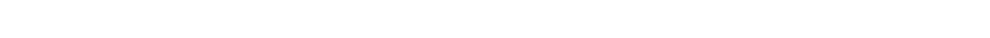

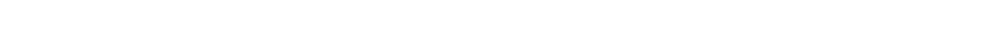

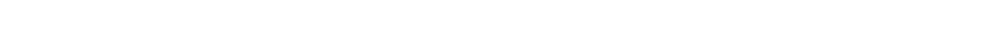

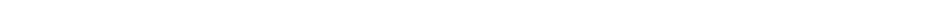

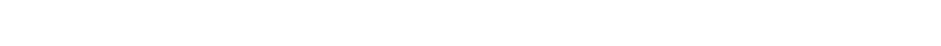

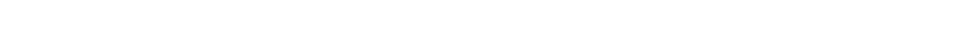
an

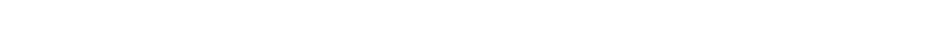

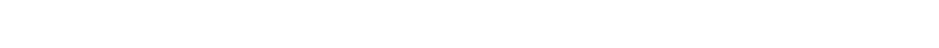




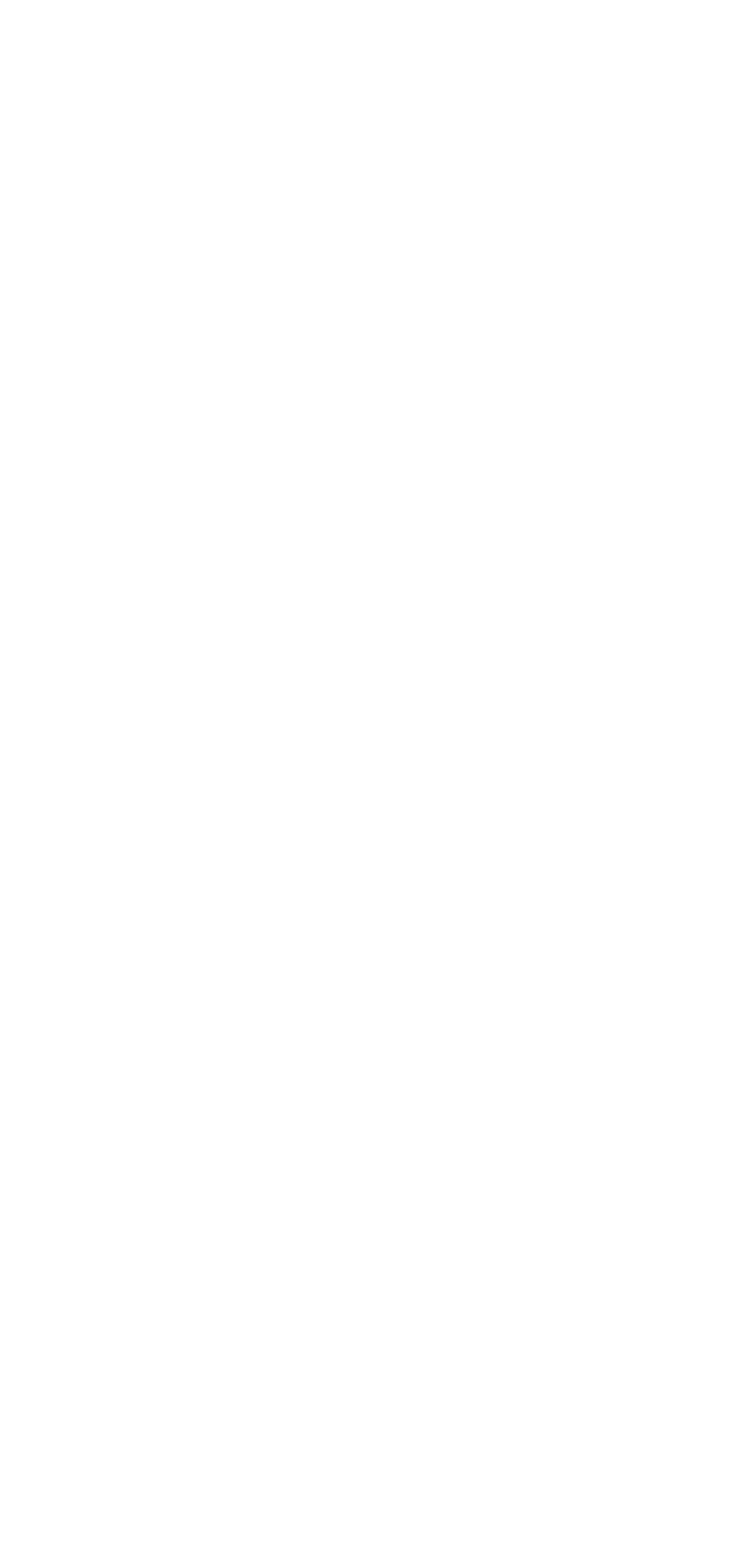




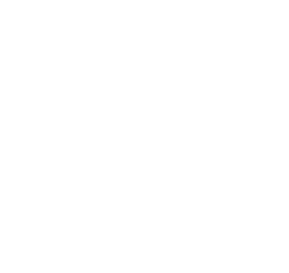

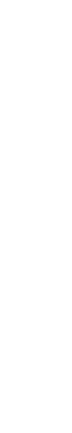

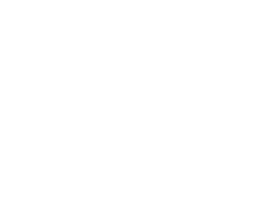

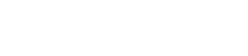

is

응

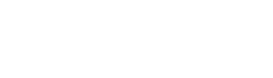

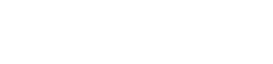

$\because$

ง

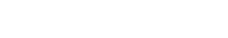

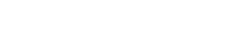
オニンーニ

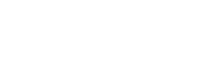

Enescosones

5

a

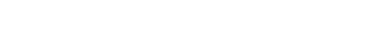

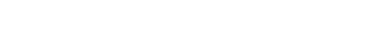

里

ï maornm DOOONANa แํํ으누은

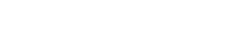

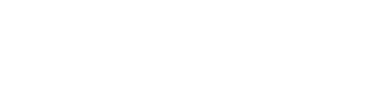

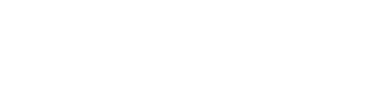

w

tosencosenos 일 究苋 E

ma ○ ïŁ世

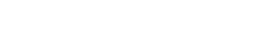
:

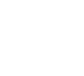
munnn 웅으으응 แニニラテニニニ

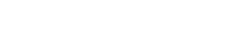

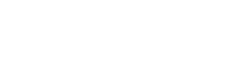




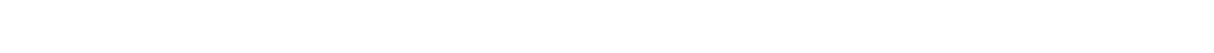

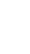

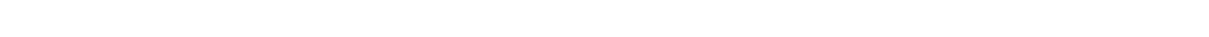
ü:

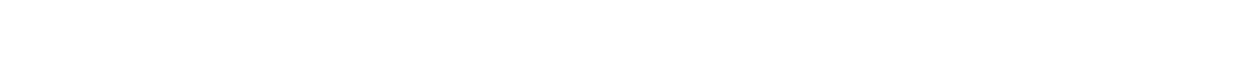

○ำก

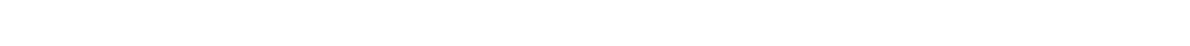

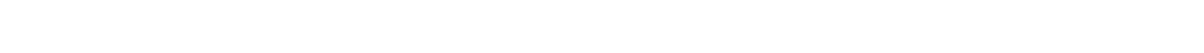

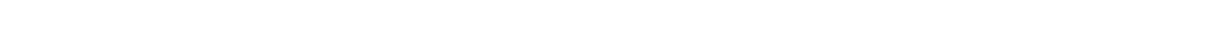

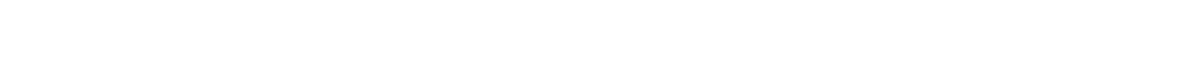

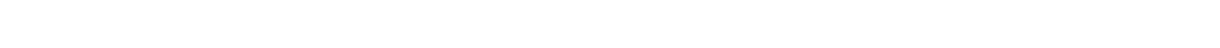
is

is

a w 《

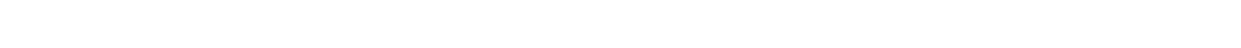

a 2 n

mmmmmmmmmmmmتี

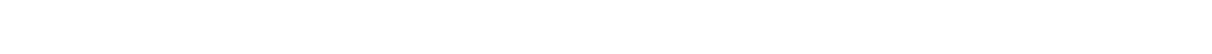

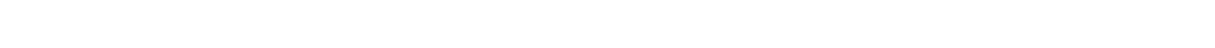

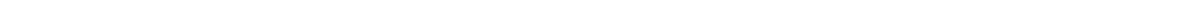

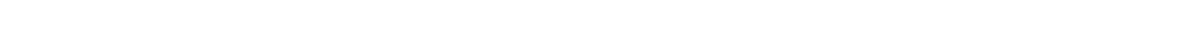

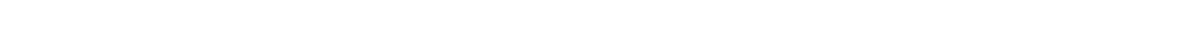

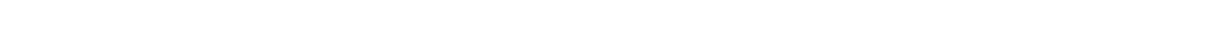
$\pm$

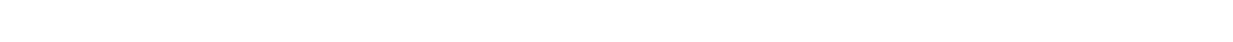
u.

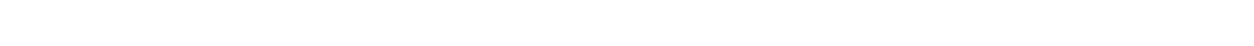

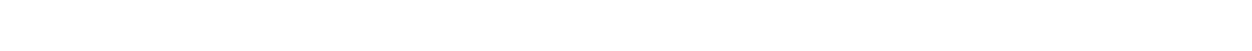

주일 .. 紊..

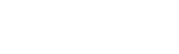
준단

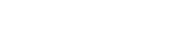

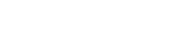

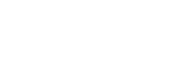
is $2 \stackrel{0}{0}$ w 0 No눙ㅇㅁ

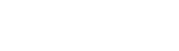
든

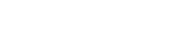

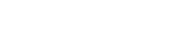
엉영ㅇㅇㅇ 亏

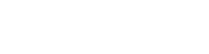

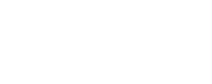

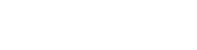
峁

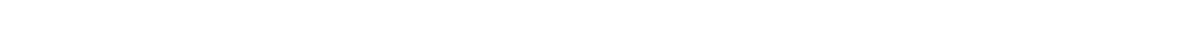

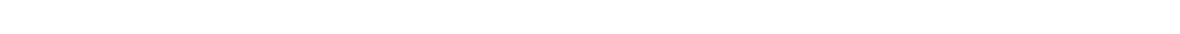

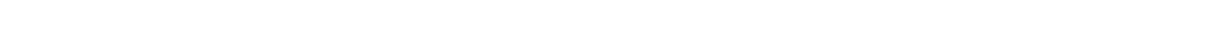

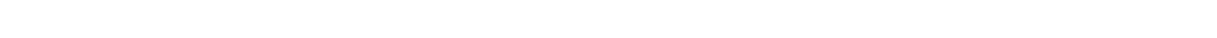

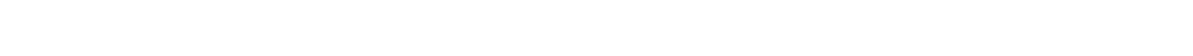

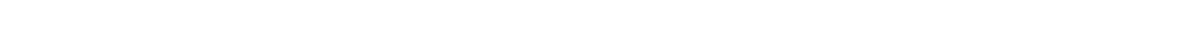

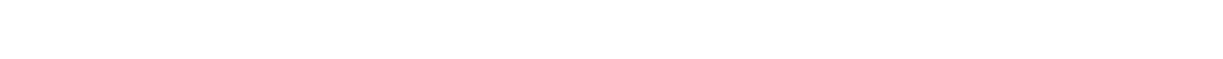

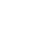

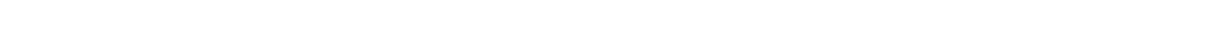

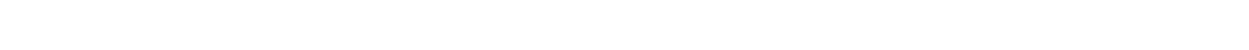

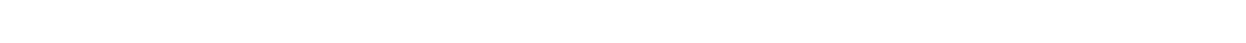

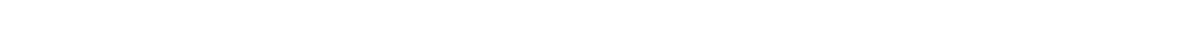

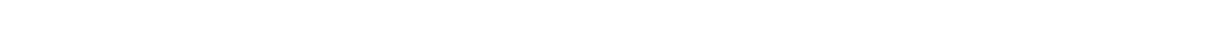

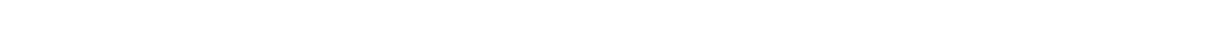

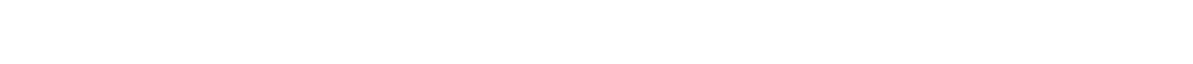




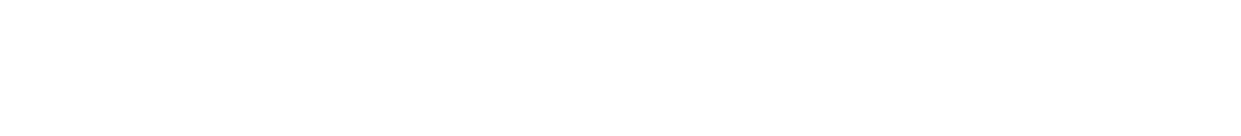

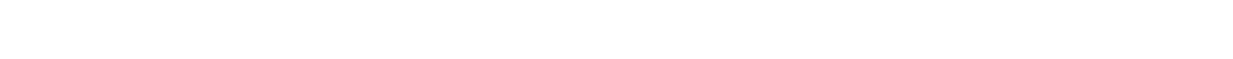

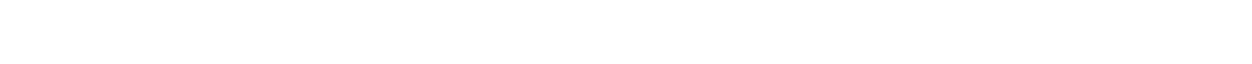

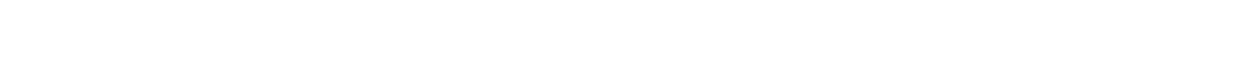

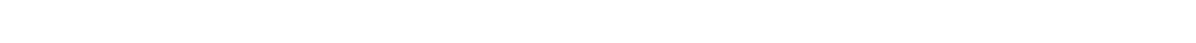

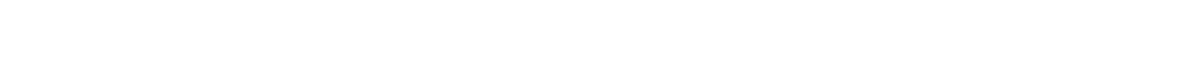

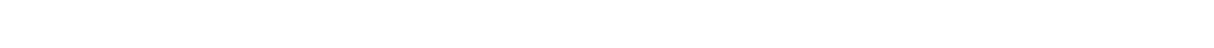

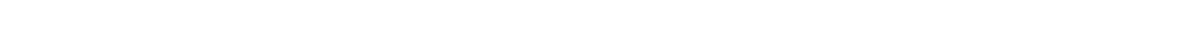

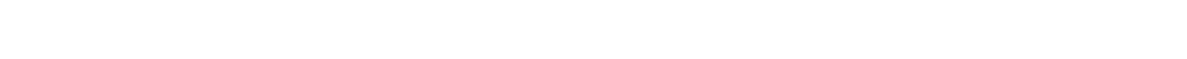

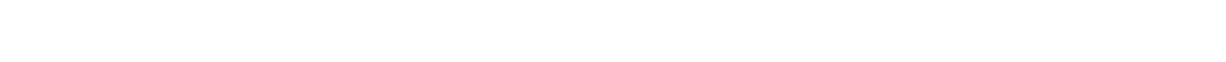
is

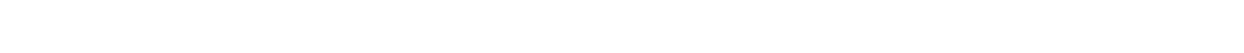
ú

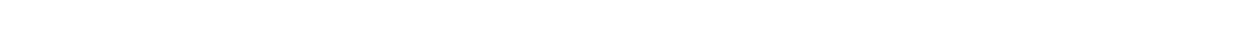

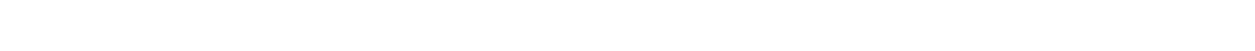

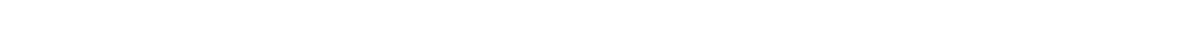

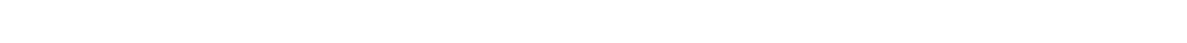

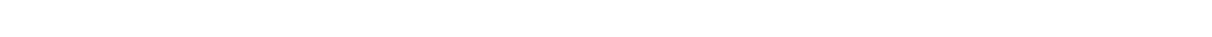

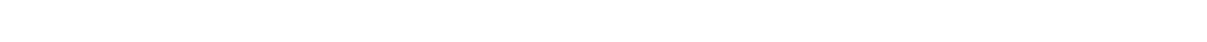

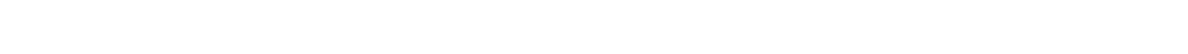

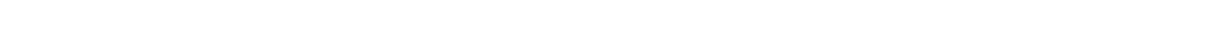

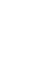

"⿻ㅡ⿱

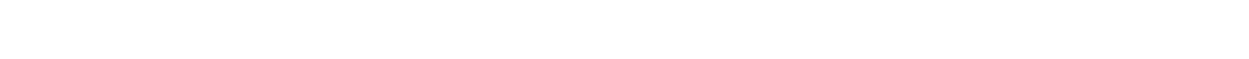

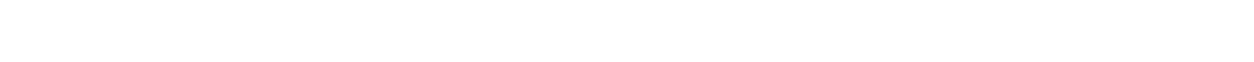

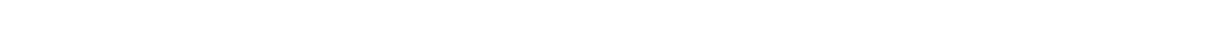
崖 $\stackrel{a}{\infty}$

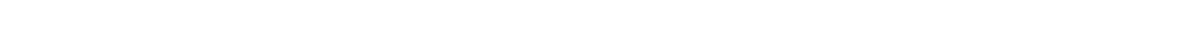

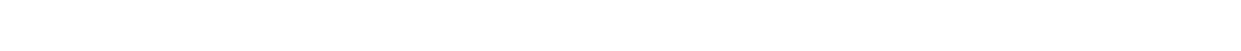

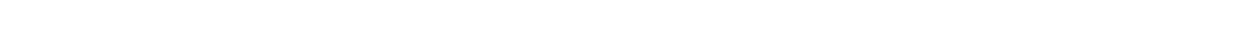

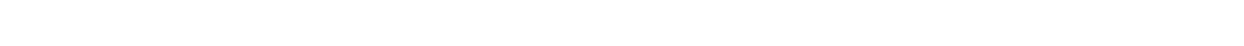

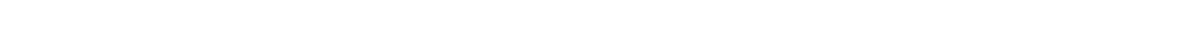

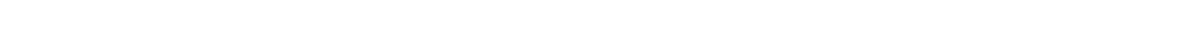

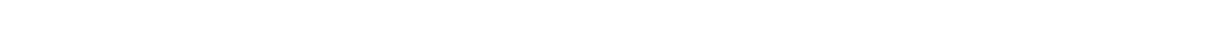

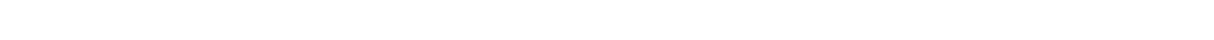

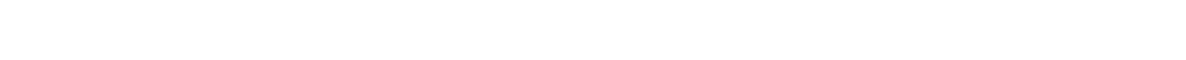




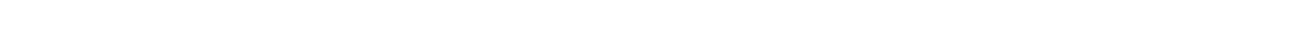
w wo

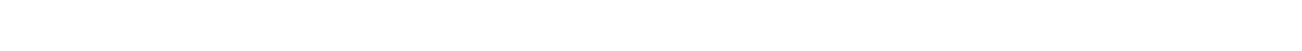

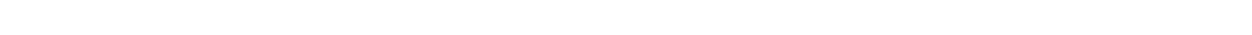
ผ幺

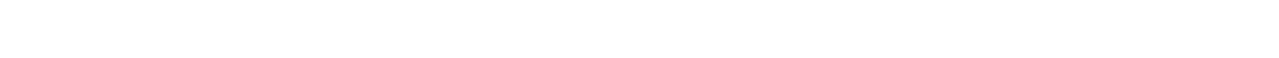

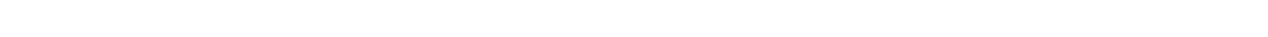

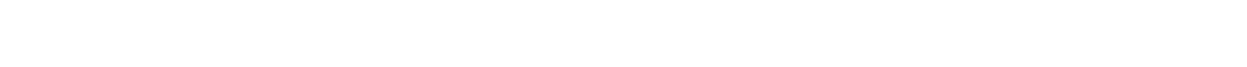

\begin{tabular}{|c|c|c|c|c|c|c|c|c|c|}
\hline $\bar{w}$ & nus & en in & $n n$ & $\sim n$ & nn & $n n$ & enes & nnes & $n$ es \\
\hline & & & & $a$ & $a$ & & a & & $a$ \\
\hline
\end{tabular}

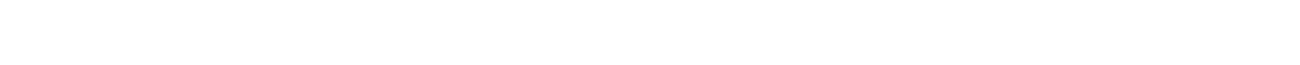

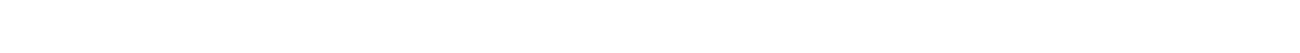

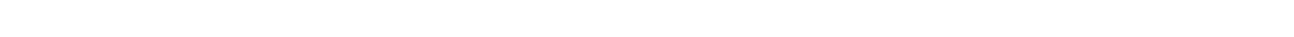

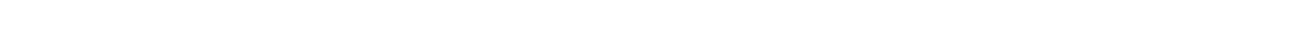

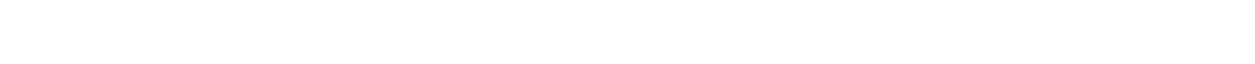

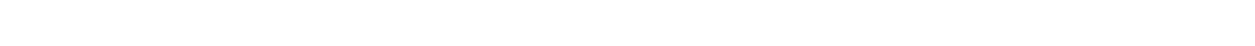

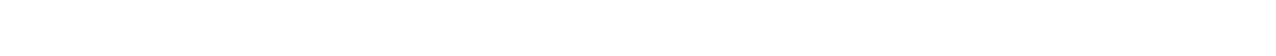

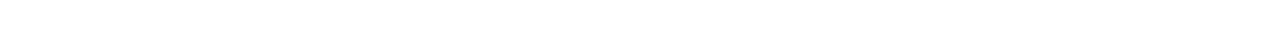

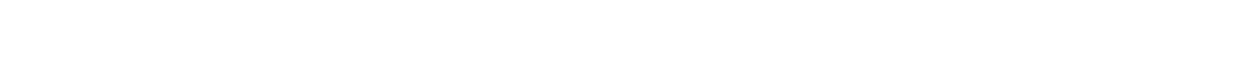

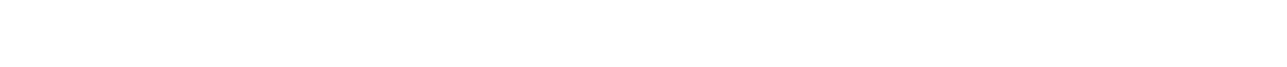
ia
a a
a a
$a$ a
a. a.
a a
a a
a a

๔ แ с

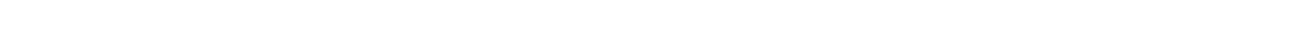

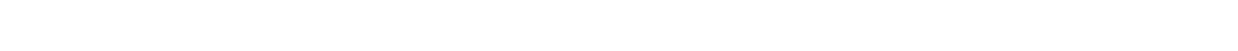

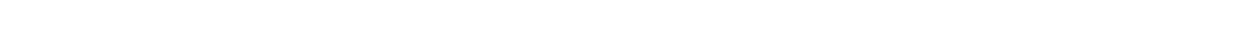

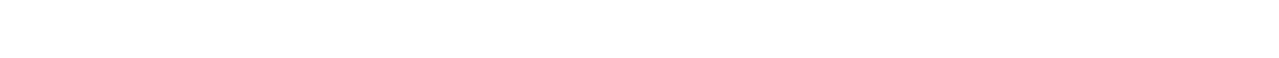

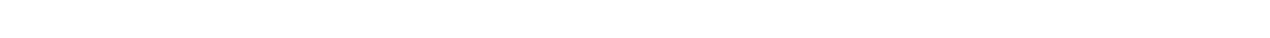

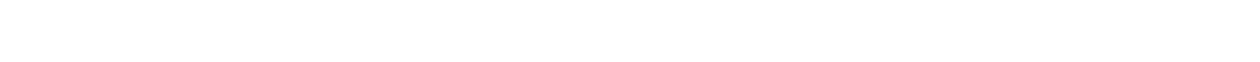
立
nos
on es
$n$
in $n$
is os
ins
$\cos n$
in
en
5
a a
a. a
a a $a$
×

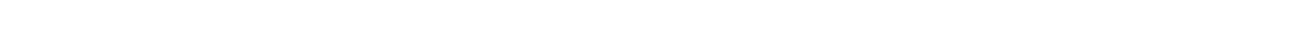

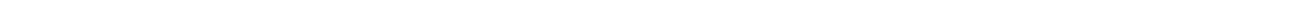

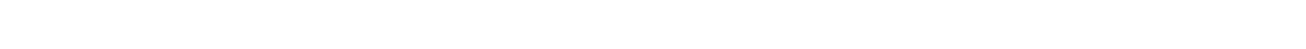

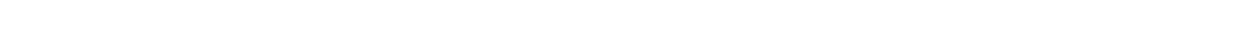

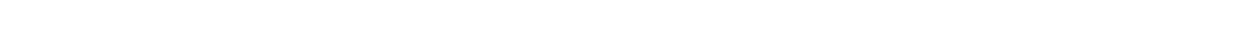

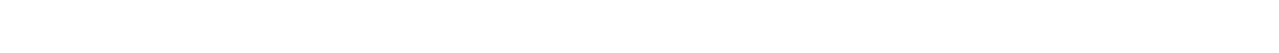

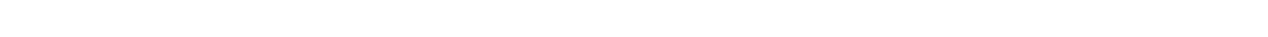

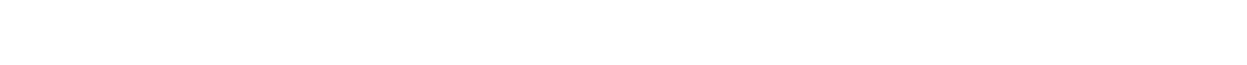




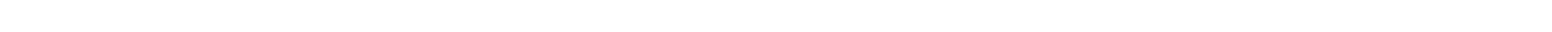

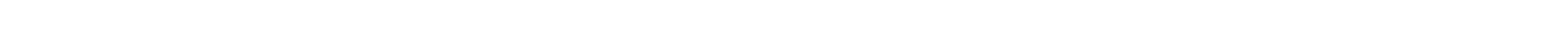

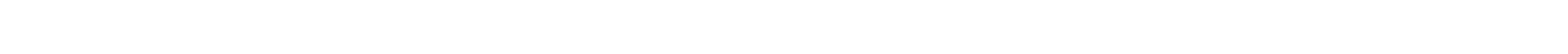

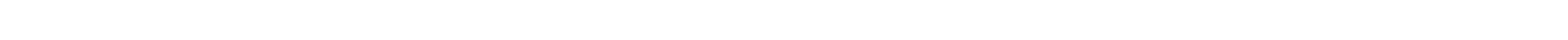

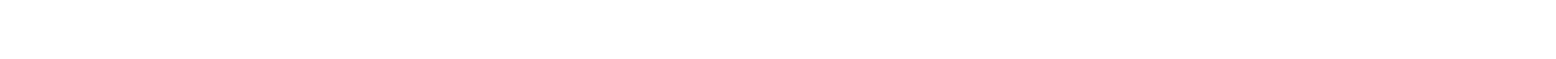

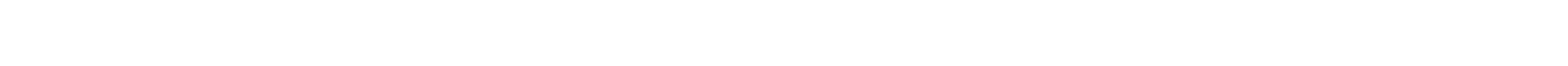
5

a a

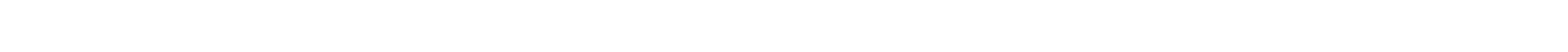

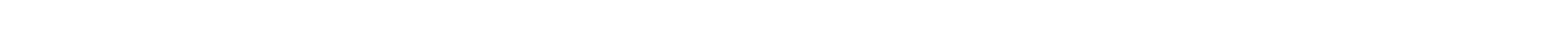
$x_{0}$

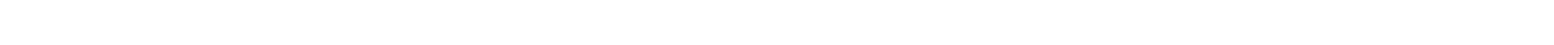

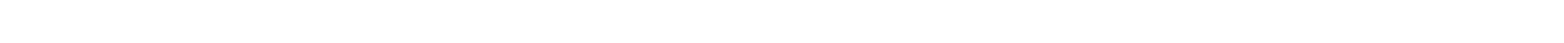

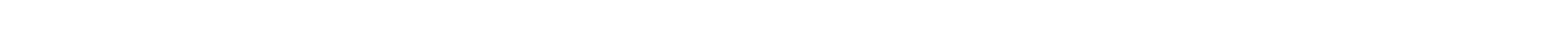

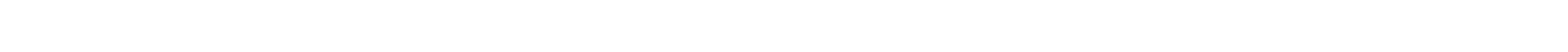

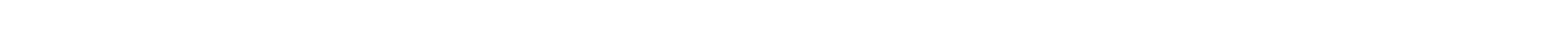

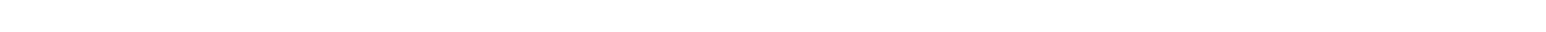

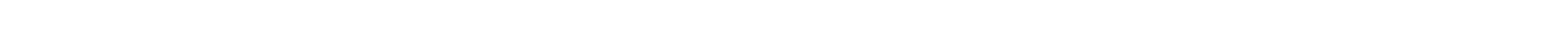
in

a. a.

a.

a.

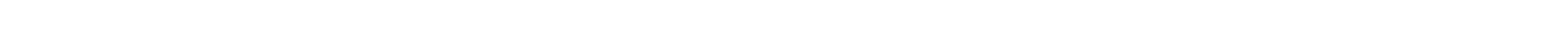
« L

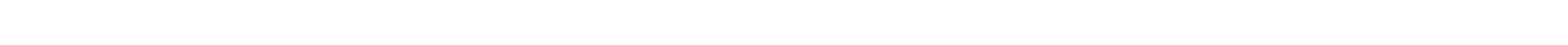

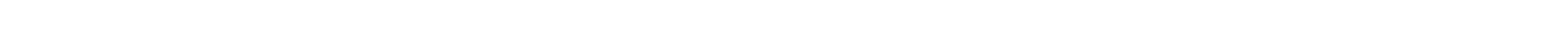

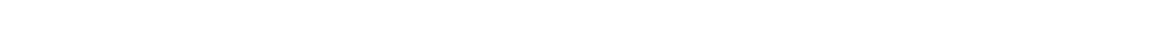

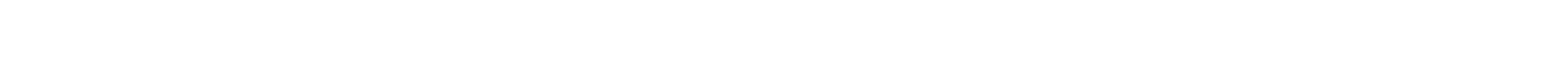

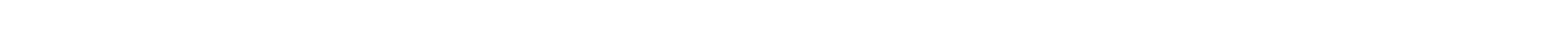

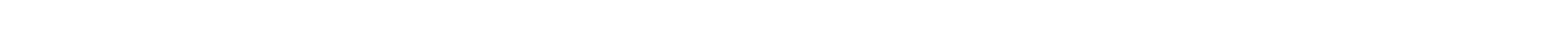

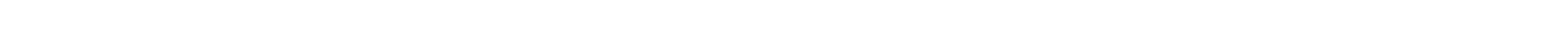
5

a. a

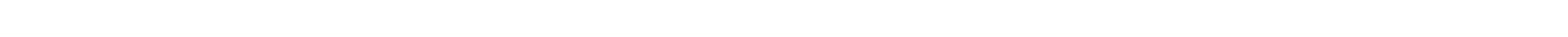
แ \begin{tabular}{cc}
3 \\
\hline
\end{tabular}

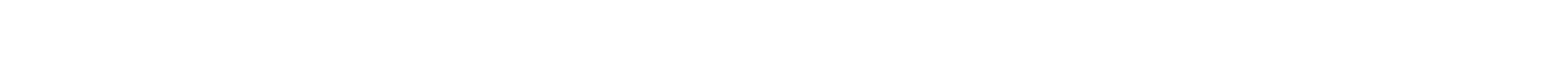

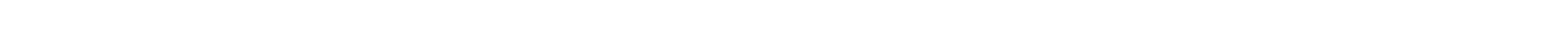

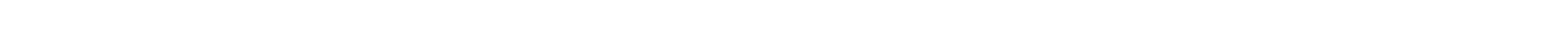

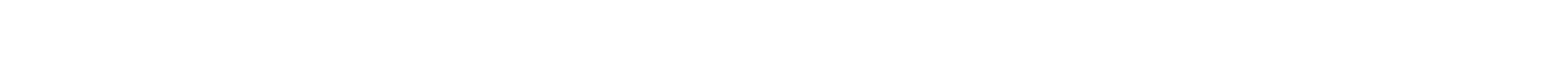



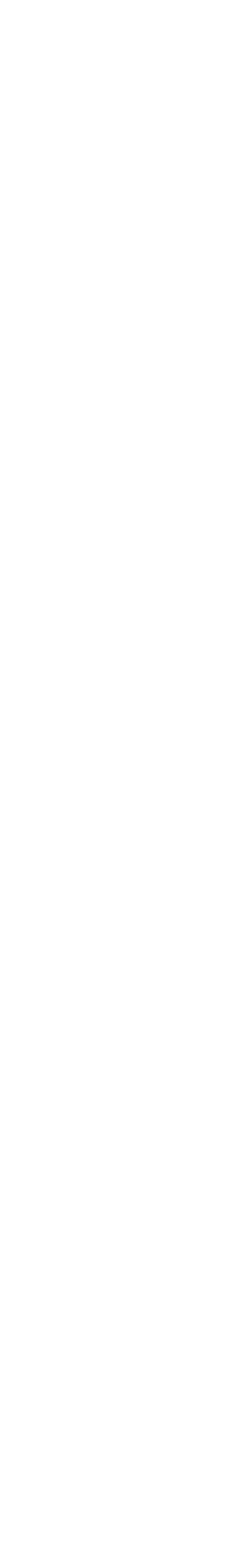


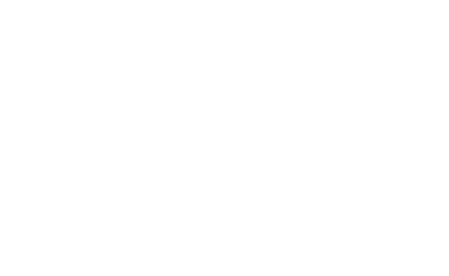

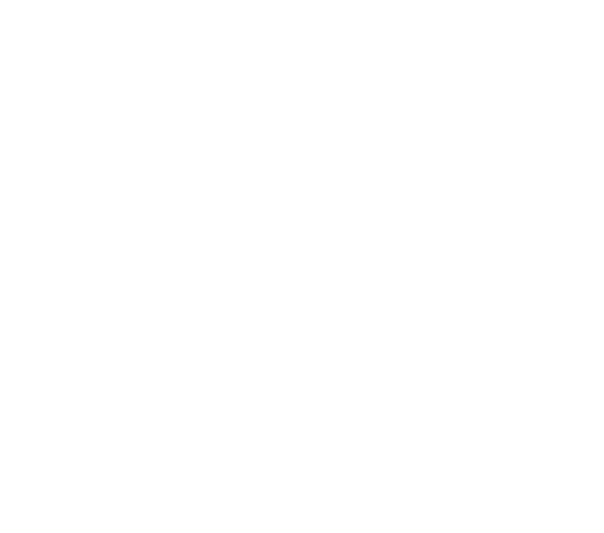

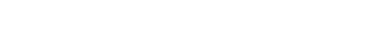

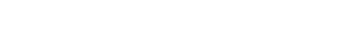

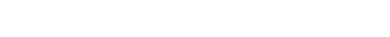

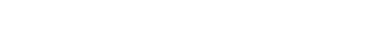

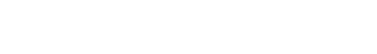

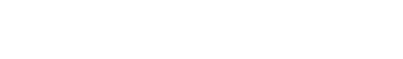
5 × NัO์

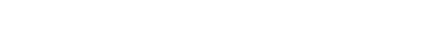

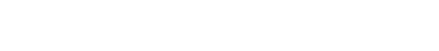

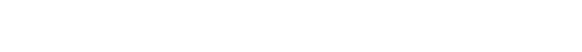

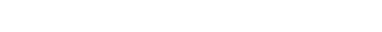

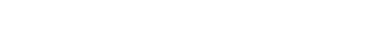

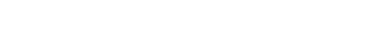

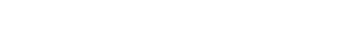

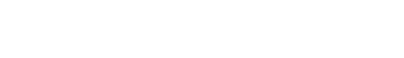
is

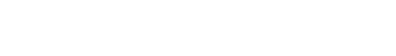

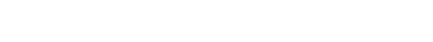

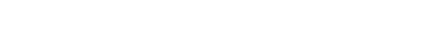

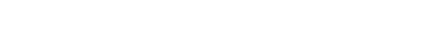

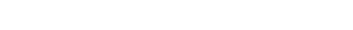

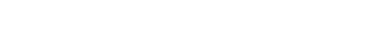

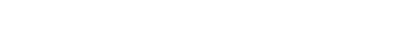

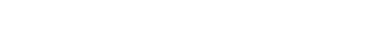

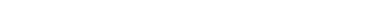

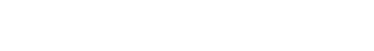



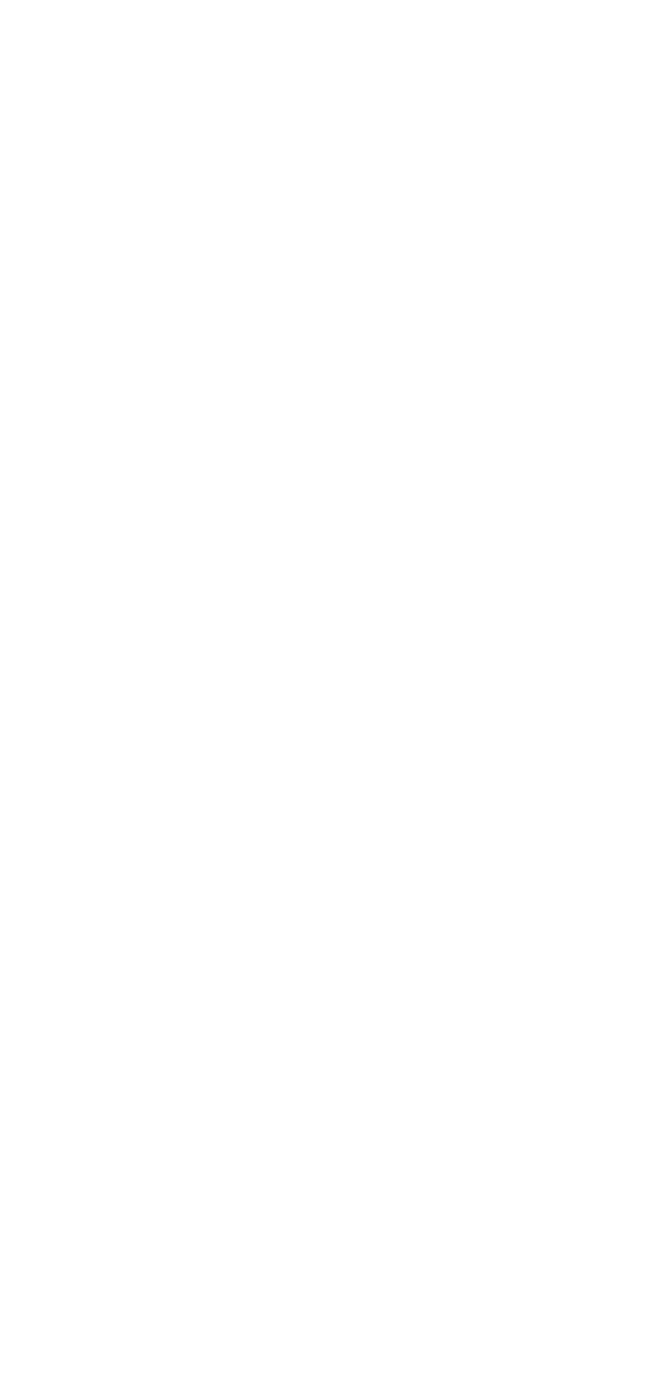

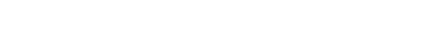
人ู

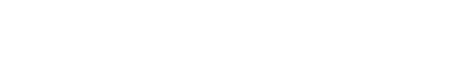

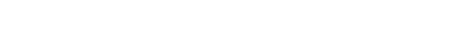

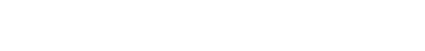

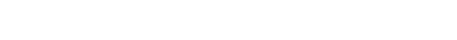

兵

กำm

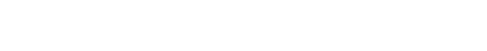

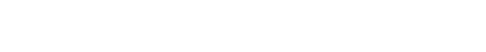

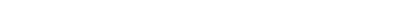
Domána

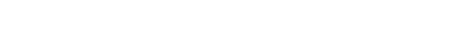

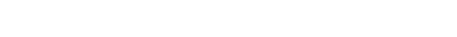

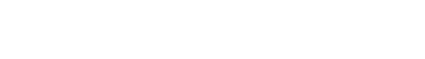




$$
\begin{aligned}
& \text { o } \\
& 0 \\
& \ddot{\Xi}
\end{aligned}
$$$$
\text { 용무 }
$$

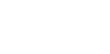$$
\text { a }
$$$$
\text { ... } \vec{a} \text {... }
$$$$
\text { 莺范 }
$$$$
\text { 劼岕。 }
$$$$
\text { 岁 }
$$$$
\text { is }
$$

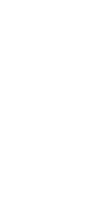

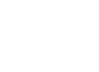

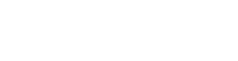

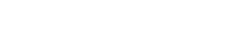

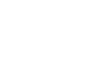

w

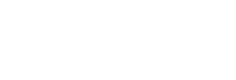

응 功出出出 的品品上的

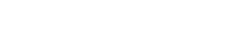

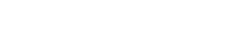

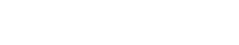

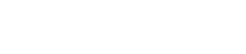

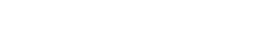

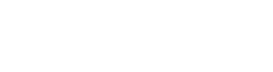
a zazazaza

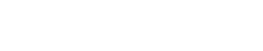

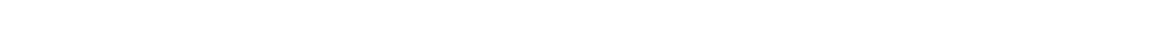

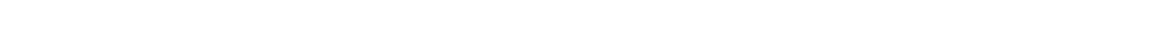
к

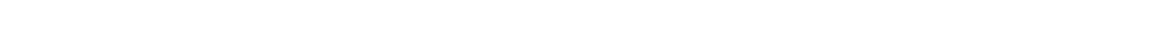

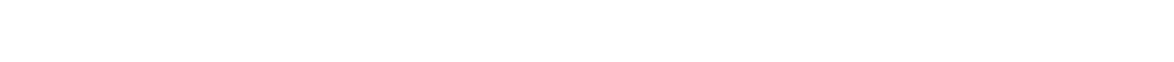
mn w

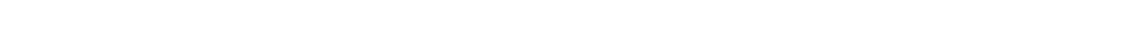

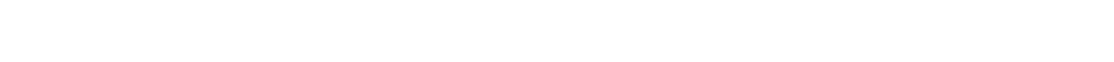

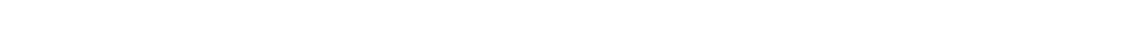
$\underset{n}{\infty}$

a a a in in

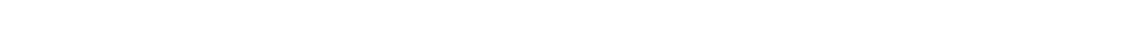
u山

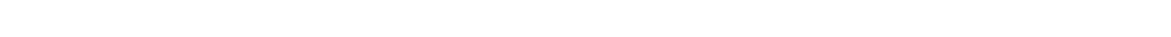

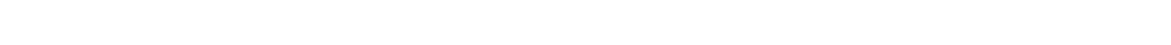

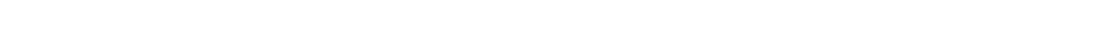

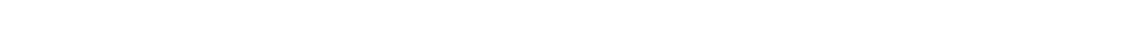

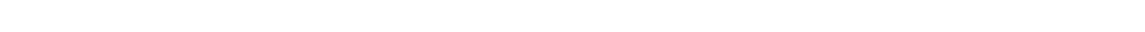

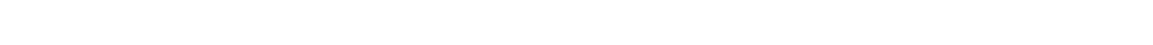

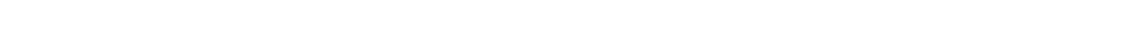

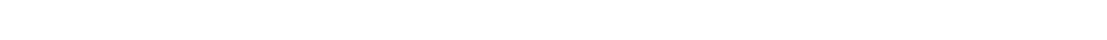

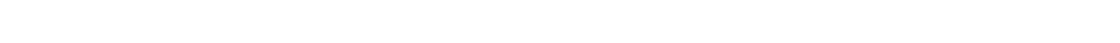

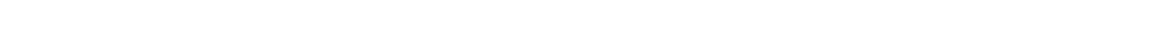

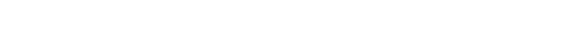

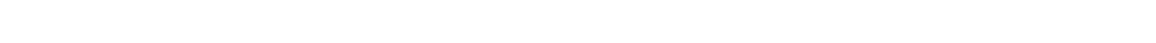
w

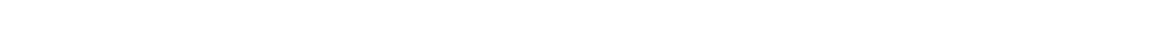

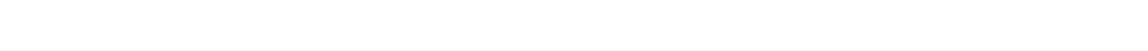

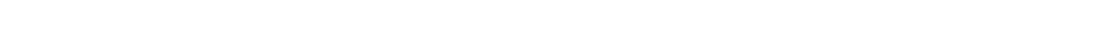

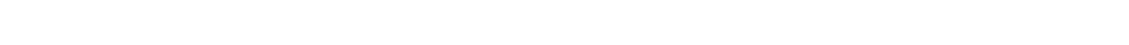
w

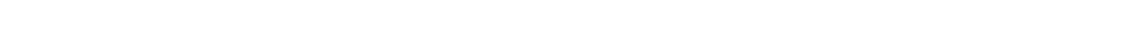
Y

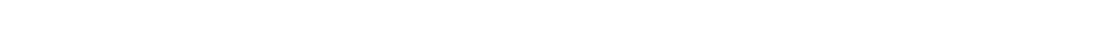

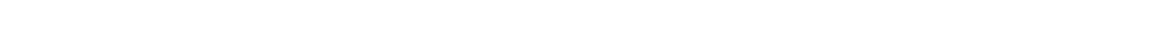
$\alpha$

- a a a a abaababaa aㅠmำ

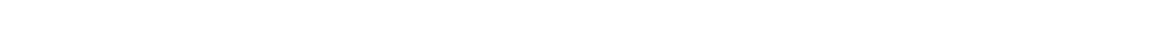

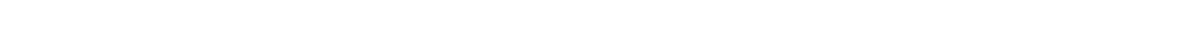

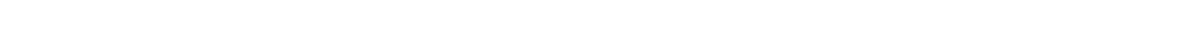

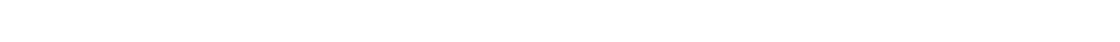
ăo

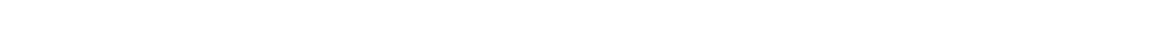

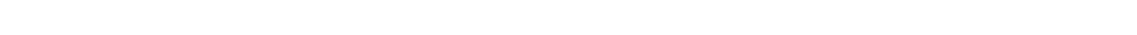

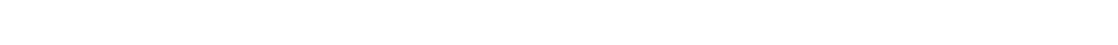

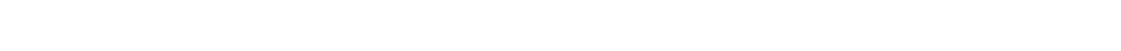




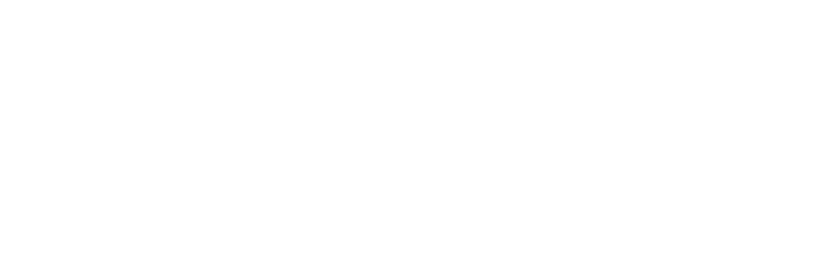

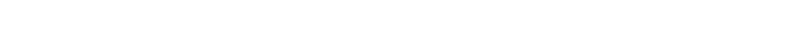

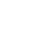

in
isaca
a a is
$a$

in

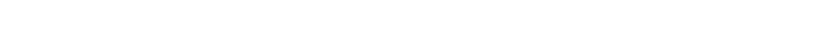
ш

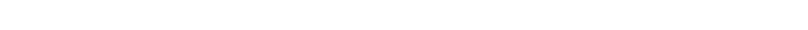

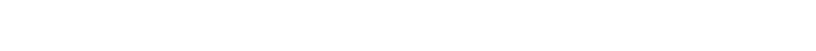

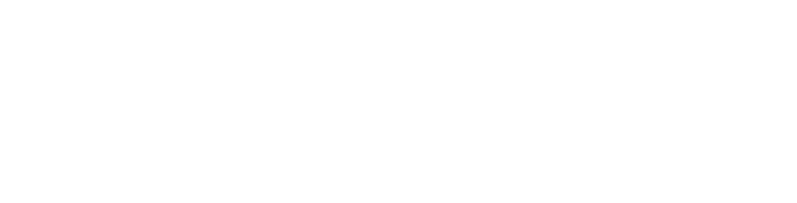

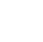

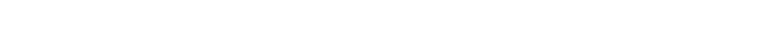

a

a a in is

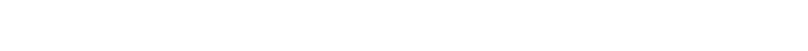

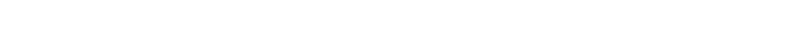

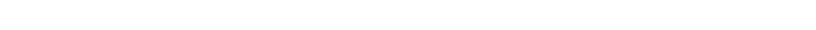

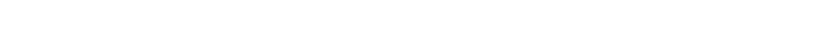

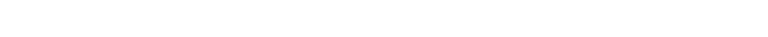

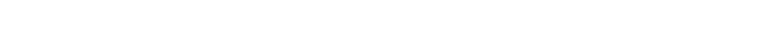

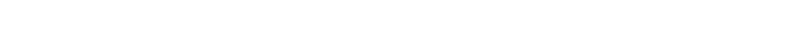

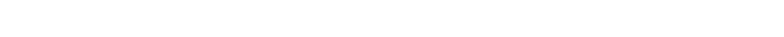

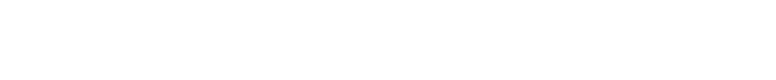

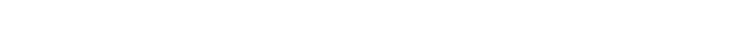

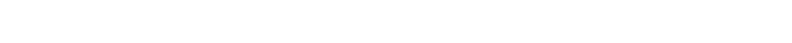

ia

ain is os

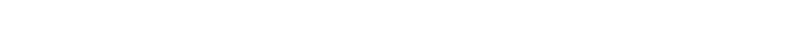
व m $>$ ن \& 1000000000 in 000000000000000

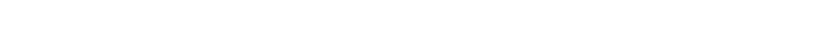

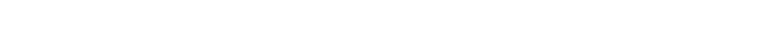

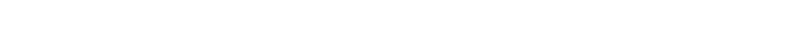

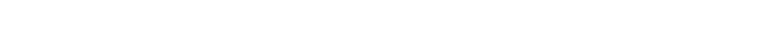

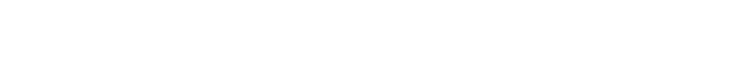




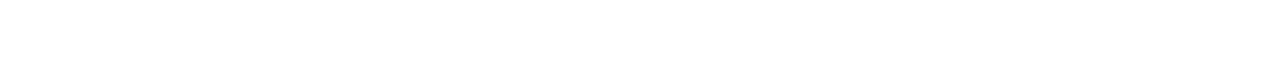
in in a a a b a a a a a a a

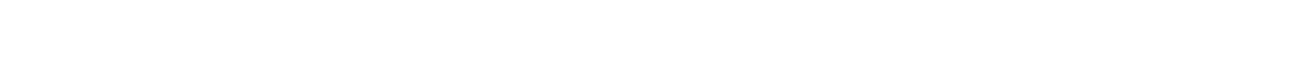
w

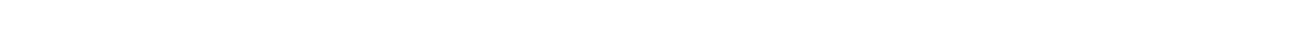

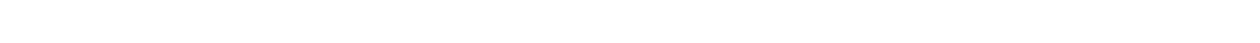

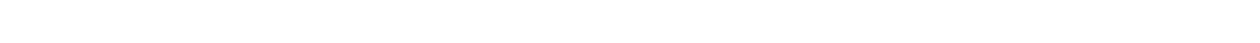

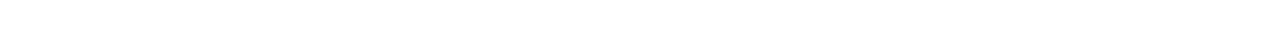

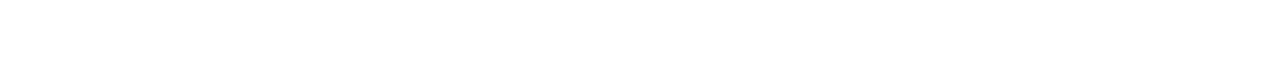

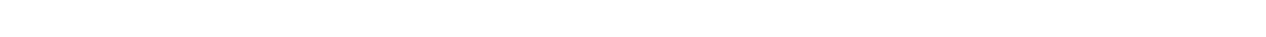

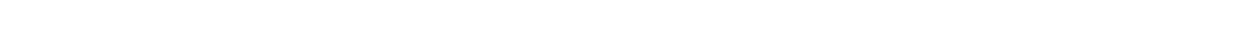

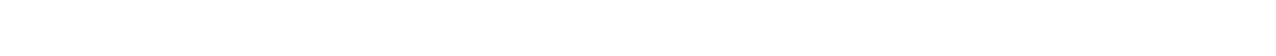

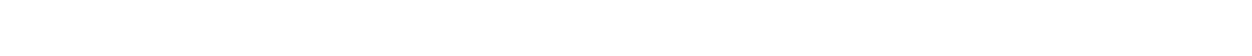

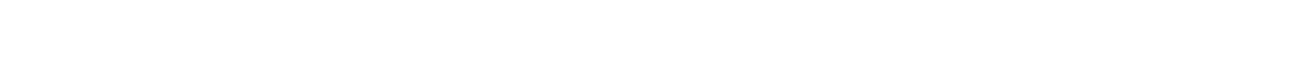
แ

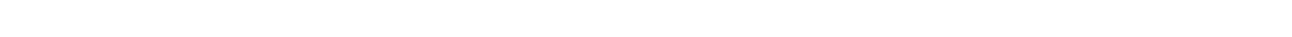

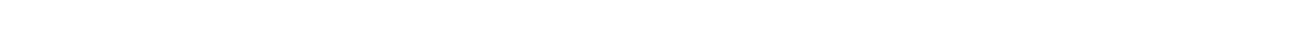

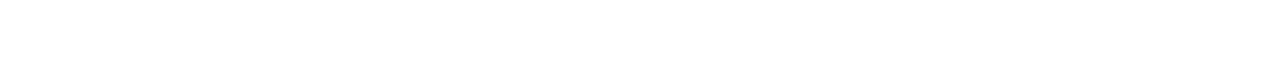

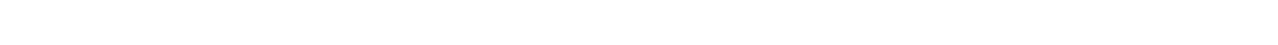

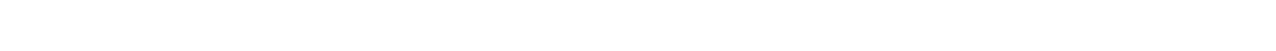

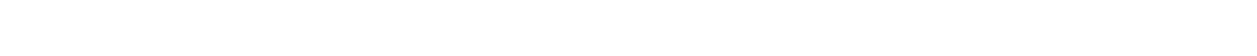

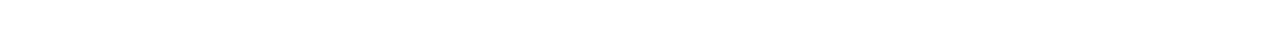

En in is a a a a a a in is

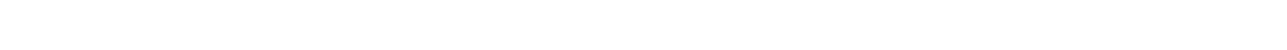

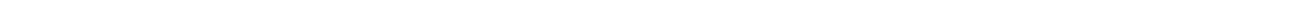

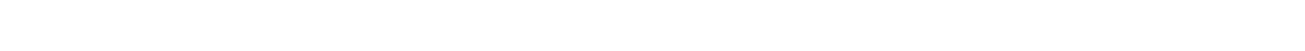

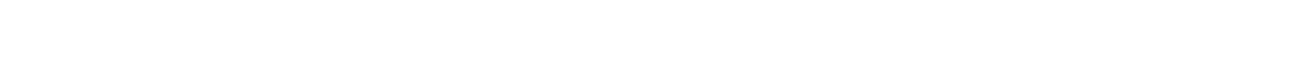

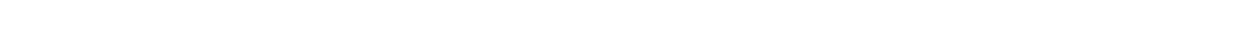

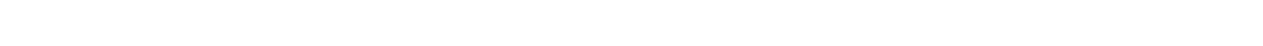

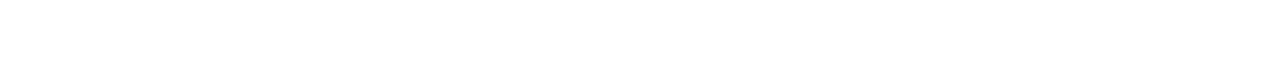

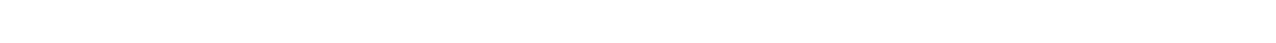
Q

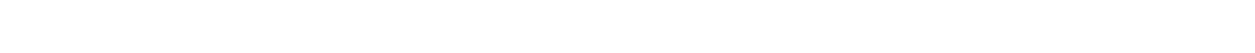

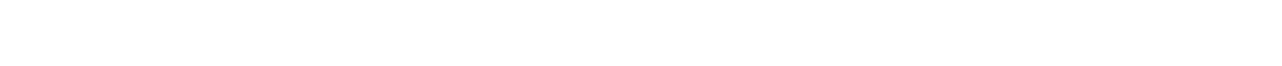
$\stackrel{4}{\infty}$
a a
a acaacaaca.
a a a

×

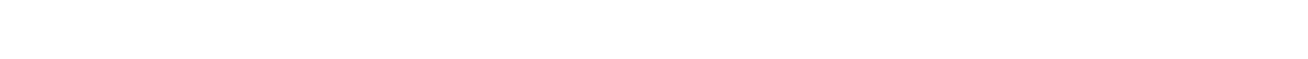
1

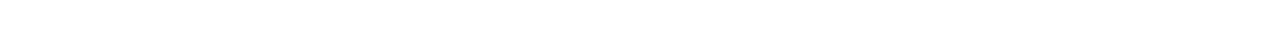

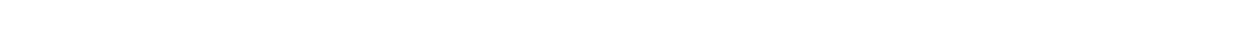

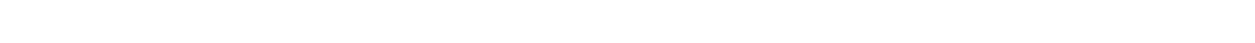
WI

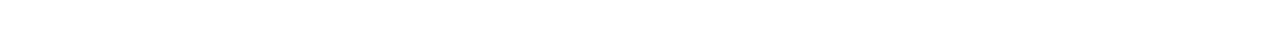

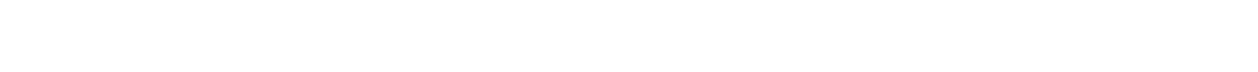




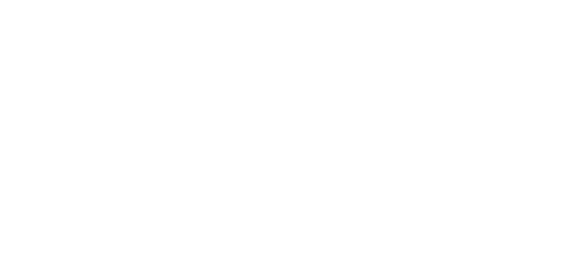

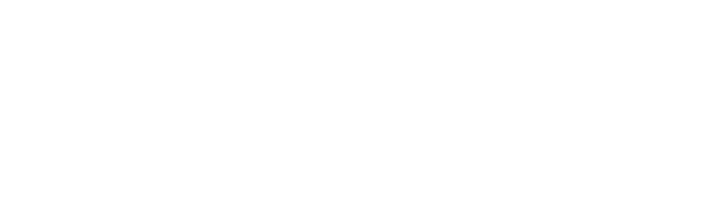

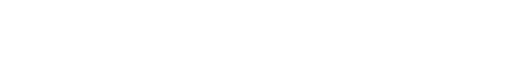

5

is

maonon-matonotar

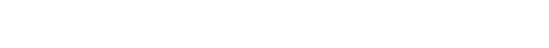
एँّنं

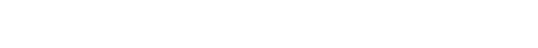

号

z

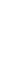

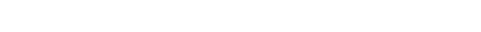

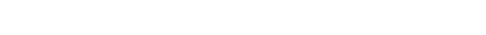

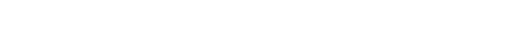

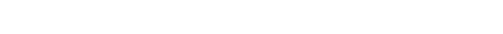

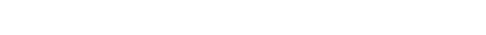

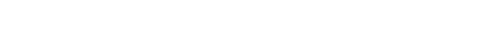

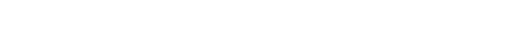
$\Sigma$

is

aces

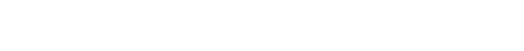

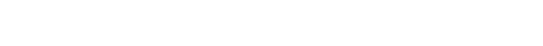

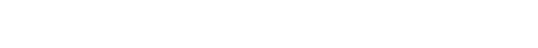

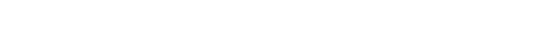

$\hat{n} \sim \hat{N} \hat{n} \infty \infty^{\infty} \infty \infty_{\infty}^{\infty} \infty \infty \infty m$

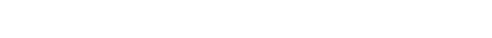

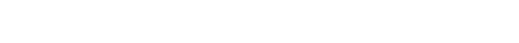

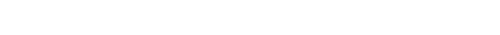

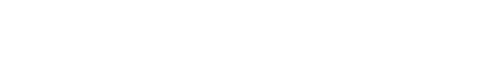

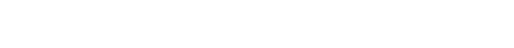
$\Sigma$

E aacon in o

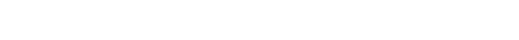
ü $\dot{1} \dot{0} \dot{0} \dot{0} \dot{0} \dot{0} \dot{0} \dot{0} \dot{0} \dot{0}$

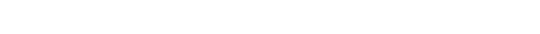

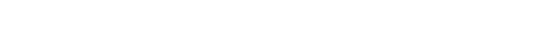

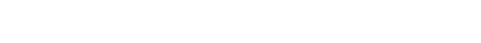

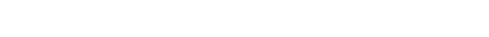

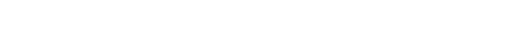

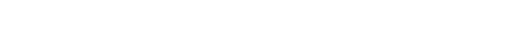

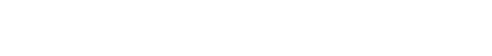


옹ำ

我上

w号告

$\sum_{0} \frac{\pi}{a} a$

z

$\rightarrow \geq \frac{1}{\alpha}$

w้

mà

waำ

a) 1 in

1.

wis

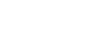

$x+\frac{1}{1}$

$\dot{2} \frac{1}{2} \dot{2}$

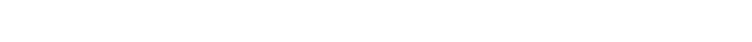

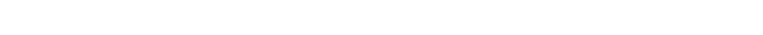

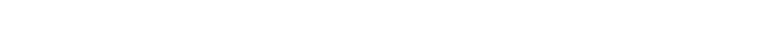

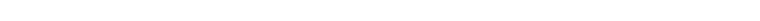

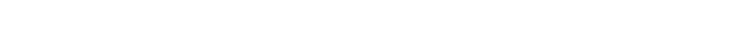<smiles>[131IH]</smiles>

w

$\frac{a}{2}$

×

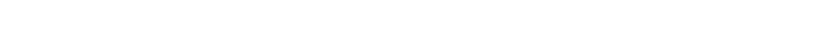

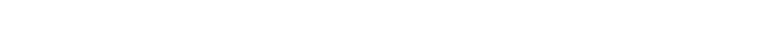

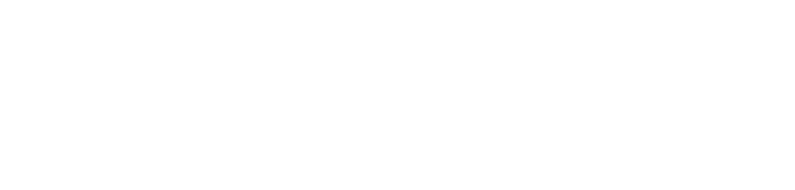

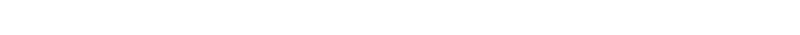

$a$

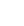

$=\mp \omega-$

용

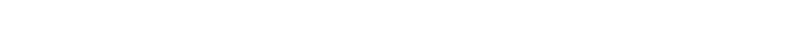

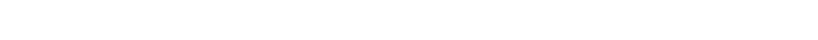
\&u

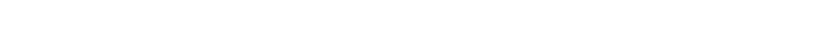

i.

ï $\ddot{w} \stackrel{\alpha}{\circ} \ddot{z}$

a

$\sum_{w} \sum_{w} \geq$ $\sum_{i}^{m} \geq$

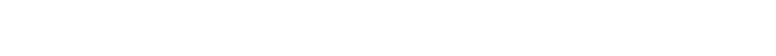

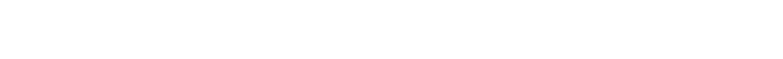

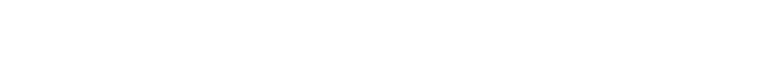

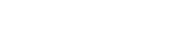

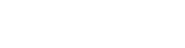
エー一⿻上丨

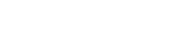
áa 웡둥으은 . r $\ddot{w}-\ddot{w} \sim \ddot{w}$

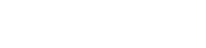

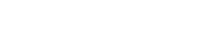
$a=a$

แ⿻

tin

응뭉

$z_{0}^{2} z z^{2} z^{2}$

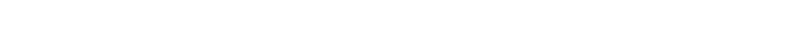

in

๔

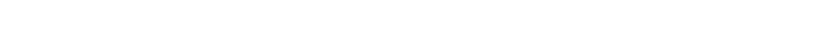

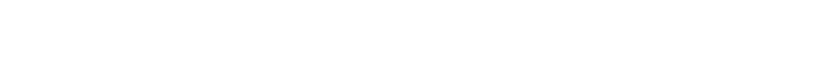

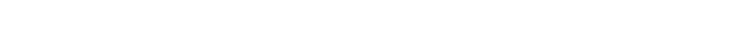

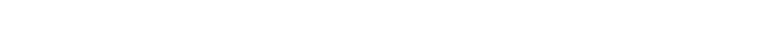

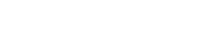

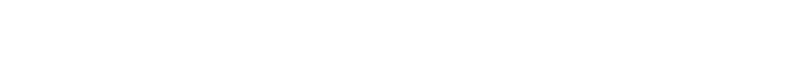

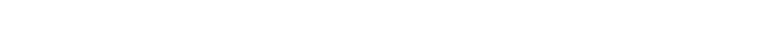

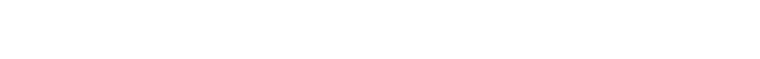


$=u$

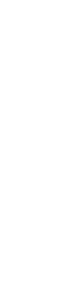

贸

孯

a 4 o

on

옹.

哭范

a s

岗음

$<\leftleftarrows$

这崖

น.

둥요

品

zm.

$05 \frac{a}{10}$

$=$ ? ?

¿

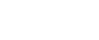

站艺

ए人

ow

a

ㄴㅇㅇ

w-

는

वே?

응

罂

屯娄

$\geq . \infty$

各乏的

- $-0 \%$

兄

ธำ

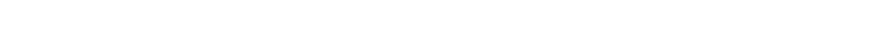

is

is $\ln \alpha$

* *

๔

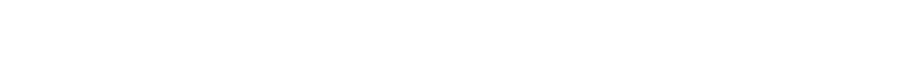

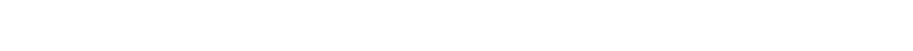

$z m o \tilde{\circ}$

훙응

แேN

")-

x

언단

woon

3 ⿰冫

= 하 i

z" n

F淀

넘의

ㄴ:

돌

㟔的=

J4

둥ㅇㅇ

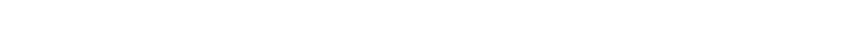

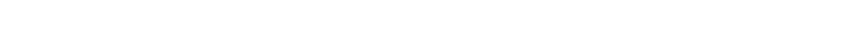

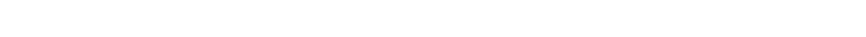

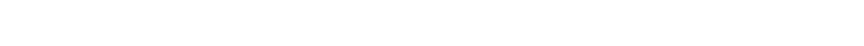

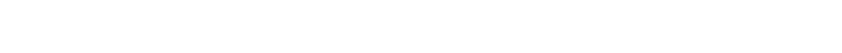

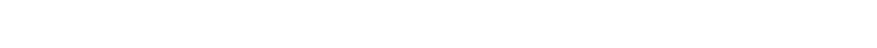
$\pm a$ 0

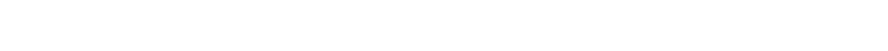
แய

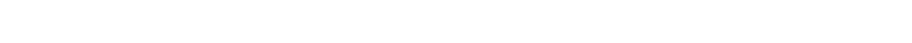

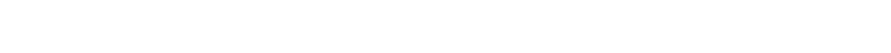

잉 $\because \frac{0}{0} \cdot \frac{1}{1}$

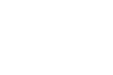

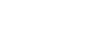

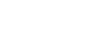

I.

in

ñ

$\begin{array}{ll}x & z \\ x & 2\end{array}$

in $z-\frac{\alpha}{\alpha}$

$\sum_{i=1}^{\infty} \sum_{i=1}^{a}$

要

.. 定...

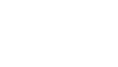

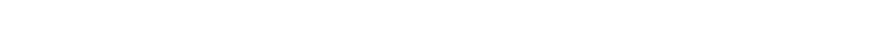

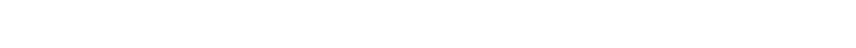

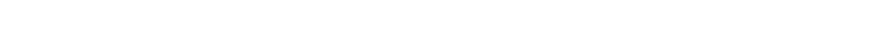

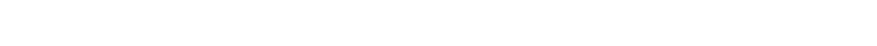

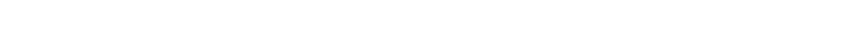

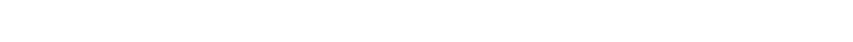

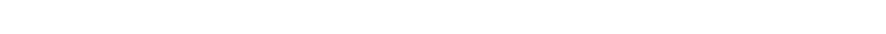

is

a

$a$

a

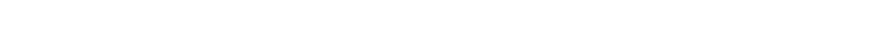
uै

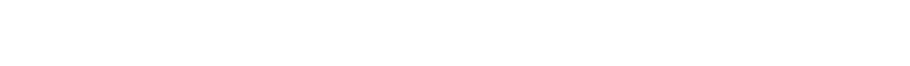

คค

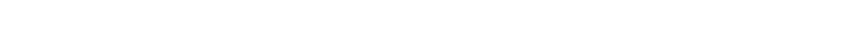

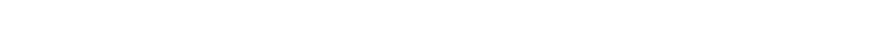

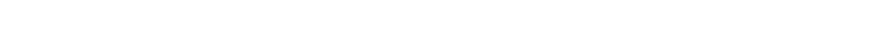

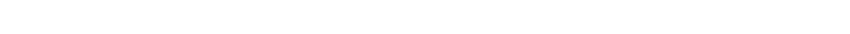

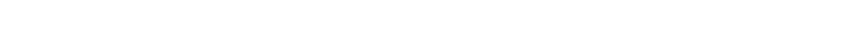
$\ddot{0}$

$\forall w n \hat{1}$ a. 은 min $\simeq 000$ 등ㅇㅇㅇ $\ddot{w}-\ddot{m} \sim$ rw口 这客: $a z_{z} z$ $z_{0} z_{0}$

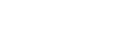

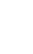

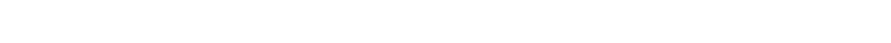
范

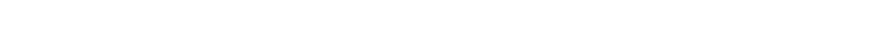

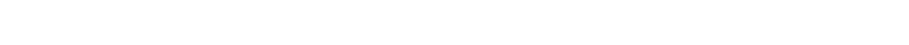

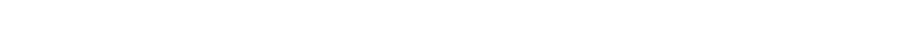

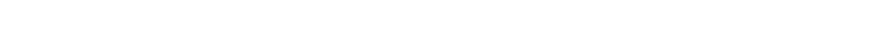

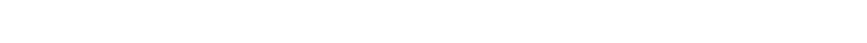

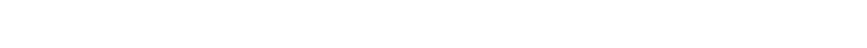

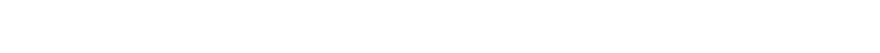

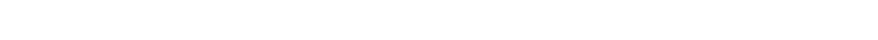

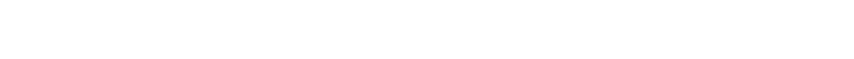



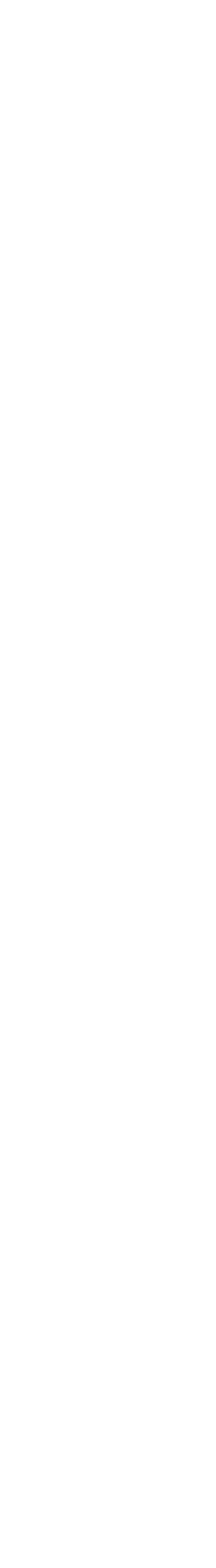


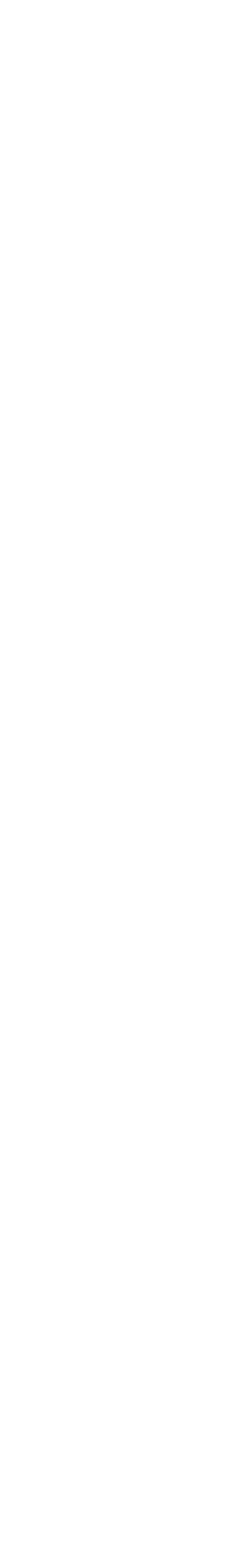



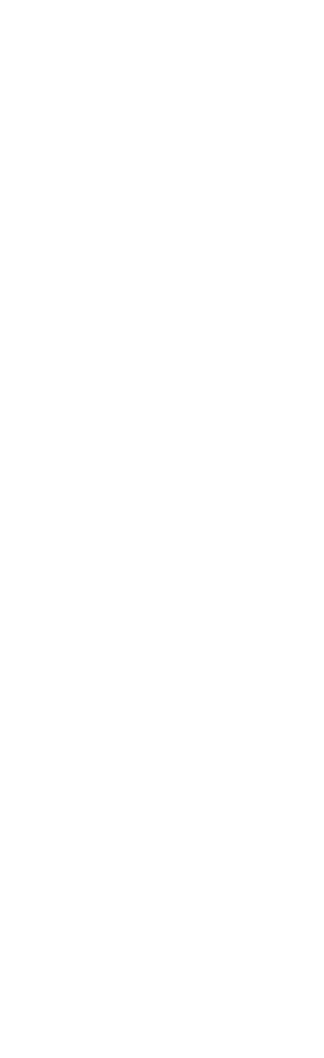

企

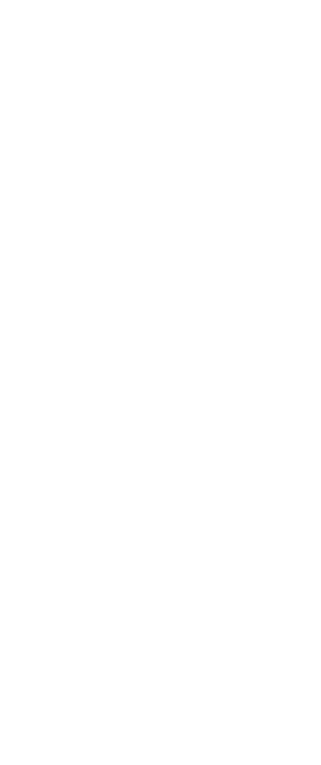



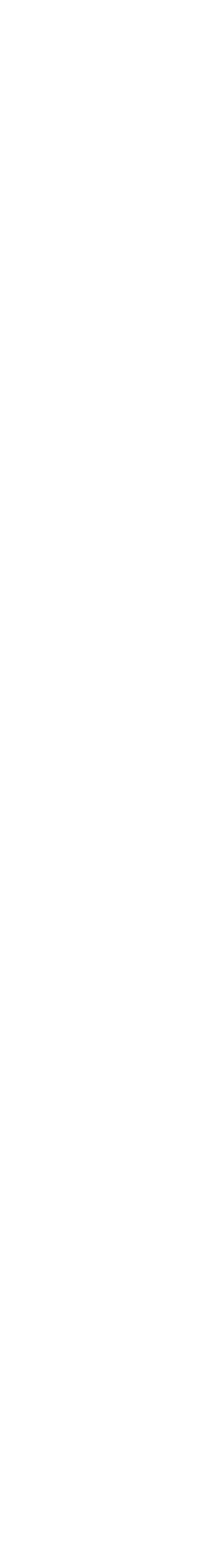

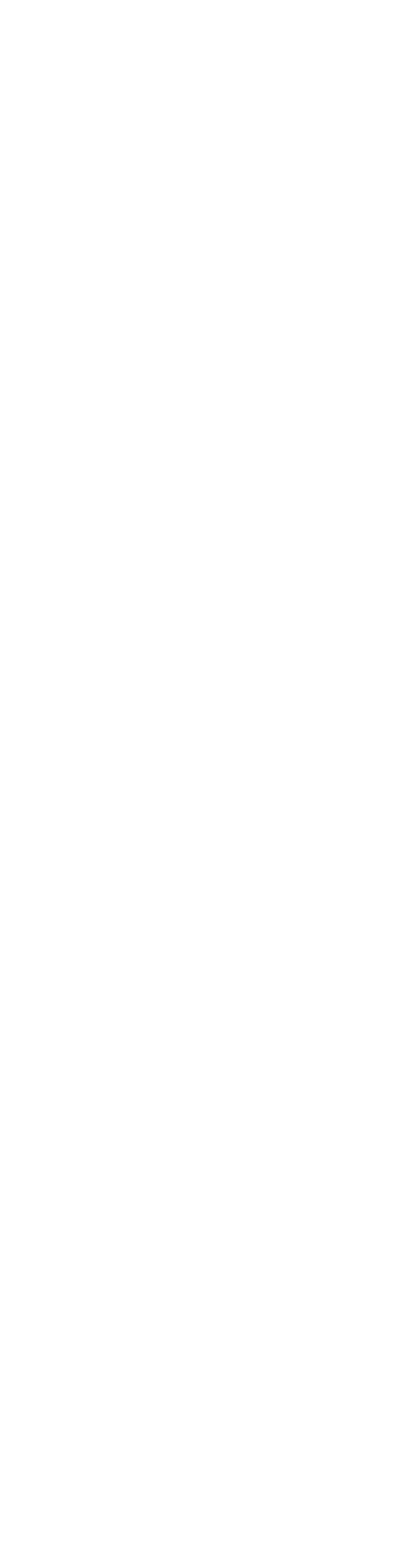

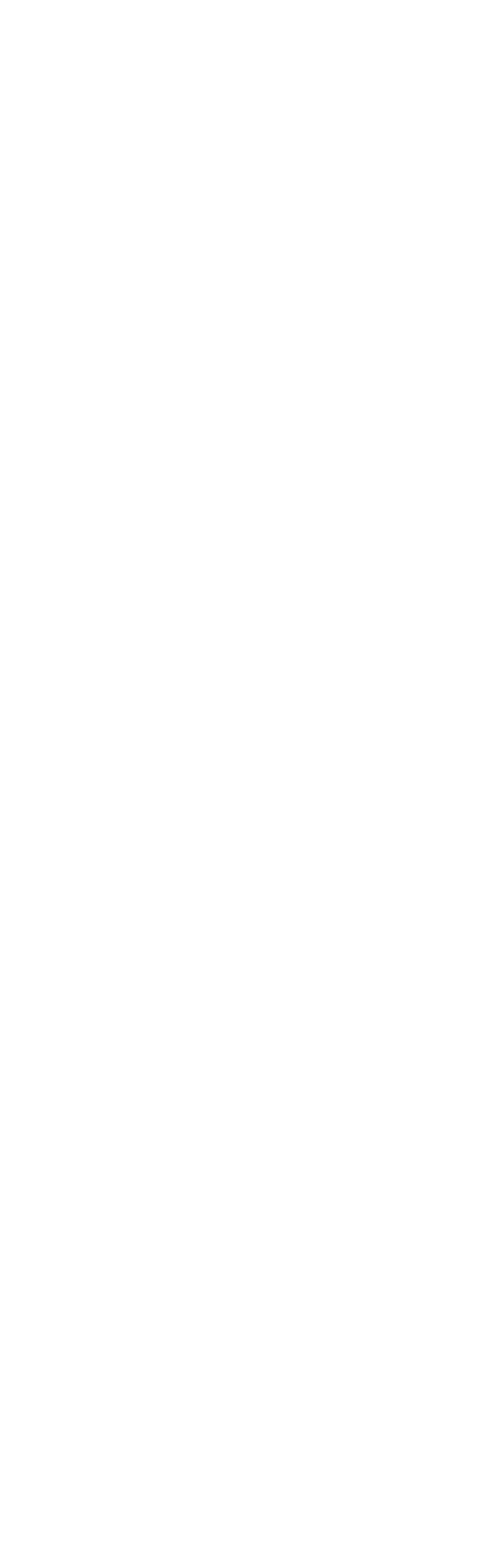

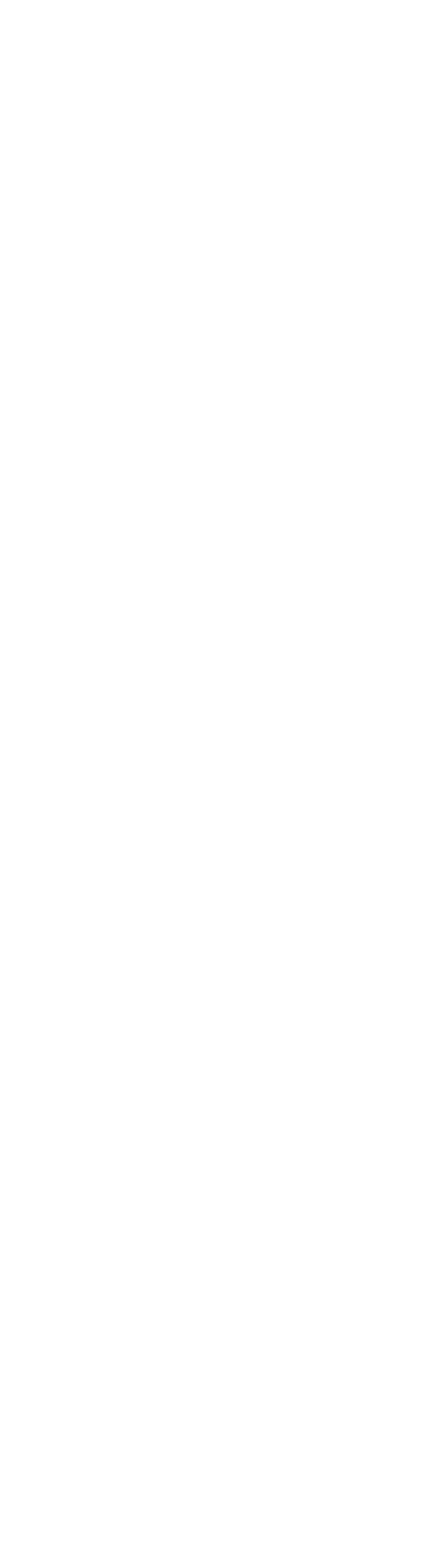


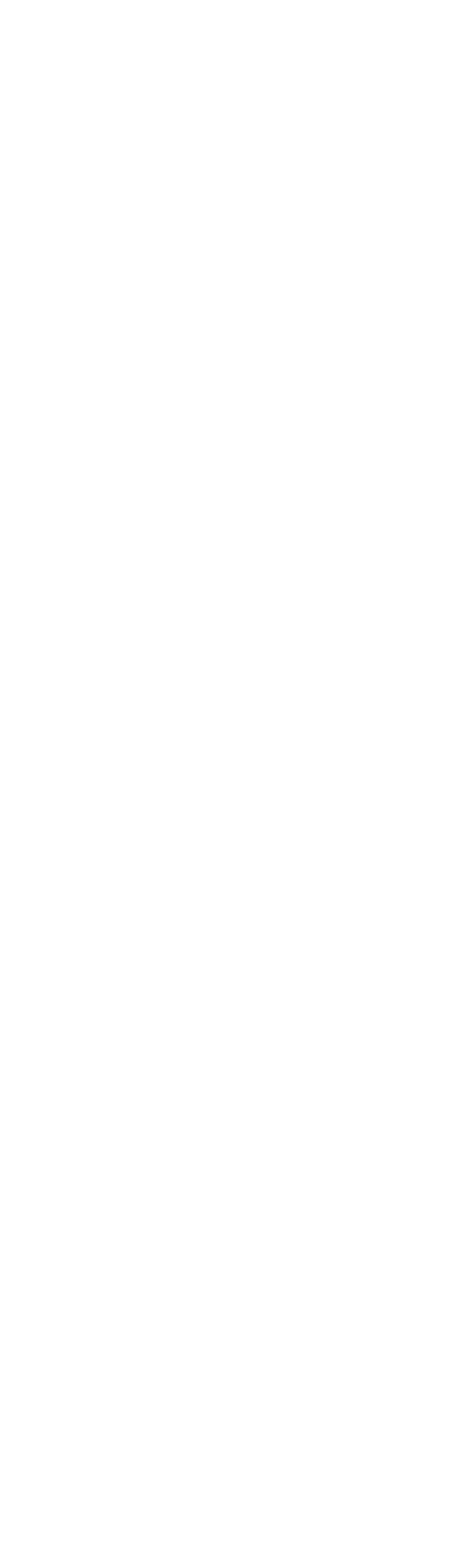

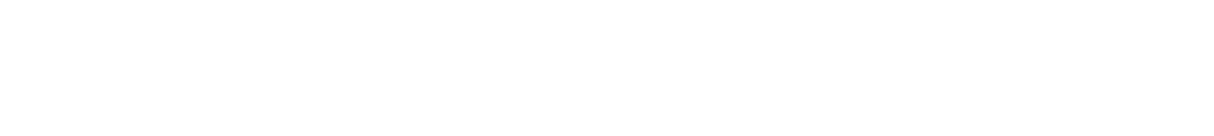

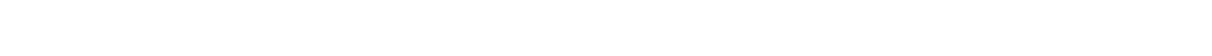
w

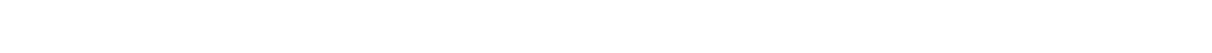

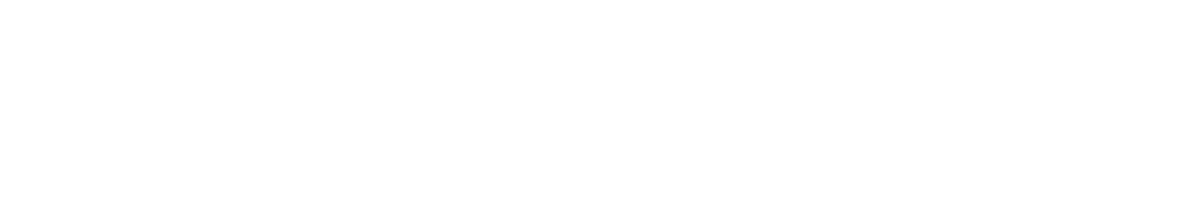

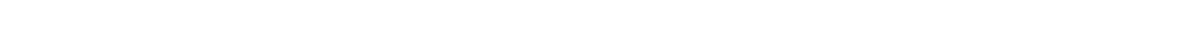

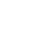

"ำm

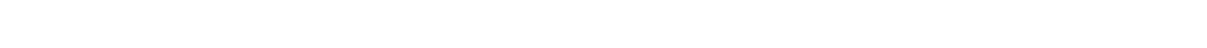
₹

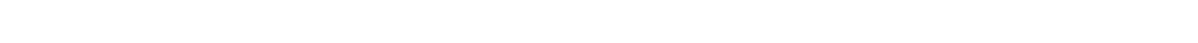

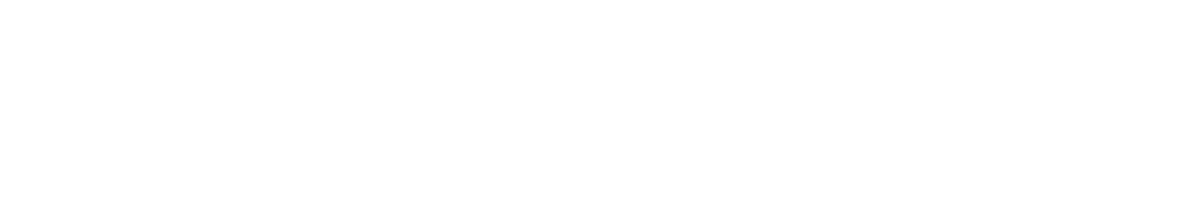

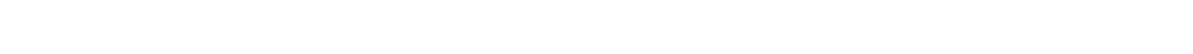

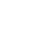

noN-rnmo⿺ a <

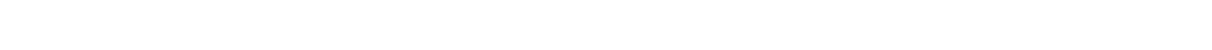

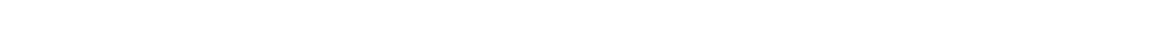

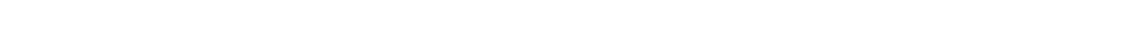

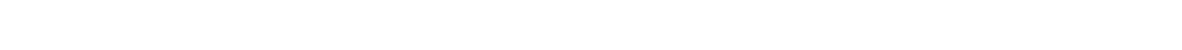

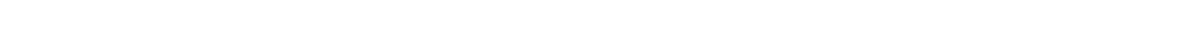

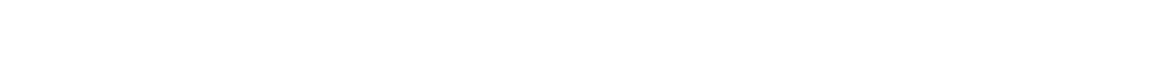

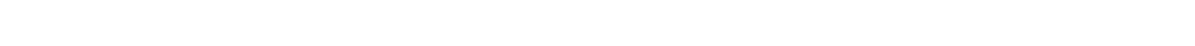
E

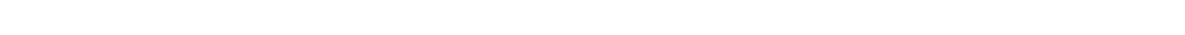
« 世 zug

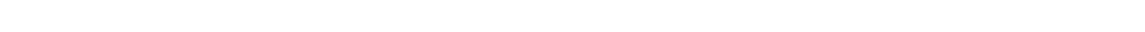

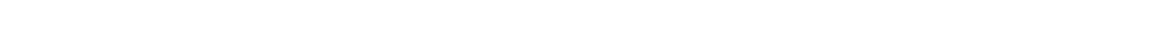

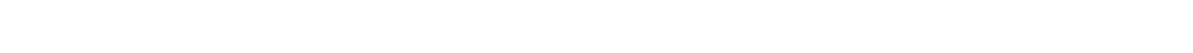

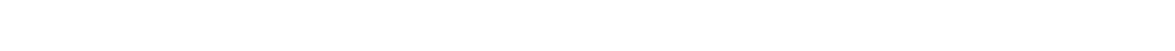

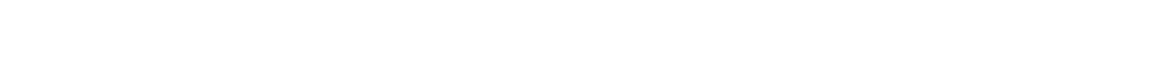




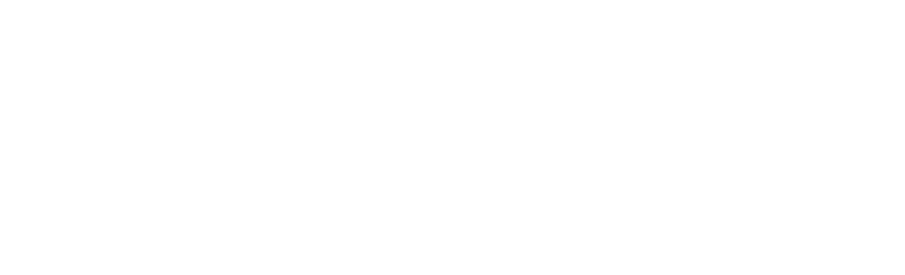

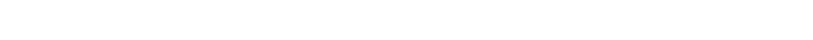

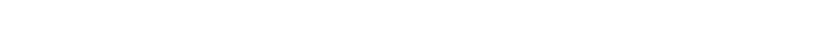

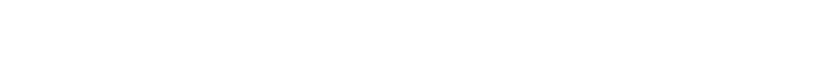

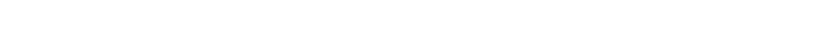

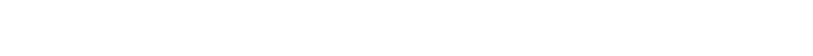

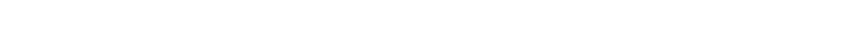
a

is

"

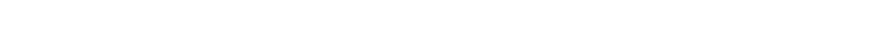

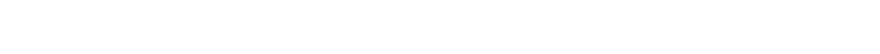

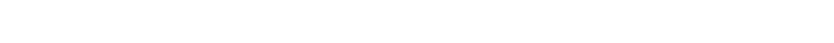

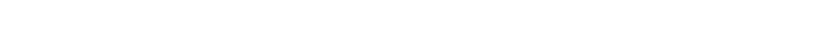

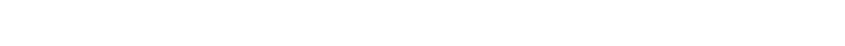

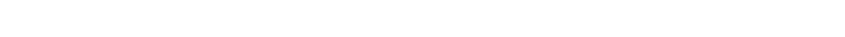

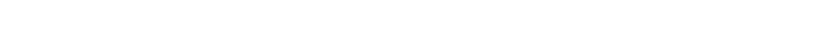

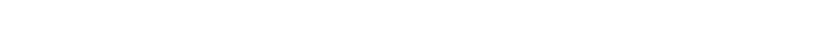

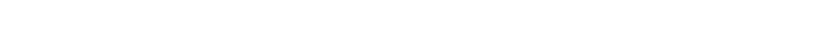

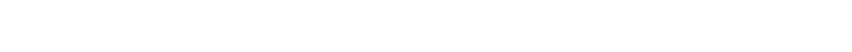

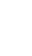

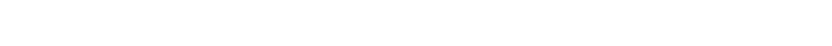
แ山山

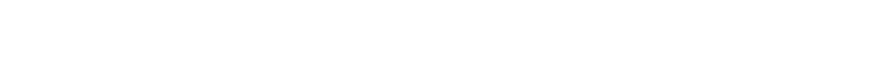

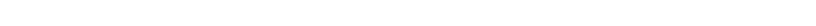

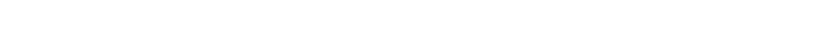

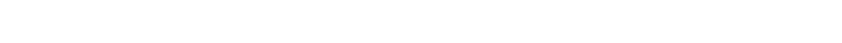

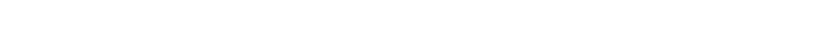

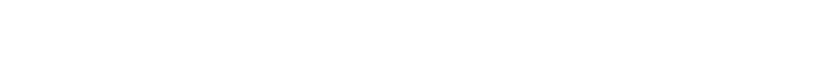

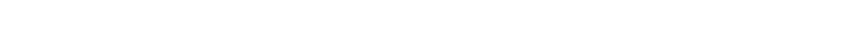
a

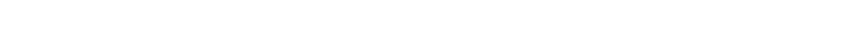
$x-10 \% m$ ш

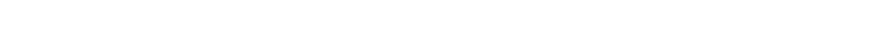

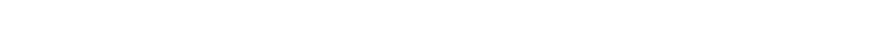

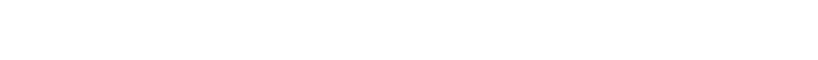

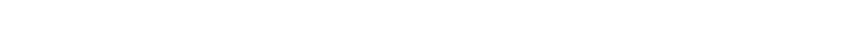
aa a

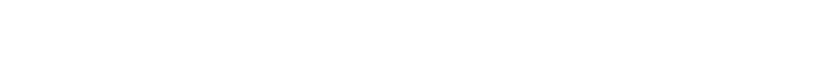



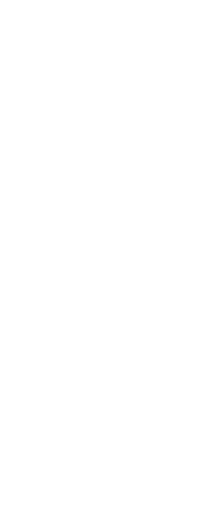

wenen es

in

in

ผ แ山

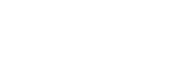

$x-g y=5$

究

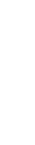

- $\quad \sim \sim \sim \sim$

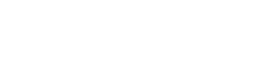

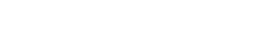

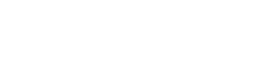

空点

I出

㟧eneson

崖

क क

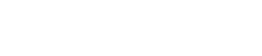

-

- 出

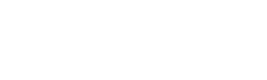

渵

ㄹ.

部

"艹

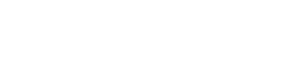

3

$\Xi$

$=w$

당 क

웅 a

Lunra

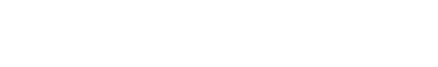

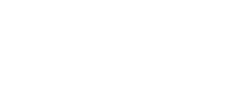

向 $\quad a z z=$

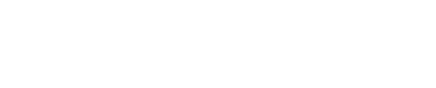

ङ के

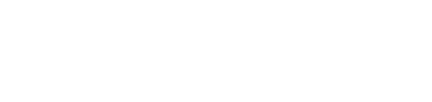


焉, is

á $=$

$=\frac{1}{4}$

$z \stackrel{2}{\alpha}$

茂上专乏

的此崖

次盗出。

s. $\div a$

○次要

ロே゙ニロ

药占二出

-

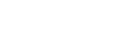

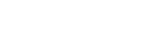

대워

的落。

x的的

Iñ. U⿺乚一匕

a $\simeq a$ a

ज紊

战方京

도은

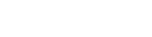

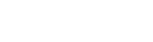

-0워

4

누ㅇㅛㅛ

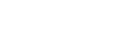

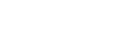

준

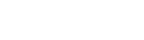

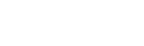

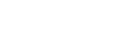

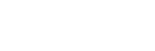

xis

แज山上

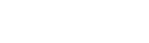

\& on

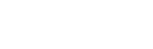

u保之

anin.

$3 \div \div 0^{2}$

$2-0 n=0$

-

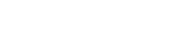

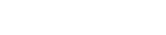

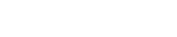

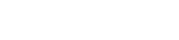

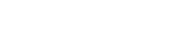

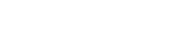

แ

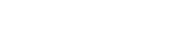

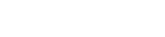

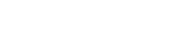

5

in

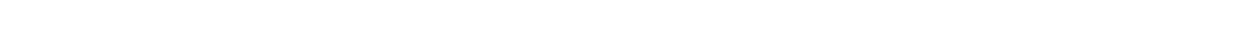

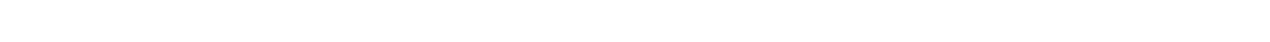

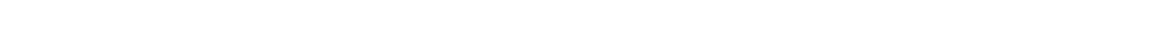

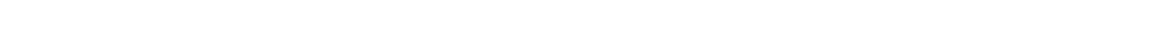

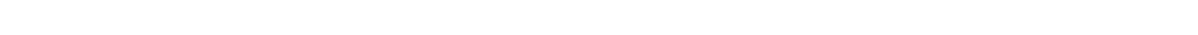
200

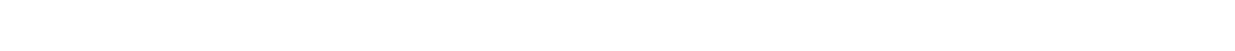

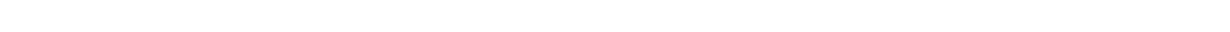

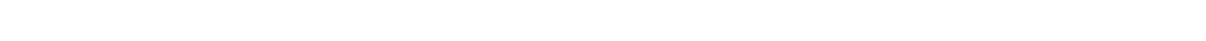

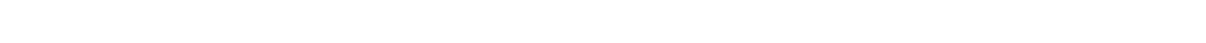

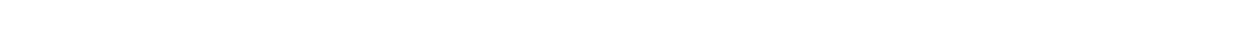
\&

" $⿻$ nnm

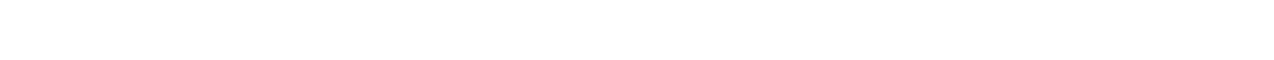

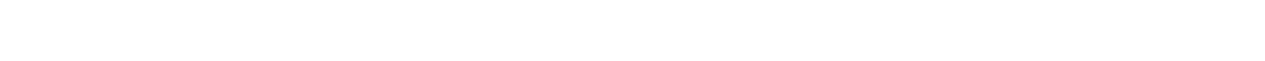

$\stackrel{\infty}{-}$

$\div$

폻

is

- in

占

w

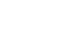

중을

Q5

$\sum_{w} z_{n}$

छ

임ำ

$=-$

No

ㅇ․ㅇㅇㅡ.

草-

元。

$\therefore<$

능을

जै

w

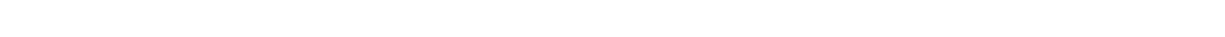

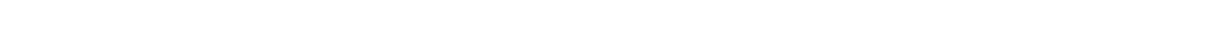

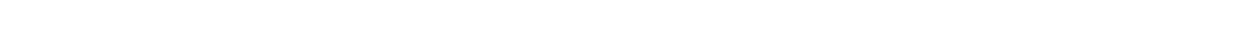

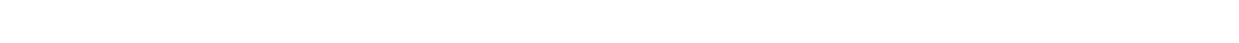

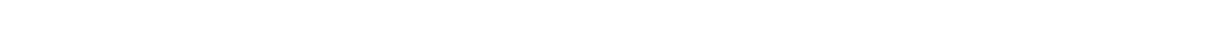

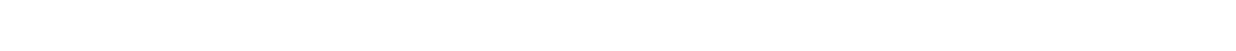
is

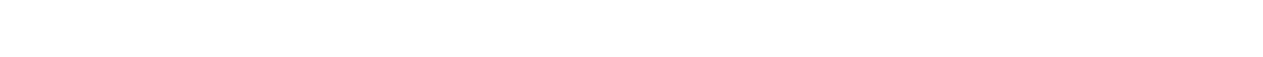

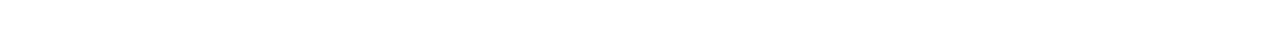

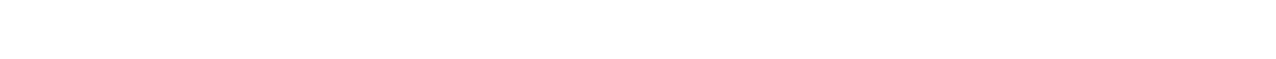

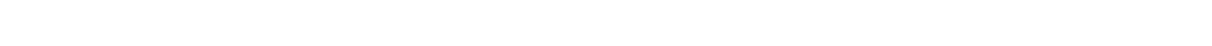

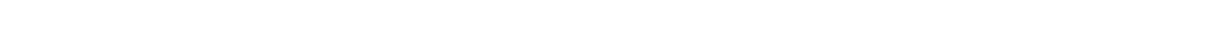

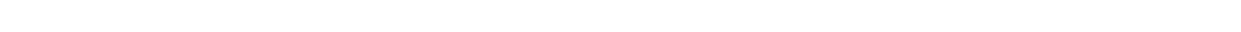

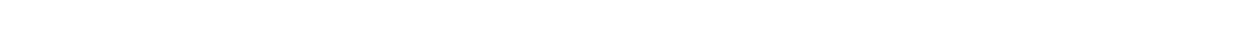

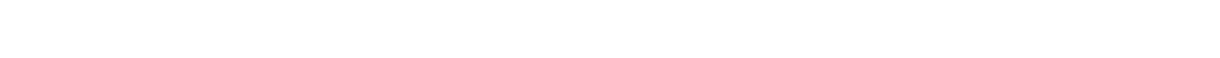

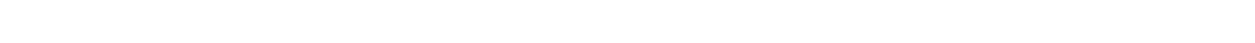
5

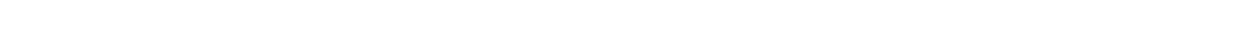

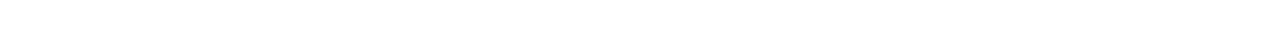

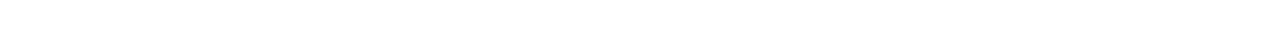

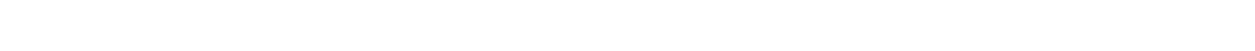

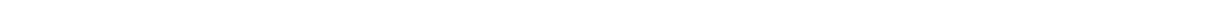

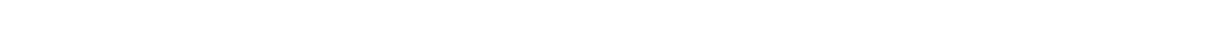

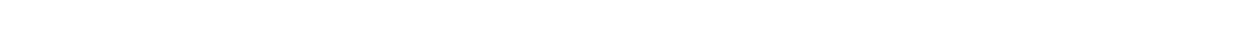

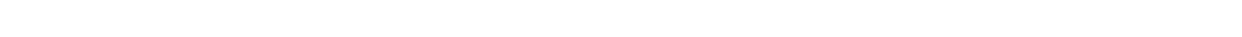

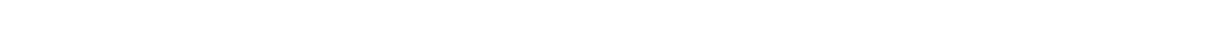




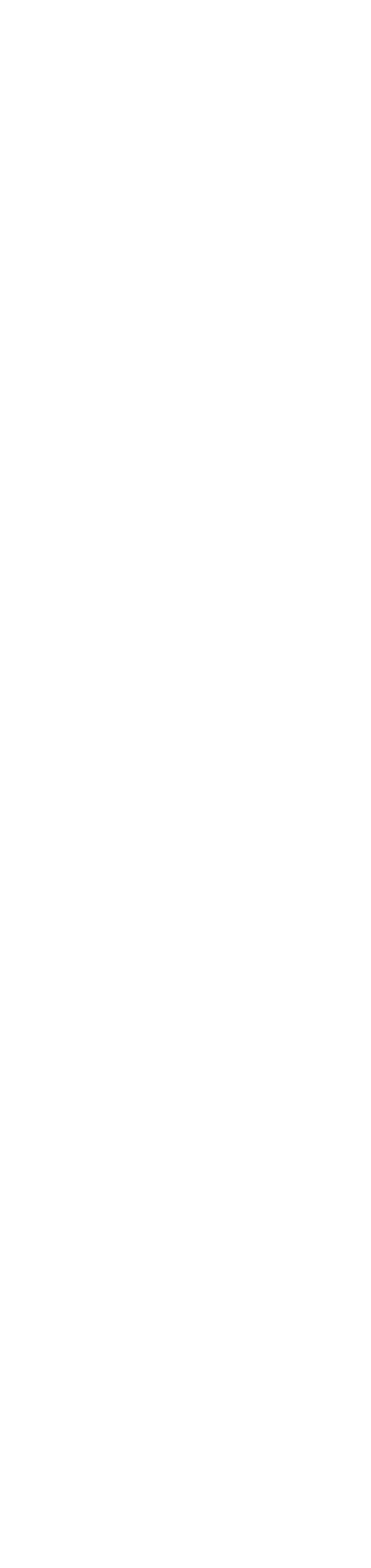

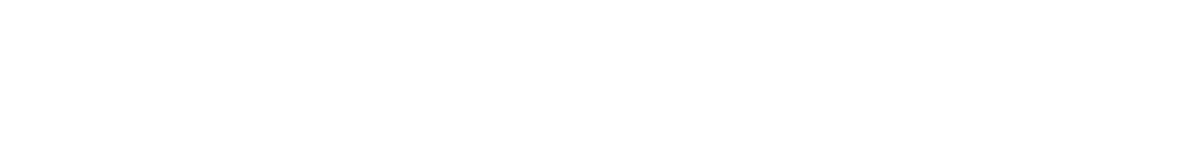

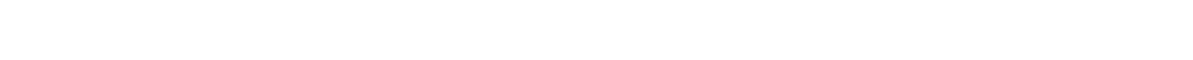

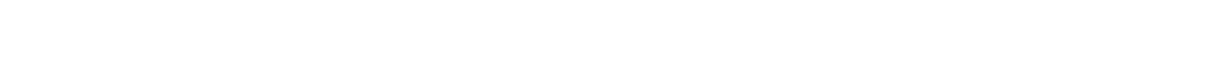

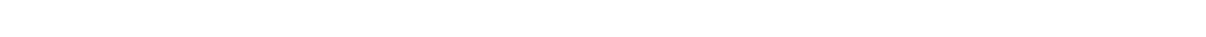
쓴

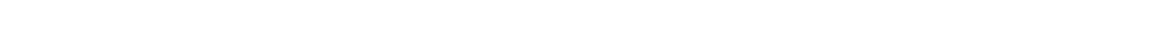

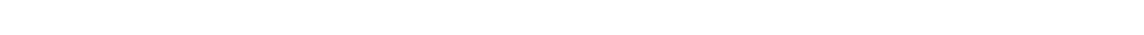

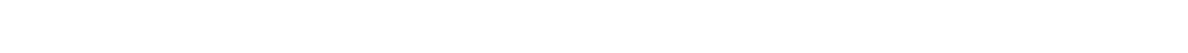

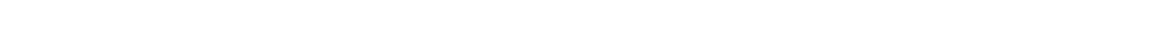

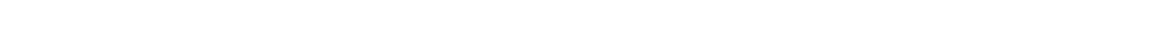

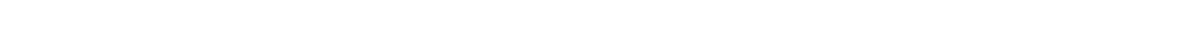
$\approx$

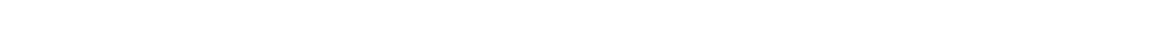
<

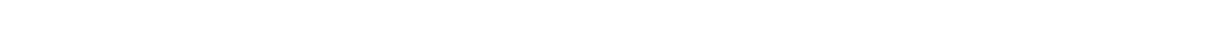

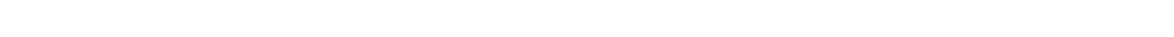

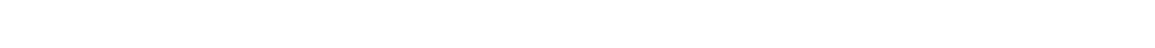

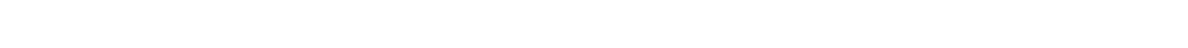

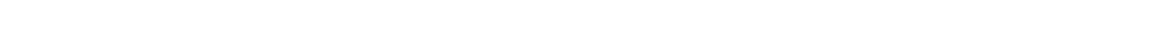

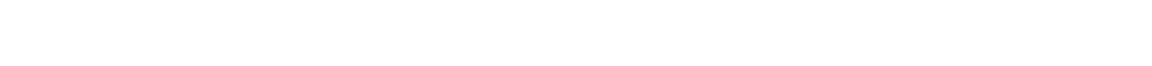

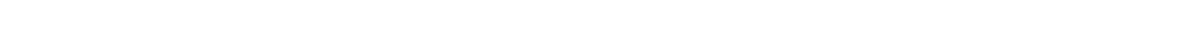

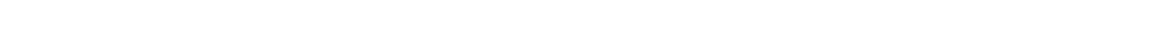

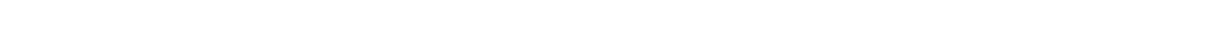

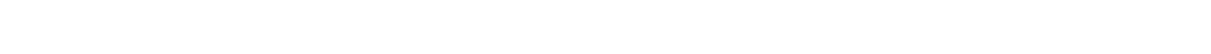

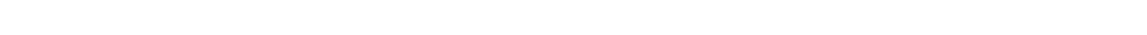

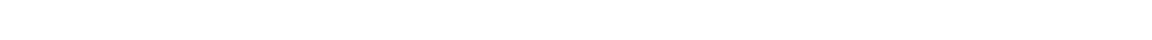

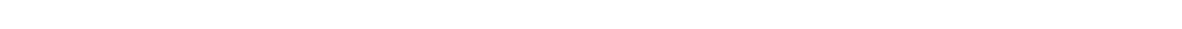

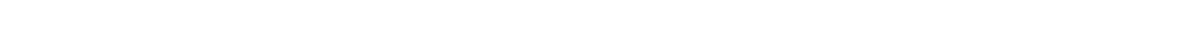
NNNAN

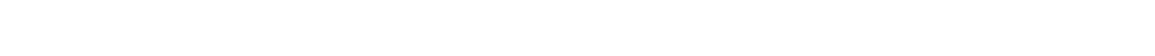

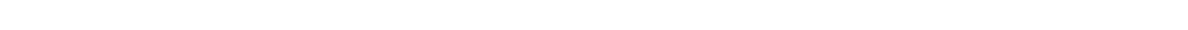

$\approx$ ๔ w wádíj:

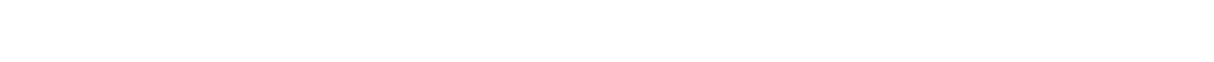

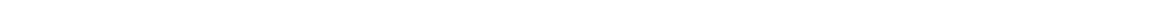

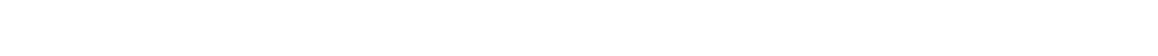

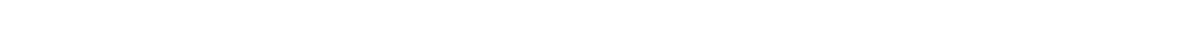

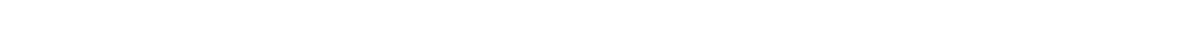

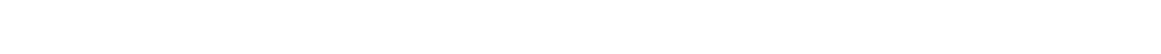


5
4
3
$x$
$x$

늠음

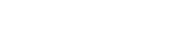

نut wa m心 는

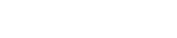

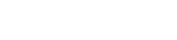
w.

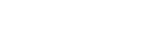

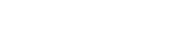

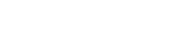

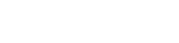
ㅇํㅇํํㅇํㅇ $20_{0} \tilde{n}$

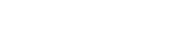
을요

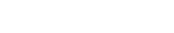
品w 분드 动出出! an $0 \geq n$

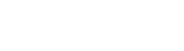

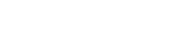

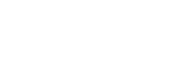

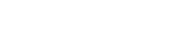
$\sum_{\infty} m^{\mathbf{3}}$ J

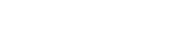

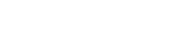

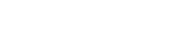
noaำ zis oc 可:

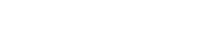
$=0 \sum \frac{a}{2}$ manc!.

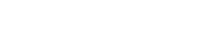
- in 1่ง 뿐돌

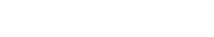
50 .

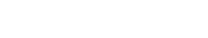
은ํำ =영

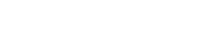

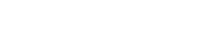

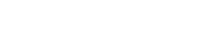

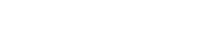

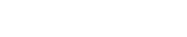

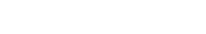
Jă

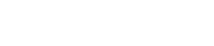

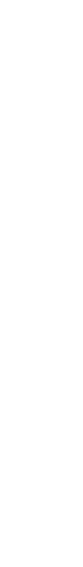

웅

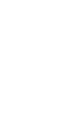
.

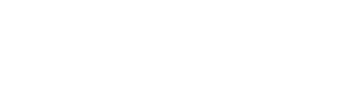
b a b a .

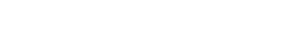

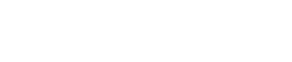
I

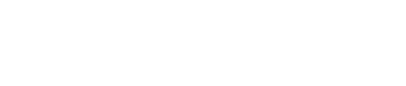

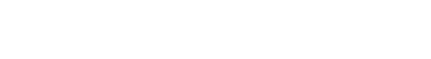

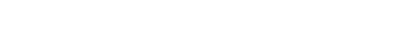

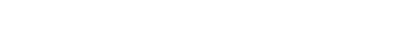

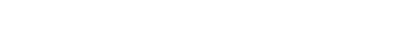
.

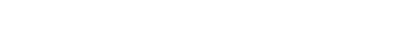

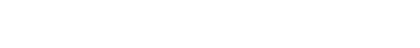

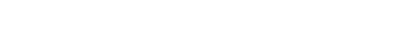

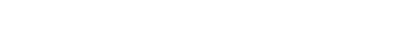
a s w o w a a ¿ $z z$ z $z z$

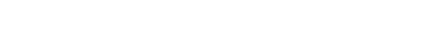

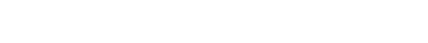

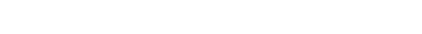

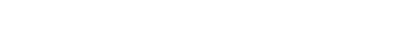
¿a =añama的a

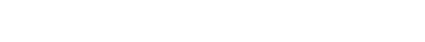

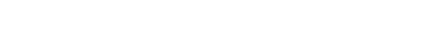

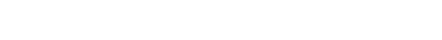
ல்

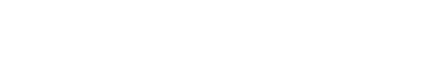

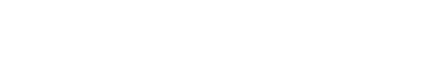
$\stackrel{\Sigma}{\Sigma}$

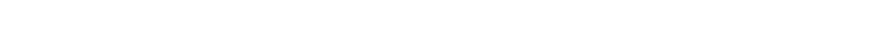

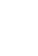

ONONOaO

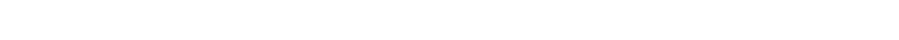
a w 0000 은

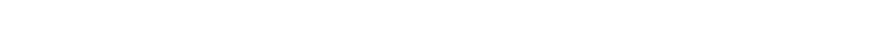

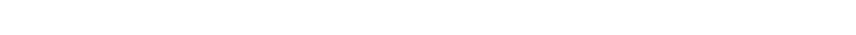

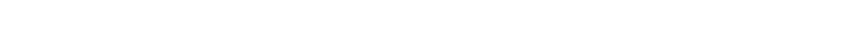
แ5

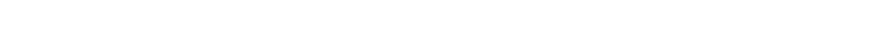

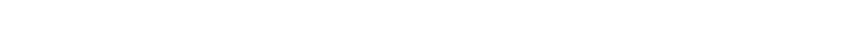

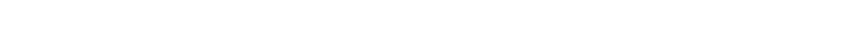

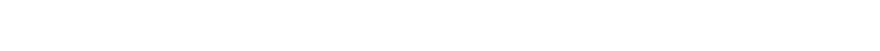
in

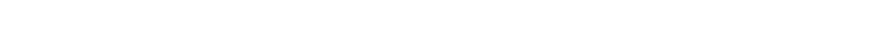
a d

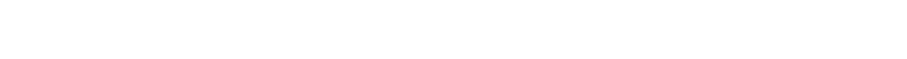
三丨

ODODON

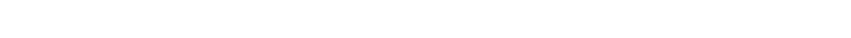

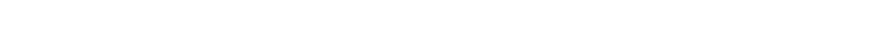

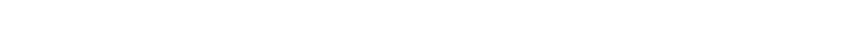

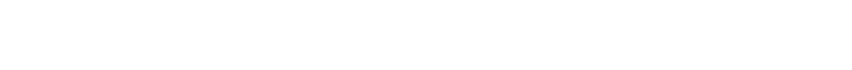

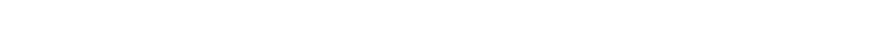

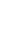

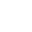

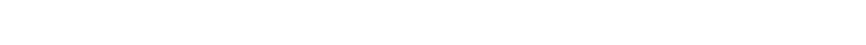
ш

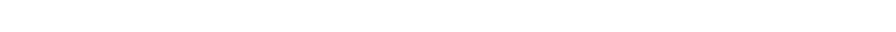

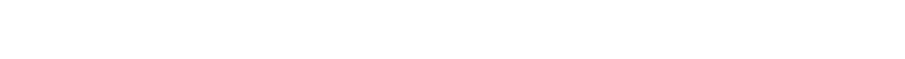

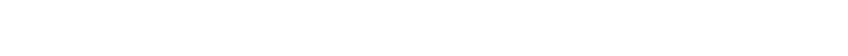
WEI

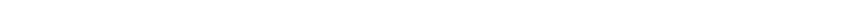
佂

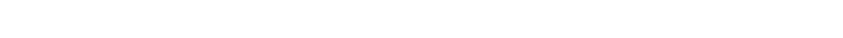

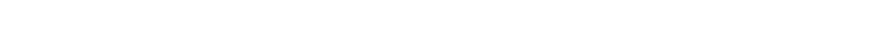

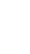

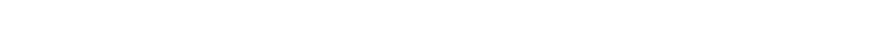

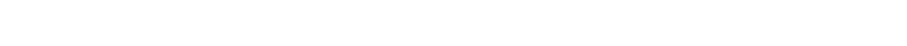
는

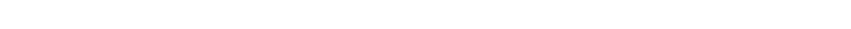

OO-

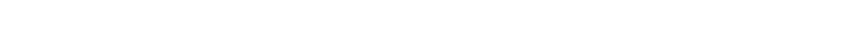
แ

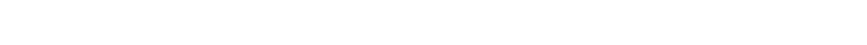

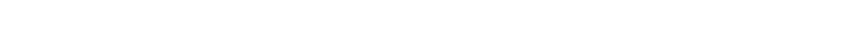




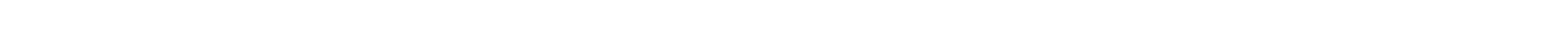

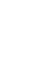

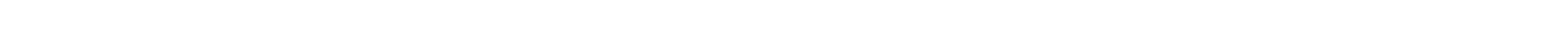

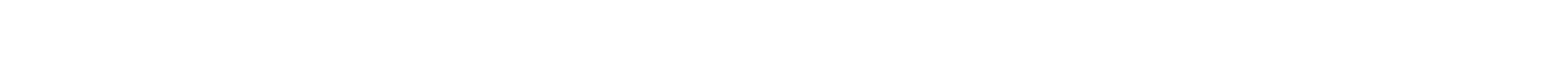

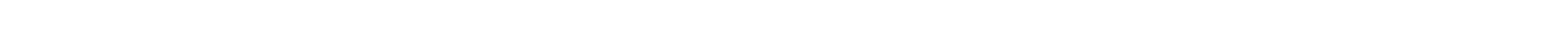

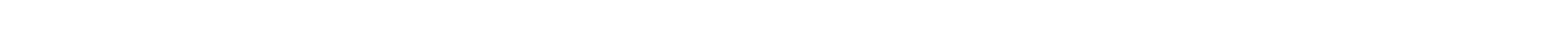

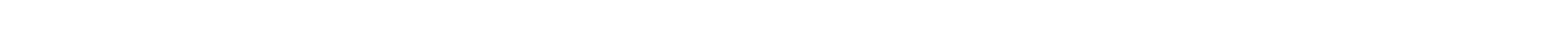

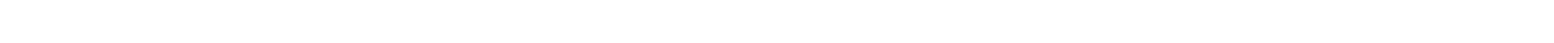
ய

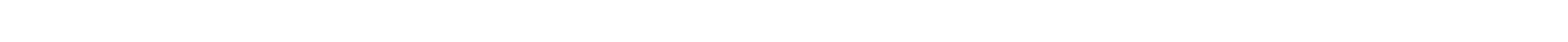

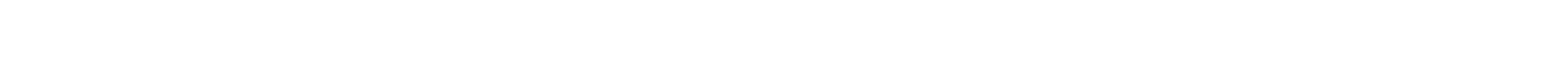

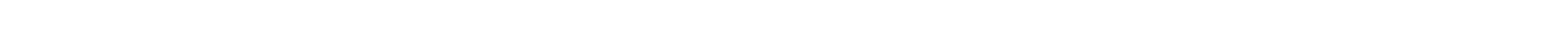
$\leftarrow$

a

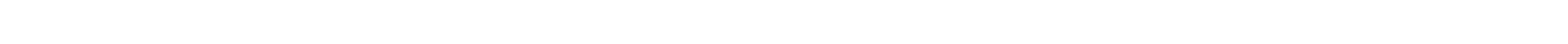
«

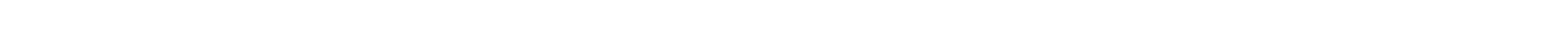

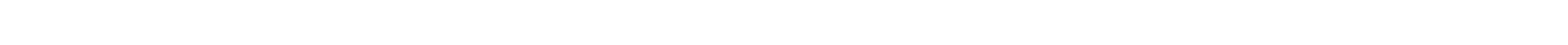
ogogogos

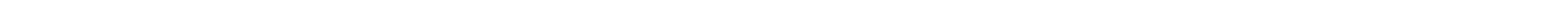

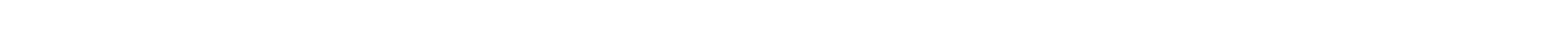

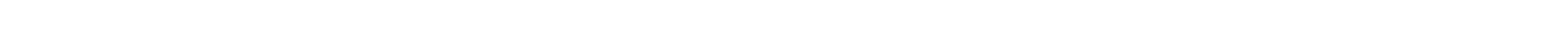

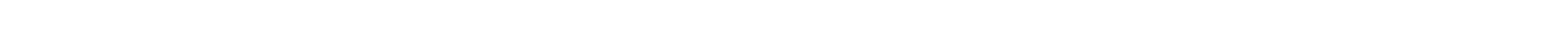

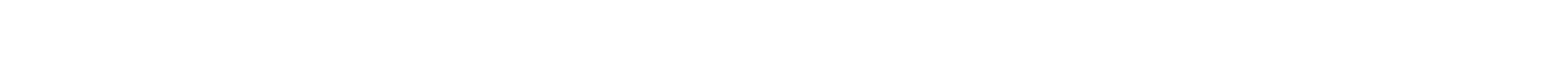

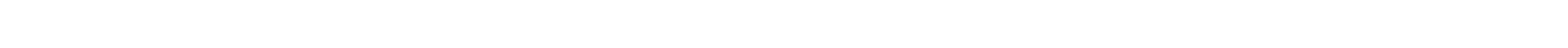

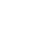

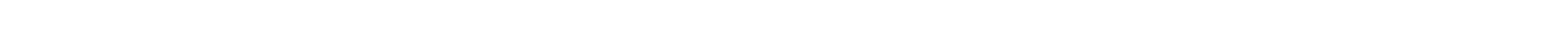

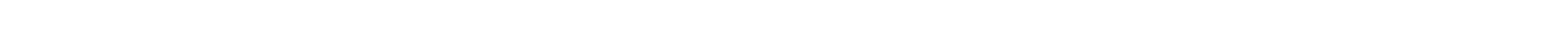

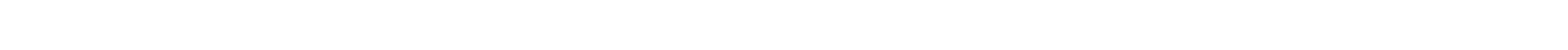

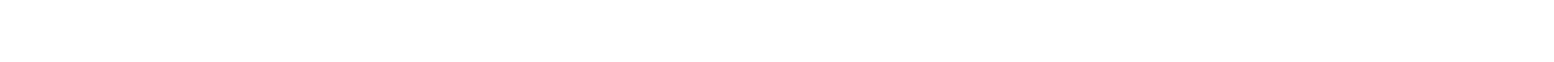

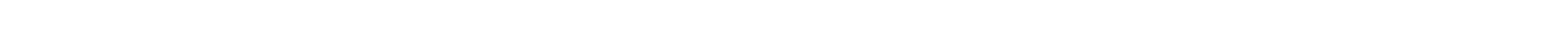

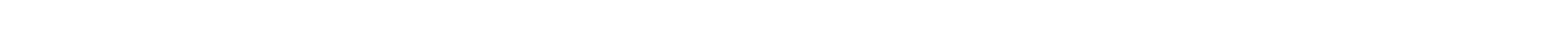

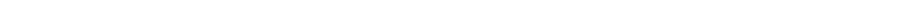

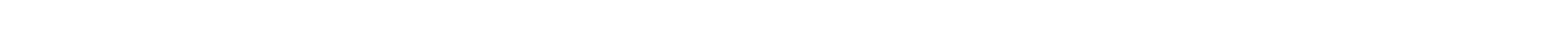

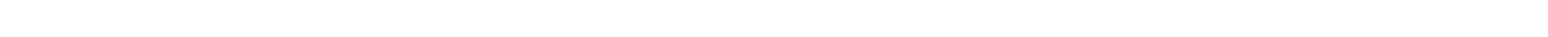

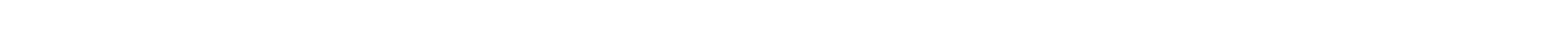

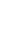

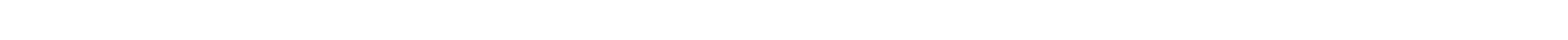
« แ

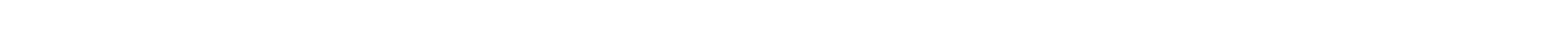

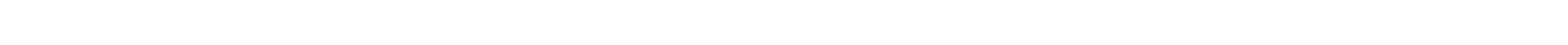

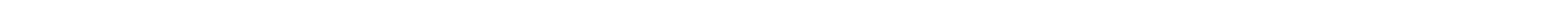

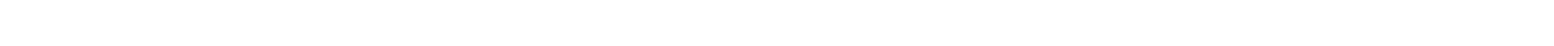

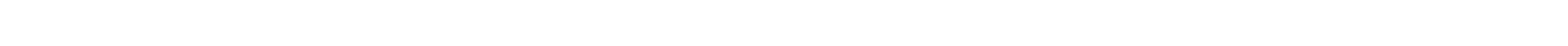

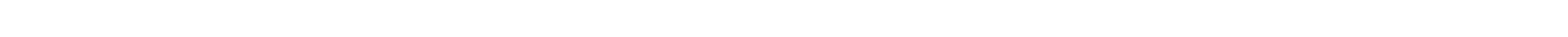

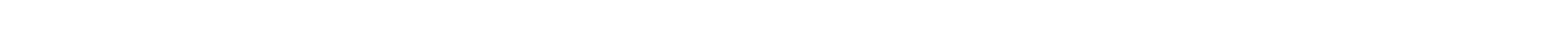

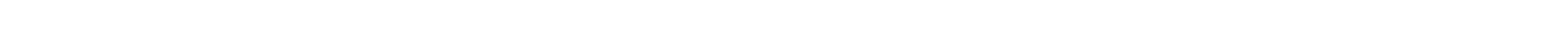



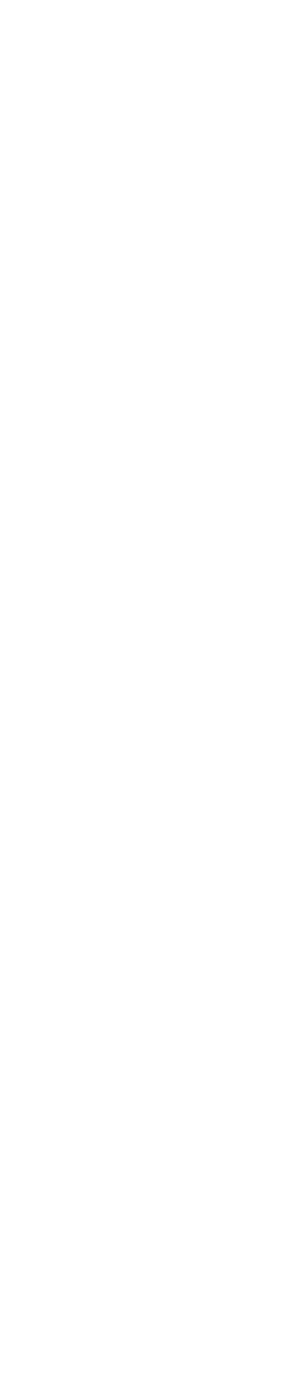

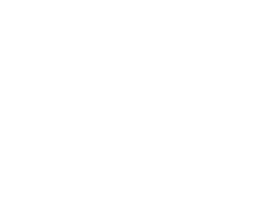

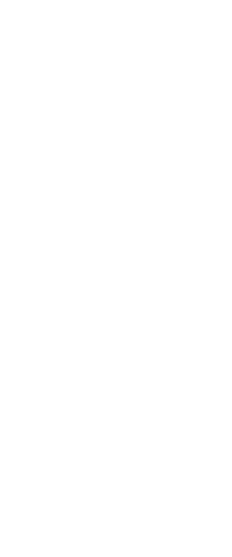




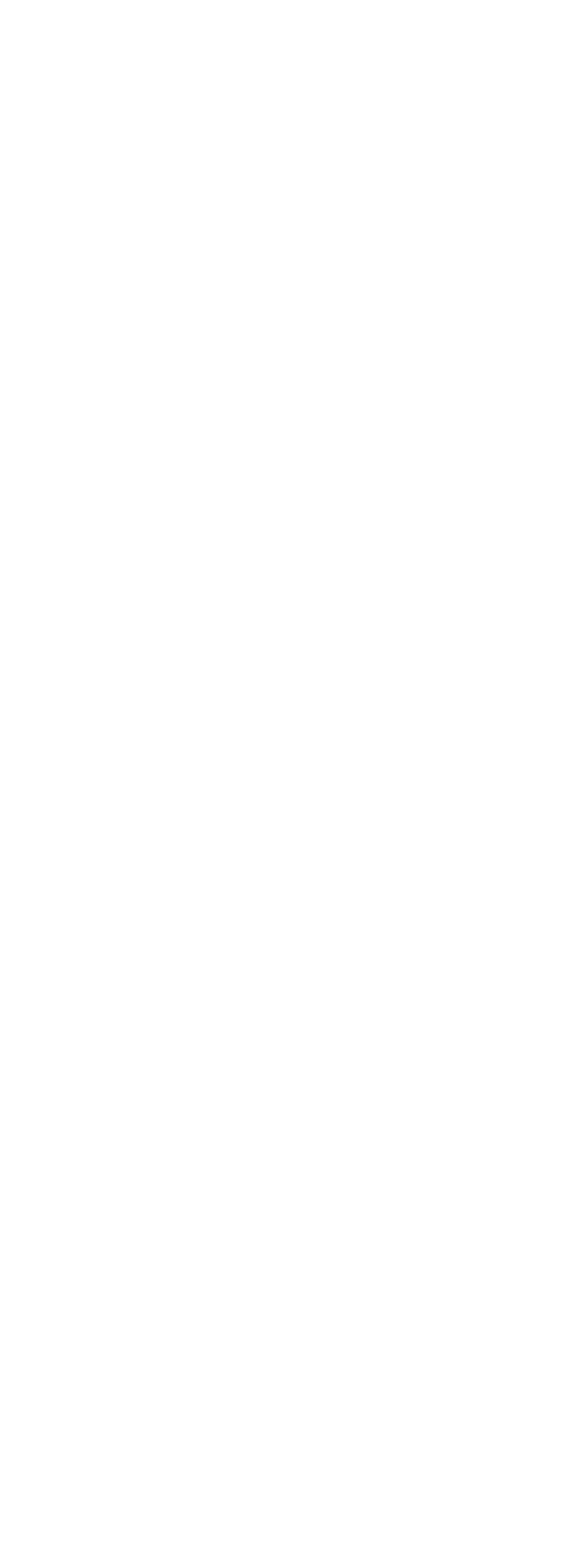

5
w
$a$
5

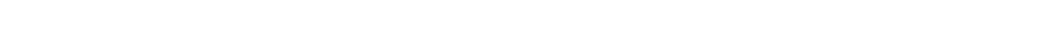

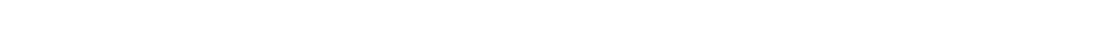

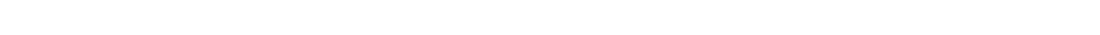

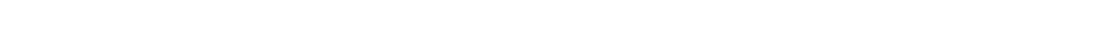

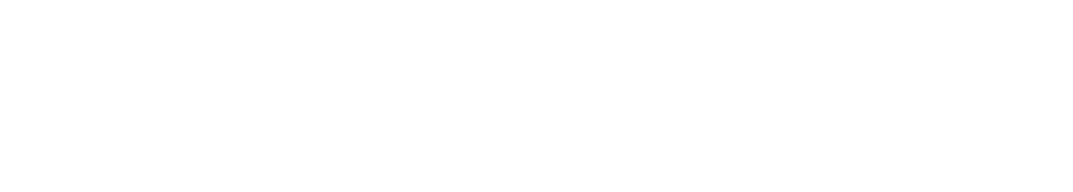

$\leftarrow$

¿

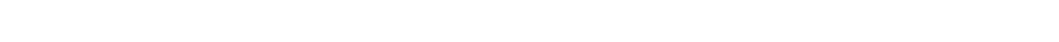

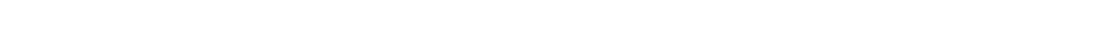

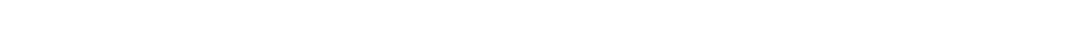
-

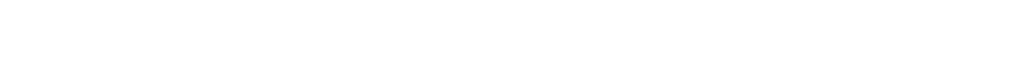

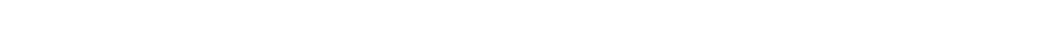

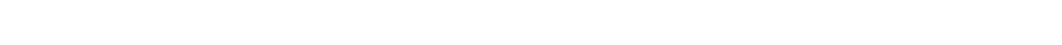

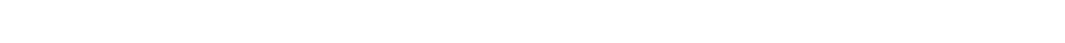

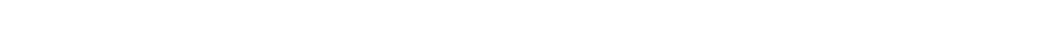

$\stackrel{5}{\Sigma}$

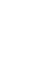

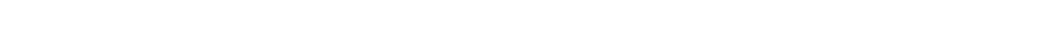

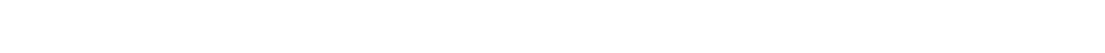

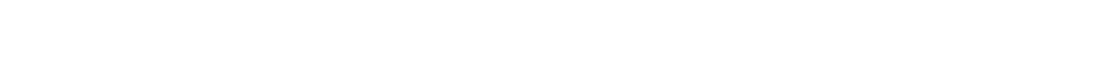

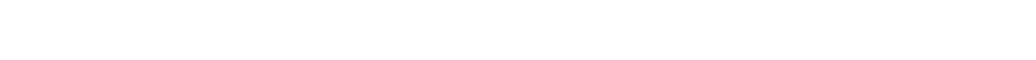

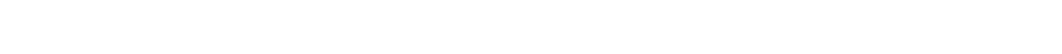
QOO-

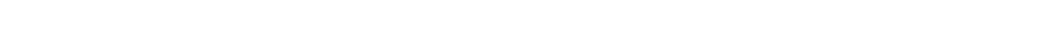
0000000000000000000000000000000000

twascosencosises on

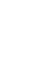

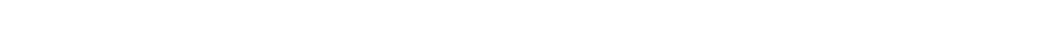

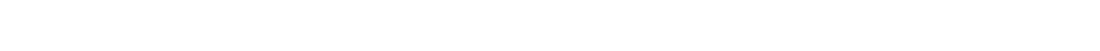

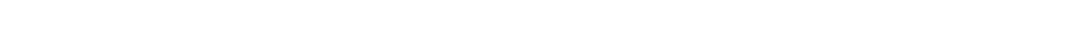

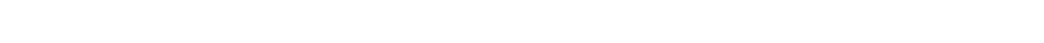

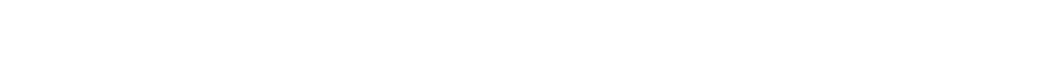

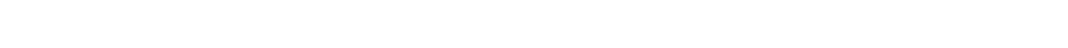

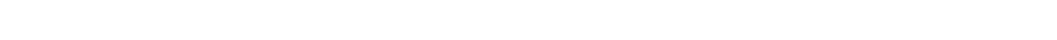

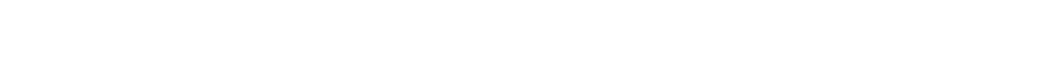


$\underline{m}$

is

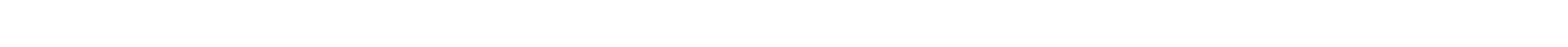
ж

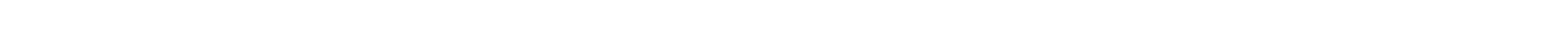

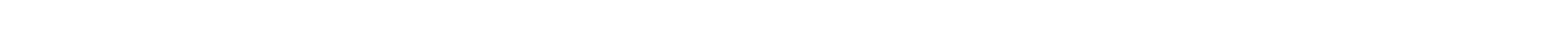

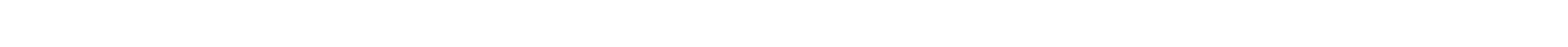

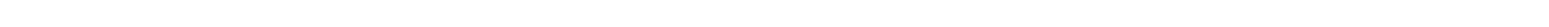

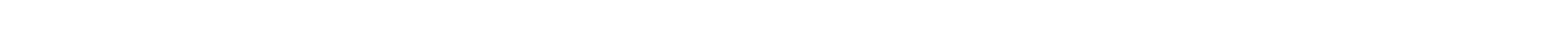

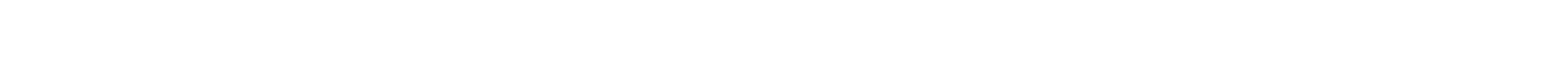

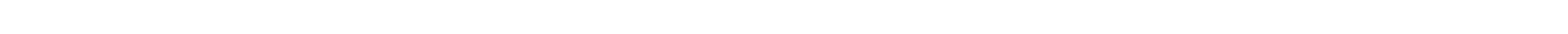

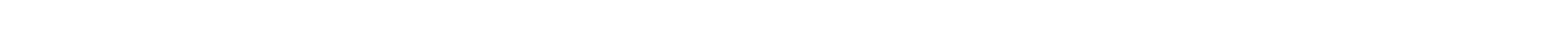

$\stackrel{5}{\Sigma}$

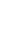

운 w

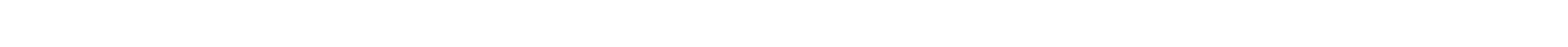

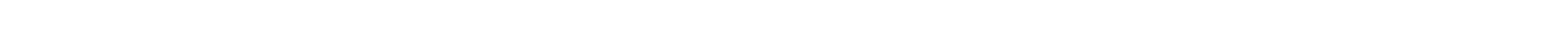

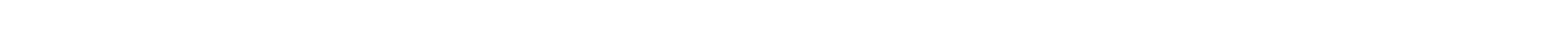

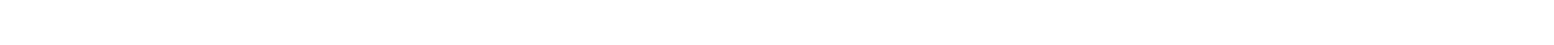

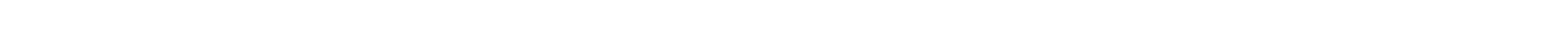

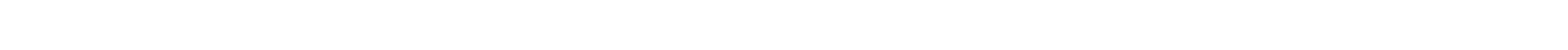

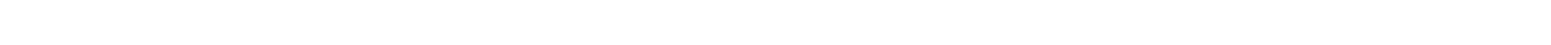

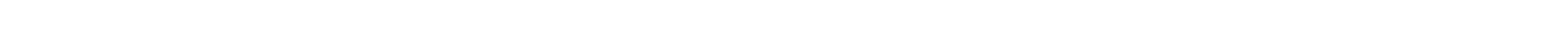

w

$\leqslant$

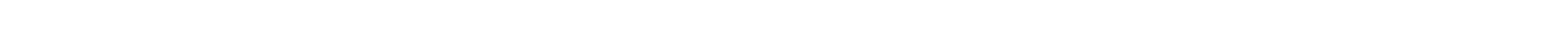
ж

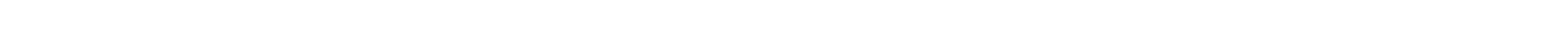

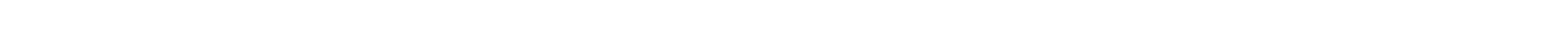

ח M N

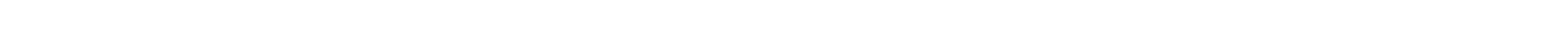

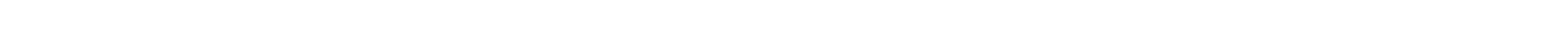

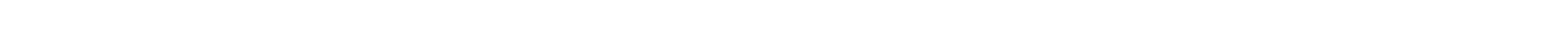

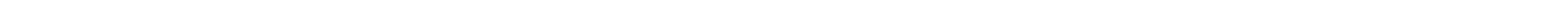

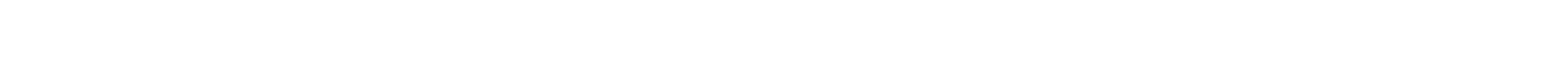

$\stackrel{\leftarrow}{\mathbf{w}}$

$\underline{\mathbf{x}}$

s

$\backsim$

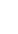

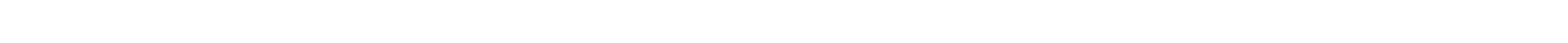

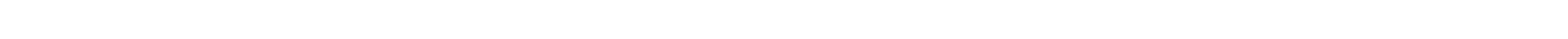

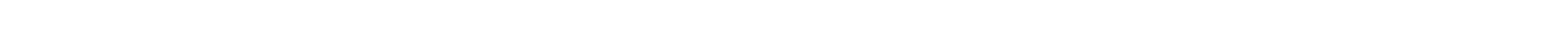

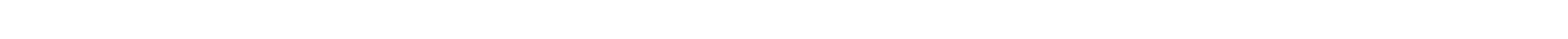

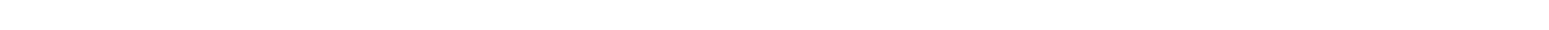

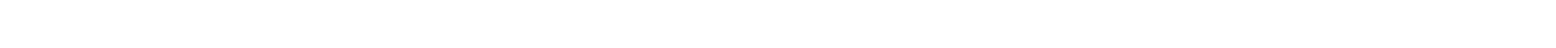

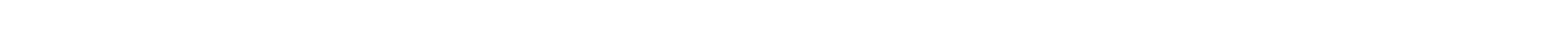

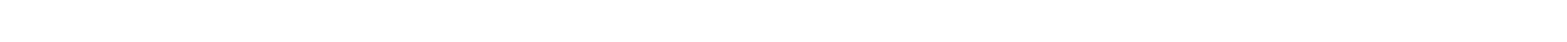

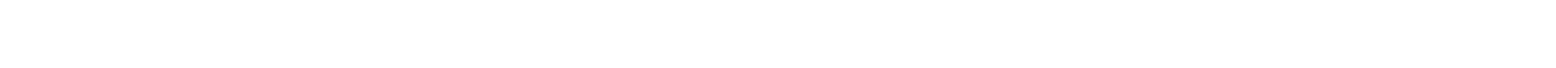


is

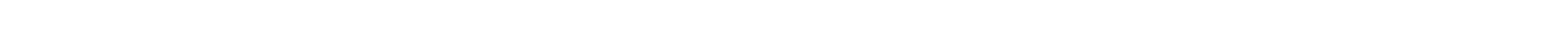
w w

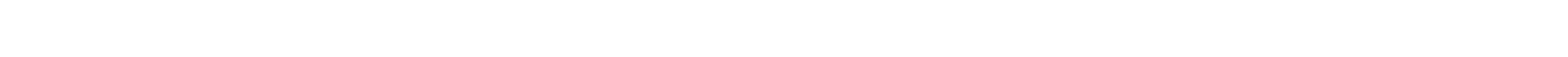

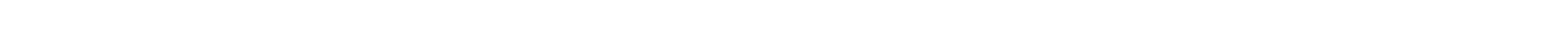

NNN NMmmmMmmmm

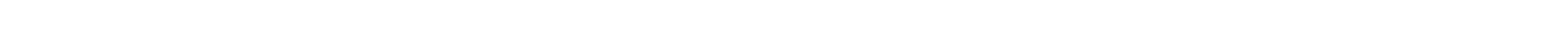
แ

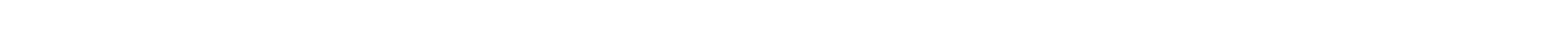
๗

点

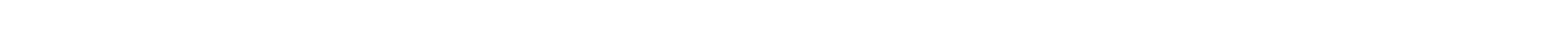
w w

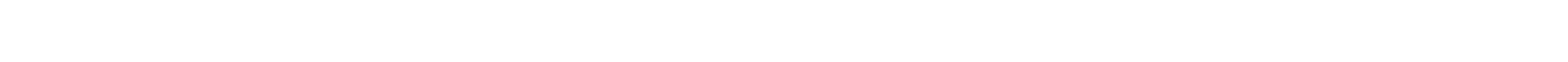

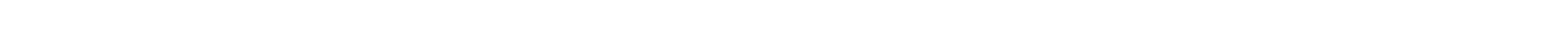

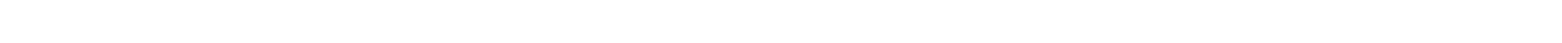

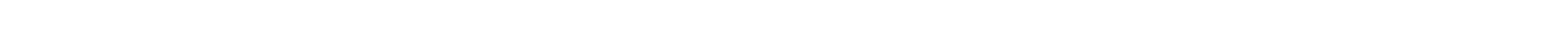

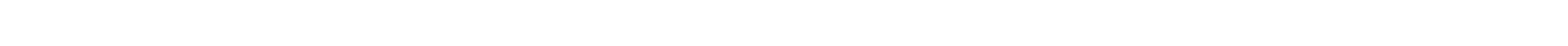

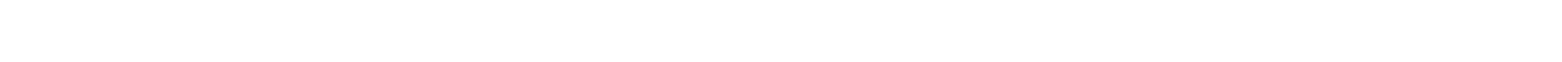

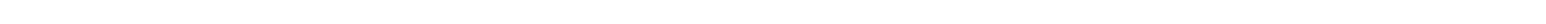

\section{$\stackrel{5}{\frac{5}{2}}$

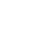

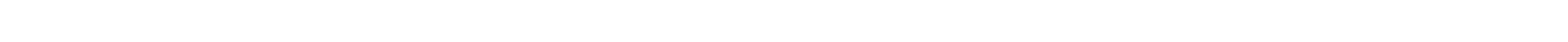
a

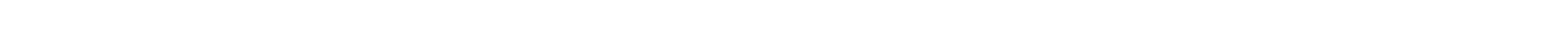

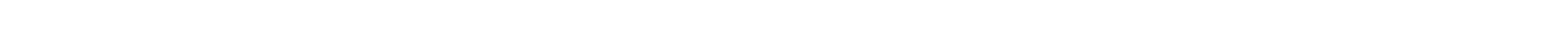

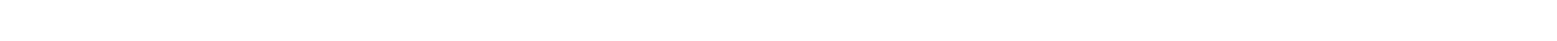

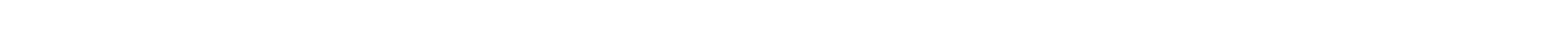

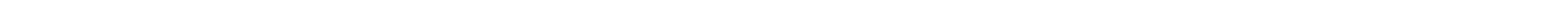

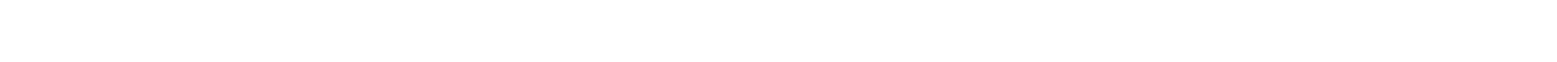

$\stackrel{5}{w}$

$\underline{\Sigma}$

$\pm$

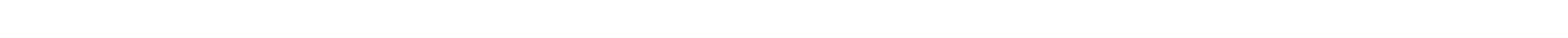

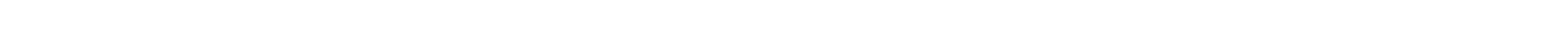

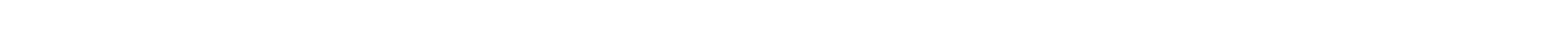

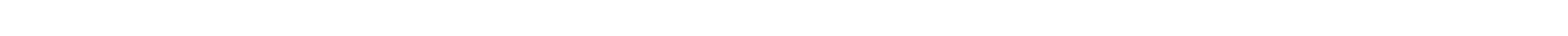

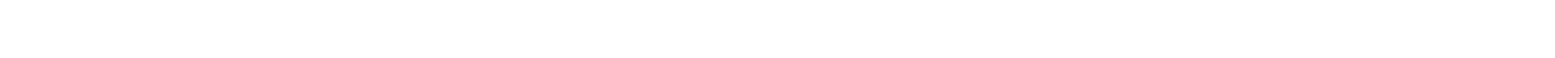

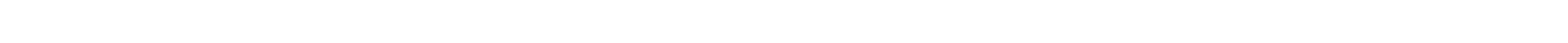

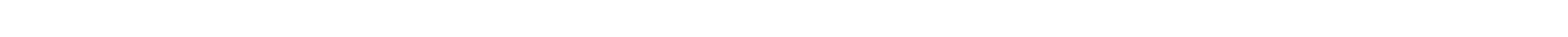

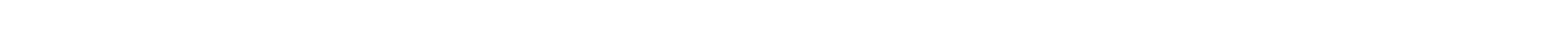

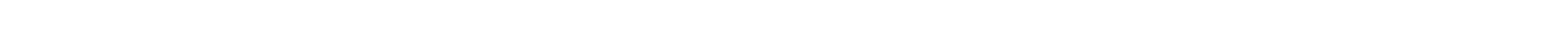


E

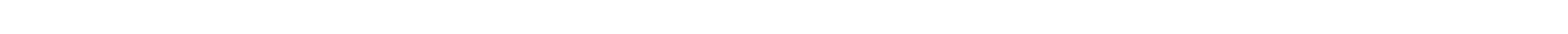

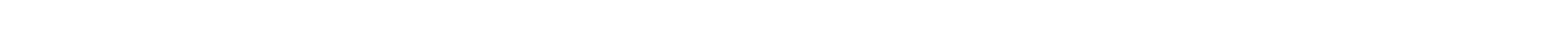

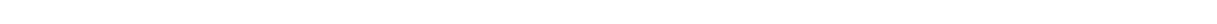

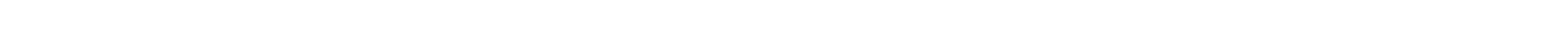

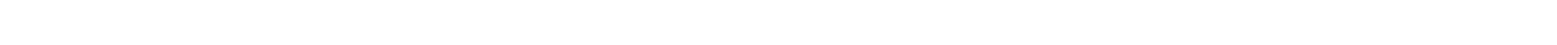

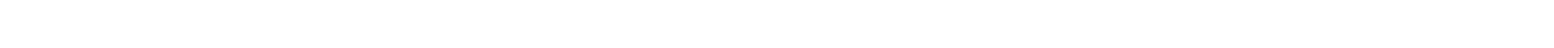
แЕラ

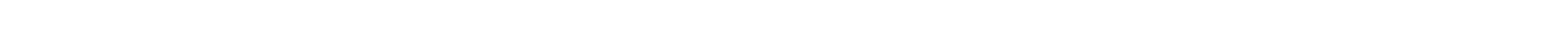

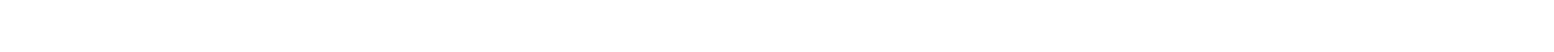

凰 х wááán்

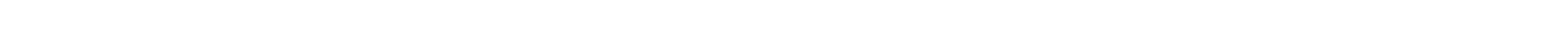

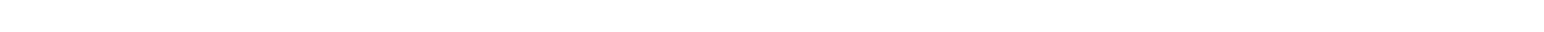

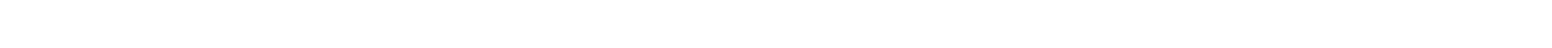

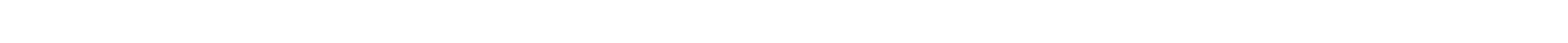

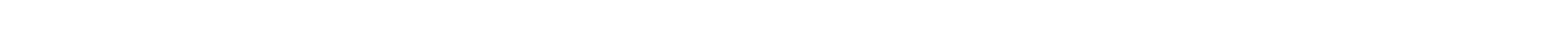

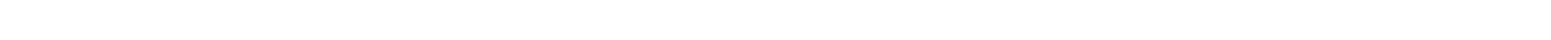

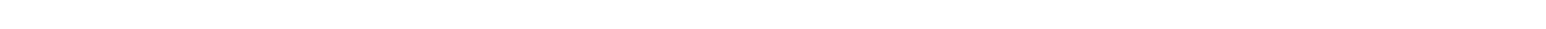

㭊

으은 un

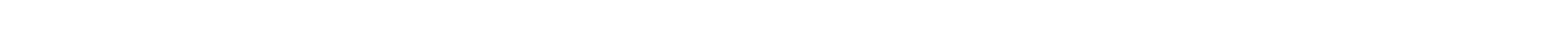

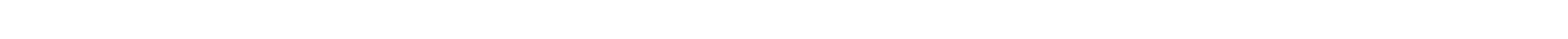

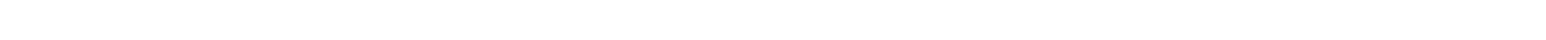

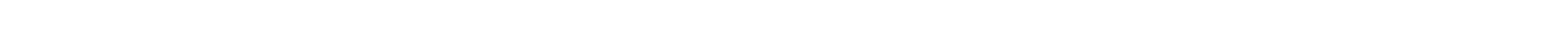

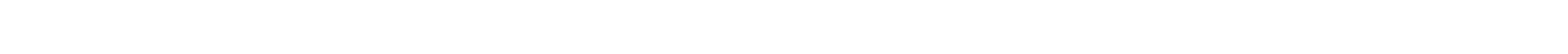

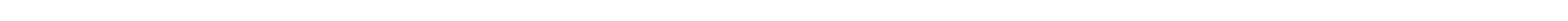

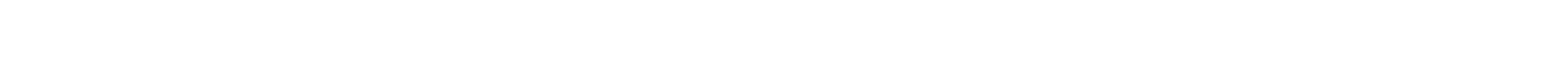

w

en

enes

n

$\stackrel{a}{n}$

- MN "ú

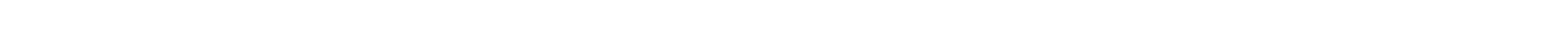
mต้ w tá

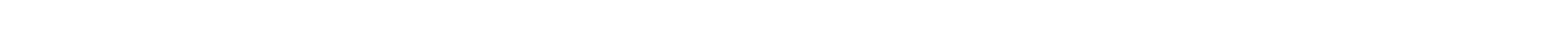

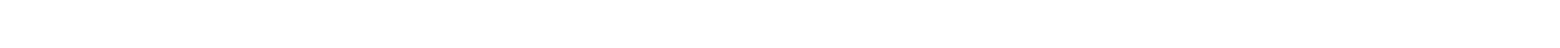




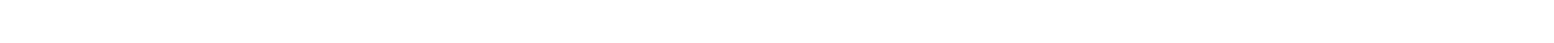

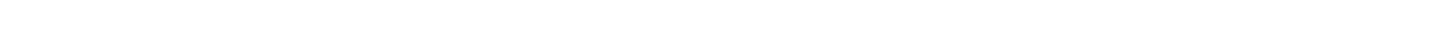

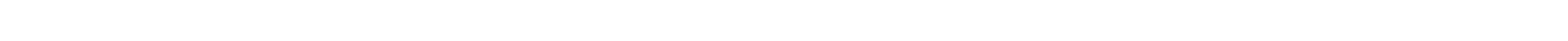

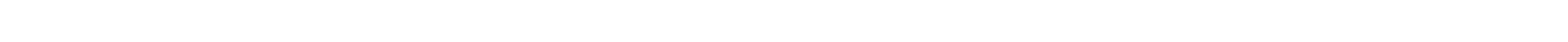

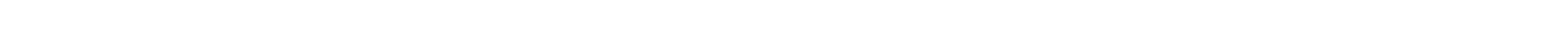

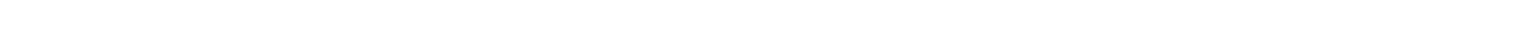

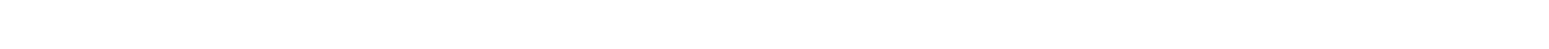

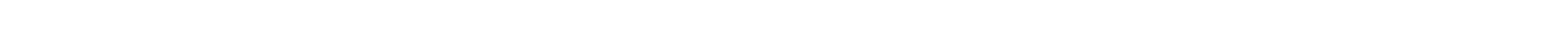

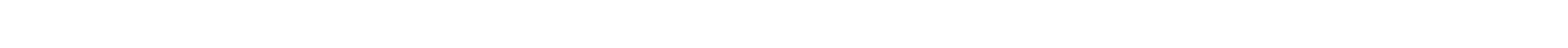

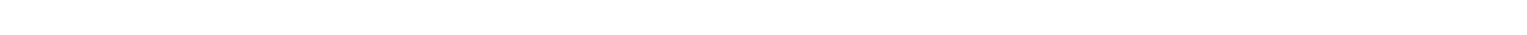

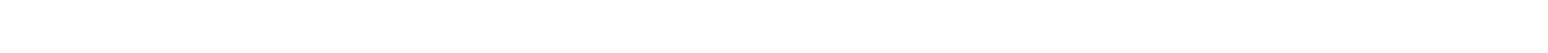

$$
\text { 岕 }
$$

n

no

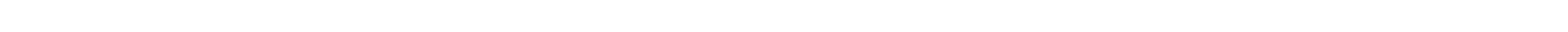

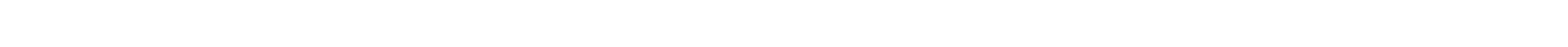
世ú

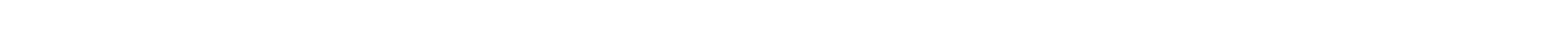

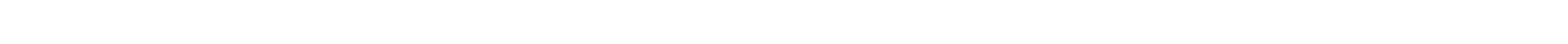

"

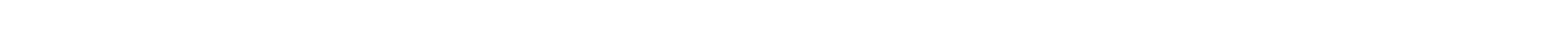

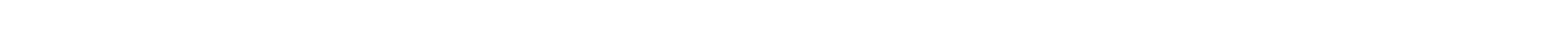
¿nanomon

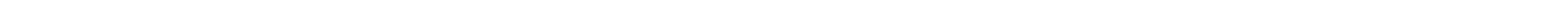

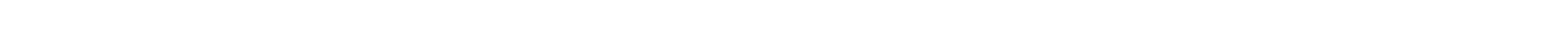

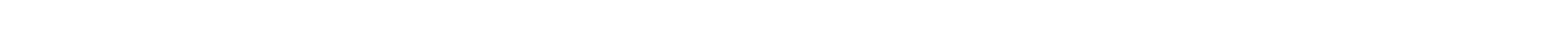
点

n

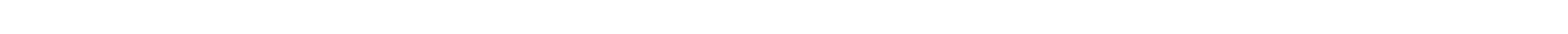

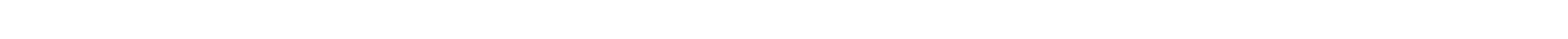
Q

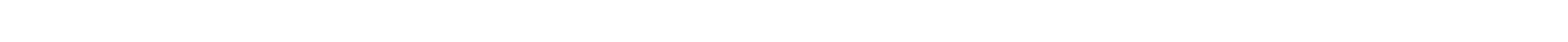

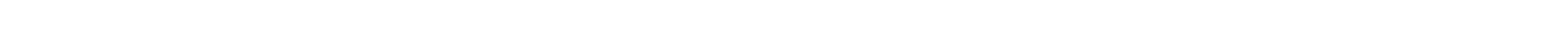
แ FЕ

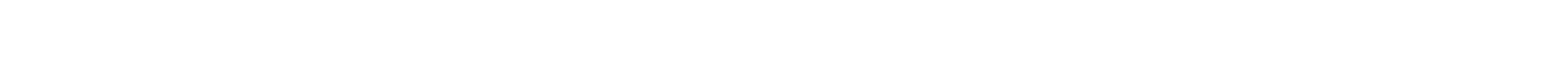

$\stackrel{+}{u}$

$\stackrel{\omega}{\Sigma}$

n

is

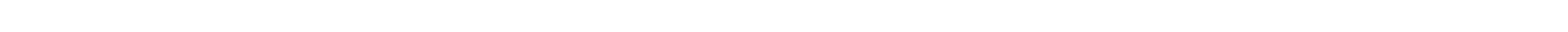

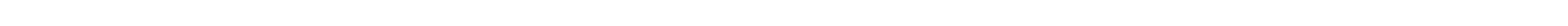

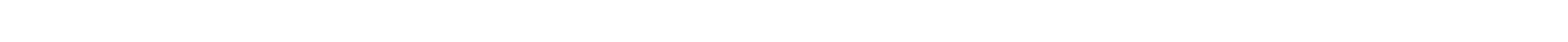
a

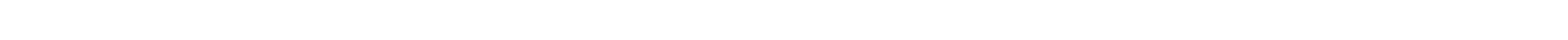

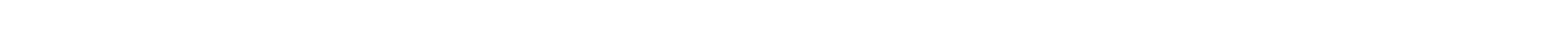

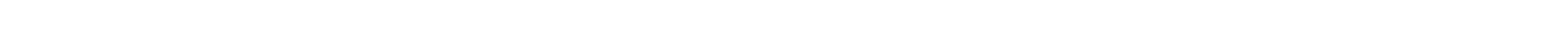

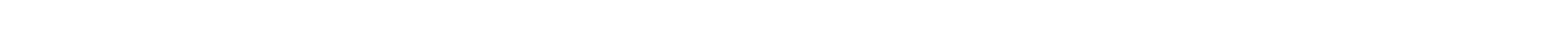

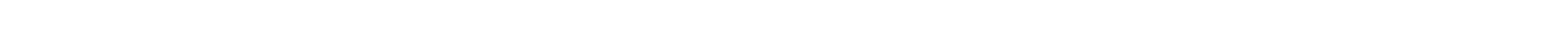

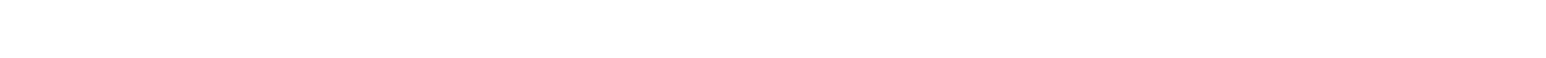


in

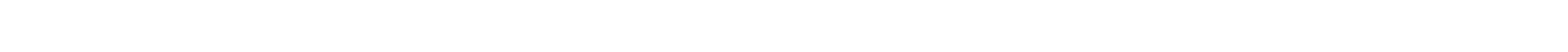

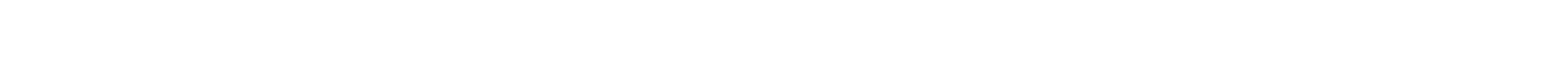

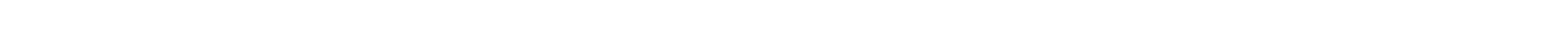

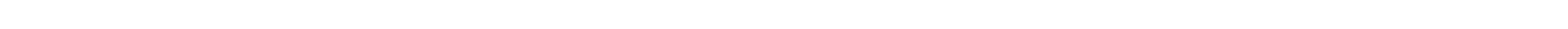

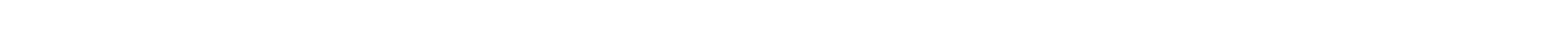
แ

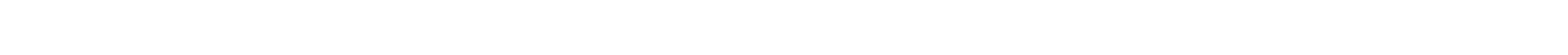

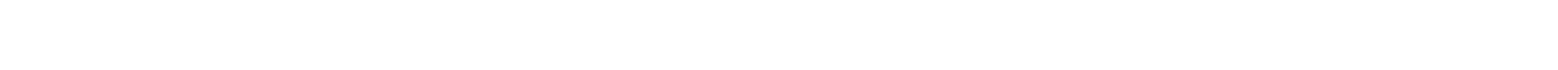

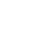

w

n

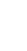

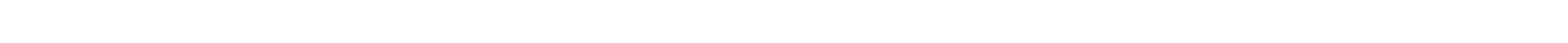
w w —

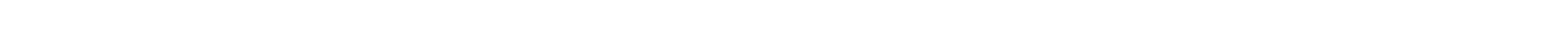

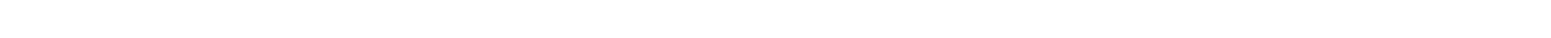

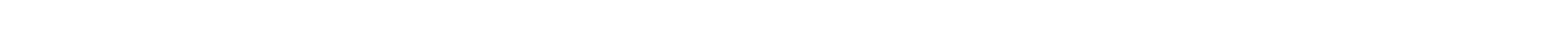
HFЕ

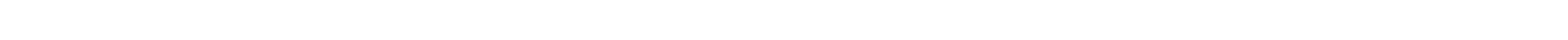

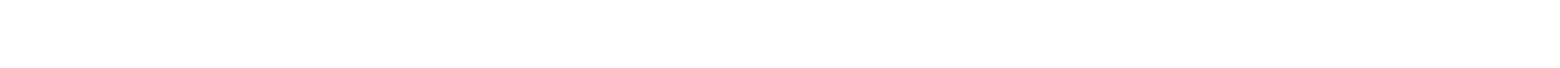

$$
\text { 步 }
$$

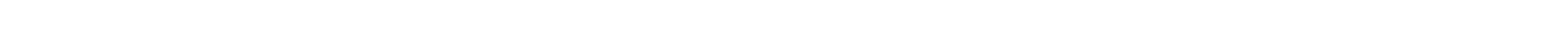

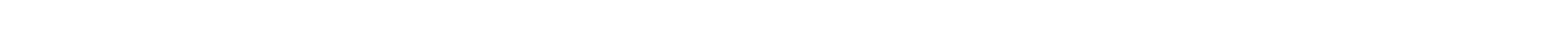

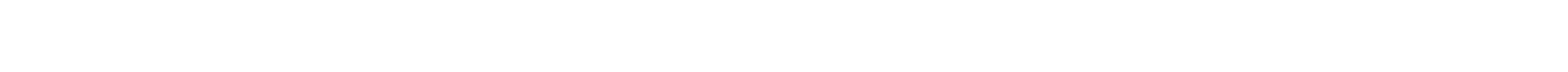

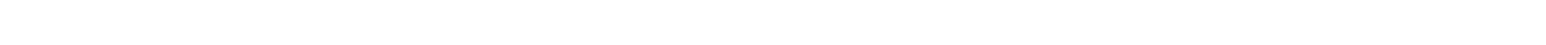

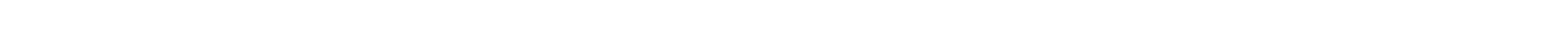

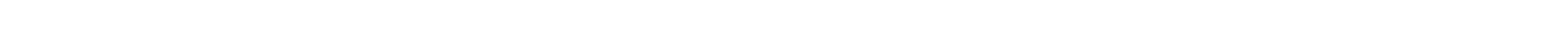

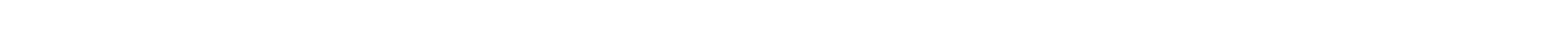

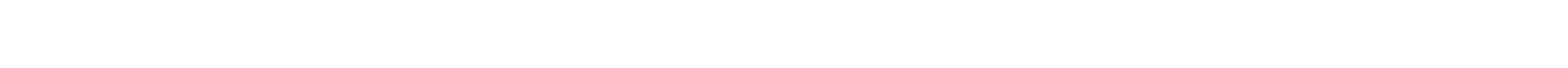

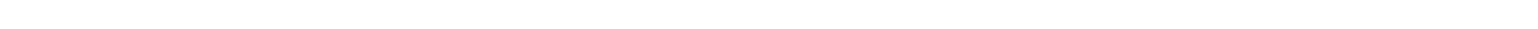

$$
\stackrel{5}{5}
$$

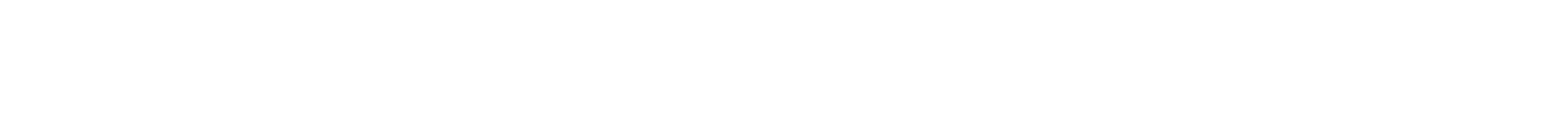

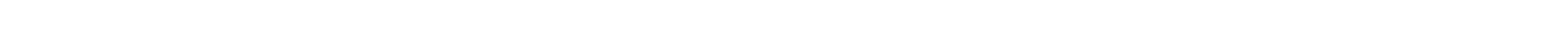

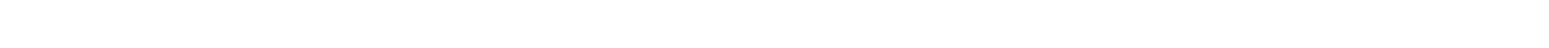

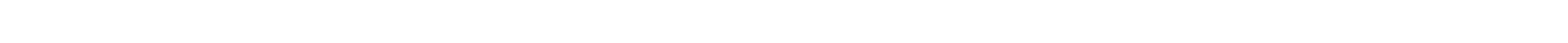

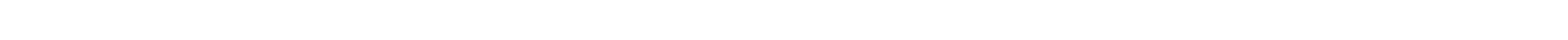

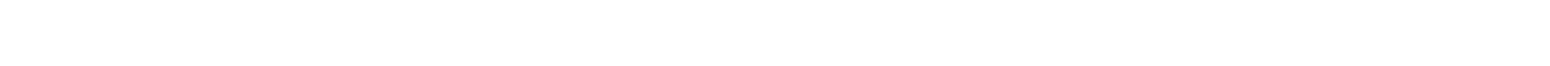




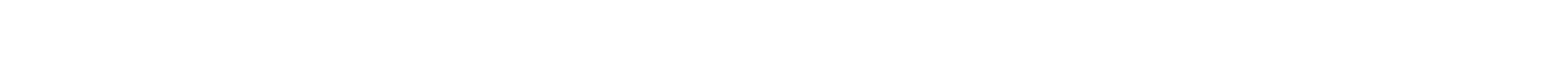

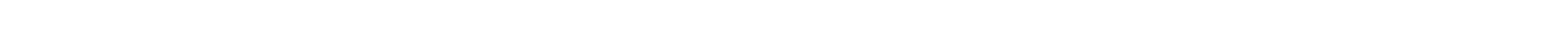

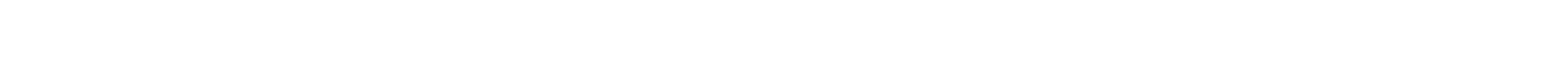

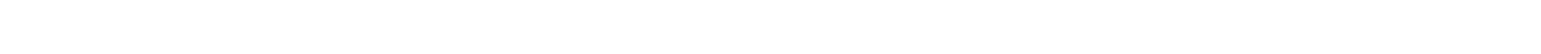

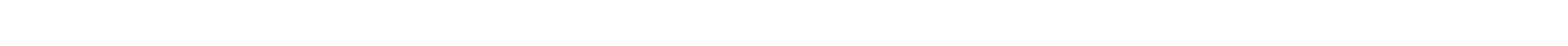

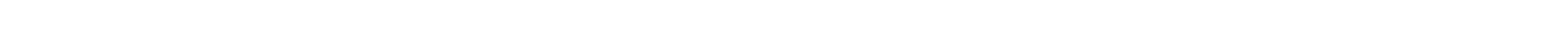

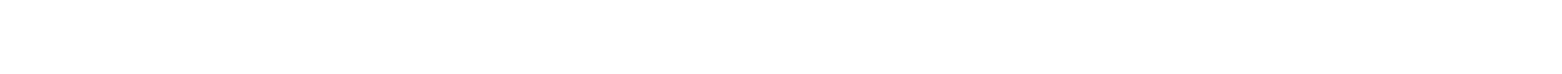

$\stackrel{\leftarrow}{\longleftarrow}$

a

5

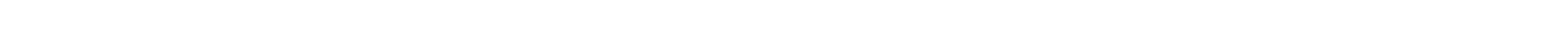
Е ×

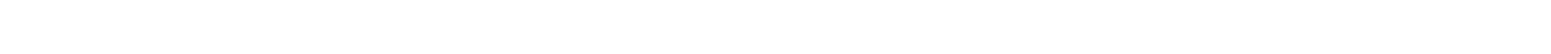

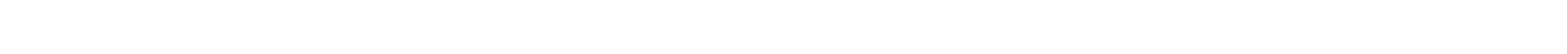

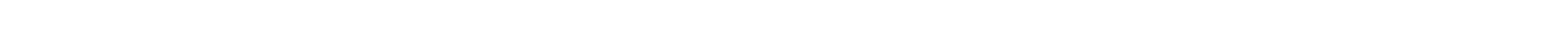

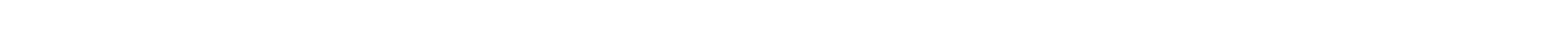

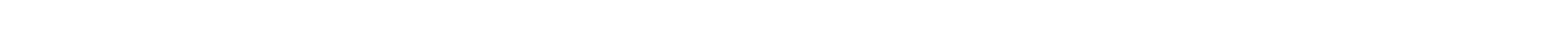

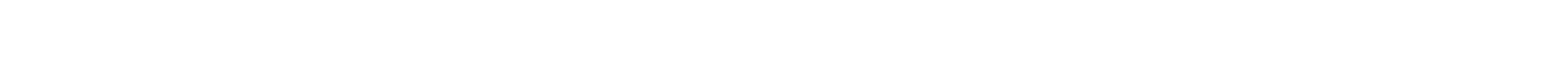

5
5
5

․ แ

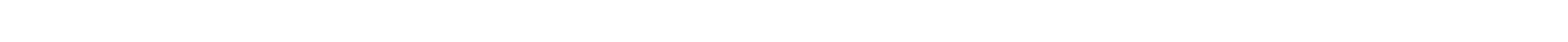

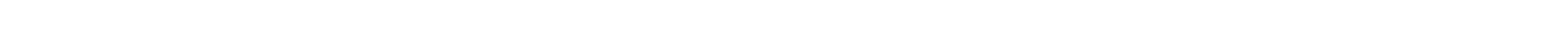

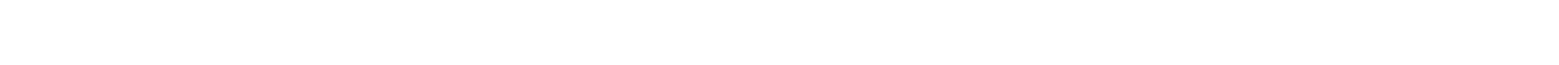

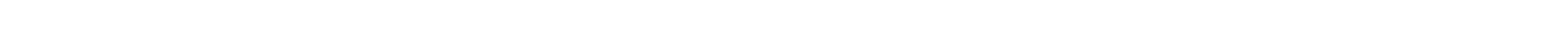

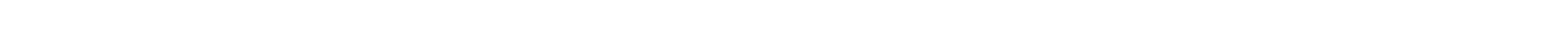

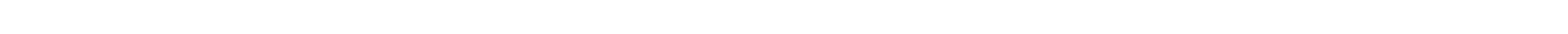

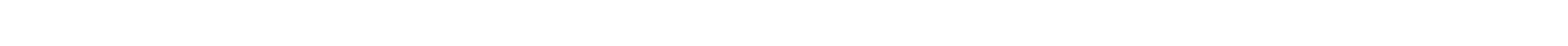
-

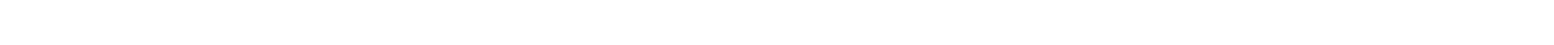

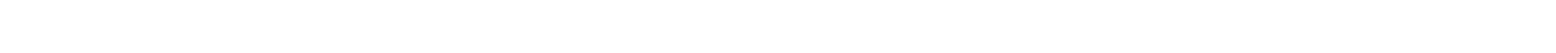

w

2

is

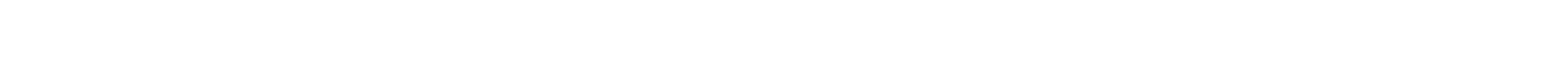
H-

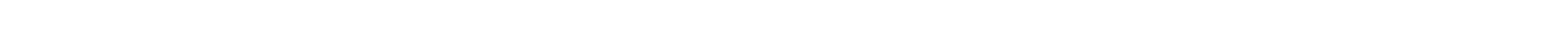

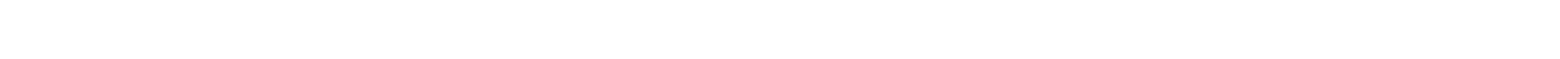

$\simeq \sim \sim \sim N N \sim N N \sim m m m m m m m m m m m m m m m m m m m m m m m m m m m m m m m m m m m m m m m m m$

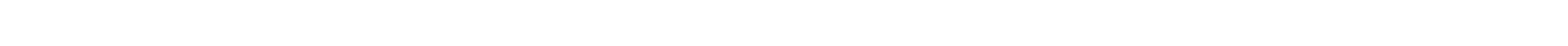

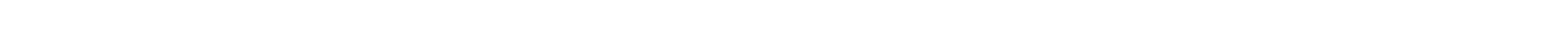

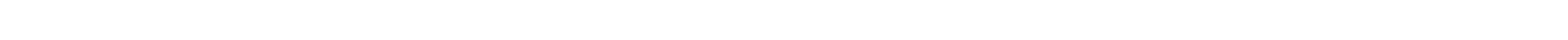

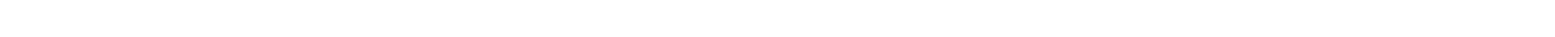

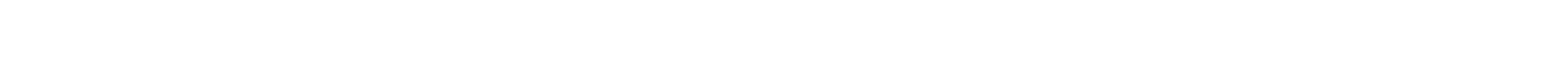


点

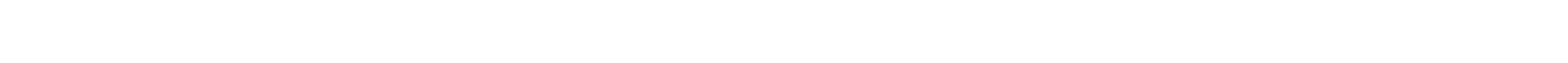

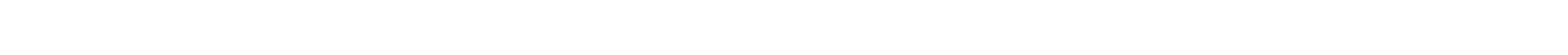
×

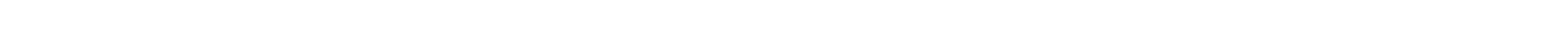

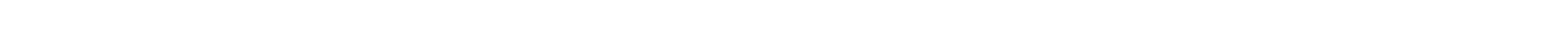

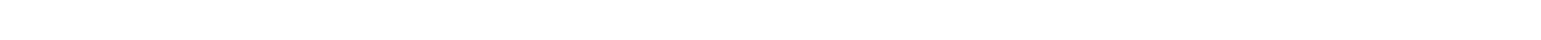

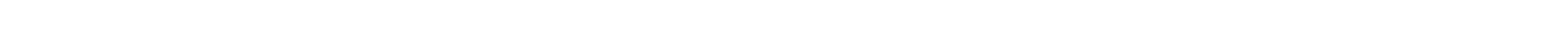

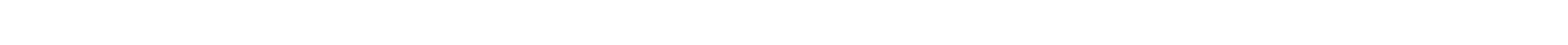

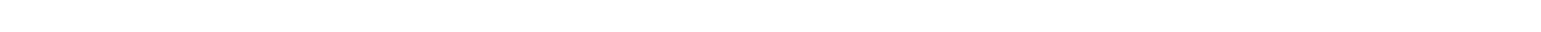

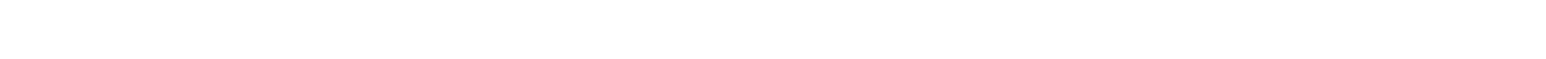

$$
\text { ș }
$$

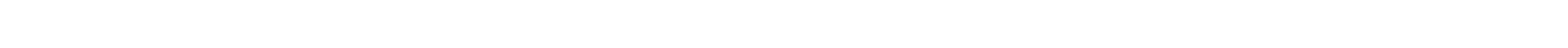
w = w

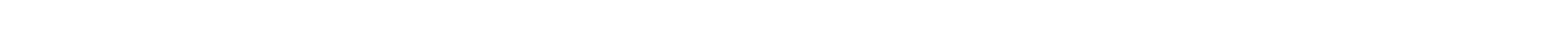

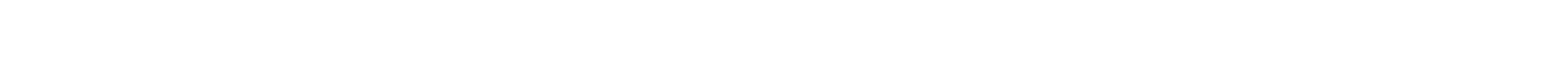

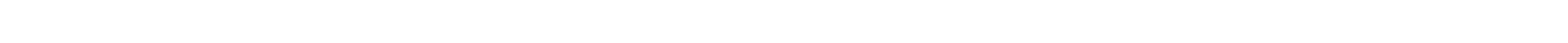

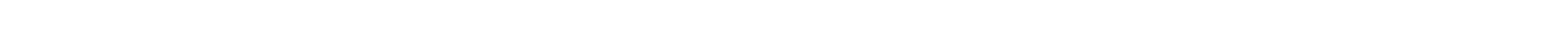

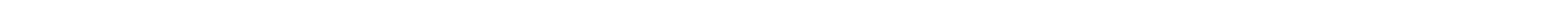

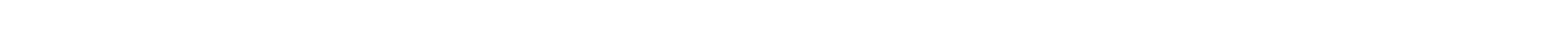

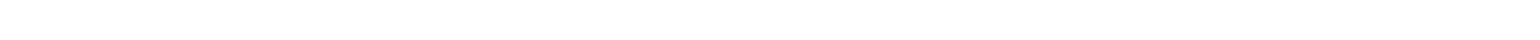

$$
5
$$

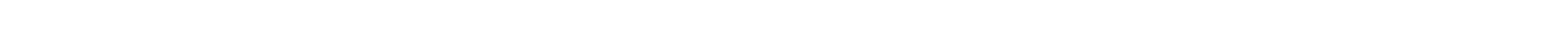
w

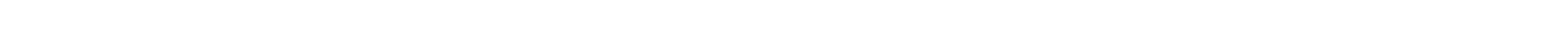

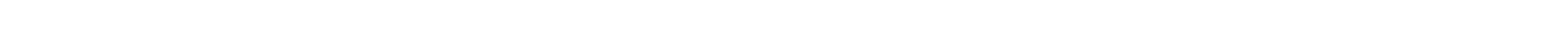

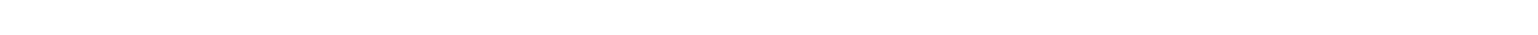

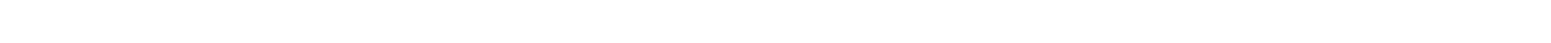

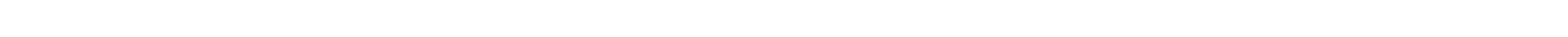

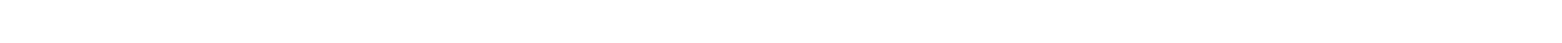

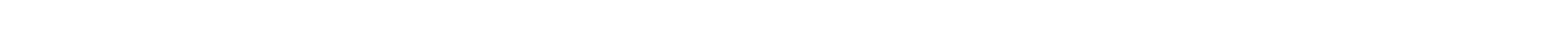

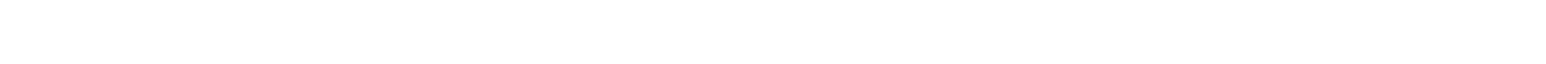

$$
\text { 㭊 }
$$

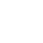

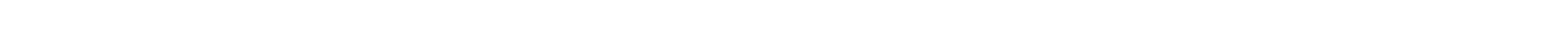

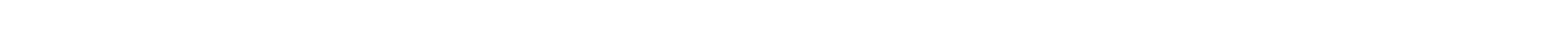

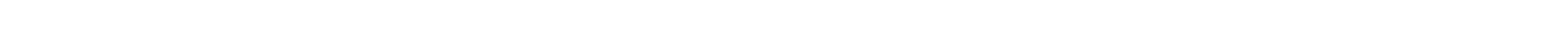

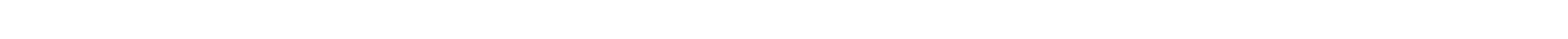

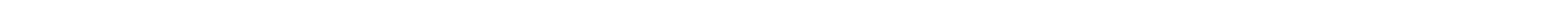

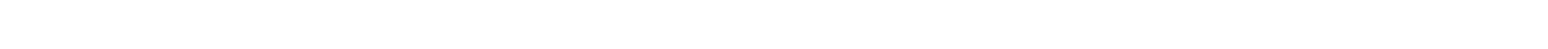

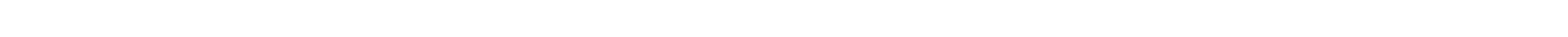

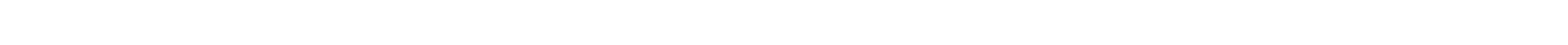

NMㄴ

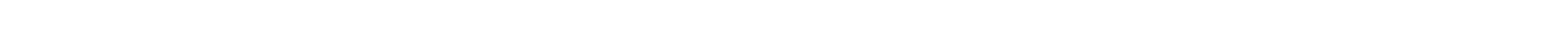


㟧

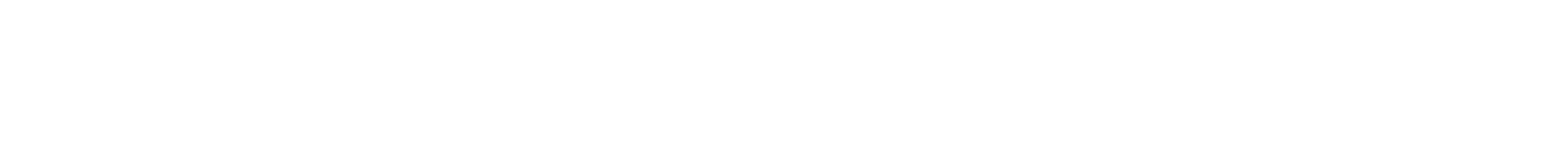

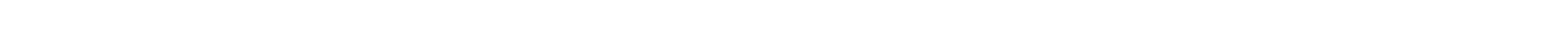

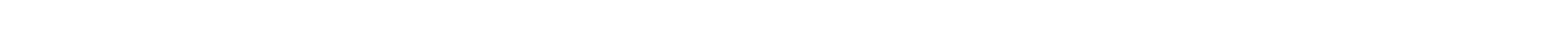

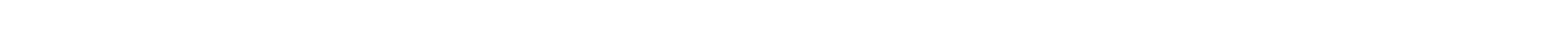

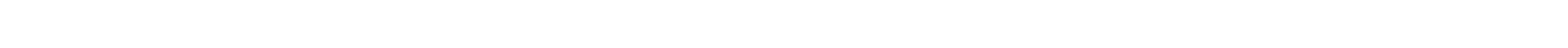

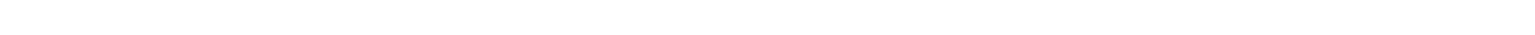

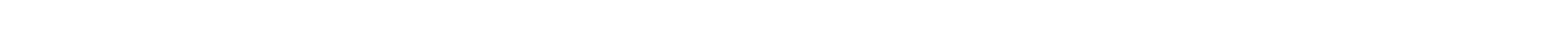

至

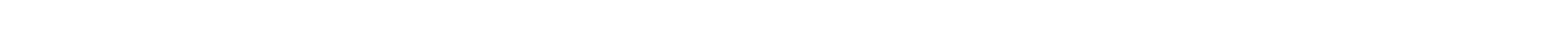
ư

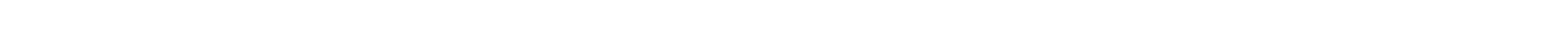

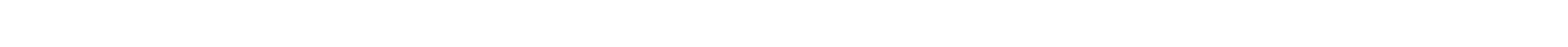

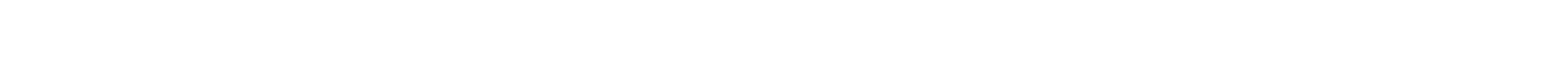

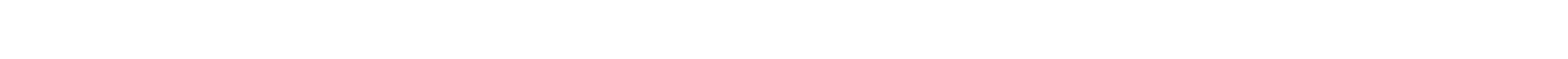

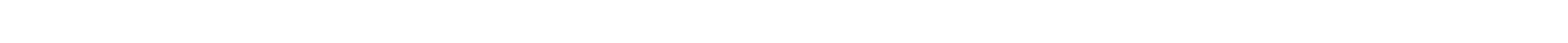

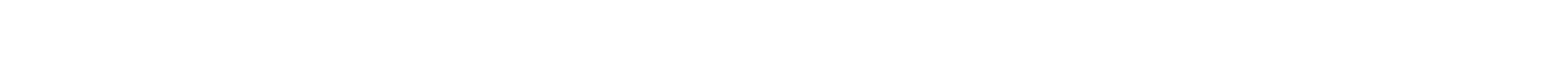

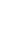

$\stackrel{w}{\Sigma}$

is

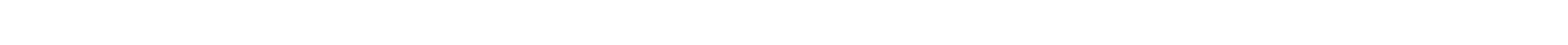

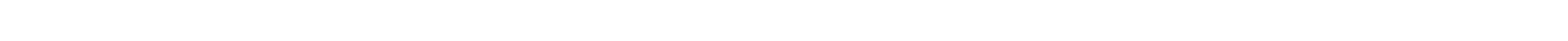

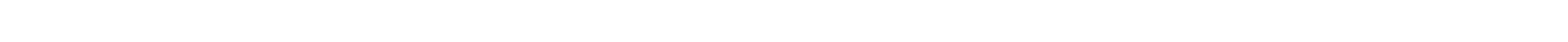

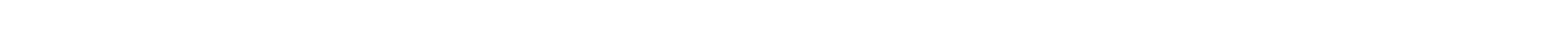

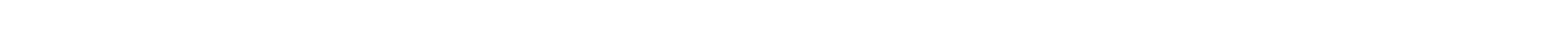

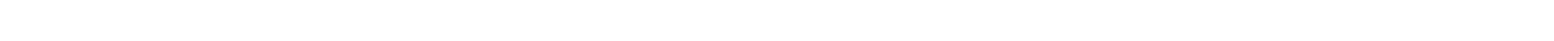

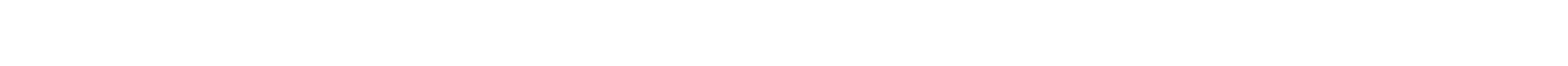

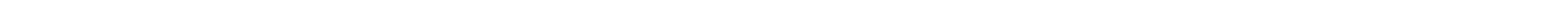

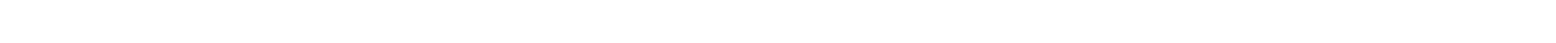

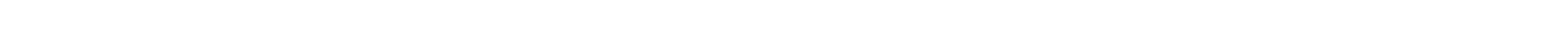

a

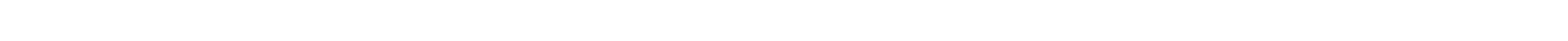
س山ل

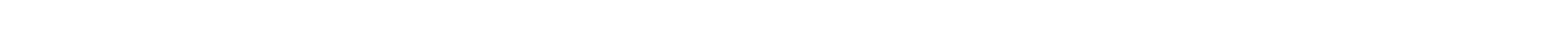

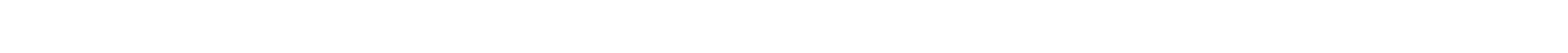

W

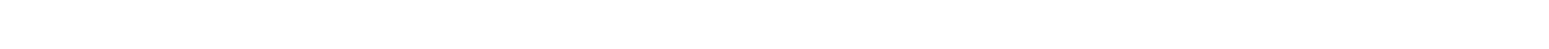

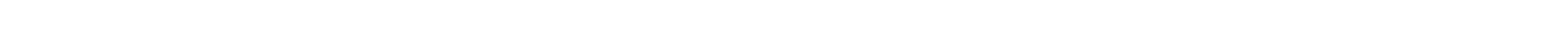

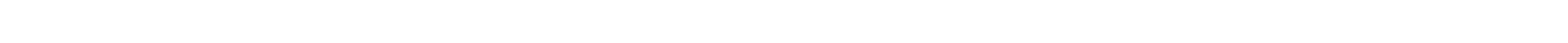

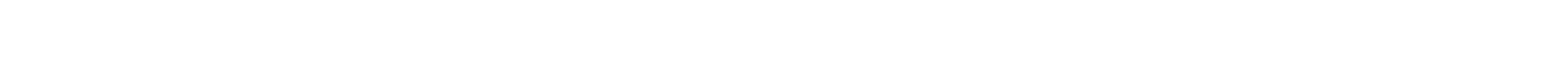




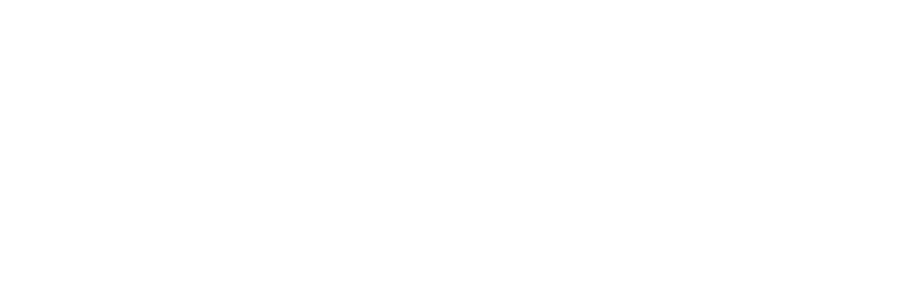

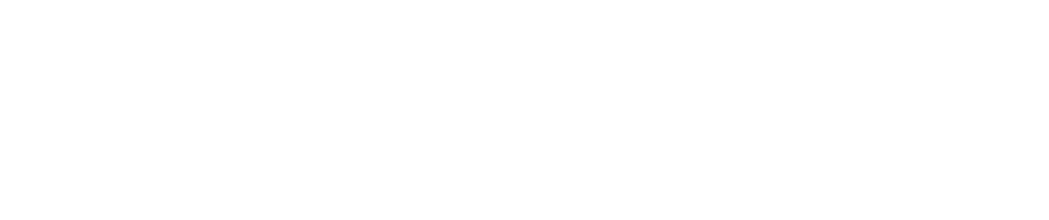

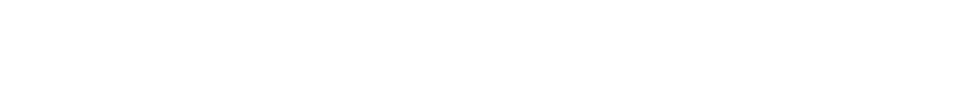

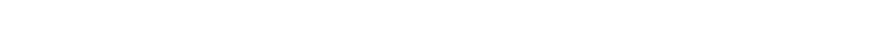
taz? Бळ w - -5- -

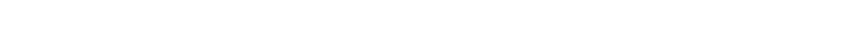

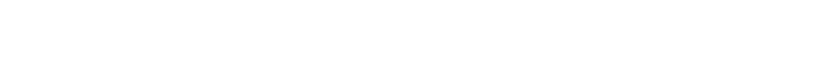

$$
\begin{aligned}
& 5 \\
& 5 \\
& 5
\end{aligned}
$$

× 世山

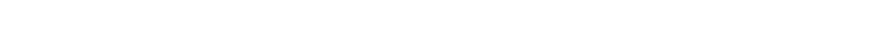

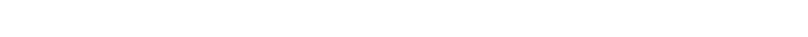

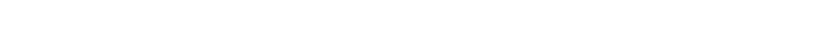

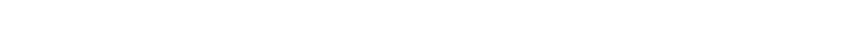

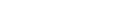

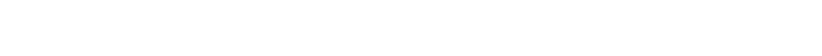

录

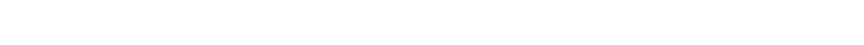
w w

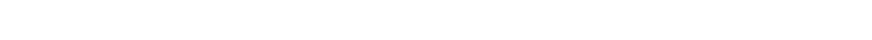

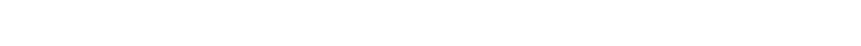

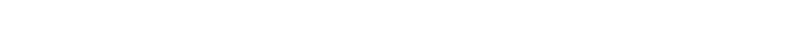

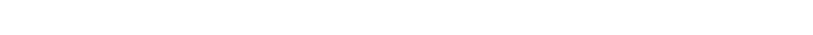

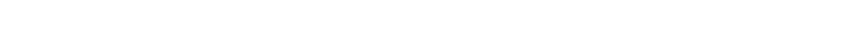

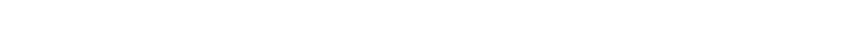

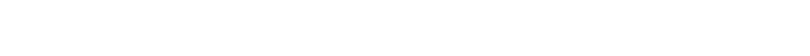




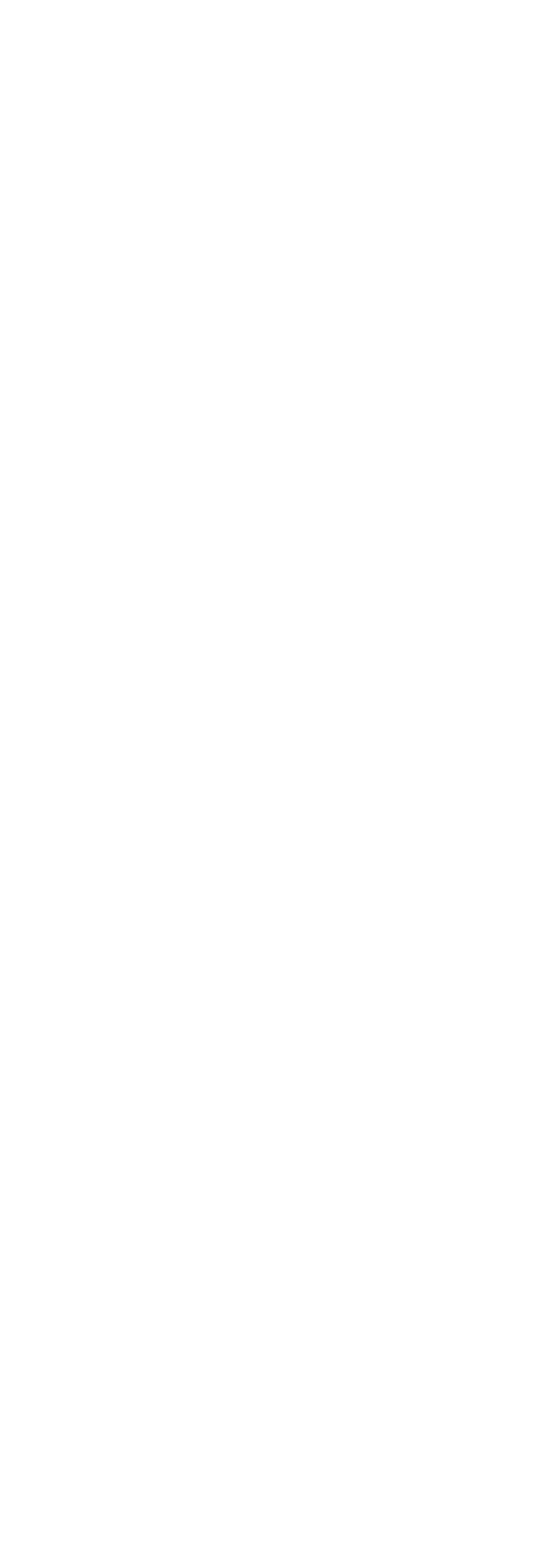




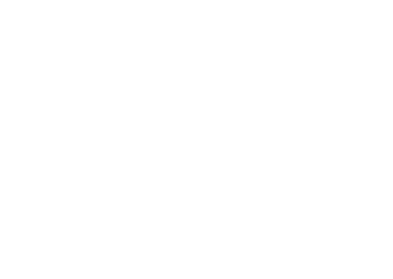

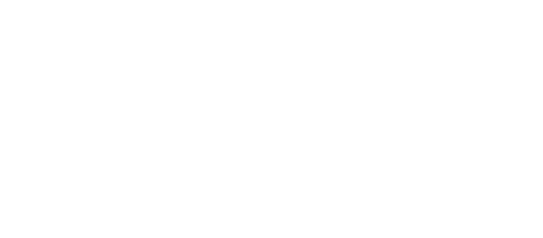

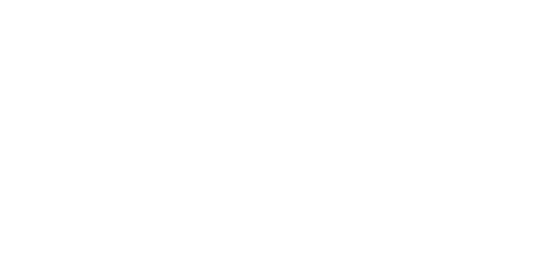

To

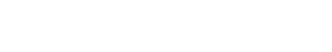

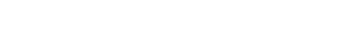

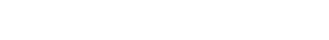

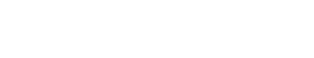

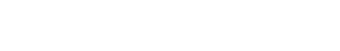

$\Sigma$ is

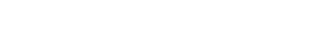
แ

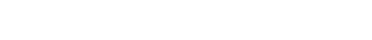
×

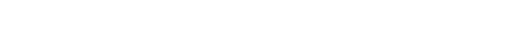

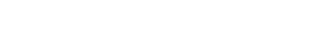

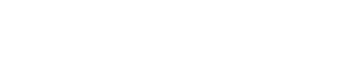
D- KNNG-TON5

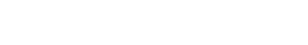

w<smiles></smiles>

is

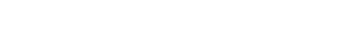
ผ w

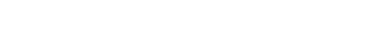

araagaoa00

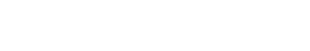

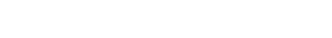

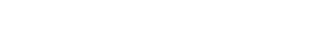
NúñN

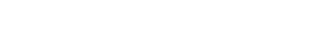




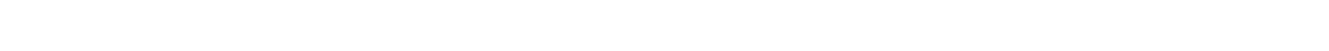

$\leftleftarrows$

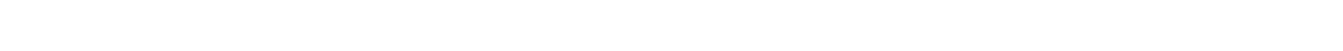
w w

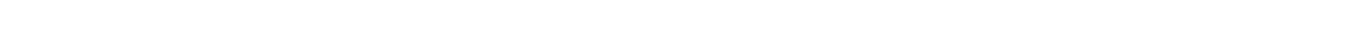

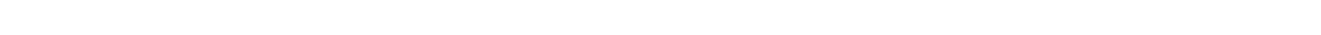

$\stackrel{\ln }{\sim}$

m

in:

范

용

$\stackrel{\omega}{\longrightarrow}$

ख.

的的

000

an-

造

被工。

$=$ 它a

- \pm 0.5

Jౌo a

w 35

or

w us

a.

× 워

a.

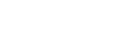

mio 2

언을

U一

可先

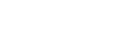

0.0

$\exists \pm \bar{a}<$

$\vec{x}=\frac{\alpha}{2}$

a

- a 证

$\therefore 0 \sim m$

$m: \geq 0$

o $2 \frac{5}{2}$

m‥

=웅

$\overline{2} \simeq \approx 0$

$a=-\div$

m.

$+\infty$

- $2 \prod_{0}^{2}$

c. 50

c

은.

w $\because \ddot{0}$

Ex r

"à in

$>\approx n-$

ब्रa

는

(2)

은

$\div$

$\underset{\check{I}}{\ddot{O}}$

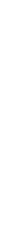

$\frac{0}{2}$

-

ï

둥

으

wis

w

$\sum_{m}^{0}$

in

aㄹ

जั

क)

00

与世

녕영

$a=z$

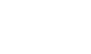

虫

u.

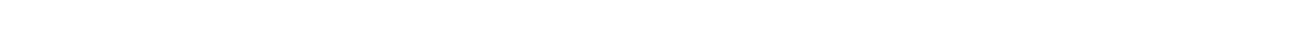

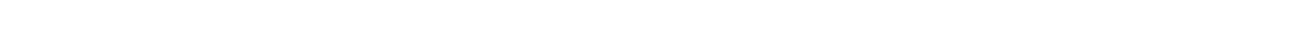

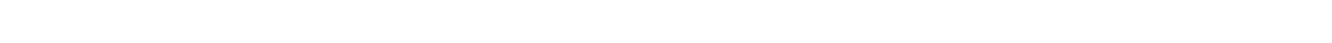

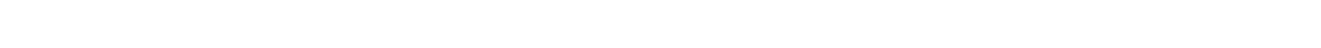

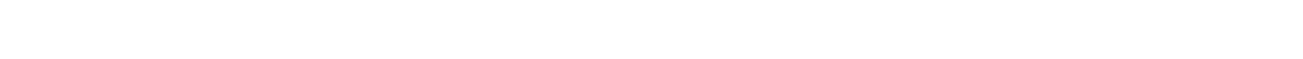

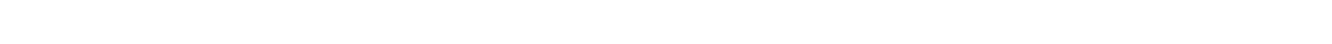

$\alpha$

is

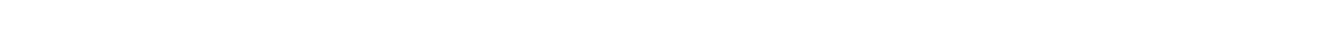
\& แ

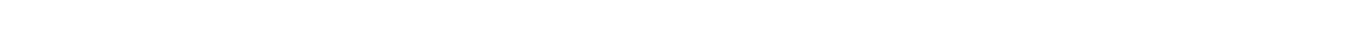

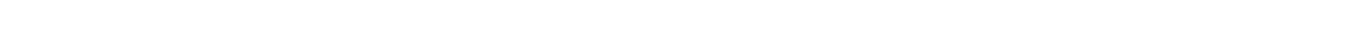

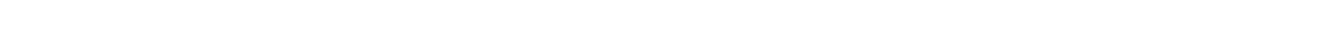

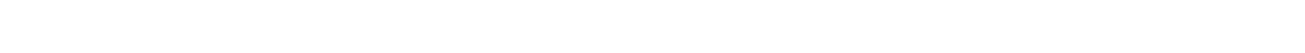

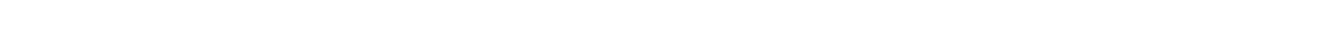

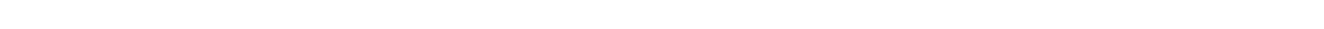

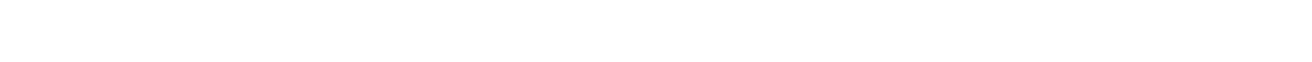

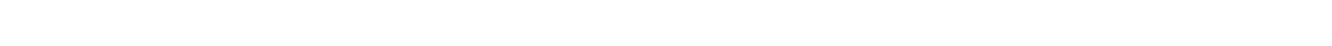

a

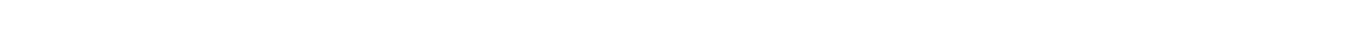
a

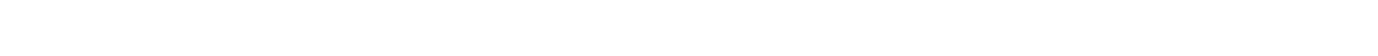
× -

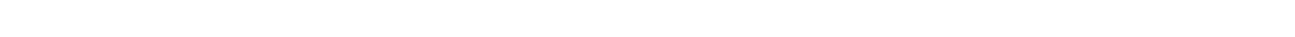

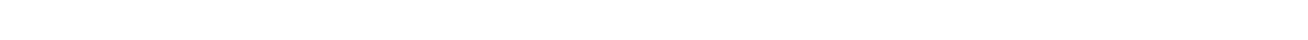
w

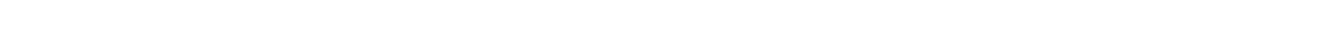
O NOOFNMOFNNOF-NOF-NOO-NOO-NNO-NNOFENMOENNOFE

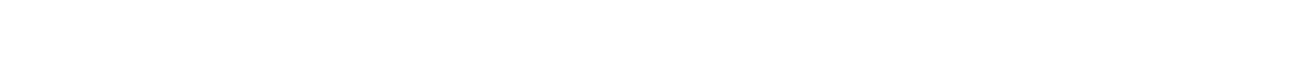

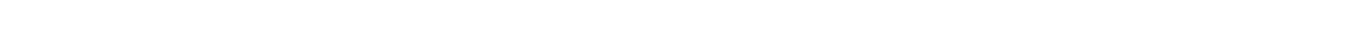

点

х w w

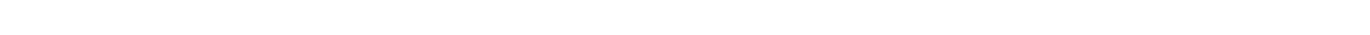
-

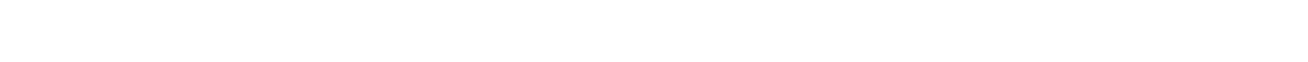

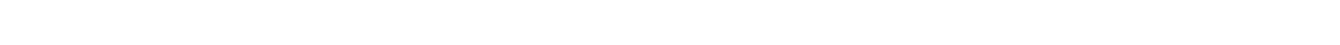

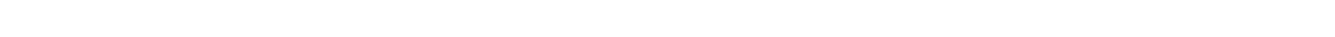
-

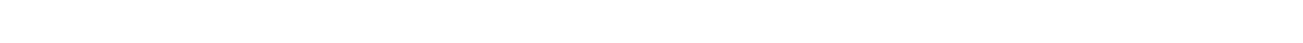




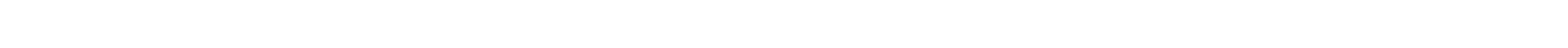

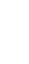

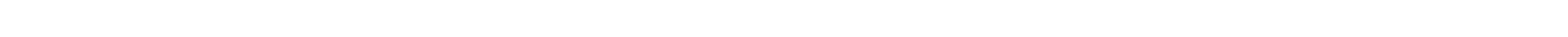

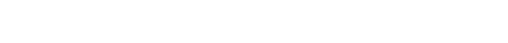

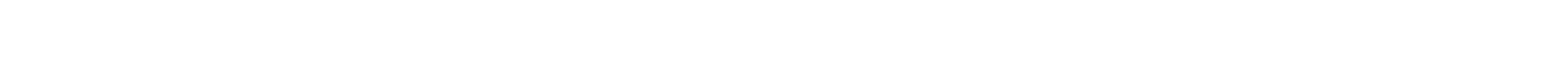

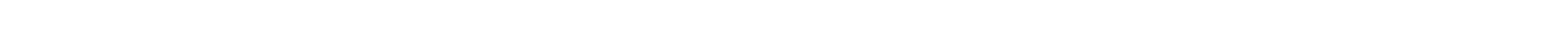

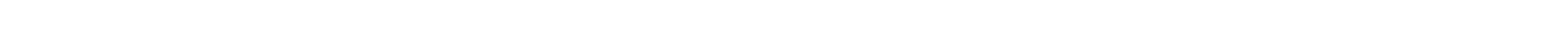

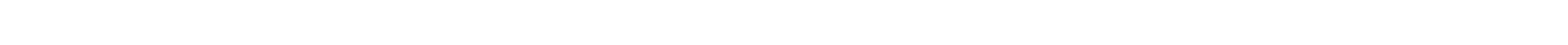

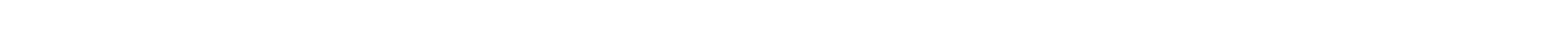

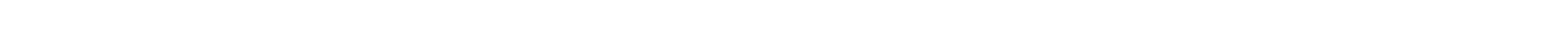

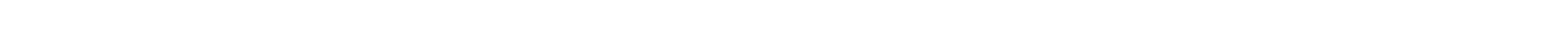

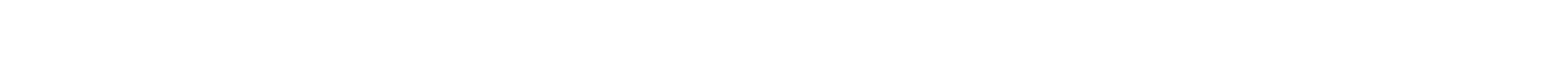

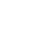

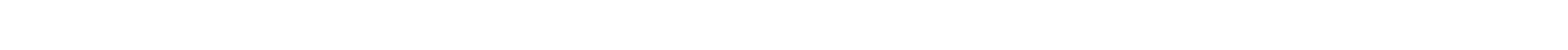
$a$

兵

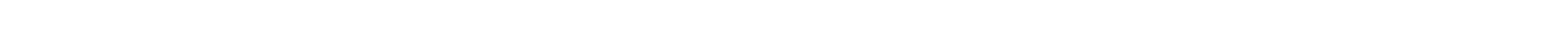
u w Ł

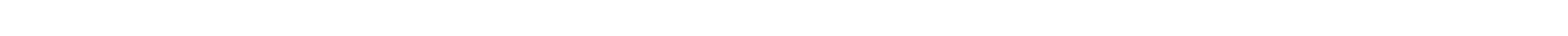

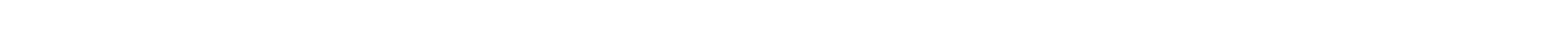

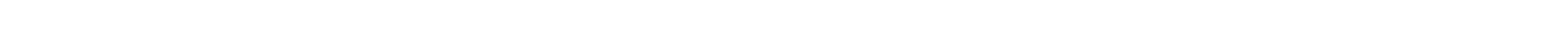

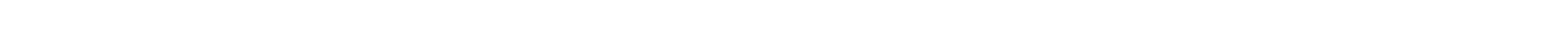

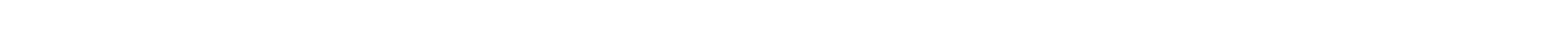

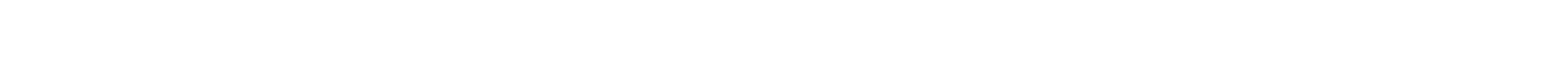

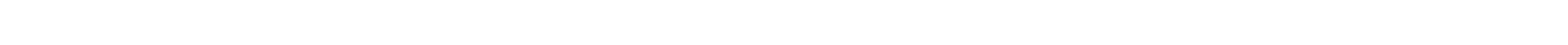

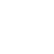

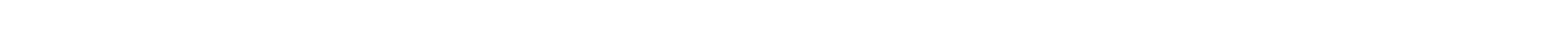
w एँ

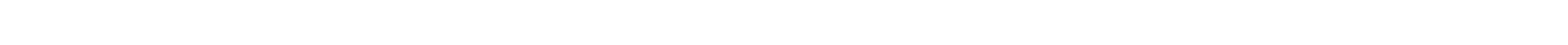

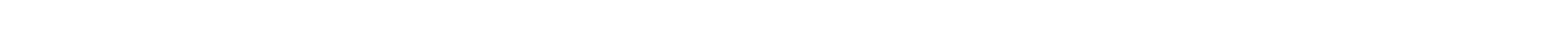

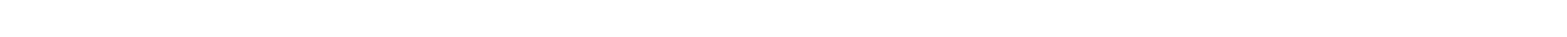

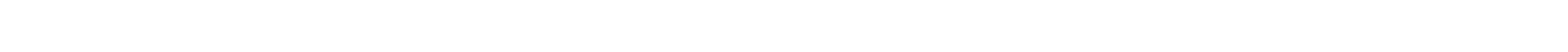

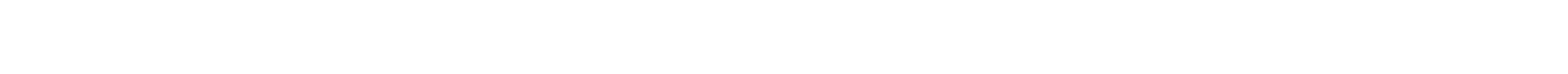

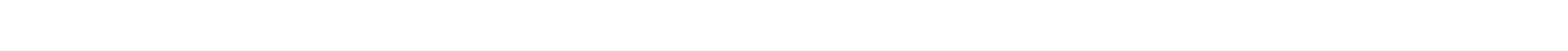

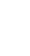

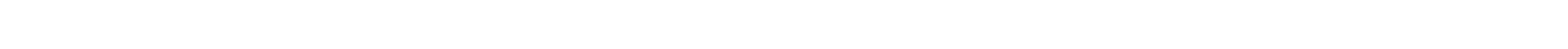

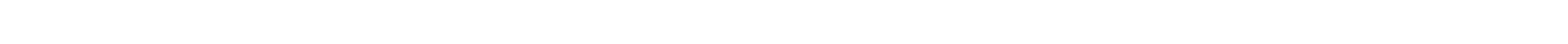

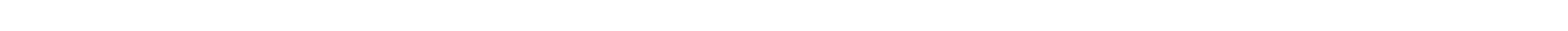

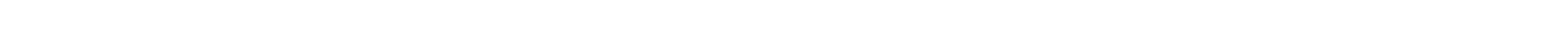

mmmmmmmmmmmmmmmmm

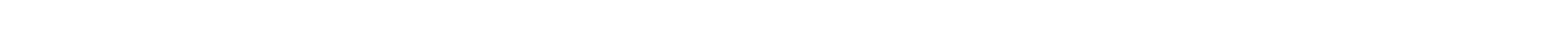

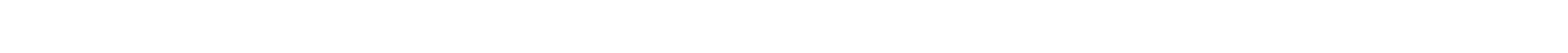

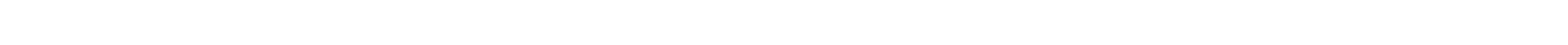

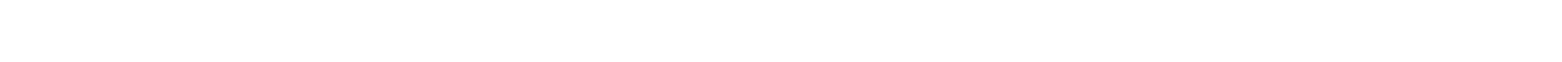



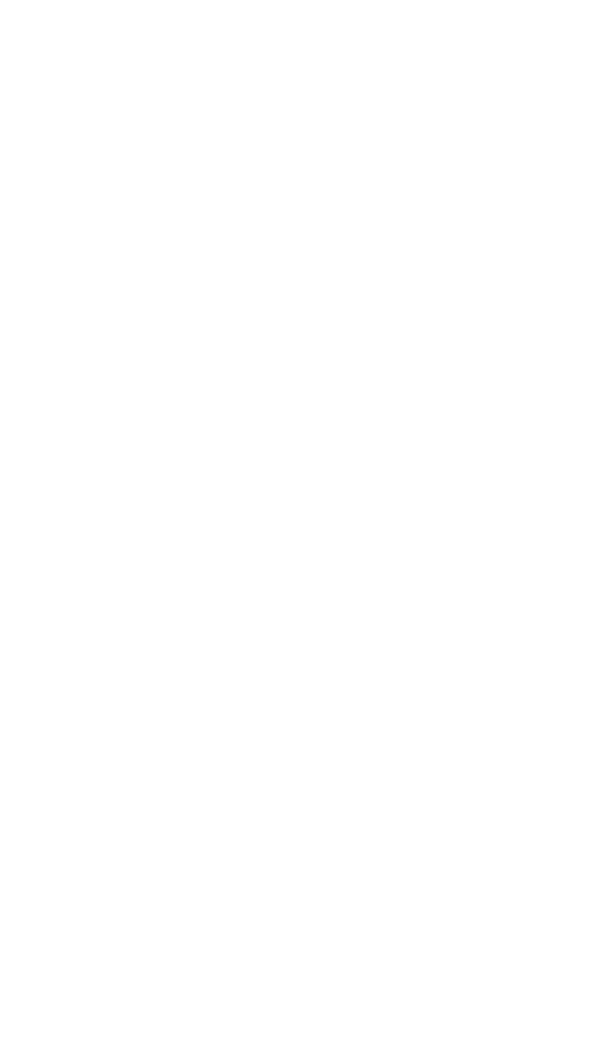

$$
\text { twesciosencosencescoses }
$$

N

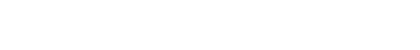
t

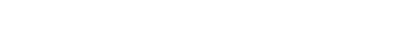

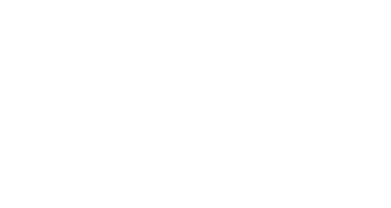

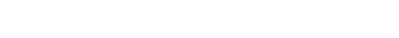

is

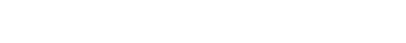

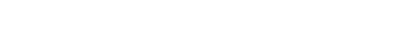

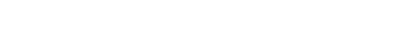

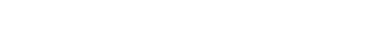

NNMMNaNMMOON

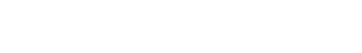

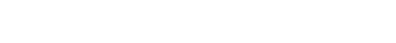

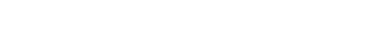

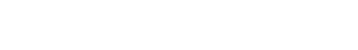




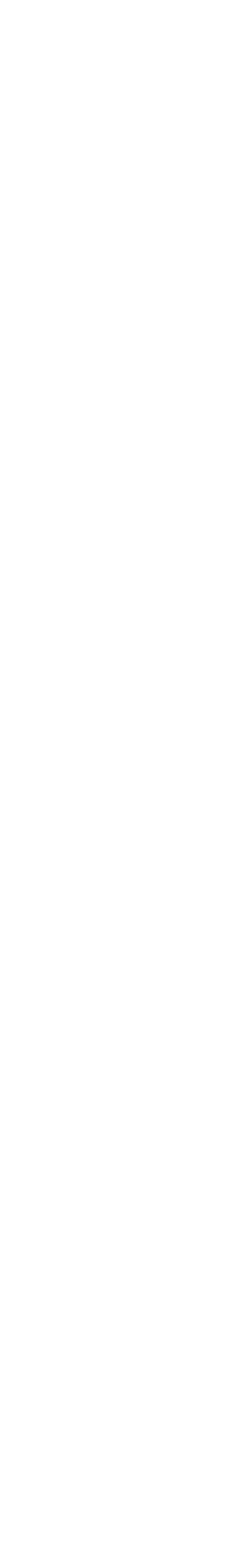




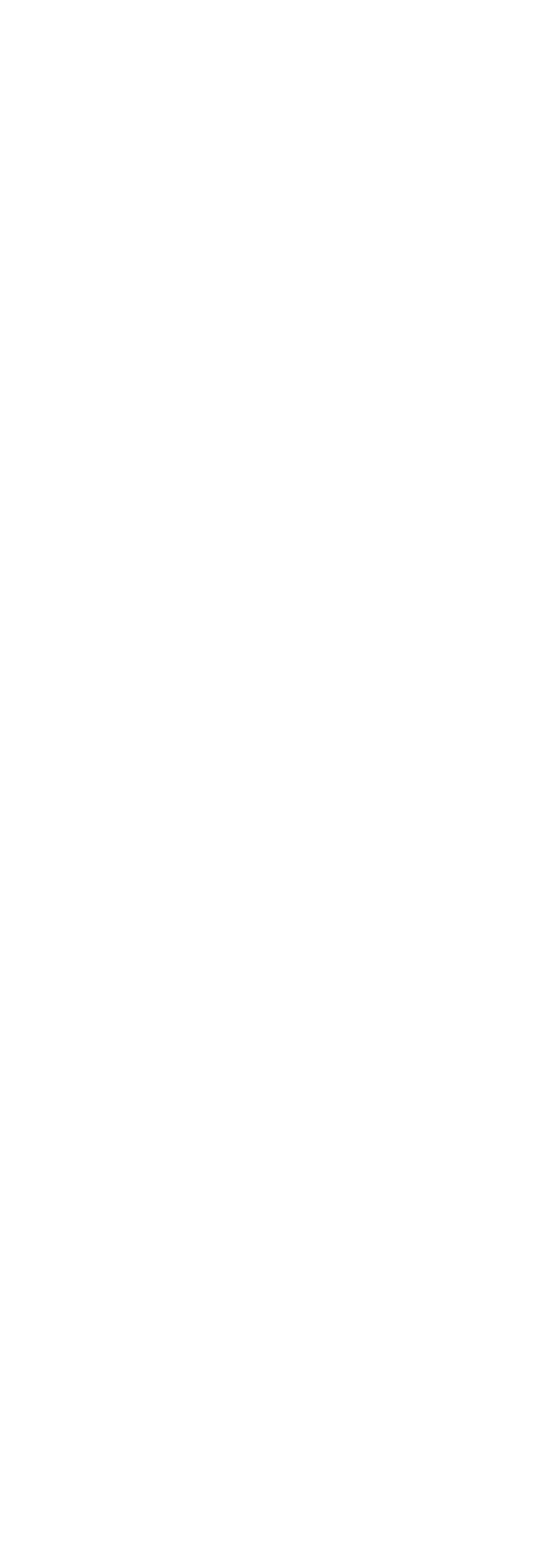

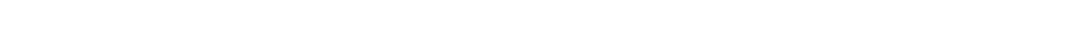

i

n uा ?

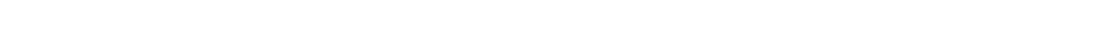

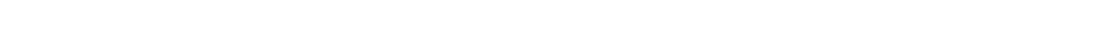

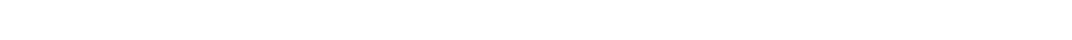

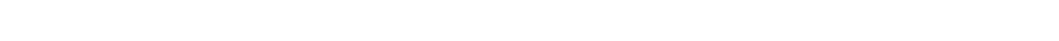
س

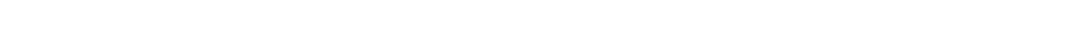

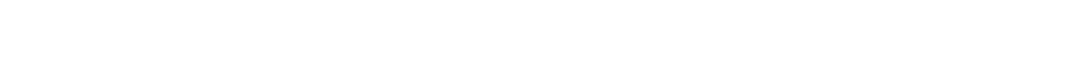

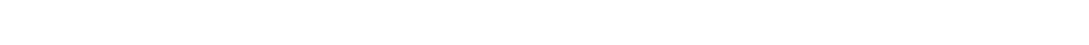
in

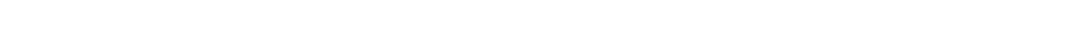

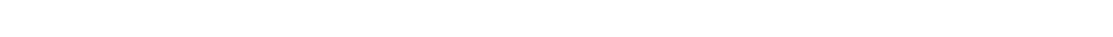

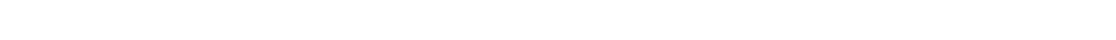
×

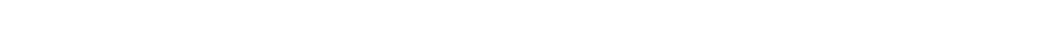

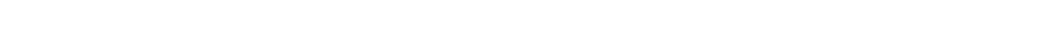
แ

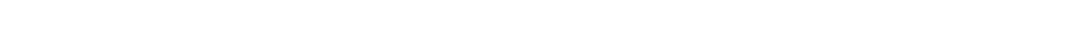

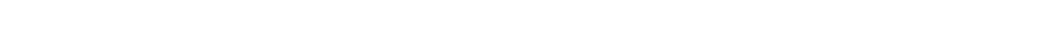

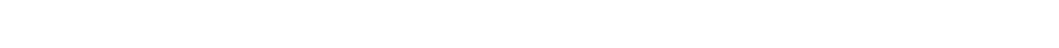

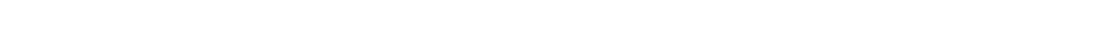

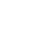

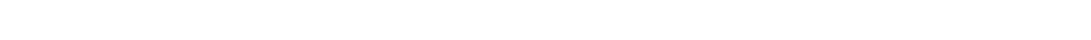
$\leftarrow>$ n

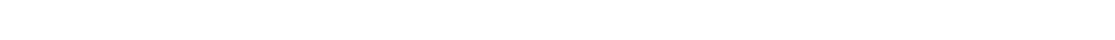

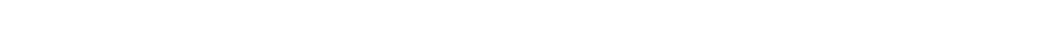

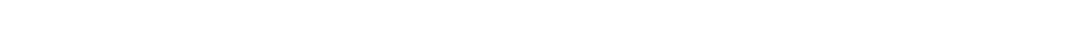

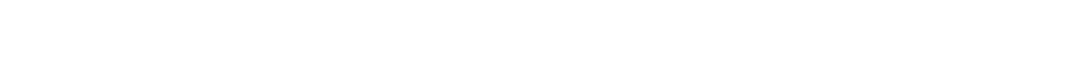

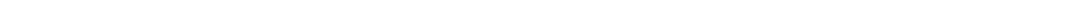

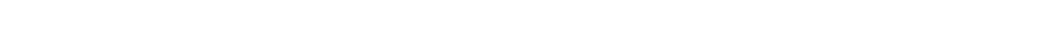

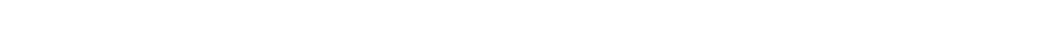

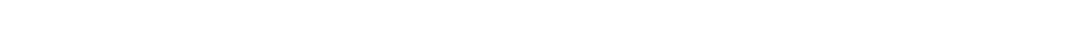

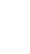
๔

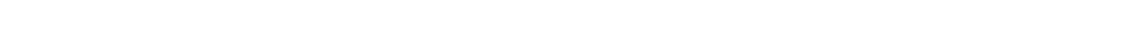

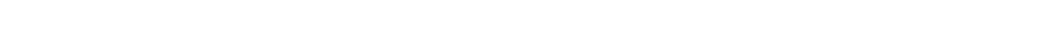

a a a 0000000000000000000000000000000

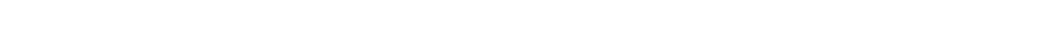
แ an

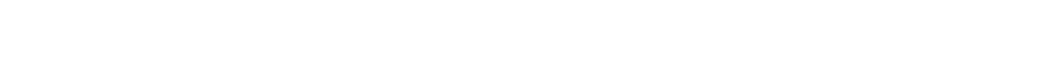




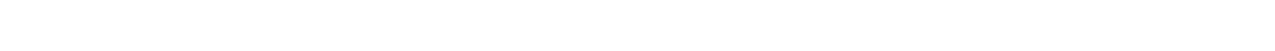

$\stackrel{4}{i}$

a

a. $\propto$

$a, a>$

a.

a.

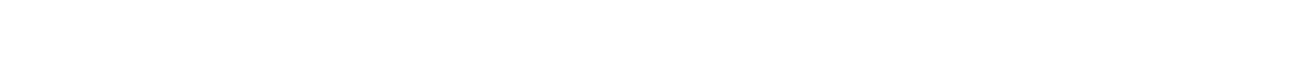
"ا

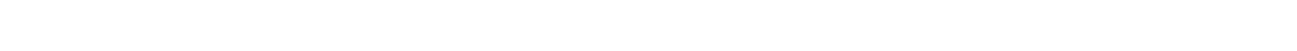

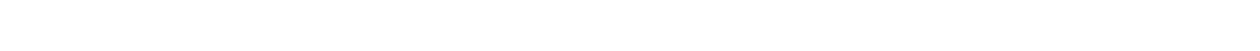

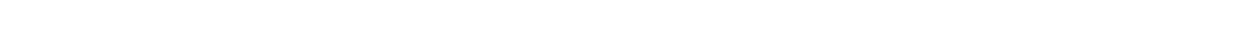

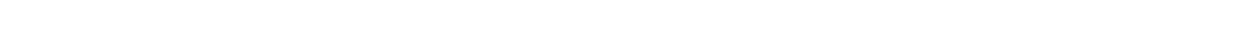

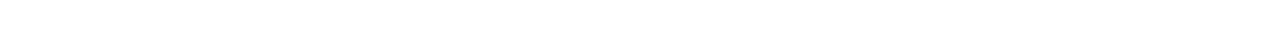

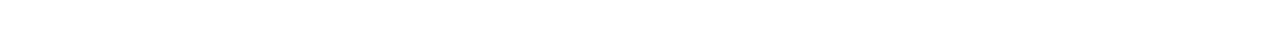

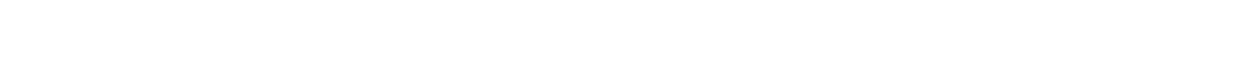

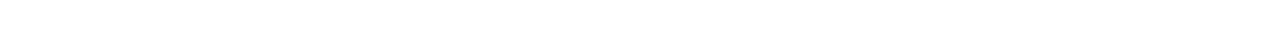

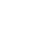

a. a

a.

a.

a.

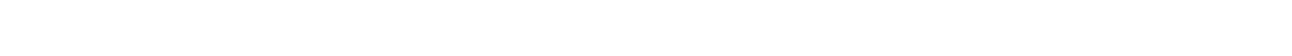
$\omega$ ú द

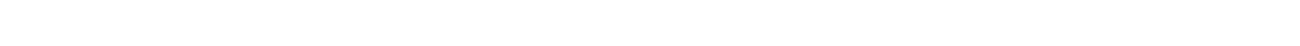

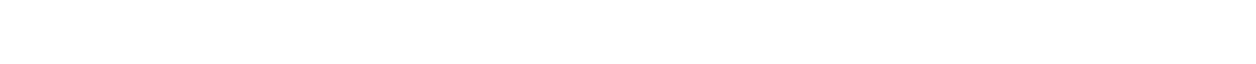
w5

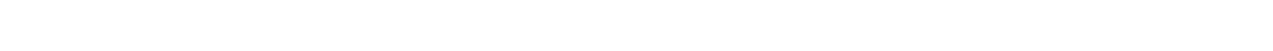

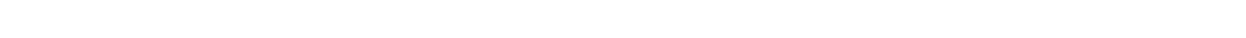

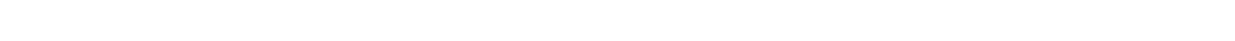

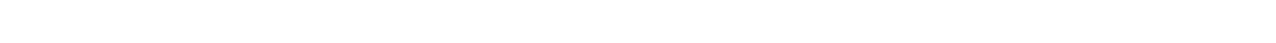
ב.

$\leftarrow$

mom

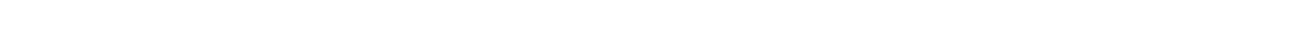
«

붕ํํำฟ

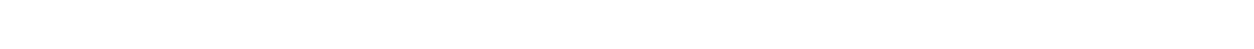
LES

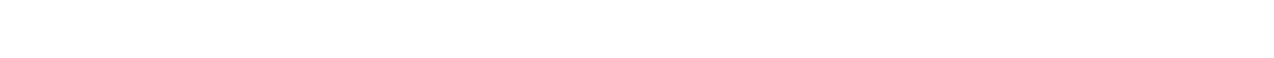

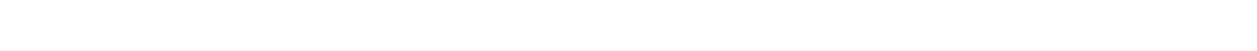

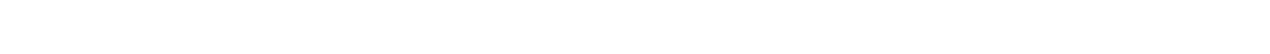
5

×

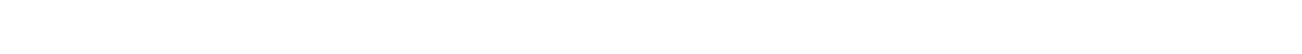

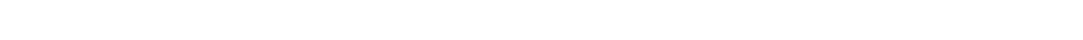

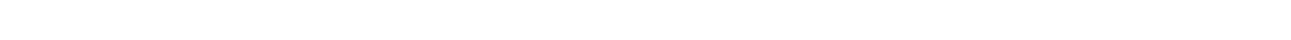

$00000-55-555-55-5 \sim N \sim \sim \sim N \sim \sim N \sim N \sim m m m m m m m m m m m m$

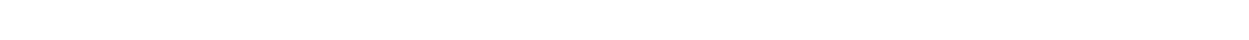

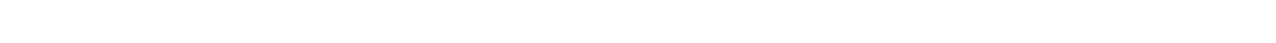

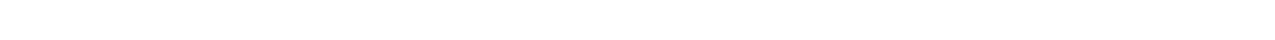

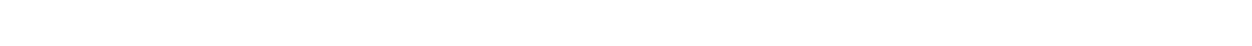

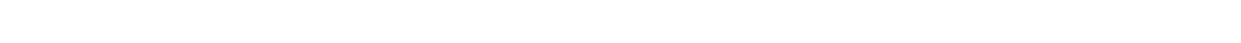




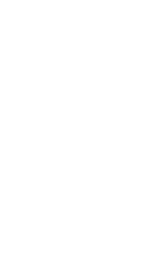

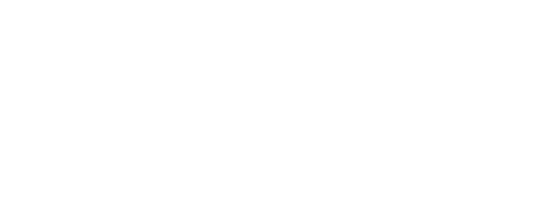

过向

实.

$\overrightarrow{0}$

$: \sum \hat{\alpha}$

min:-

些

这

号

土柴方

4. 2

$\therefore$ 4

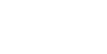

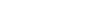

tosen

is

Nom

뜬ำ

=

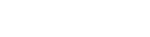

z m

告 -

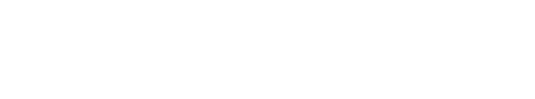

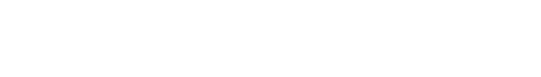

v.

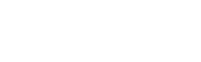

tusen

$c \mathrm{c}$

药

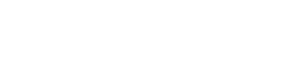

*

as-

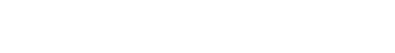

$\geq \infty \frac{\pi}{a}$

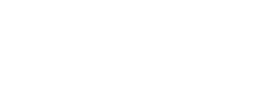

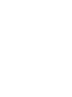

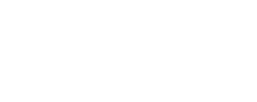

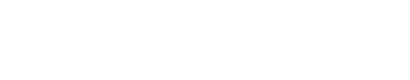

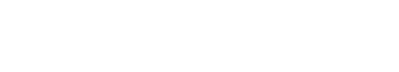

- 100

4 的.

$=\quad 30$

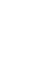

$\frac{a}{2} \sim \frac{0}{5}$

点点这

s=

20

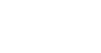

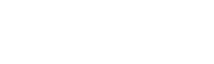

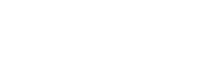

品

as

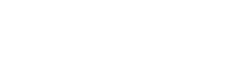

능

Fa $\alpha+\infty a \hat{a}$

$m=\dot{m} \dot{0}$

a wan

$\forall \rightarrow$

$\ln _{\infty}^{\infty} \infty$

$\omega \div \div=$

모웜

un $\quad$ I 

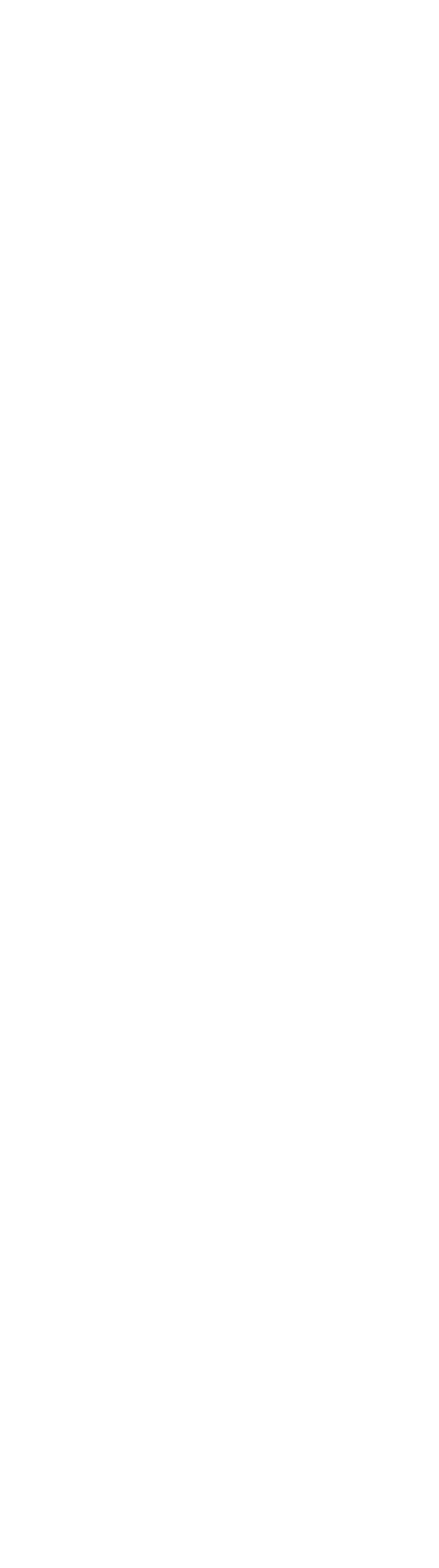

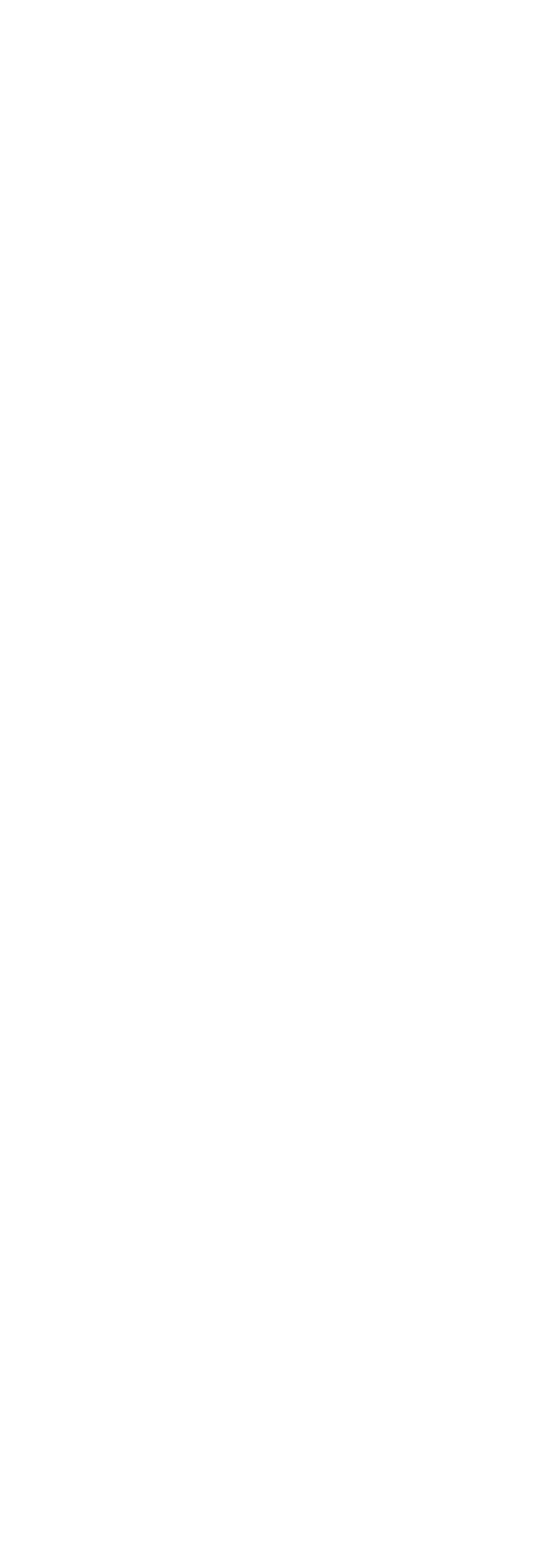

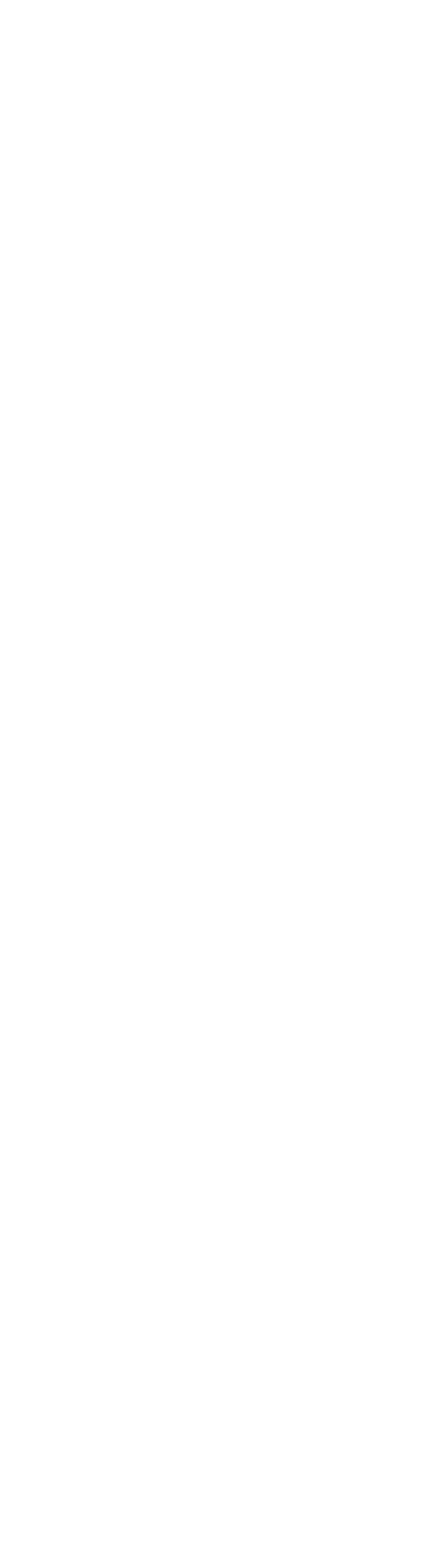


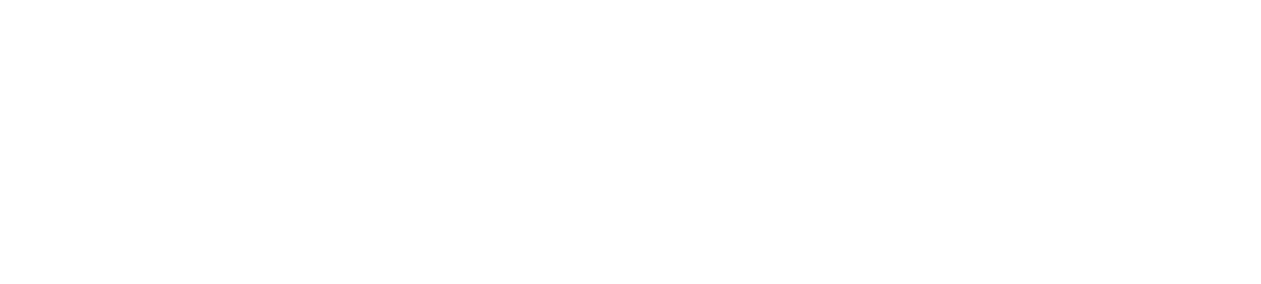

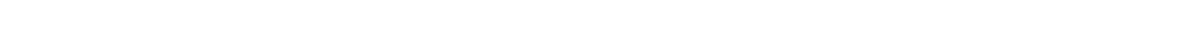

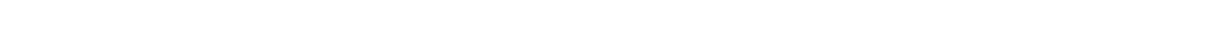

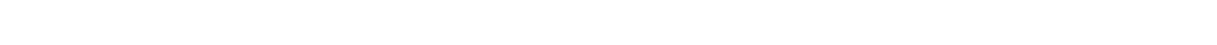

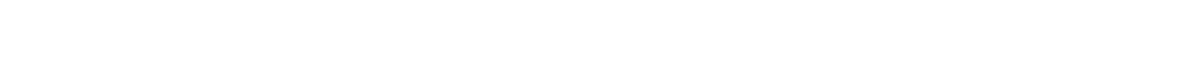

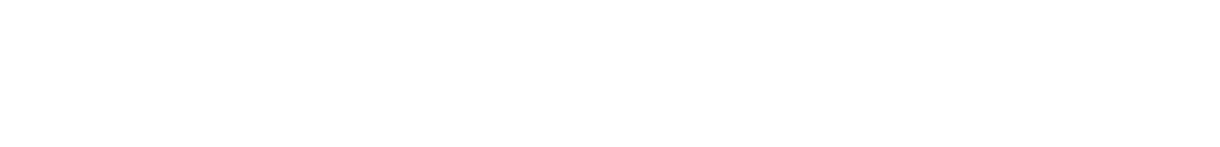

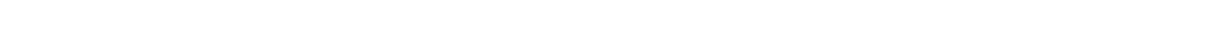

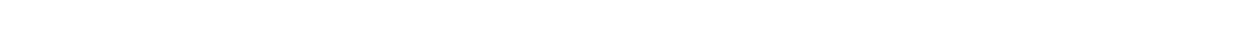
س

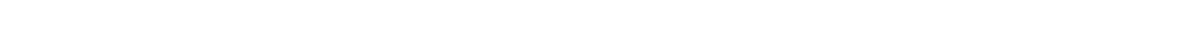

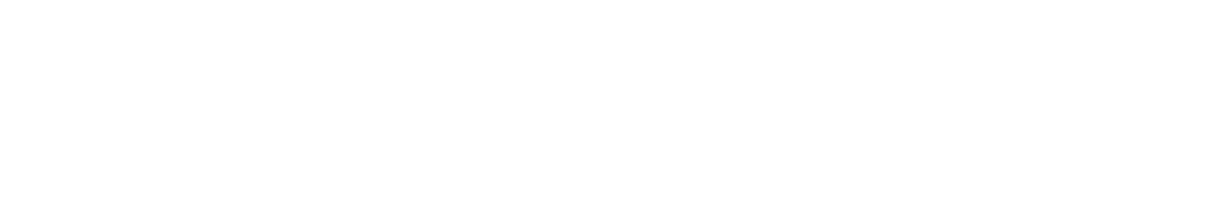

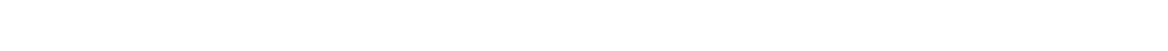

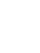

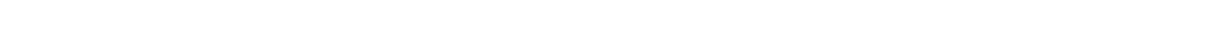

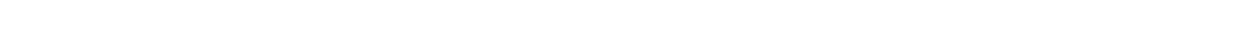

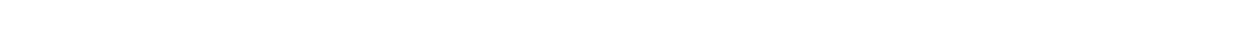

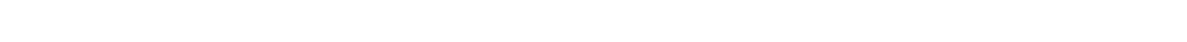
a

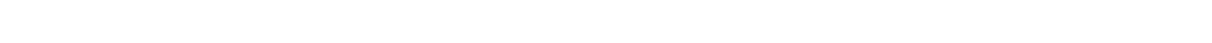

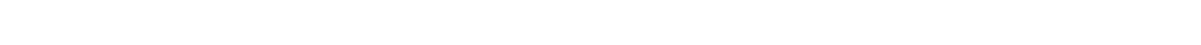

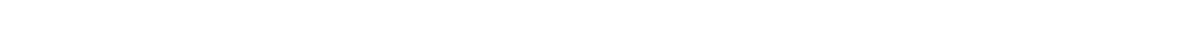

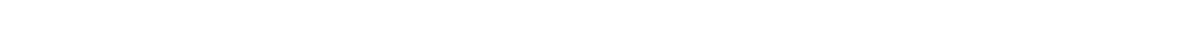

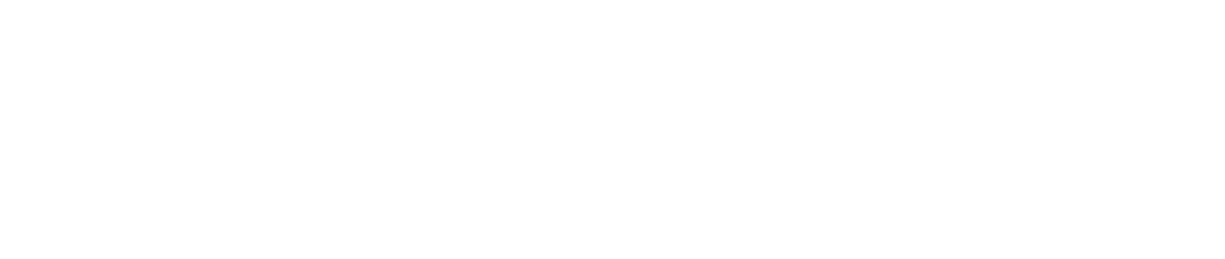

aaaa00000000000000000000000000000000000

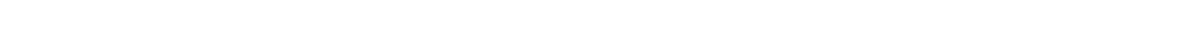

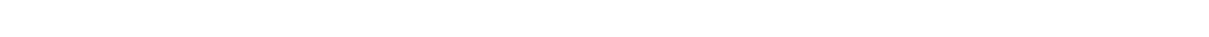

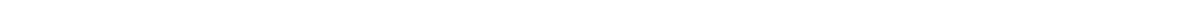

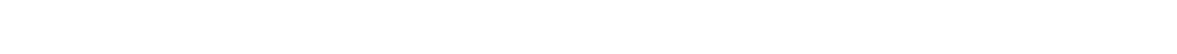




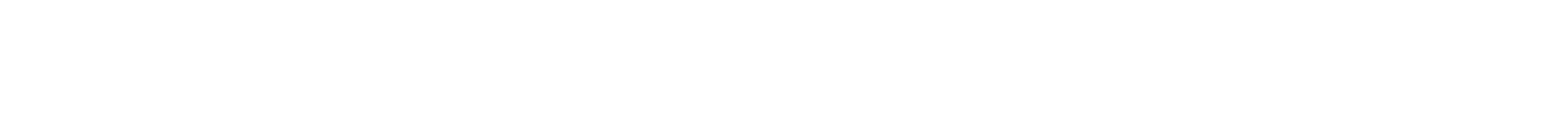

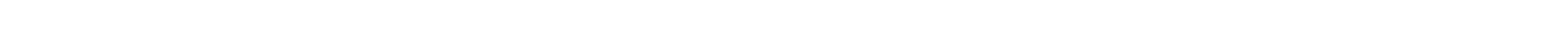

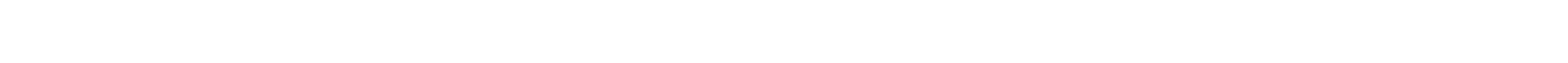

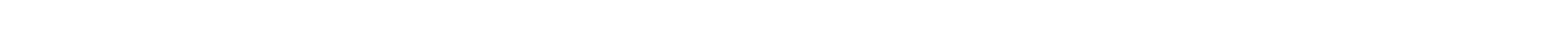

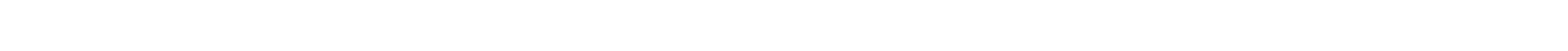

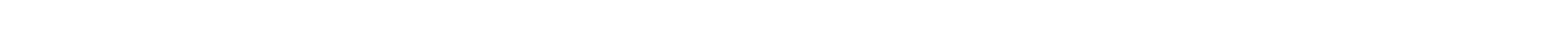

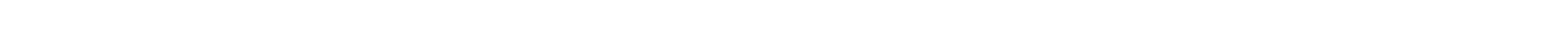
w

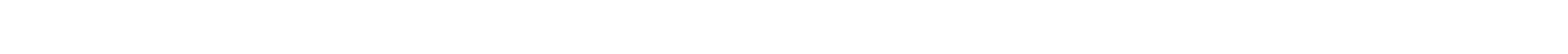

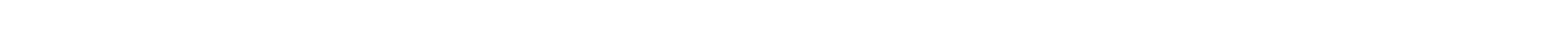
‘

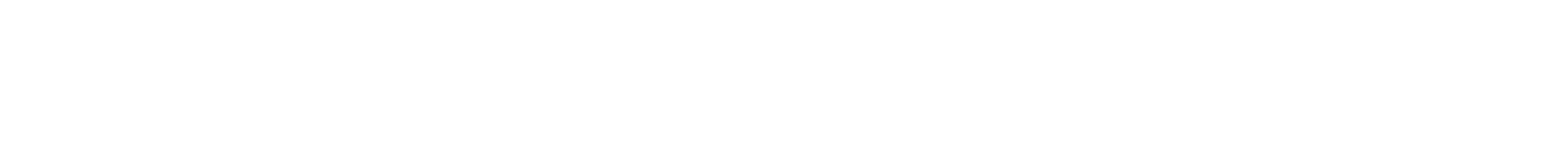
x

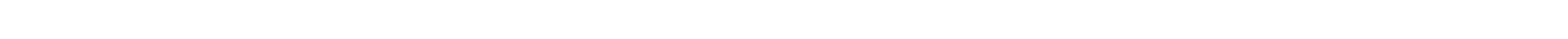

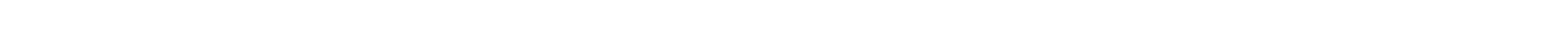

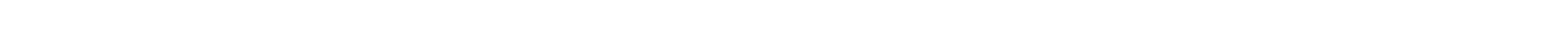

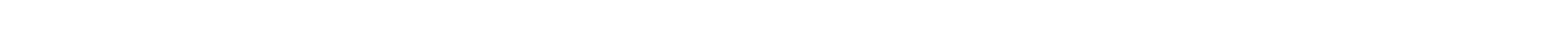
й LE

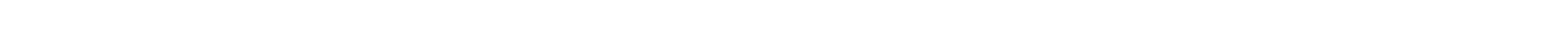

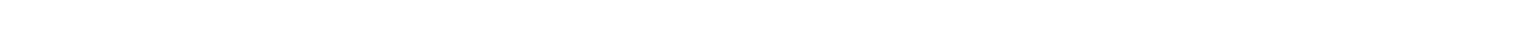

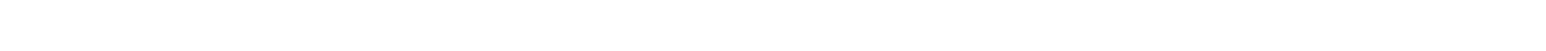

wNN N

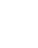

고의

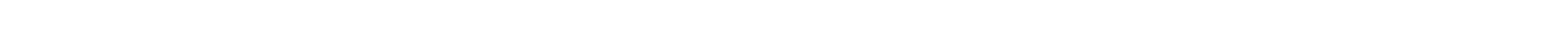
L

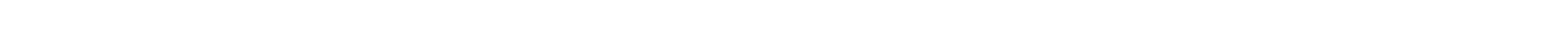

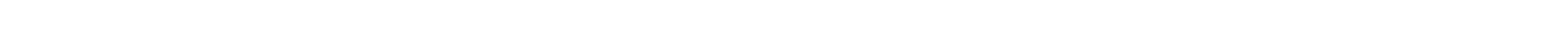

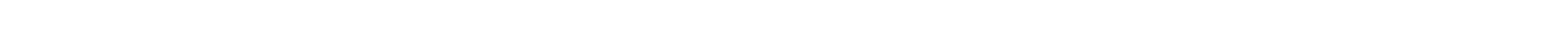

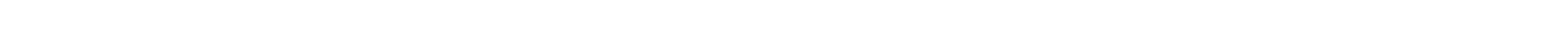

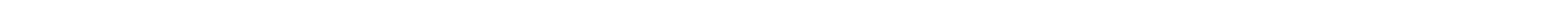

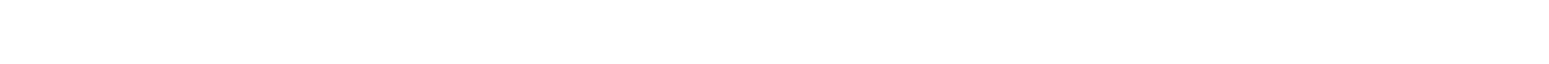

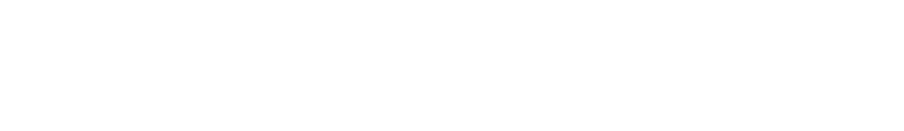

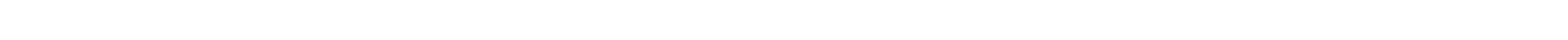
w a

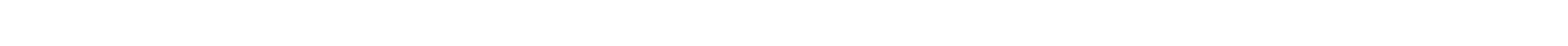

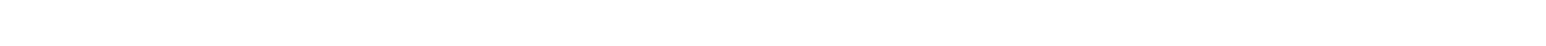

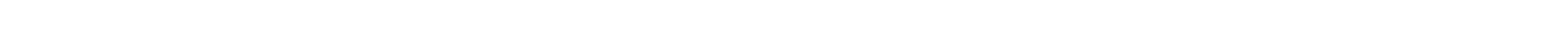

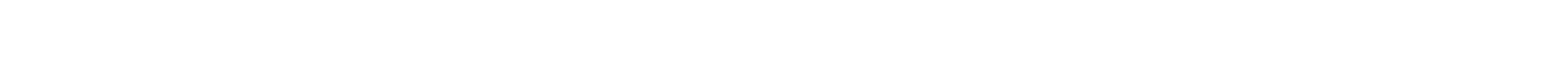

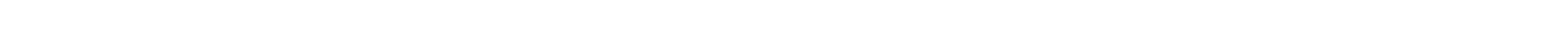

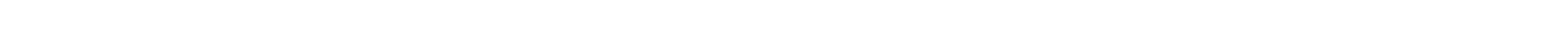




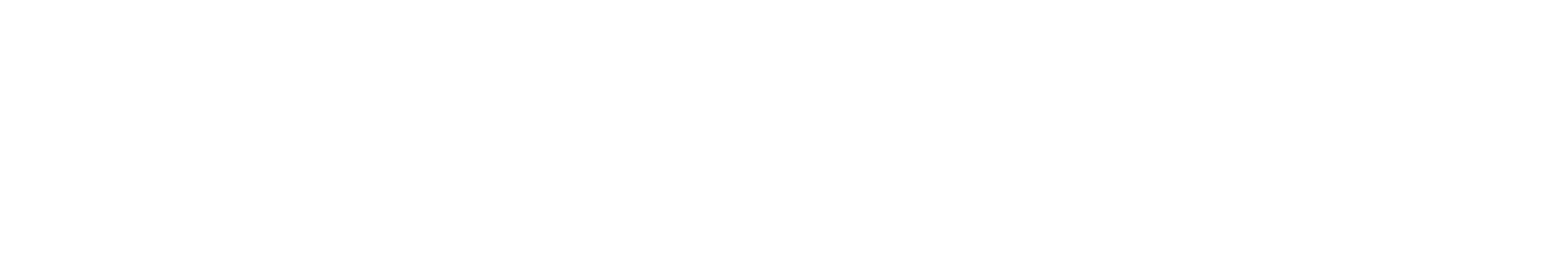

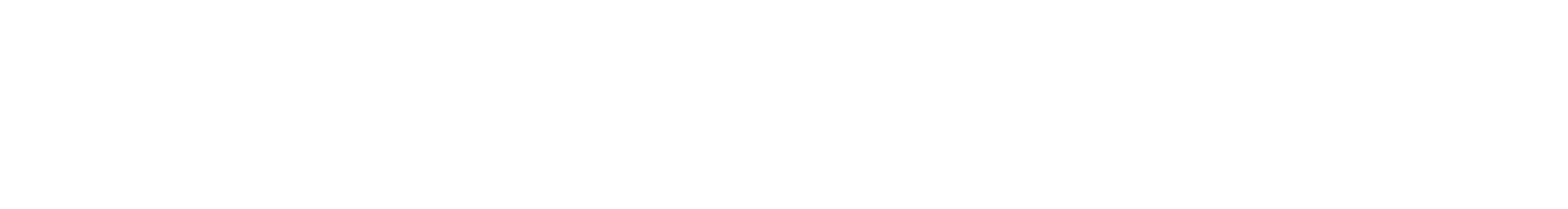
$\stackrel{w}{\Sigma}$ is
es
is
is
n
n
is
is
$\stackrel{5}{5}$

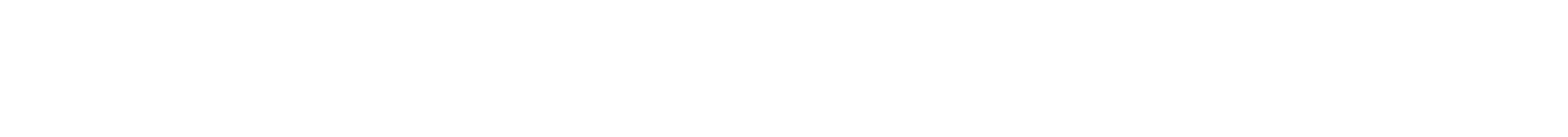

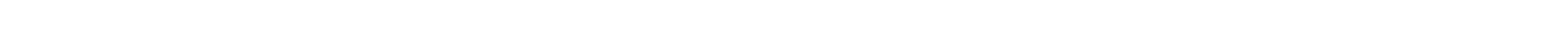

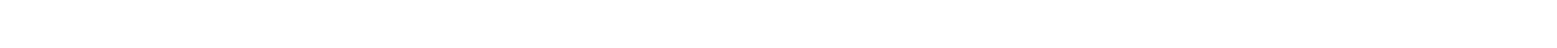

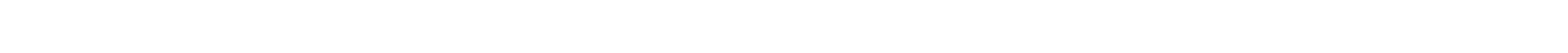

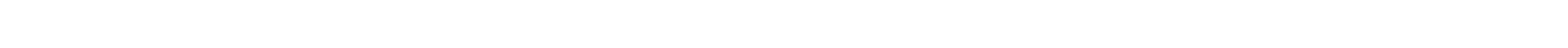

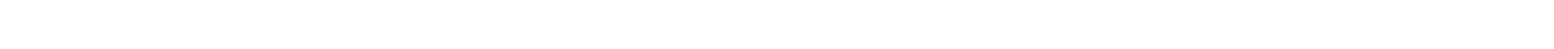

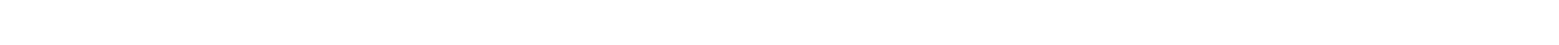

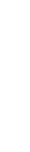

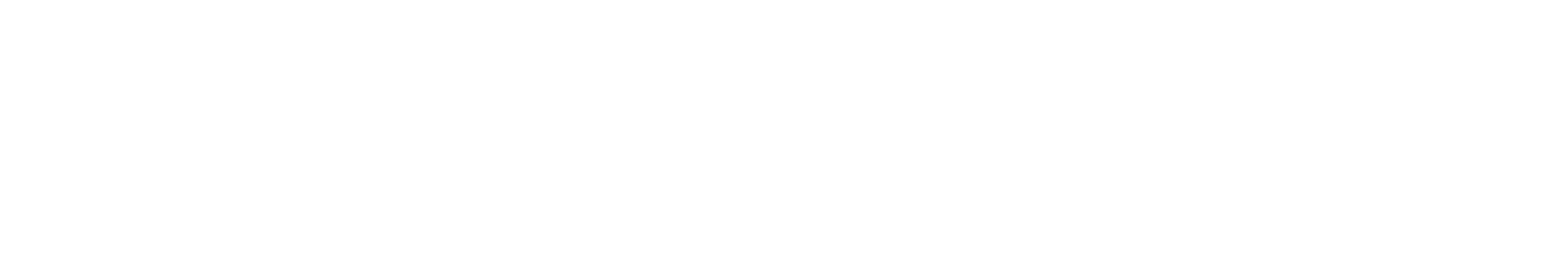

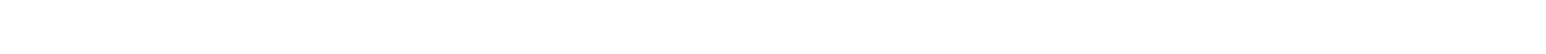

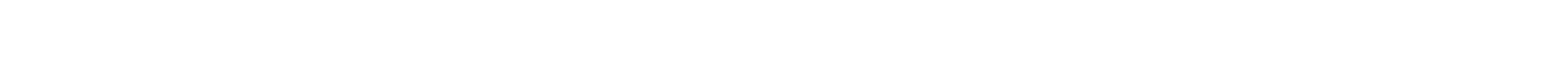

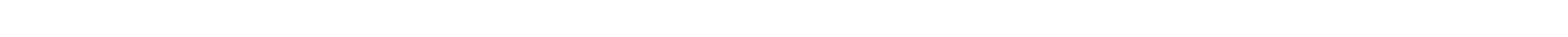

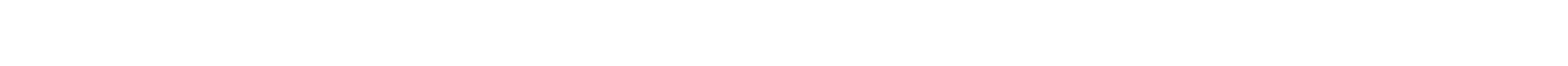

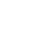

is

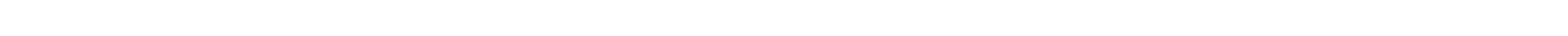

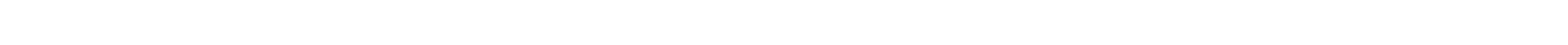

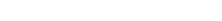

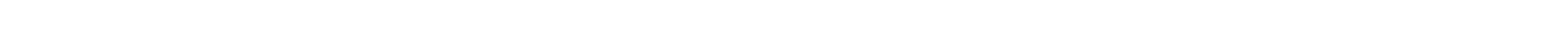

MMMMMMMMMMMMMMMMMMNMMMMMMMMMMMMMMMMMMNMMMMMMMMMMMMM

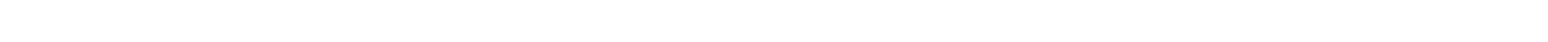

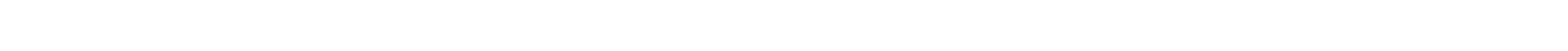
\&

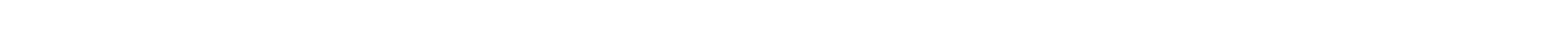

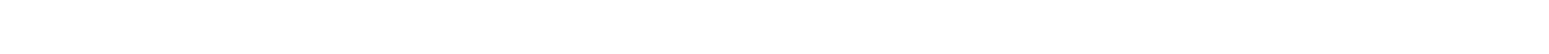




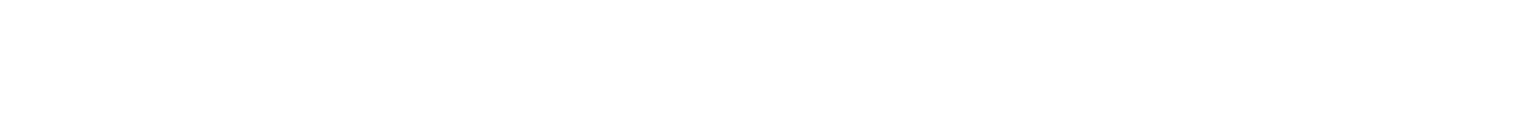

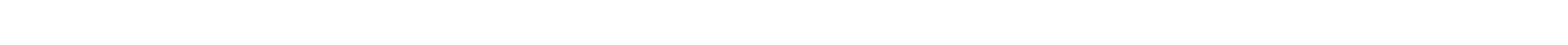
ü

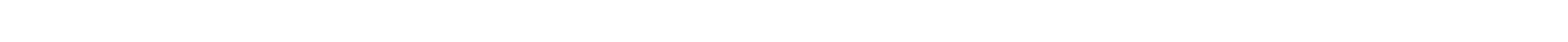

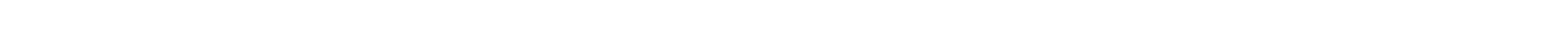

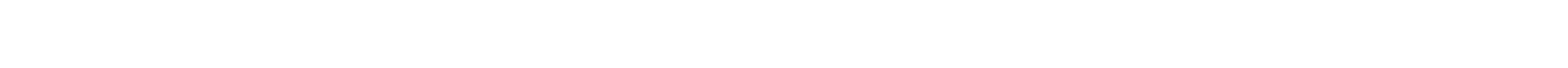

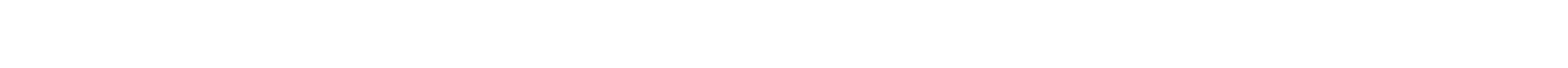

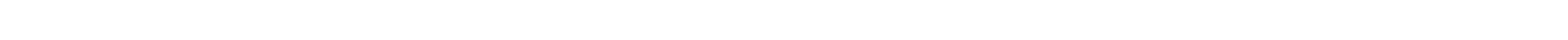

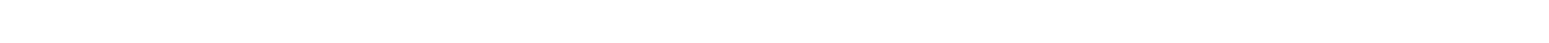

㭊的

n

๔

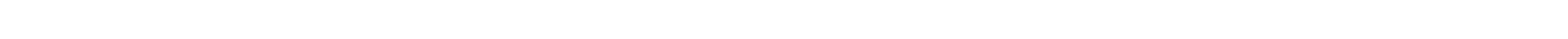

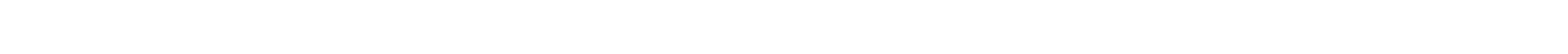

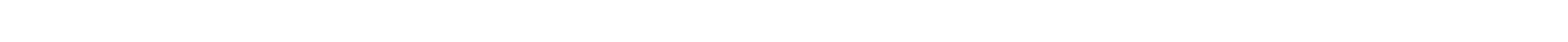

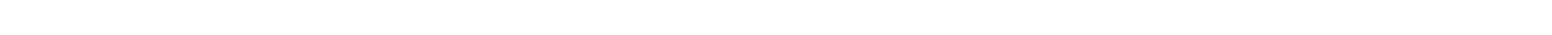

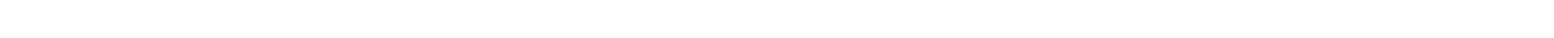

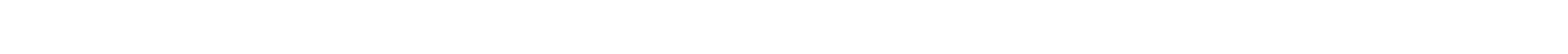

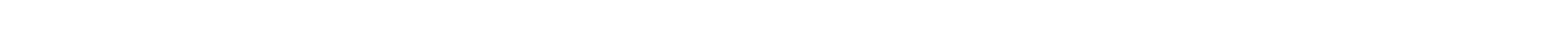

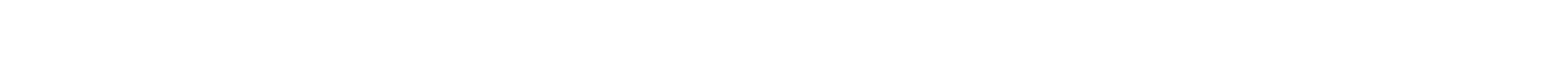

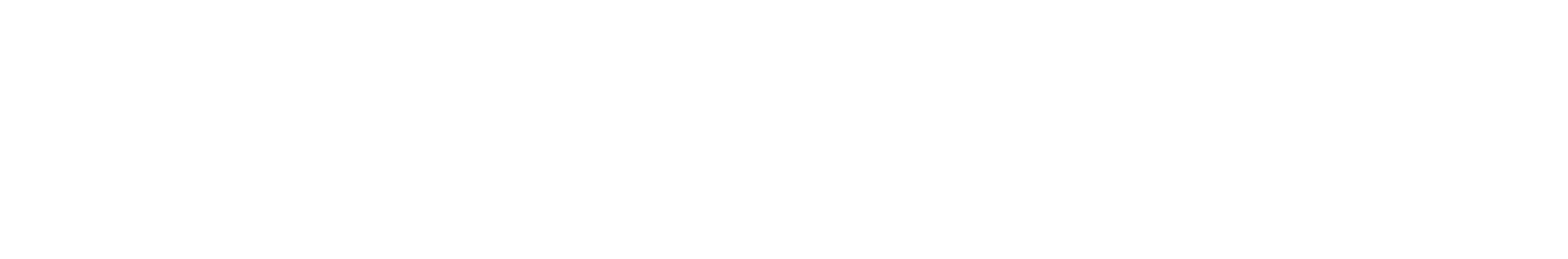

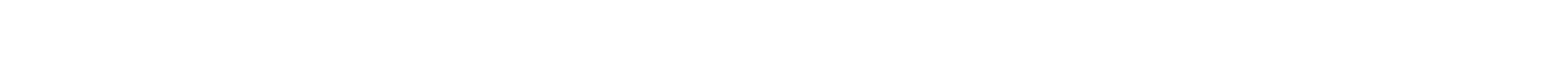

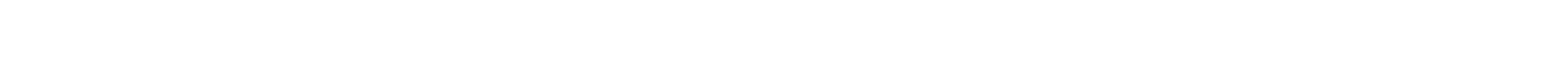

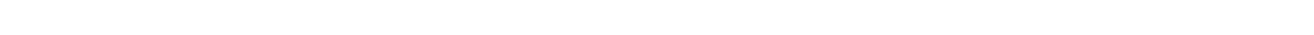

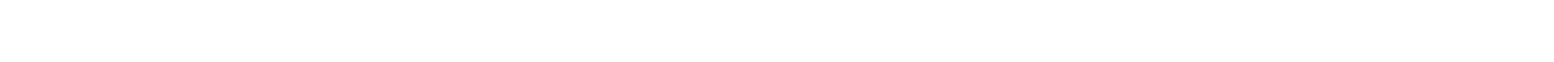

¿

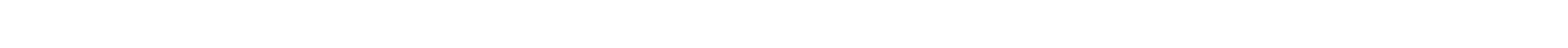

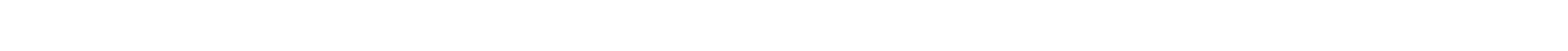

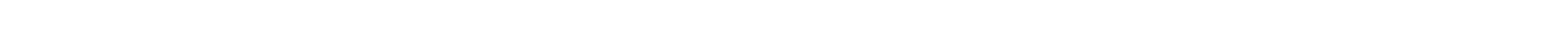

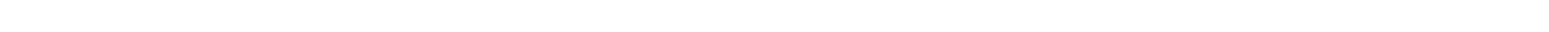

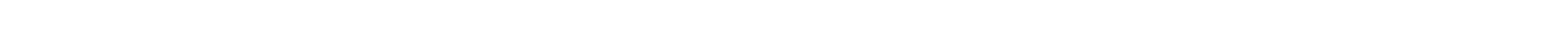

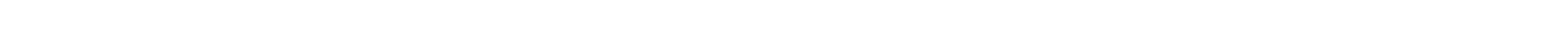

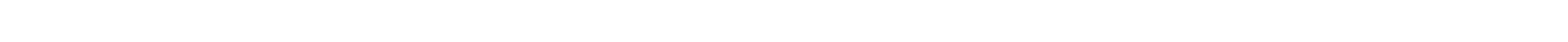

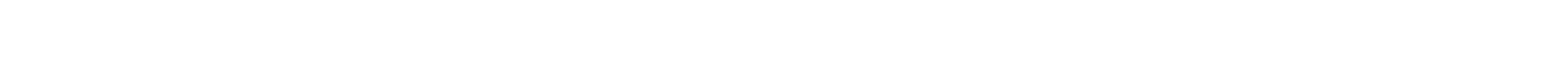




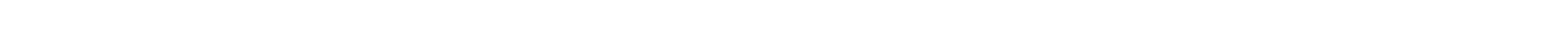
a d

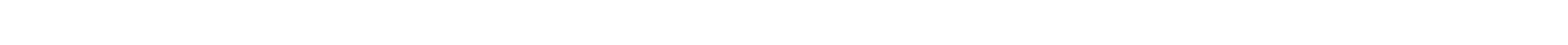

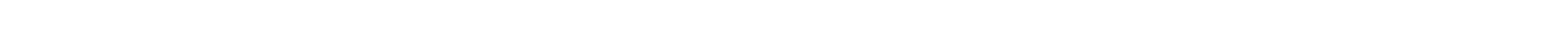

a a a a o a ma00000000000000000000000000000000000000

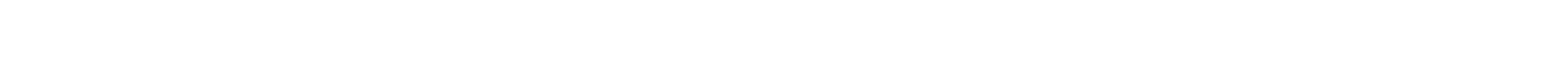

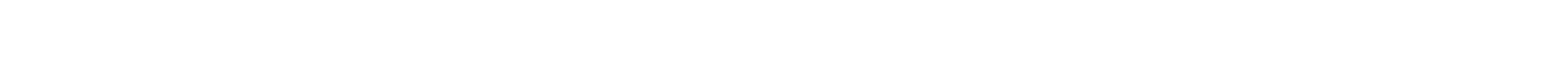

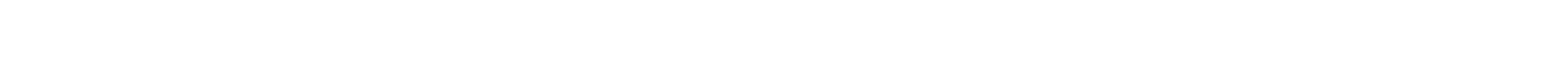

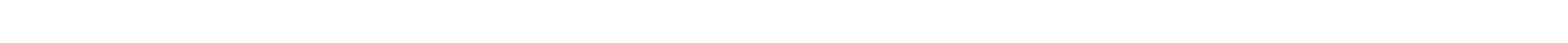

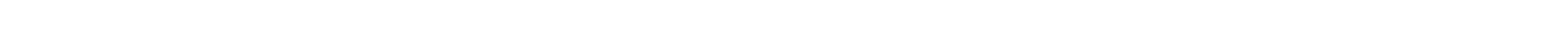
\begin{tabular}{cc}
$x^{3}$ \\
\hline
\end{tabular}

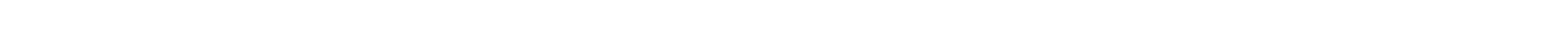

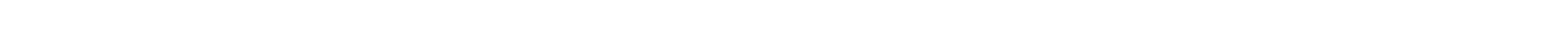

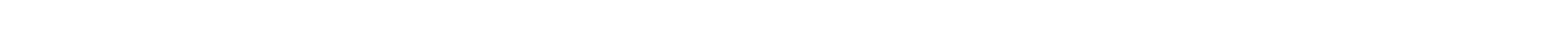

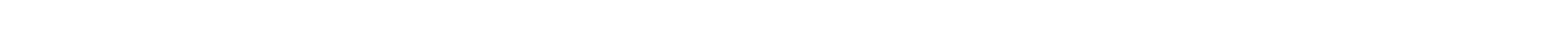

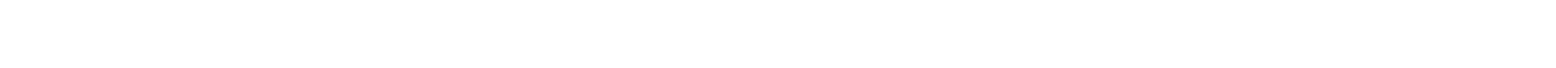

n

n

is

$\stackrel{5}{5}$

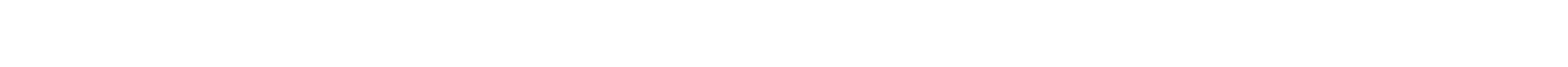
ul

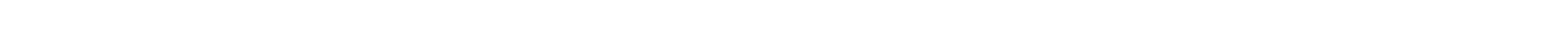

\% no

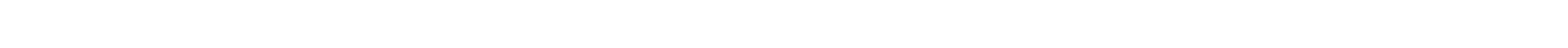

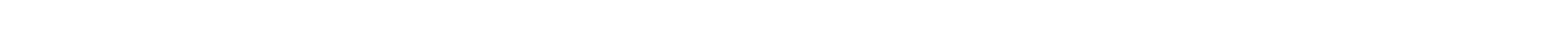
- - NNNMOF-NNNMO-FNNNNMOF-NNMO-FNNMOF-NNMOF-NNNNNMOOOE

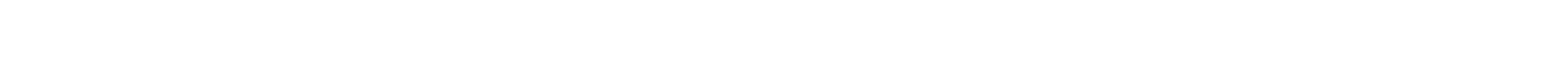

r

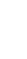

c

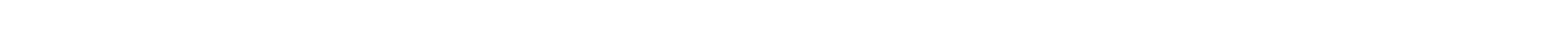
แ

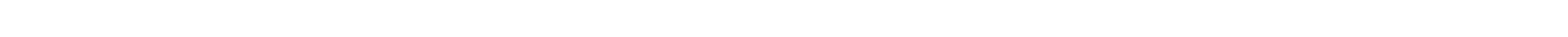
व

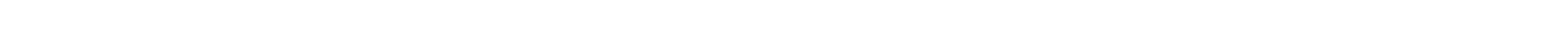

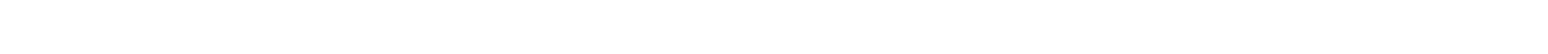

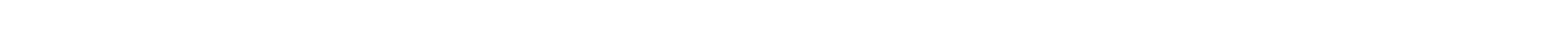
-

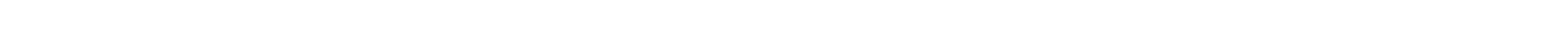

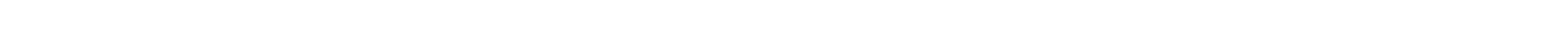

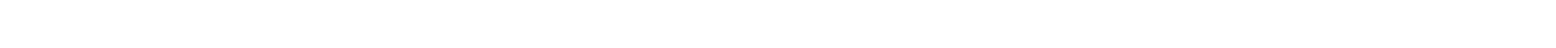




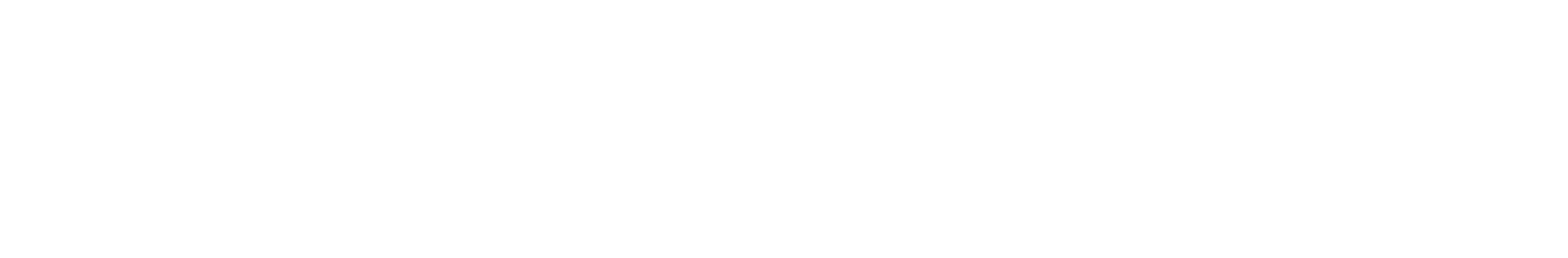

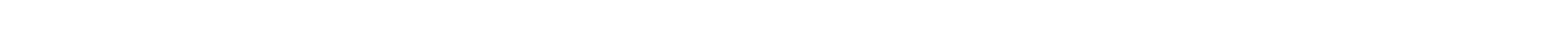

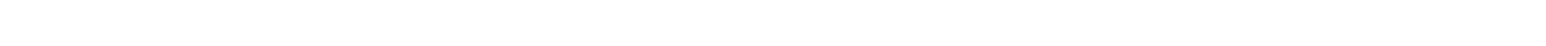

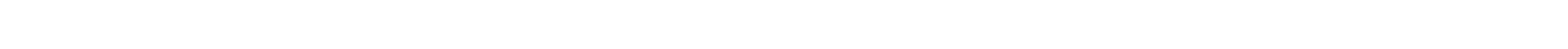

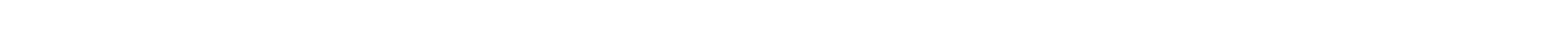

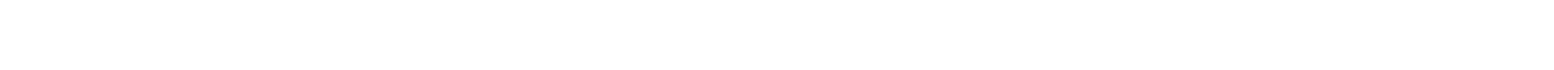

r

$\frac{w}{2}$

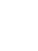

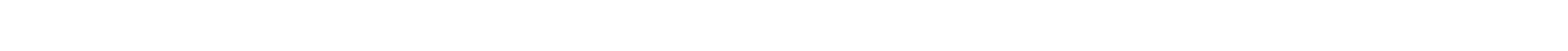

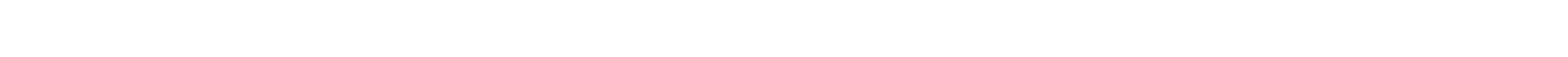

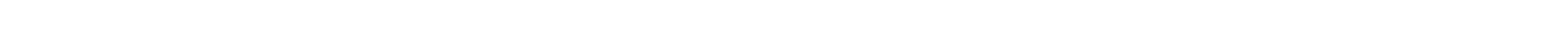

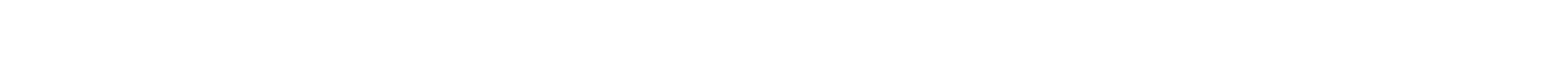

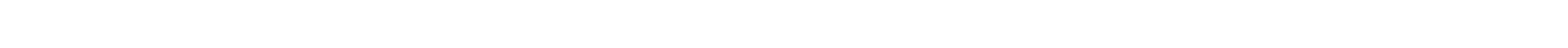

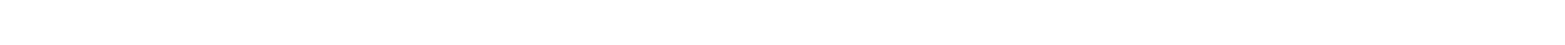

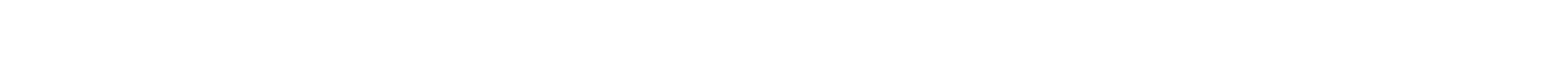
$\stackrel{5}{\stackrel{5}{2}}$

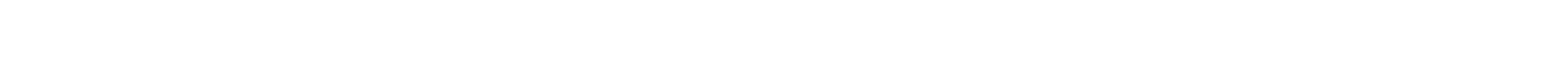

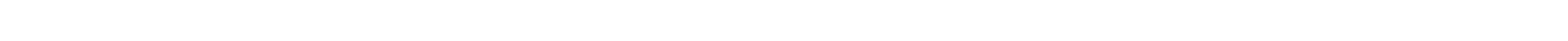
< w ino

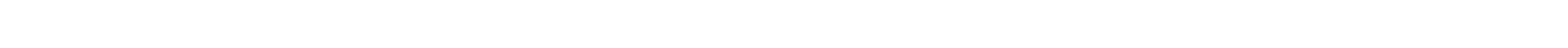

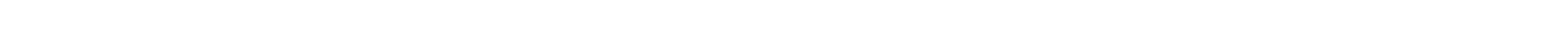

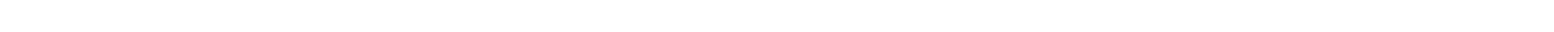

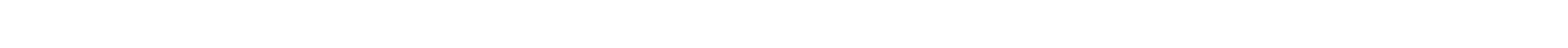

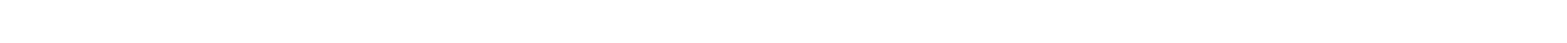

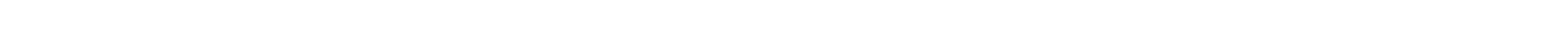

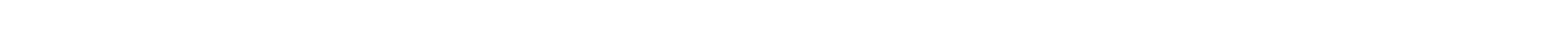

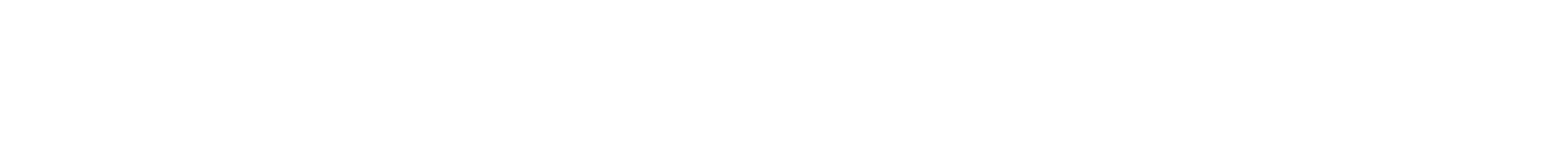

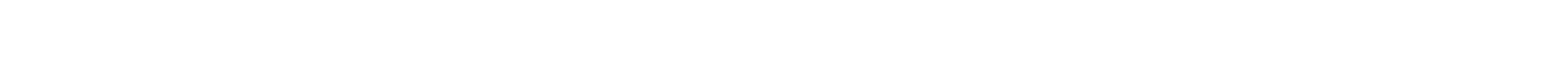
w

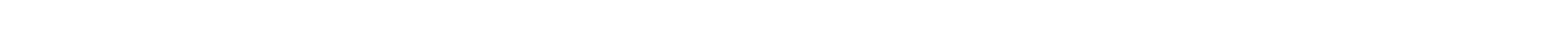

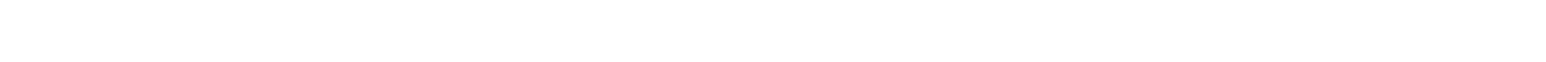

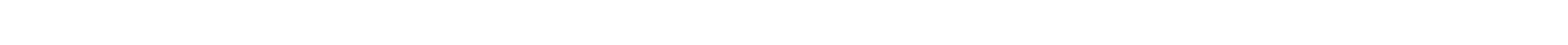

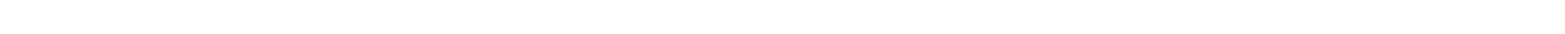

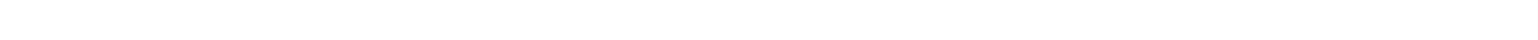

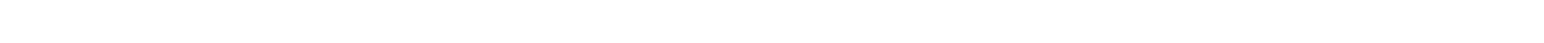




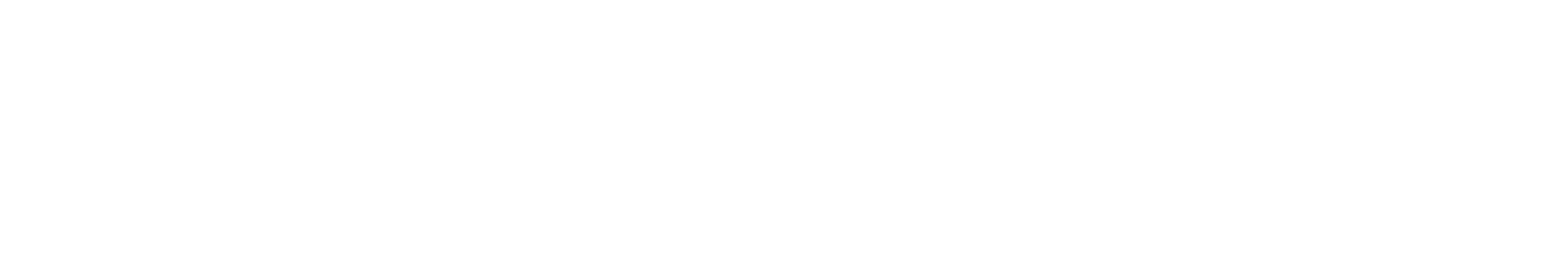

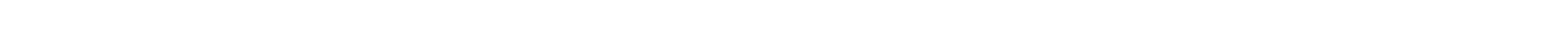

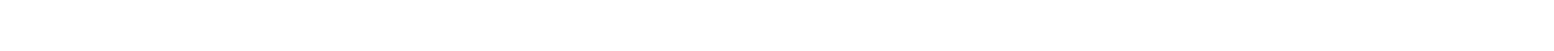

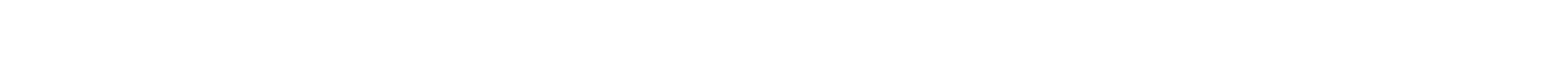

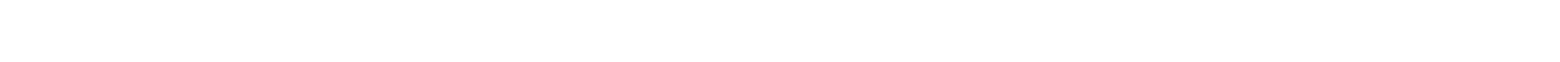
$\stackrel{\text { is }}{ \pm}$
n
n
n
is
n
n
s

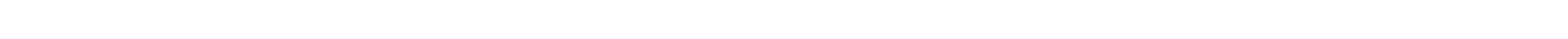 w

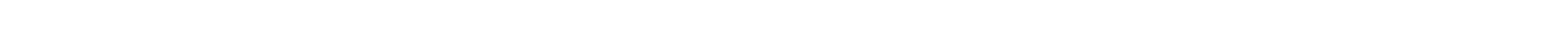

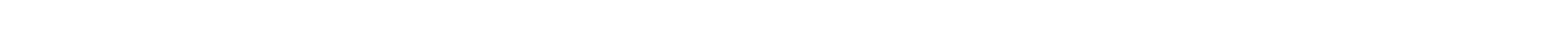

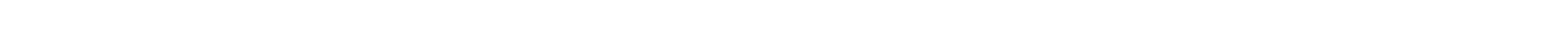

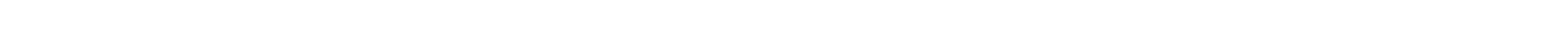

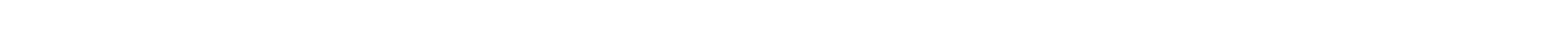

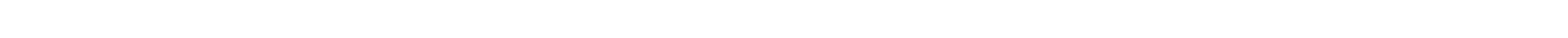

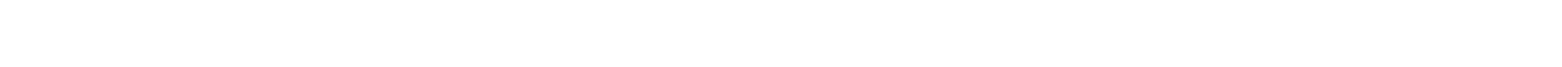

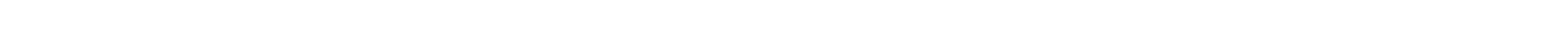
แ山

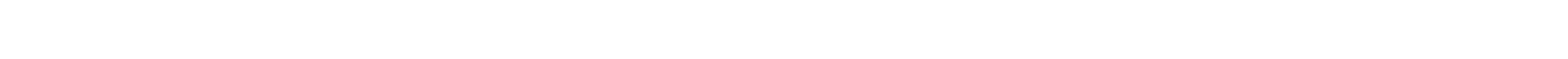

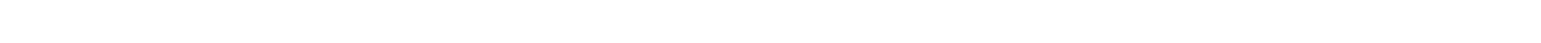

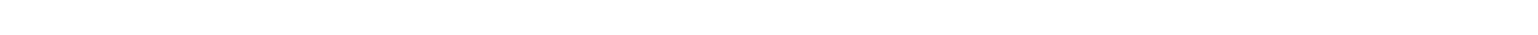

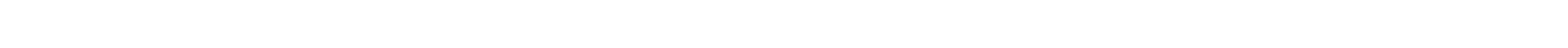

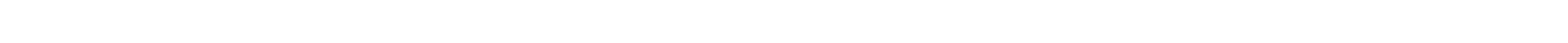

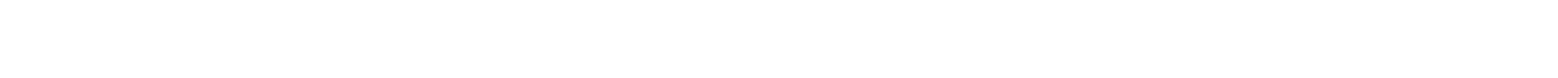

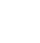

$\Sigma$

$\vdots$

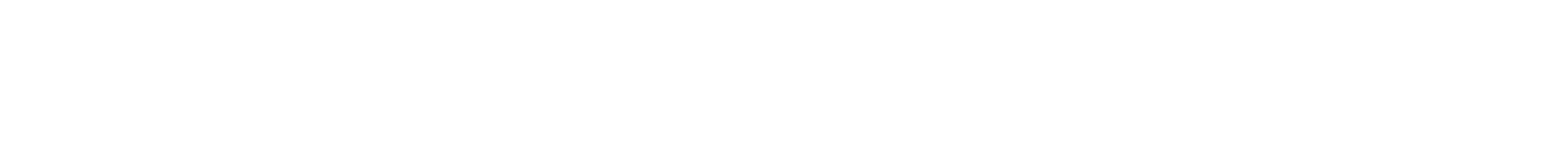

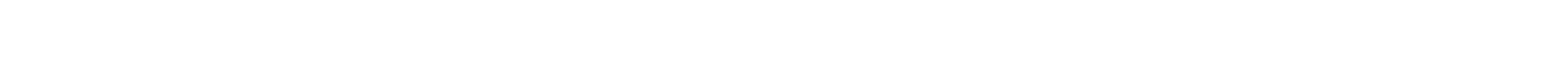

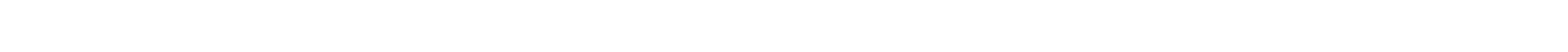

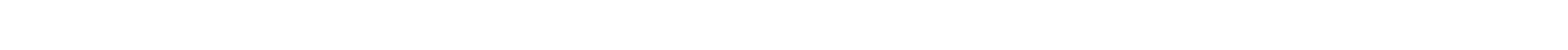

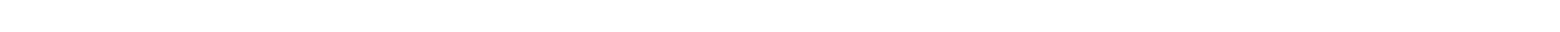

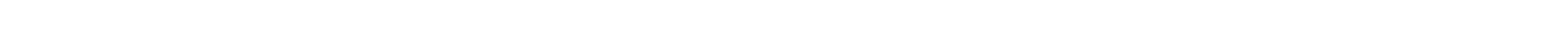




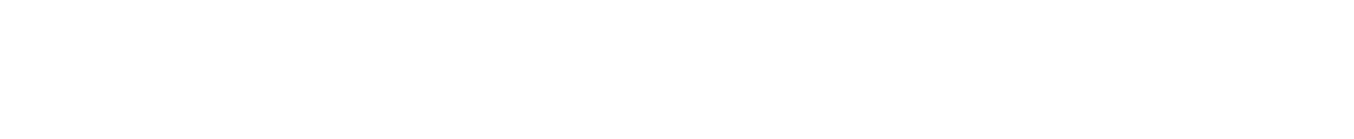

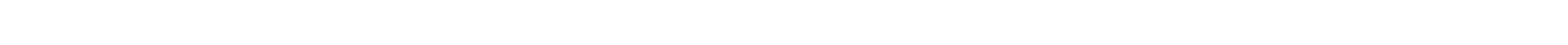
ผ

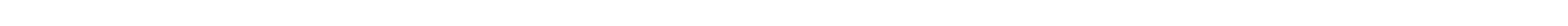

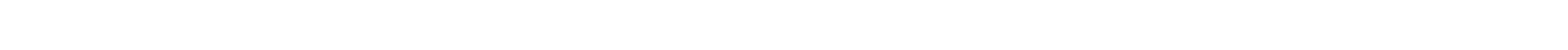

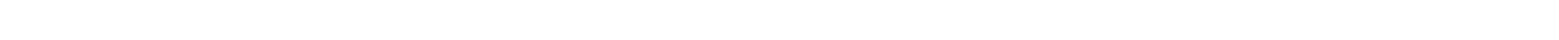

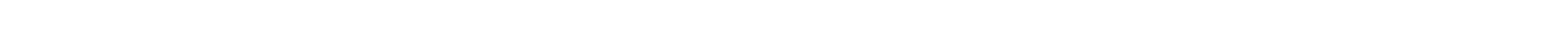

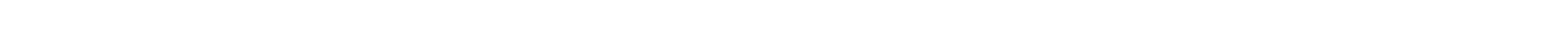

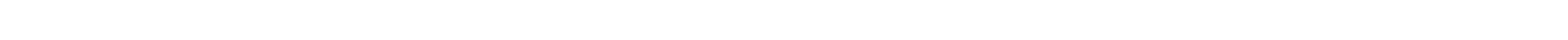

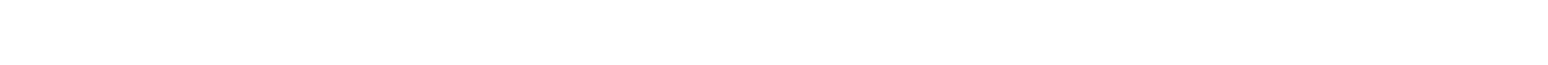

๘ $\infty$

E

$\stackrel{4}{5}$

х 世 ш 娄药

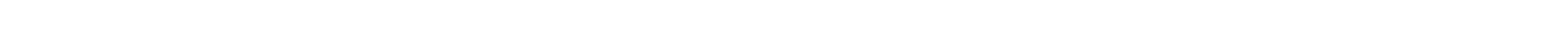

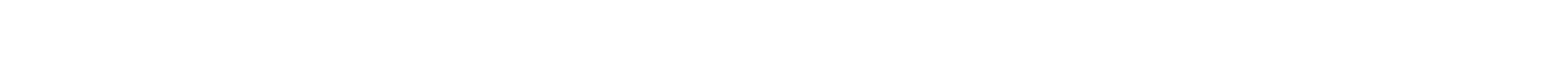
W

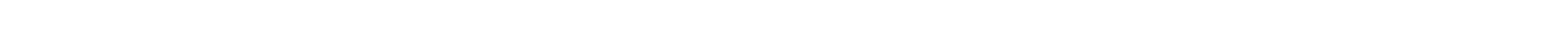

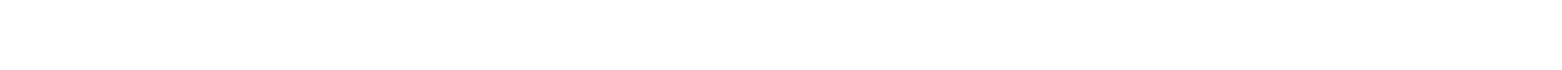

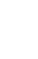

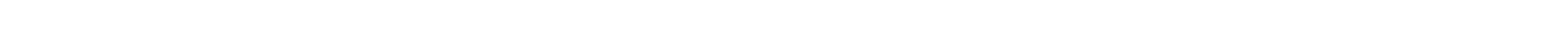
u

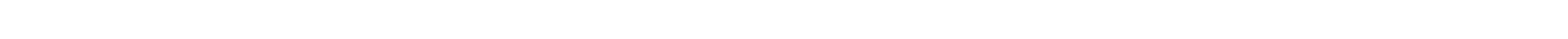

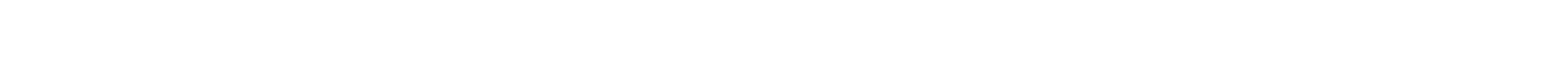

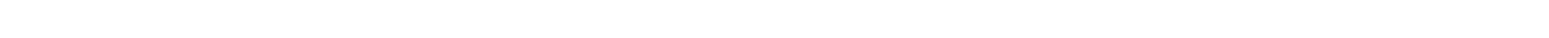

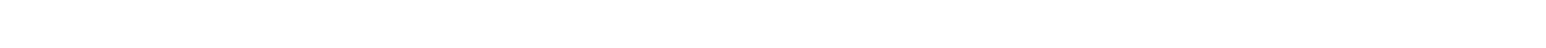
世

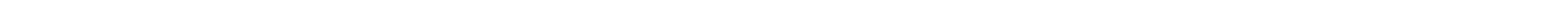

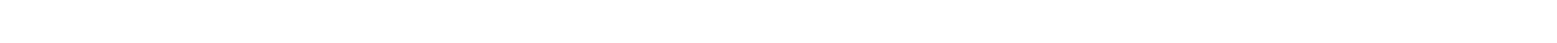

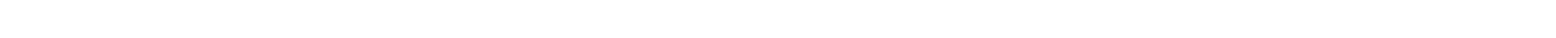
in
n
n
is
is
es
n

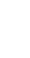

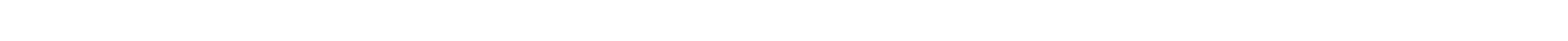

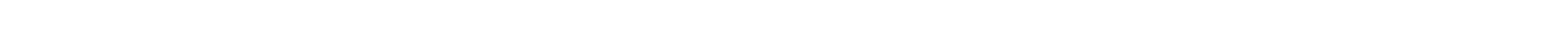 उ世,

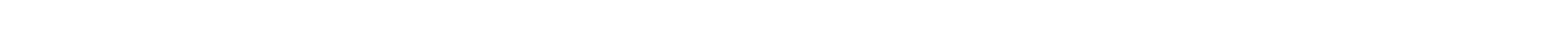

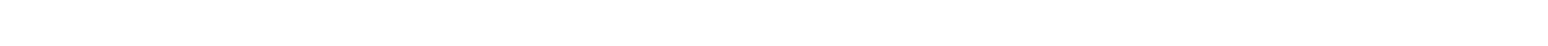

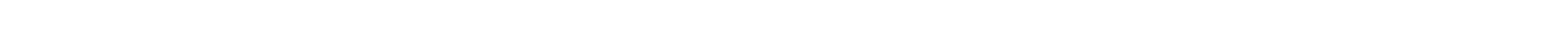

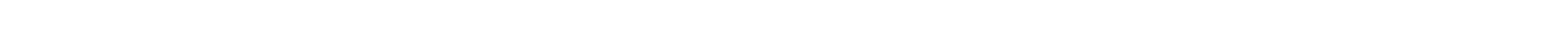

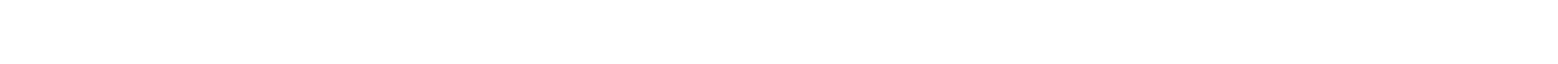




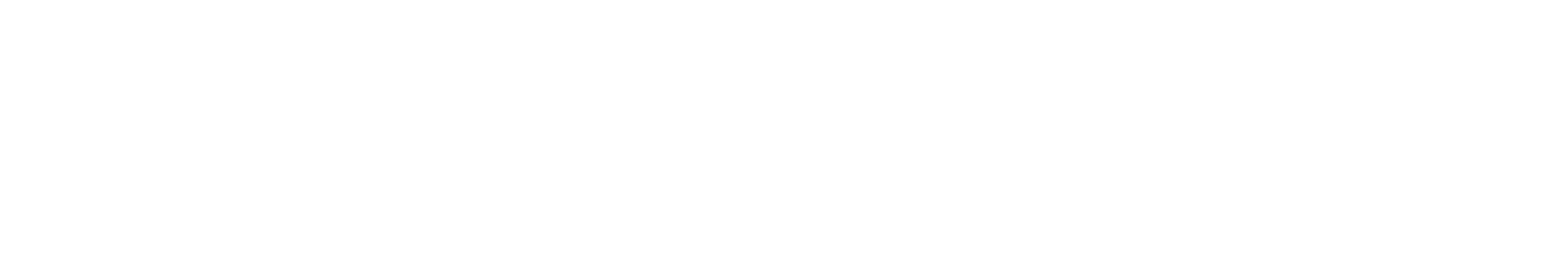

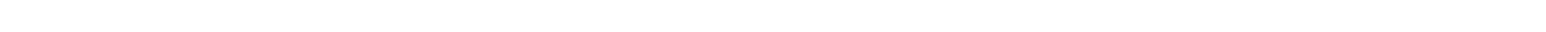

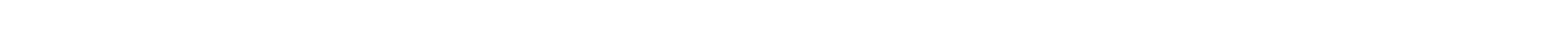

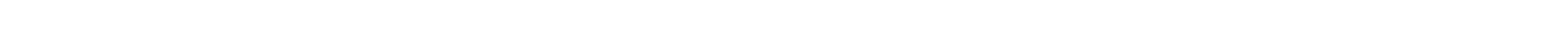

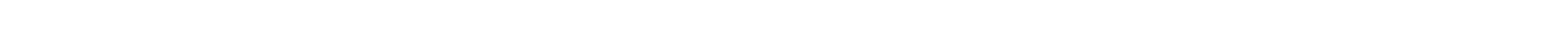

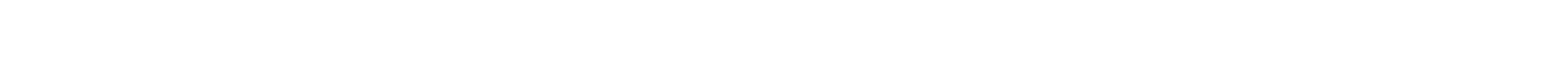

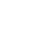

$\underline{\mathbf{x}}$

s

es

n

u

is

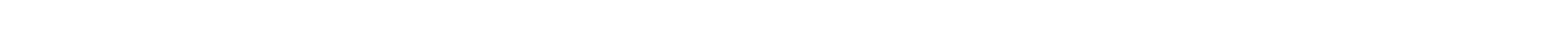
w

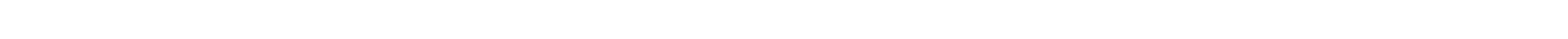

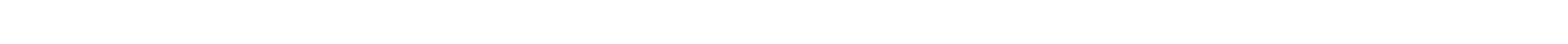

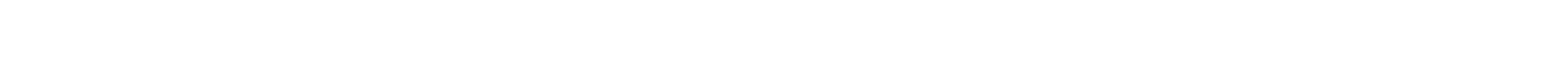
W

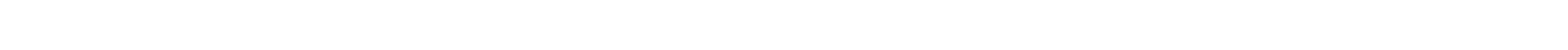

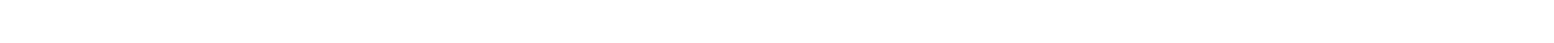

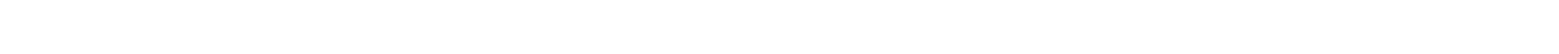

崖的

n

n

$\infty$

n

n

is

in

x

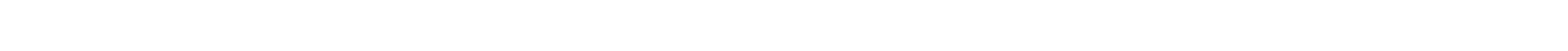

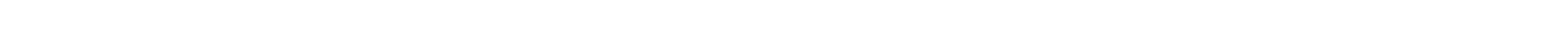

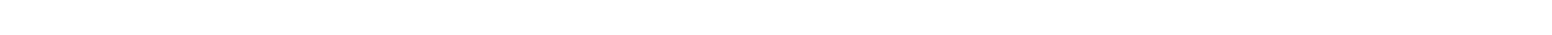
ăóa 山-

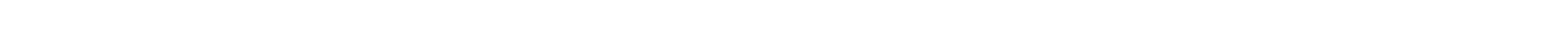

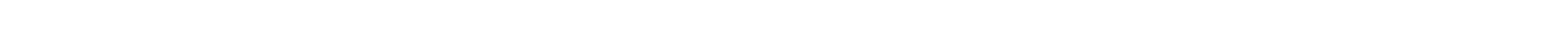

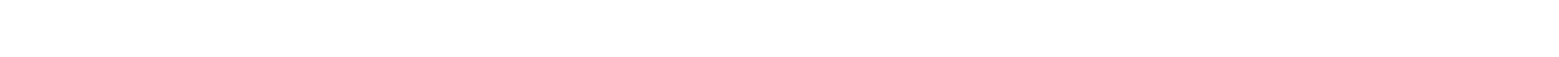

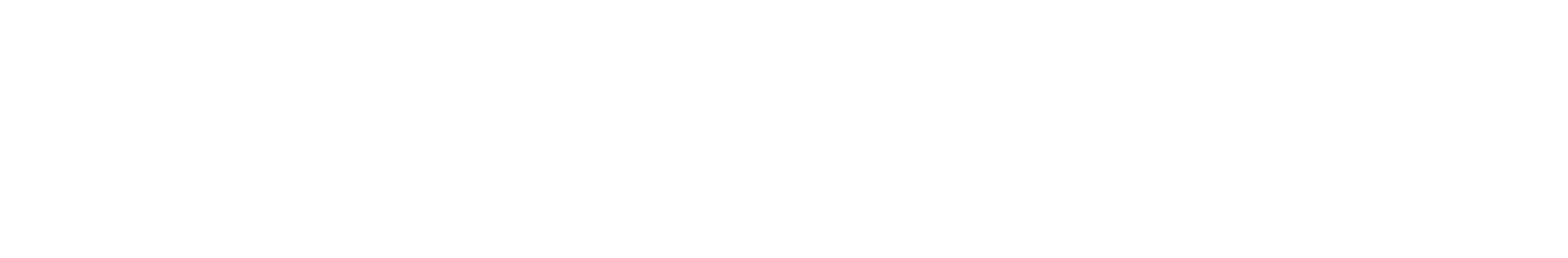

ㅇㅇㅇㅇㅇ으즈ス

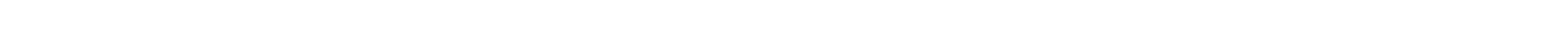

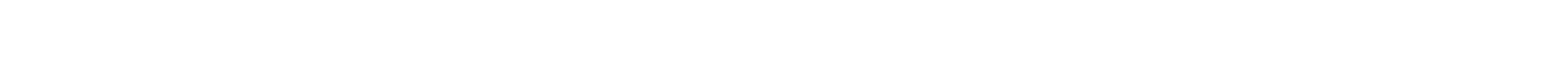

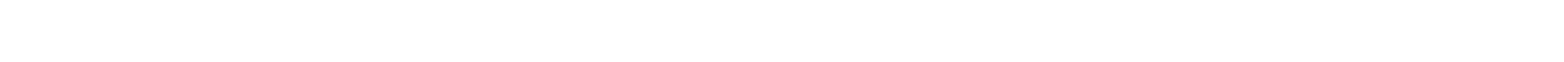




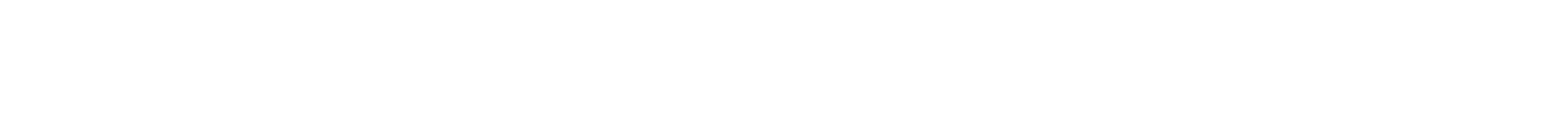

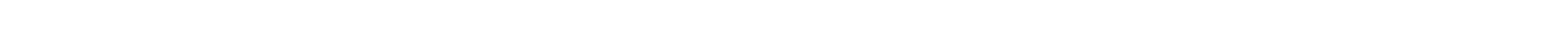
ש

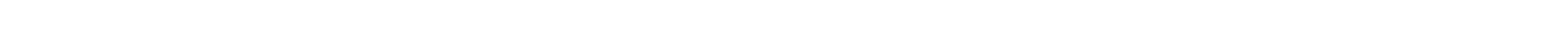

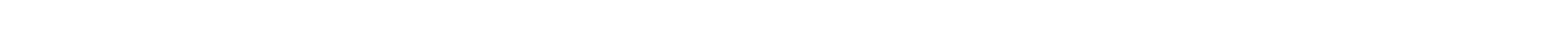

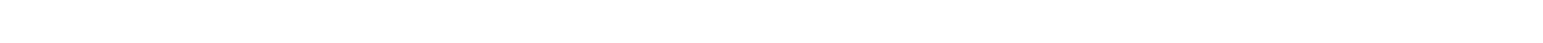

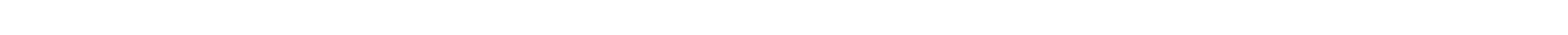

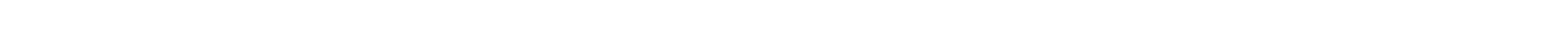

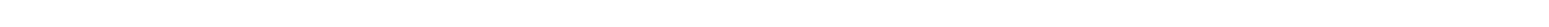

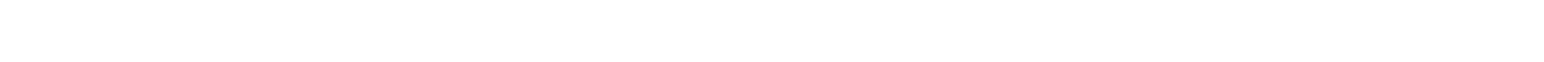

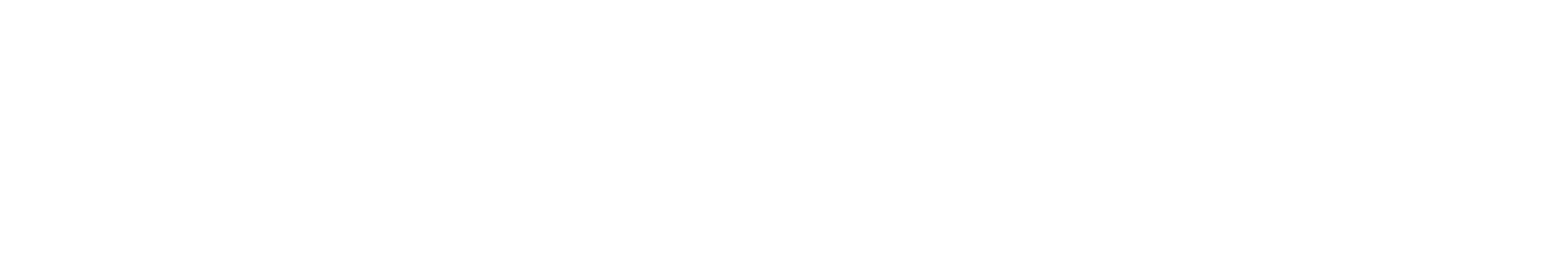

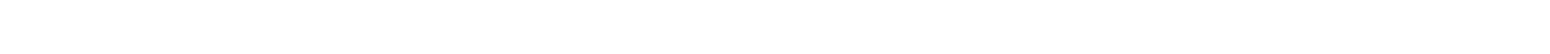

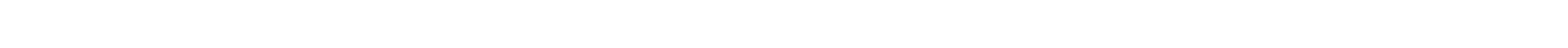

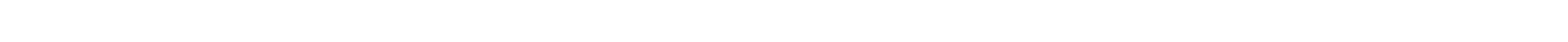

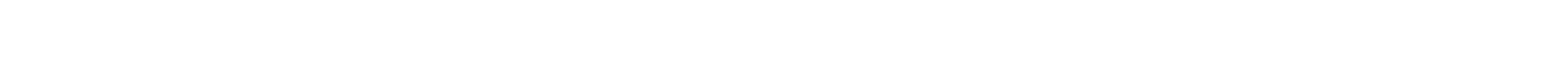

出 is

es

is

is

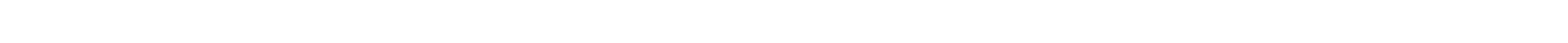
w w 3
5
5

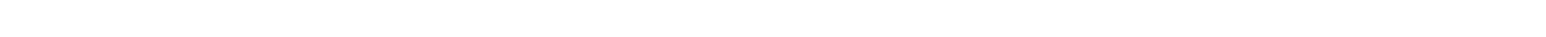

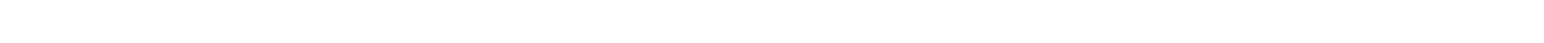

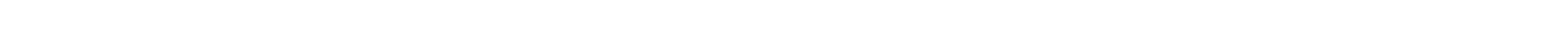

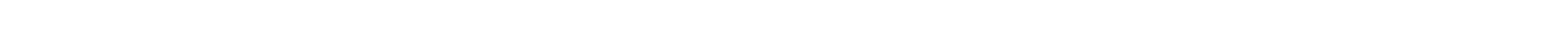

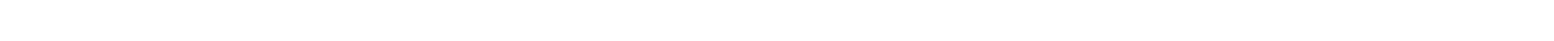

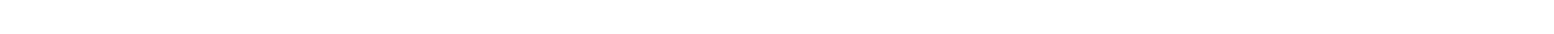

is

n

is

in

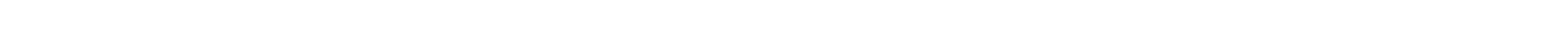

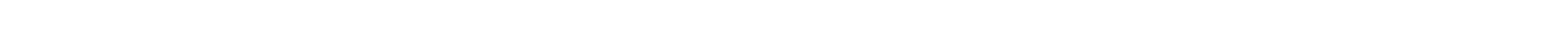

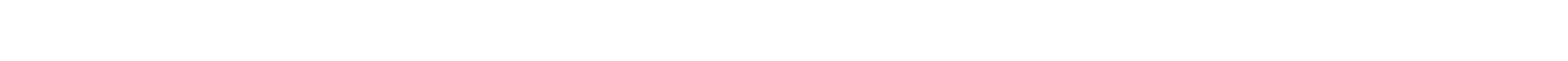

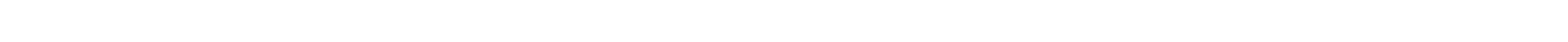

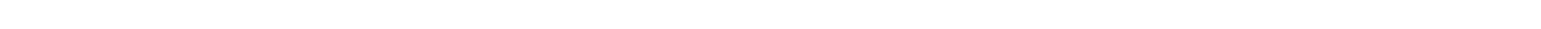

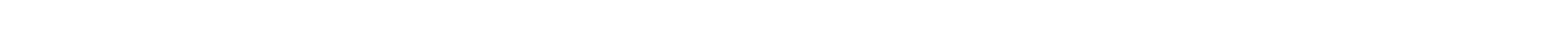

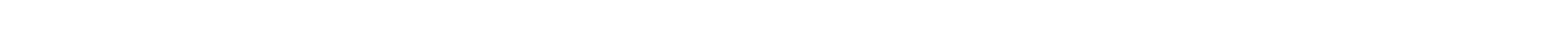

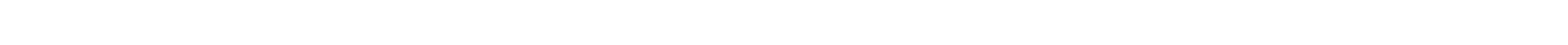

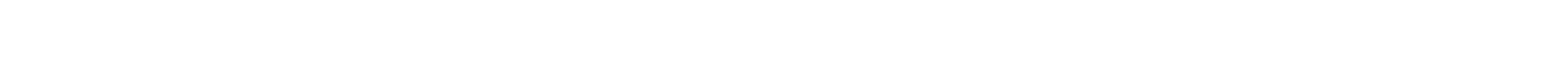




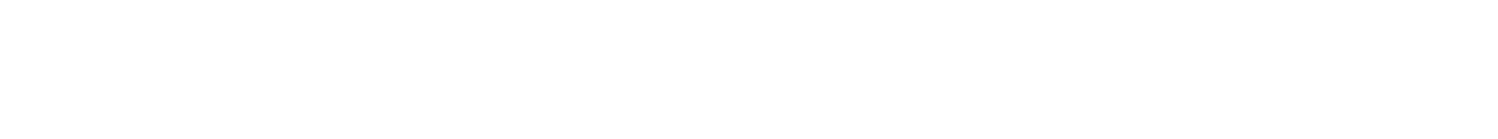

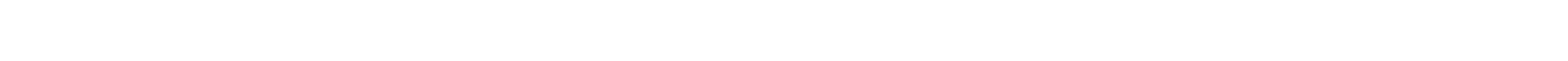
w F

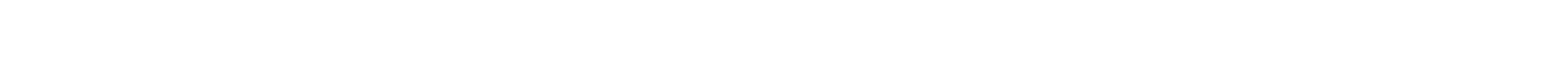

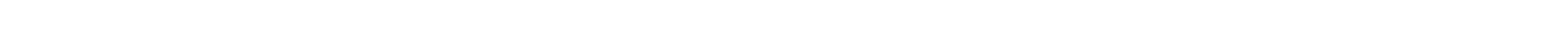

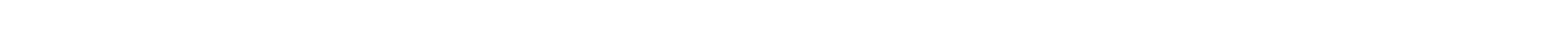

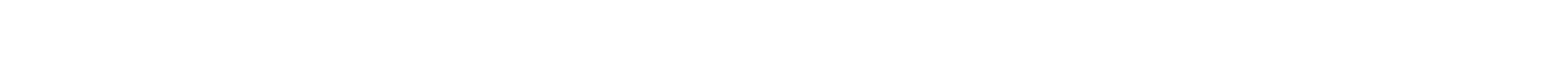

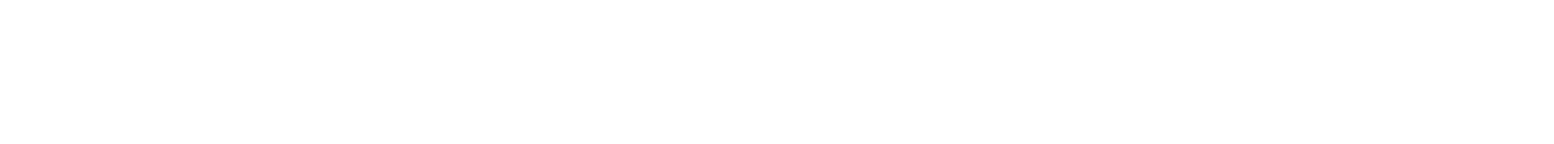

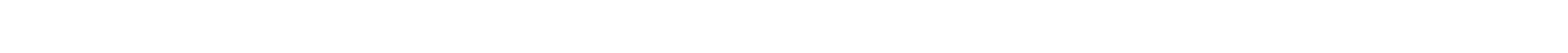

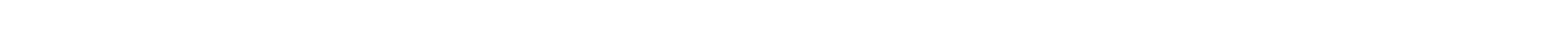
\begin{tabular}{lll}
5 \\
\hline
\end{tabular}

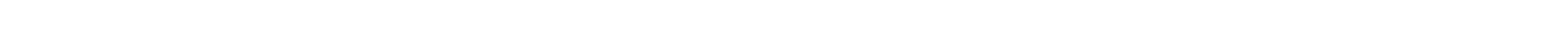

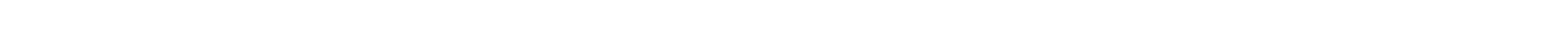

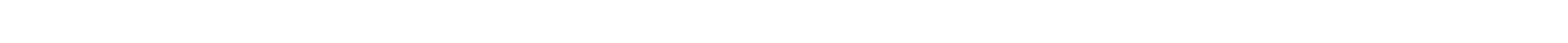

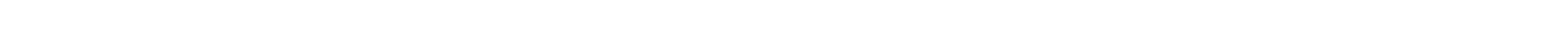

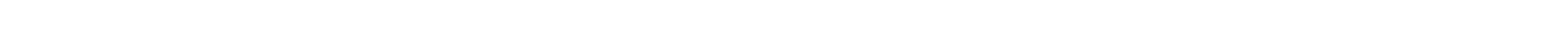

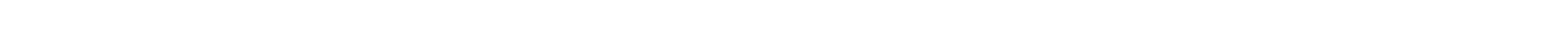

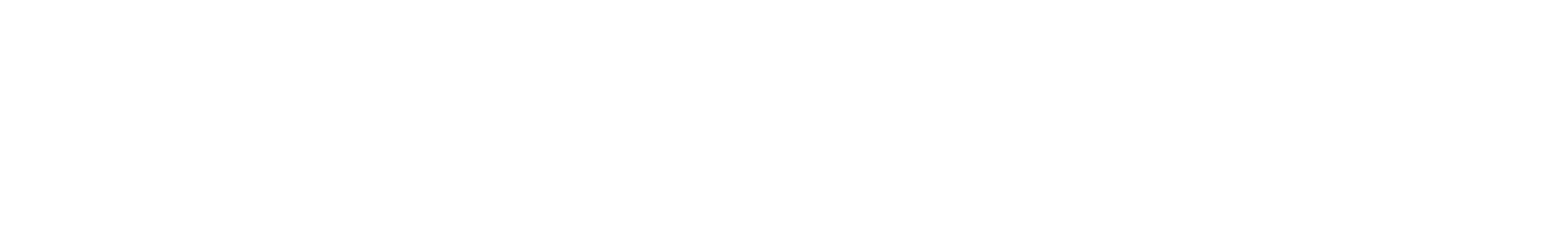

60006

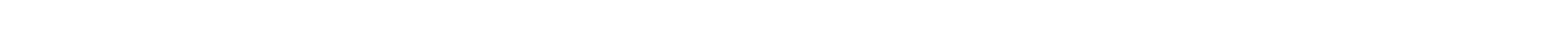

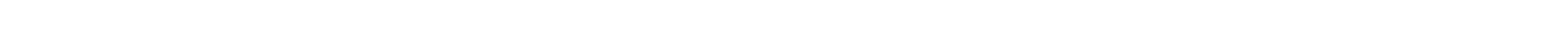

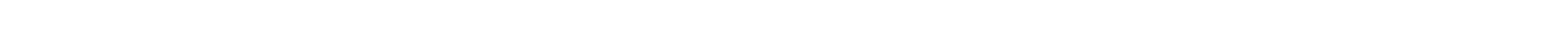

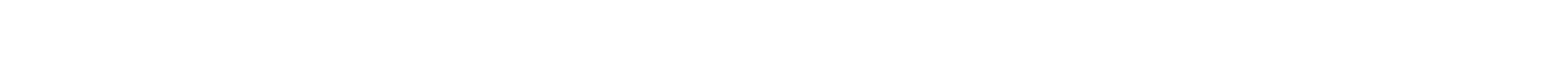

$\underline{\mathbf{x}}$

is

n

in

is

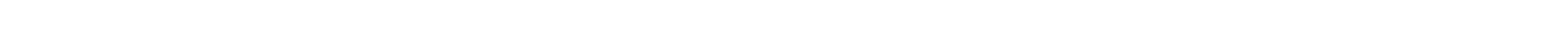

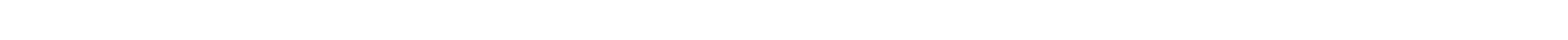
a 3
$x^{2}$

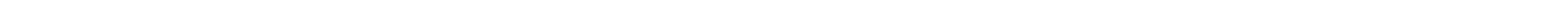

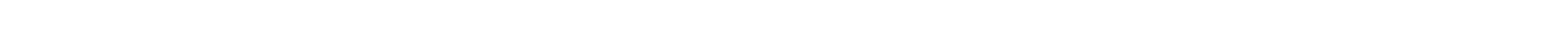
W

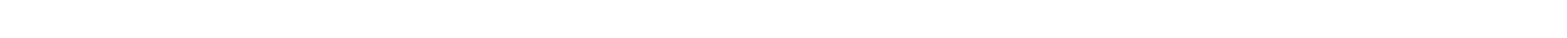

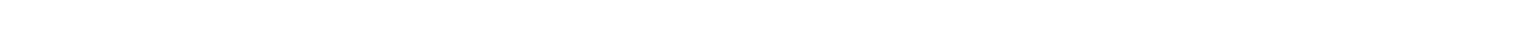

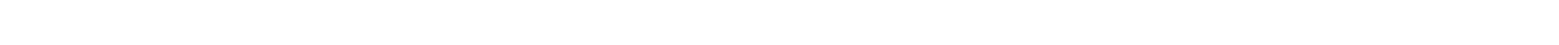




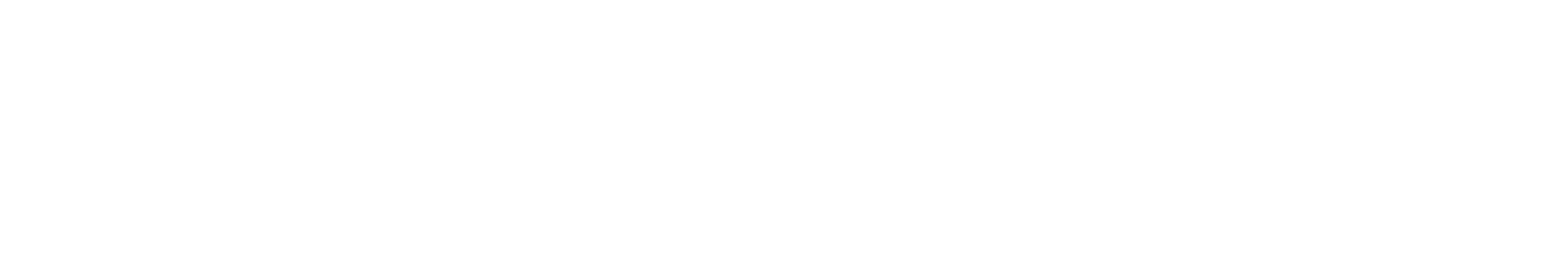

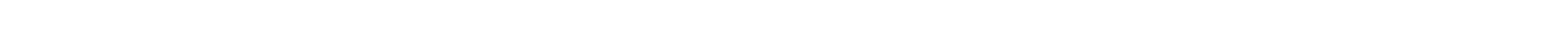

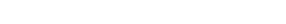
แ

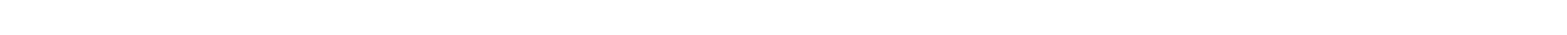

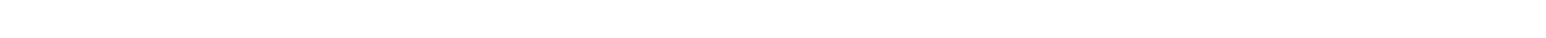

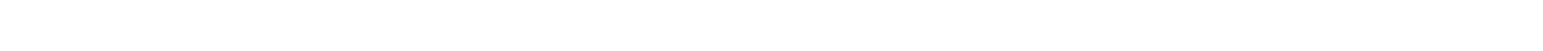

+

$\$$

s

๔

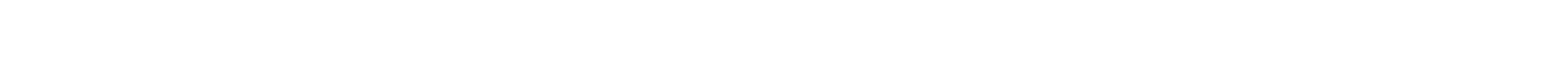

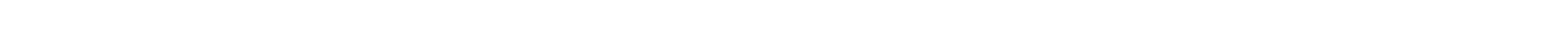

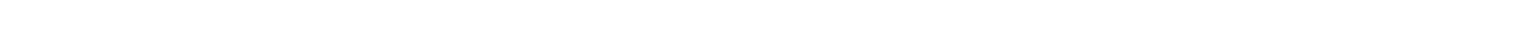

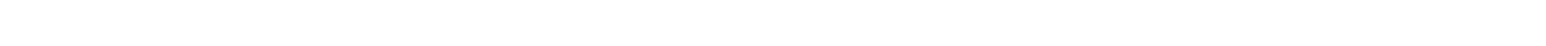

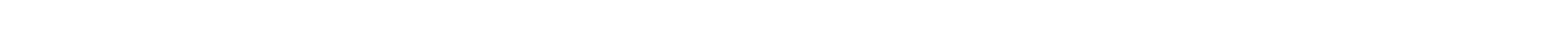

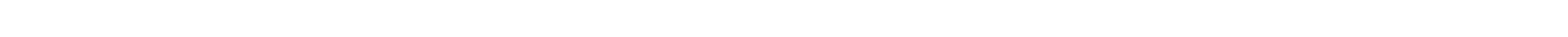

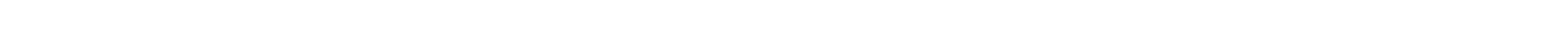

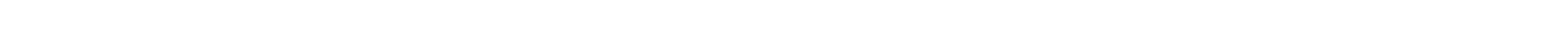
เ
n n
in en
n
$\infty$
$\infty$
$\pm$

n

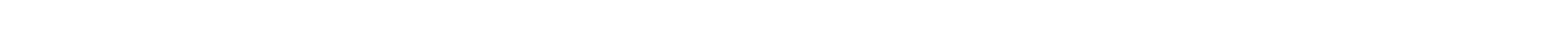

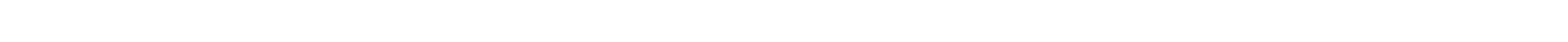

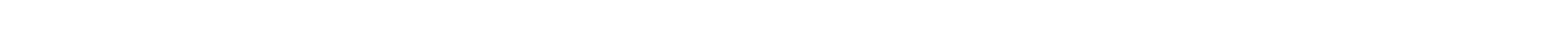

mm

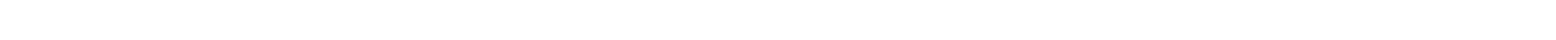
W

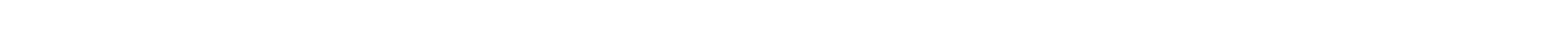

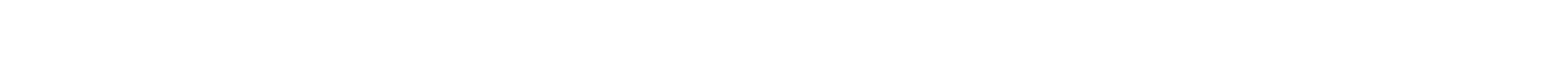

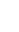

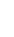

$<$

5

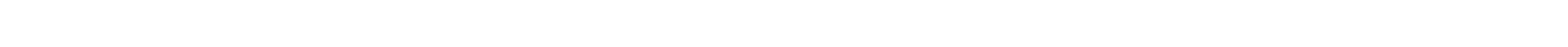

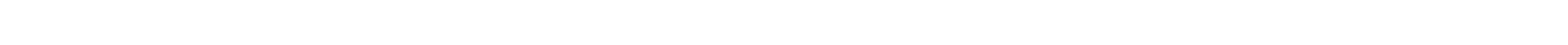

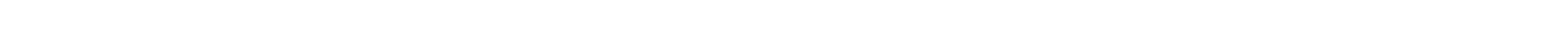

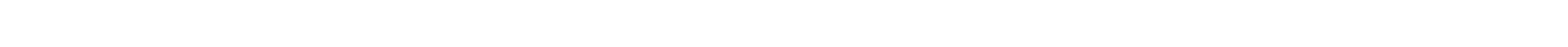

nמm

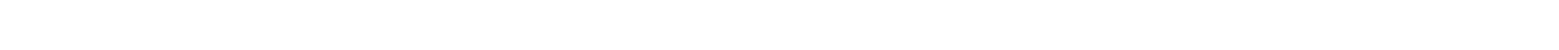

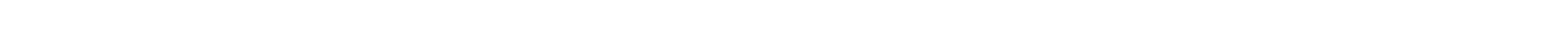

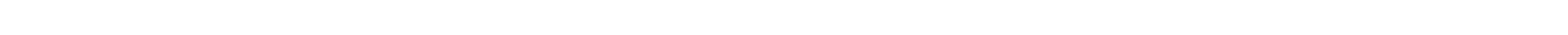

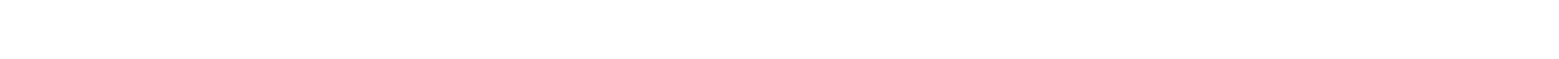




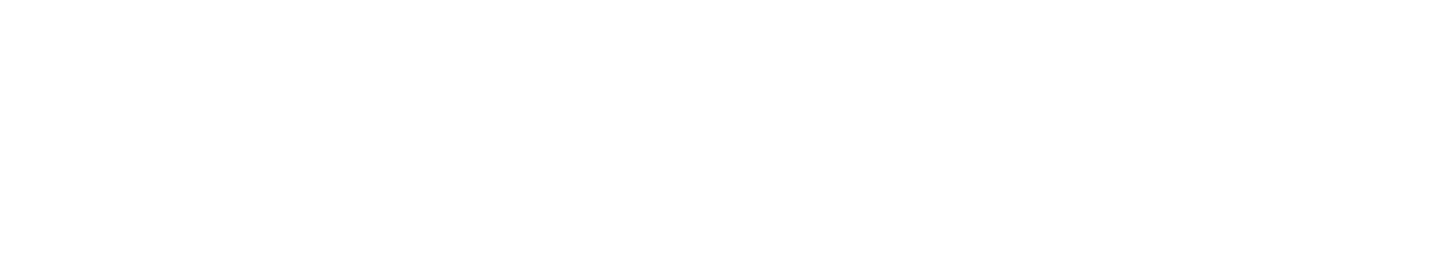

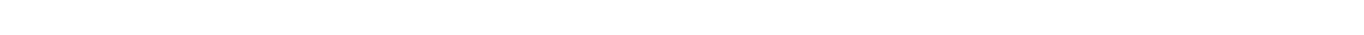

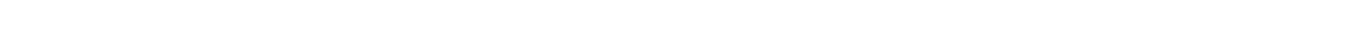

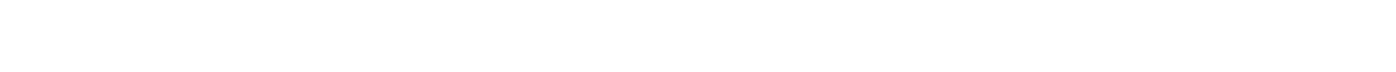

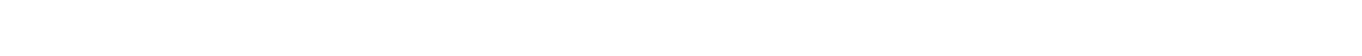

$\Sigma$

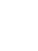

«

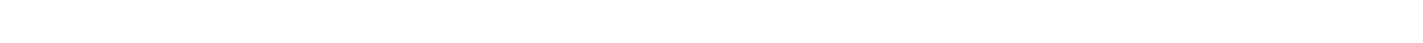

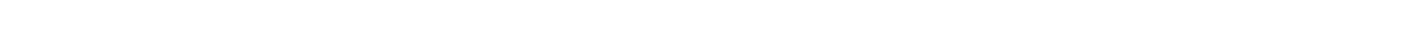

伤

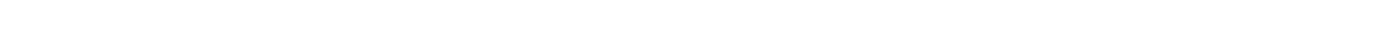

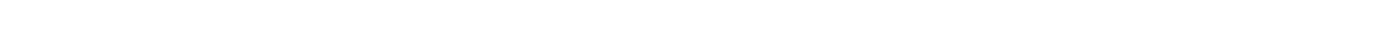

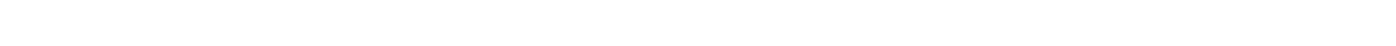

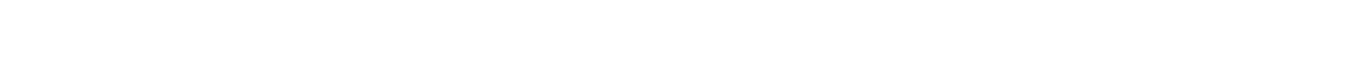
5

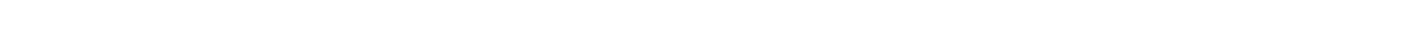
ш

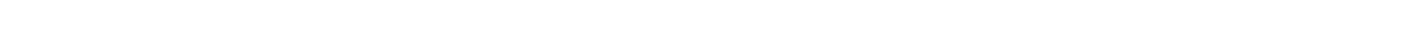

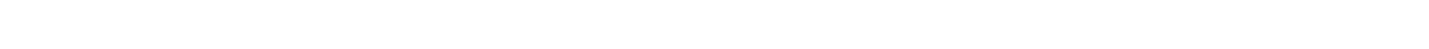

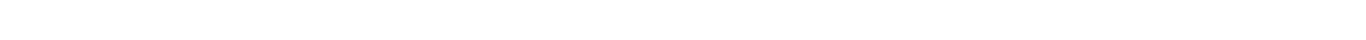

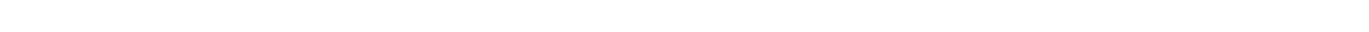

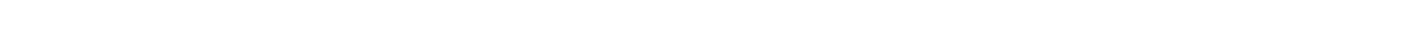

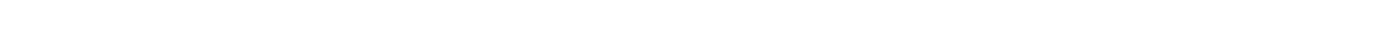

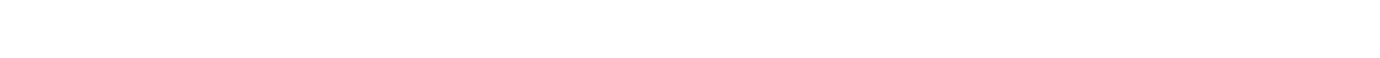
rom

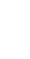

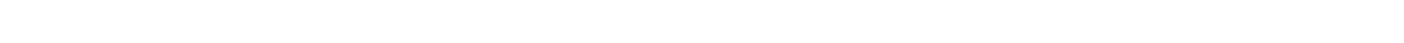

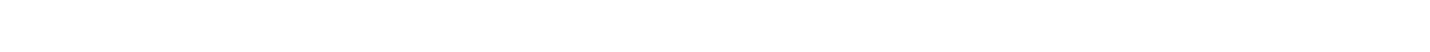
б w

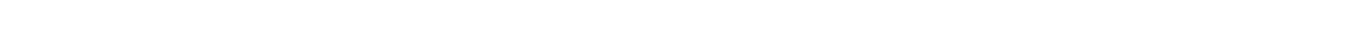
แ

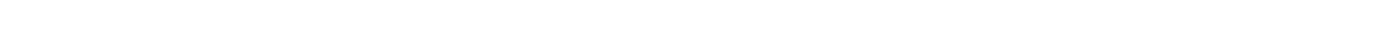

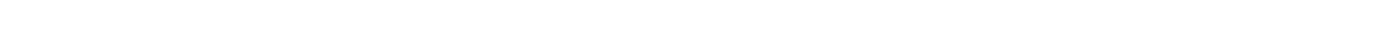

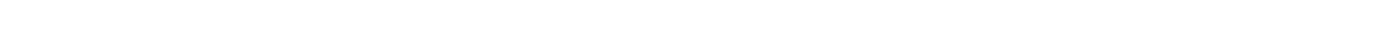



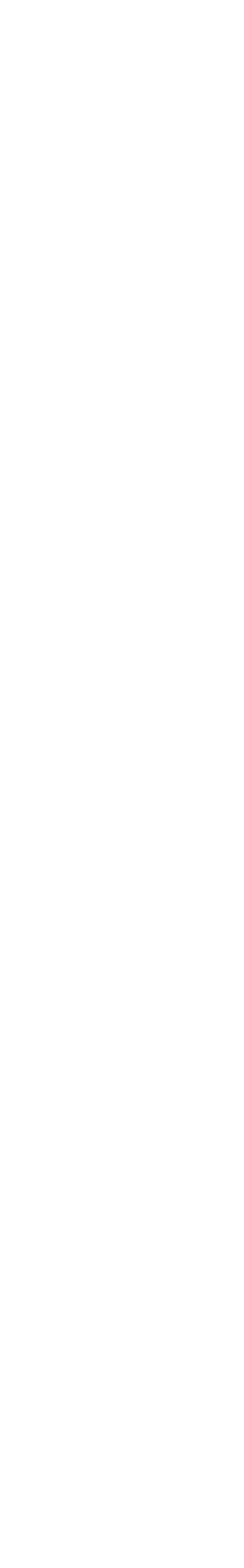
$\geq$ 声。完 แ w a $\dot{a}=$

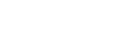
언은 든 ㅎํㅇํํ z 닌 高的 wom mon m는 등

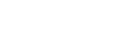
贻"上" - iñ ㅍำ 地: 采上下

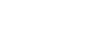
u. mo ลับ. ํํㄹㅇㅛ 20 $\exists \dot{0}$ 岁<smiles>C1CC2CCC(C1)O2</smiles>
든 mar m的茨 mon 3 โw? io $m<n a$ $m>5$

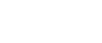
$\sum n i$ :

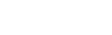
cat 겅응 는년 $\vec{n} \leftarrow=$ a

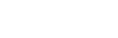
要>0

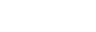
운동 is

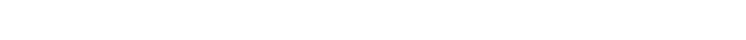
w ×

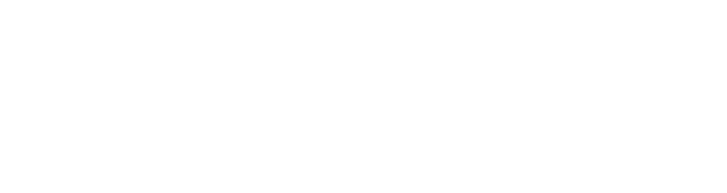

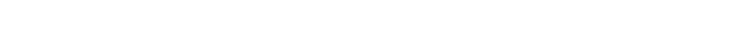
s

"

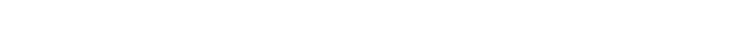

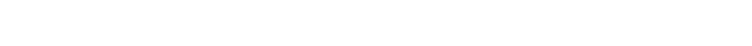

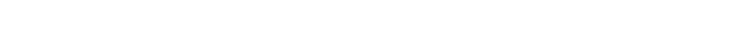
$\infty_{\infty}^{0} \hat{a}$

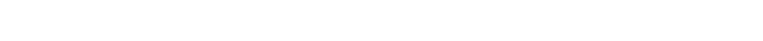

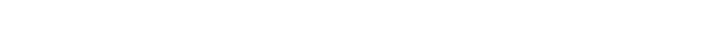
ய

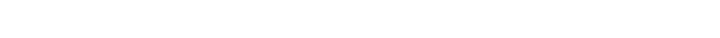
ดกNO-

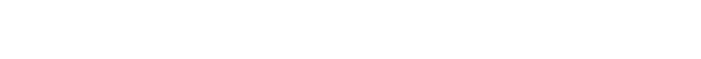

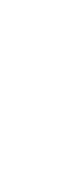

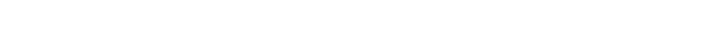
w

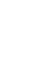

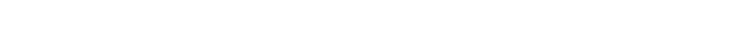
шس

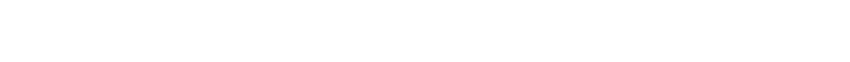

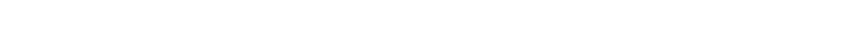

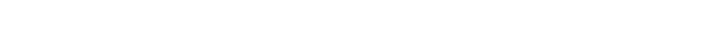

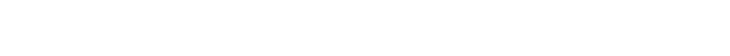

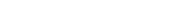

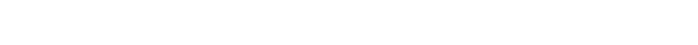

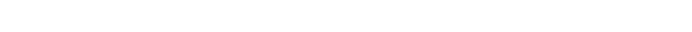

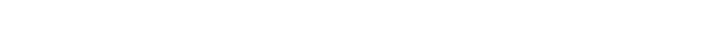

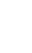

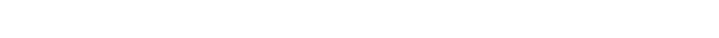

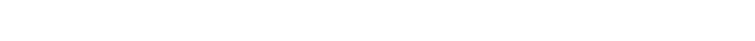

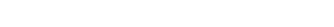

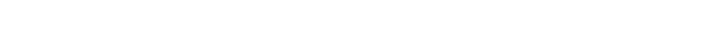

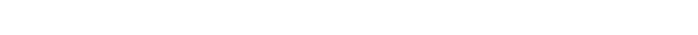

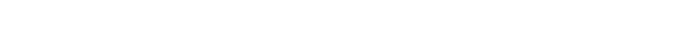

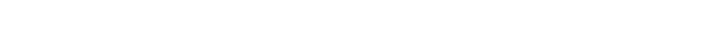

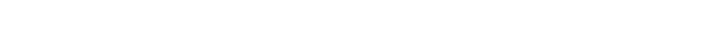

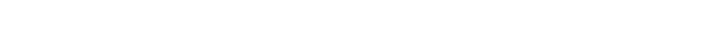


눈은

and

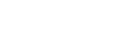
$z-0$ 求范岕 范上

a

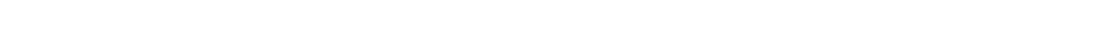

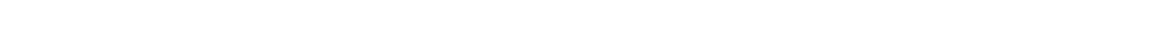
L

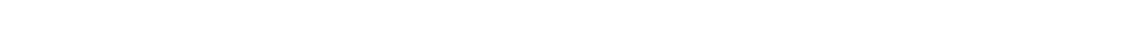

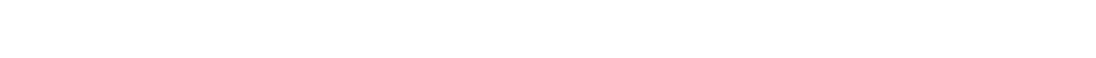

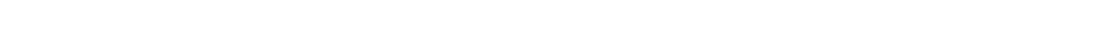

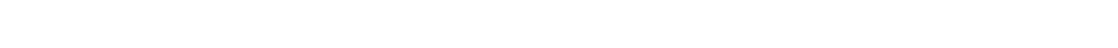

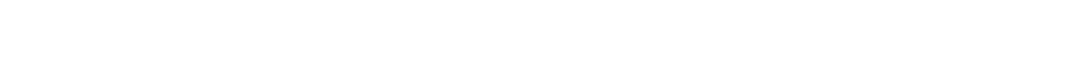

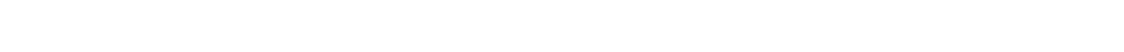

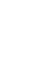

"น

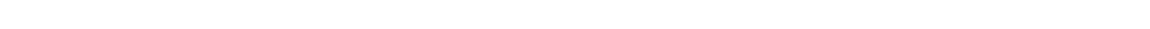

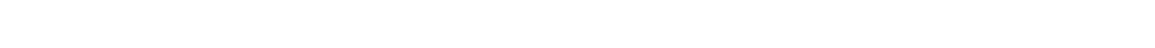

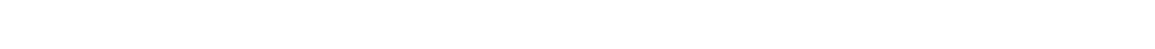

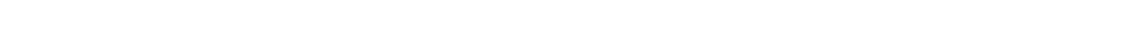

$\underset{5}{m}$

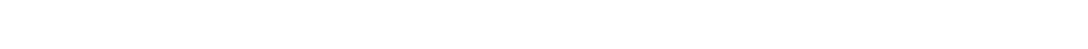

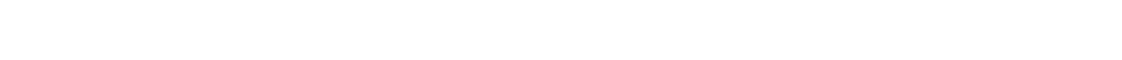

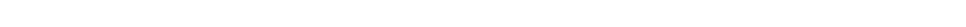

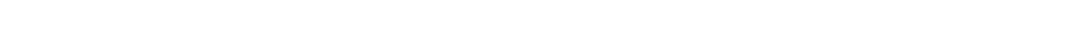

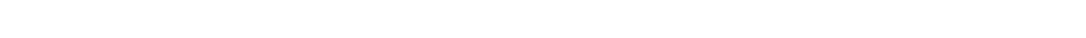

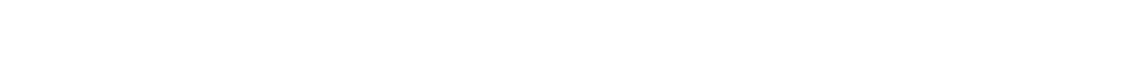

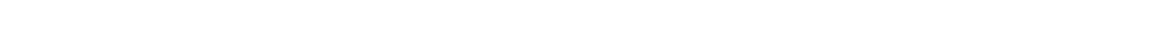

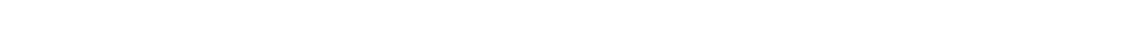

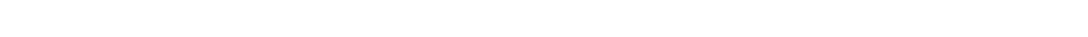

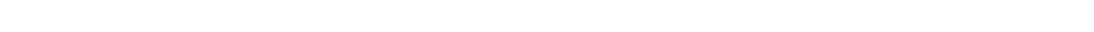

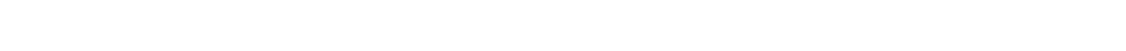

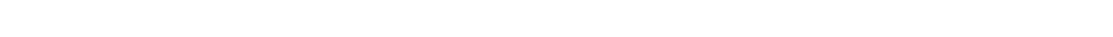

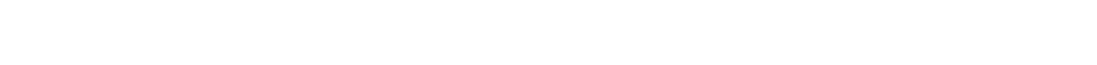
읭

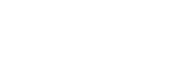
$\because 2 \div 2=0$

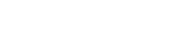

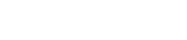
m:

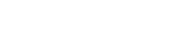
ケッレแーแ

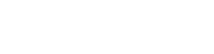
$z^{2} z^{z} z$ 4 出 w a

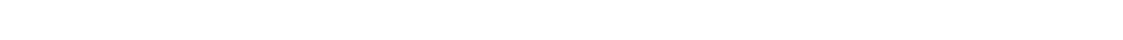

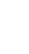

a a.

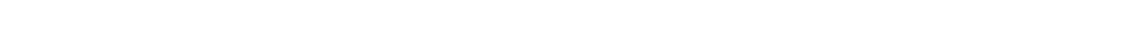
ü T \begin{tabular}{ll}
\hline \\
3 \\
3
\end{tabular}

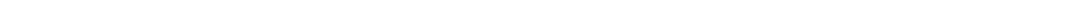

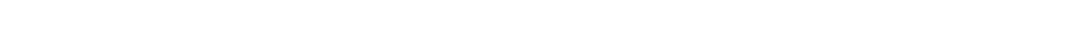

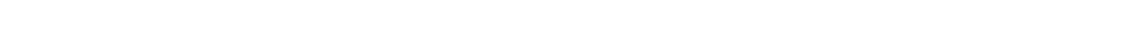

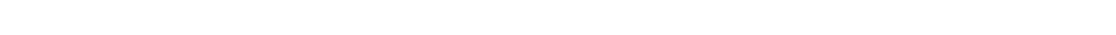

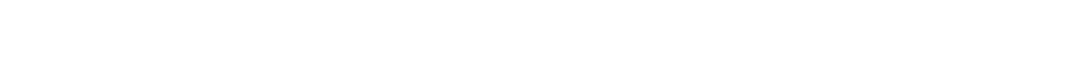




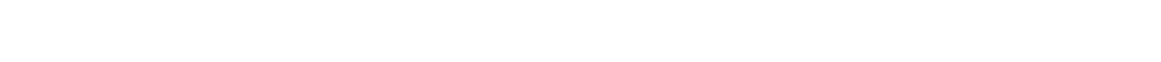
in

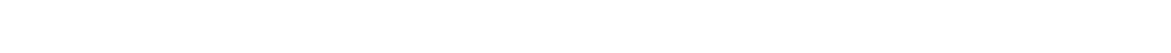
. - =

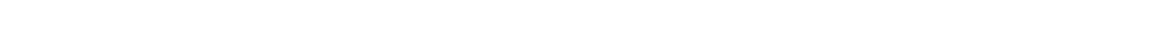

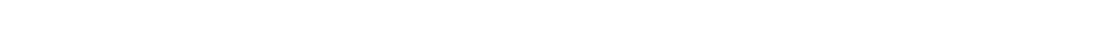

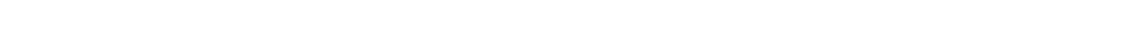

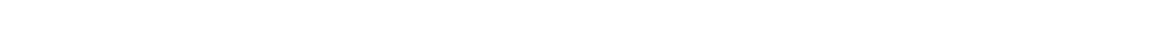

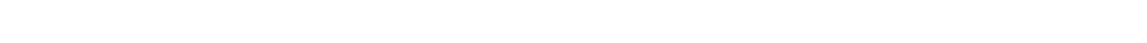

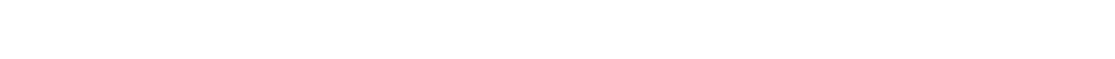

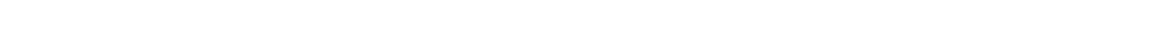

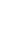
is a a n

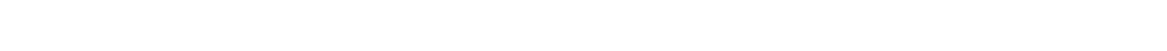
ж. -

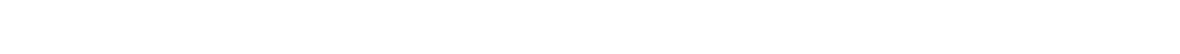

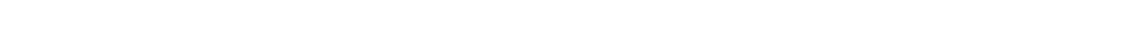
w

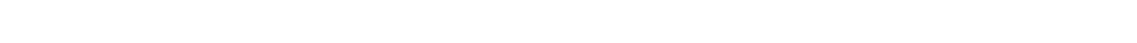

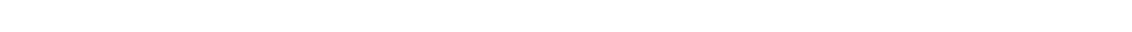

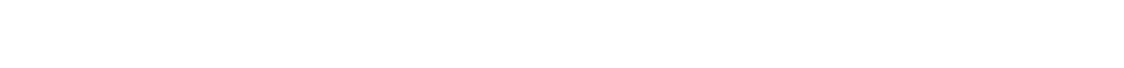

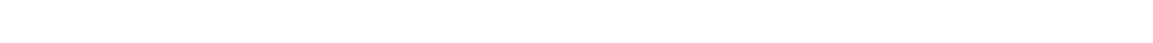
in

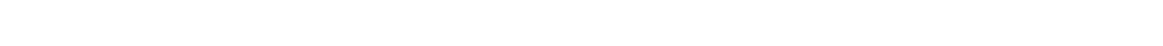

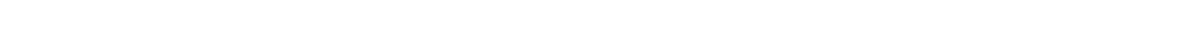
w \begin{tabular}{lll}
\hline \\
\hline
\end{tabular}

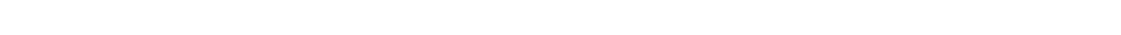

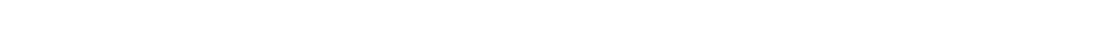
w LED

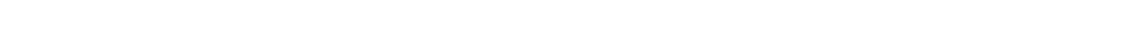

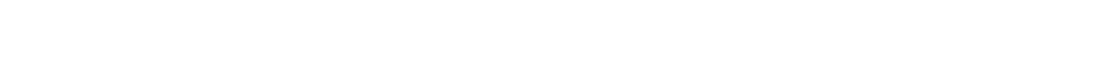

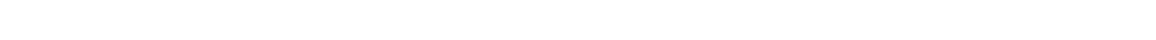
$\alpha$

$\sim$

a

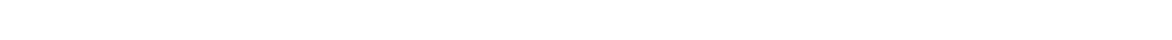
tü

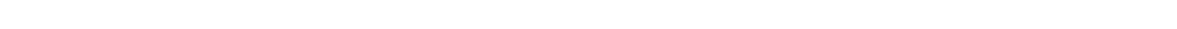

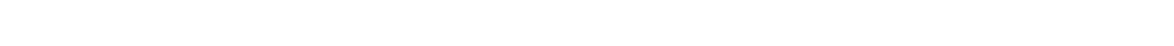

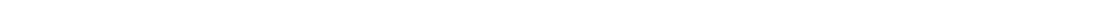

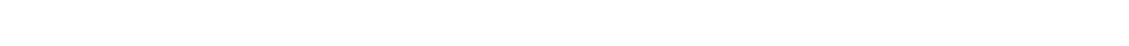
แ

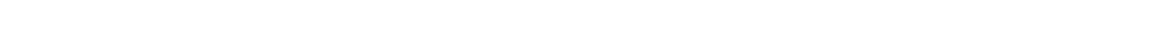
in minn 

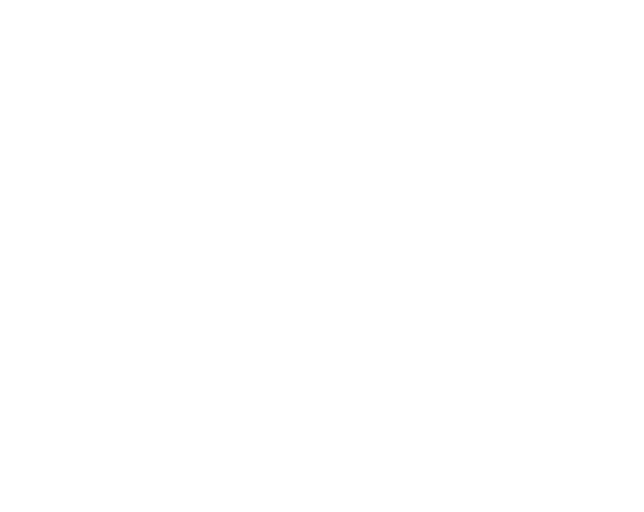

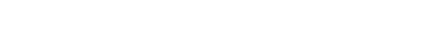

$\leq$

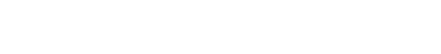

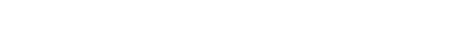
\# Oncinom

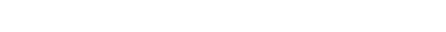

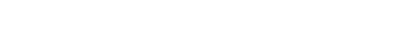

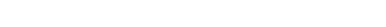

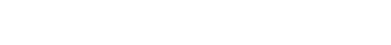

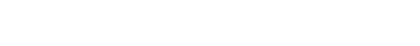

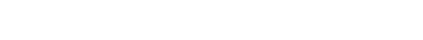

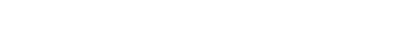

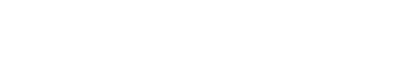

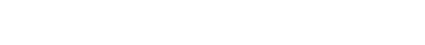
$\sum$

$\stackrel{a}{i} a$

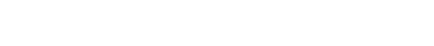
w $\dot{0} \dot{0} \dot{0} \dot{0} \dot{0} \dot{0}$

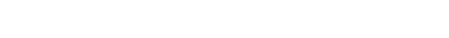

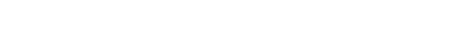

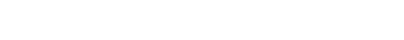
幺幺 n w 2022220202202 L5 QNTF-TNNTNFTOC

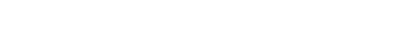

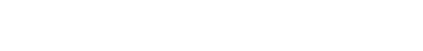

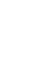
o

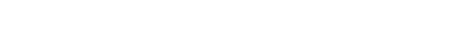

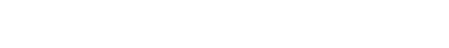

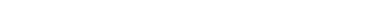

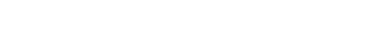

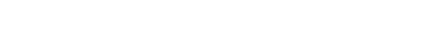
anmardoto -

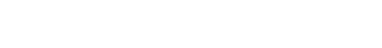



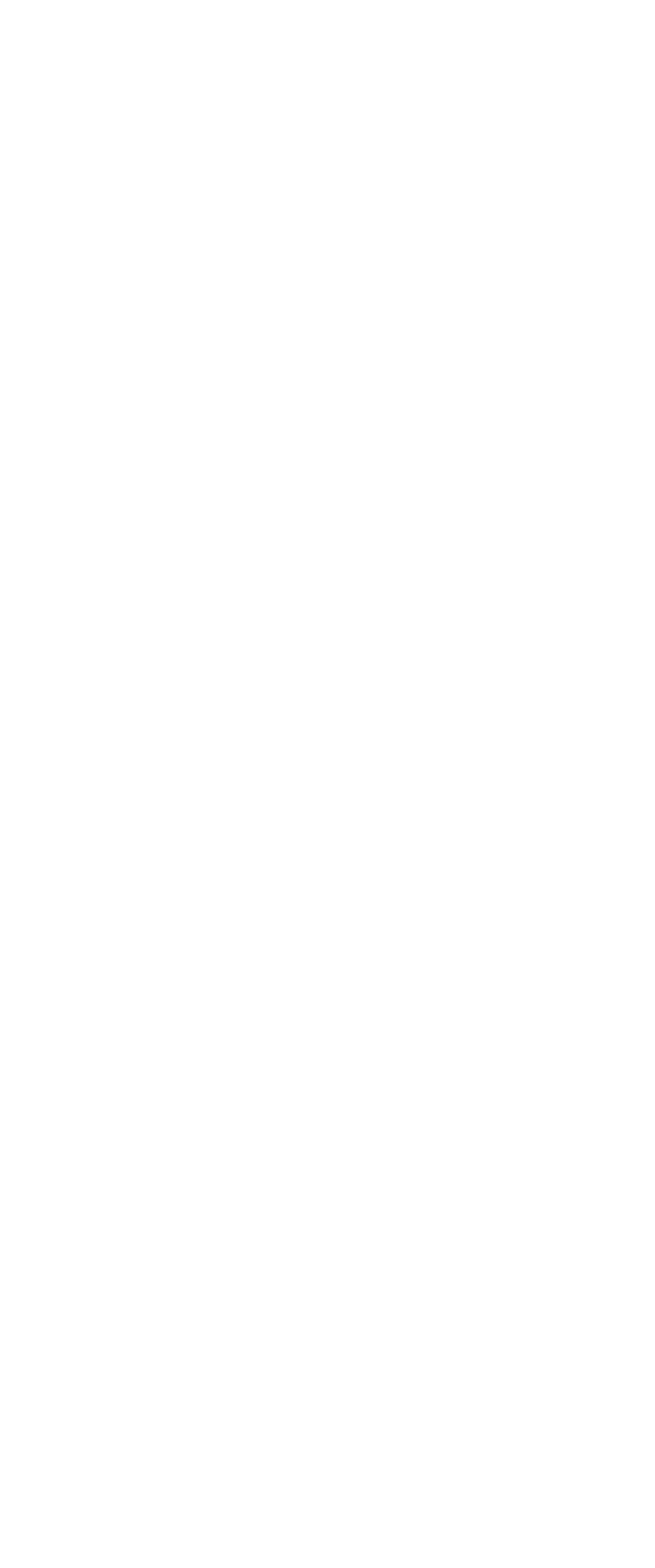

twescosenesenes

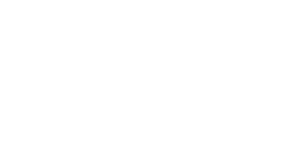

ํㅡ슷

w

$\infty 0=0=\infty \infty \omega$

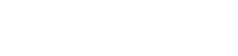

wesesenesen es as

t $20-500$ ш

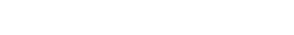

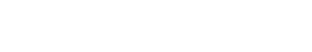

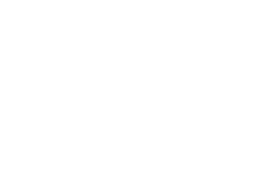

Eusescososeseses 5

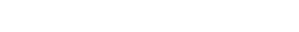
w 等

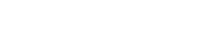

wazaza

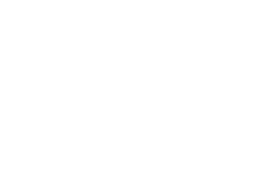



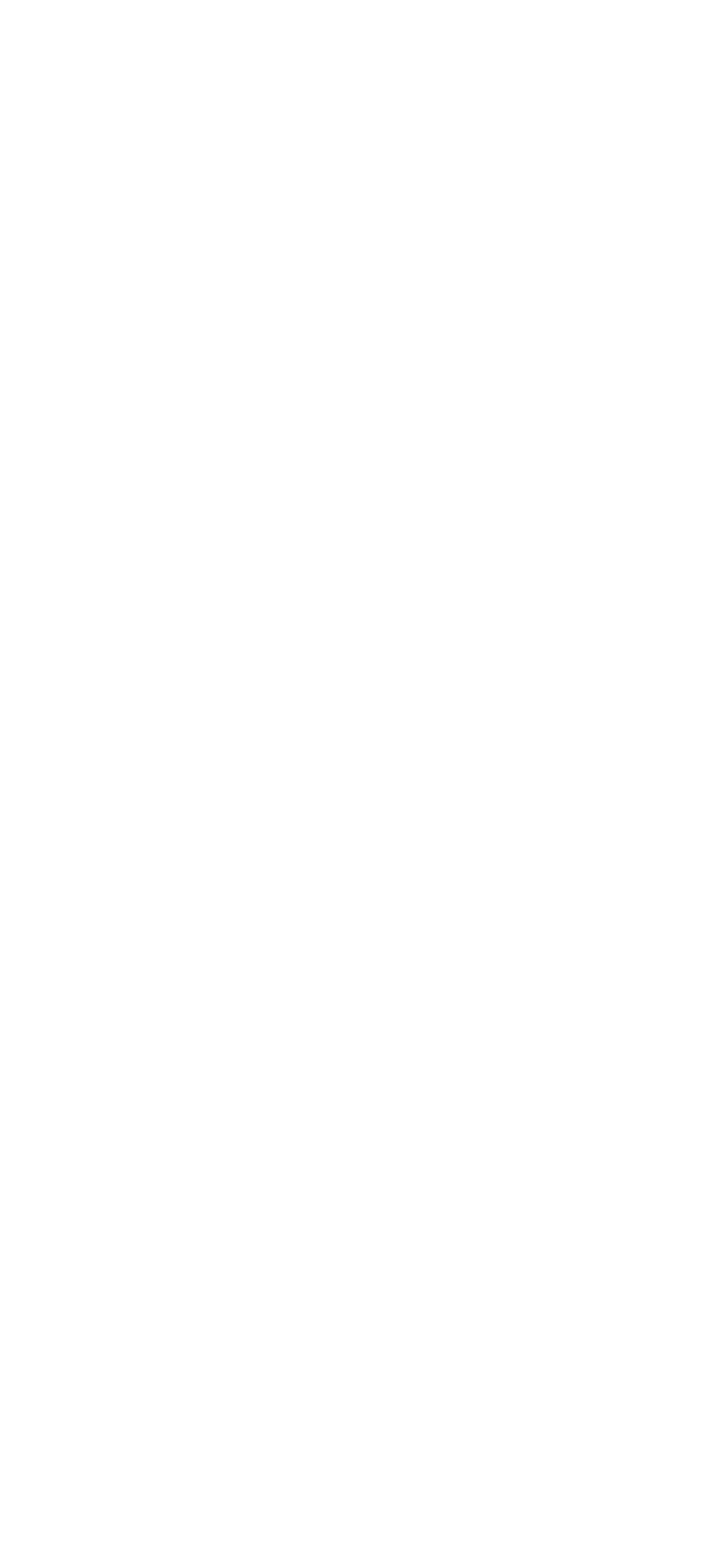

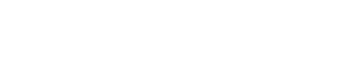

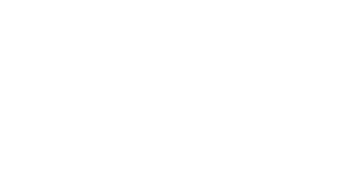

ผ人ั

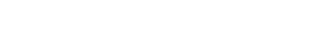

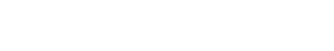

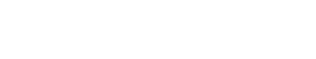

$5 \cos \operatorname{sen} \operatorname{ses} c \cos$

is a

- a a

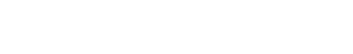

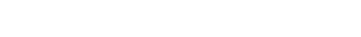
a

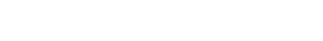

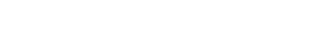

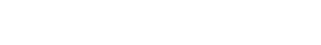

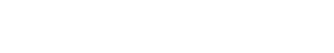
ニニ ニ E

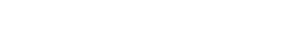

Exescososencososos

is

$a$

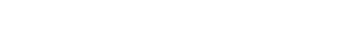

ü

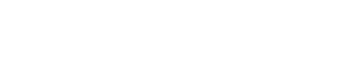

Dodonarara

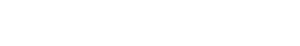

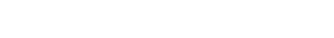

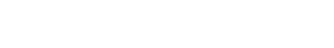

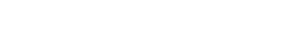



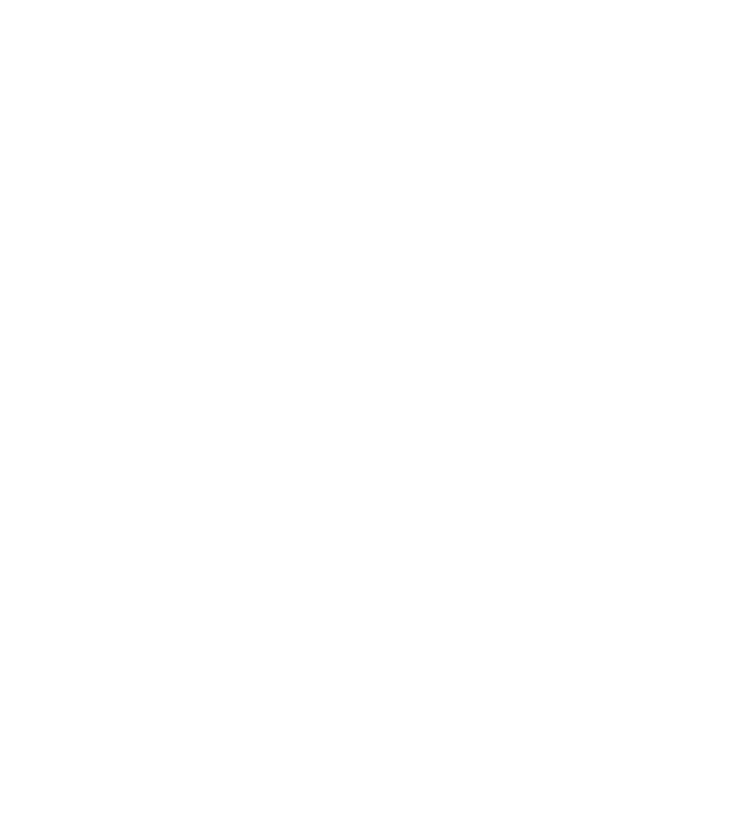

$z-1$

$=\frac{m}{\sim}$

$\frac{5}{\pi}$

空

a.

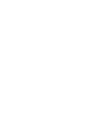

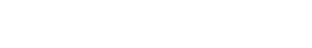

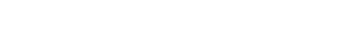

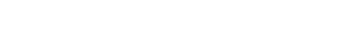
$\triangle \sim 5 \cong-N 5=-ー ニ \cong$

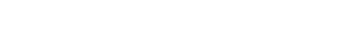

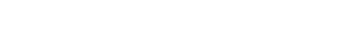
in

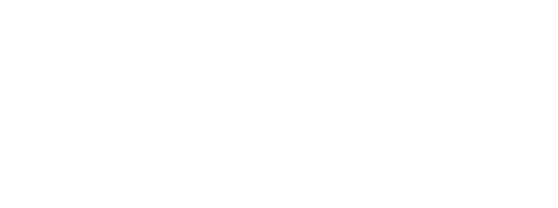

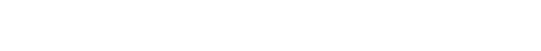

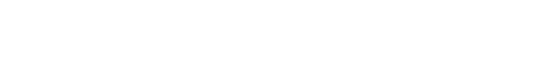

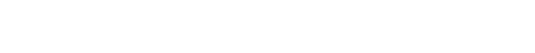

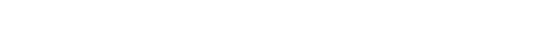

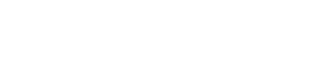

$\ln _{n \rightarrow \infty}$

ำ

으으은

$=-5$

ํํ으ํ

승ํㅇㅇㅇ

$\ddot{u}-\ddot{w} \sim$

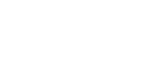

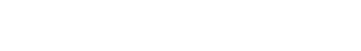

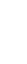

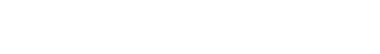

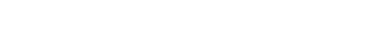

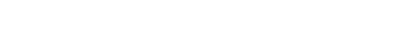
I

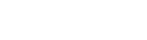

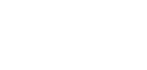

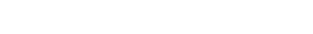

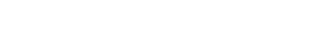

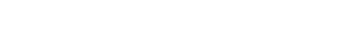

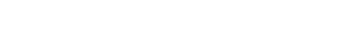

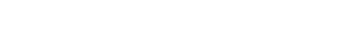



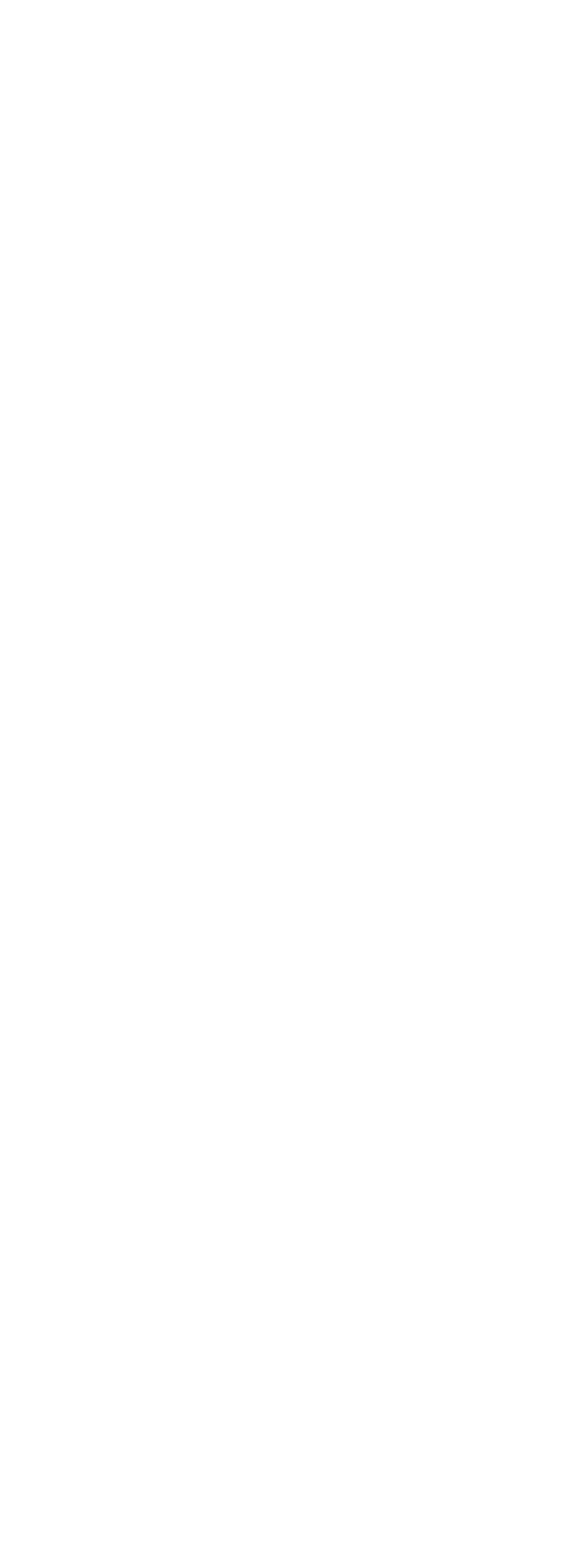

잉요

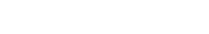

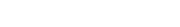
Imña twescosenes 5 - $N$ m O 促

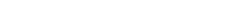
nin in m n w

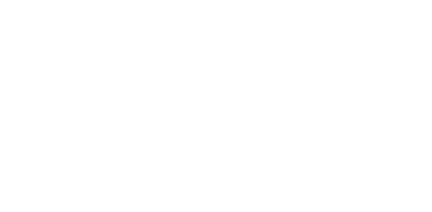
$\underline{\underline{x}}$

os on mesen in

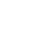
舟 on a

$\rightarrow-1$ w吅

w :

m

땐

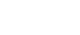

$\sum \frac{m}{m}$

에

$\propto 0$

जo

은

w

w口

anis

$z^{2} \geq 2$

nnmm

แ2:0

onññ

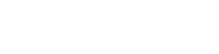



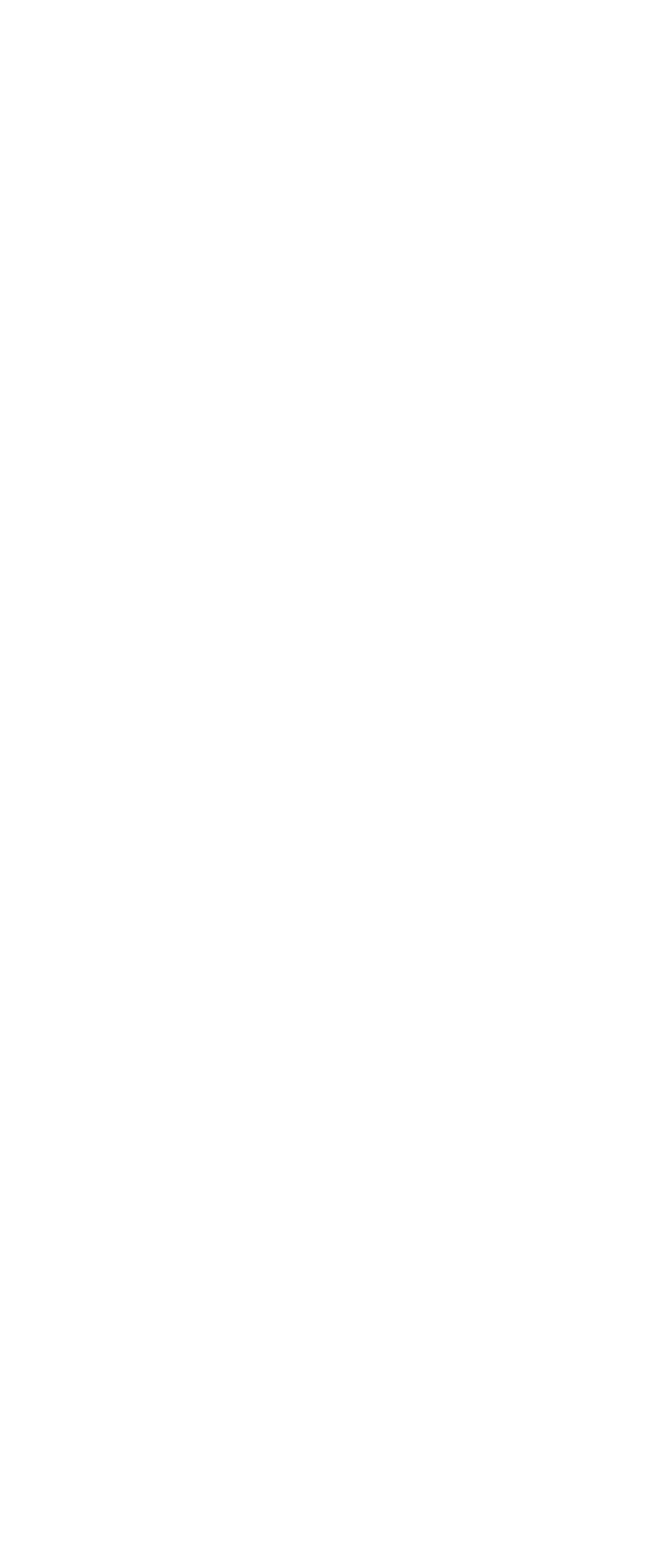
is

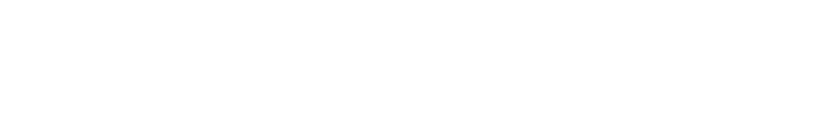

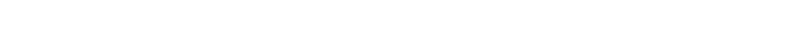

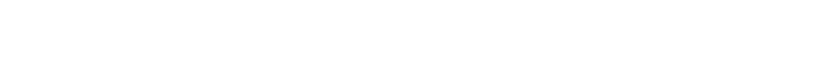

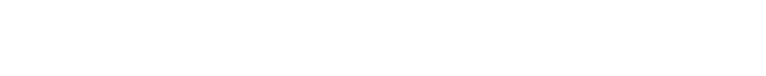

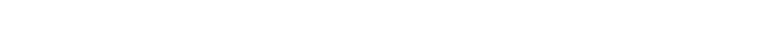

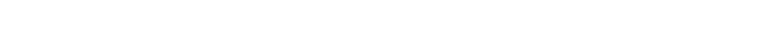

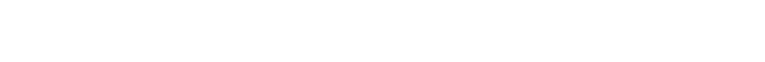

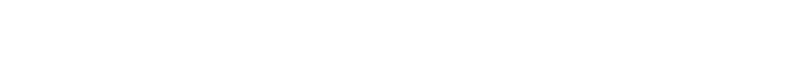

$\leq \alpha$

a a

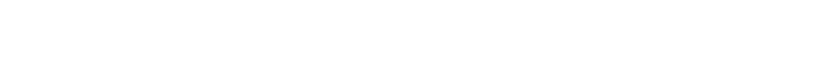

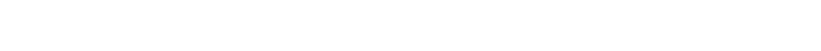
I

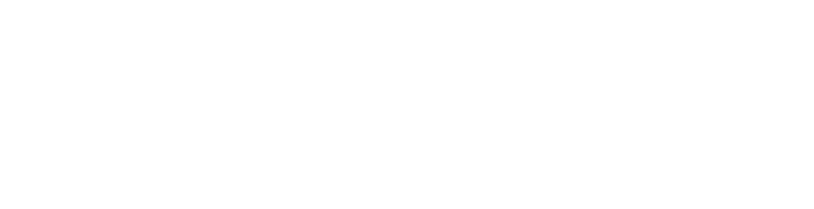

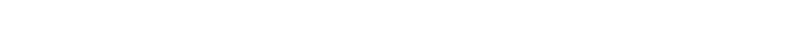

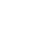

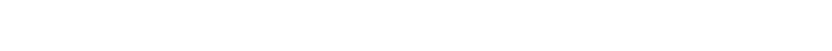

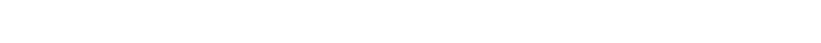

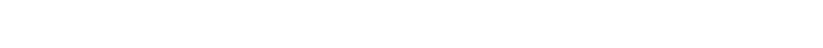

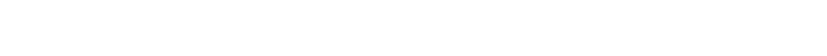

iits $\quad$ nNmmmmmmmmmmmmatadatanunm

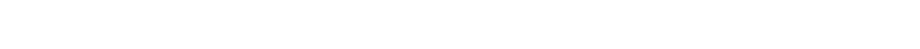
\&

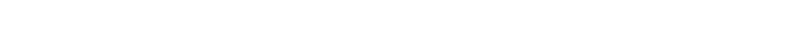

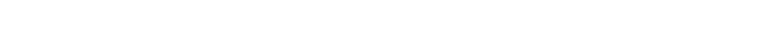
NーN NNOFーONOFOーNF

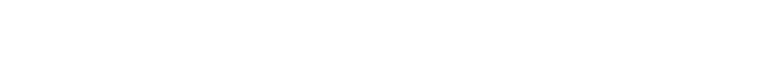

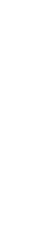

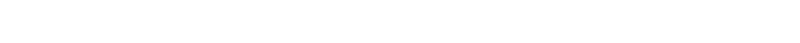

is

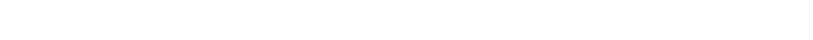
u山 úa

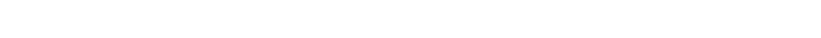

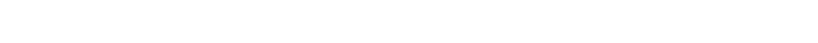

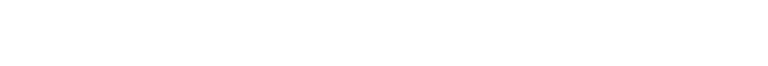

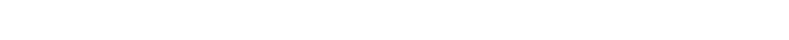

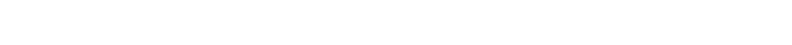

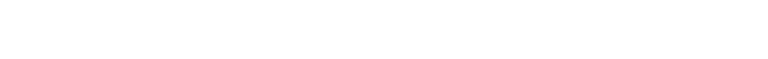



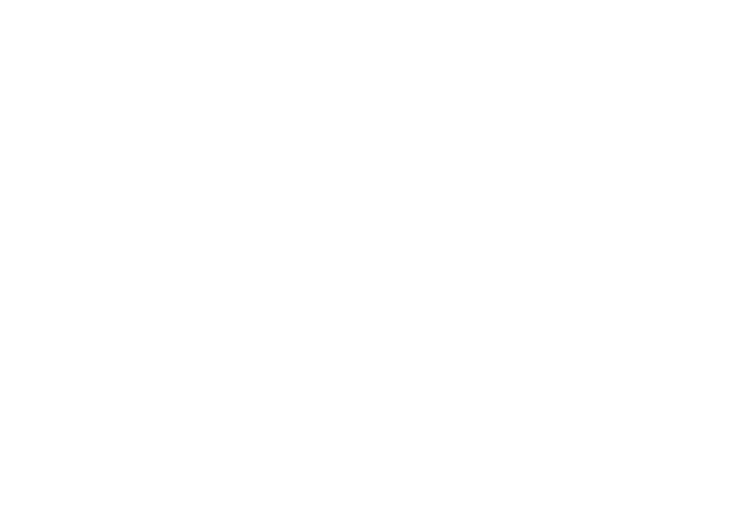

twencososonesos

is

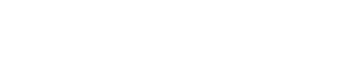
ช

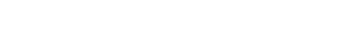

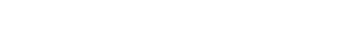

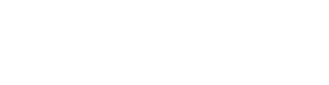

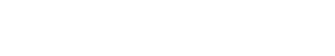
\&

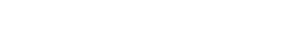

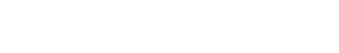
: $\quad$ a

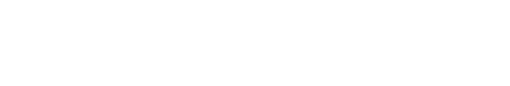

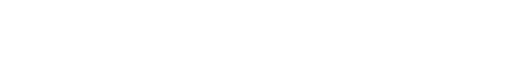

0

is

$\ddot{w} \approx \ddot{w}$

岂岌客

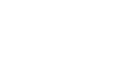
a. $n \geq n z$

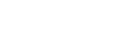
2100 ă 느은 aํㅇ응

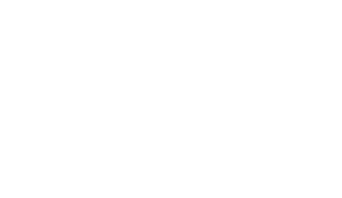

Euscosescosonos is

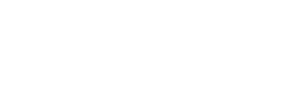

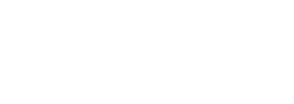
品

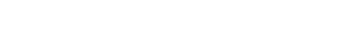
a - >aaaaㅇㅇㅇ

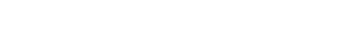
ज

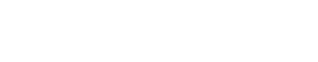

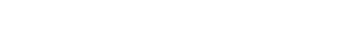

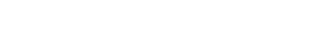

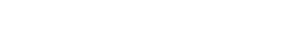




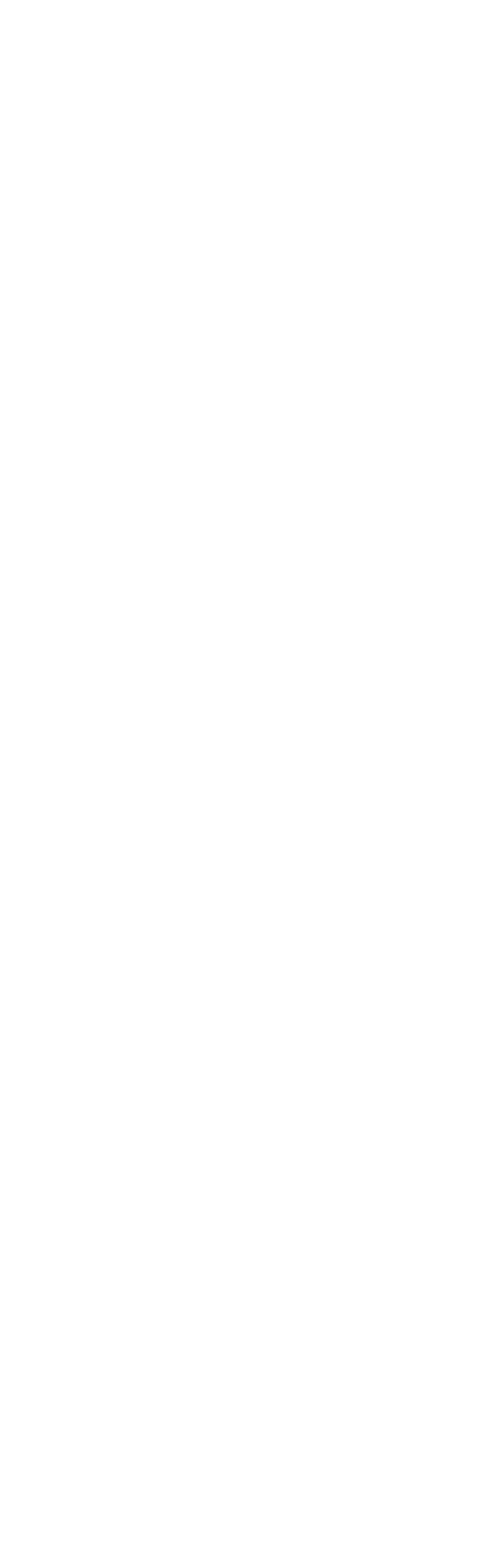

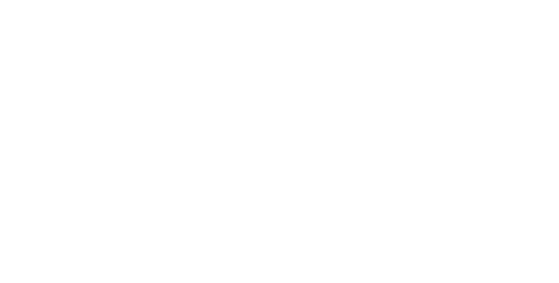

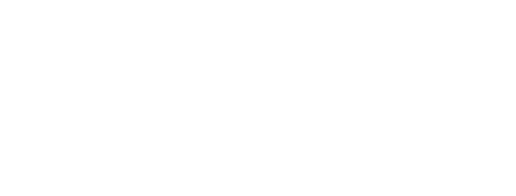

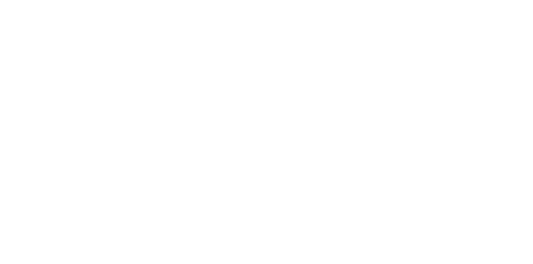

a)

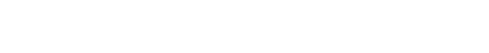

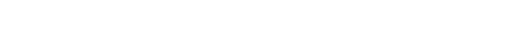

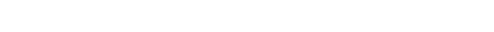

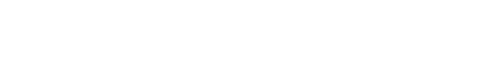

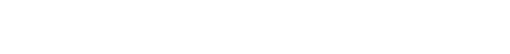
in in

$\infty n$

๑ O w -

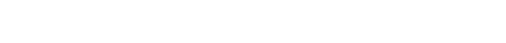

Бธธธ㇒ธ

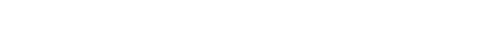
WE Nㅜ№m FNNEEE

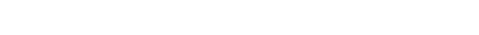

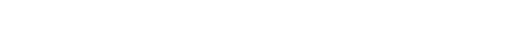

\pm in enes

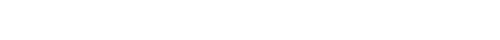
少

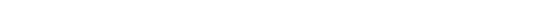

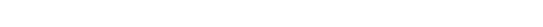

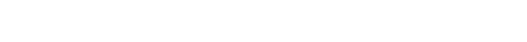

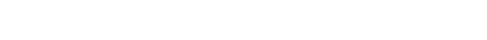

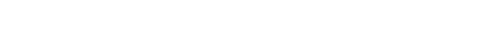
แ

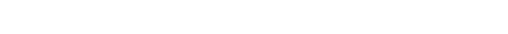

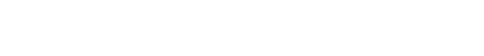




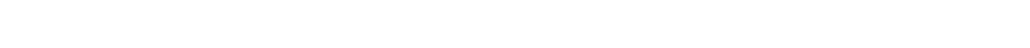

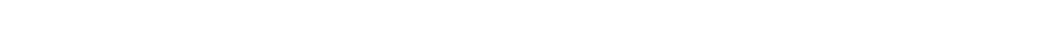

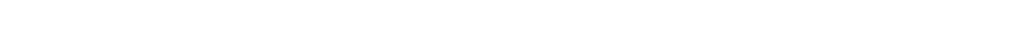

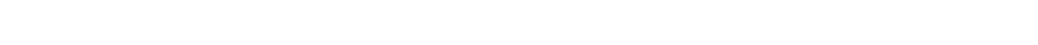

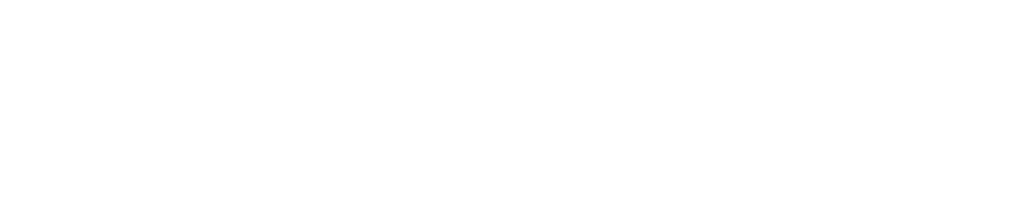

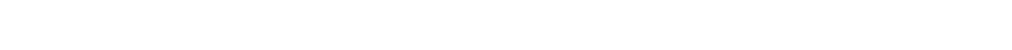
is 년

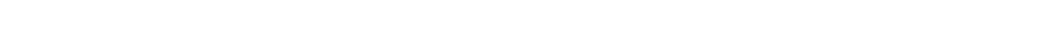

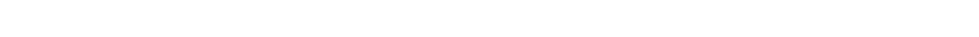

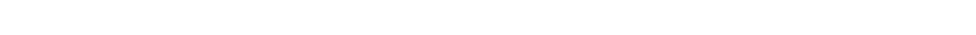

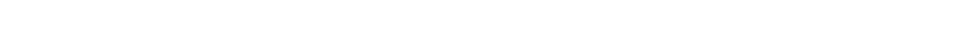

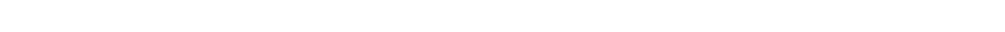

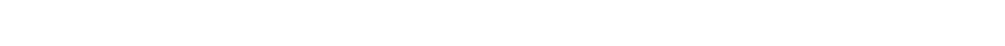
O- N-NMOFMNE-OFNONONONOFNNF-ーNFNN

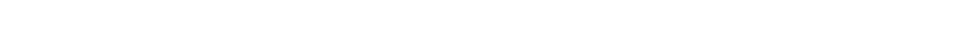

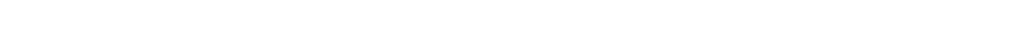

in

х w

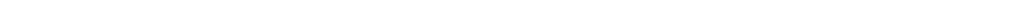

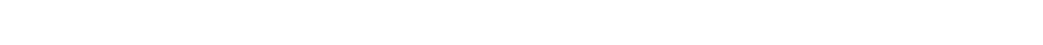

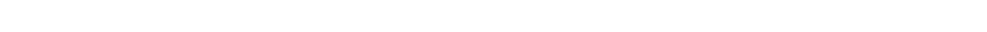

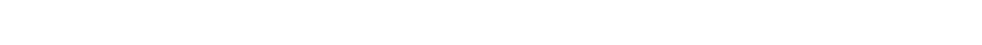

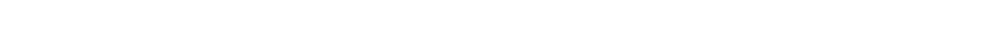

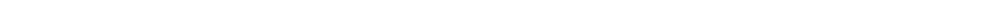

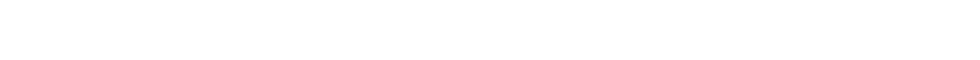

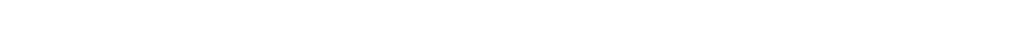
正

a

幺иmo w山 在

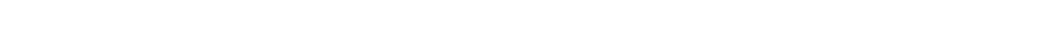

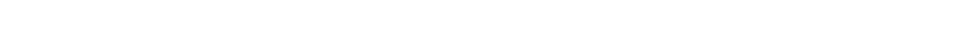

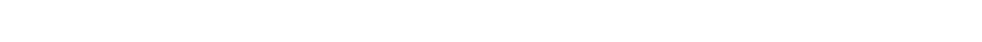

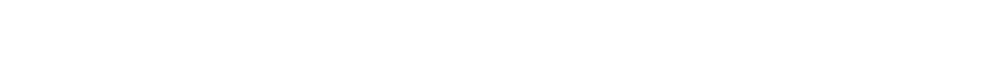

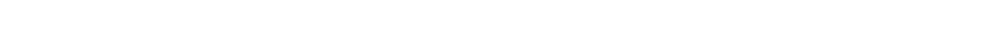

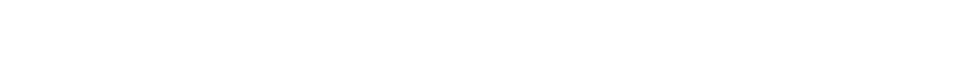



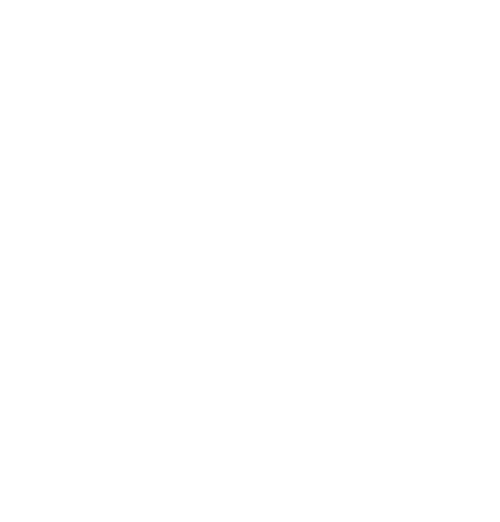

twen en $\sum_{i}>$

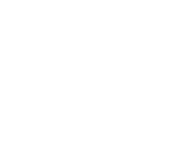

$\therefore \quad$ 모요 แะニ

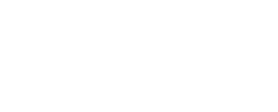

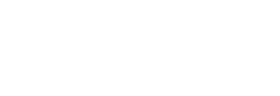
II

Exesen is

nnoo

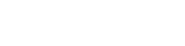

-

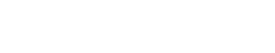

- $\ddot{w}$ noor 푸 무 의

w थ कून

w

$\underset{z}{2}$

\&

닐.

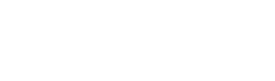

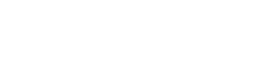
ob w

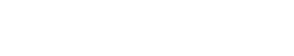

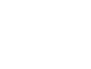

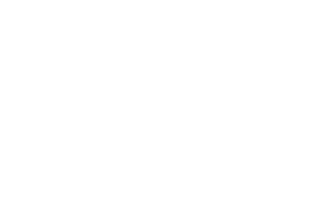



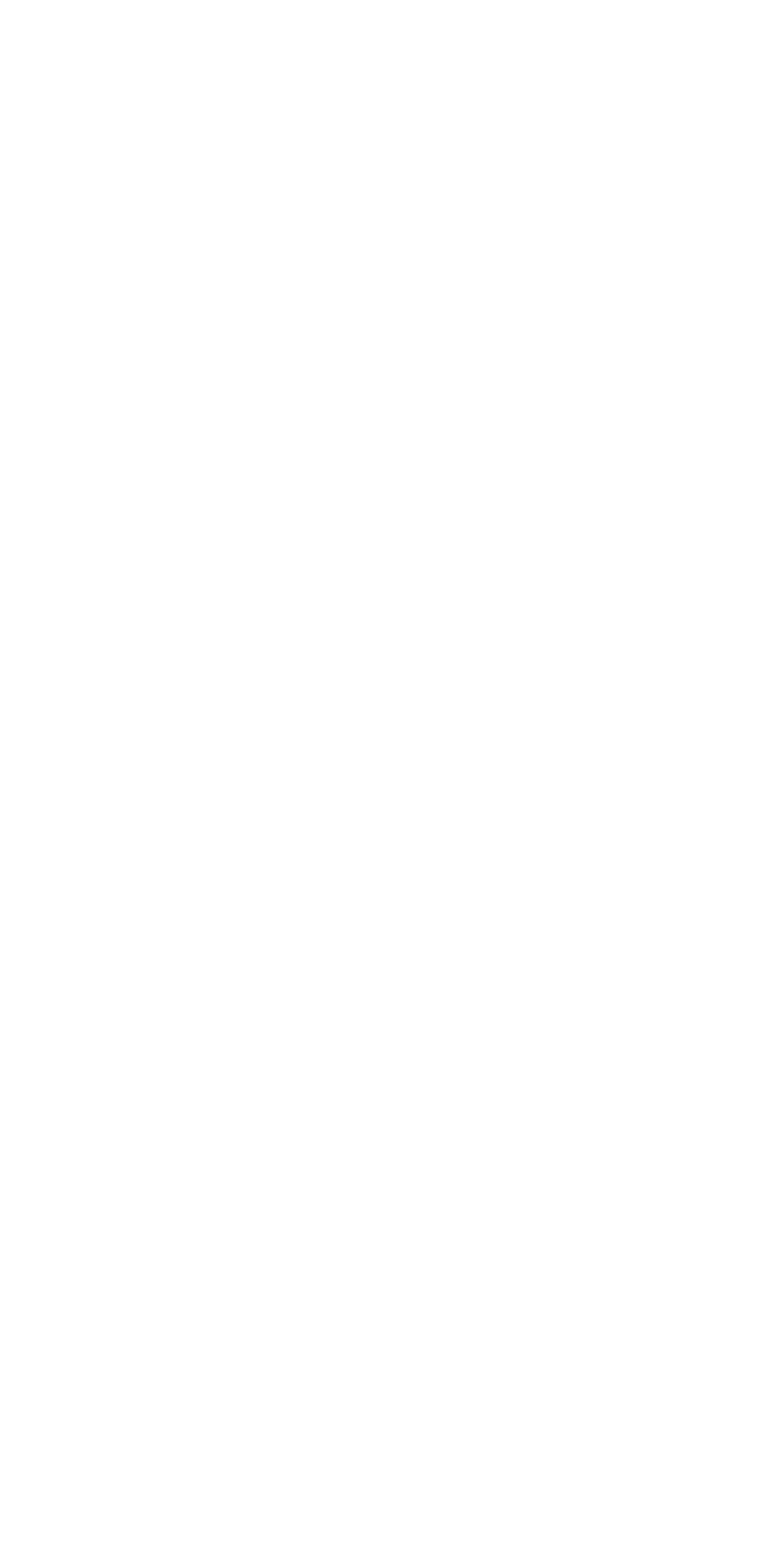

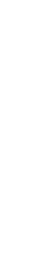

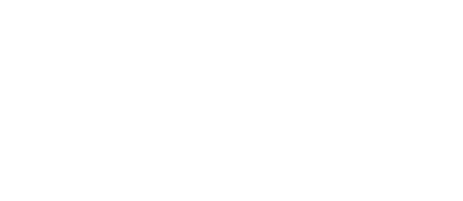

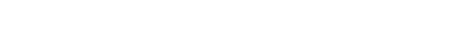
$\Sigma$

in

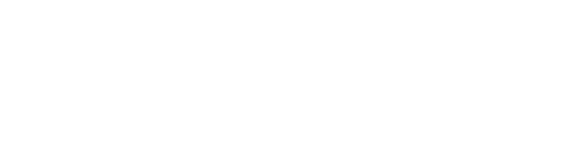

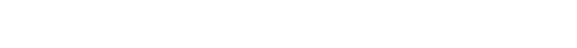

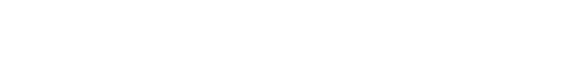
$\circ$

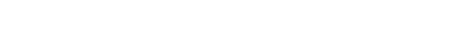
-

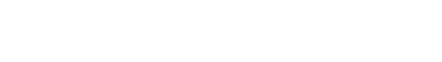

w

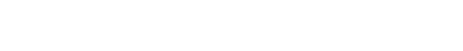

$=2$ $=-$ 응

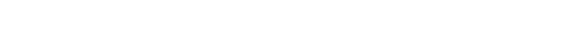
응

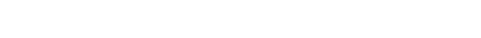

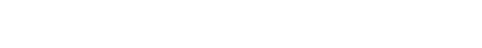

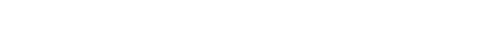
z

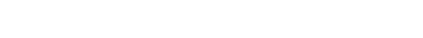

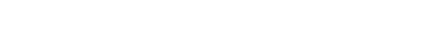

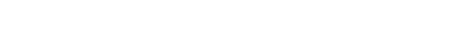

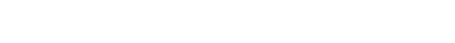
$\Xi \cong N \cong N \cong=E N O E=E$ 


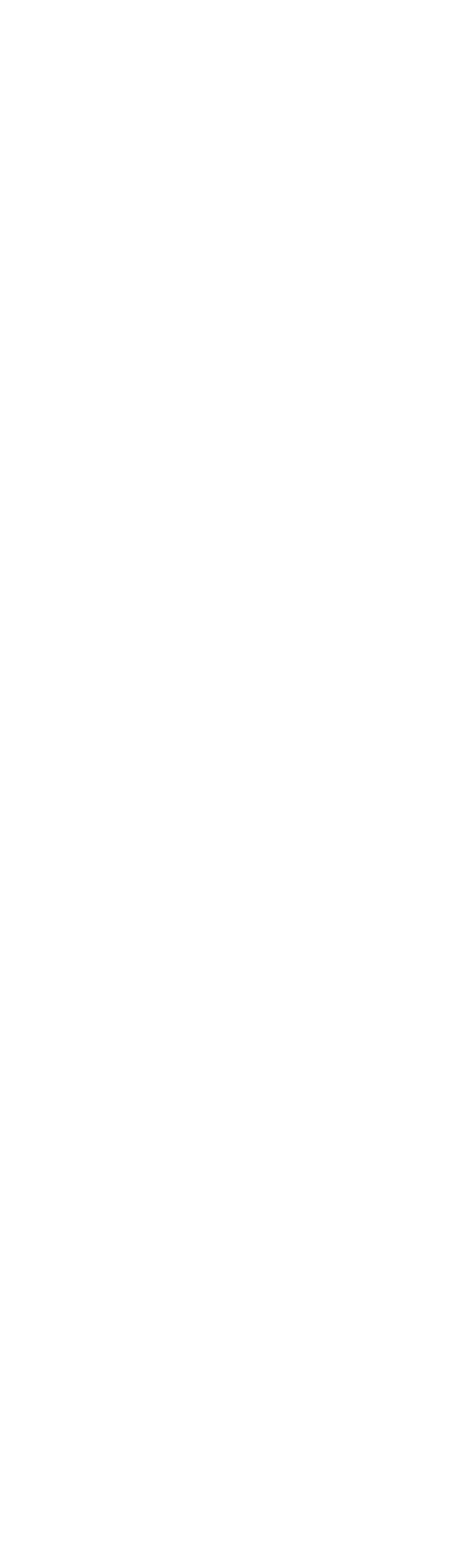

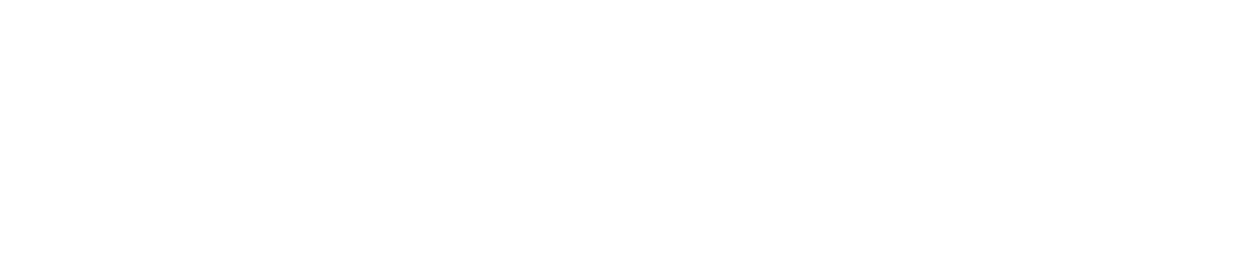

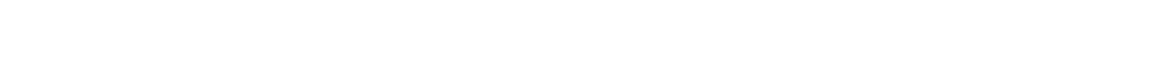

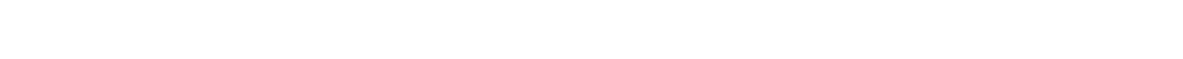

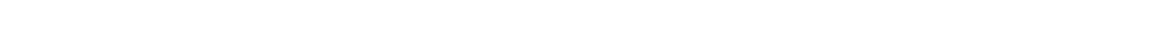

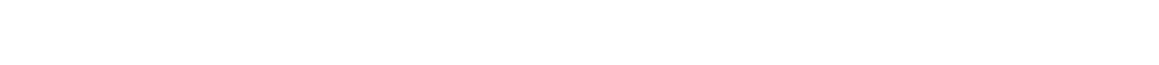

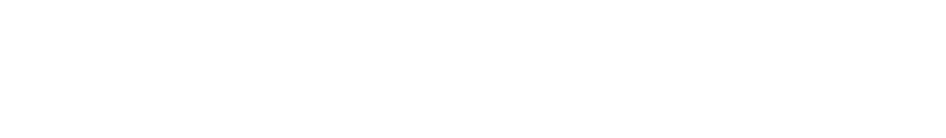

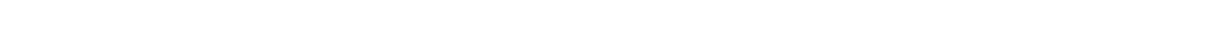
క

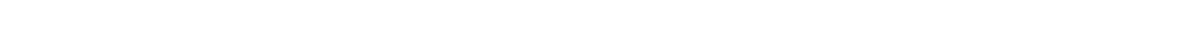

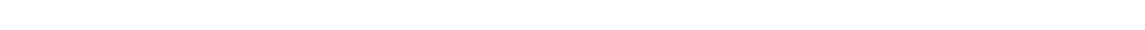

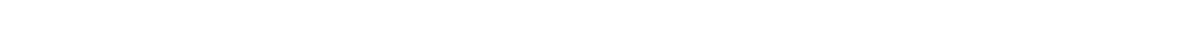 is
is

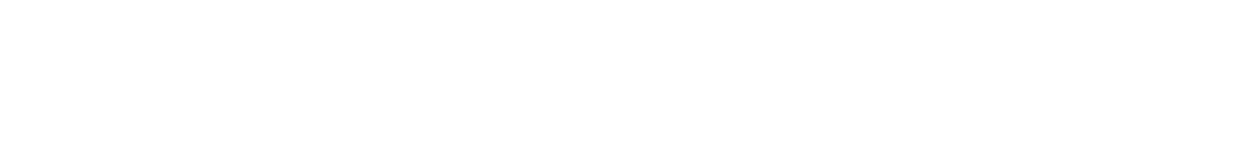

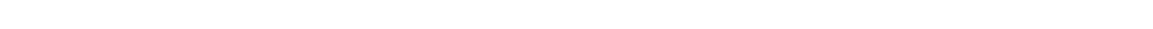

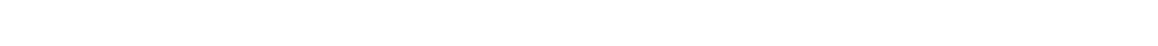

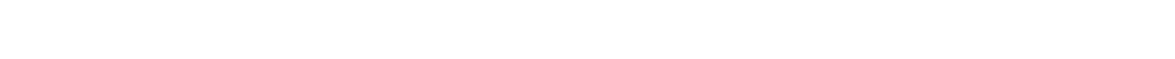

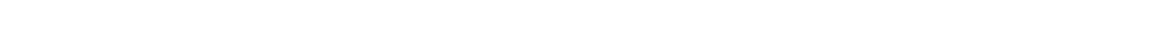

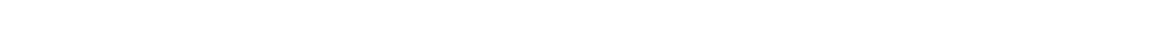

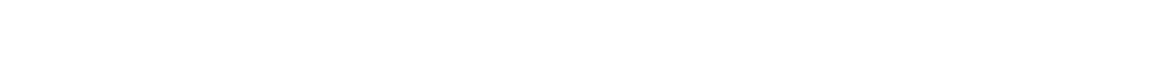

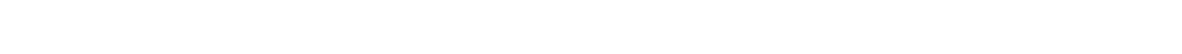
a

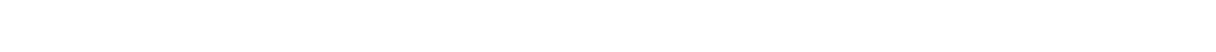

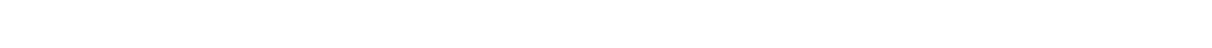
w

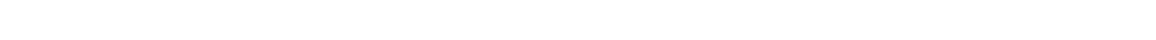

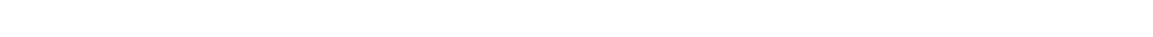

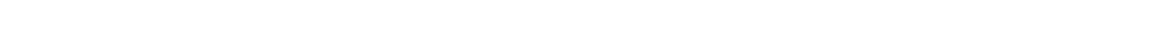

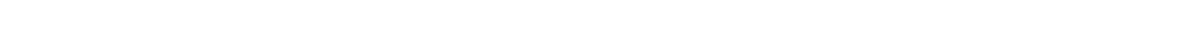

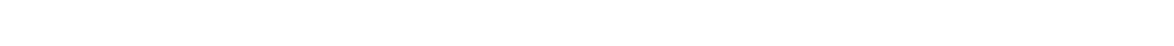

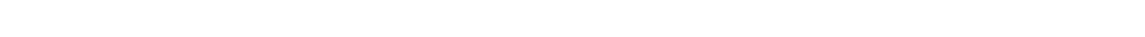

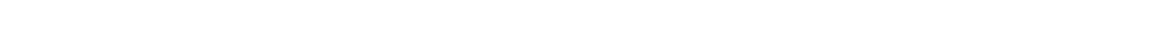




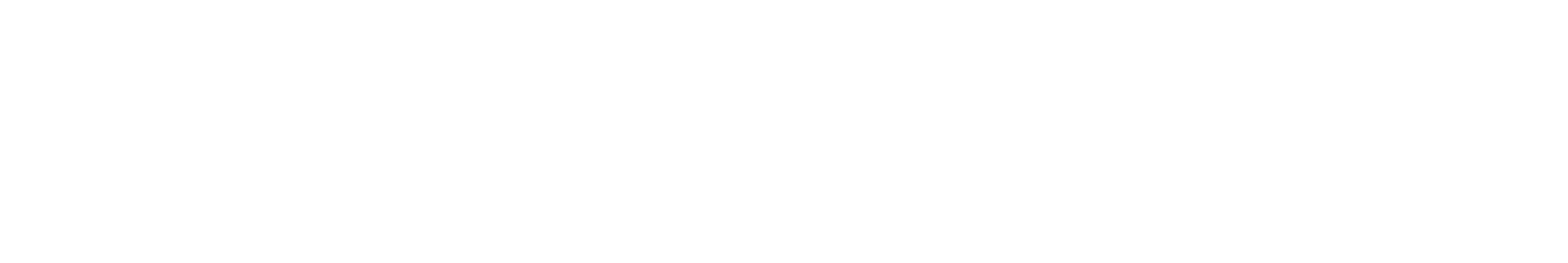

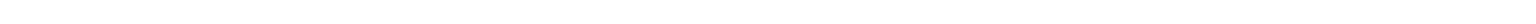

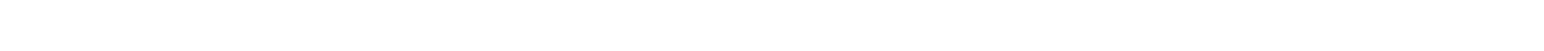

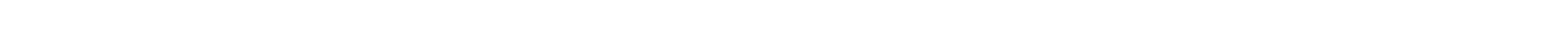

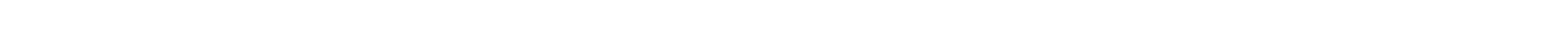

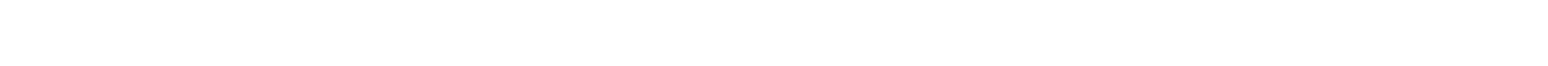

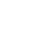

" а шим

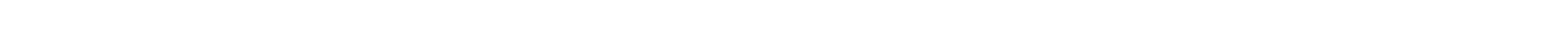

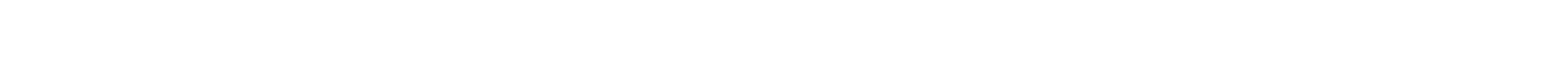

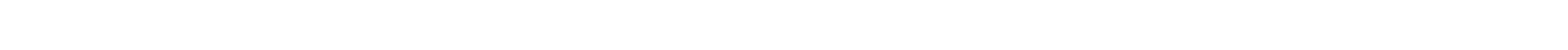

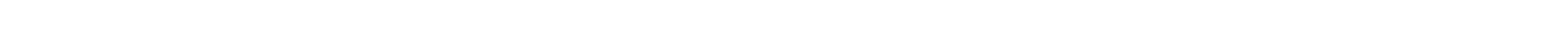

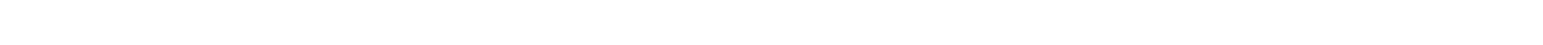

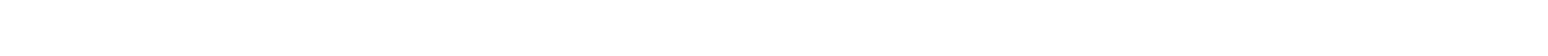
: es
in
en is
is
n

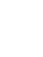

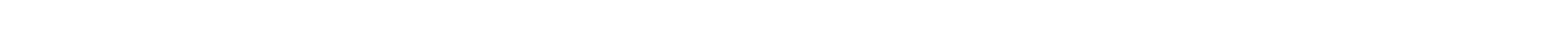

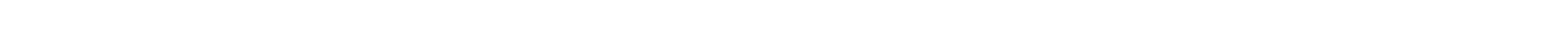

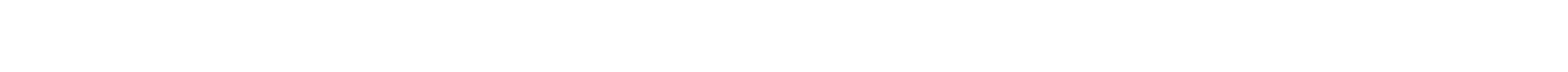

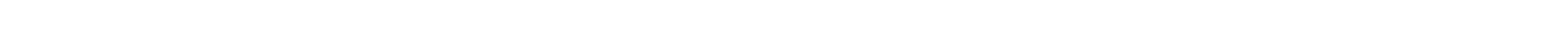

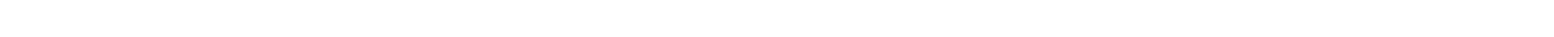

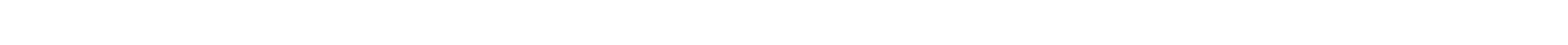

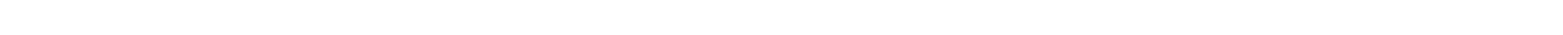
OENN N

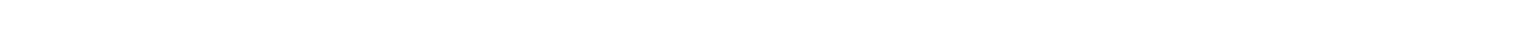

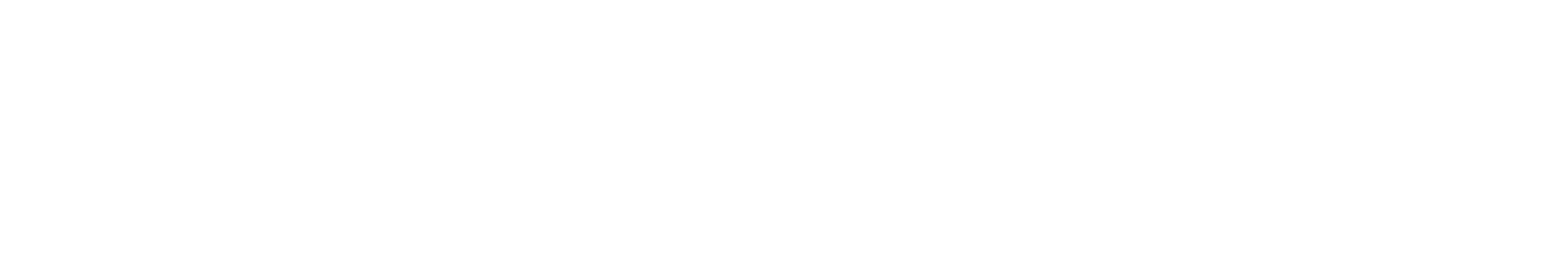

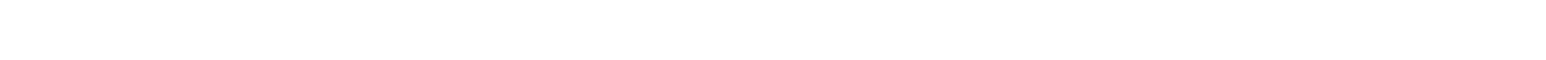

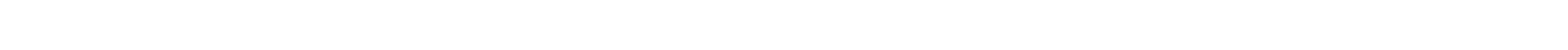

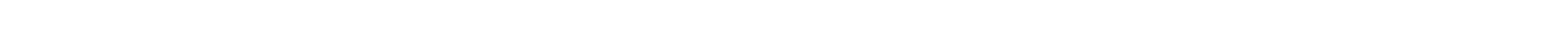

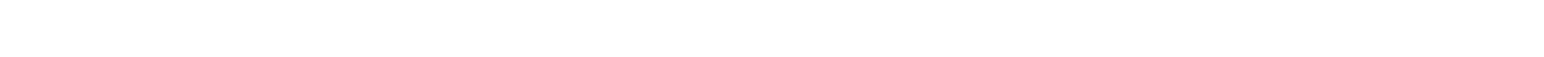




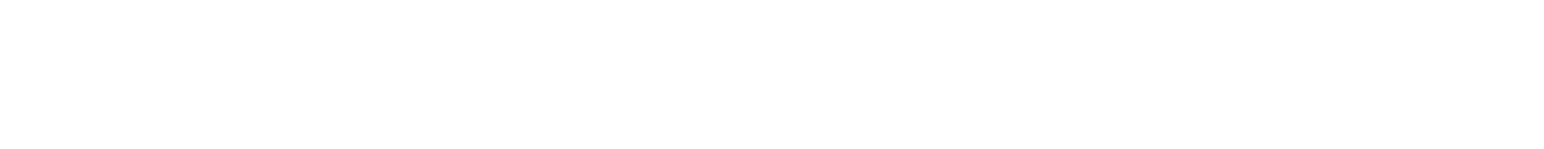

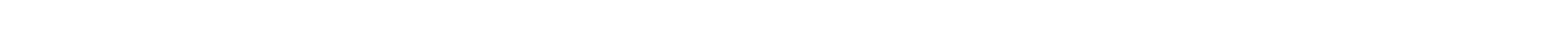
«

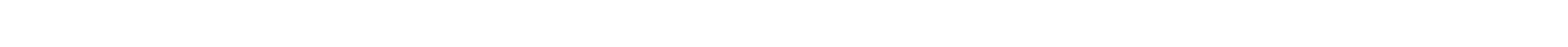

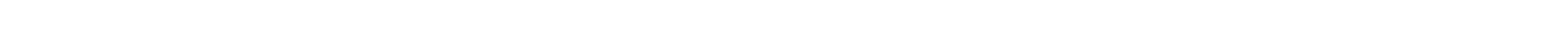

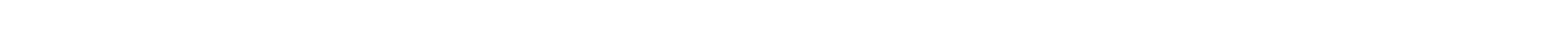

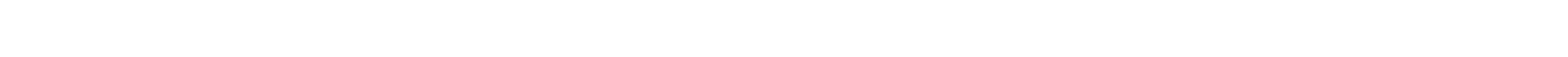

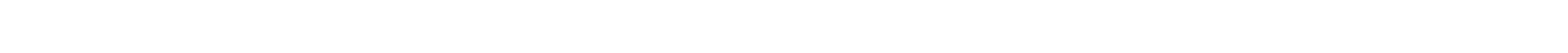

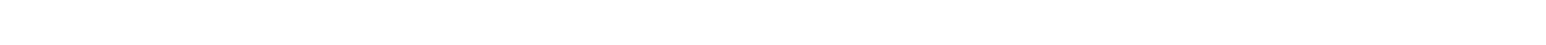
2
$\stackrel{\omega}{\omega}$
o
as
is
u
is
is

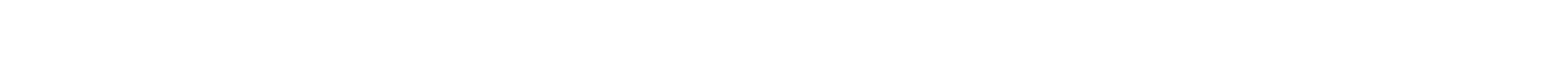

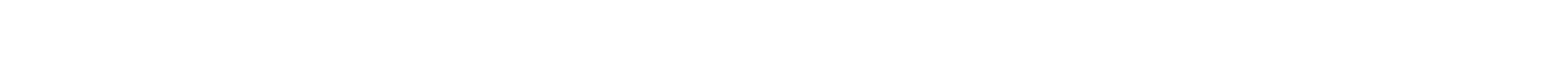 ×

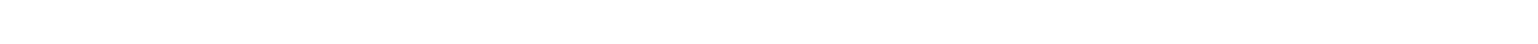

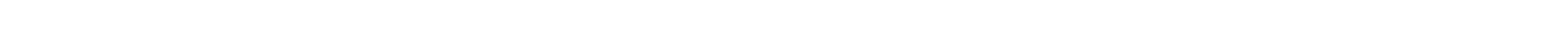

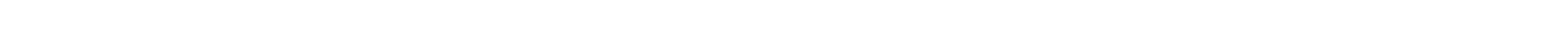

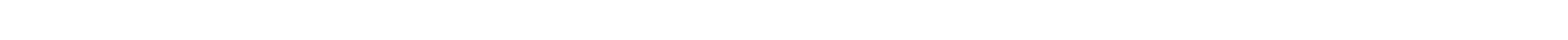

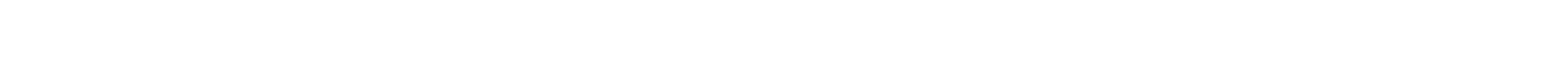$$
\text { twon }
$$

is

is

n

in as

in is

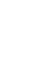

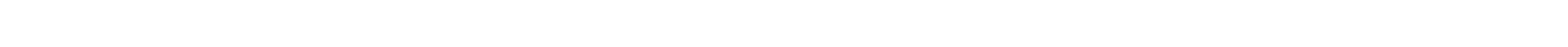

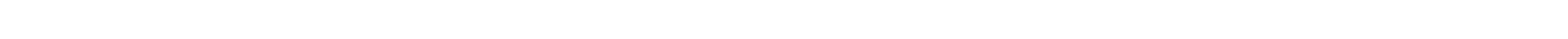

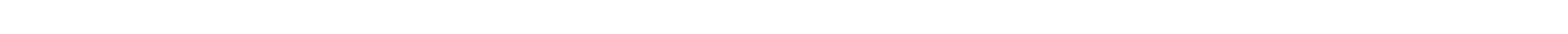

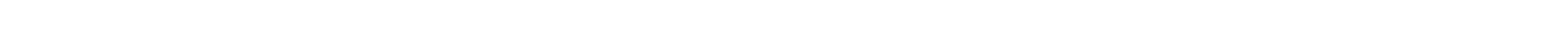

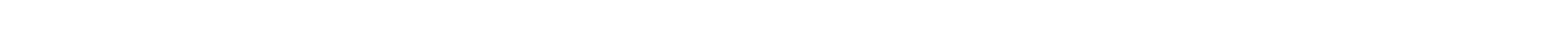
แ

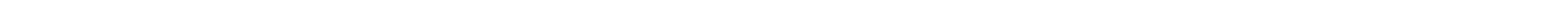

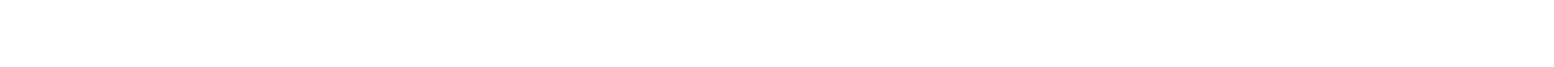

w

$\lesssim$

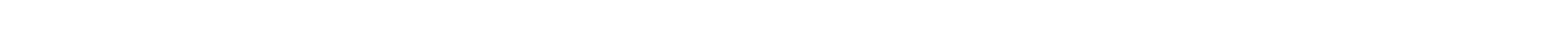
แ

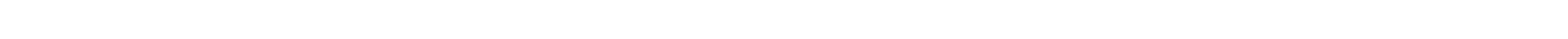

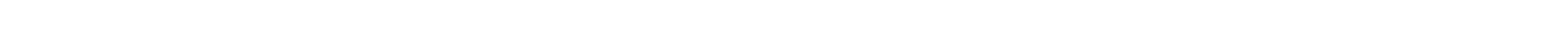

W

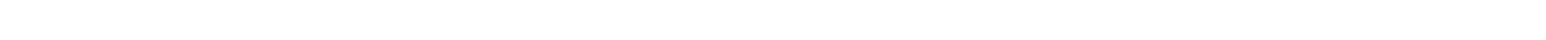

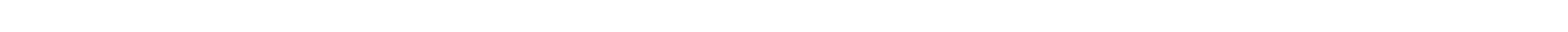

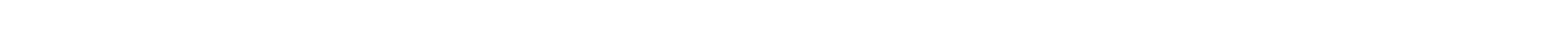

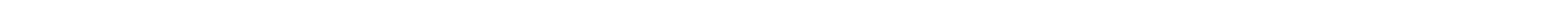

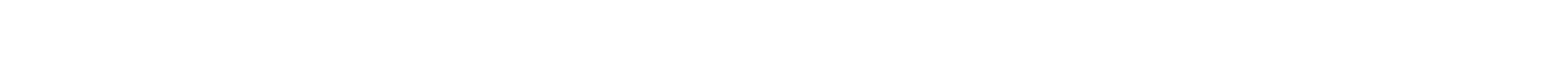




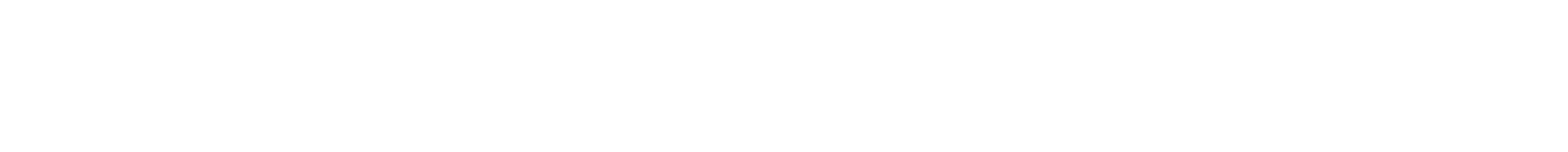

w

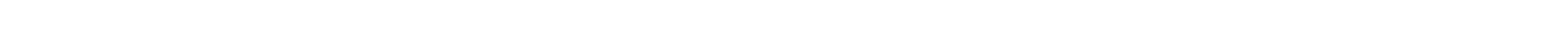
4

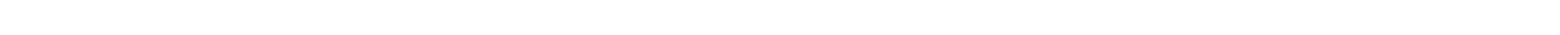

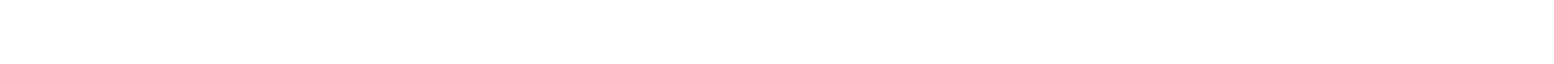
س5 ñ
is is
en is
is
u
a

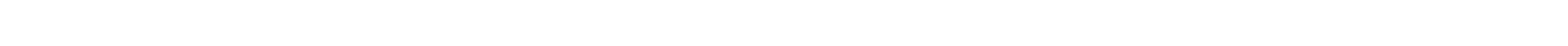

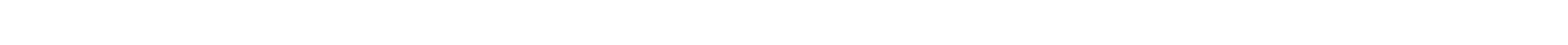 a -

- - - F- -

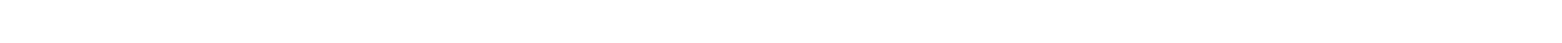

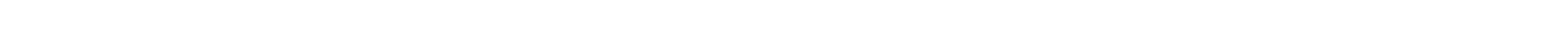

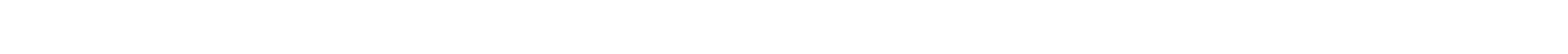

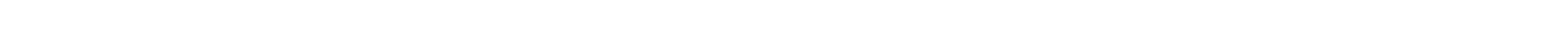

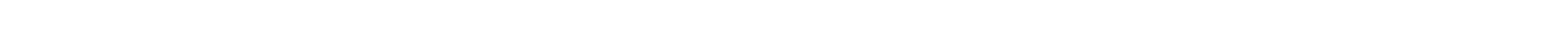
\pm in
is
es
on
is
is
" -

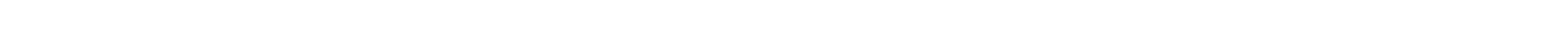 แ

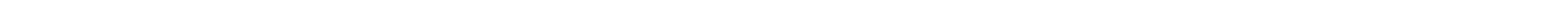

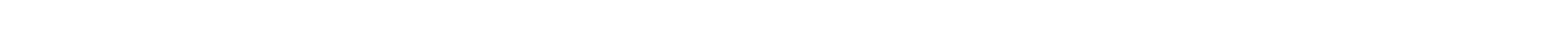

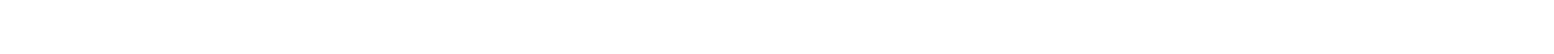

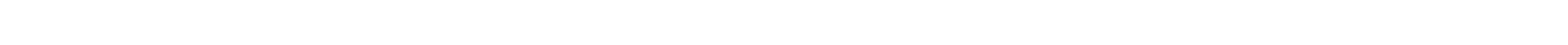

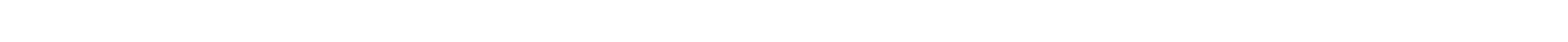
O $\simeq$ ฯ

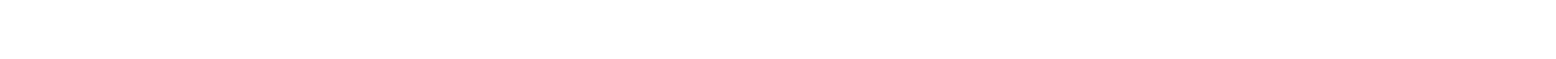

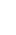

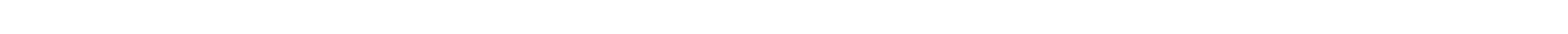

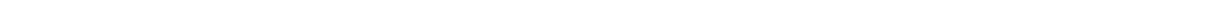
แ山

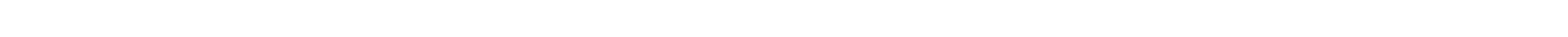

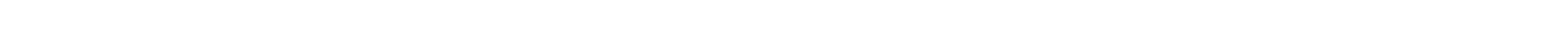

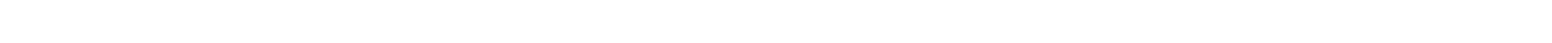

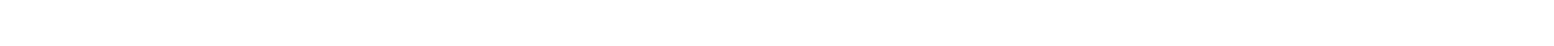

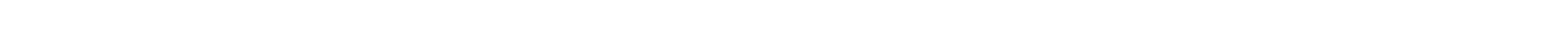

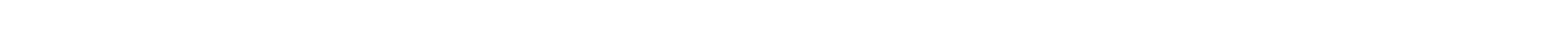

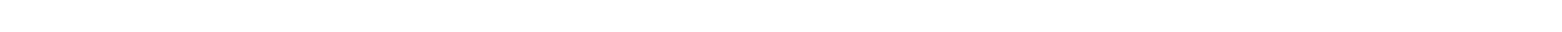

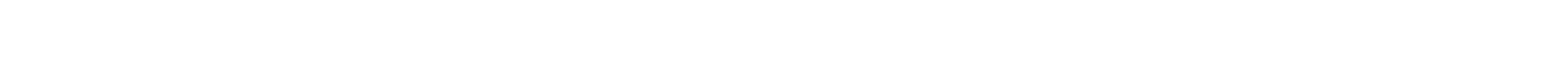




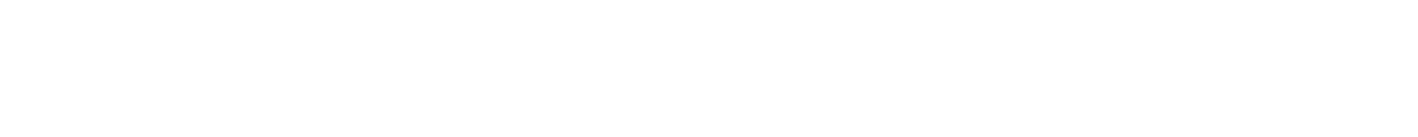

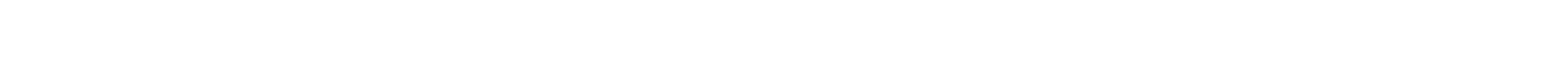
w

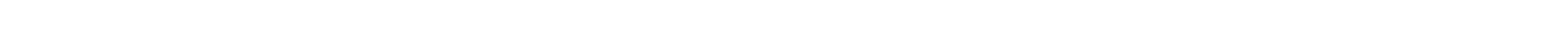

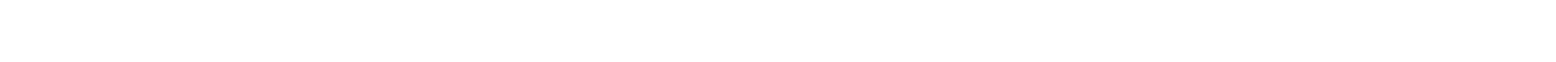
แ

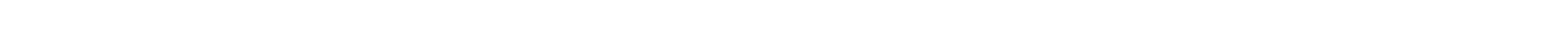

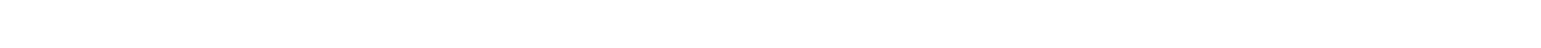

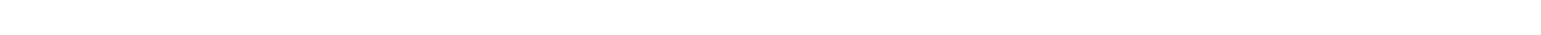
$\stackrel{\leftarrow}{\Sigma}$
en
in
n
u
is
s
n

$\stackrel{5}{5}$

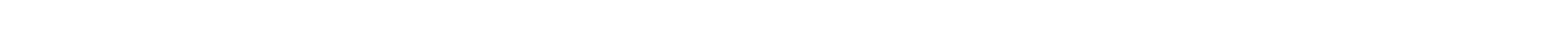

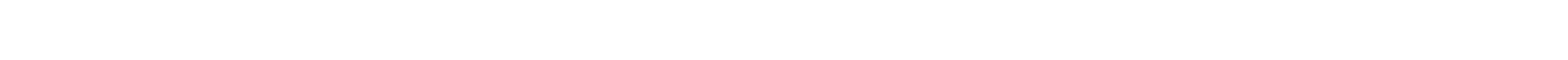
з

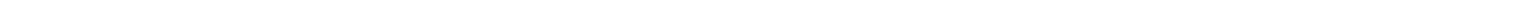

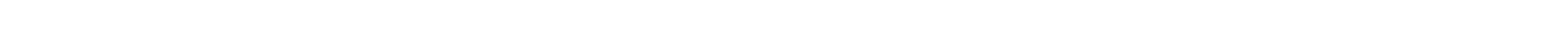

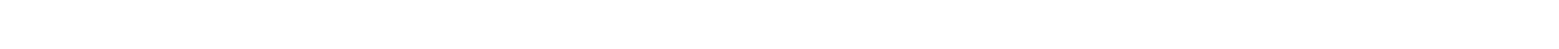

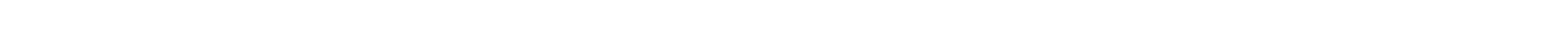

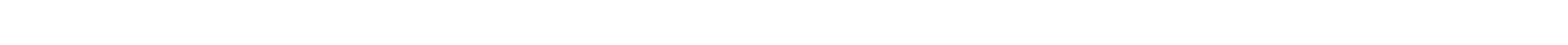

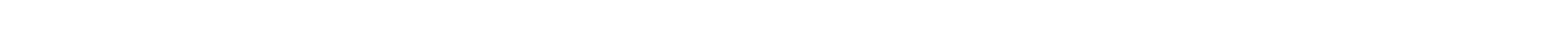

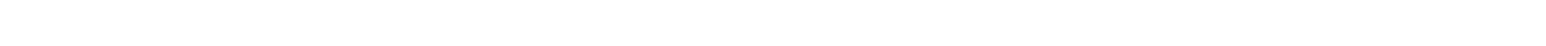
w n
is
n
$\sim$
es
is

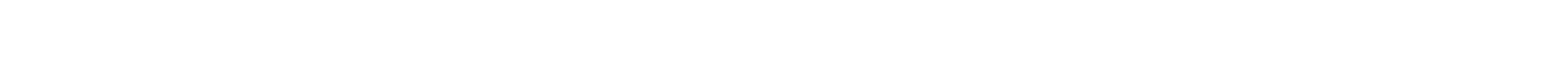

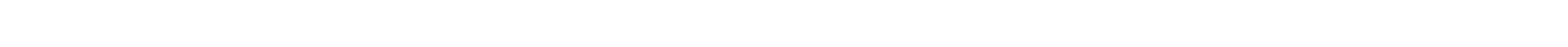

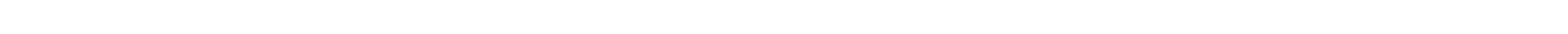
-

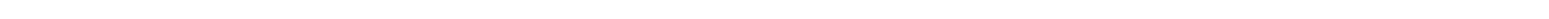

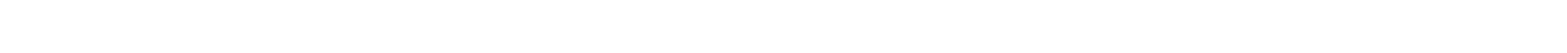

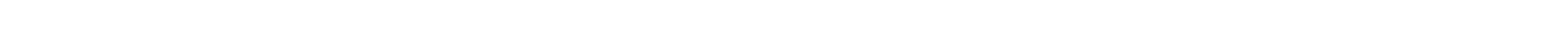
은

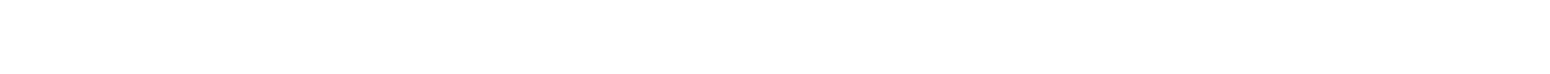

in

cimmómmonN بs

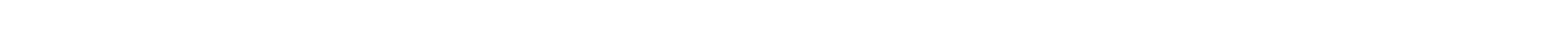

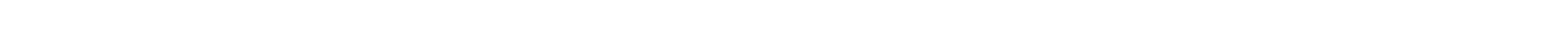

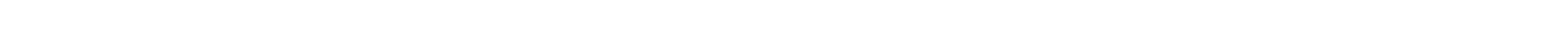

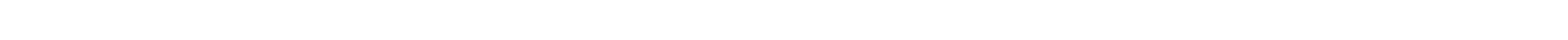

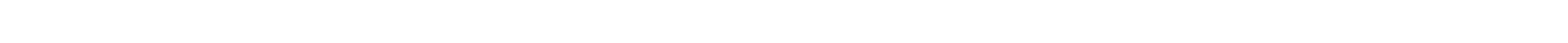

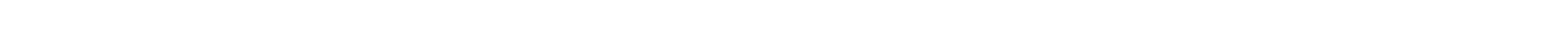



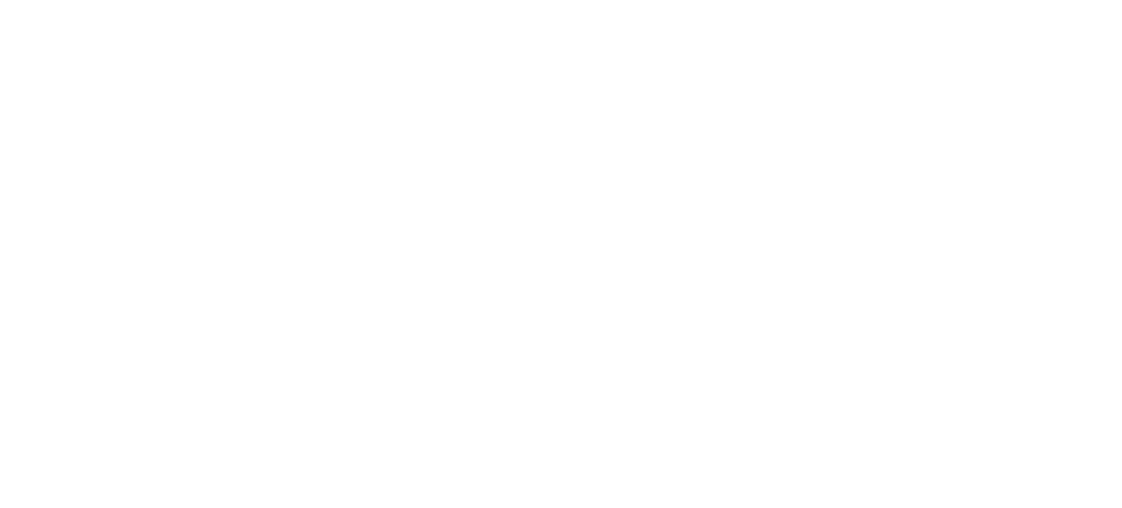

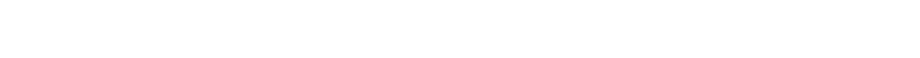
$<$ is

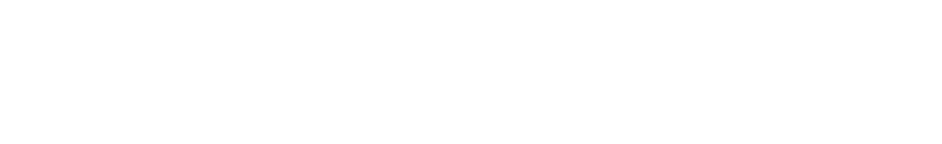

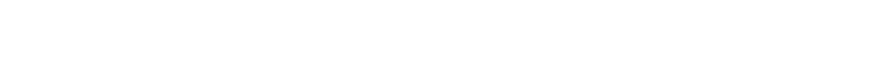

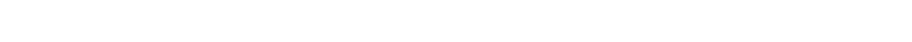

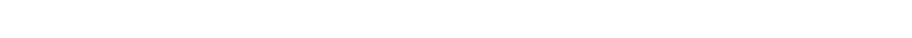

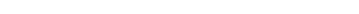

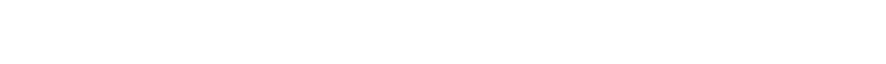

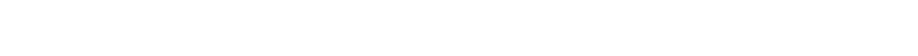

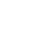

a

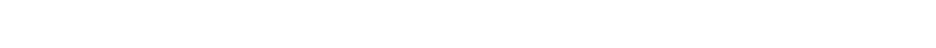
a

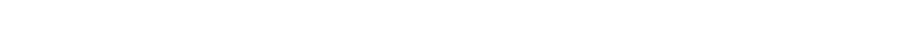

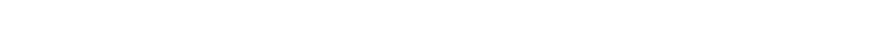

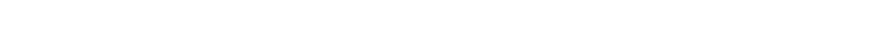
w

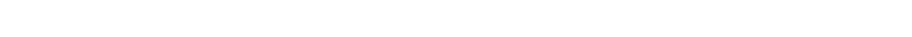

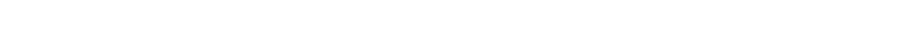
エ

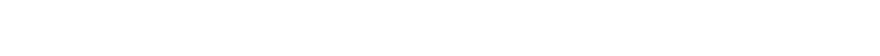
Wi

㟧

es

is

is

is is

is

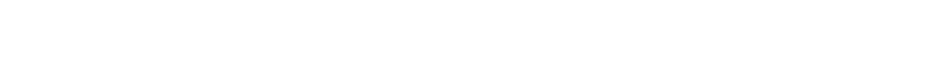

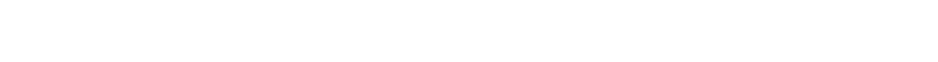

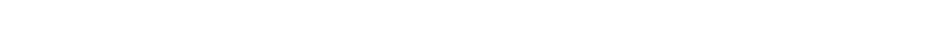

nen $n$ m

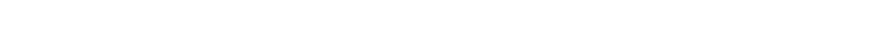
W

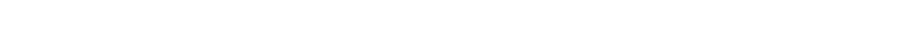

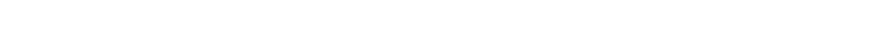

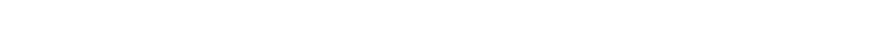



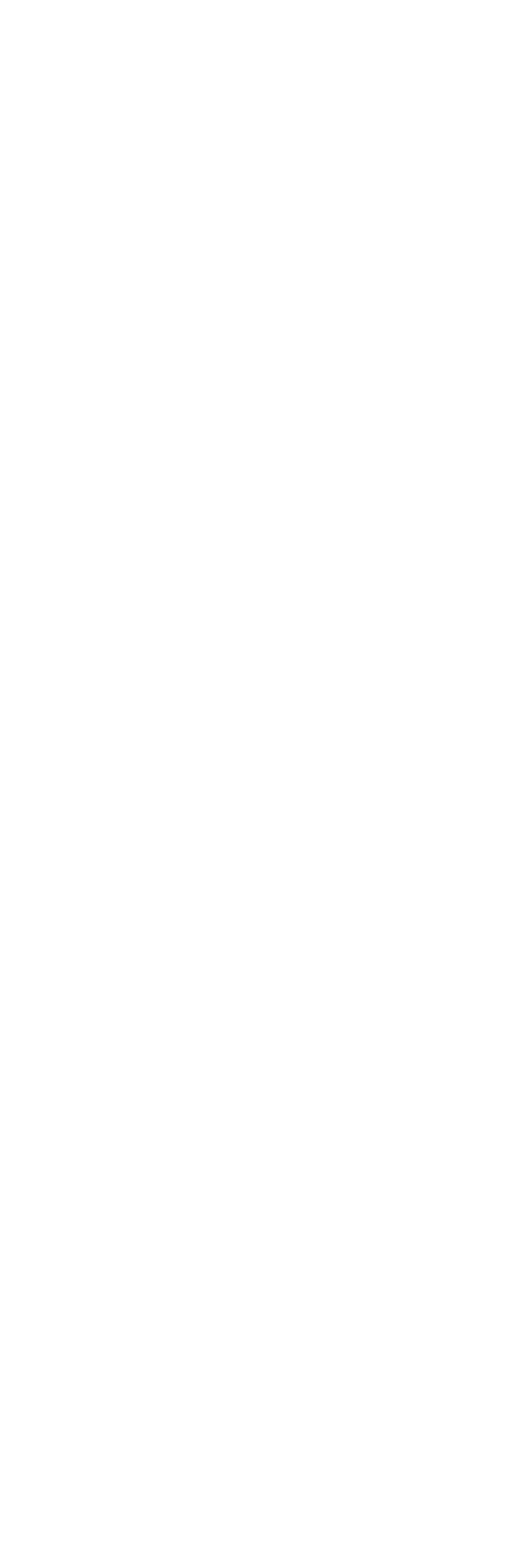


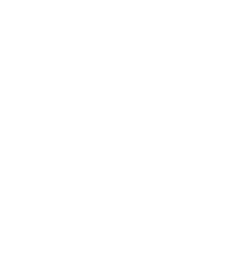

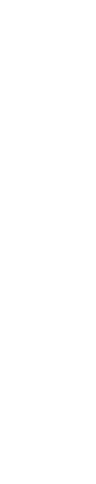

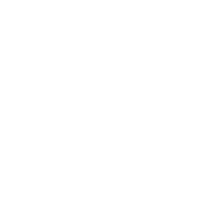

twencosen

点

운ำㄴำ

aun.

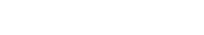

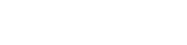

- $\quad n \infty \pi \infty$ แลํํำ

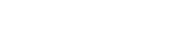

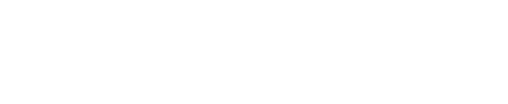

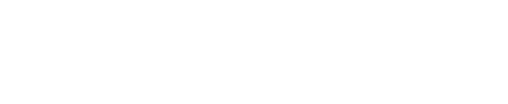

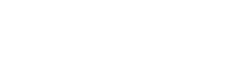

뜬osonos 5

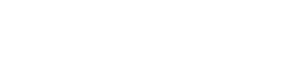

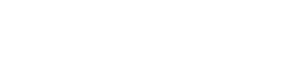

in

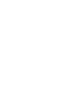

ä

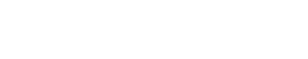
至去合

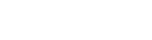

w

2

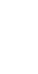

난

in

")

$1 \quad$ a 0

告 娄它衣

जै ल

tisenesen

는

wesenes os

a

So n

ํㅇㅇ w

更

西-

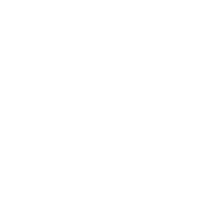



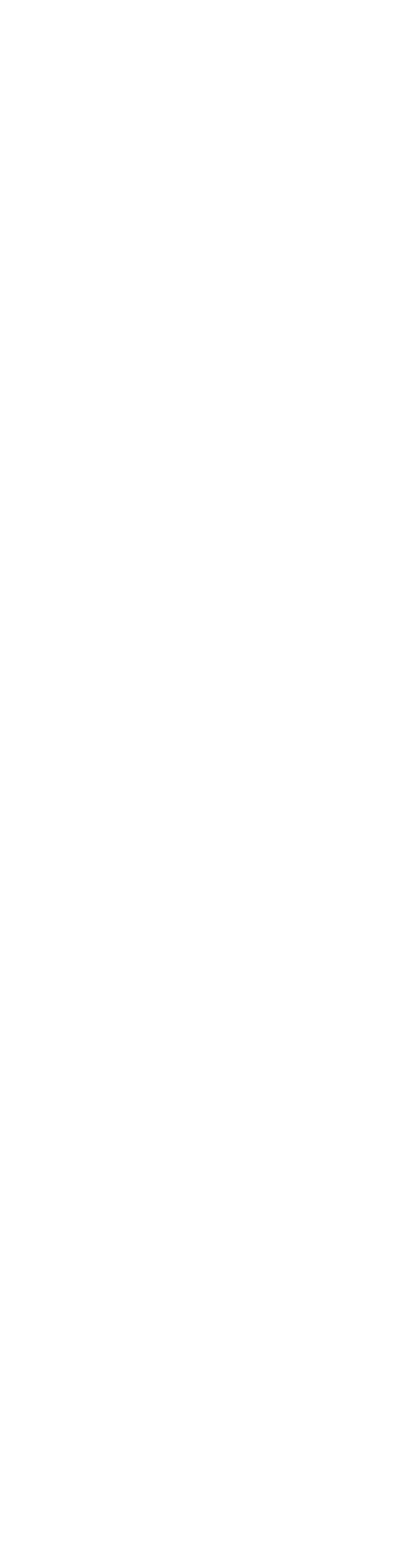

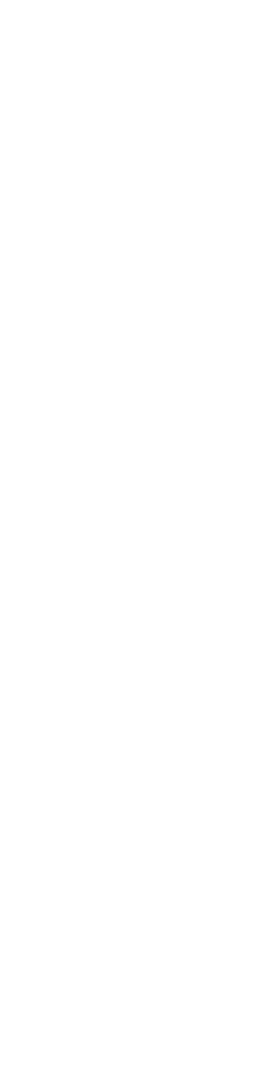

它

产导的

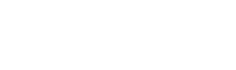

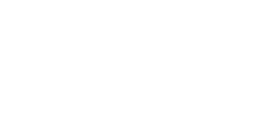

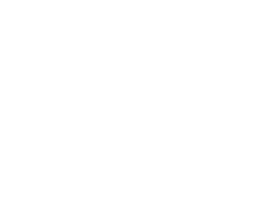

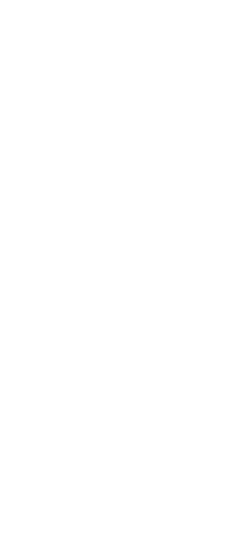



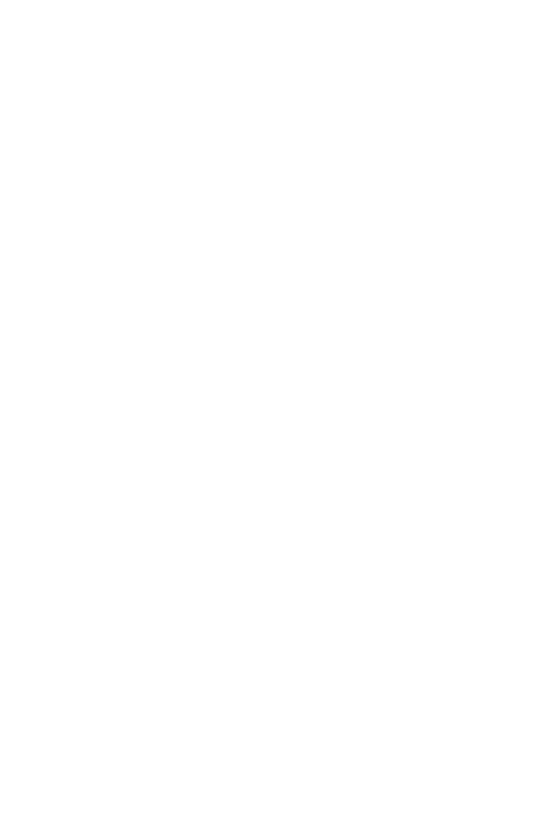

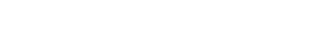
แลํํํํํํำล

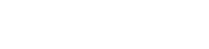

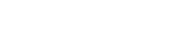
twencosenos is

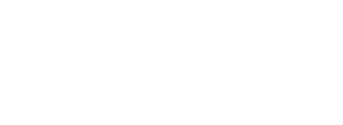

i⿱ $\dot{0}$ $z z:$ ơmoñ்

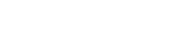

wennenen Non

$=-$

à 2 is

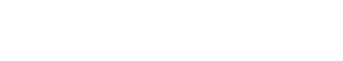

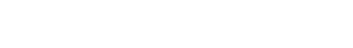

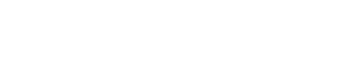
å

w更是

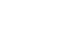

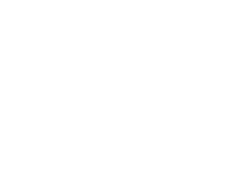




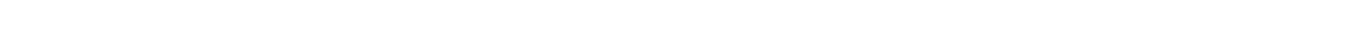

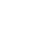

ONOㅁำ $\alpha$ स

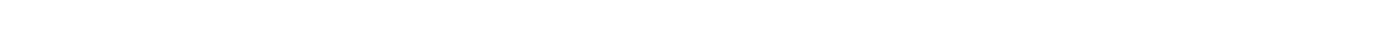

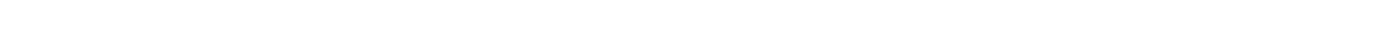

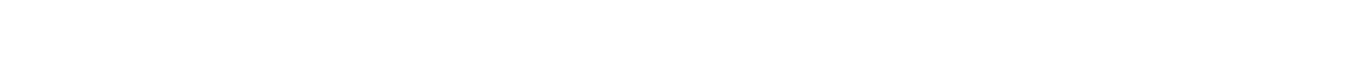

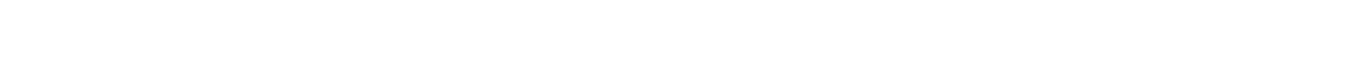

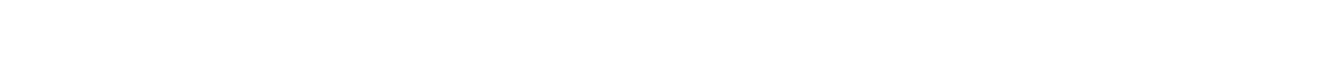

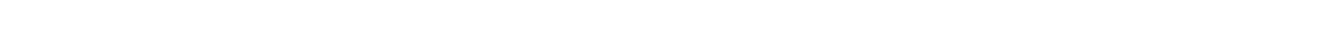

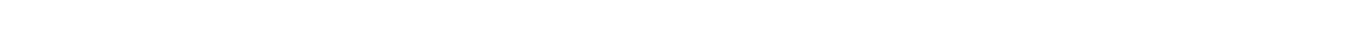

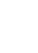
×

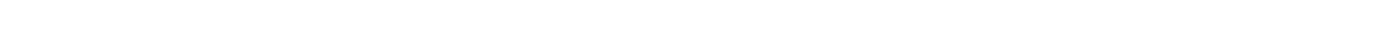

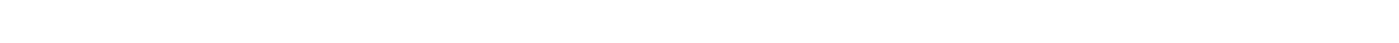

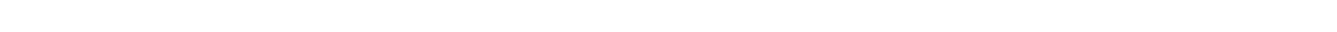

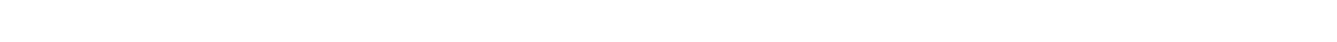

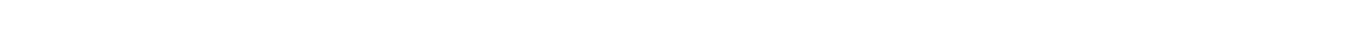

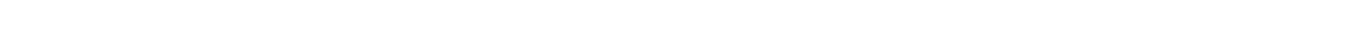

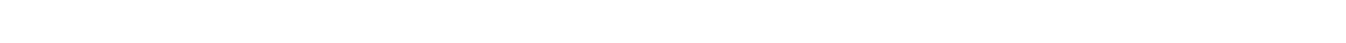
mñó =

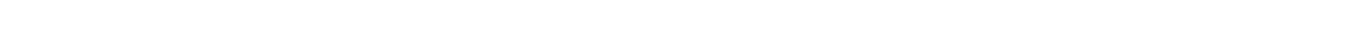
$\underline{\Sigma}$

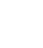
" "

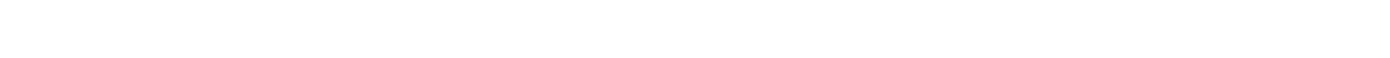

Ob

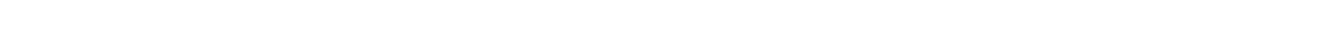

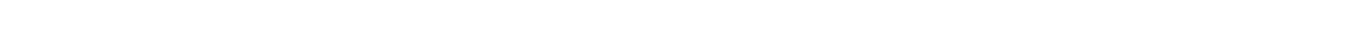

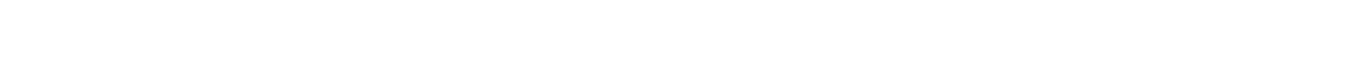

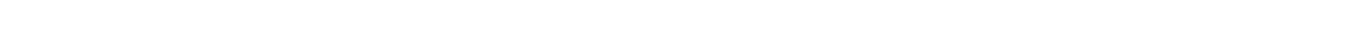

$\underset{1}{2}$

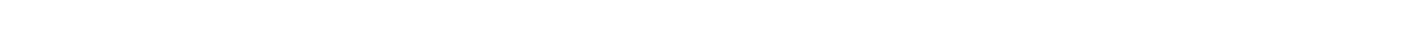
แu \begin{tabular}{lll}
$x$ \\
\hline
\end{tabular}

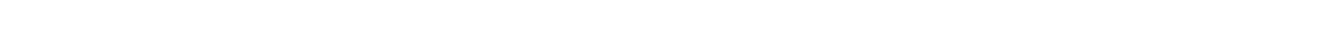
w

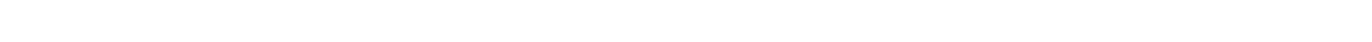
a

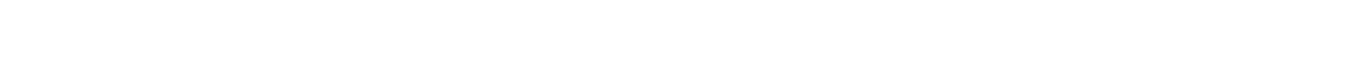



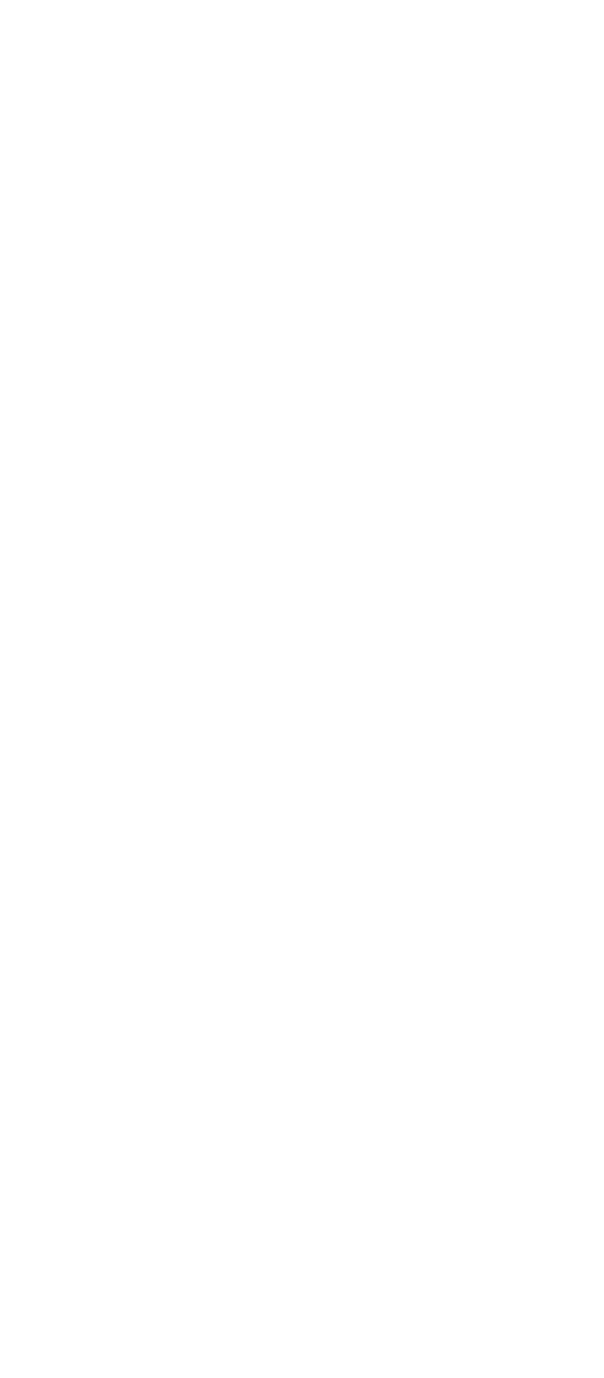

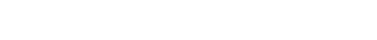

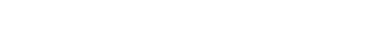

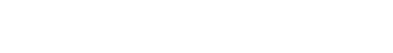

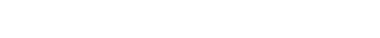

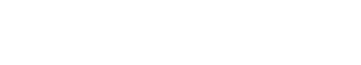

twoscosesescososesos a is

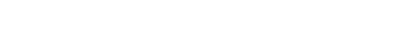
uس

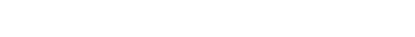

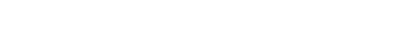

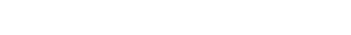

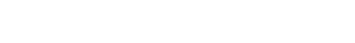

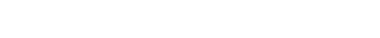

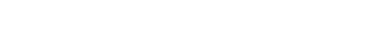

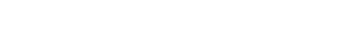



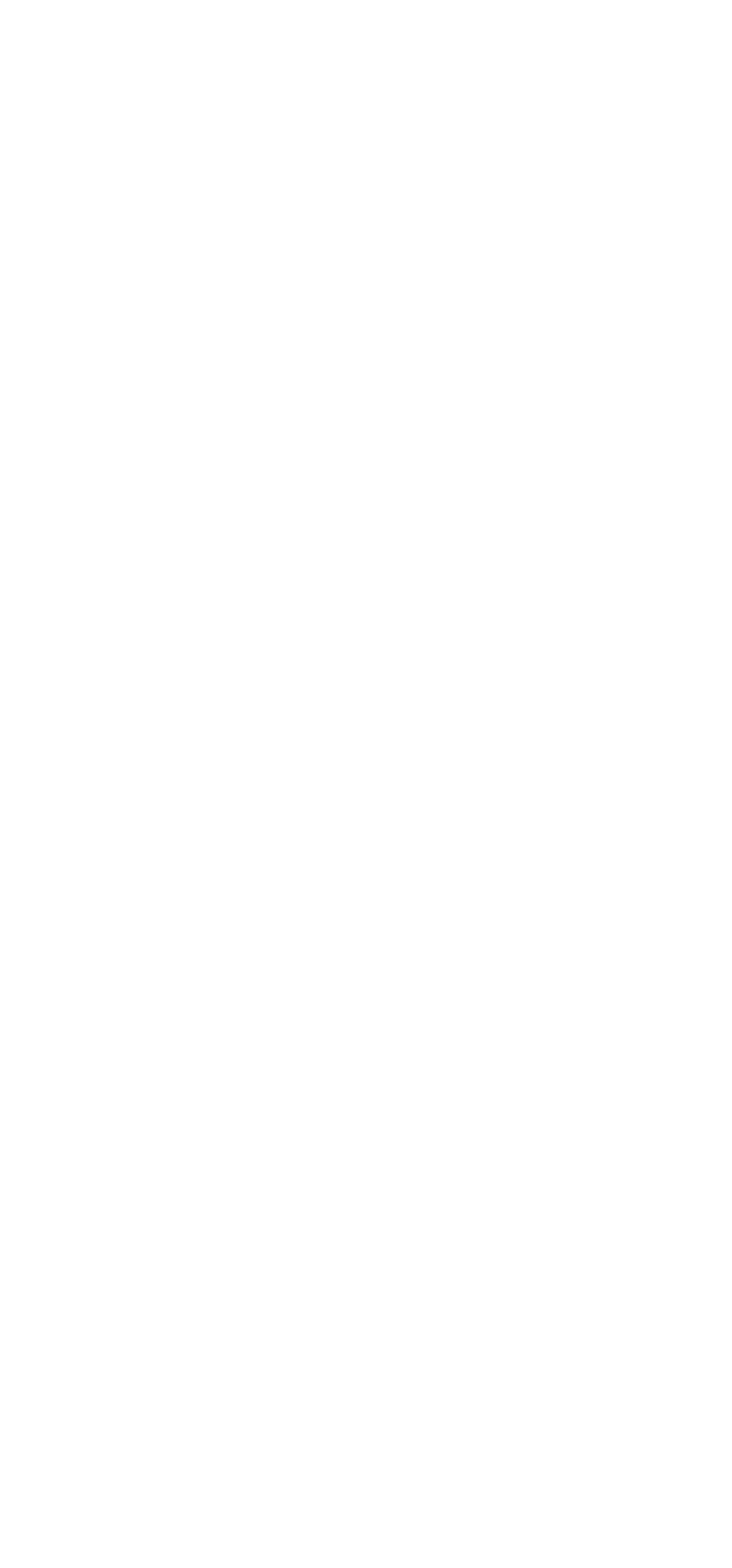

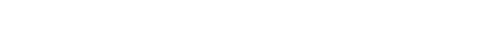

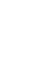

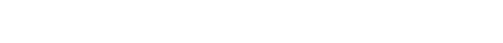
шسن

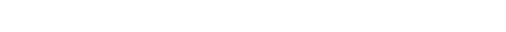

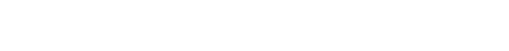

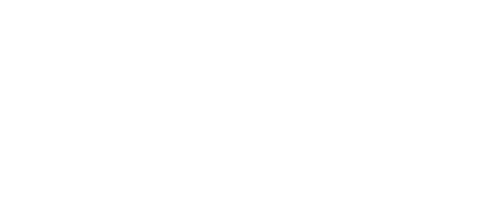

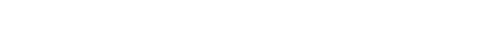
E 叫

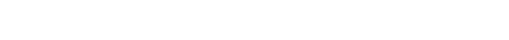
या 000000500000

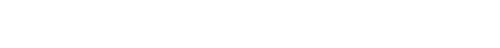

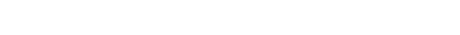

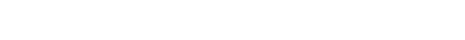

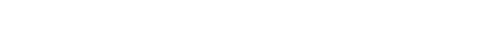

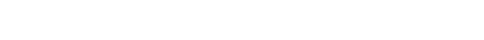

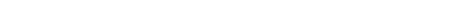

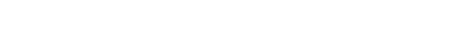

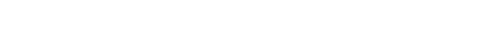
$\therefore$ a a a a

mmnna $0 \geq m \alpha \infty \infty n n n$

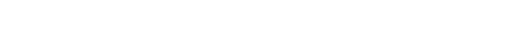
$\Rightarrow$ or का पय

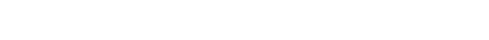

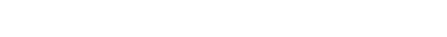

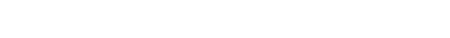

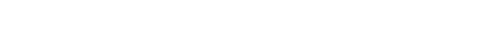

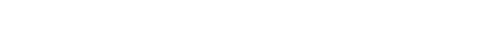

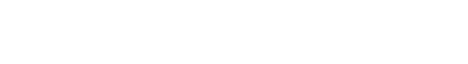


응 둥 숭요 崩 造这 으음 $\infty$

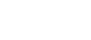
ละก z的告 픙응 눙 안둥 w

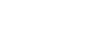
wm $10^{1}$ แ a $=$ 는을 EDin 뜬몽 天坛 吠产 0 050 แ웜 $\ddot{\alpha} x 0 "$ 담 的穴 - u.

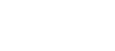
ป气上 능요 $=\sim 20$

둔우 은용 m. ○宇号 0ंख 2 민. 은

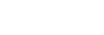
wo 的㙄 na 年 व 뜽응

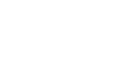

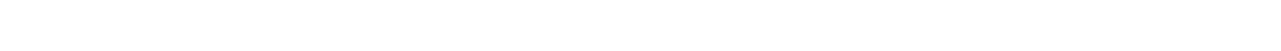
$\frac{2}{n}$

w

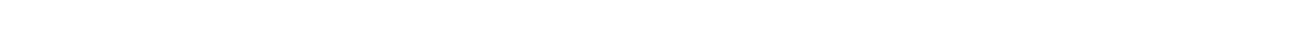
з

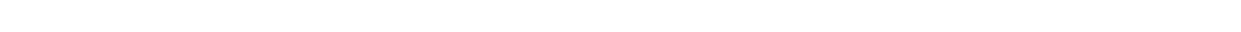

Nmmmmmm-S+4

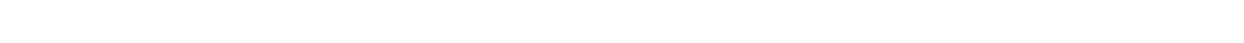
w

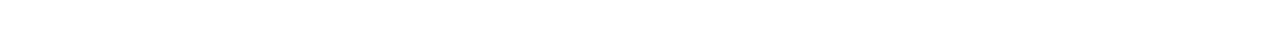
000--

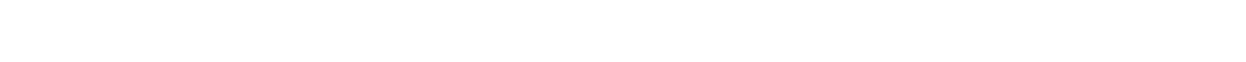

D̃ is

에 $\leftarrow$ L " U一,

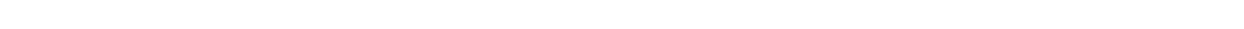

요용

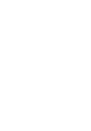

m

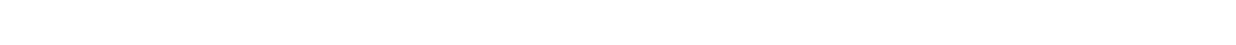

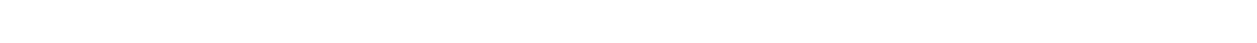

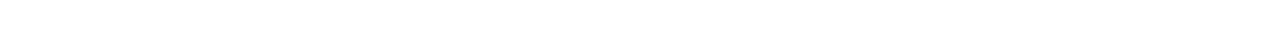

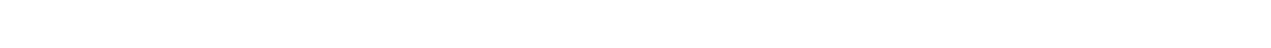

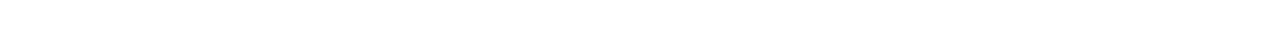

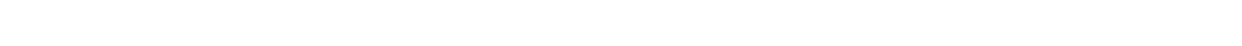



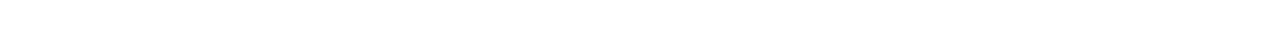

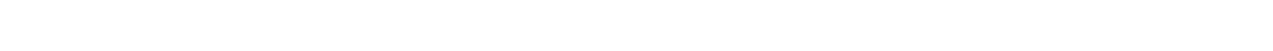

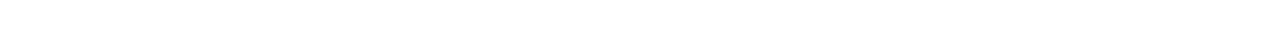

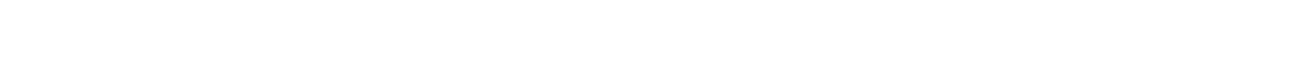

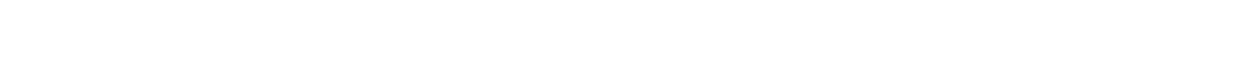

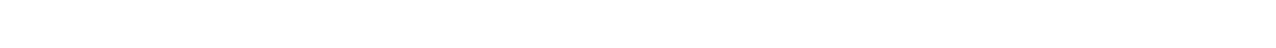

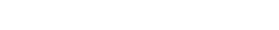

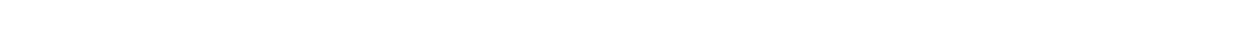

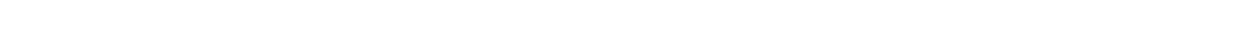

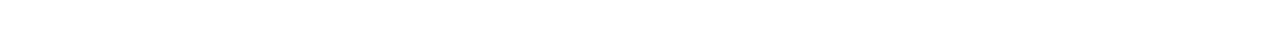
$\Sigma$

in

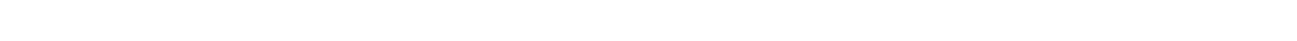

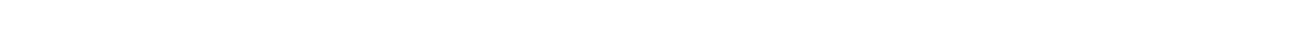

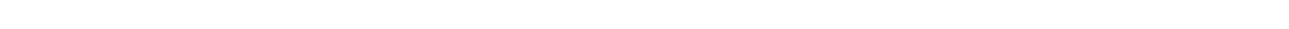

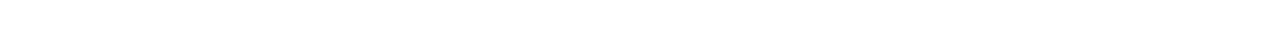

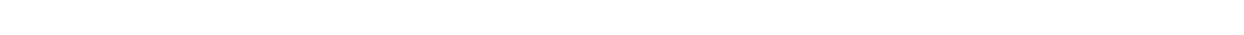

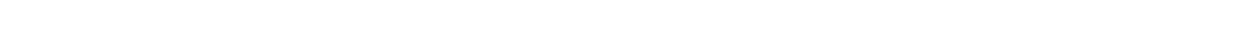

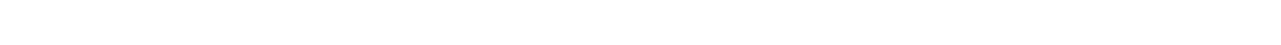

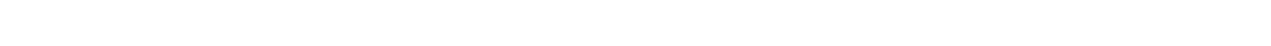

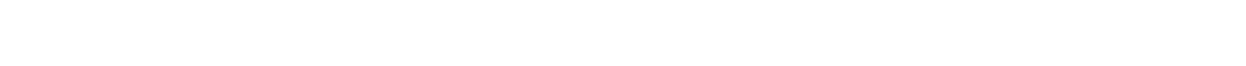




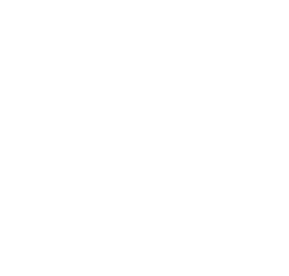

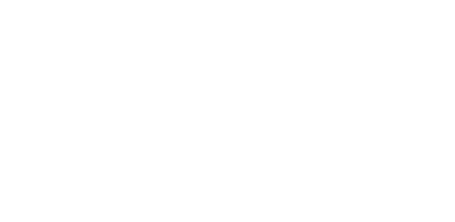

twenesosiones

in

m nmanos क या

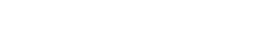

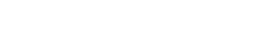

品㱒的的

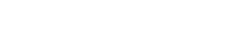

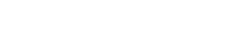

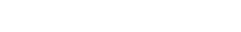

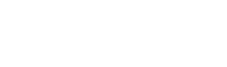

Euseseseneseses

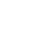
玄

sornun ü $\rightarrow 0 m n a n \infty$

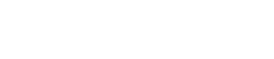

$\prod^{3} \bar{a}$

压

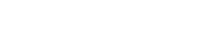

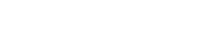

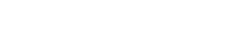

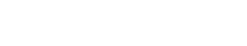

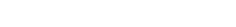

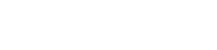

논

w.

$\sum$

tuescosososes $\Sigma$

a $n$

á

inmorman

a

"1)

× जo

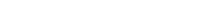

0000000

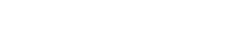
แニラミニニミう

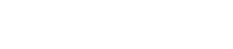
的括和的的 

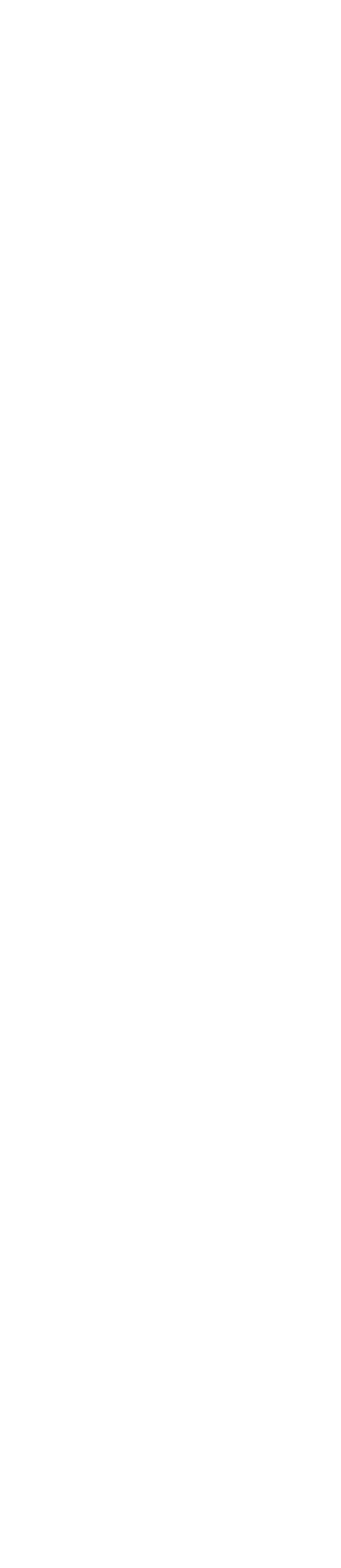

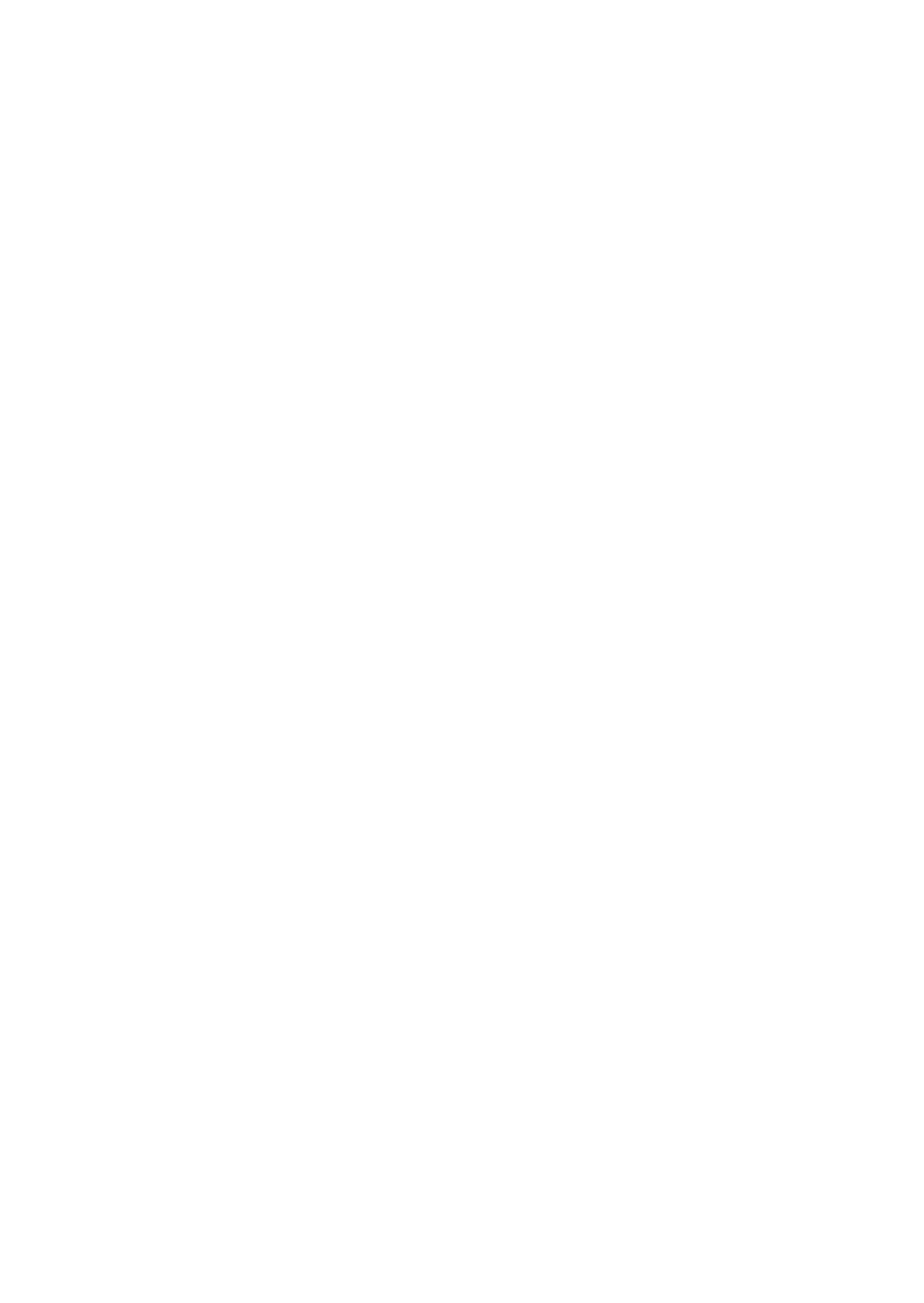

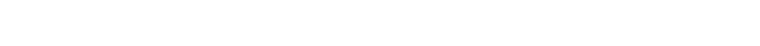
uแ

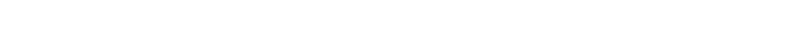

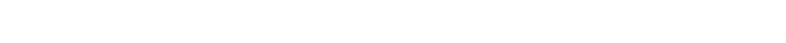

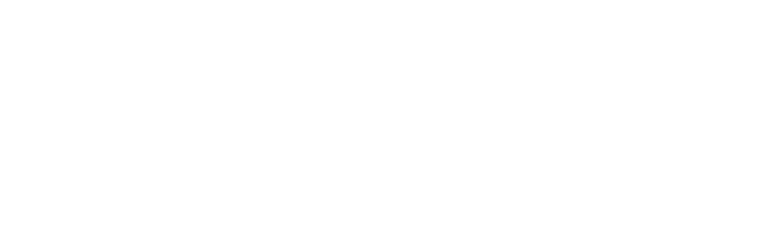

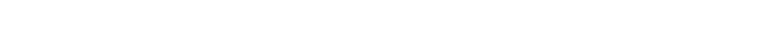
$z$
$\stackrel{a}{5}$
a a a

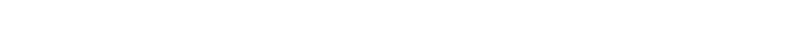

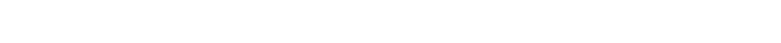

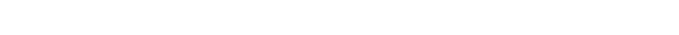
$x-\mu$ m n

mmmmmmmmats

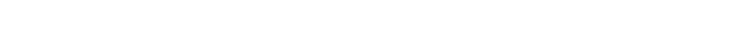
แ বm

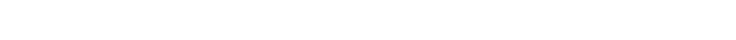

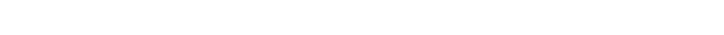

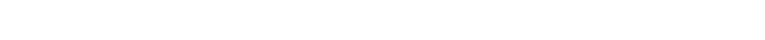
$a$

is

$a$

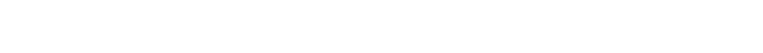
w w a w on m n

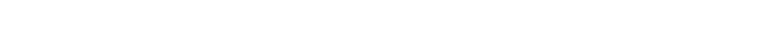

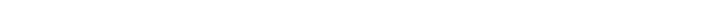

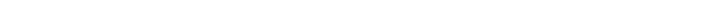

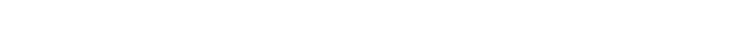

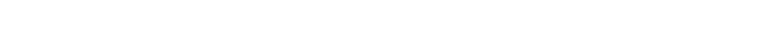

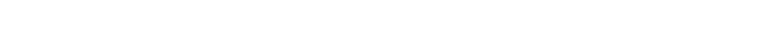

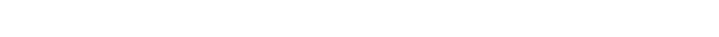

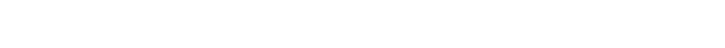



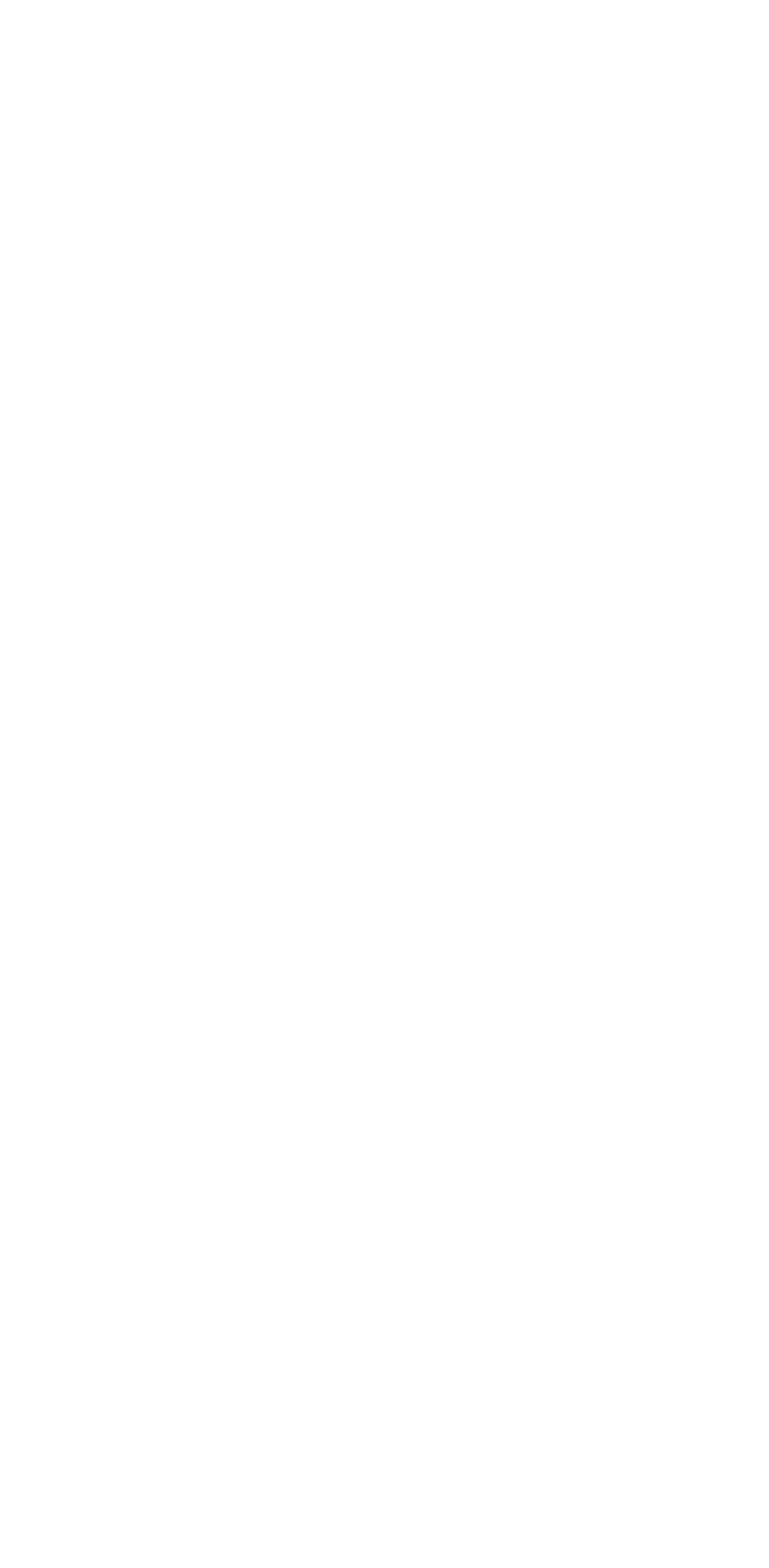

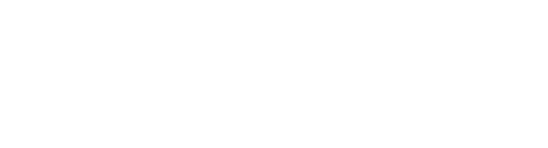

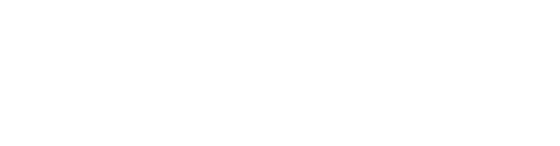

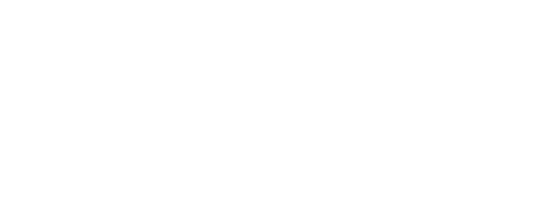

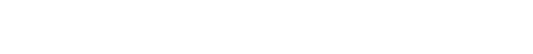
$\underline{x}$ s a a

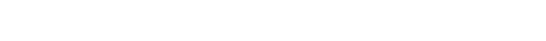

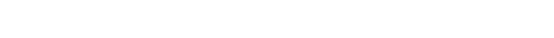

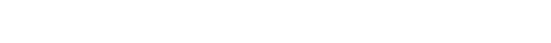

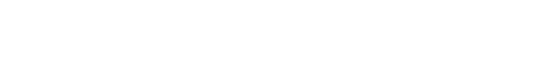

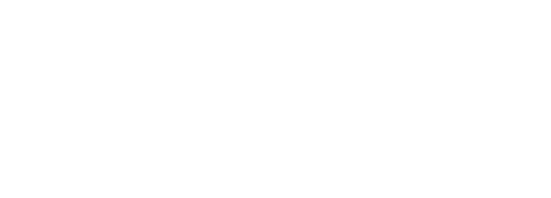

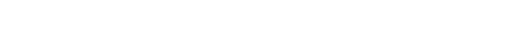
年 a nes

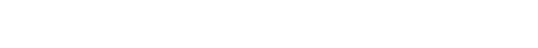
w

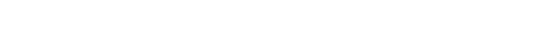

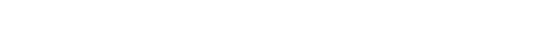

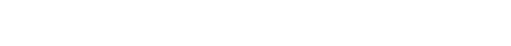

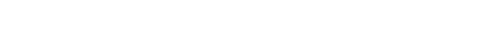

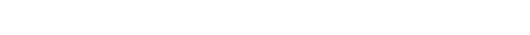

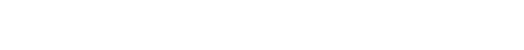

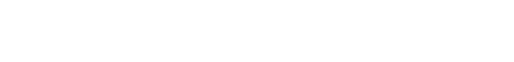



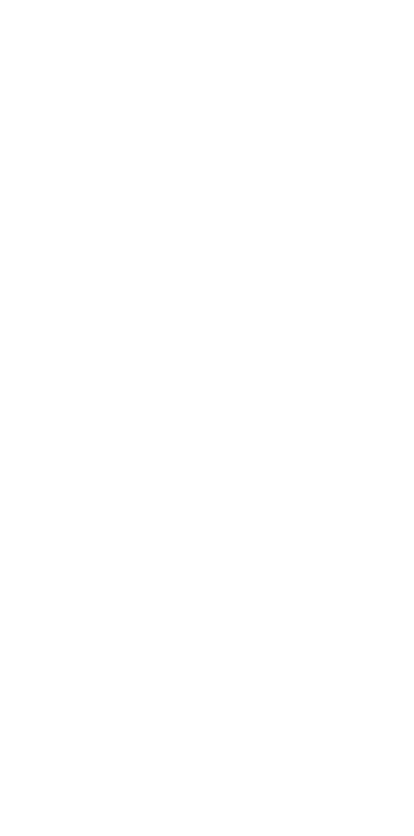

z

$\frac{5}{3}$

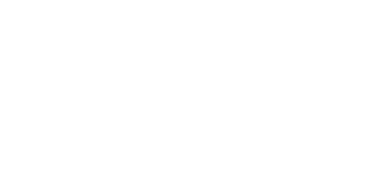

空

Ea

åñ

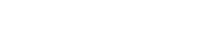

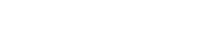

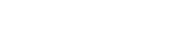

- ज) inningo

และேะே

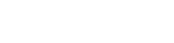

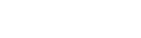

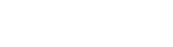

tenesenses

3

$=$

\& क a a

- $\quad \simeq \quad$ a

$t>0+50$

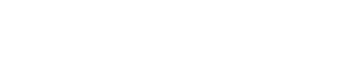

$\stackrel{w}{a}$

a 을

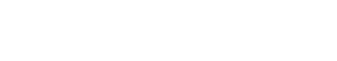

更正余方

is $\quad 0 \simeq N \cong N O$

3 กักักิ์ 


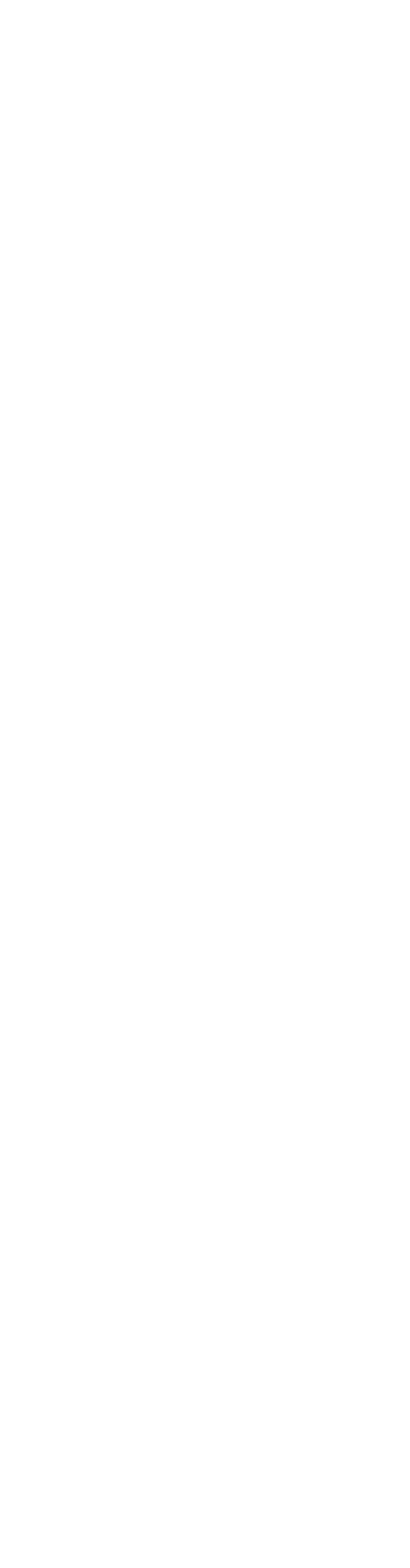



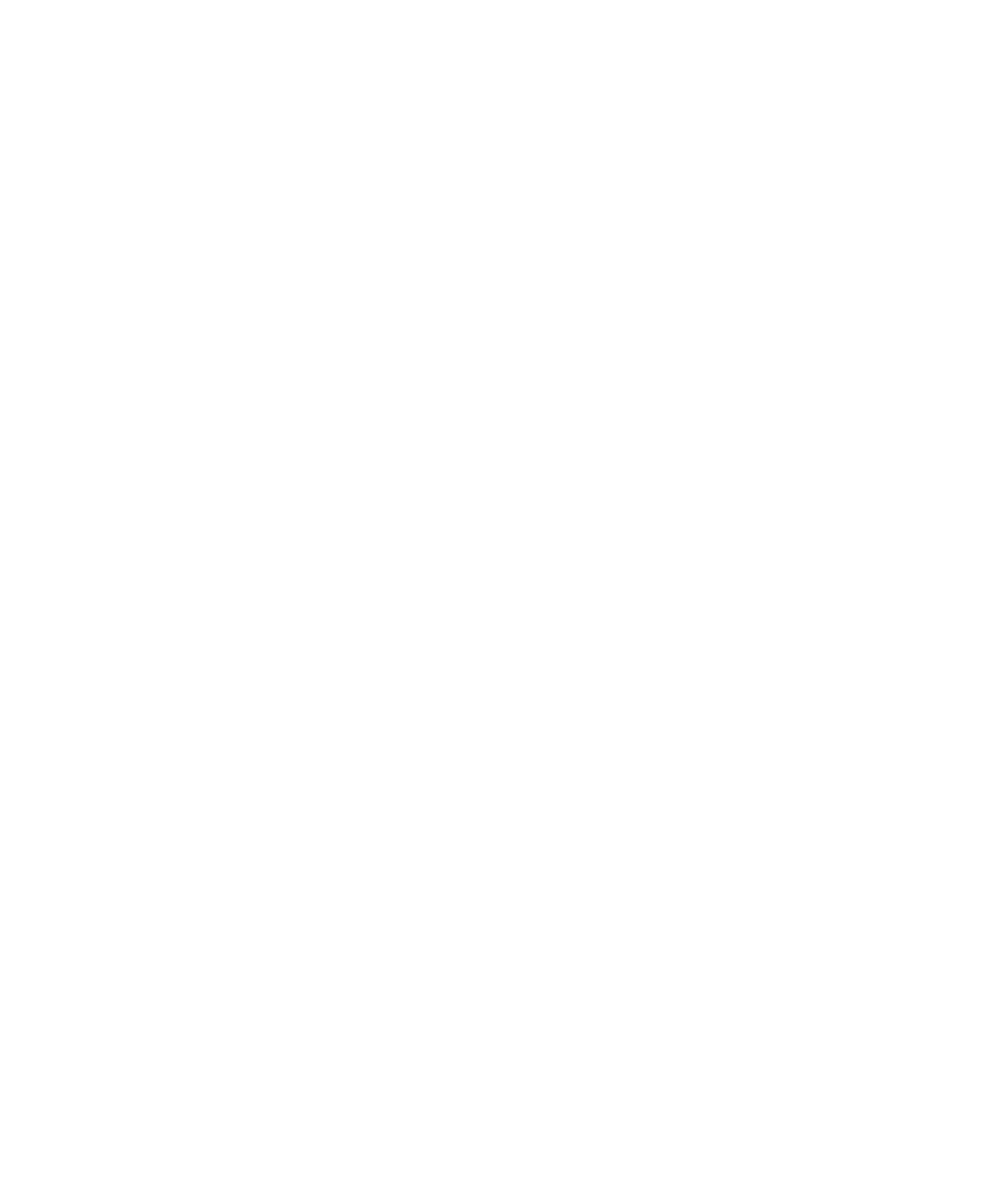

n n

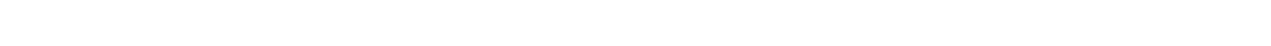

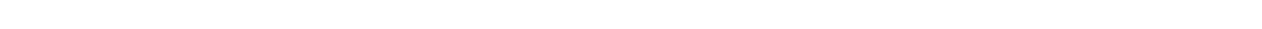

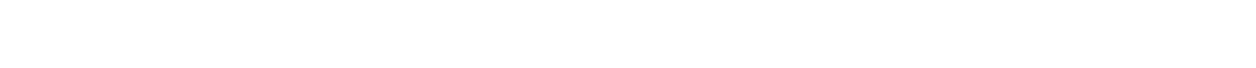

$\frac{1}{2}$
5
5

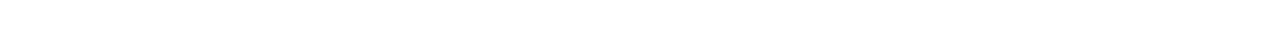
w

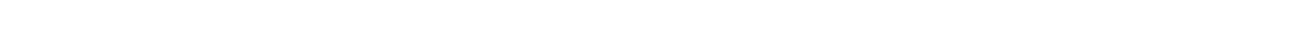
g

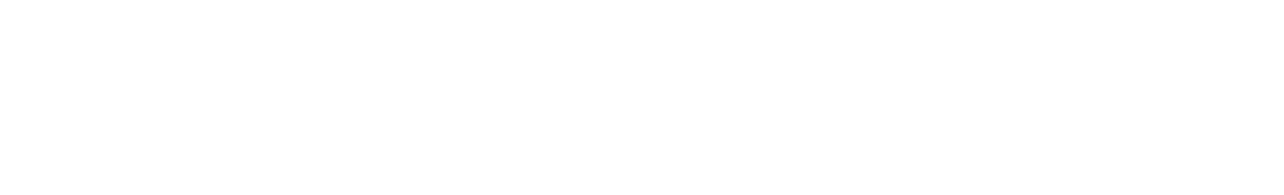

$\stackrel{\longleftarrow}{\mathbf{\Sigma}}$

$\stackrel{2}{5}$

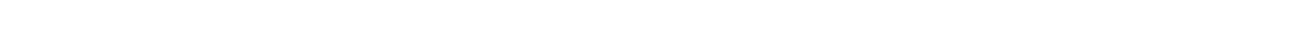

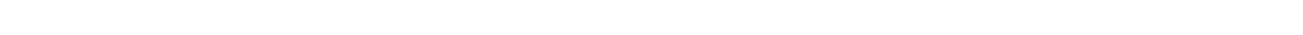

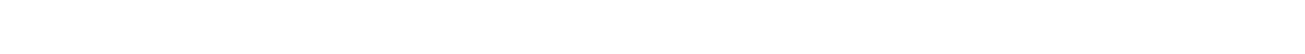

Nヘ

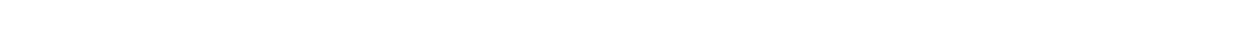
w

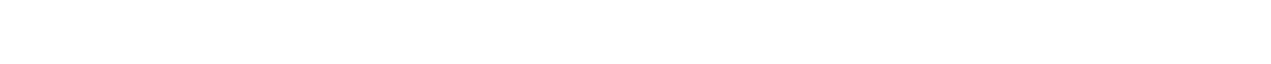

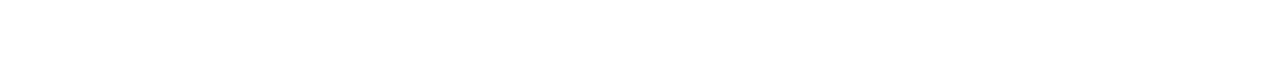

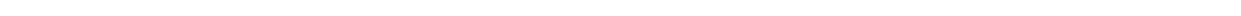

$\stackrel{t}{2} \sin \sin n$

is

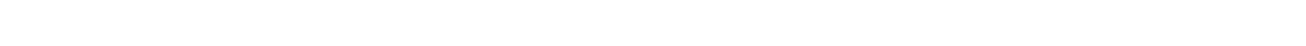

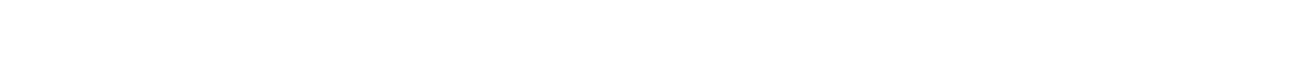

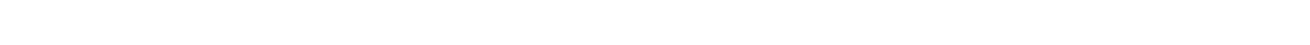

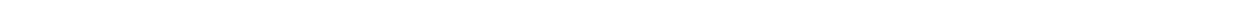
in w w w แ a

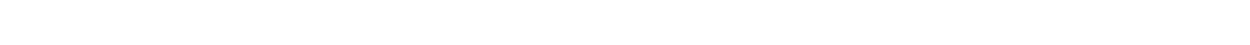

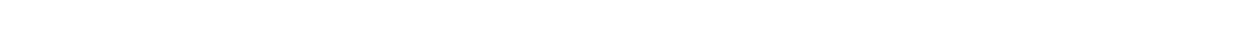


5
$\vdots$
5

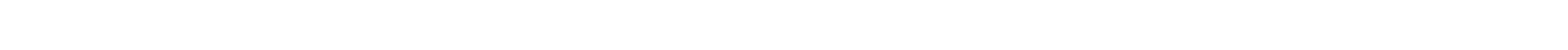
แ

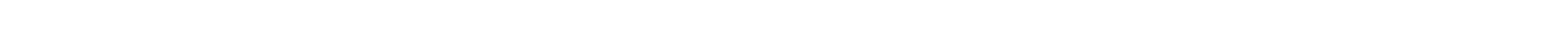

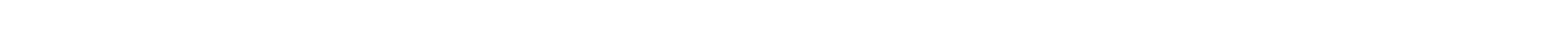

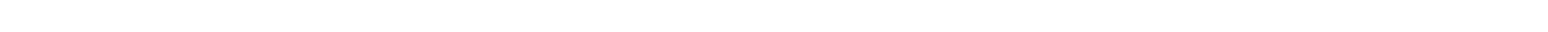

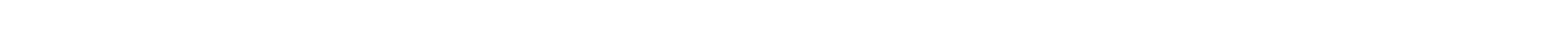

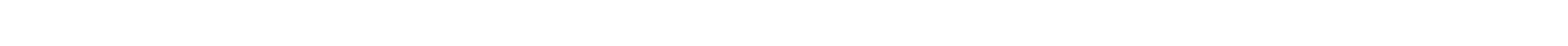

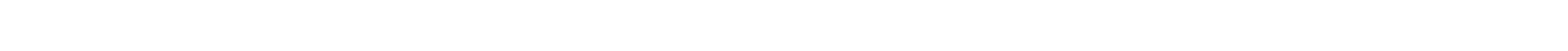

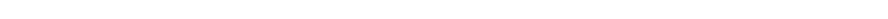

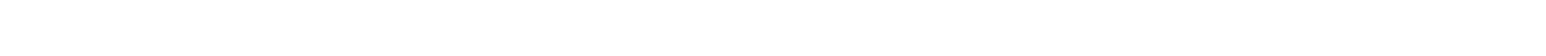

$\leftarrow$

$\frac{w}{5}$

es

in en

is

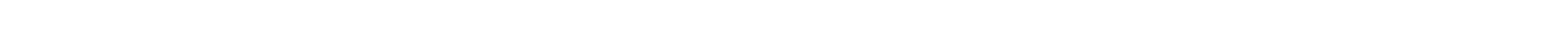

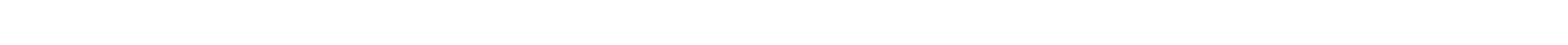

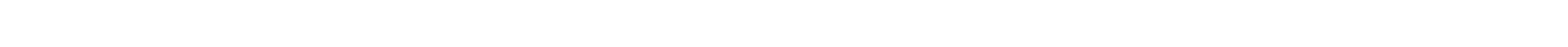

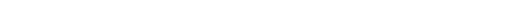

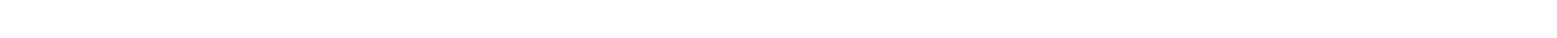

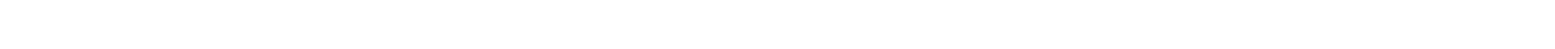

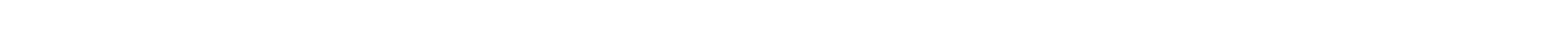

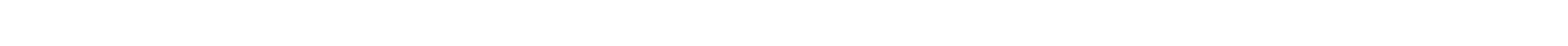

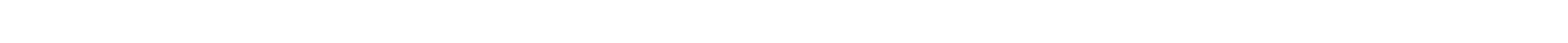

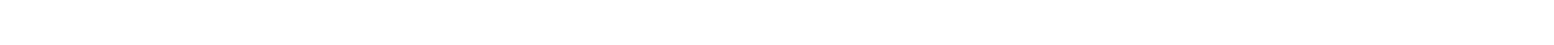

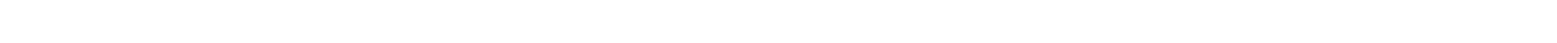

$\stackrel{\leftarrow}{\Sigma}$

us

n n

in

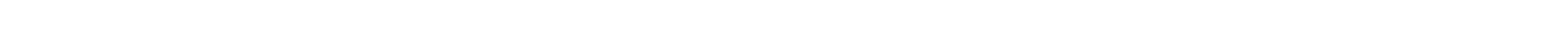
ш

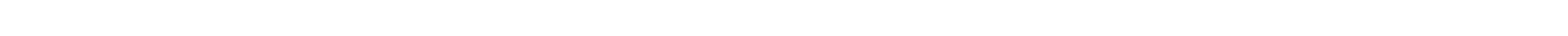
世

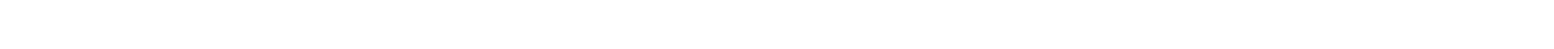

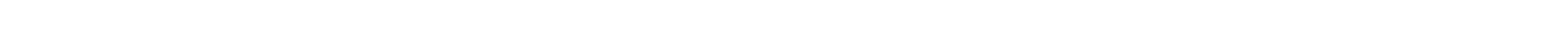

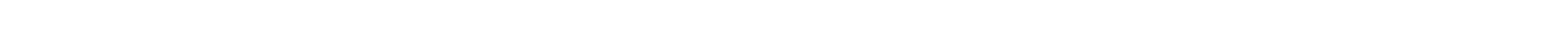

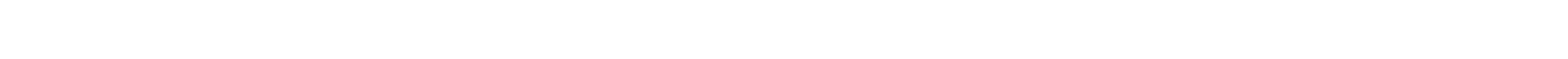

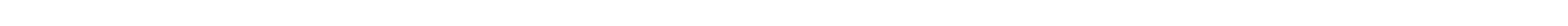

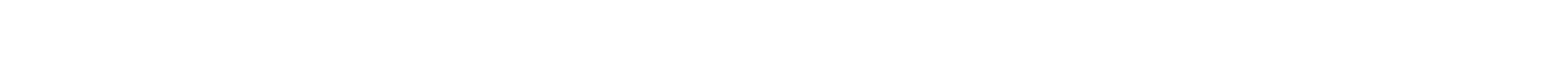

$\stackrel{\leftarrow}{\Sigma}$

es

is

en

is

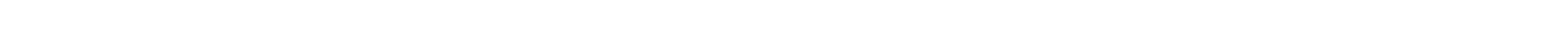

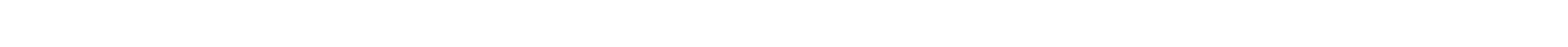

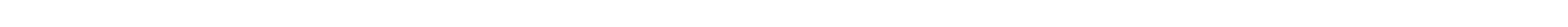

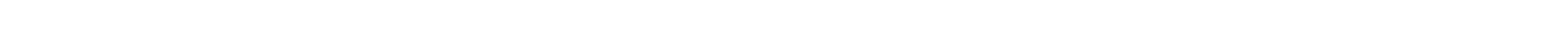

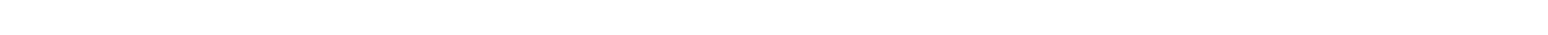

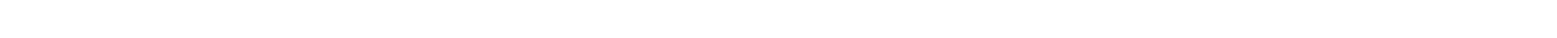

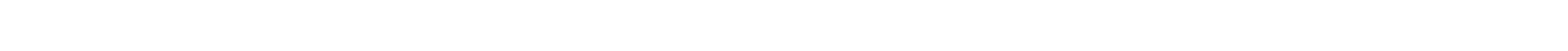

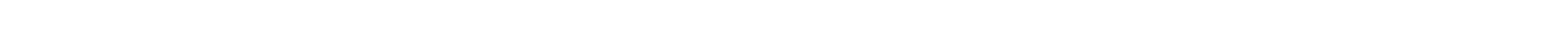

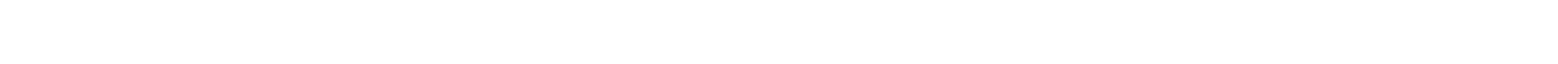




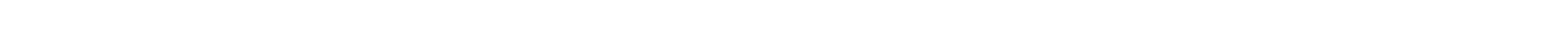

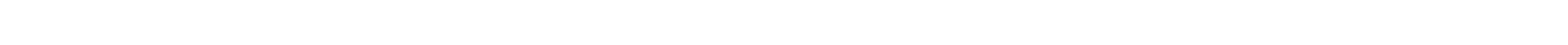

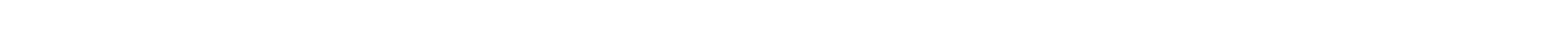

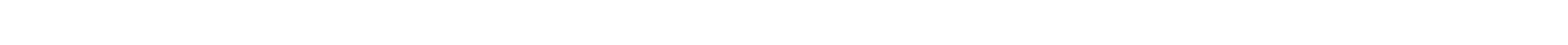

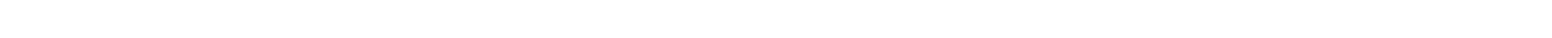

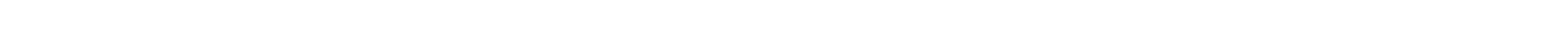

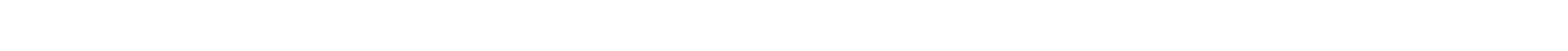

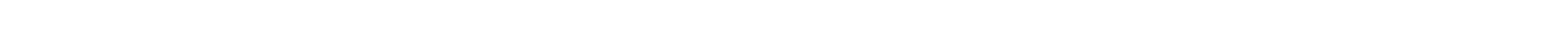

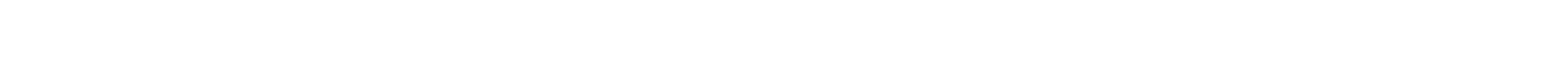

立

"

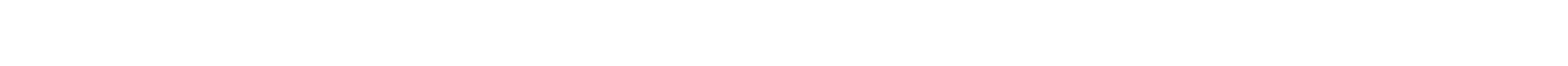

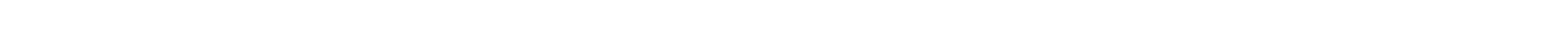

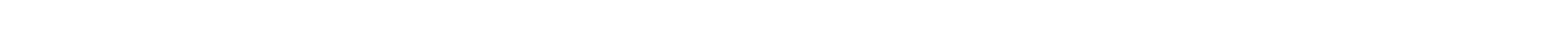

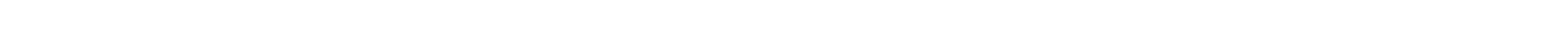
แ

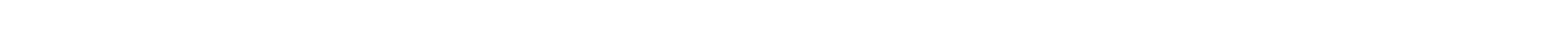

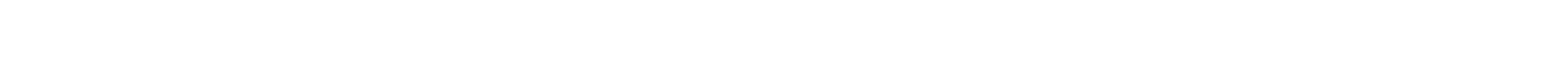
5

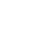

is

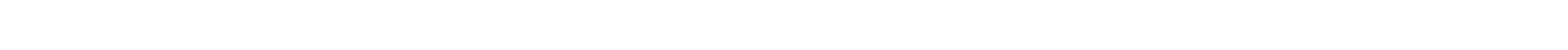

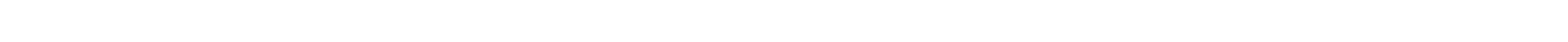
L य

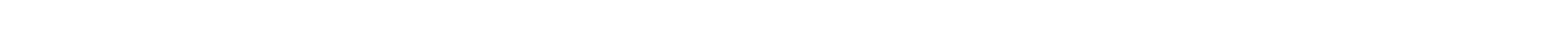

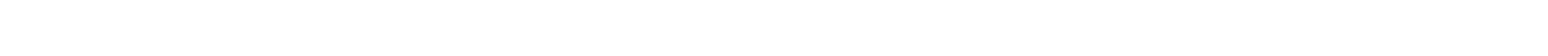

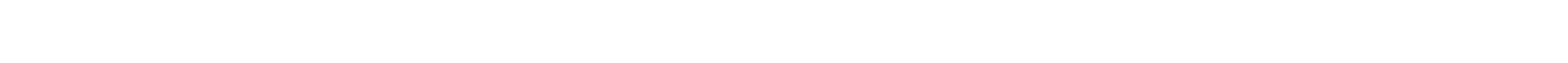
- N

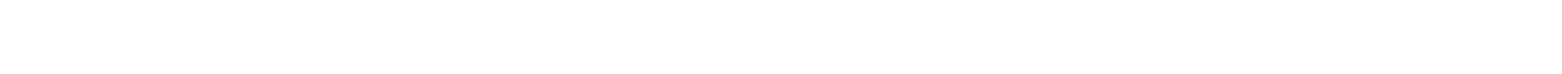

w

a

is

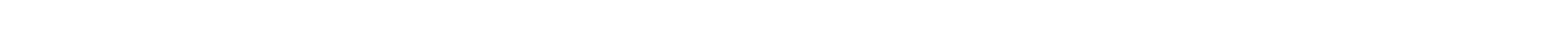

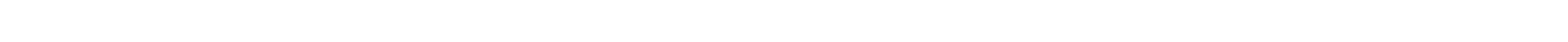

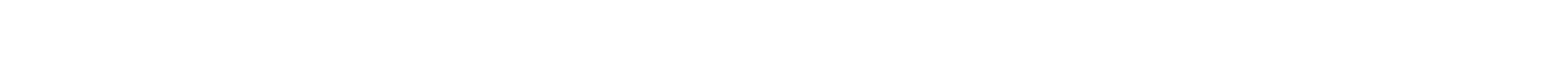

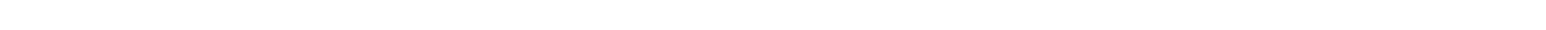

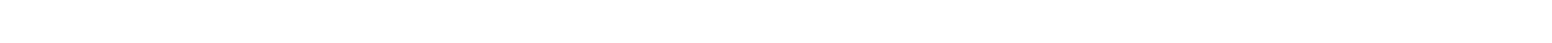

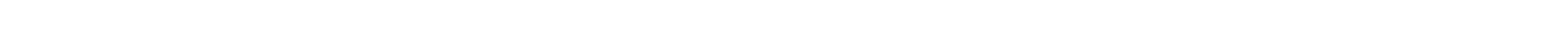

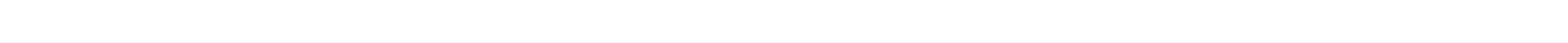

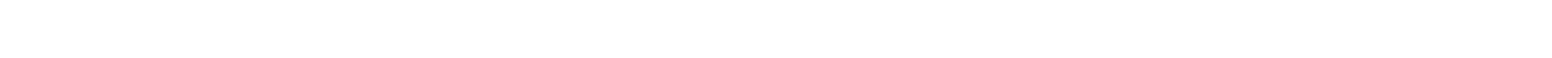


in

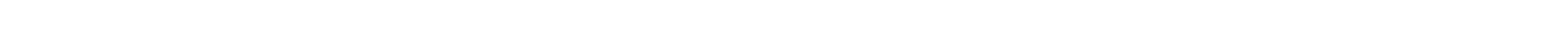
แm

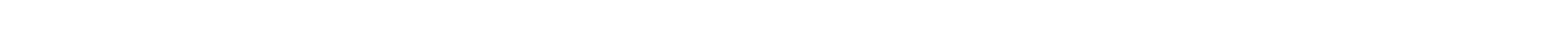
7

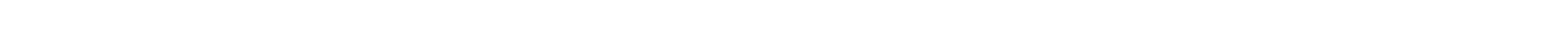

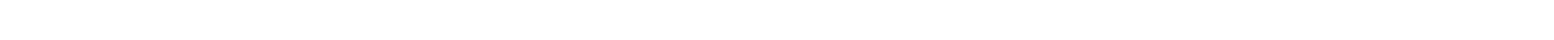

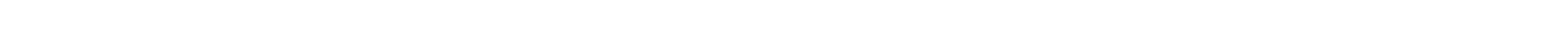

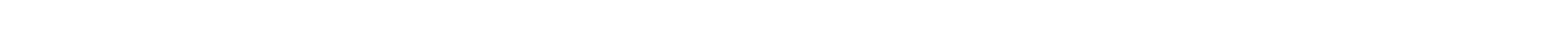

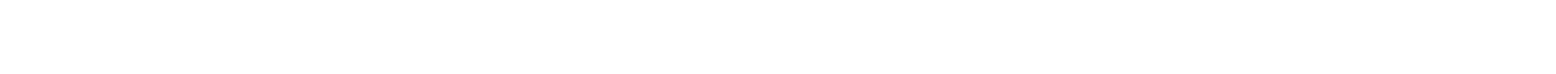

$$
\text { 立 }
$$

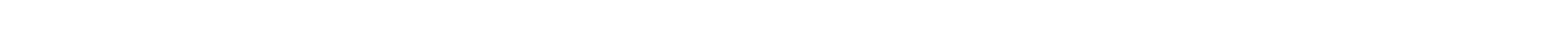

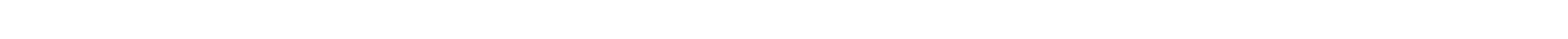

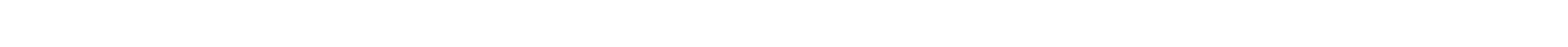

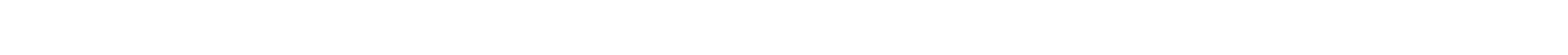

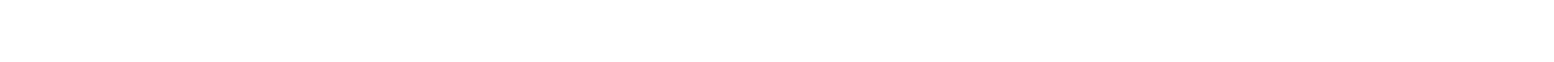

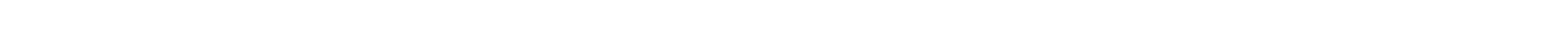

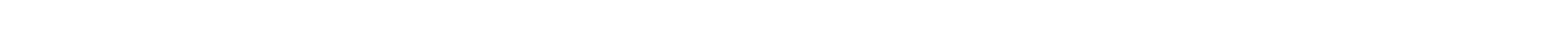

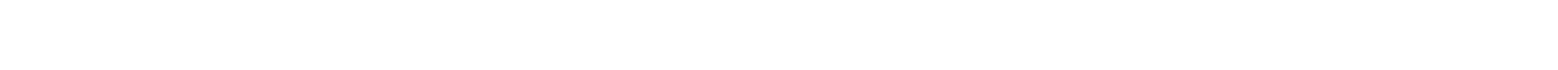

$\stackrel{5}{\Sigma}$

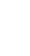

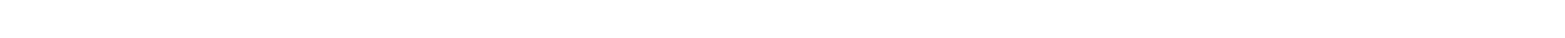

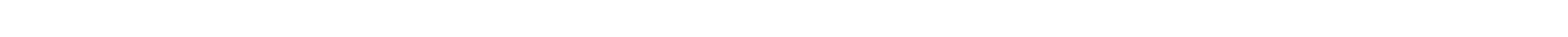
- यरำ

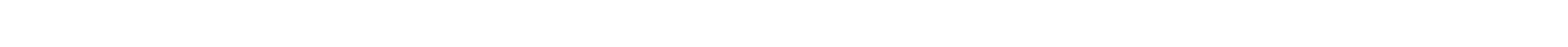

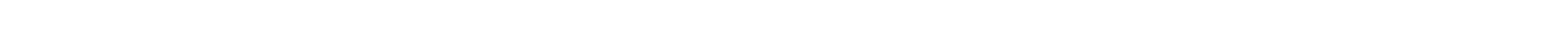

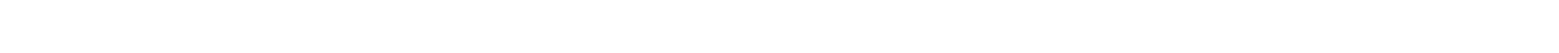

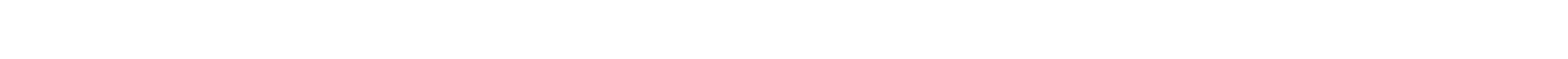

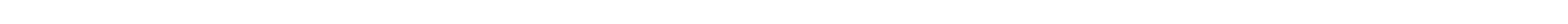

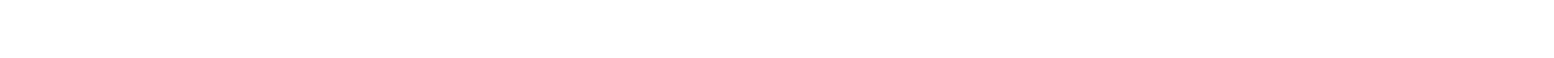
亲

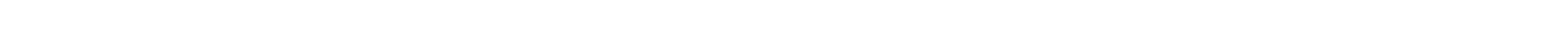

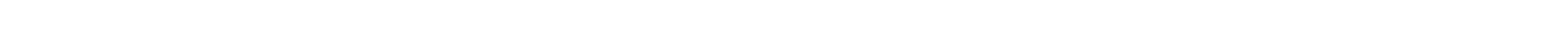

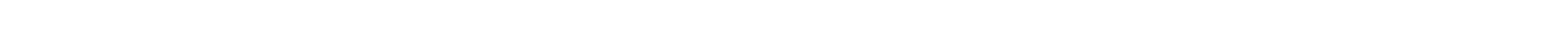

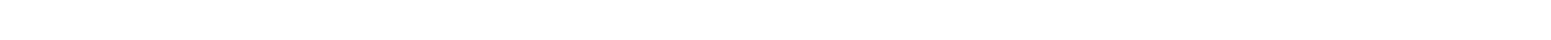

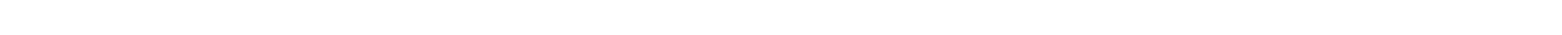
แ

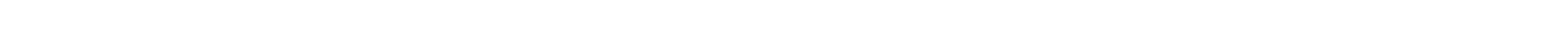

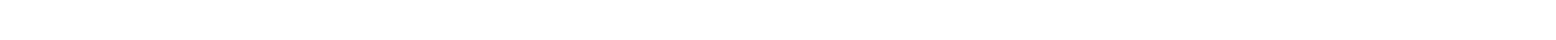

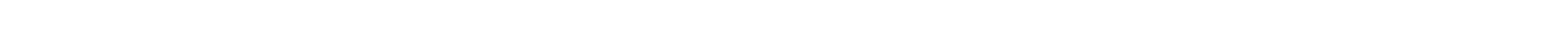




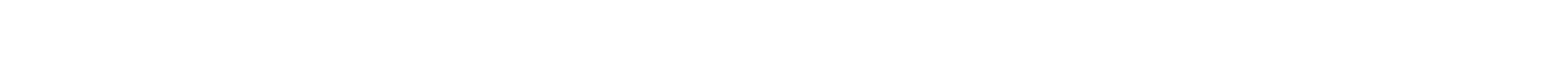

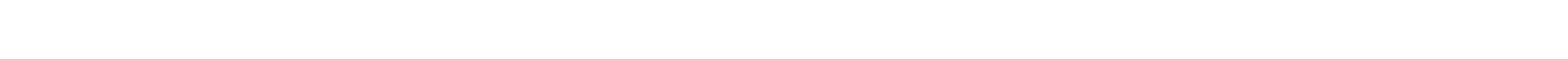

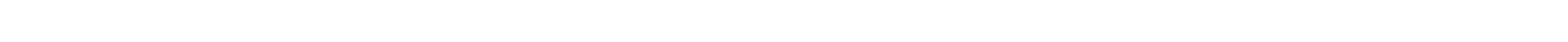

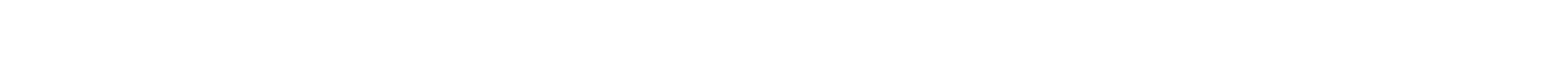

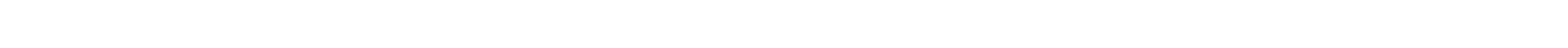

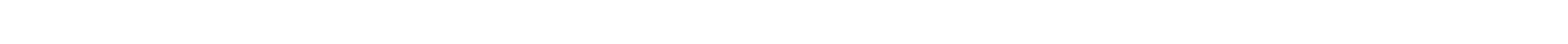

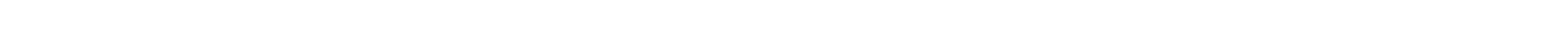

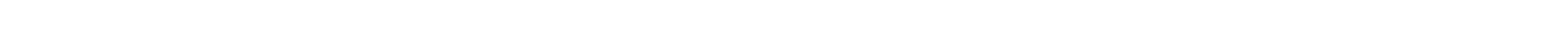
mm

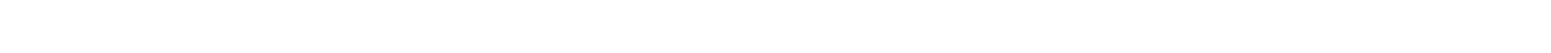

$\underset{\mathbf{w}}{\mathbf{z}}$

is

a

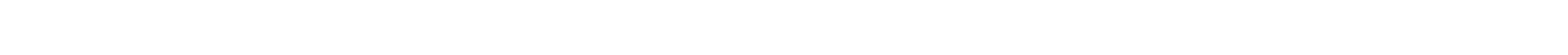
< उ

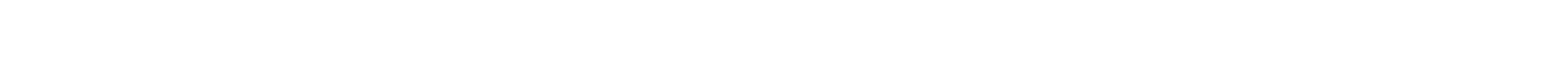

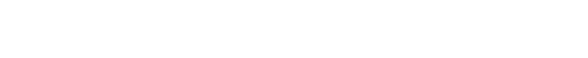

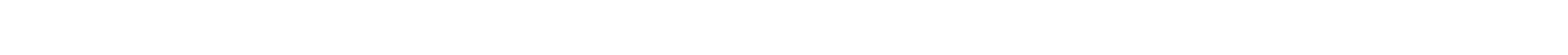

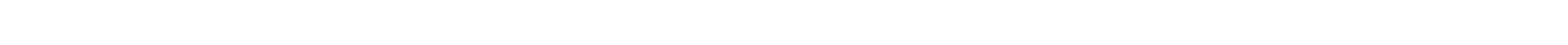
o- $\approx$ Nmo

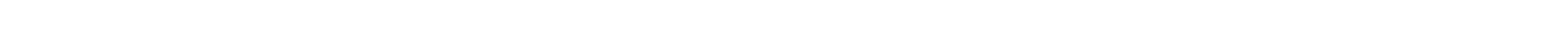

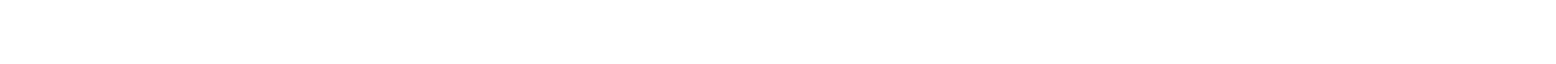
5

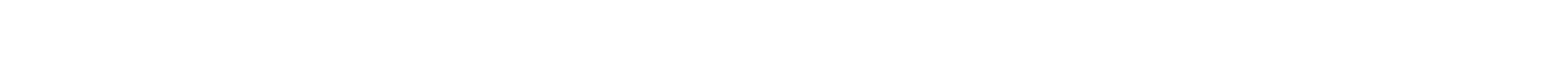

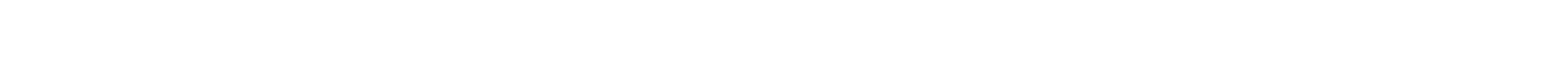

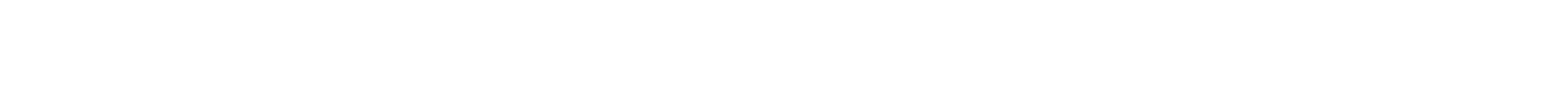

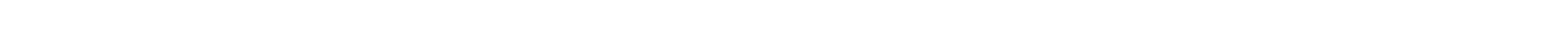

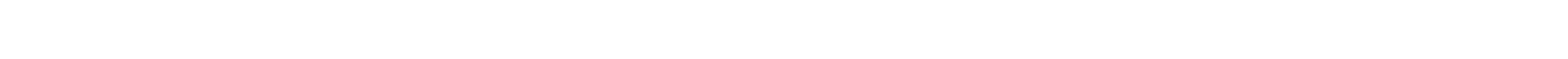

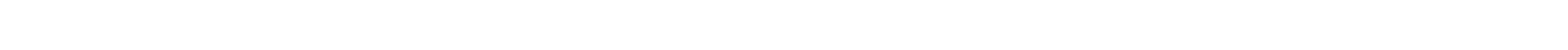

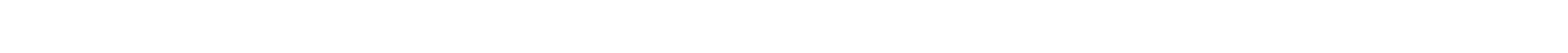

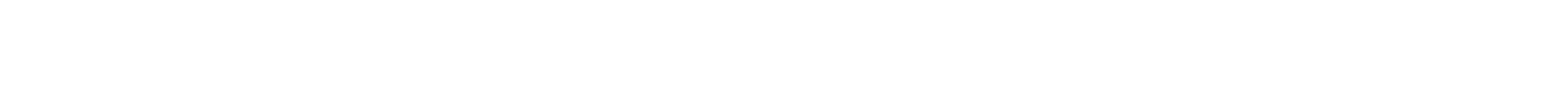
出
榇
n
is
n

in

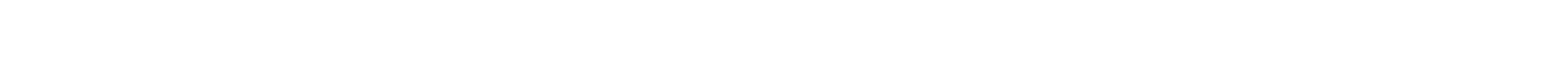

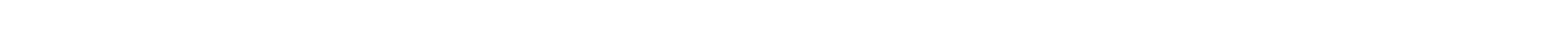
उ งทำง

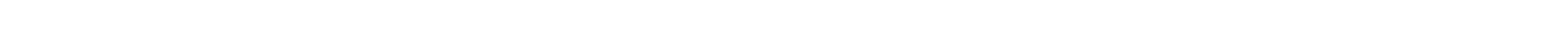

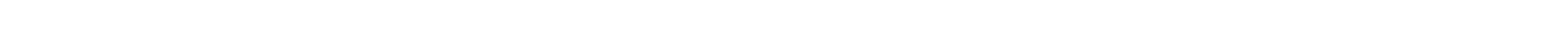

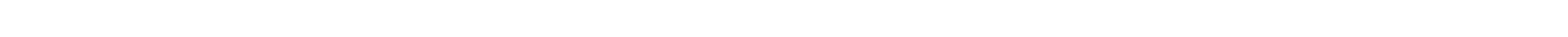

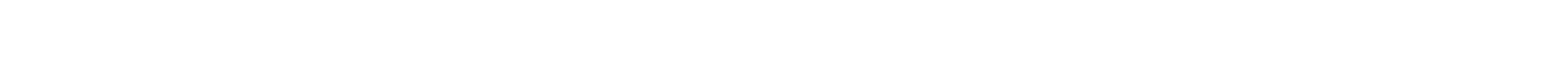



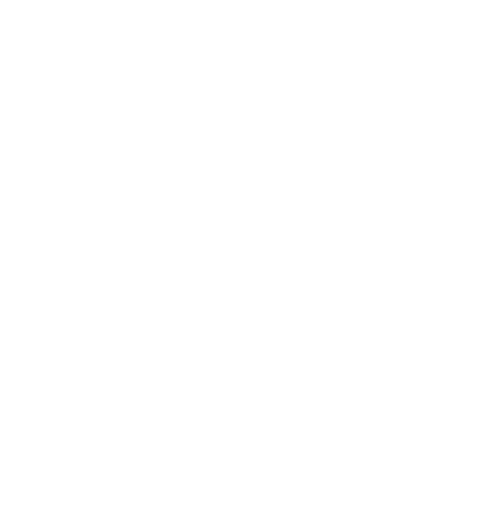

Eusesesosesesos a

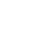

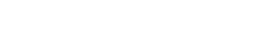
Hus. a waroró

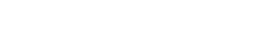

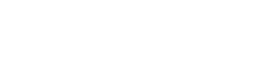

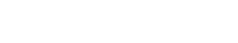

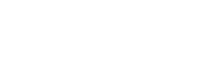

Euscosososeses

5 ตㄴำ แ山े

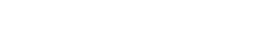
s.7.5.

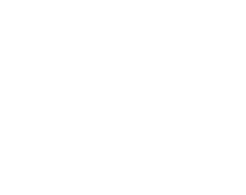

twenes es usenes

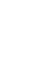

\# nNonomn a $\rightarrow m m \sim 0 n$ in $\stackrel{m}{\longrightarrow} \dot{0} \dot{\sim} \dot{a} \dot{a}$ a wámano - a

mooroor 슴ํaa

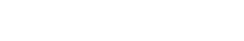

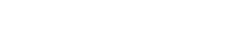

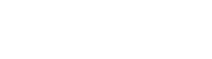




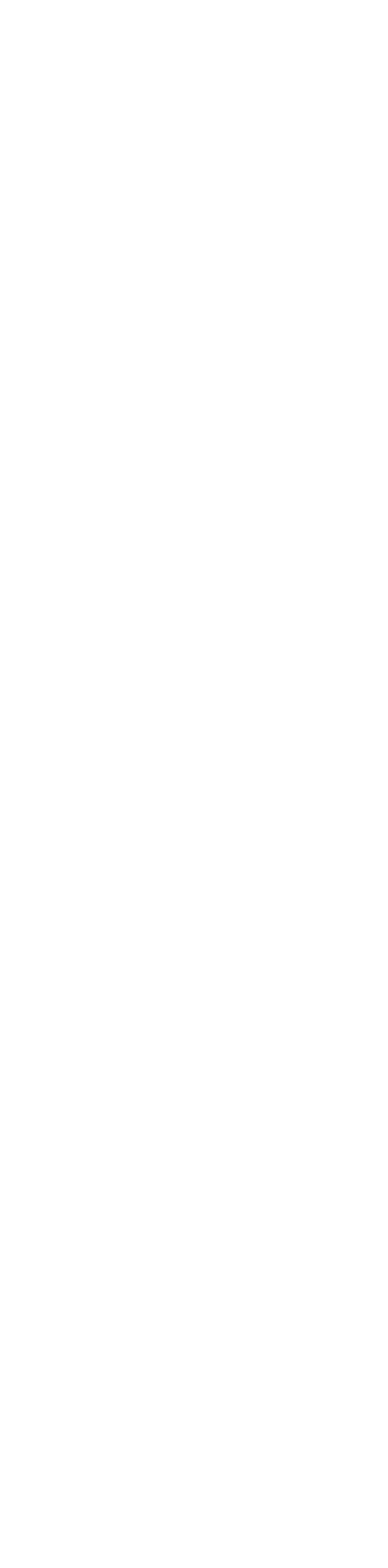

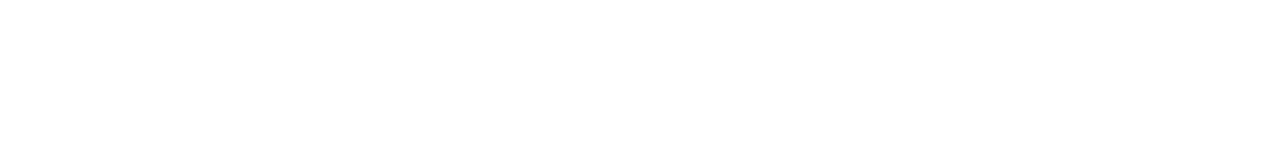

"

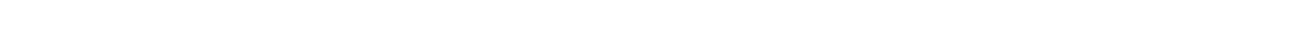
а w

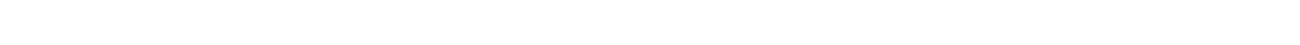

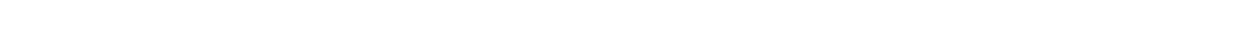

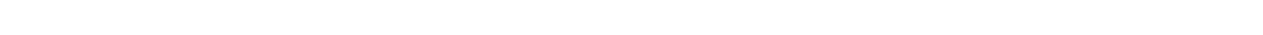

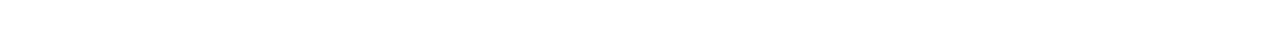

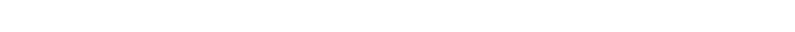

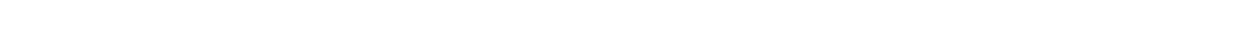

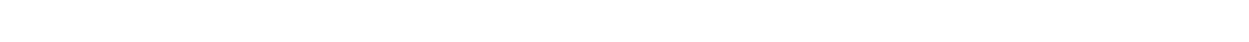

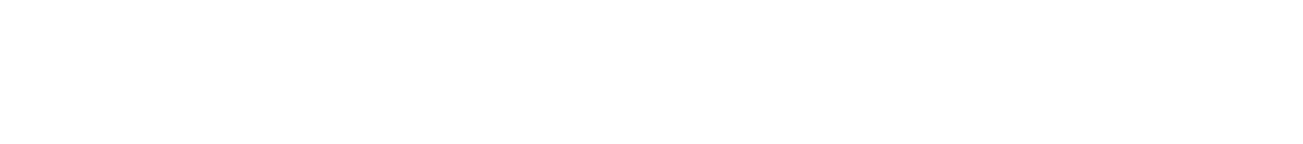

"

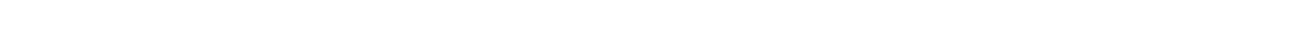

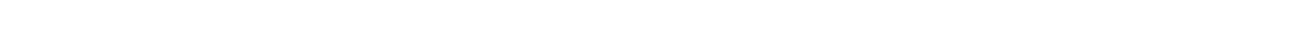

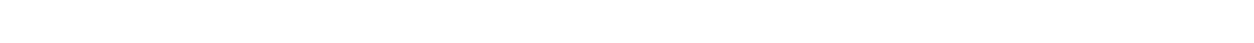

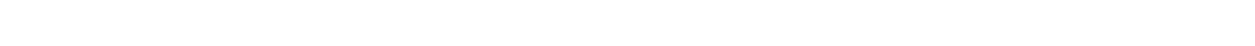

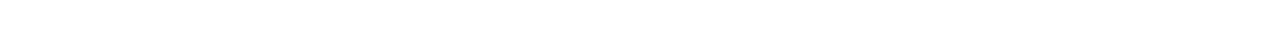

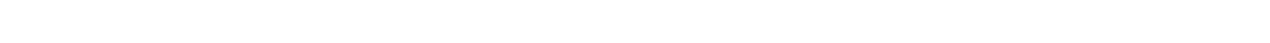

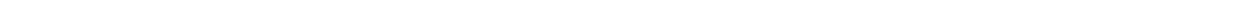

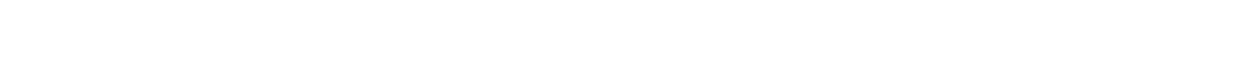

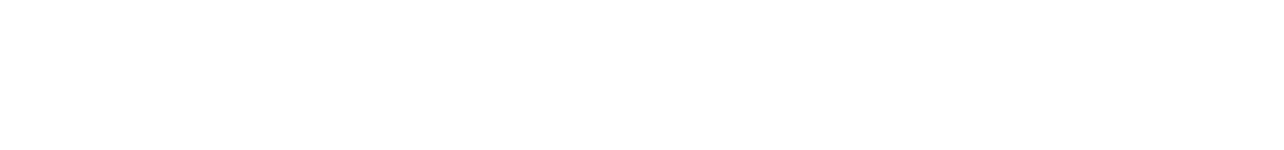

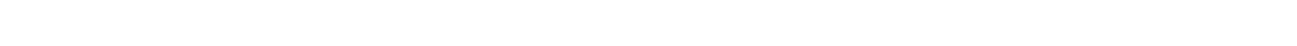

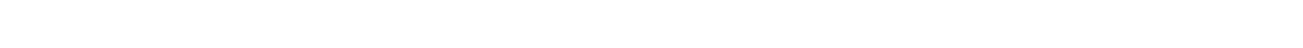

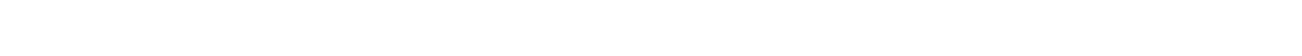

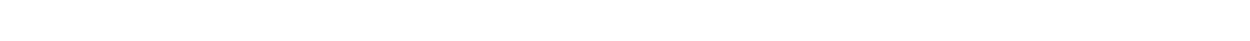

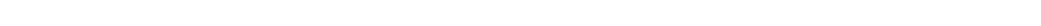

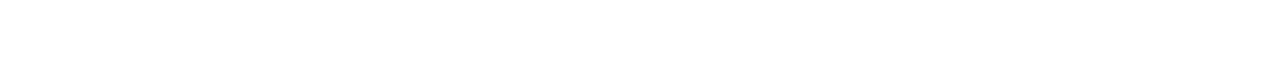

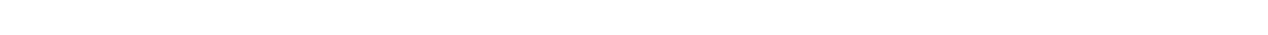

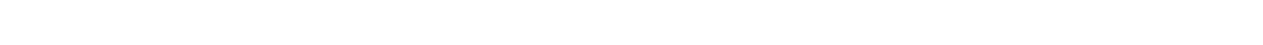

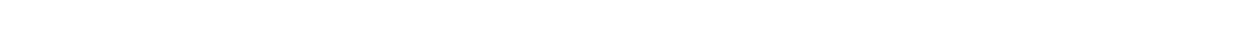

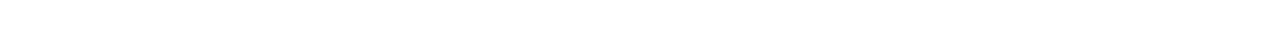

$\stackrel{a}{2}$

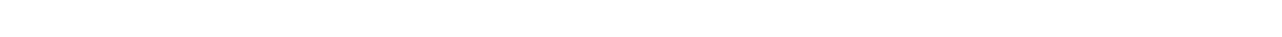
a d n

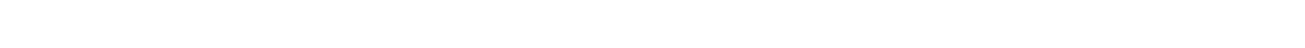

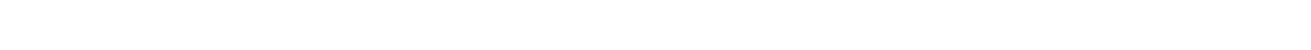

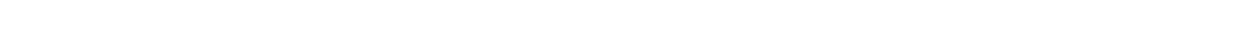

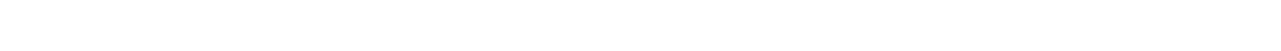

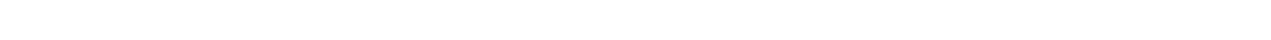

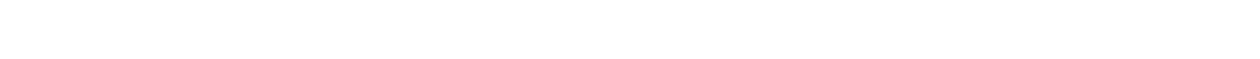




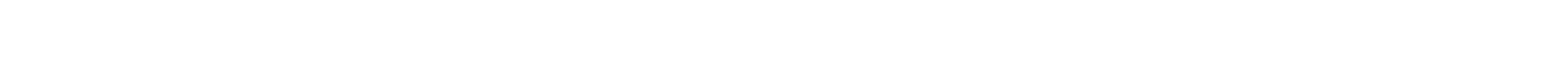

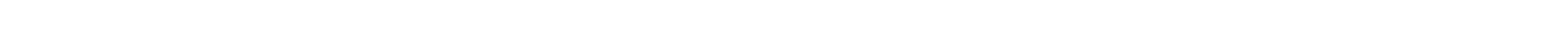

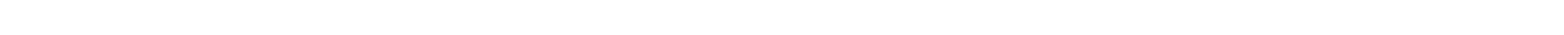

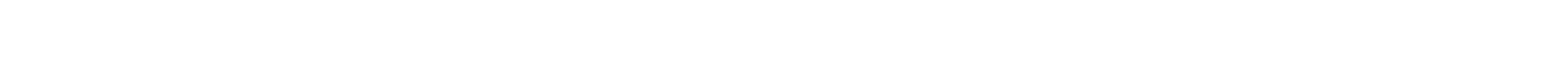
im is
nn
is
$\sin \operatorname{sn}$
n $n$
Non in a

$\bar{c}$

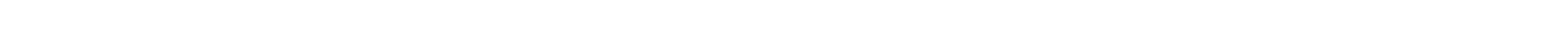
w

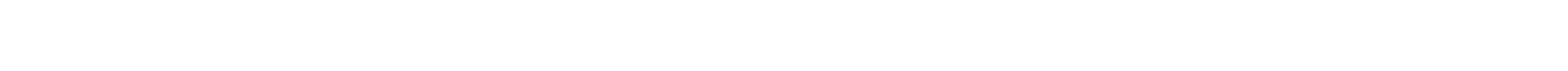

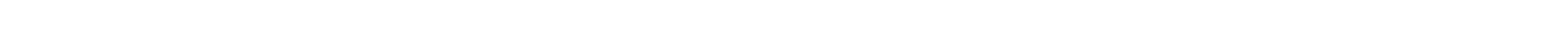

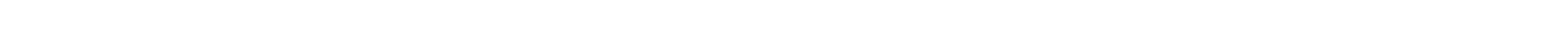
W

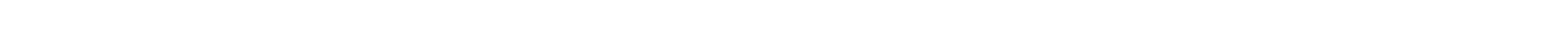

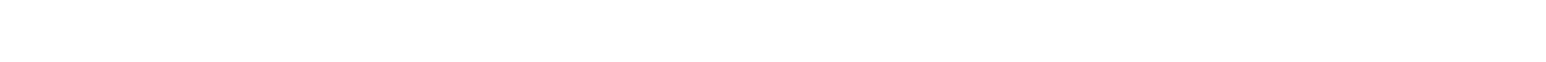

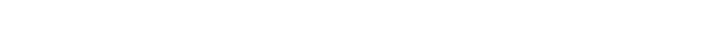
n
$\infty$
is
is

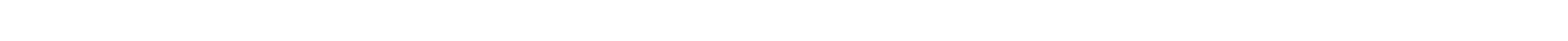

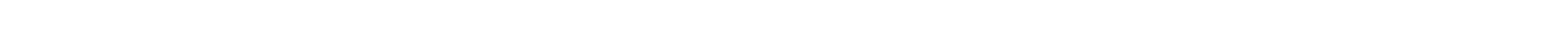

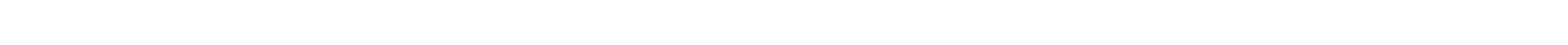

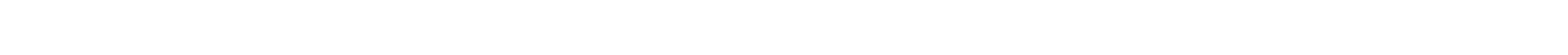
Бй

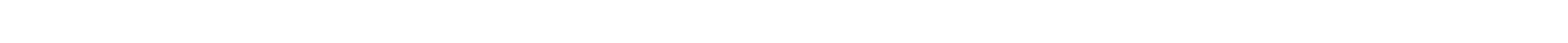

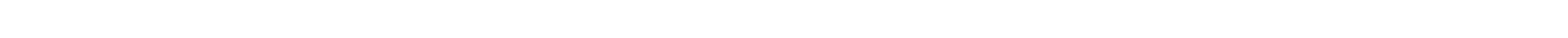

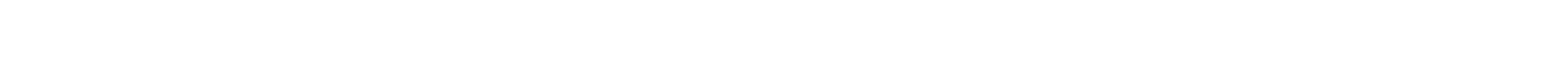

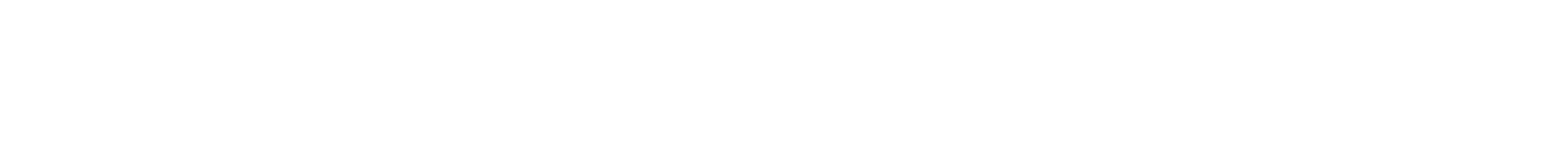

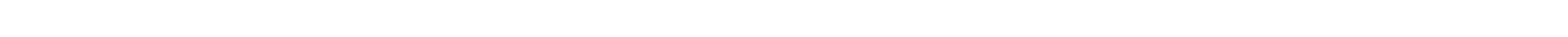

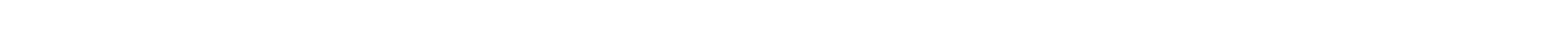

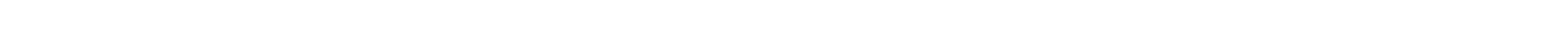

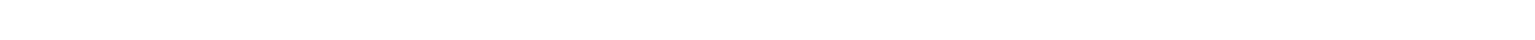

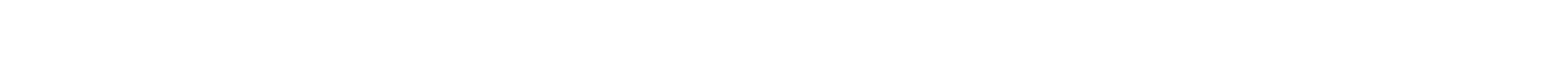

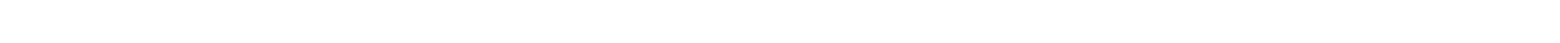

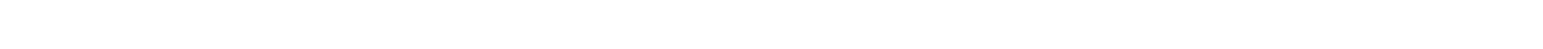
ㅇํำดั 


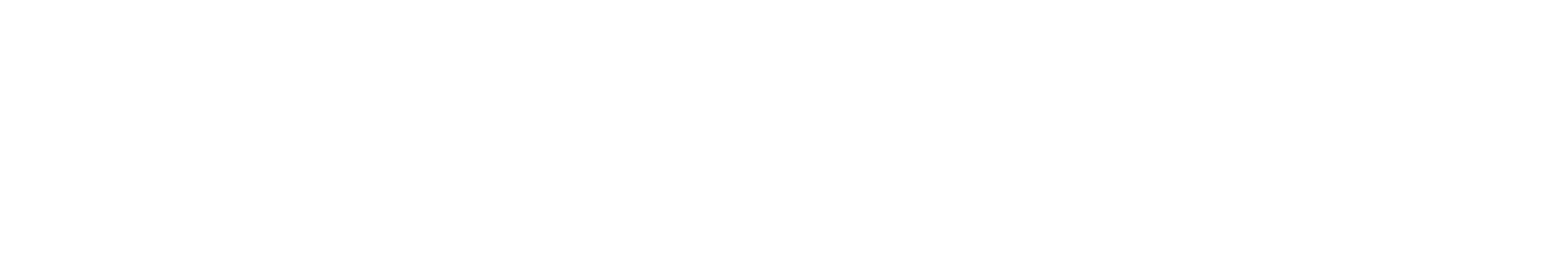

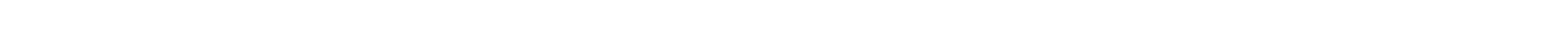

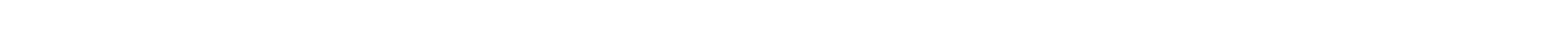
แ

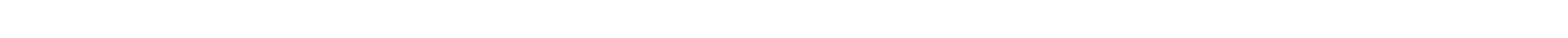

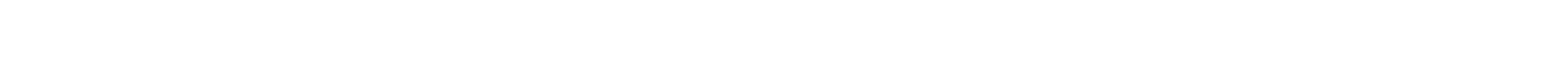

$$
\text { t. }
$$

崩

n

u

n

$\sin N$

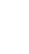

" a ш

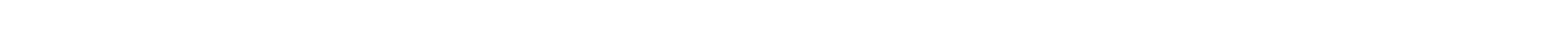

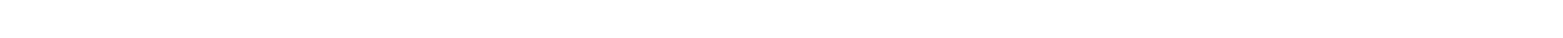

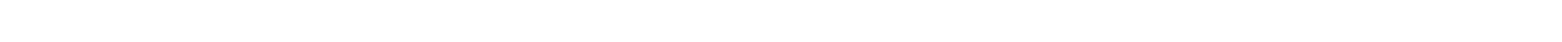
ш -

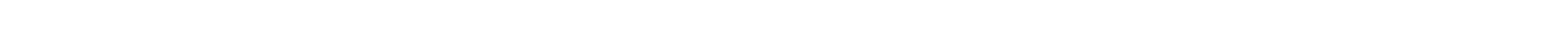

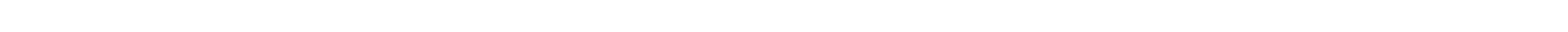

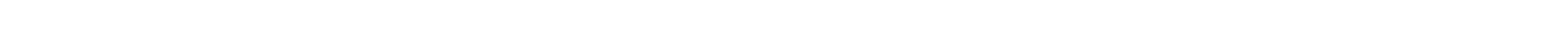

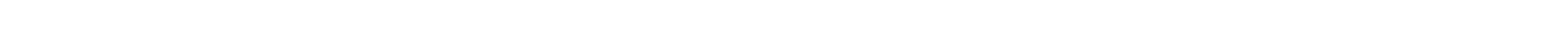

$\overline{\underline{w}}$

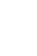

in к

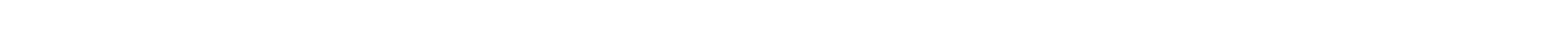

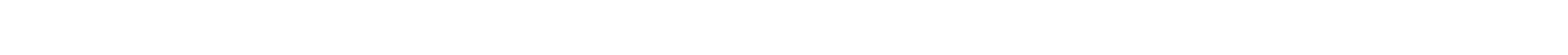

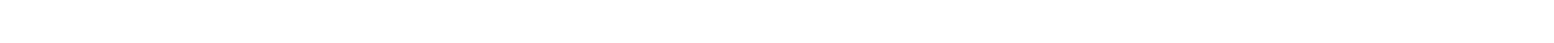

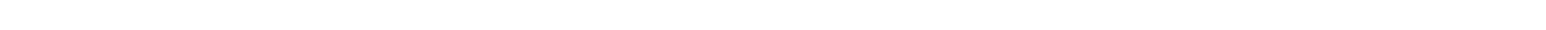

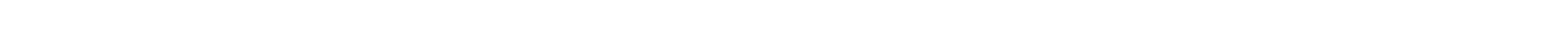

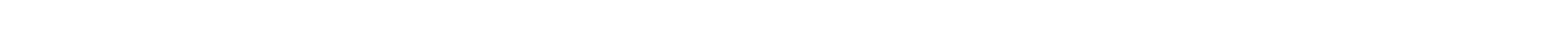

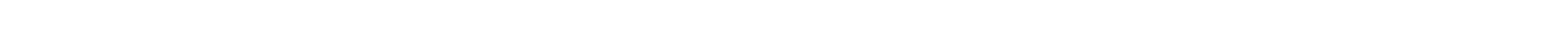

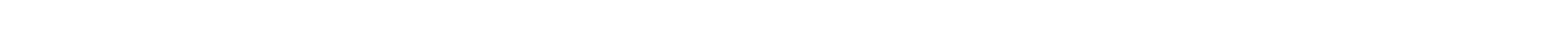

$\leftarrow$

21

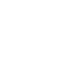

皮

๘

n

en

in

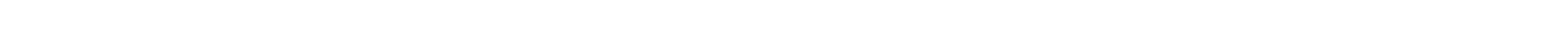

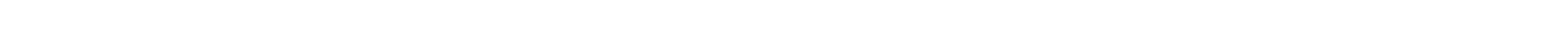
5
5 3
5

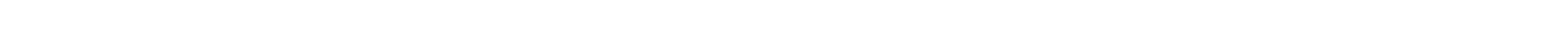

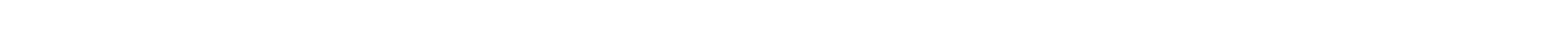

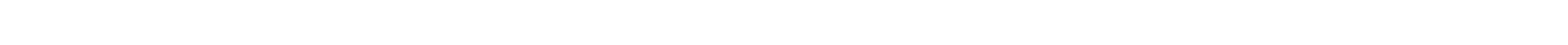

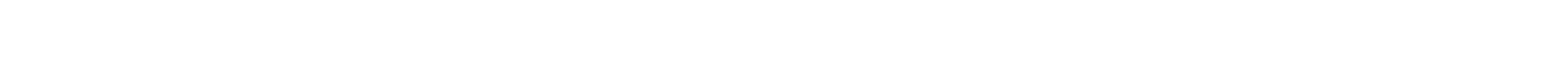




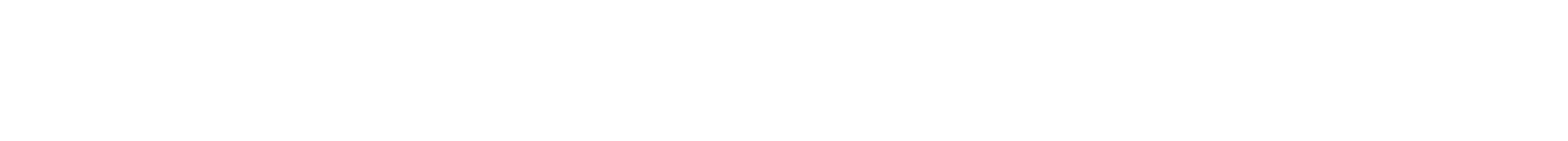
๙

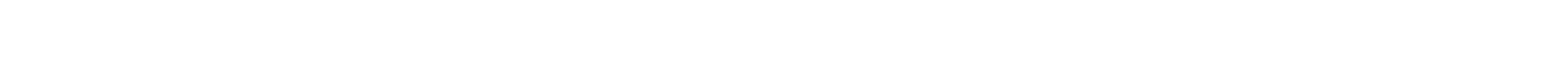

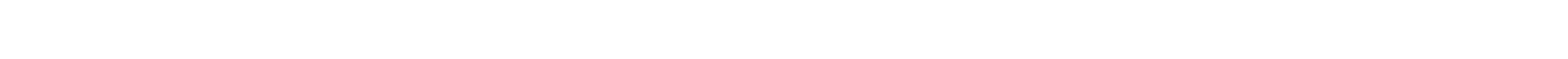

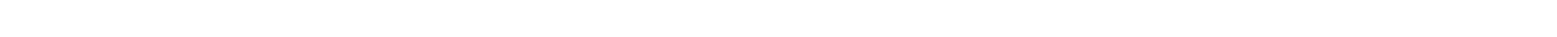

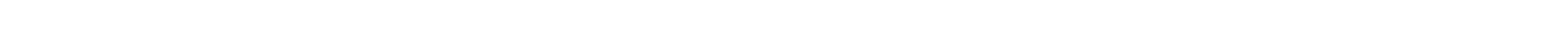

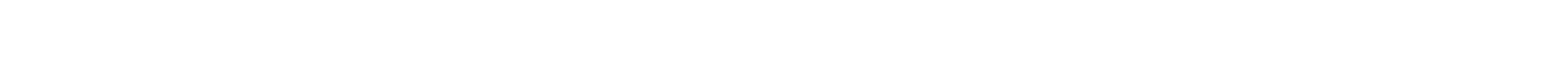

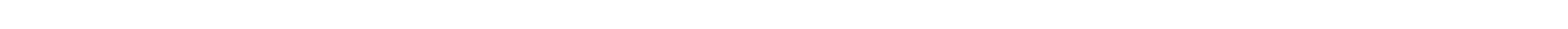

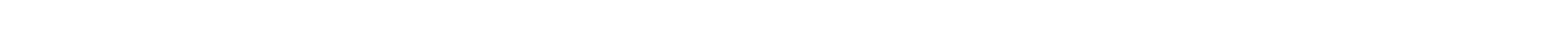

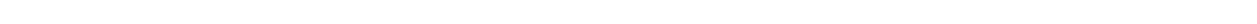
in

o I u u. ŁI

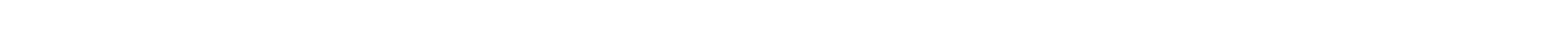

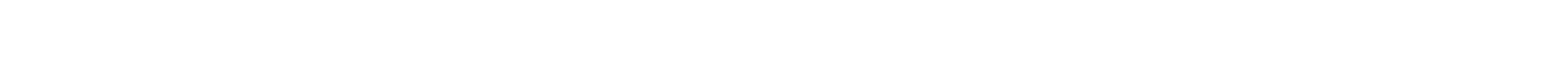
WE

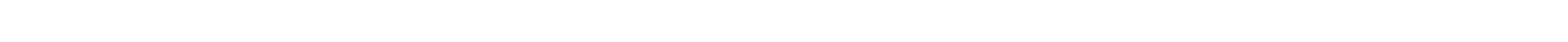

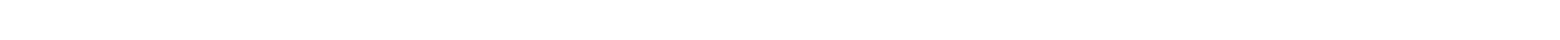

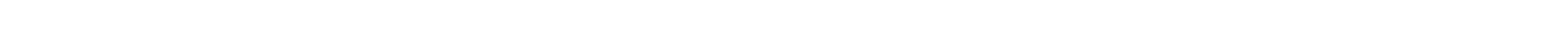

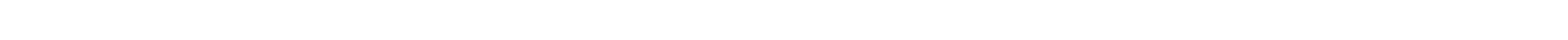

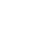

m mom $m$ m w w

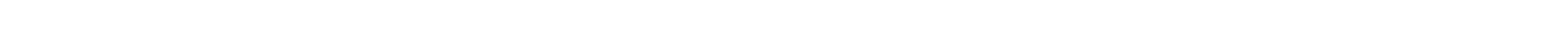

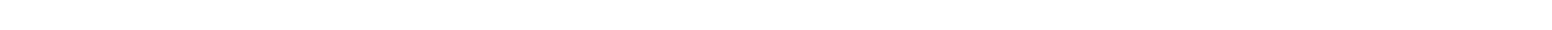
๙

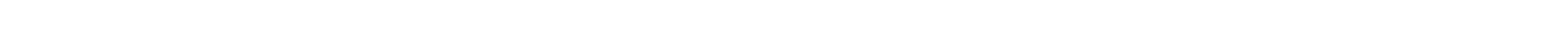

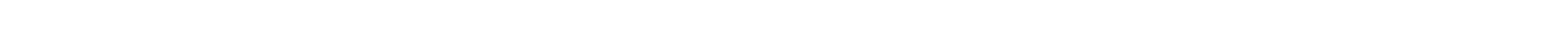

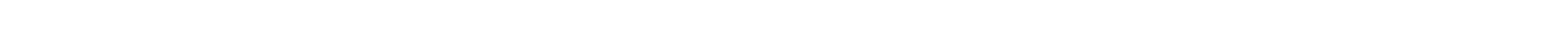

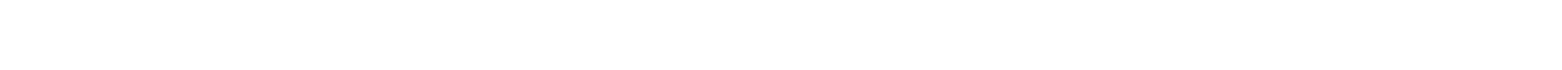

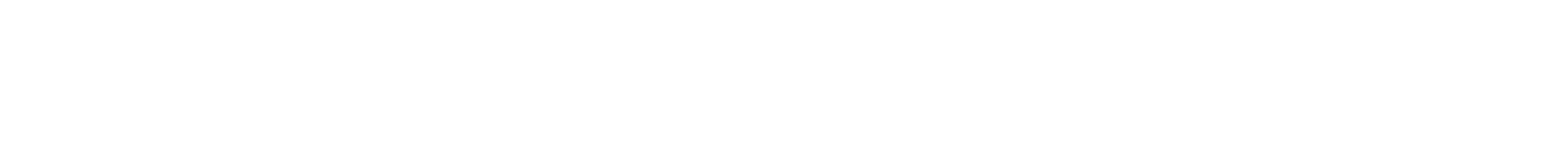
a "ú

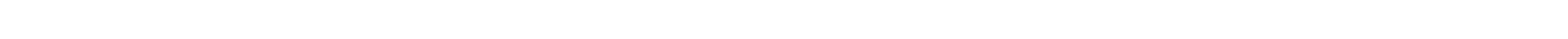

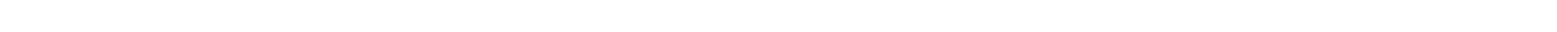
ง

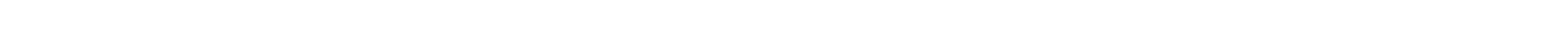

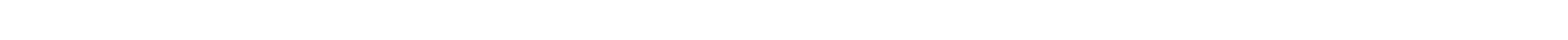

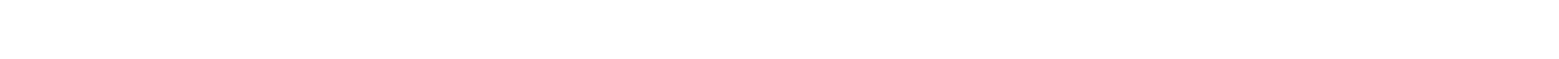




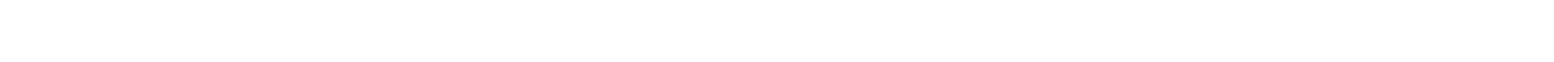

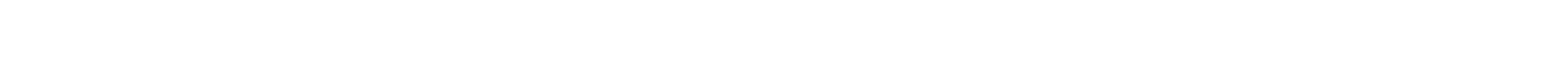

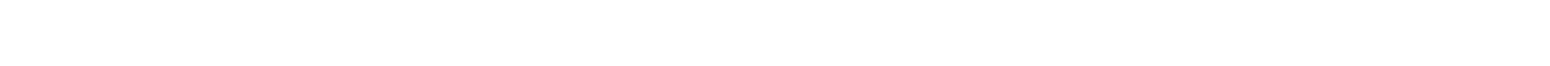

之

a

$\sim$

n

is

in in

is

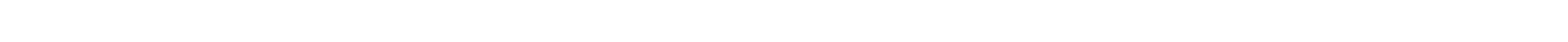
u ú

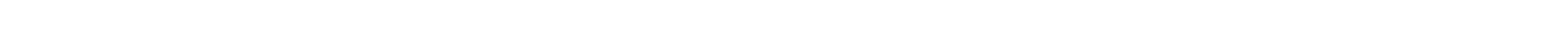

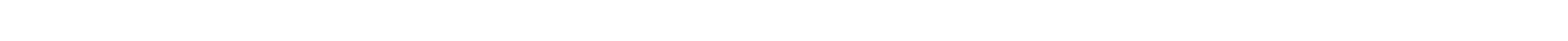

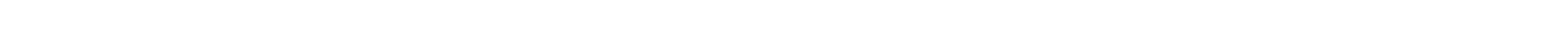

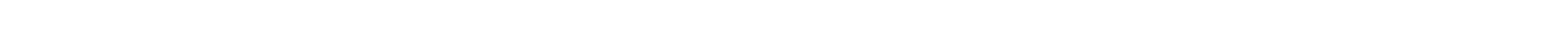

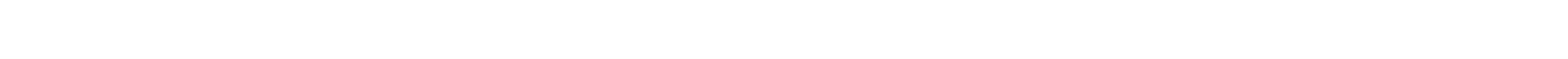

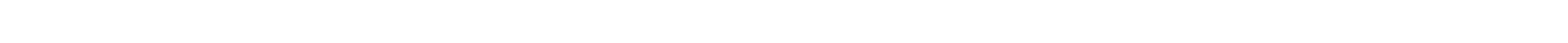

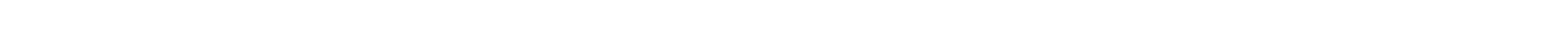

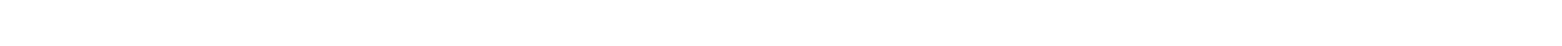
竝的 х

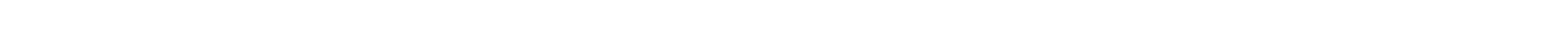

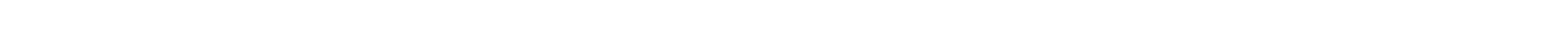

ตй

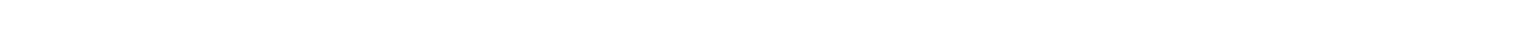

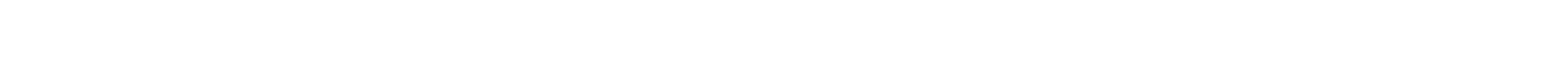

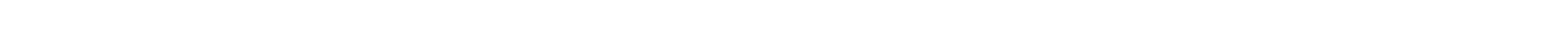

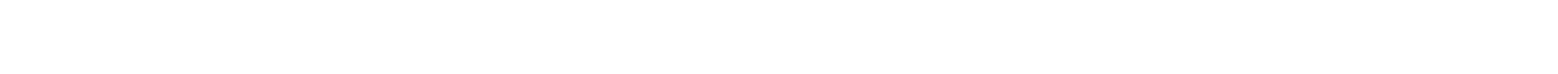

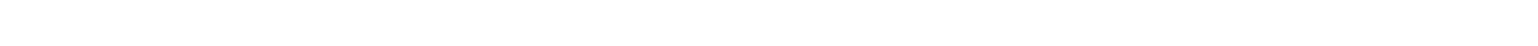

in

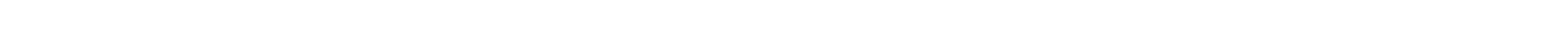

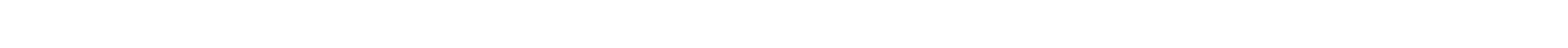
\begin{tabular}{ccc}
\hline \\
\hline
\end{tabular}

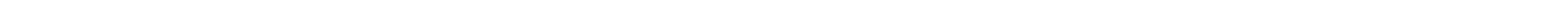

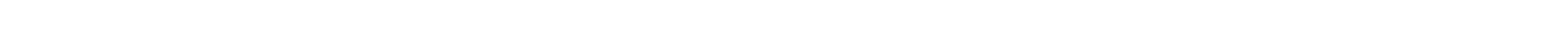

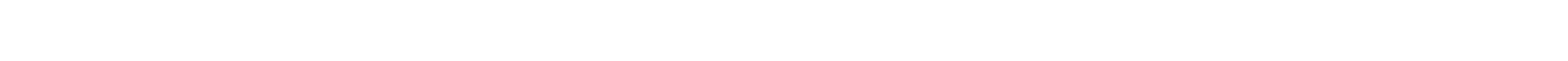

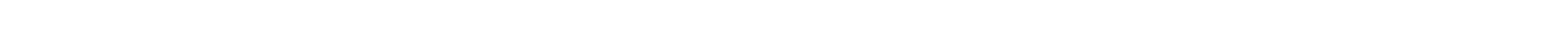

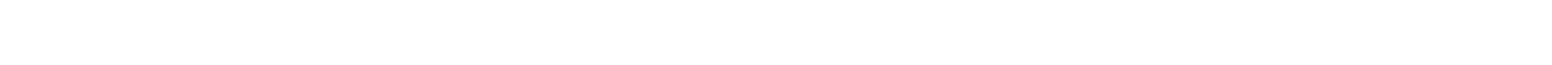




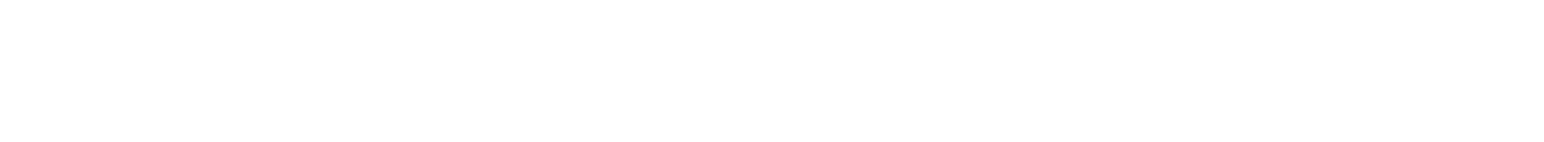

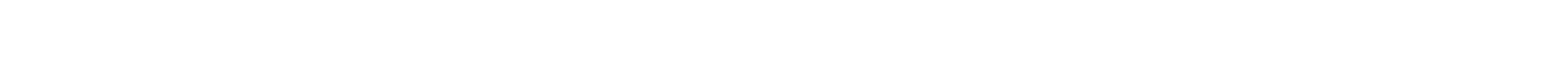

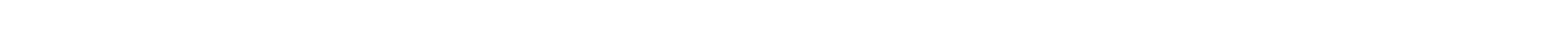

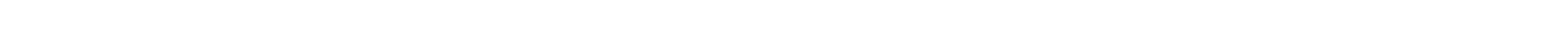

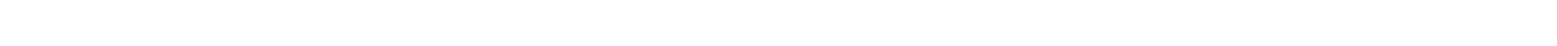

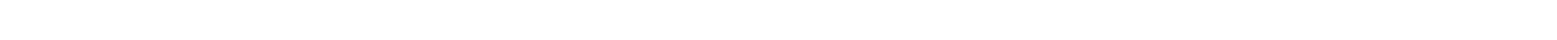

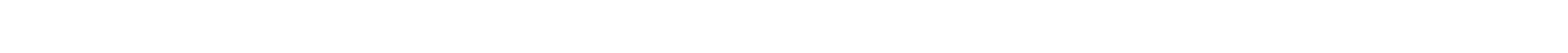
등으은

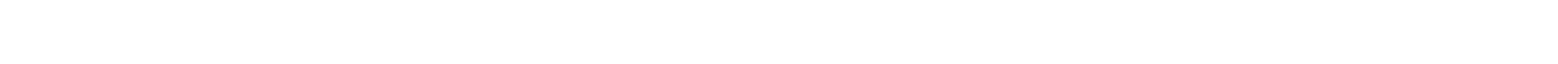

崖

in u

en

is

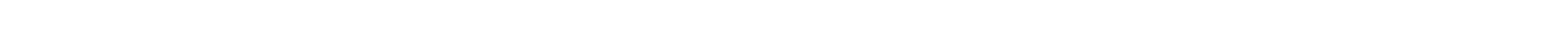

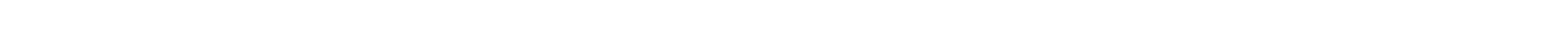

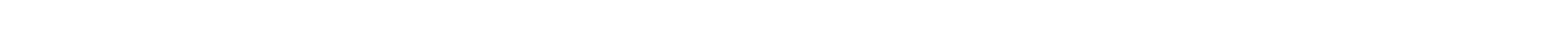

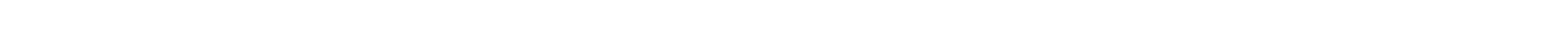

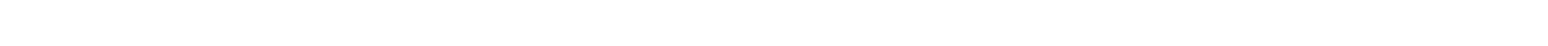

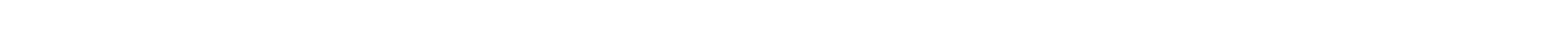

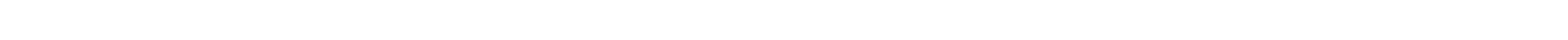

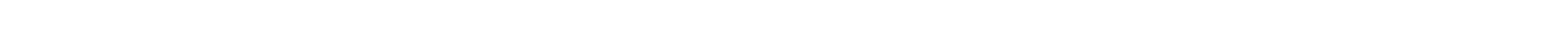

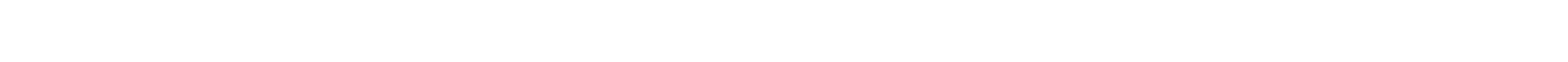

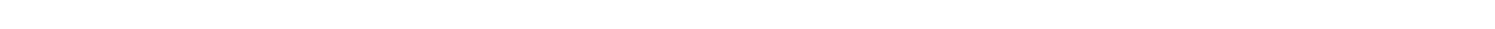

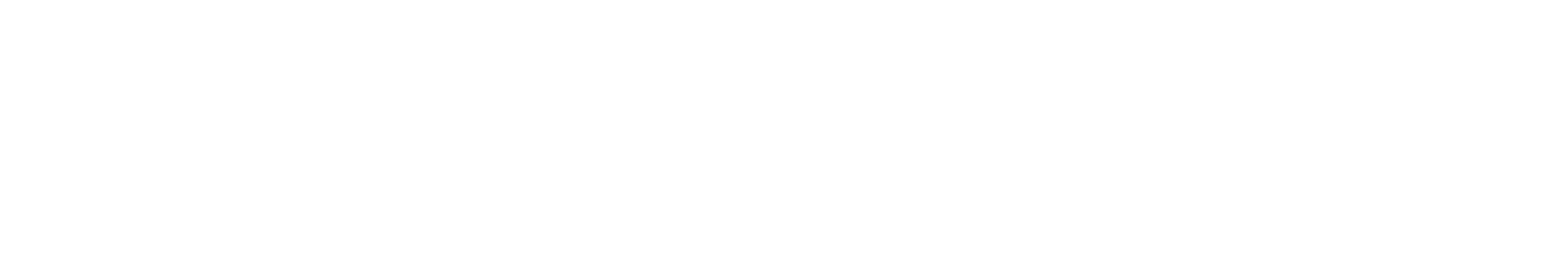

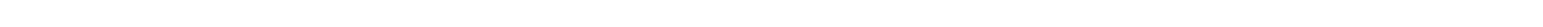

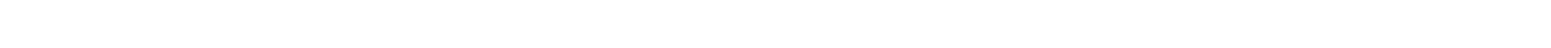

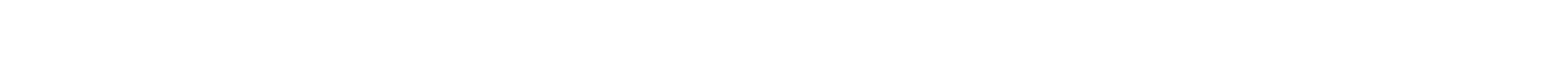

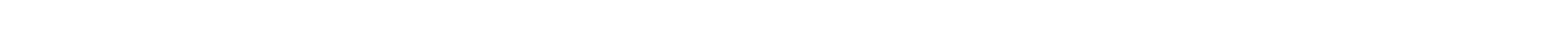

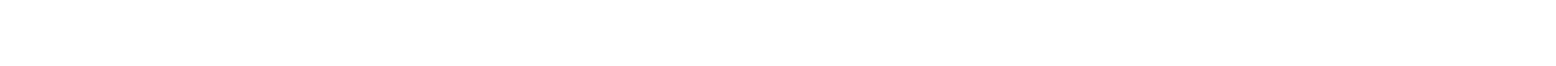

in $n$

u

$\sin$

$\stackrel{a}{t}$

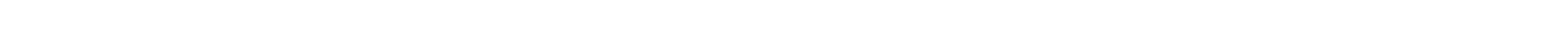
a An

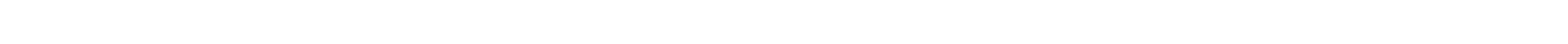
×

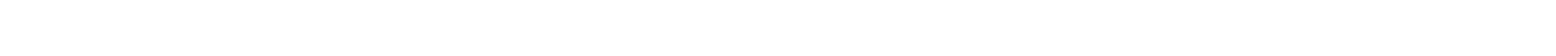

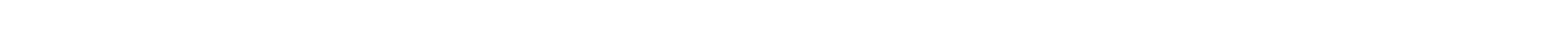

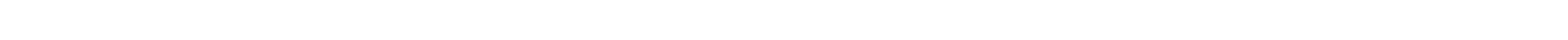

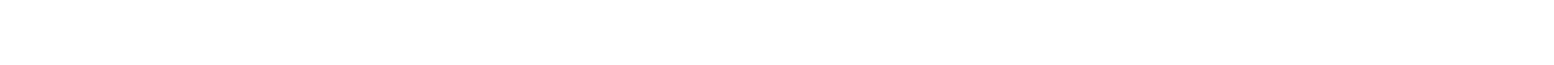




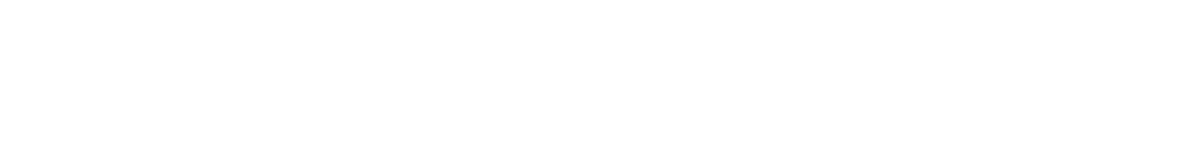

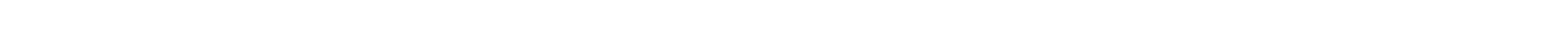
ǘó

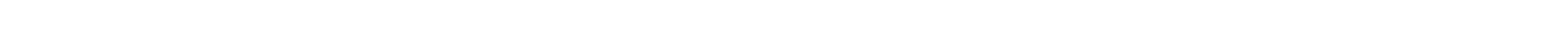

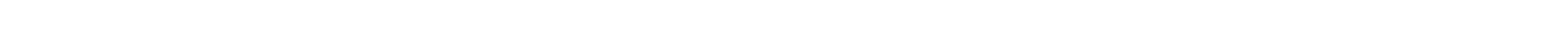

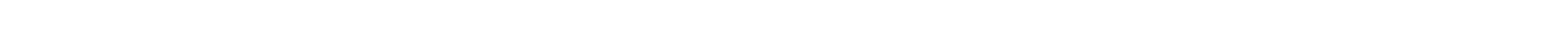

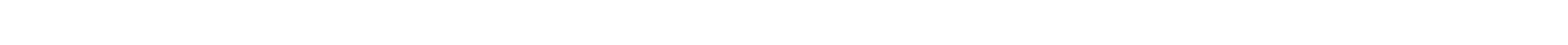

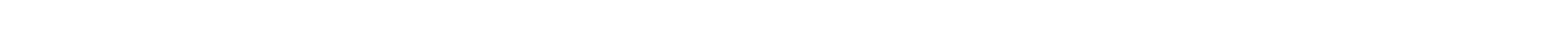

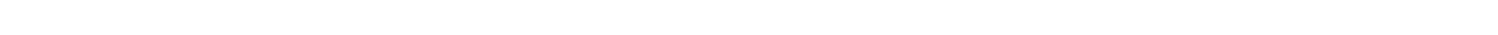

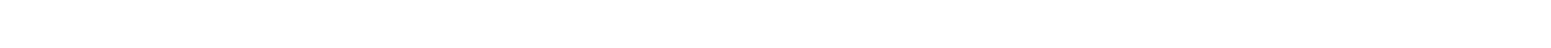

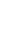

$\stackrel{w}{2}$

in

is

皮

s

s

is

E

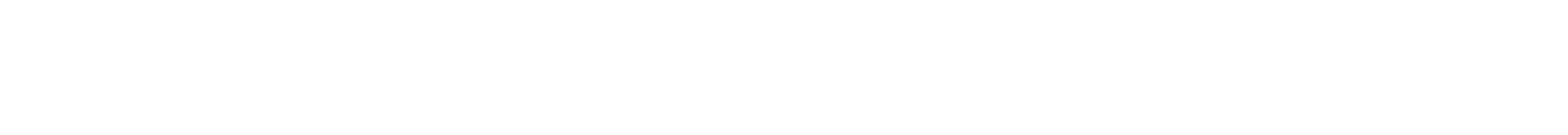

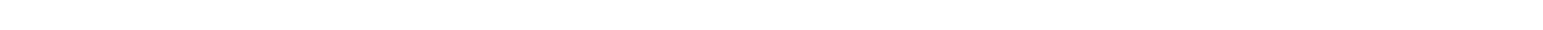

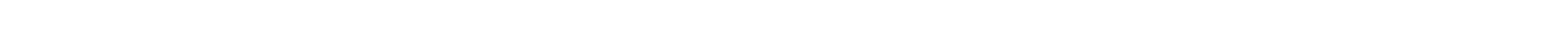

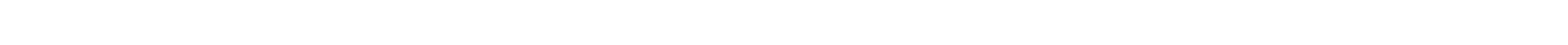

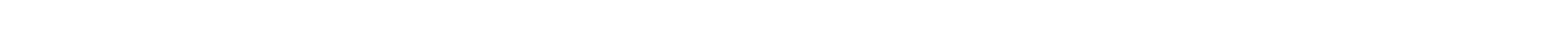

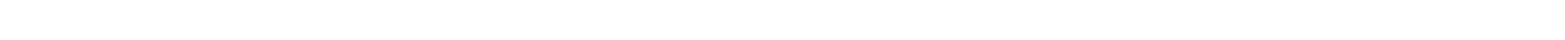

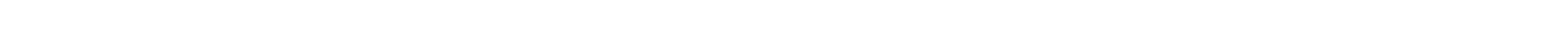

$\stackrel{w}{\mathbf{z}}$

is

is

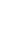

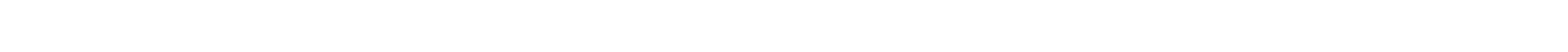
w山

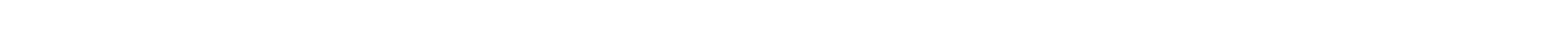

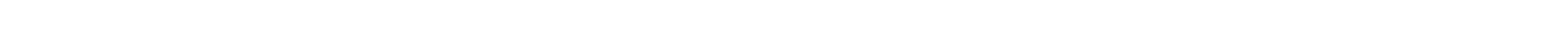

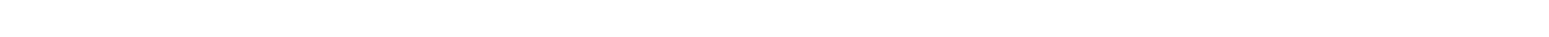
แ

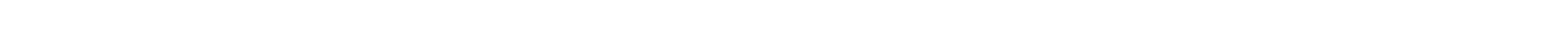

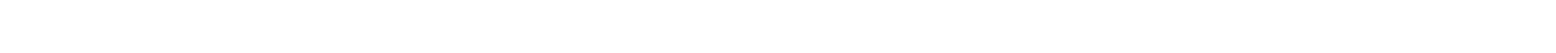

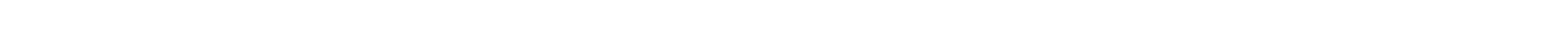
$\stackrel{5}{w}$
sn
in
in
s
n
us
$\omega$

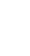

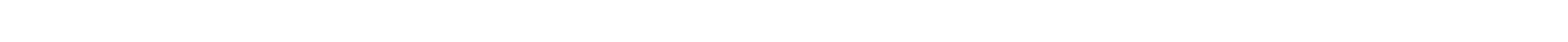

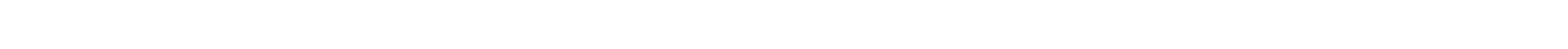

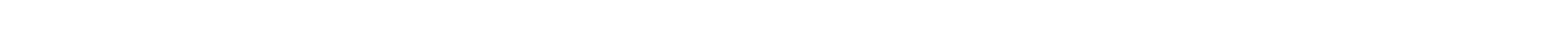

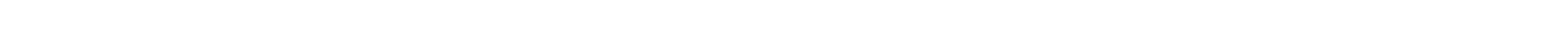

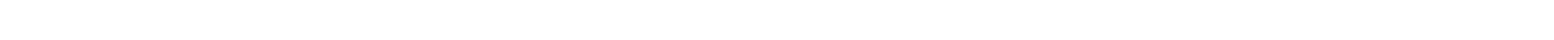

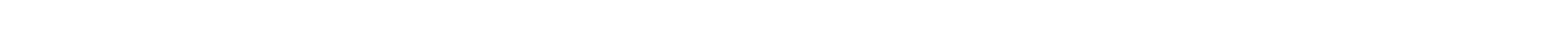

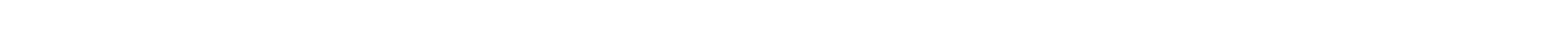

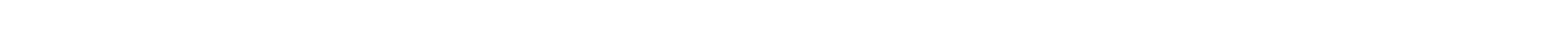




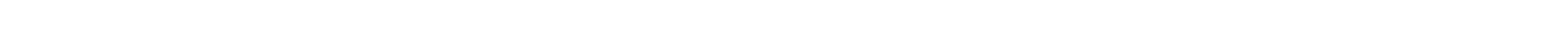
5 ผ r 3
3

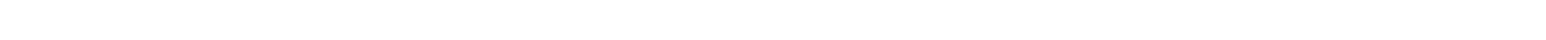

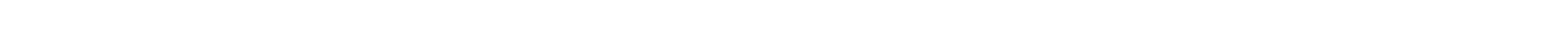

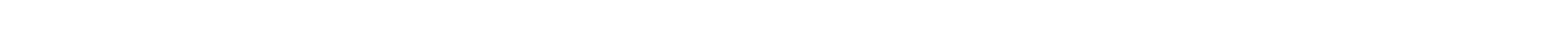

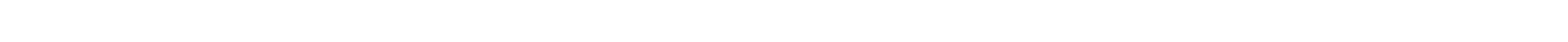

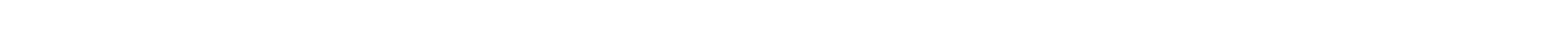

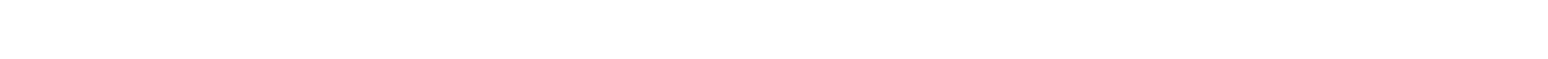

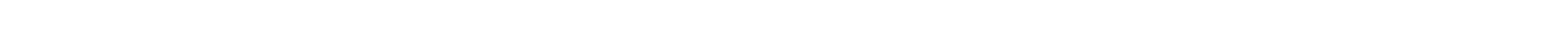

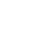

in

a แ1

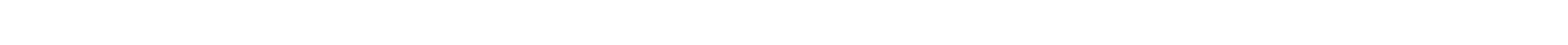

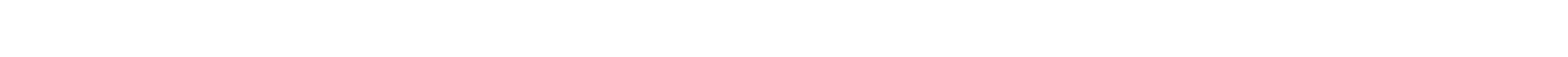

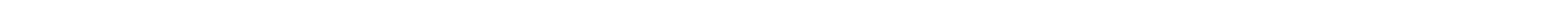

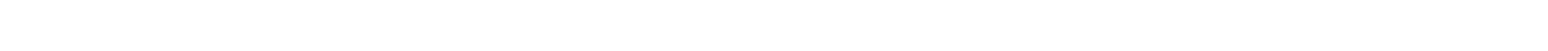

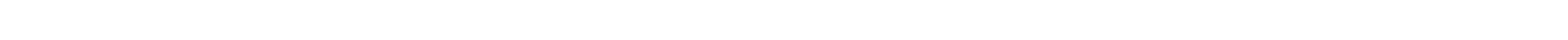

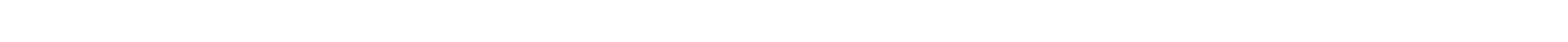

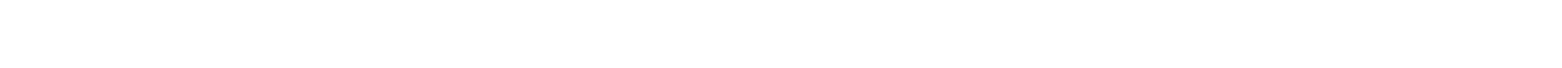

$$
\text { II }
$$$$
n
$$$$
\text { es }
$$

$\sim$

-

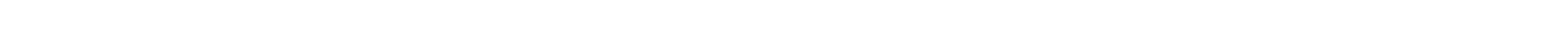

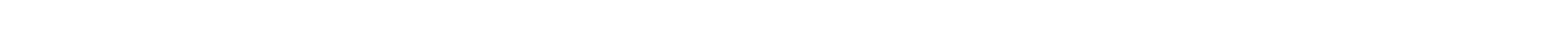

mmmmm:

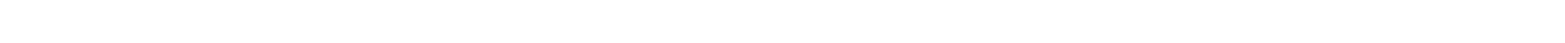

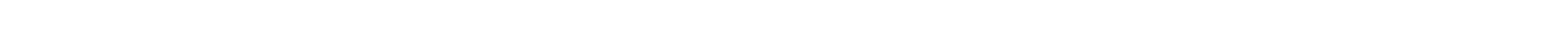

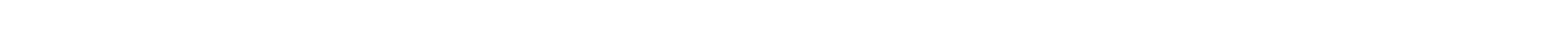

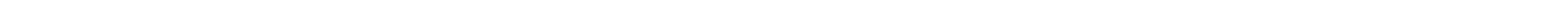

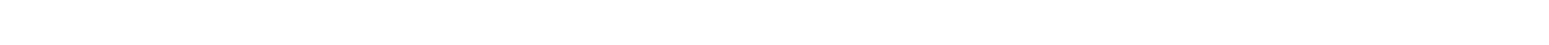

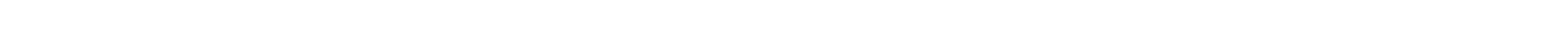

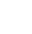

in

n "1

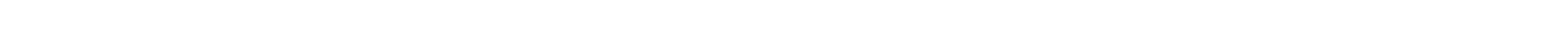

MMMMMMMMMMMMMMMMMMMMMMMMMMMMMMMMMMMMMMMMMMMMmMMMMMM

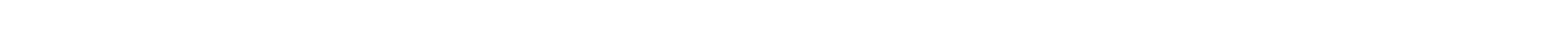
WE

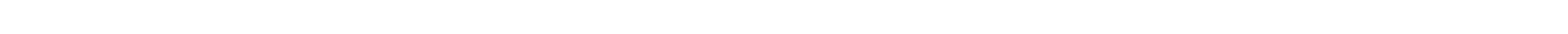

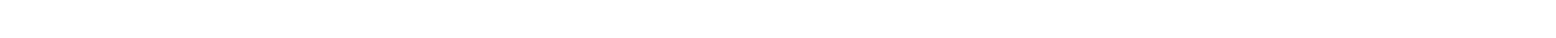

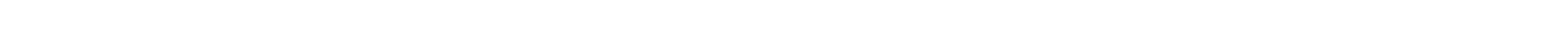




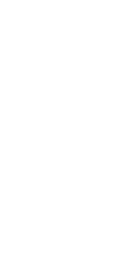

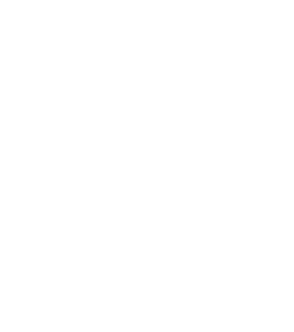

$\ddot{0}$

究

을

$\ddot{w} \dot{m} \dot{x}$

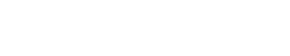

$\frac{5}{3}$

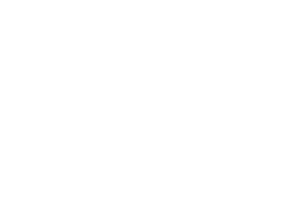

$\cong .$.

焉

㸃 n n

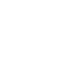

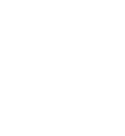

$m$

突

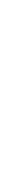

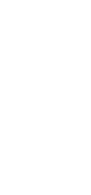

心

$\stackrel{5}{2}$

2

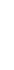

$\therefore$ en

c a uto

a

a

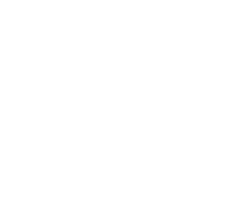



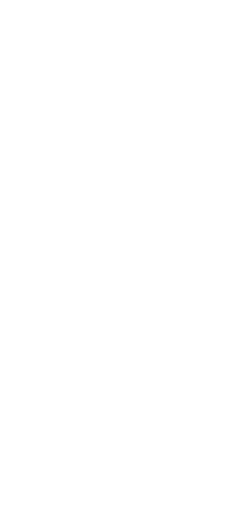

twesesosos is

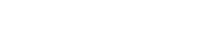

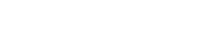
$t>a m m i s$

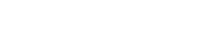

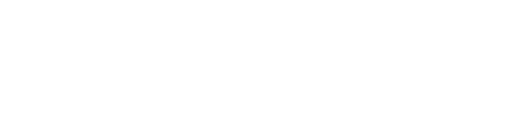
I I 㟧岌 twonenes

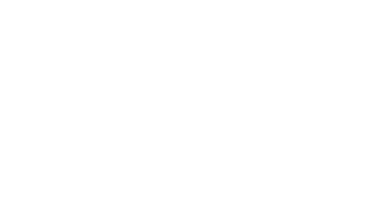

ii) üi mwon a

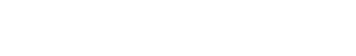

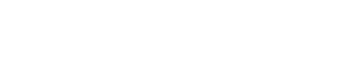
¿ $\tilde{u}$

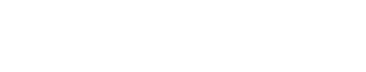

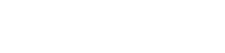

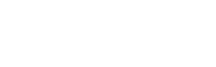
몽 a 2 윤응

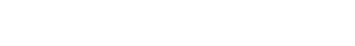
$\ddot{w} \ddot{\omega} \sim$ a $\sim 20 m \tilde{a}$ 5正造造向

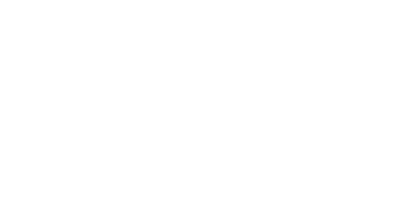




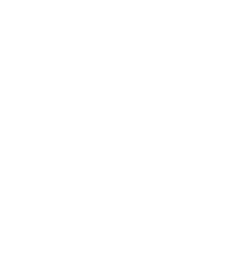

tusenesen

is

in $\sim 0 \sim m$

w山.

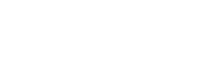

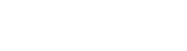

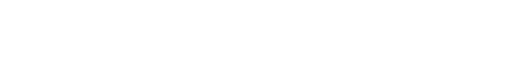

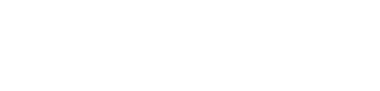

Lesesenos

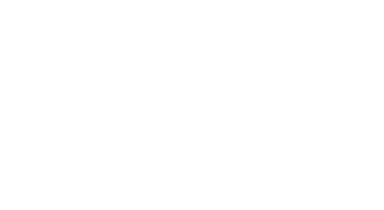

ï $\ddot{0}$ ininn

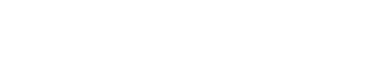
せミこミミミ

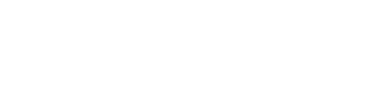

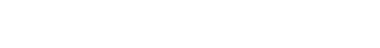
$\therefore \therefore=1$ 才u的的 知然

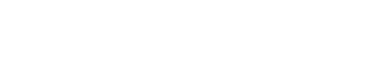
coct

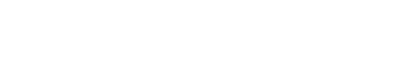
영요영

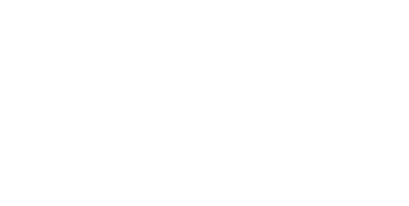



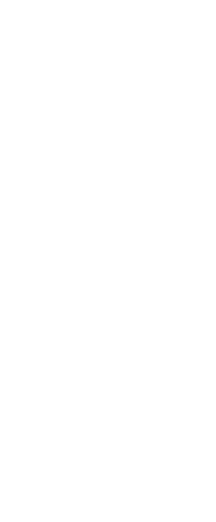

mencosen

5

in 0 a $\therefore-10000$

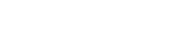

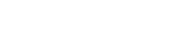

$\therefore \quad$ aूकव

-

붕

Túneses

is

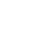

- 담요

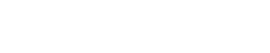

-

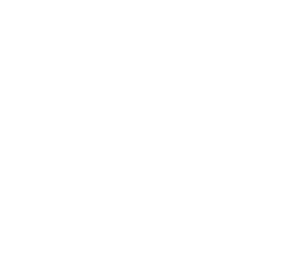

coz tosenesos

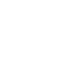

\pm E

$=0$ 므느ำ

-

范-

웡

눌

w- $-\frac{2}{2}$

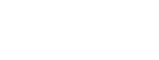

シェ்̃̃ 

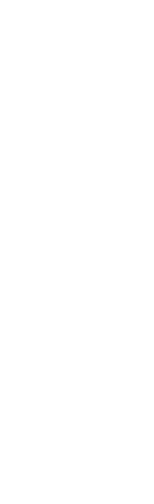

țisen

is

añ

a

出的的的

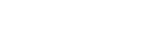

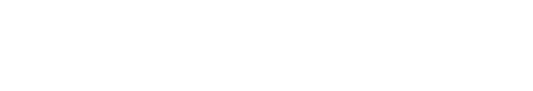

i $\quad$ 는

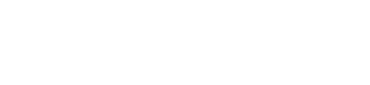

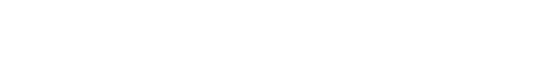

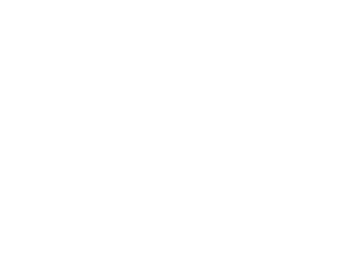

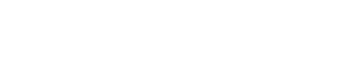

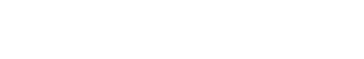

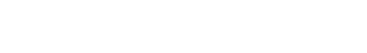

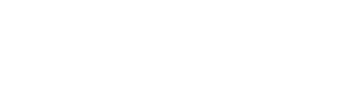

เ $\quad$ a a

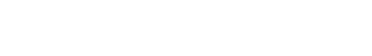

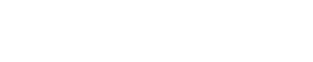

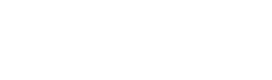

$\stackrel{a}{a}$

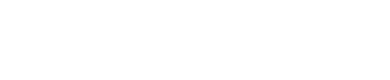

㟧

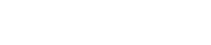

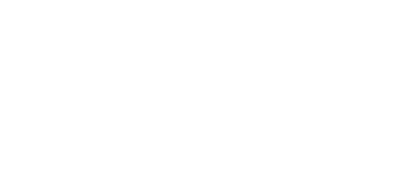




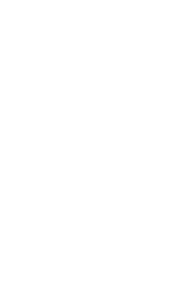

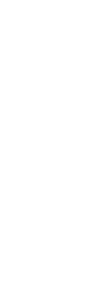

点

뜽

z

곤

气

돈

茖

E

空

密

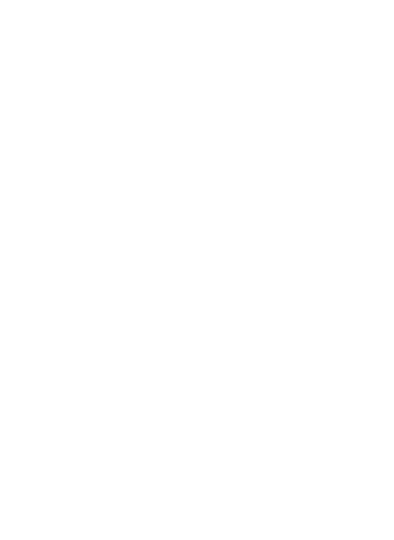

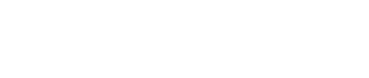

wละ์

..

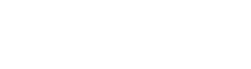

岸

늘

5

a

- Lvivin

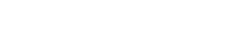

蕰

우

यू

品

舟

\pm 0 品

a a

แ w?

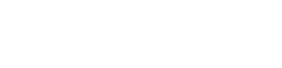

远宫

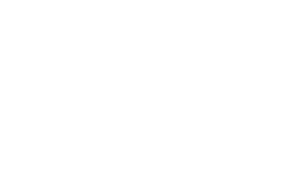



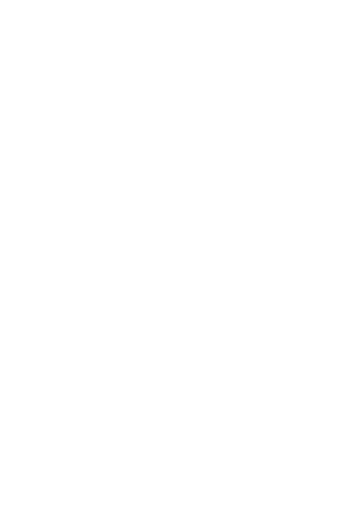

Tyescososencosos

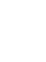

mmo ํㅜㅇํำ u

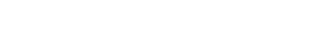

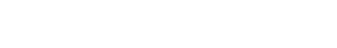

$\sim \sim \sim \sim \sim \sim N N M$

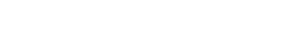
u-

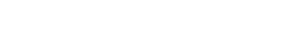

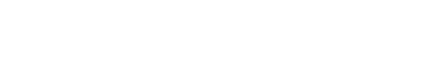

tusencosencosenes

is

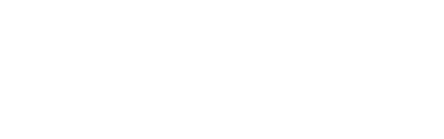

i. $\quad$ and munn

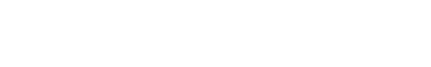

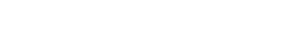

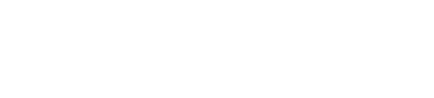

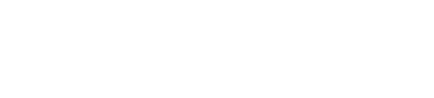

w

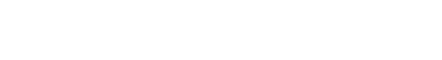
$2 x$

in :

in

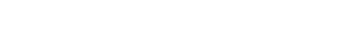

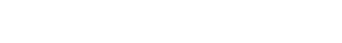

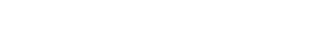

3 a

$\geq 0$

이웅 แ $20 \div \div \div \div \div \div \div$

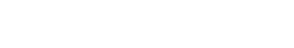
อ-c $\sim \sim \sim \sim \sim ⿻ \sim$

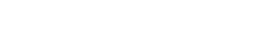



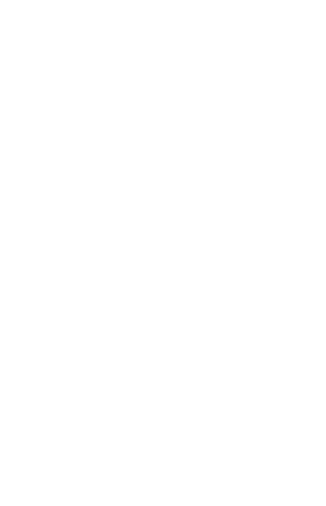

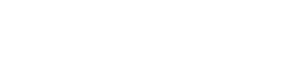
a

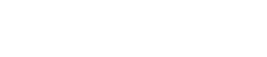
แ x

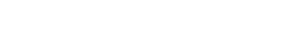

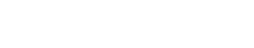

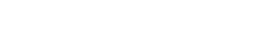
-

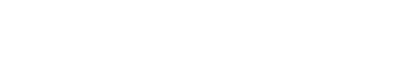

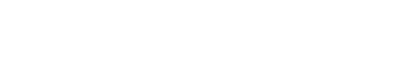
$\underline{m}$

tuscosesencos is

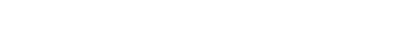

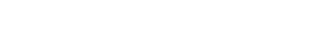

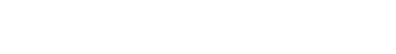

ii $\quad--n n N$ ตू⿻ แำำำำ

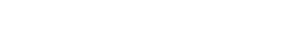

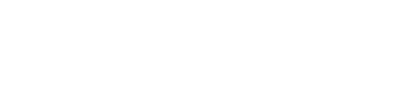

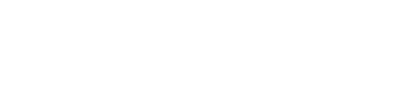

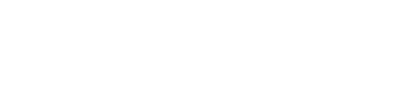
士5 ह

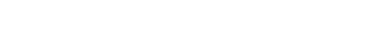

=а w .

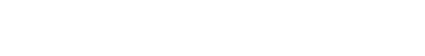
:용

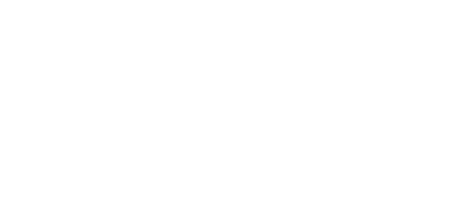




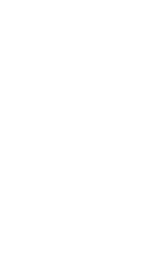

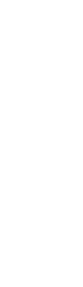

些

委点

ดูำ

ำ

twann

E

ㄸํㅇำ

$\rightarrow \infty n$

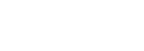

r $\sim \pi N$

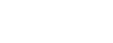

要

密

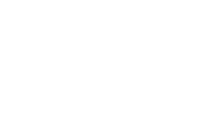

乩充

㟧

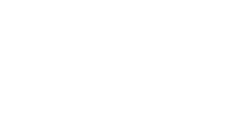

崩

tusen

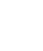

- a $a+n$

w

-

$\underset{5}{5}$

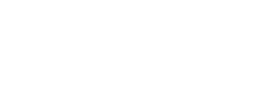

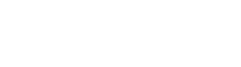

는

z $\quad$ o. 2 tones

z

离

u

u.

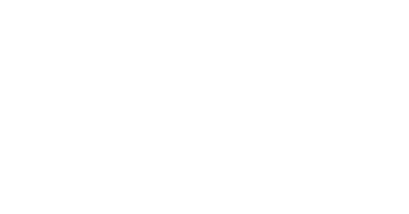



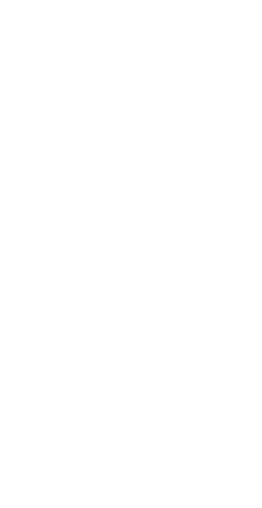

toncsesenes

is

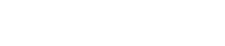
แ山แ? DMmM̃Na

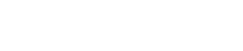

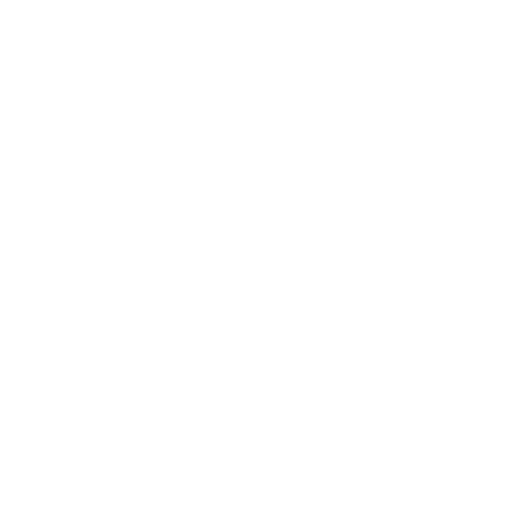

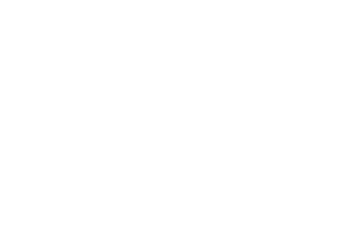

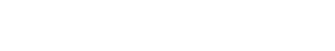

$2 x \quad \sum$

=

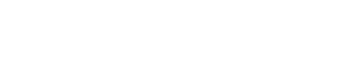

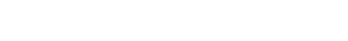

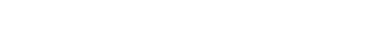

ज造这

룰

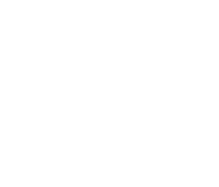




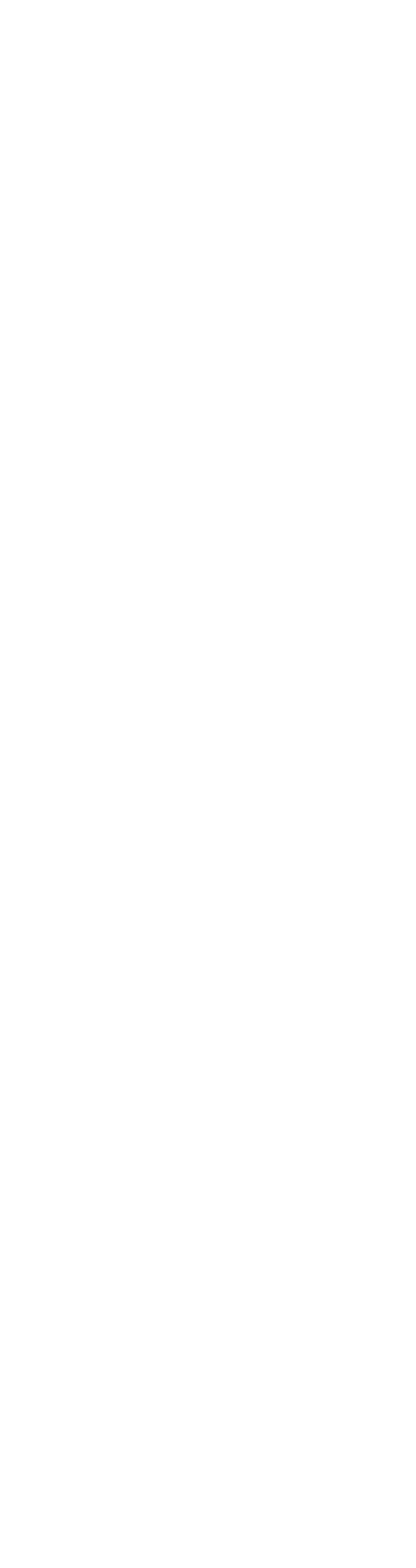

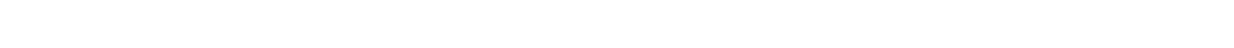

؛

운

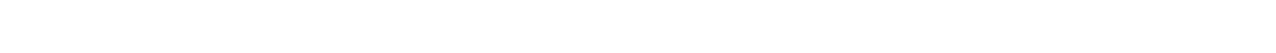
5

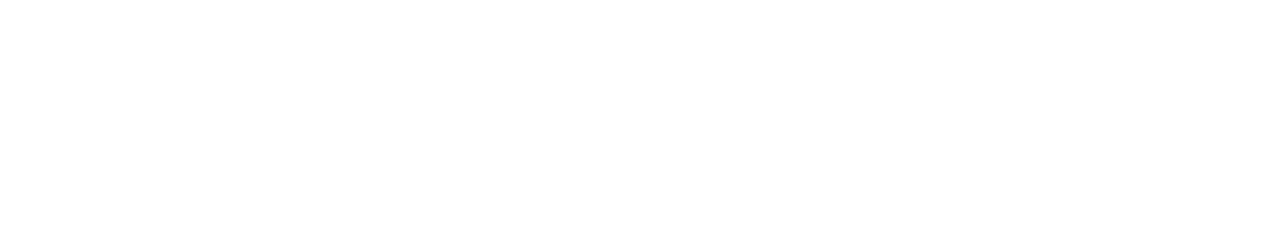

$$
\begin{aligned}
& \text { w }
\end{aligned}
$$

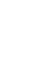

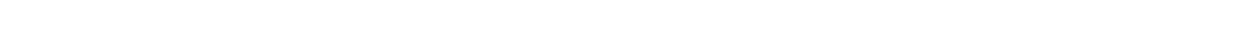
w w 곤

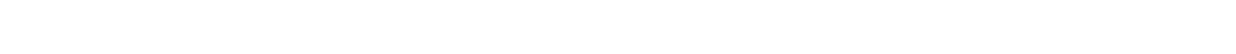

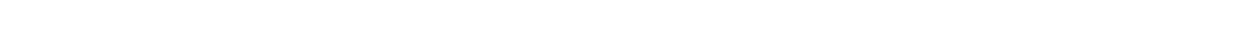

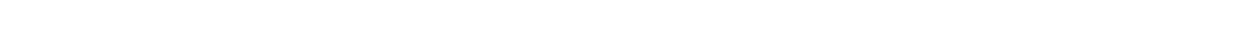

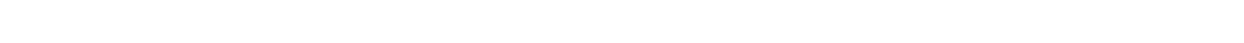

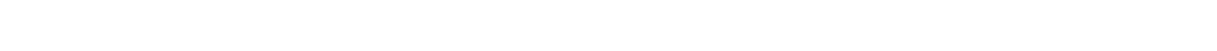

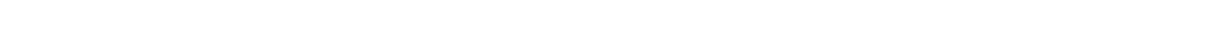

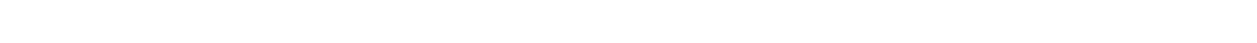
is

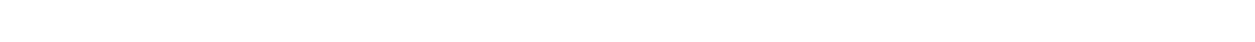
แ

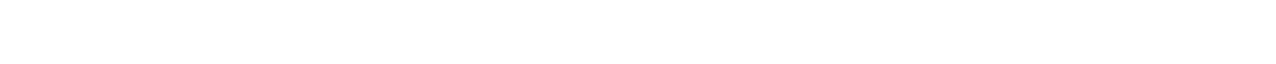

-F-F-F-F- - NNN "ñ

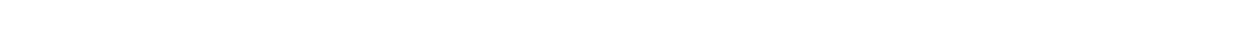

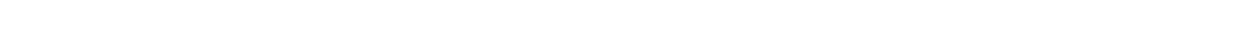

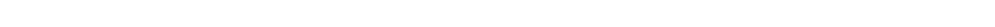

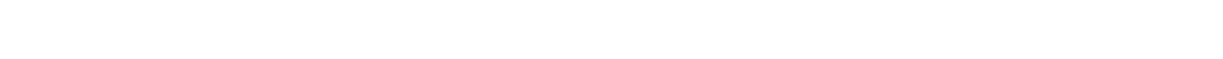

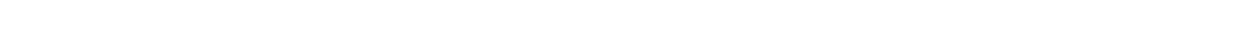

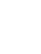

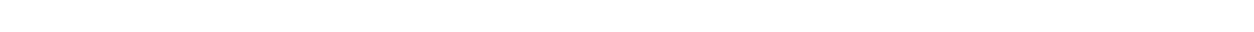
ǘ 3

$0000000000000000-\bar{n}$ -

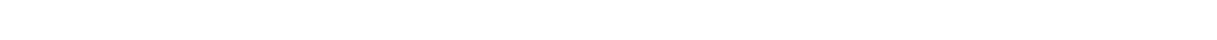

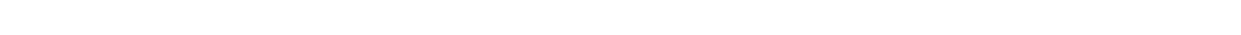

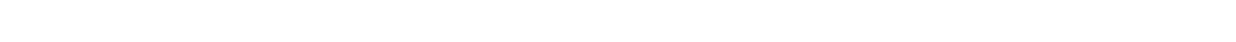
O=N

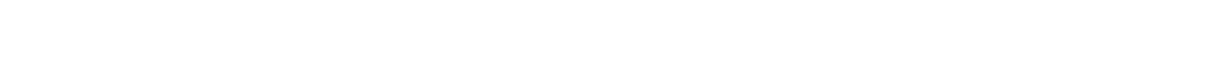




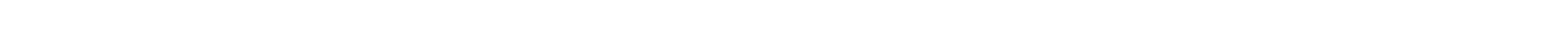

$\stackrel{a}{\infty}$

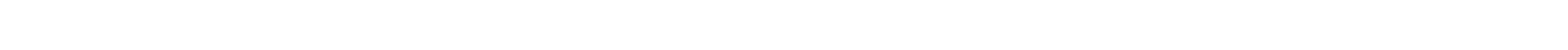

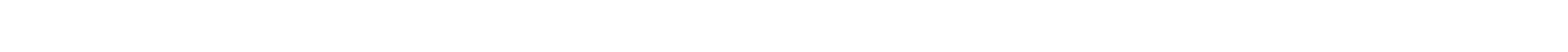
\begin{tabular}{ll}
2 \\
\hline
\end{tabular}

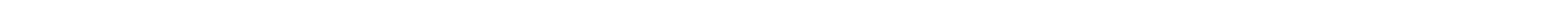
Nâñ

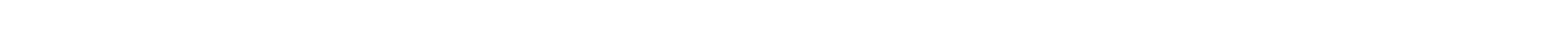

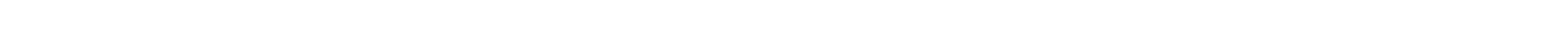

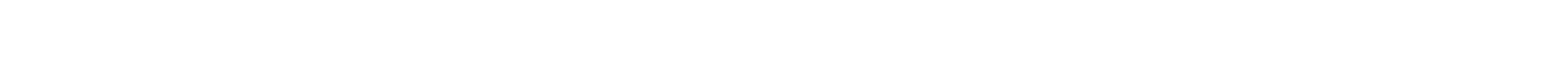

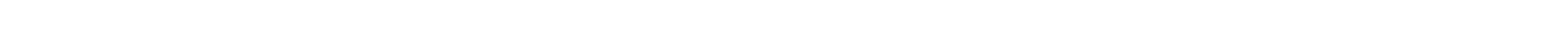
a

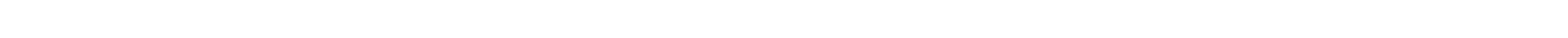

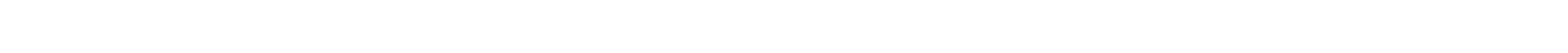
3
0

수의

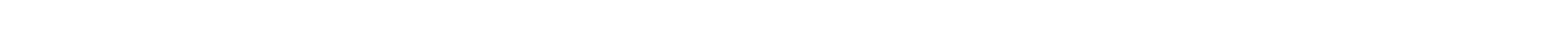

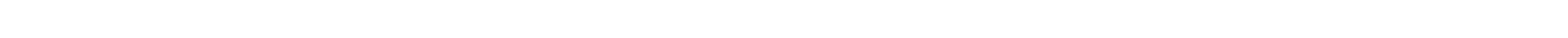

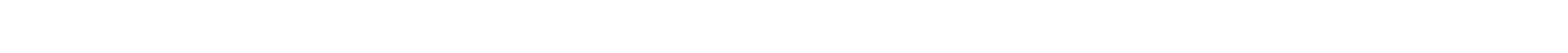

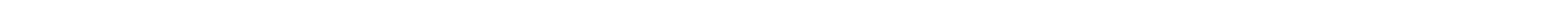

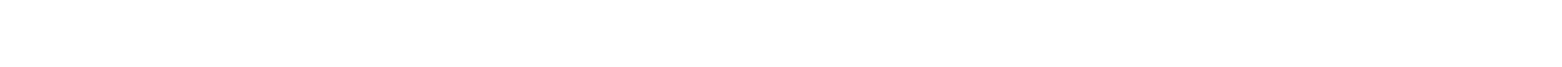

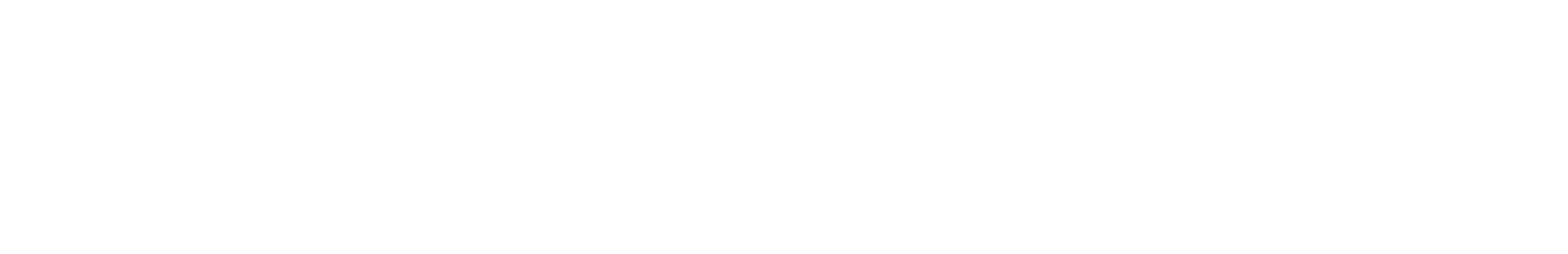

ดำ

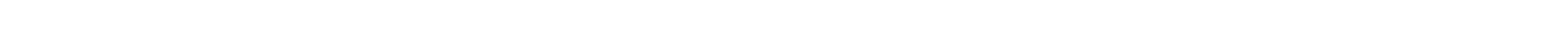

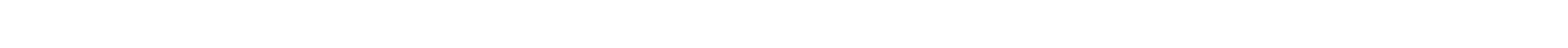

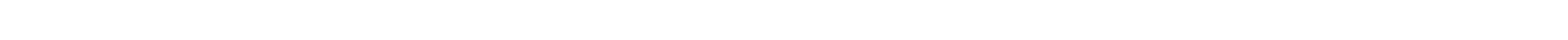

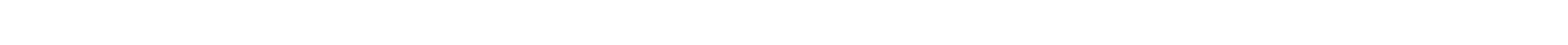

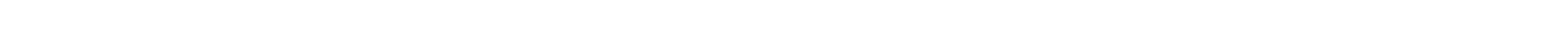

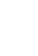

is

$$
\text { sis }
$$

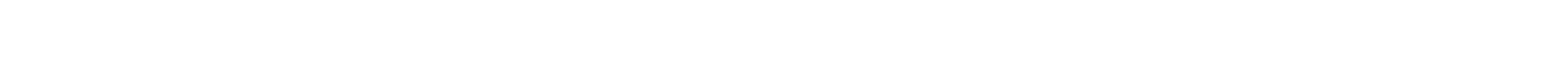

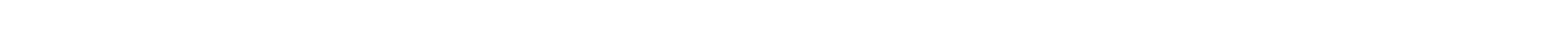

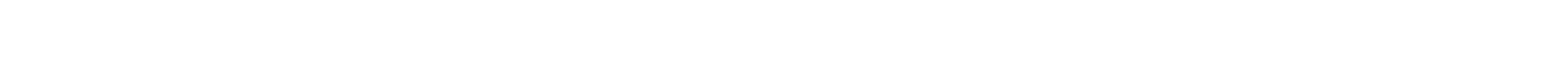

meniningmin

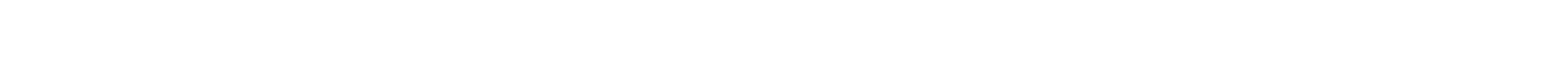
แ

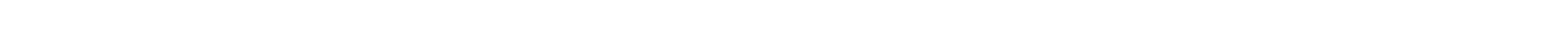

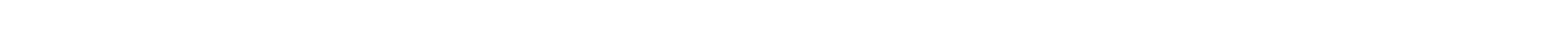

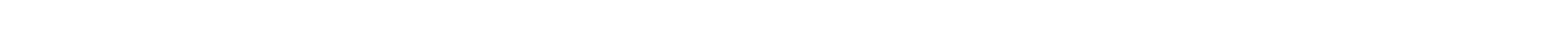



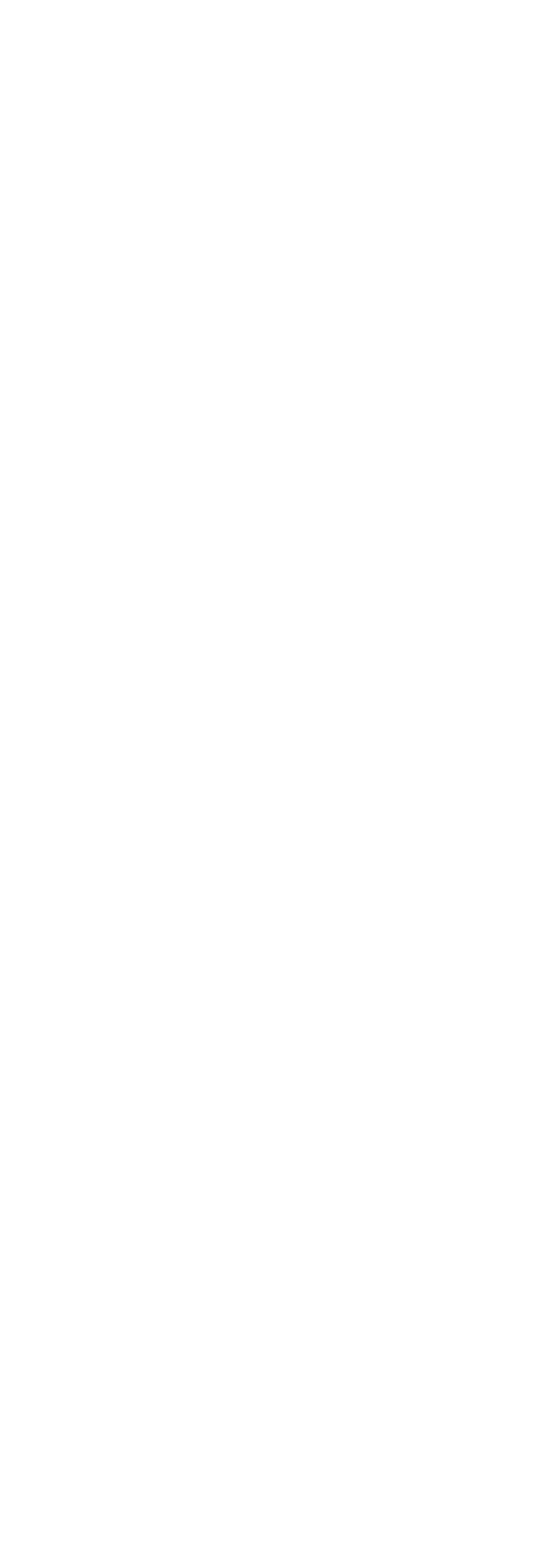

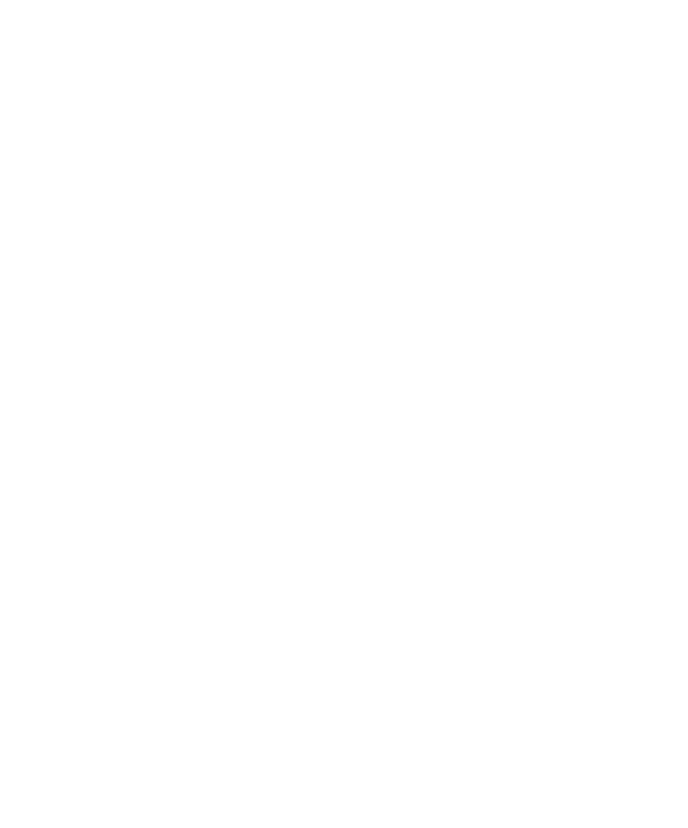

$z$

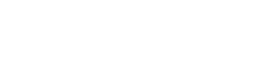

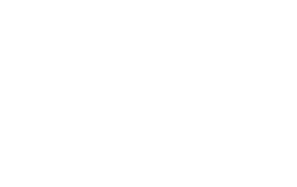

$\sim \sim \sim \sim \sim \sim \sim \sim \sim \sim 乛 N$ 잉

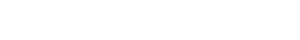

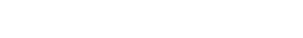

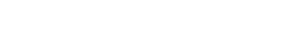

世的里 on

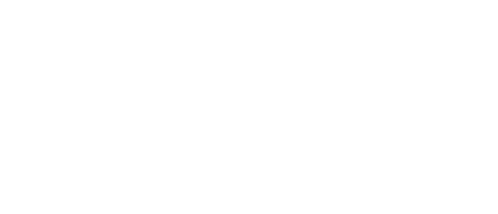

is

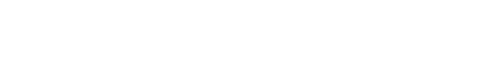

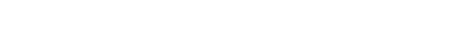

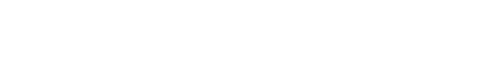

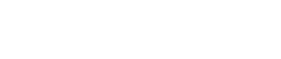

it 4 s

पอ

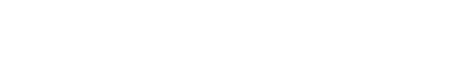

$2 \quad 0151$

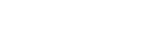

응

응요

is

x

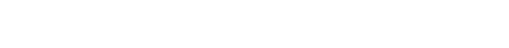

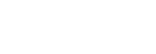

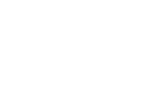

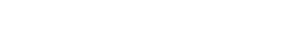

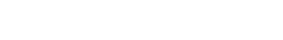

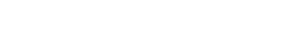

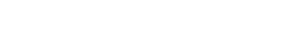




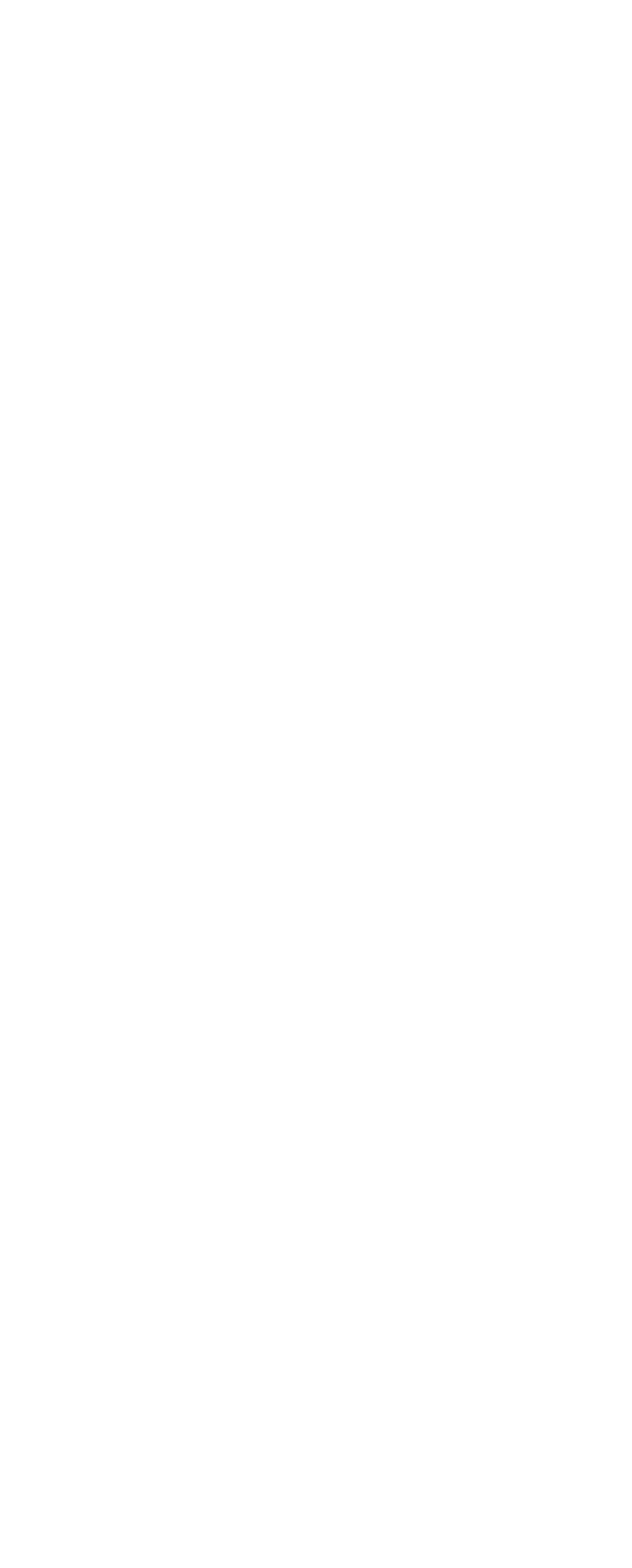

- -

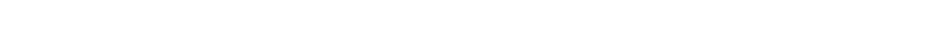

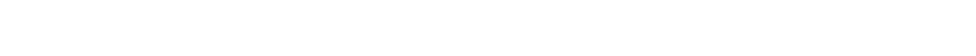

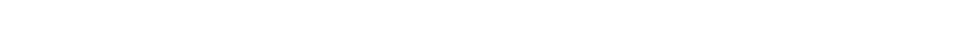

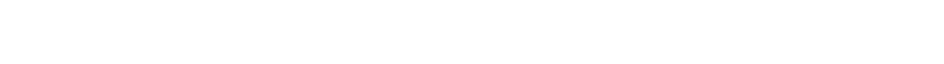

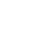

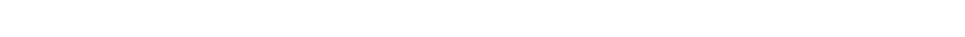

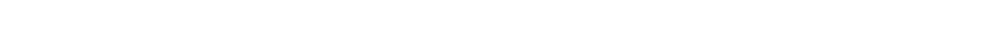

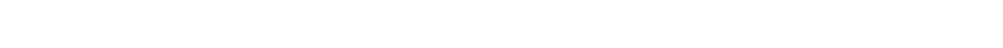

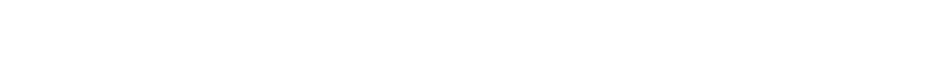

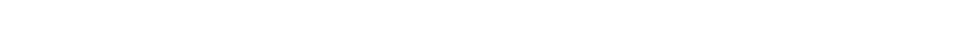

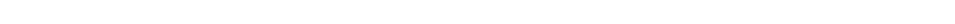

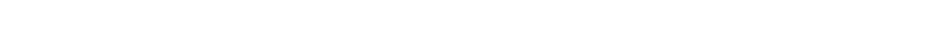

w en en en in os on on

$\stackrel{4}{\leftarrow}$

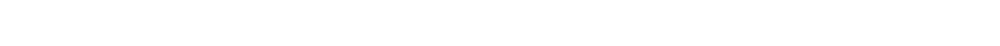
" بر

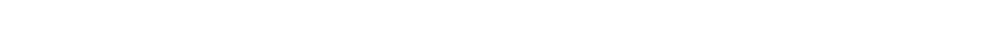

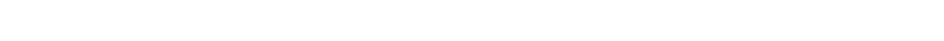

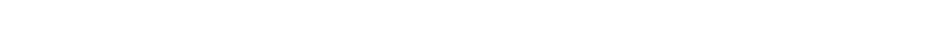

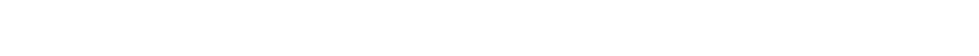

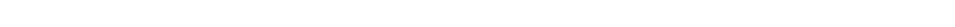

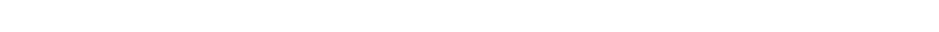

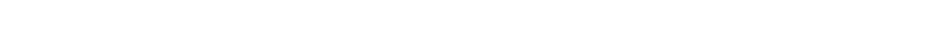

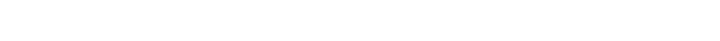

en en

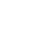

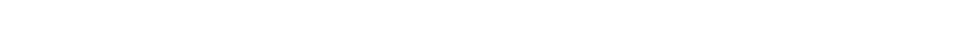
a - >а

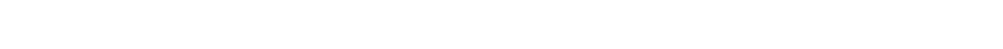

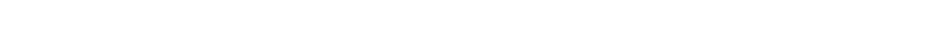

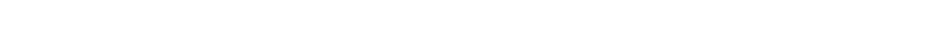

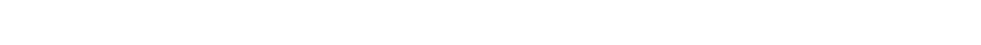

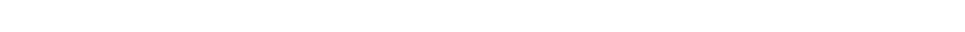

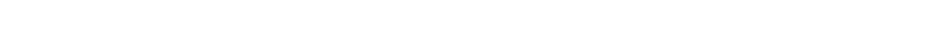

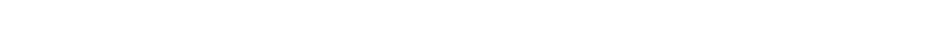




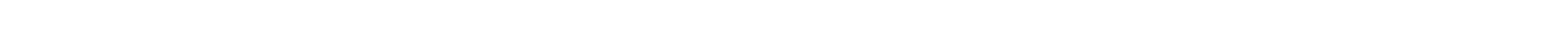

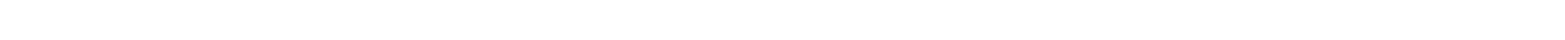

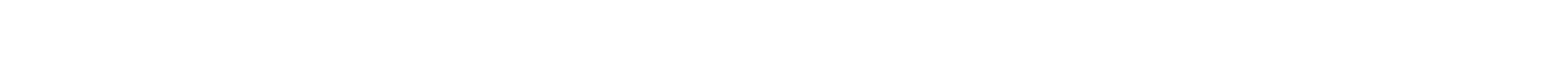

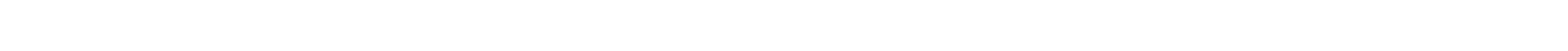

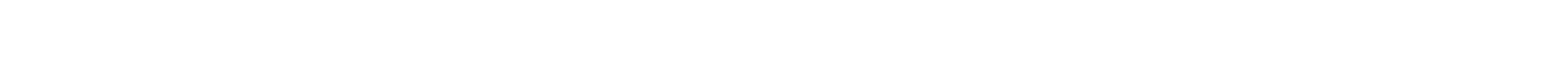

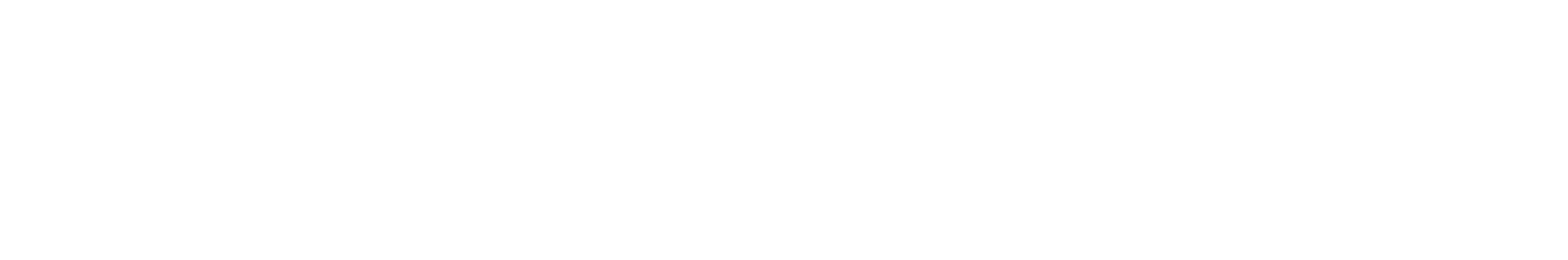

mmMmmmmmmmmmmmmmmmmmmmmmmmmmmmmmmg

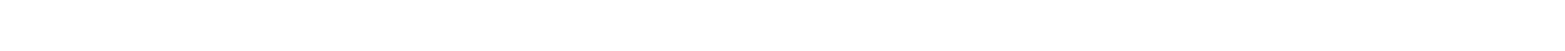
w-5 -

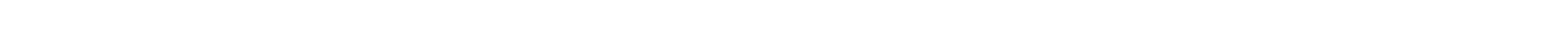

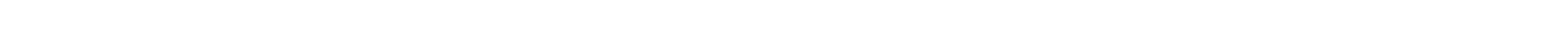

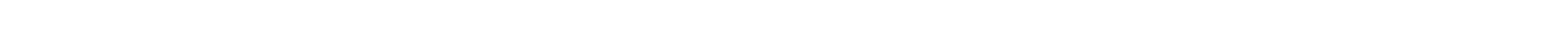

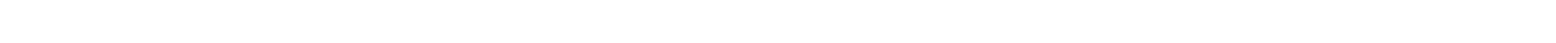

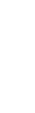

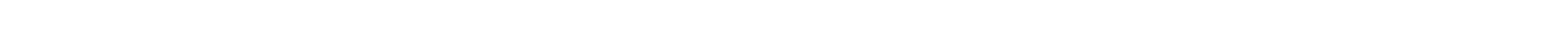

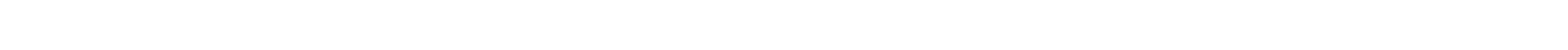

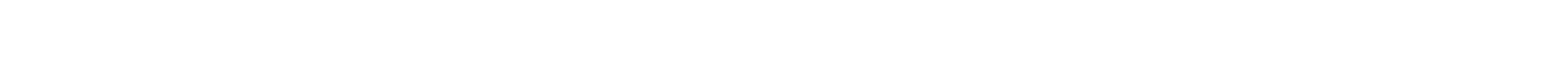

mmmmmmmmmmmm

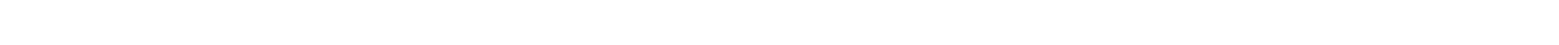

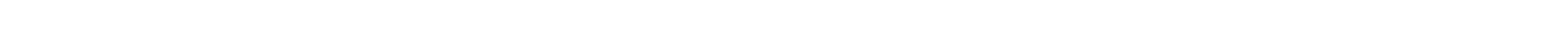

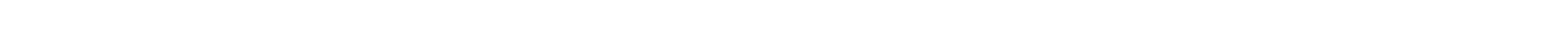

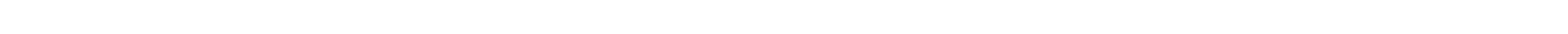

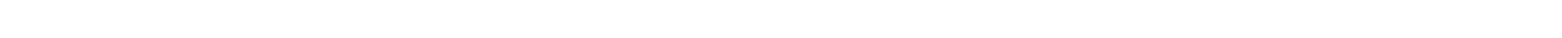

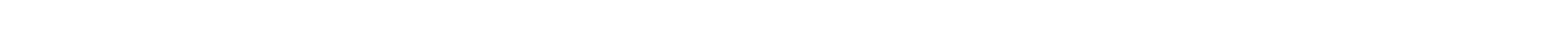
in
$\cos \cos n$
is
s
in in in
in on
in
皮
$\stackrel{a}{\llcorner}$
x- 世山

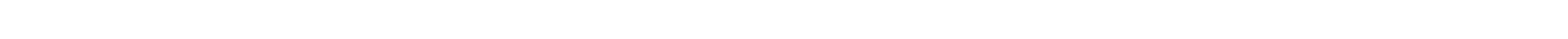

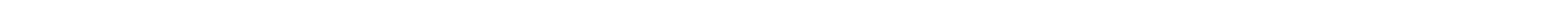
й แ

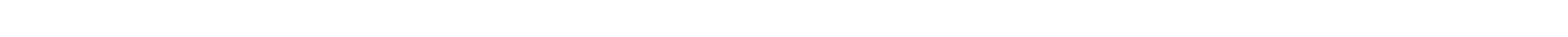

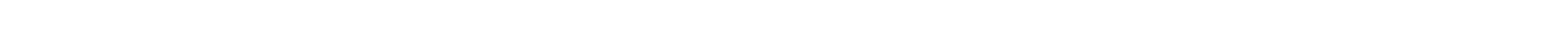

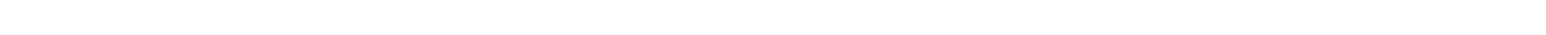




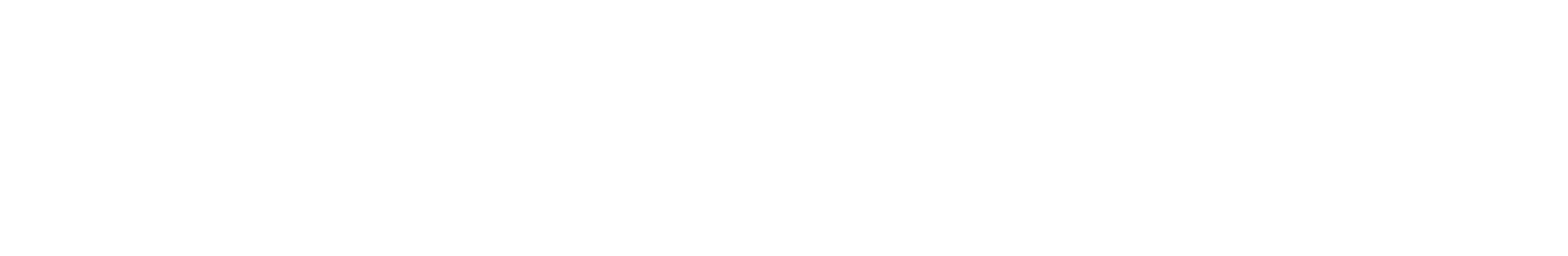

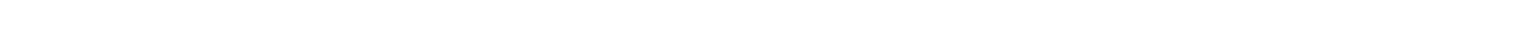

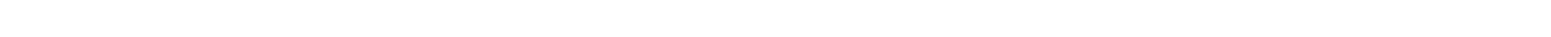
แ

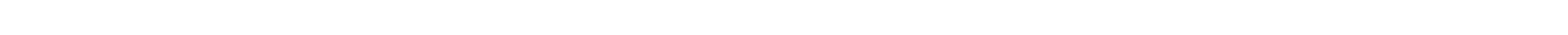

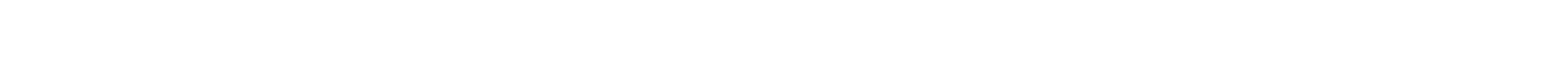

$$
\text { 垔 }
$$

$\underline{\mathbf{z}}$

a

"-

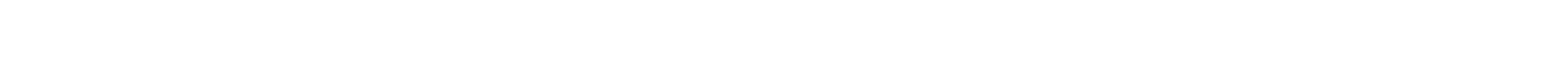
\begin{aligned} 3 \\ \hline\end{aligned}

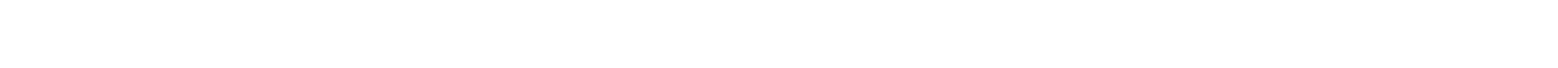

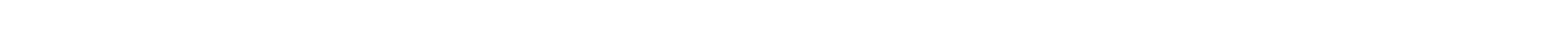

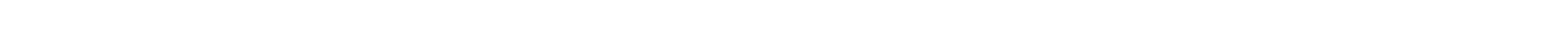

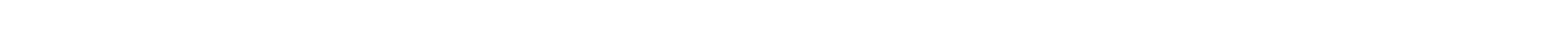

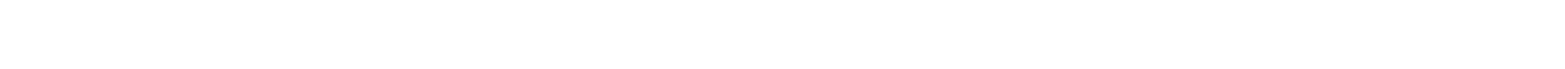

in

n

in

is

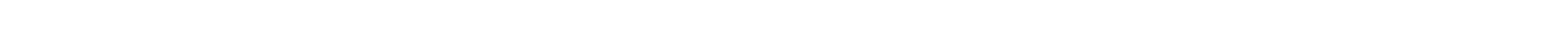

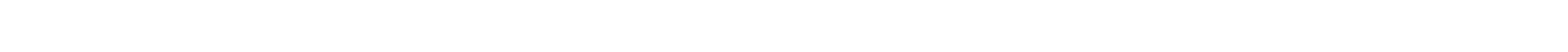
I

mиn

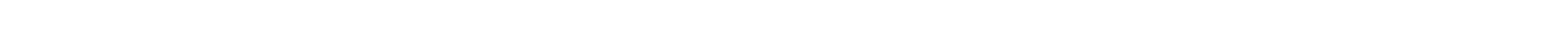

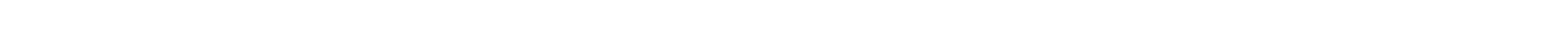
世

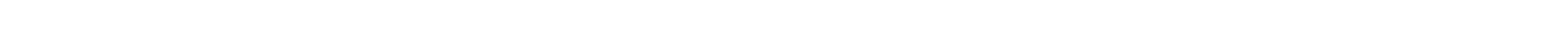

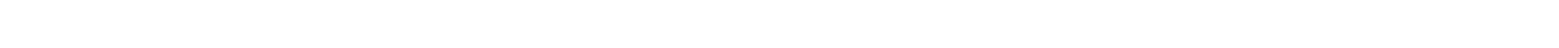

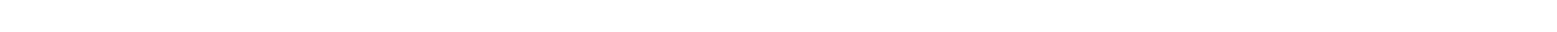

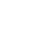

is

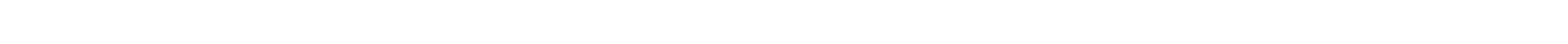
ш

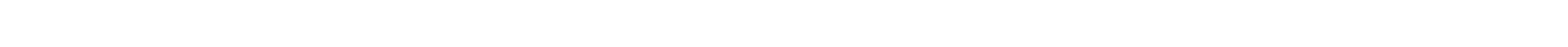

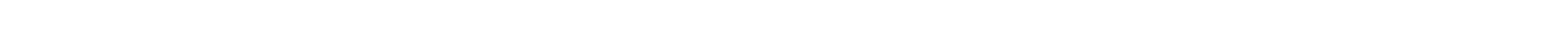
แ

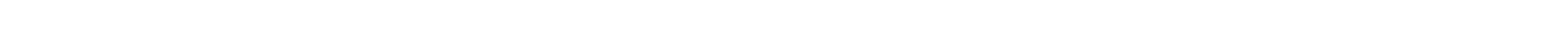

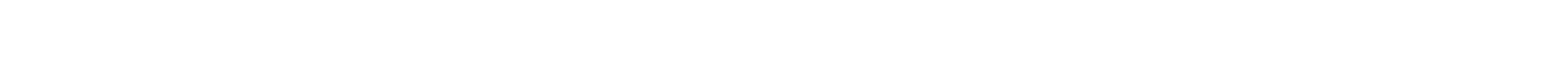




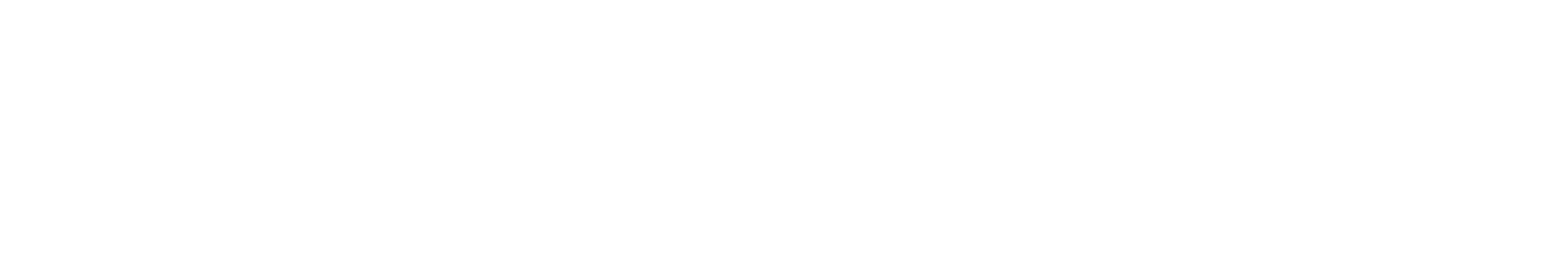

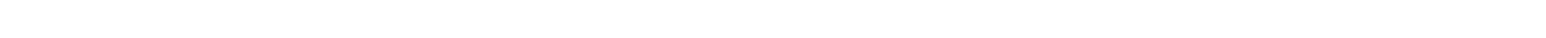

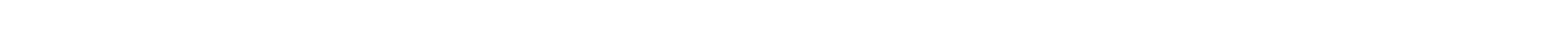

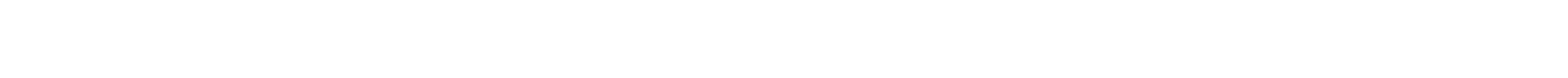

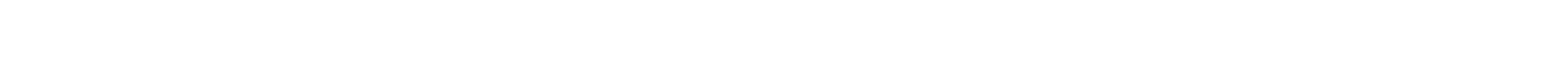

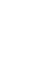
is
$\infty$
is
in
es
is

is

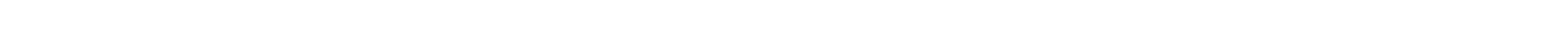
"

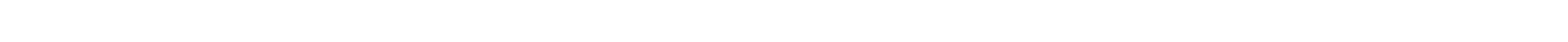

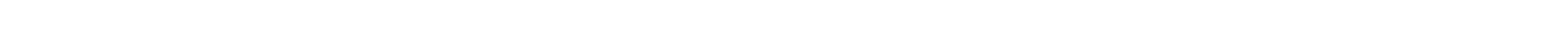

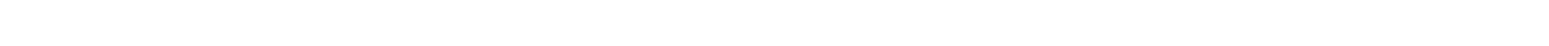

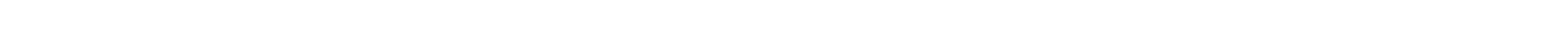

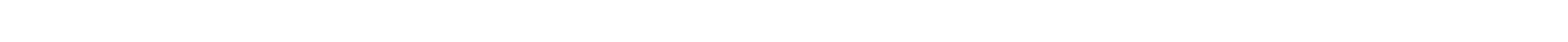

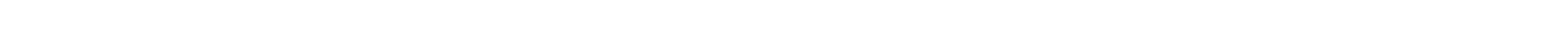

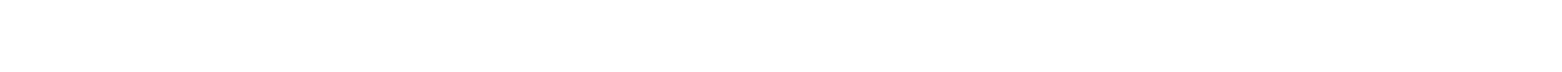

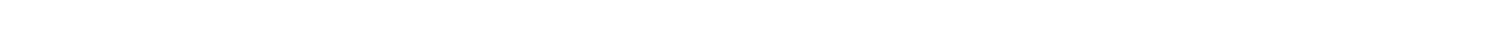

is

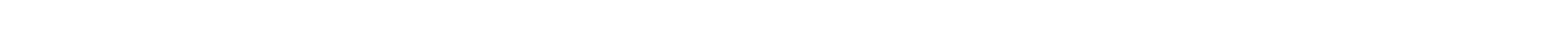
un

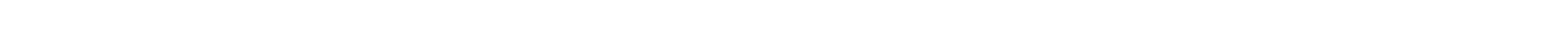

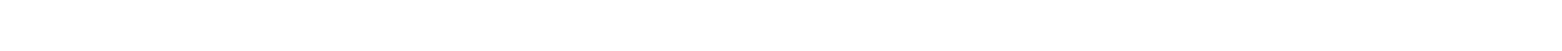

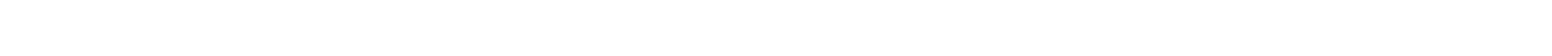
LEF

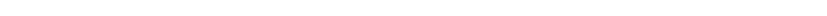

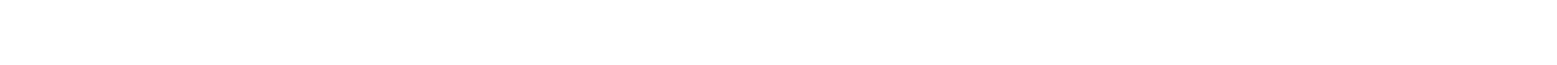

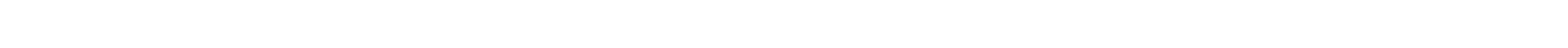

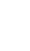

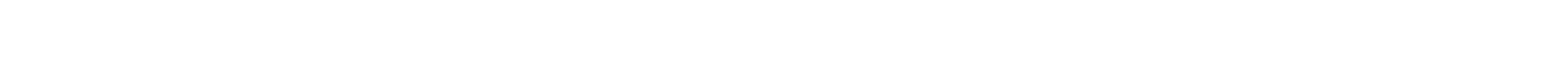

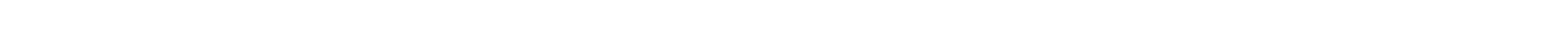

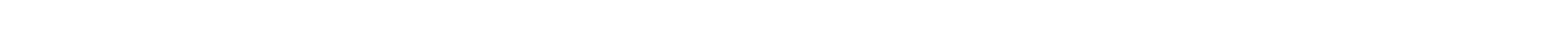

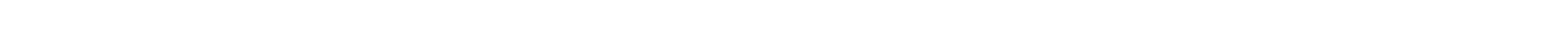

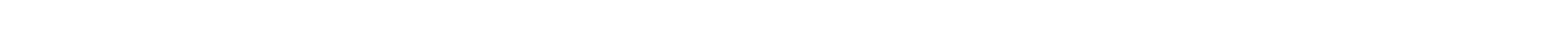
w

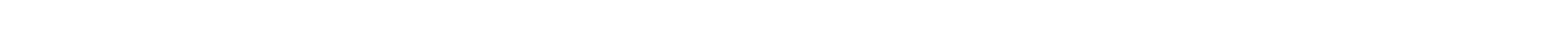

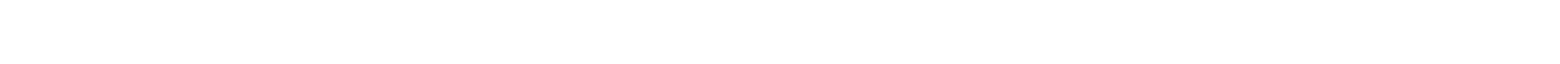




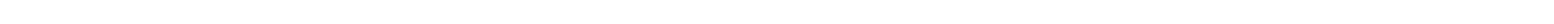

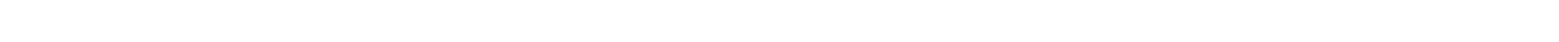

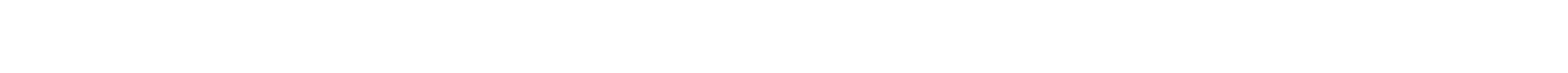

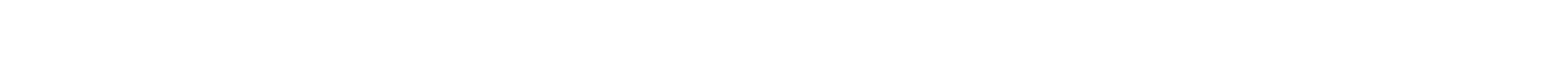

亲 $n$

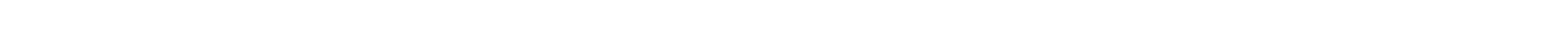
Hu

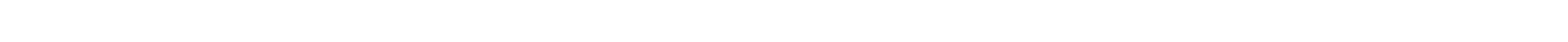
З

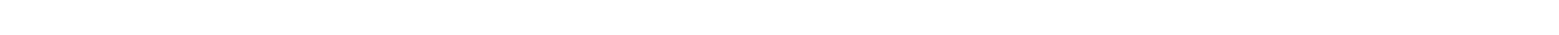

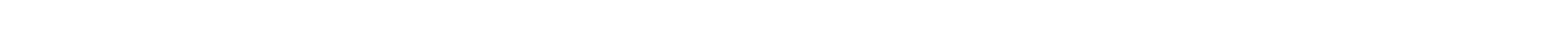
แ

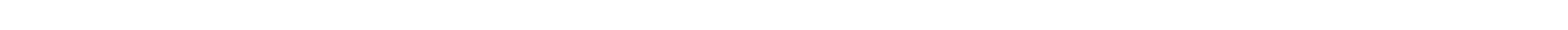

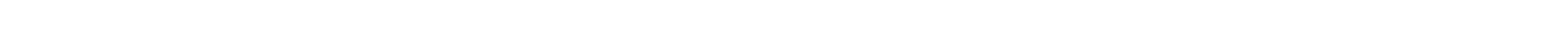

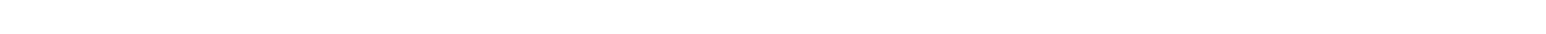

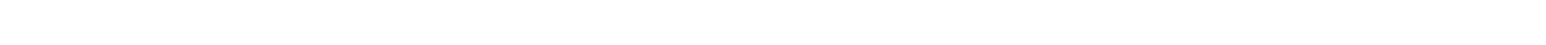
w n
s
n
n
n
in es
\&

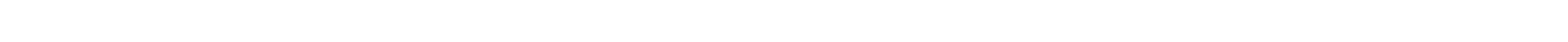

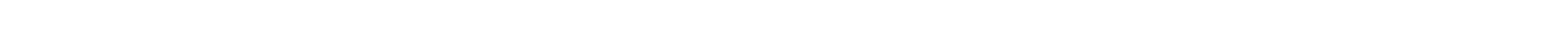

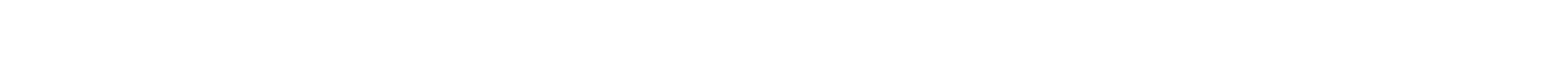

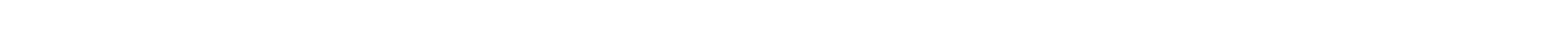

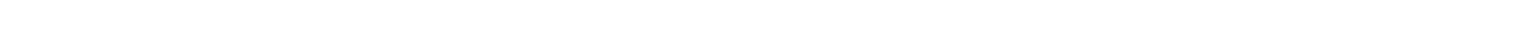

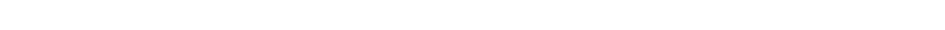

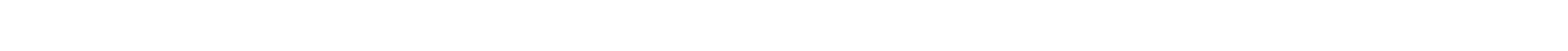

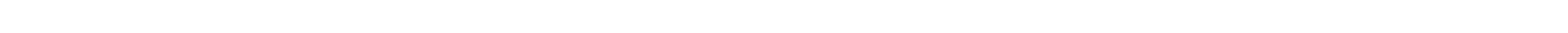

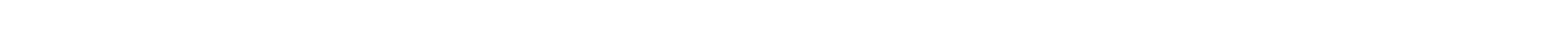

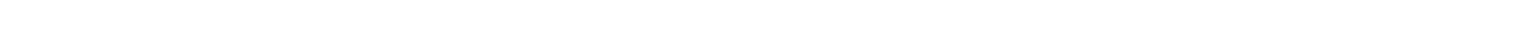

5
is
n
n
↔
皮
es

in

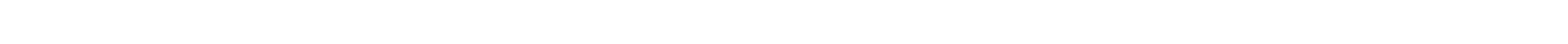

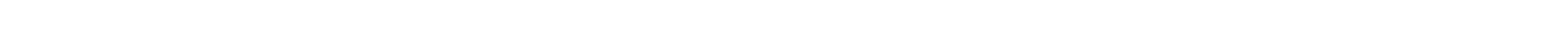

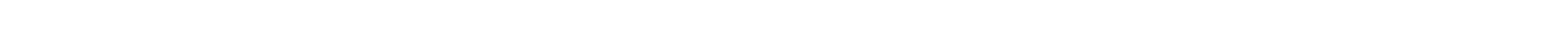

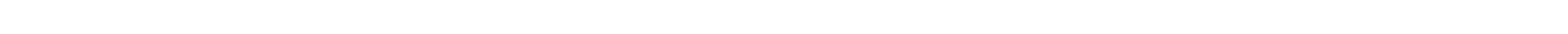

ג

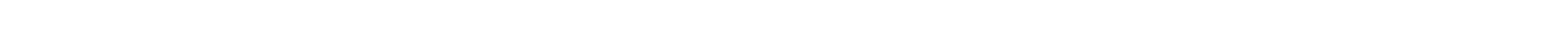

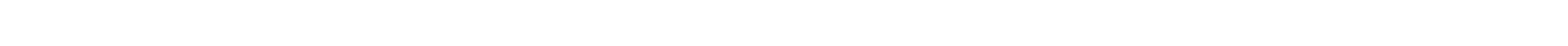

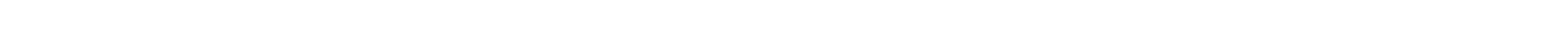

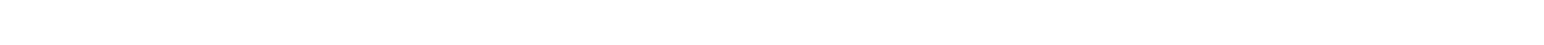




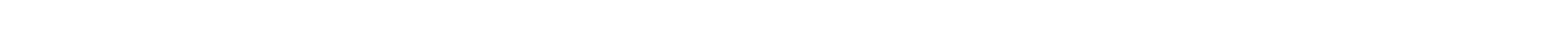

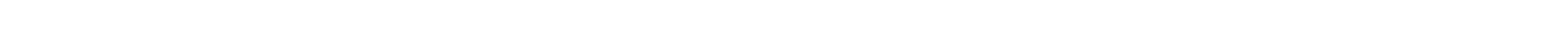

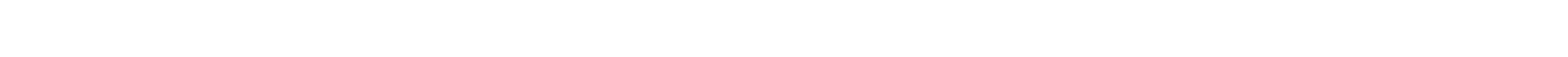

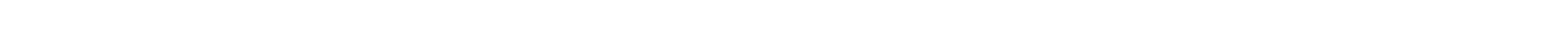

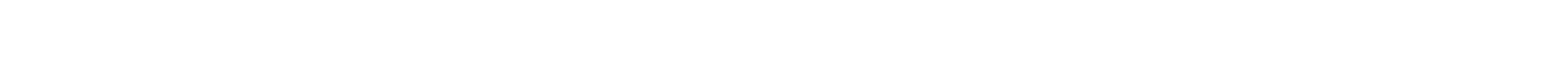

$\mapsto$

悲

en

n

is

n

is

"

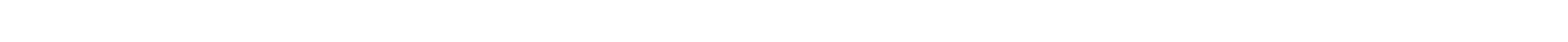
Е

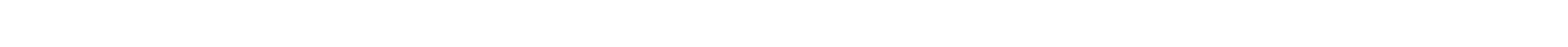

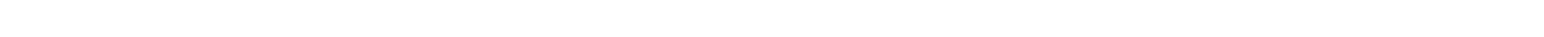

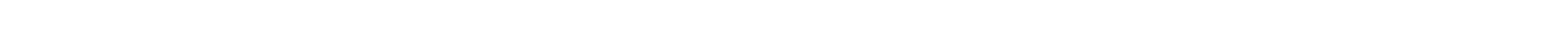

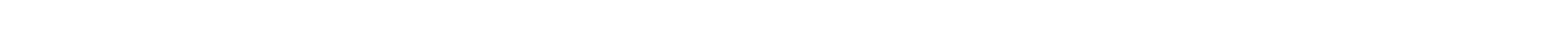

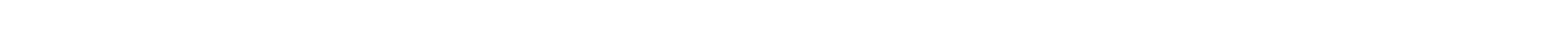

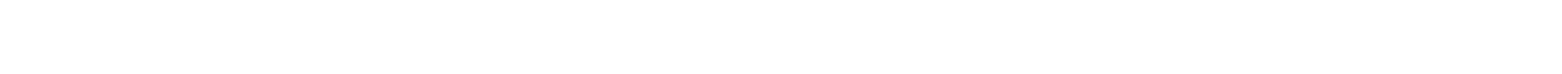
$\stackrel{\omega}{m}$ o
n
n

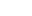
is
n
$\stackrel{2}{5}$

"o에 出

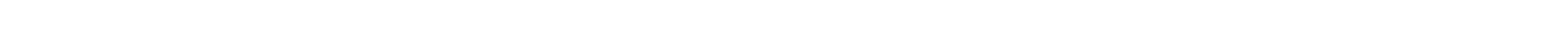

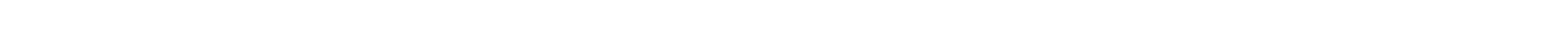

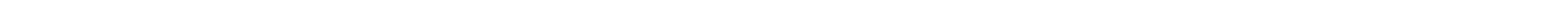

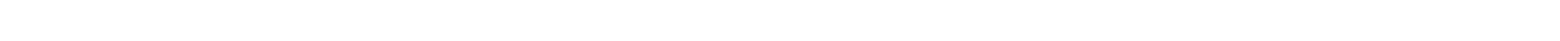

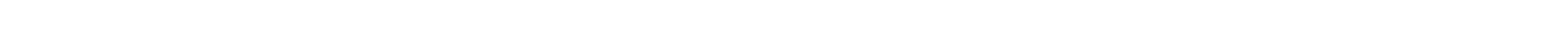

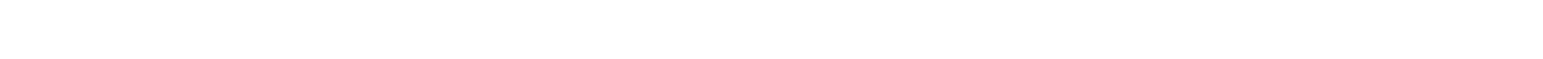

出

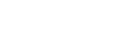

en

n

is

n

n

n

a

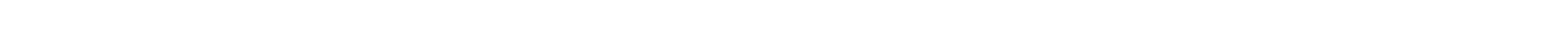

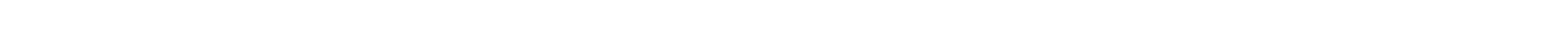
"

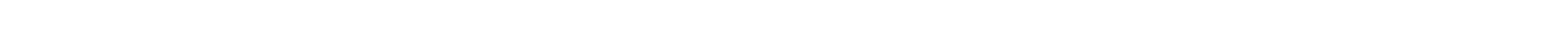

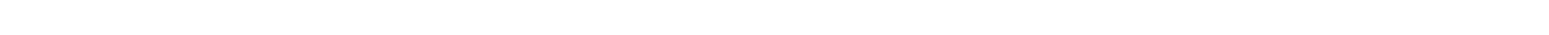

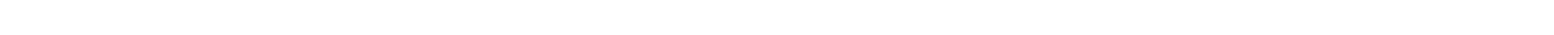

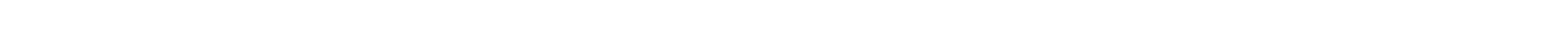

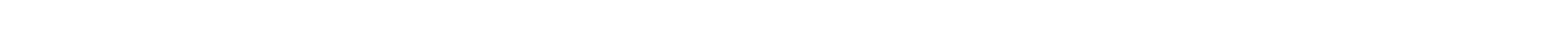

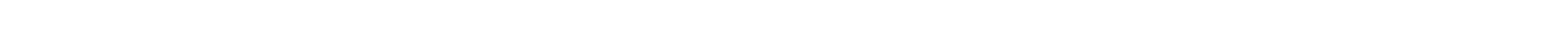

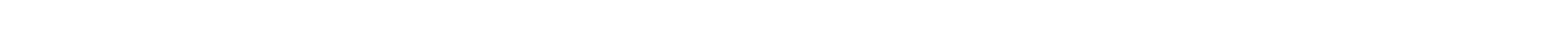

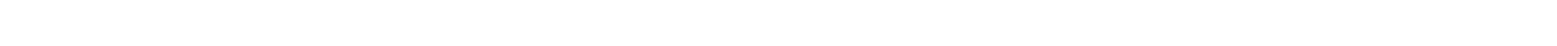




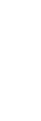

$\infty \quad n$

an on en

s.

$\sim$

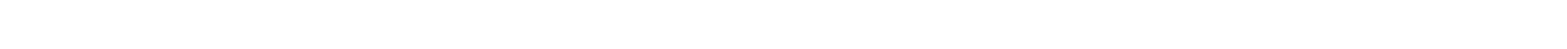
« L Jம்

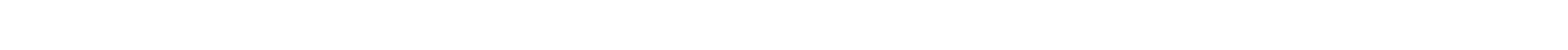

W

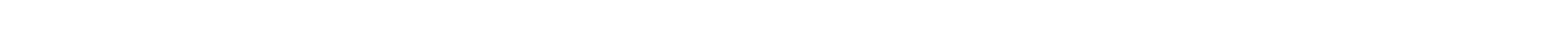

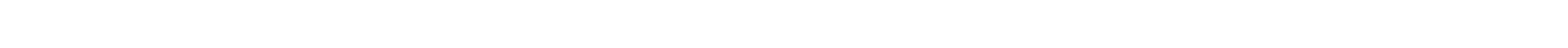

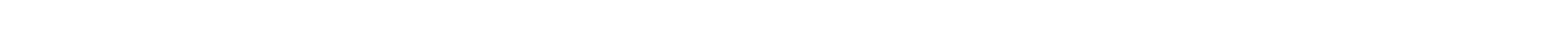

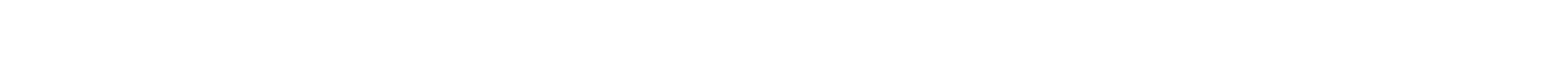

$\underline{\Sigma}$

$\stackrel{a}{2}$

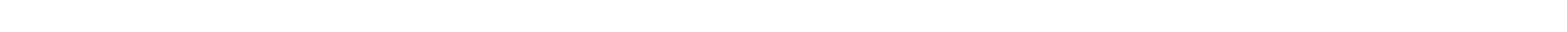

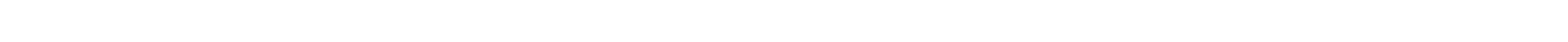


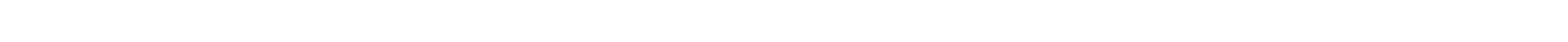

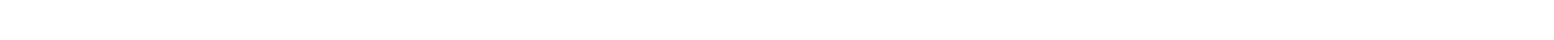

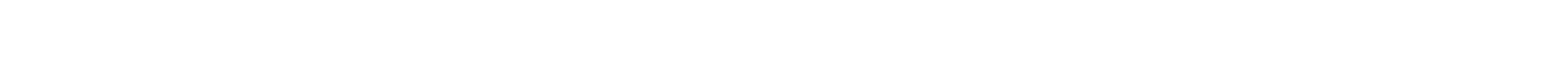
แ

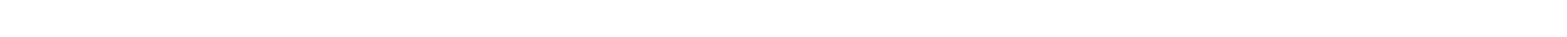

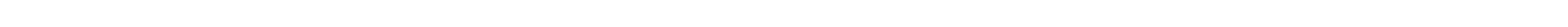

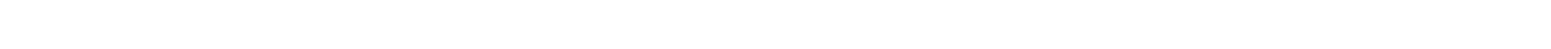

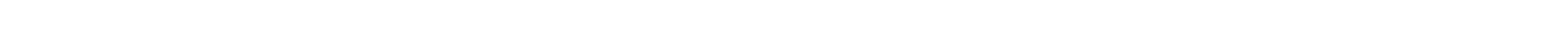

an in en

u.

in

is

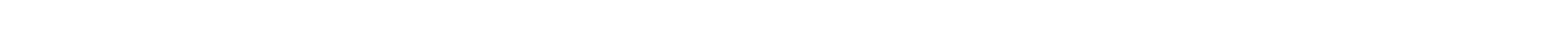
U - w5

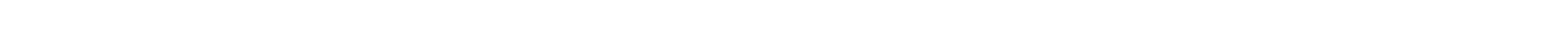

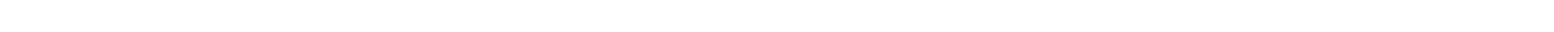

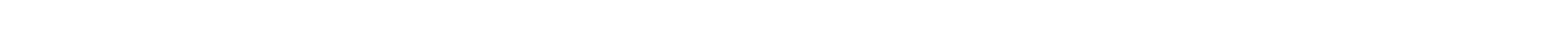

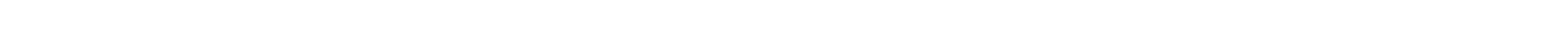

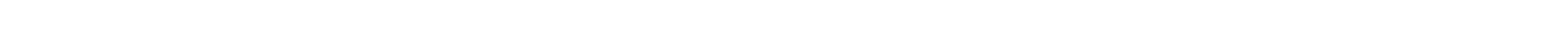

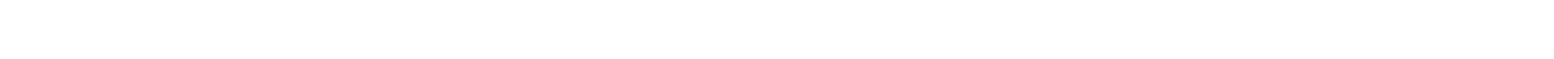

$a$

is

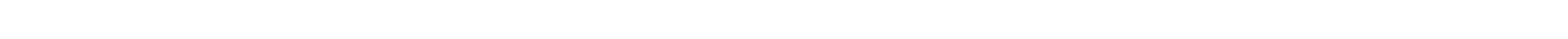

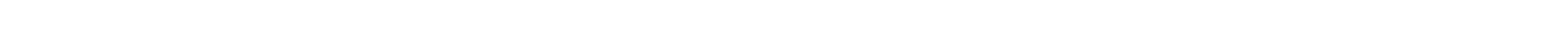

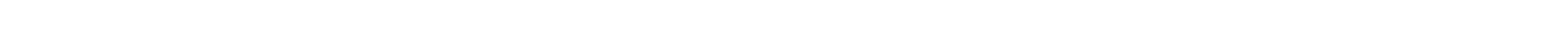
«o

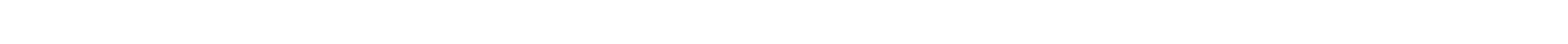

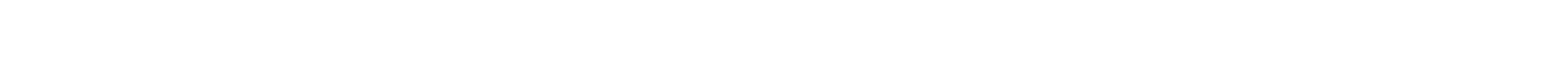

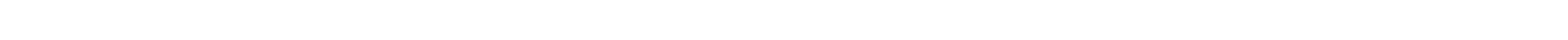
a

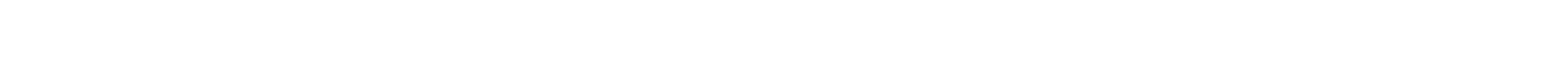




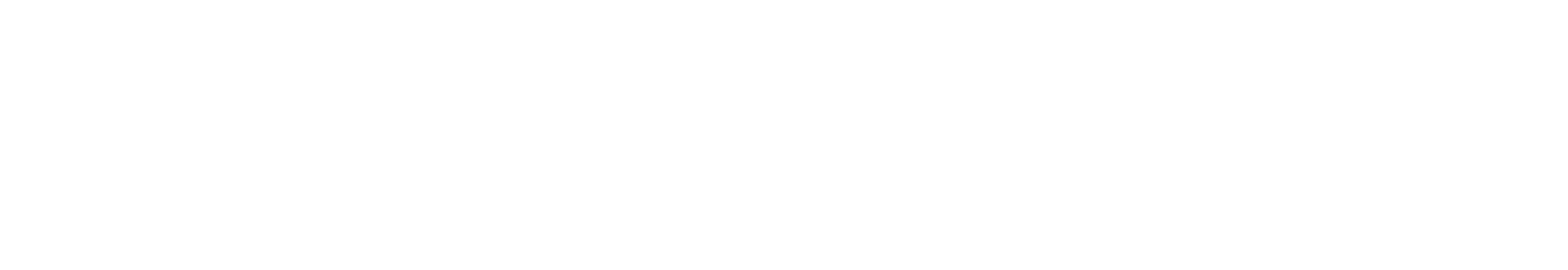

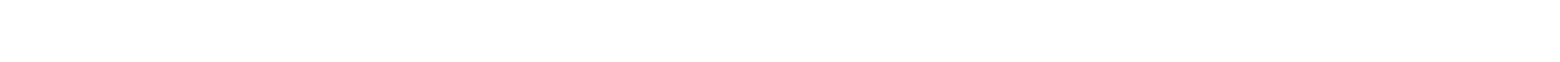

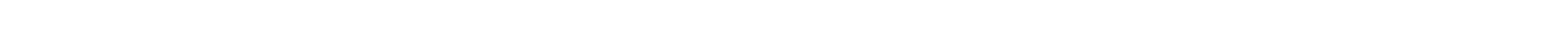

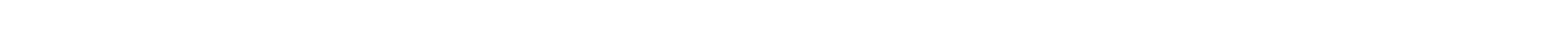

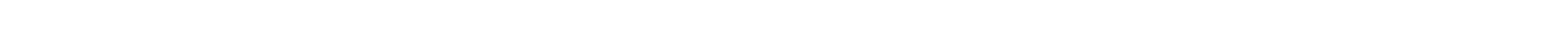

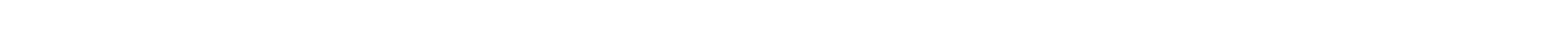
tomes
n
n
in
s
n
is
is

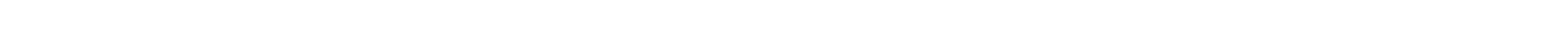
แ山 †

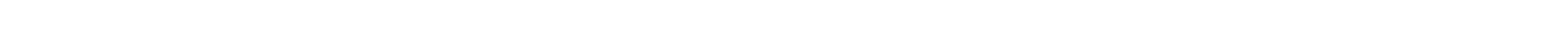

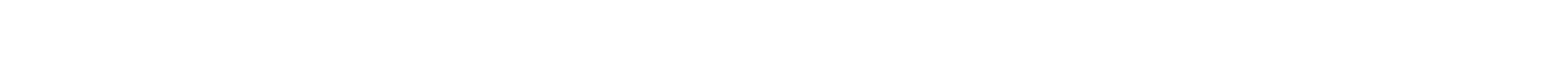

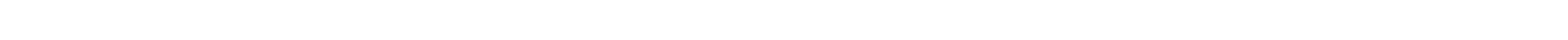

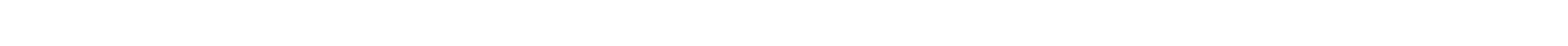

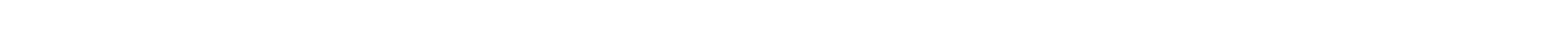

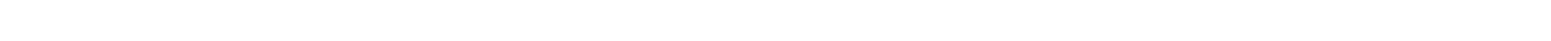

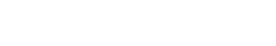

秘 n

n

n

on

n ж

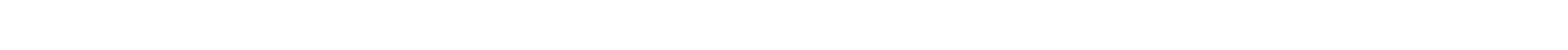

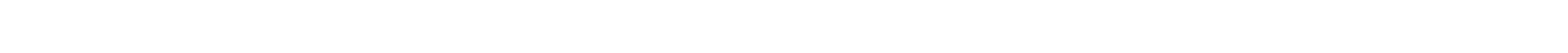

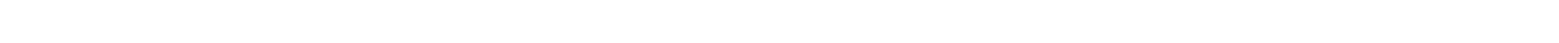

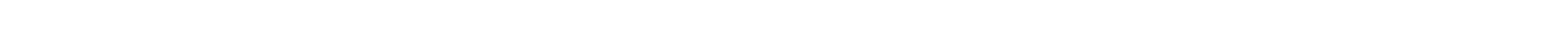

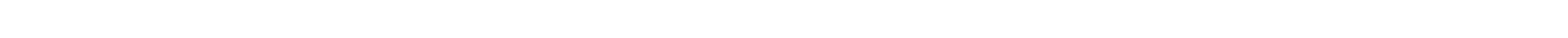

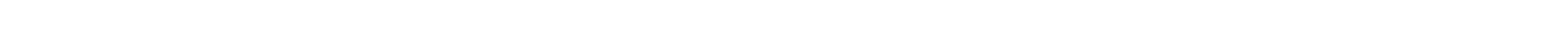

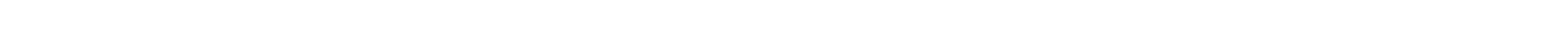

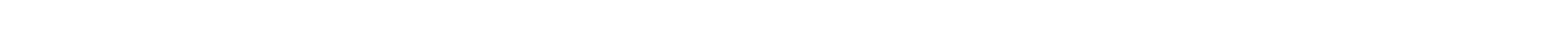

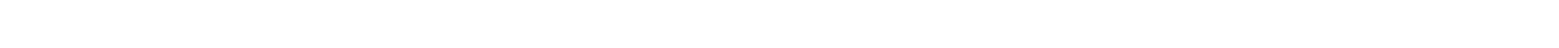

m $n$

$\backsim$

n

in en

u

in

is

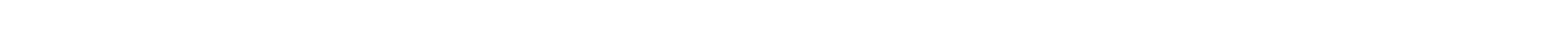

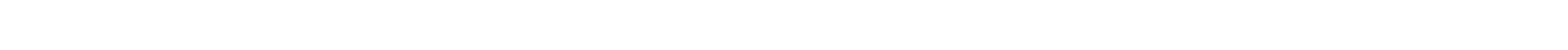

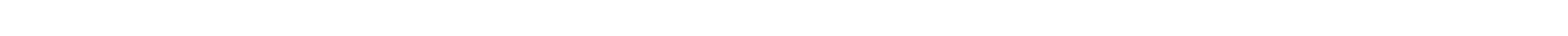

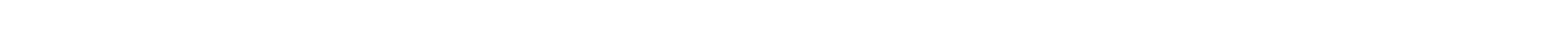

Nom

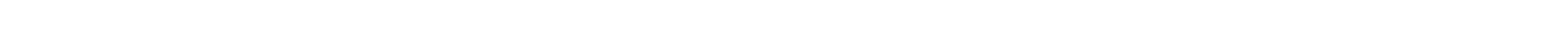

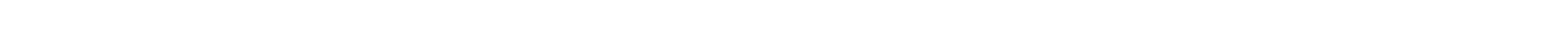

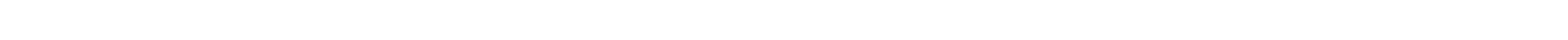

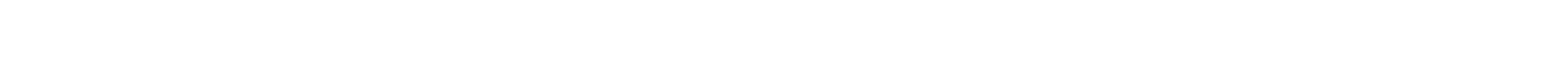




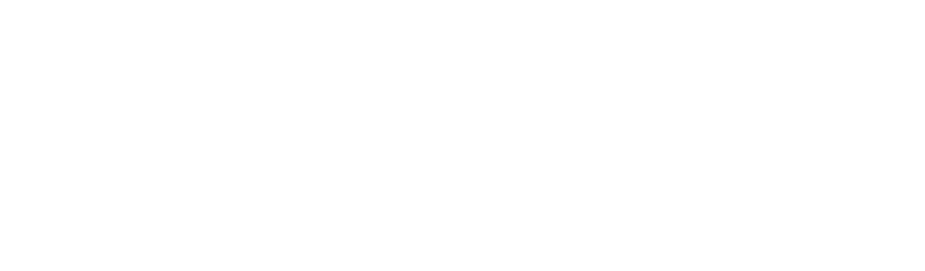

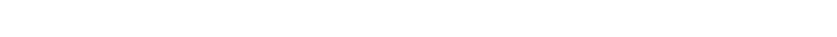

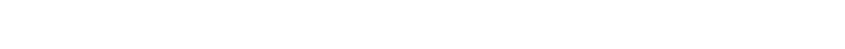

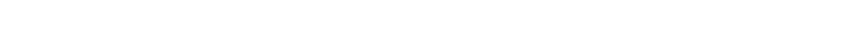

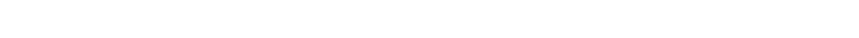

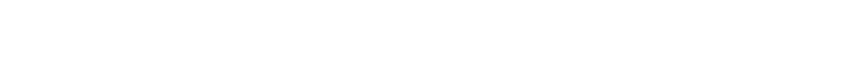

is

is

is

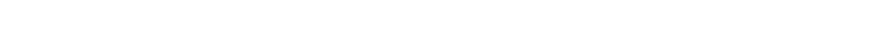

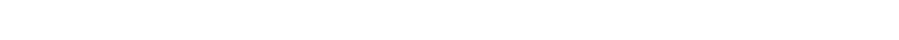

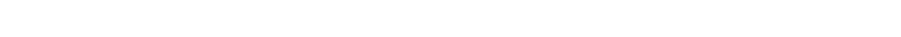

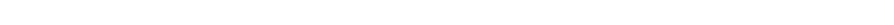

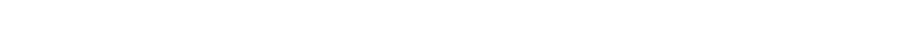

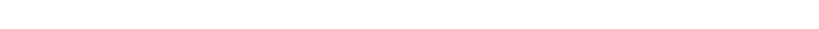

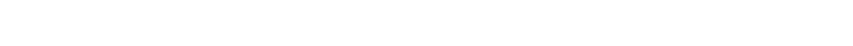

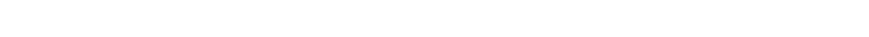

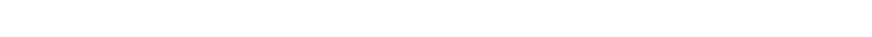

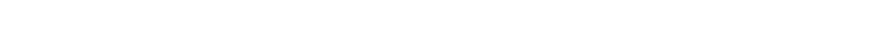

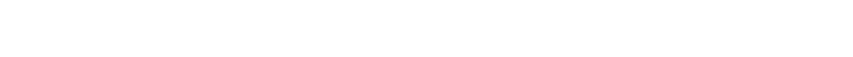

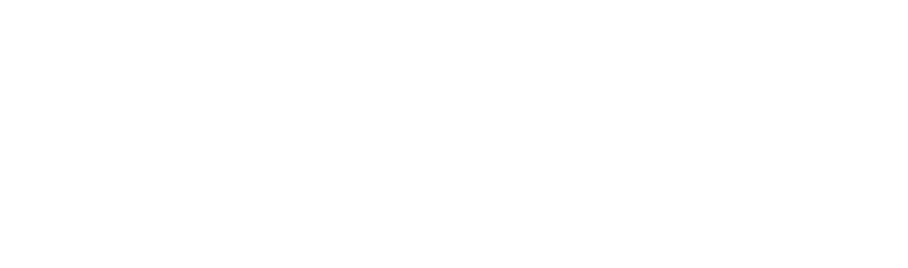

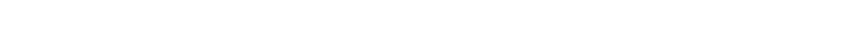

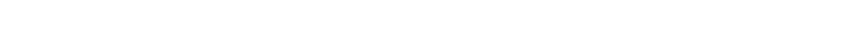

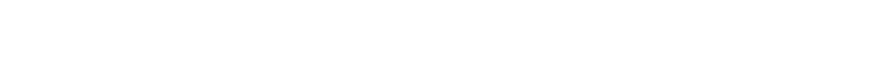
-

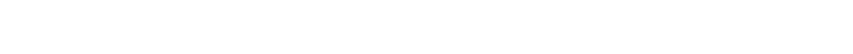

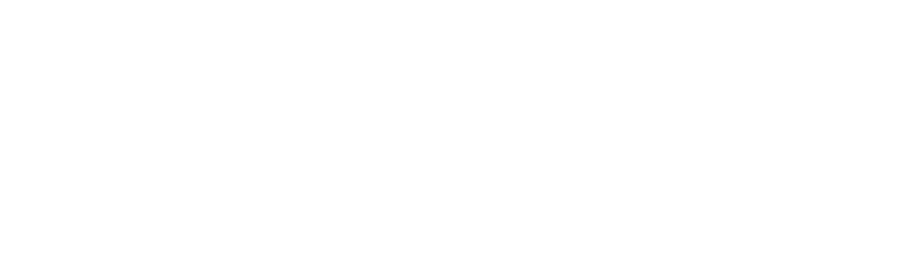

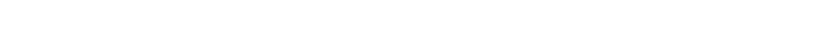

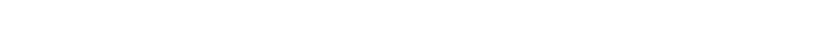

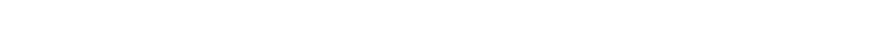

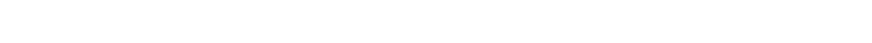
NN NmoO

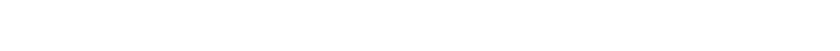




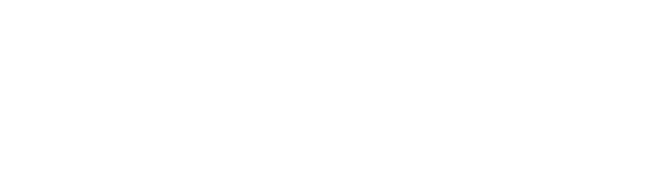
o

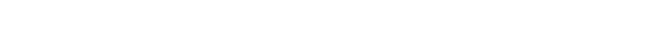
$\alpha$ w

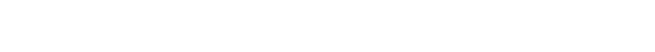

nnm $n$ แn

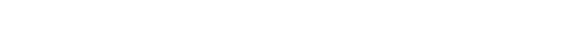
w- - - -

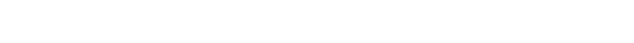

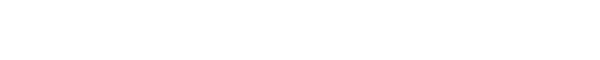

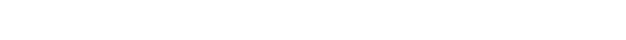
III

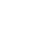

"ñ⿱

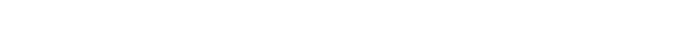
- ul mon on

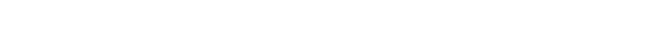

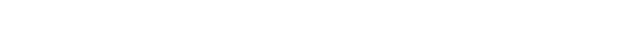

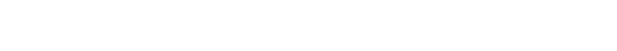

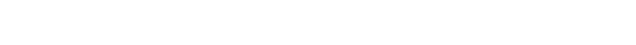

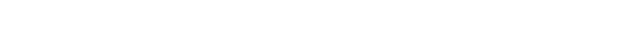

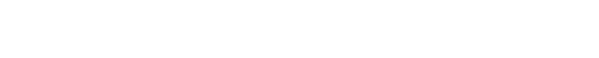

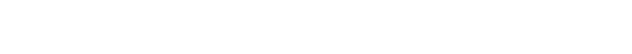

i

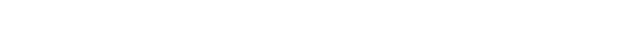

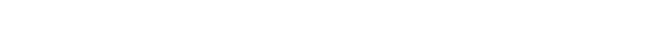

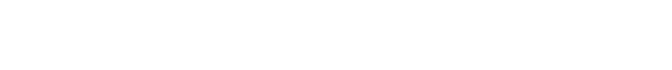

ง

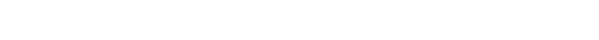
u

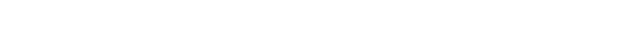

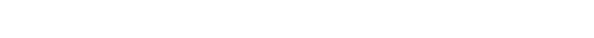

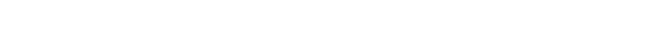
5

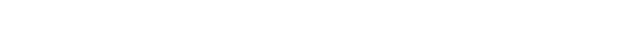
uै - ш

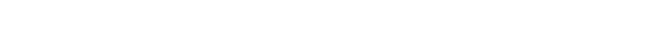

NNNNNNNNNMmMmMMmMMMm

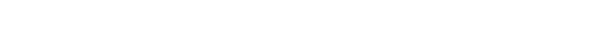

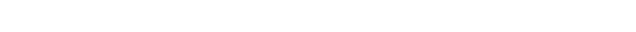

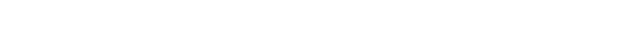

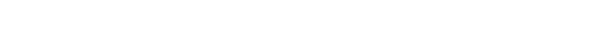



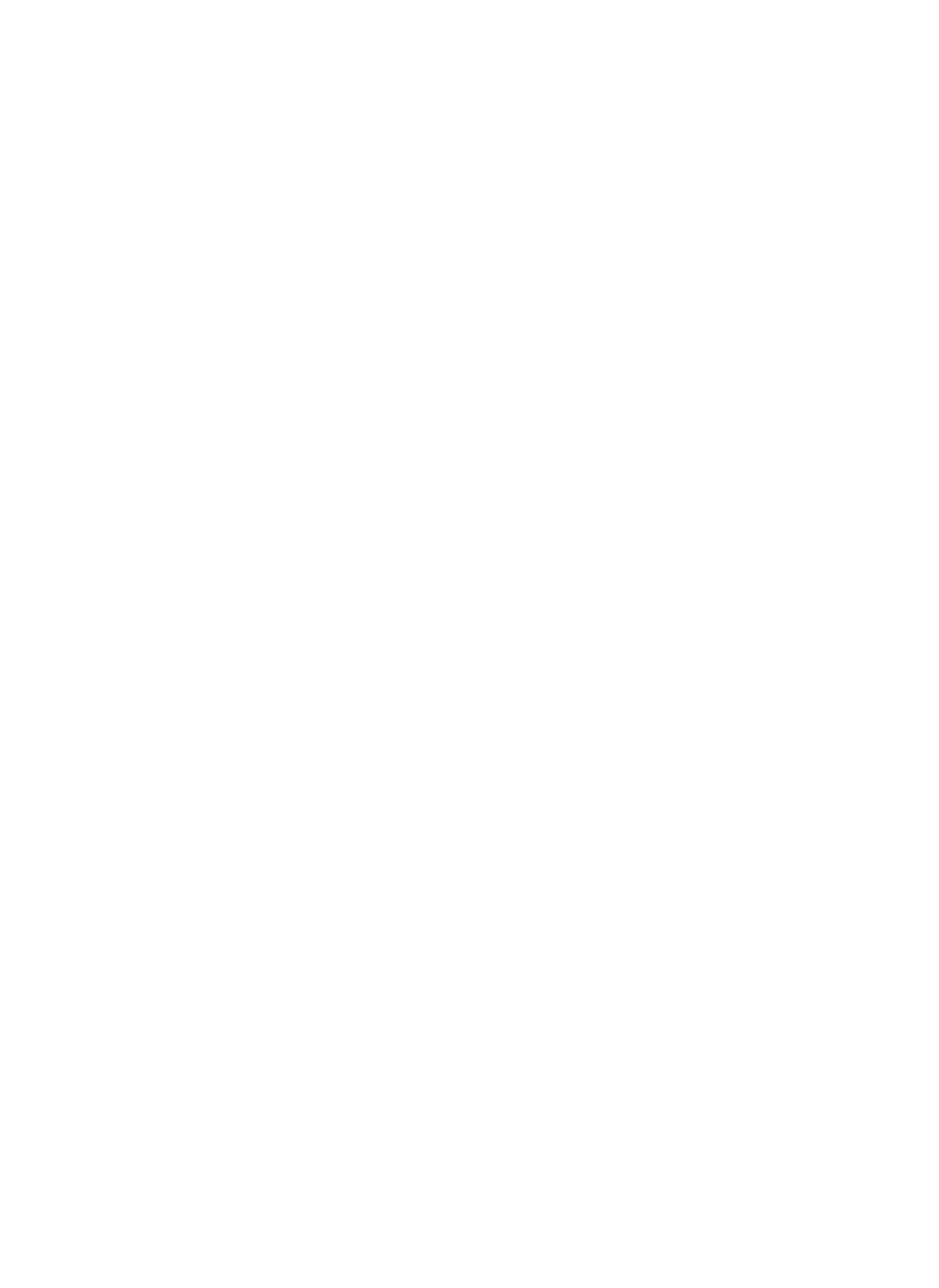

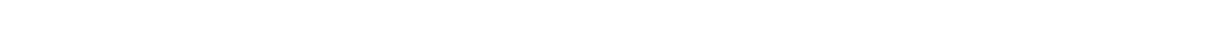
山யن

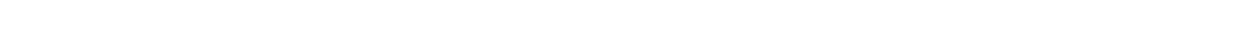

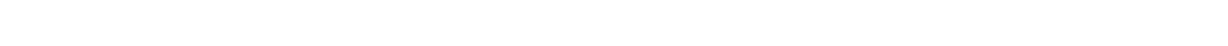

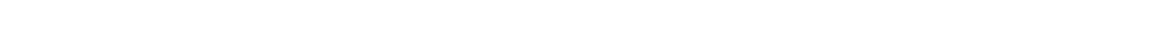

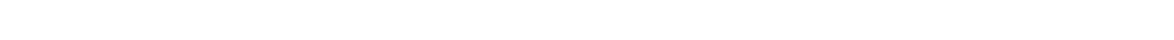

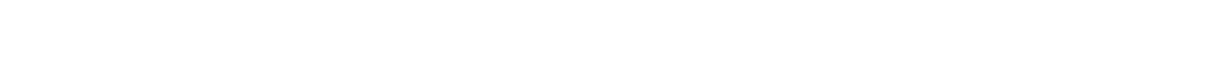

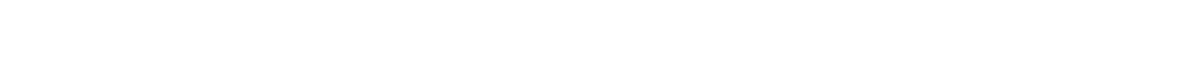

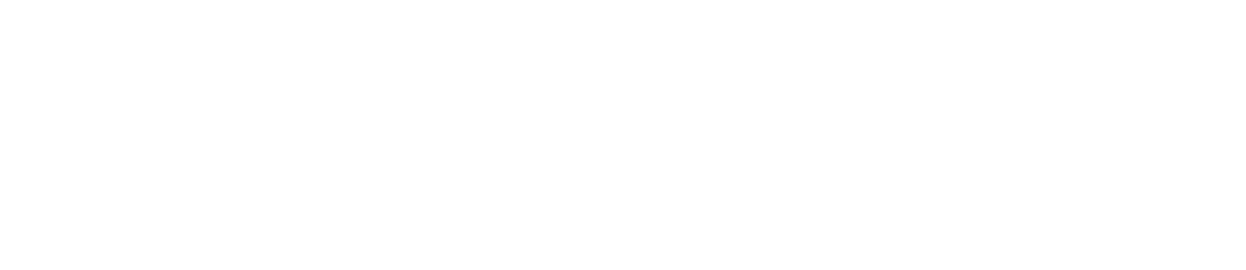

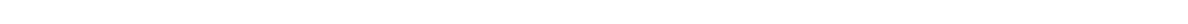
๙

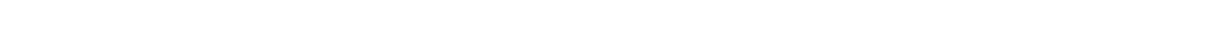

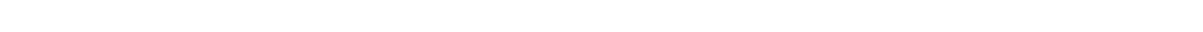
m0 -

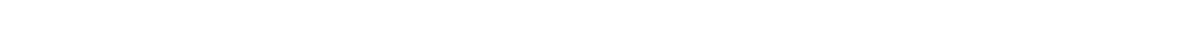

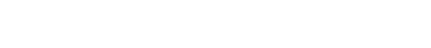

a

is

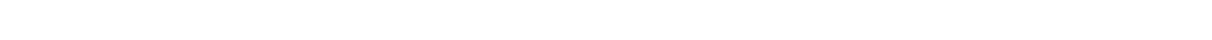
$\alpha \vec{\omega}=0 . \cdots \cdots$

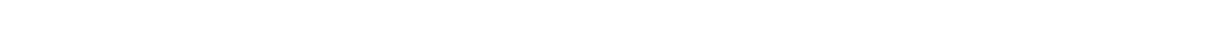

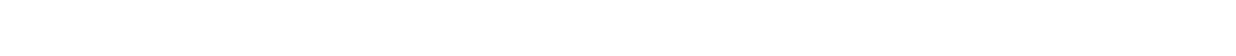

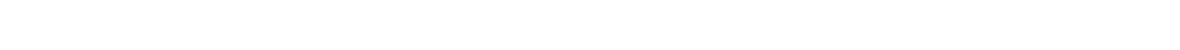

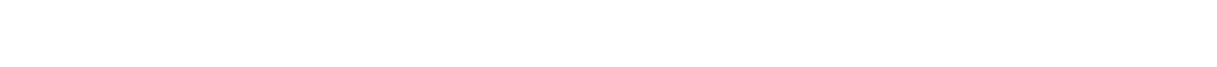

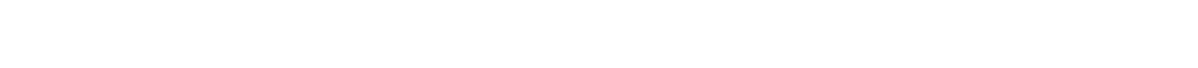

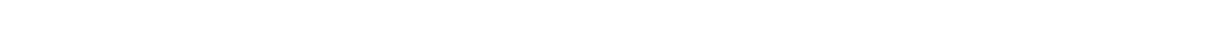

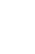

ม

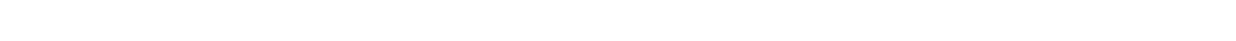

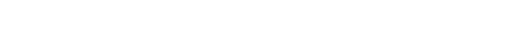

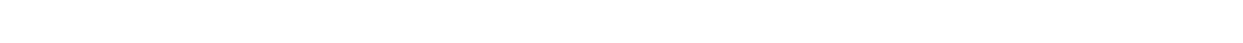

in й

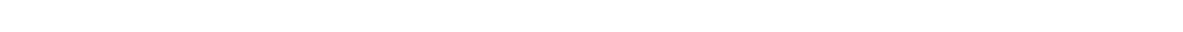
WE-

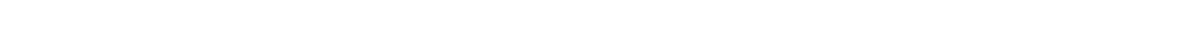
by 
is

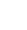

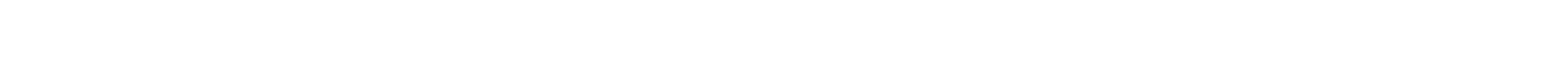


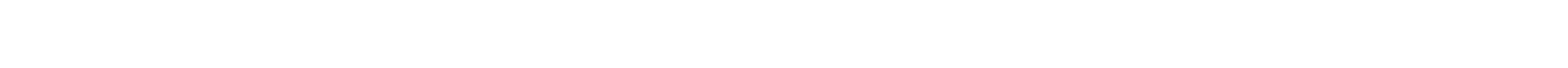

nm

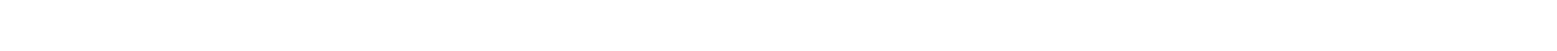

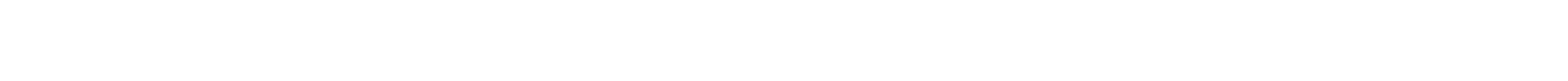

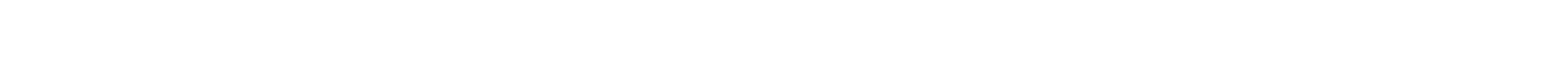

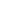
il.

nn

as us

n

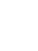

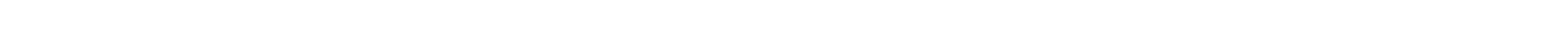
w u.:-1:t

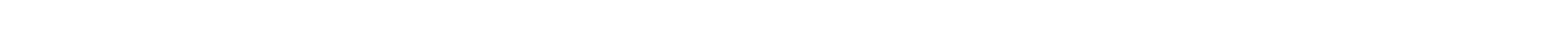

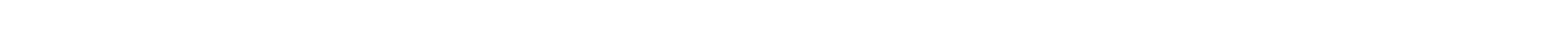
nпำ

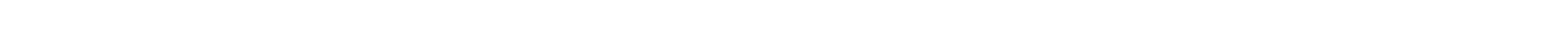
anom

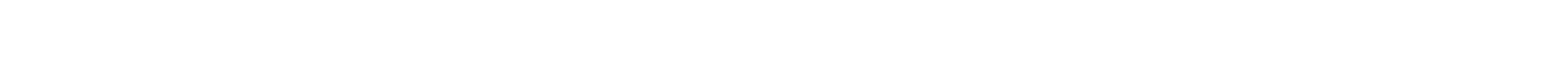
w
w
n
s
is
is

is

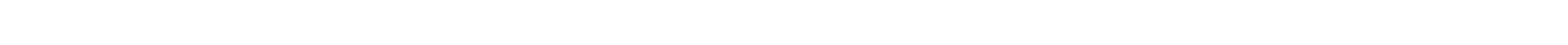
a d

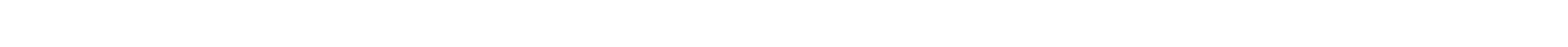

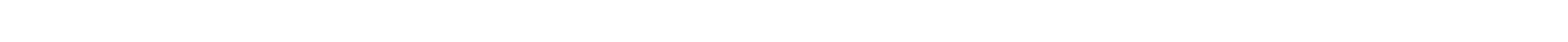
mm

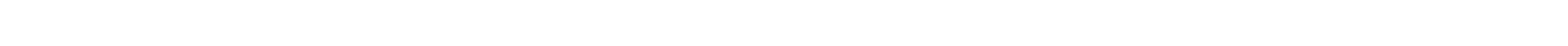

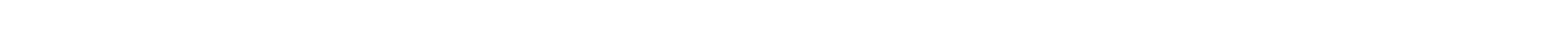

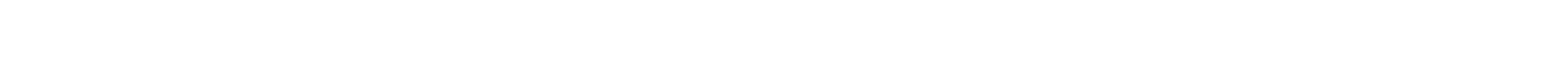

盛

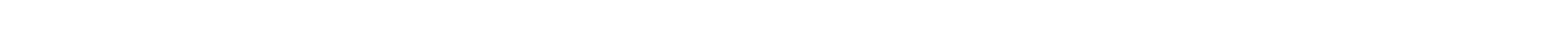

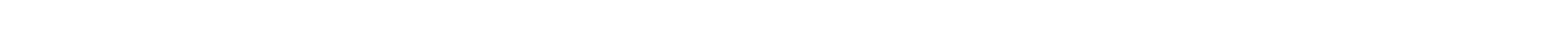

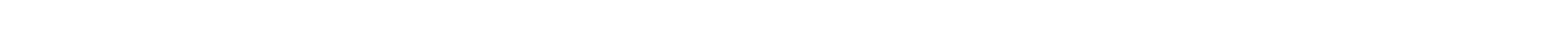

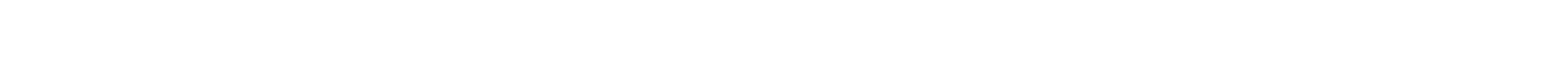

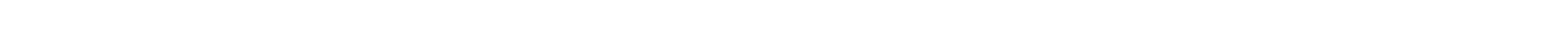
w

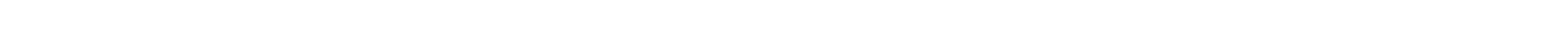

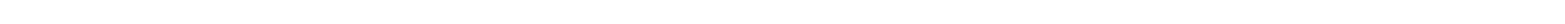

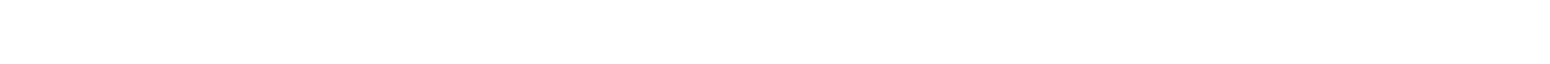




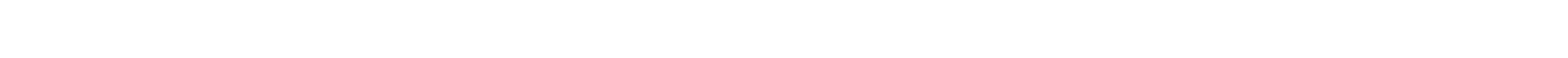
بै

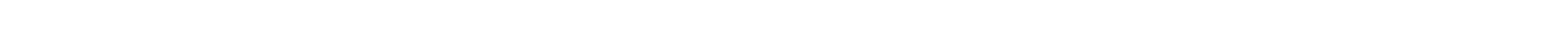

nnd

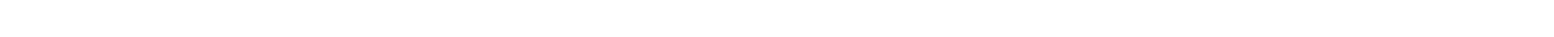

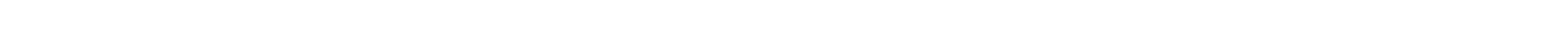

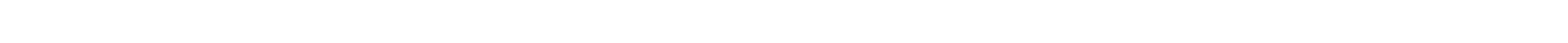

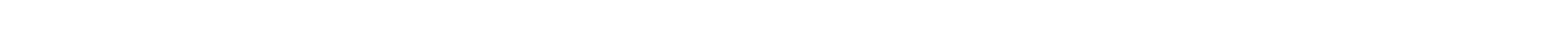

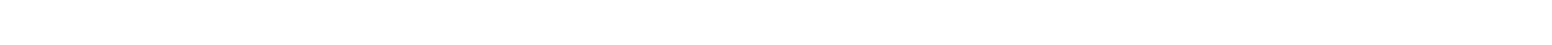

$\stackrel{w}{\Sigma}$

$\stackrel{+}{\leftarrow}$

党

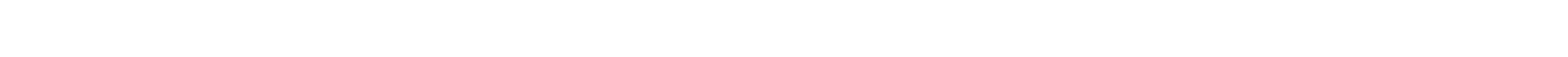

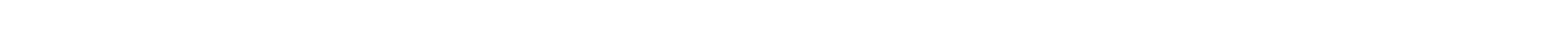

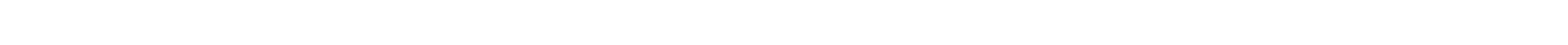

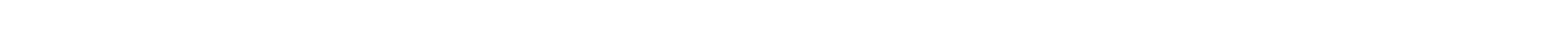

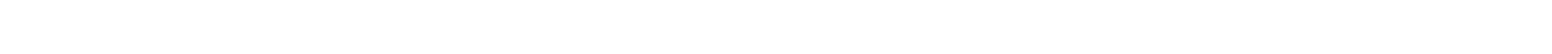
w

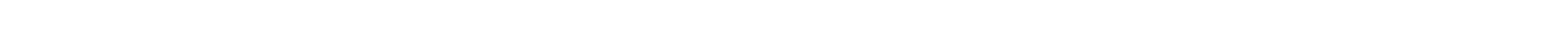

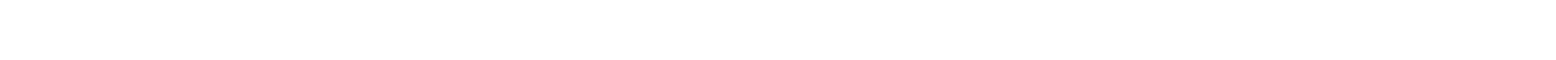
is

$\Sigma$

No

in

$\therefore$

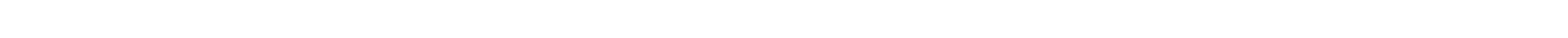

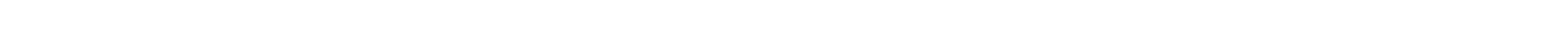

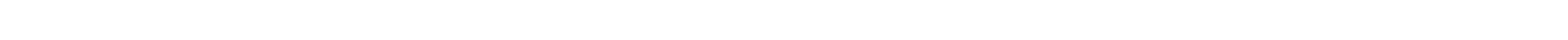

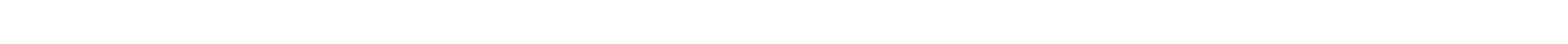

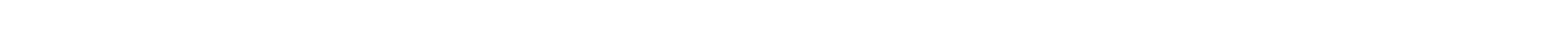

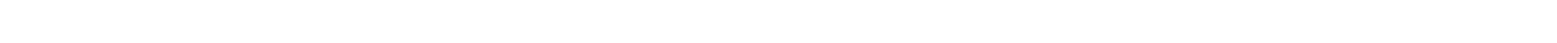

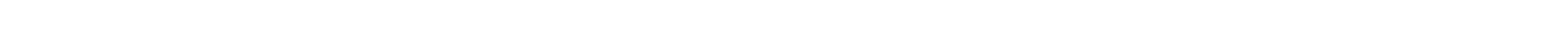

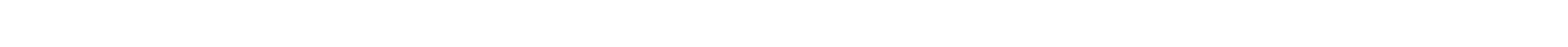

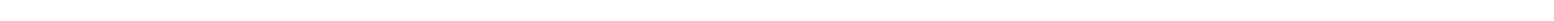

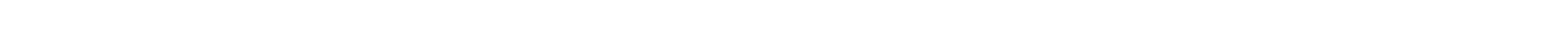

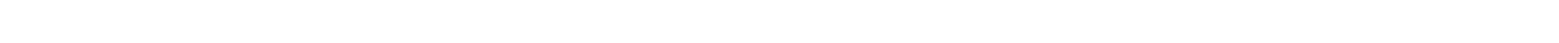

r

is

n

n

is

$\stackrel{5}{t}$

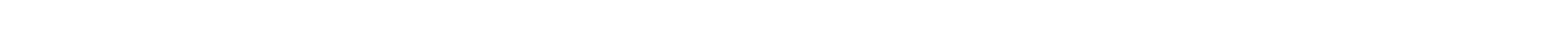
w

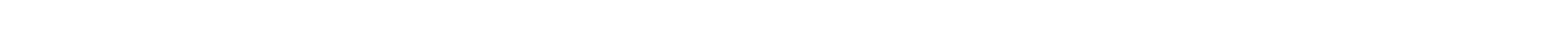
3
3

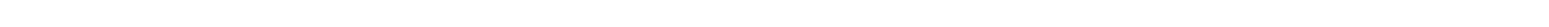

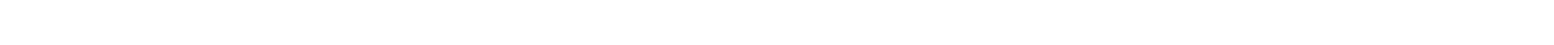

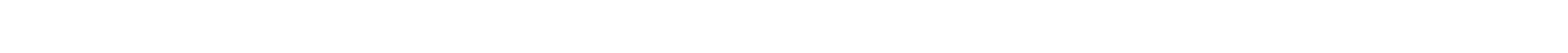

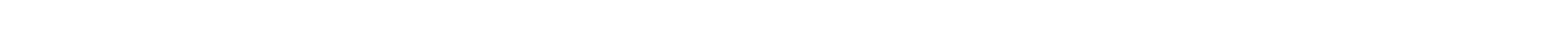

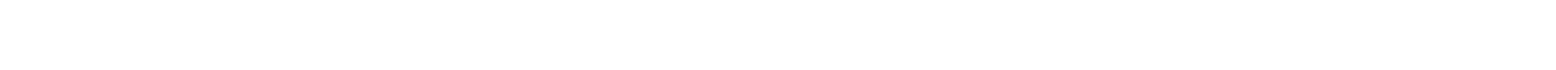




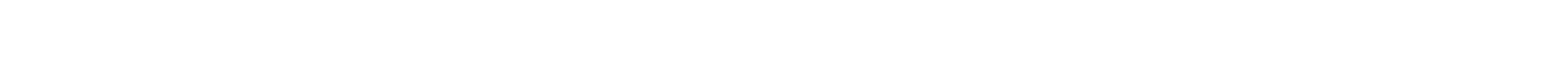

5

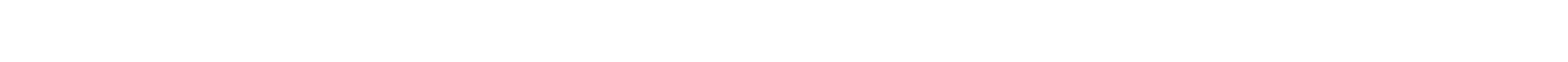

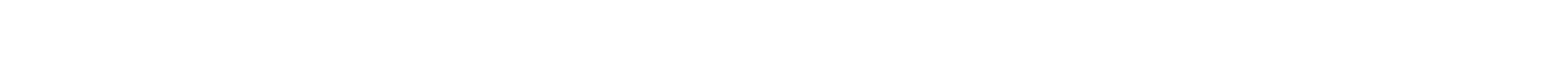

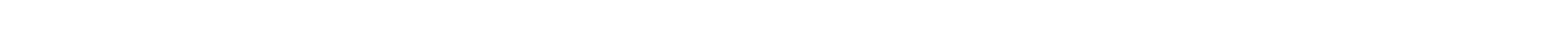

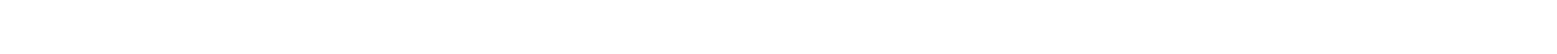

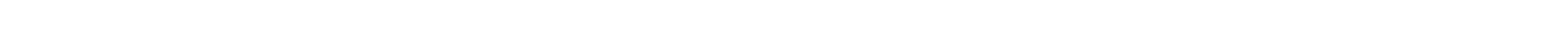

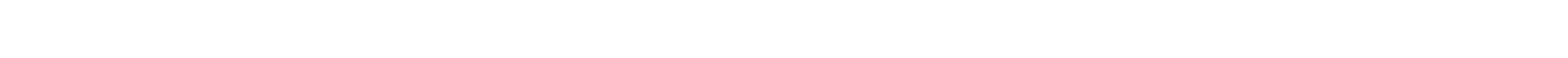

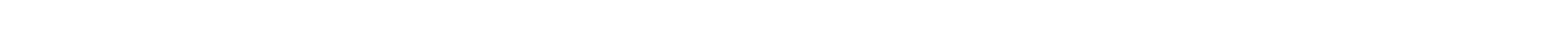

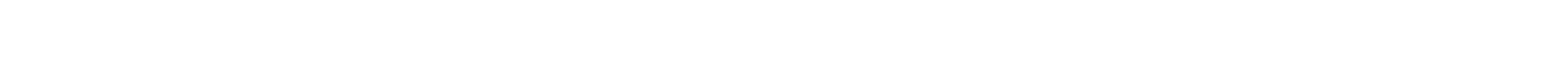

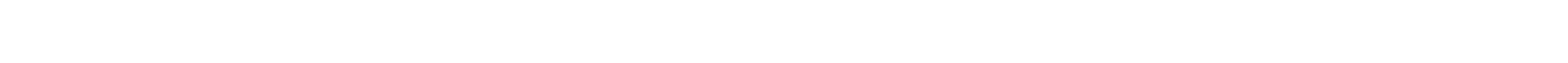

\&

$\cos$

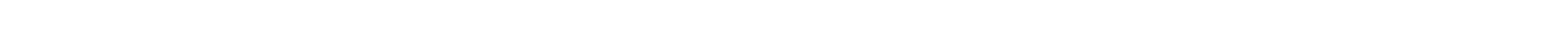

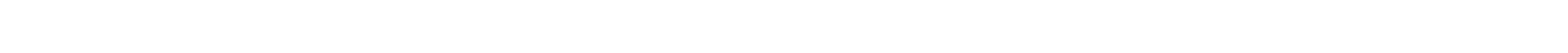

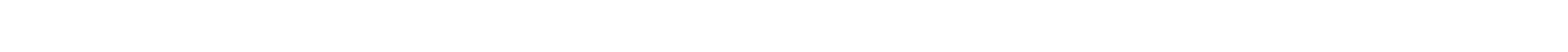

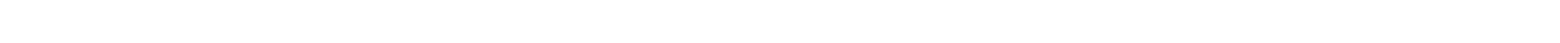

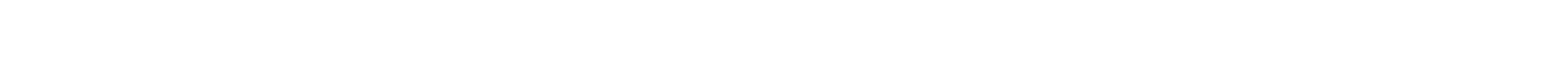

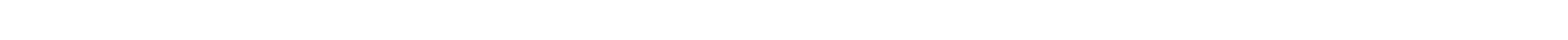

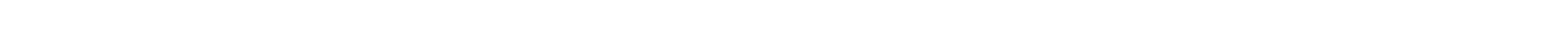

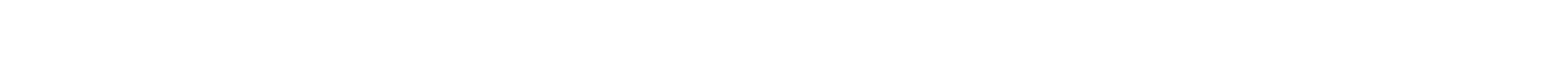

$\stackrel{\text { LU }}{\Sigma}$

$\infty$

$\$$

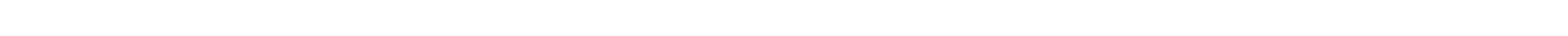

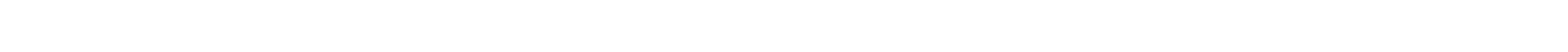
J

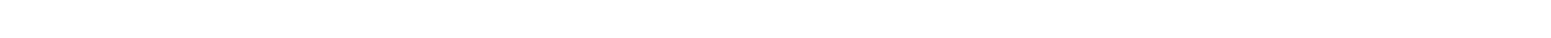

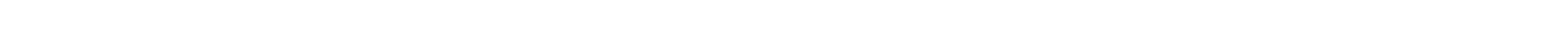

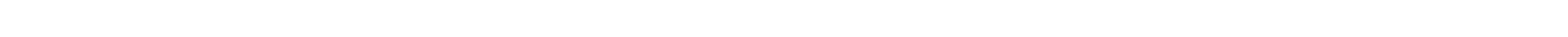

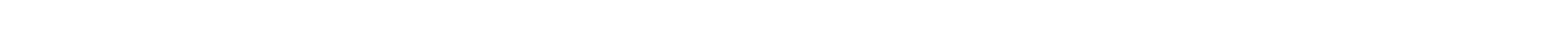

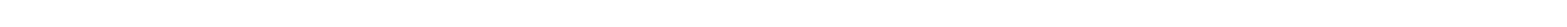

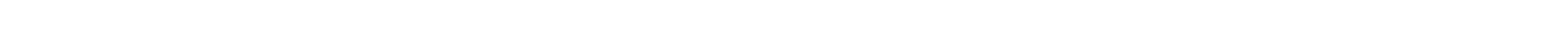

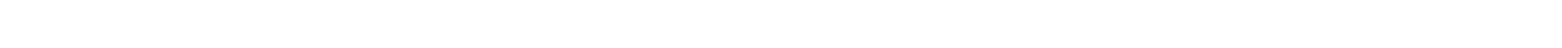

\footnotetext{
$\stackrel{w}{2}$ is

$\lesssim$
}

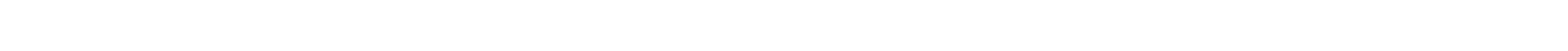

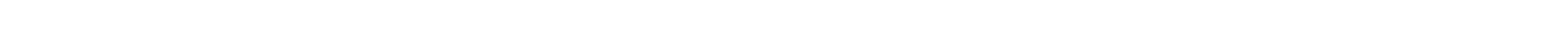
แ

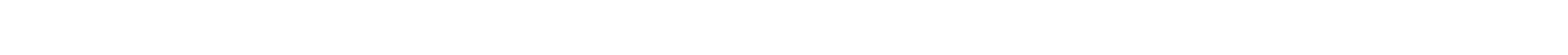

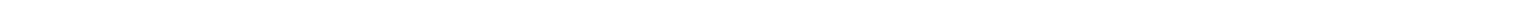

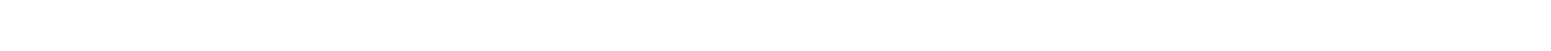
แ

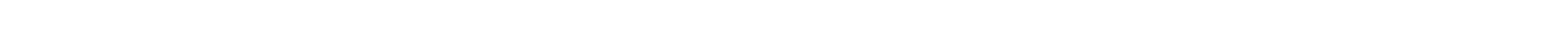
mmmmm m mommmm 


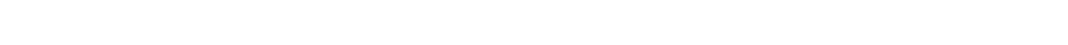

is

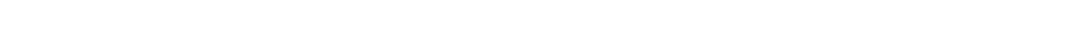
Uu

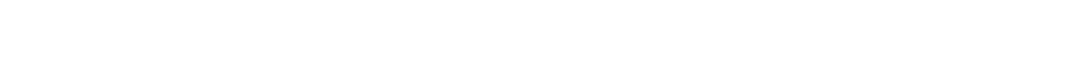

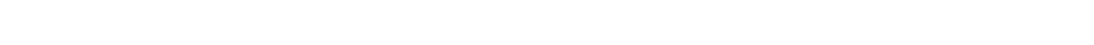

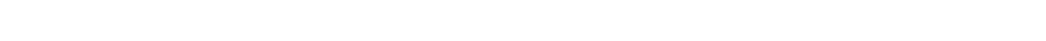

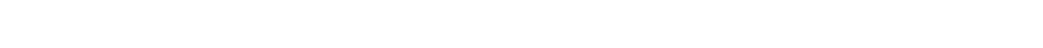

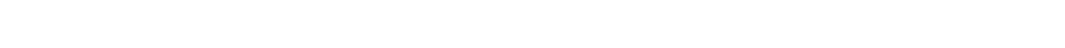

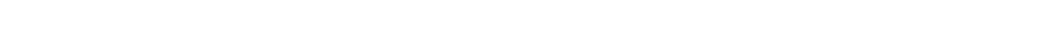

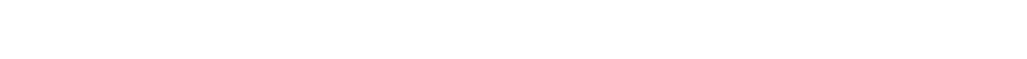

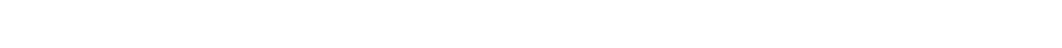

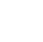

is

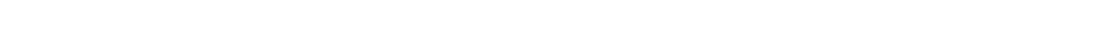

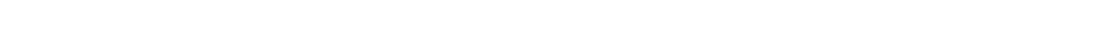
3
4

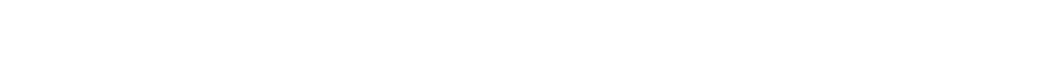

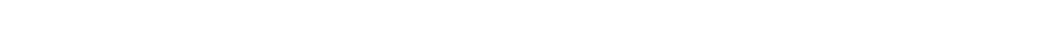

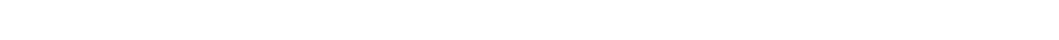

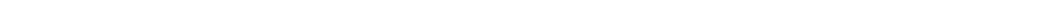

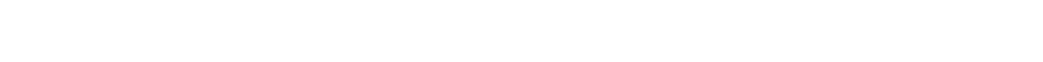

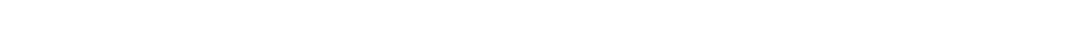

$\stackrel{2}{\text { in }}$

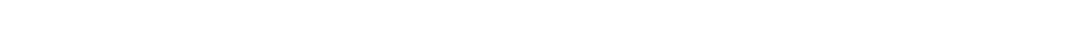

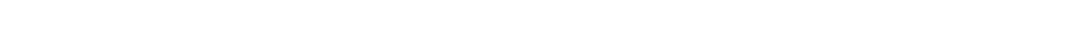

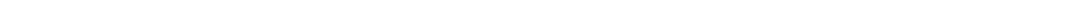

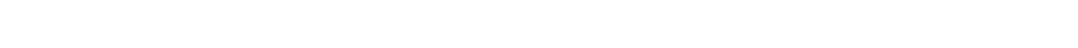

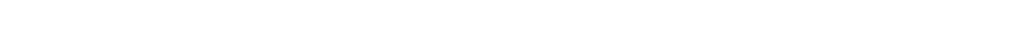

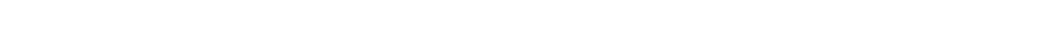

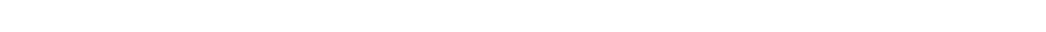

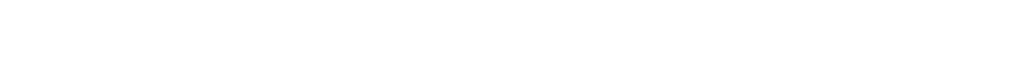

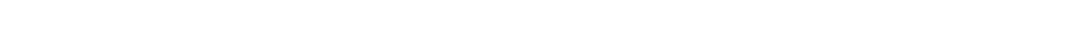
$\Sigma$

$\frac{10}{2}$

ง w

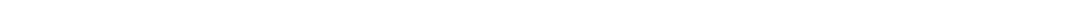

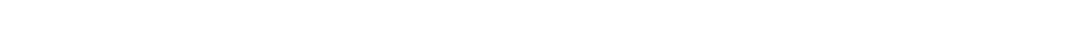

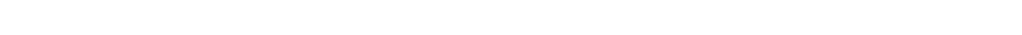

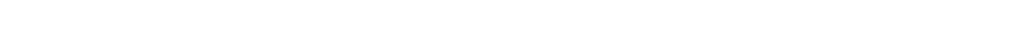

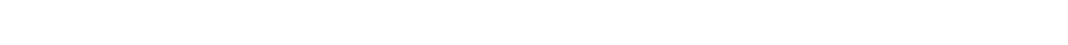

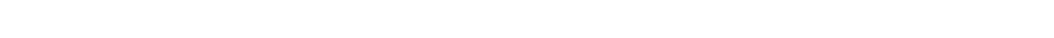

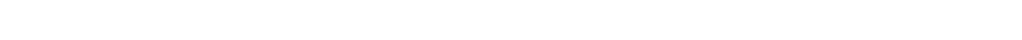




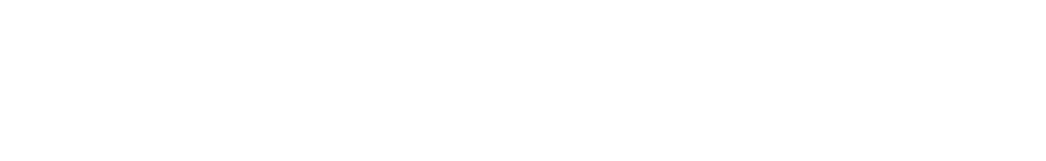
ผ

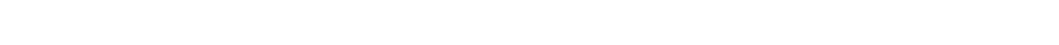

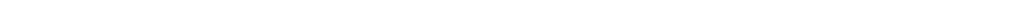

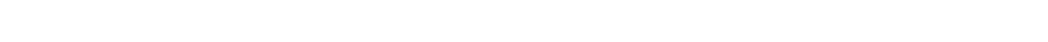

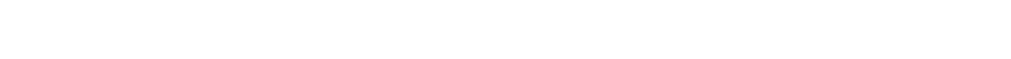

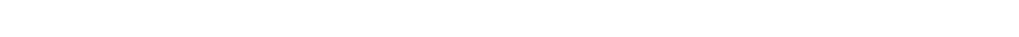

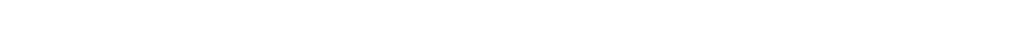

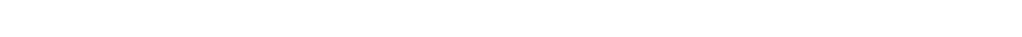

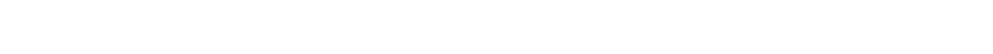

$\therefore \sum_{0}$ $\sum \cos 0$ ㄴำ n.:안도일. m 음 =

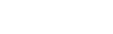
z幺i $\sin \pi$ $\infty \div$ 은든 ona

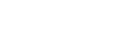
202 in

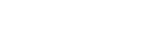
of 얼 次些品 西以

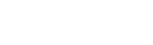
$m$ ns 2.

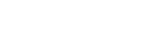
땐 造=下 依的 药 $z$ 过? in: 른 o w in $N \rightarrow$ in

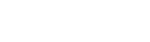
안: :

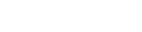
当的金 注2 $\dot{0}=$ 的定

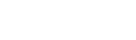
ㄷー 3 แ

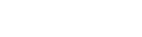
nom

: : : aㄴ.

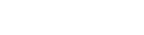
론ำ 이은

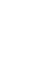

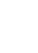

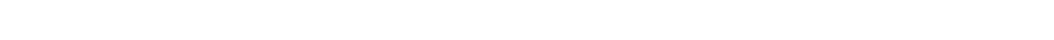

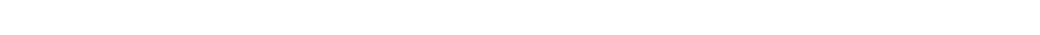

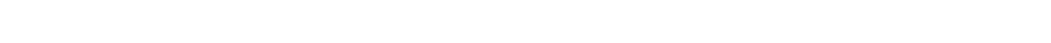

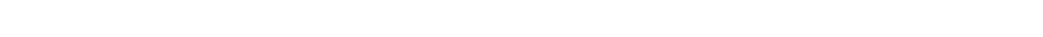

$\because \dddot{m}$

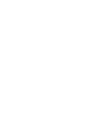

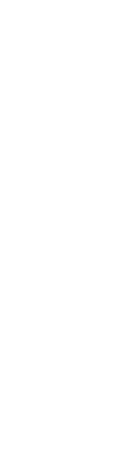
$-2 n \stackrel{z}{2}$ ñ

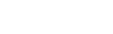

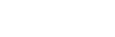
ì 잉요 $\mapsto \ddot{u}-\ddot{u} \sim$ $>$

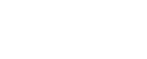

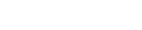

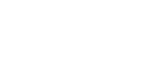
号品焉: $\underset{\Sigma}{\mathbf{w}}$

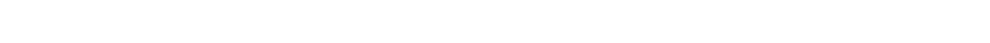

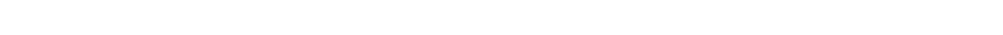

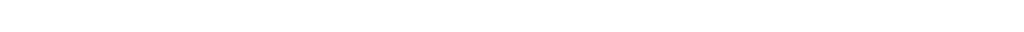

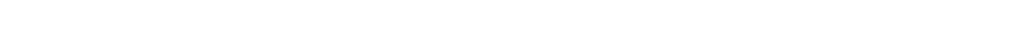

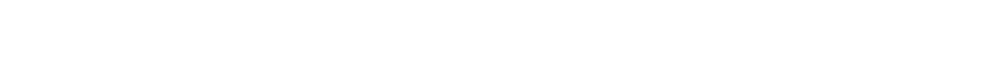

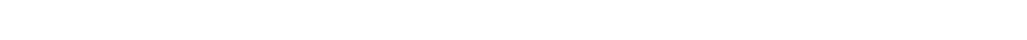
is

๔ 虫

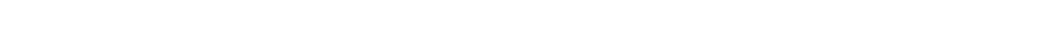

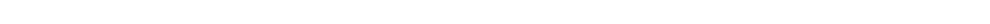

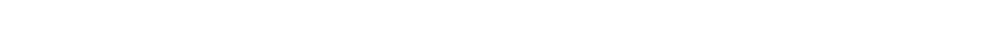
20000

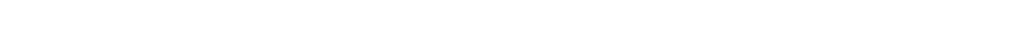
-

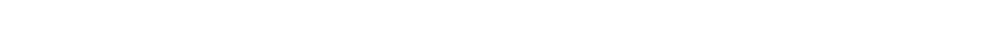

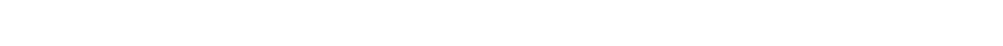

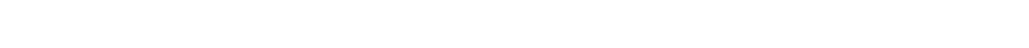
ț ส

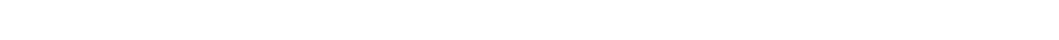

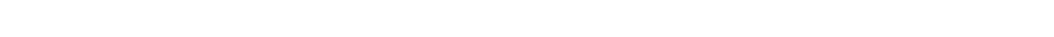

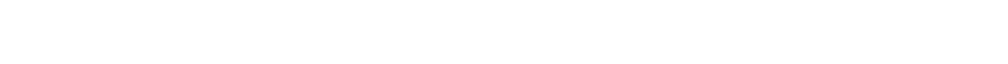

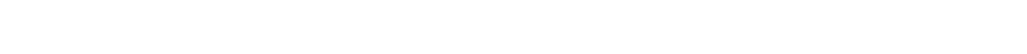

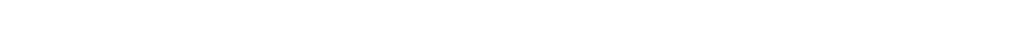

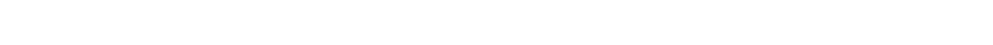




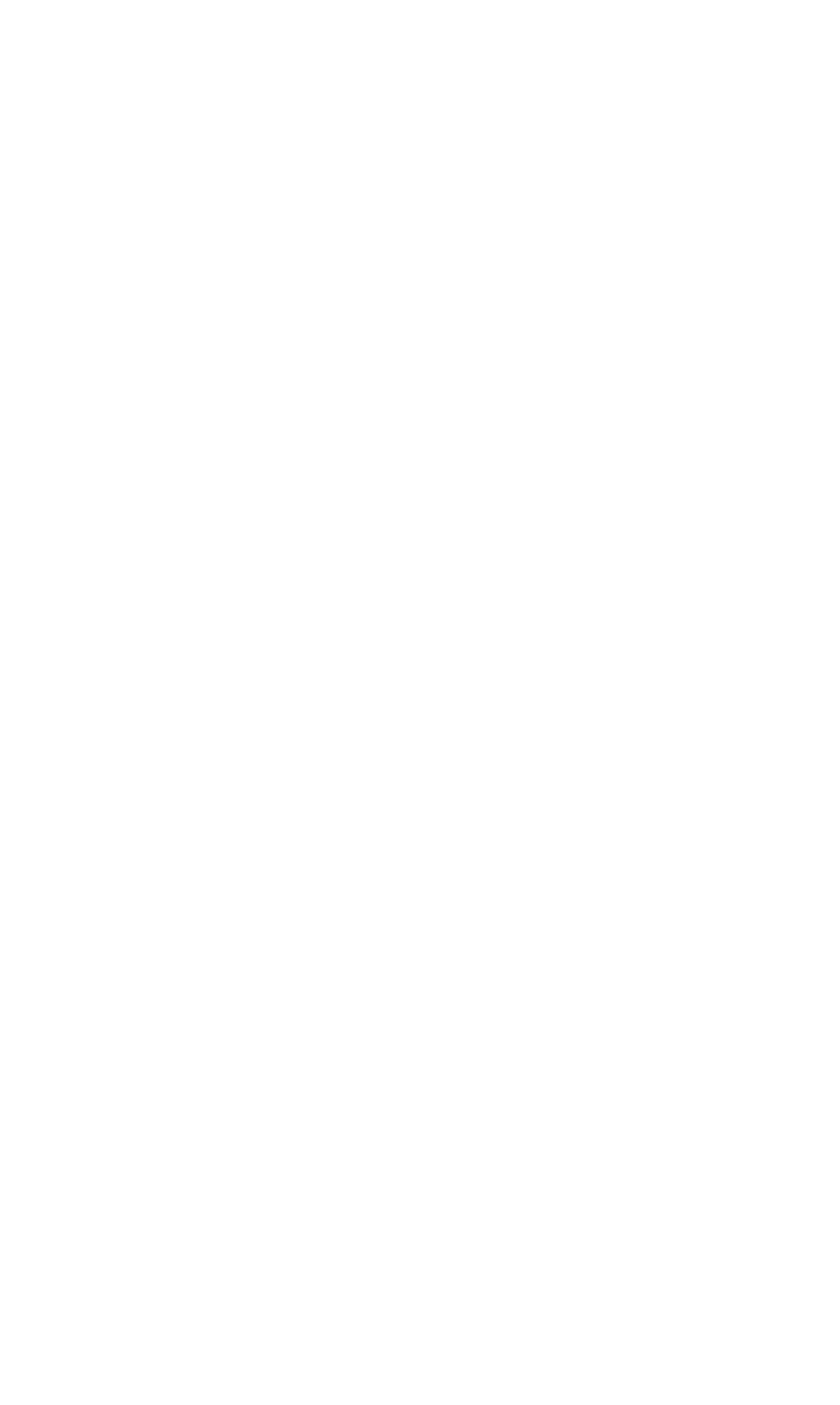

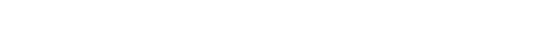
\& 20 * ง wrmm: $\dot{0} \dot{0} \dot{0} \dot{0} \dot{0} \dot{0}$ r

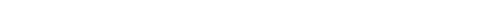

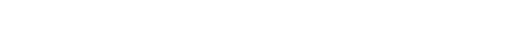
$\vdash$$$
\alpha
$$

" anmarmnmoonmonoson

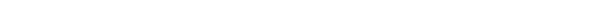

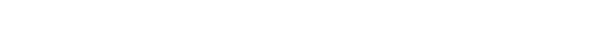

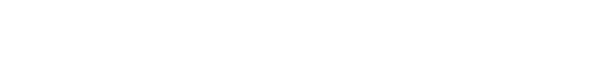

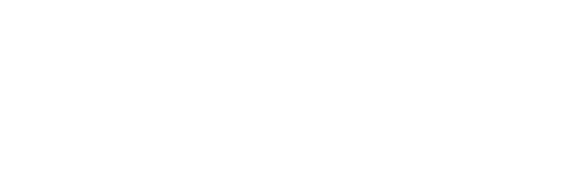

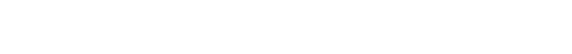
$\sigma$

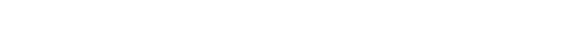

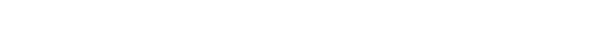

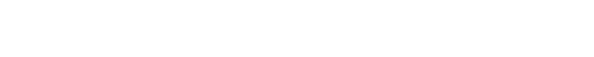

$\sim \sim \sim m m m m m m m m m m m m m s$ ज no 0 o

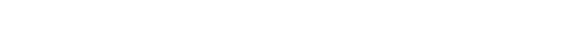
C5

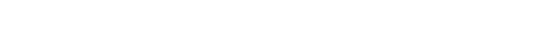

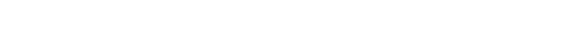
in ๙

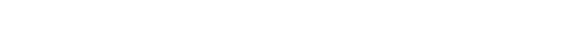

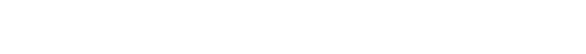

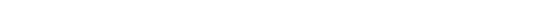

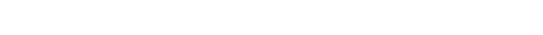

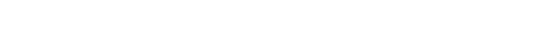

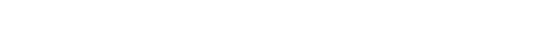

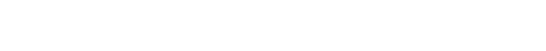




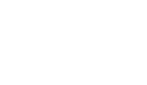

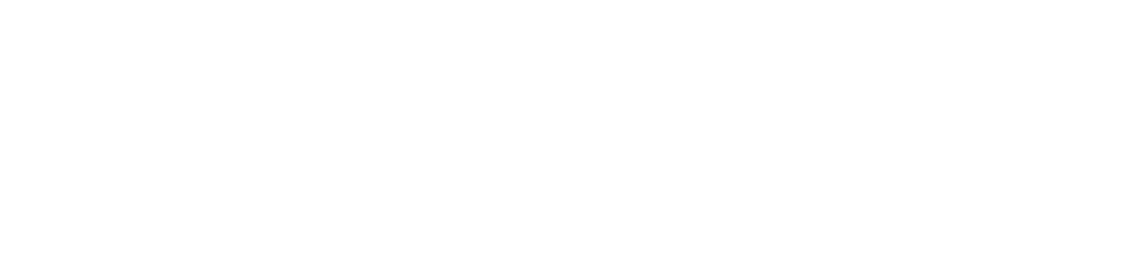

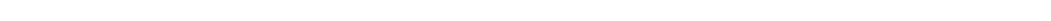

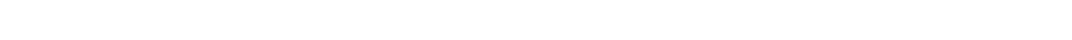

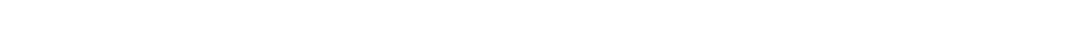

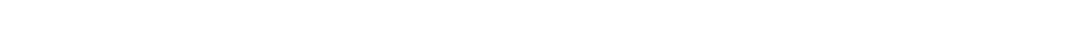

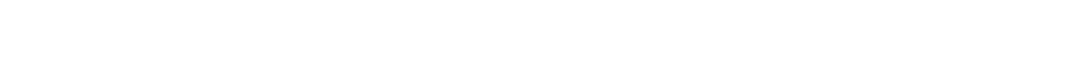

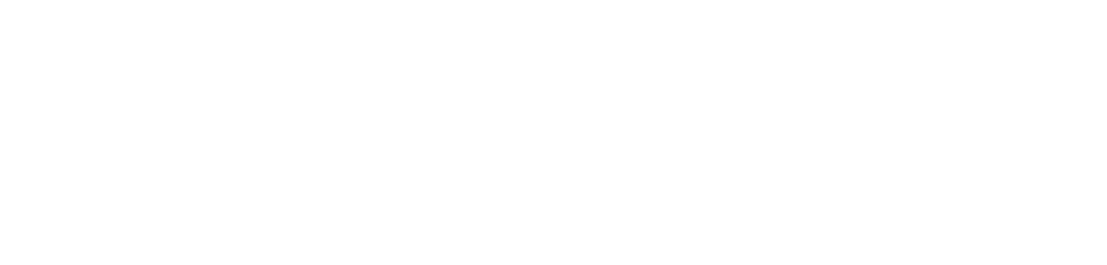

$\because \check{i} \cong \bar{m}$

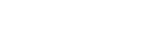

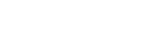

주의

200 열

임

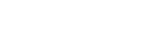

$\sum \sum a+\infty$

$\sum_{\infty}=\sum x$

w-

ỡ

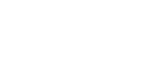

는

$\alpha \dot{0}-\dot{\Sigma}$

$00=0$

$\therefore=\pi \frac{1}{0}$

$\cos ^{2}+2$

牙幺

乞

$\simeq \therefore 9 z$

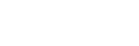

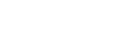

mm $\sim \Sigma=$

$m-\ln 0 \hat{n}$

om๊นณ

믈.

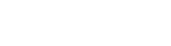

- z岀亡。

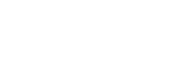

- ㅇ́ma z

मำำ

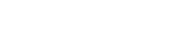

arian in

os in $: 2$ a

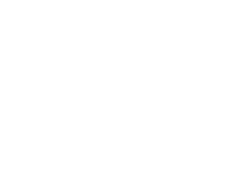

드

$=\frac{2 \pi}{5}$

$=0 \cos$

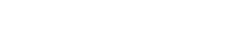

0 正品

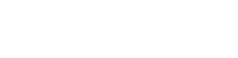

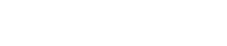

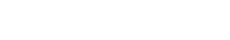
ono noino in

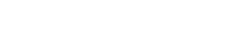

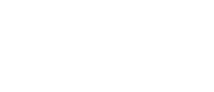

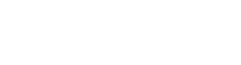

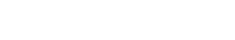

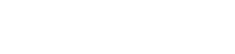

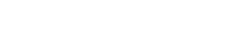

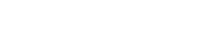

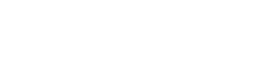
z七. 2020

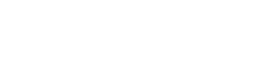
出 $w$ w w !ct

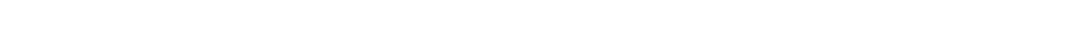

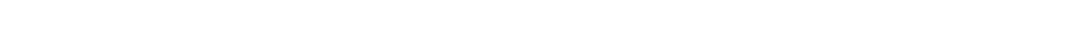

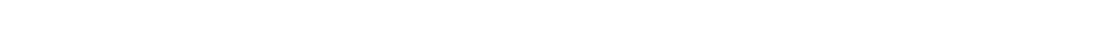

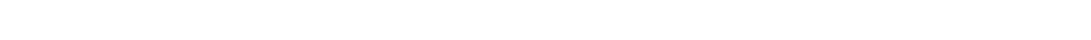
QCF-F

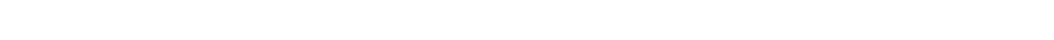

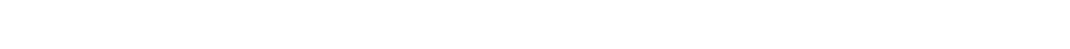

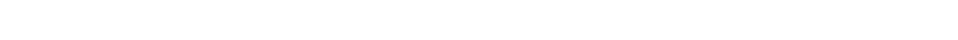

$$
\begin{aligned}
& \text { is }
\end{aligned}
$$

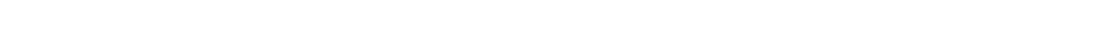

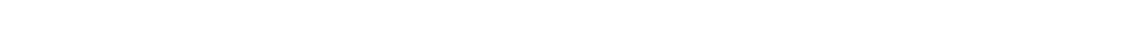

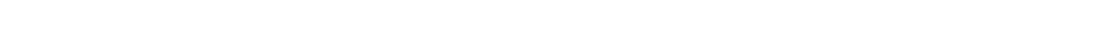

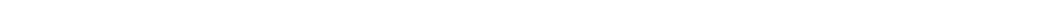

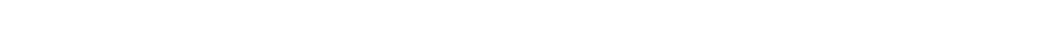

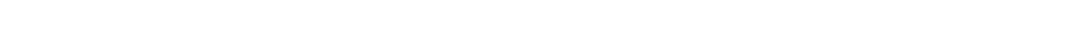

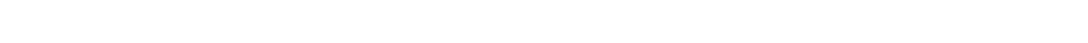

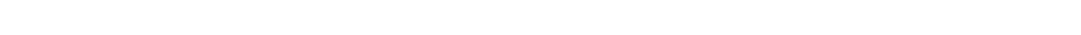

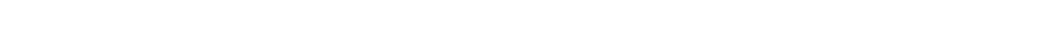

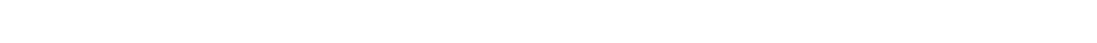
is

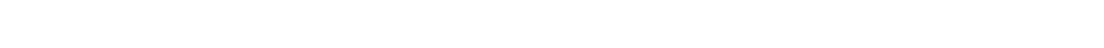
แ प w

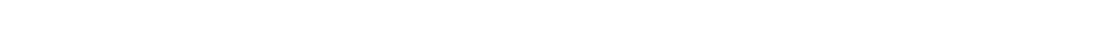

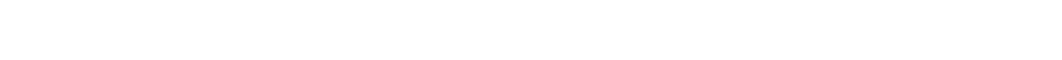

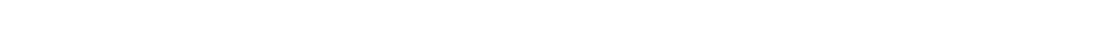

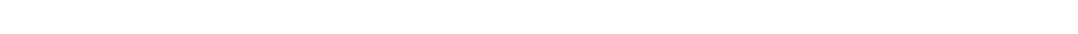

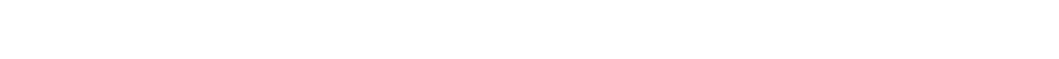




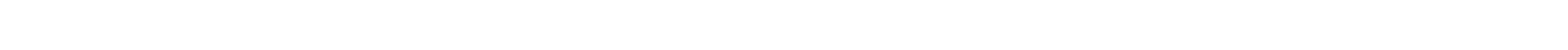

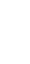

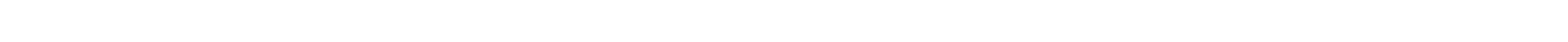
w a w

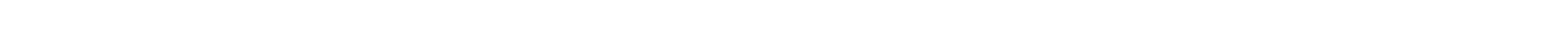

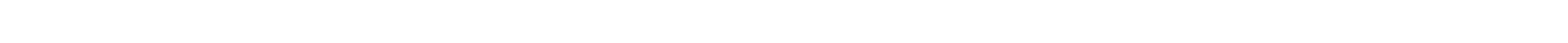

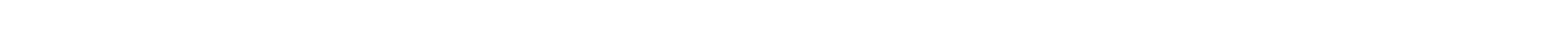
ᄂ oN NN

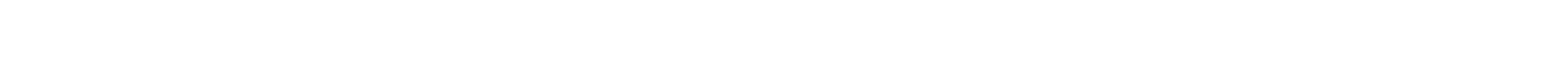

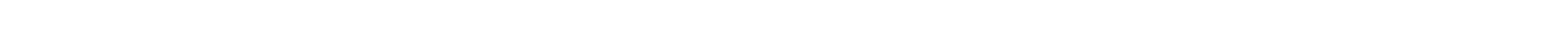

a жш 니

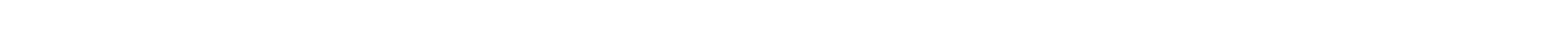

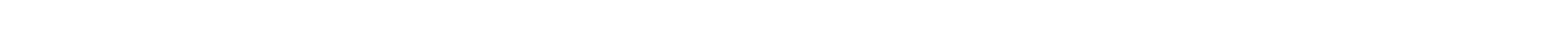
ñ แ

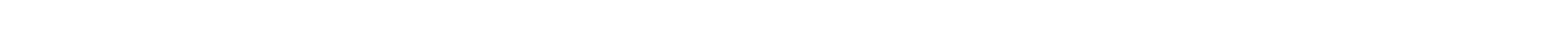

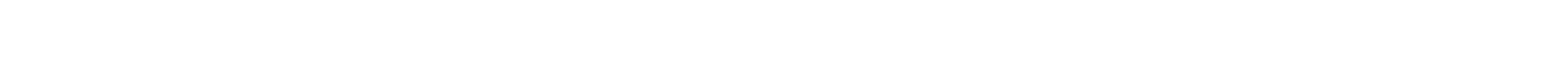

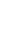

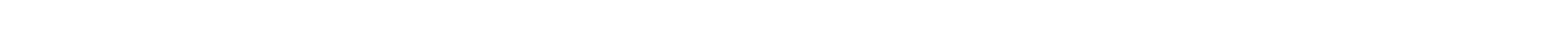
w

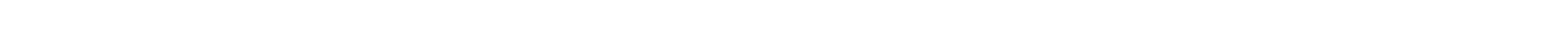

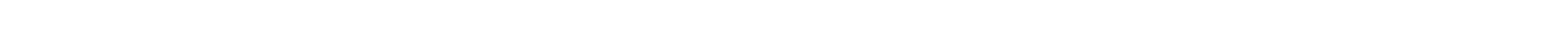

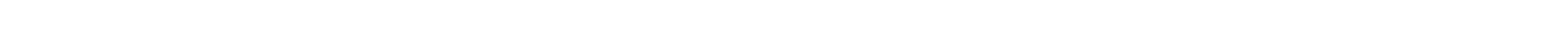

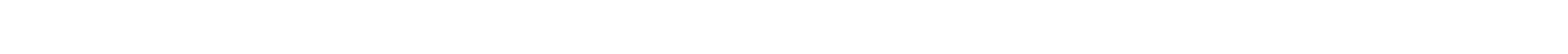

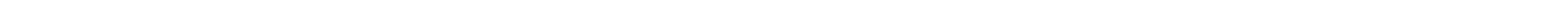

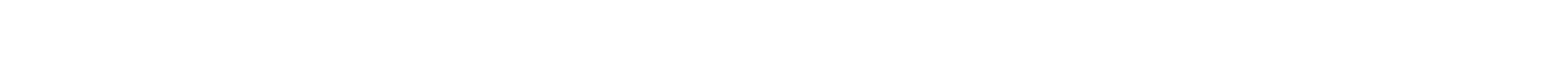

as

in en en in en

$\stackrel{a}{\leftarrow}$

nomm H L

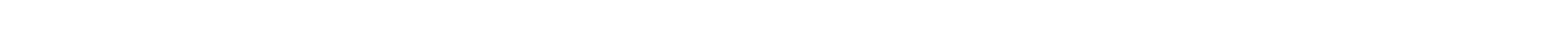

mmmmmmmmmmmmmmmmmmmmmmmmmmmmmmmmmmm

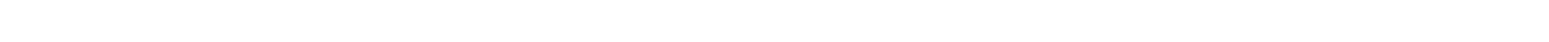
w -

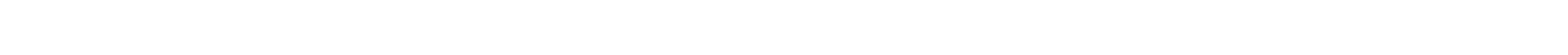

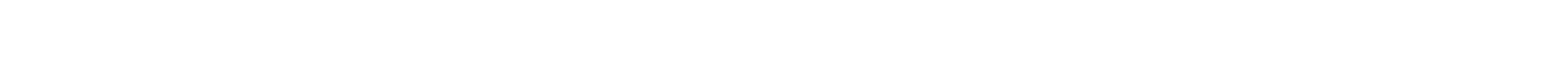




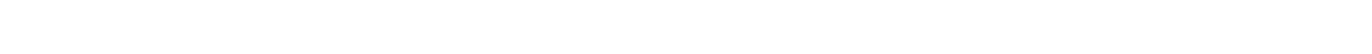

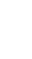

к-

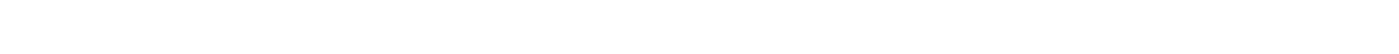
a un on or o o or

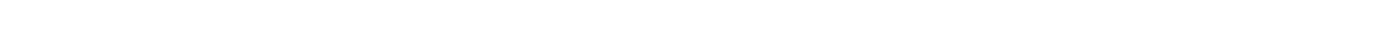

OOO 20 이의

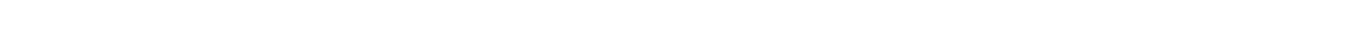
a

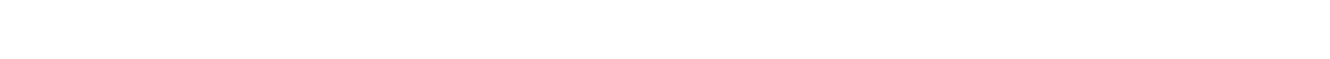

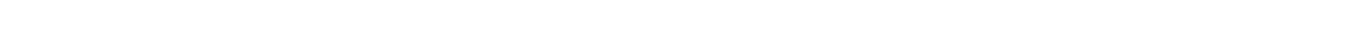

$\approx$

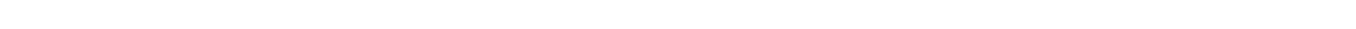

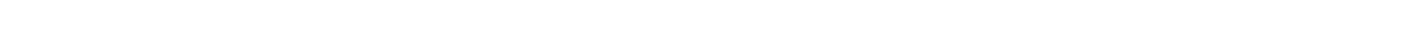
$\omega$

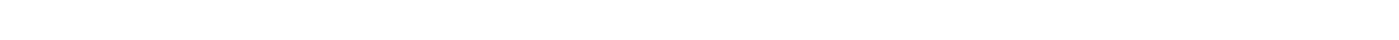

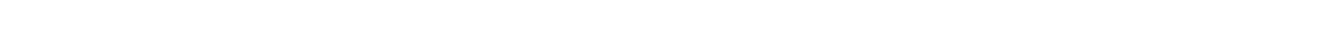

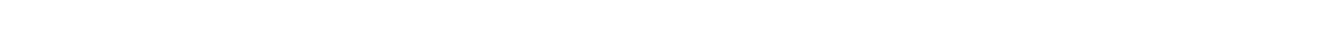

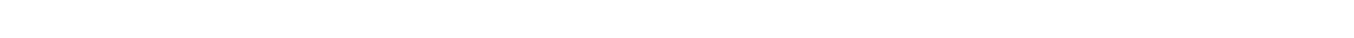

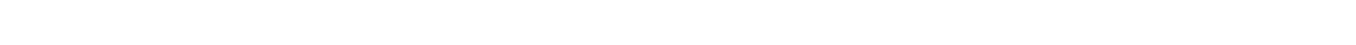

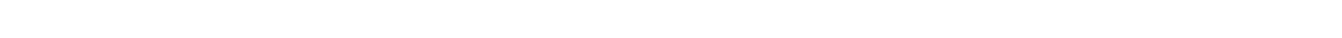

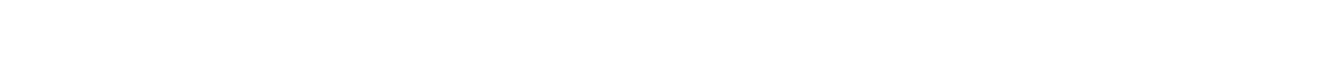<smiles>[111In]</smiles>

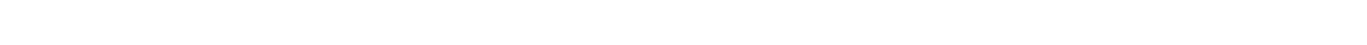
$\leftarrow$ in

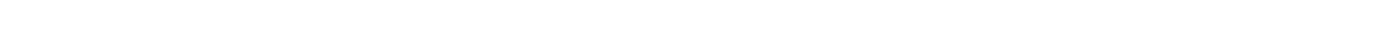
ж山 "M

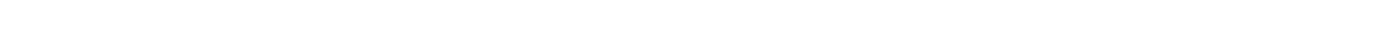

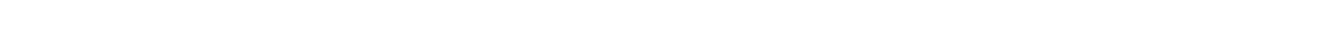

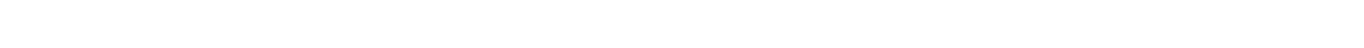

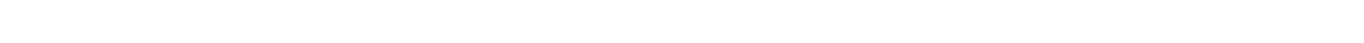

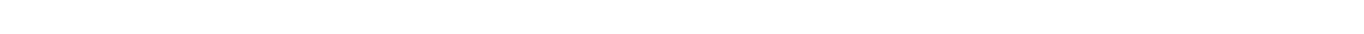

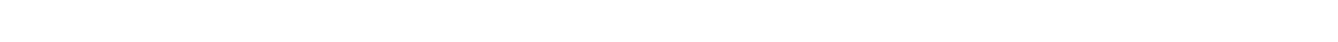
స̃ó

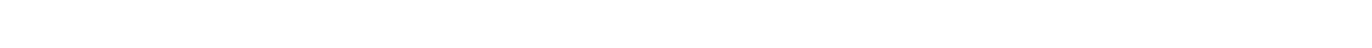
is

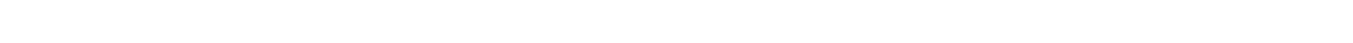
o 2 m ü wm a w w

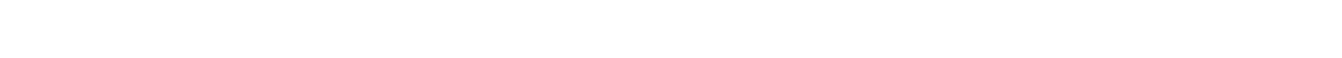

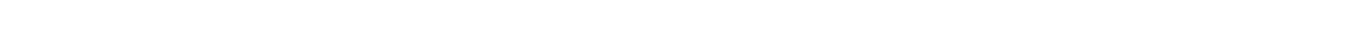

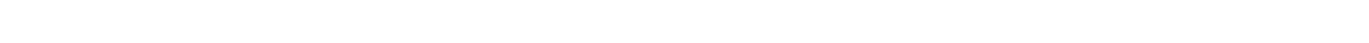

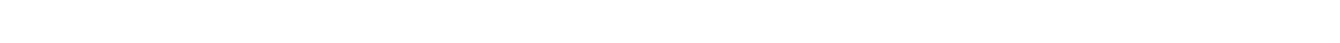




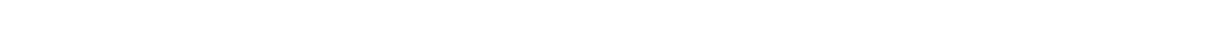

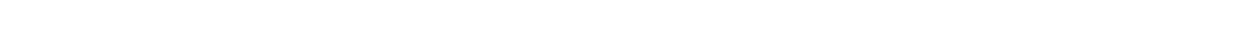
-

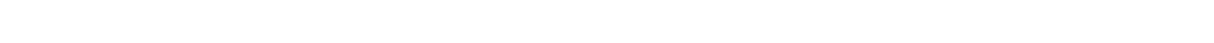

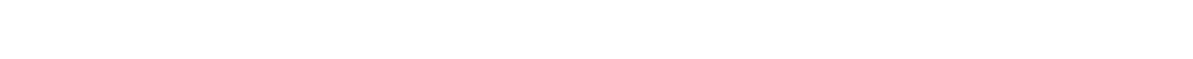

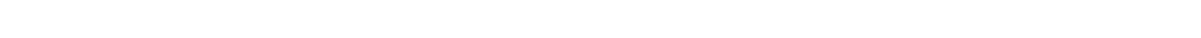

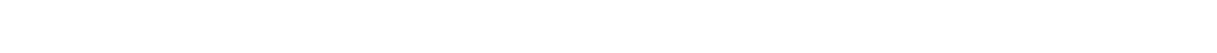

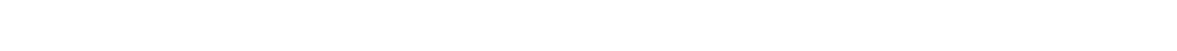

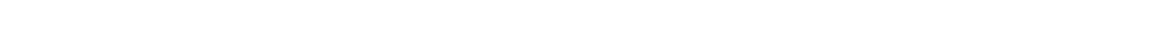

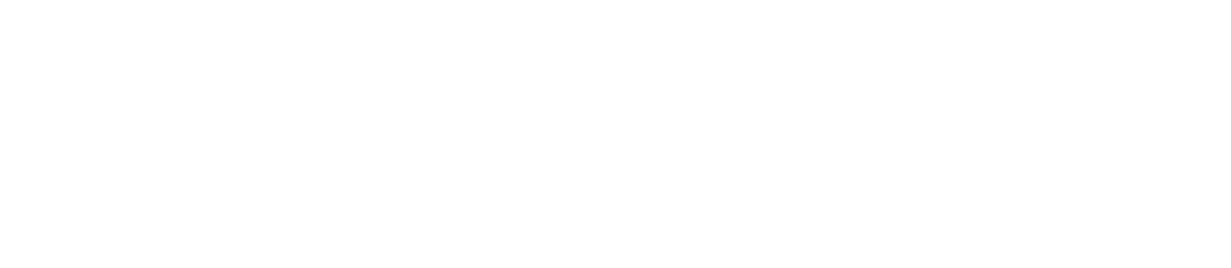

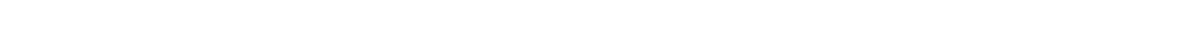

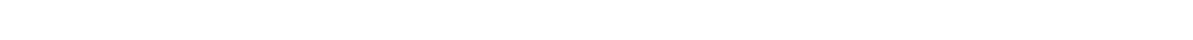

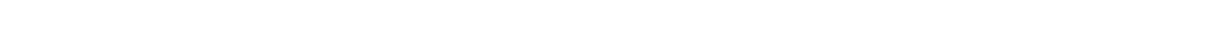

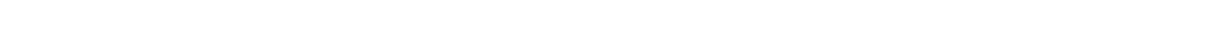

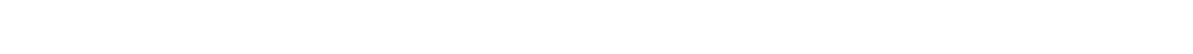

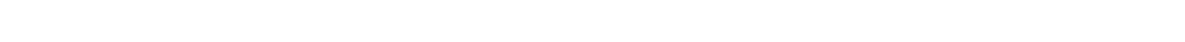

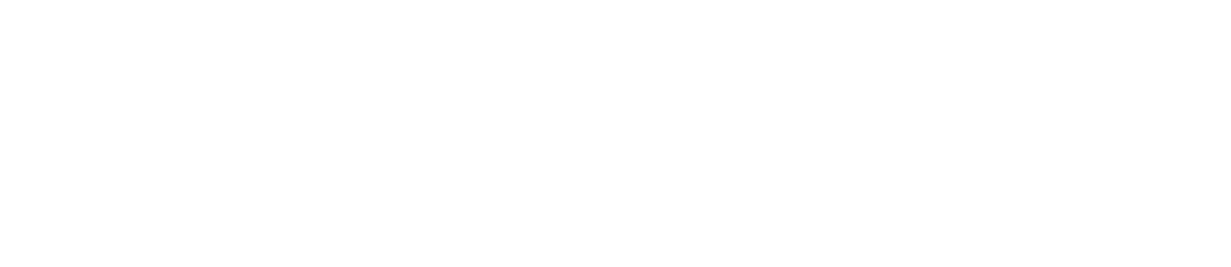

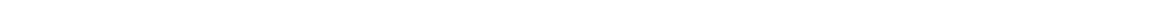
un w in in

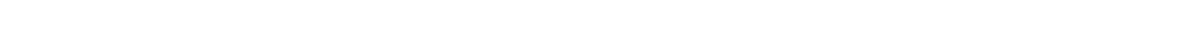

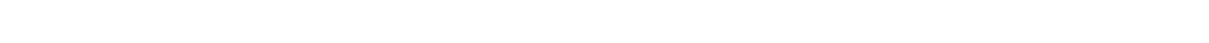

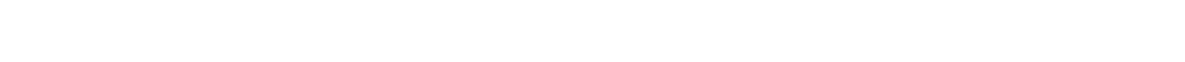

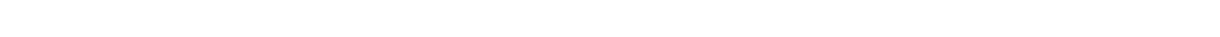

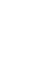

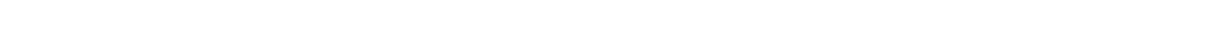

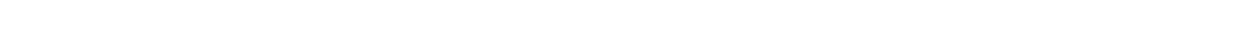

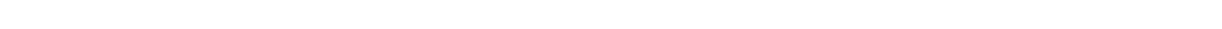

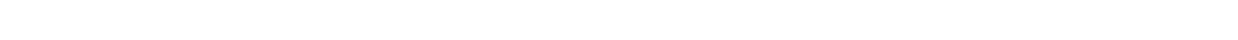

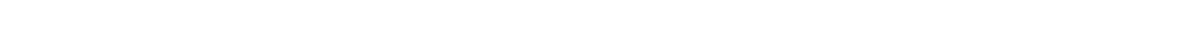

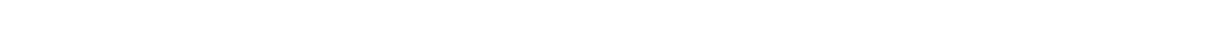

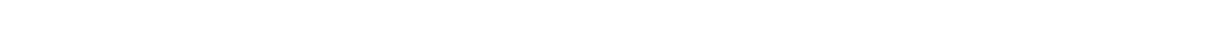

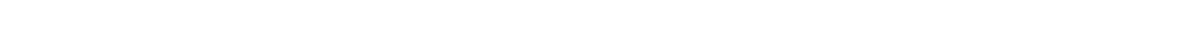

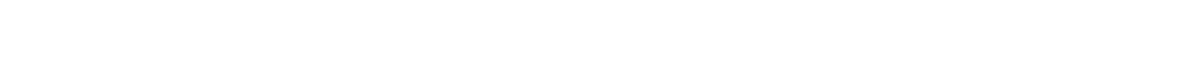




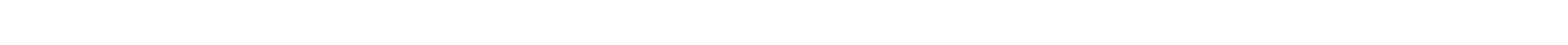
is

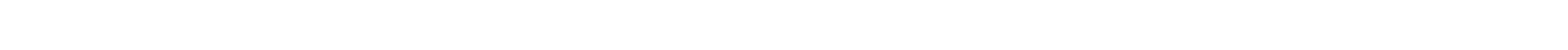

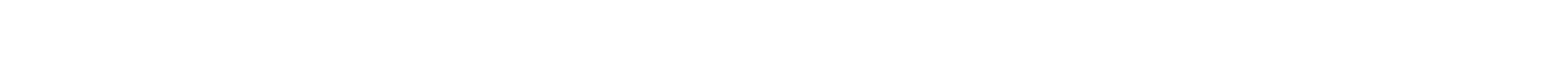

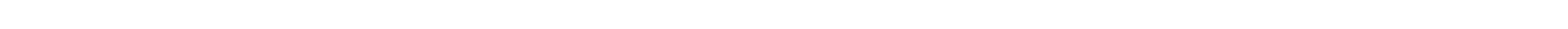
sing

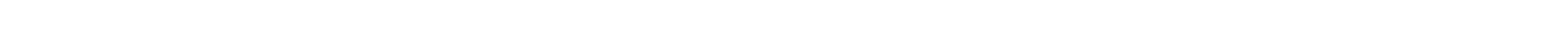

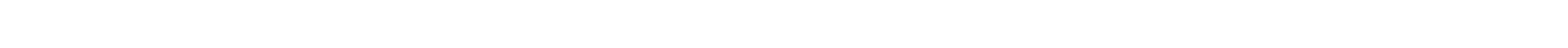

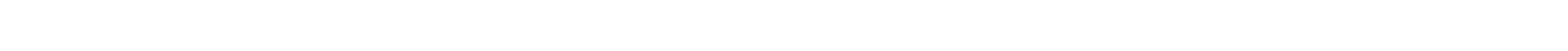

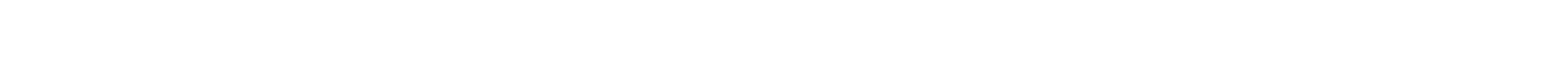

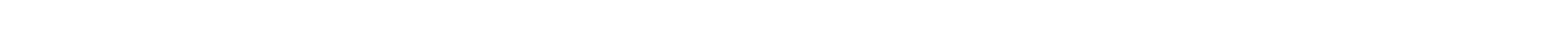
a

s

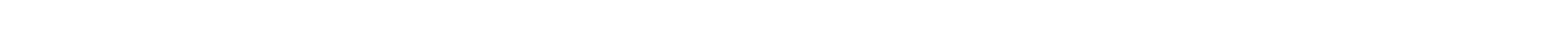

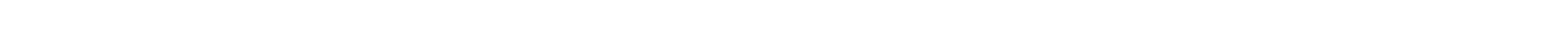

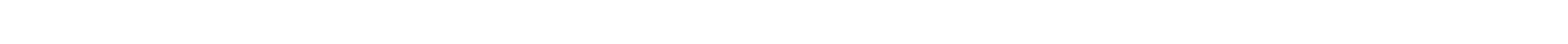

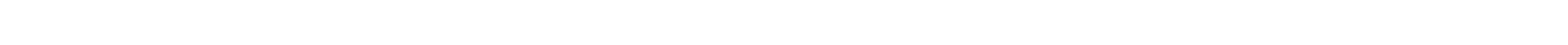
-

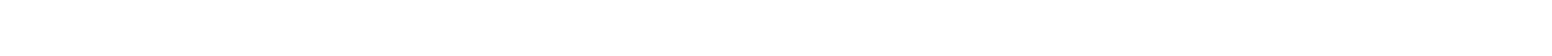

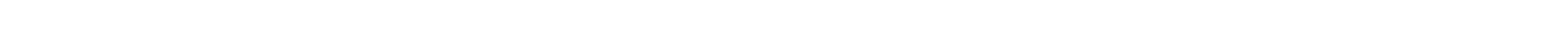

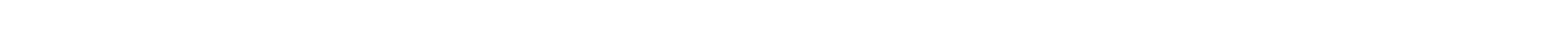

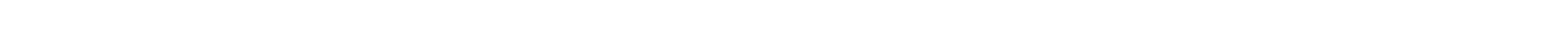

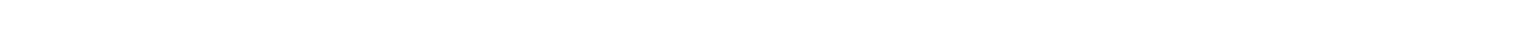

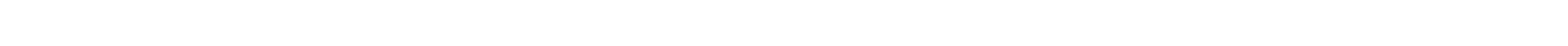

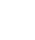

in

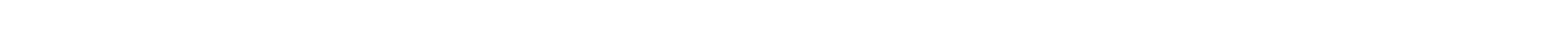

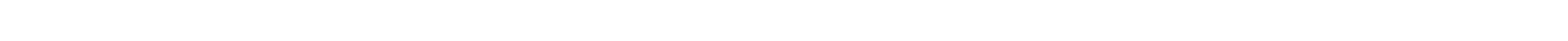

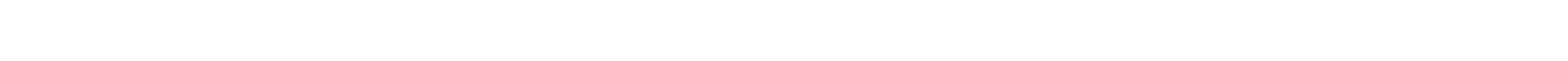

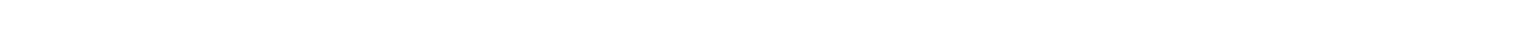

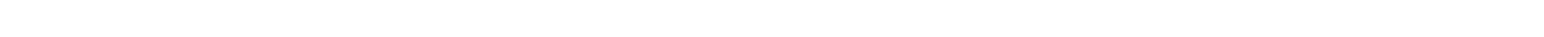

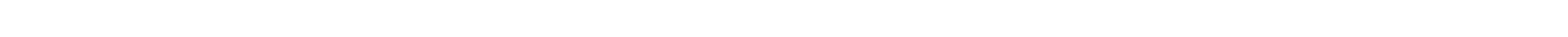

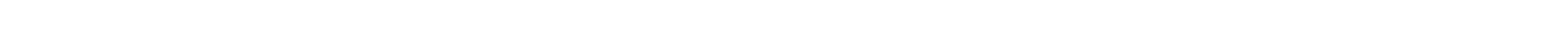

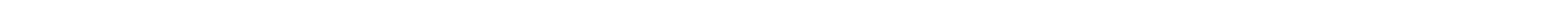

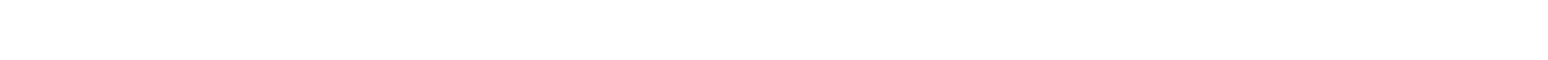

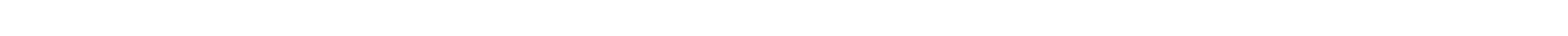

$\underline{5}$

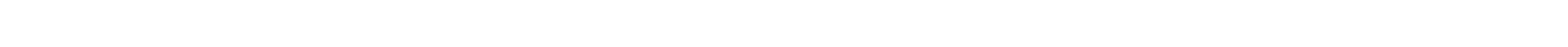

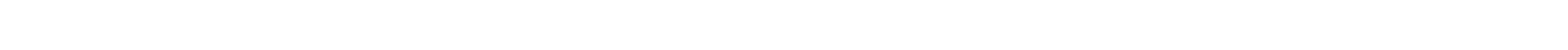
a

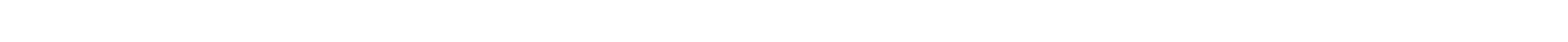

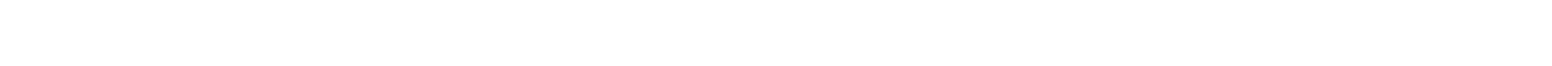

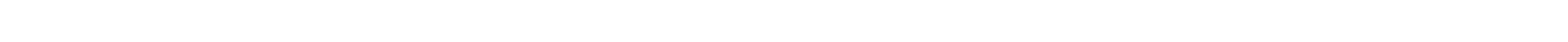

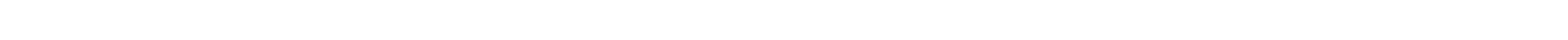

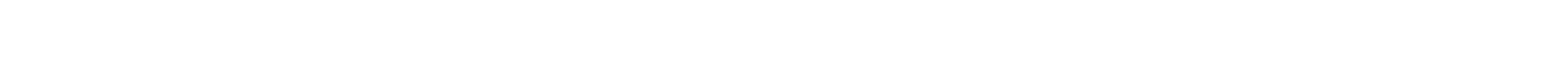




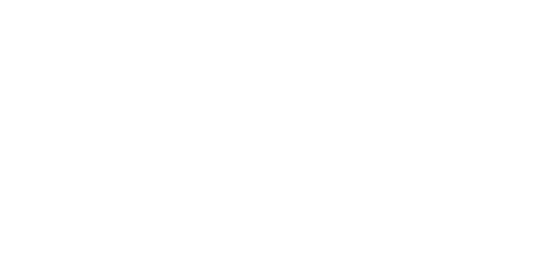

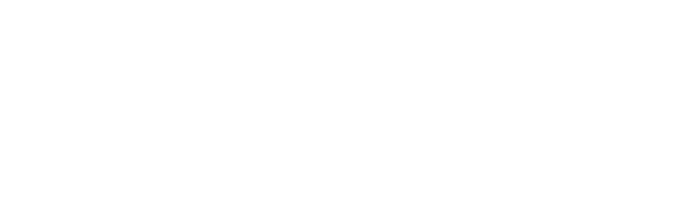

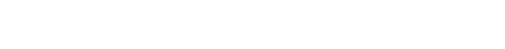

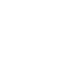

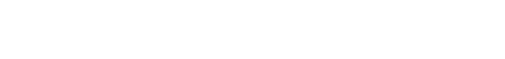
ow

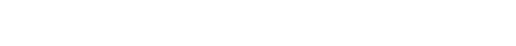

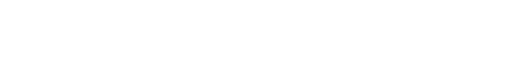

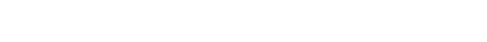

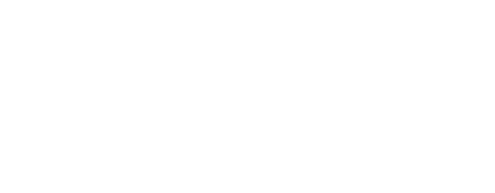

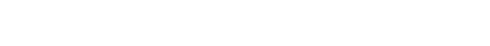
$\Sigma$

\&

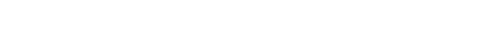

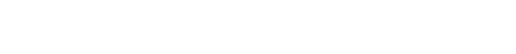

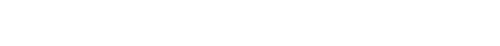

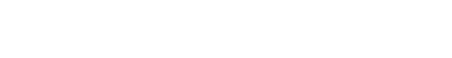

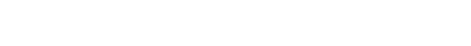

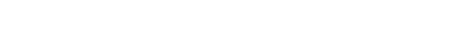

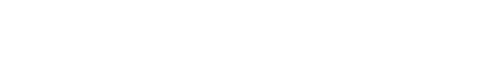

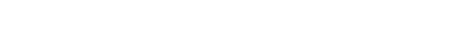

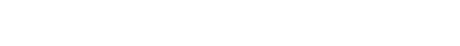

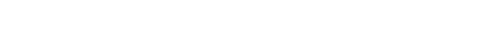

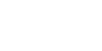

व

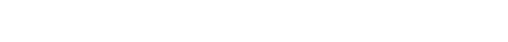
ungmmm s

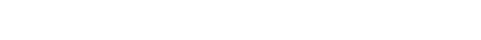

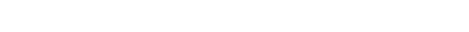

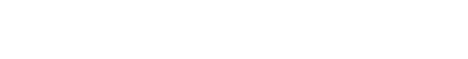




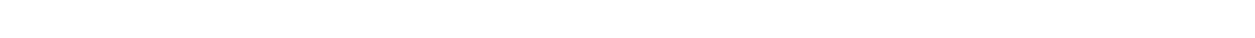

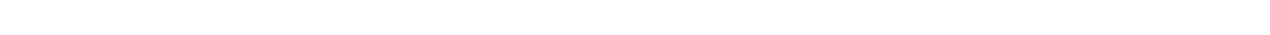

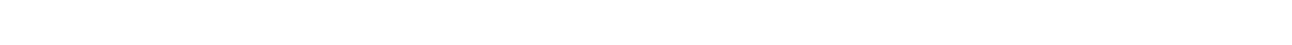

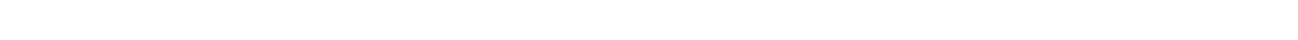

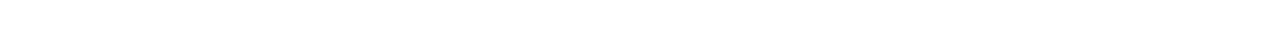

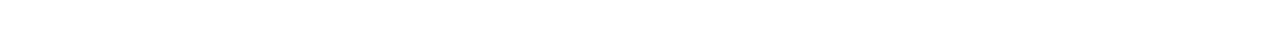

$\stackrel{-}{2}$

$\underset{2}{2}$

$\stackrel{x}{\sim}$

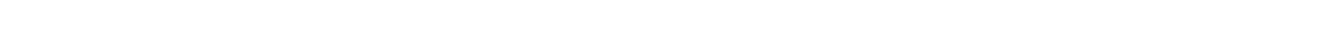
w

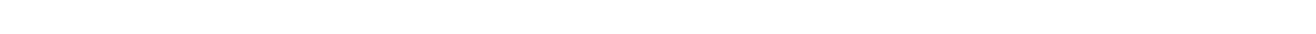
30 ind

ion

$-\Sigma$

$\underline{w}=\underline{m}$

는

זm⿻

20

$\rightarrow 0: 09$

든

和는

"1.

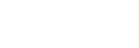

등ㅎㅇ.

ב.․․․

正这行

品。立上

더울

종요

4 이요

䵝上是

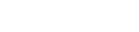

$20 \%$

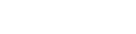

zm保少

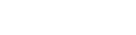

.

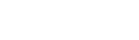

$20 、 .1$

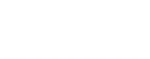

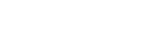

J $2 \dot{a}$ in

a

₹ $\geq 0=$

인

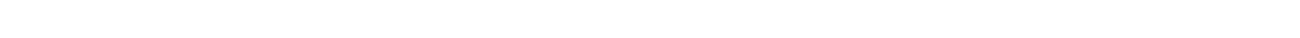

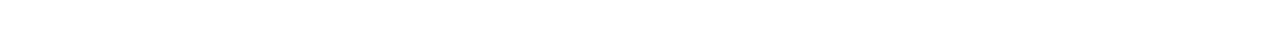
แ士

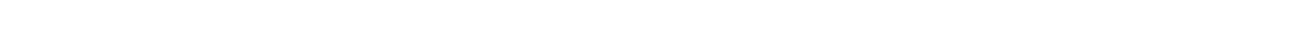

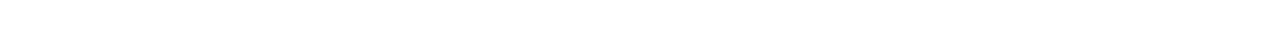

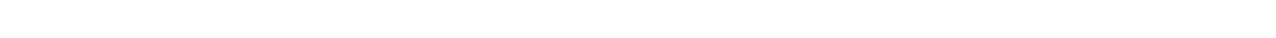

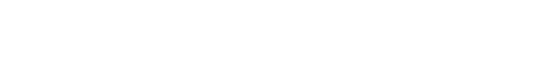

$\stackrel{\square}{\square}$

$\therefore$

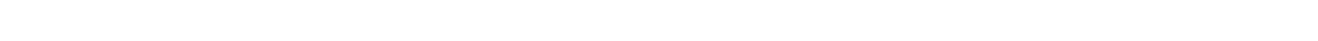

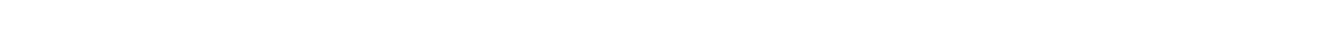

-

$\ddot{i} \stackrel{\sigma}{z}$

in

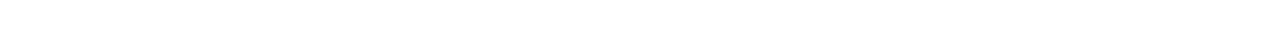

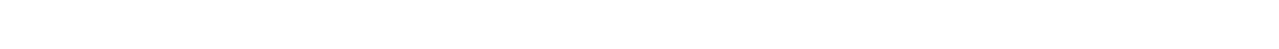

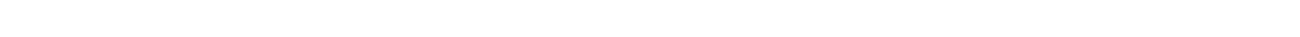

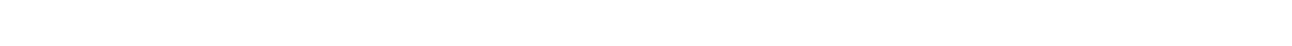
O

o

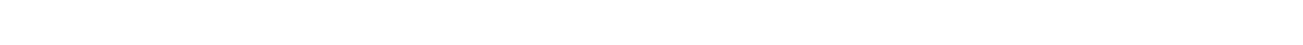

$<$

in

눙

a:

"O- 0 ommong:

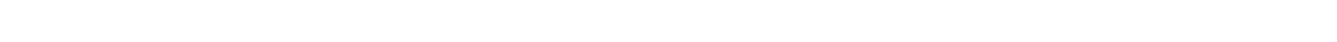
L $\sim \ddot{w}-$ उั:

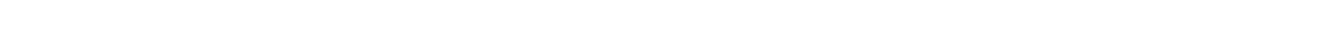

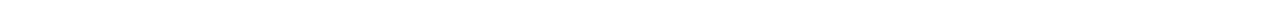

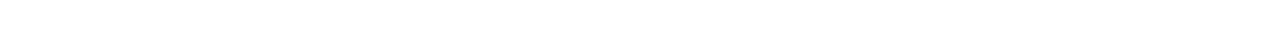

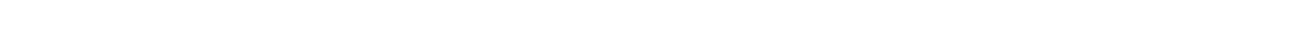

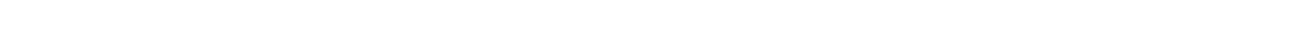

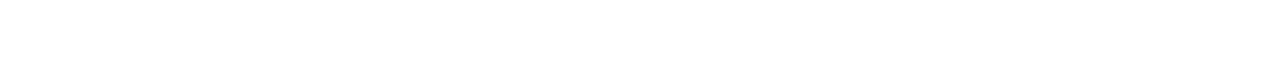




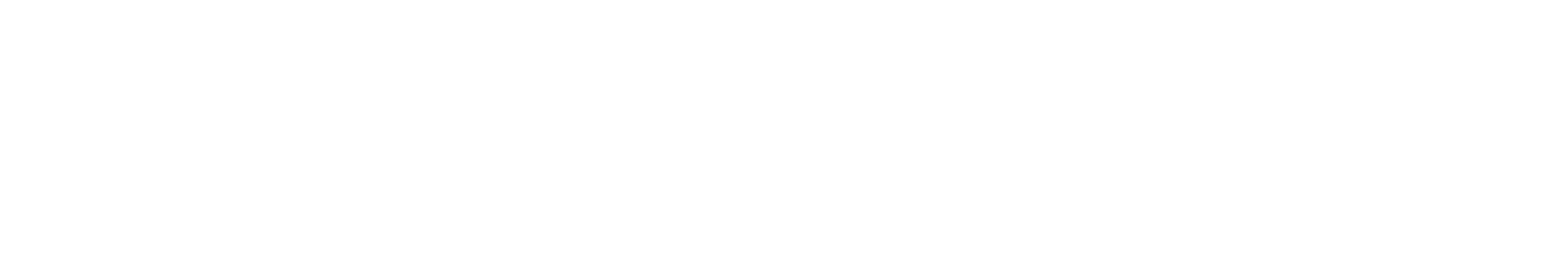

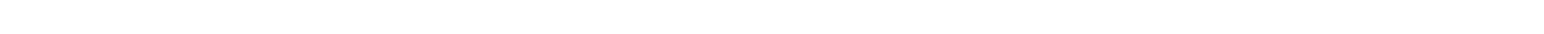

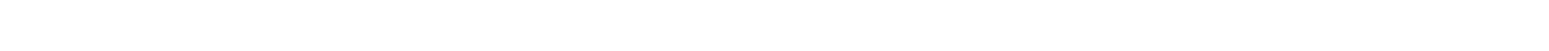

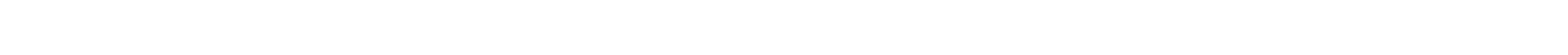

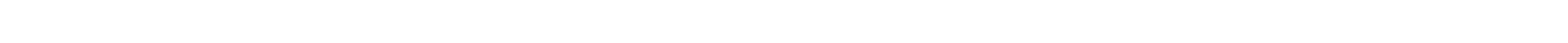

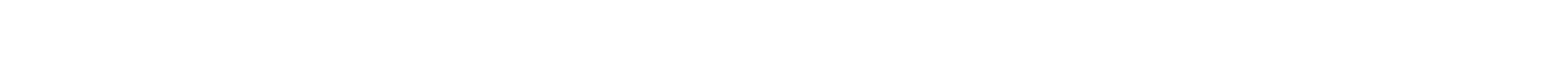

-

荘:

os

n

en

os

en

a

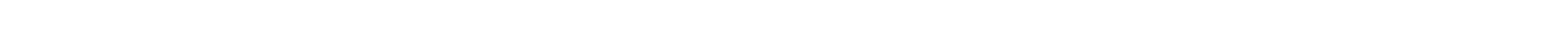

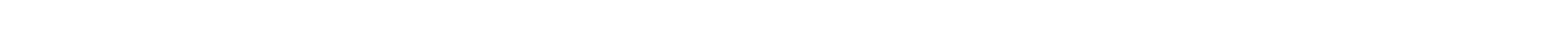

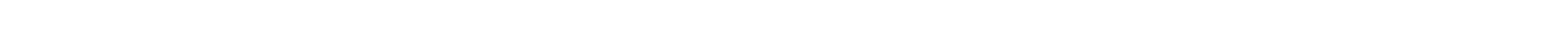

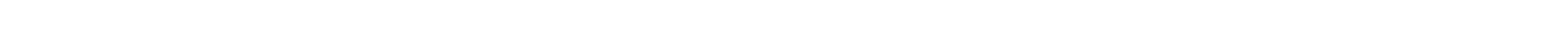

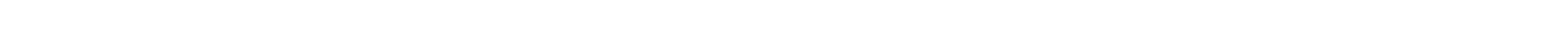
แ

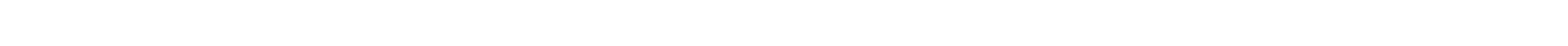

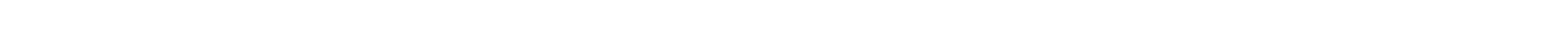

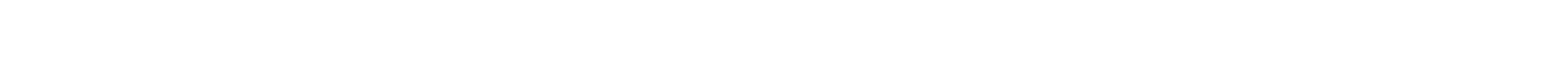

亲

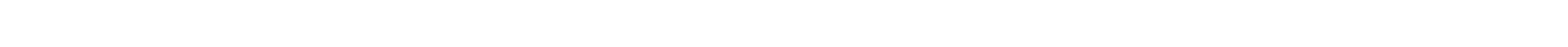

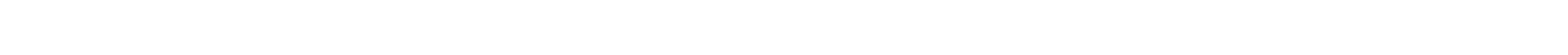

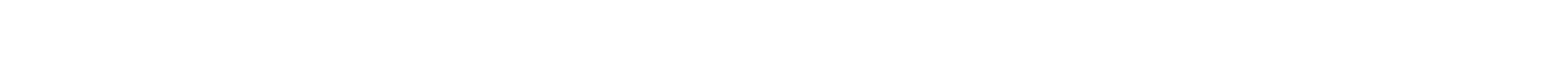

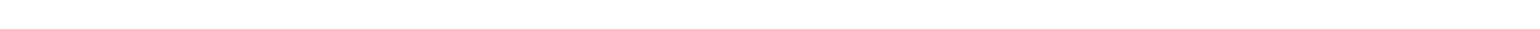

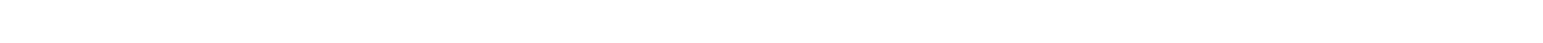

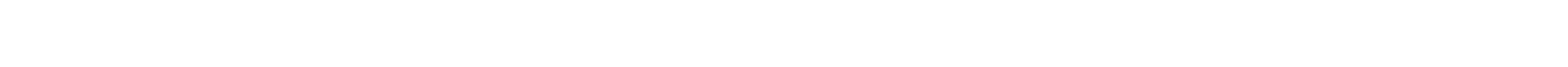

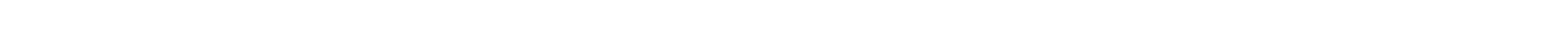

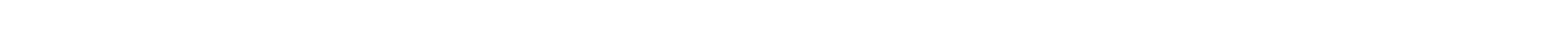

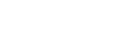

5
is
n
in
n
en
en
is
¿

on

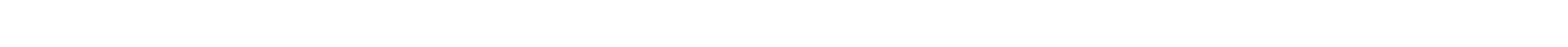
แ

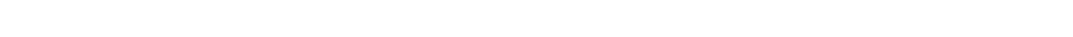
a

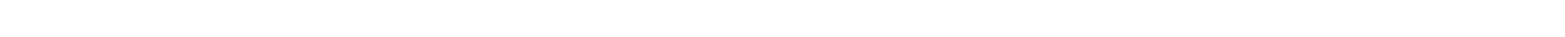

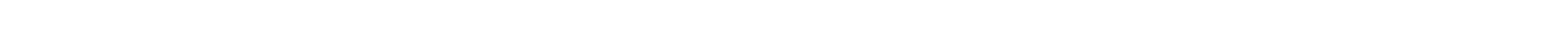

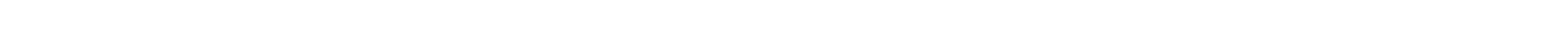

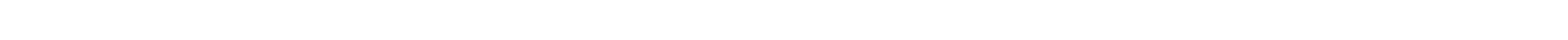

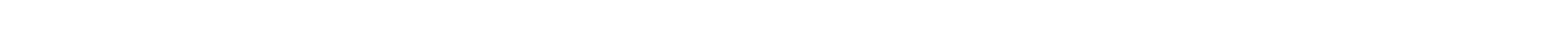




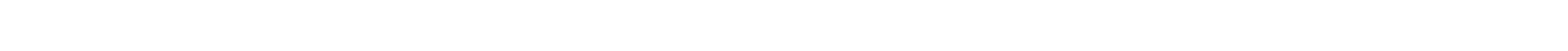

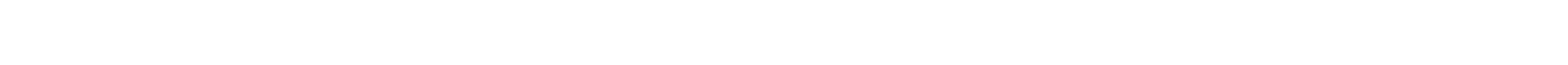

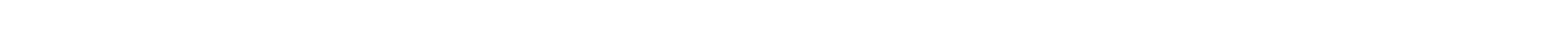

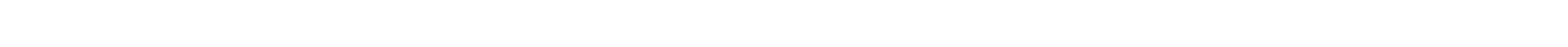

mon m W

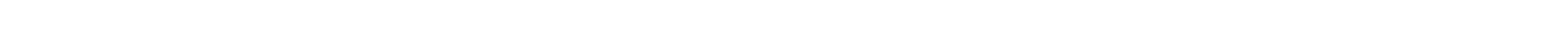
wE

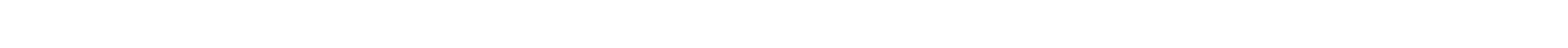

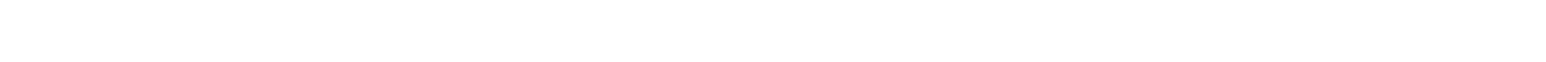

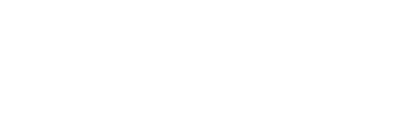

๔

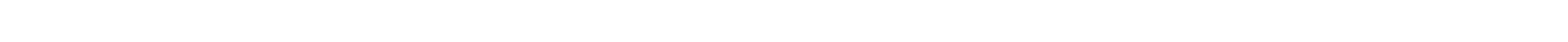

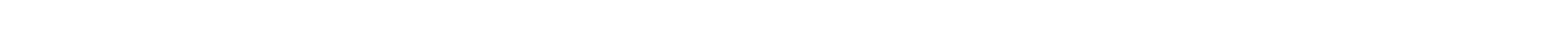
然

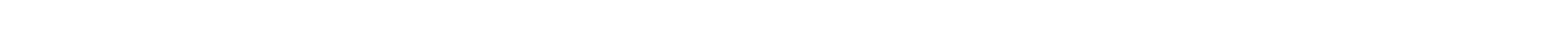

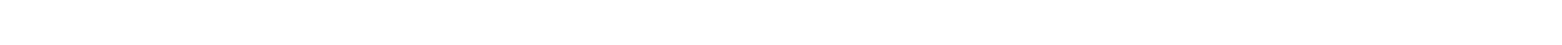

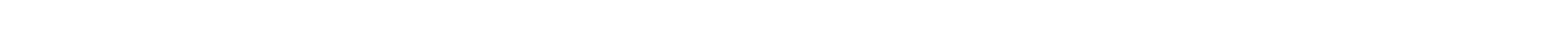

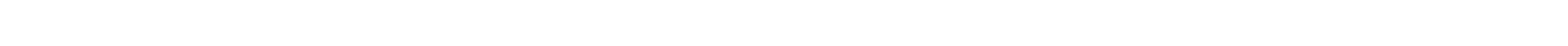

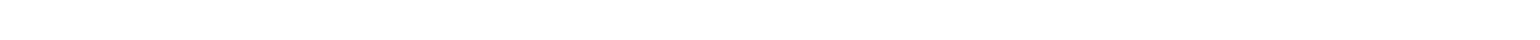

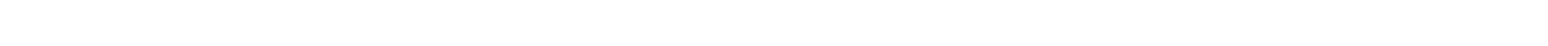
w n
a
n
es
n
es
is

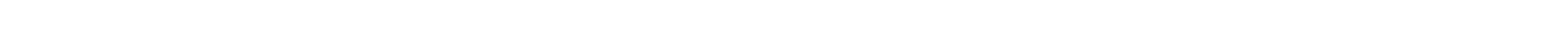

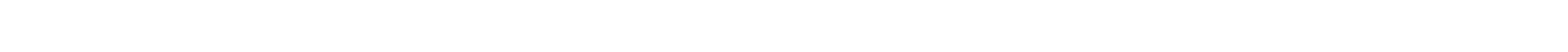
व ×-

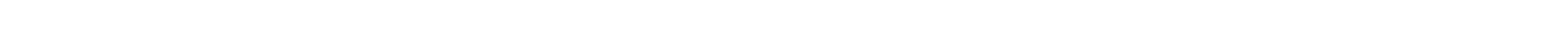

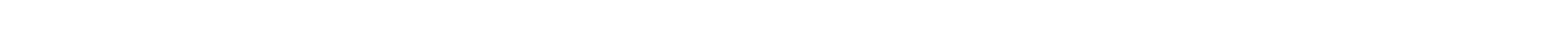

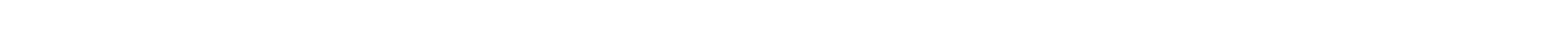

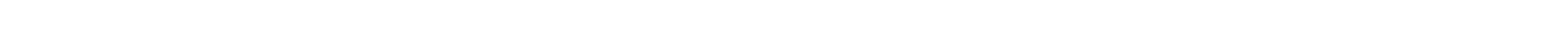

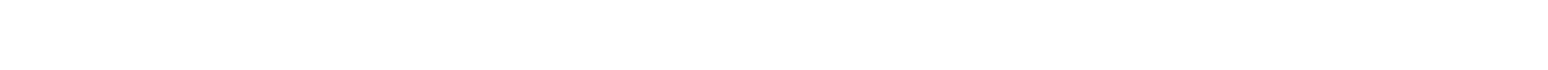

$\approx$

" u. $\dot{0} \dot{0} \dot{0} \cdot \dot{0} \cdot \dot{0} \cdot \dot{0} \dot{0} \dot{0} \dot{0} \dot{0} \dot{0} \dot{0} \dot{0} \dot{0} \dot{0} \dot{0} \dot{0} \dot{0}$

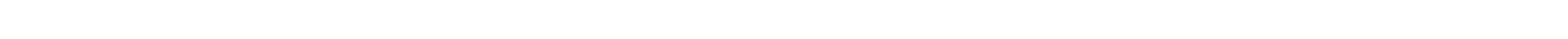

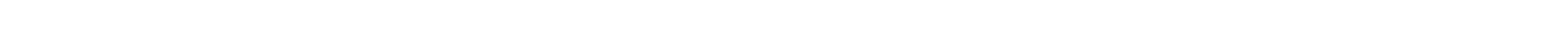

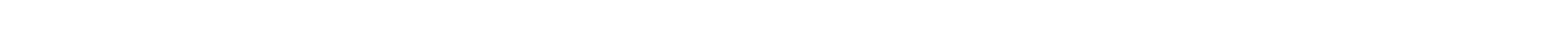

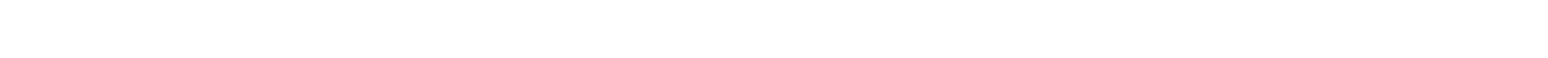

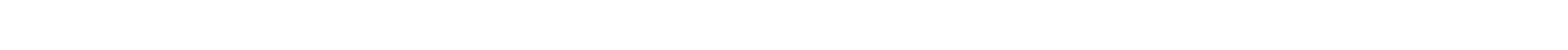

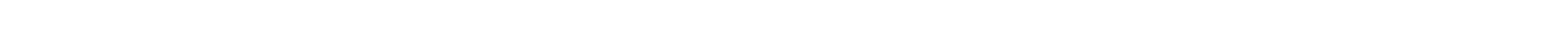

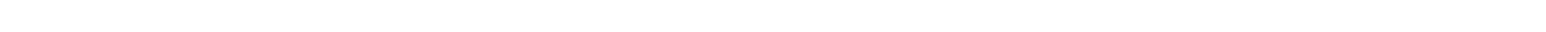

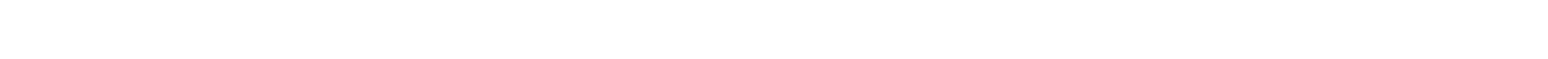




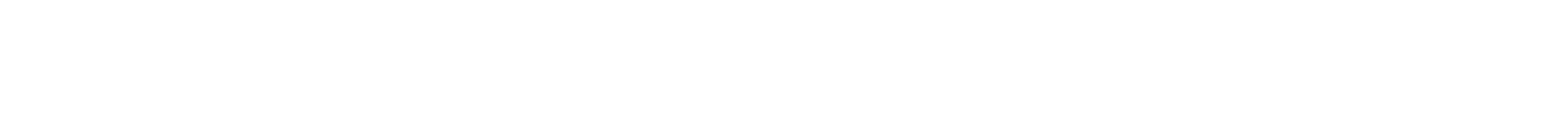

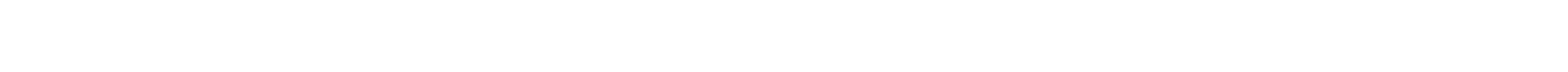

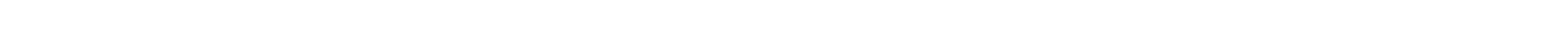

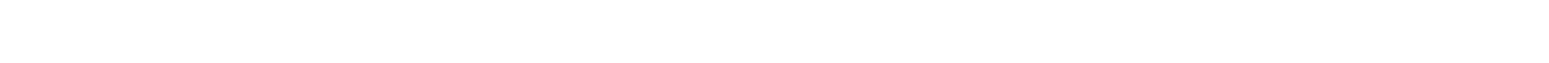

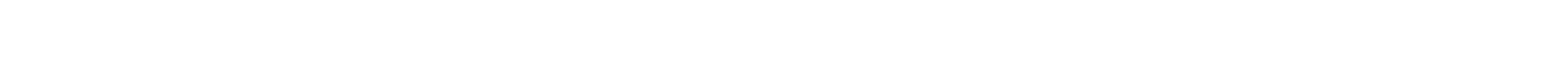

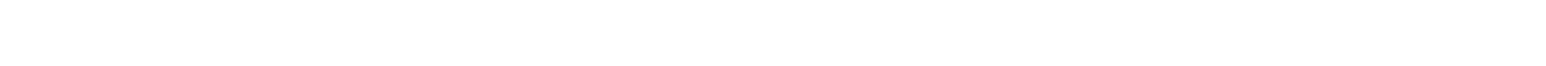

is

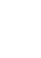

in

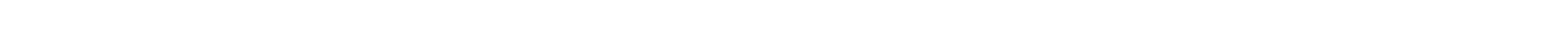
a

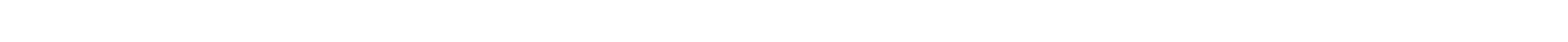

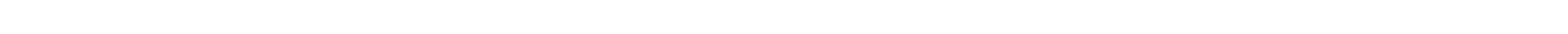

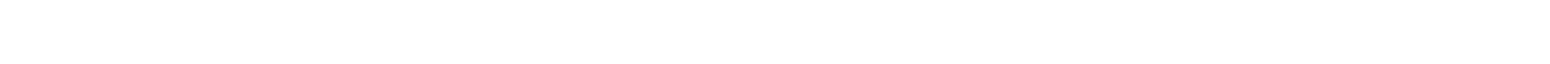

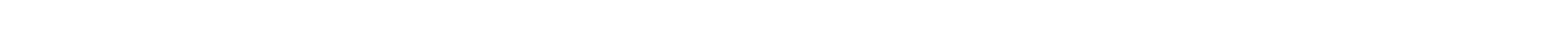

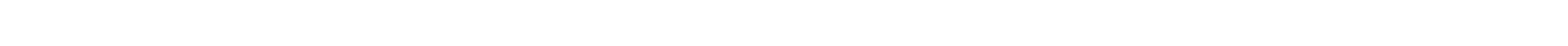

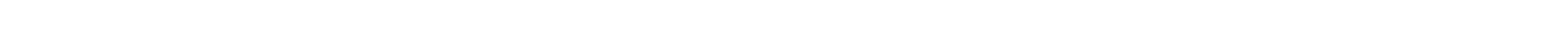

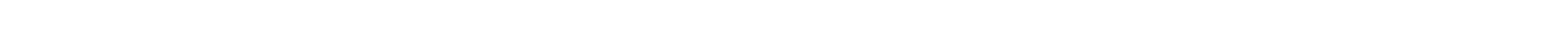

is

an n n

a

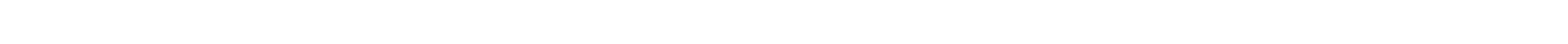
\&

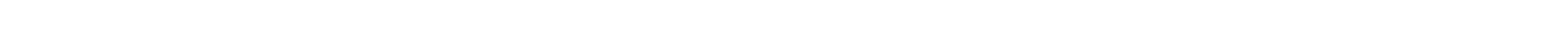

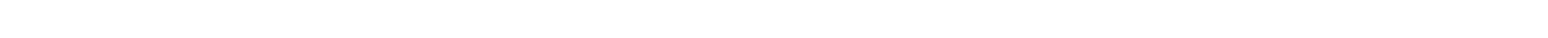
a a 0 a

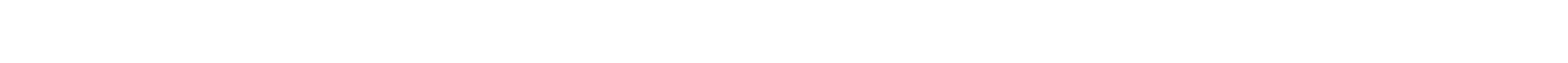
a

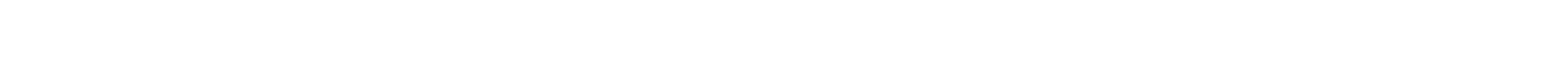

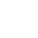

$\mathbf{z}$

$\leftarrow$

แn

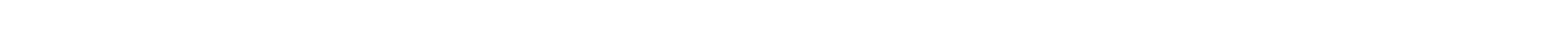

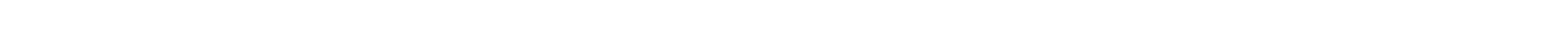
व

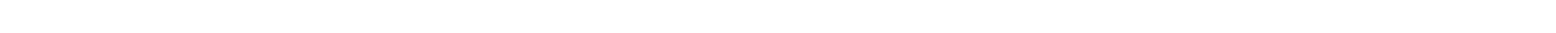

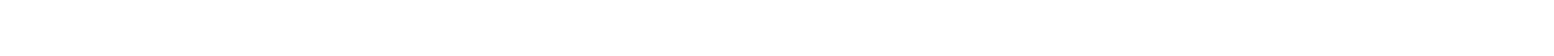
แ a

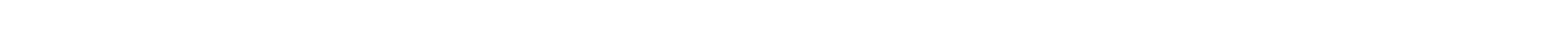
ำ 


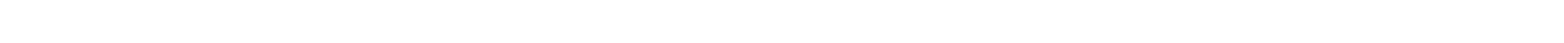

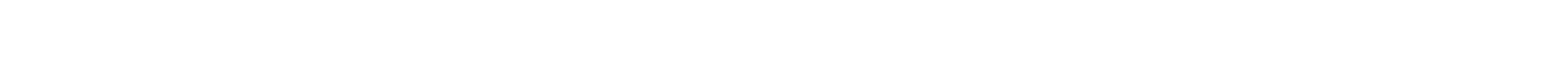

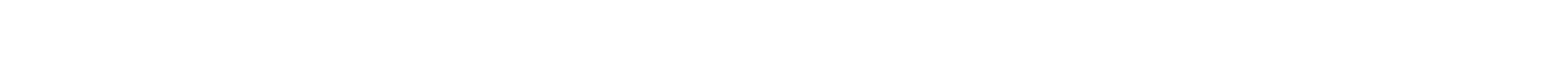

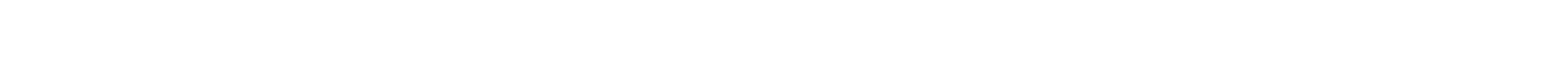

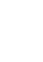

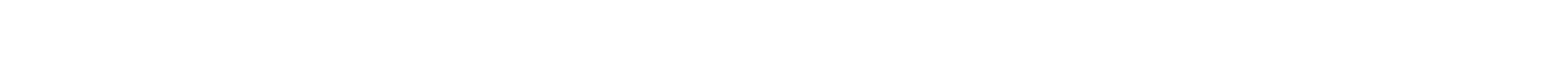

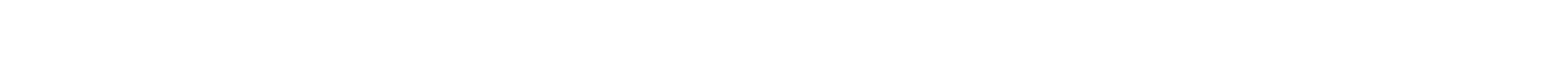

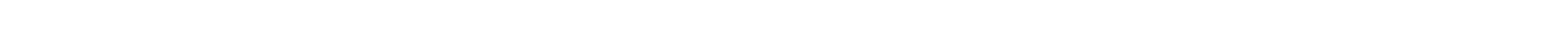

mimmmmmmmmmmmmmmmmmmmmmmmmmmmmmmmmmmmmmmmmmm

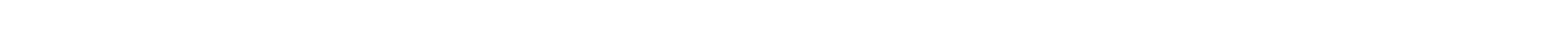

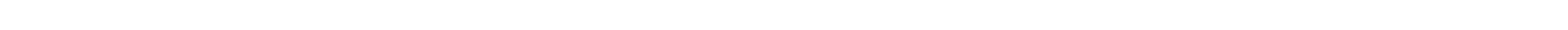

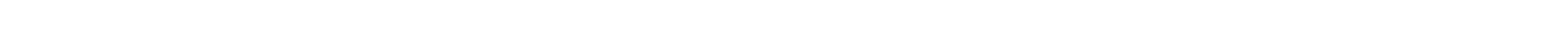

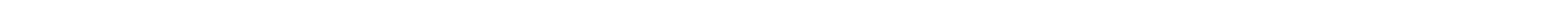

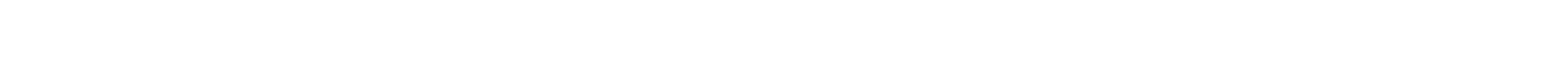

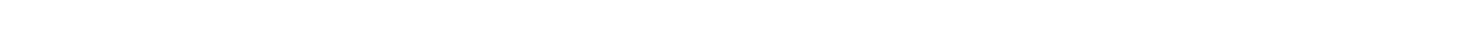

$\stackrel{\pi}{n}$

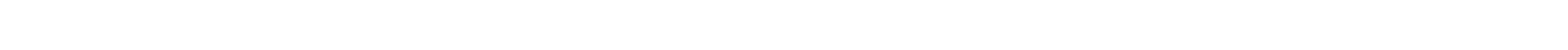

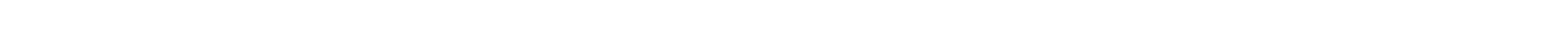

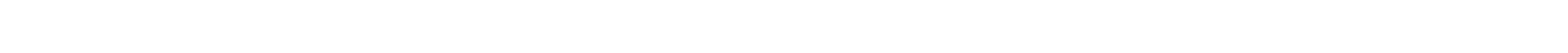

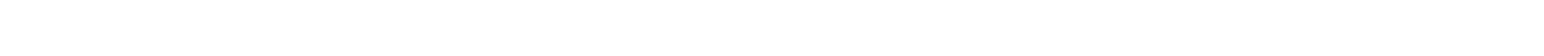

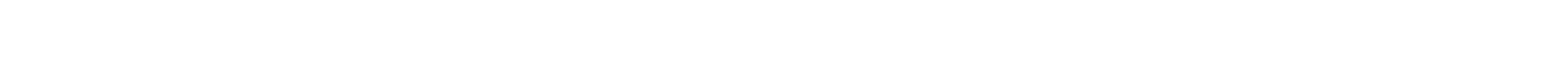
O

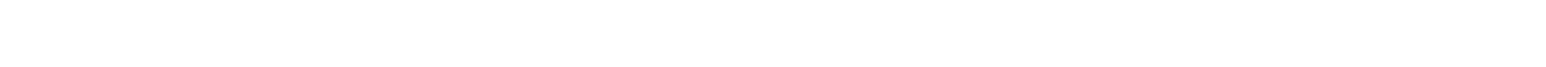

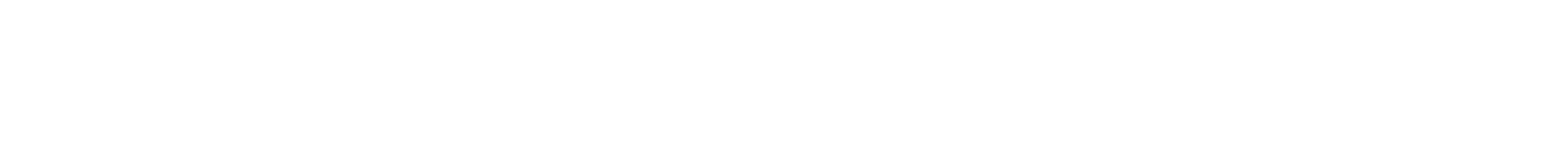

๔

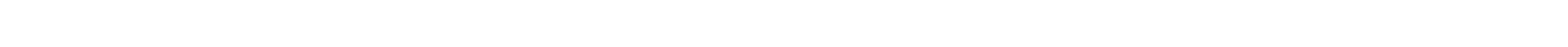

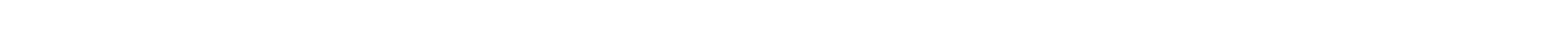

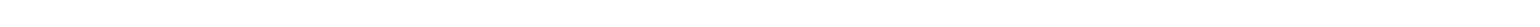

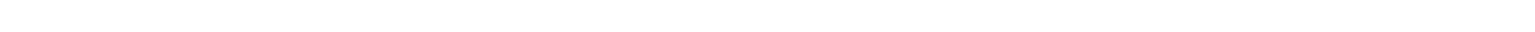

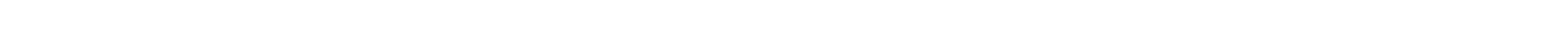
WE-

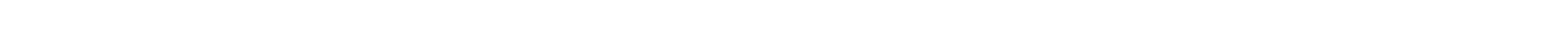
-

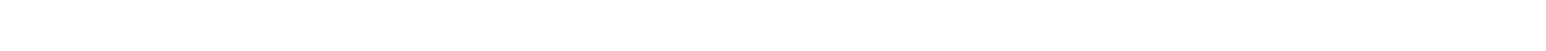




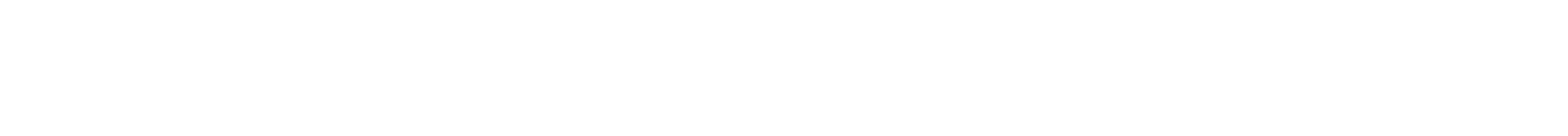

ํำ "

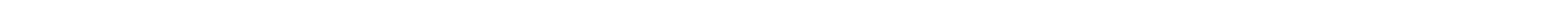

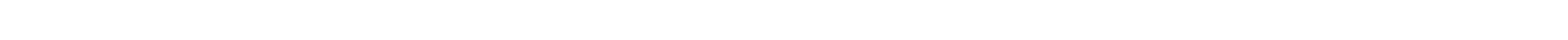

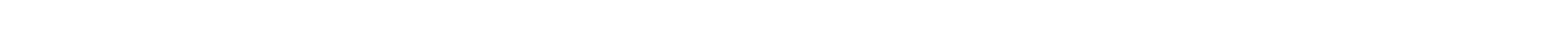

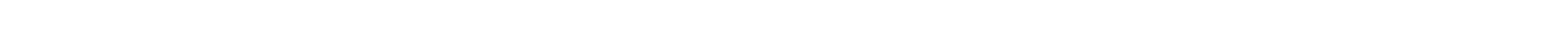
แ

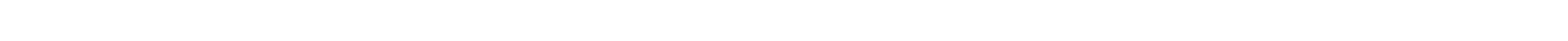

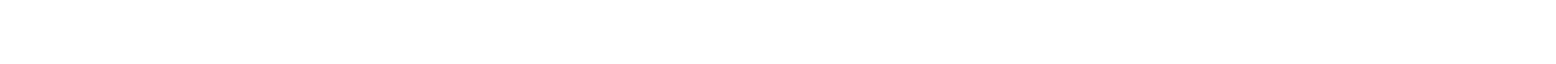

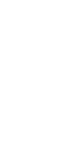

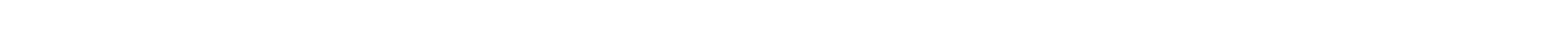

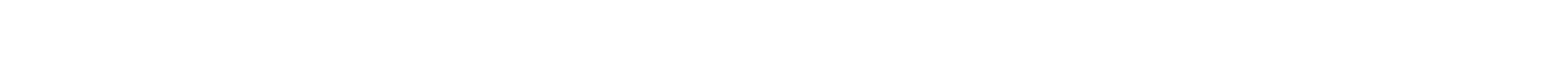

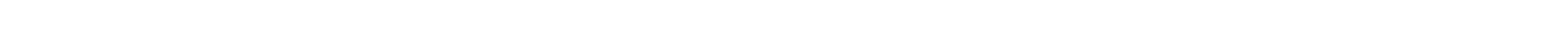

000000000000000000000000000000000000000000000000000

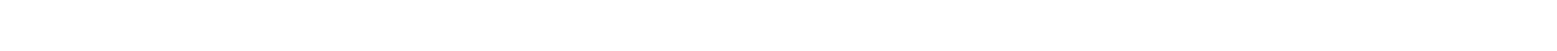

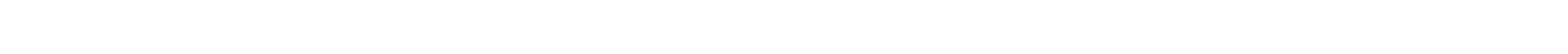

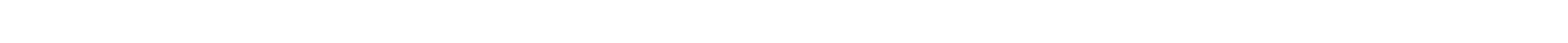

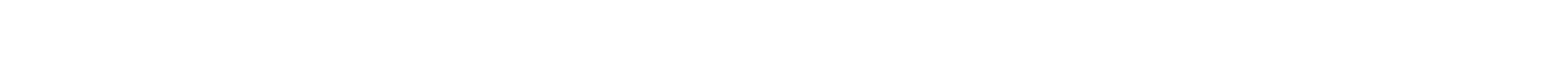
絫的

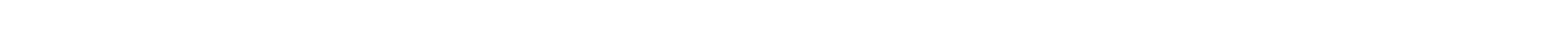
L

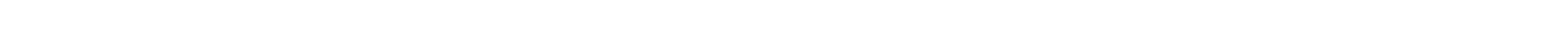

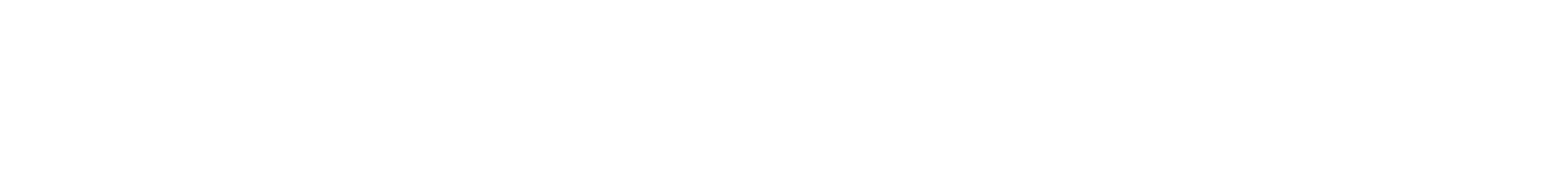
$+$

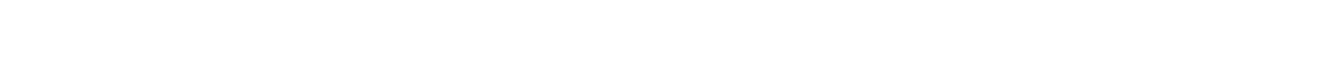

a

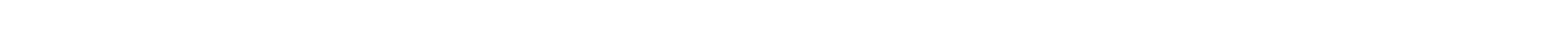

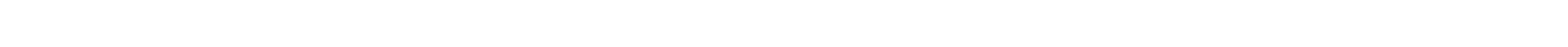

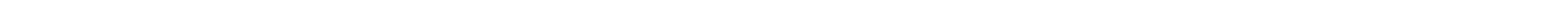

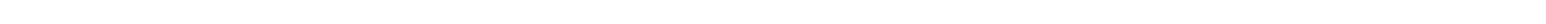

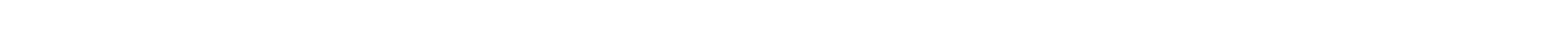

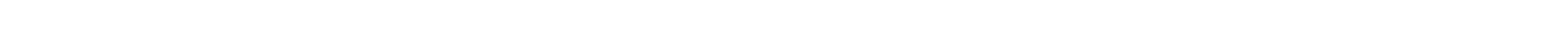


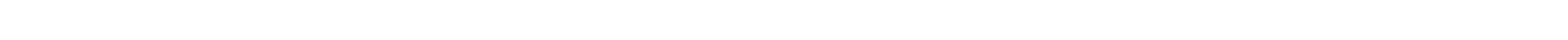

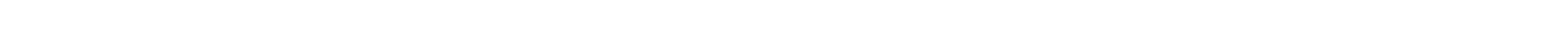

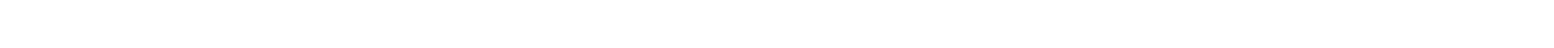




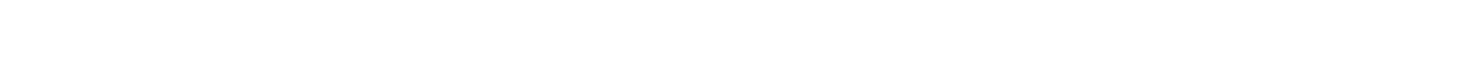

i

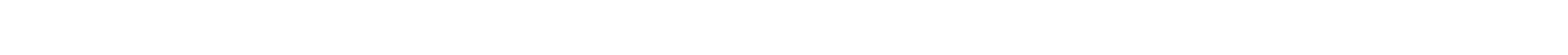

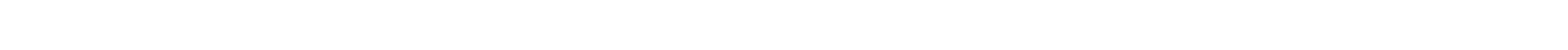
作

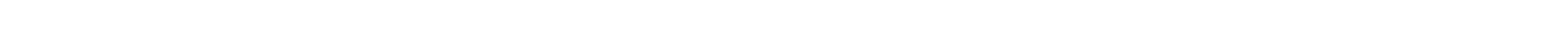

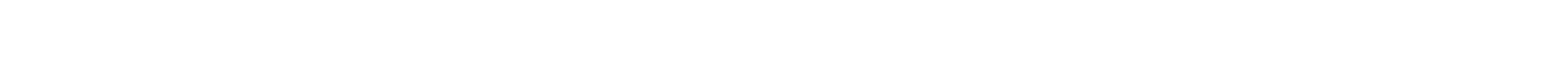

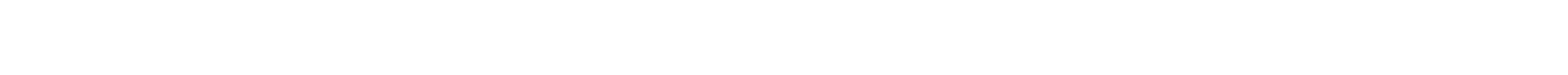

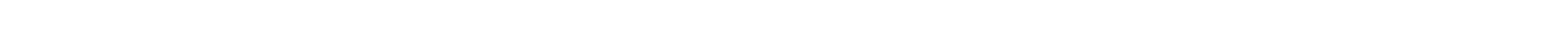

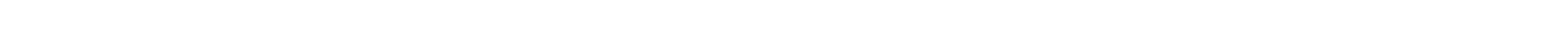

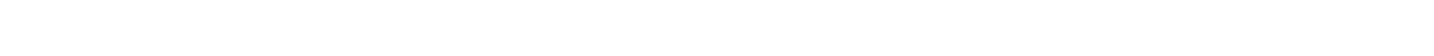

is

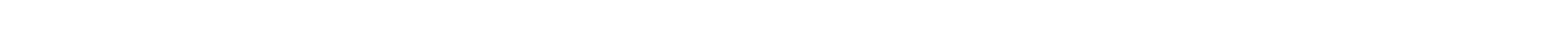
แ

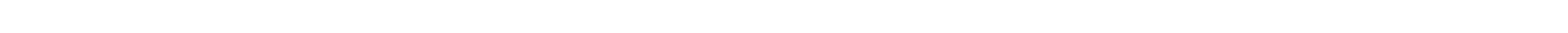
= -

W

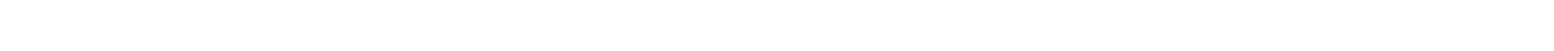

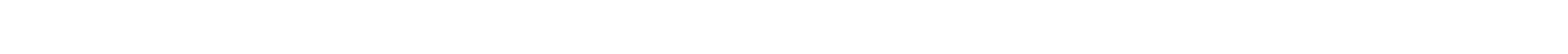

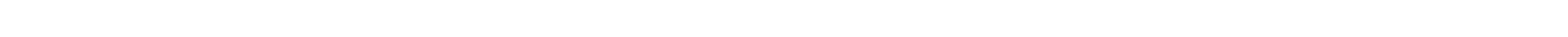

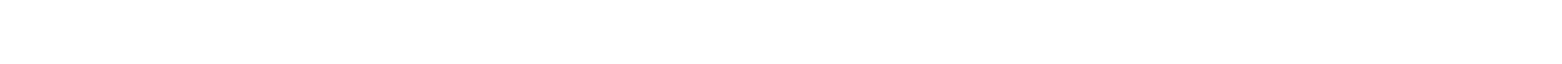
w

盛

is

en

n

in

is

in

en

is

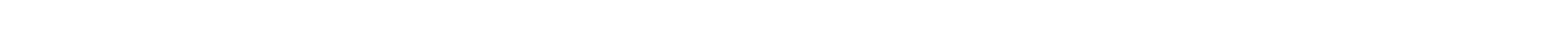
w

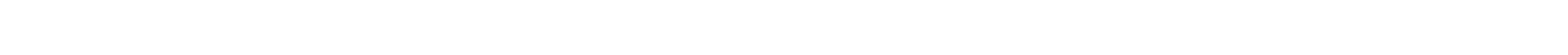

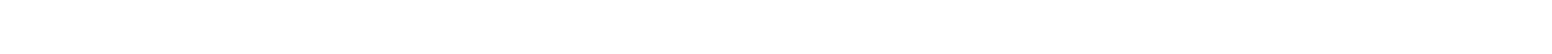

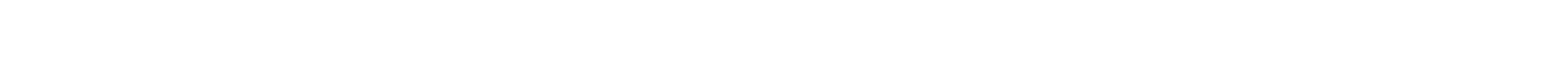

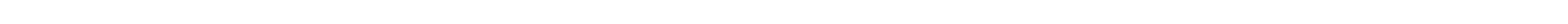

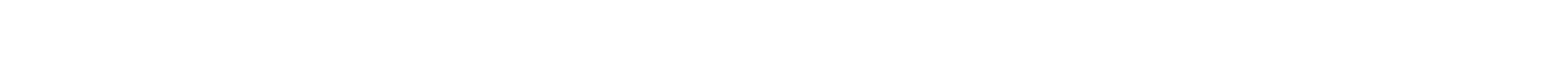

s

$\stackrel{a}{\llcorner}$

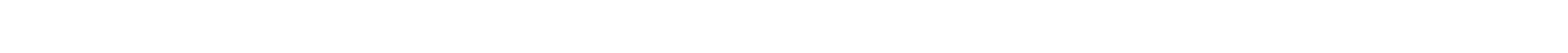

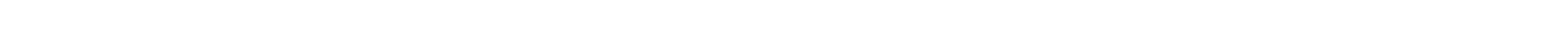
"

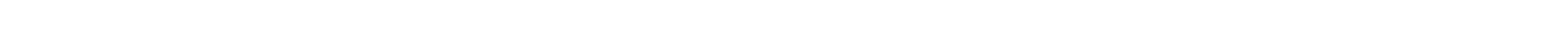

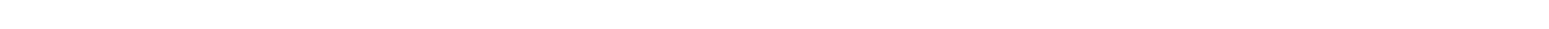

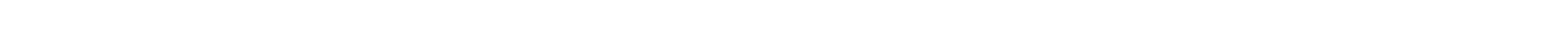

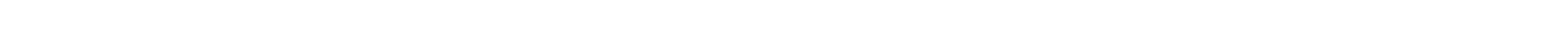

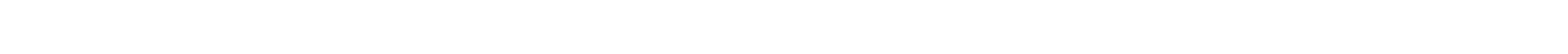

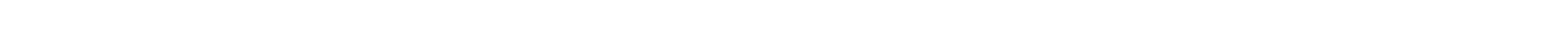




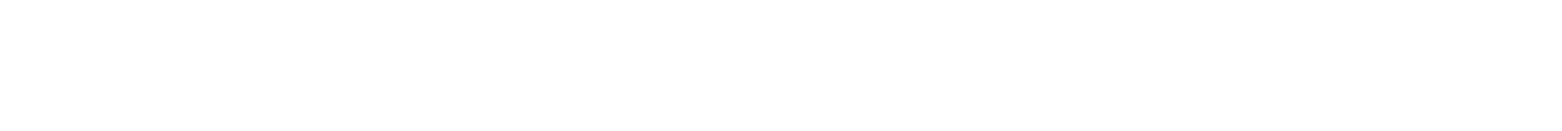

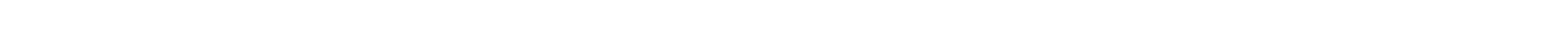

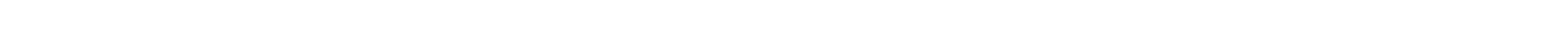

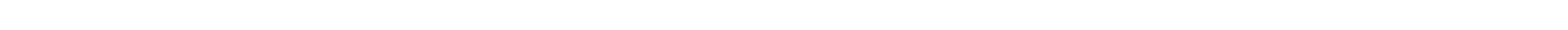

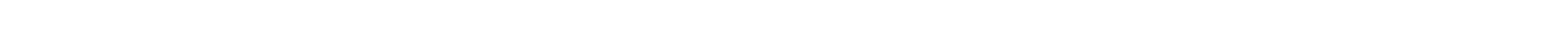

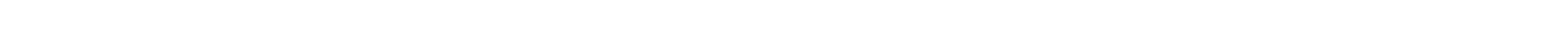

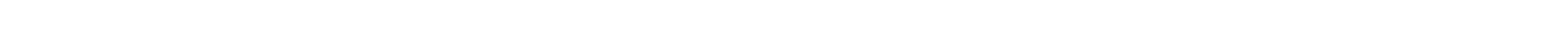

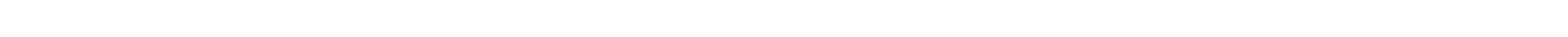

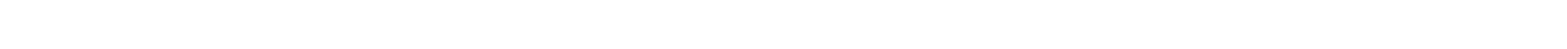
-

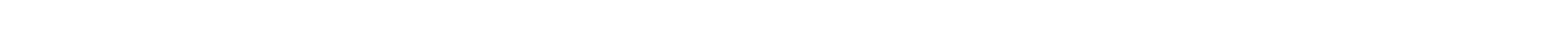

wen

$\Sigma$

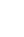

is

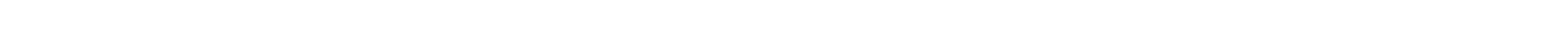
a da. w s d

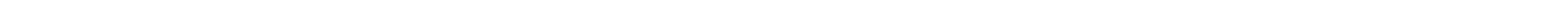

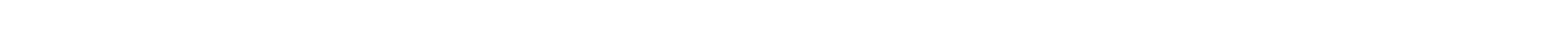

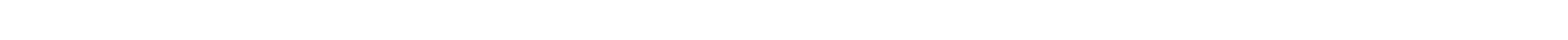

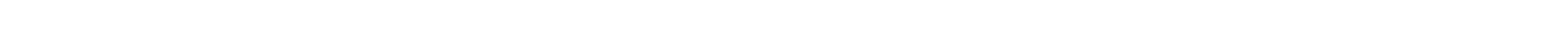

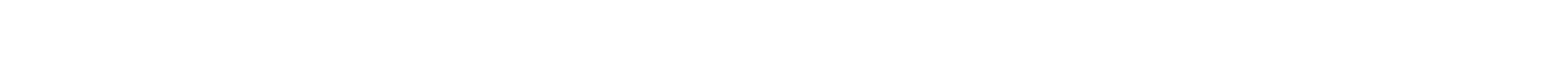

n

n

is

n

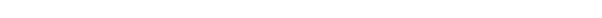

is

"ำกำ

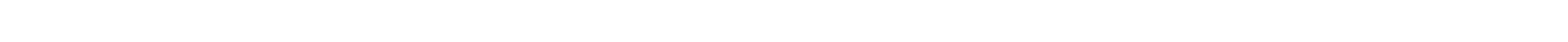
- $>$ N

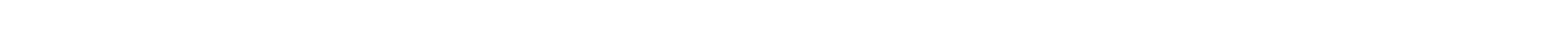

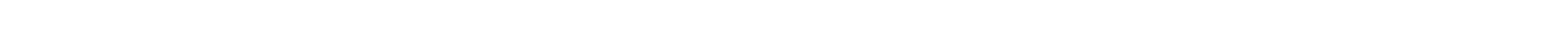

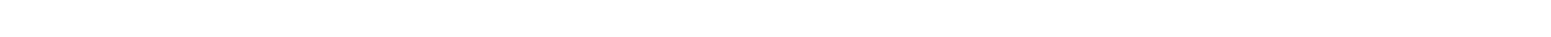

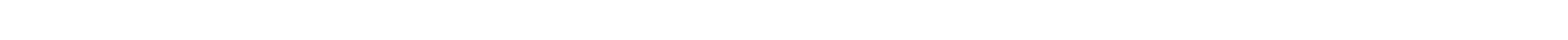

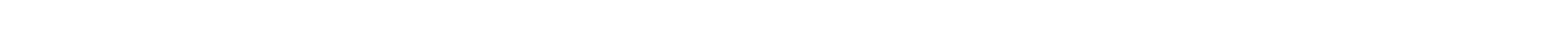

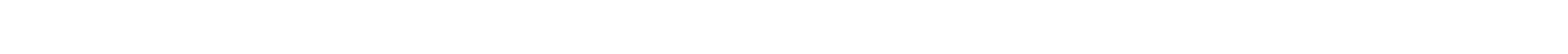

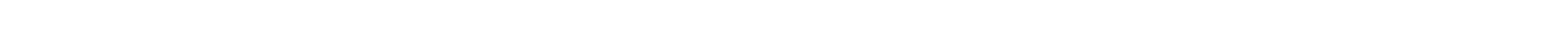

$m$ n

in

n

n

as

u

is ๔ "ú বL

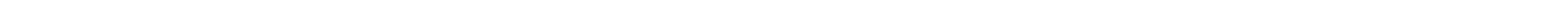

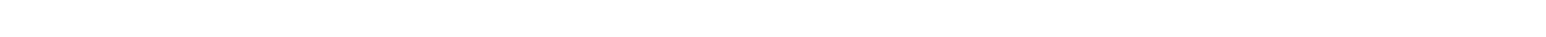
:

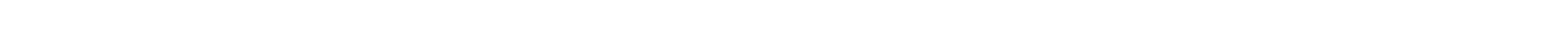

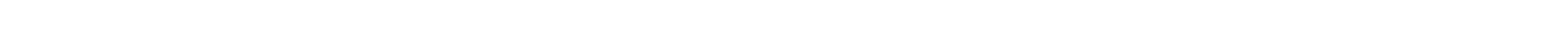

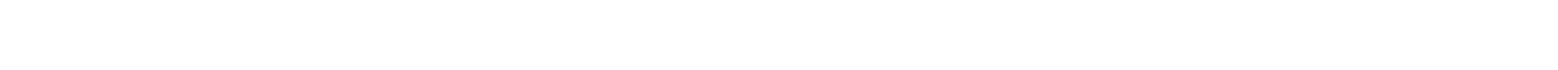




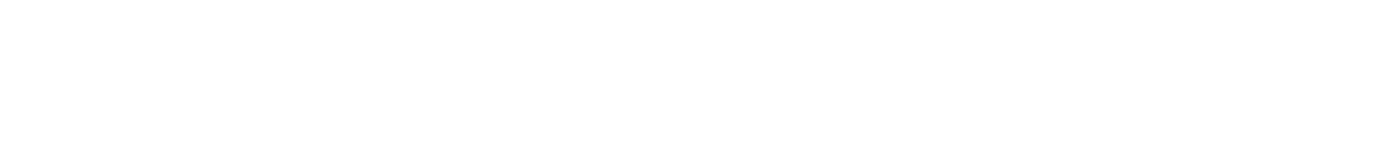

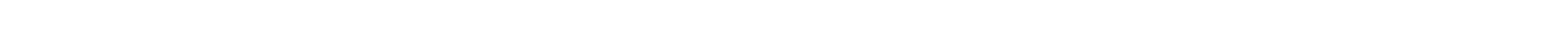

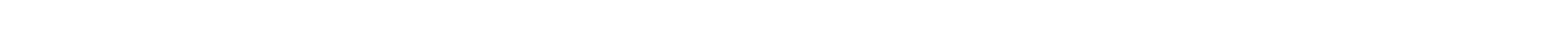
- m.

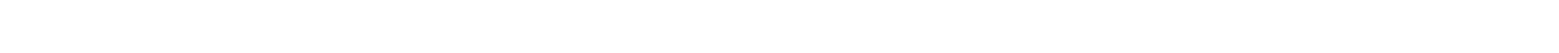

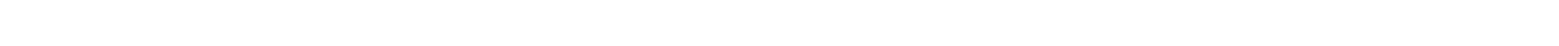

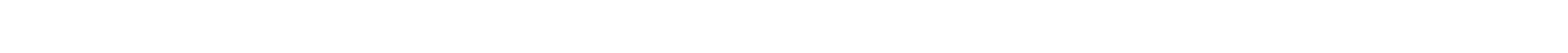

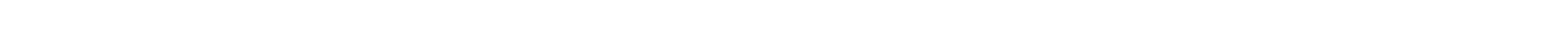

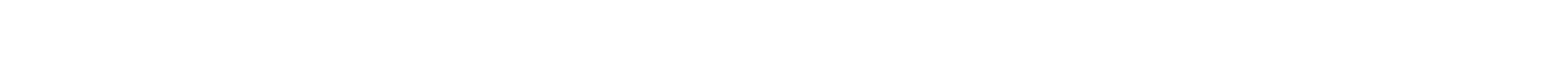
it os eseses
i
en
$\backsim$

in

Х щ -

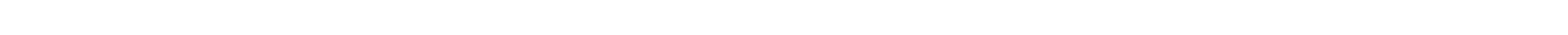

NANRNA

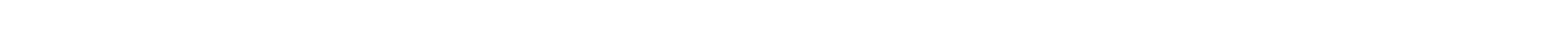

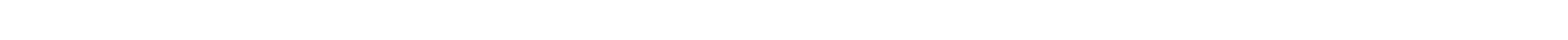

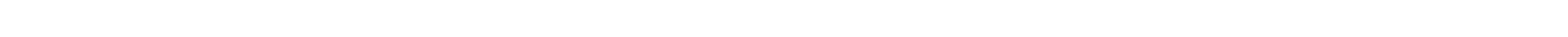

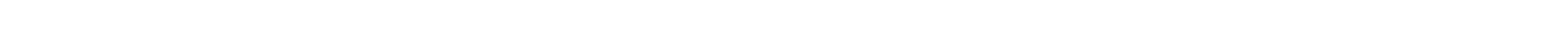

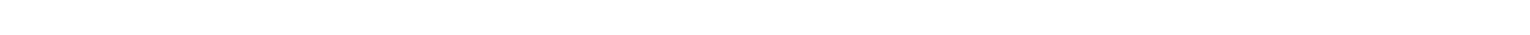
$\leftarrow$ in
s
n
$\sim$
en
is
n

in

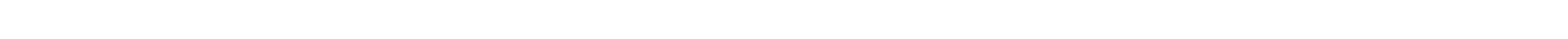

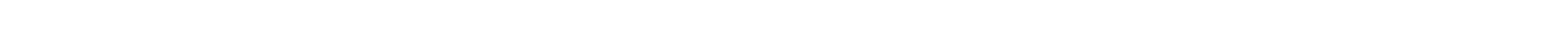

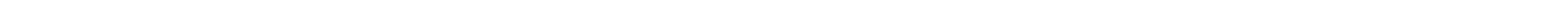

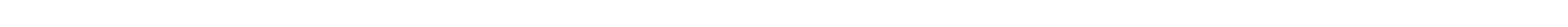

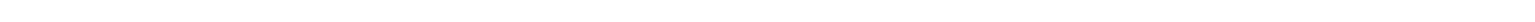

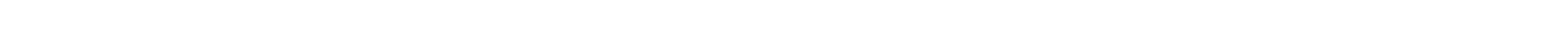

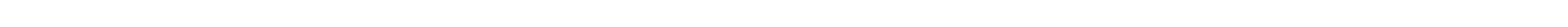

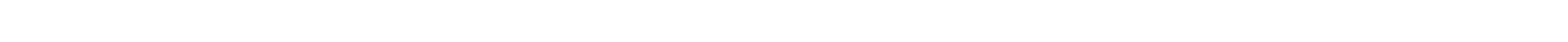

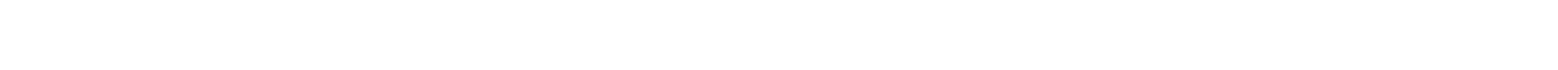

es

es

is

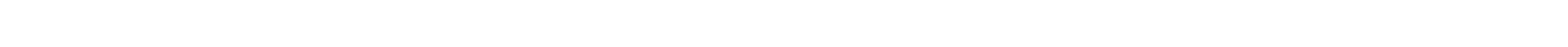

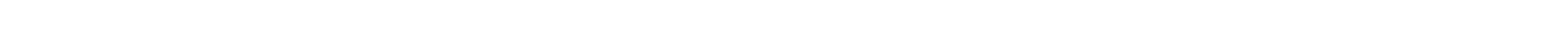

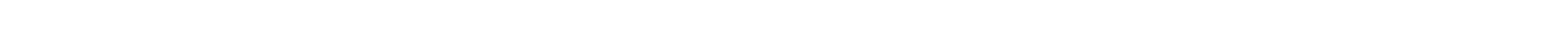

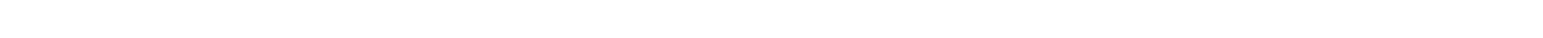

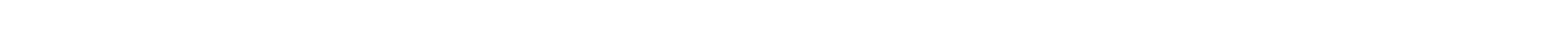

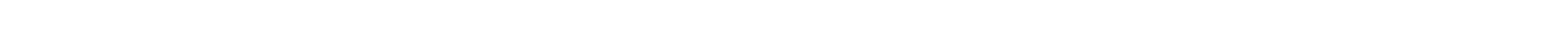

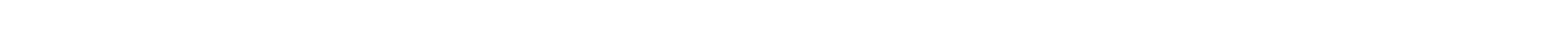

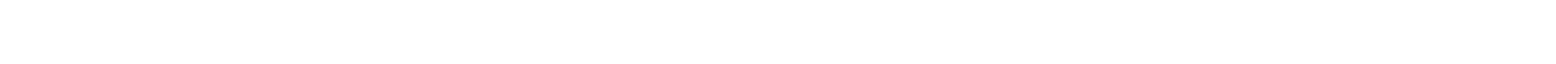




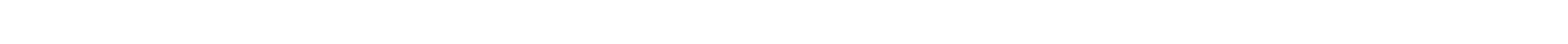

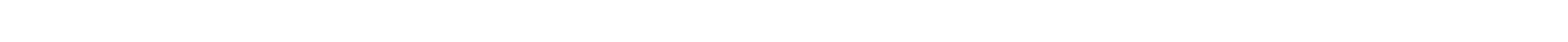
- UNN

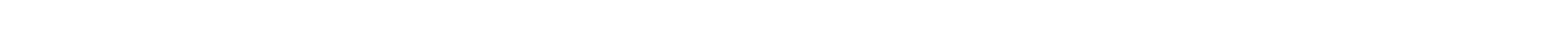

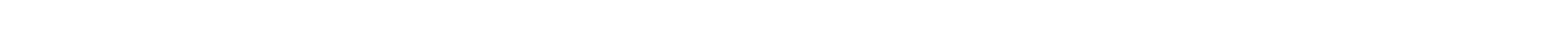

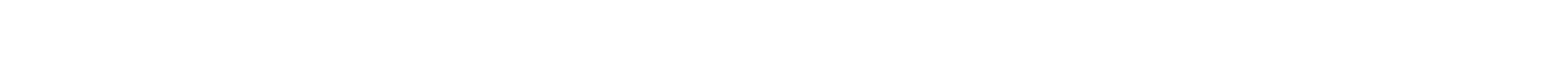

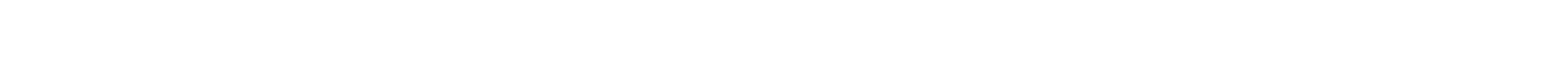

$\Sigma$

n

is

$n$

is

is

↔

a

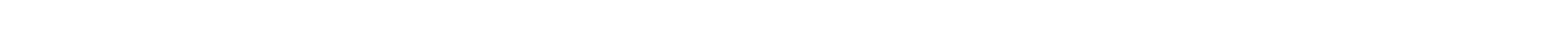

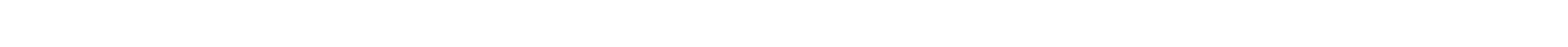

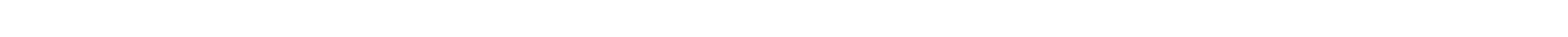

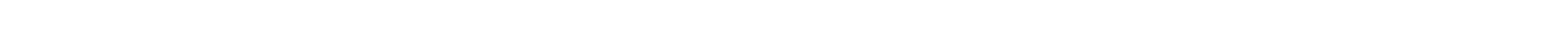

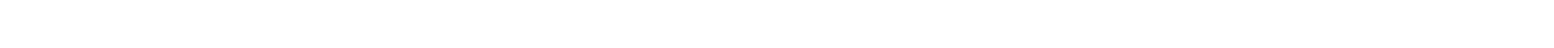

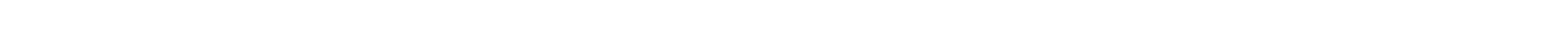
แ

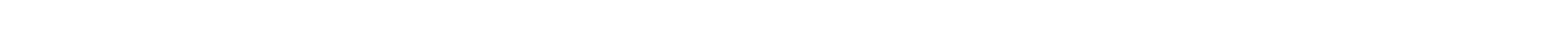

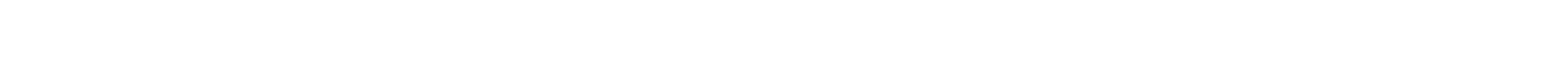

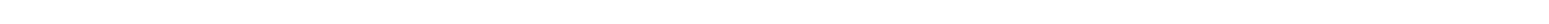

$$
\frac{1}{5}
$$

is

is

E

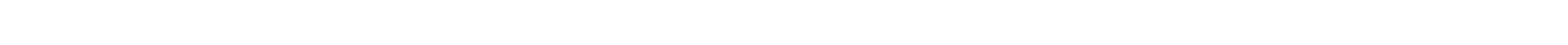

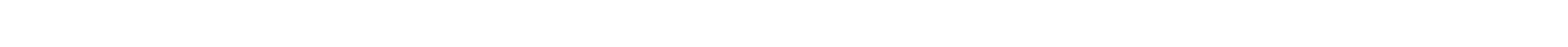

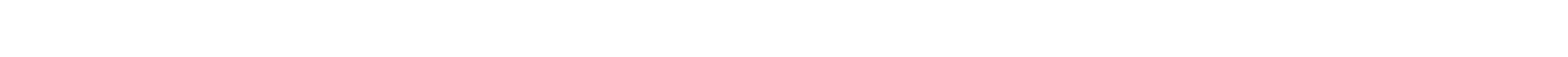

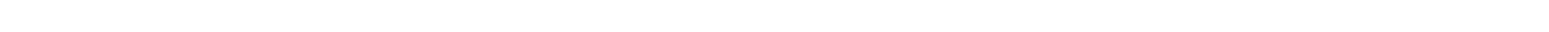

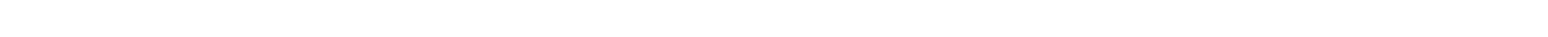

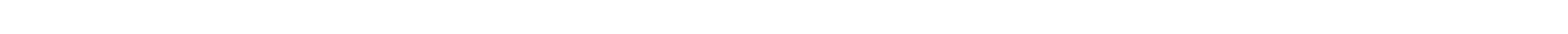

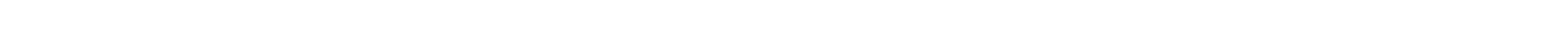

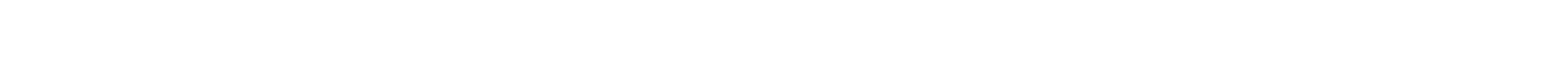

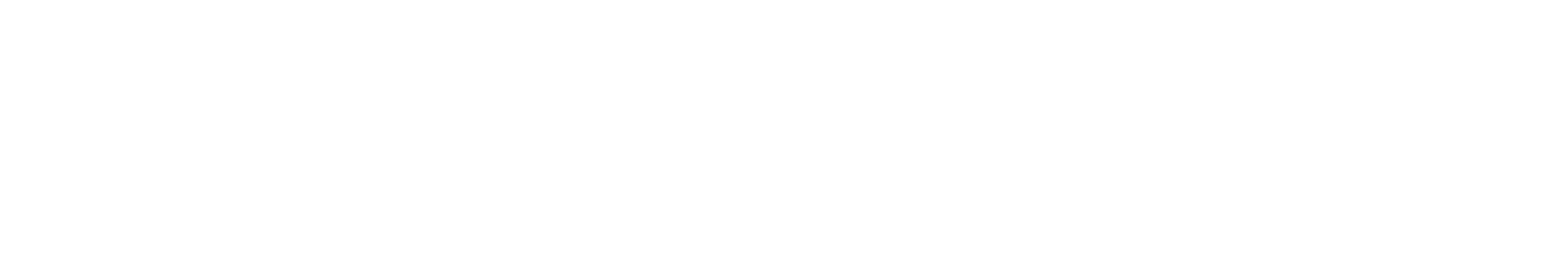

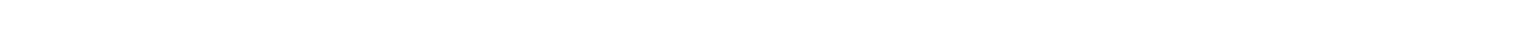

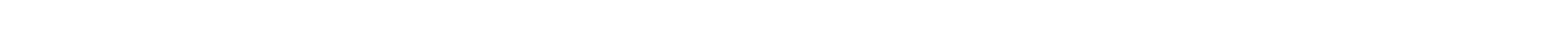

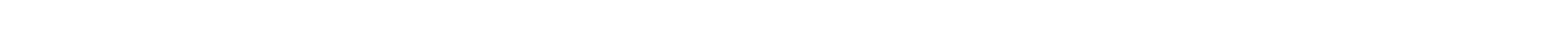

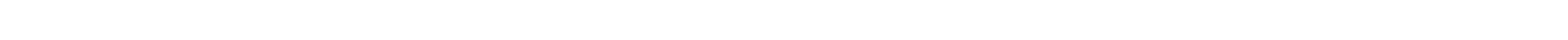

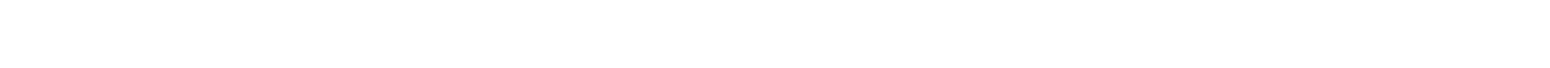




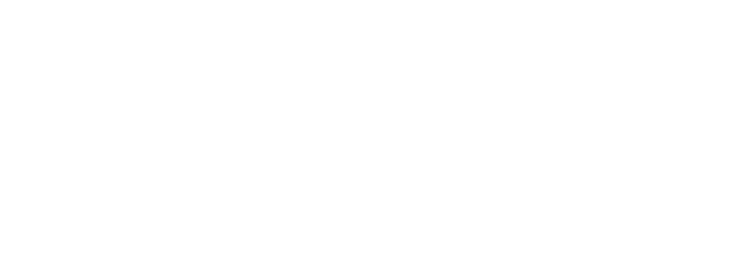

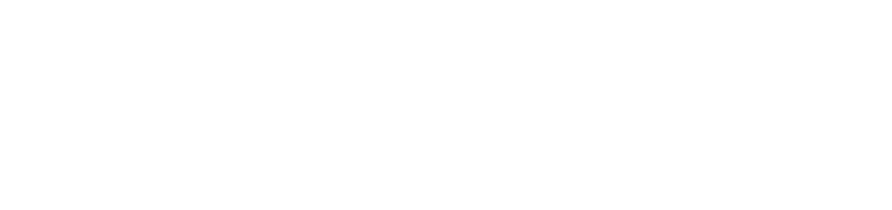

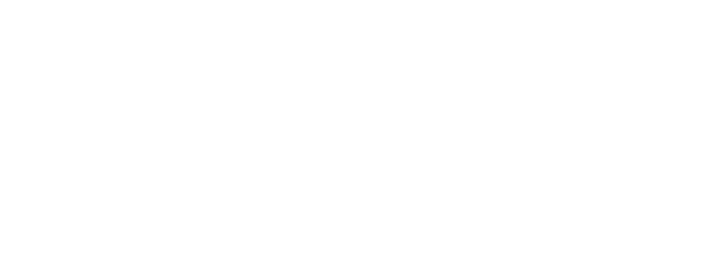

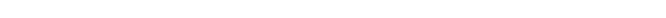

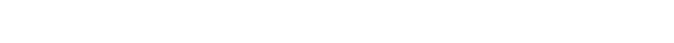

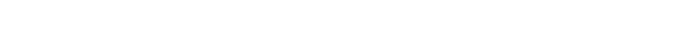

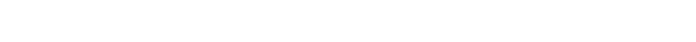

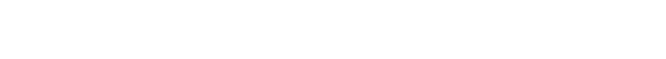

点出

m

n

n

n n

$\therefore$

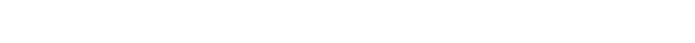

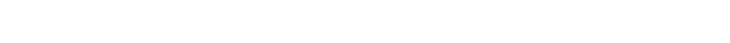

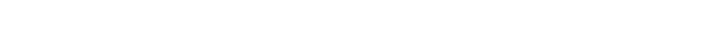

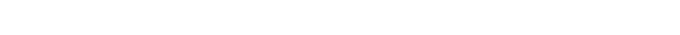

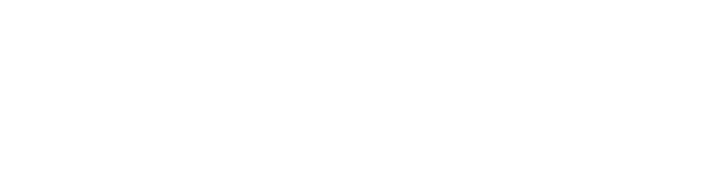

$\stackrel{5}{2}$

m

n

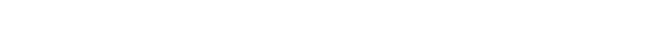

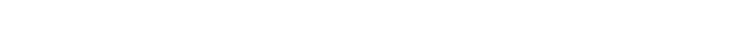

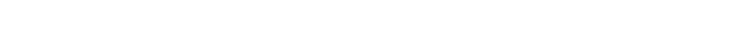
J

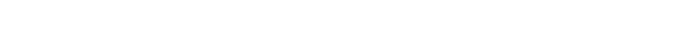

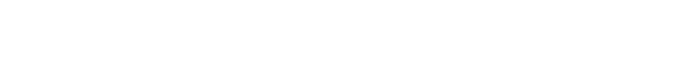

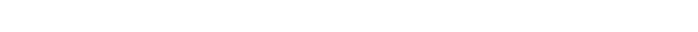

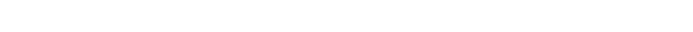

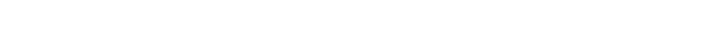

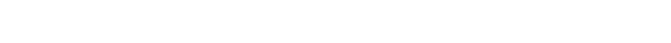




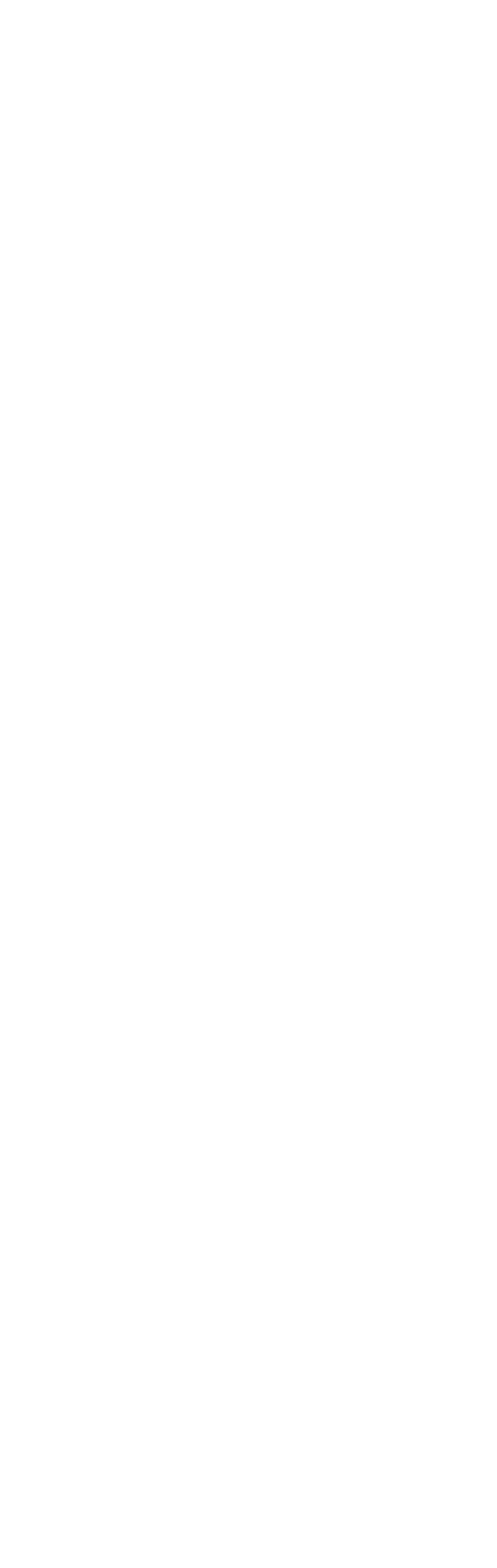

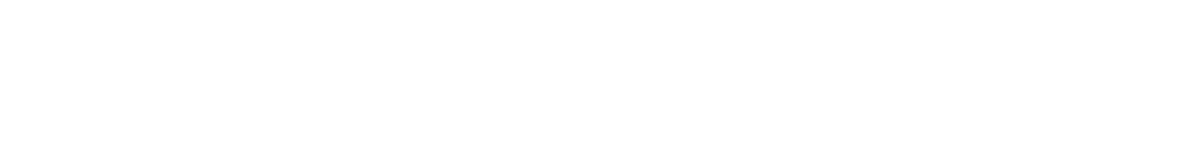

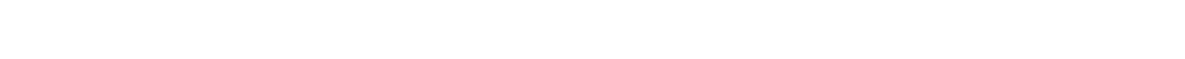

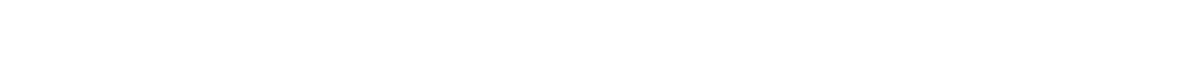

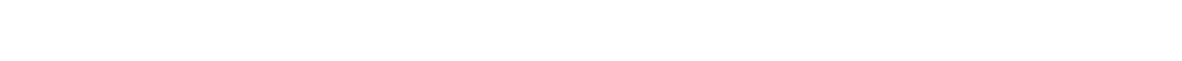

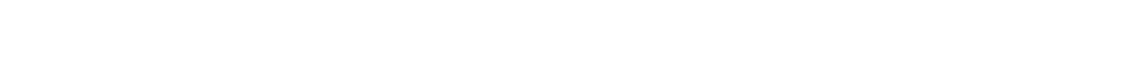

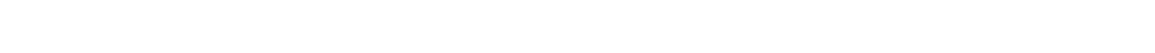

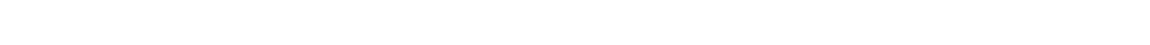

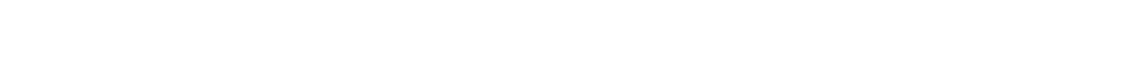

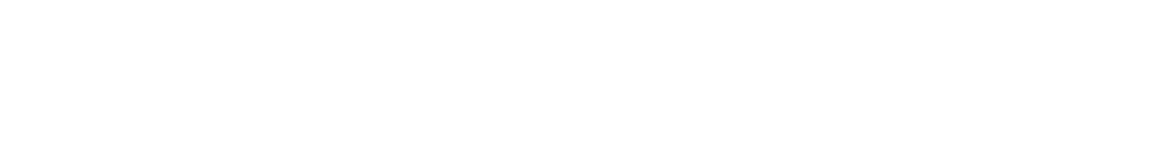

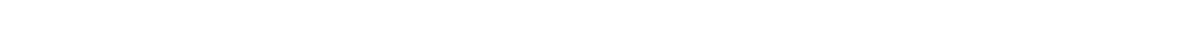

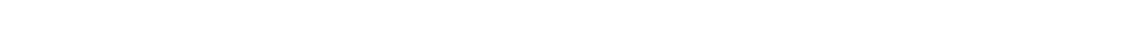

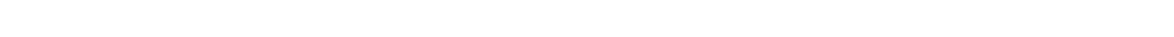

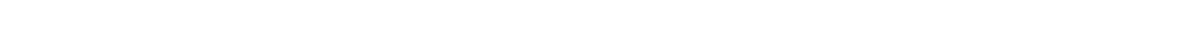

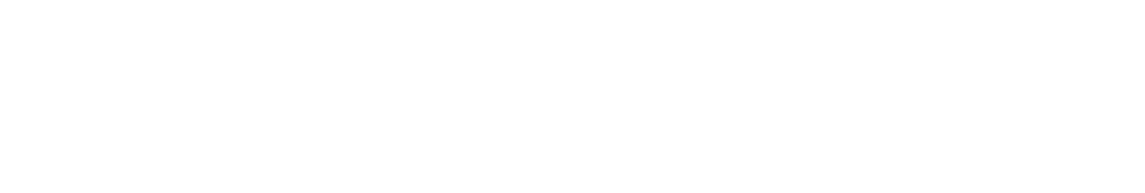

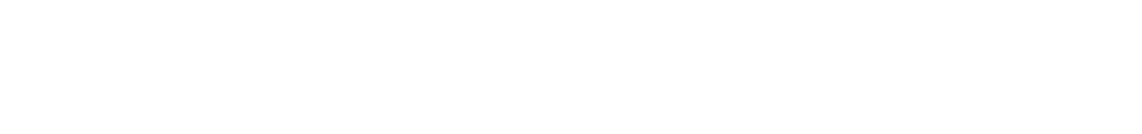

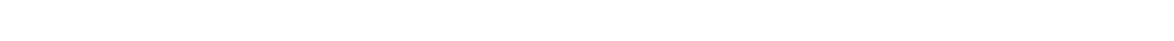

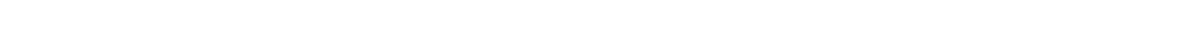

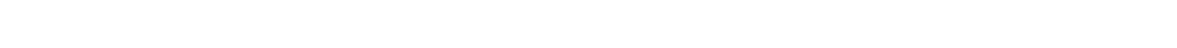

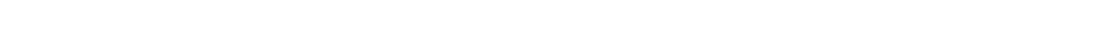

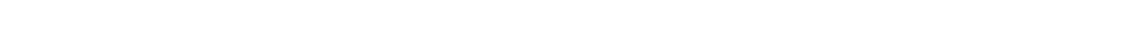

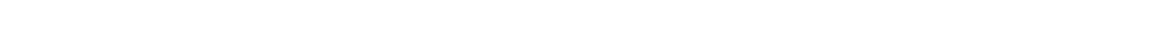

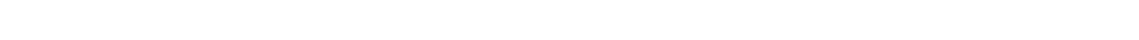
a

$\mathbf{w}$

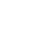

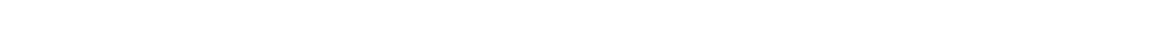

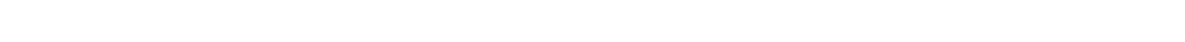

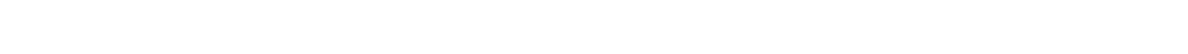

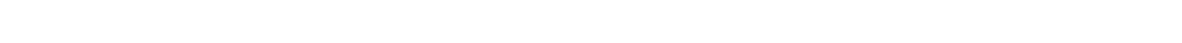

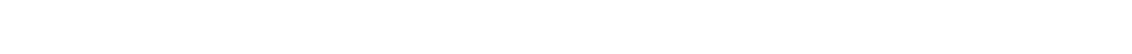

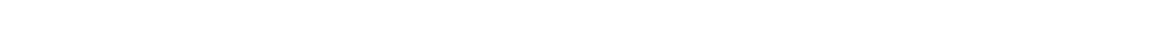
and-m=nms

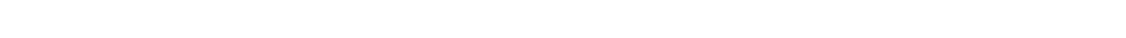

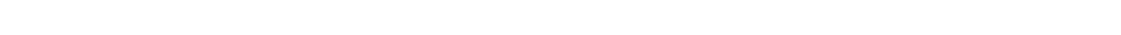




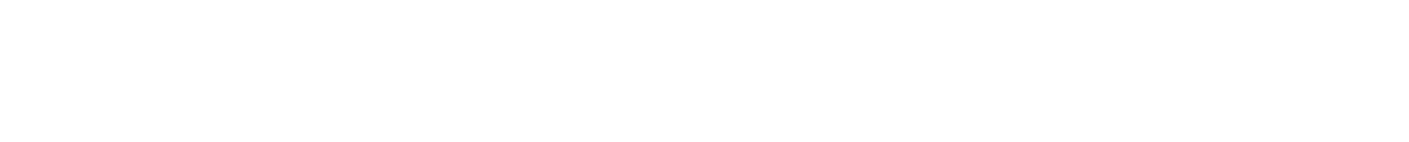

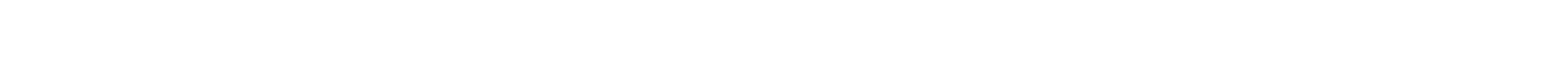
แ

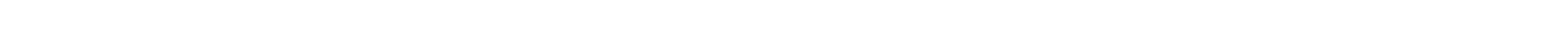

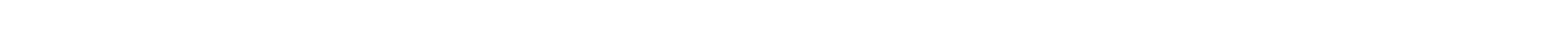

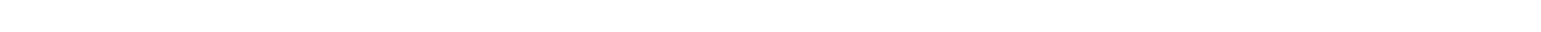

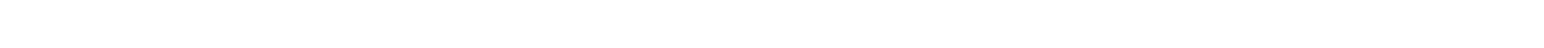

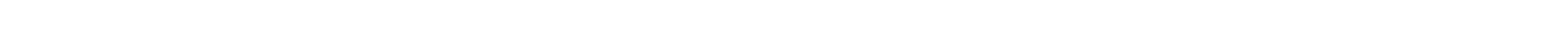

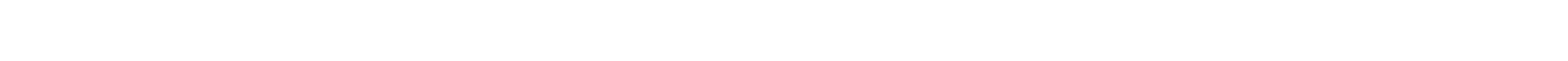

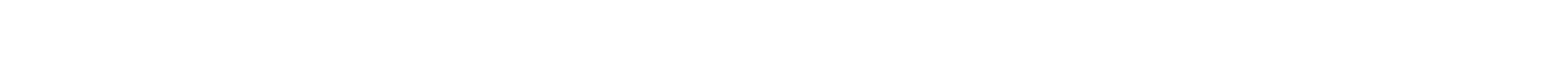

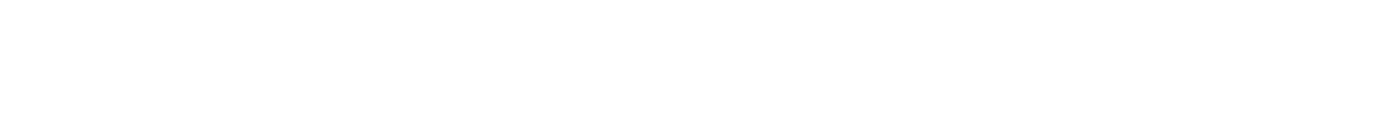

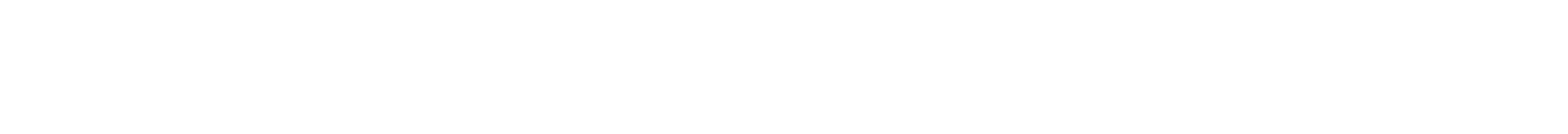

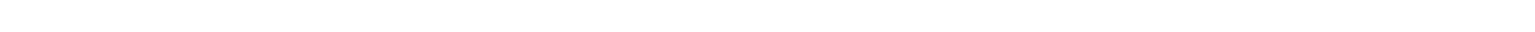

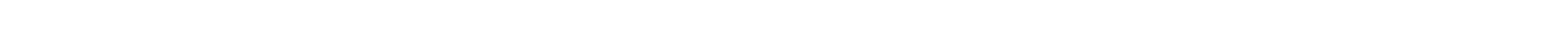

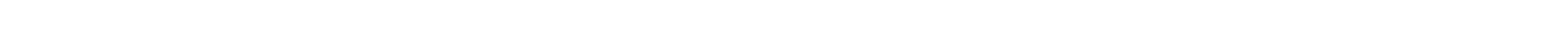

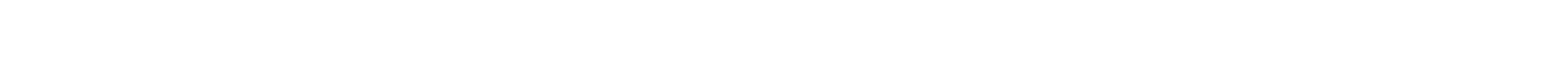

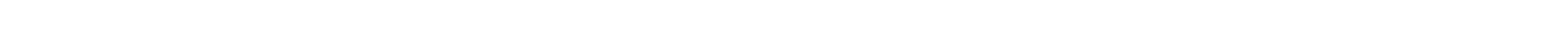

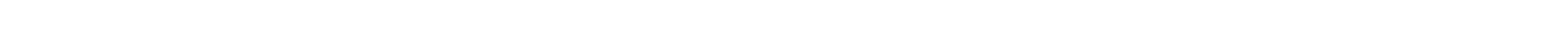
$\stackrel{5}{\mathbf{s}}$
nos
in
un
is
in is
ines en
$\infty$ n
is

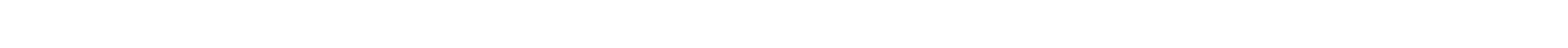

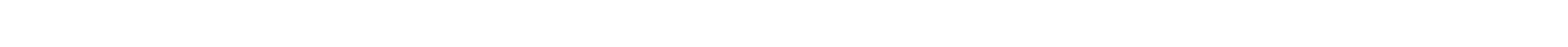

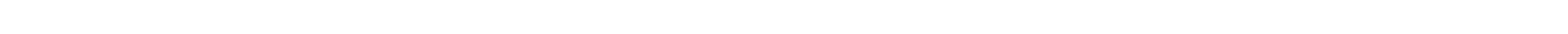

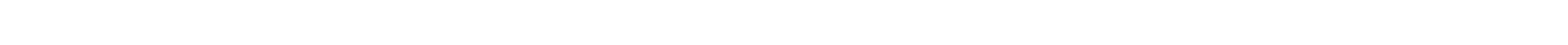

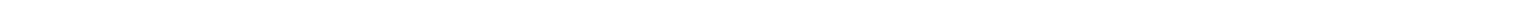

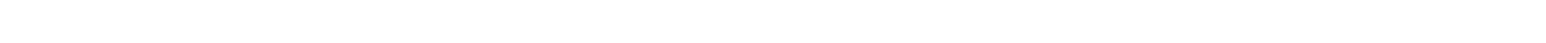

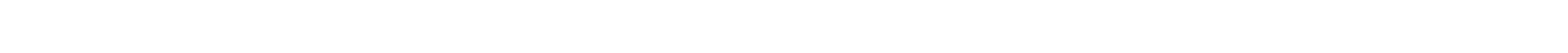
acintingorion

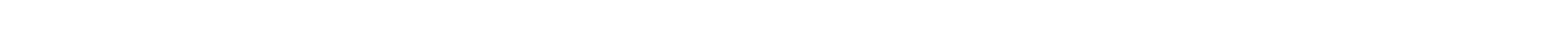

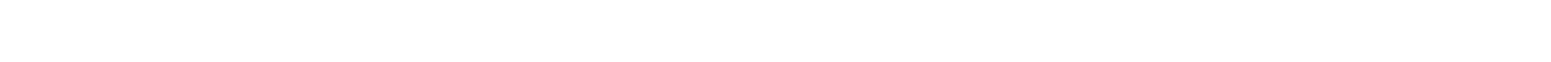

w

is

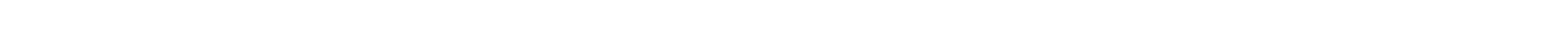
ul ú

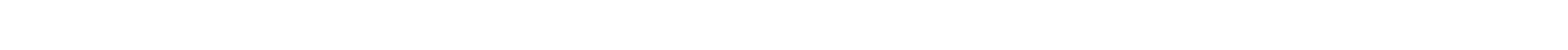

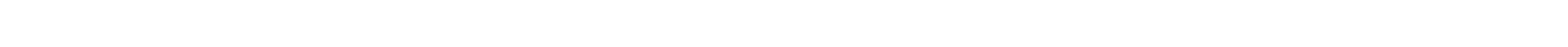

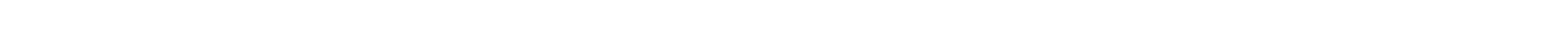

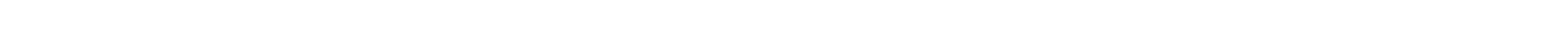

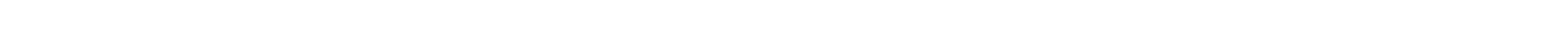

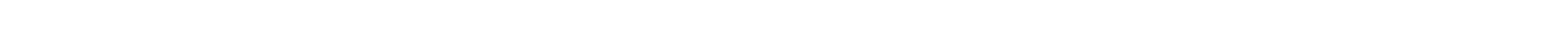

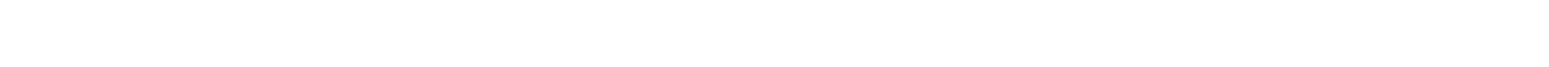




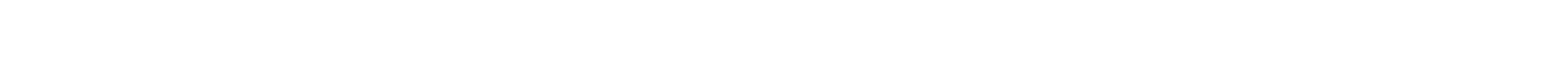

is

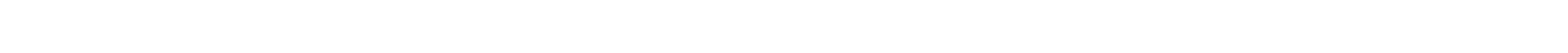

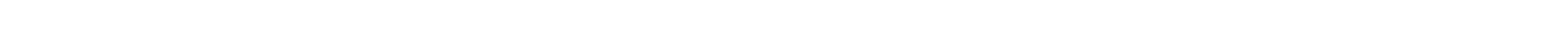

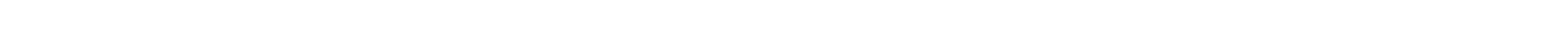

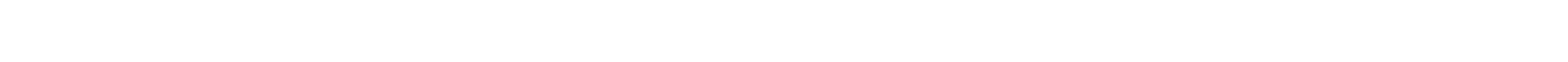

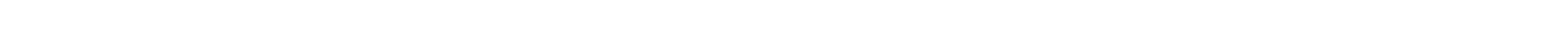

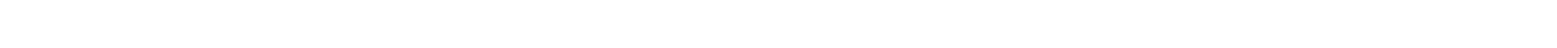
世

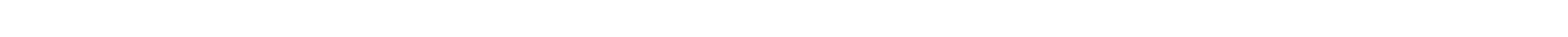

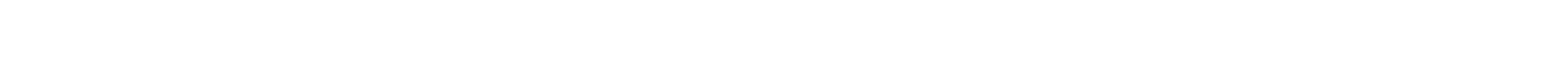

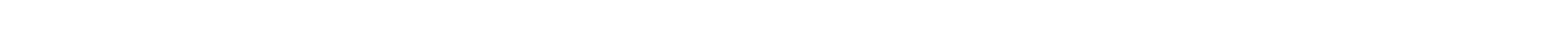
in

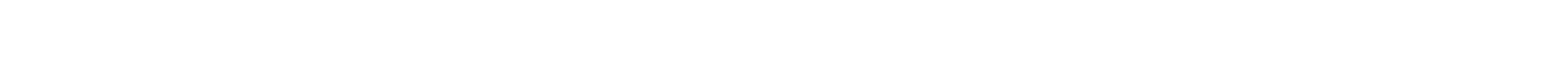

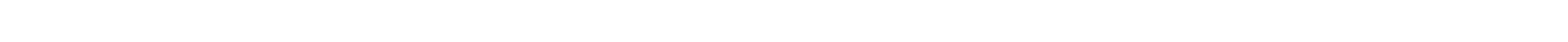
a

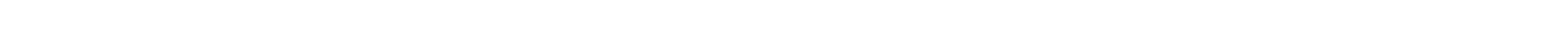

mmmmmMmmm tี

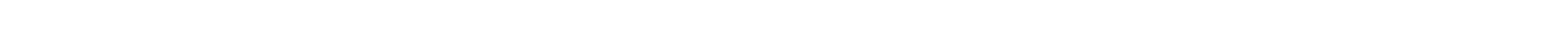

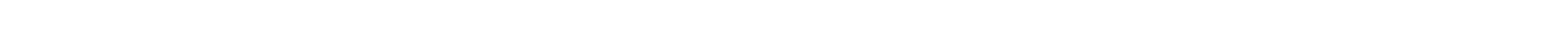

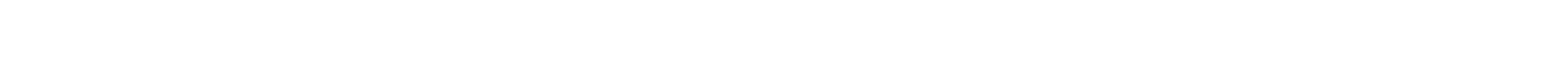

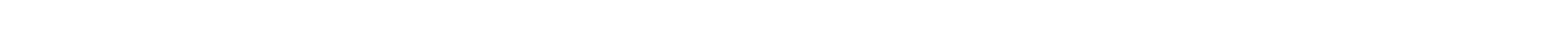

w

$\sim$

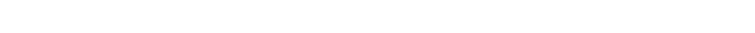

$\underline{5}$

m ü

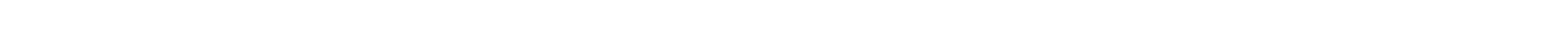

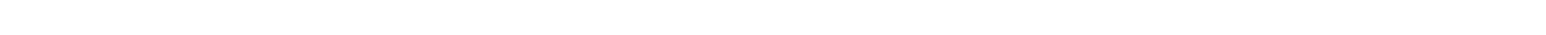

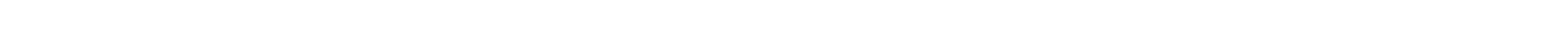
แ 促

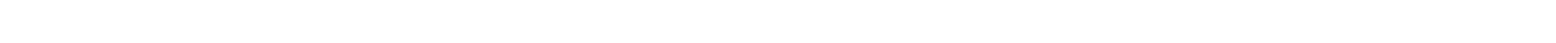

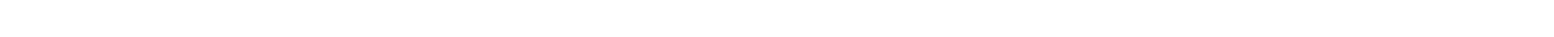

I

$\stackrel{2}{2}$

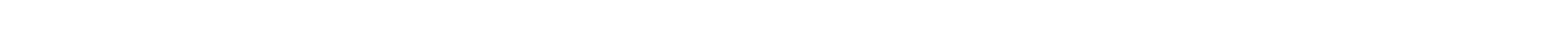
a d

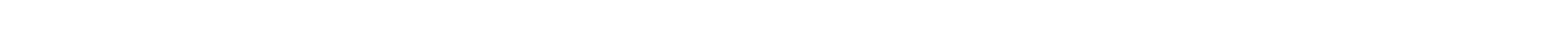
《uा

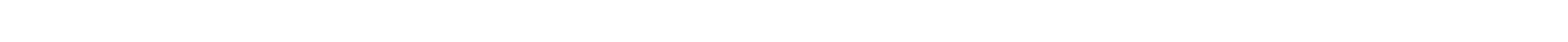

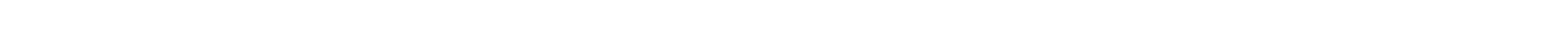
w

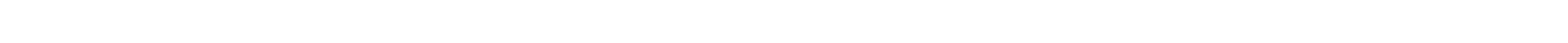

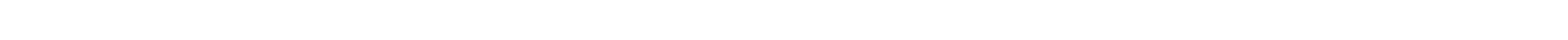

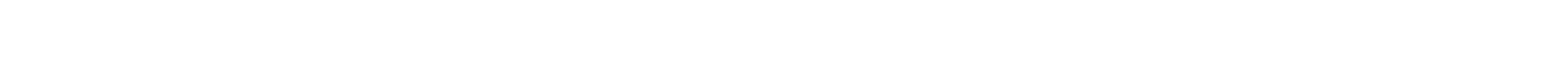




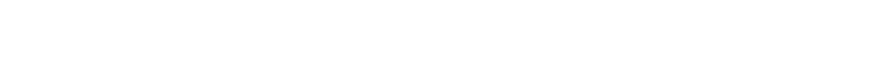

in

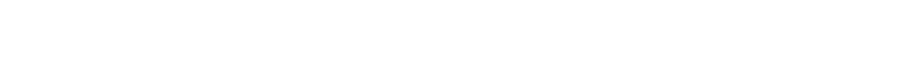
$\infty \pi \infty n$

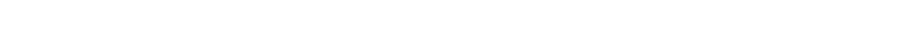

مீ丨 nᄄm w

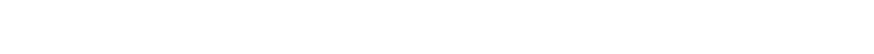

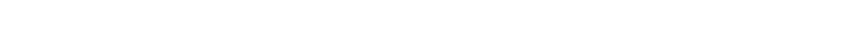

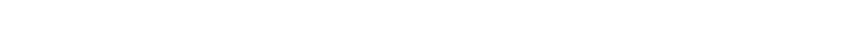

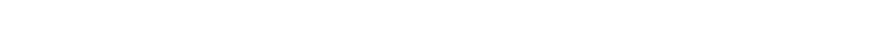

en

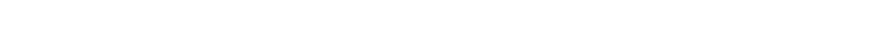
umo 4 w20030

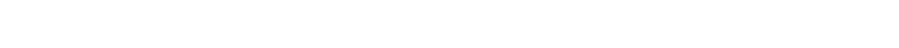

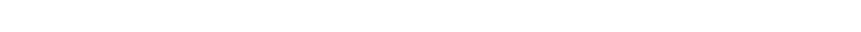
ลิน

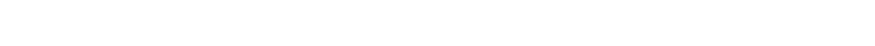

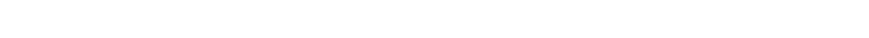

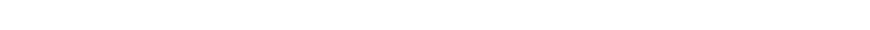

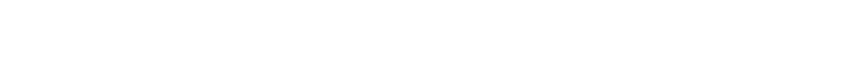

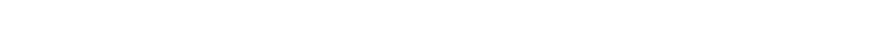
E

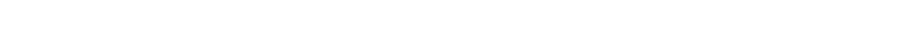

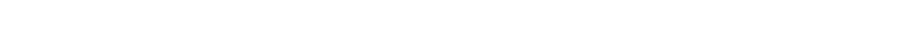

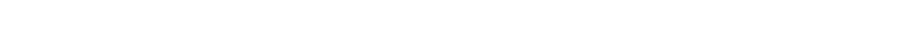

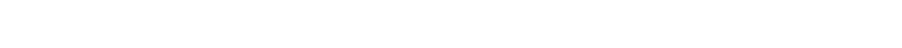

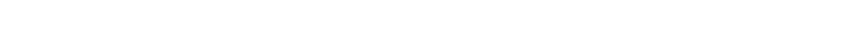
баă

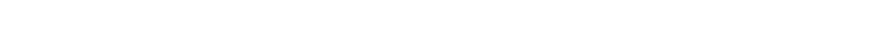

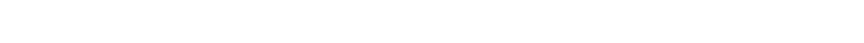

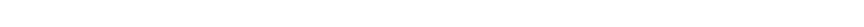

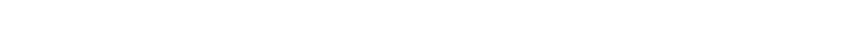

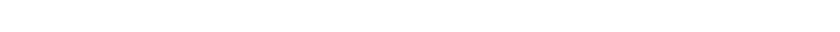

ㄴ.

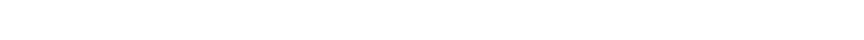

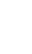

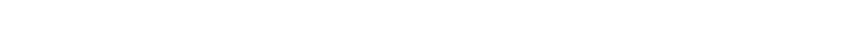

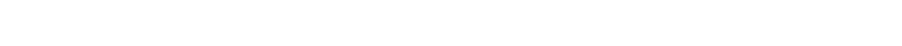

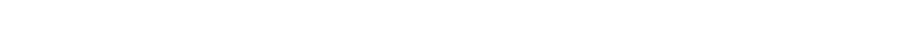

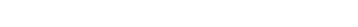

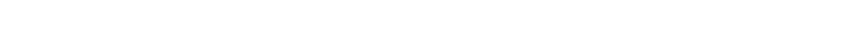

ก N N N N N

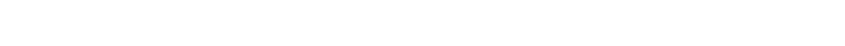

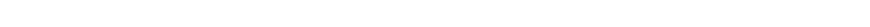

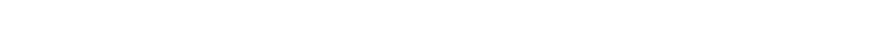

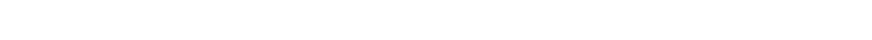

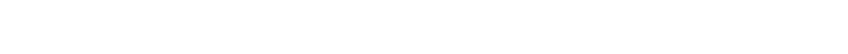

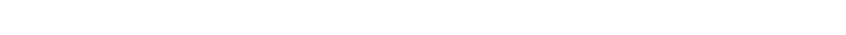




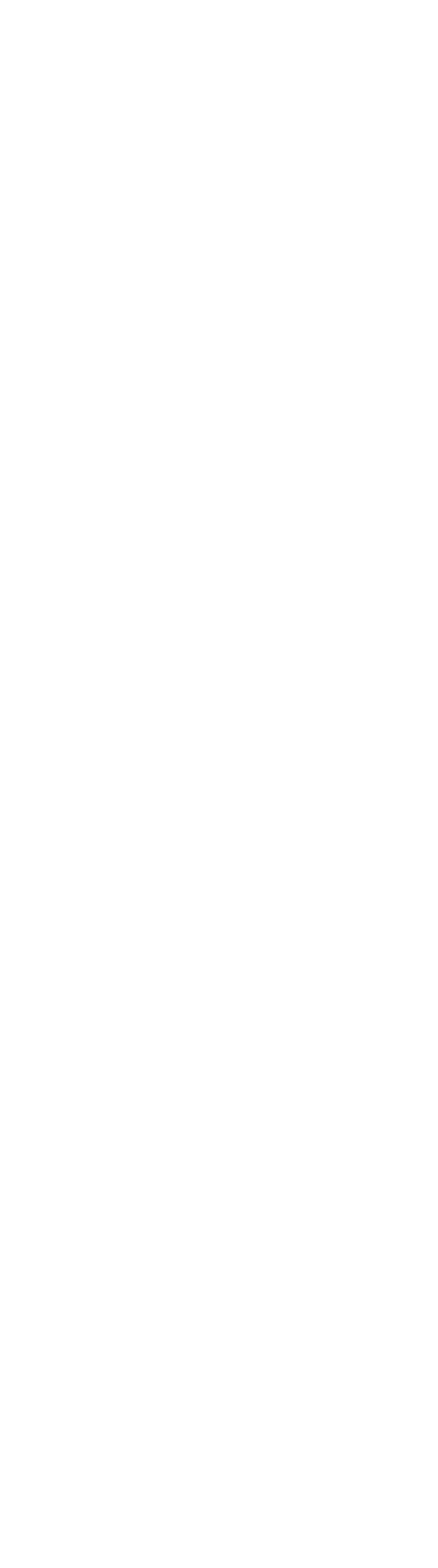

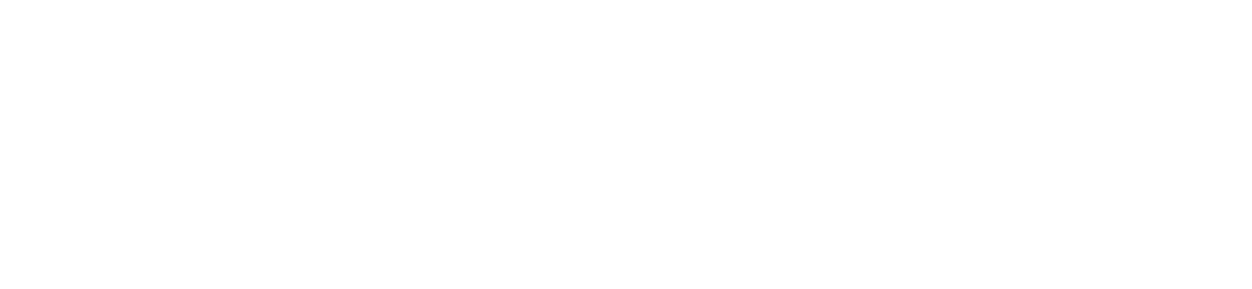

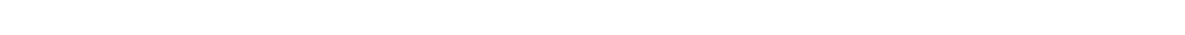

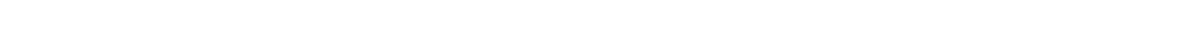
แ

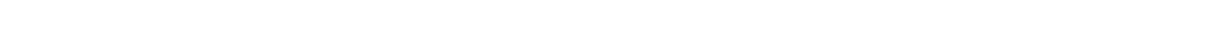

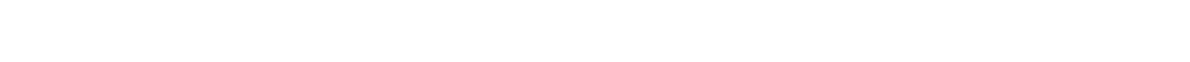

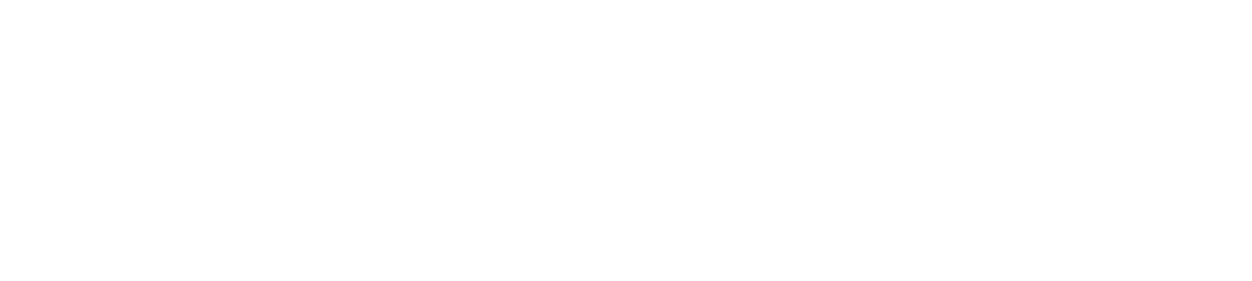

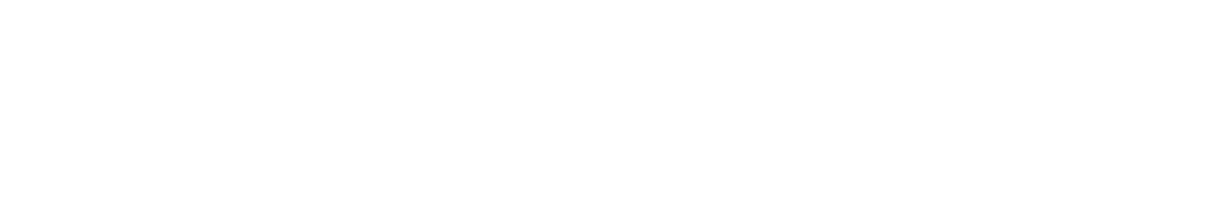

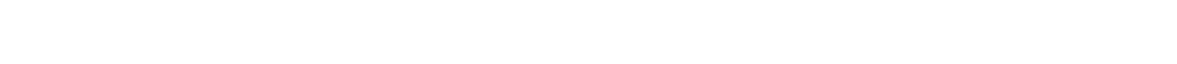

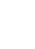

ำ w Е

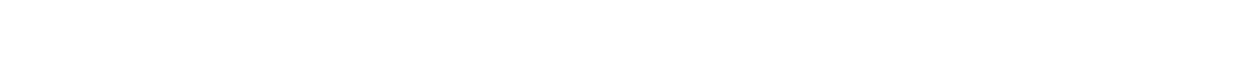

MN minnmimm

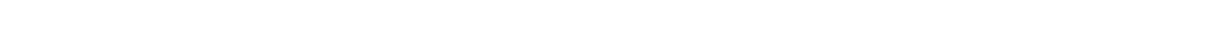

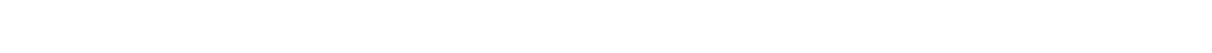

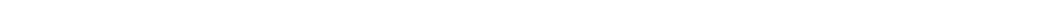
>

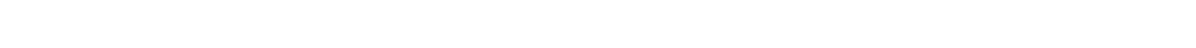

ty $\vdots$ ж

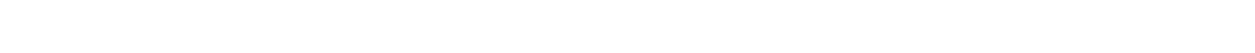

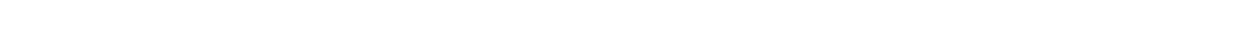

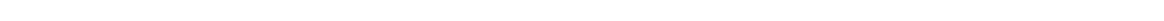

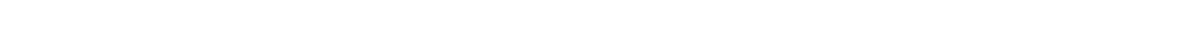

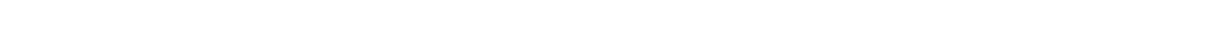
-

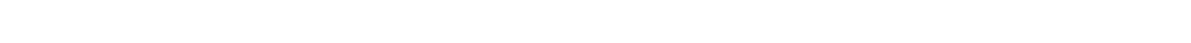

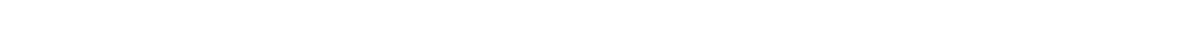




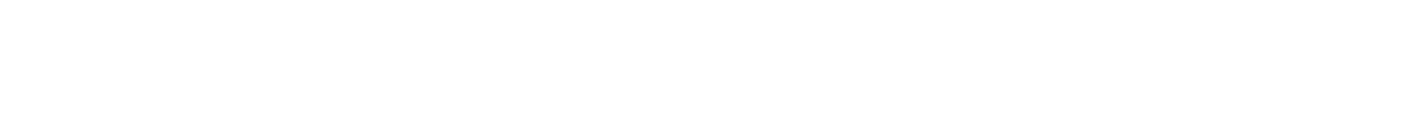

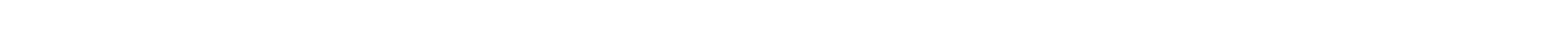

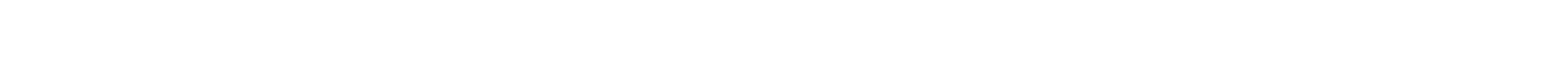

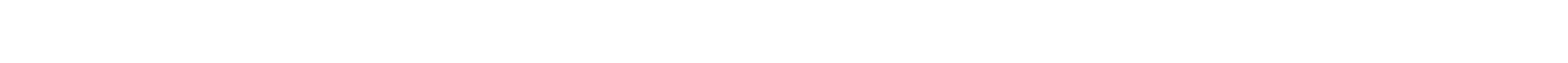

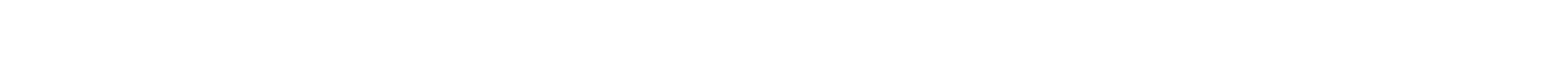

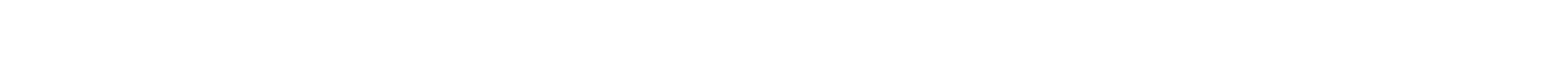

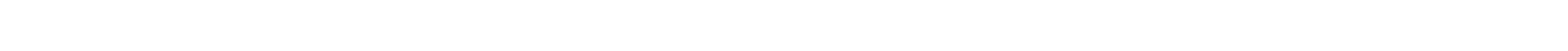

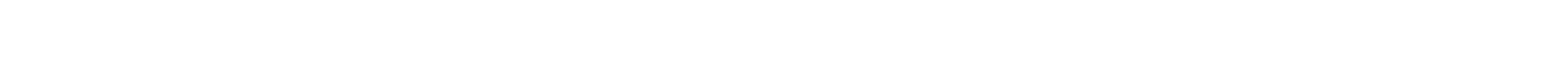

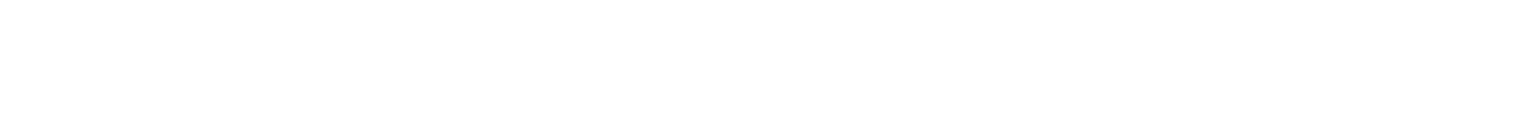

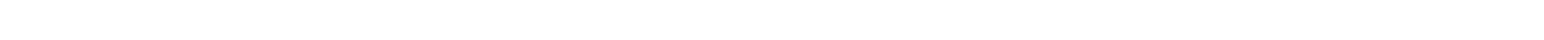

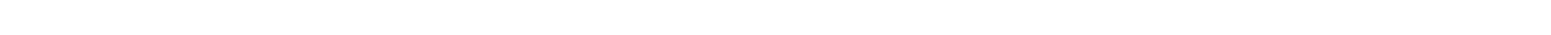

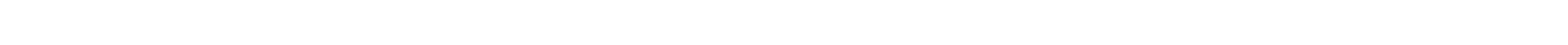

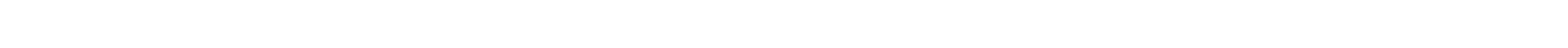

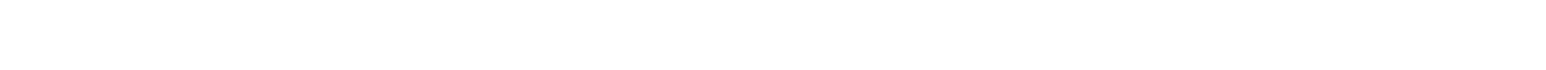

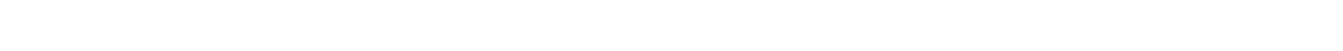

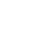

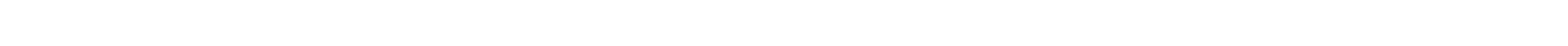

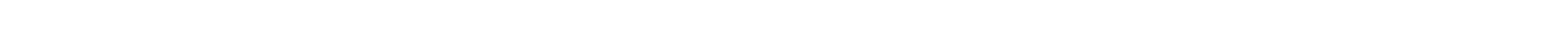

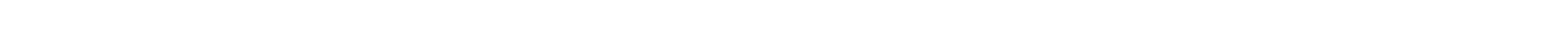

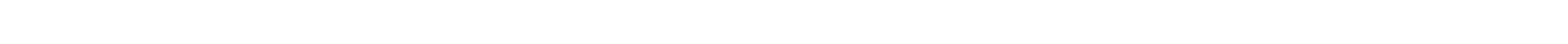

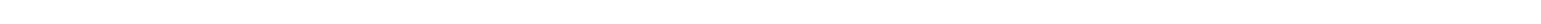

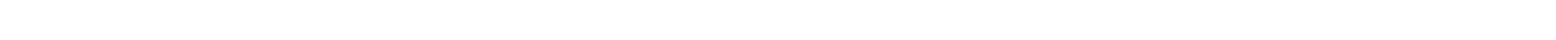
w Е

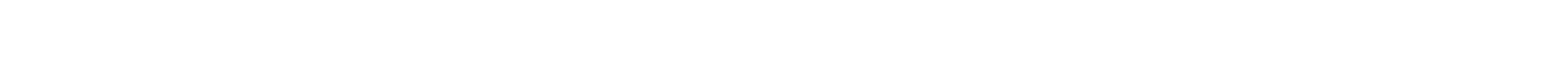

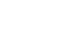
n
m
in is
is
n
in
n n

$\stackrel{\square}{10}$

-

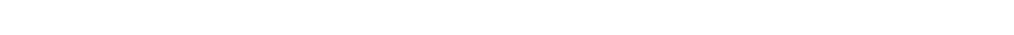

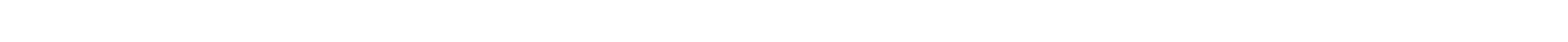

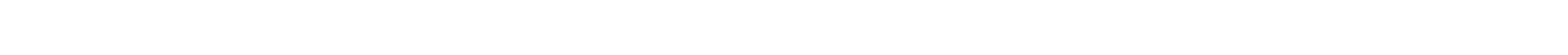

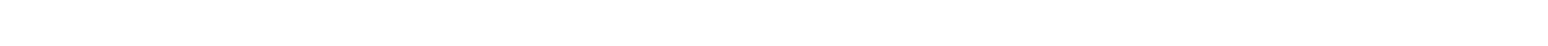

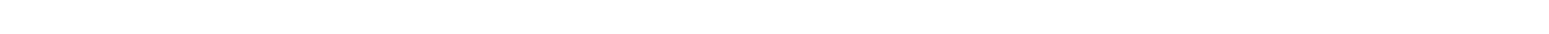

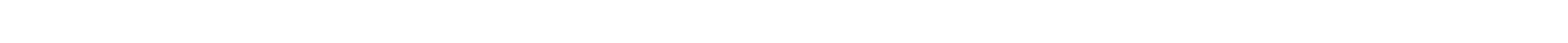

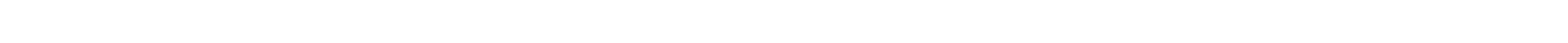

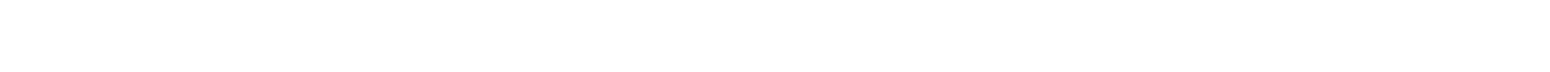




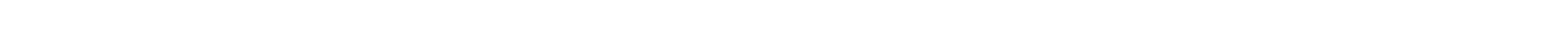
is

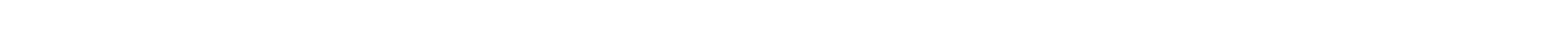

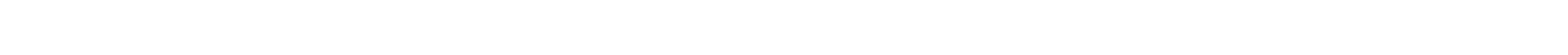
"

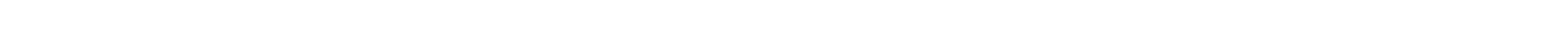

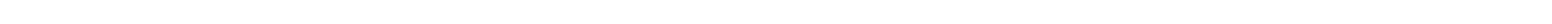

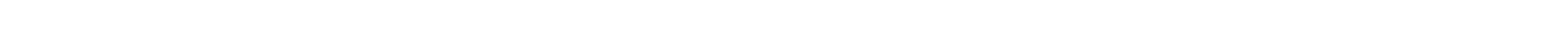

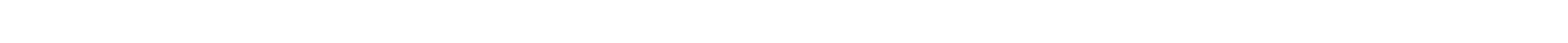

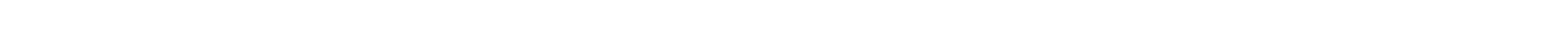

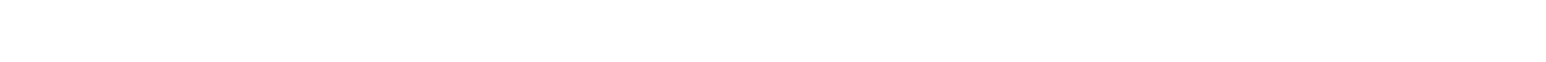

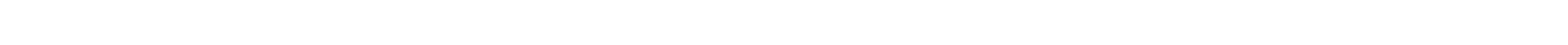

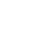

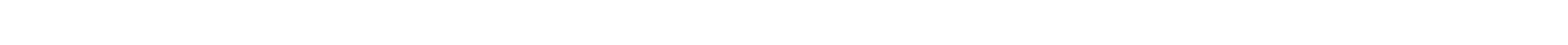
ú -

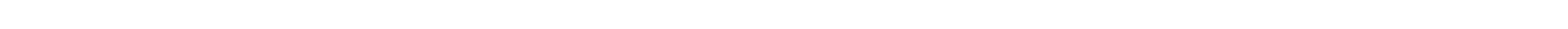

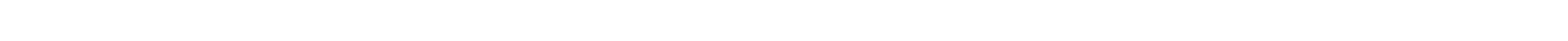

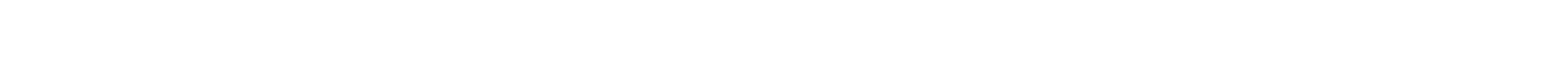
แே

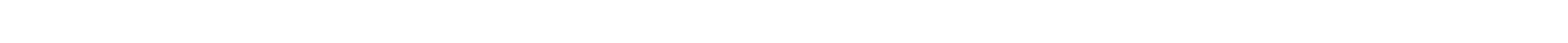

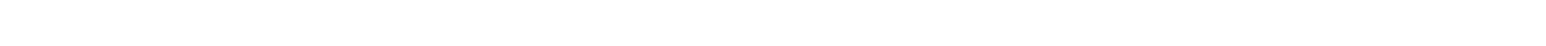

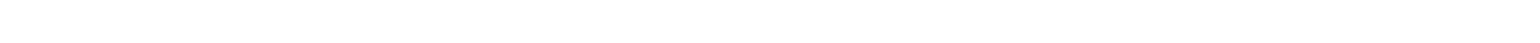

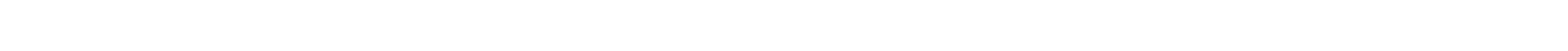

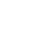

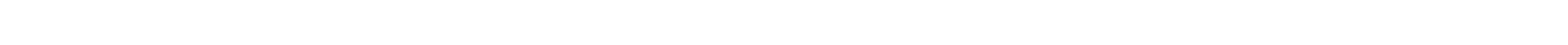

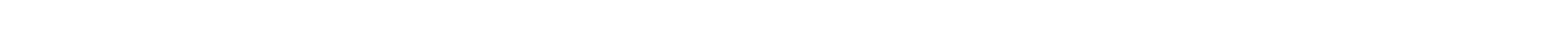

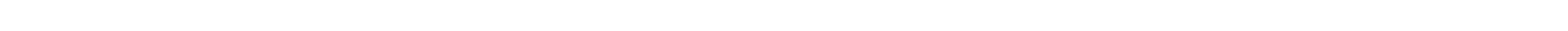
\begin{tabular}{ll}
3 \\
\hline
\end{tabular}

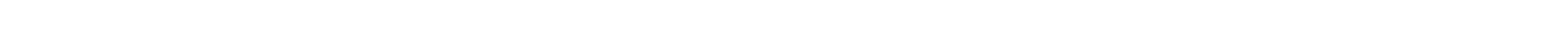

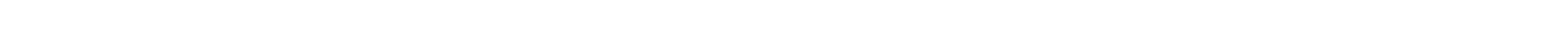

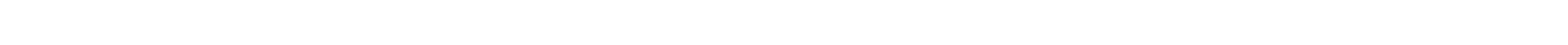

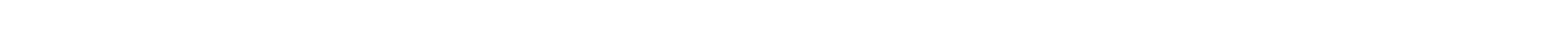

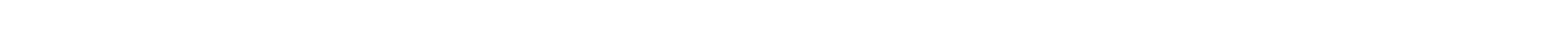

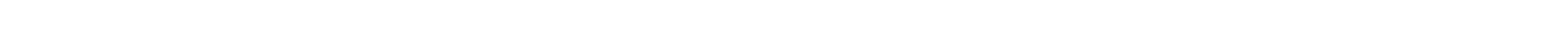

岁

in

es

$=$

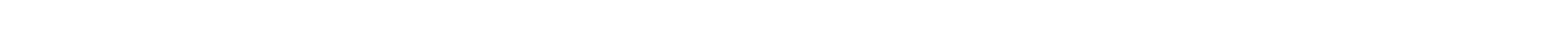

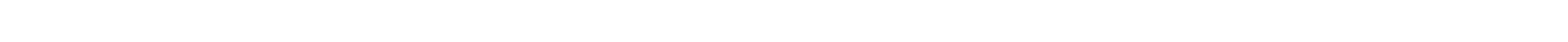

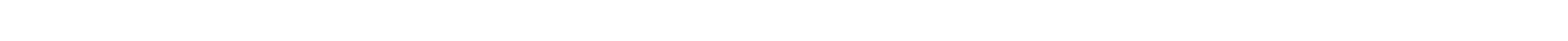

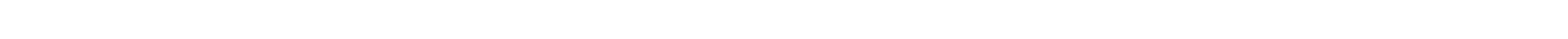

Б5555555

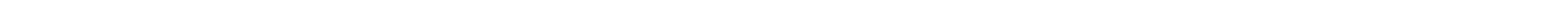

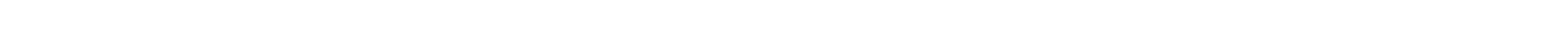

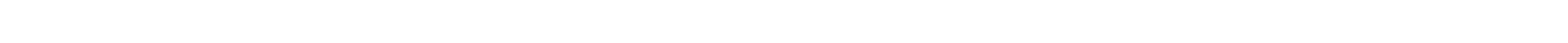

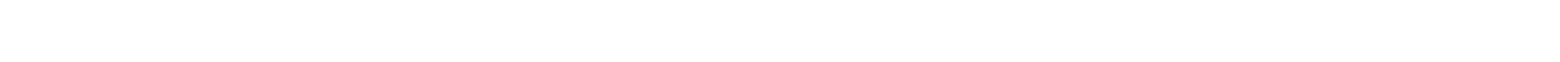




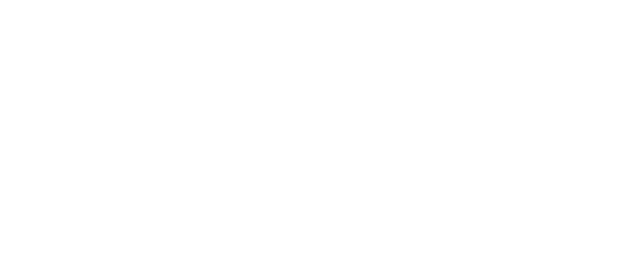

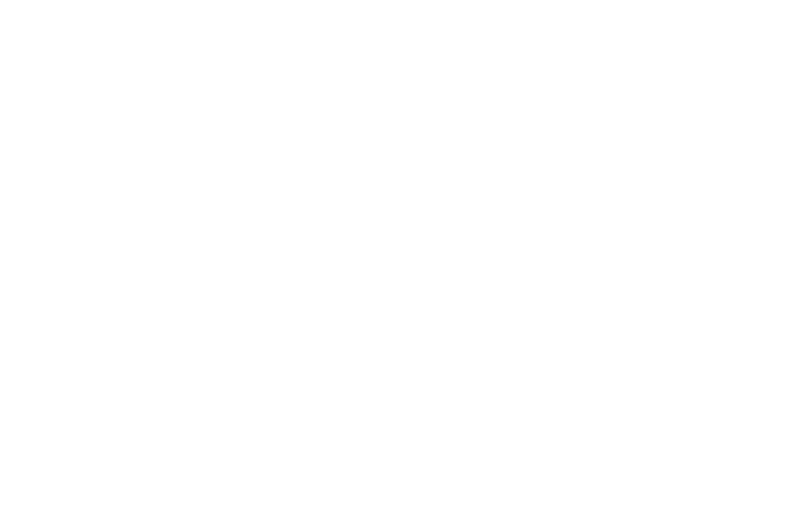

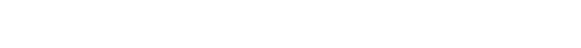

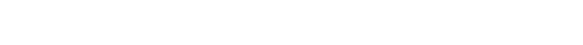

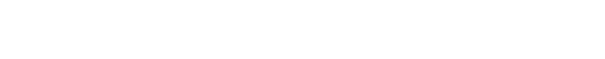

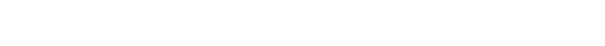

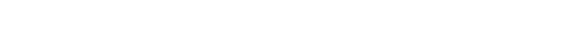

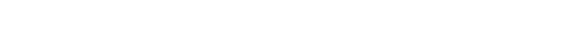

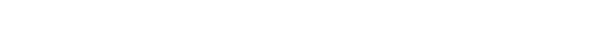

in

"aำ \& सMำ?

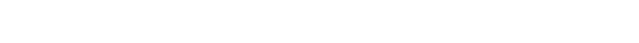

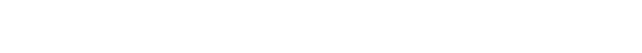

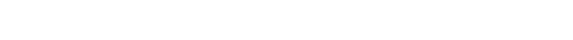

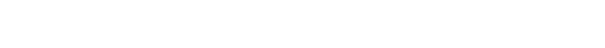

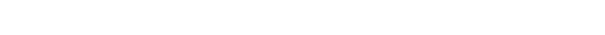

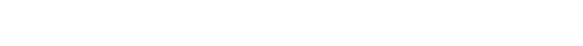

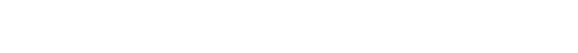

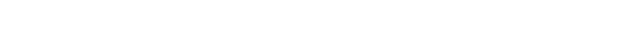

in

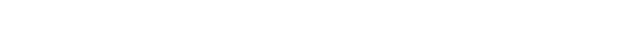
a 14.

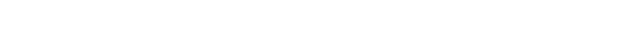

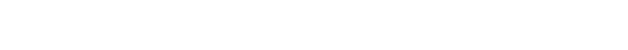

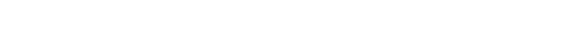

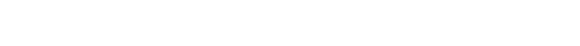

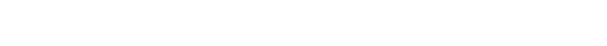

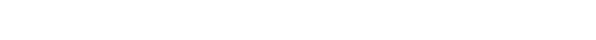

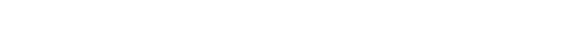




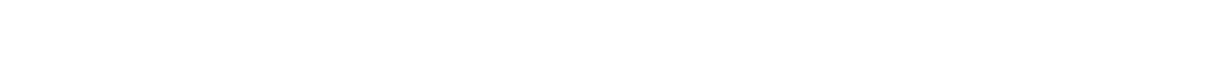

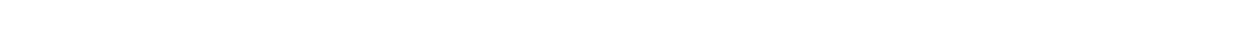

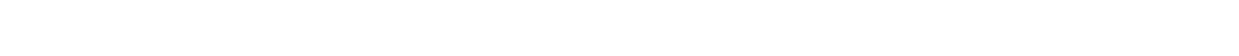

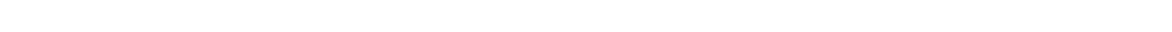

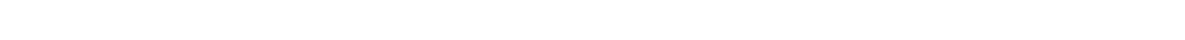

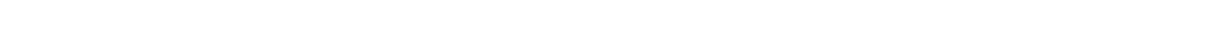

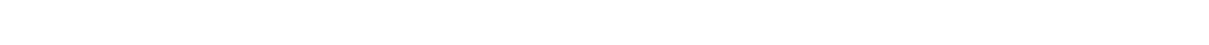

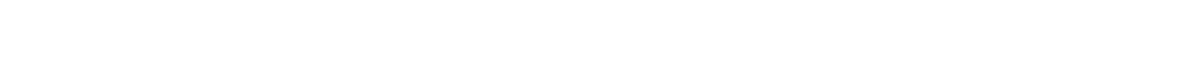

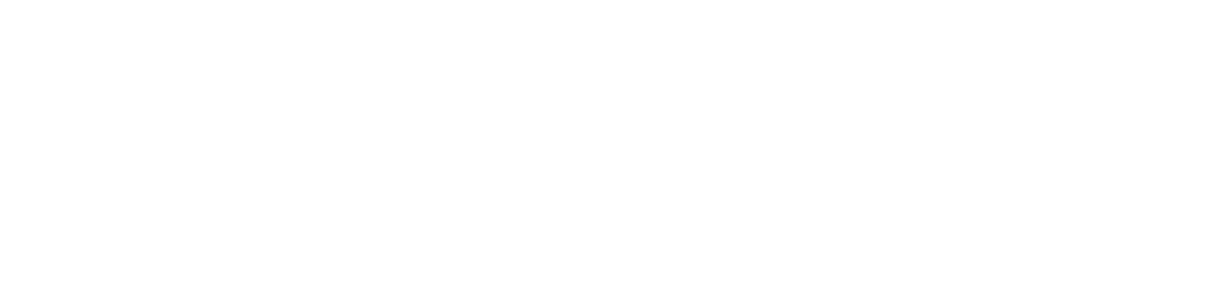
$\sigma \quad$ ui 2

Mmmmmmmmmmmmmmmmmmmmmm

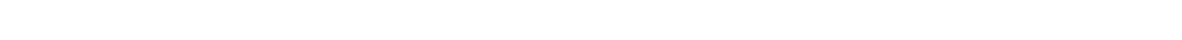

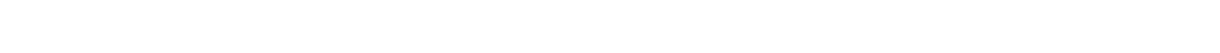

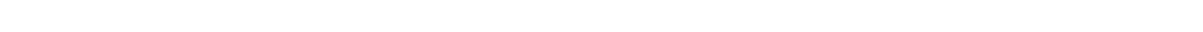

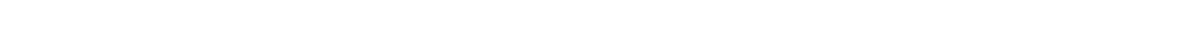

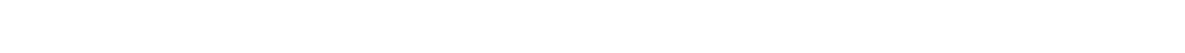

w n n on on es es en

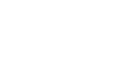

a

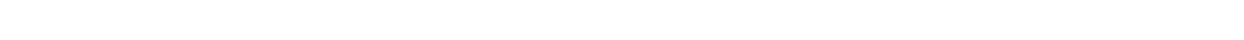

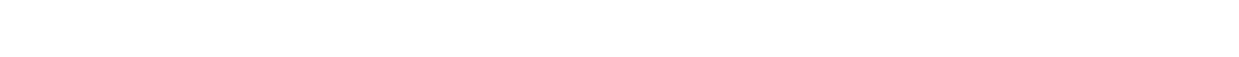

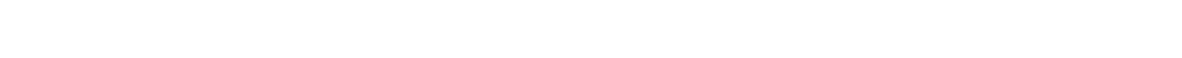

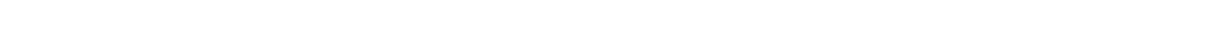

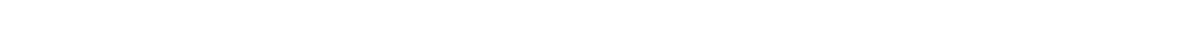

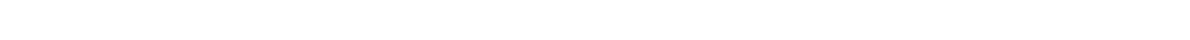

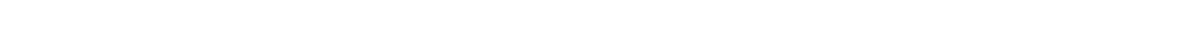
$\sim \sum_{i=0}^{\infty}$ กับ 등 느으. na $=a$ 20 - $\ddot{u}-\ddot{H}$

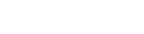
두용ㅇ

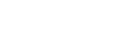

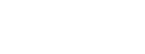

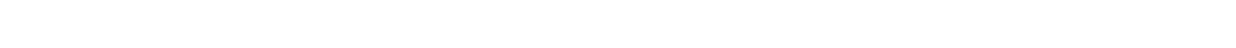

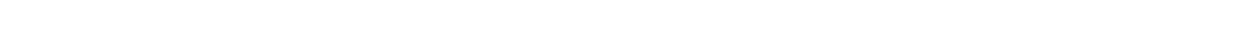
公

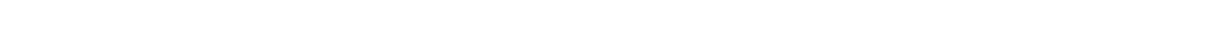

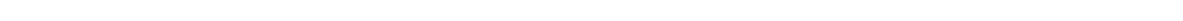

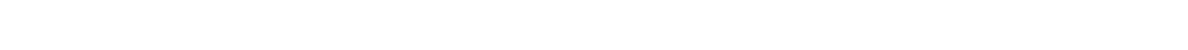

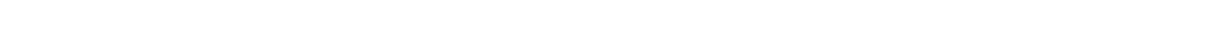

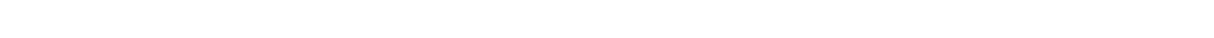

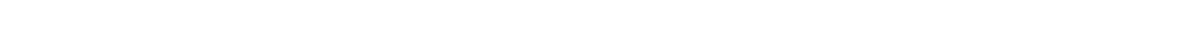

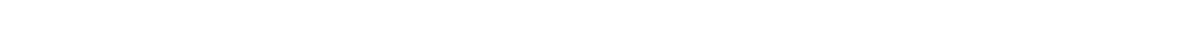




\begin{tabular}{|c|c|c|c|c|c|c|c|}
\hline 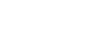 & n & $n$ & $n$ & n & $n$ & $e s$ & is \\
\hline
\end{tabular}

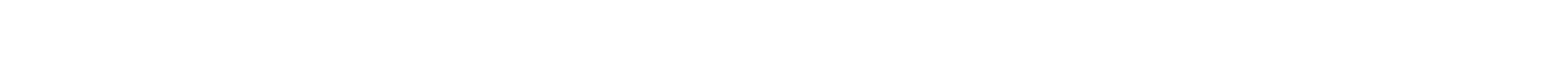

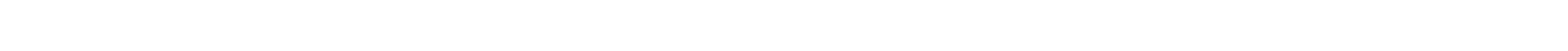

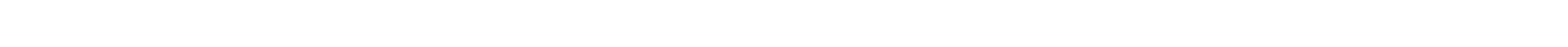

mmmmmmmmmmmmmmmmmmmmmmmmmmmmmmmmmmmmmMmm

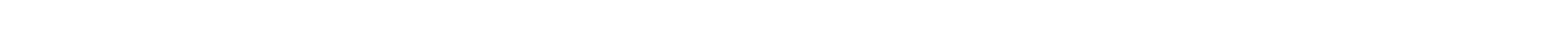
แ

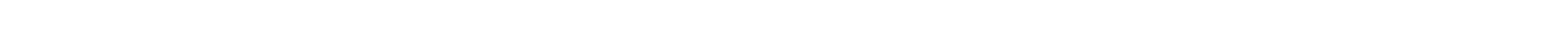
IN N-N

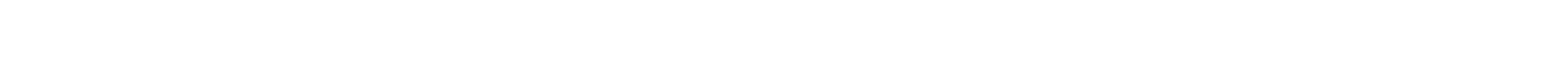
w
is
es es
is
in
n

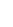
in

๔

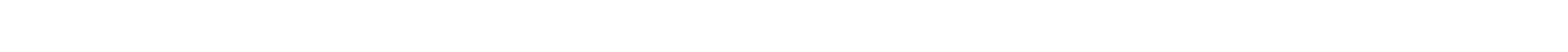

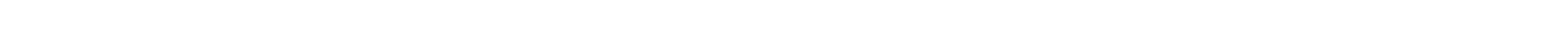
年

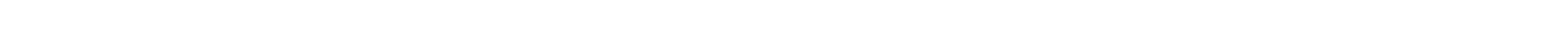

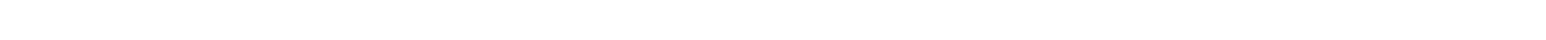

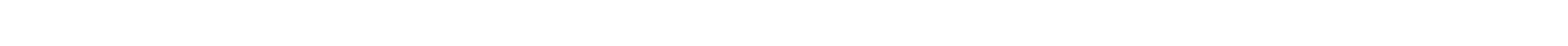

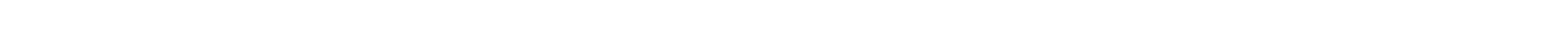

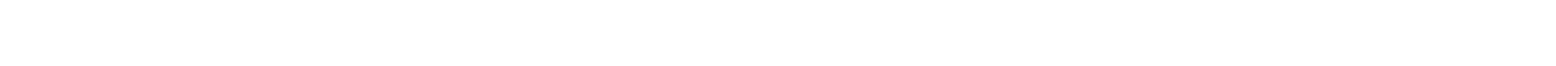

$\pm$

"ำ

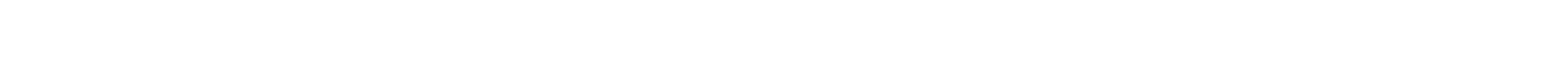

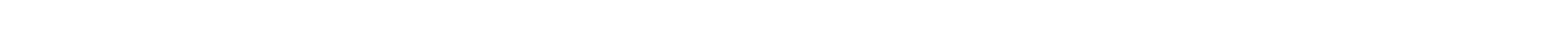

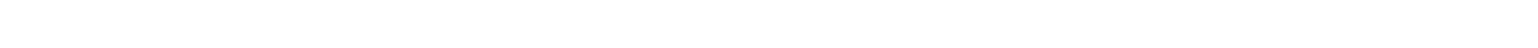

으으ำ

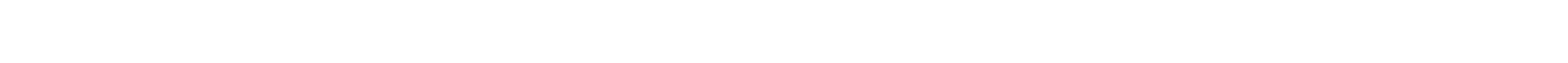

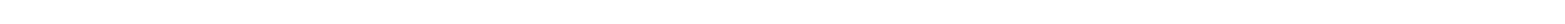

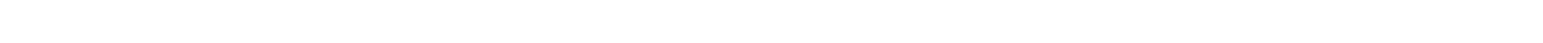

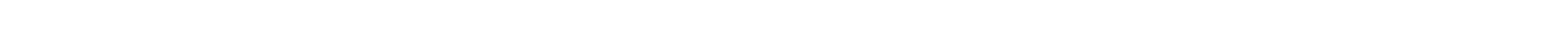

$\leftarrow$ is

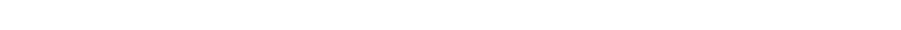

2

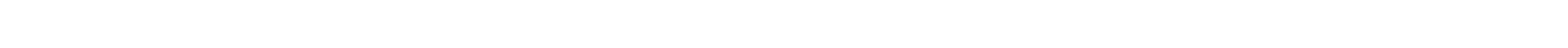

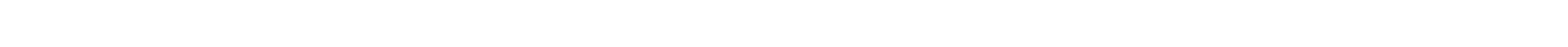

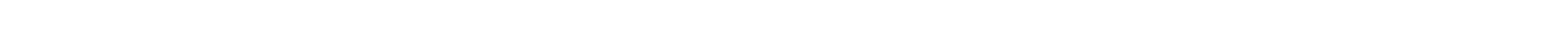

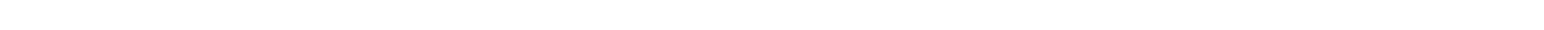

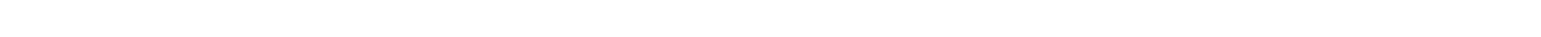

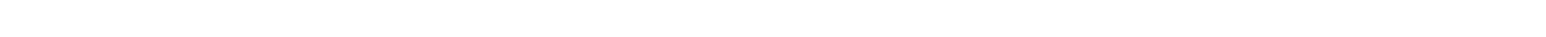

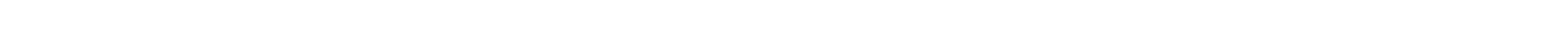

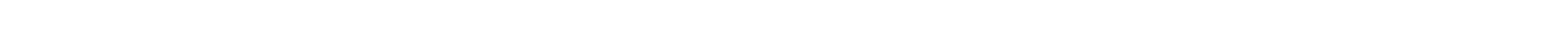

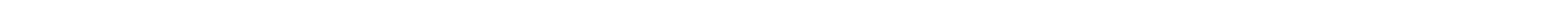

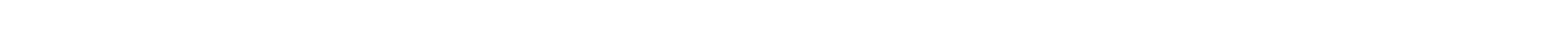

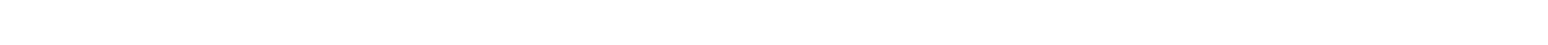




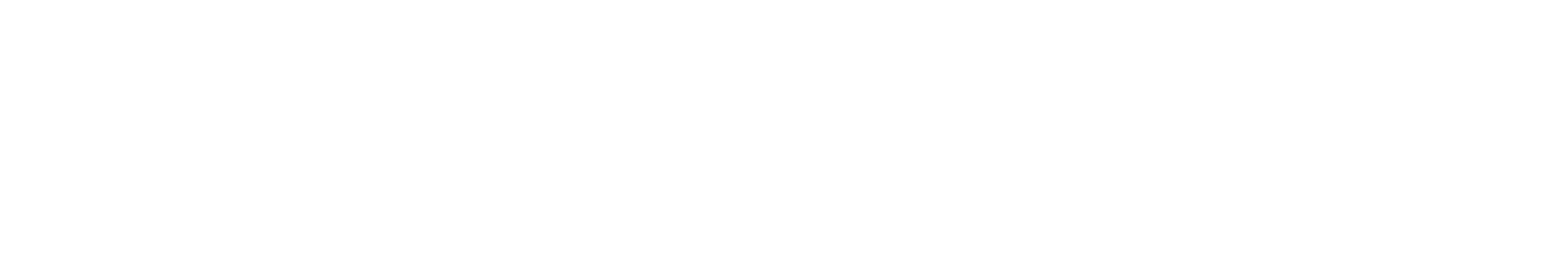

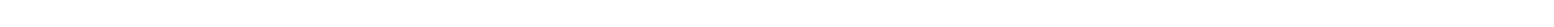

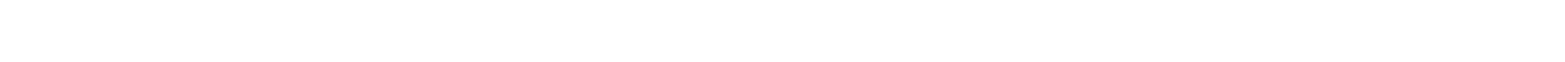

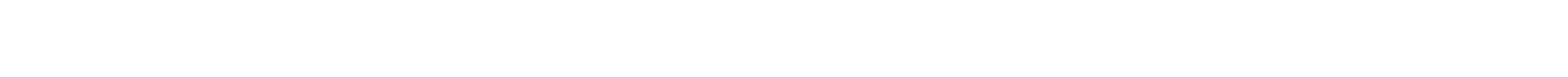

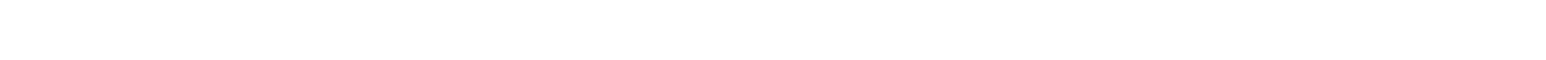
F

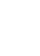
n n
n
皮
n
事
$\stackrel{a}{5}$

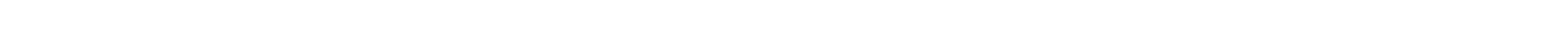
t

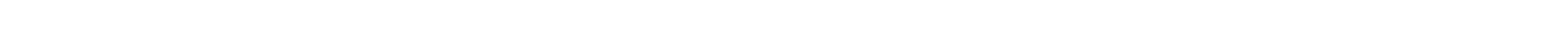

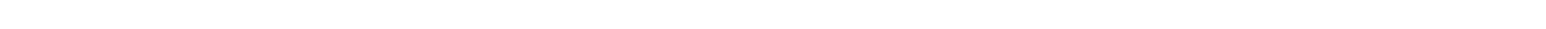

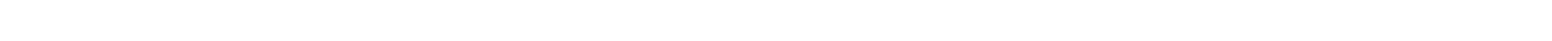

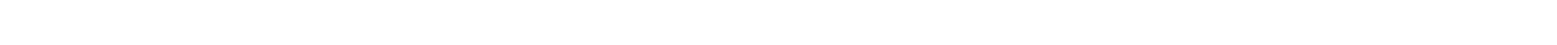

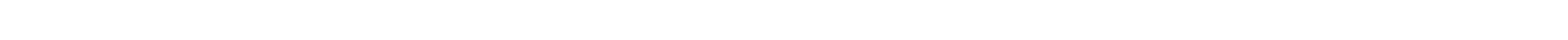

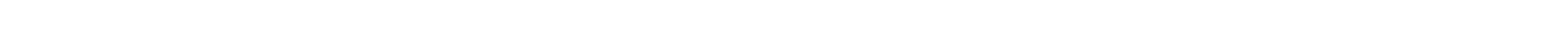

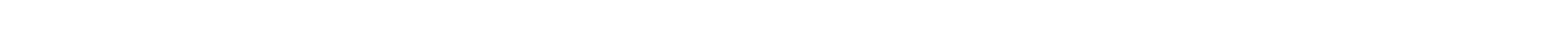

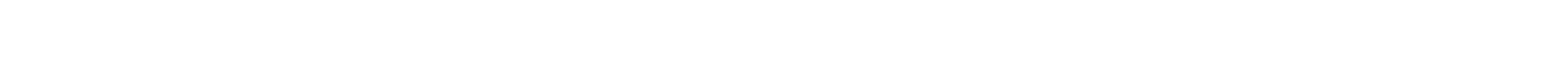

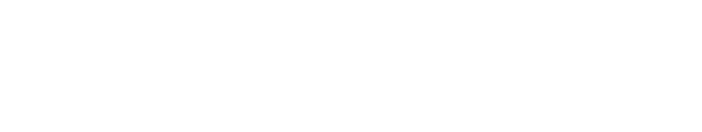

(n)

is

n

en

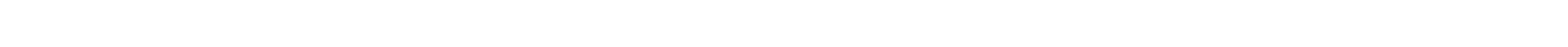

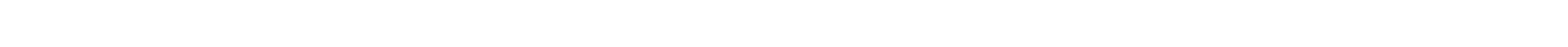

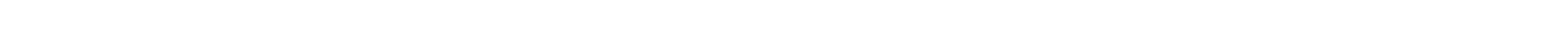

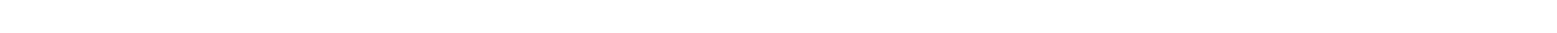

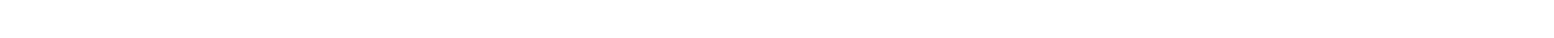

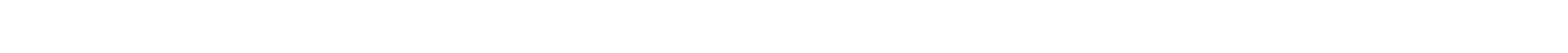

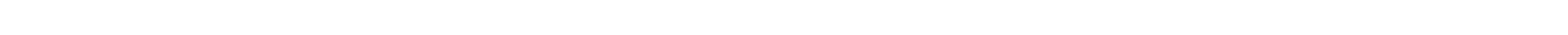

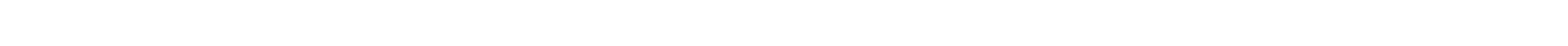

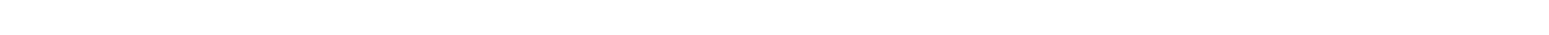

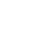

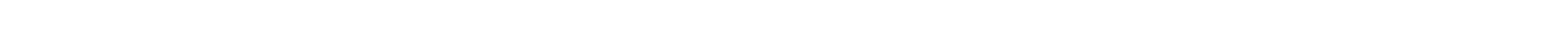
moonotonoguna in

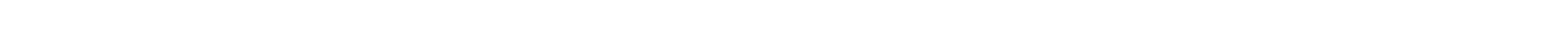
“

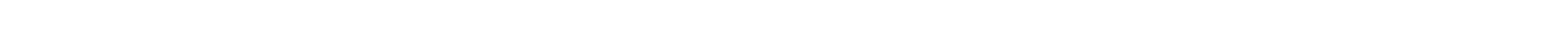

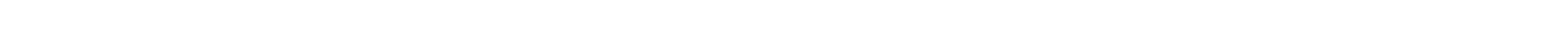

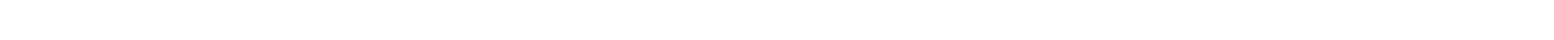

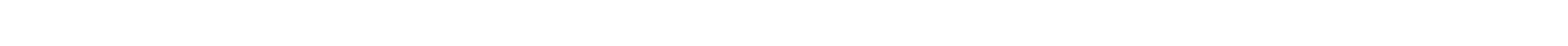

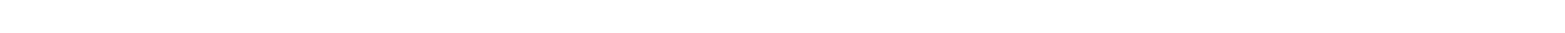

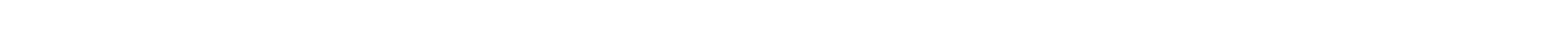

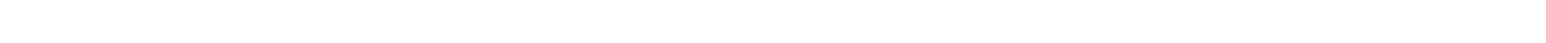




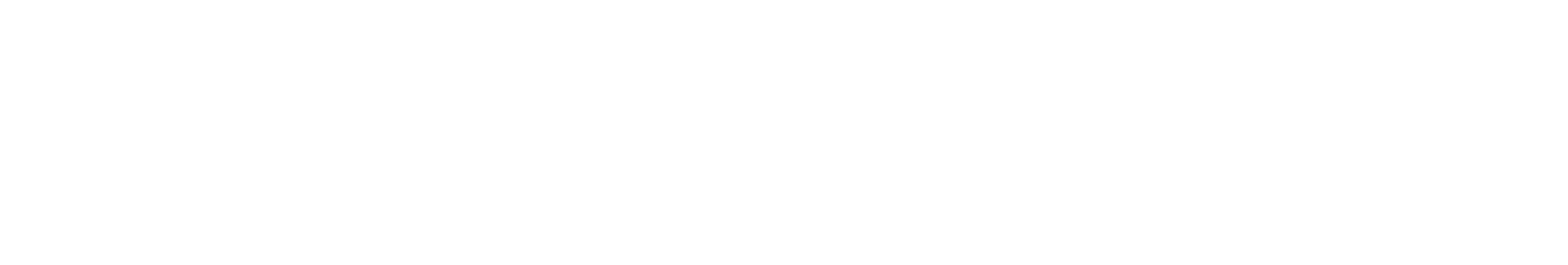

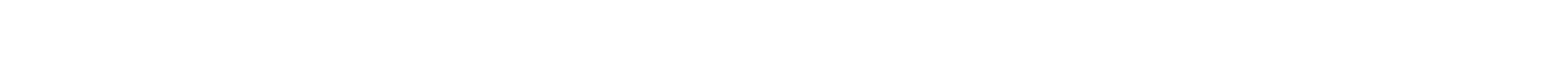

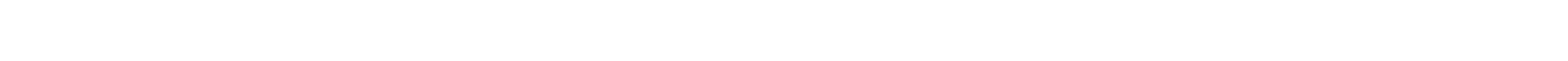

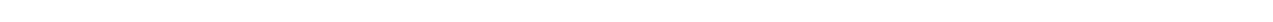

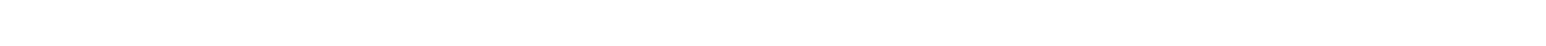

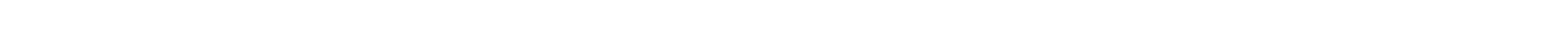

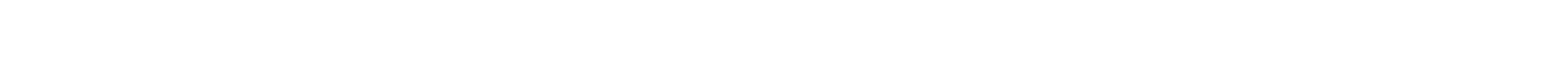

a

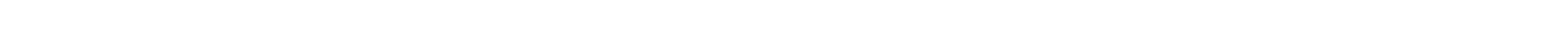

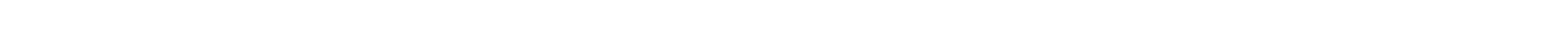

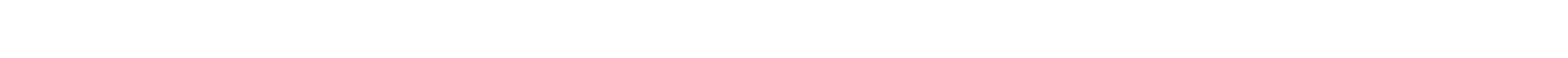

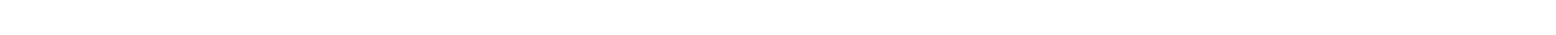

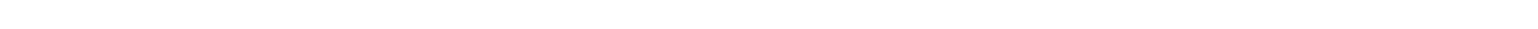

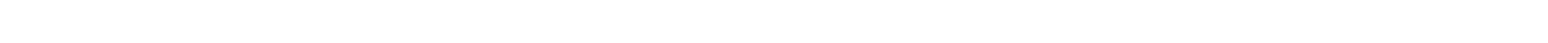

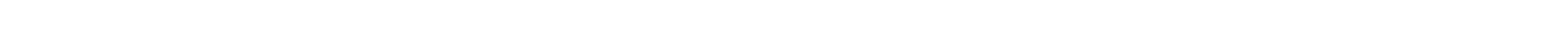

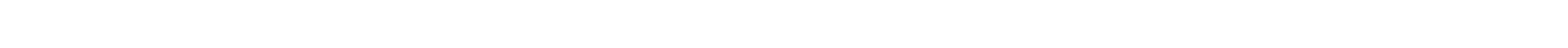

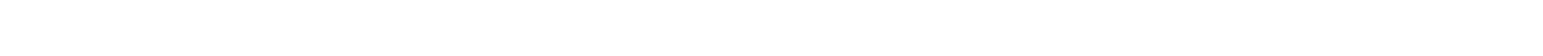

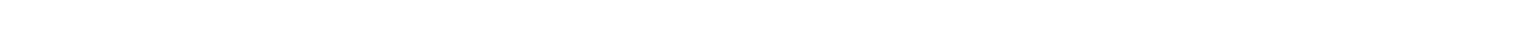
is

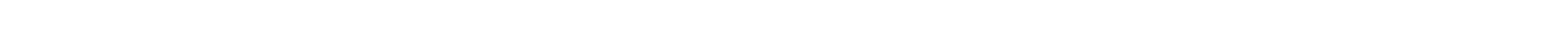
ứ 出

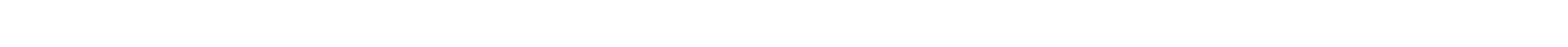

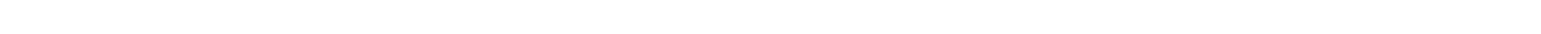
200000000000000000000000000000000000000000000000000

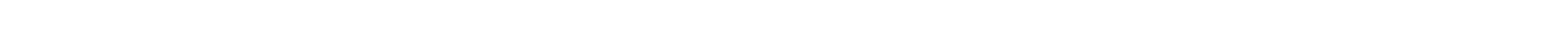

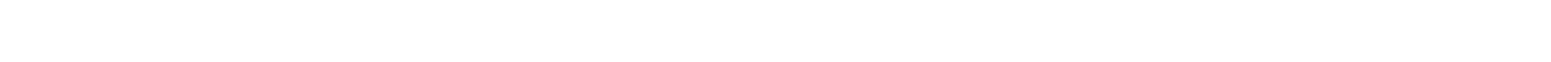

$\stackrel{\leftarrow}{\Sigma}$

is

in

$\backsim$

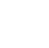

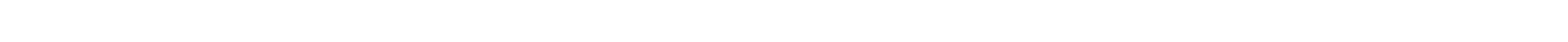

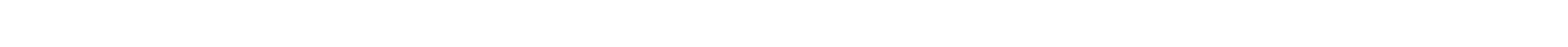

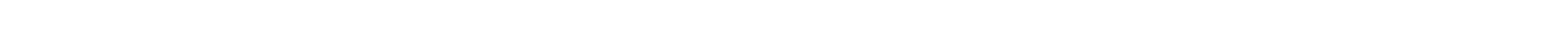

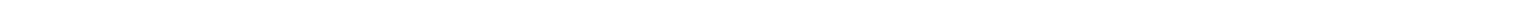

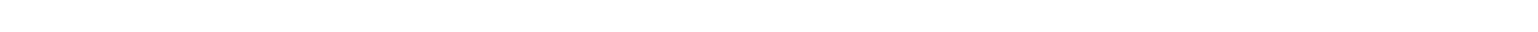

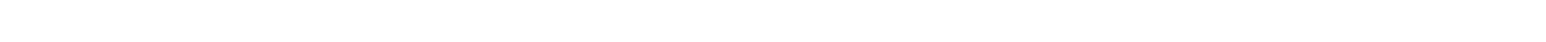

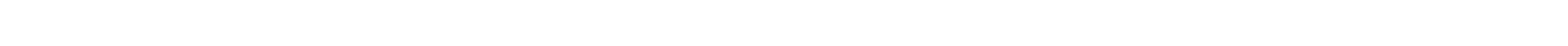
은 -

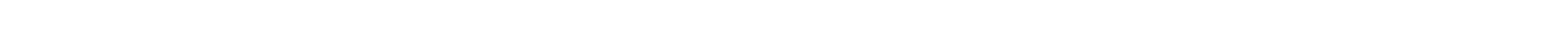




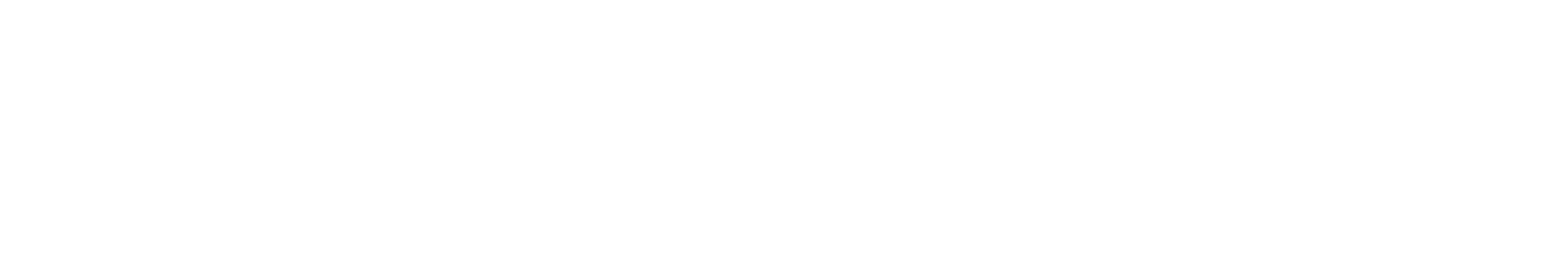

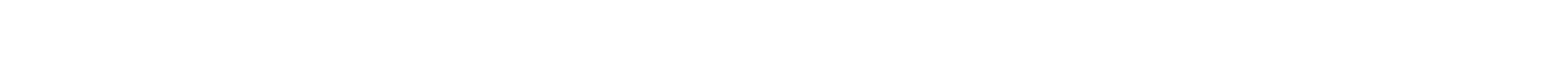

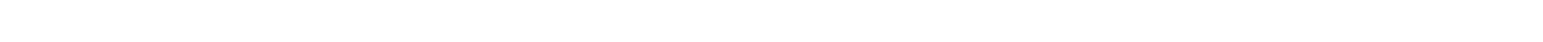

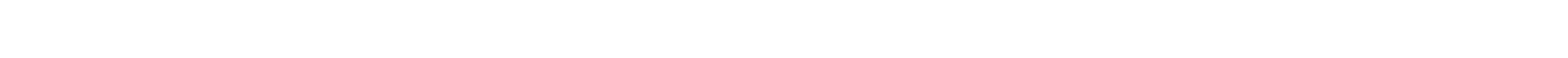

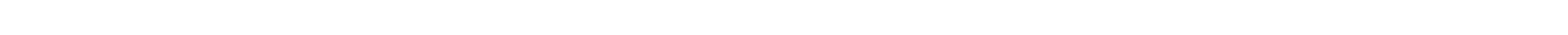

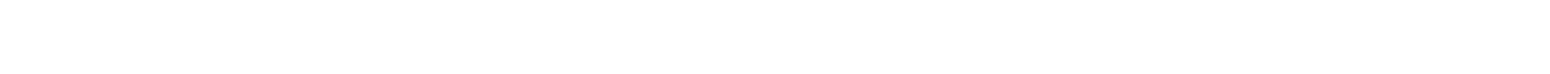
wis
m
u
in
in

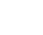

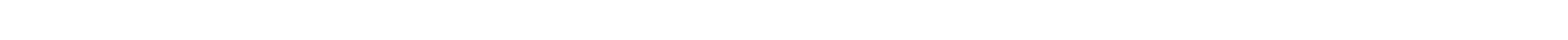

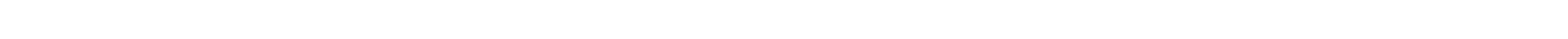

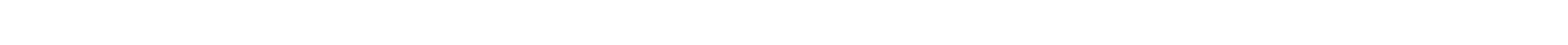

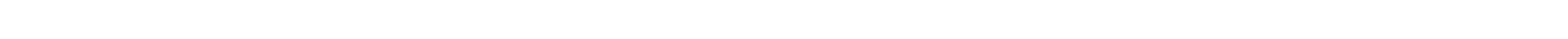
mann

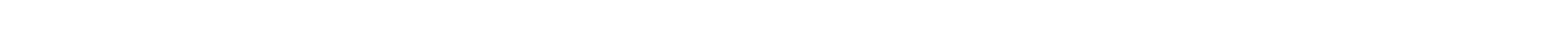

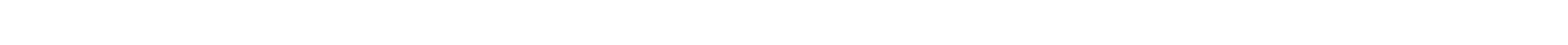

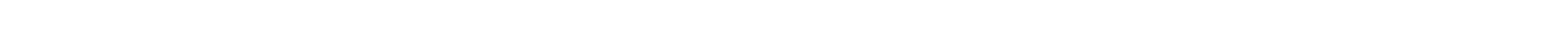
ヘNmQ

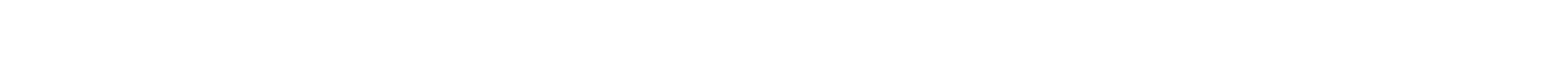

\pm is

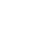

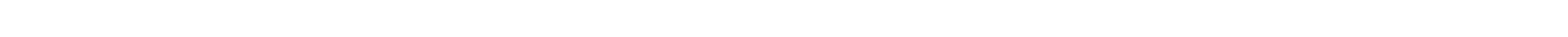

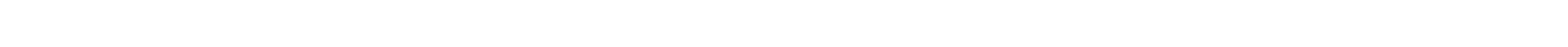

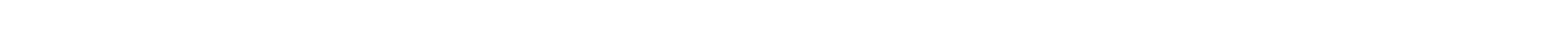

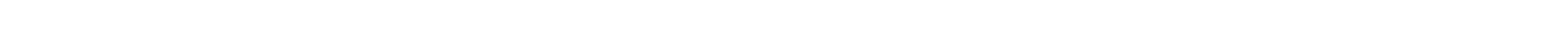

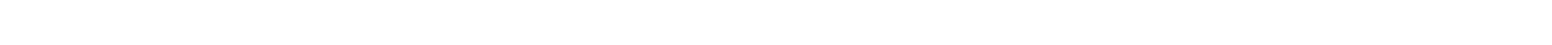
แ

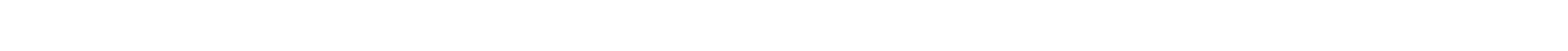

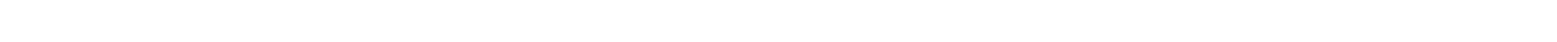

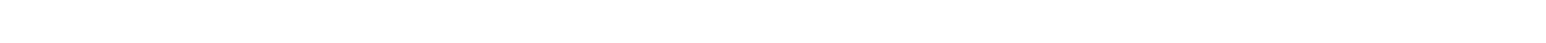

$\stackrel{n}{1}$ o

o

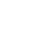

n

$\infty$

in

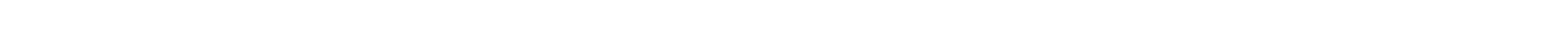

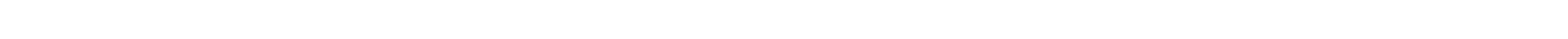

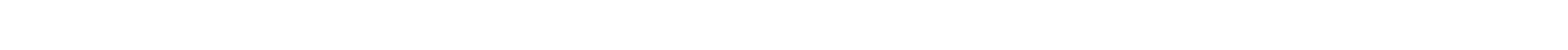
a u उ

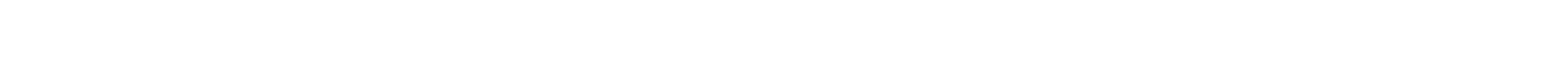

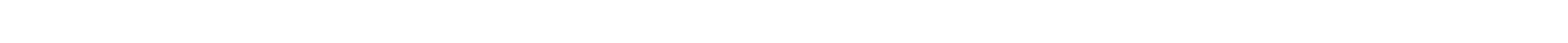

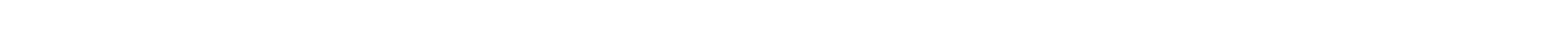

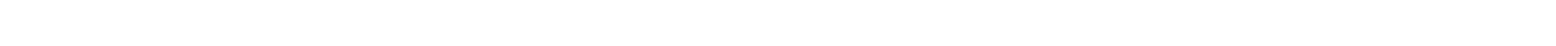

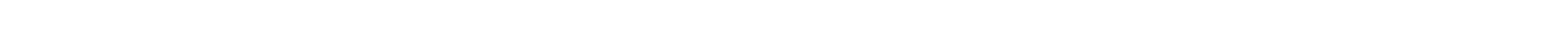




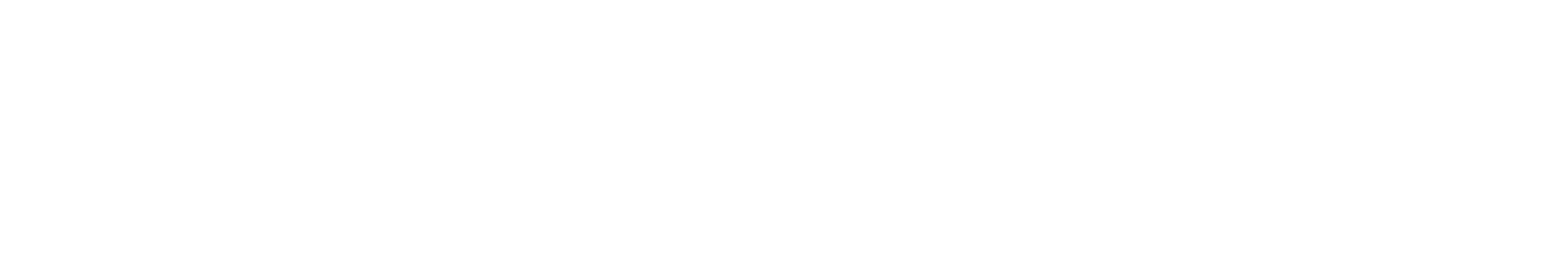

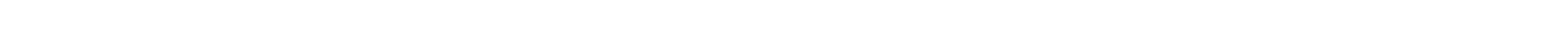

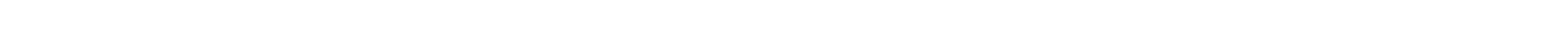

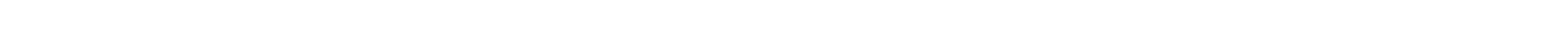

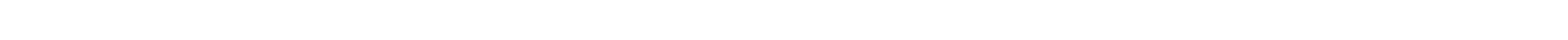

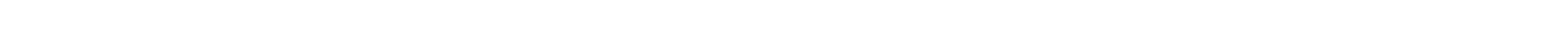

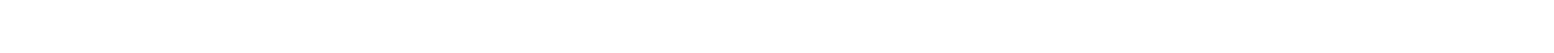

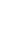

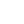

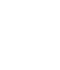

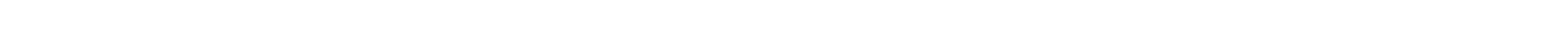

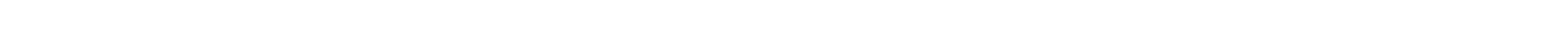

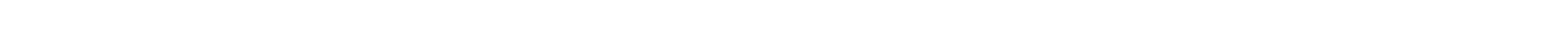

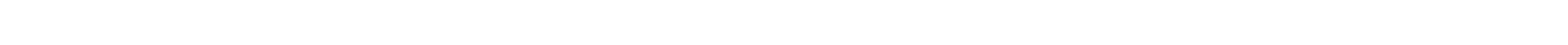

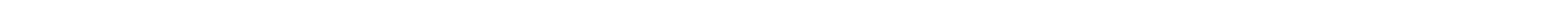

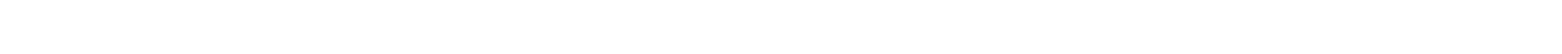

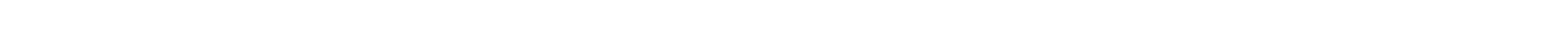

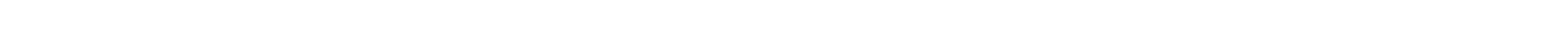

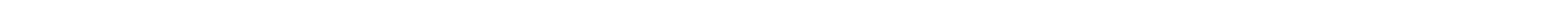

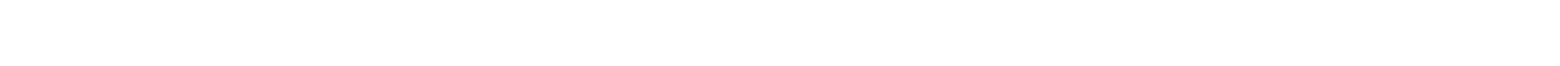

$\stackrel{\leftarrow}{\Sigma}$

us

s

n

is

$\pm$

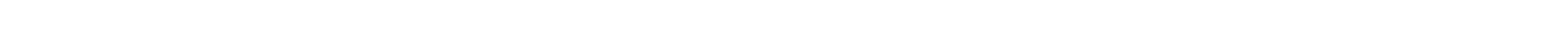

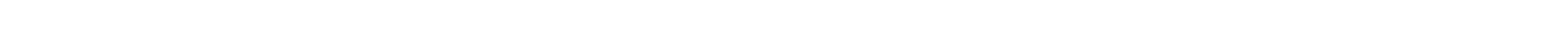

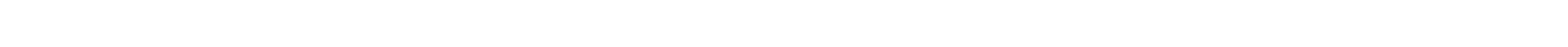

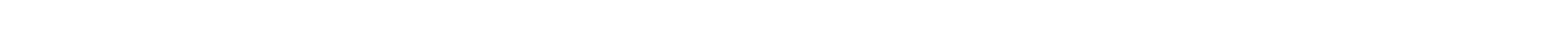

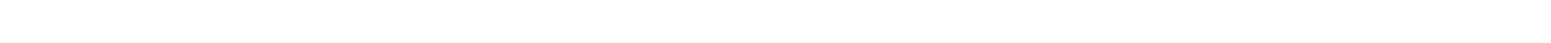
W

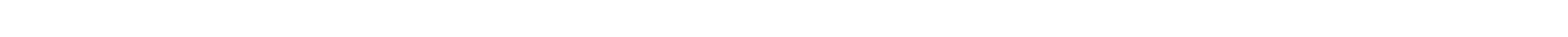

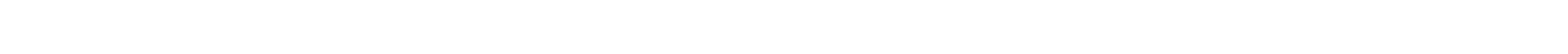

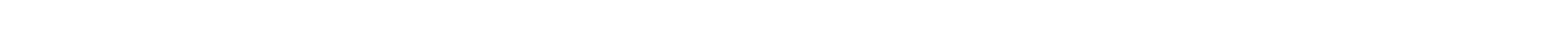

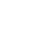

is

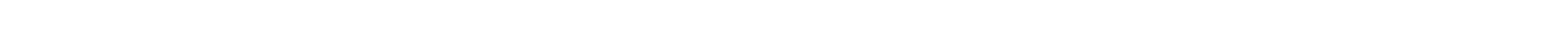
жым.? ?

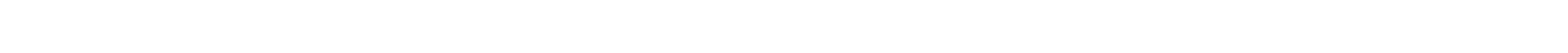

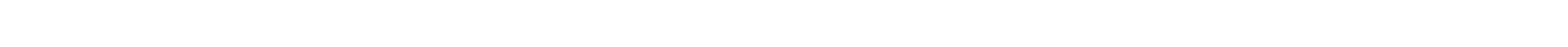

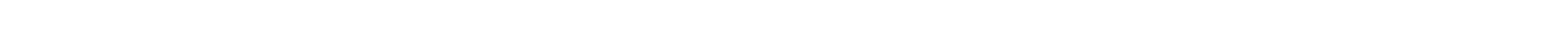

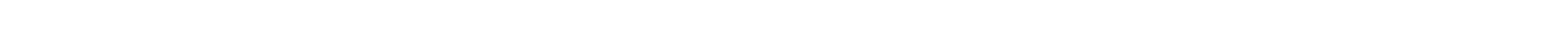
añ

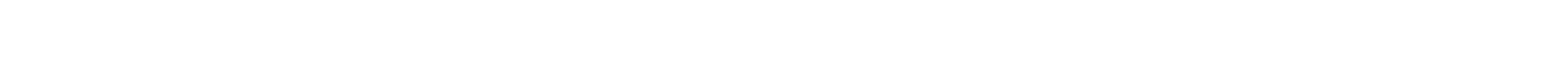




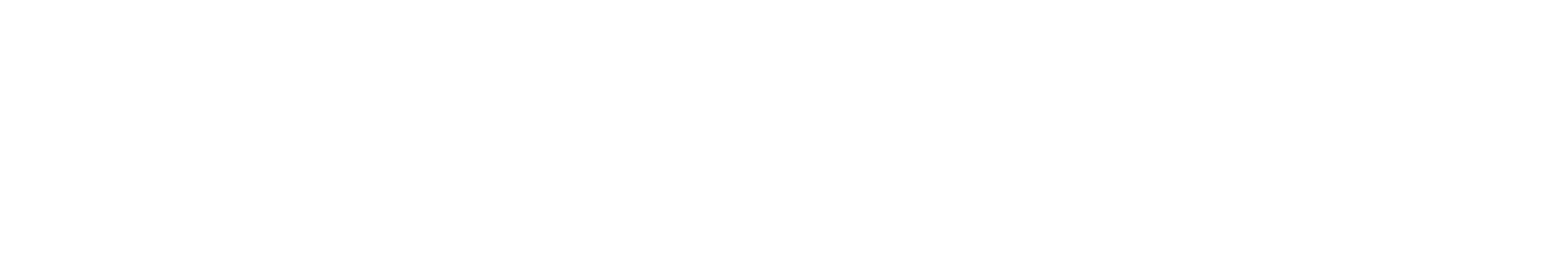

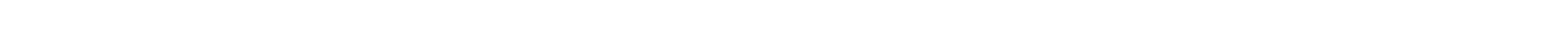

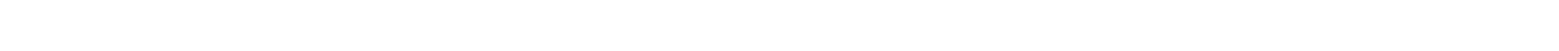

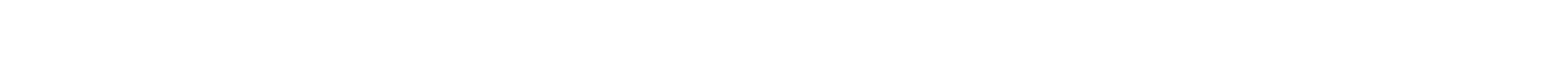

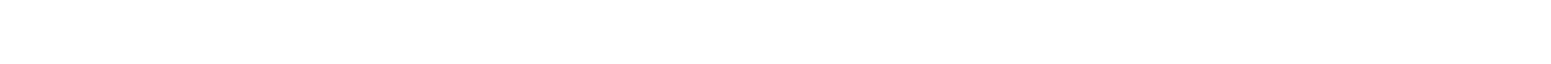

z

in $n$

n

as is

en

n

is

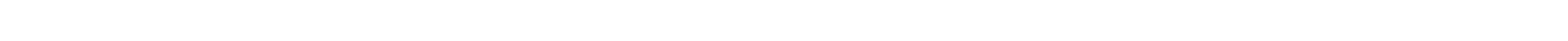
H

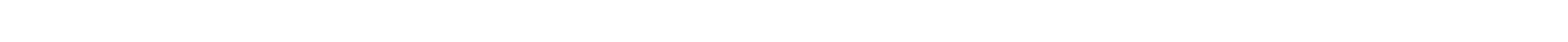

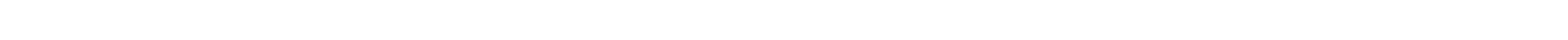

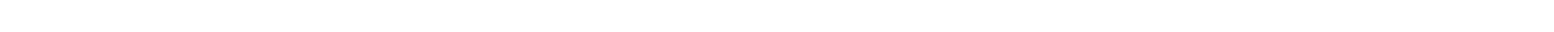

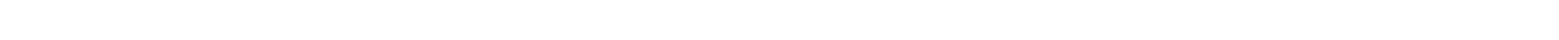

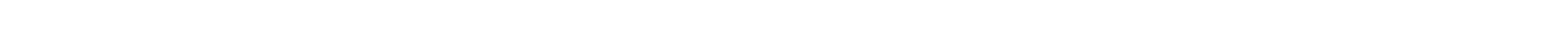

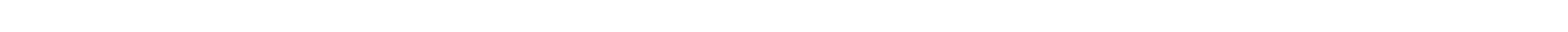

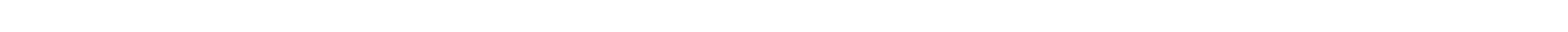

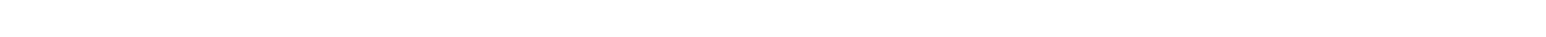

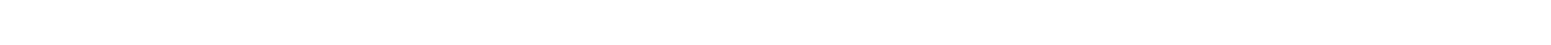

5
a
is
n
in
in
is
ด =

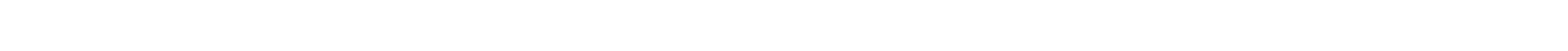

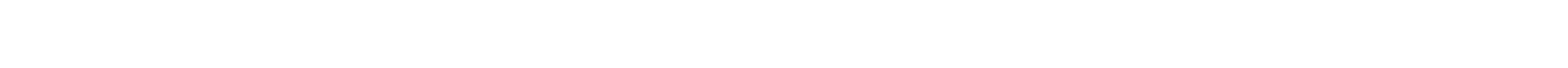

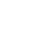

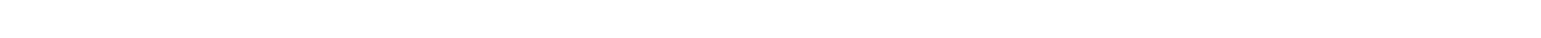
Oñ

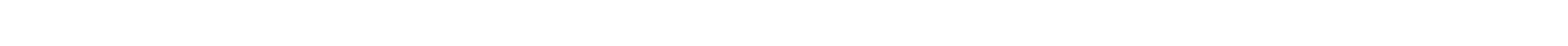

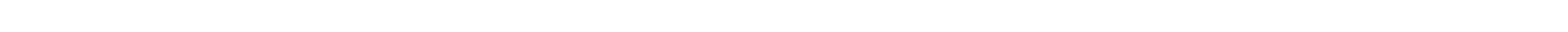

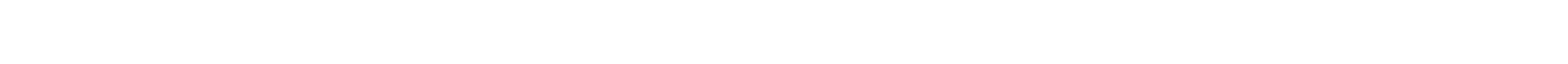

a

in

en

n

is

is

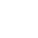

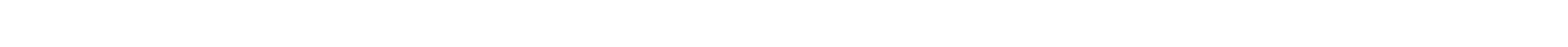
ü

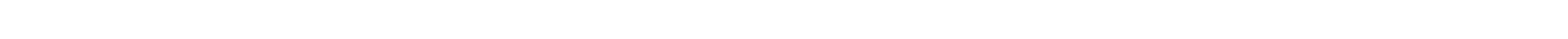

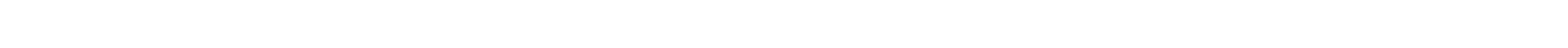

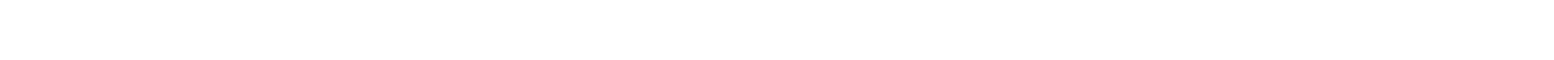

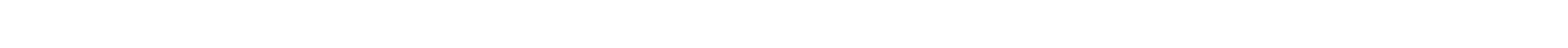

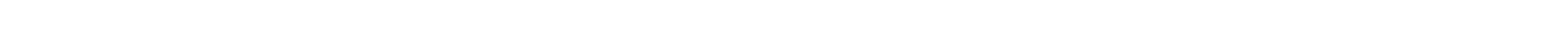

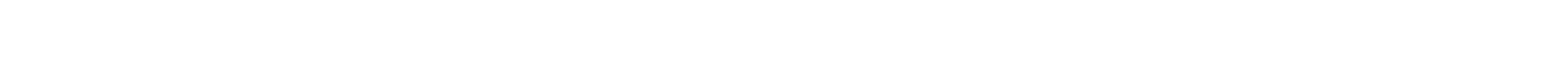




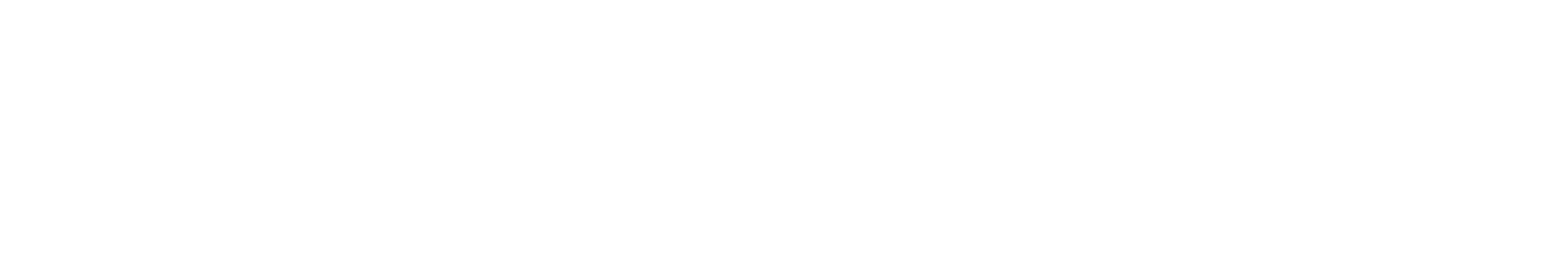

w

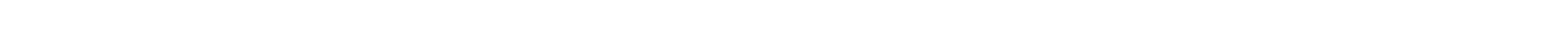

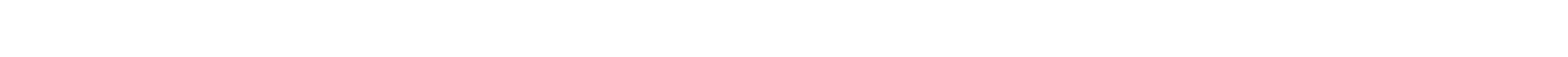

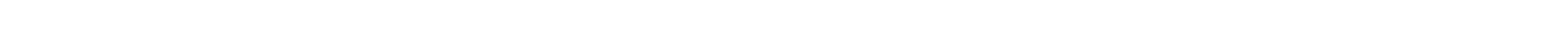

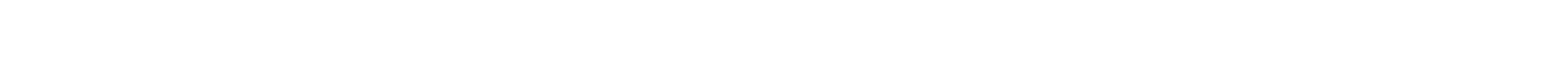

เ

s

n

n

in

i

os

is

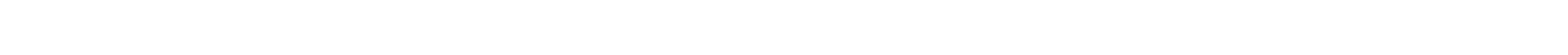

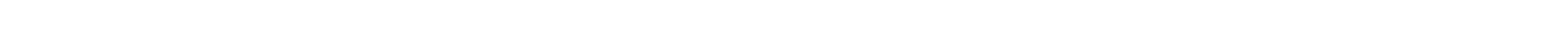

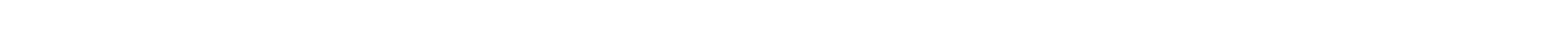

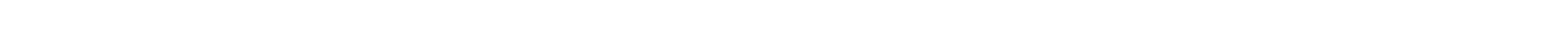

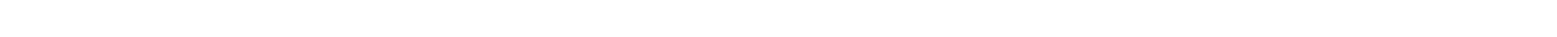

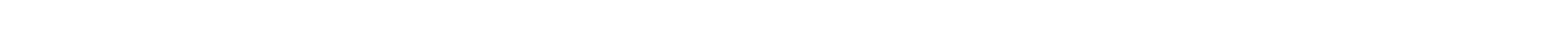

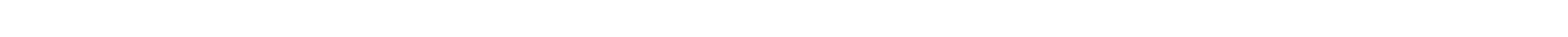

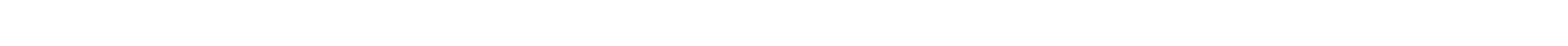

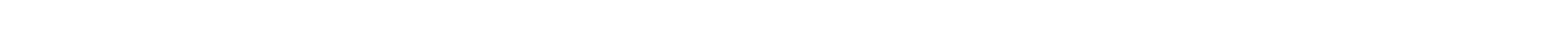

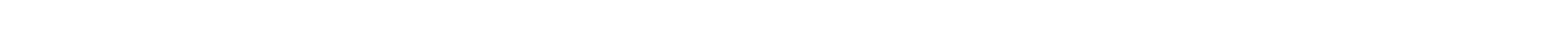

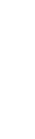
is

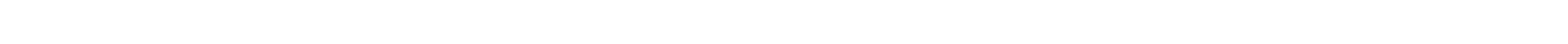

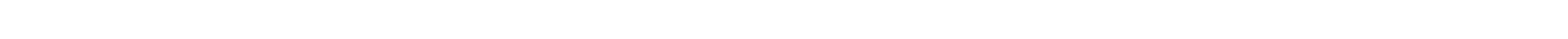

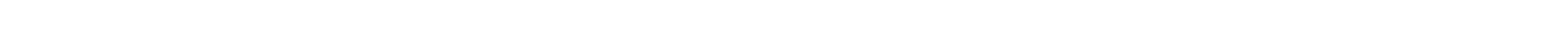

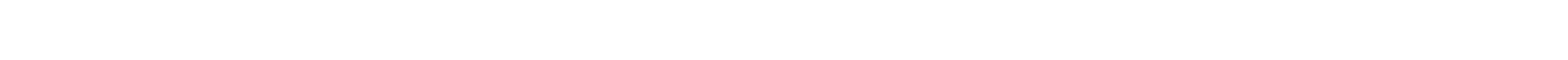

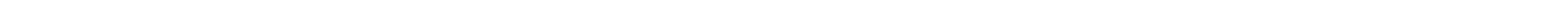

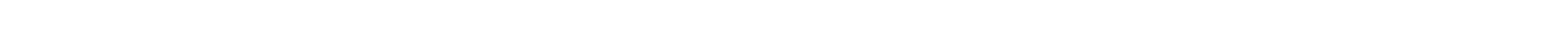

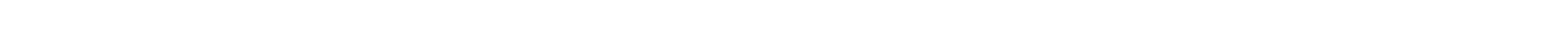

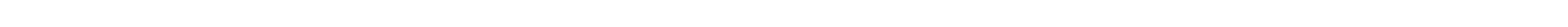

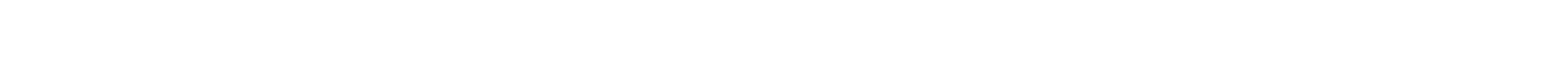

岕

n

i

is

n

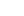

n

is

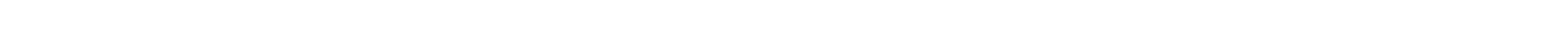

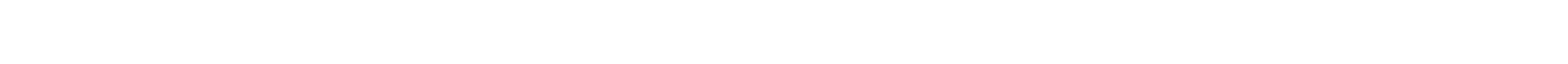
×

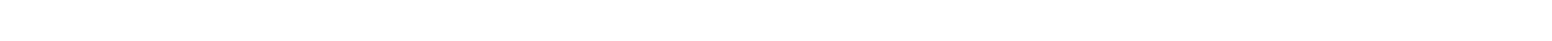

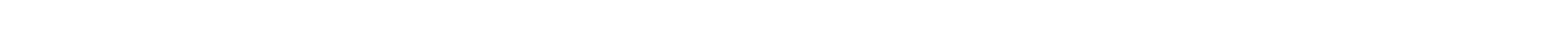

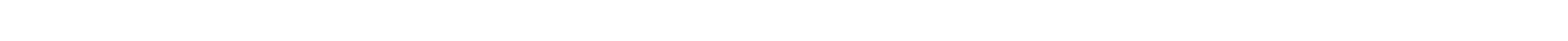

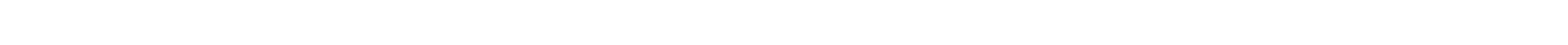

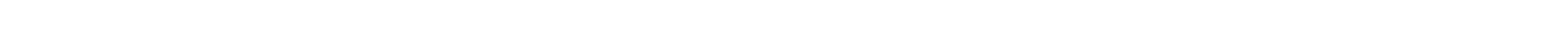




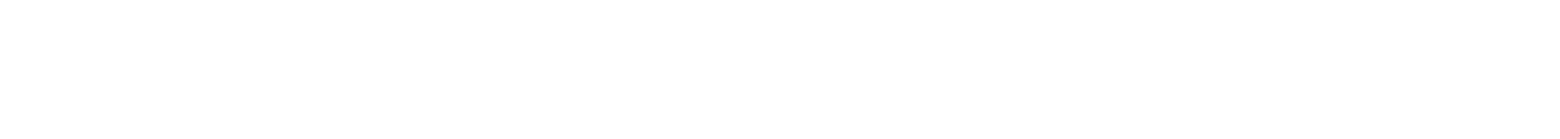

"

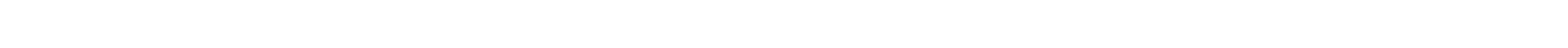
< й

-

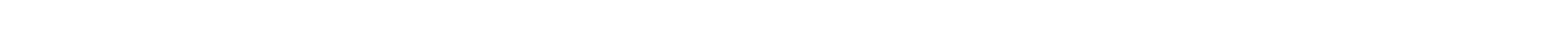

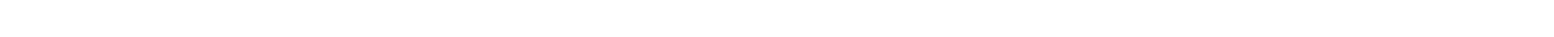

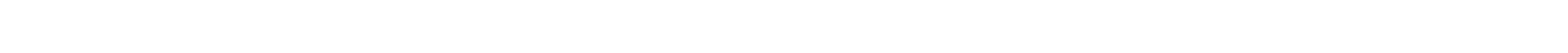

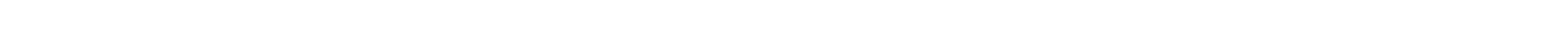

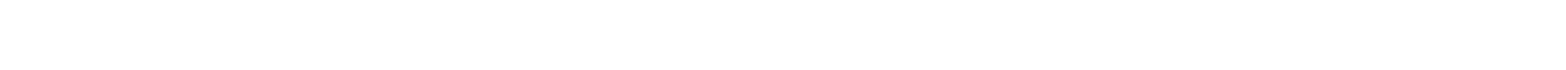

$$
\begin{aligned}
& \text { L } \\
& \square \\
& 5
\end{aligned}
$$

is

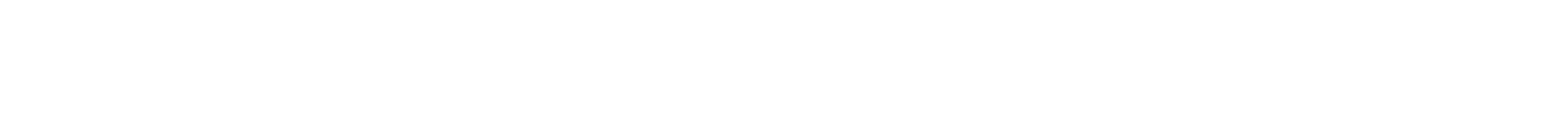

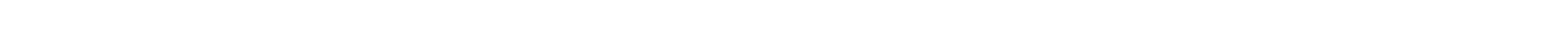

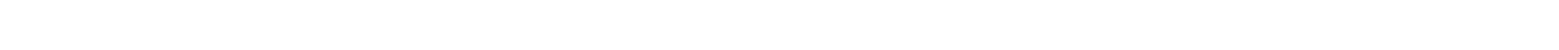

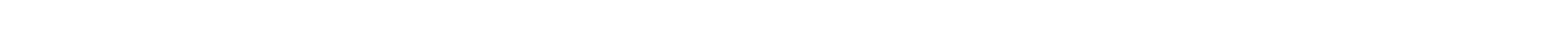

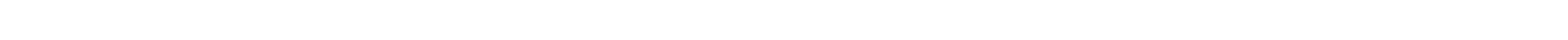

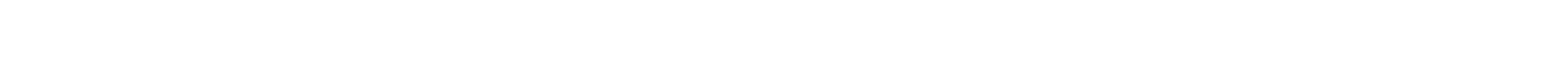

$\stackrel{\leftarrow}{\omega}$

$\underset{\mathbf{s}}{\mathbf{s}}$ n

is

n

n

n

n

en

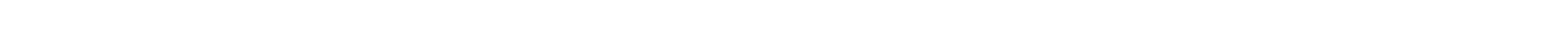
世 w w

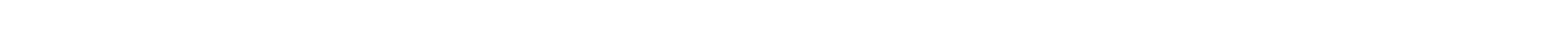

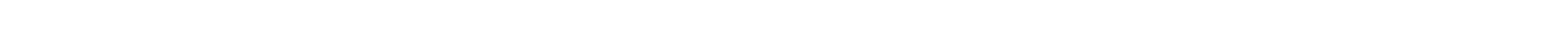

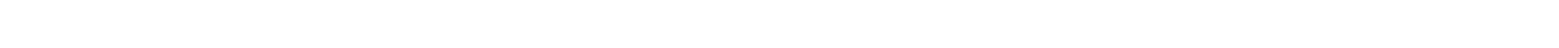

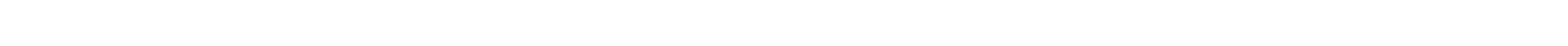

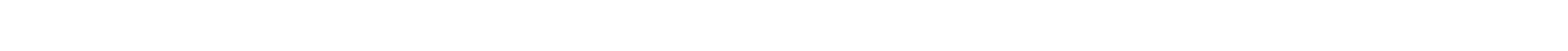

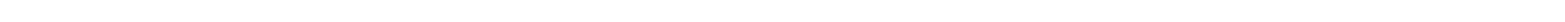

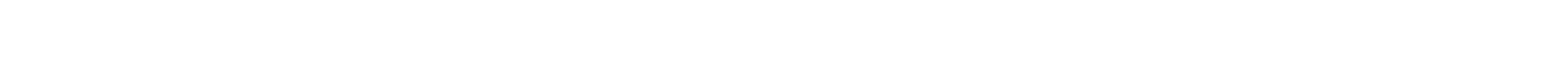

to e

n

n

es

n

in

in

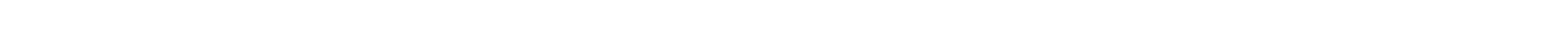

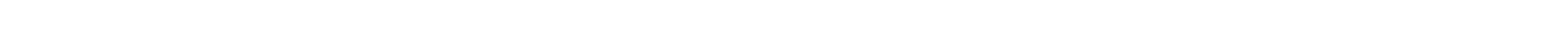
un in w.

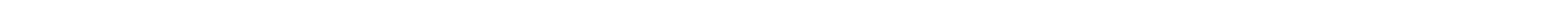

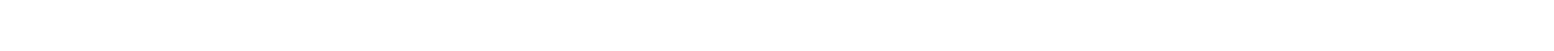

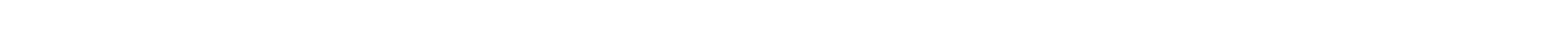

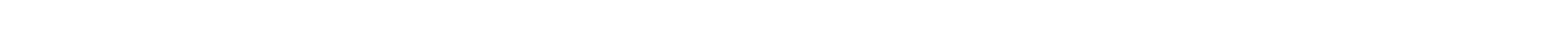

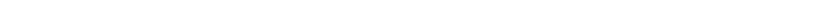

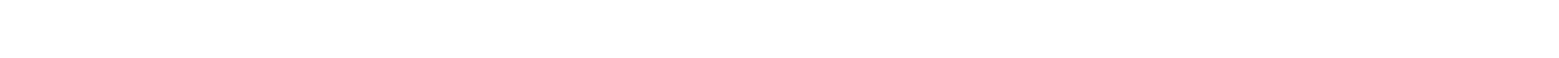



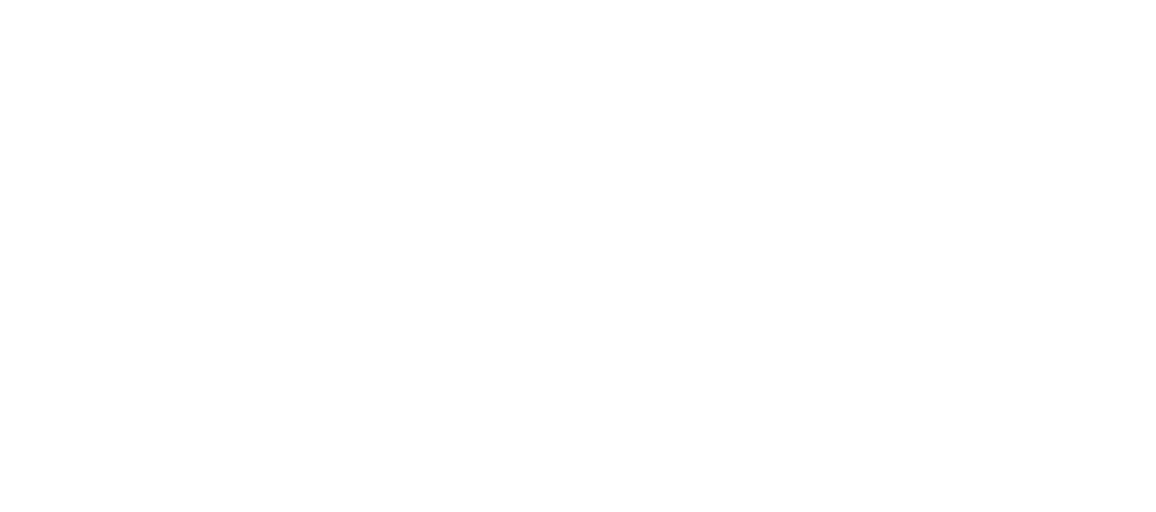

$$
\text { 秥的 }
$$$$
\text { is }
$$$$
\text { is }
$$$$
\text { n }
$$

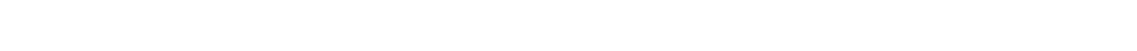

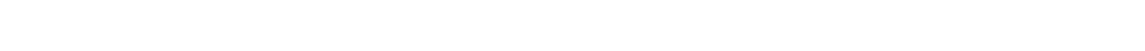

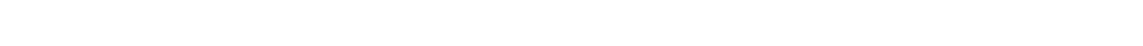

-

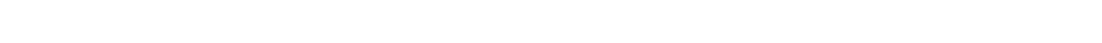

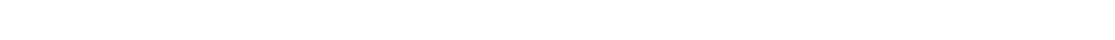

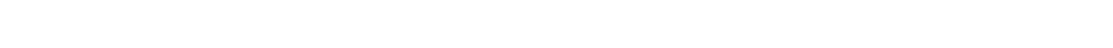

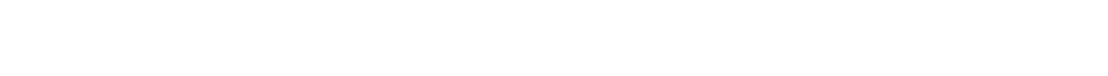

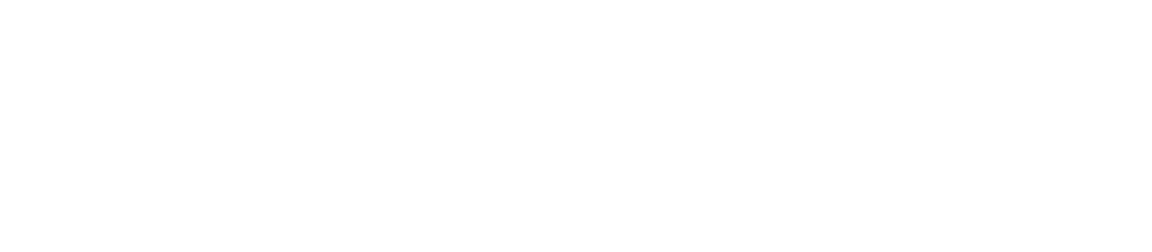

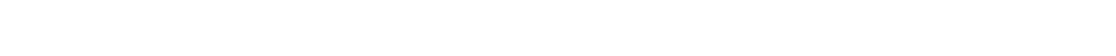

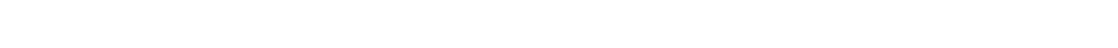

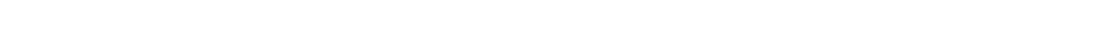

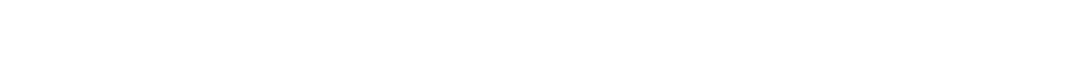<smiles>[10BH]</smiles>

崖

in

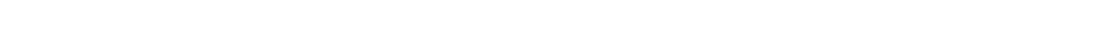

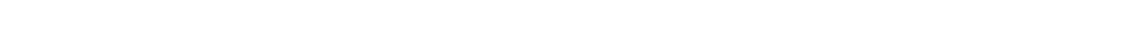

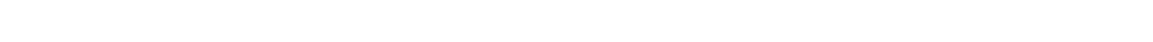

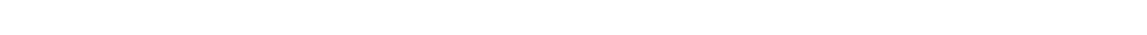

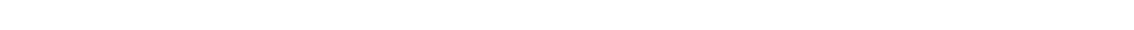

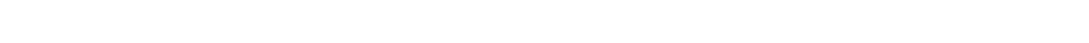

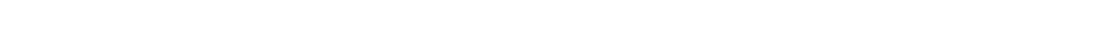

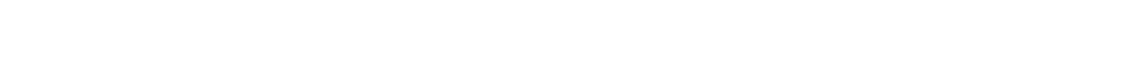

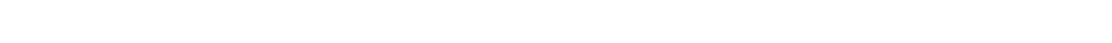

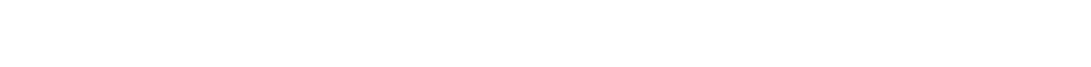




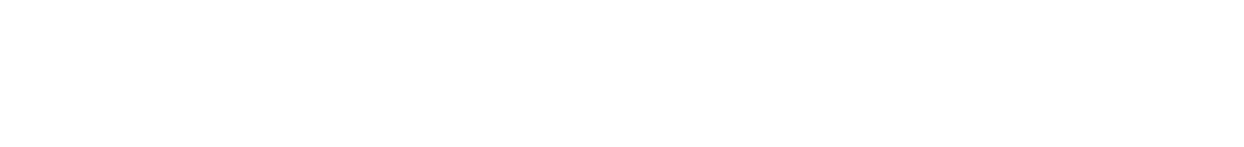

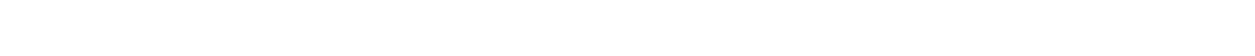

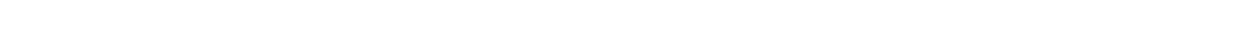

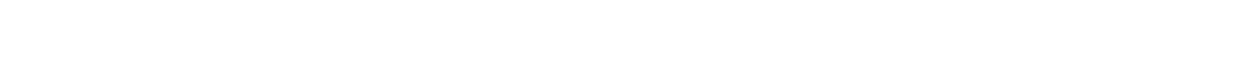

nnmumm n

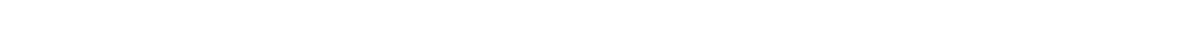

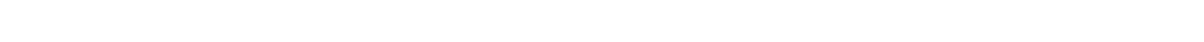

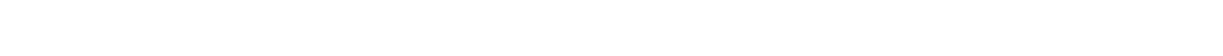

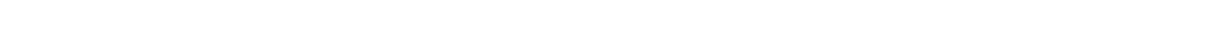

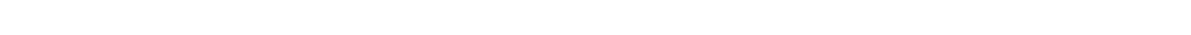

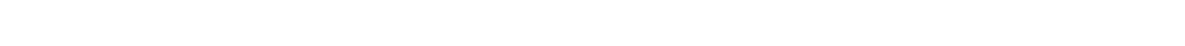

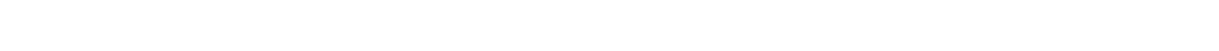

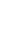

mo-n w L

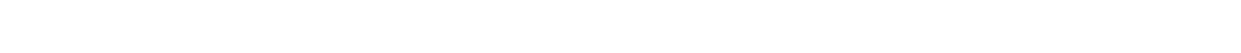

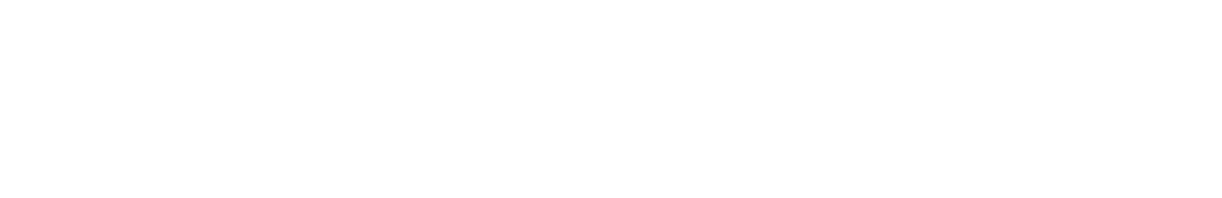

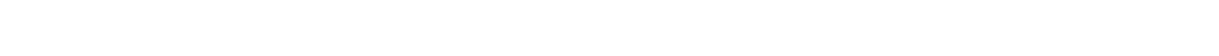

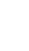

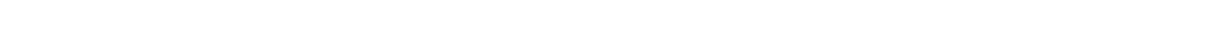

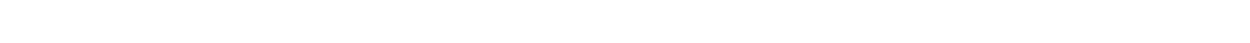
\begin{tabular}{rll}
\hline \\
\hline
\end{tabular}

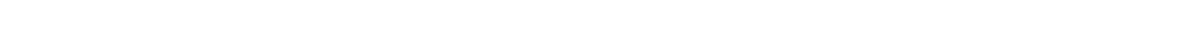

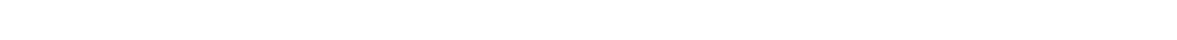
ய a แก $00000000000000000-2-7-7-7-2-20000000000$

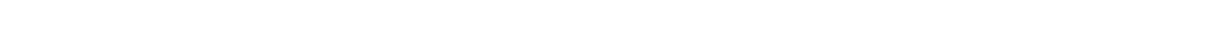

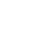

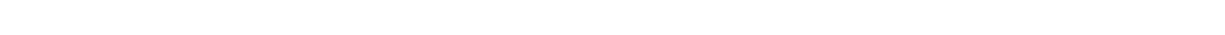

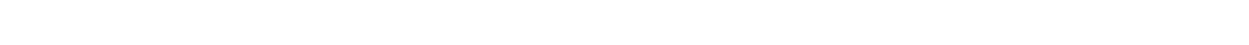
ז $>$ - -

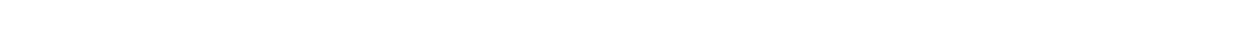

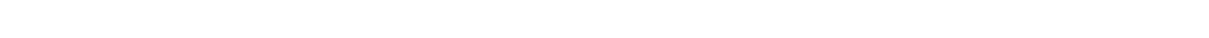

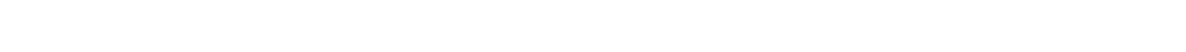

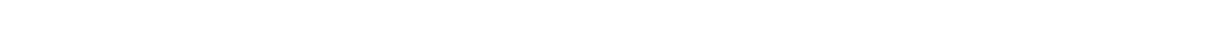

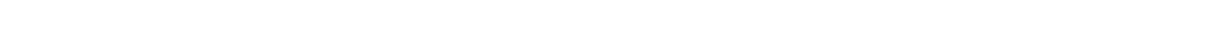

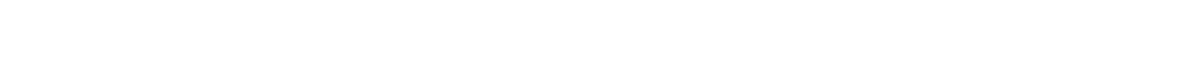




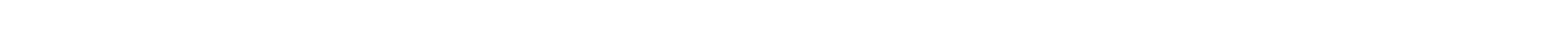

in

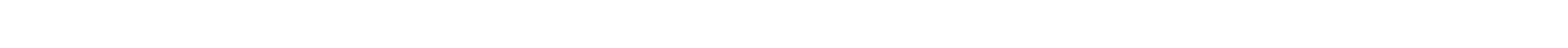
жй

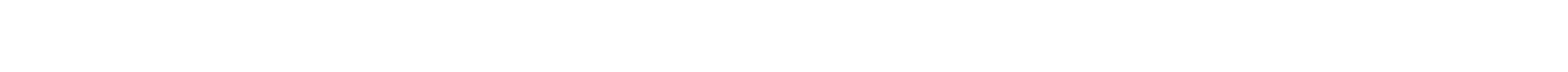

ఒั

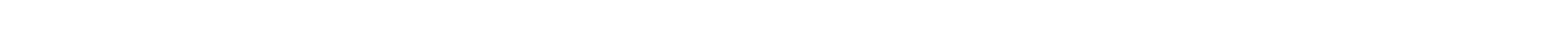
แ

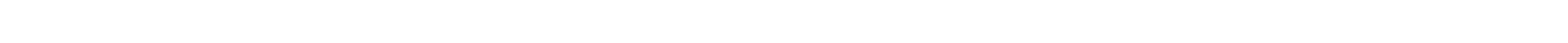

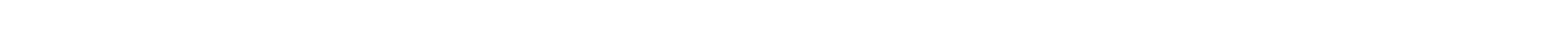

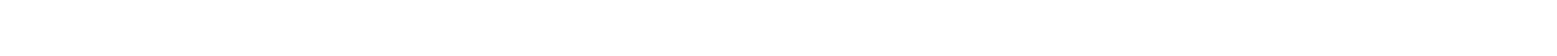

$+$

$\underline{\underline{z}}$

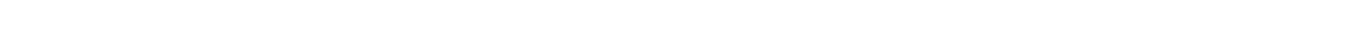

$\pm$

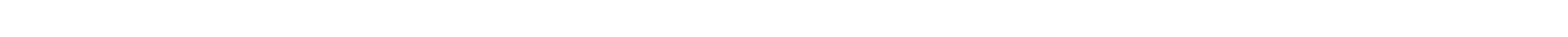

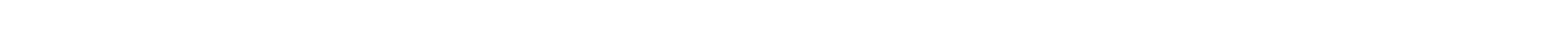

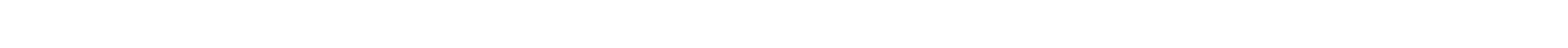
\begin{tabular}{ll}
3 \\
\hline
\end{tabular}

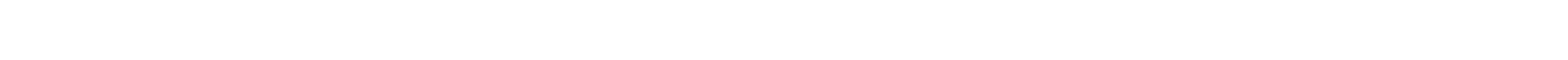

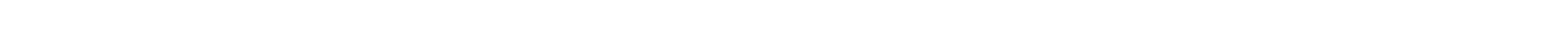

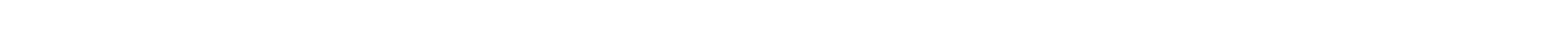

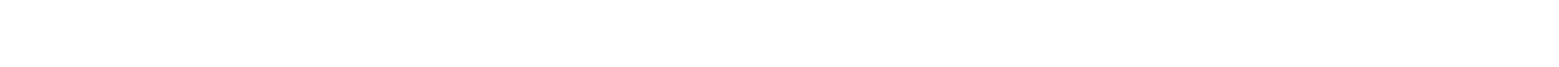

$\stackrel{2}{2}$

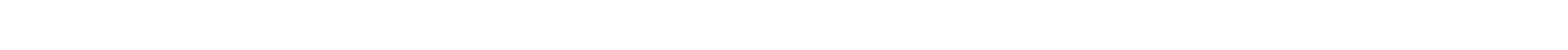

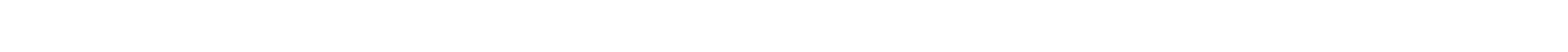

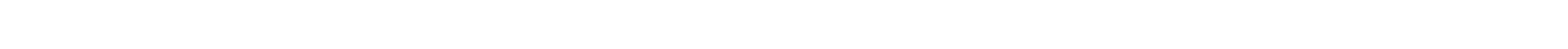

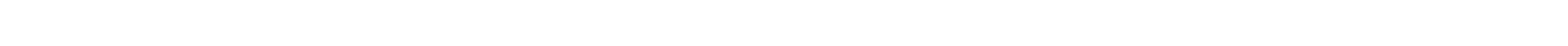

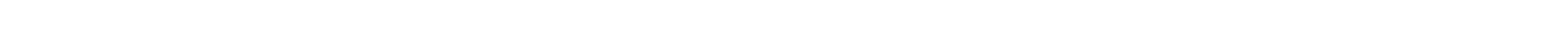

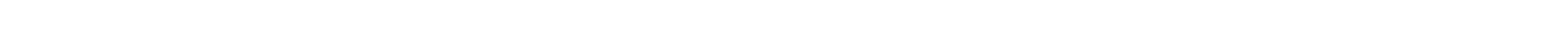

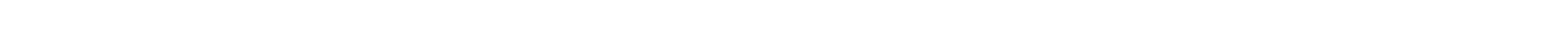

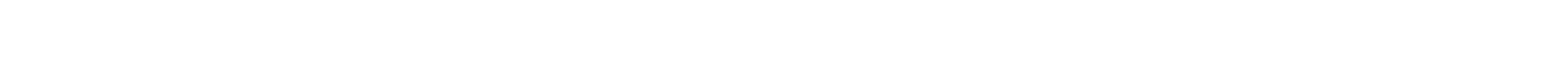

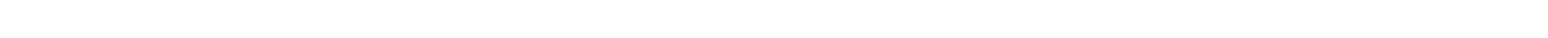

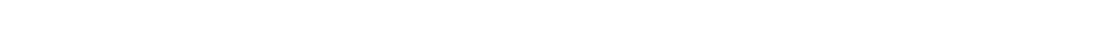

in

in

к

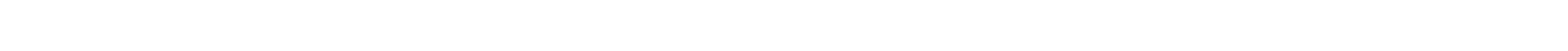

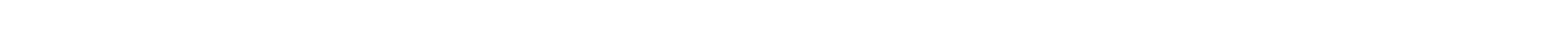

ammommon mana

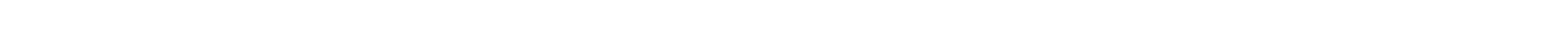

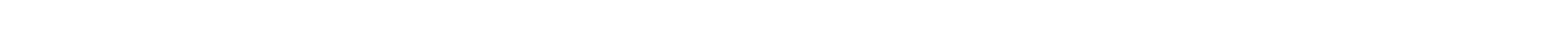

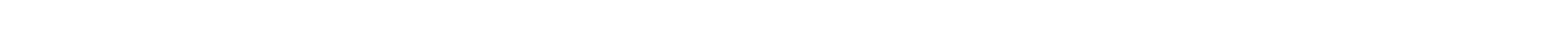

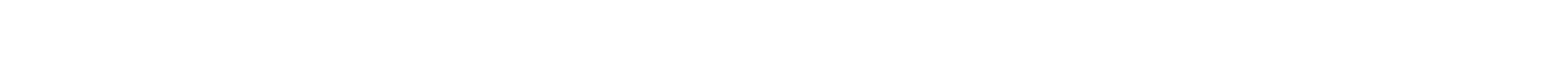




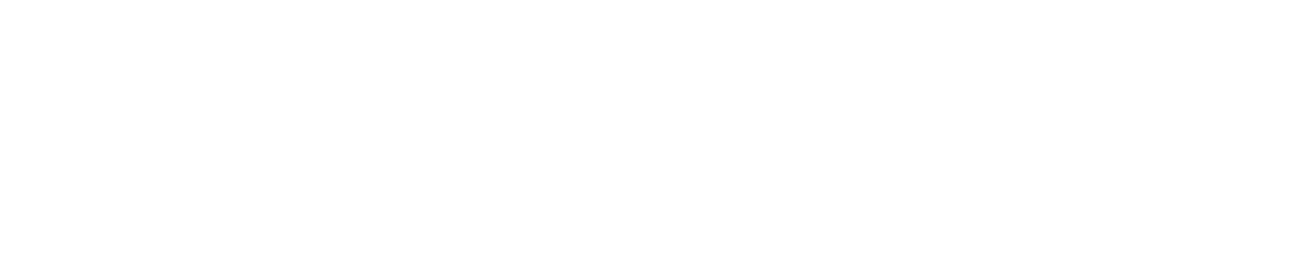

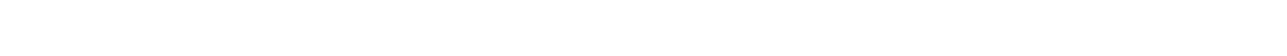
in

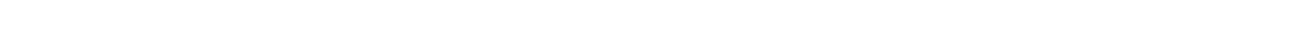
w

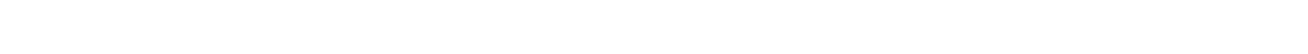

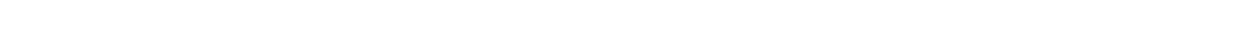

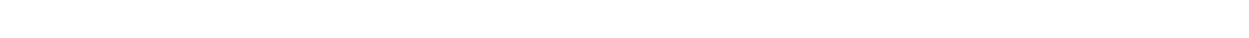

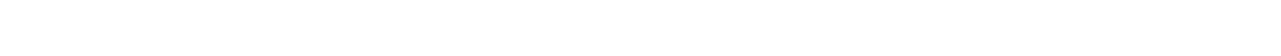

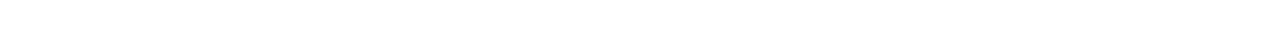

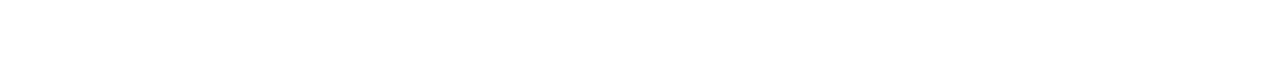

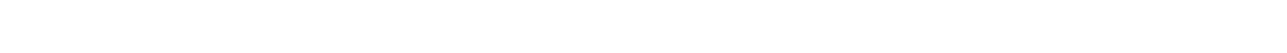
is

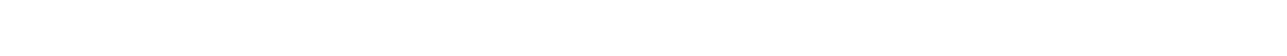
w

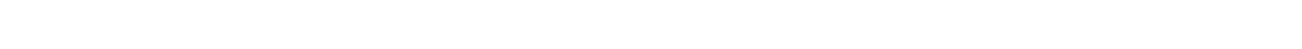

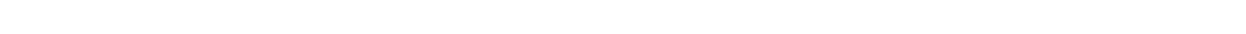

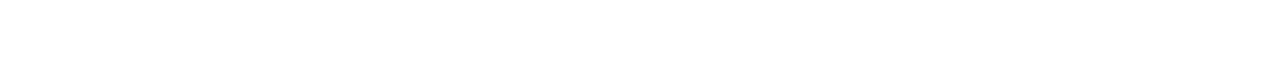

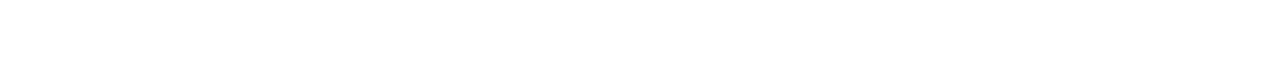

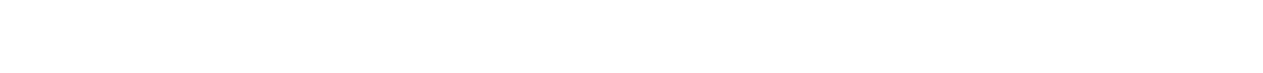

एँ

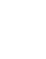

- 囟

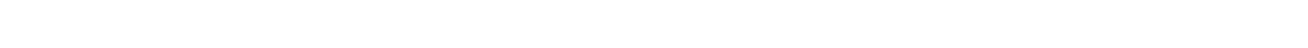

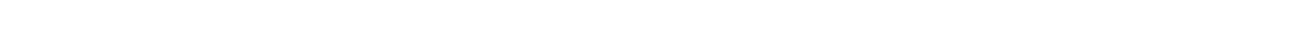

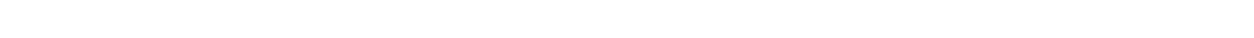

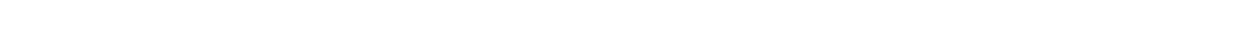

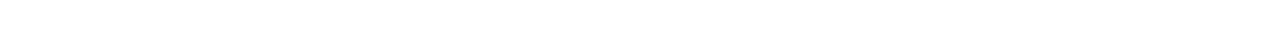

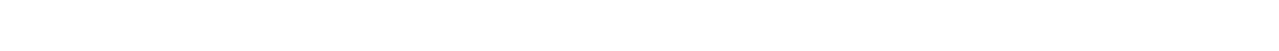

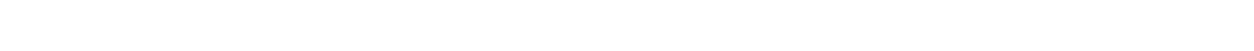


m 0 ?

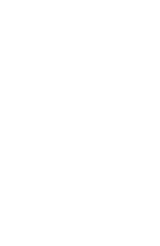
ozo $=m=0$ 징ㅇ $5 \geq 00-\frac{1}{x}$ 뚱요

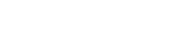

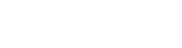
○ा z山 의

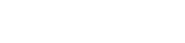
氺的

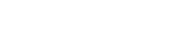

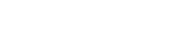
․․ㅇ $n$ 디는

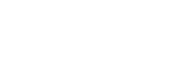
u되. ज: ニํำ 늠 J. - m 0 aำ은

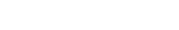

芯一

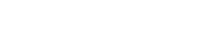
a $z \geq z$

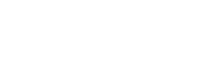
운

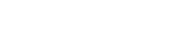

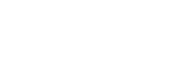
뜨에

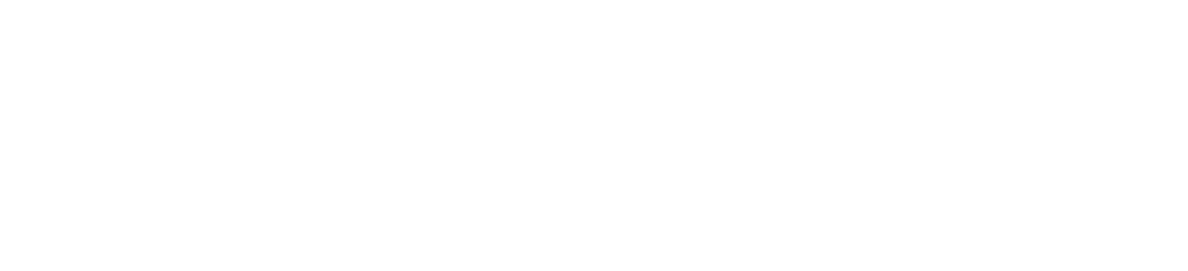

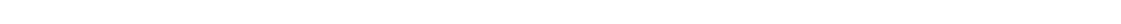

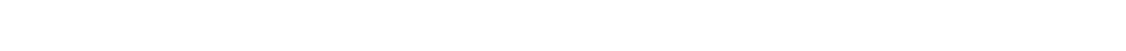

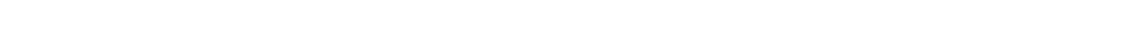

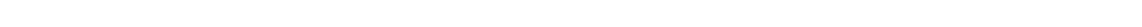

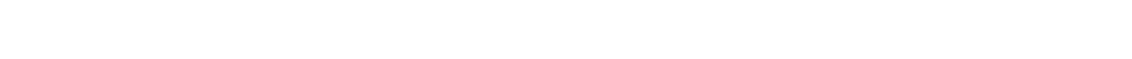

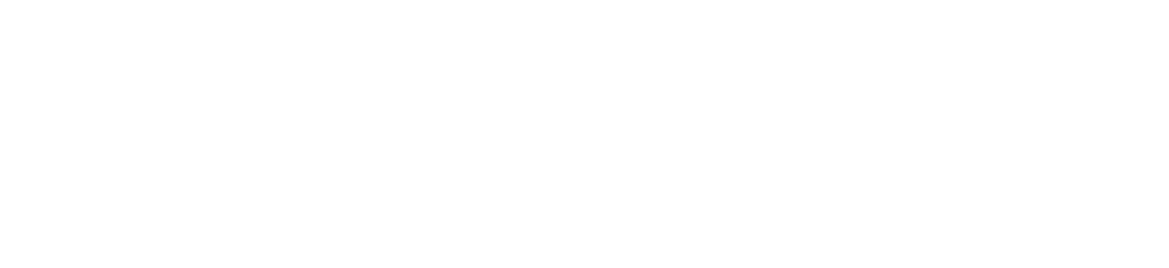

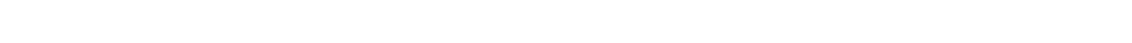

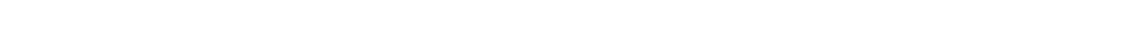

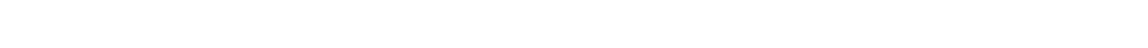

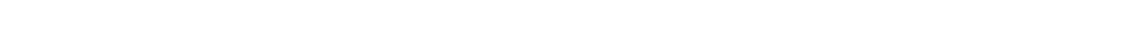

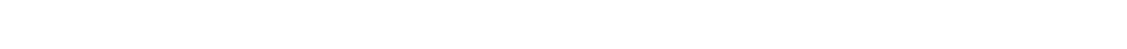

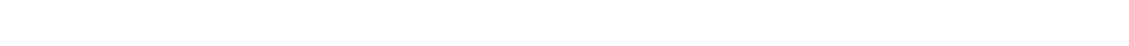

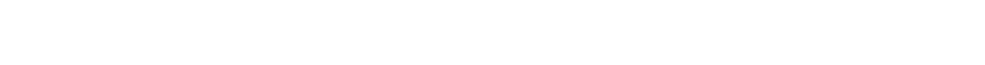

in

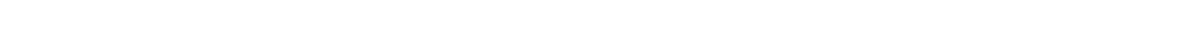

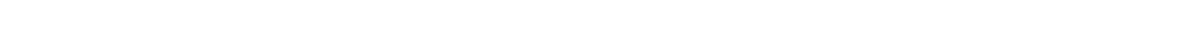

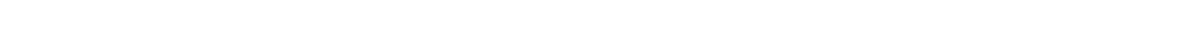

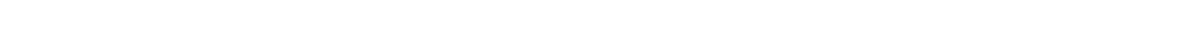

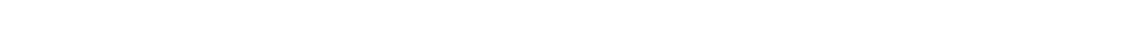

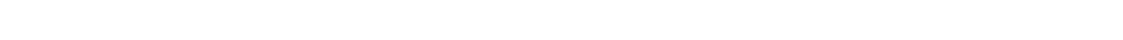

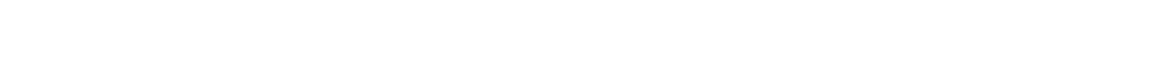

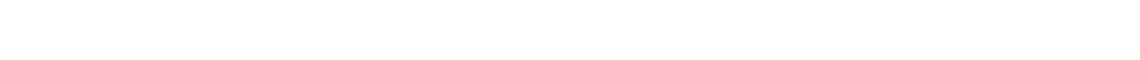

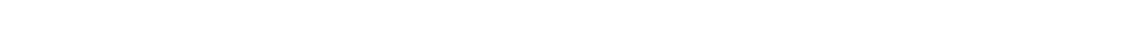

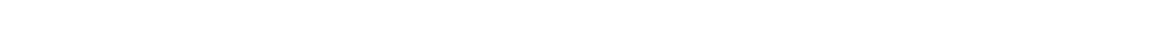
点

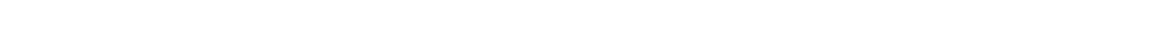
山ै

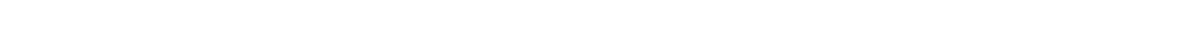
\begin{tabular}{cc}
3 \\
3 \\
\hline
\end{tabular}

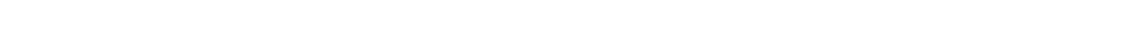

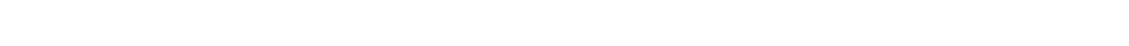

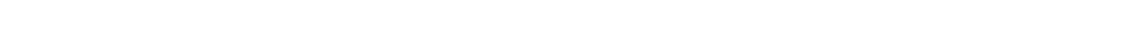

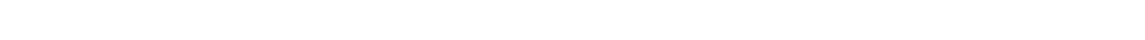

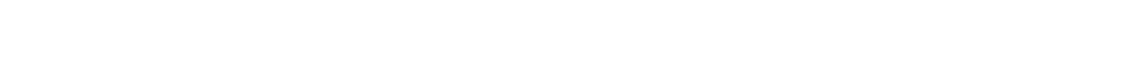




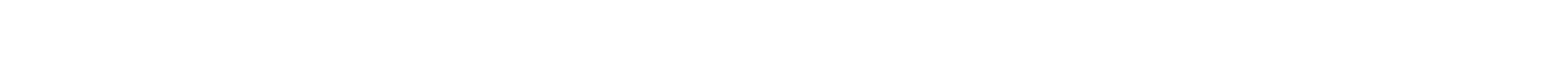

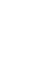

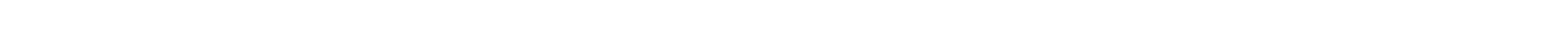

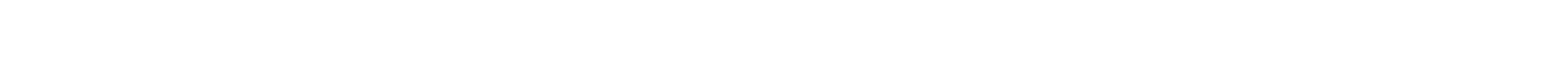

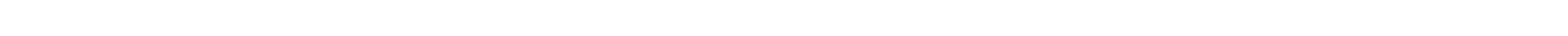

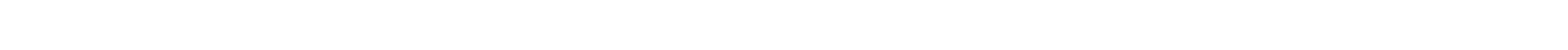

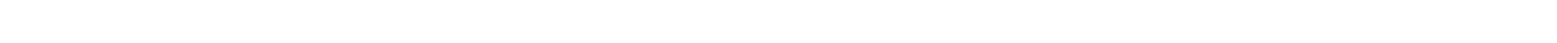

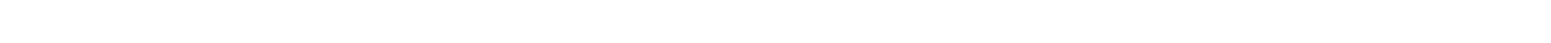

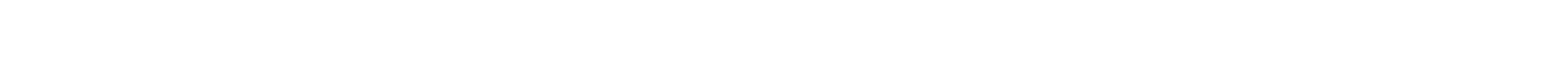

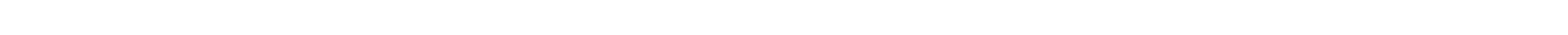

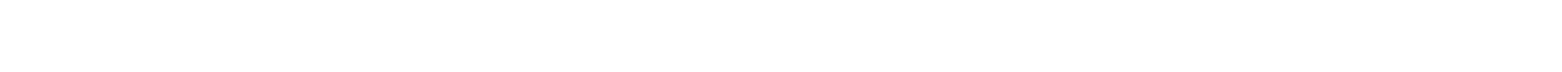

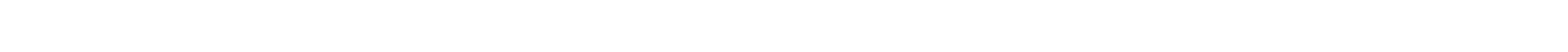

$\therefore$ к

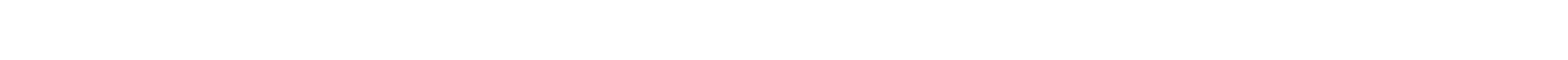

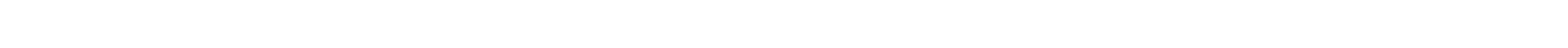

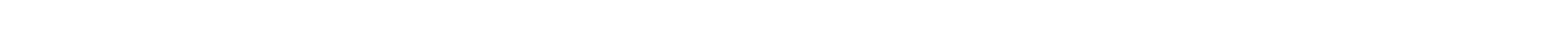

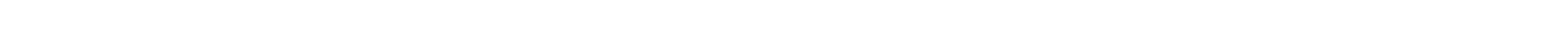

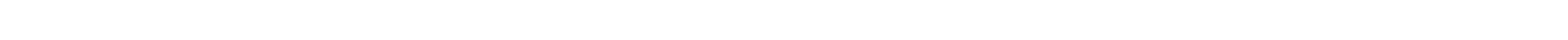

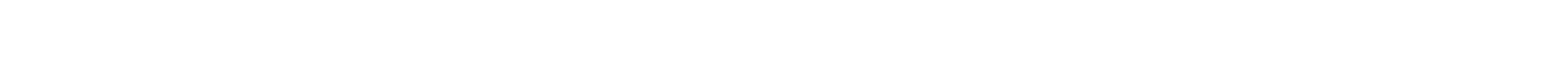

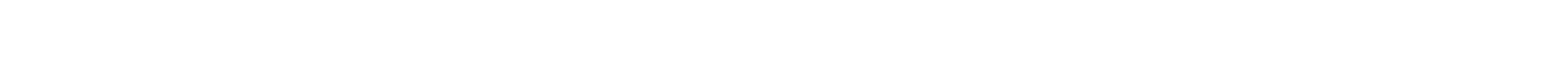

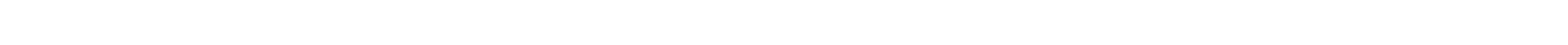

$\stackrel{a}{c}$

б व

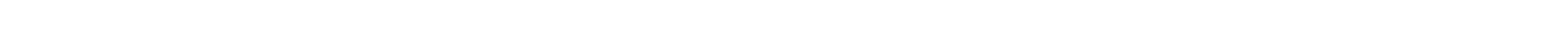

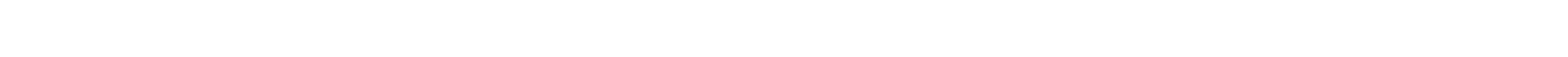

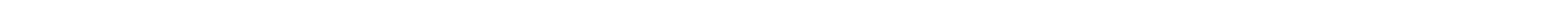

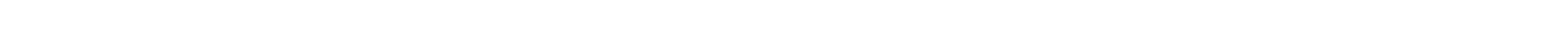

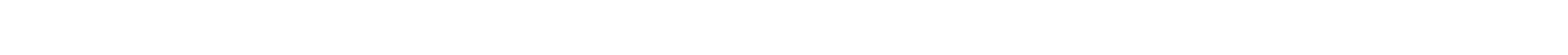

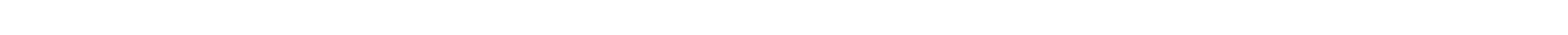

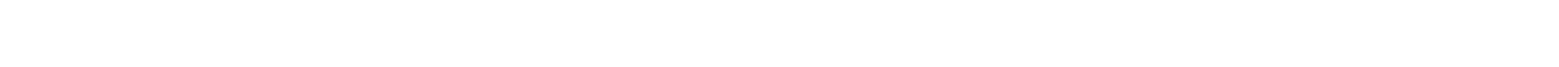

n

N

n

is

n

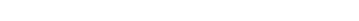

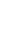

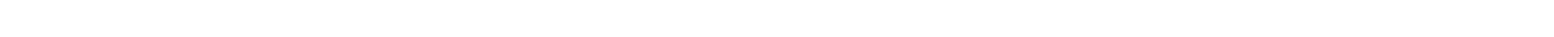

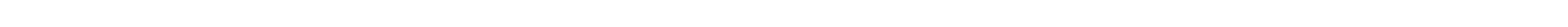

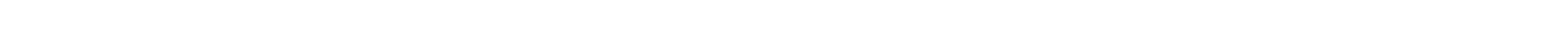
\begin{tabular}{lll}
\hline \\
\hline
\end{tabular}

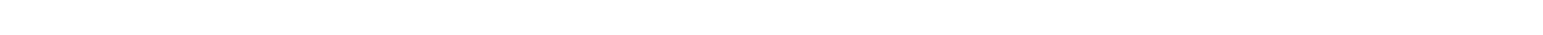
й W

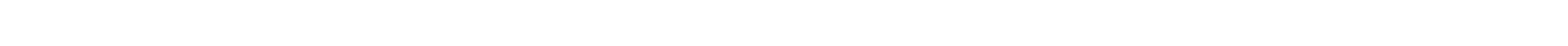

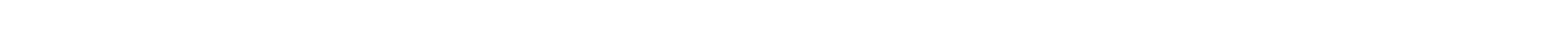

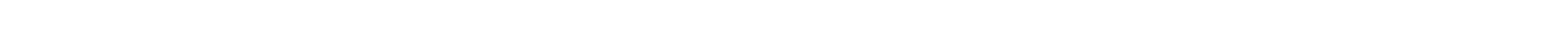




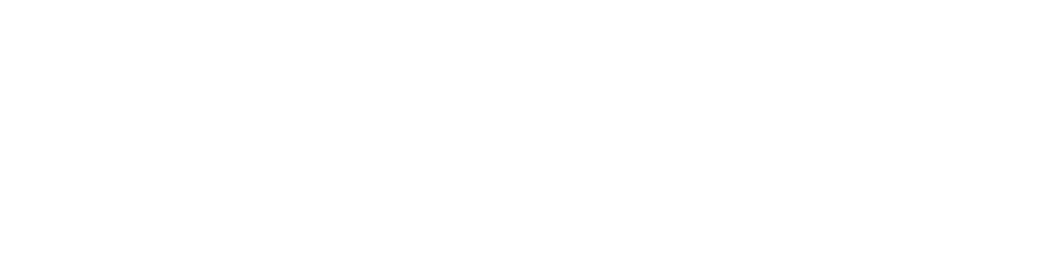

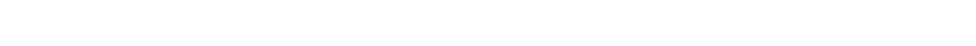

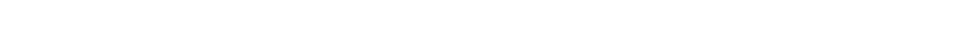

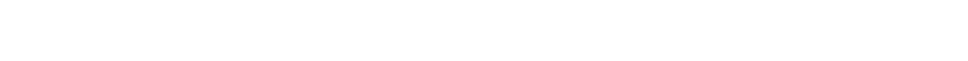

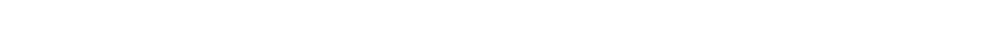

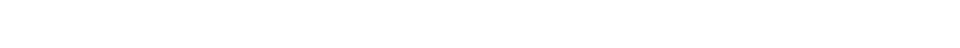

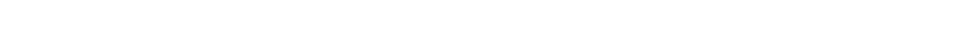

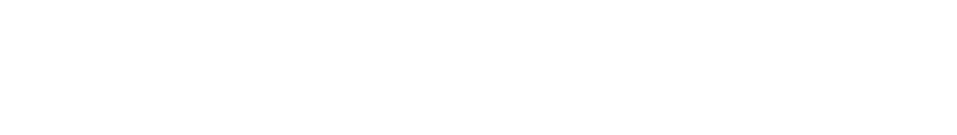

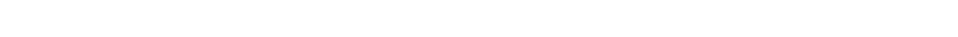

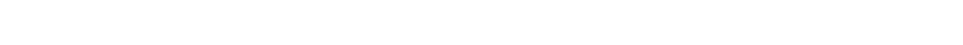

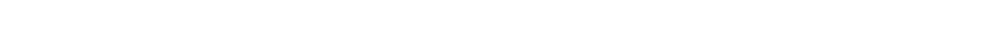

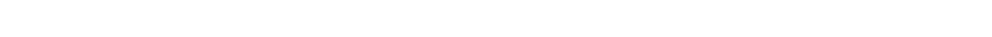

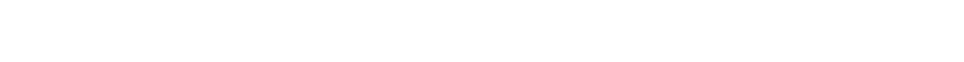

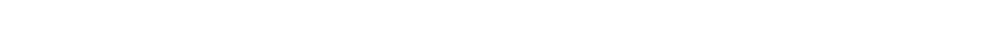
$\Sigma$ a

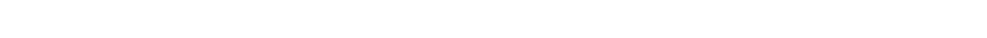

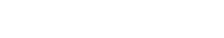

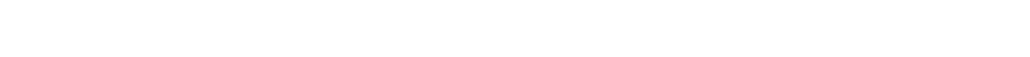

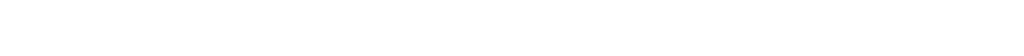

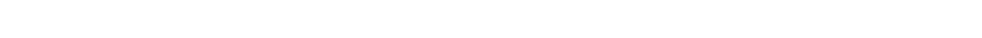

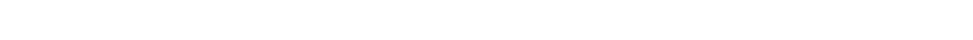
แ

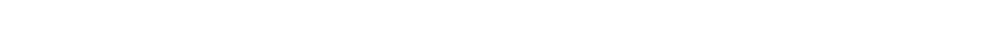

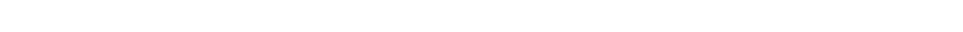

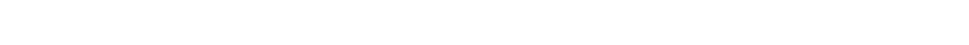

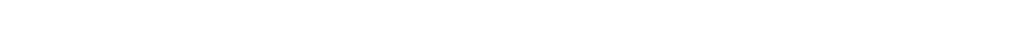
ร.

is

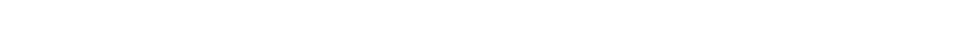

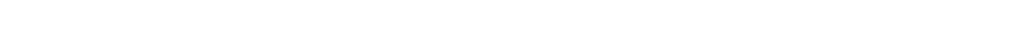
"ü

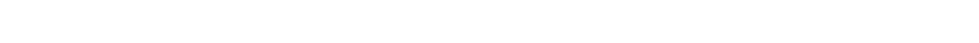

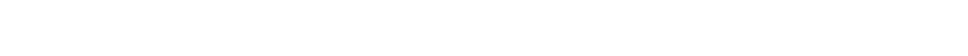

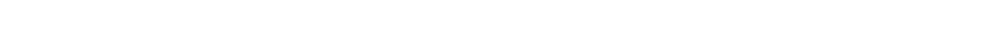

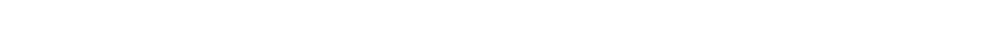

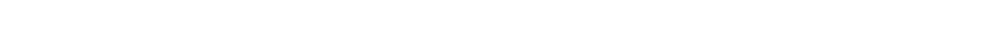

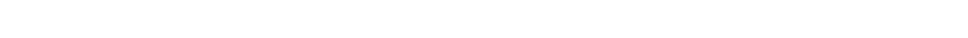

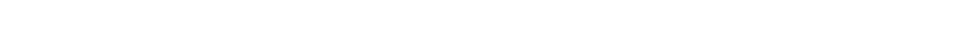




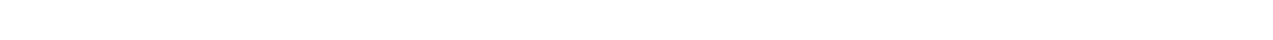
w

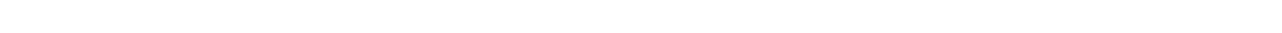

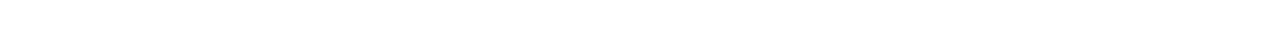

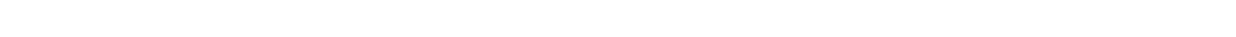

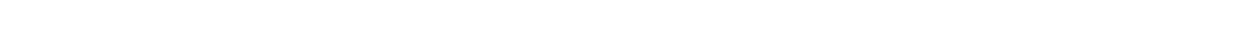

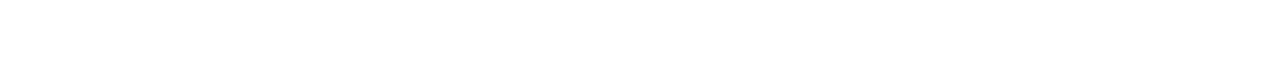

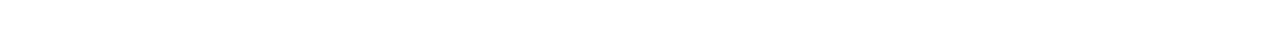

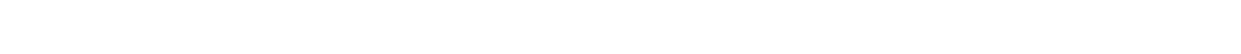
w
in
is
is
$\omega$
es
is

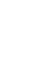

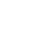

" uw.

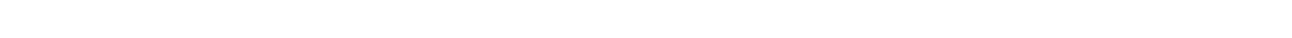
x - ing

$\therefore$ o in

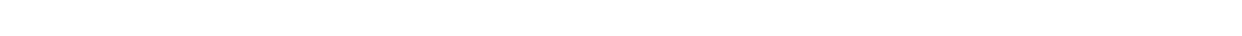

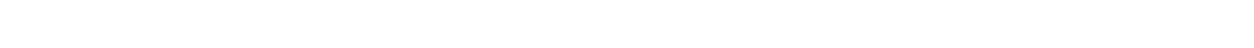

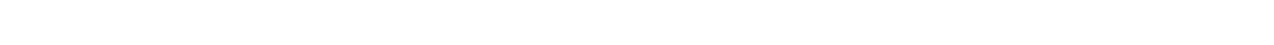

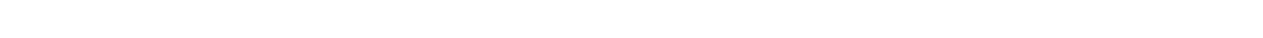

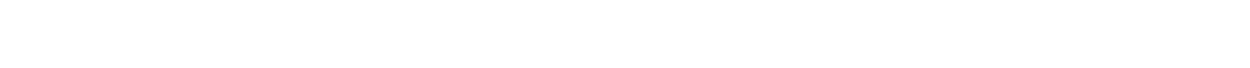

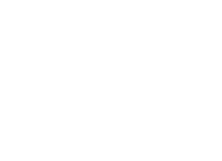

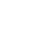

mom

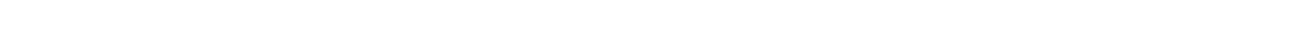

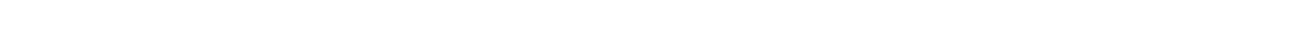
×

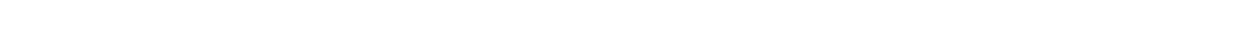

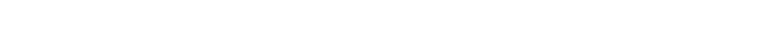

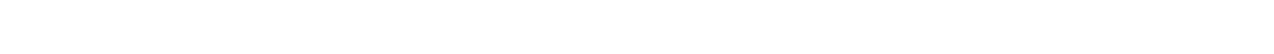

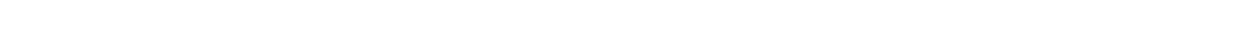

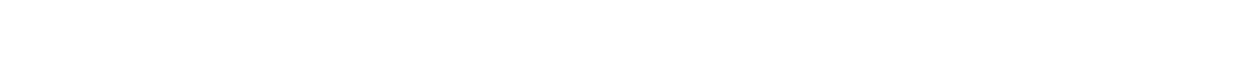

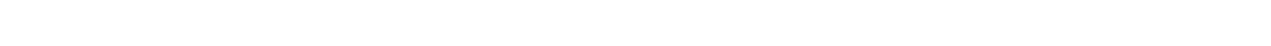

in

a. $\alpha \propto \alpha$

acoo a a a a a acab -

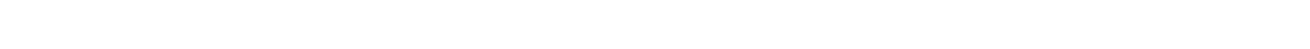
a J

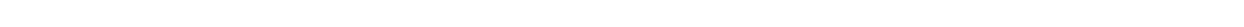

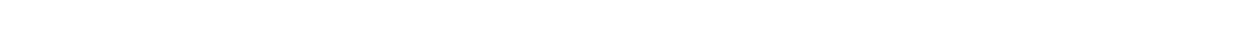

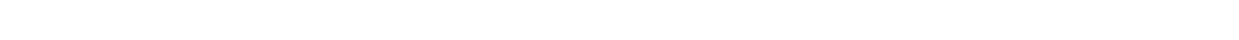

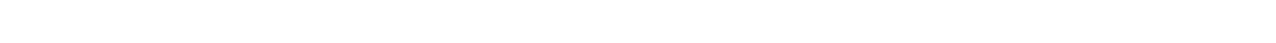

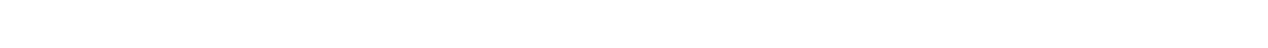

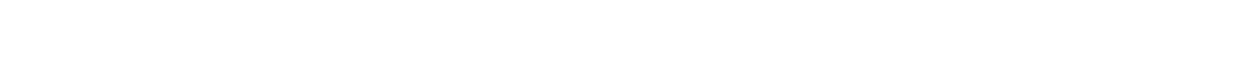




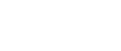
es
is
is
es
u
is

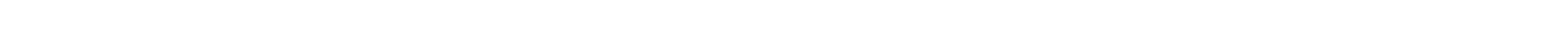

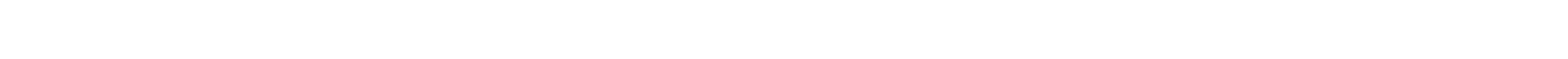

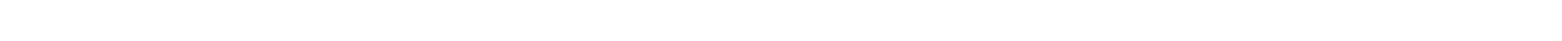

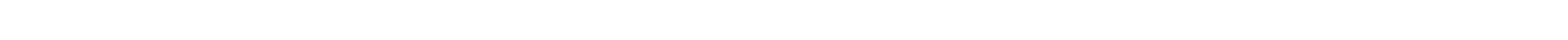

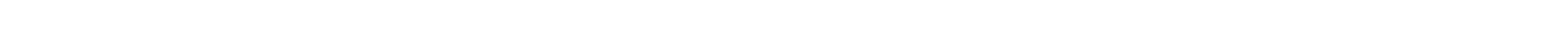

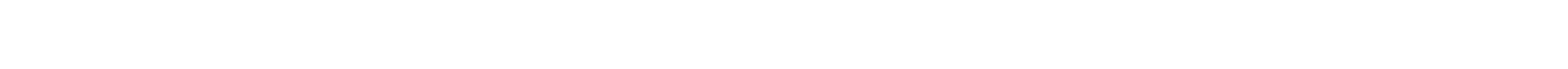

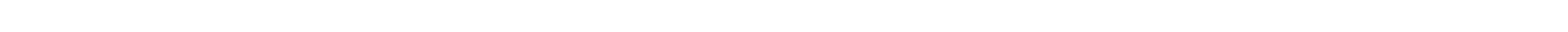

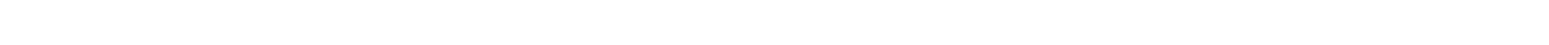

$\stackrel{m}{m}$

$\underline{\Sigma}$

ns $\quad \sin$

n

n

in

in

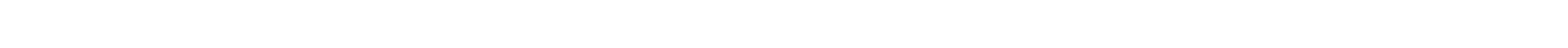
น

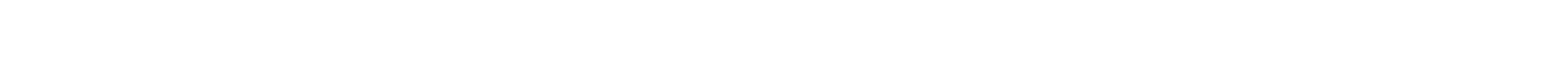
$=4$ wñ

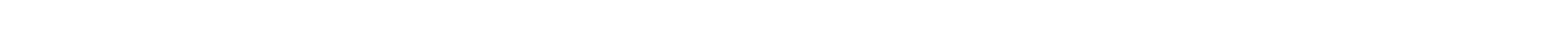

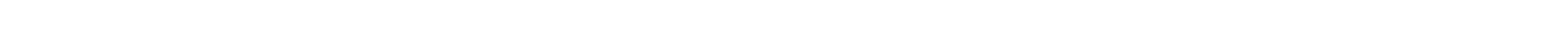

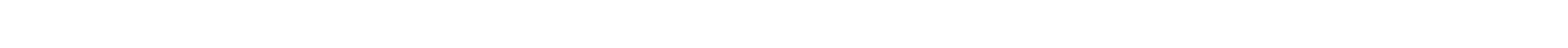

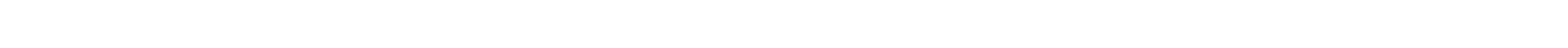

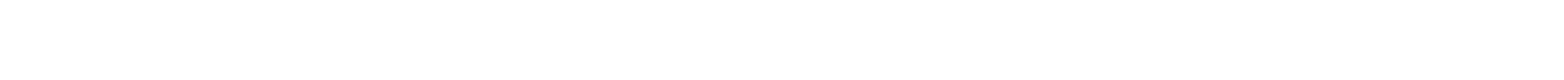

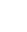

n

u

n

n

u

in

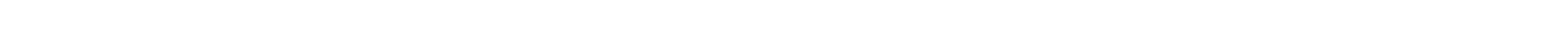

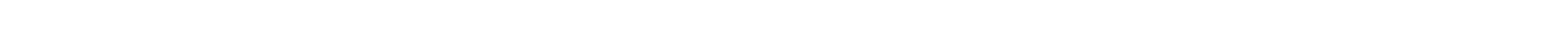
-

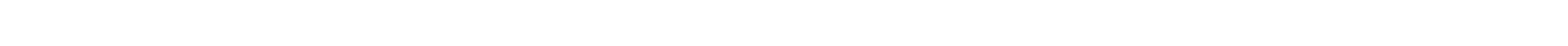

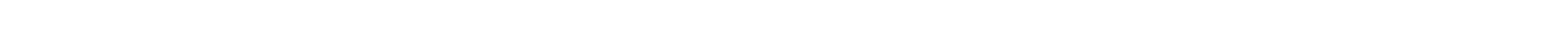

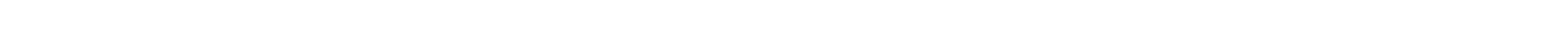

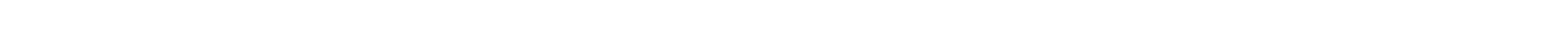
Y

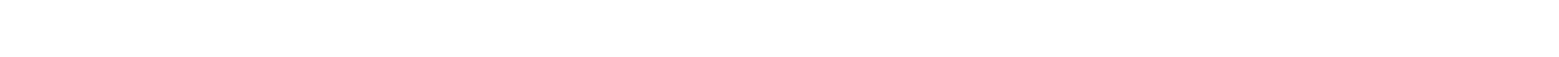

L

$n$

s

n

n

n

E

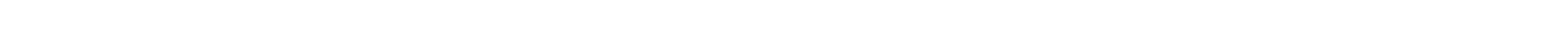

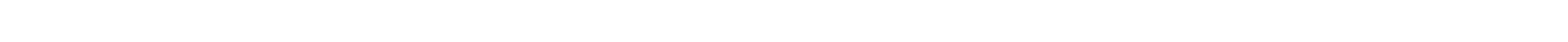

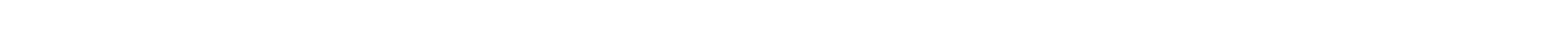

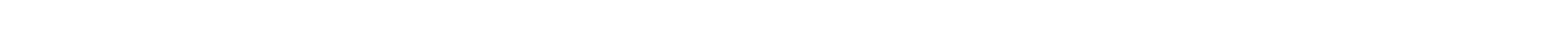

แ

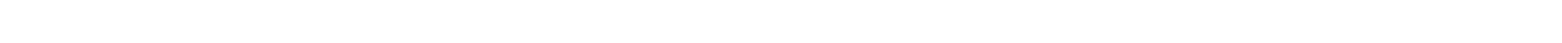

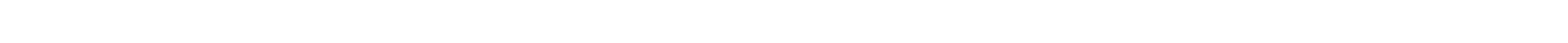

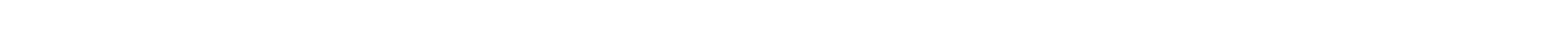

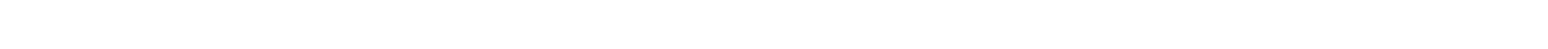

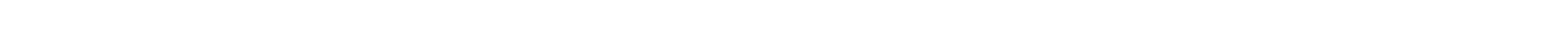




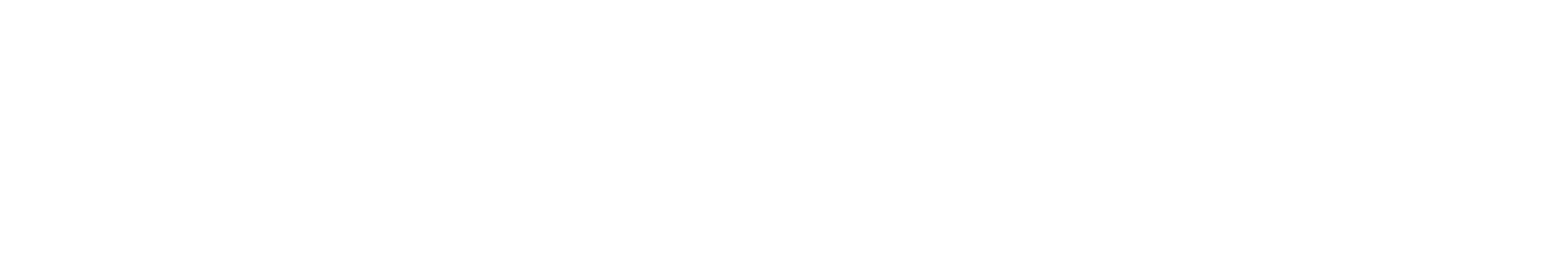

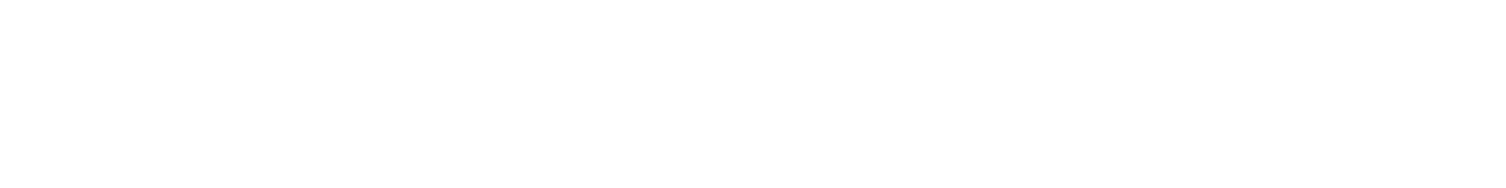
$\stackrel{5}{u_{i}}$ in
n
a
n
is
is
in
in
$n$

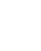

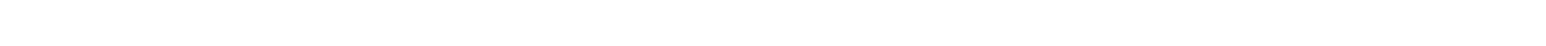

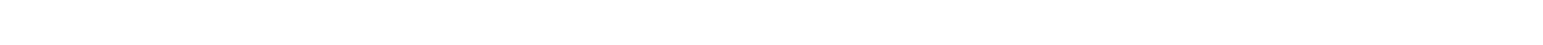

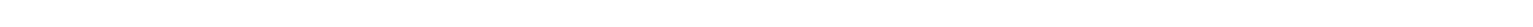
> d

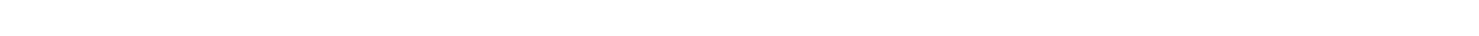

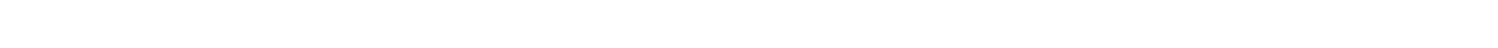

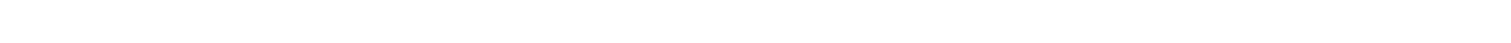

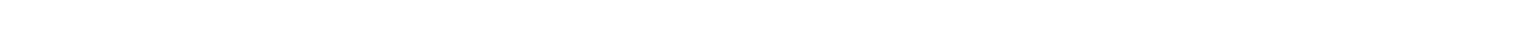
OC-FNNMOOF-NNMOCF-NNMOOFF-NNNT-F-FNNF-FNNMOOF-NNME

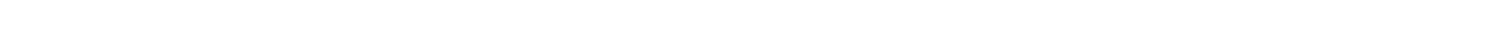

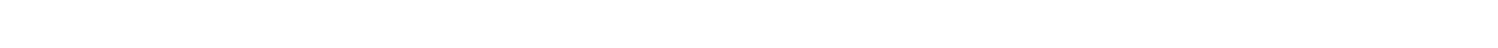

$$
\text { in }
$$

เ. en

i

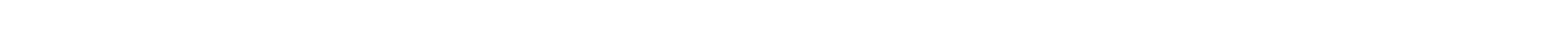
ب s

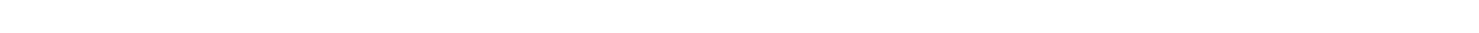

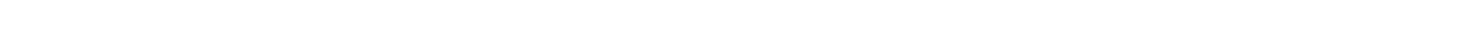

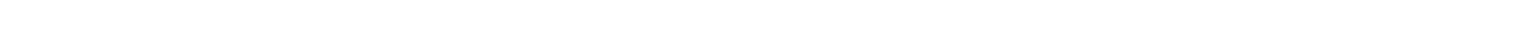

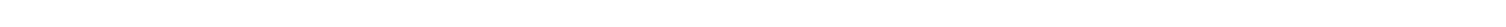

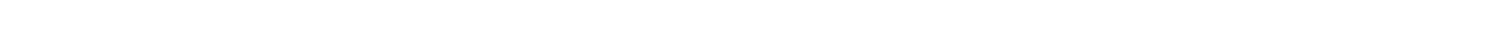

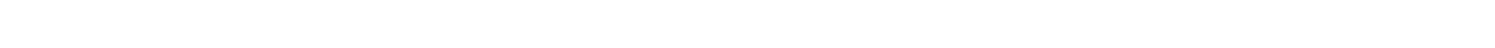

twen

n

es

n

n

en

$n$

$\leftarrow$

a-mot a " un

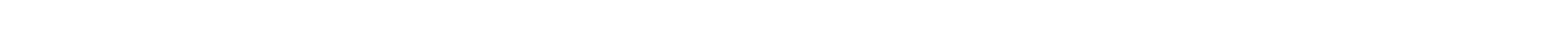

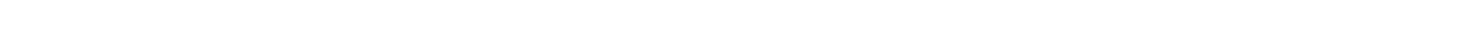

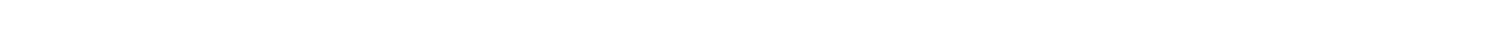
w ¿ COO- - N N

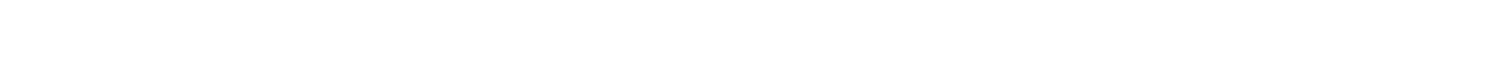


正舟早

$=0 \dot{0} \dot{0}$

कीजिए.

도은

a dún

د๐:

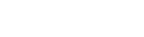

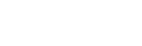

는

万ूn

$\checkmark=$ wi

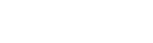

즌질

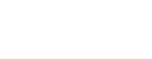

a $=x$

m这立的

mo gris

. 능

zวa =

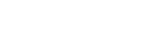

-axiz

แับa!

무의

> u.

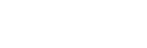

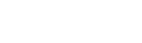

$\sum_{0}$ 品宓a

운쬬

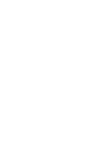

ณ.

$=-5$

它势

… I...

웅

$0 \geq 0 \geq$

$\sum_{u=1}^{0} \sum_{n=0}^{0}$

a

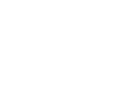

$\sim ⿻$

an 0

它出的

으옹응ㅇㅇ

.

L

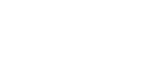

$a=z=2$

点 n

in in

$n$

$\infty$

nosenenes

is

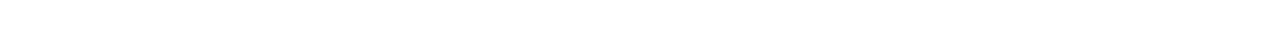

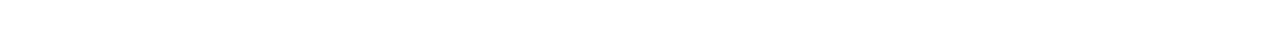

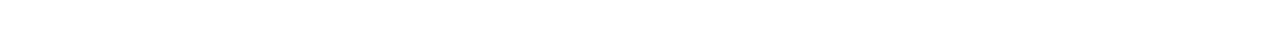

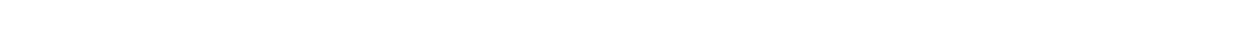

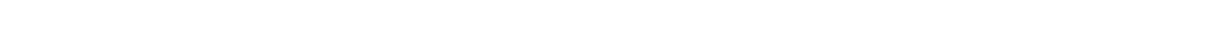

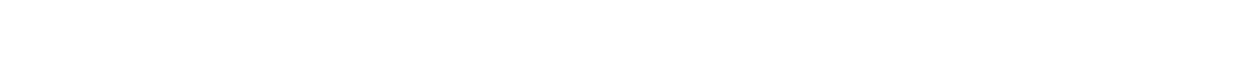

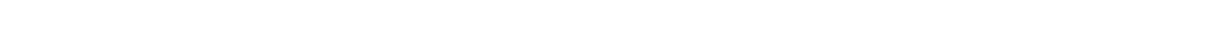

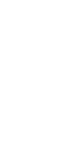

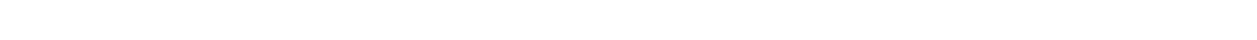

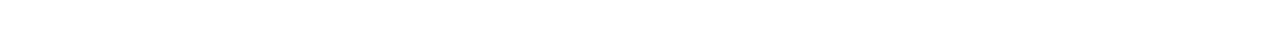

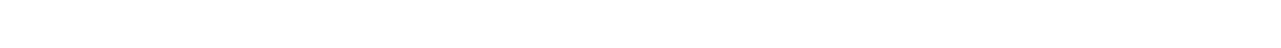
×

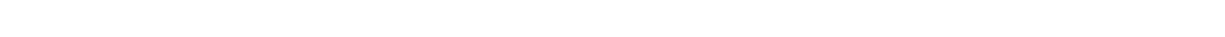

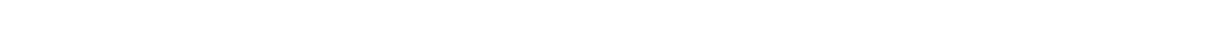

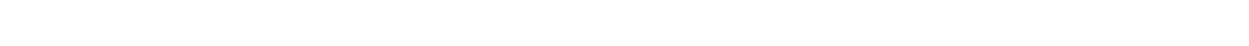

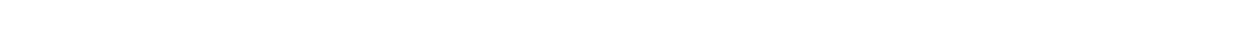

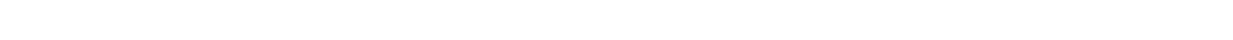

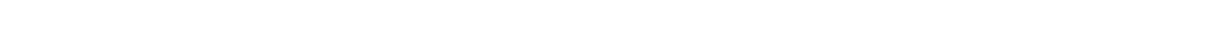

$\stackrel{5}{\mathbf{z}}$

(n)

in

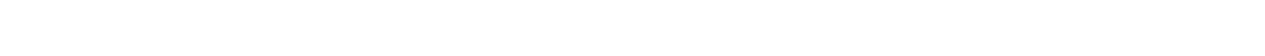
w

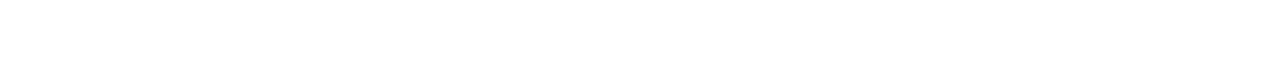

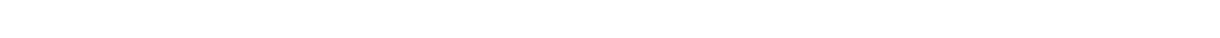
алода

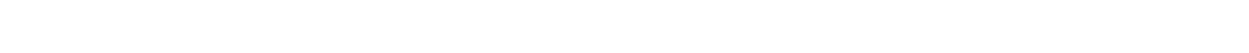

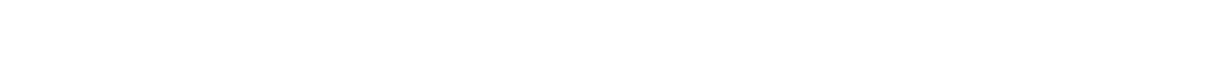
tis in
s
is is es
is
ension
n $n$
n

$\stackrel{s}{5}$

-

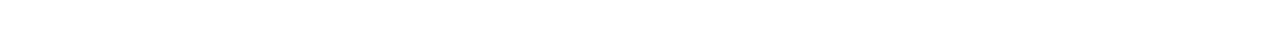
पu

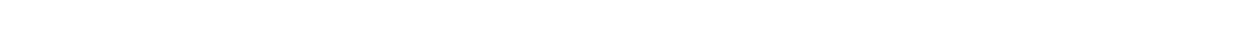

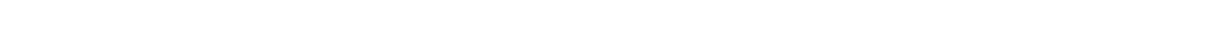

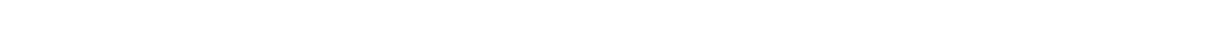

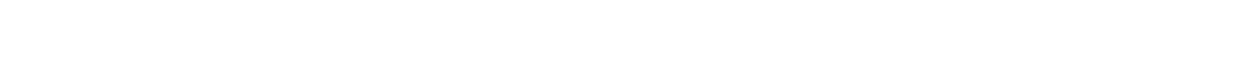

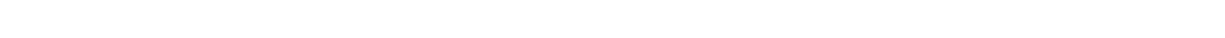

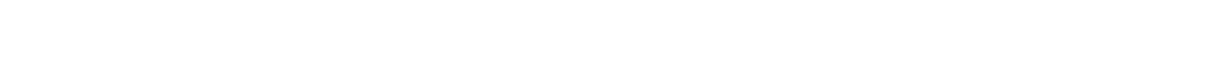



Li n
n
n
n
is
s
m
en
市

omoñ

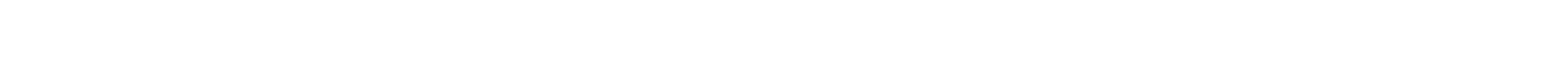

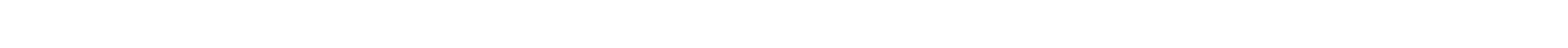

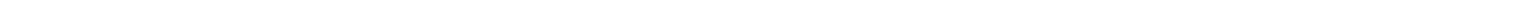
గñ

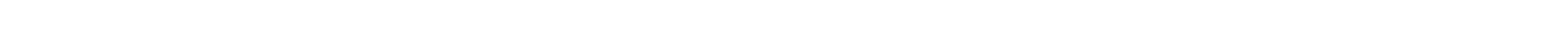

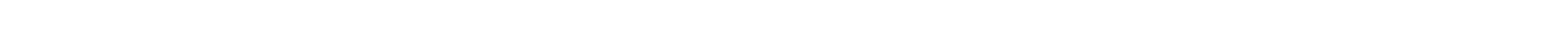

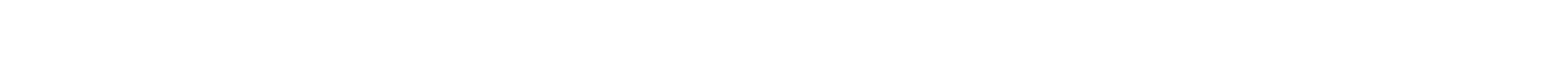
$\div$
$\sim$
in m
is $n$
n n
$\stackrel{a}{\leftarrow}$

" a

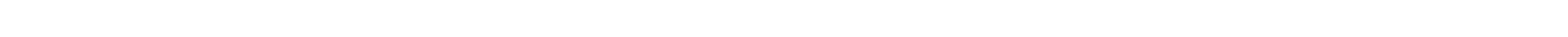
एक

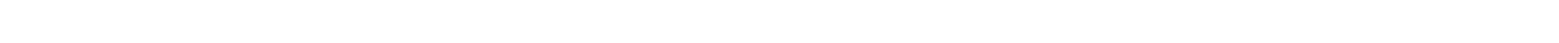

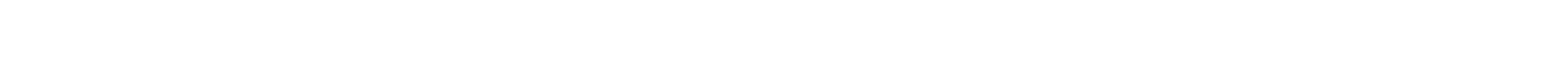

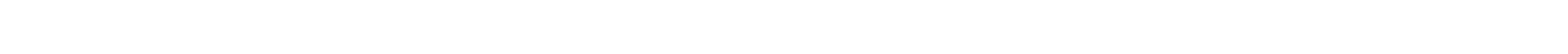

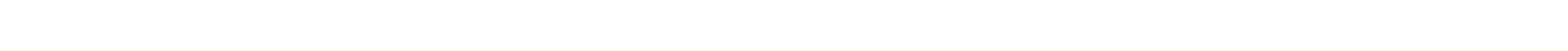

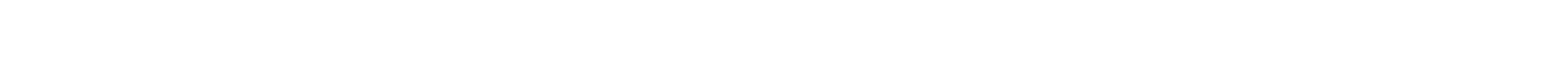
is o
$\sim$
a

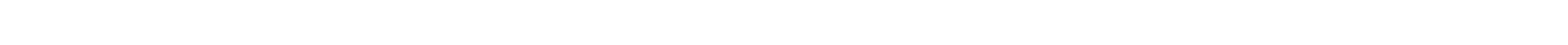

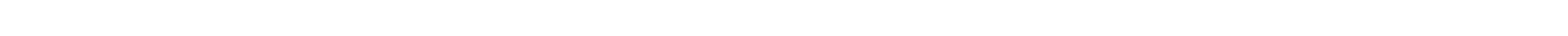

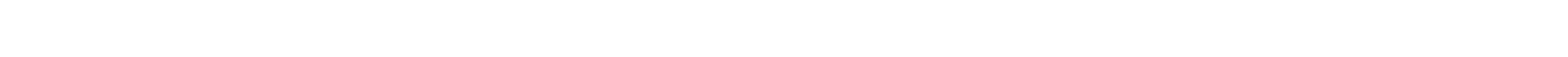

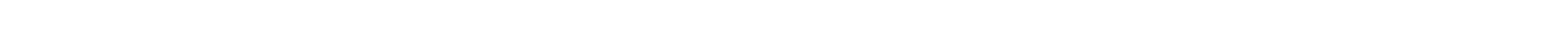

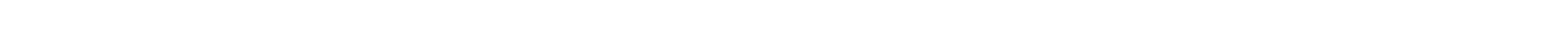

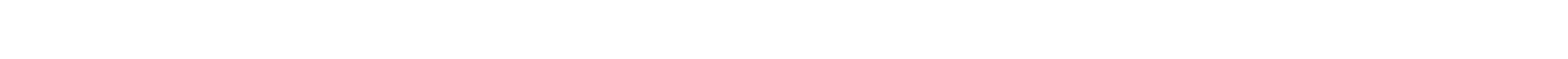

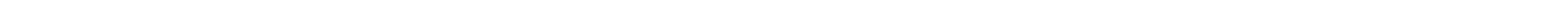

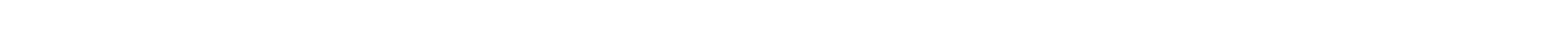

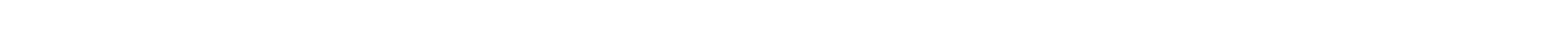
Wus unesen
in m

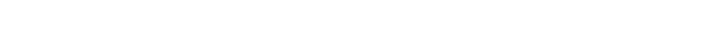
n
is

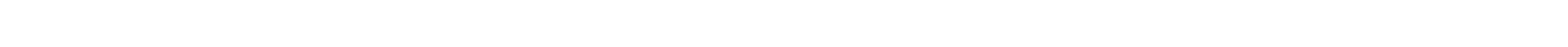

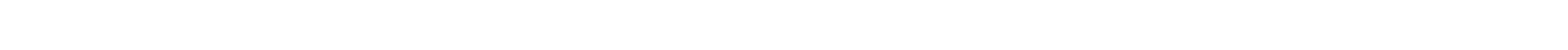

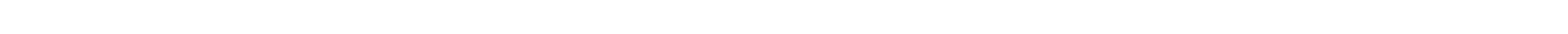

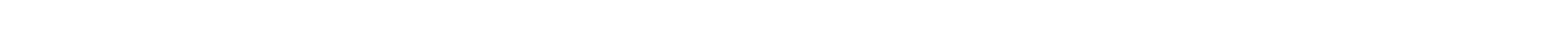

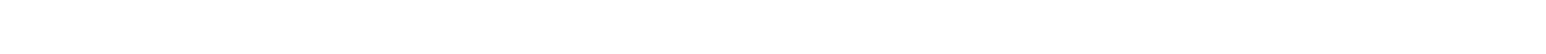

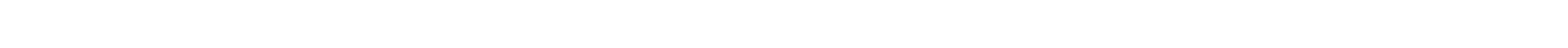

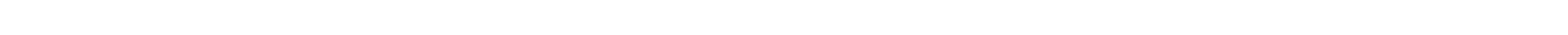

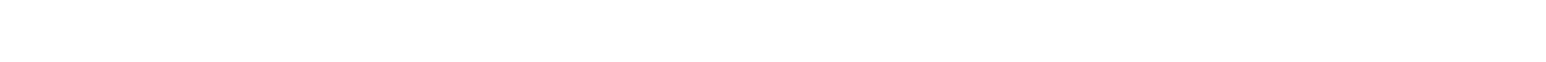




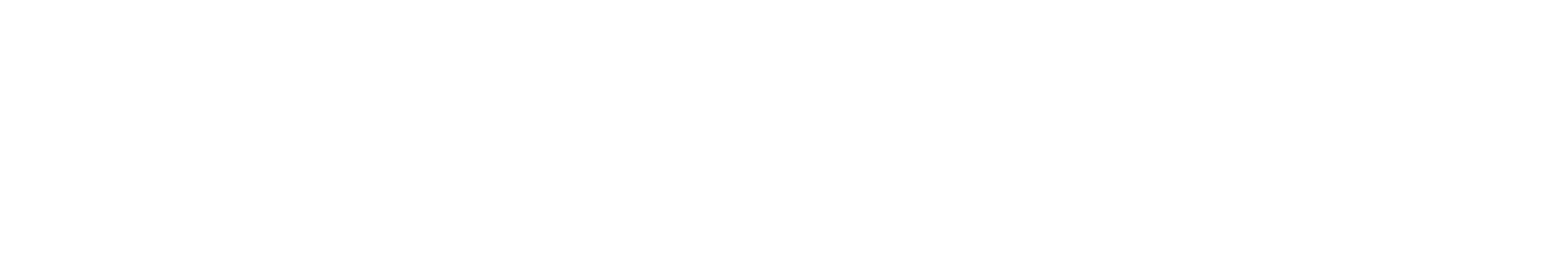

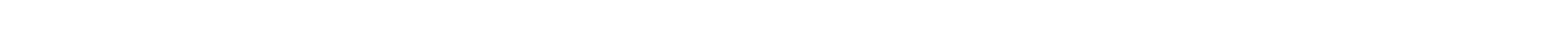

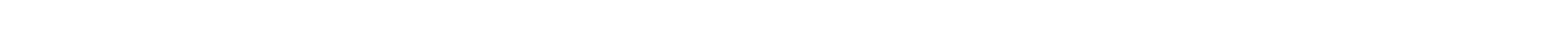

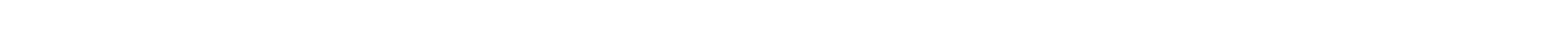

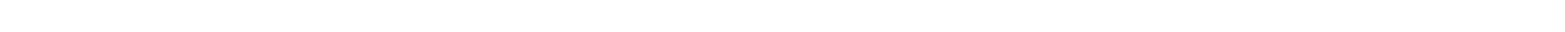

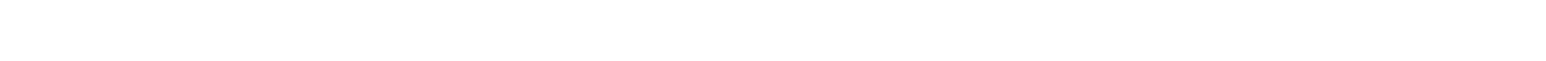
㟧
$\infty$
in
is
$\sim$
n

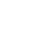

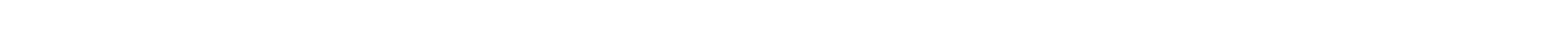

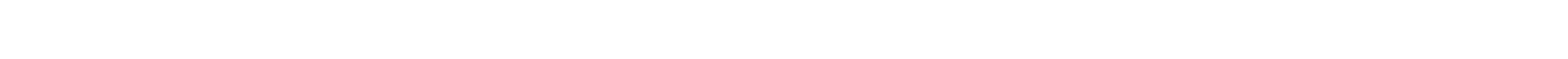

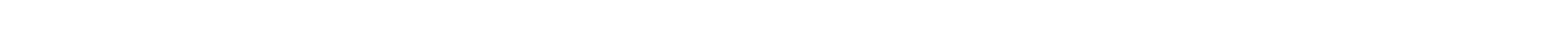

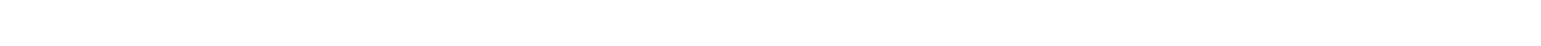

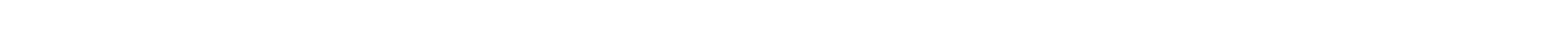

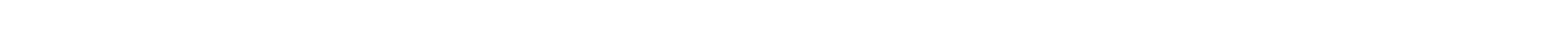

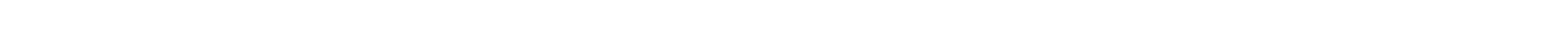

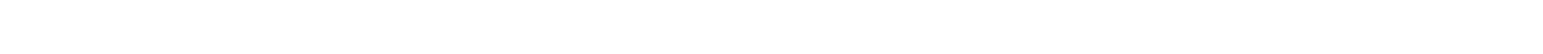

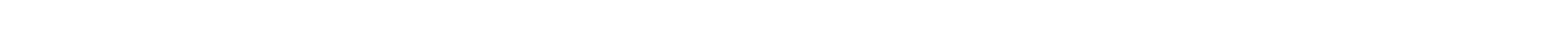
w.
n
in
os
n
$n$
is

5

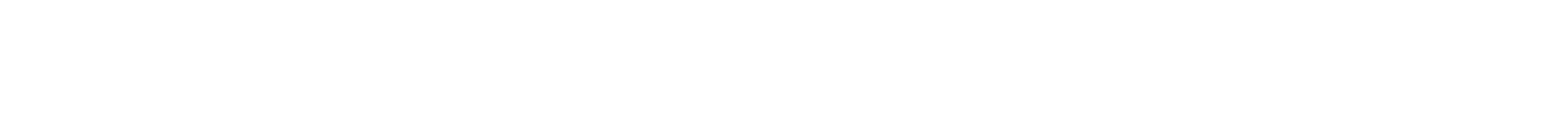

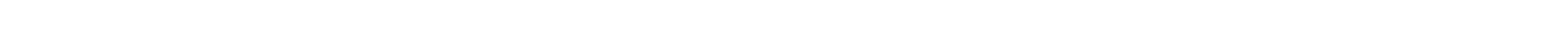

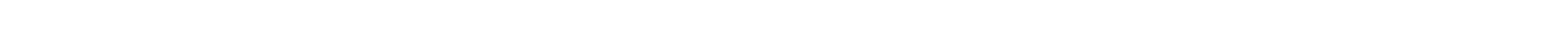

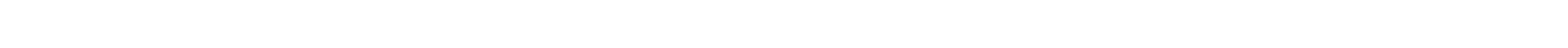

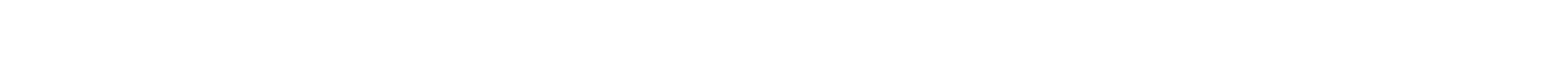

n

un es

n

$n$

is

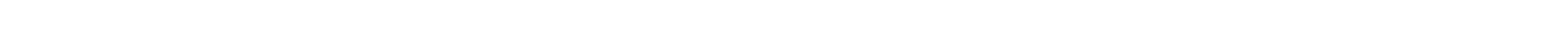

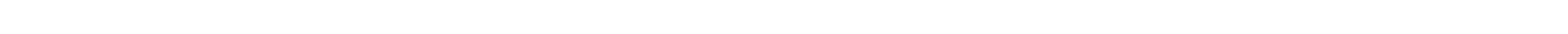

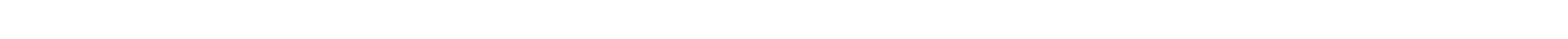

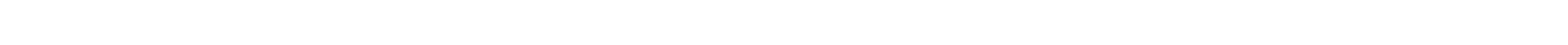

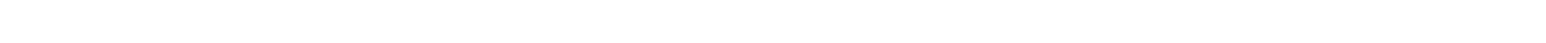

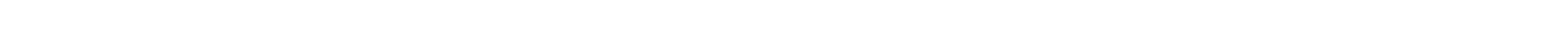

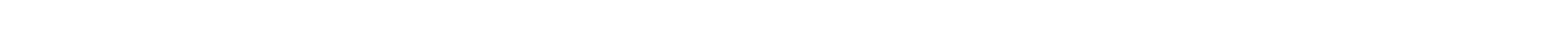

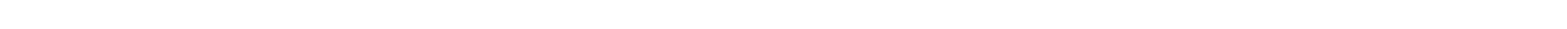

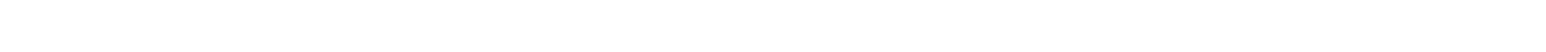




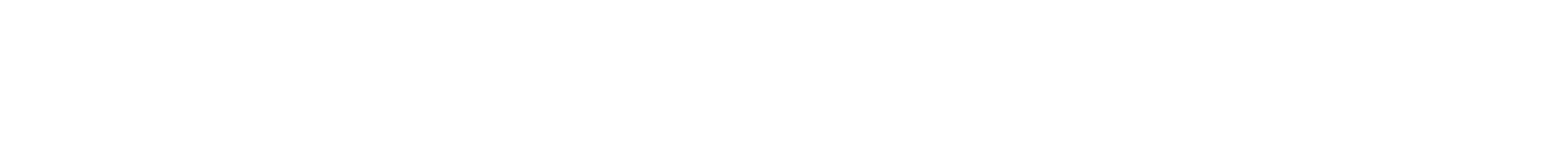
o

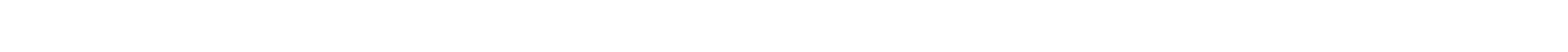

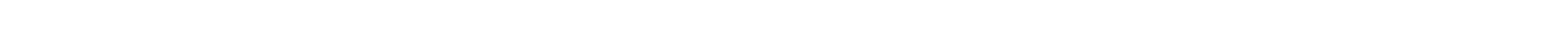

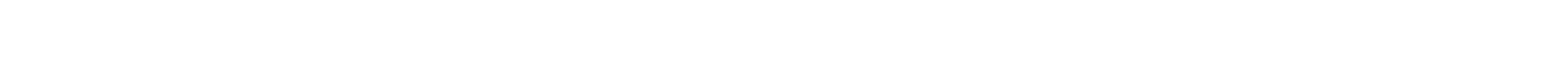

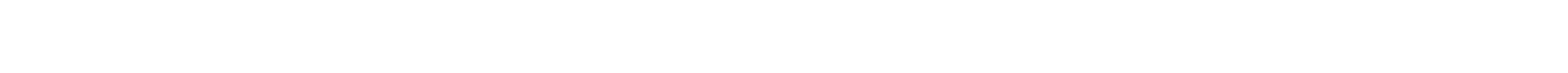

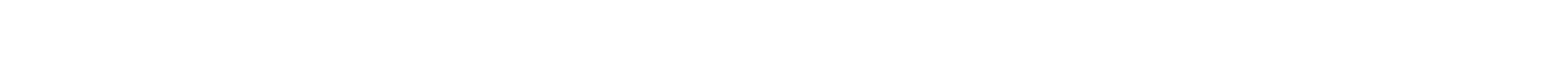

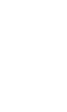

约

n

is

a

n

๔ w

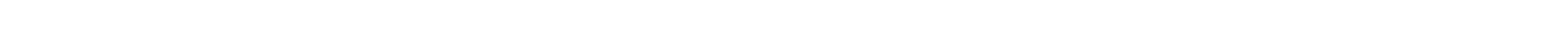
3 J

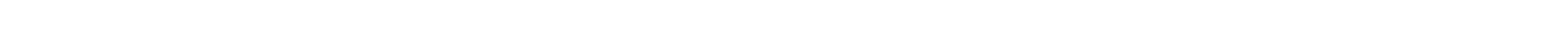
แ000000000000000000000000000000000000000000000\%

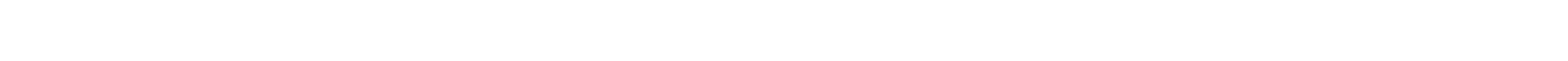

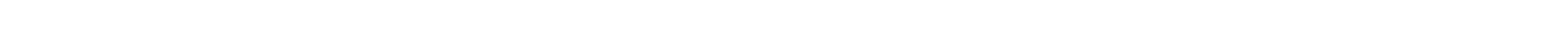

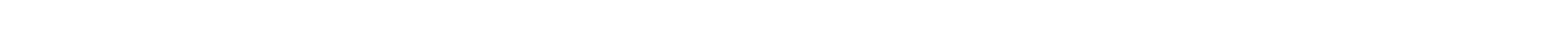

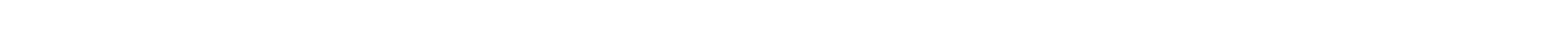

$\underline{m}$

in

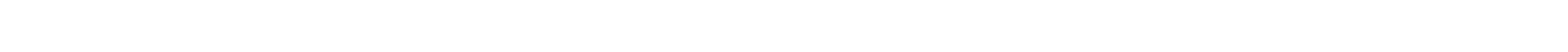
u u-

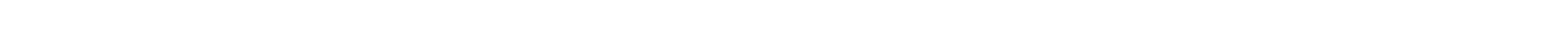
x

గొ

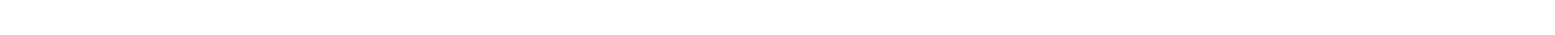

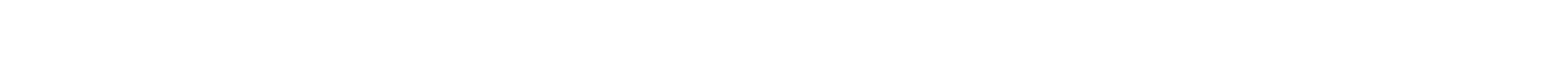

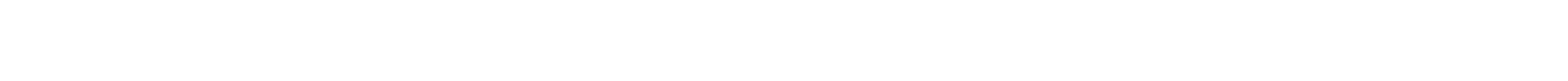

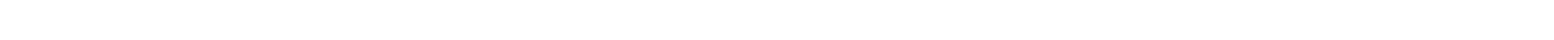

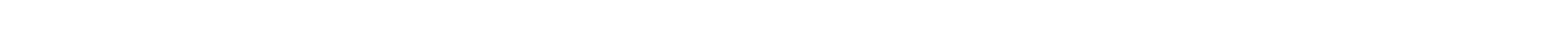
4. 了 thy ำn

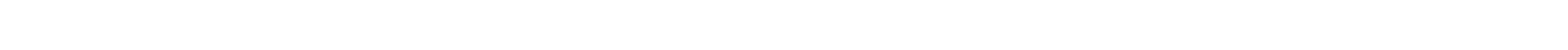

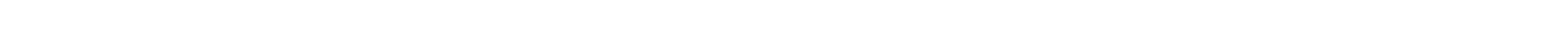

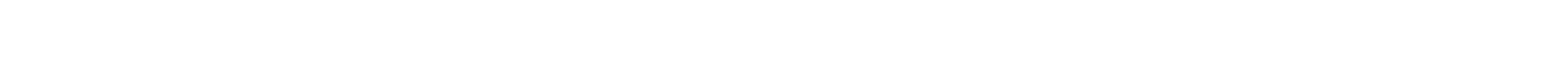




\begin{tabular}{|c|c|c|c|c|c|c|}
\hline$\underline{\Sigma}$ & $\infty$ & $n$ & $n$ & us & $n$ & es \\
\hline
\end{tabular}

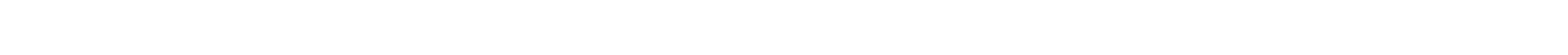

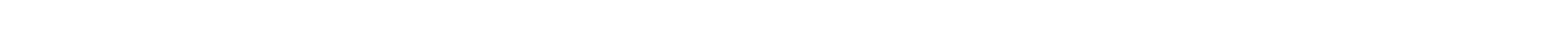

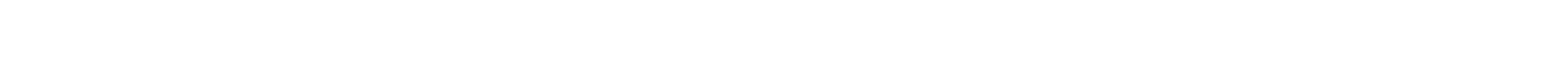

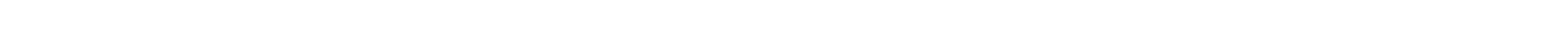

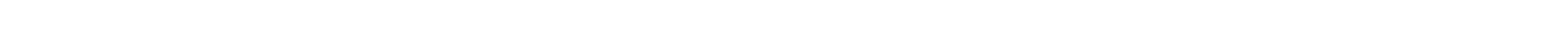

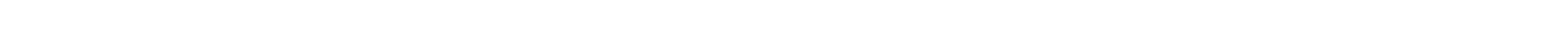

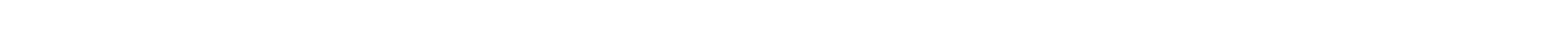

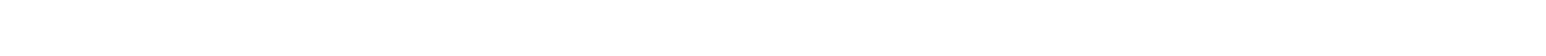

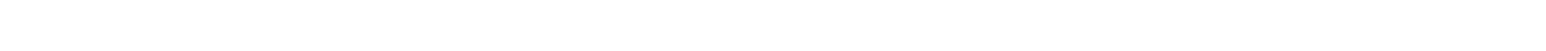
$\stackrel{4}{ \pm}$
$\infty$
is en en
@
n

$\alpha$

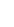

๔

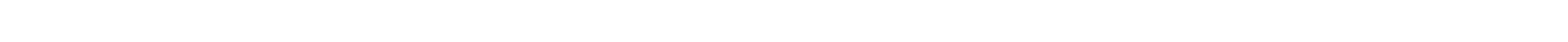

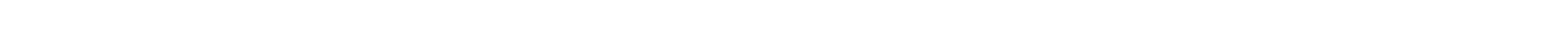

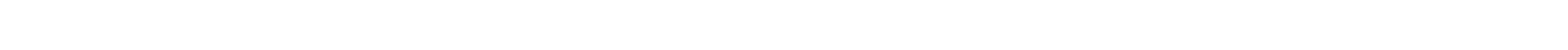

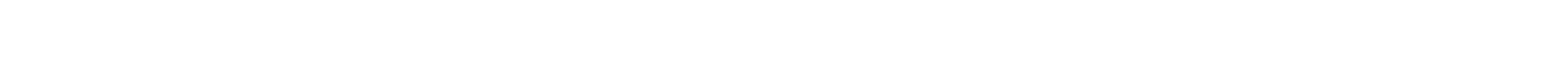

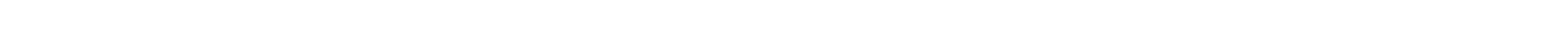

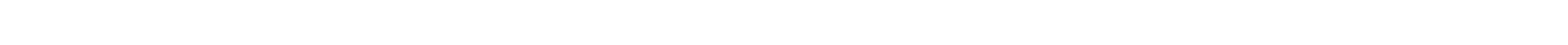

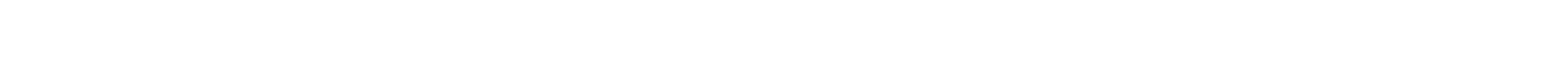

11
5
5

n

n

n

n

$\omega$

n

moknanmon--

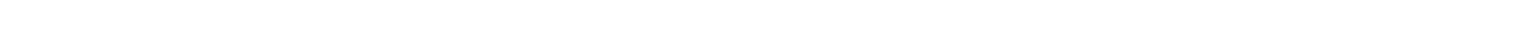
« य Z J

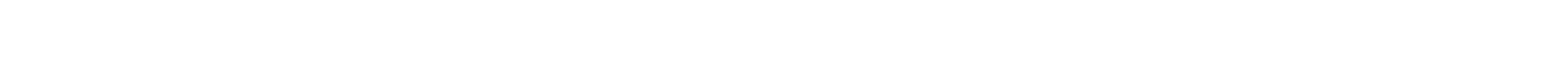

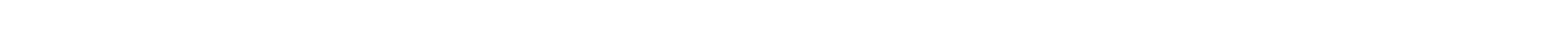

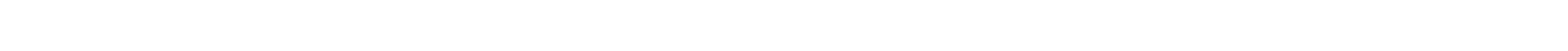

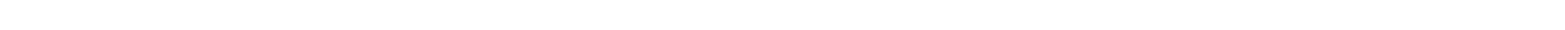

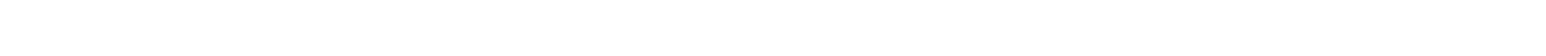

is

ג的O स

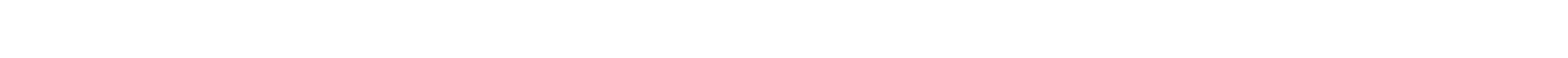

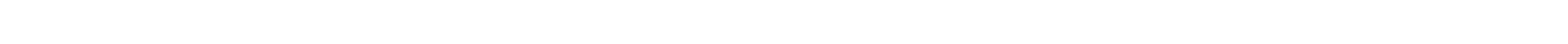

W 응 WF

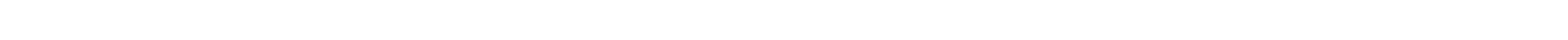

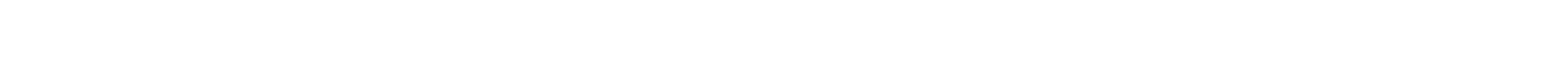




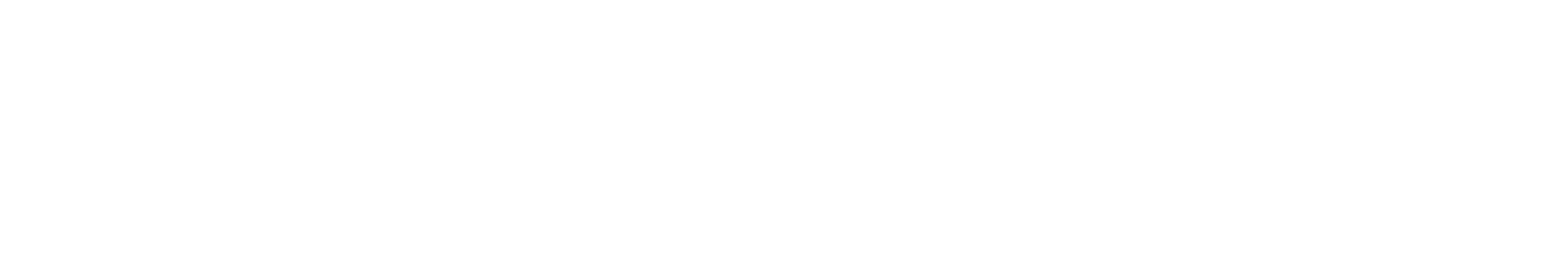

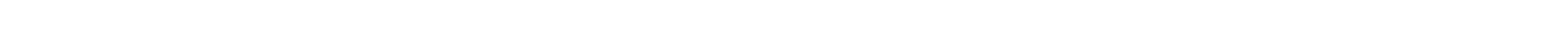

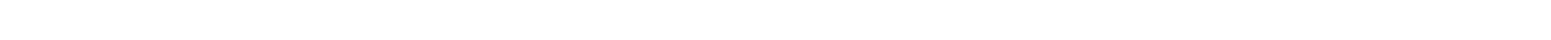

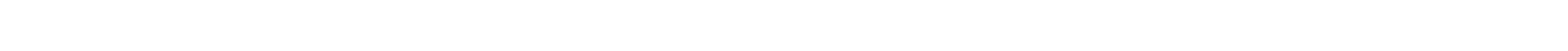

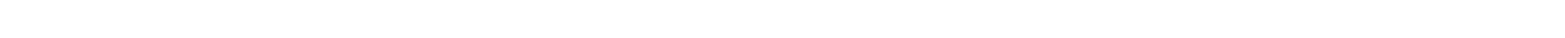

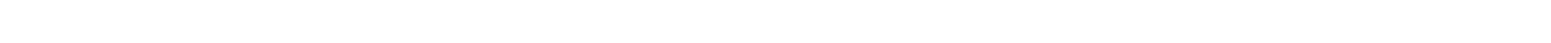

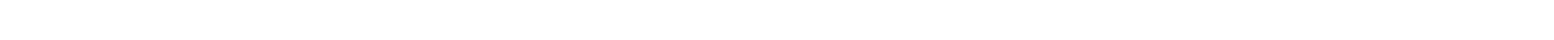
is en
$\stackrel{w}{2}$ is
$\sim$
n
is
m
in

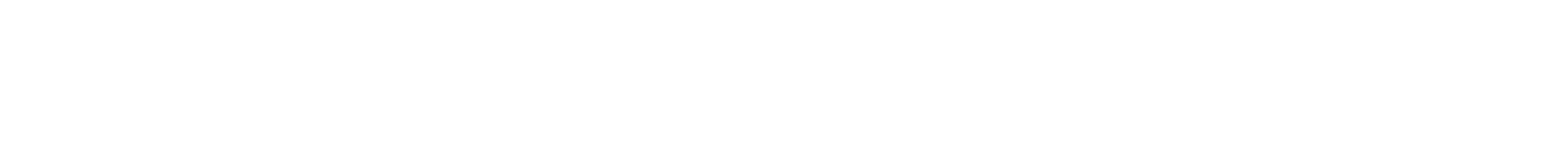

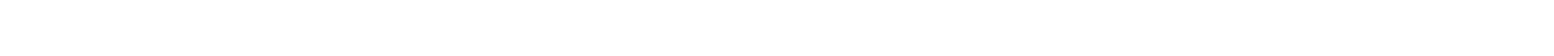

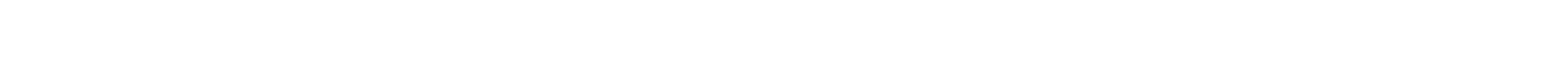

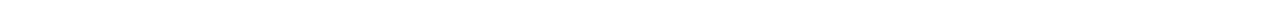

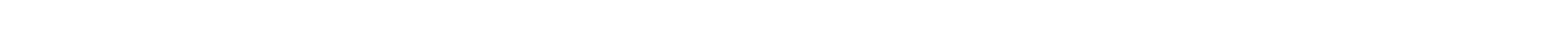

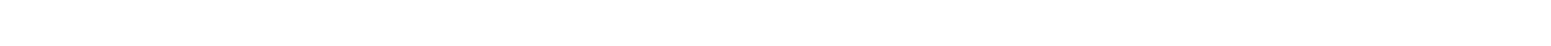
is
is
en
en
is

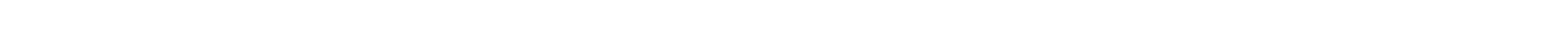

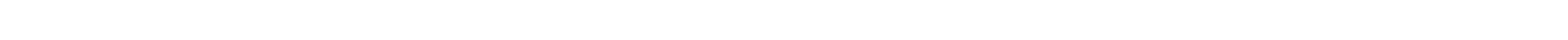

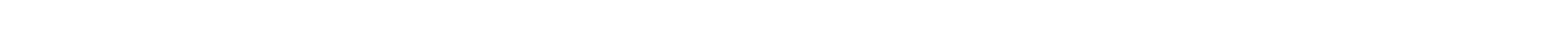

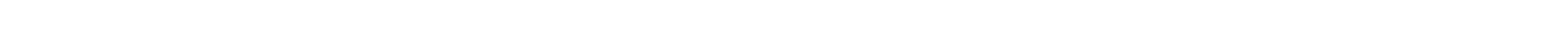

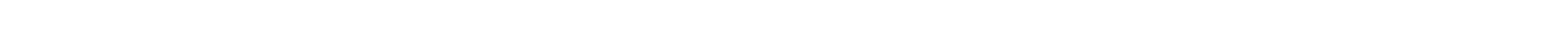

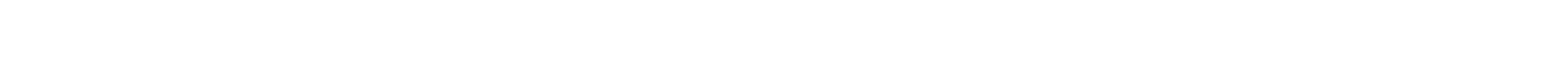

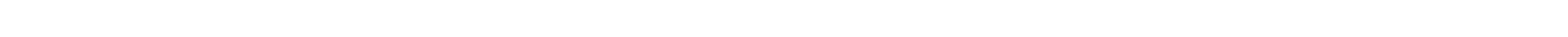

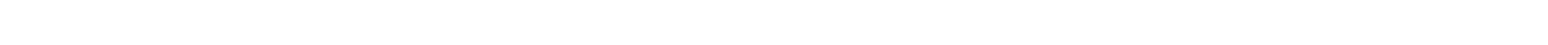

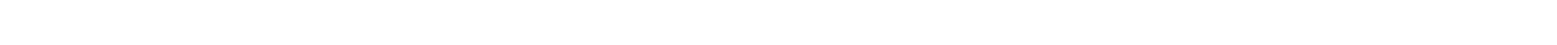

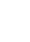

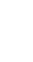

is

1 य.

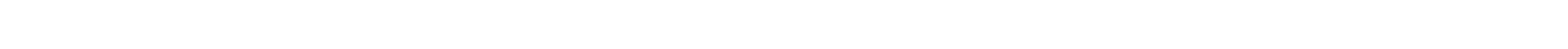

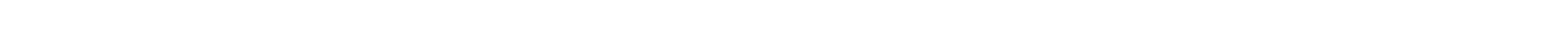

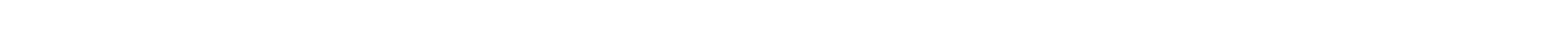

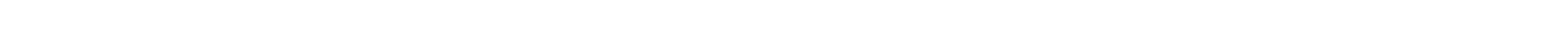

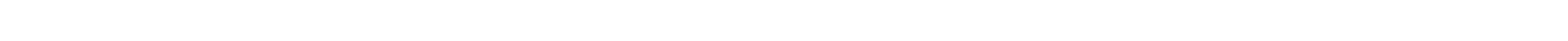

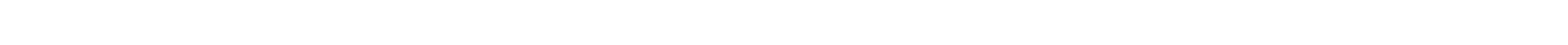

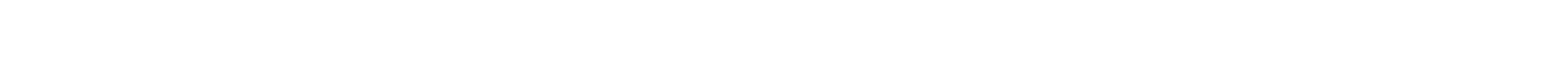




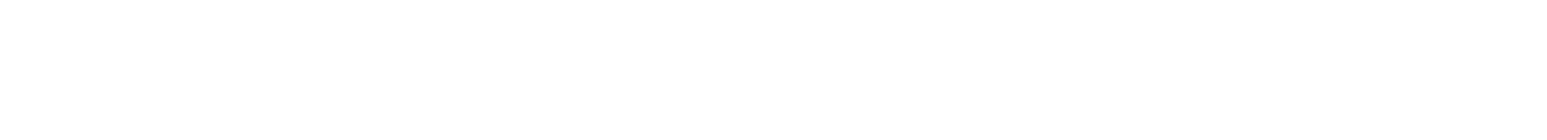

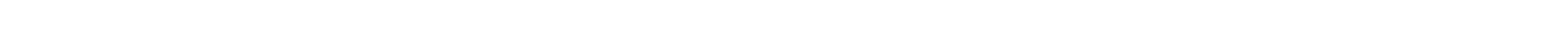
ш

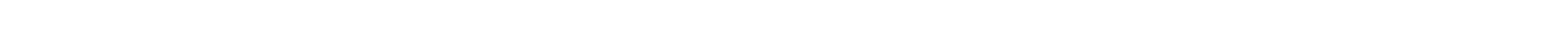

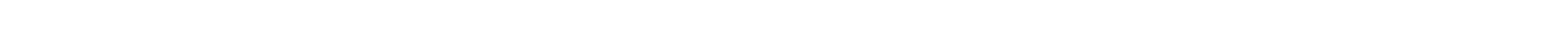

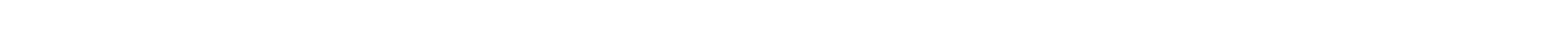

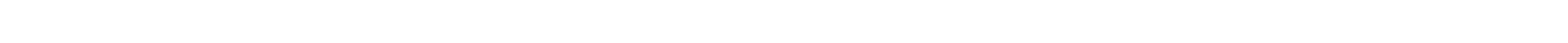

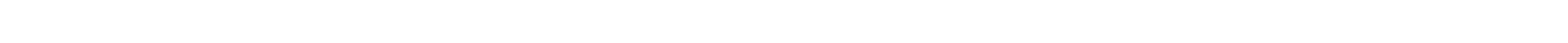

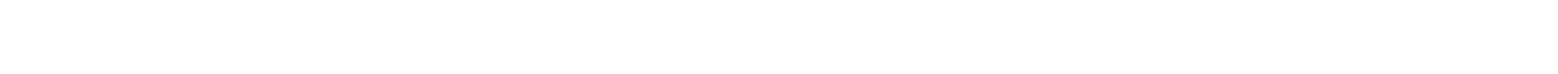

os

n

$\sim$

n

n

$\stackrel{4}{2}$

иนก х

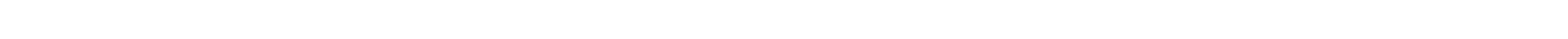

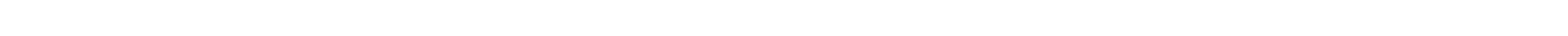

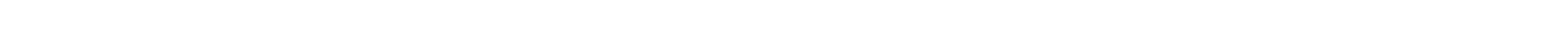

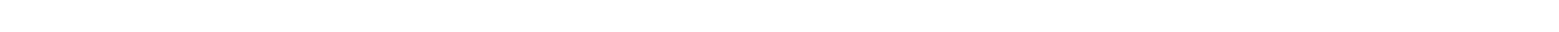

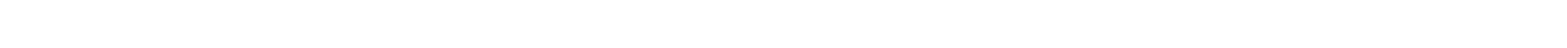

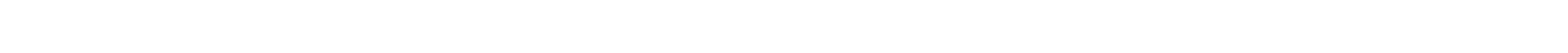

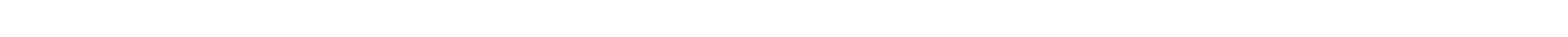

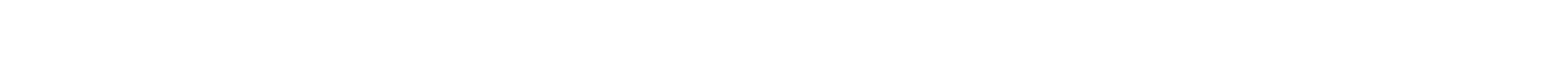

$+$

$\stackrel{\leftarrow}{\longrightarrow}$

$\therefore$

a

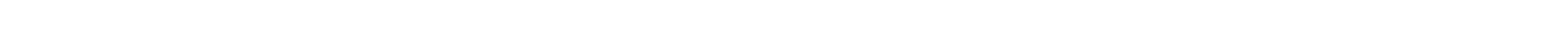
×

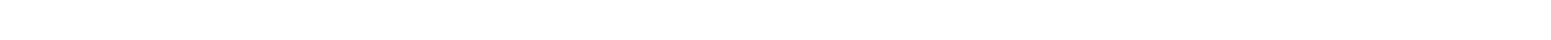

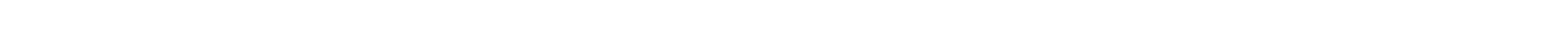

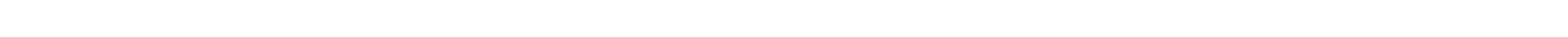

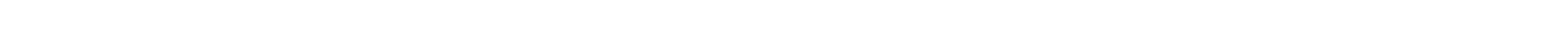

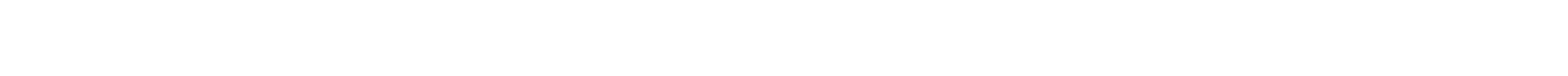
is
a

Ł

en

n

in

n

$\infty$

离

"O-nOA o

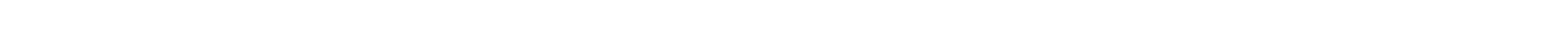

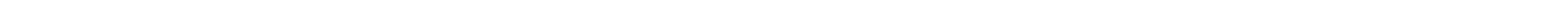

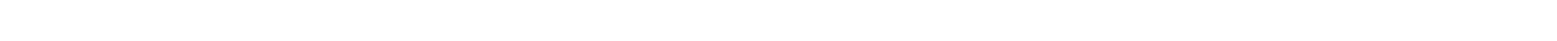

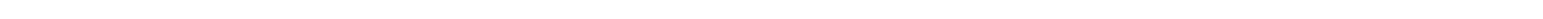

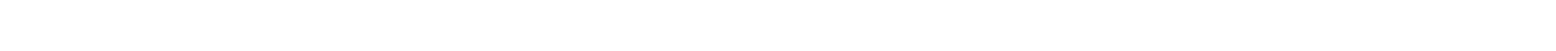

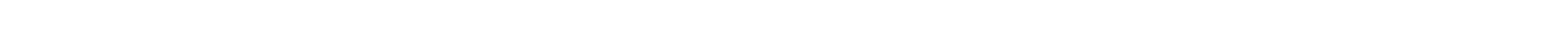

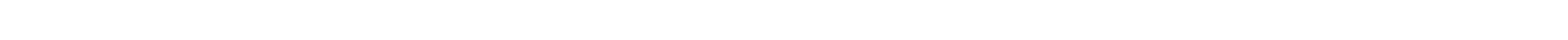

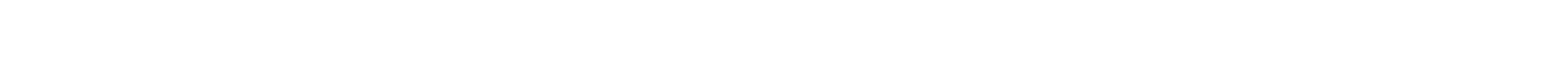




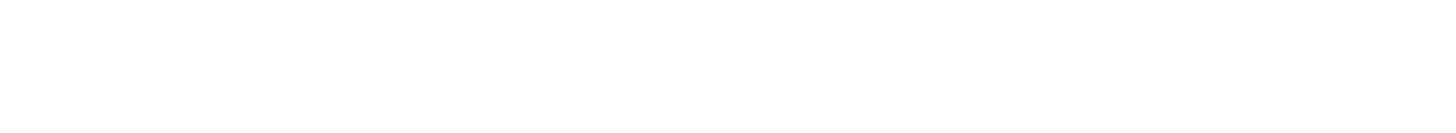

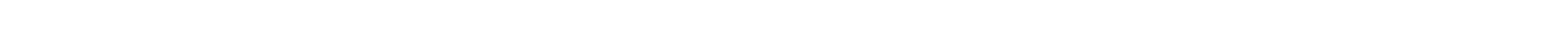

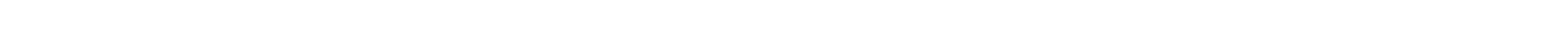

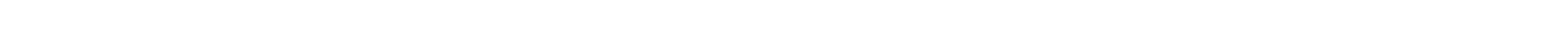

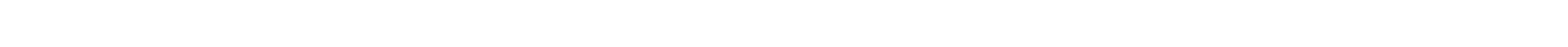

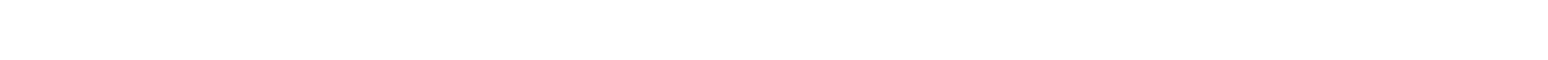

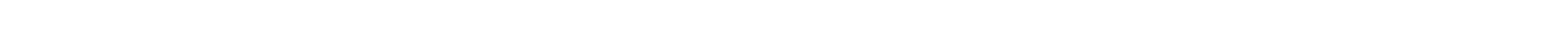

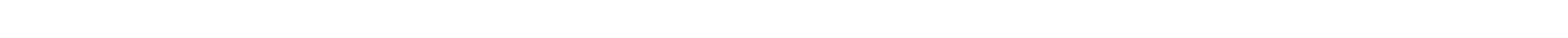

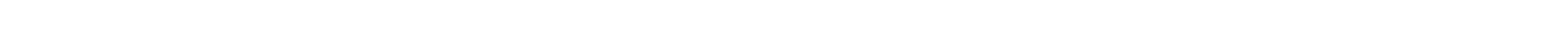

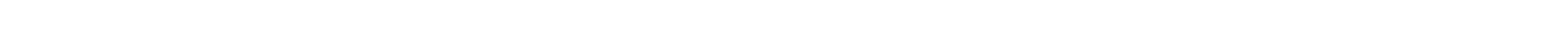
产 o
is
n
in en
n
n
in

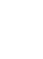

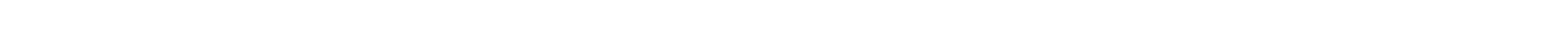
แ山

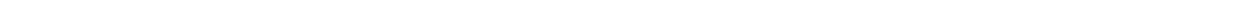
v几n

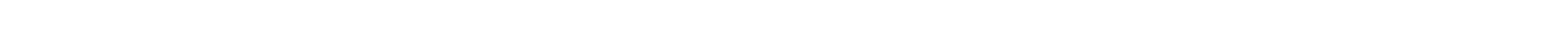

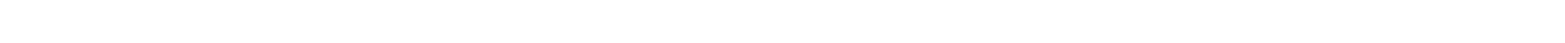

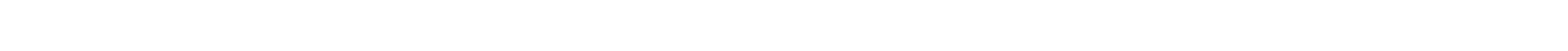

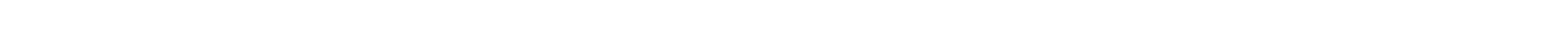

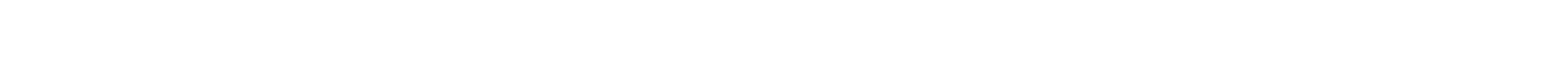
is a c a
en
as
a
n

点

а

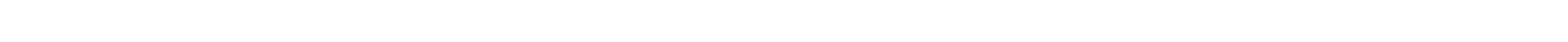

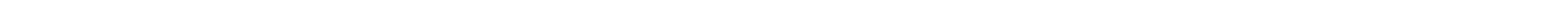

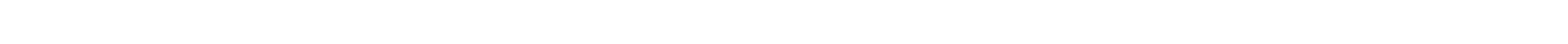

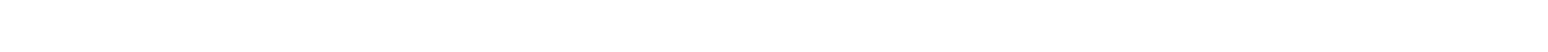

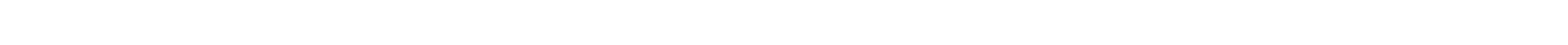

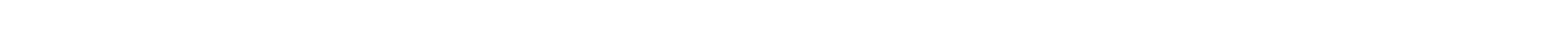

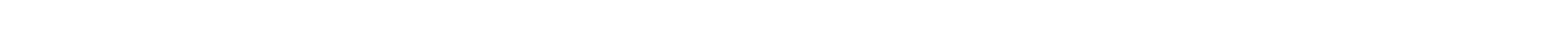

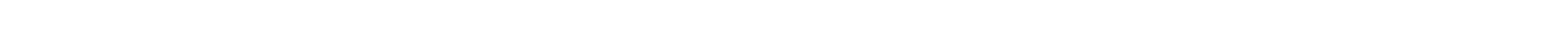

$\frac{21}{2}$

$\backsim$

ur

en

is

min a ammosa a d t แU

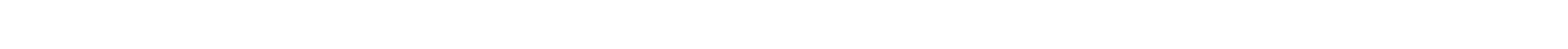

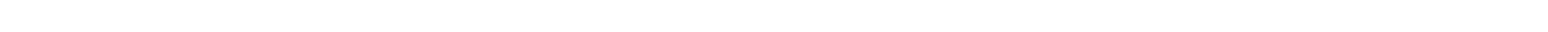

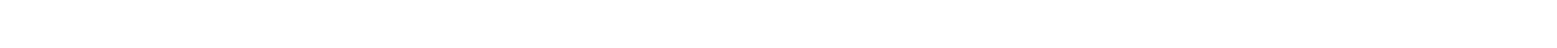

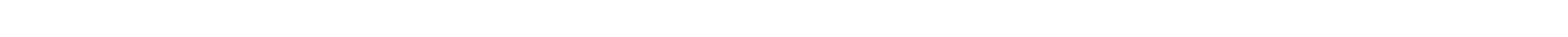

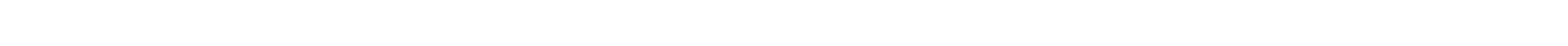

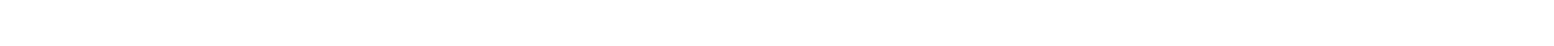

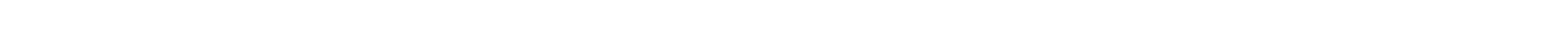


i

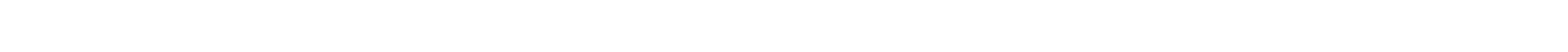

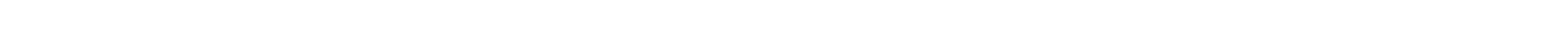

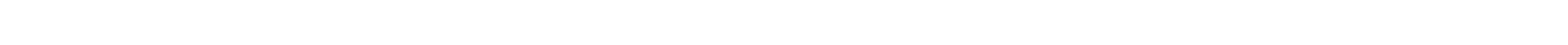

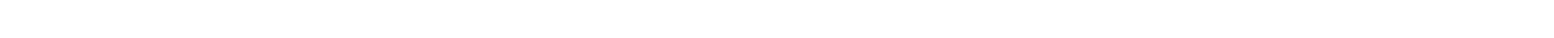

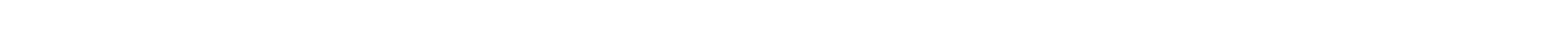

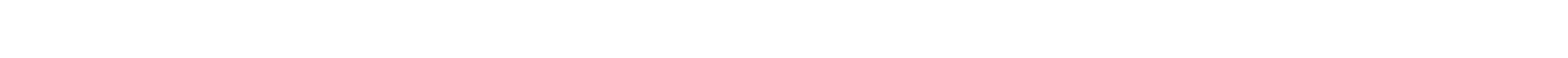

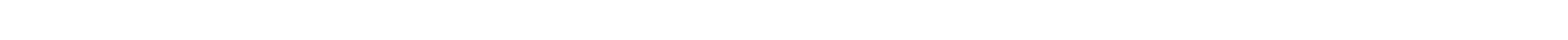

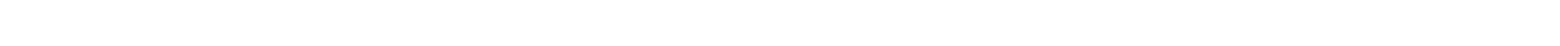

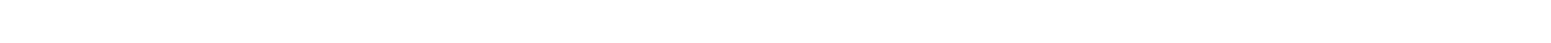
is
$\infty$
n
n
n

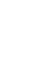

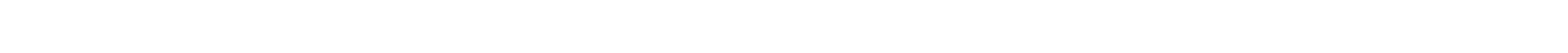

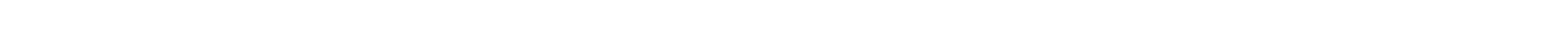

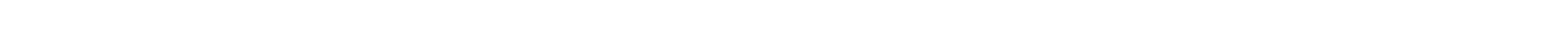
3 J

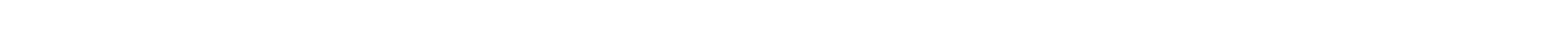

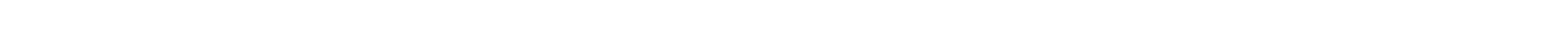

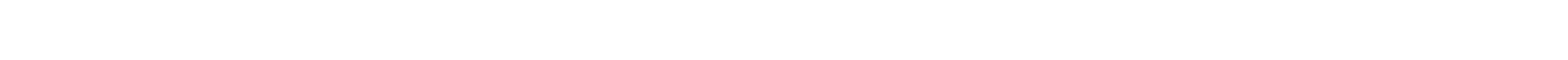

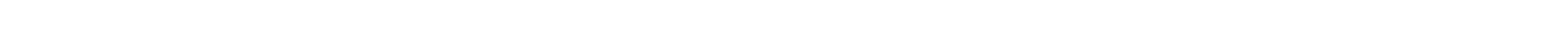

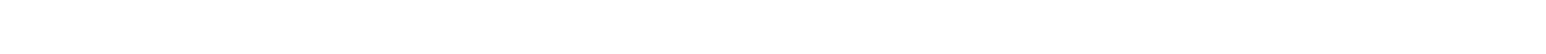

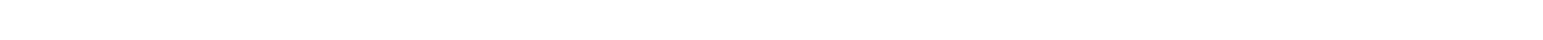

$$
\begin{array}{lll}
5 & n & n
\end{array}
$$$$
\text { n }
$$$$
\text { is }
$$

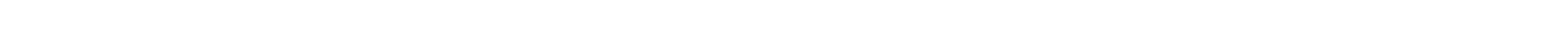

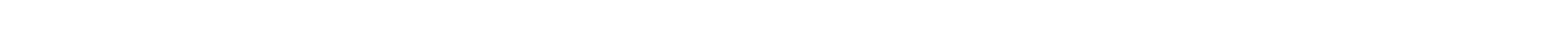

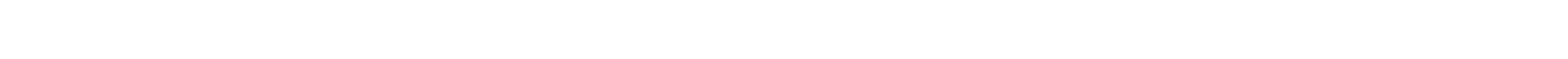

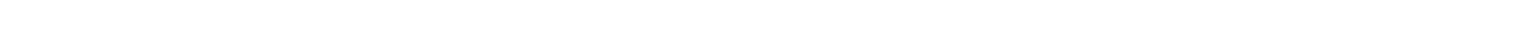

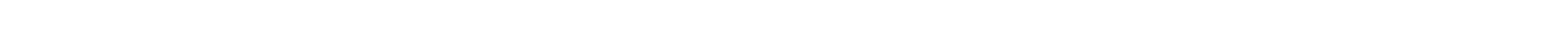

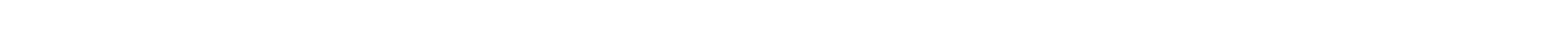

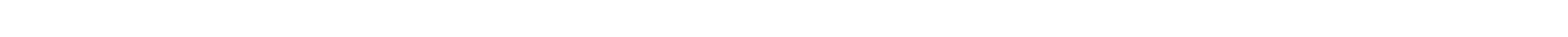

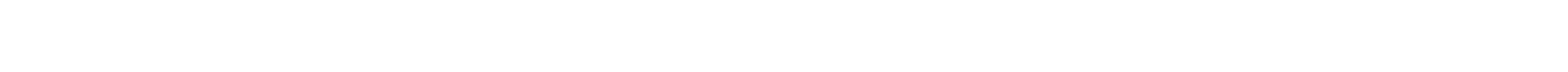

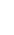

a

a 수 a

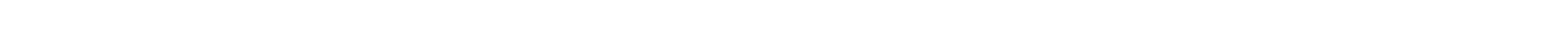

व

MD

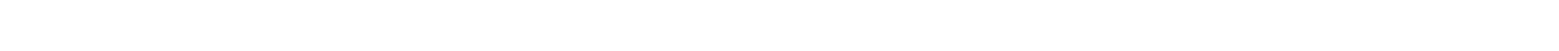

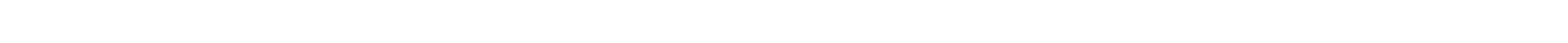

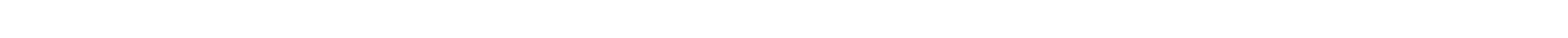

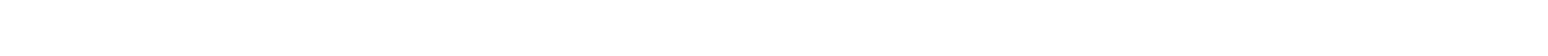


is

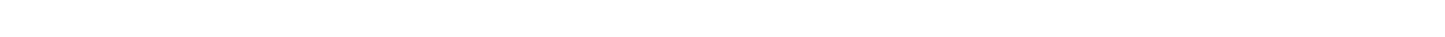

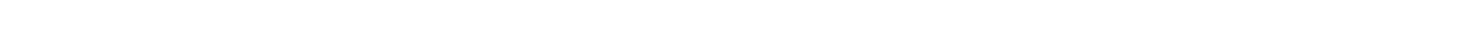

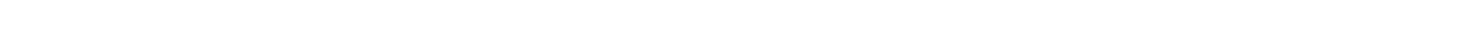
4"

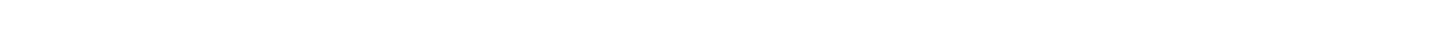

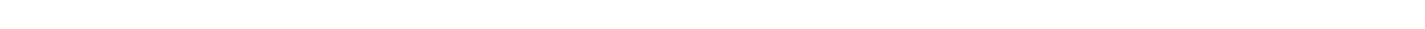

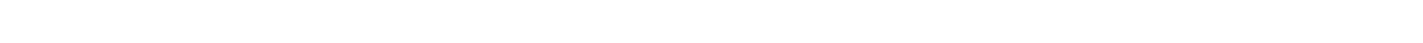

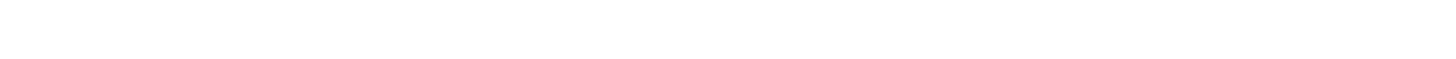

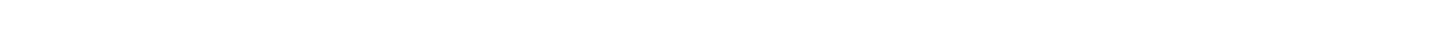
え̃̃mmmmmmmm

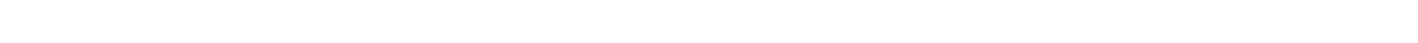

$$
\text { ॅ̄ }
$$

a

in

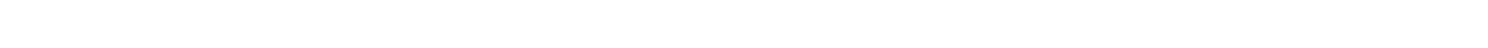
แ.

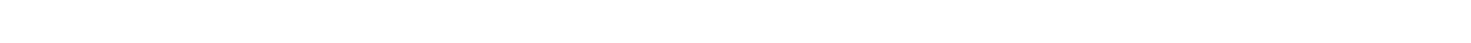

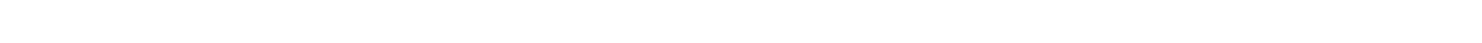

- कूa

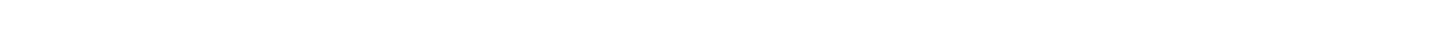

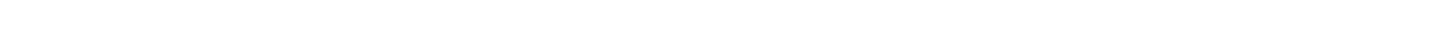

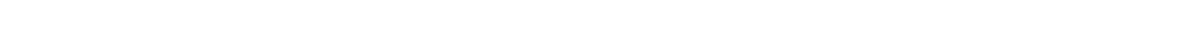

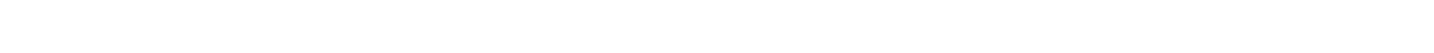

$\stackrel{5}{\Sigma}$

in

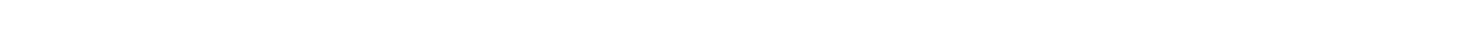
u u

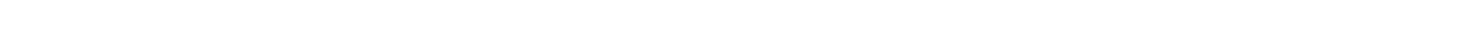

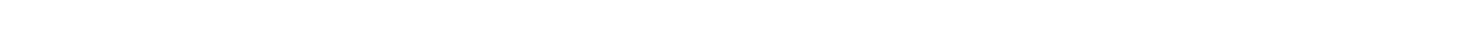

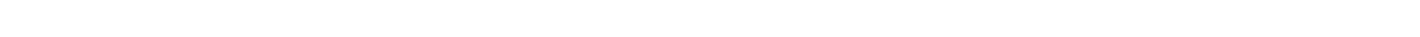

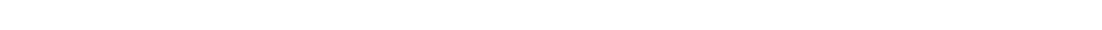
แ

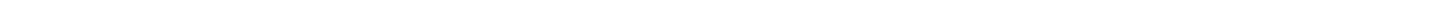

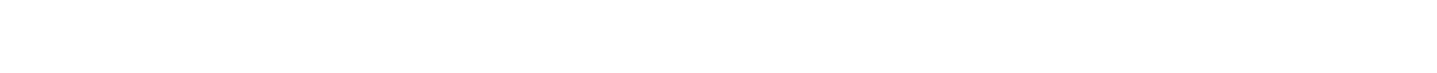

w

s.

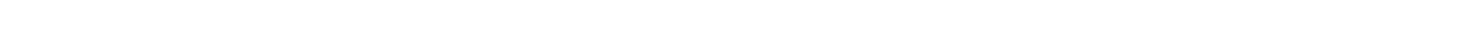

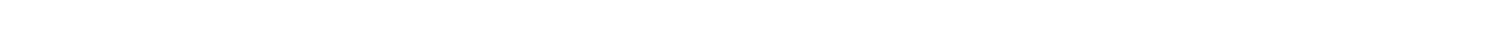

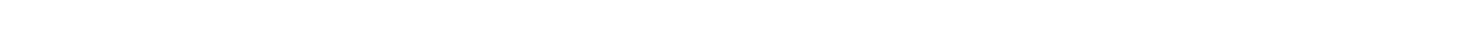

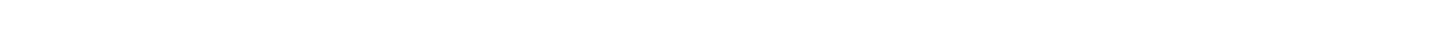
w

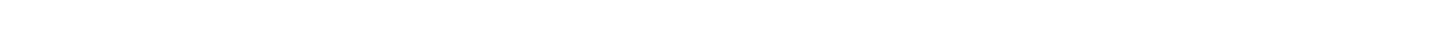

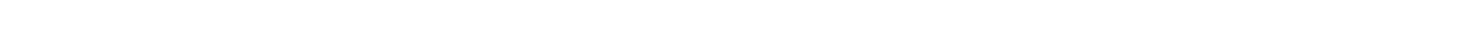

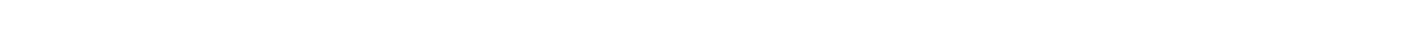

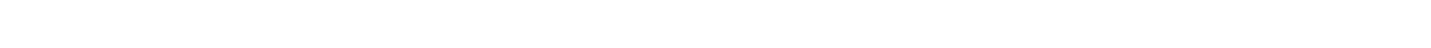



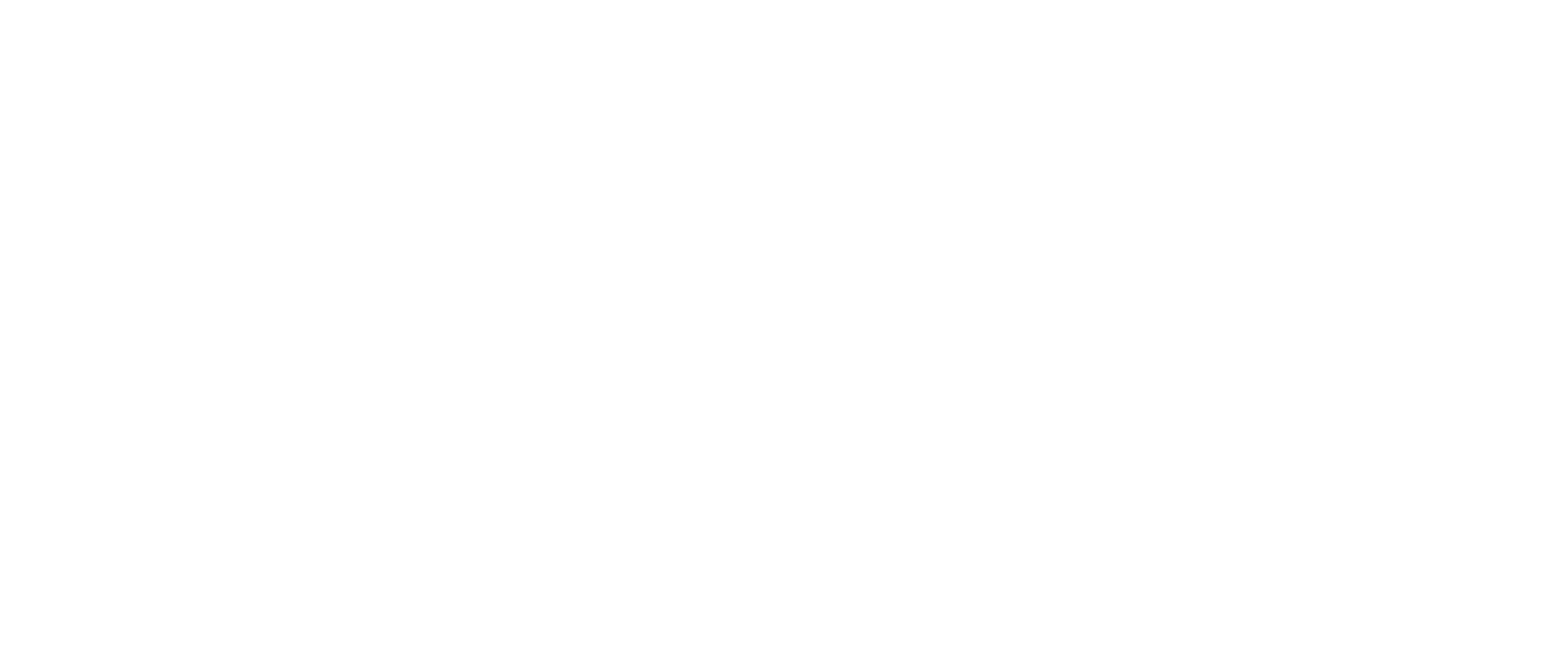

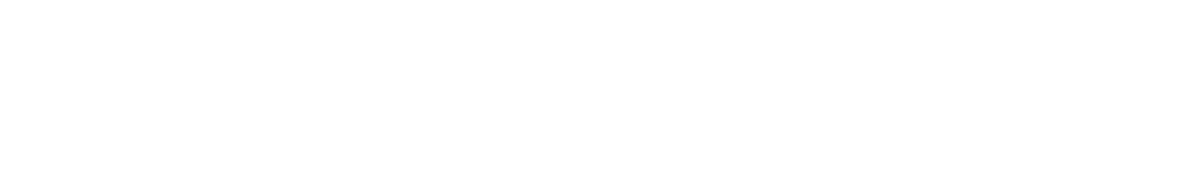

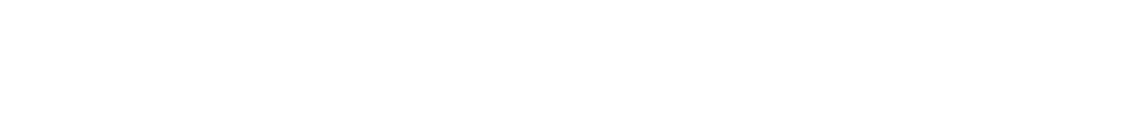

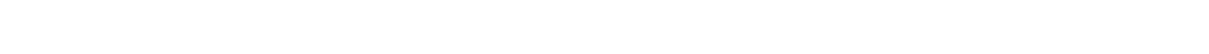
w

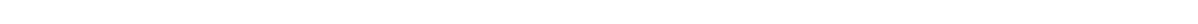
J

$\omega: u$

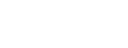

T-

$\because \stackrel{\leftrightarrow}{\circ} \ddot{\circ}$

war 20

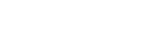

$3 \sin$

ำ 웅영

EDáa

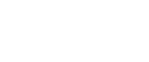

我눙

m

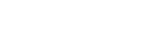

品呇出

ปละ์

ar:ais

$\therefore \dot{0} \div$

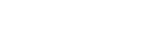

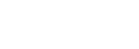

$\stackrel{m}{\sim} \dot{\Sigma} \dot{\Sigma}$

중ㅇㅇ응

№toin

行品出。

红。잉

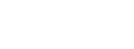

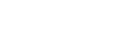

紧的

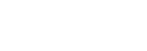

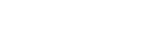

แニะ出占

n $=\div$ i

$z \dot{\sim} \dot{\sim}$

눙

崖产走

运尘

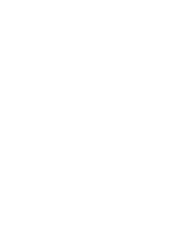

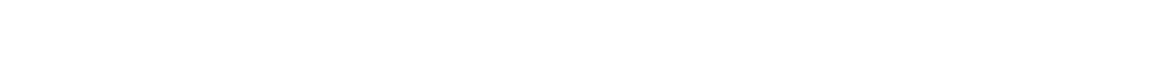

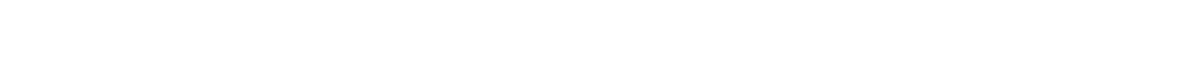

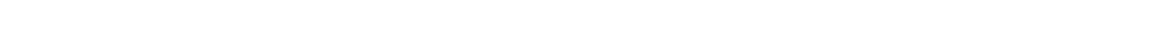

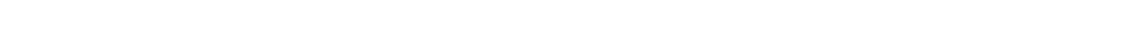

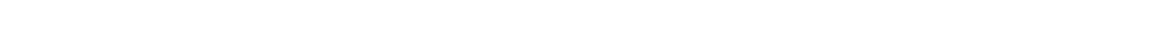

点

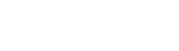

$\sum \frac{0}{2}=\sum$

ㅁํㄴ

aㅡ는

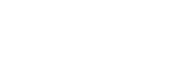
듬ㄴㄷㄴ

중하 的ón

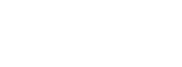
은

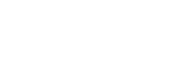

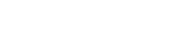

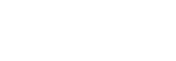
뚜을 $\ddot{w} ّ \ddot{w}-\ddot{u}$

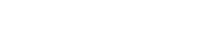
$a z z z z$

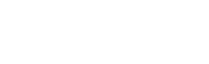

$w_{w} w_{w}$

in in

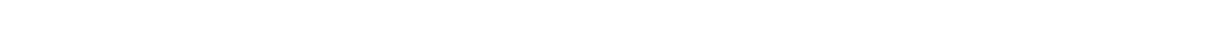

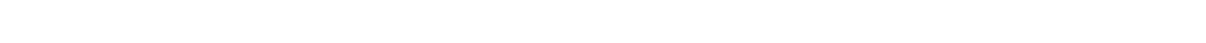

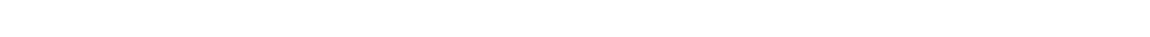

mMmmmmmmmmmmmmmmmmmmm

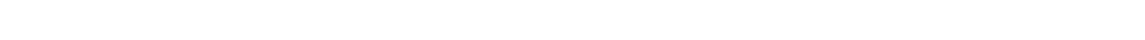

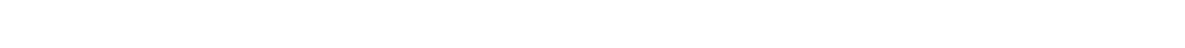

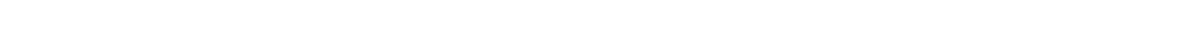

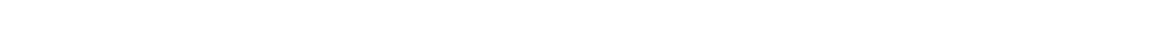

$5 \sin \infty \operatorname{sen}$

n

en

i

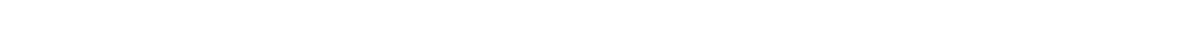
w

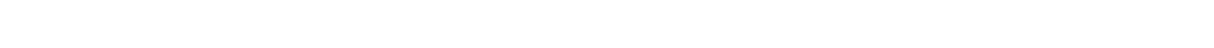

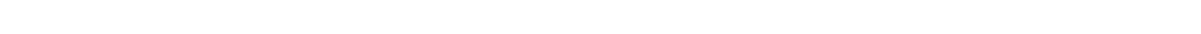

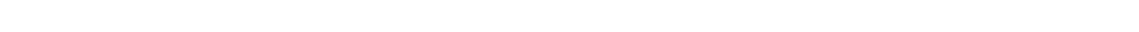

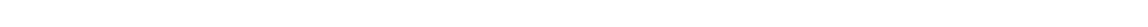

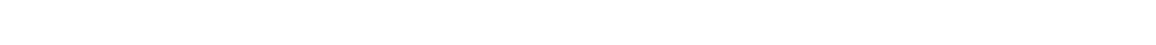

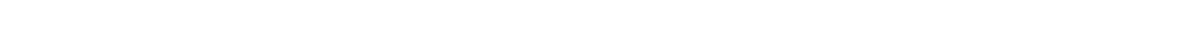

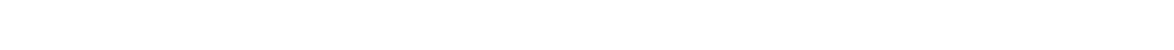

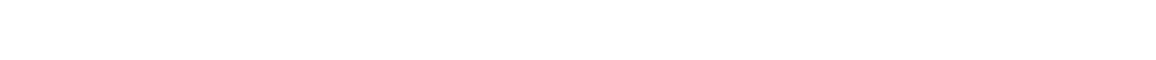




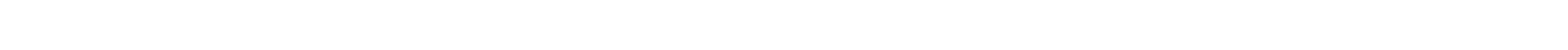

is "

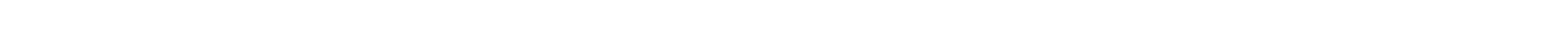

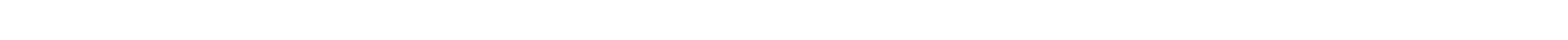
gog

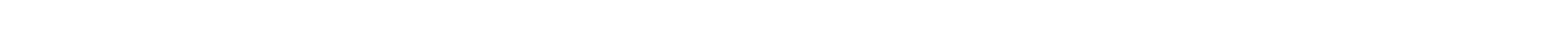

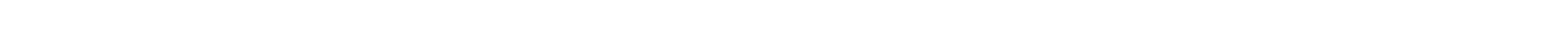

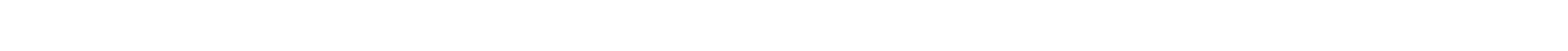
-

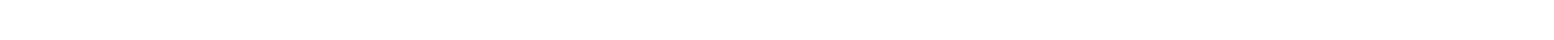

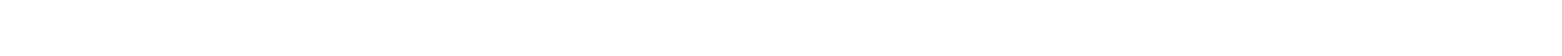

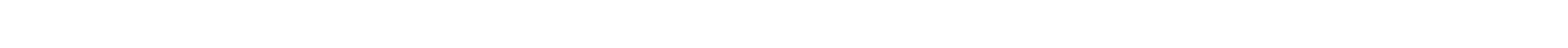

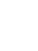

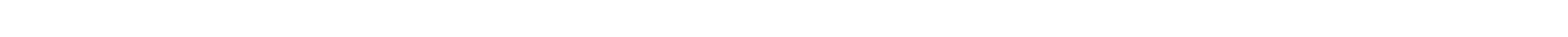

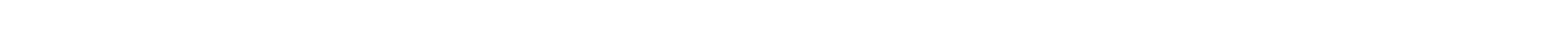

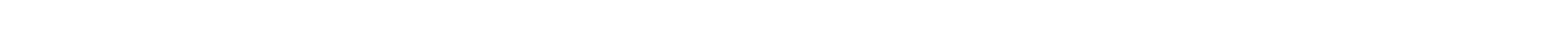
了

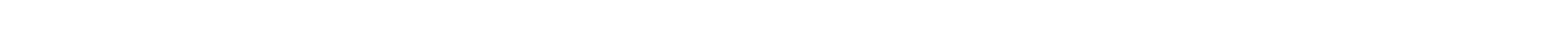

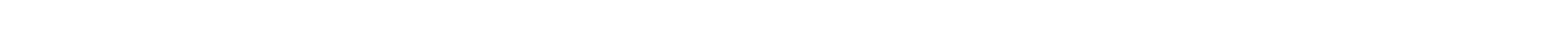

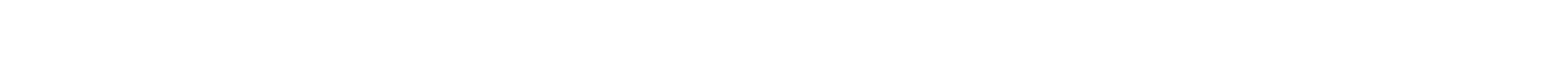

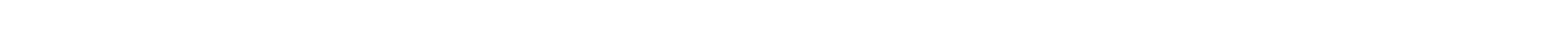

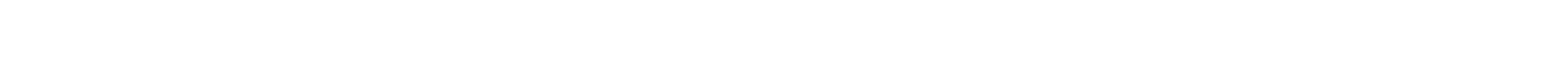

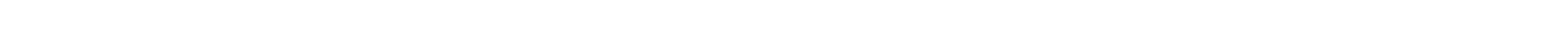

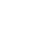

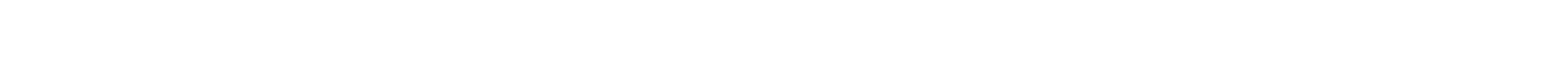

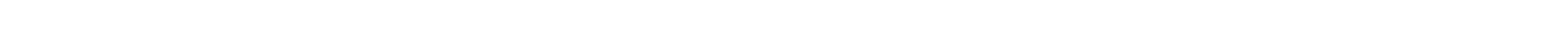

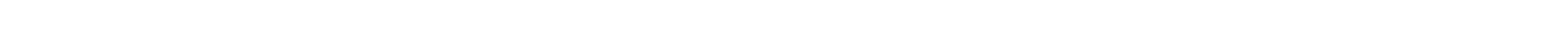

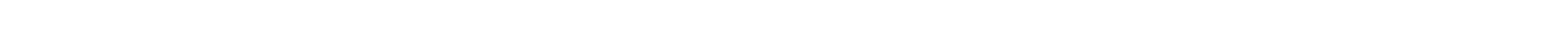

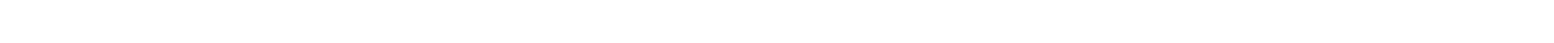

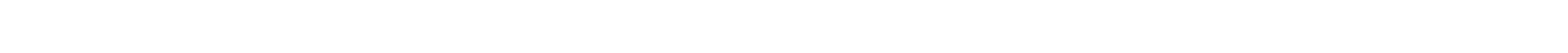

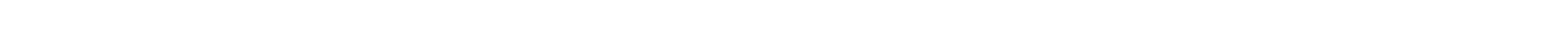

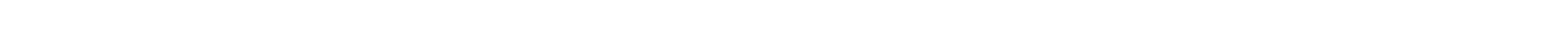

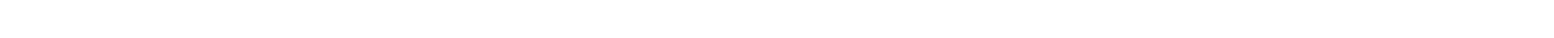

is

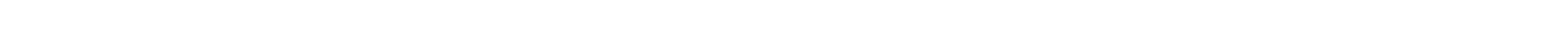

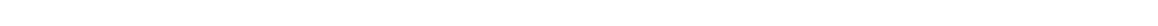

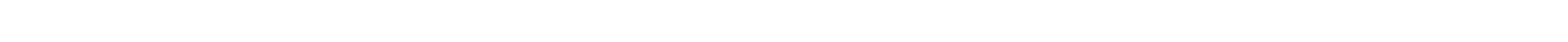
a wa

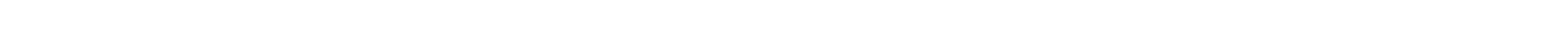

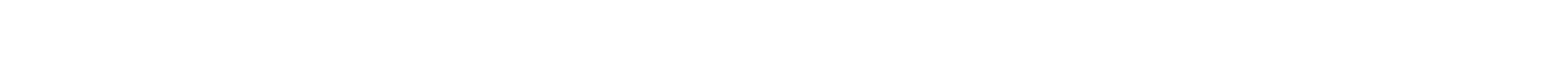
แ

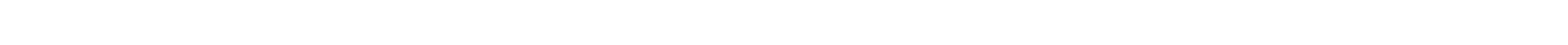
añ

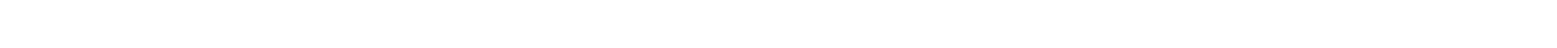




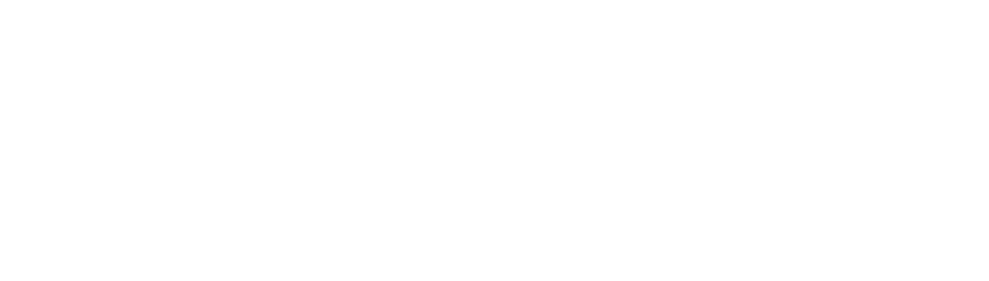

-

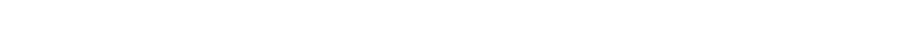

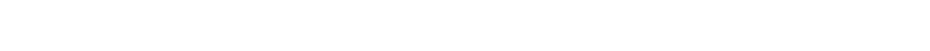

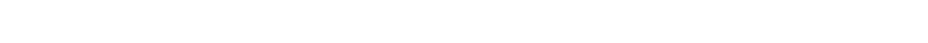

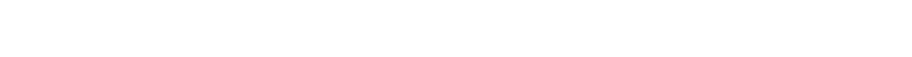

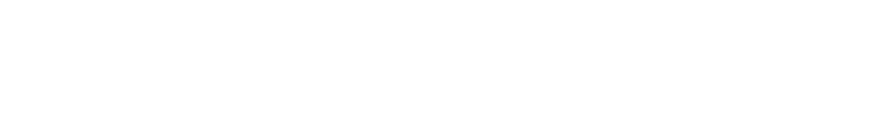

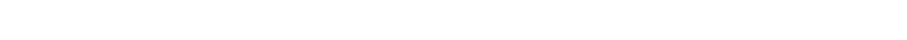

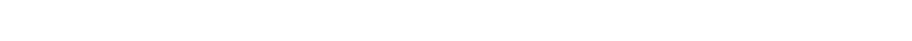

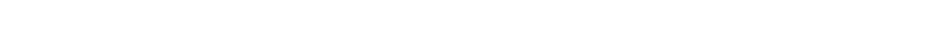
ON-NTNNT-MNNTUMTOOOONNNNNN-F-TN

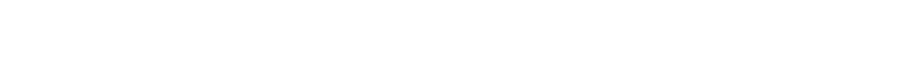

㟧

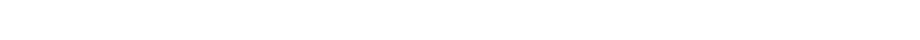
in

๔ úm

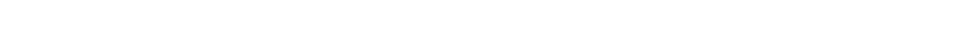

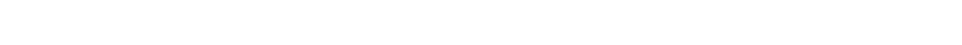

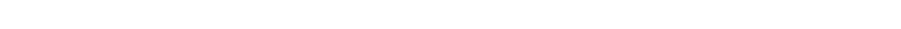

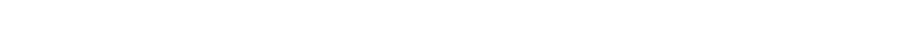

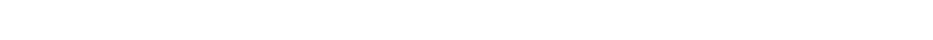

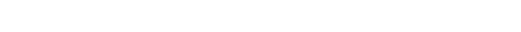

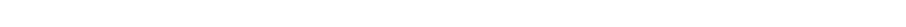

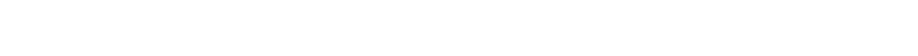

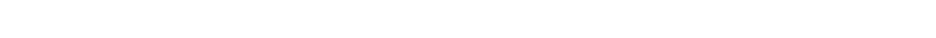

$\stackrel{\square}{\leftarrow}$

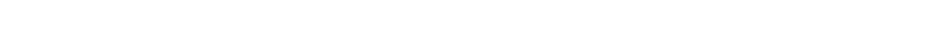

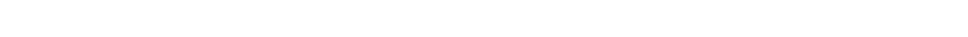

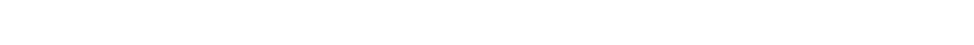

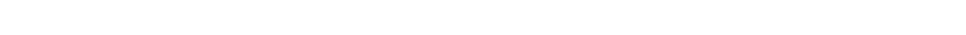
os

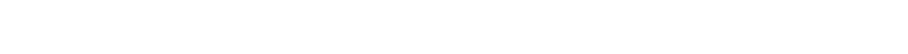

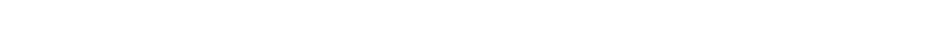

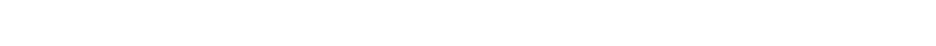

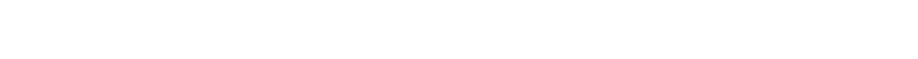




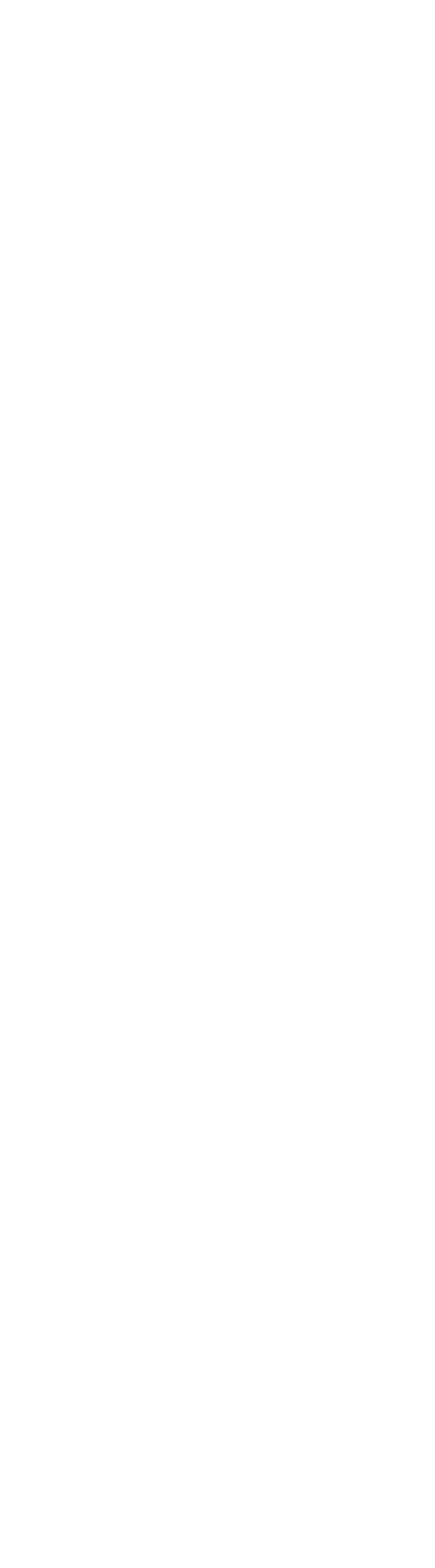

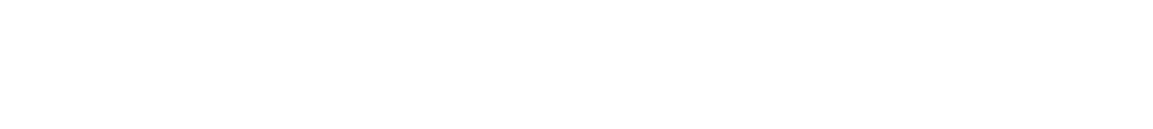

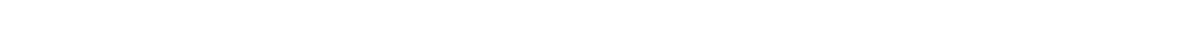
w山M ᄂ

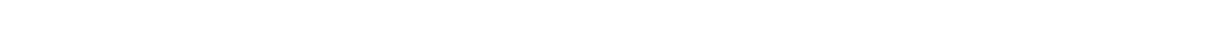

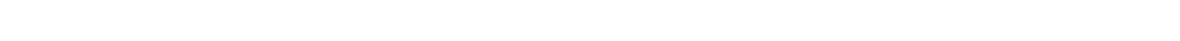

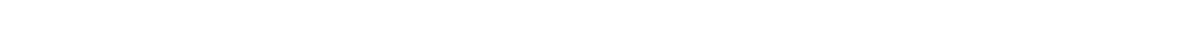

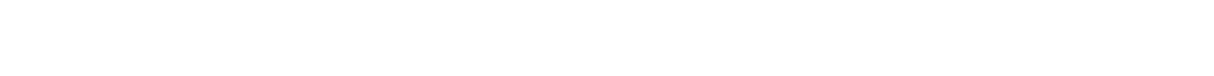

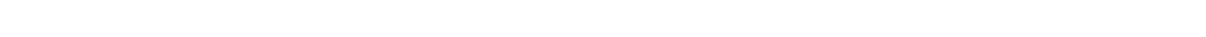

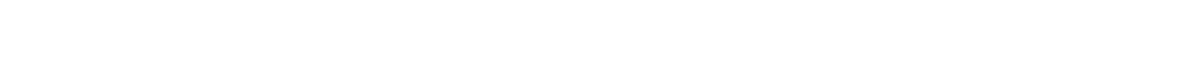

$$
\stackrel{w}{\Sigma}
$$

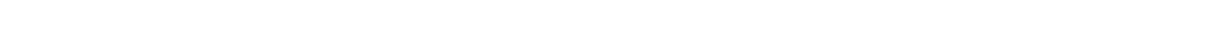
ot

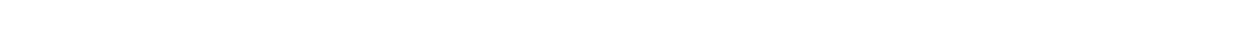
ay ong

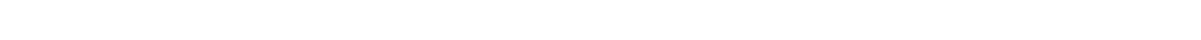

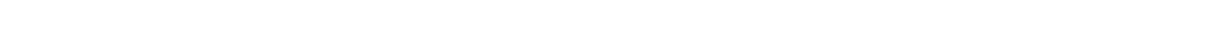

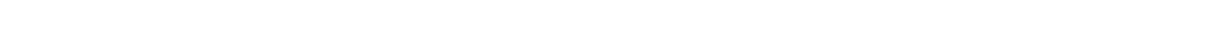

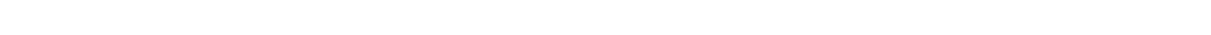

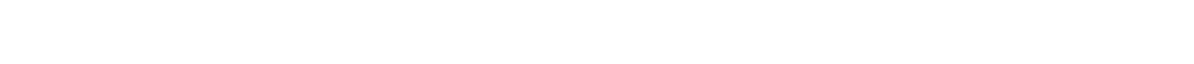

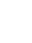

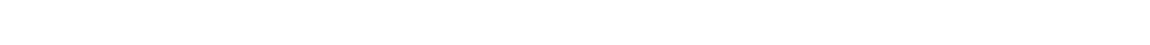

is

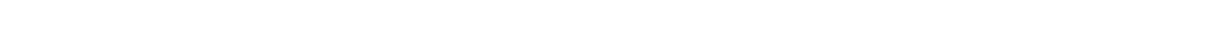
w

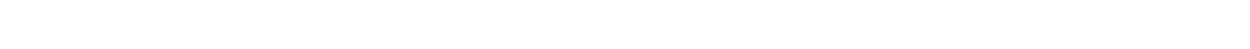

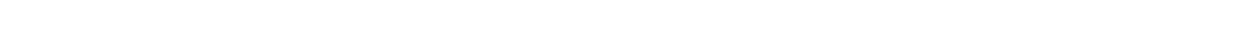

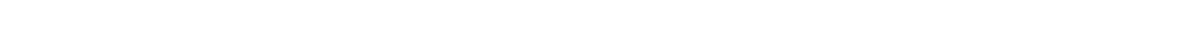

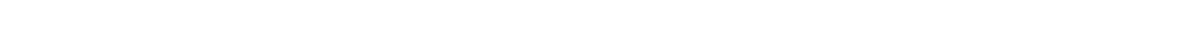

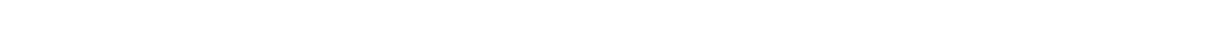

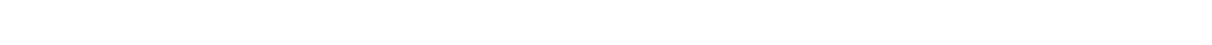

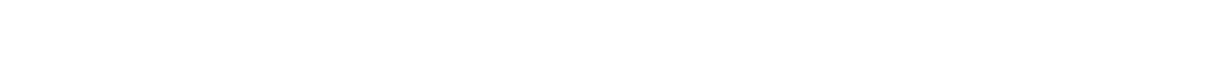

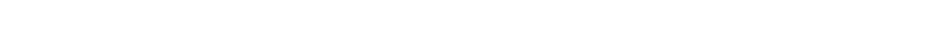

en

$\stackrel{a}{\llcorner}$

งำน

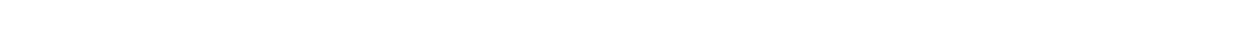

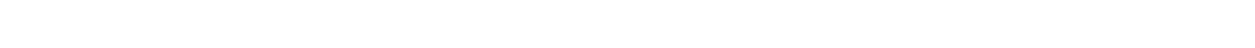

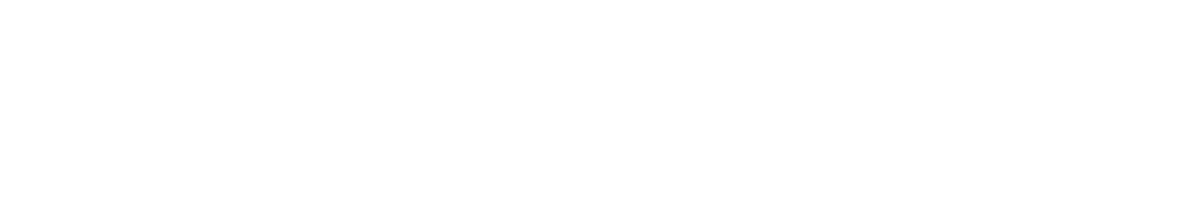



岕
n
n
n
is
a
n
i

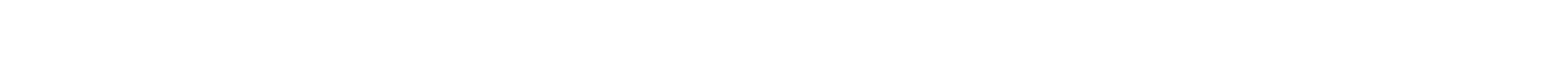

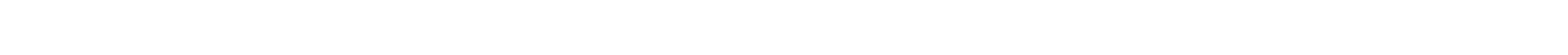
- wo

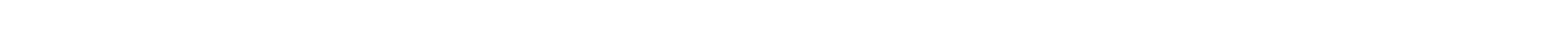

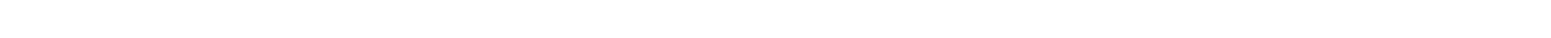

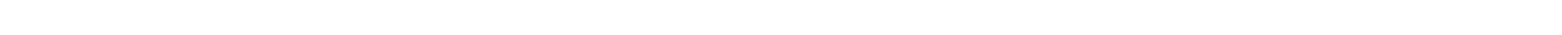

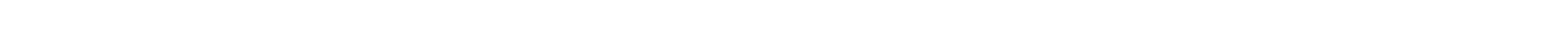

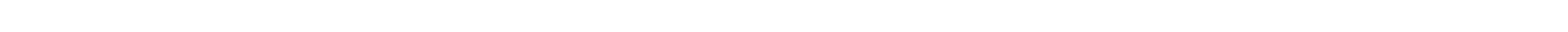

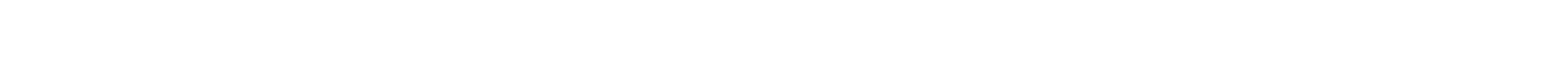

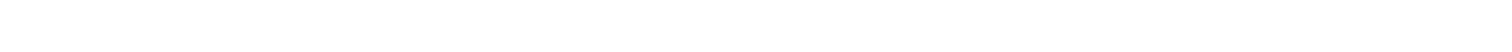

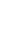

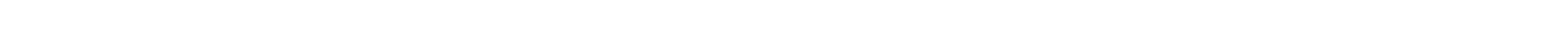
w

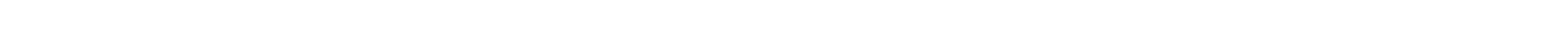

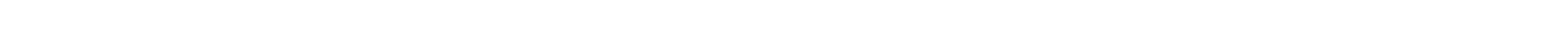

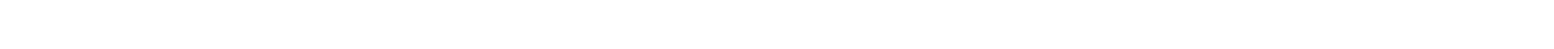

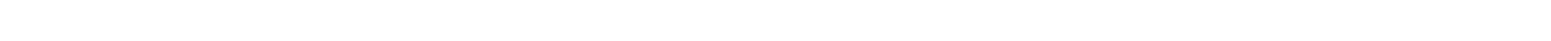

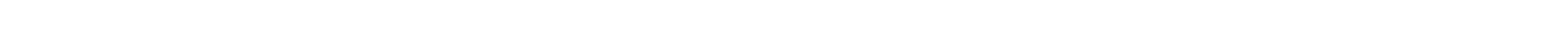

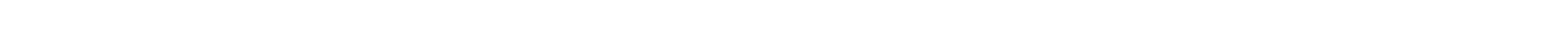

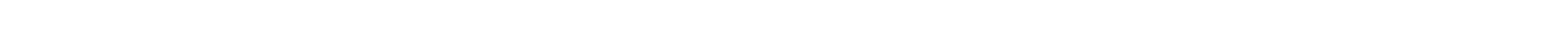

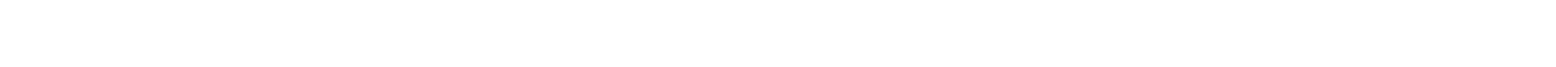

$$
\text { 㭊的 }
$$

is

n

n

n

en

os eseneses

๔ щ

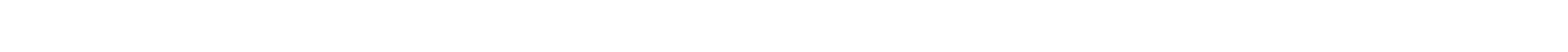

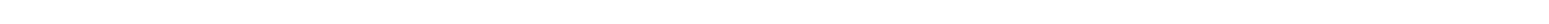

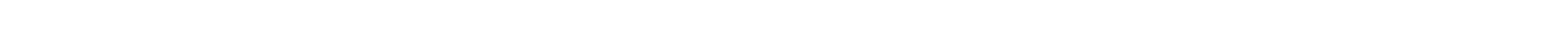

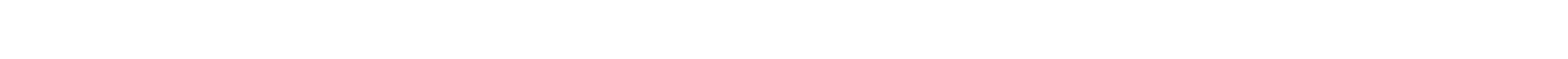

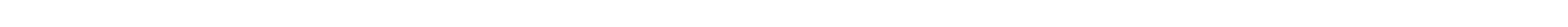

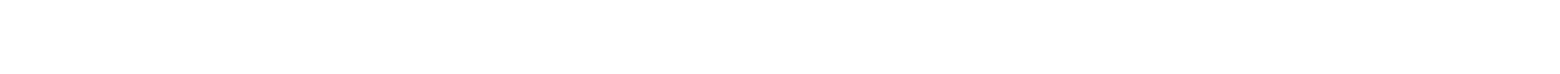

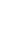

$\frac{\omega}{z}$

$\stackrel{a}{2}$

กテ- แ

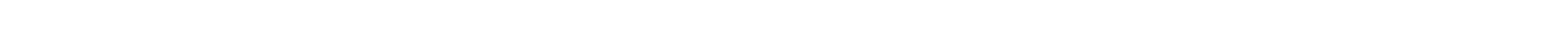

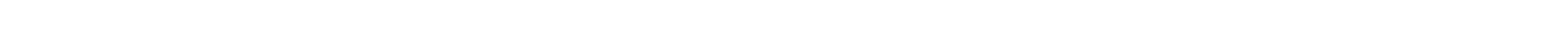

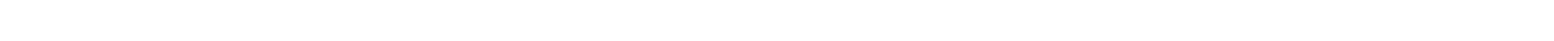

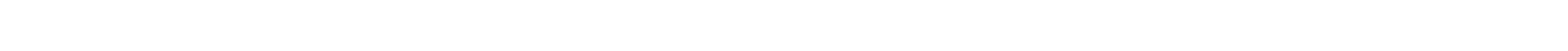

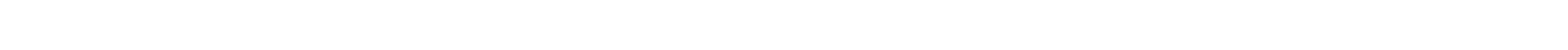

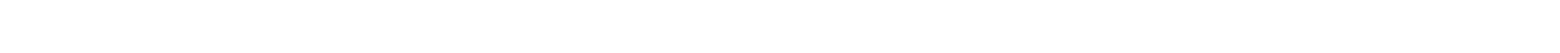




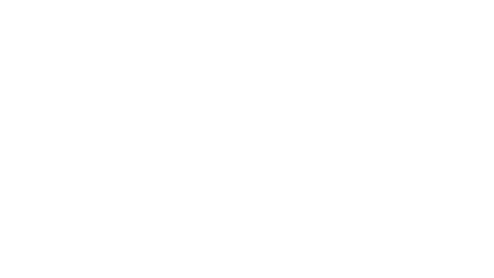

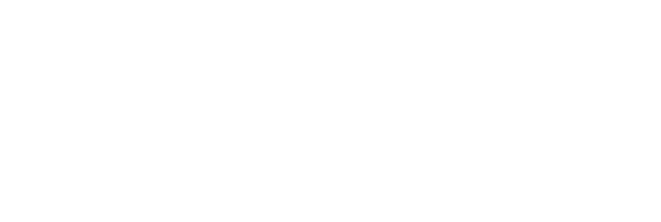

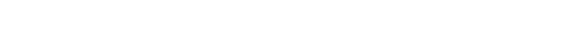

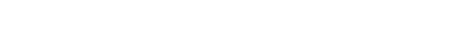

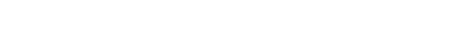

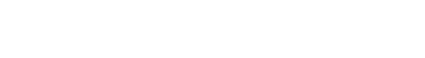

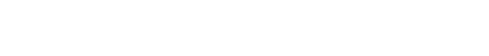

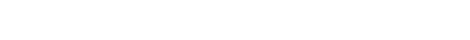

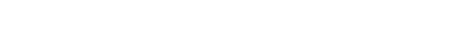
d nnnumnnnwunnum

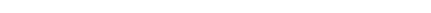
ㄴ.

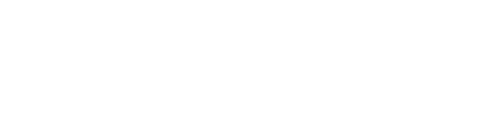

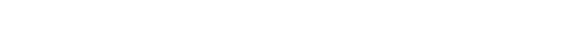
a.

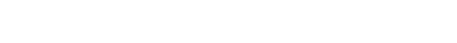

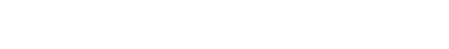

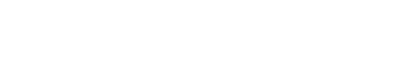
เ u.

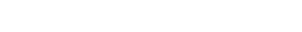

$$
\begin{aligned}
& \text { w n on } \\
& \text { is }
\end{aligned}
$$

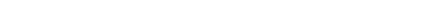
시에

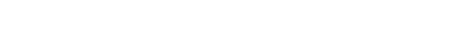

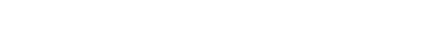

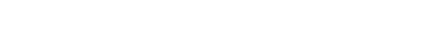




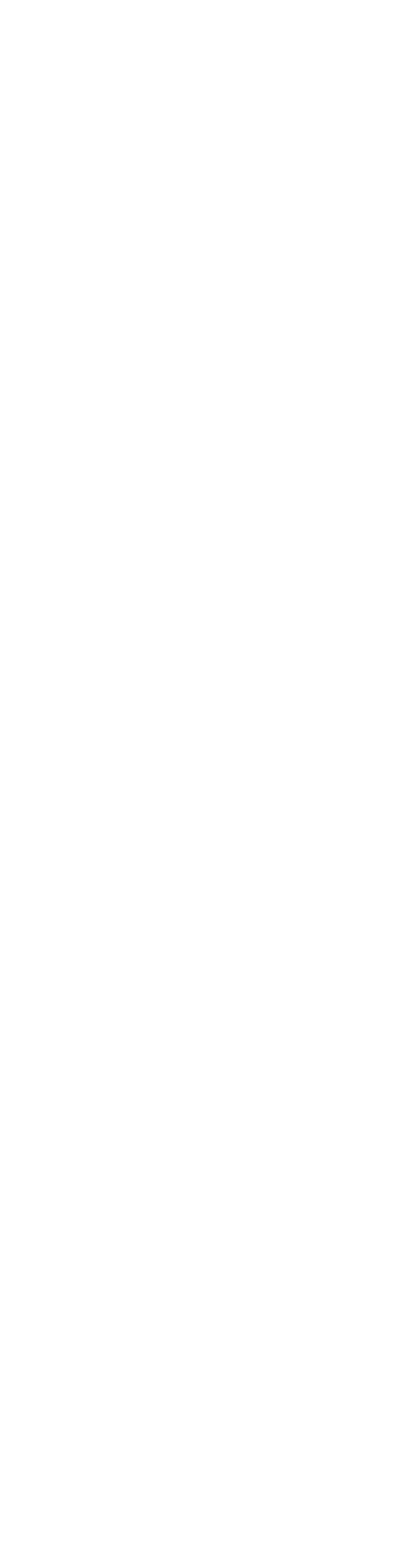
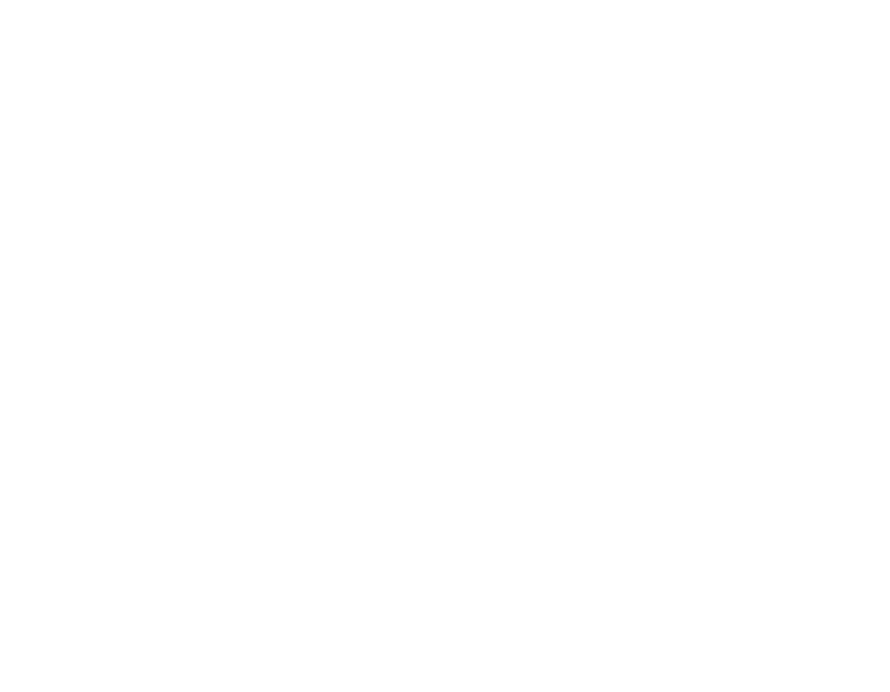

×

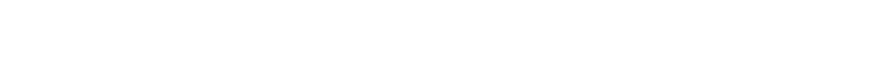

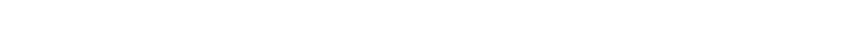

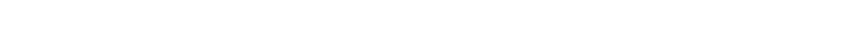

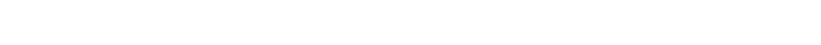

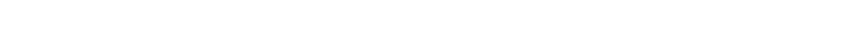

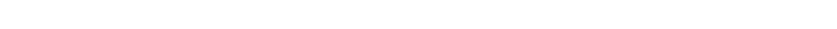

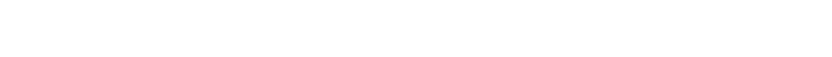

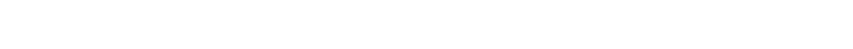
a

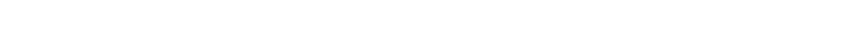

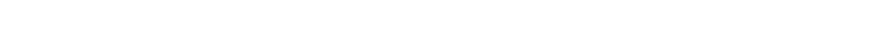

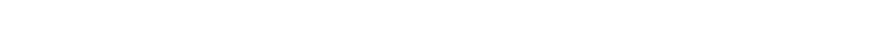

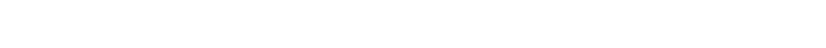

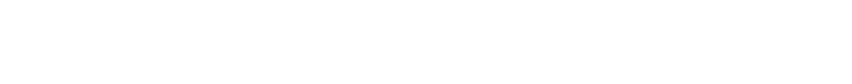

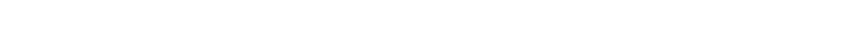

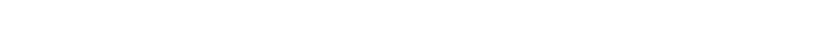

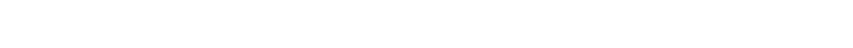

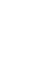

๙

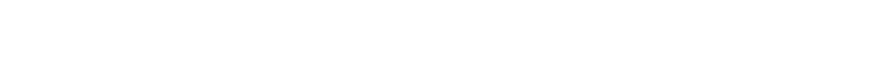

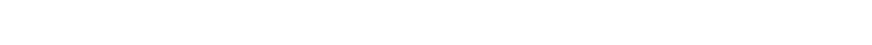

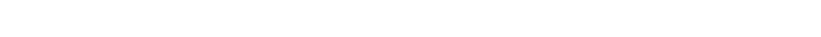

nмmmmm

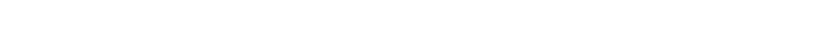
w

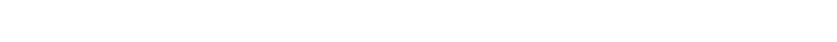
a กัง 


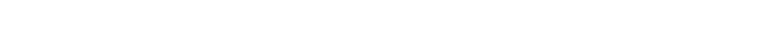

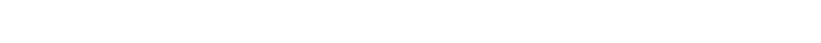

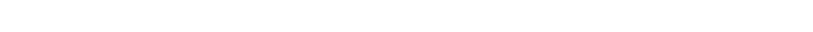

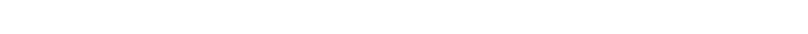

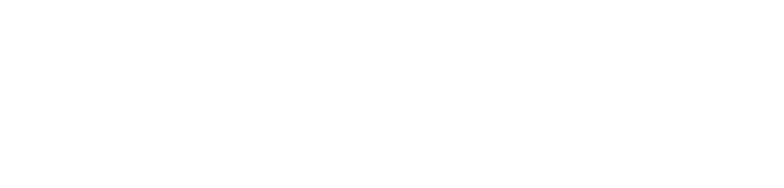

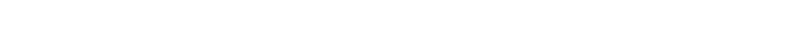
is

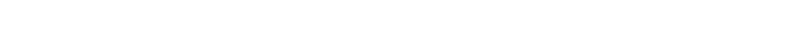

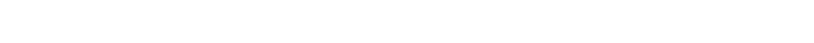

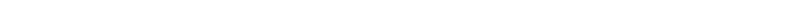
з

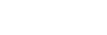

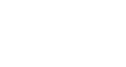

$x \div-\infty$

wo

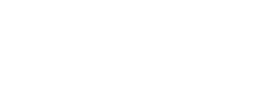

mi

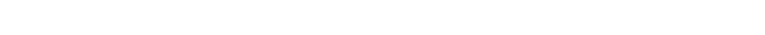

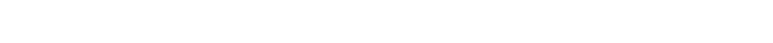

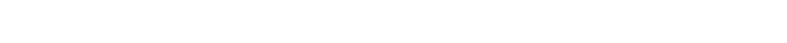

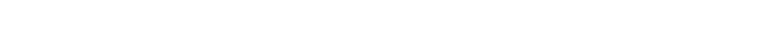

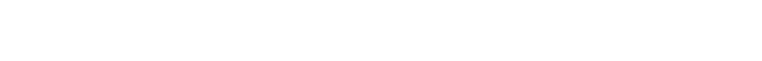

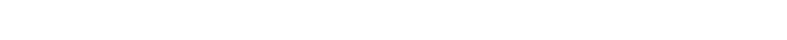
$\$$

-

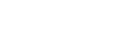

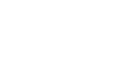

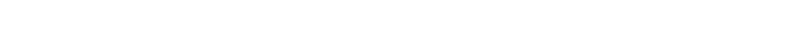

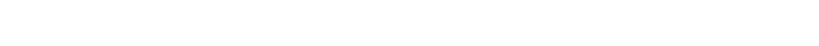

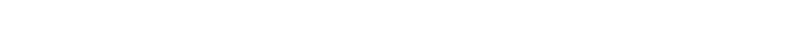
-

s. $\ddot{z} \ddot{z} \ddot{\omega} 0$ $\exists \dot{C} \infty$ ㄴำ

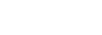

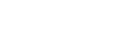

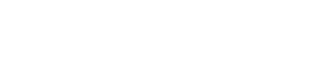
1)

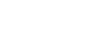
世约:

$\pm 1:$

$>\sqrt{z} \bar{c}$ 出

a. on $0: \frac{1}{0}$ $\omega \sim 0$. 두을 응 $m m=$

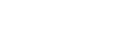
$=2=n$ is $\begin{array}{ll}40 \\ 2\end{array}$ 学的。 o 0 in $\sum_{m i} m^{2}$ ?w+ 造造客 뜬은

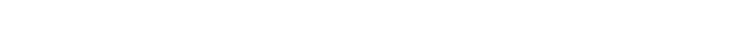
й

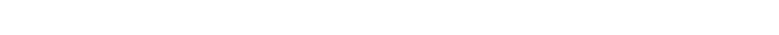

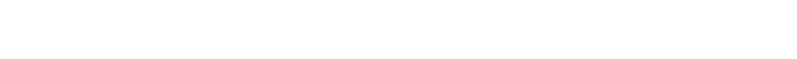

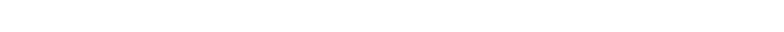

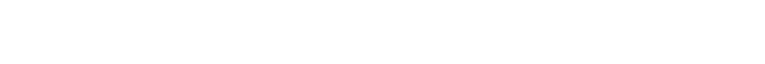

$w$

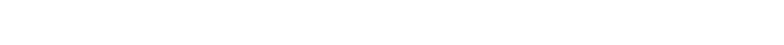
is

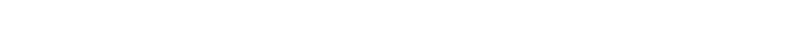

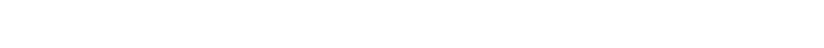

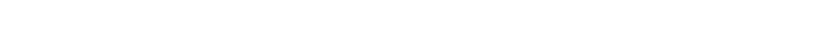
>

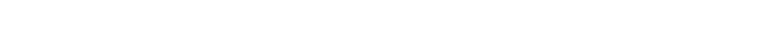

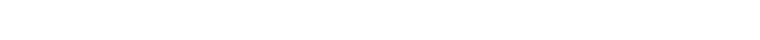

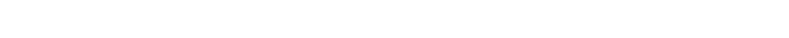

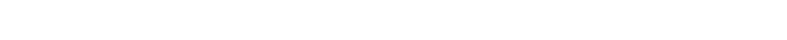

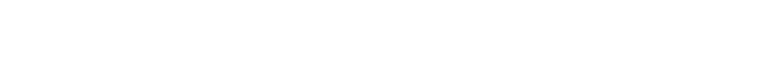



« ๔

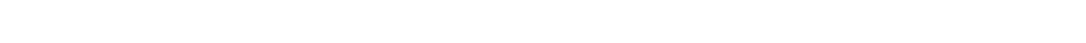

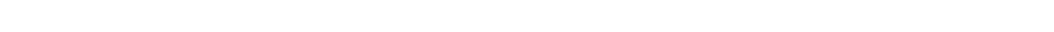

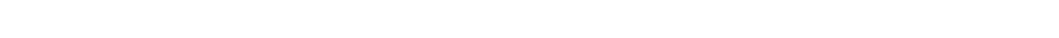

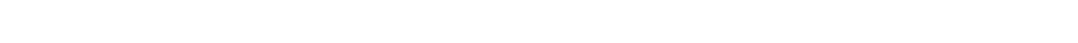

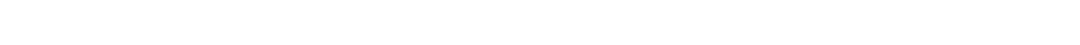

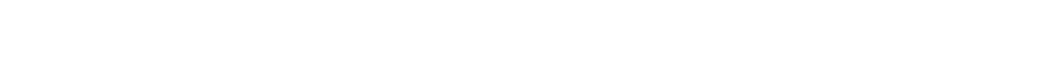

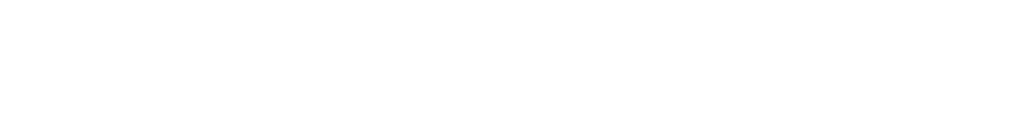

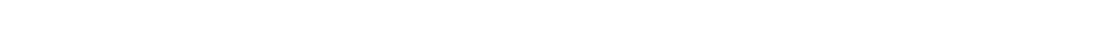
แ u.

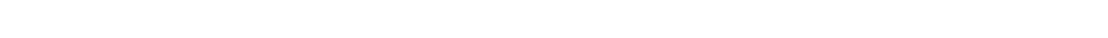
子小n

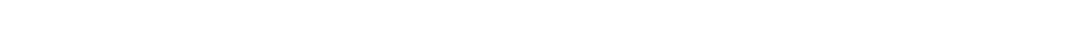

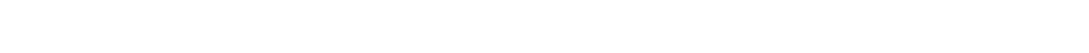

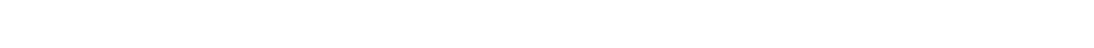

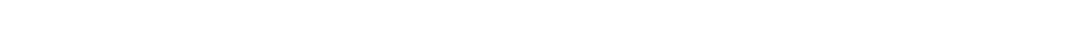

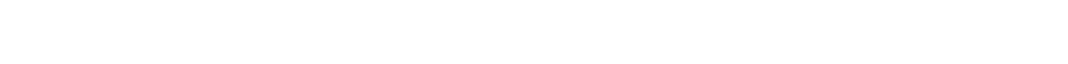

는

$a$

品

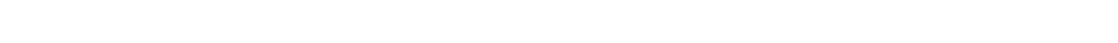

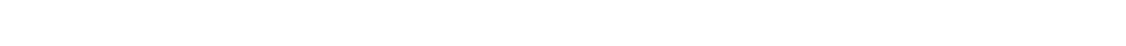

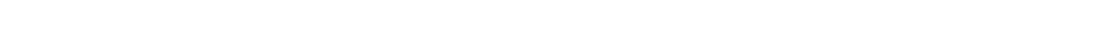

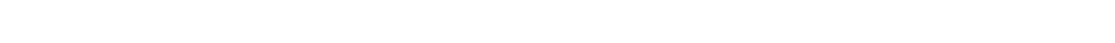

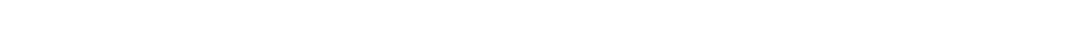

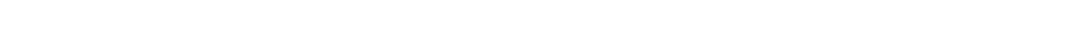

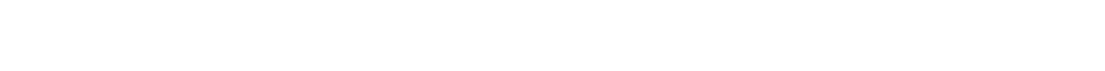

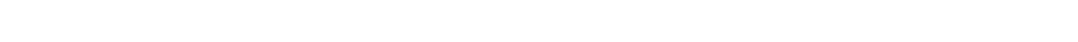

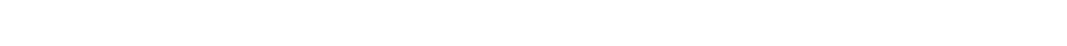

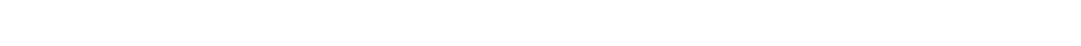

ㄴํํ

in

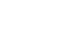

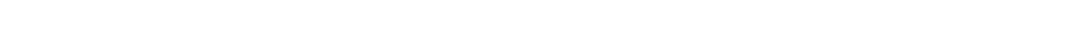

is

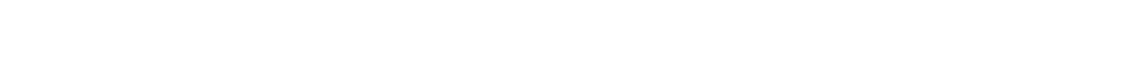
แU

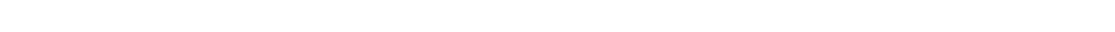
$-\ddot{w}-$ :

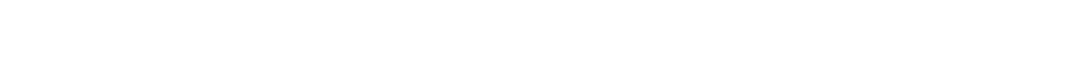

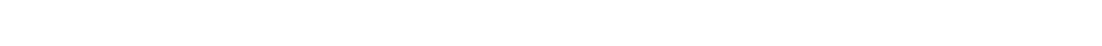
颉은

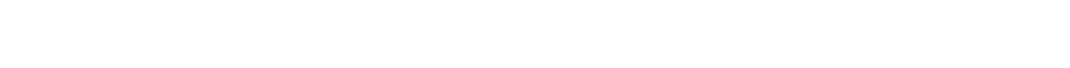




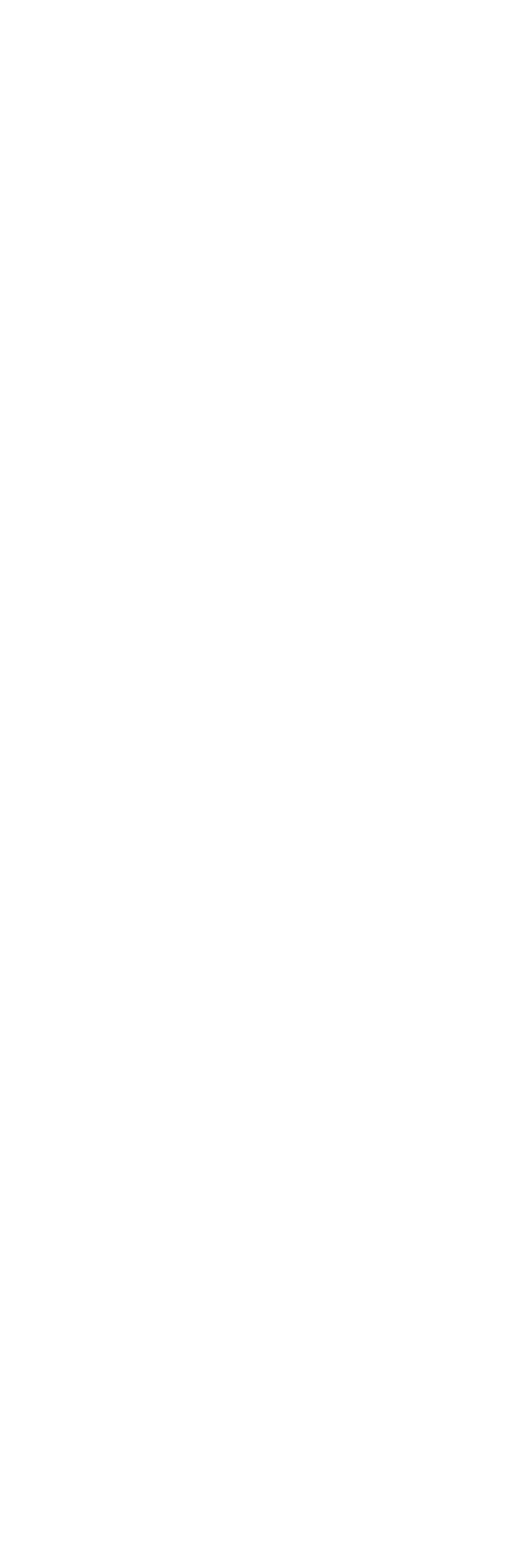

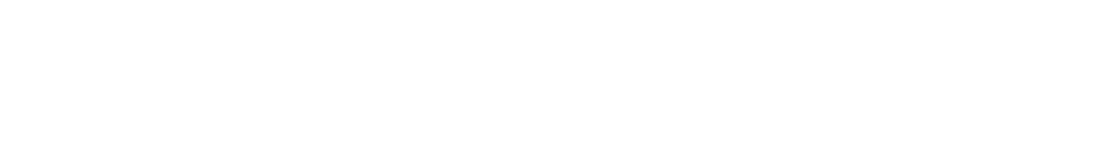

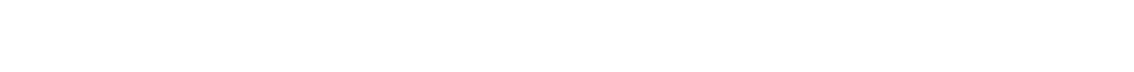

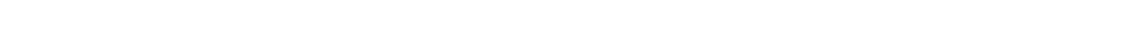
×

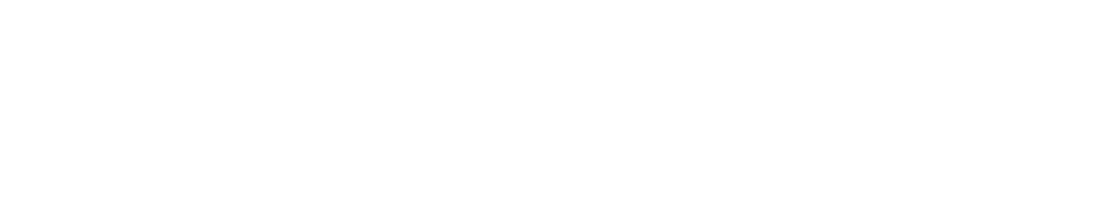

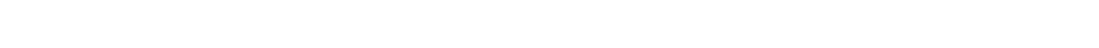

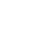

m m w a

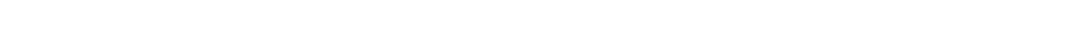

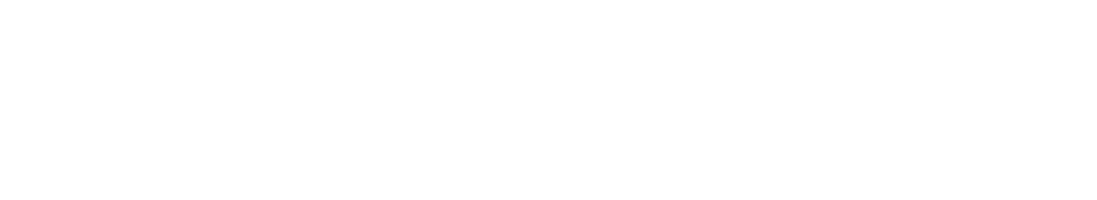

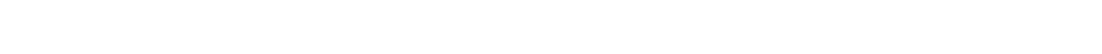
$\stackrel{2}{i}$

"

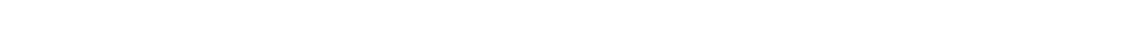
a

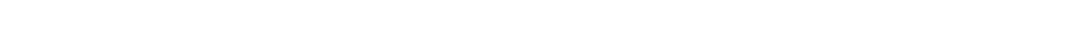

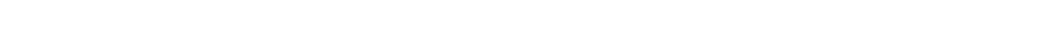

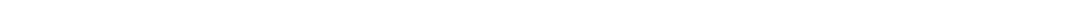
แ-

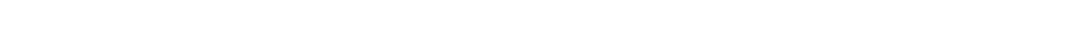

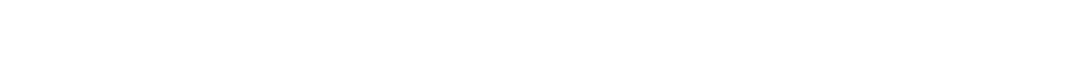

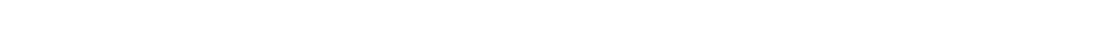
5

$\stackrel{a}{\leftarrow}$

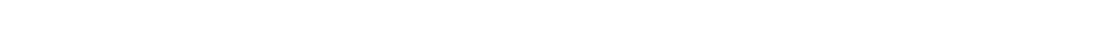

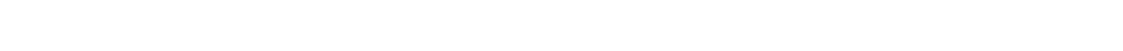
ব ×

mmmmmmmmmmmm on แ

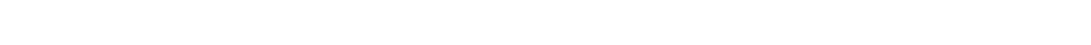

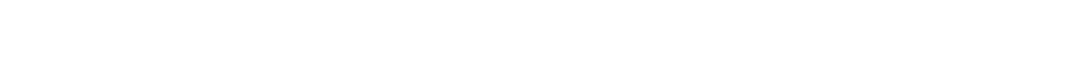




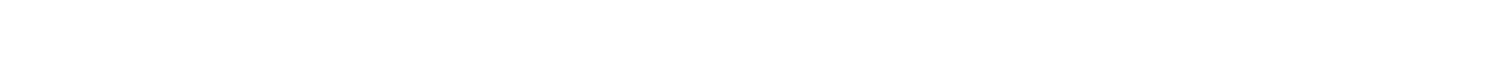

in

"

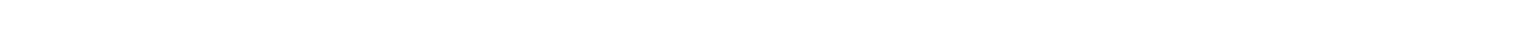

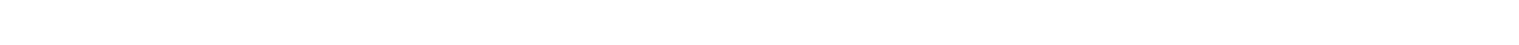
a

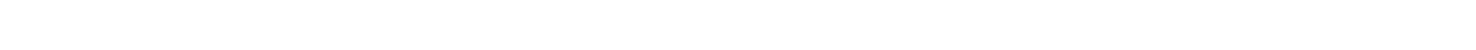

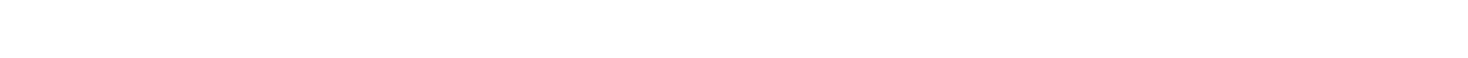

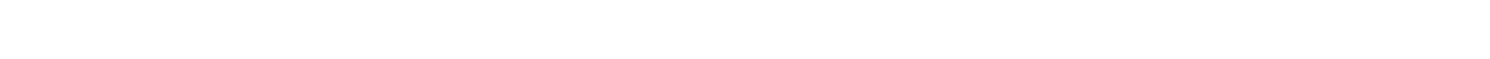

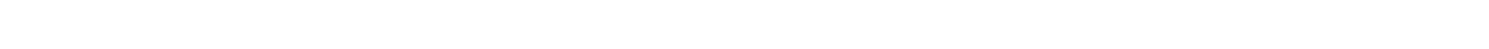

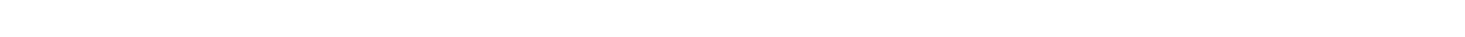

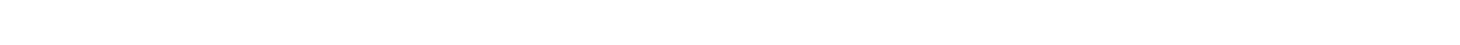

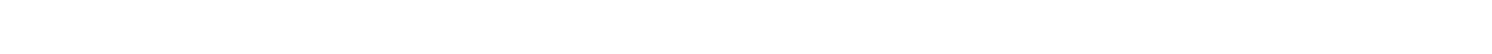

$\stackrel{5}{i}$

0

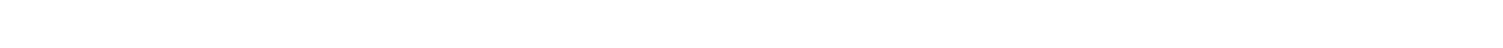
u w $\dot{\omega} \dot{0} \dot{0} \dot{0} \dot{0} \dot{0} \dot{0} \dot{0} \dot{0} \dot{0} \dot{0} \dot{0} \dot{0} \dot{0} \dot{0} \dot{0} \dot{0} \dot{0}$

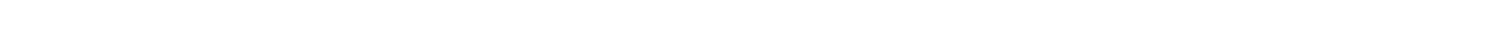
- ש

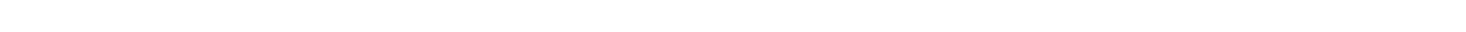

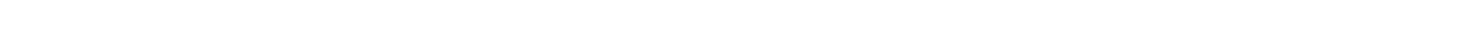

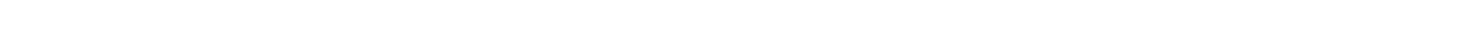
W

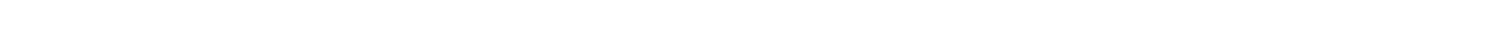

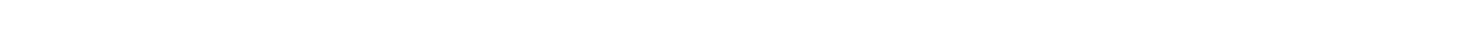

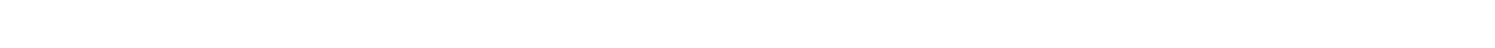

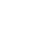

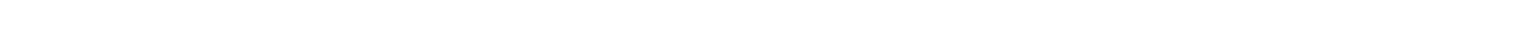

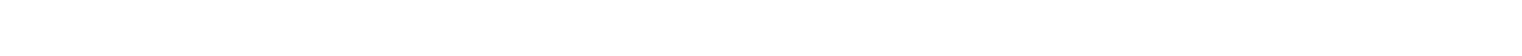
ㅆ

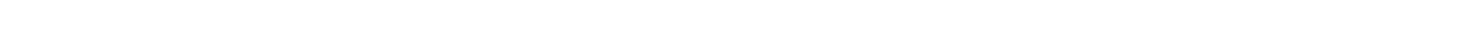

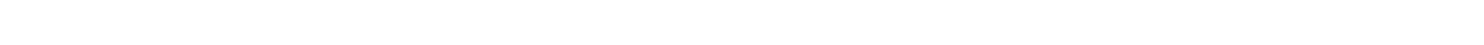

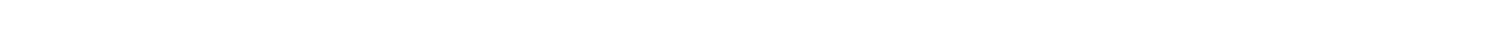

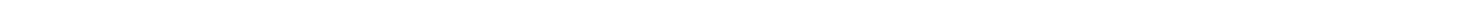

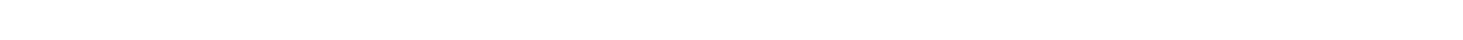
n-

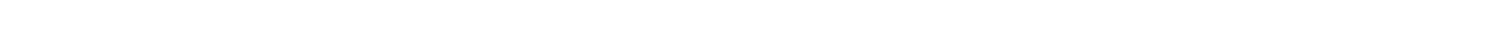
$x$

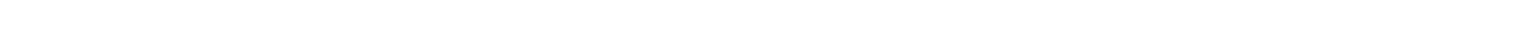

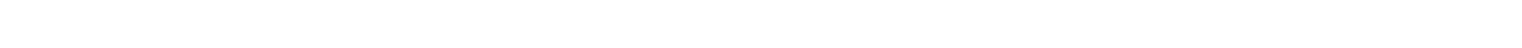
I

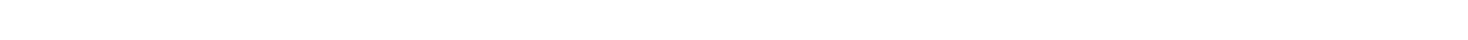

mmmmmmmmmmmmmmmanm

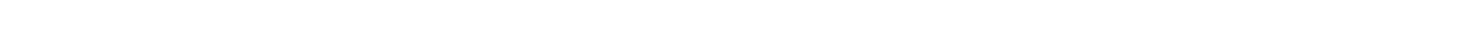

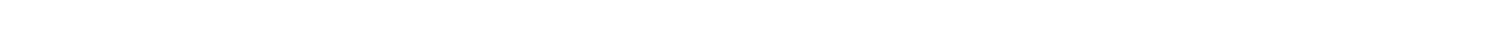

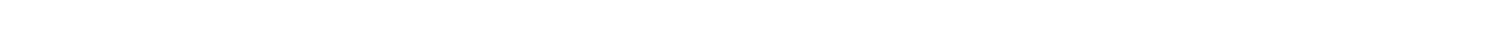

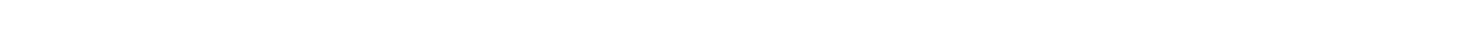

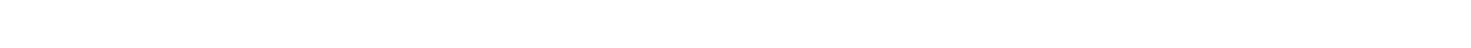




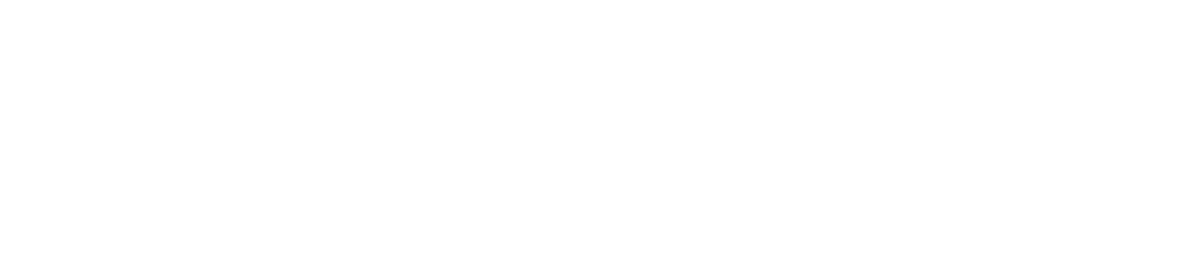

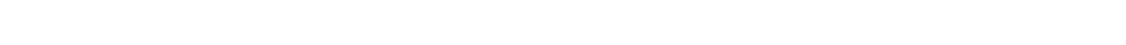

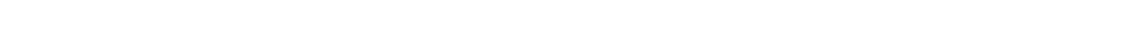

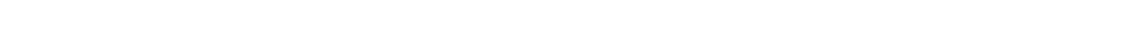

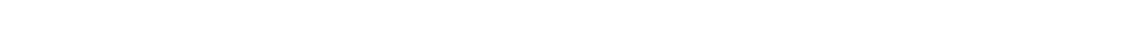

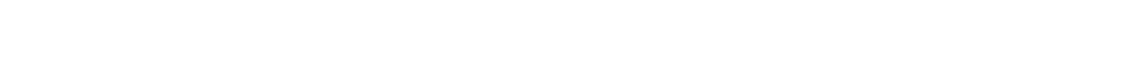

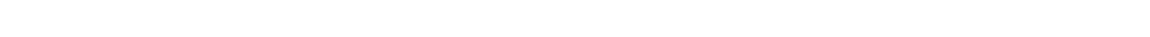
$\Sigma$

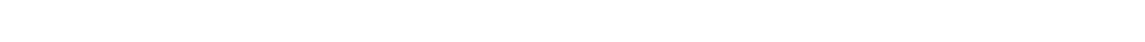

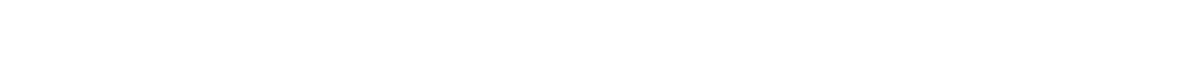

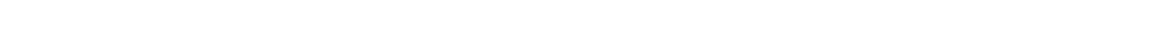

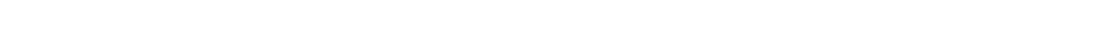

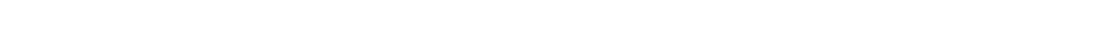

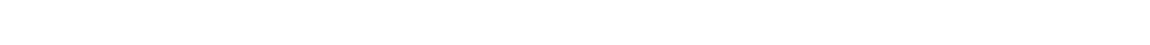

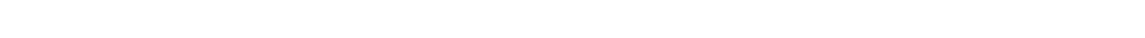

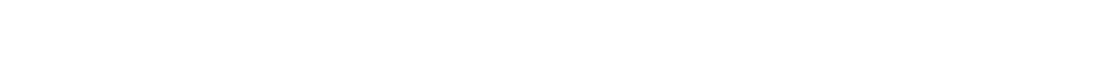

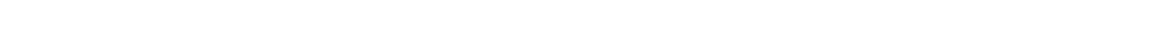
a

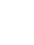

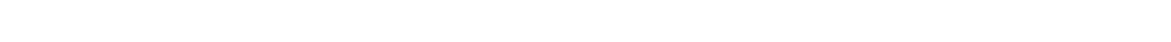

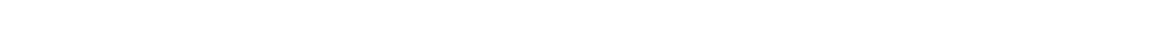

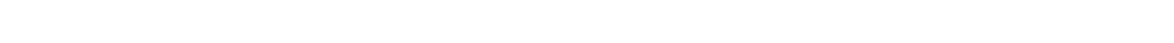

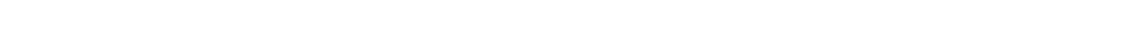

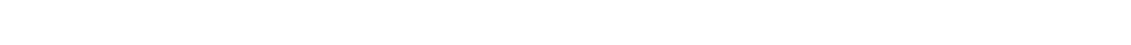

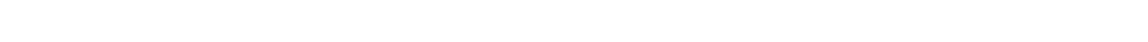

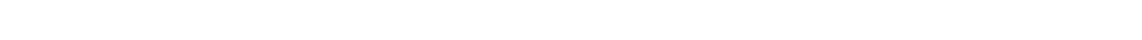

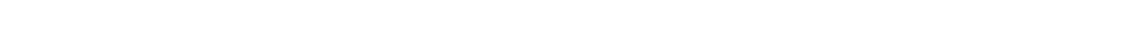

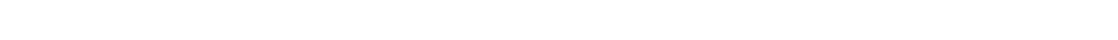

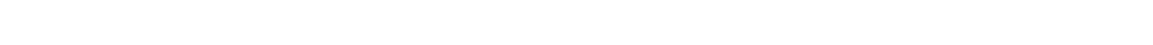
采

in

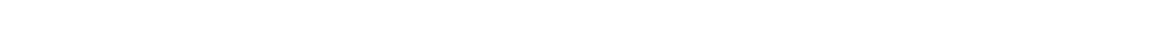

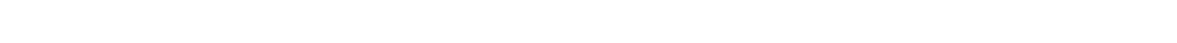
व

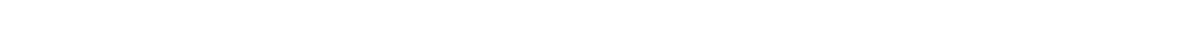

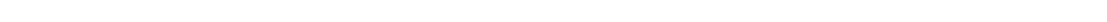

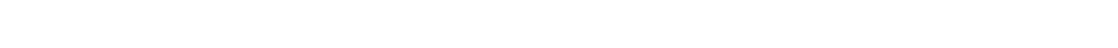

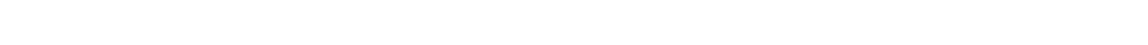

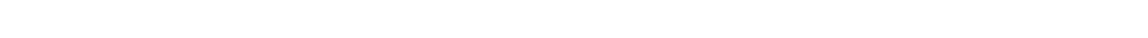

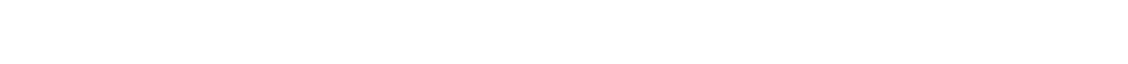




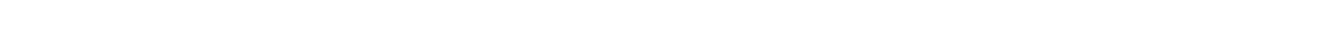

in

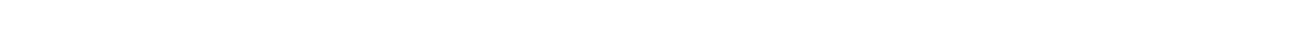

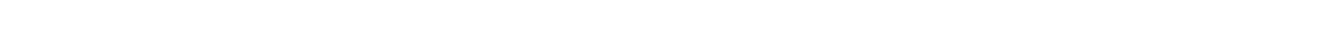
-

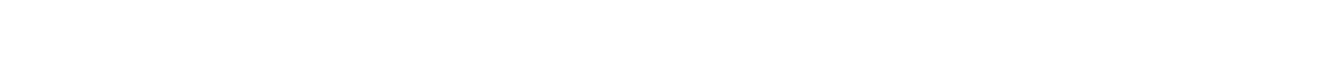

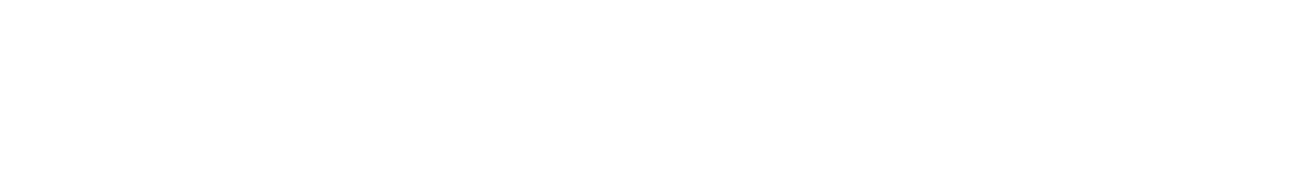

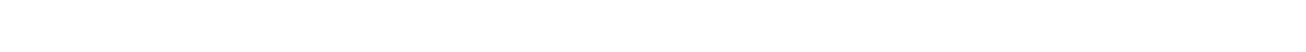

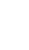

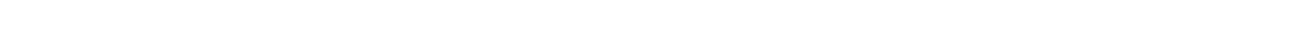

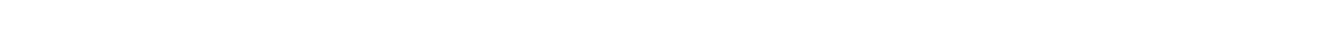
ᄂ

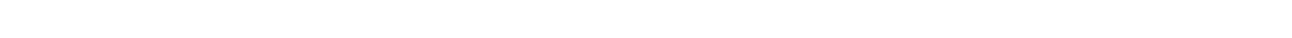

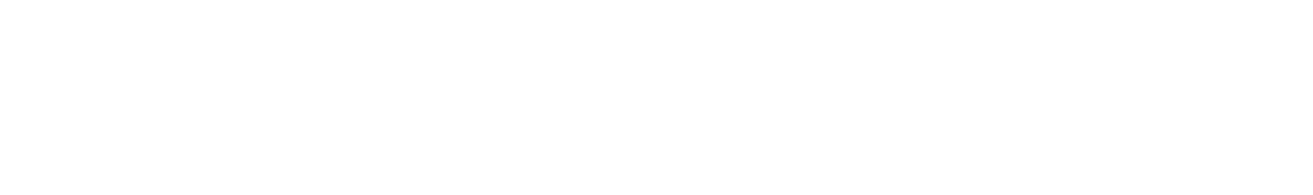

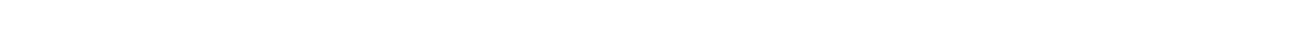

is

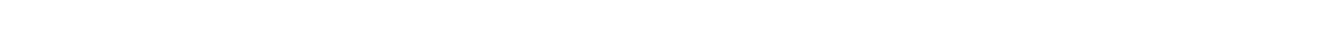
w u

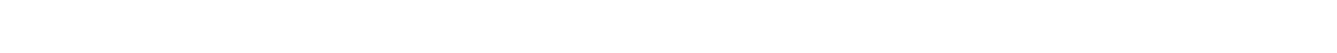

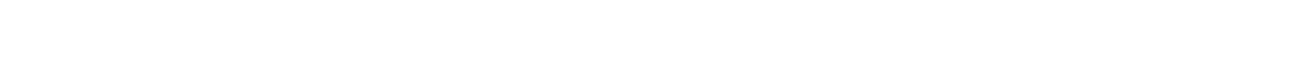

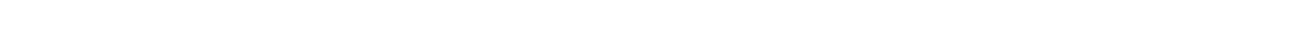

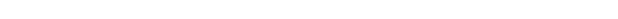

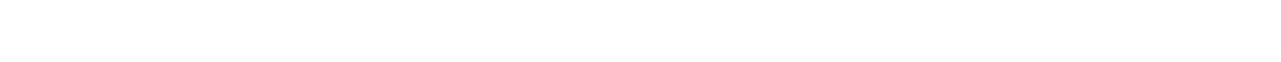

$\leftarrow$

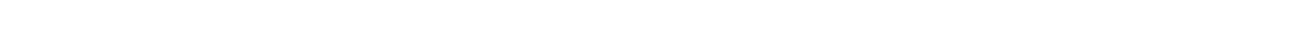

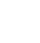

is

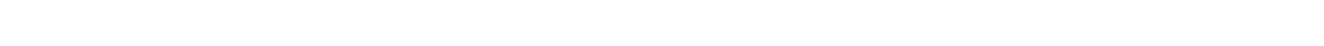
แ w

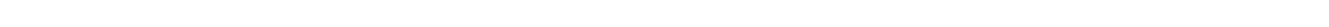

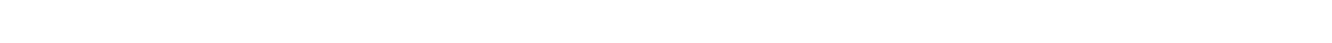

MmmMmmmmmmmmmmmmmmmmmmmm

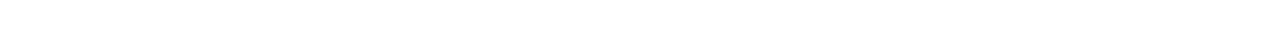

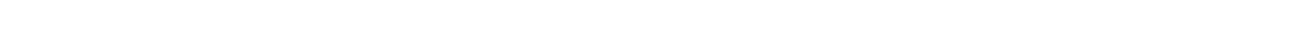

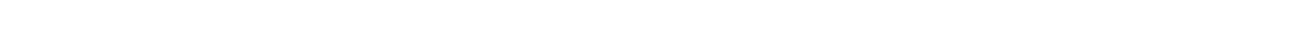

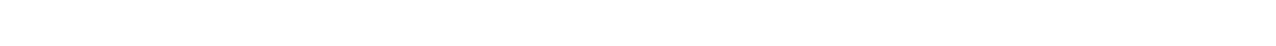

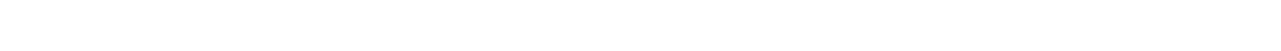




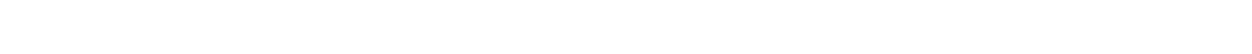

is

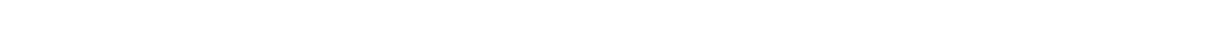

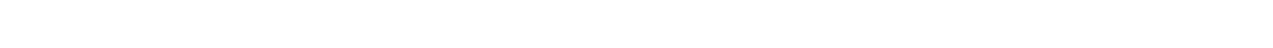

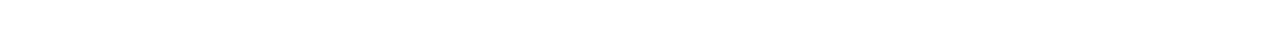

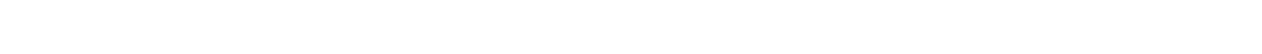

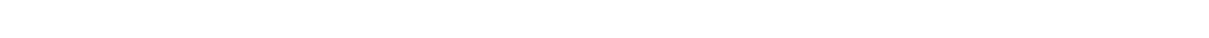

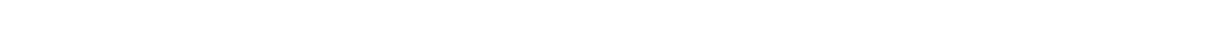

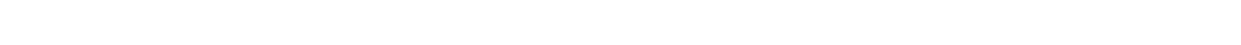

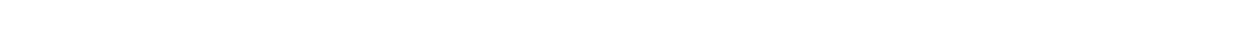

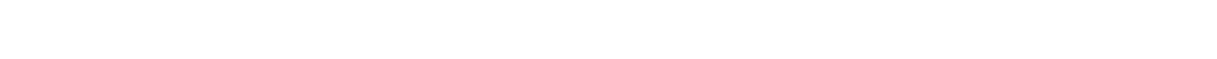

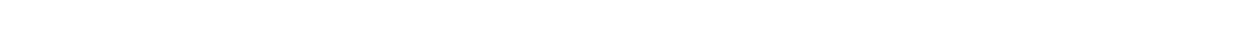
is

momm-0凸m-

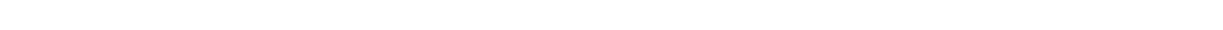

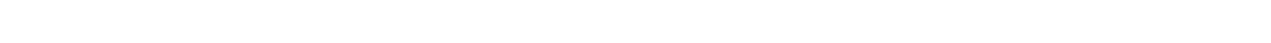

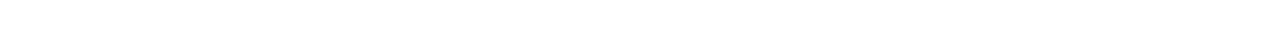

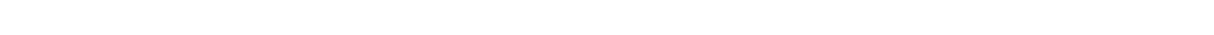

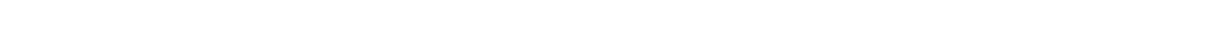

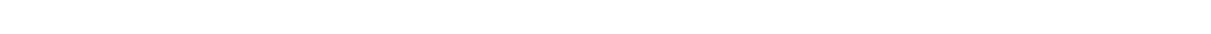

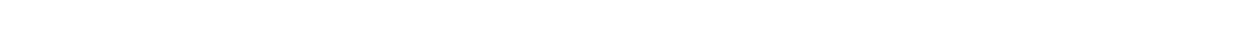

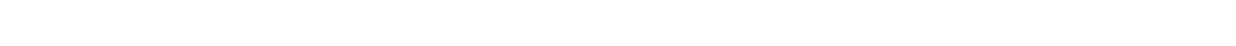

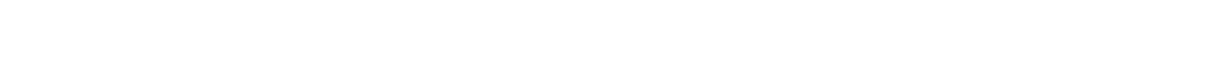

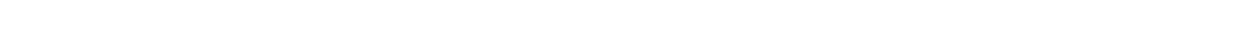
is

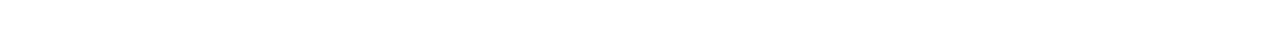

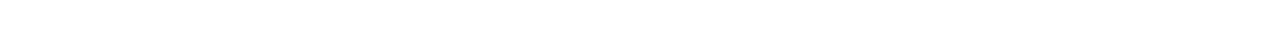

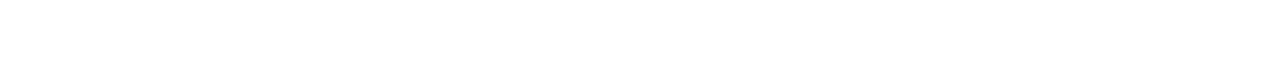

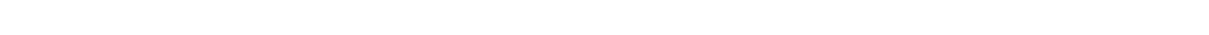

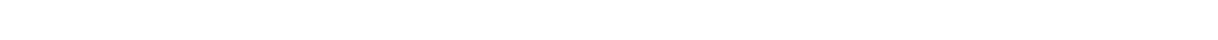

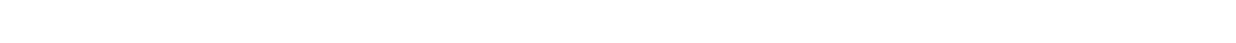

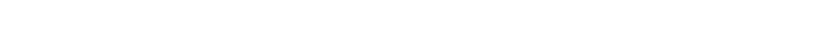

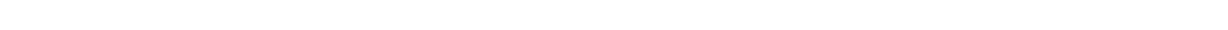
mós

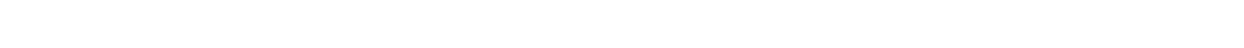

is

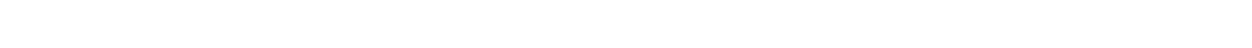

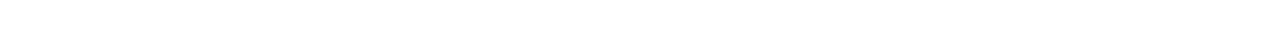

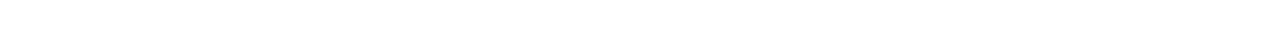

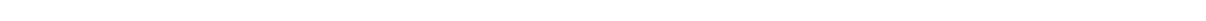

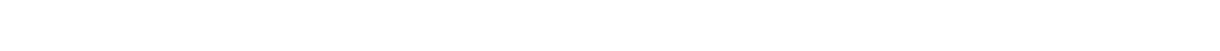

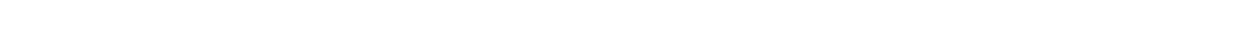
a d o in iñ

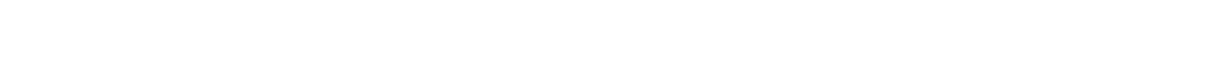




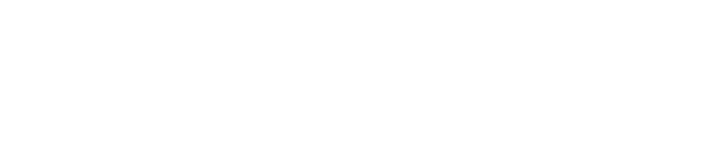

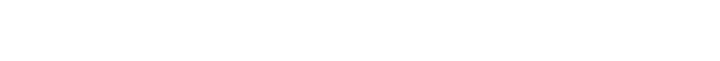

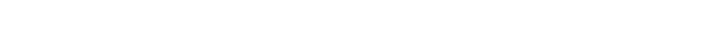
ב o

\section{(1)}

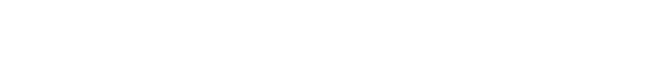

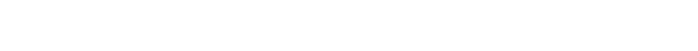

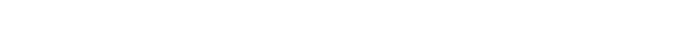

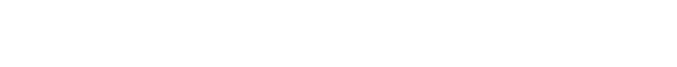

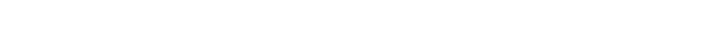

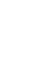

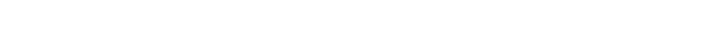

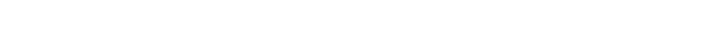

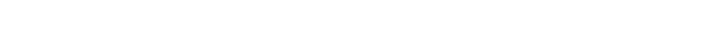
10000000000005000000006

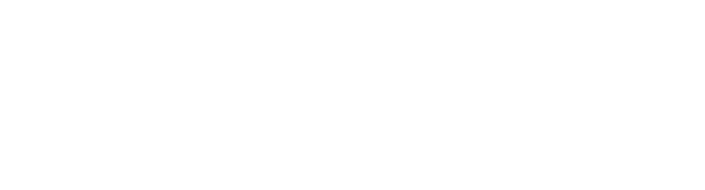

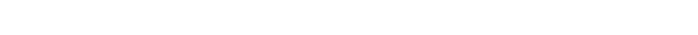

$\stackrel{a}{n}$

" a

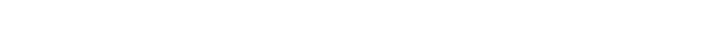

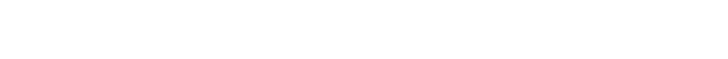

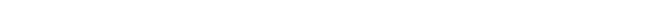

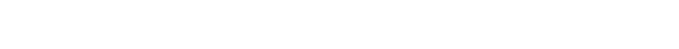

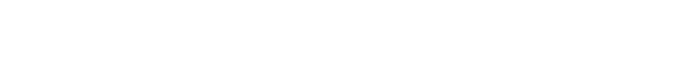

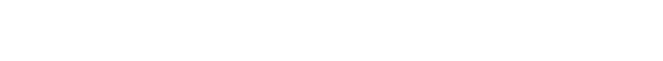

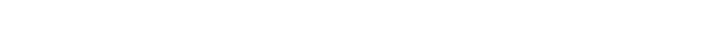
문

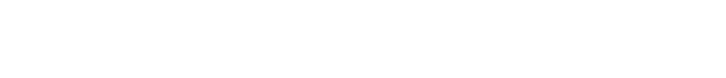

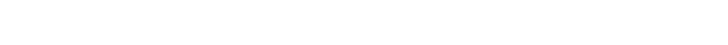

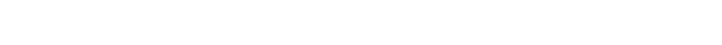

MMMMMMMMMNMMMMMMMMmMm .

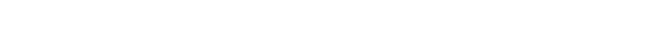

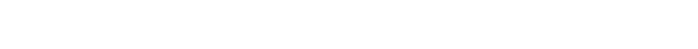

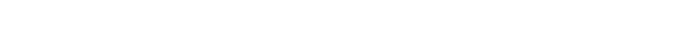

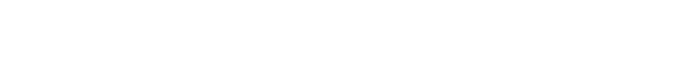



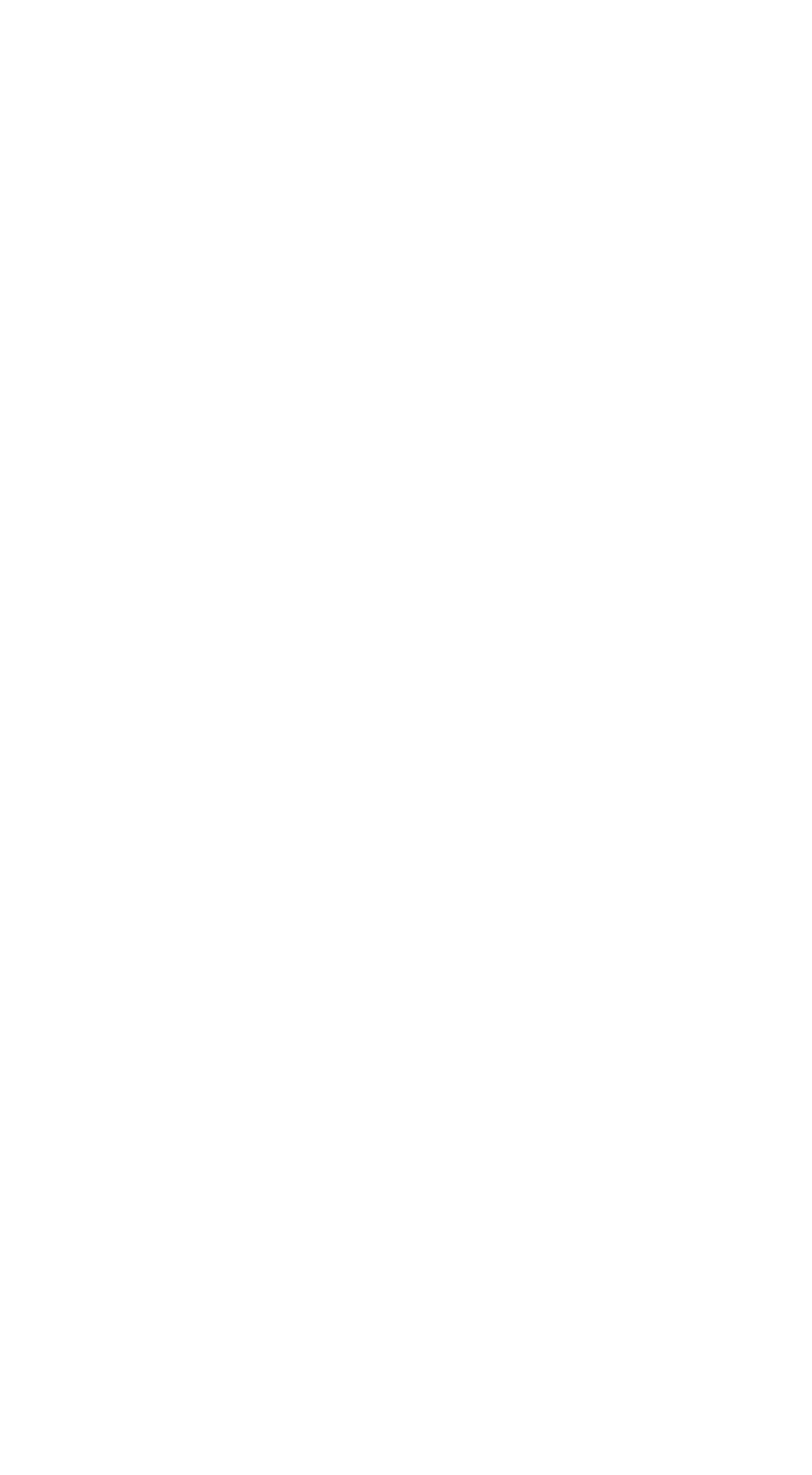

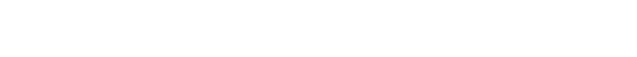
w $\because \dot{0} \dot{0} \dot{0} \dot{0} \dot{0} \dot{0} \dot{0}$

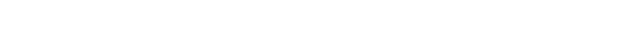

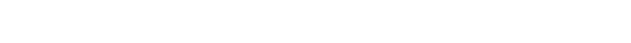

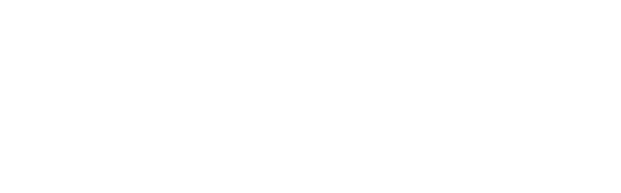

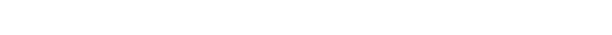

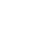

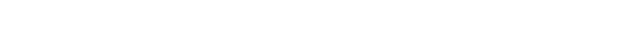

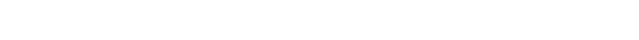

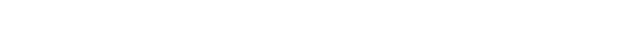
แ

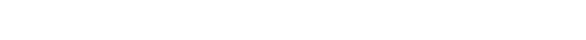

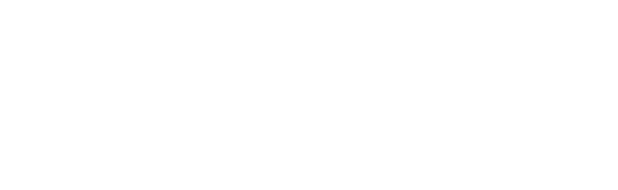

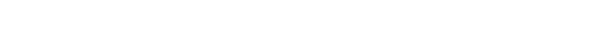
党 is mก⿻ำ $\ddot{\omega} \dot{0} \dot{0} \dot{0} \dot{0} \dot{0} \dot{0} \dot{0} \dot{0} \dot{0} \dot{0} \dot{0}$

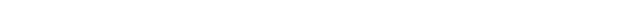

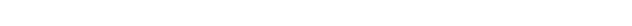
ข

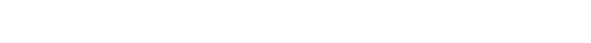

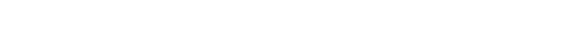

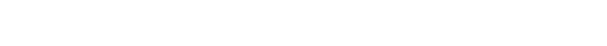

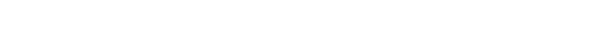

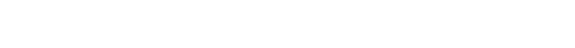




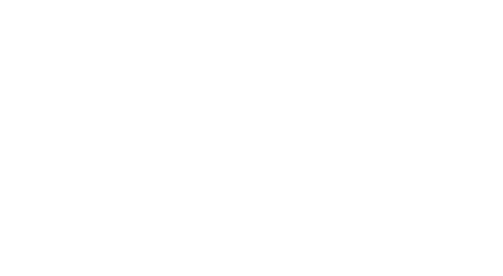

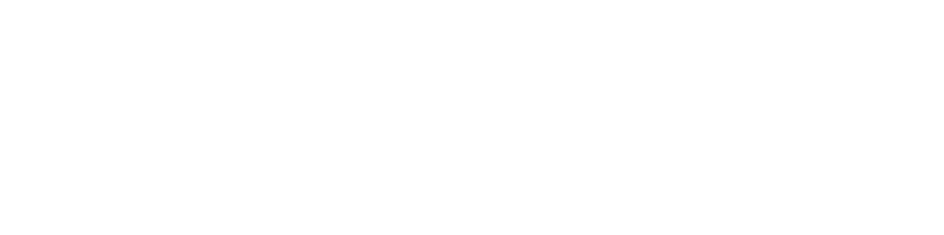

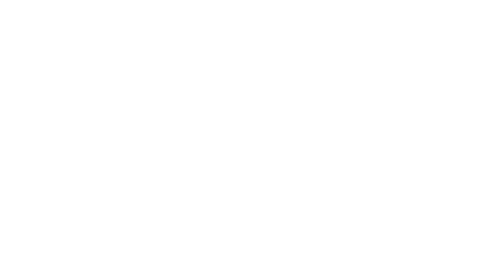

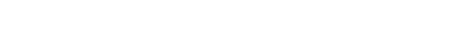

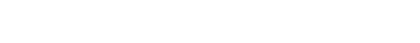

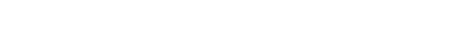

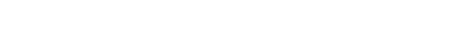

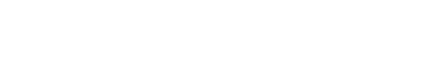
造出 志至 in

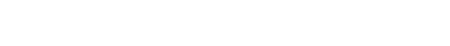

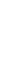

음ำ

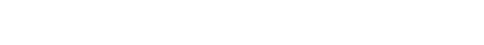

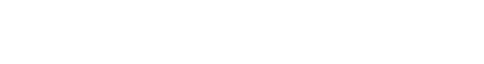

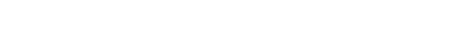

은

$$
\div
$$$$
\text { - }
$$

ï iii

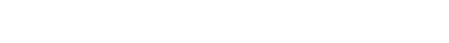

in

जmmn น

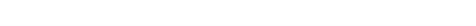

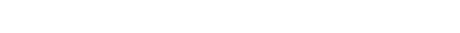

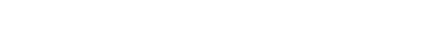

mmmmmmmmmmmmmm

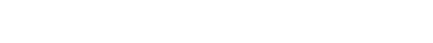

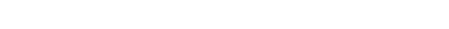

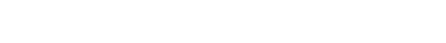

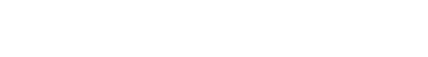



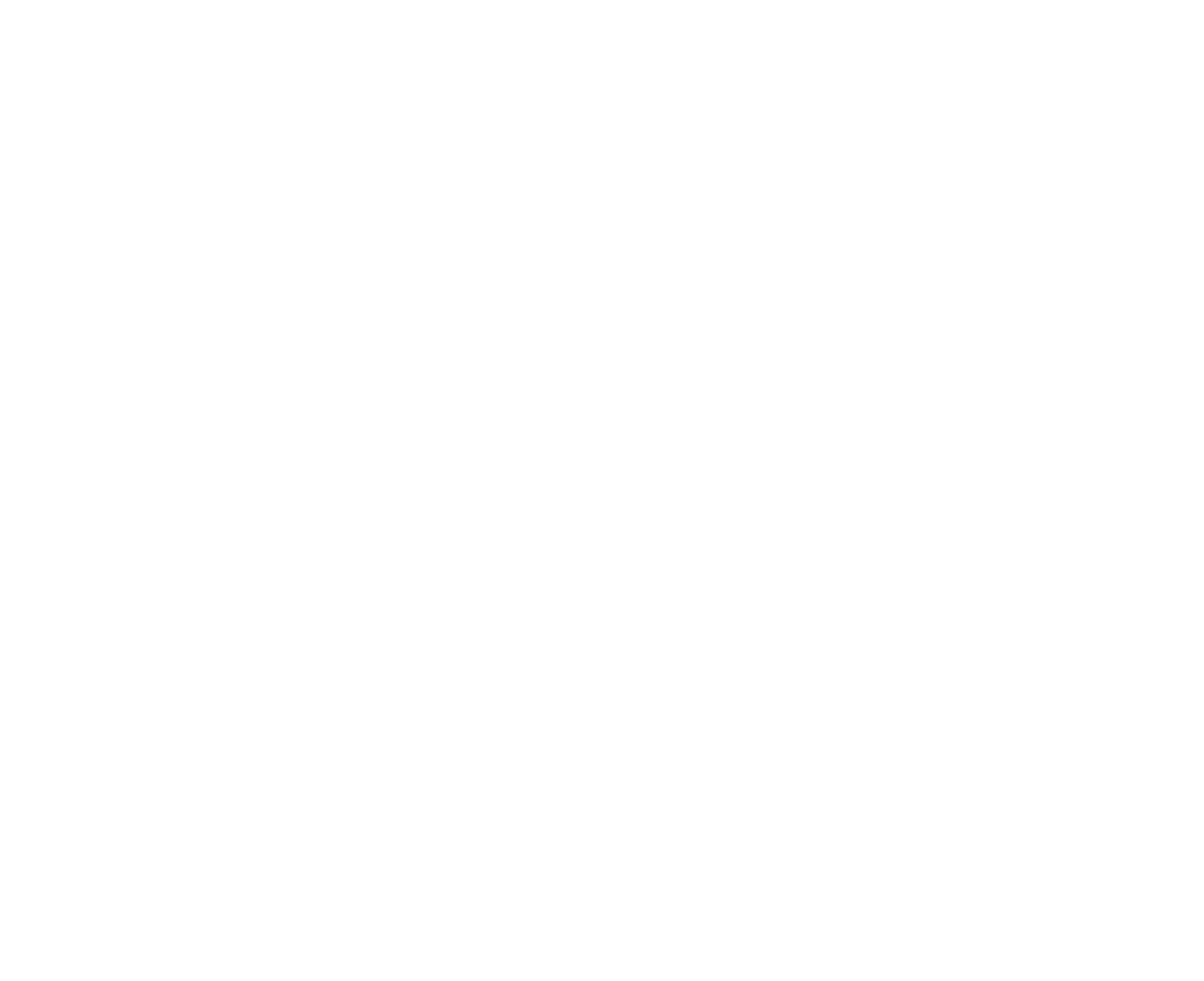

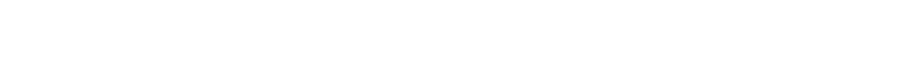

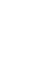

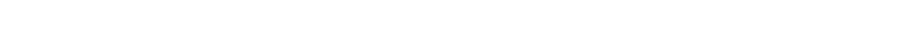

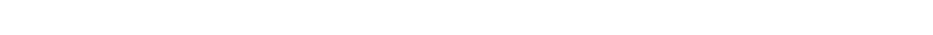

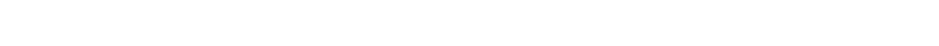

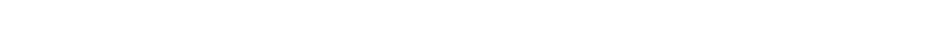

in in

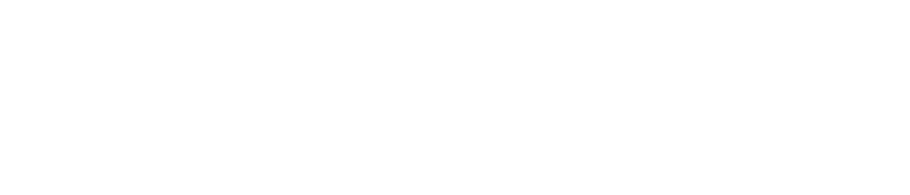

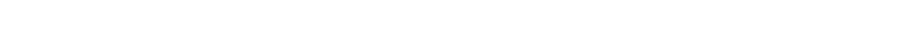

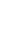

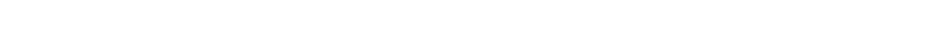

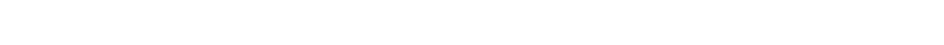

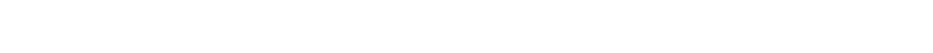

$\cong \sum_{0}^{\infty}$ ก

$\ddot{u} \quad \ddot{r} \leftarrow \ddot{m}$

¿

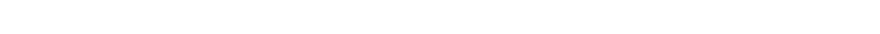
nin in

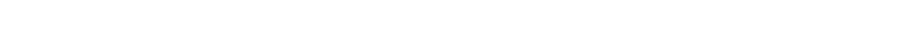
自-

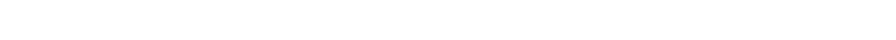

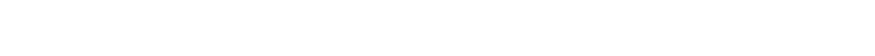
-

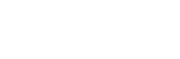

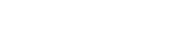
$\div \because \frac{1}{2}=-5$

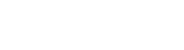

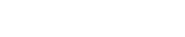
t大ancona

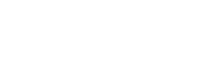
$2+\frac{a}{a}$

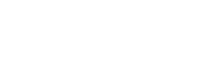

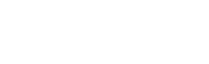
更 5

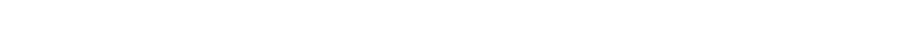

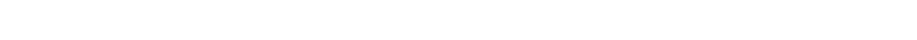
ot

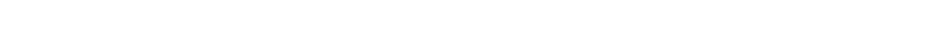

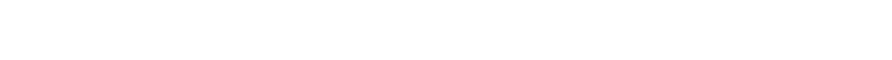

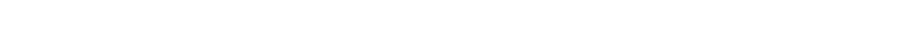

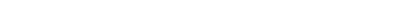

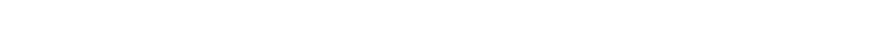




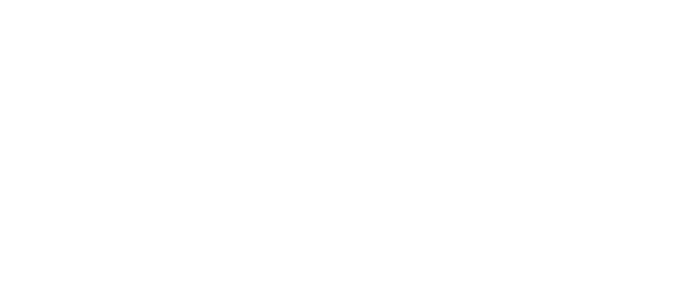

wi

范茫

เD心

w

는

3 인?

zo

$0=\frac{5}{2}$

50

$>_{n \rightarrow \infty} \infty$

und 2

a)

인 (.)

궁

뜨능

정은

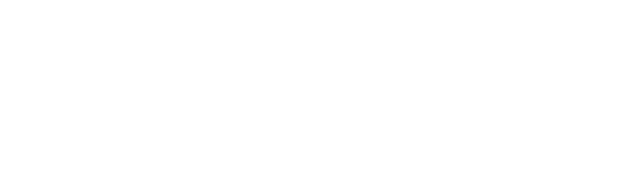

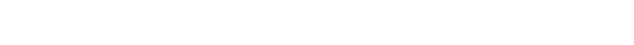

$\stackrel{2}{5}$

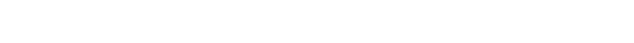

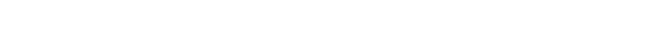

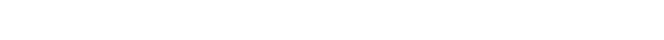

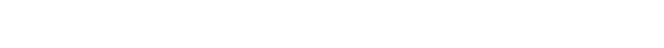

$\stackrel{\circ}{i}$

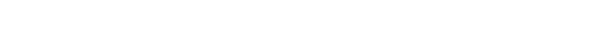

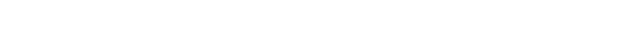

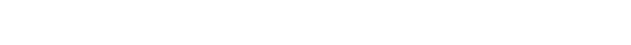

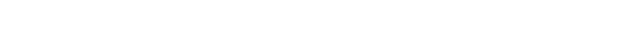

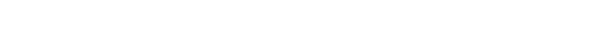

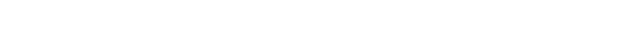

is

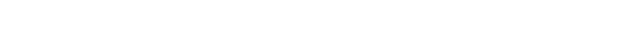

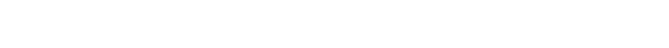

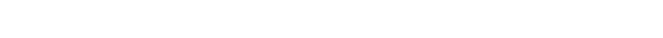

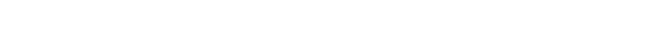

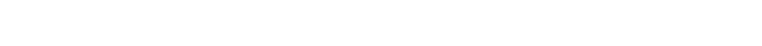

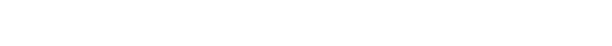

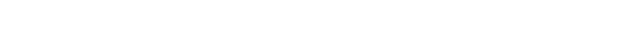

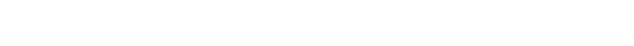

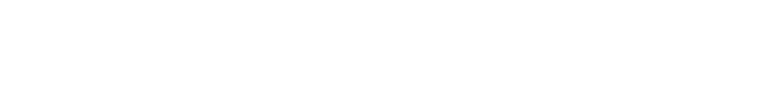

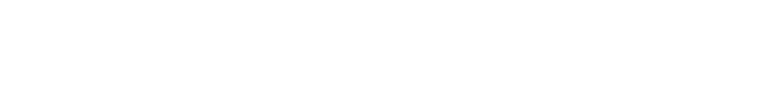
s

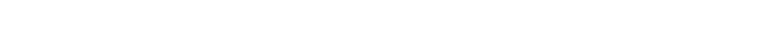
$\ln _{\substack{n \\ \infty}}^{2}$ का

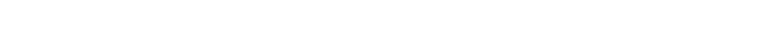
ma a ㄴ $=\ddot{\ddot{w}-}$

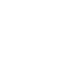
3
3
0

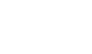

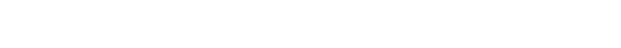

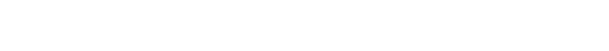

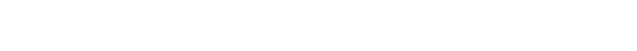

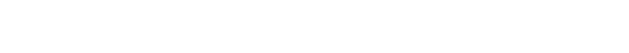

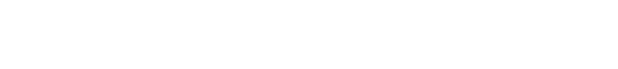


in

ผ

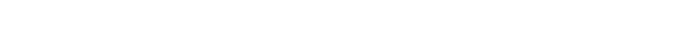

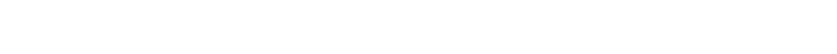

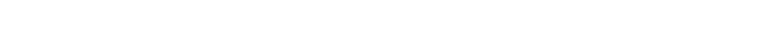

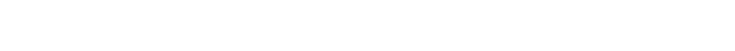

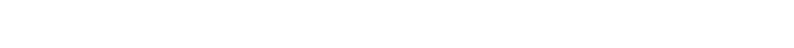

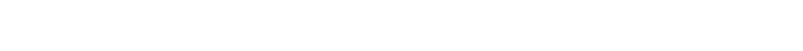

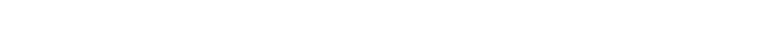

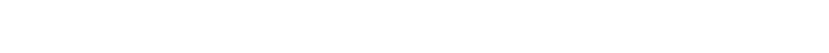
is

in oO

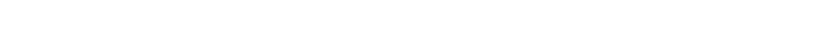

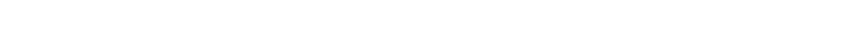

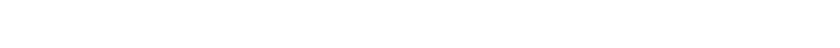

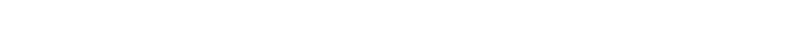

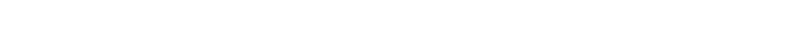

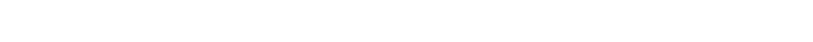

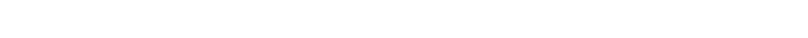

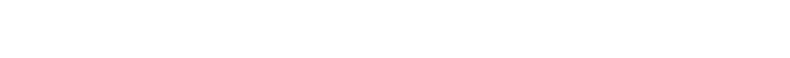

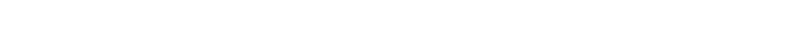

a

$\stackrel{a}{2}=$

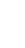

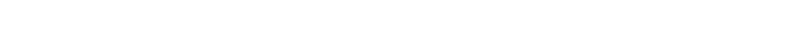
w เ

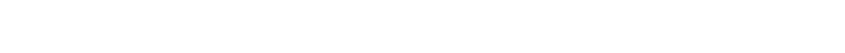

is

$\sum \pm z$

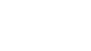$$
\text { (a) }
$$

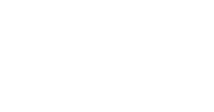

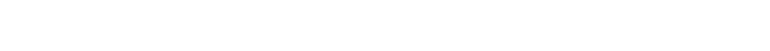

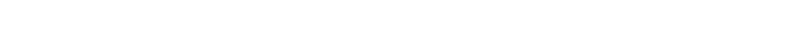

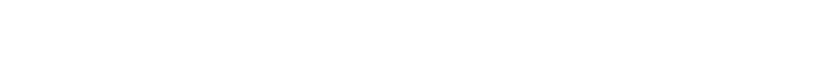

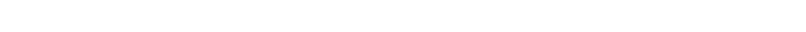

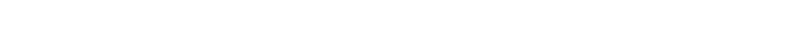

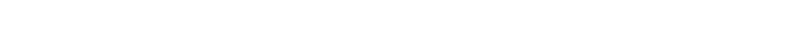
$\stackrel{\alpha}{\circ}$

กัน

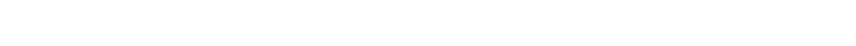

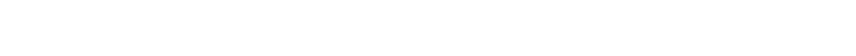

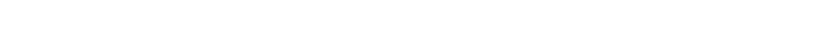

แnแun

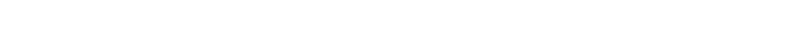

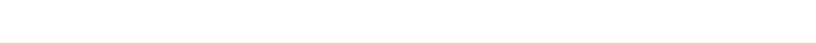

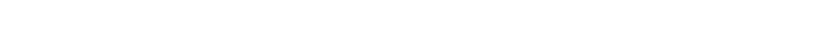

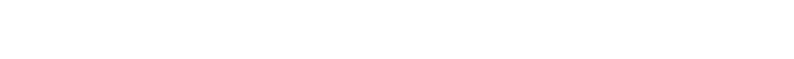




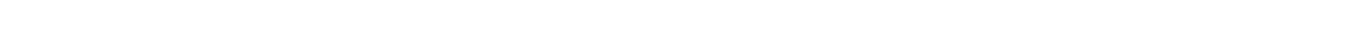

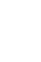

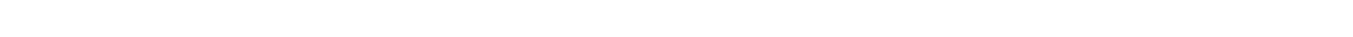

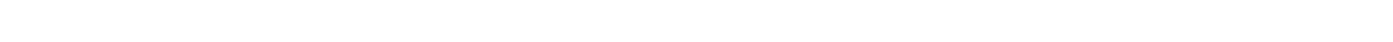
"

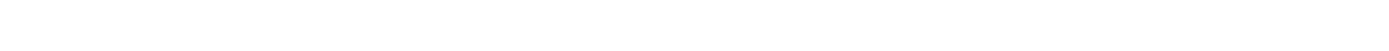

$\ddot{c}$

$\stackrel{a}{0}$

un

n 둔응 in a $x^{2}$ as

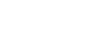
$-\frac{a}{a}$ รัด

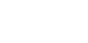
noㅡ 둔 총ㅇ nnin $\sum$ 능 ㅂov -ox $\because ニ 5$

รニ ㄴำ un 뜽응 $>$ ? aaพับ $y$ 的 $\frac{1}{z} \alpha$ inazง 10 ฐฺ: un is wis

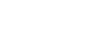
$5+\frac{\alpha}{0}$ 的之 등 운 zos 안 포을 出等 वथ แ山 少 = 䙲: 엑. s.s

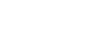
。

21 它员

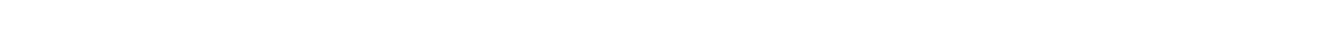

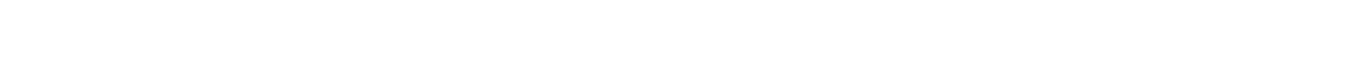

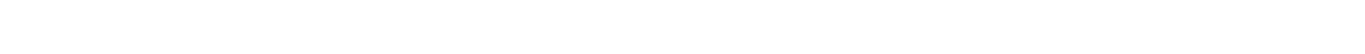

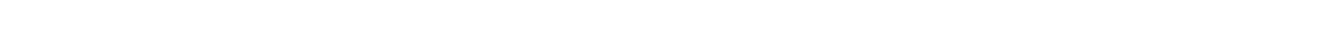

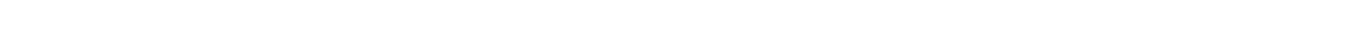
$a$

is

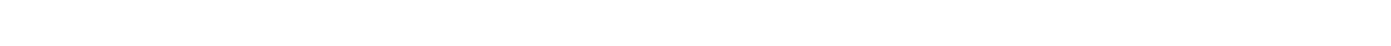

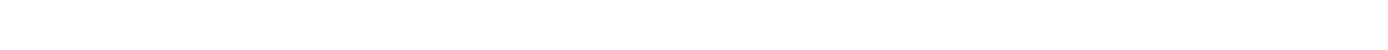

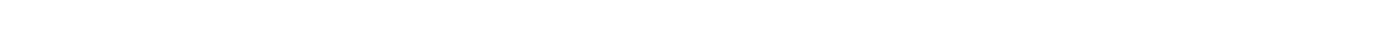

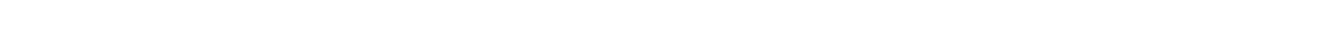

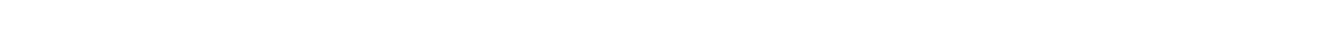

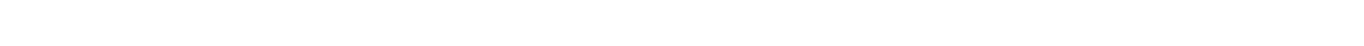

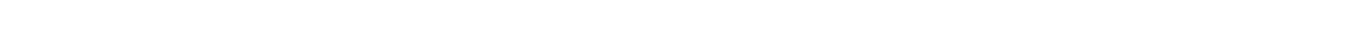

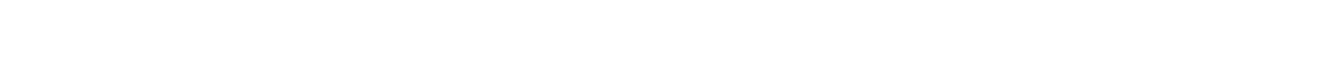

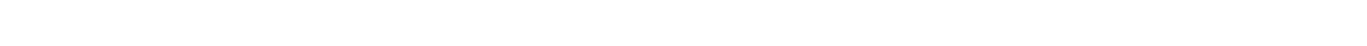

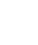

ำ w山́ 《

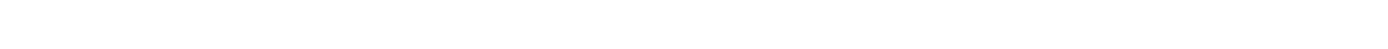

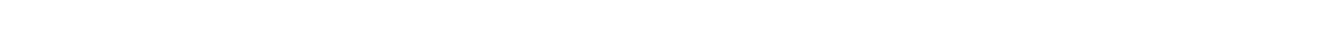

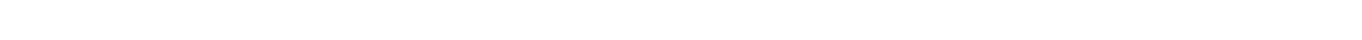

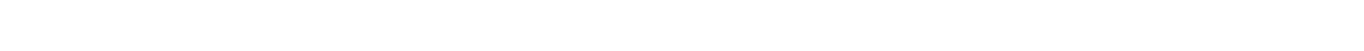

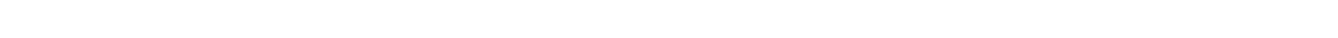

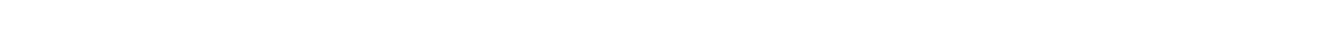

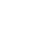

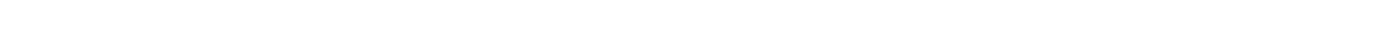

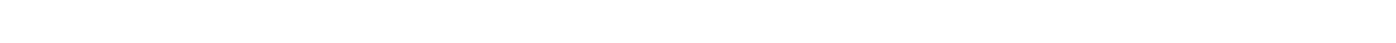
4 11

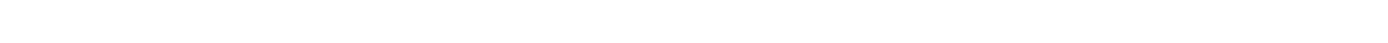

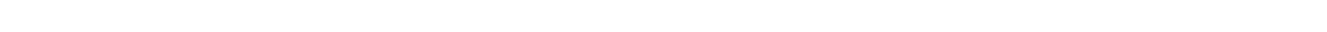

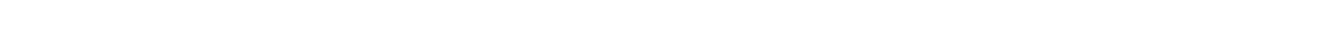

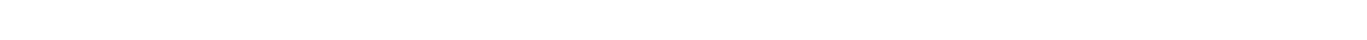

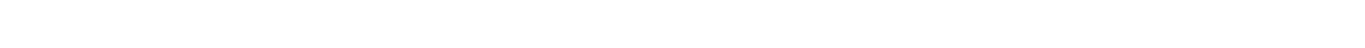

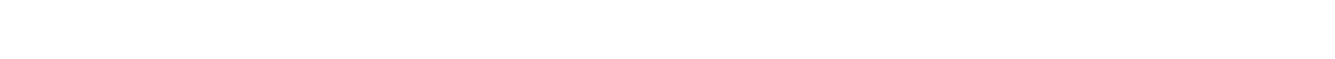



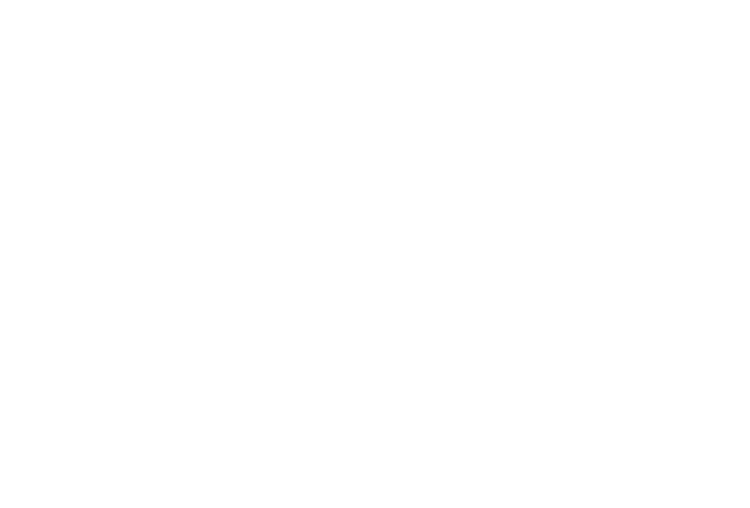

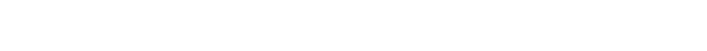

$a$

in

๔

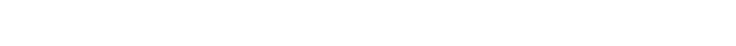

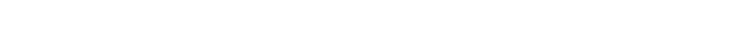

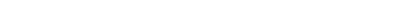

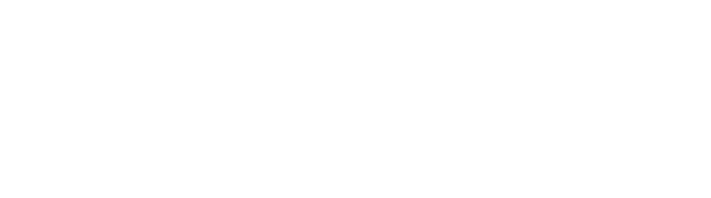

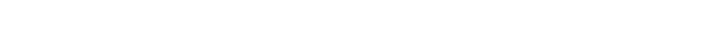
in

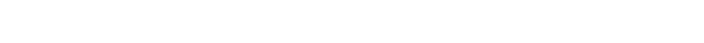

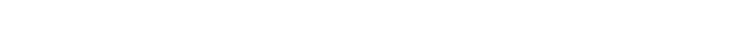

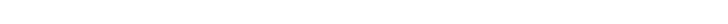

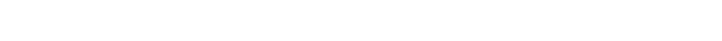

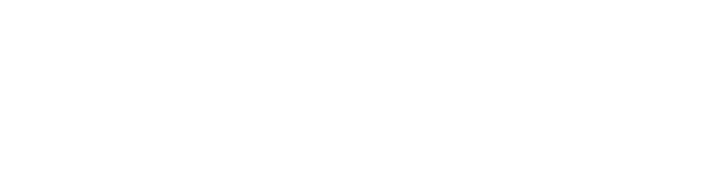

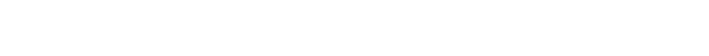

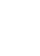

a

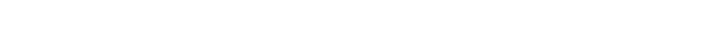

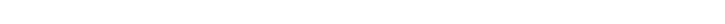

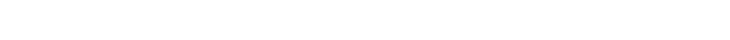

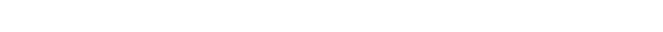

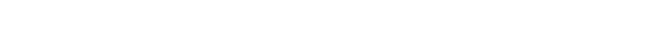

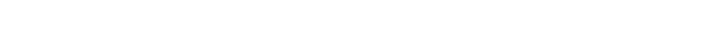

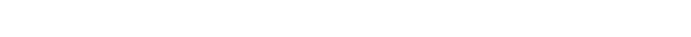

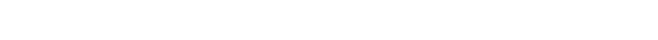



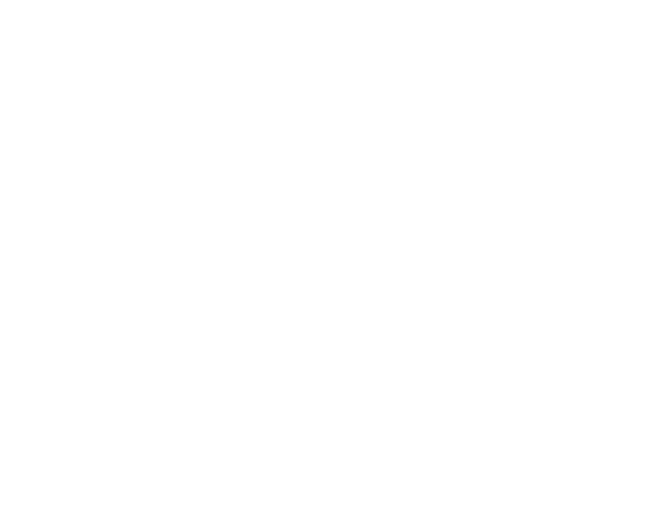

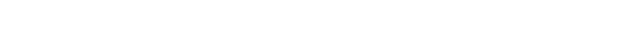
ז

๔ ш

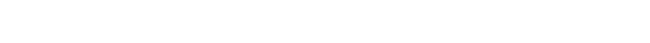

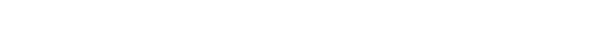

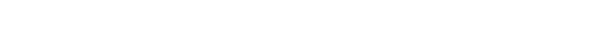

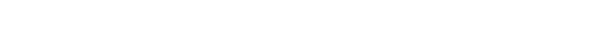

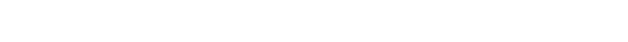

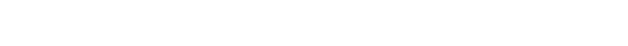

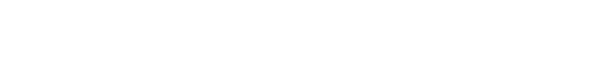

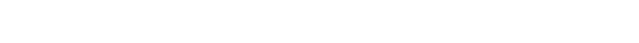
$\Sigma$

in $z$

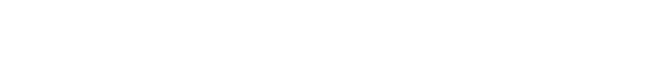

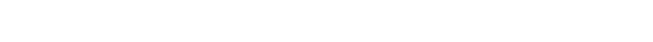
यद

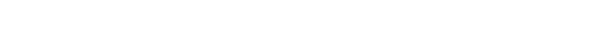

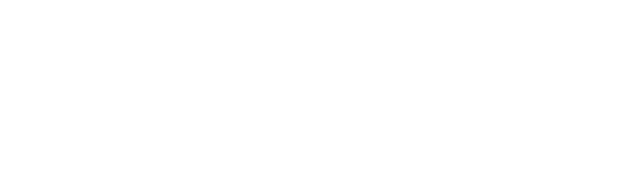

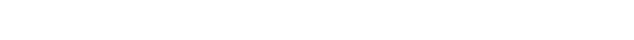

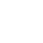

$\leq$

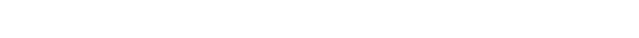

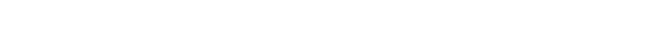

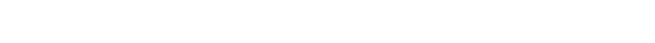

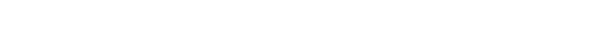

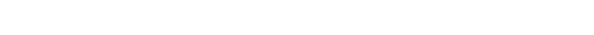

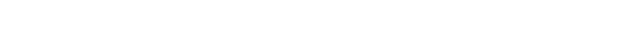

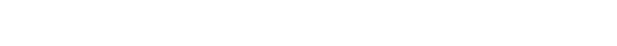

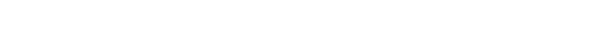




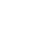

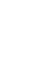
is

잉

$\leftarrow \div u$

a

事

mo

"

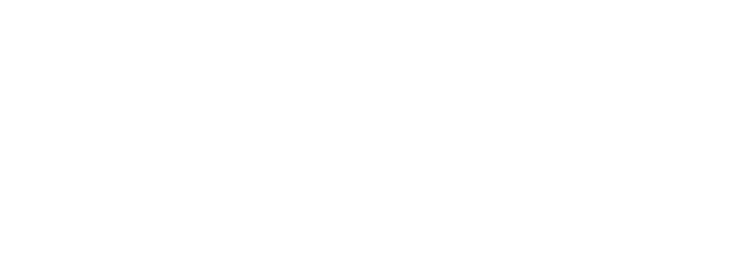

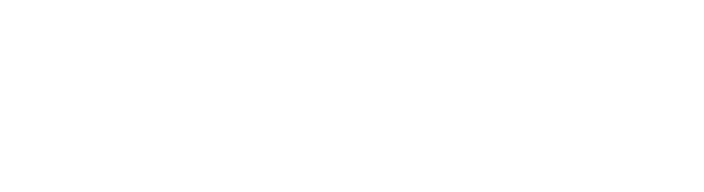

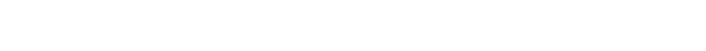
\&

m

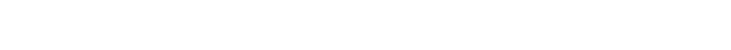

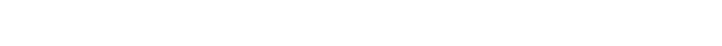

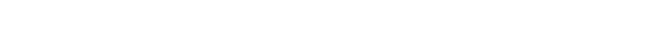
幺ำ

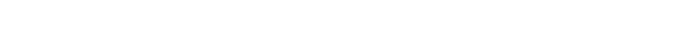

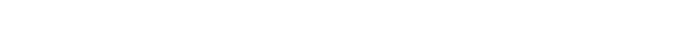
O- - OUN NNO

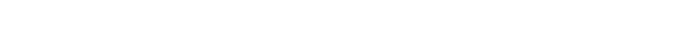

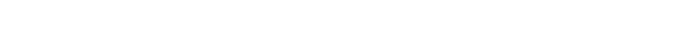
is

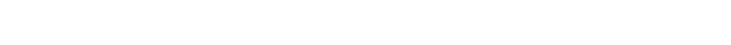

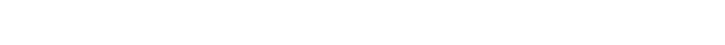

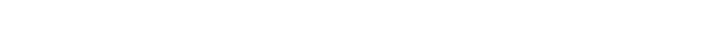

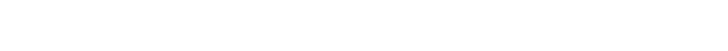

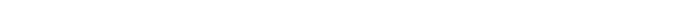
Wn

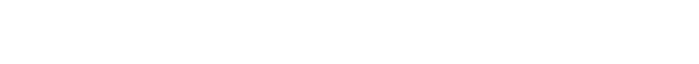

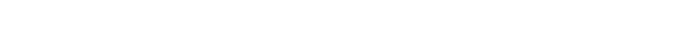

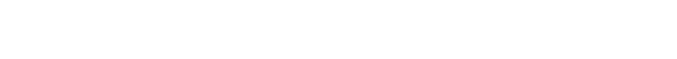

点点

$د \dot{r} \sim m a$

$\min _{x \rightarrow 2}^{2} \frac{a}{2}$

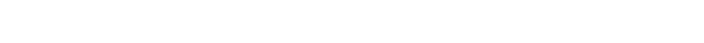

ह

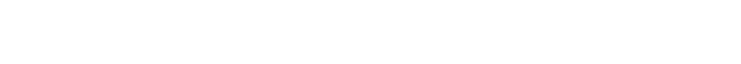
"

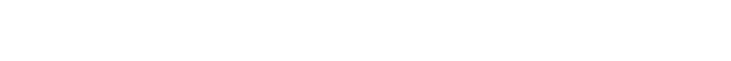

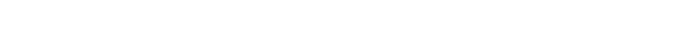

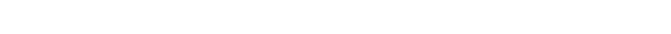

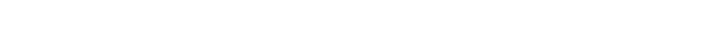

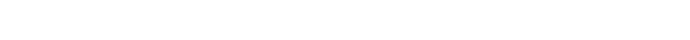

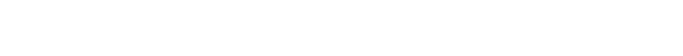




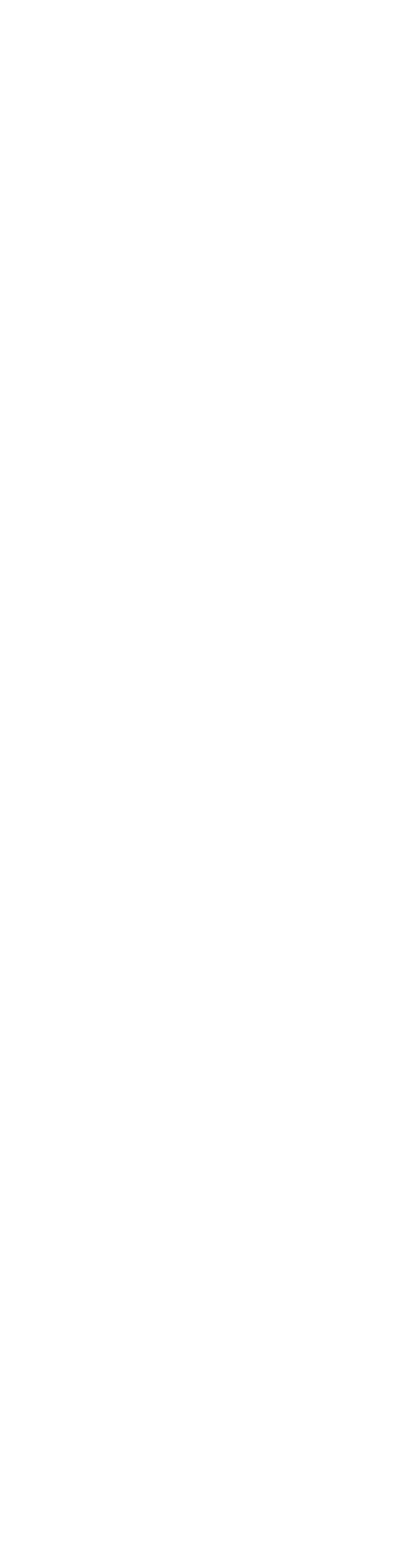

$\ddot{\sim}$

3

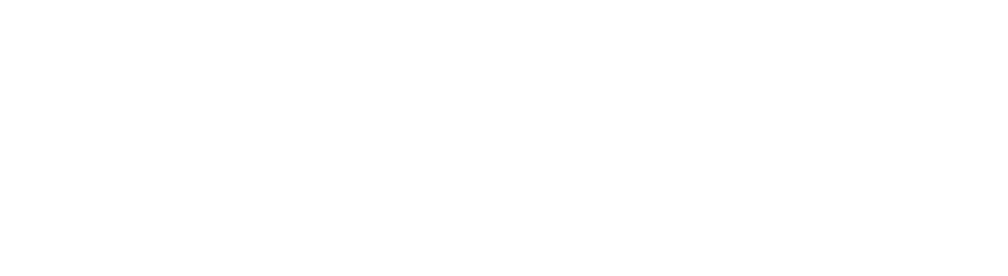

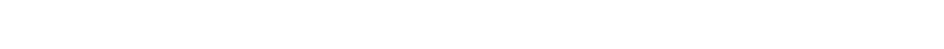

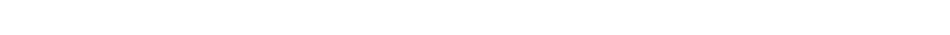

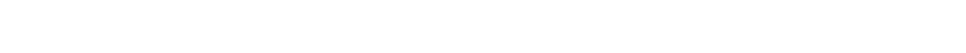

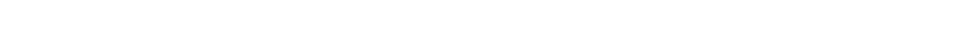

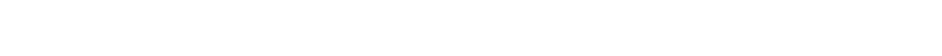

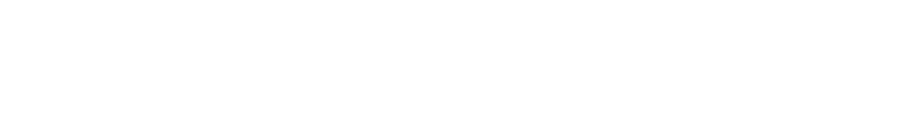

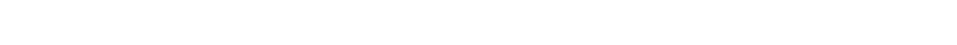
世 a wmmmmmmmmmmmmmmmmm

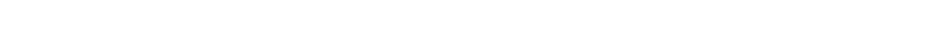

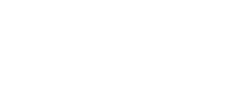

状

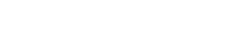

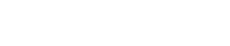

省

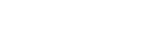

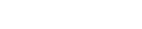

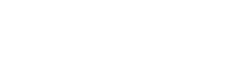

证品= 的

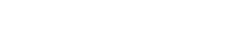

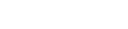

.. 弚.. 王..... I

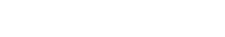

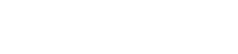

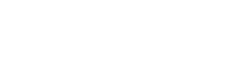
영 a

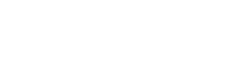
กำ

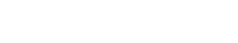

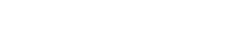
뜨눈

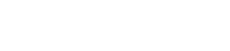
$\ddot{w} \leftarrow \ddot{L}-\ddot{w} \sim \ddot{w}-$ $\sum^{2}$

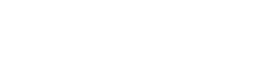

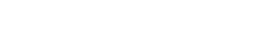

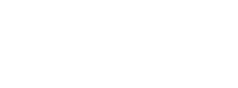

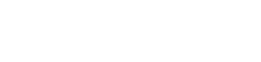

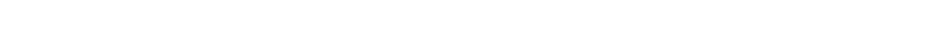

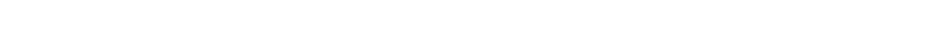

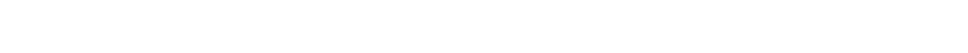

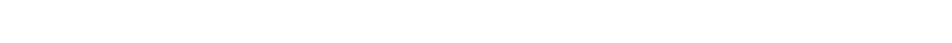

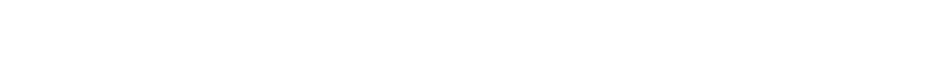

$5 N \infty$ en 2

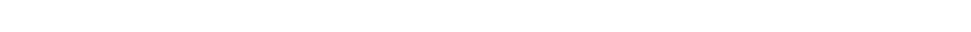

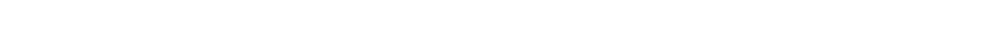

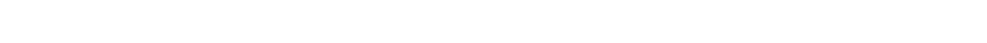

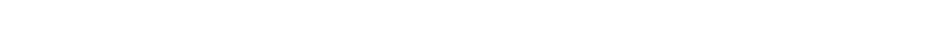

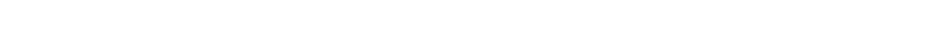

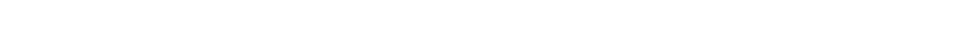

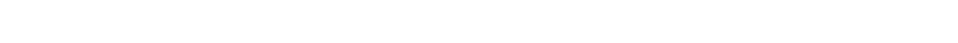

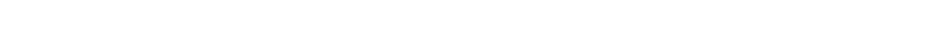

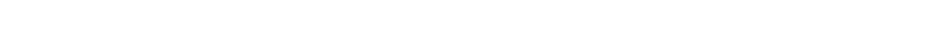

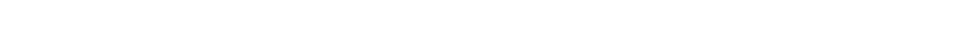
is

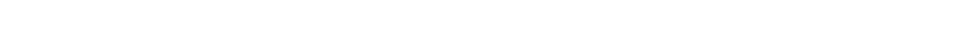

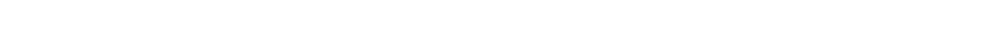

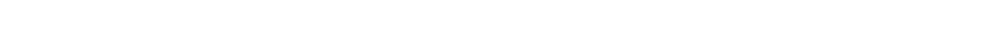

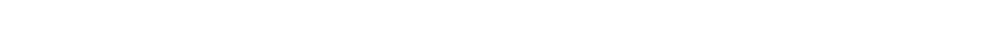

0000000000000000000000000000000

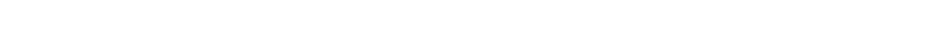

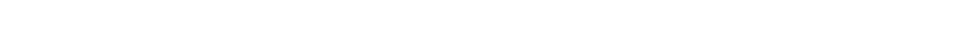

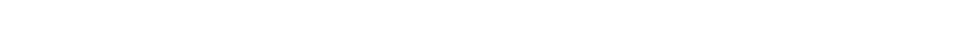

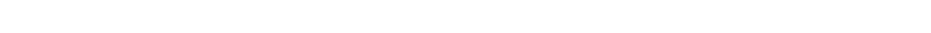

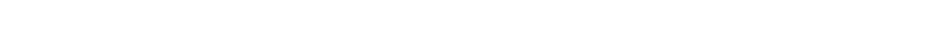




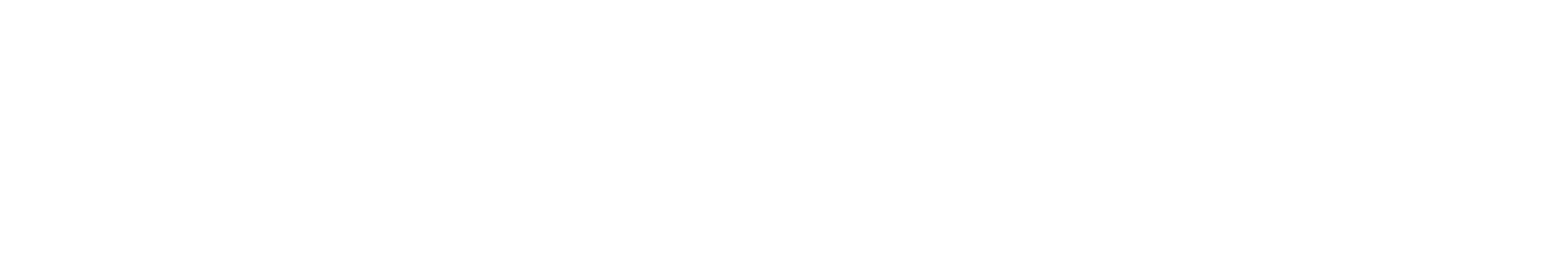

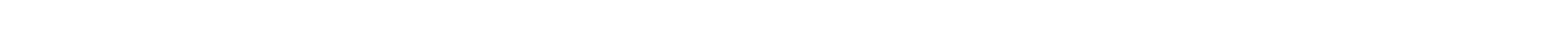

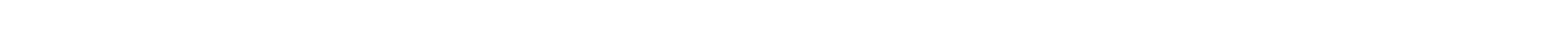

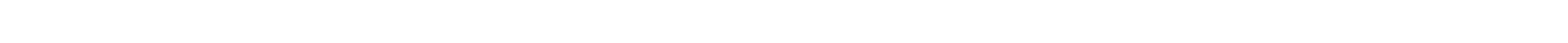

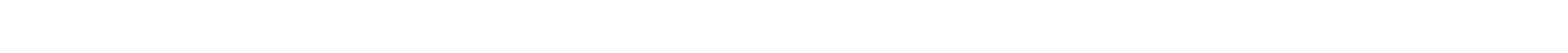

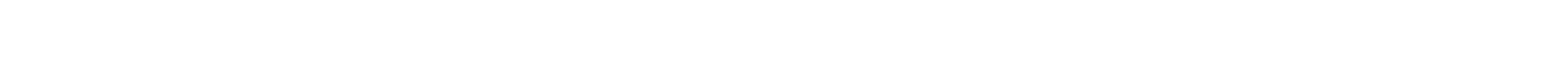

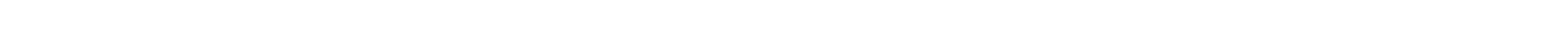

"w'

is

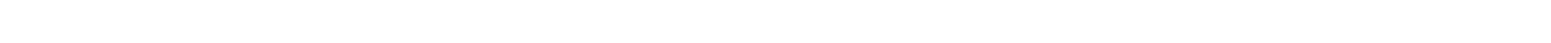

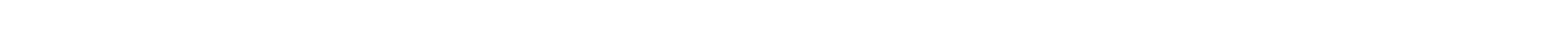

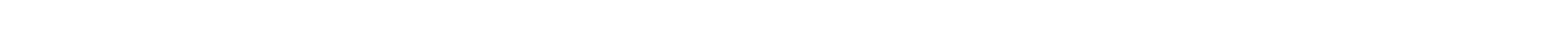
\& ш

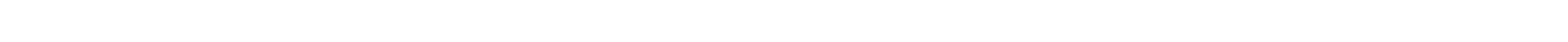

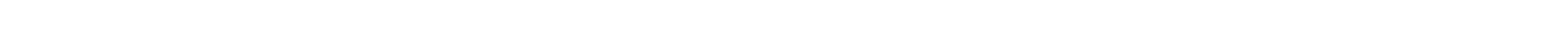
แ

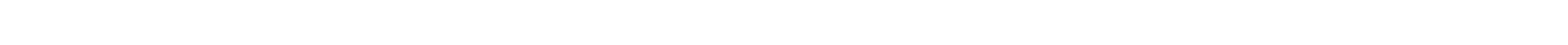

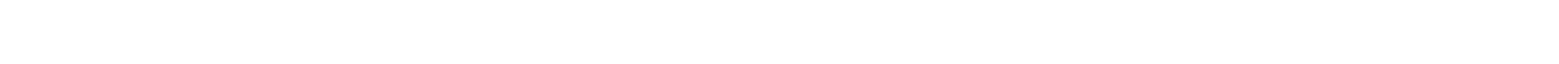

$\underline{\Sigma}$

in

is is

n

n

in is

$\sim$

in

is

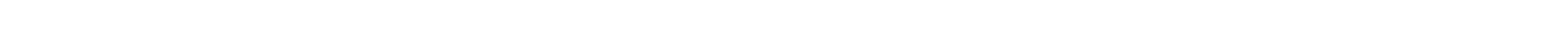

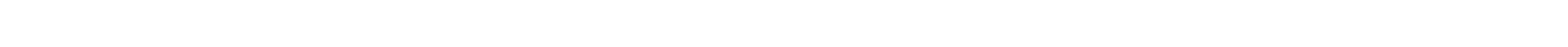

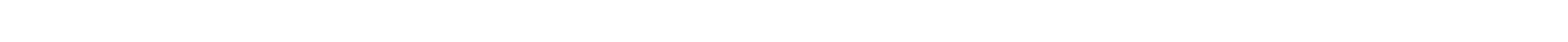

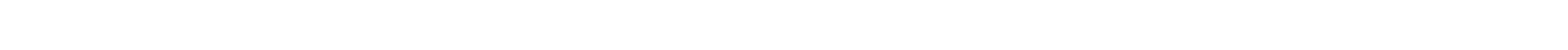

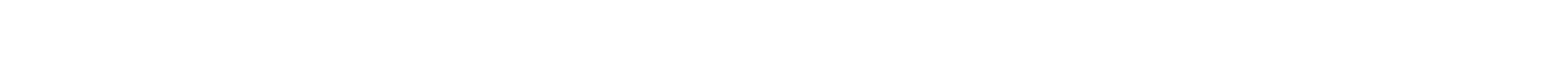

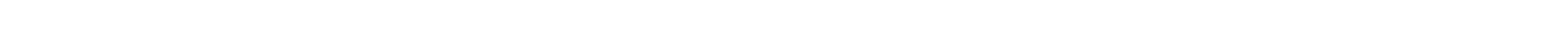

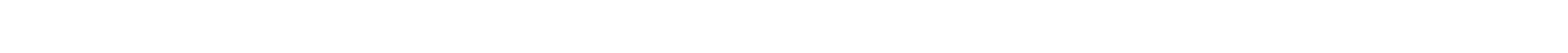

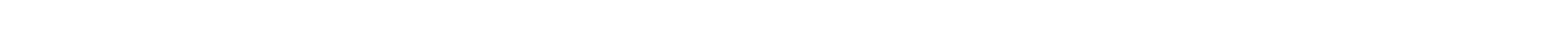

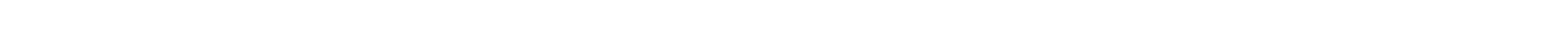

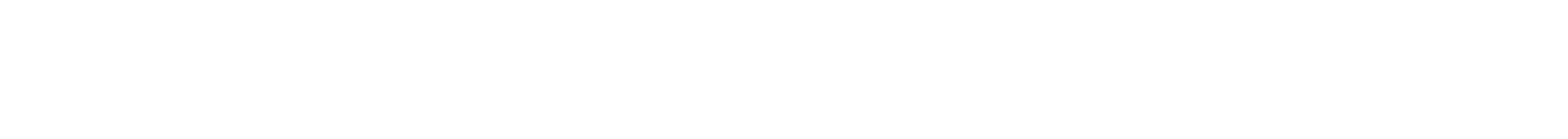

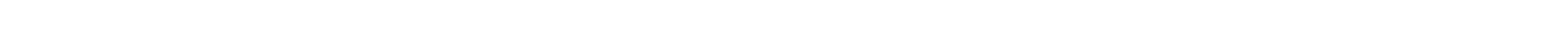

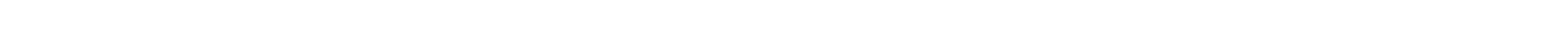

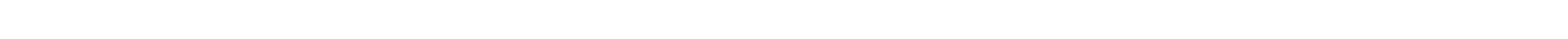

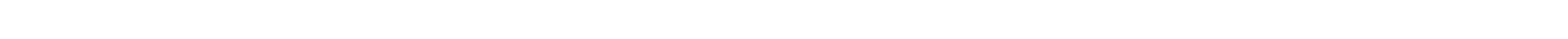

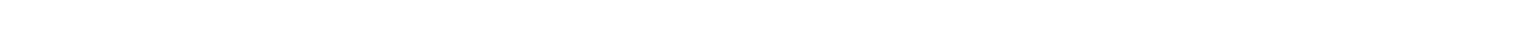

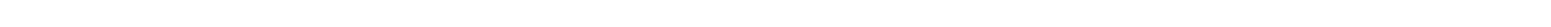

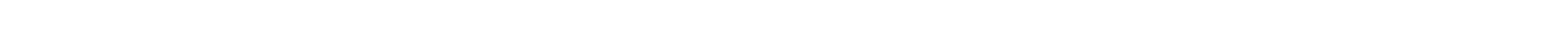

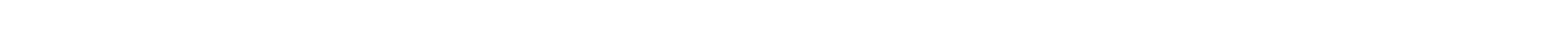

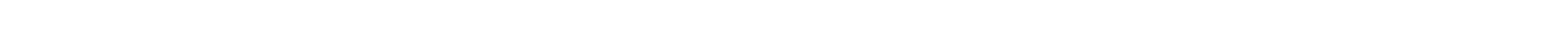

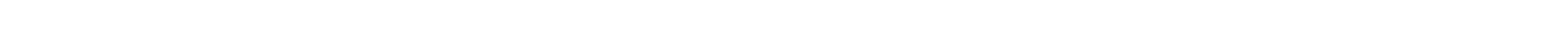




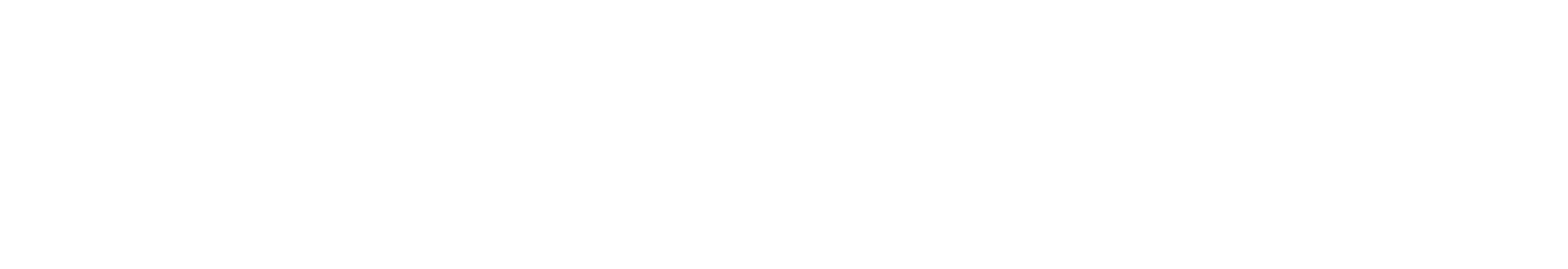

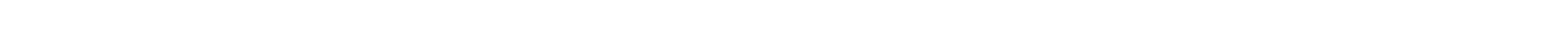

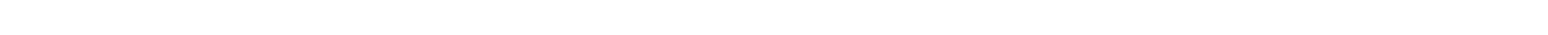

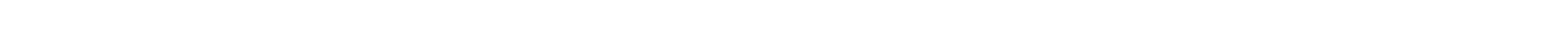

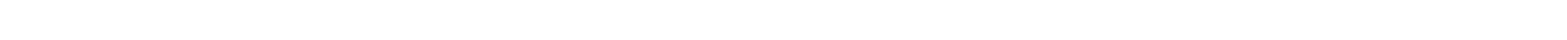

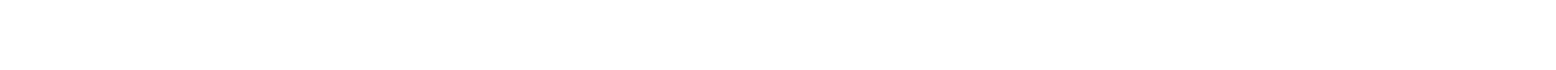

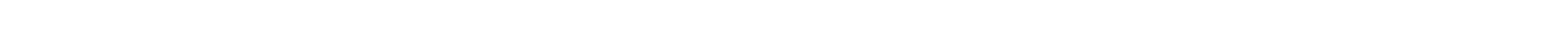

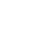

is

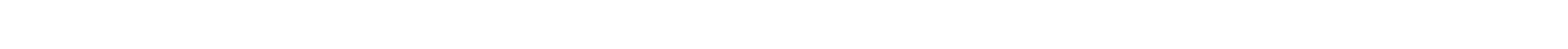
a

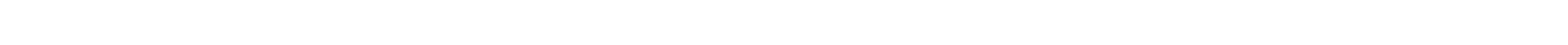

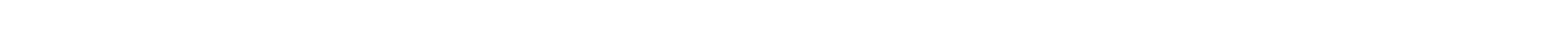

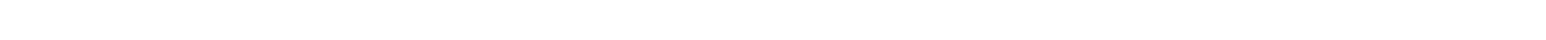

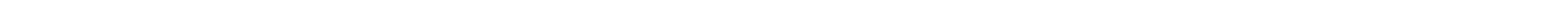

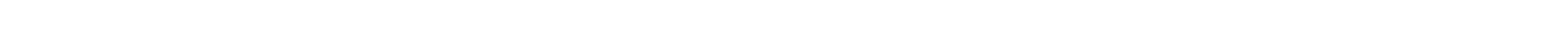

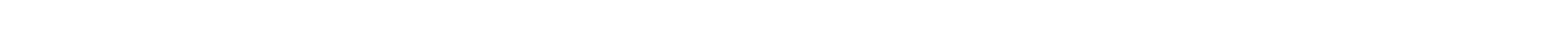

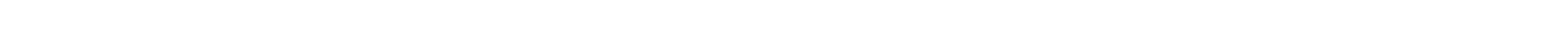

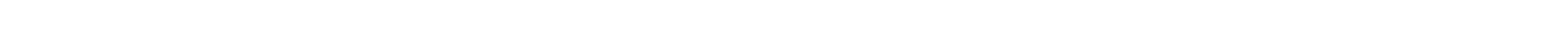

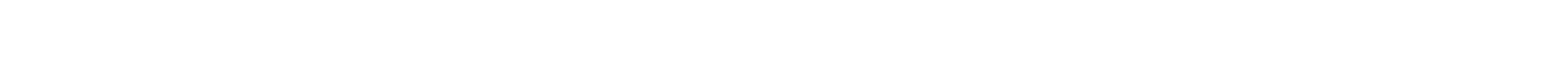

$w$
$\Sigma$
$\vdots$

WNow

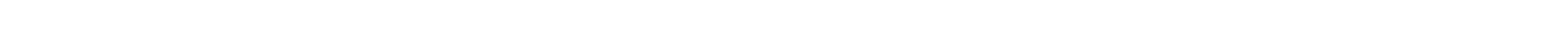

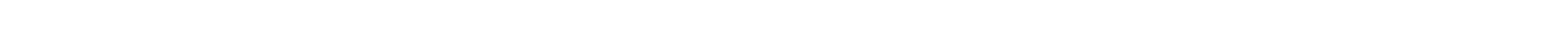

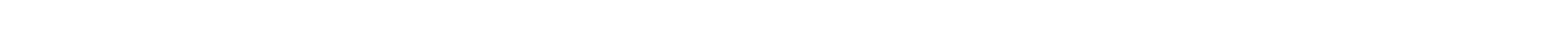

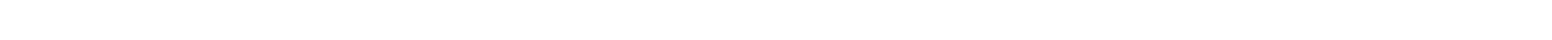

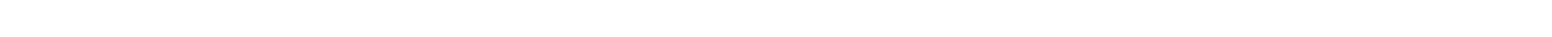

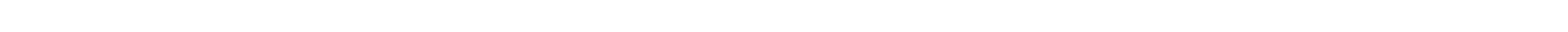

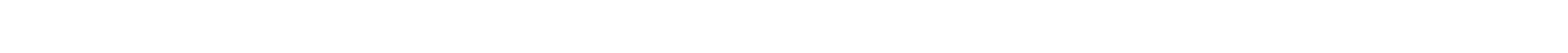

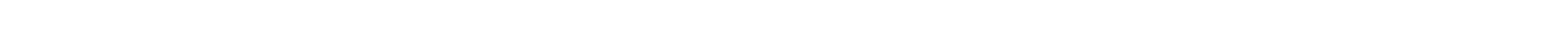

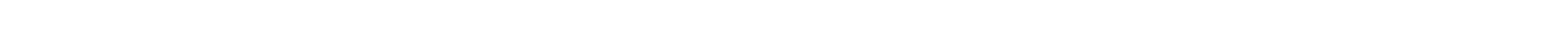

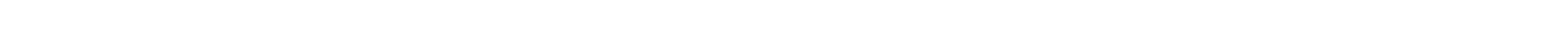

茫 en en is in

5

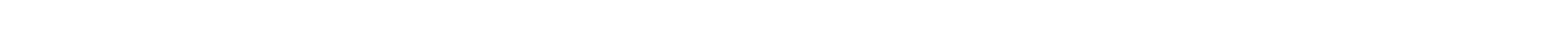

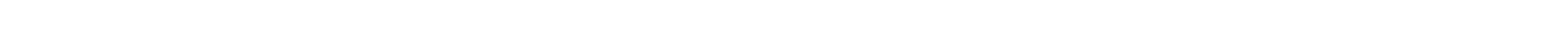
ü з

mmmmmmmm

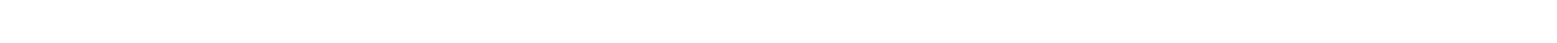

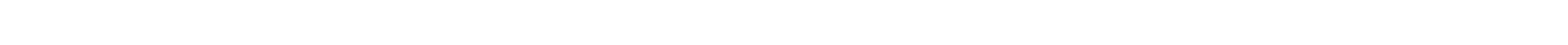

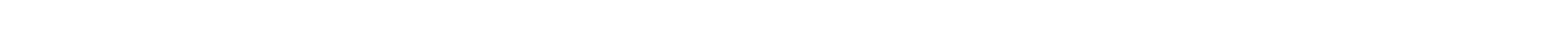

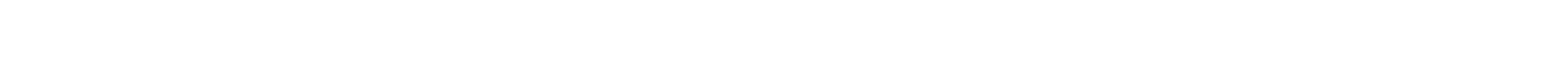


5

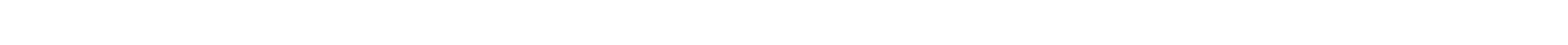

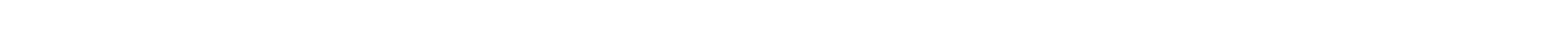

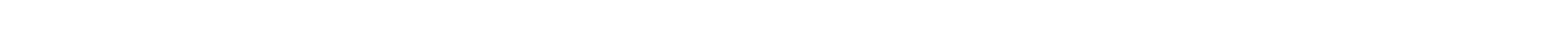

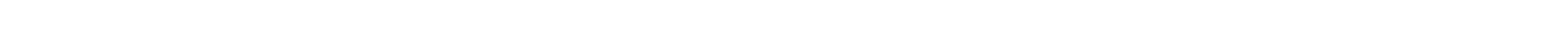

W గN

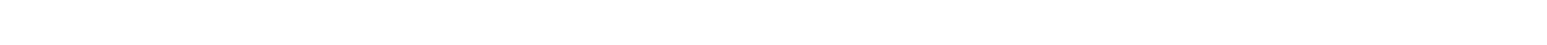

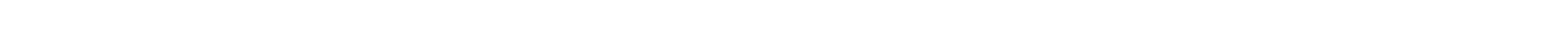

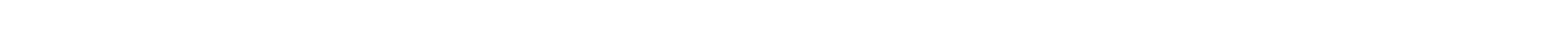

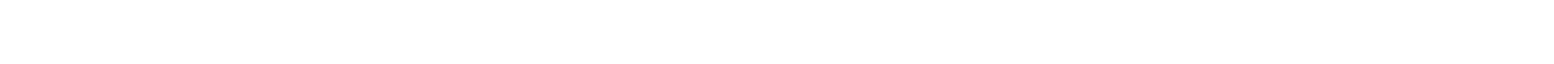
出
is
o
in
n
nn
a
n
n $n$

is

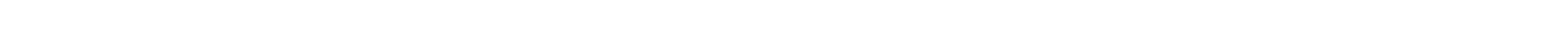

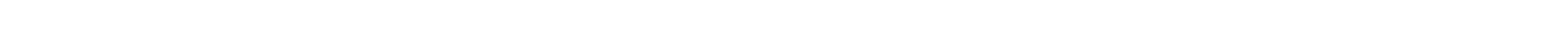

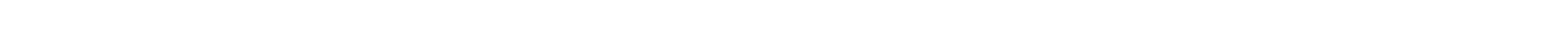

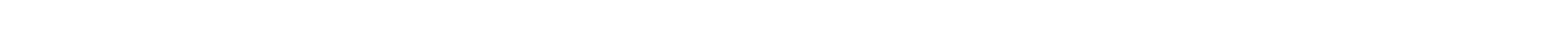

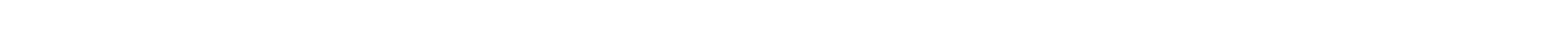

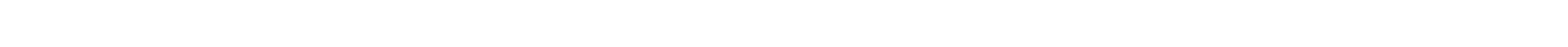

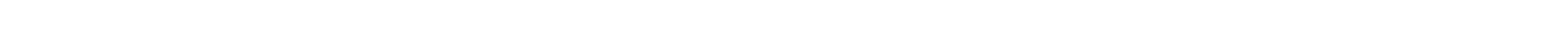

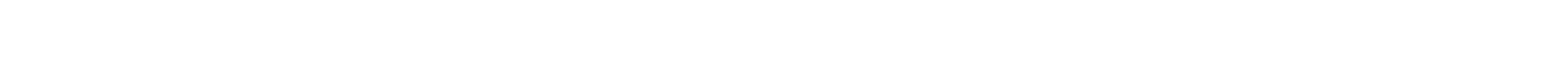
出

$\stackrel{m}{\Sigma}$

n

is

$\sim$

$\stackrel{4}{5}$

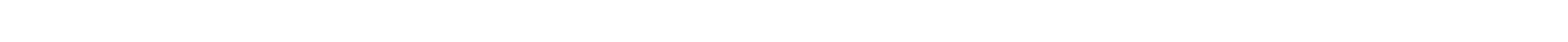
w

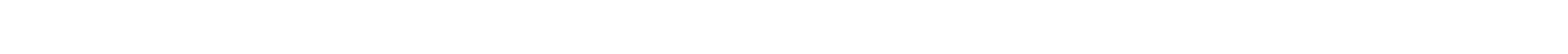
ㅆ

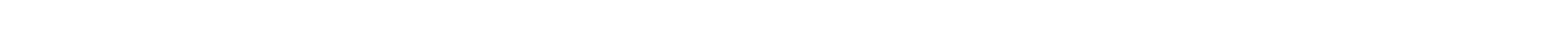

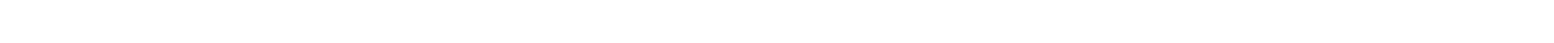

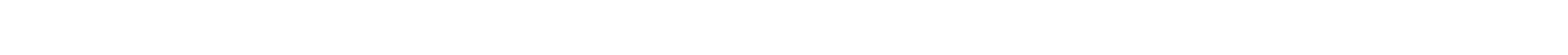

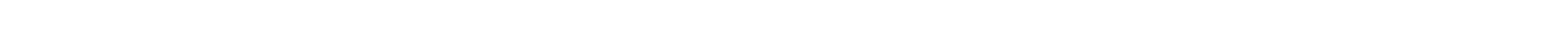

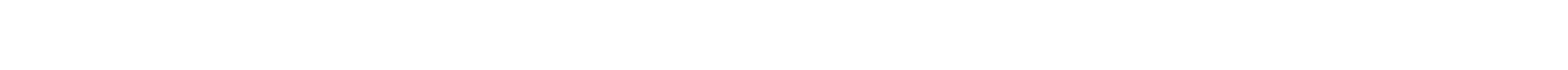

$\stackrel{5}{2}$ in on in in in

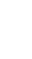

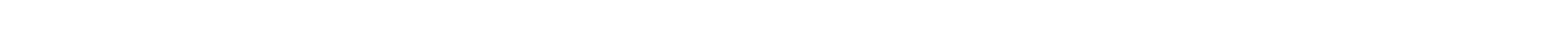

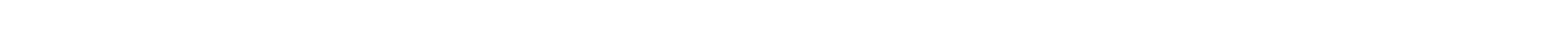
4

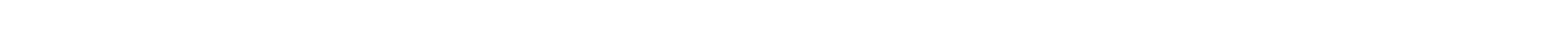

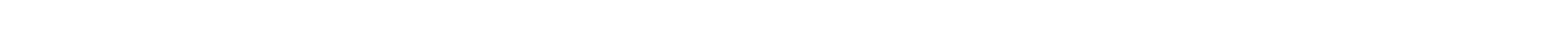

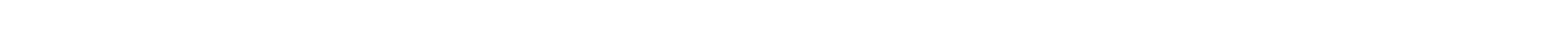

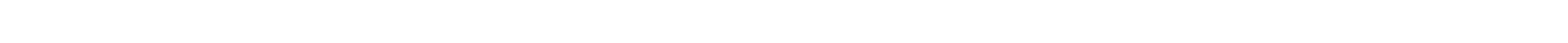

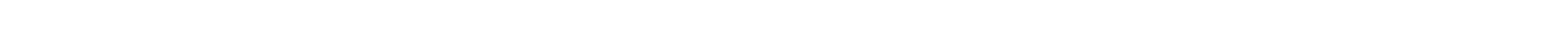

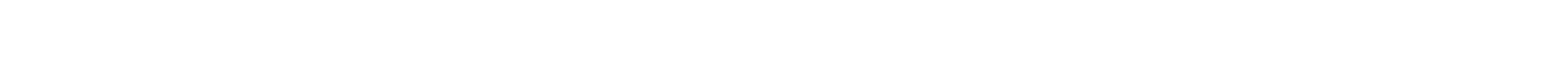




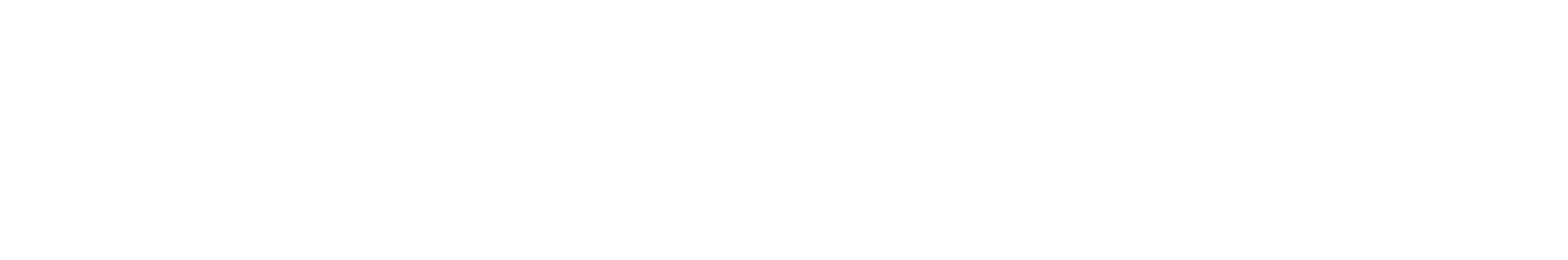

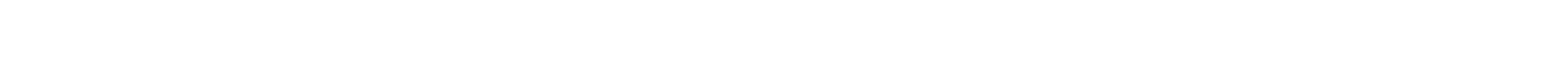

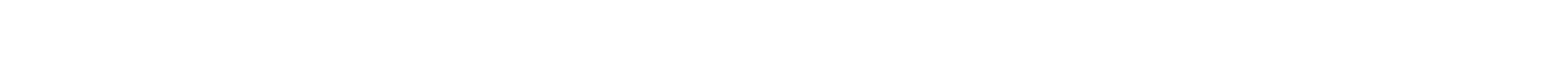

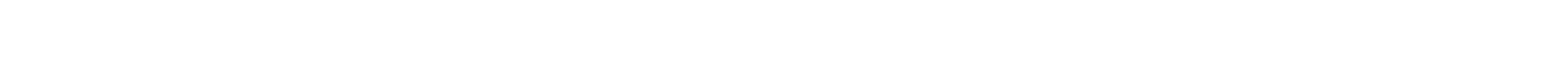

w

es

$n$

$\therefore$

J u б

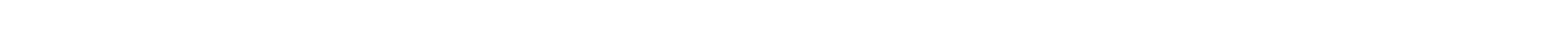

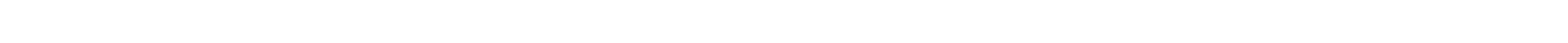

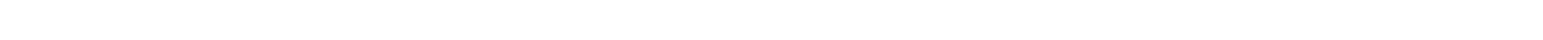

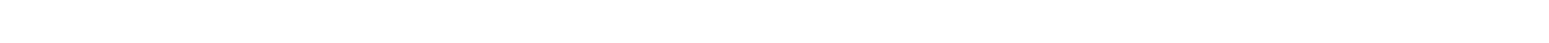

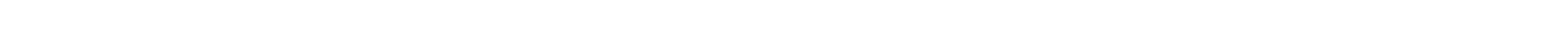

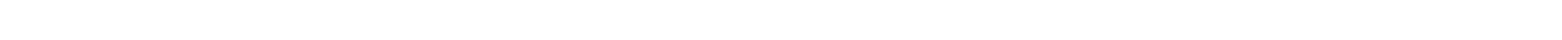

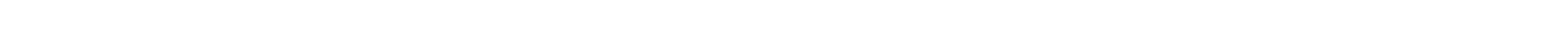

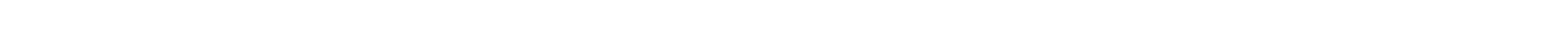

늘

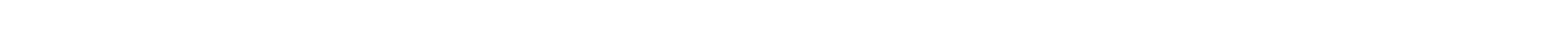
แ

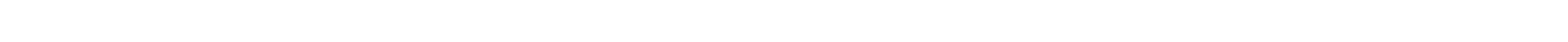

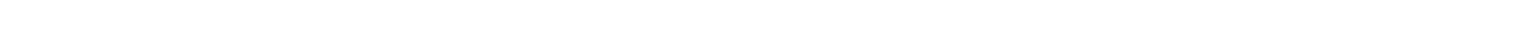

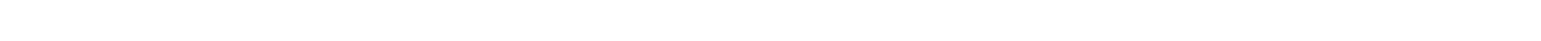

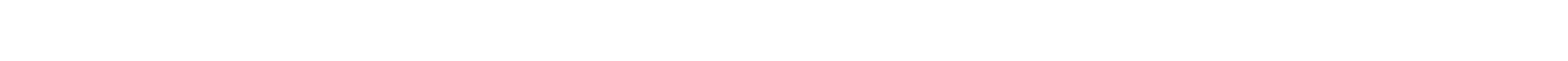

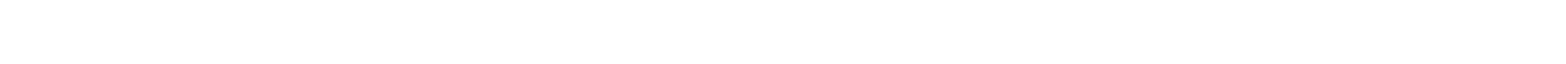

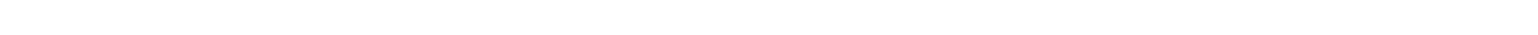

$\leftarrow$

$\stackrel{m}{\underline{m}}$

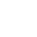

is

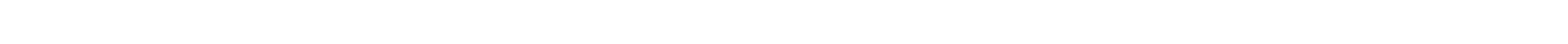

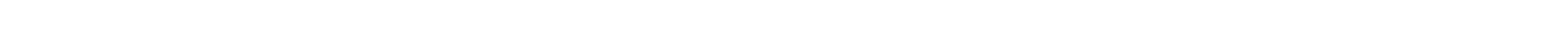

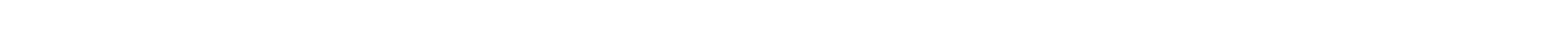
бат

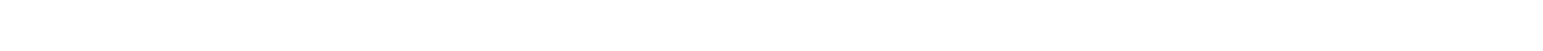

"W

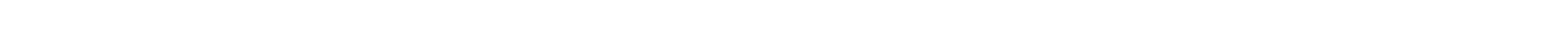

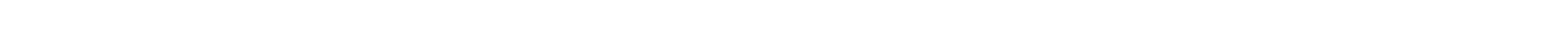

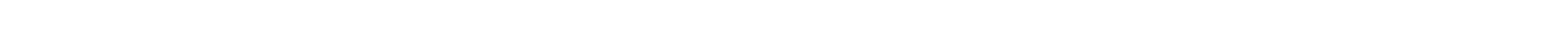

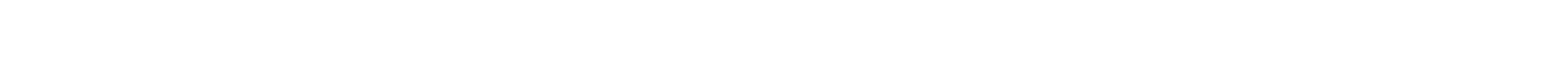




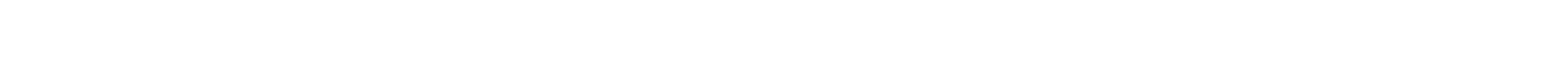

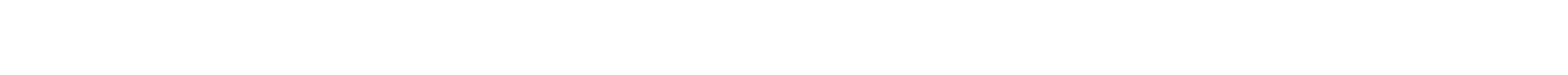

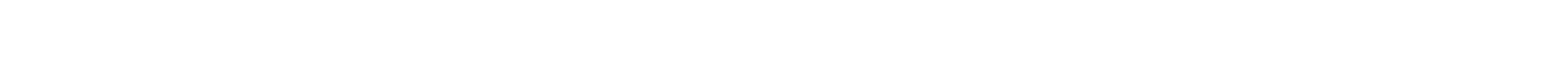

$-$

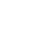

s

皮

inesen

n

n n

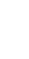

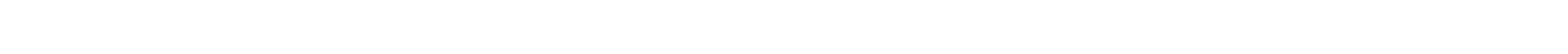

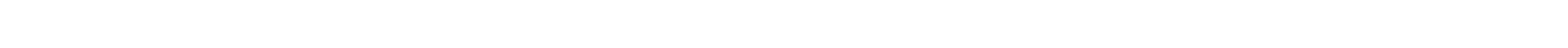

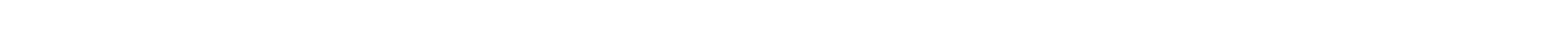

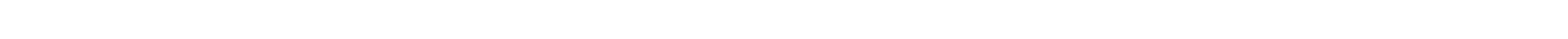

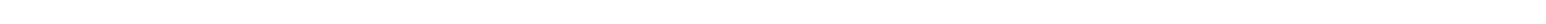

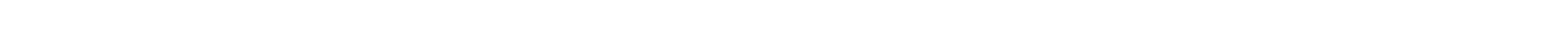

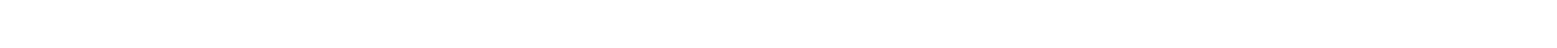

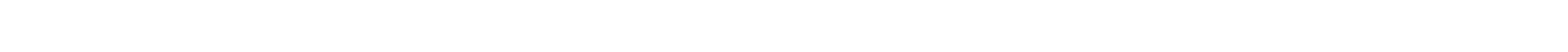

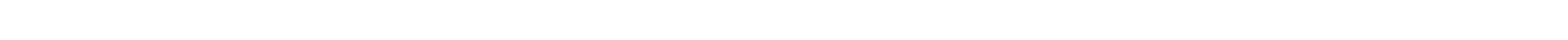
MQF-N N N

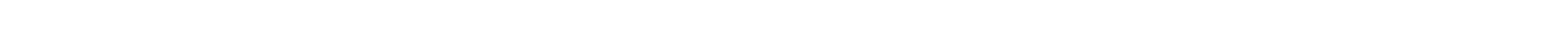

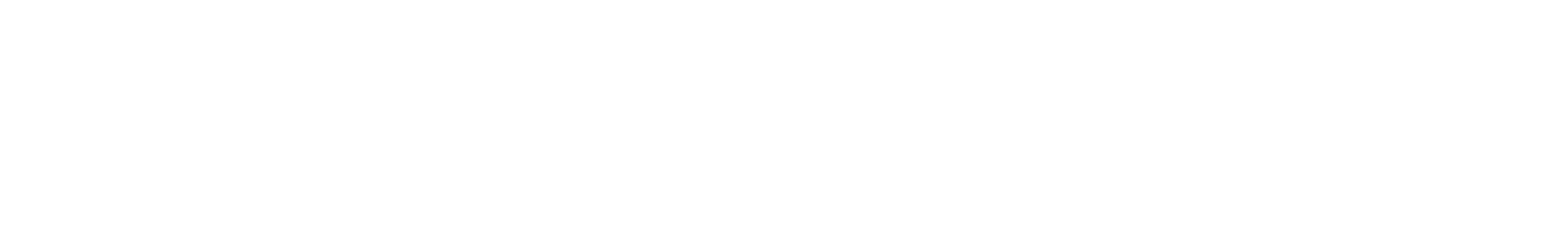

in

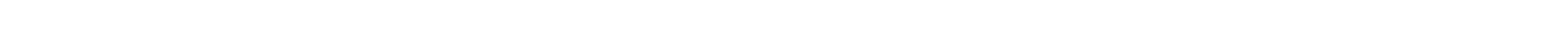

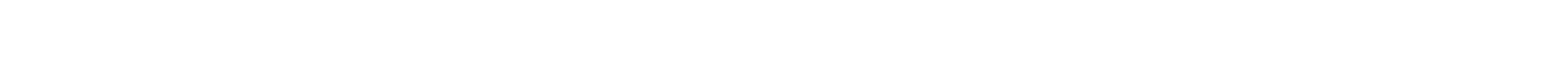

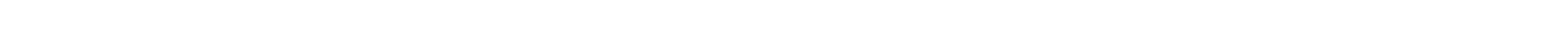

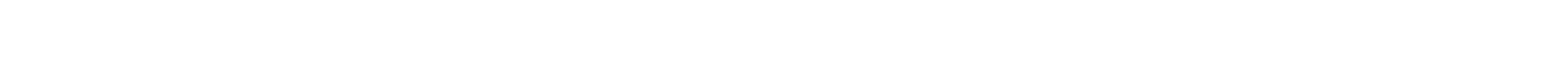

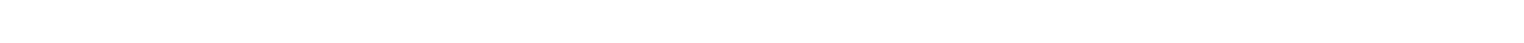

$\Sigma$

$\stackrel{2}{\sim}$

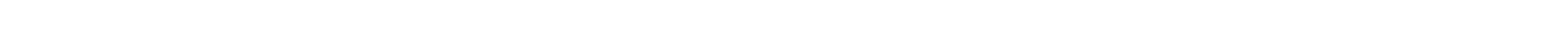
u.

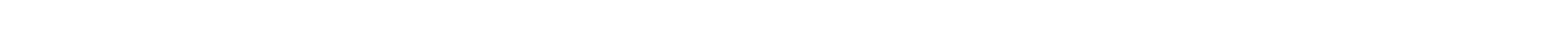

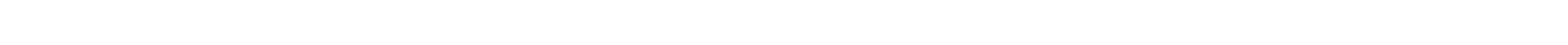

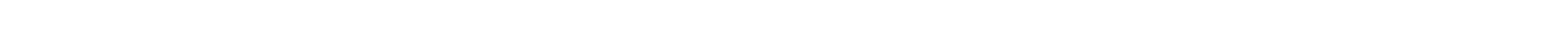

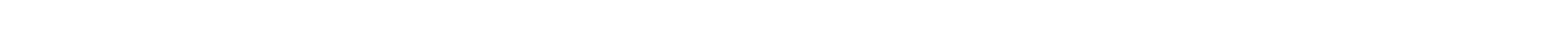

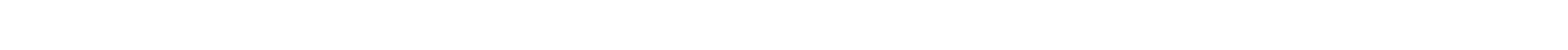

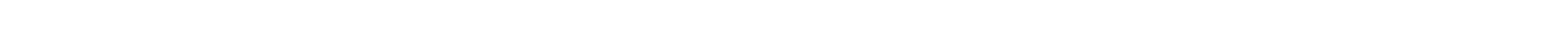

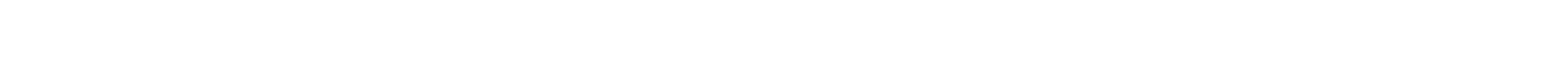




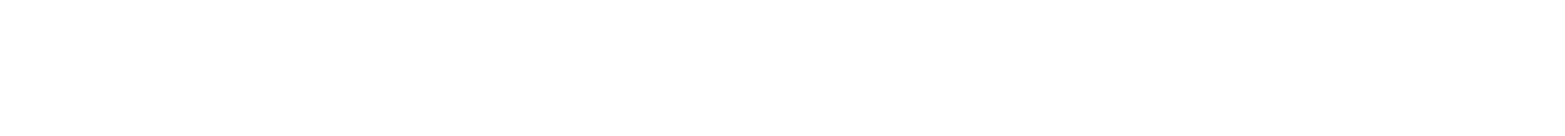

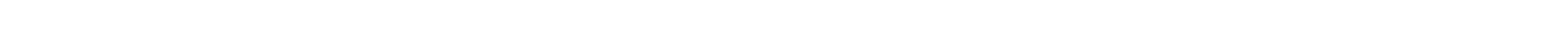

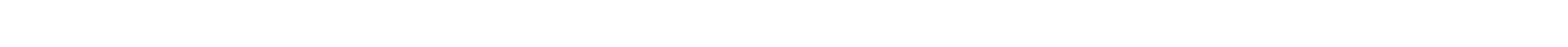

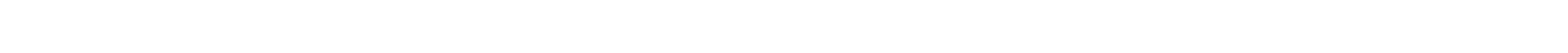

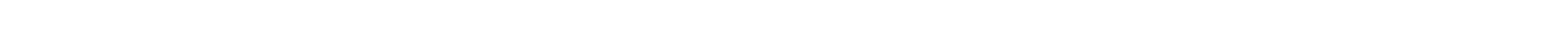

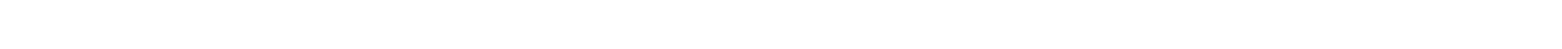

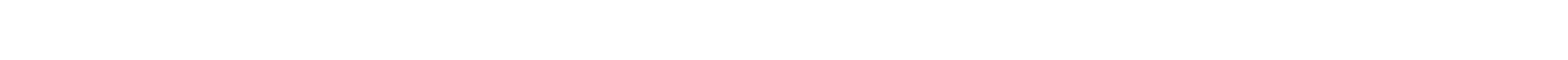

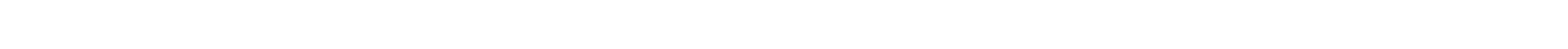

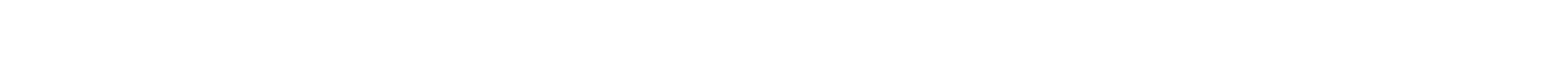

(1)

w en os

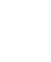

은

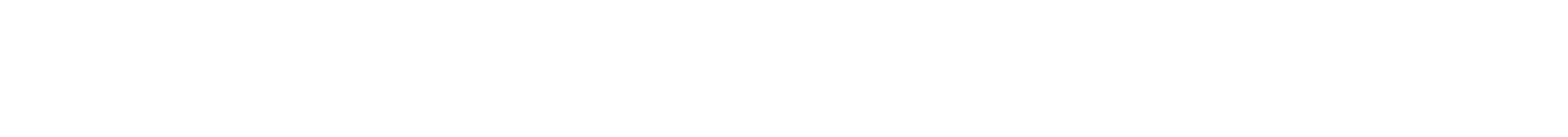

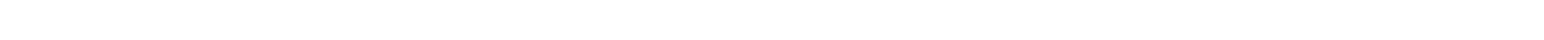

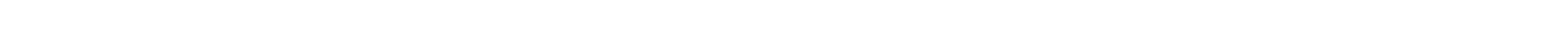

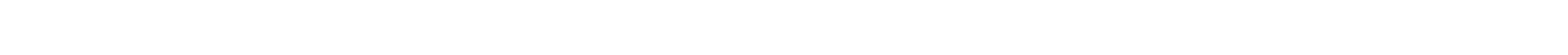

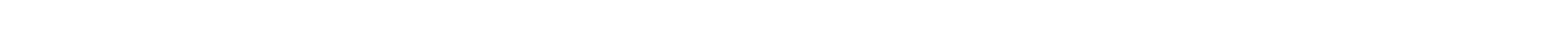

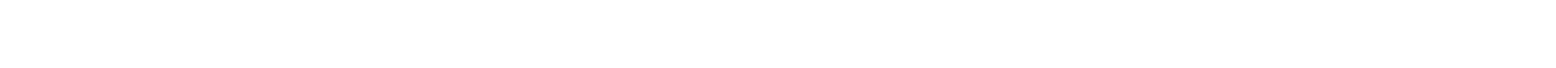

$\approx$ $\leftarrow$

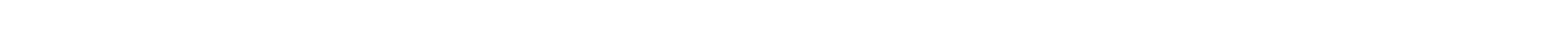

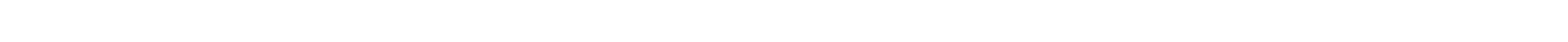

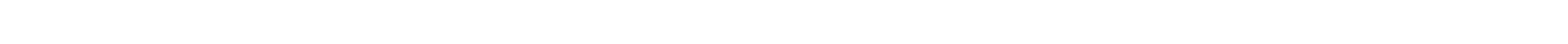

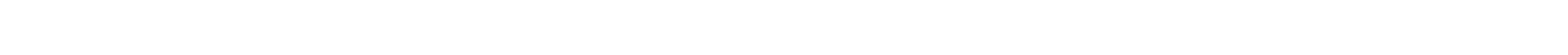

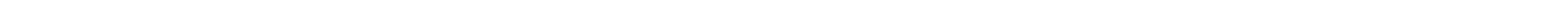
nు

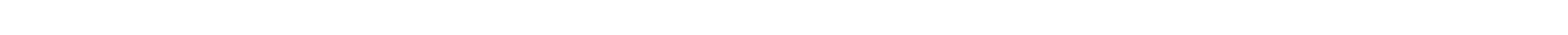

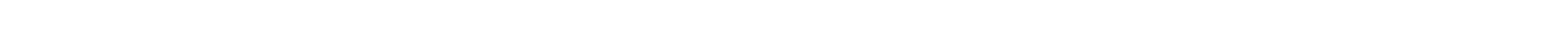

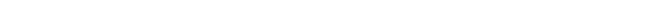

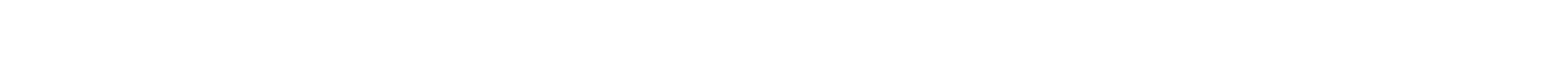

$\stackrel{15}{5}$

$\sim$

es

$\sim$

is

n

t)

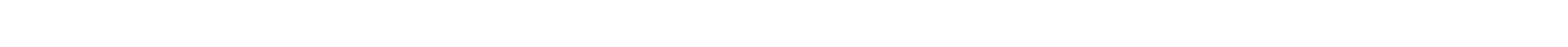

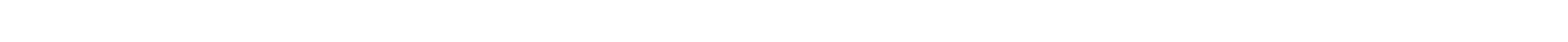

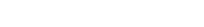

ir.

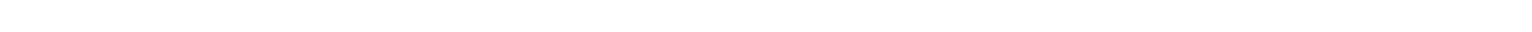

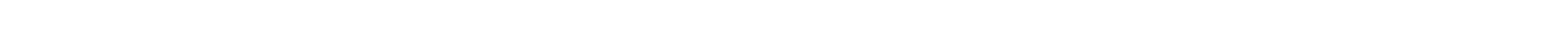

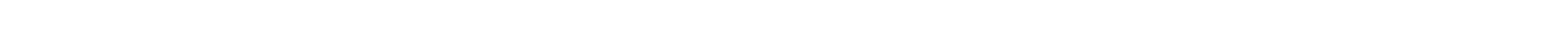

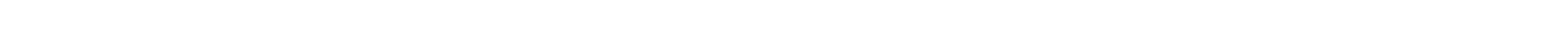

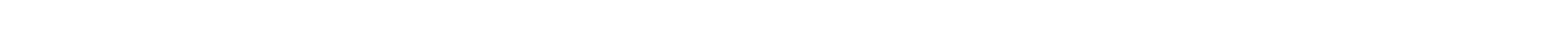

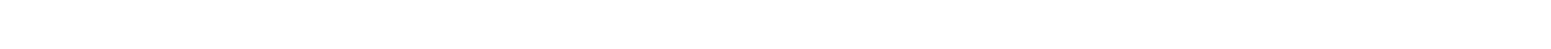




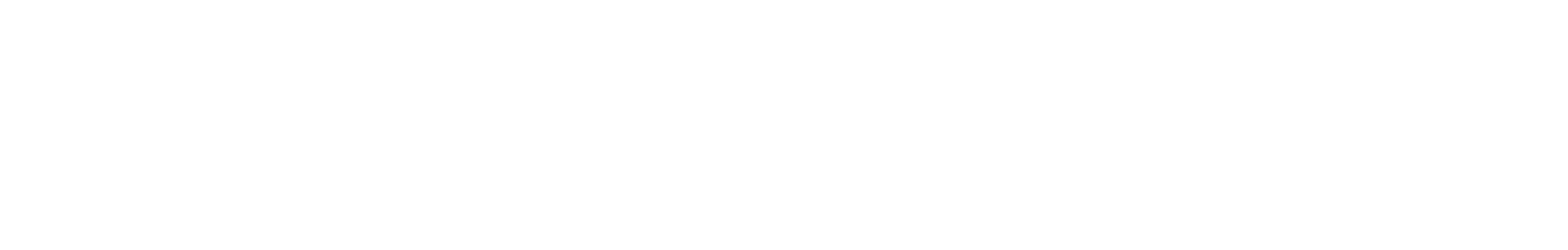

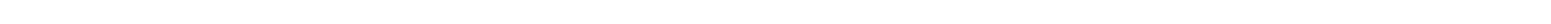

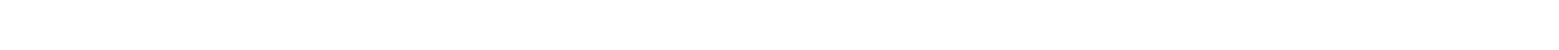

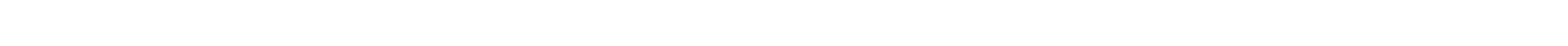

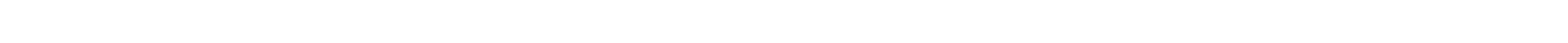

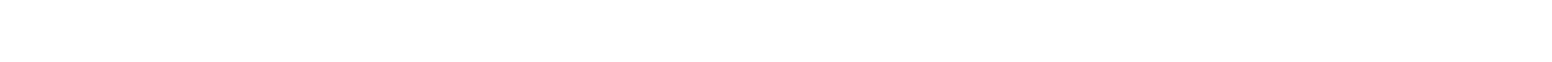

$\underline{\Sigma}$

n

$\sim$

is

is

n

is

2

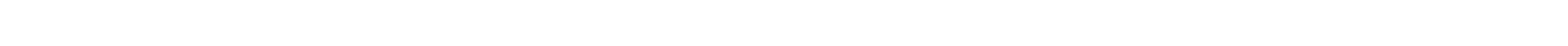

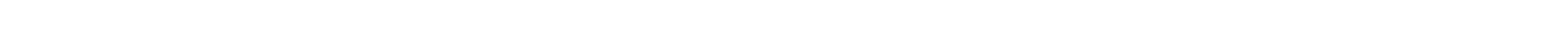

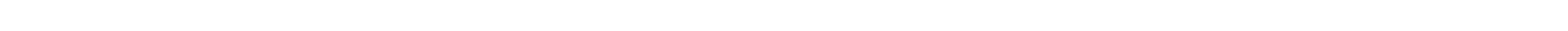

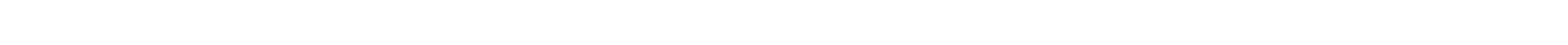

ラনスス

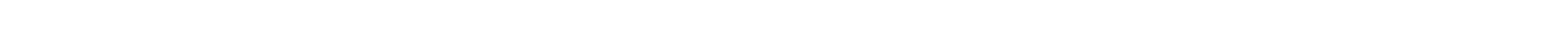

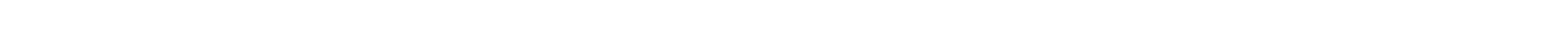

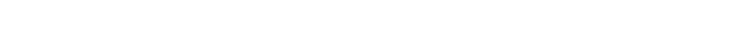

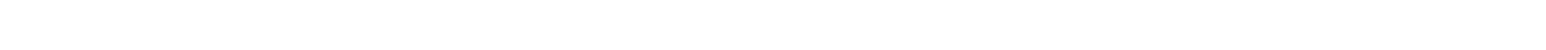

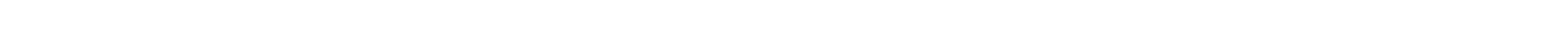
$\stackrel{w}{\Sigma} \sim$
is
is $w$
w is
is
@
in
心
$\stackrel{\pi}{\sim}$

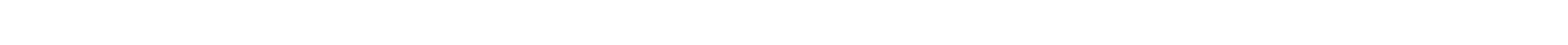

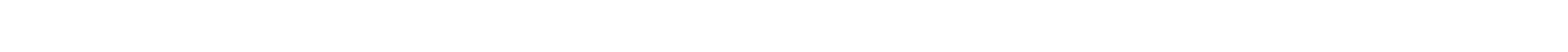

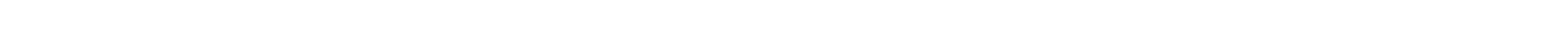

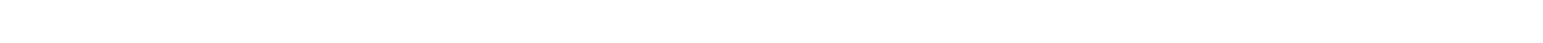

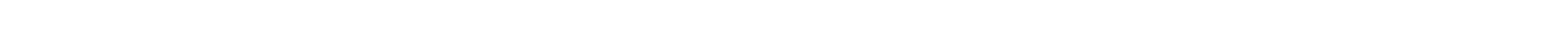

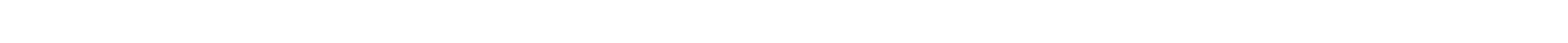

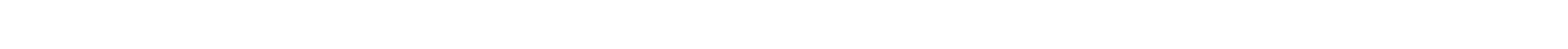

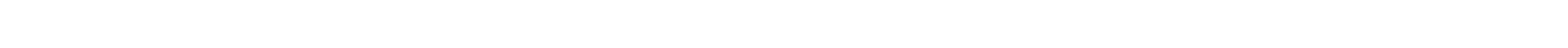

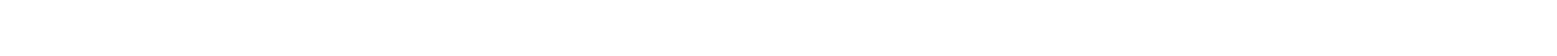

r

a

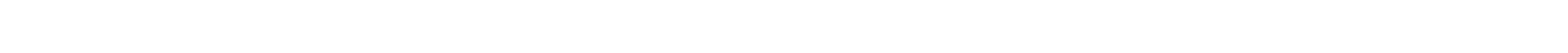
ш

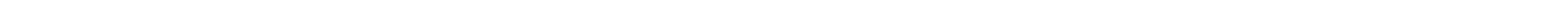

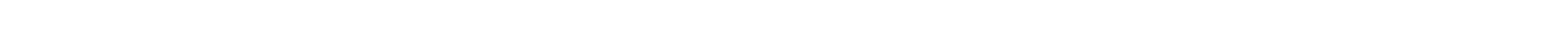

000000000000000000000000000000000000000000000000000

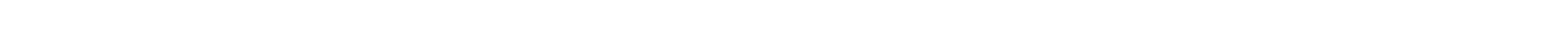

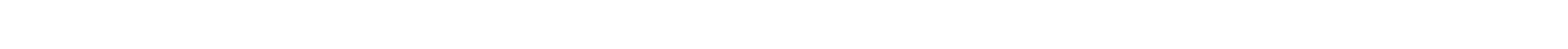

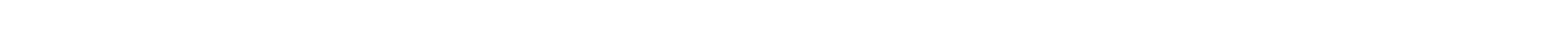

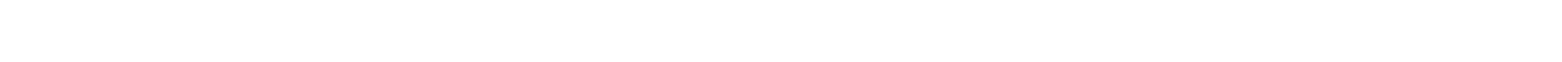




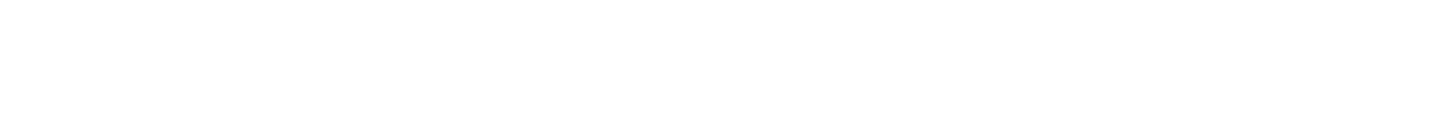

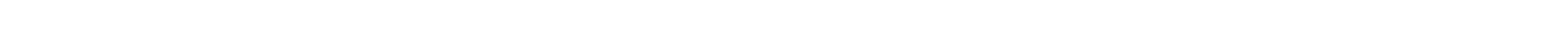
ư a

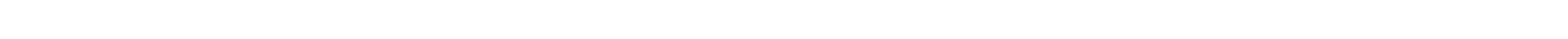

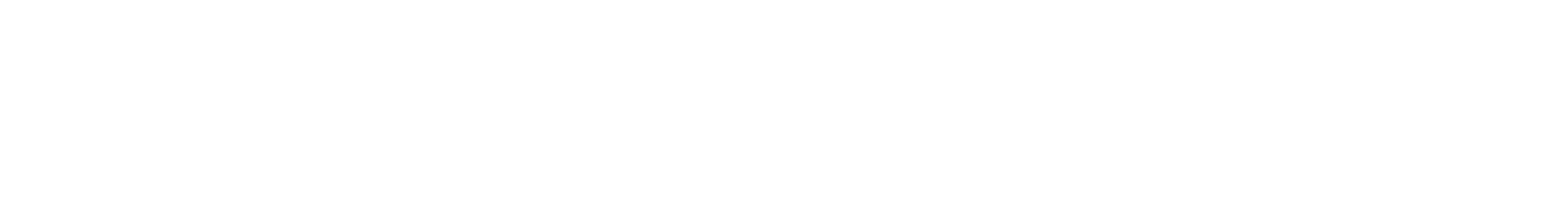

$\underset{\Sigma}{\mathrm{w}}$ en

in

is

is

n

is

is

$\pm$

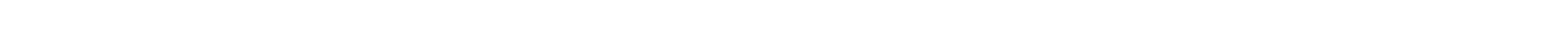
uш

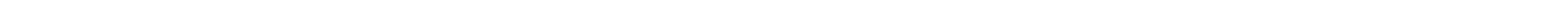
з ג

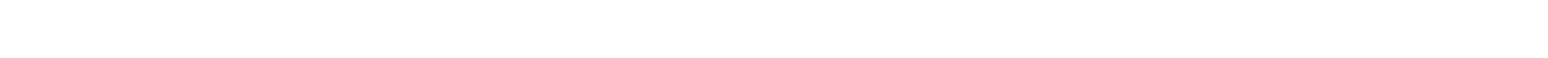

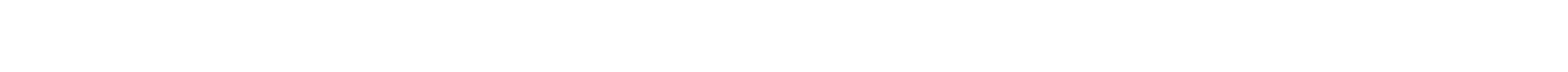

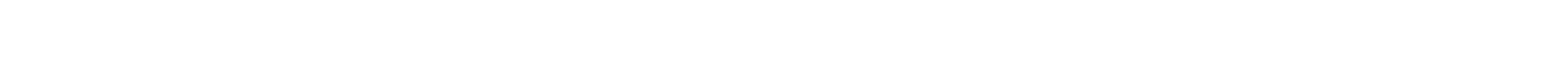
w i
en
is
en
es
n
$\stackrel{2}{2}$

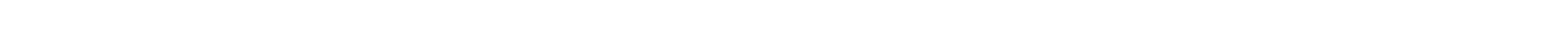
u.

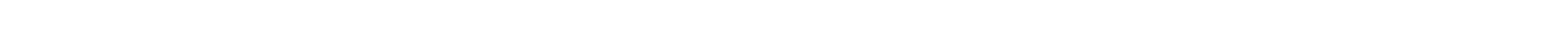
a

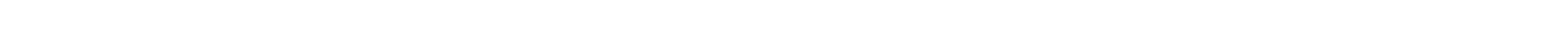

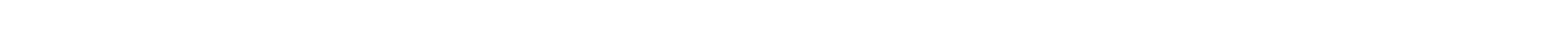

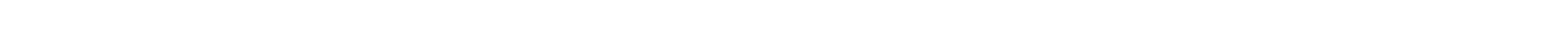

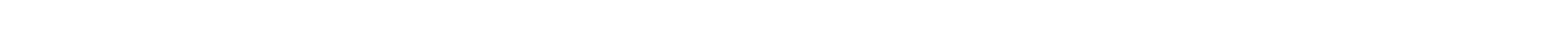

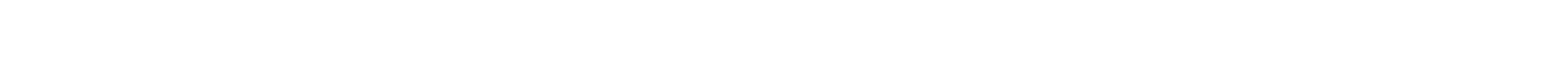

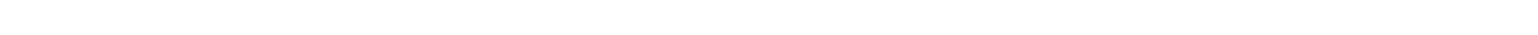
$\leftarrow$ is
n) n
e
n
is
is

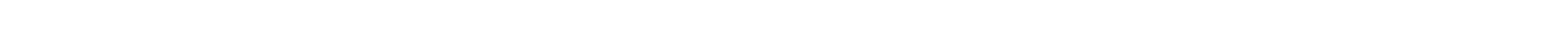

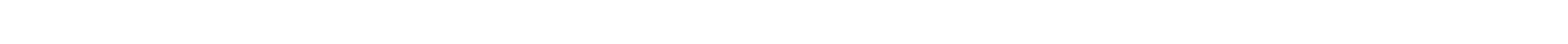
“

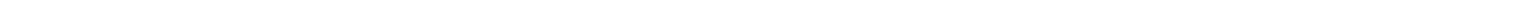

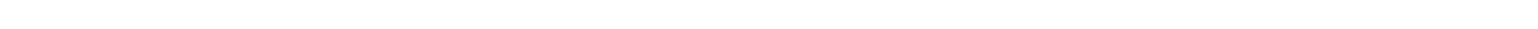

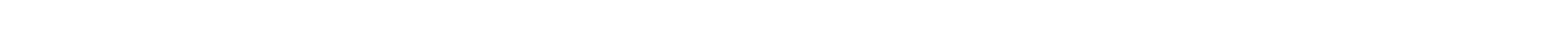

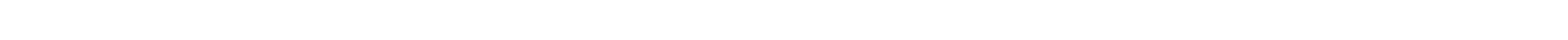

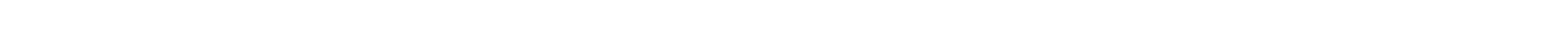

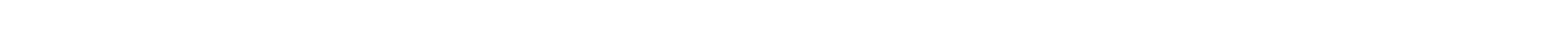


ह

in $\quad n$

$\sim$

u

in

in

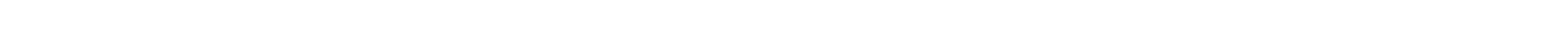
жа шы

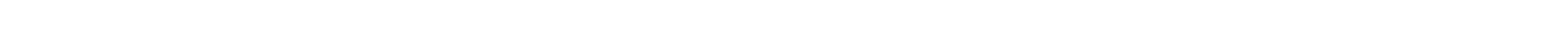

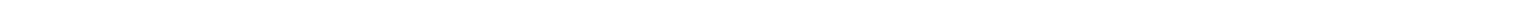

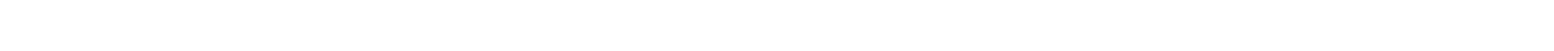

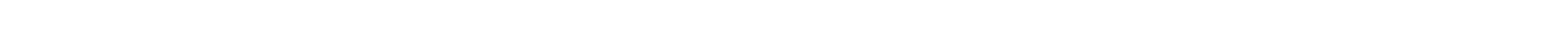

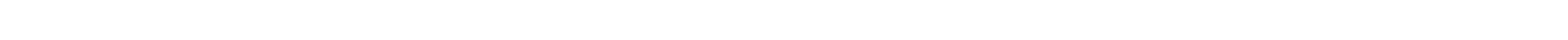

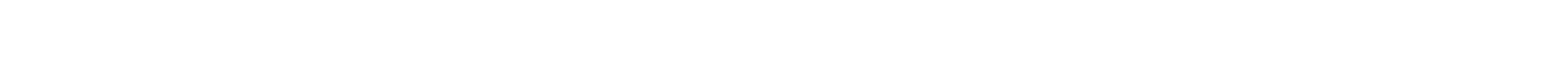

is

is

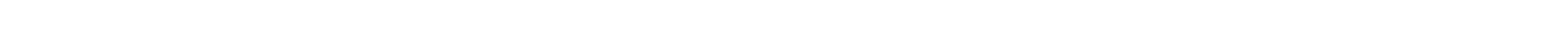

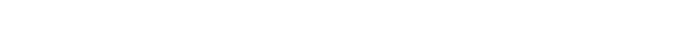
녈 d

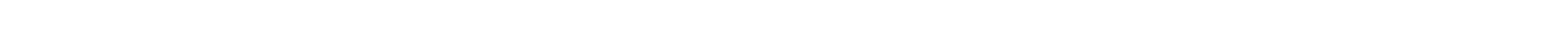

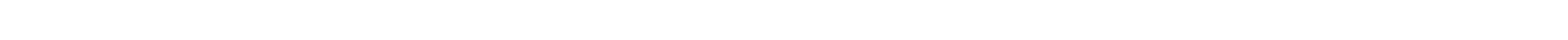

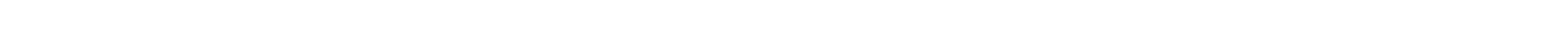

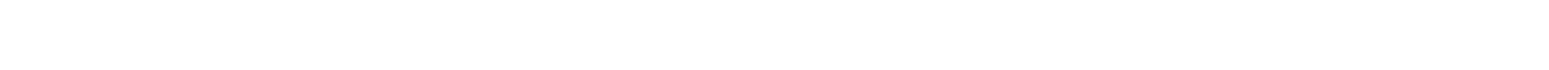

$m_{m}$

on

e

in

en

$\sim$

in

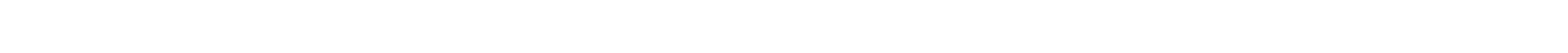

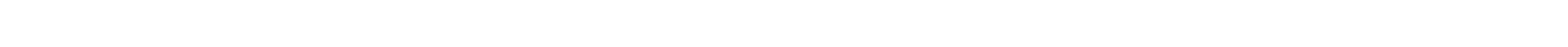

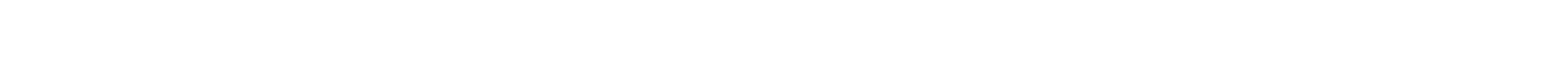

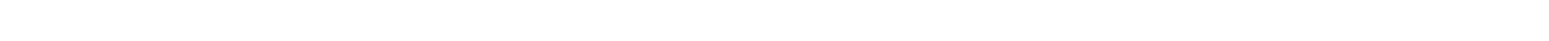

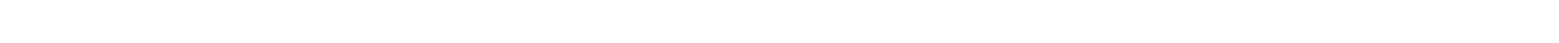

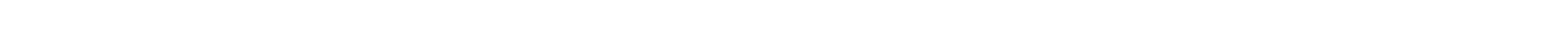

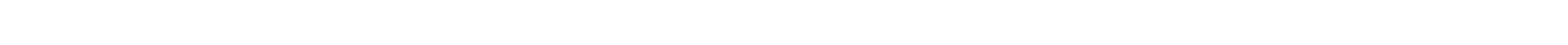

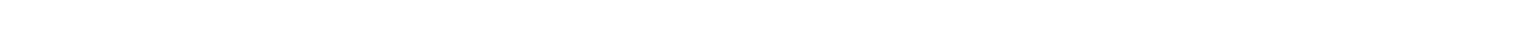

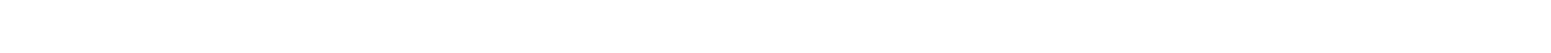

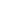

章

in

"مm" w a wínmmmmmm

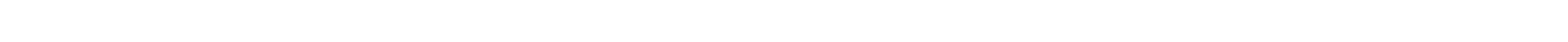

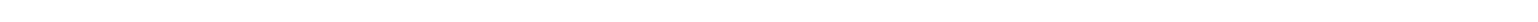

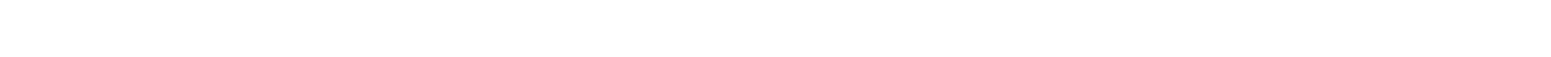

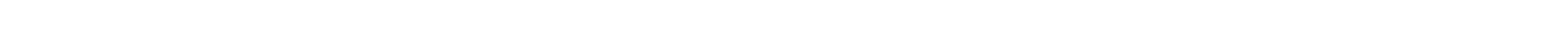

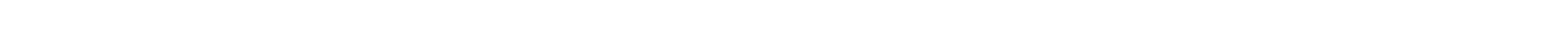

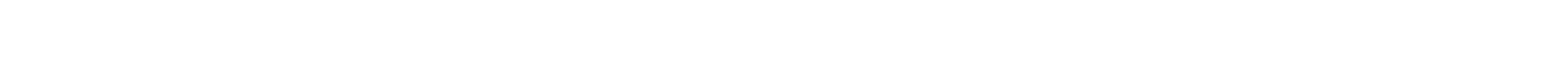




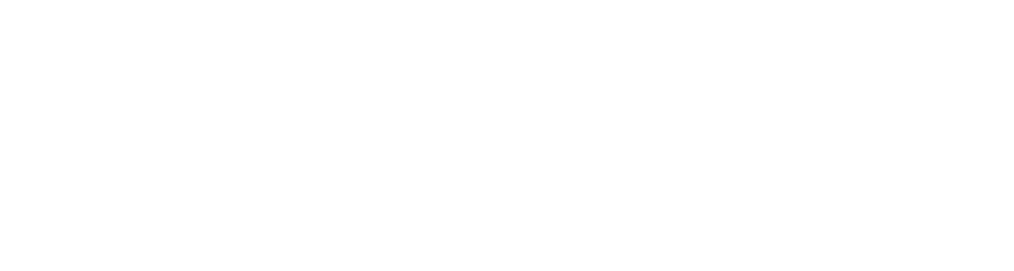

$\stackrel{a}{n}$

in

ㅆ

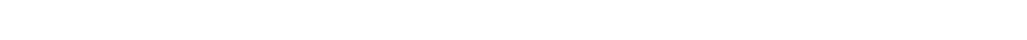

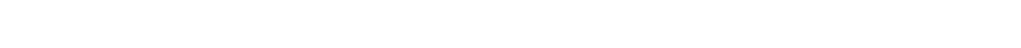

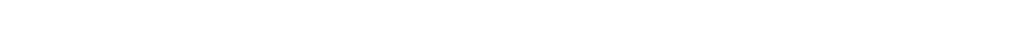

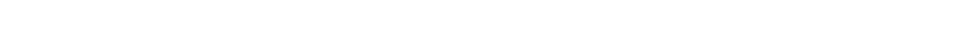

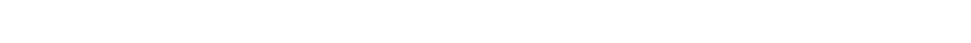
แ

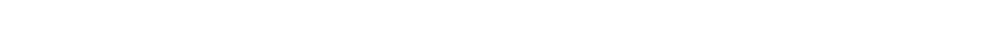

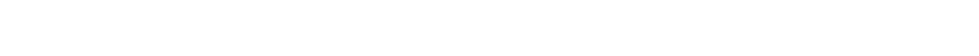

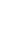

$\Sigma$

is

س ư u. ? ? - miñ

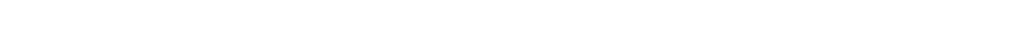

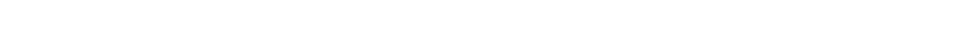

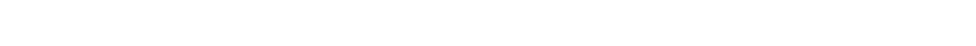

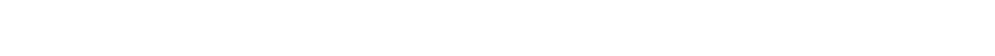

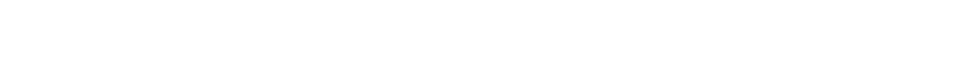

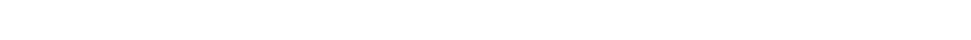

a

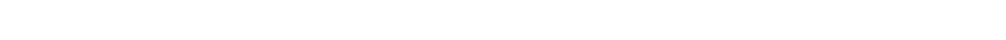
ulu

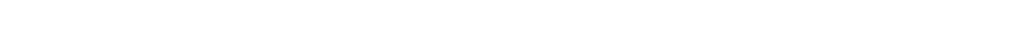

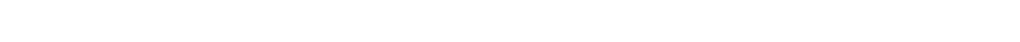

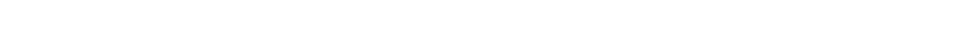

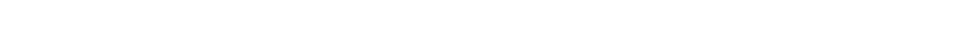

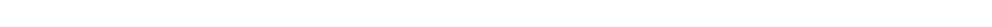

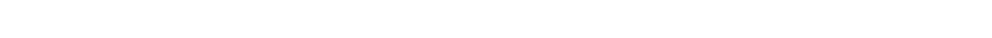

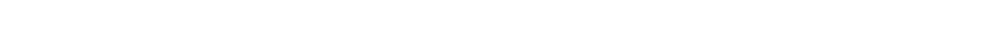

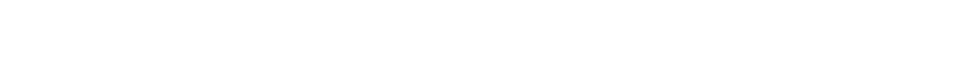




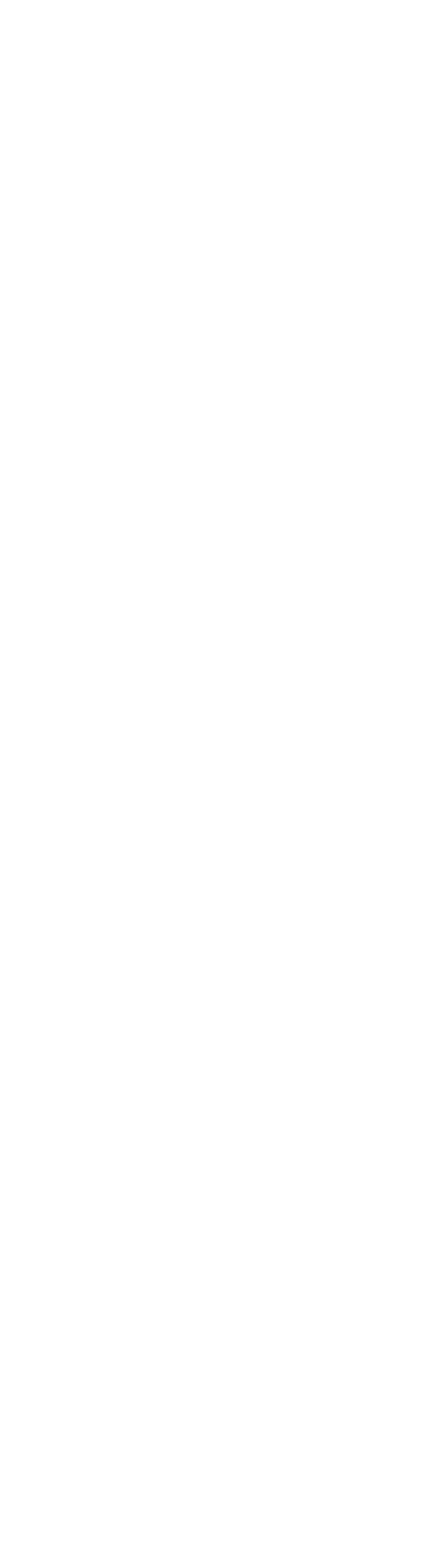

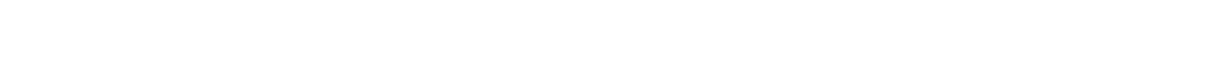

in

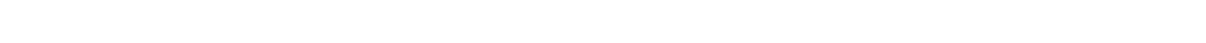
о

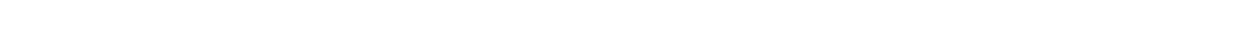

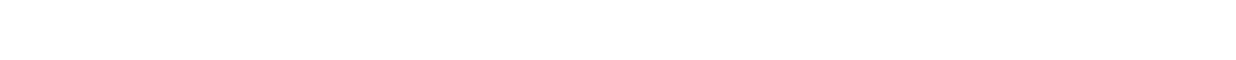

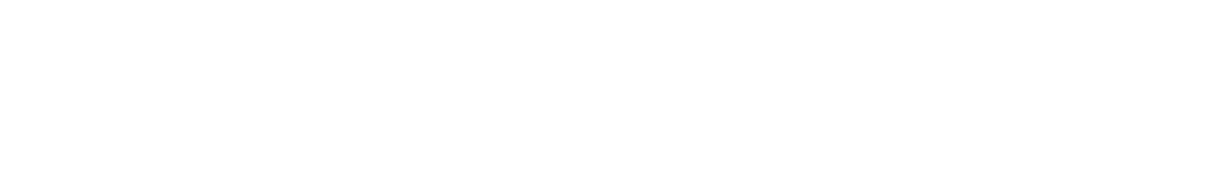

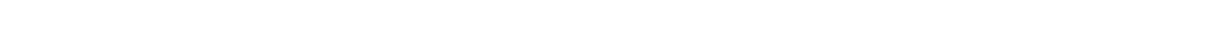

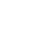

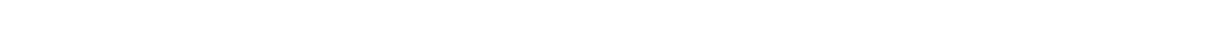
w

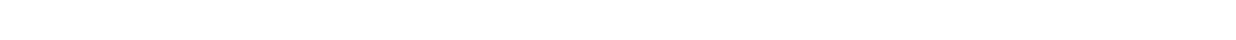

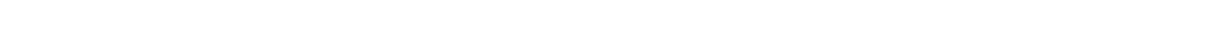

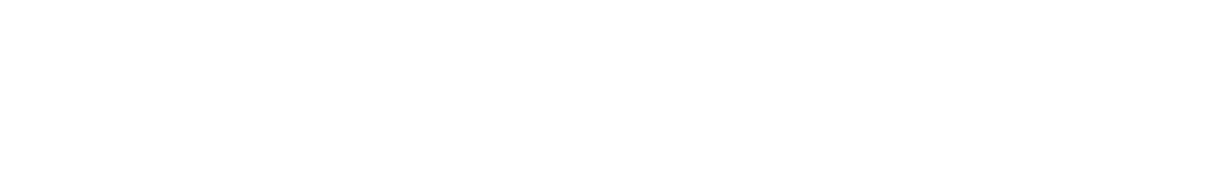

w

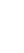

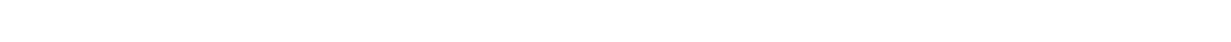

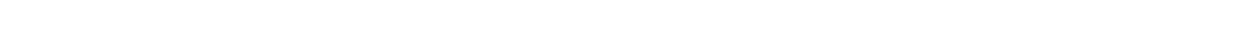

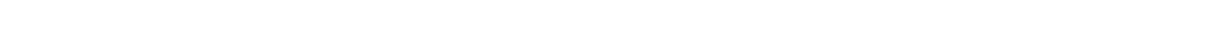

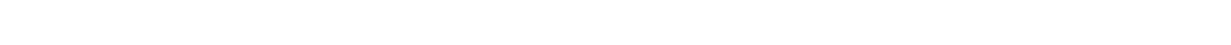

SN mom 0 m

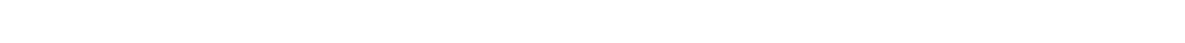

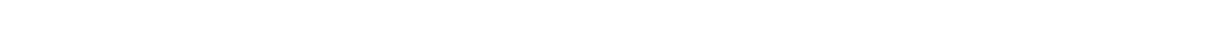

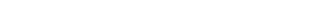

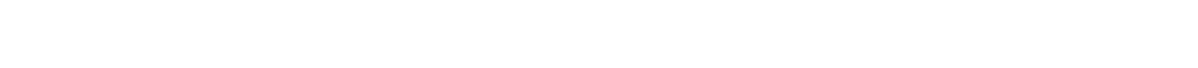

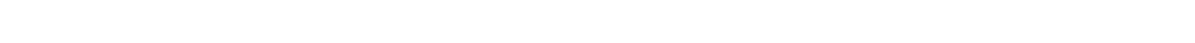

$\alpha$

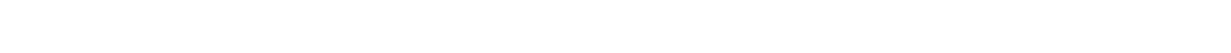
ü a

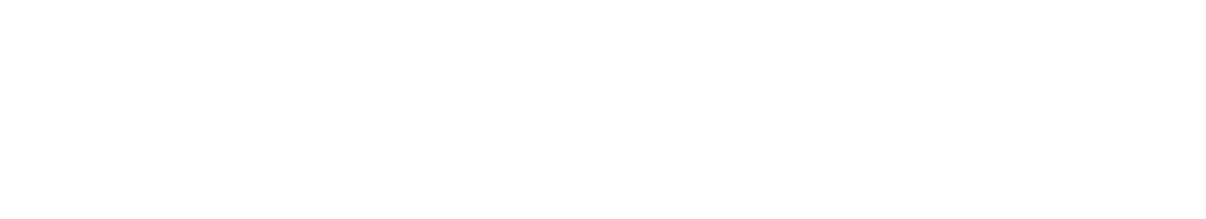



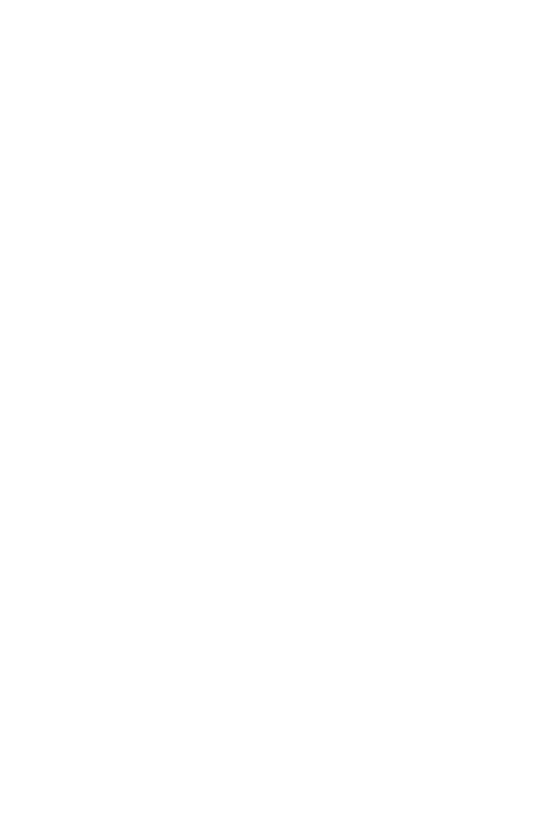

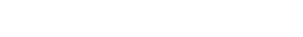
acmakanas

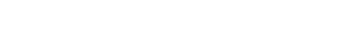

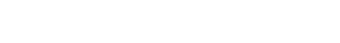
ํํำกำN

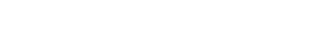

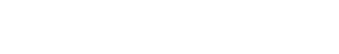
is

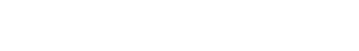
a ư

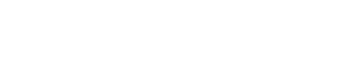

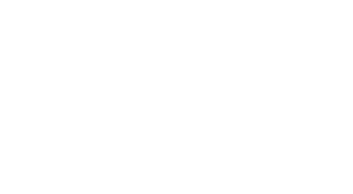

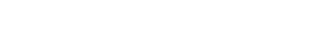
is

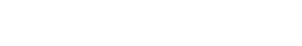

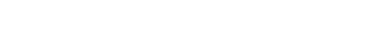

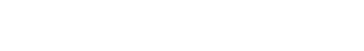

엉요있 (1) 200 แニニニニラニラニニニ

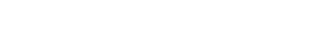

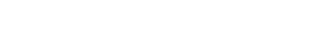


$\ddot{\circ}$

(⿸丆口

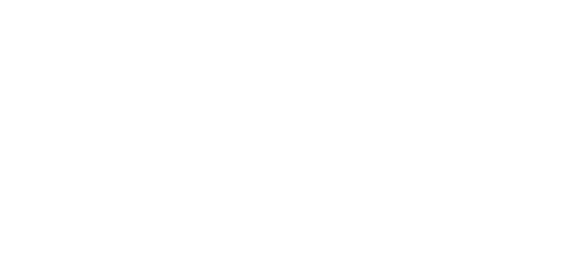

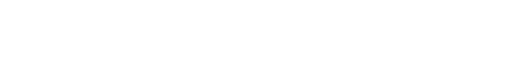

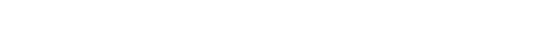

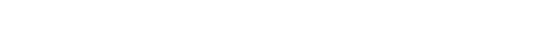

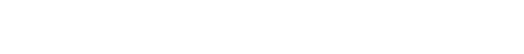

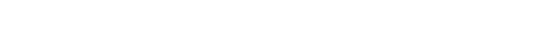
京

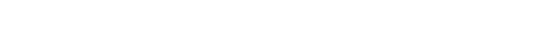

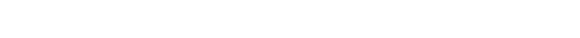

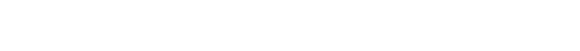

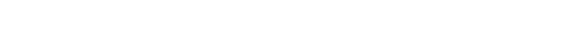
ar

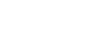

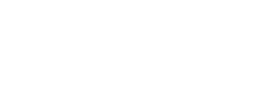

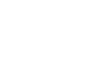

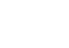
ì 可出

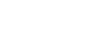
$\stackrel{m-2}{\longrightarrow}$ wio $\rightarrow \infty$ w出 a in $z \dot{2}$ जी แai แ⿻

a. 푼

它证

$a$
11

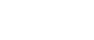

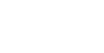

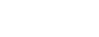

$\geqslant$

ir 2 in 2 is

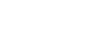

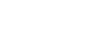
品 는

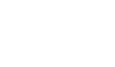

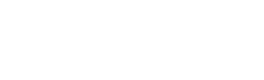

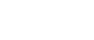

芒蓄

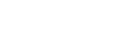

$x \quad x$ ก.

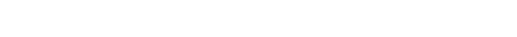

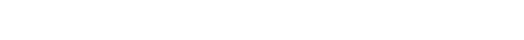

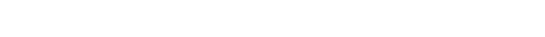

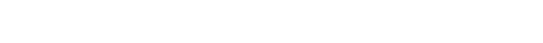
onm-OOOOONNNON-O=M

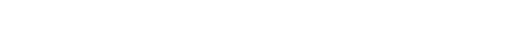

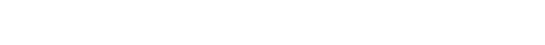
in $=\frac{1}{2}=$ ind

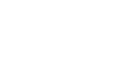
$0=2$

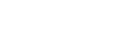
踏蕰 일

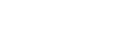
要

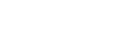

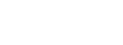

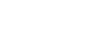

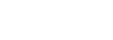
$\cong 0 \overline{0}$ 2 热出-

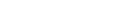
$\stackrel{5}{a}$

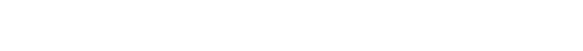
Hniñaiñ

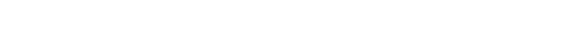

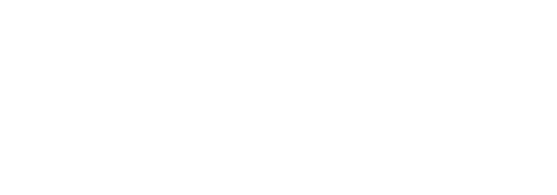

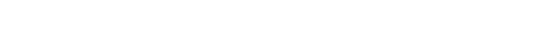
$\frac{1}{2}$

" แ艹 व

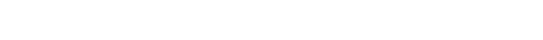

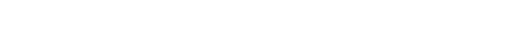

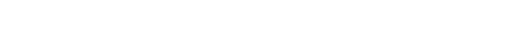

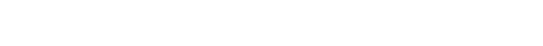

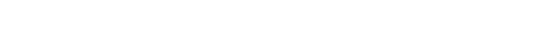

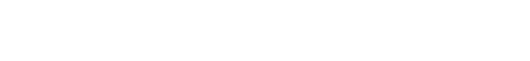



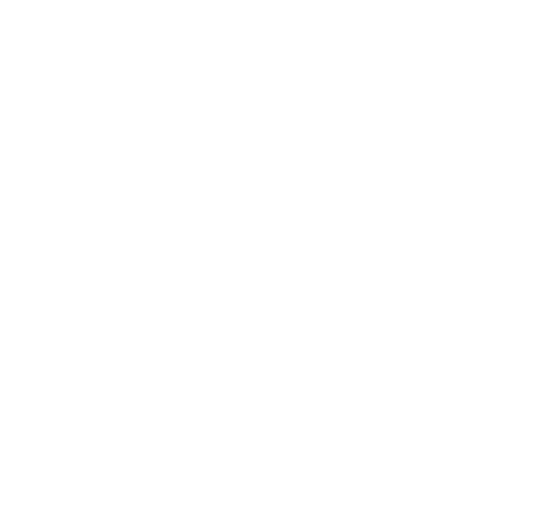

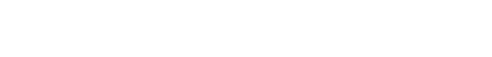

$$
\begin{aligned}
& \therefore z
\end{aligned}
$$

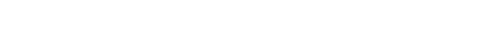
ü

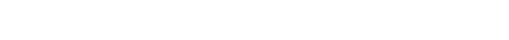

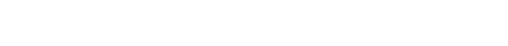

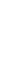

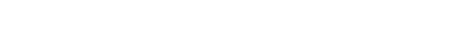

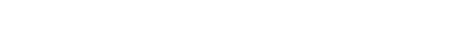

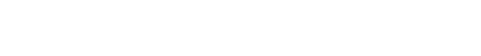

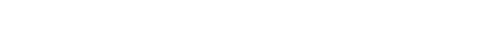

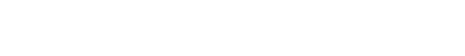

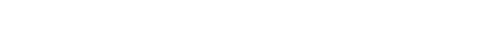
is $\vec{a} \vec{m}$. แ⿻ 宸山 -

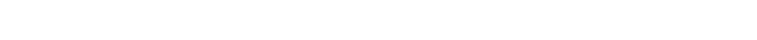

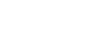

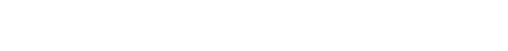

$\pi \leqslant$

Winco

$\underset{\ddot{a}}{\ddot{a}}$

30

$m=$

mooaroosoogoon

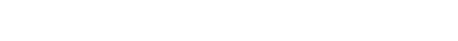

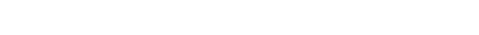

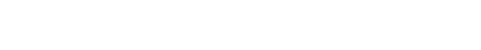

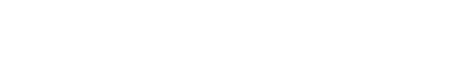

Ix

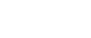

wa: in

는

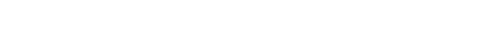

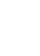

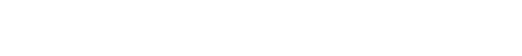

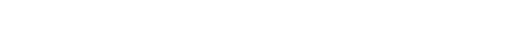

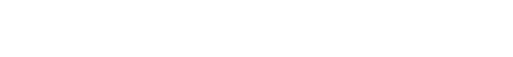

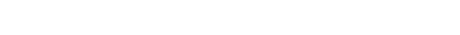

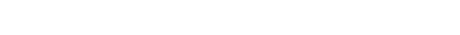

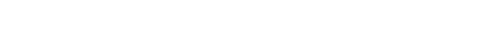

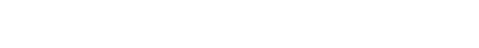

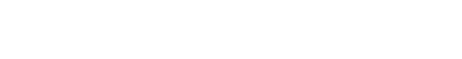



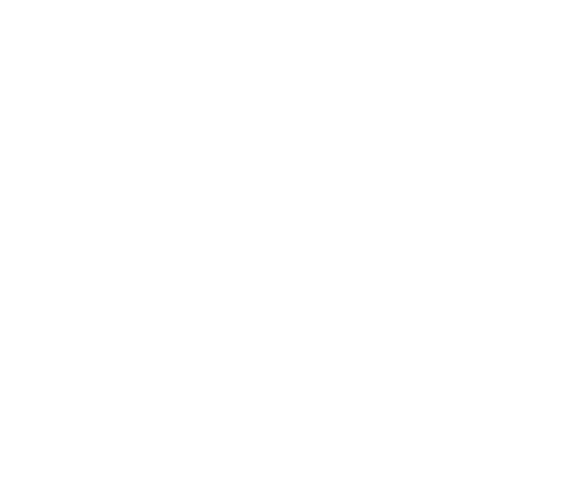

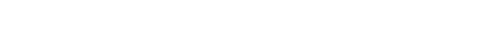

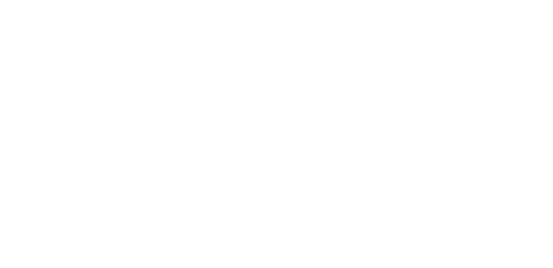

0
$\dot{0}$
$\ddot{z}$
$\ddot{z}$
$\ddot{z}$

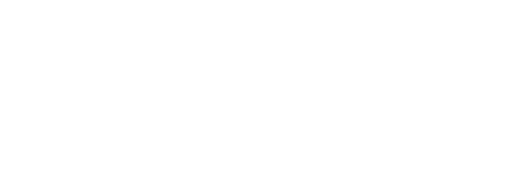

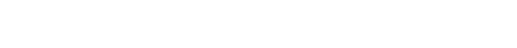

a

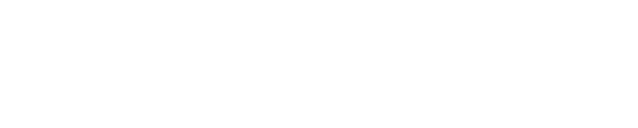

范

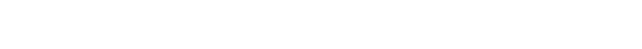

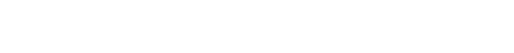

C

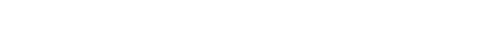

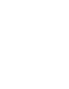

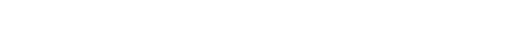

$\Sigma$

$\stackrel{5}{5}$

is

है?

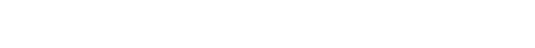

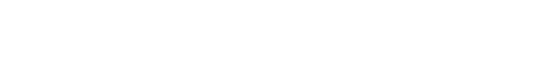

$\ddot{w}-$

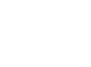

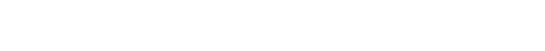

c

แ

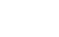

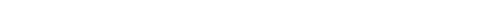

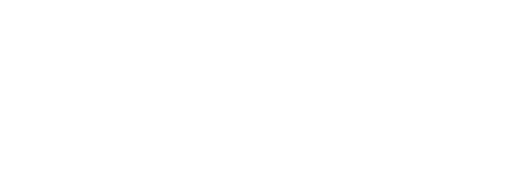



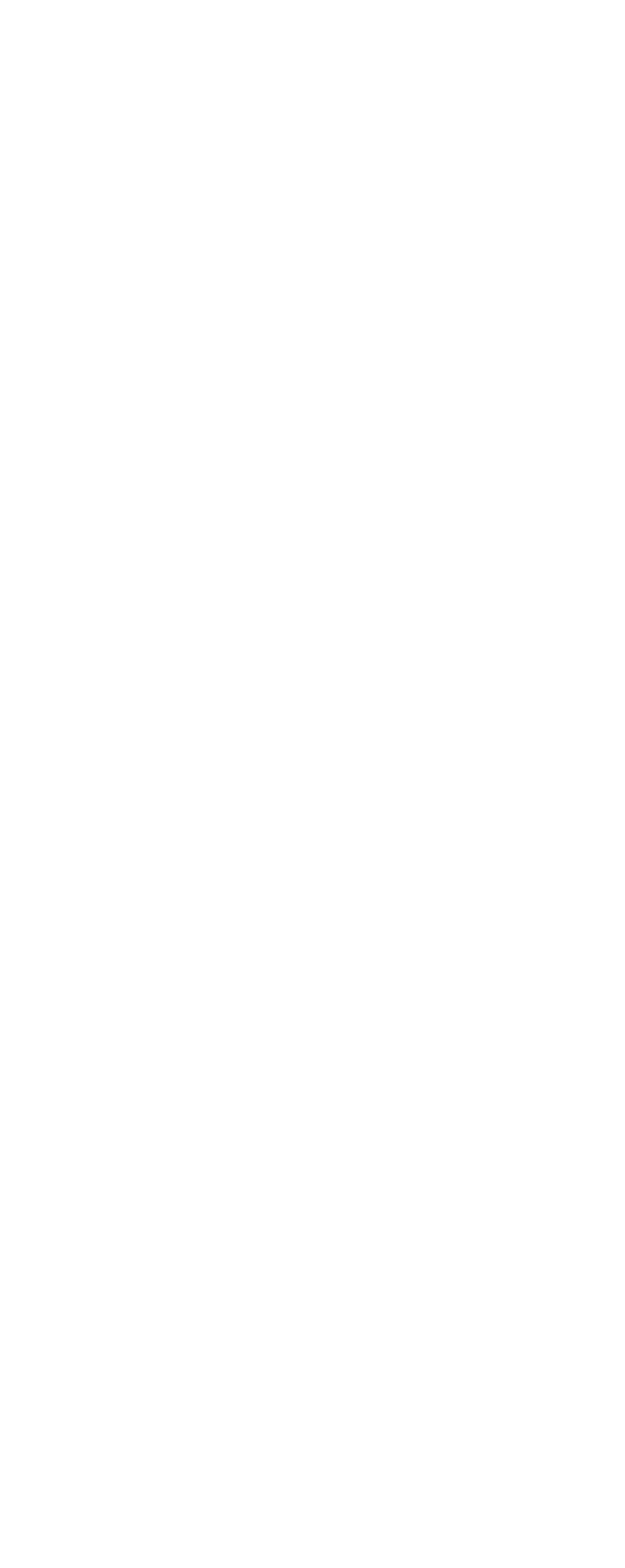

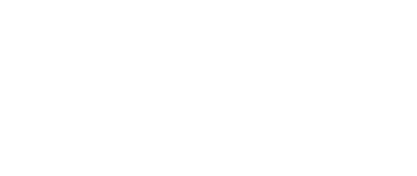

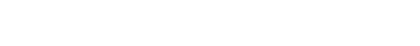
$\bar{s}=$

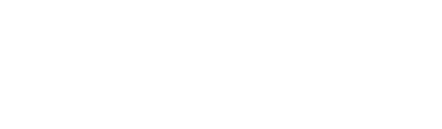

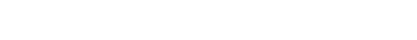

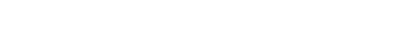

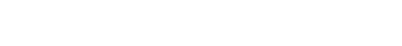

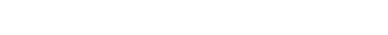

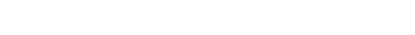
a

แñ

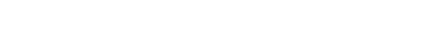

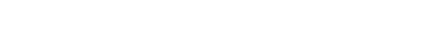
cancanaamanan

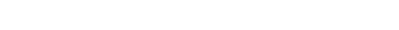
- - -

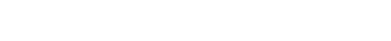

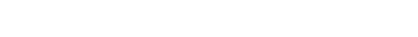

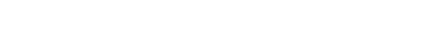

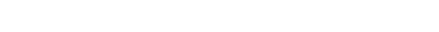
uns 둥후

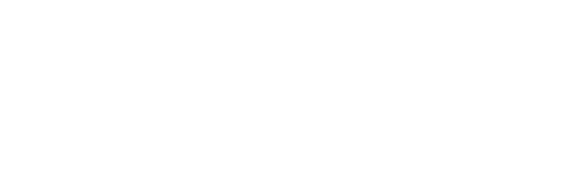




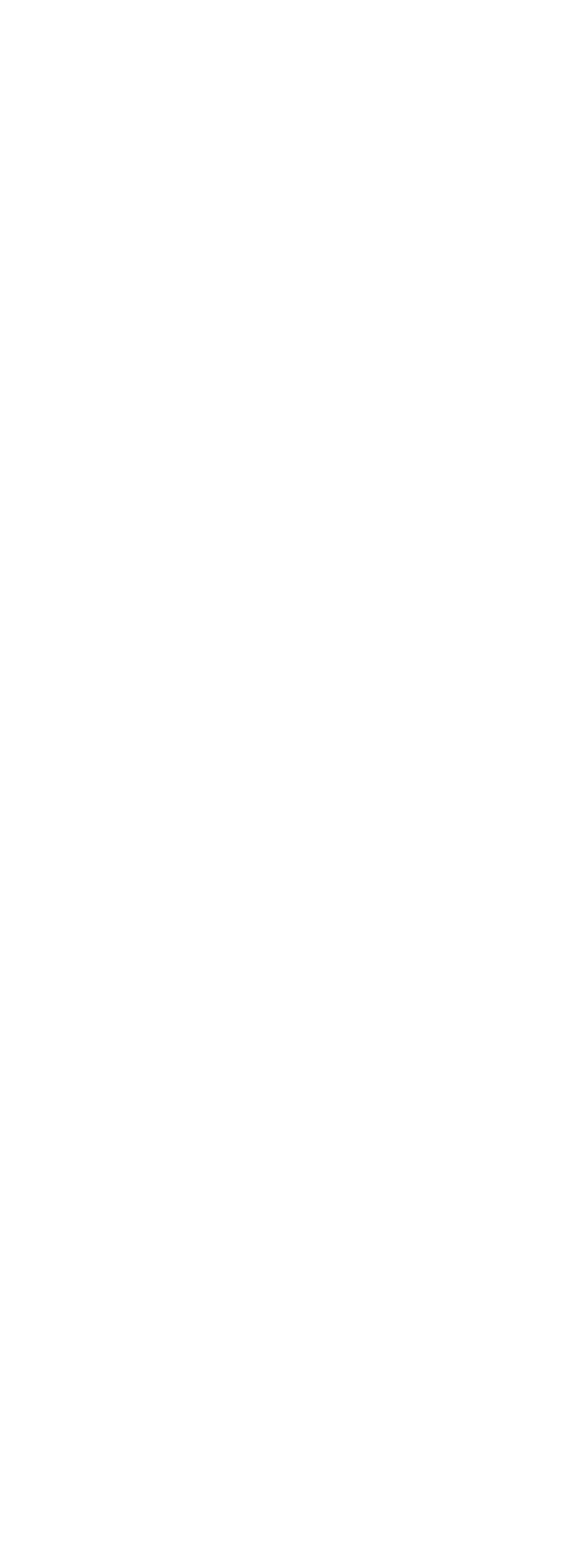




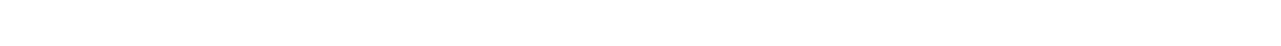
$\frac{a}{2}$

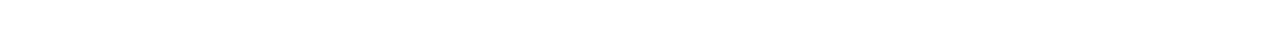
w

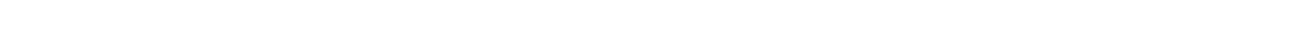
\begin{tabular}{ccc}
\hline \\
\hline
\end{tabular}

mmmmmmmmmasas

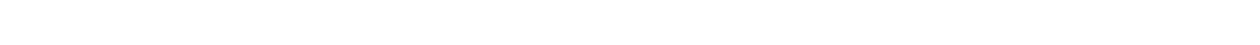

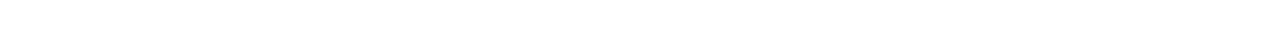

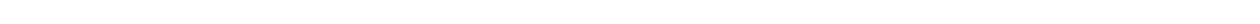

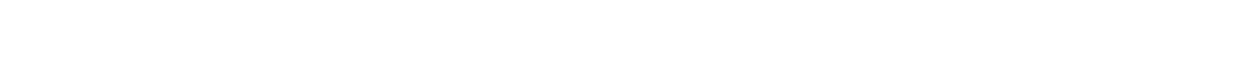

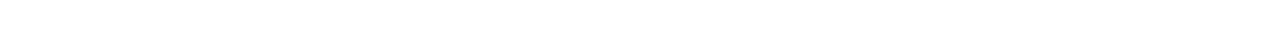
$\stackrel{a}{n}$

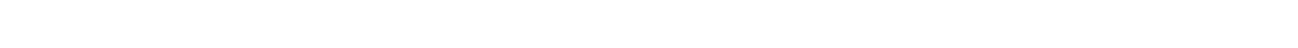

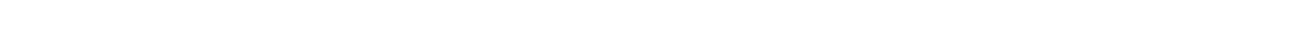

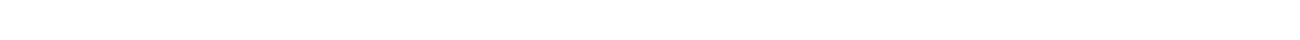

0.000000000000000-

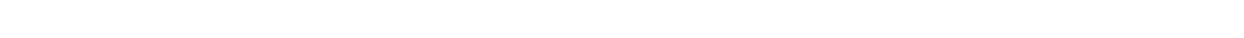

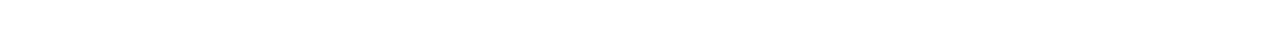

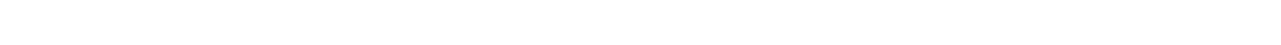

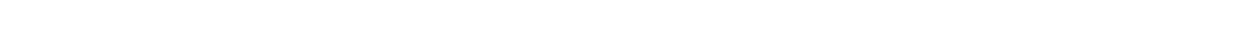

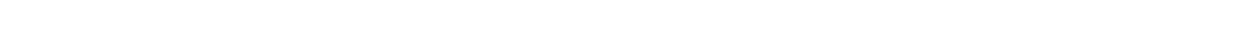

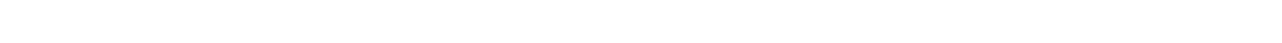

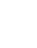

a w w w

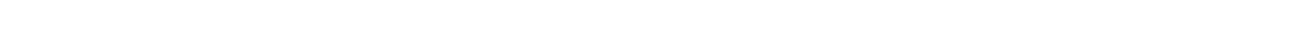

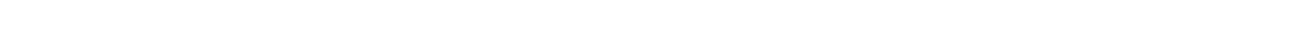

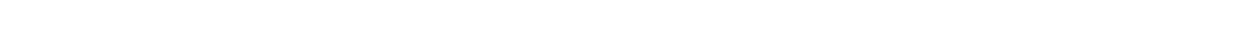
2000000000\%

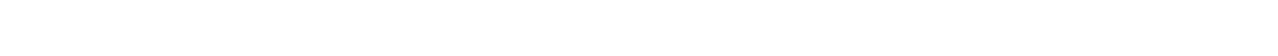

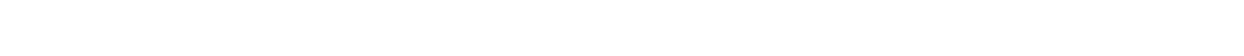

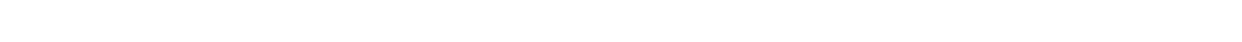

$\therefore \geq \overrightarrow{0}$ $20 \div \frac{1}{2}$ ving

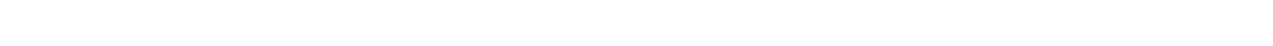

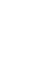

m nmm $m$ m u.

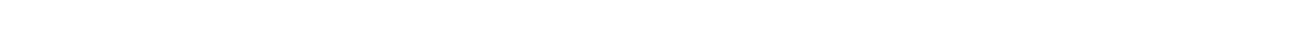

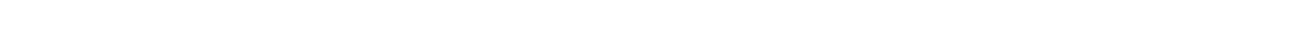

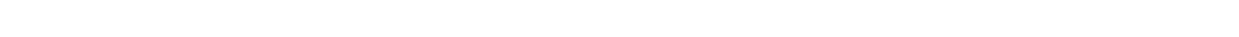

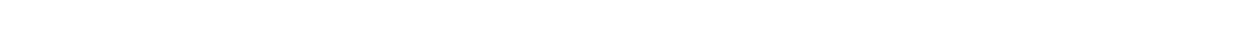

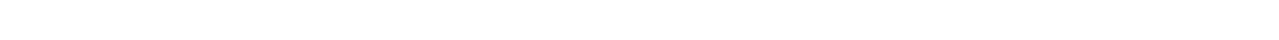

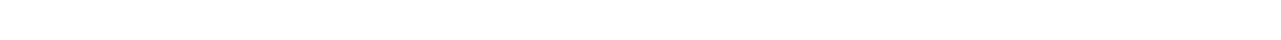

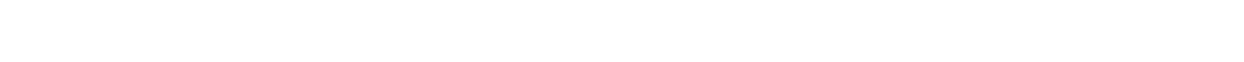



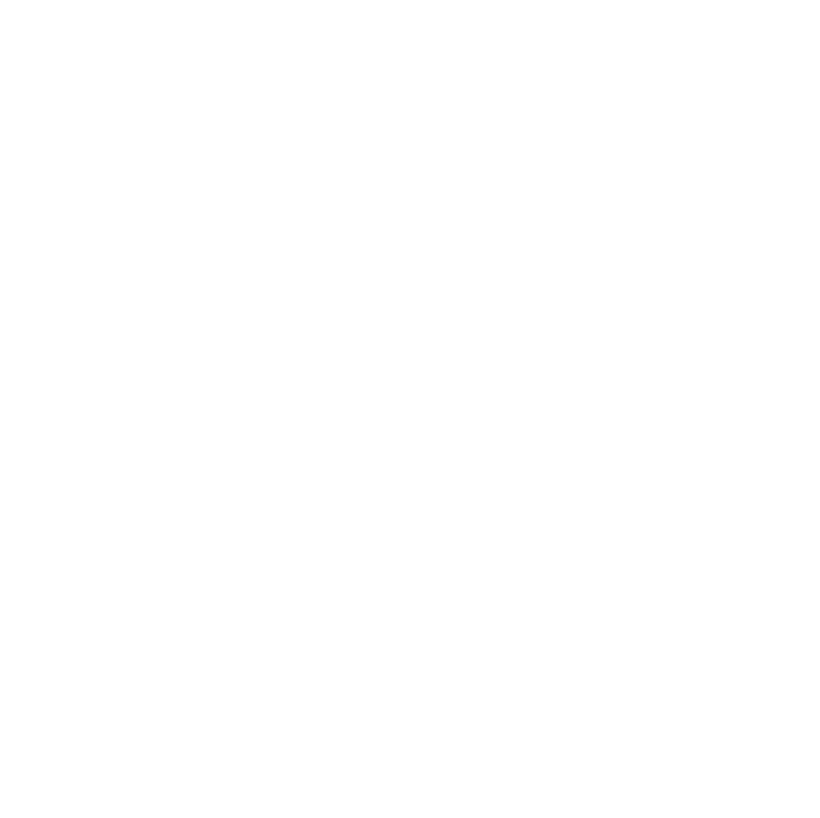

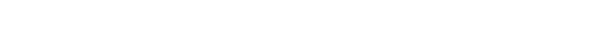

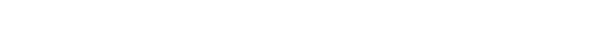

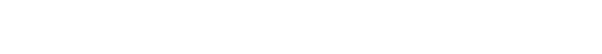

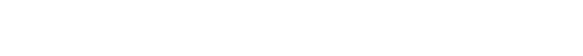

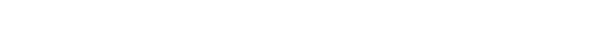

$\therefore$

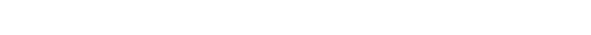

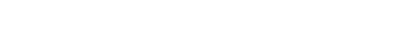

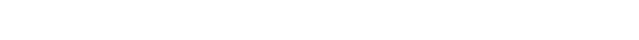

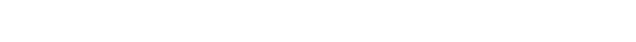

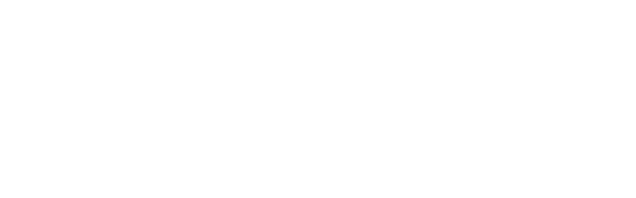

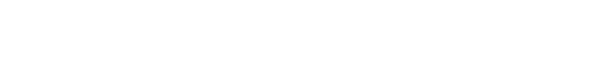
is

mmoxonmo-nnmsanmmo

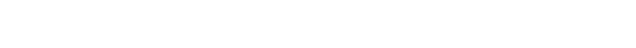

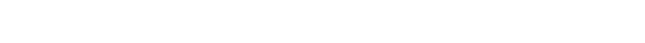

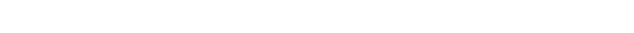

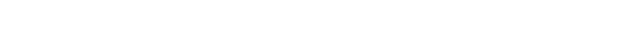

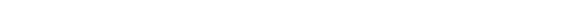

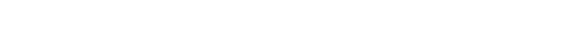

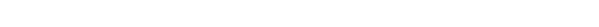

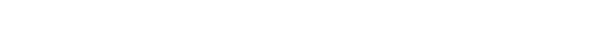

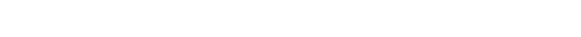

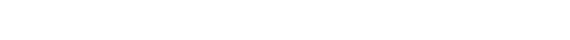




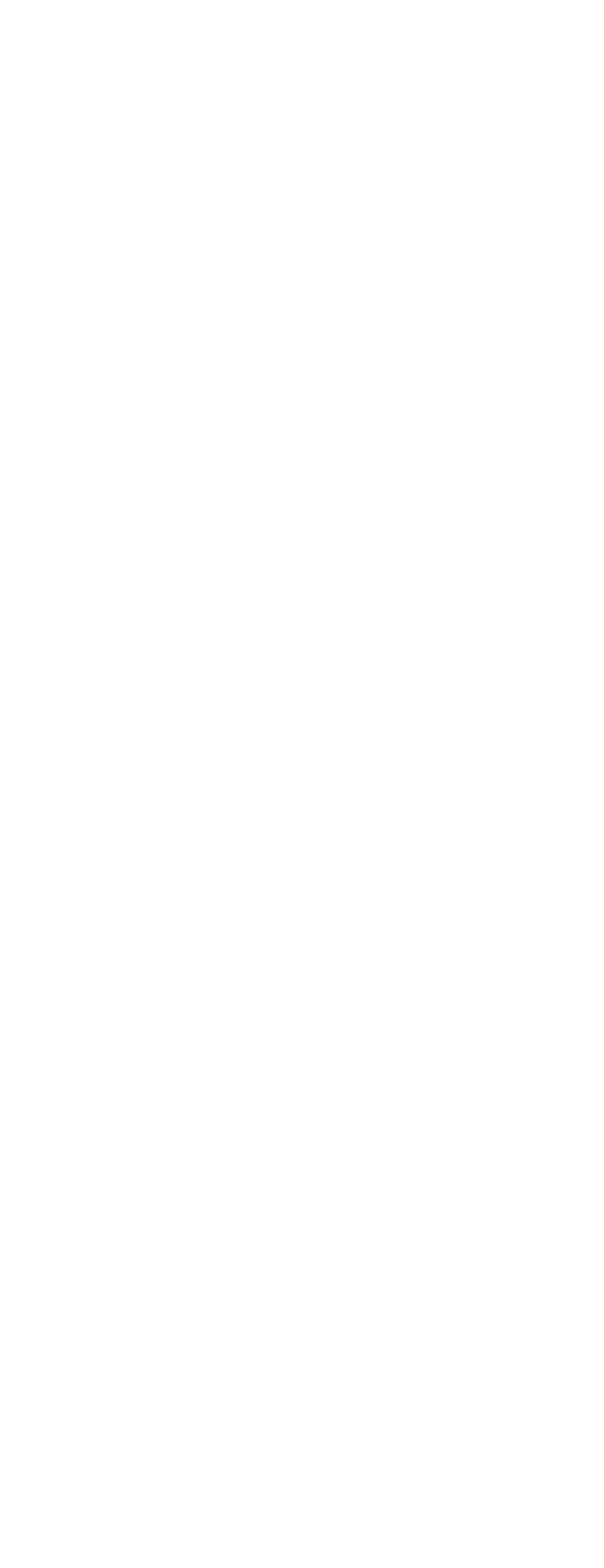




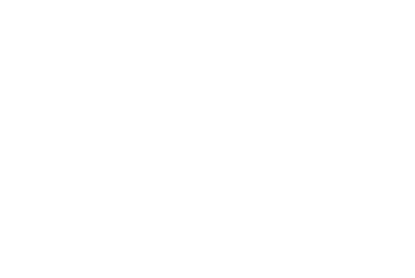

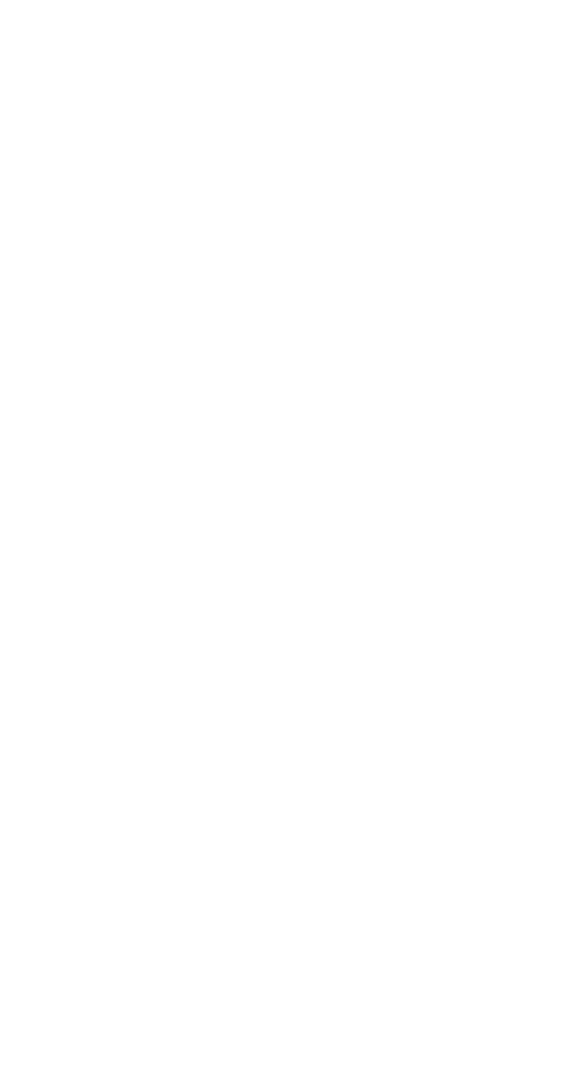

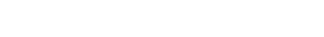

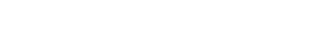

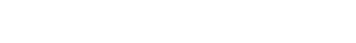

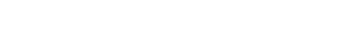
-

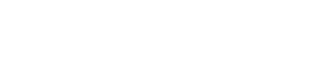

岕

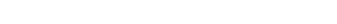

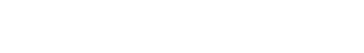
E.

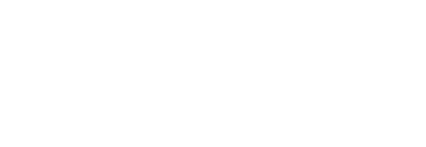
mono o o noma

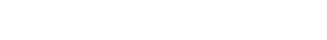

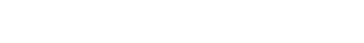

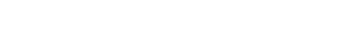

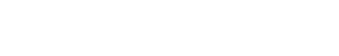



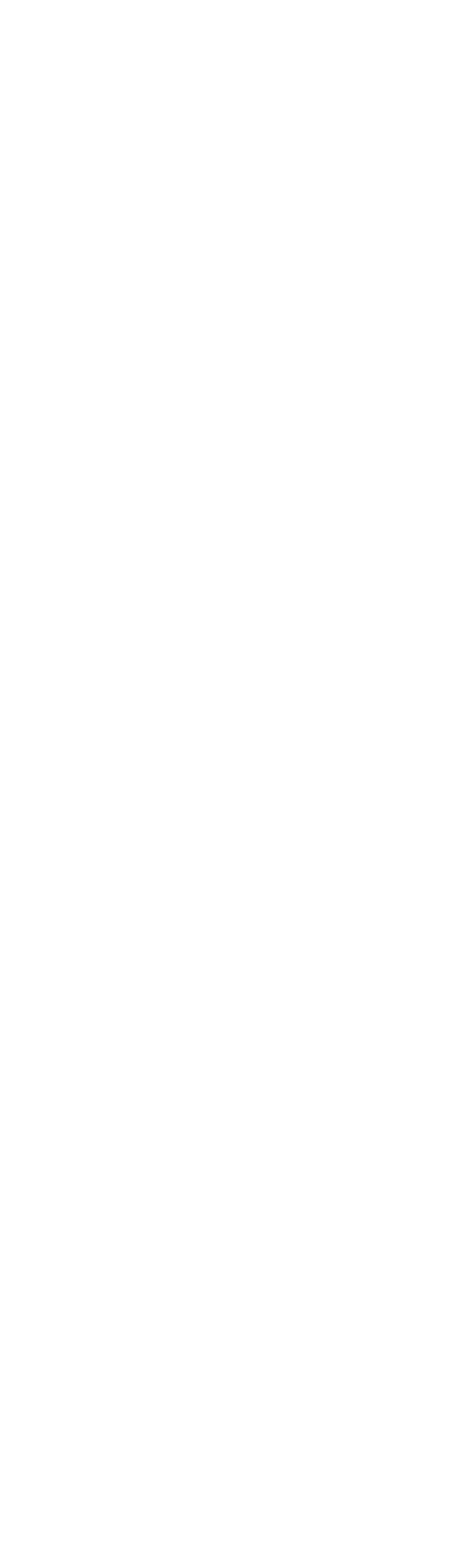


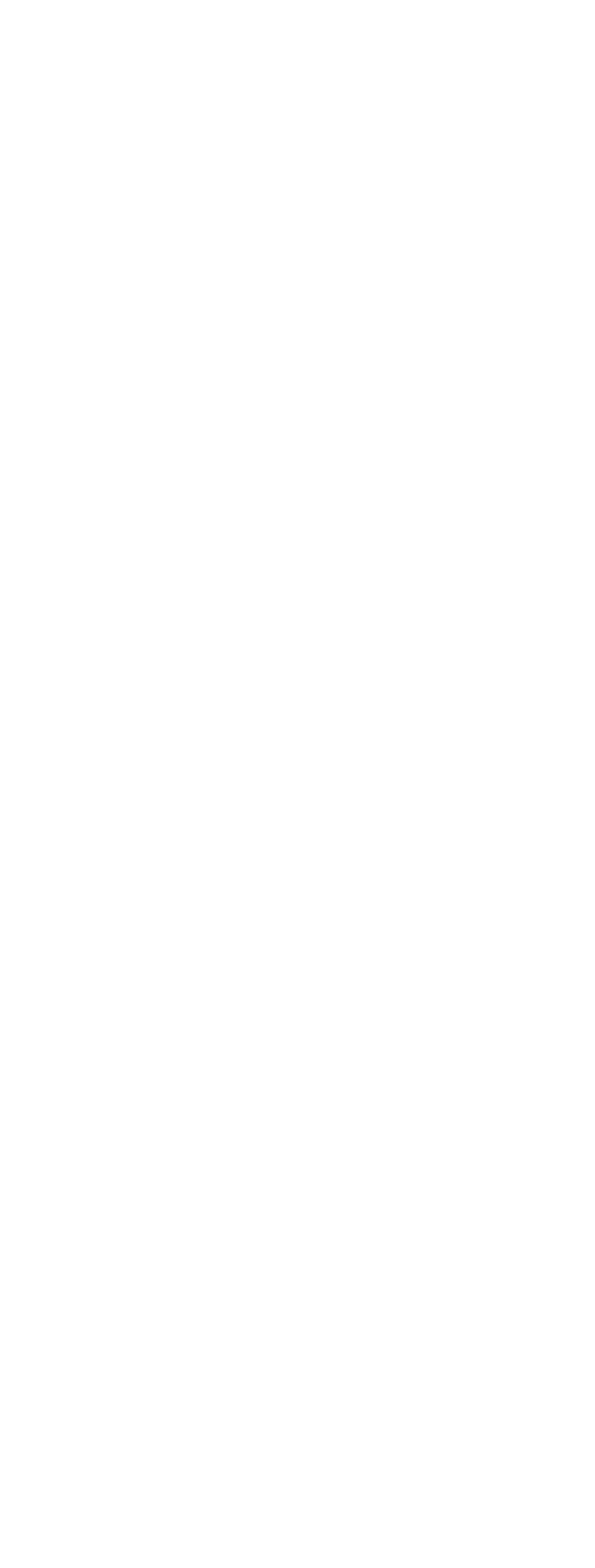

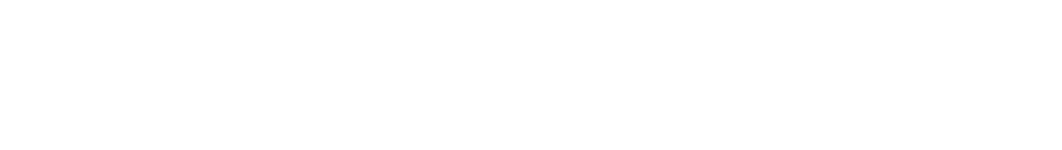

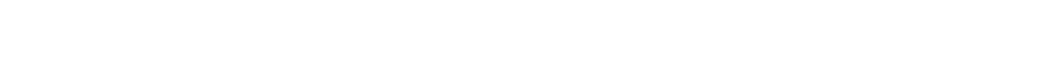

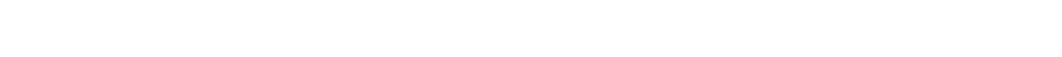

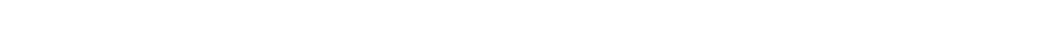

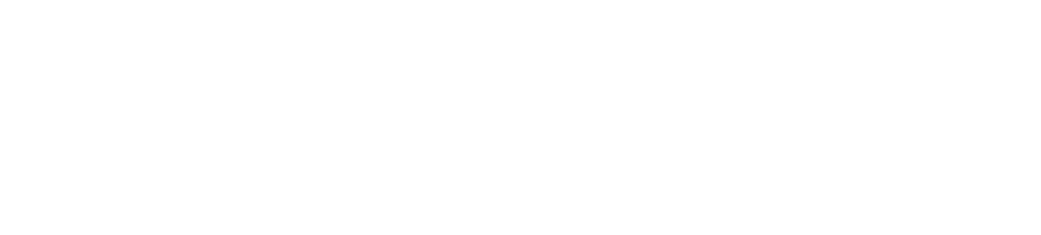

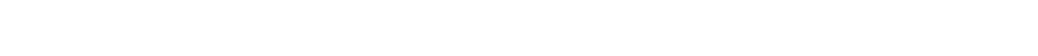

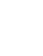

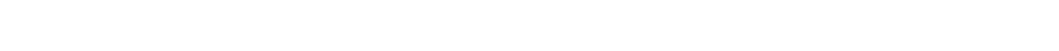

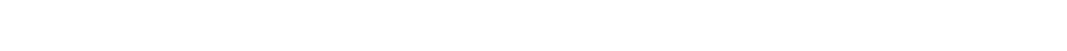

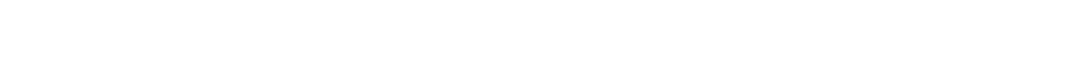

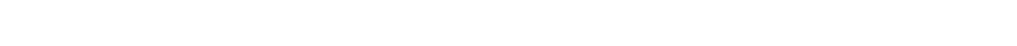

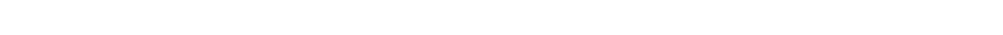

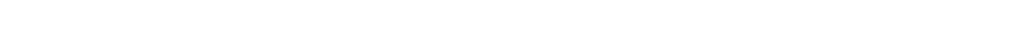

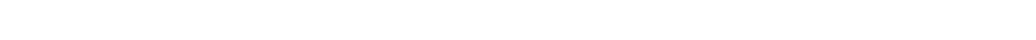

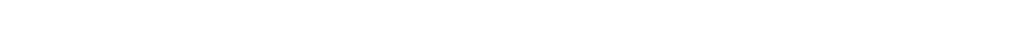

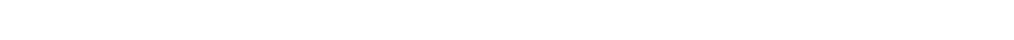

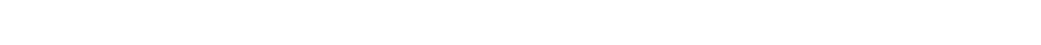
$\Sigma$

in

แ

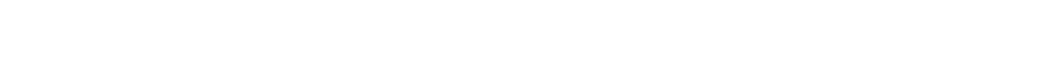

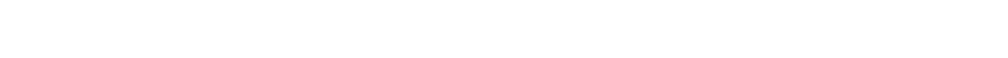

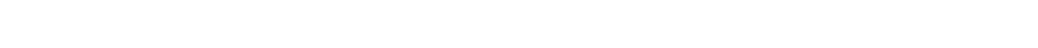

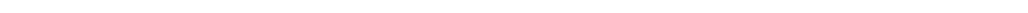

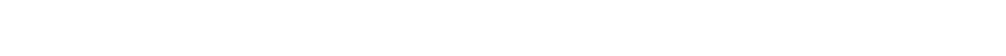

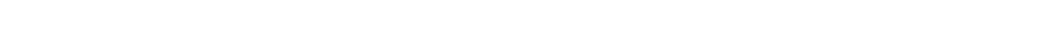
5

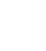

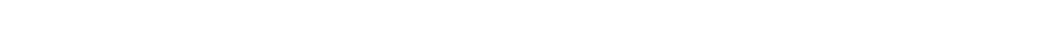
ú

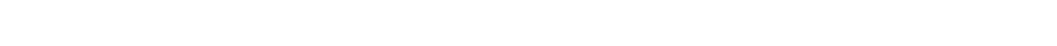

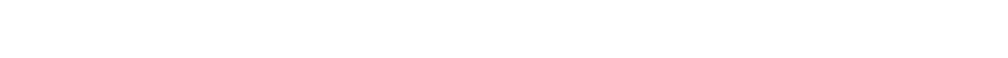

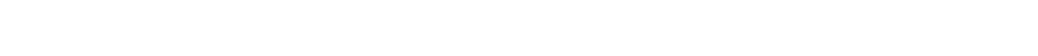

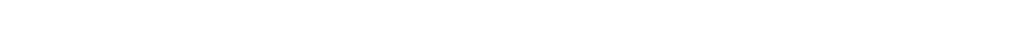

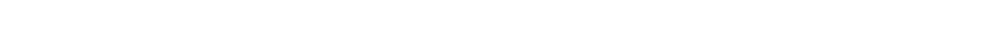


要

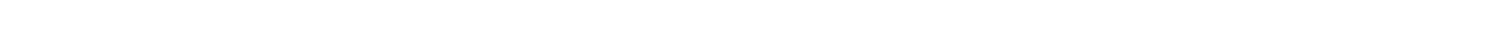
w

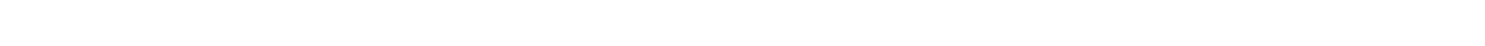

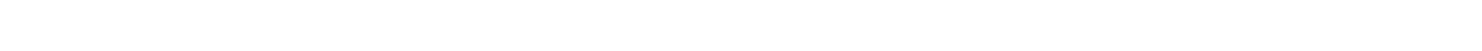
moñ

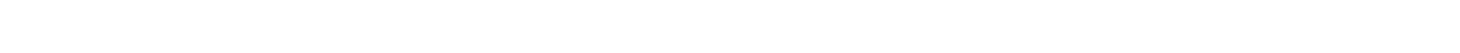

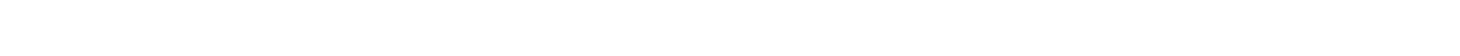

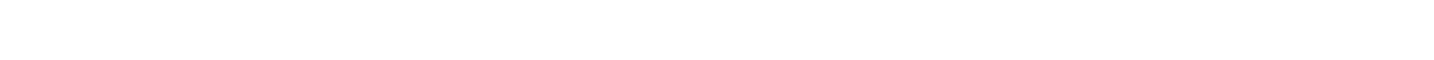

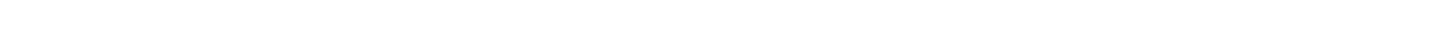
$\approx$

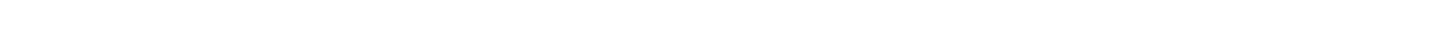
व

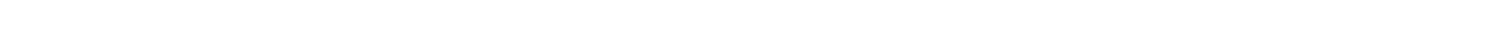

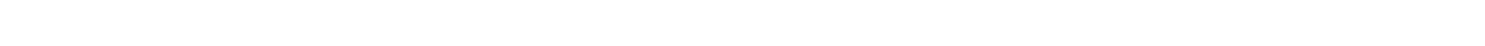

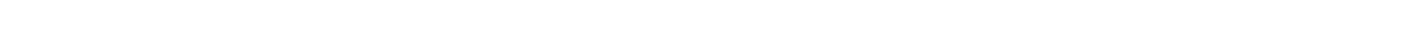

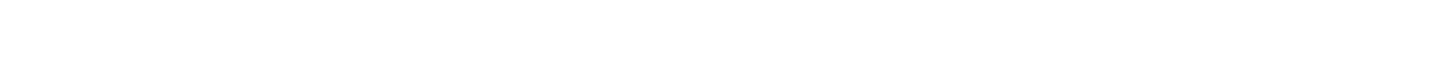

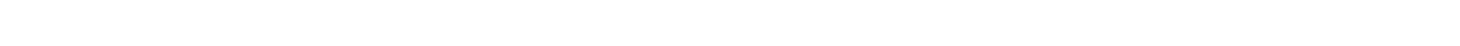

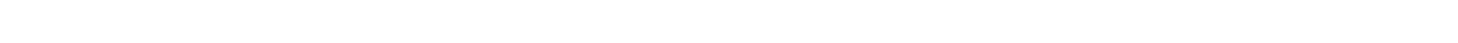

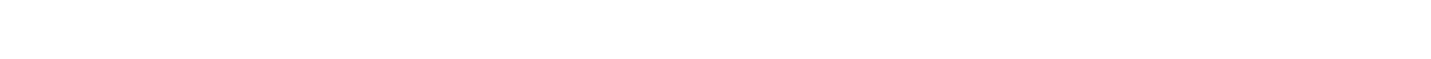

-

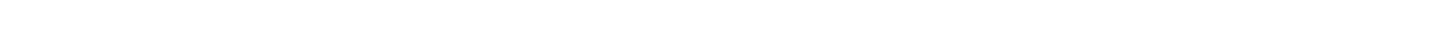
¿

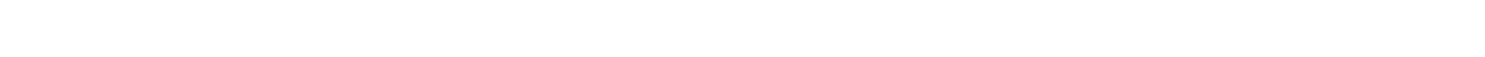

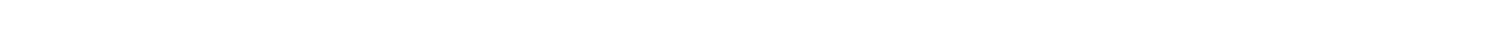

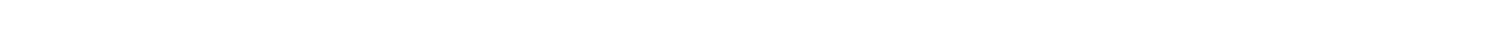

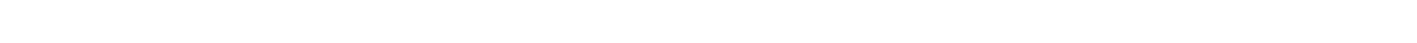

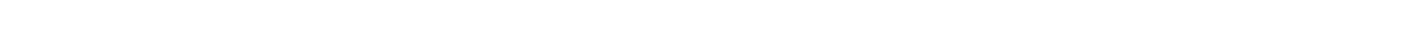

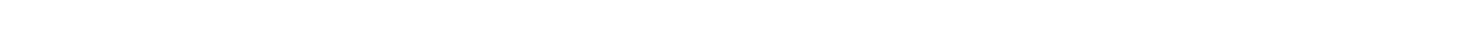

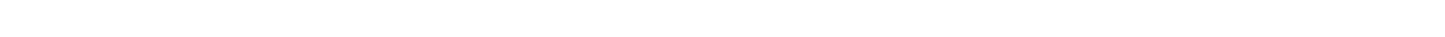

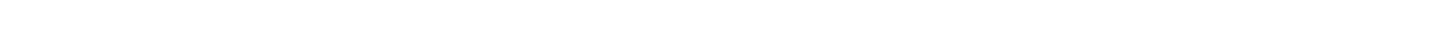

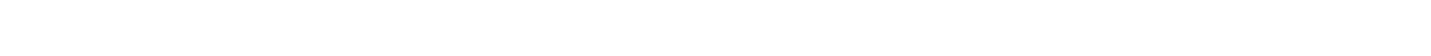

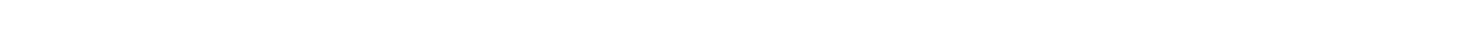

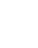

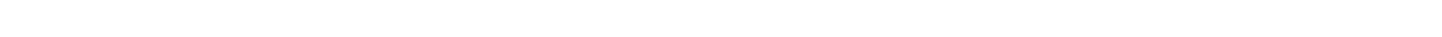

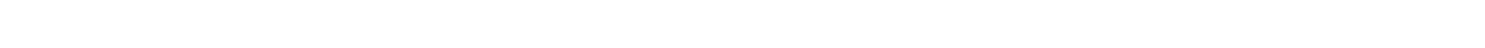

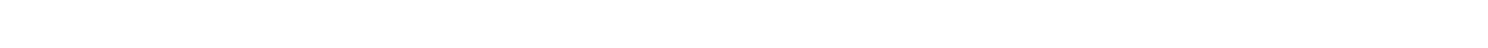

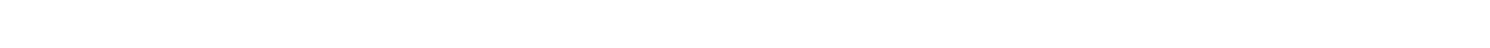
mmmmmmmm mommm

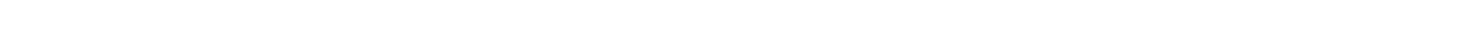

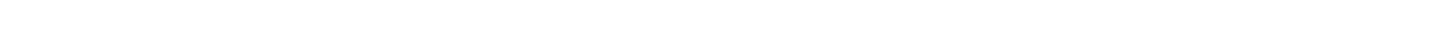

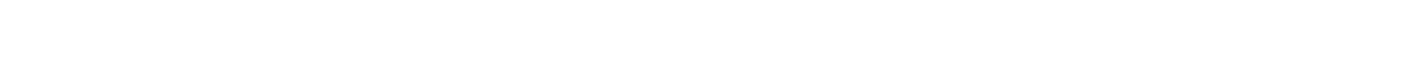




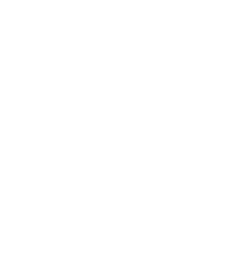

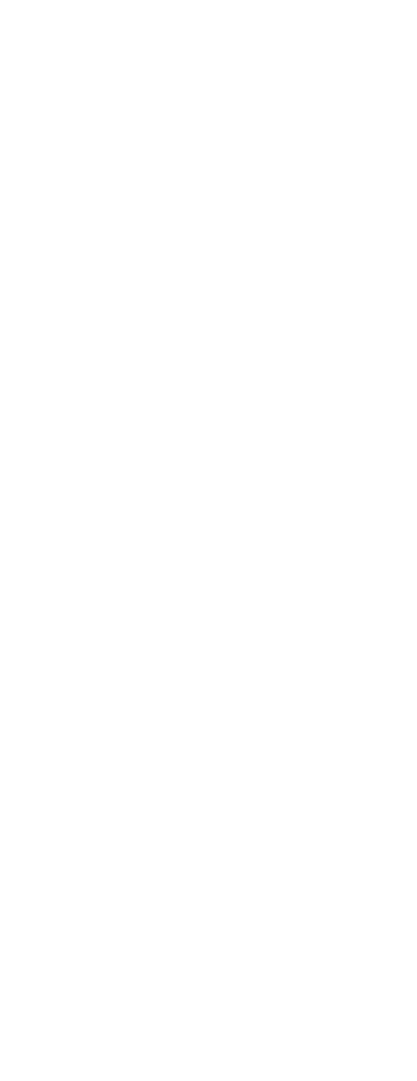

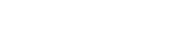
ลิกํํํํำ แேะேニะ 依合合合 Luscososonos 5 sommnam a

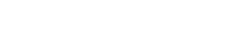

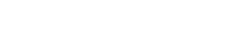

ถูำกำ

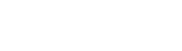

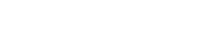
nNivisos

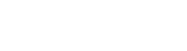



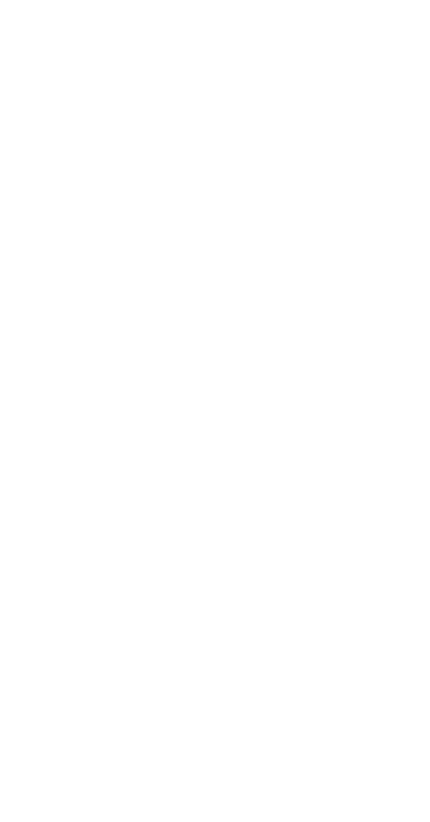

z

然

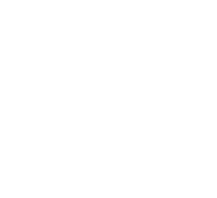

占告

웡

올

$\leqslant$

$\underline{x}$

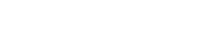

is

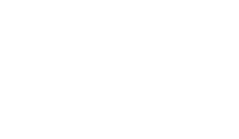

ज़

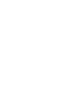

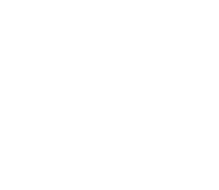

tuescosesen

$\Sigma$

$\leftarrow$

a

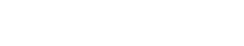

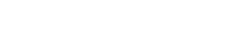

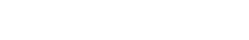

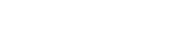
응요

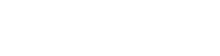
ㅇํㅇ유은 =却合的合 

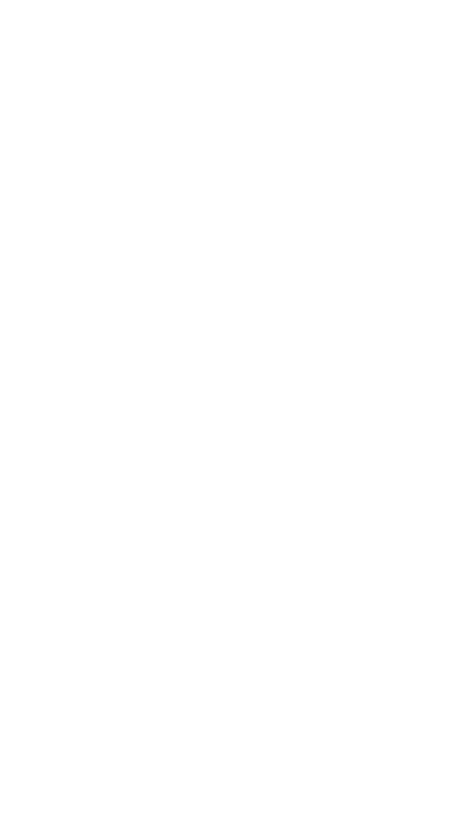

z

象

Nㅜ소웅

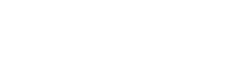

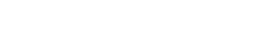
㑢去合 Tusuncenen E. 5

iñ a d a

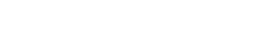

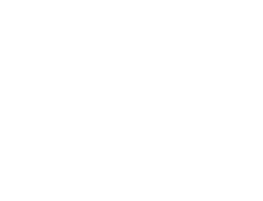
twencosicosos \& in

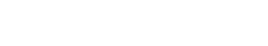

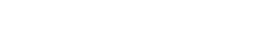

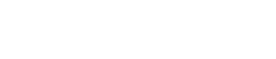

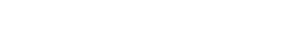

ํำ

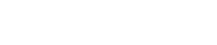
แேニニニЕニ Q NONNMON

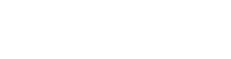




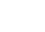

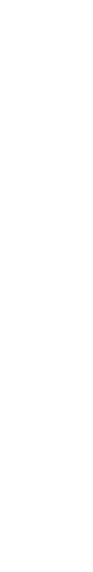
言立 ह. i: n⿺⿻一𠃋十

2上5

oxm5

E六识? EN 응

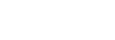

的占出

预品"

天山世

(5) 3

品。皆 >만 - : on

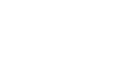
xक a in 0 的告等 zón

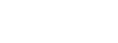
正的 uin <0 0- 0 $=1 \infty$ 인 खें 点。

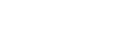
แ过 ㅇㅗㅀㅣㄴ 讪引 它. ๙ปฐ

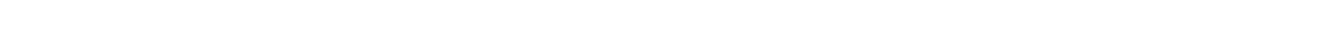

i

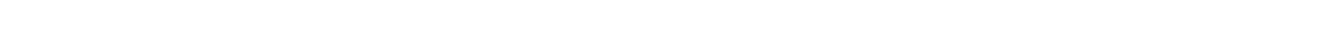
แ山.

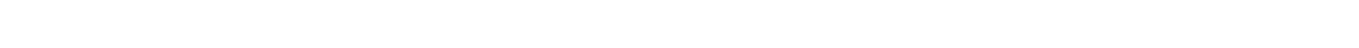

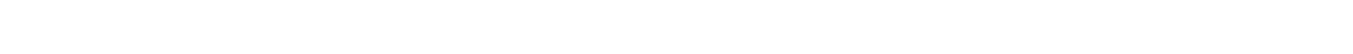

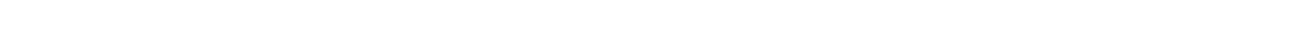

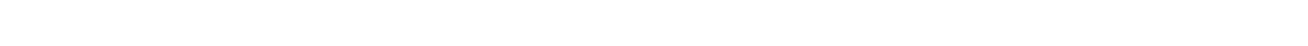

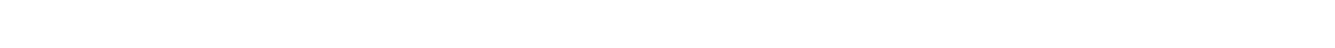

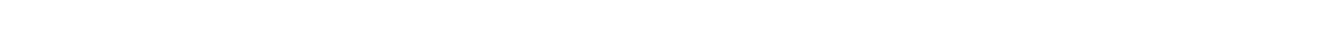

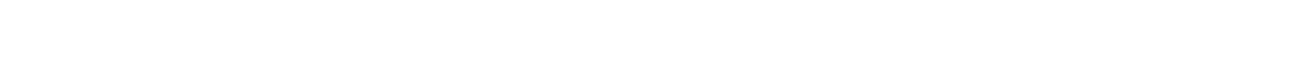

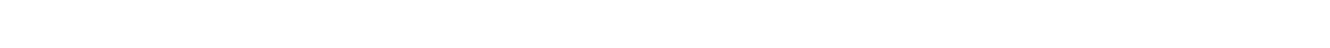

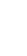

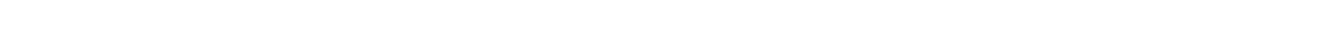
w

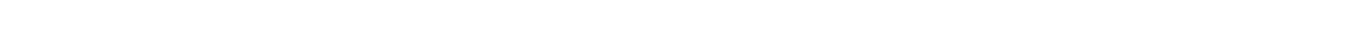
a

$\circ$

?

$\frac{1}{5}$

$\checkmark$

$\checkmark$

푼

0

운

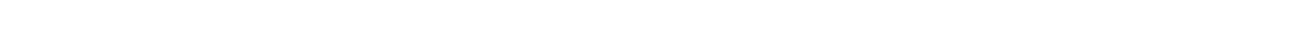

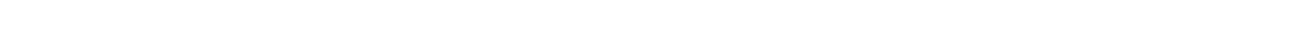

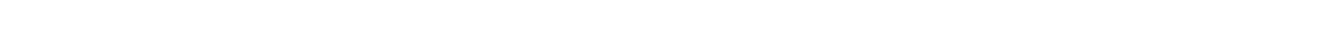
¿

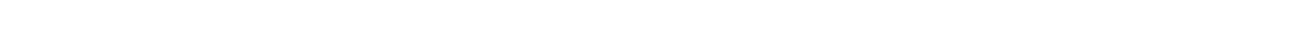

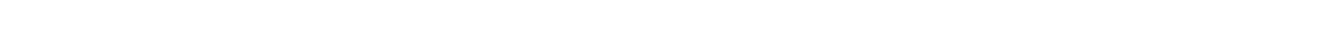

$\stackrel{x}{2}$

× ü

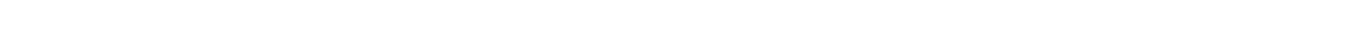

пимn ñon 世

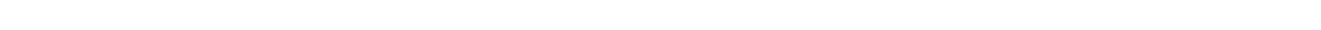
음ำm

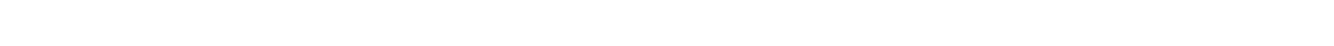
$\stackrel{-}{\leftarrow}$

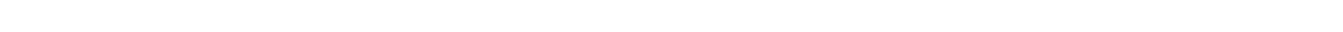
uш

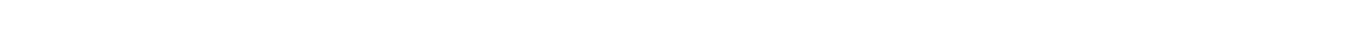

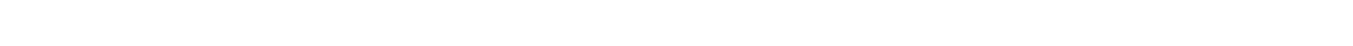

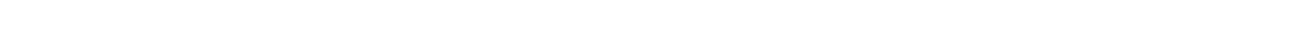

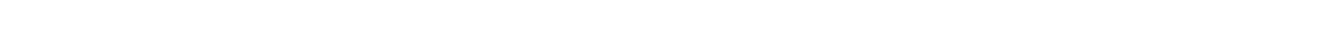

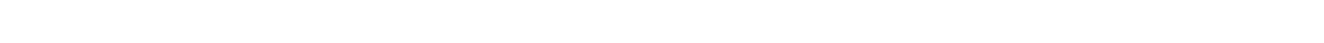

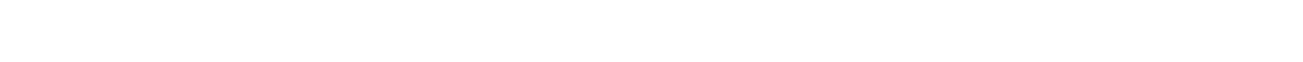



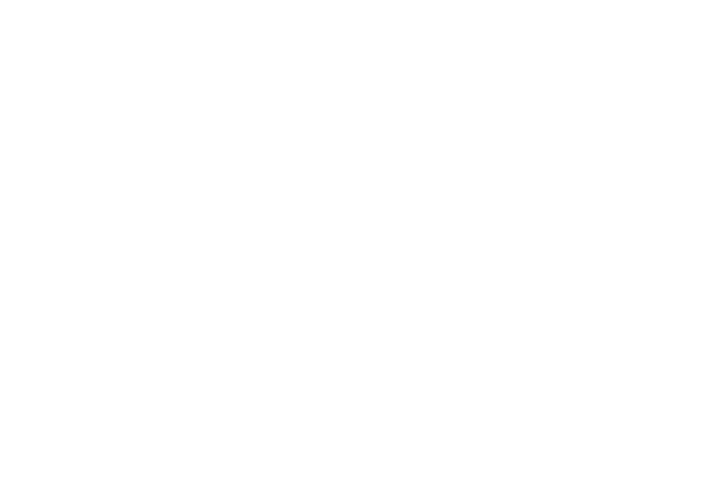

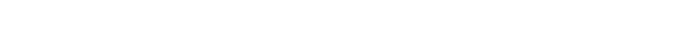
$\Sigma$

$\stackrel{5}{n}$

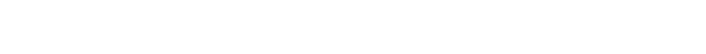


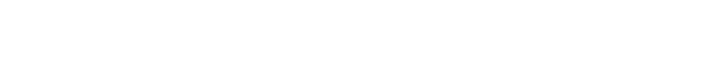

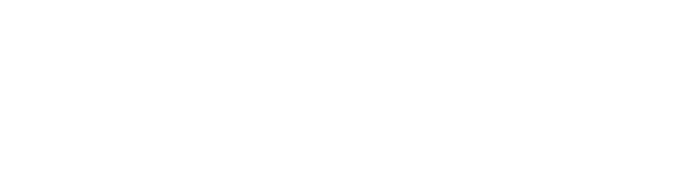

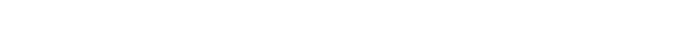
$\Sigma$

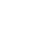

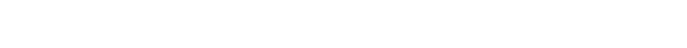

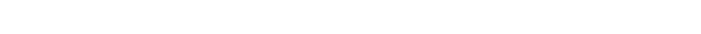

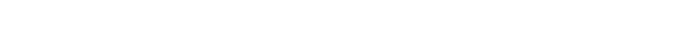

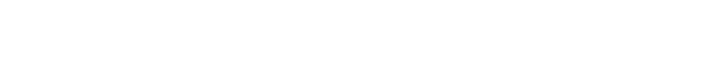

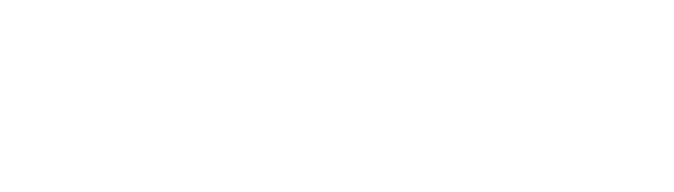

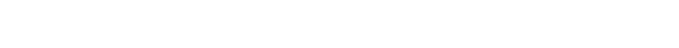

is

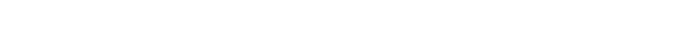

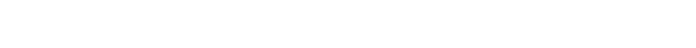
๘ 3
5

NANNANRNANRN

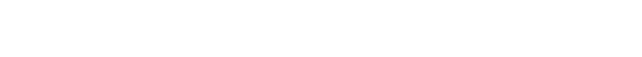

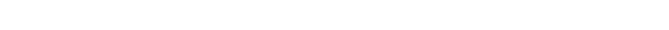

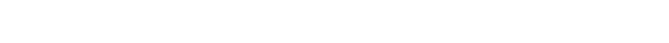

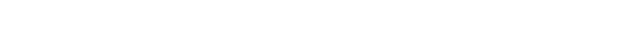



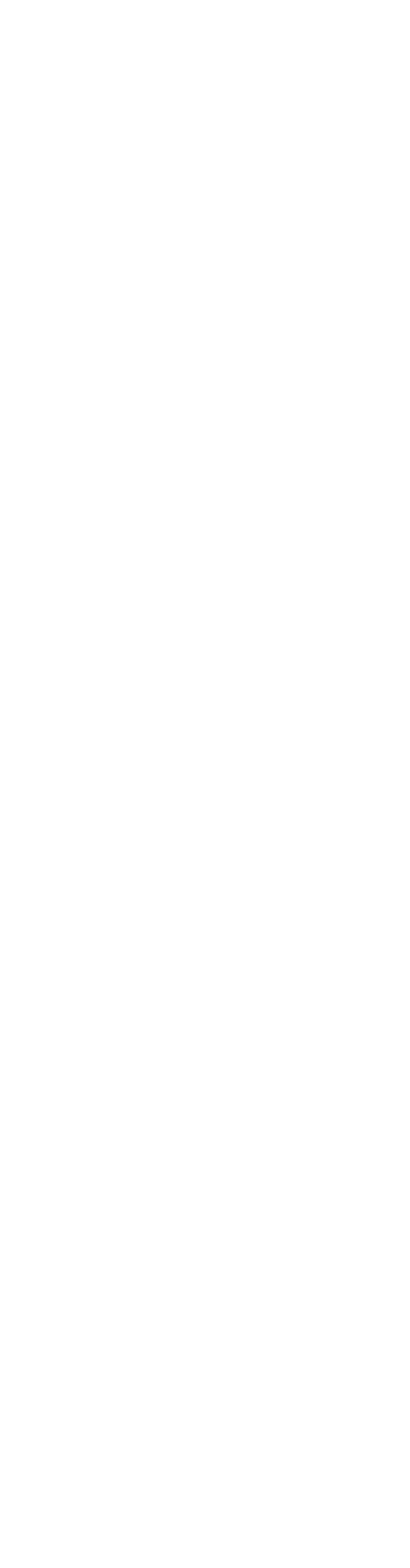

w

tusesesesenes

in

nosomn แ山山 से

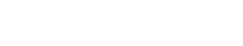

ํํㅇํำ每舟。 ํㅜㄴำ은 แ゙ニニニニニ

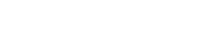

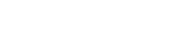




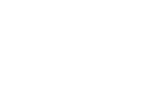

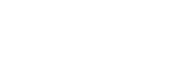

a

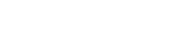

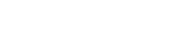

t $-n$ no

$3=\left\{\begin{array}{c}0 \\ 0\end{array}\right.$

2 200 出

단삐

-

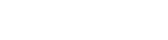

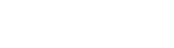

(t) $\alpha$ a 0

: - - -

느는

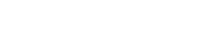

$\ddot{u}$

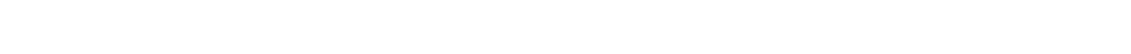

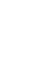

"n o ж

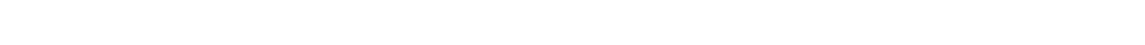
“

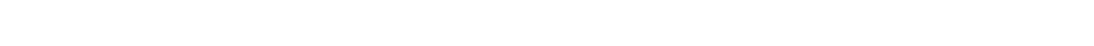

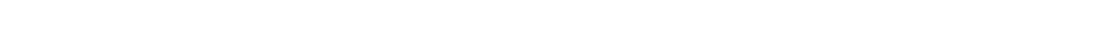

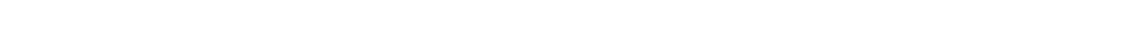

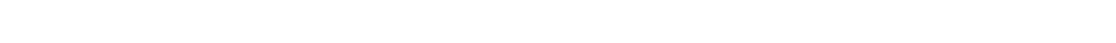

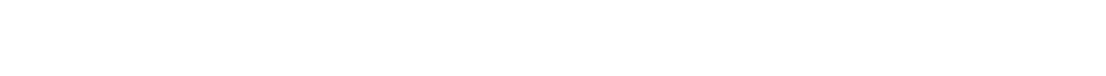

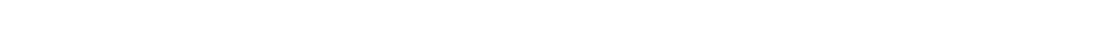

$\pm$

a

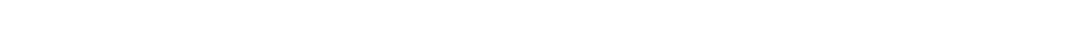
ผ " w

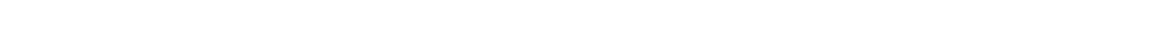

and

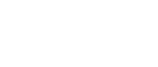

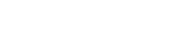

- inu.

a 00 m n

$\because=こ ュ 1$.

แ

- O幺दzu

in 응

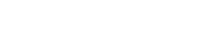

ris is a

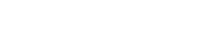

$1 \mathrm{~m}$

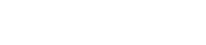

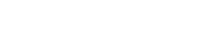

waviz.w

- o 1 a

$\overline{0}=\bar{s} \div$ c

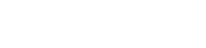

in

$=n>m i 0$ 品

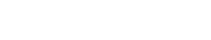

and

mo:

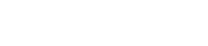

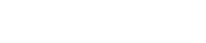

응어엉.

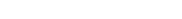

-

แ"1 9 a

4)

w.

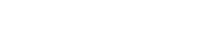

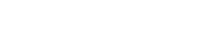

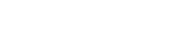

品りこ㟧李?

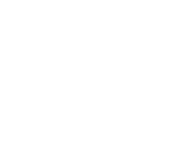

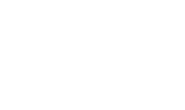

$=5-5$

$=\equiv-\frac{2}{0}$

i.

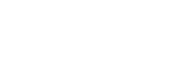

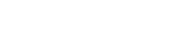

- 0 的台

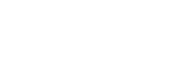
ㄴ은 i a $z$

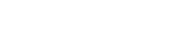

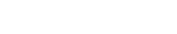
こニニュ三出

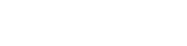
inainon 아의 - ${ }_{1}, \ddot{w}-\ddot{w} \sim$

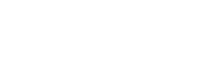

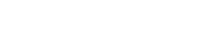

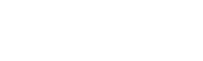
命㟧 w

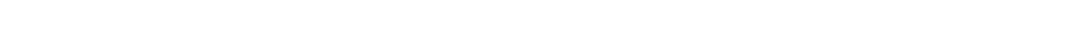

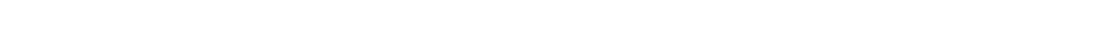

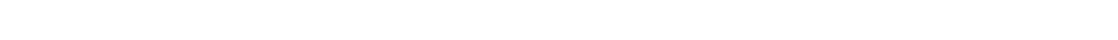

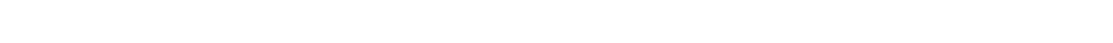

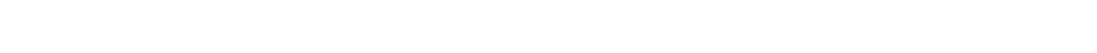

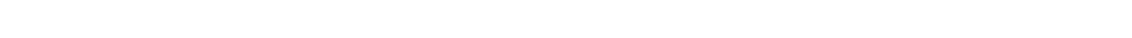
$\pm$

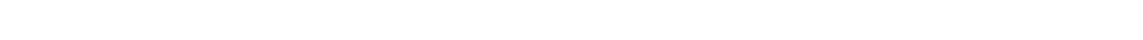
"H

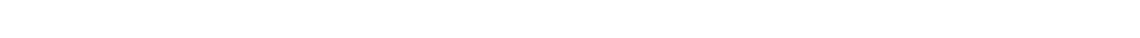

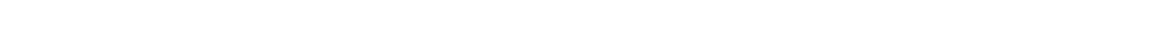

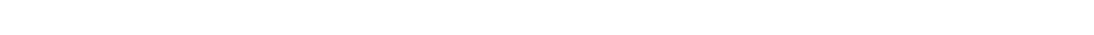

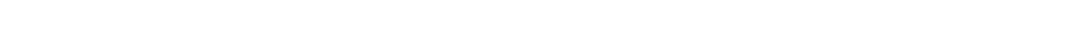

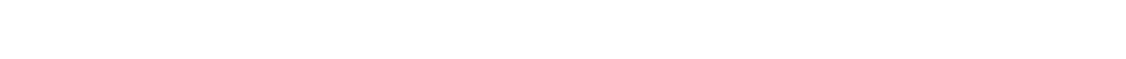
oNNRN

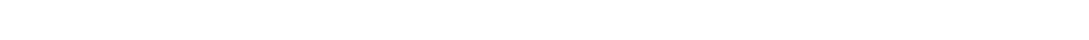

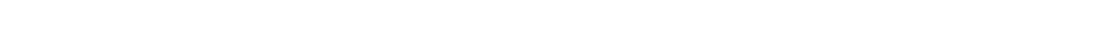
is

ำ

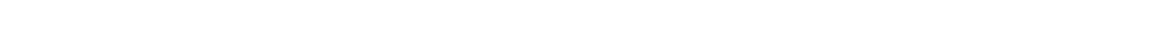

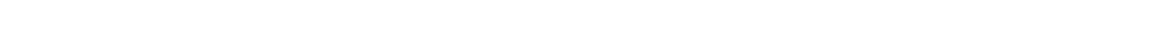

009000000000000000000000000000000000

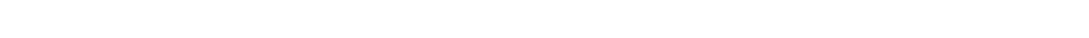

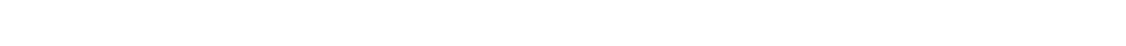

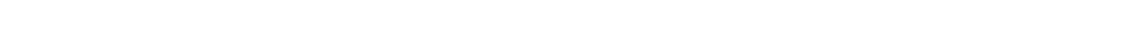

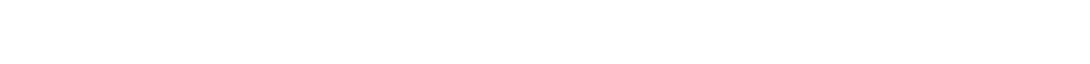




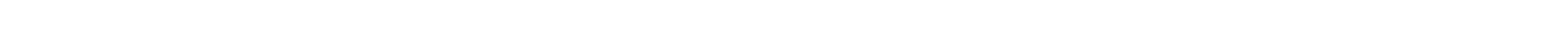

is

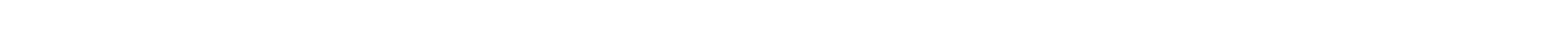

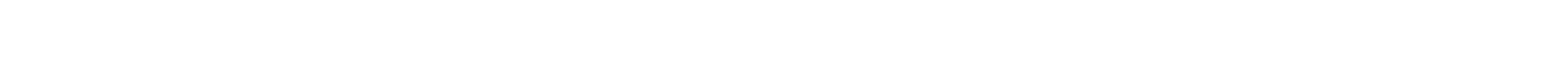

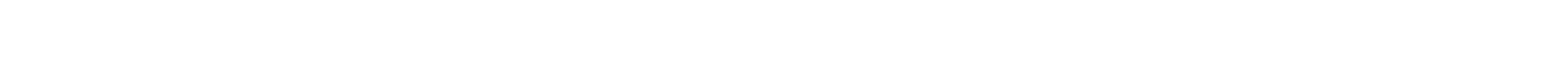

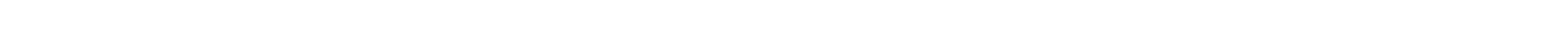

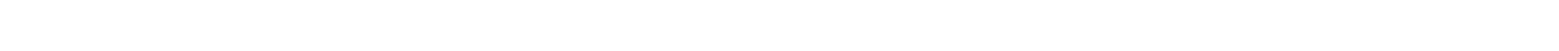

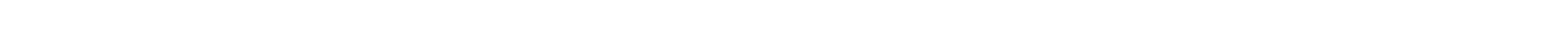

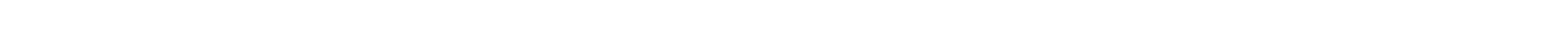

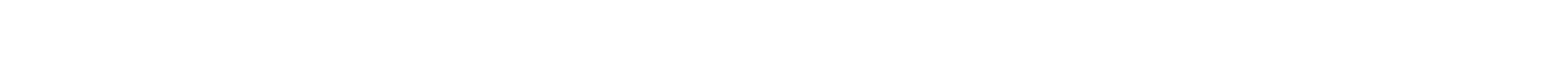

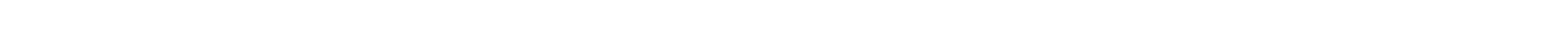

$\underset{\leftarrow}{\leftarrow}$

"은

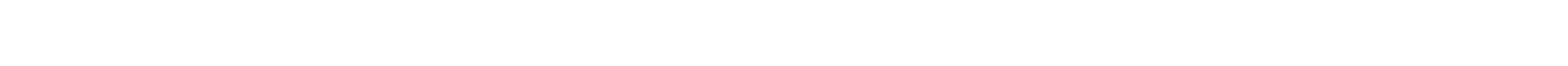
\begin{tabular}{lll}
3 \\
\hline
\end{tabular}

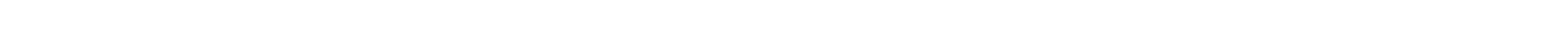

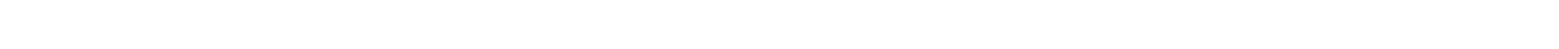

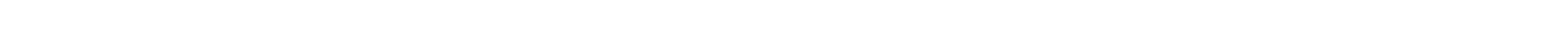

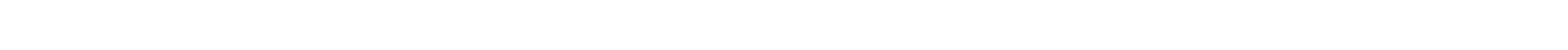

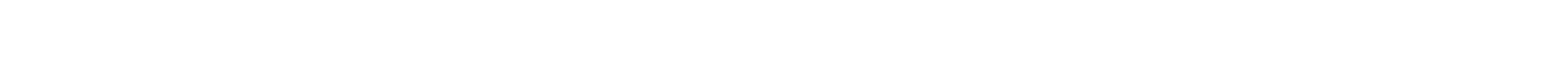

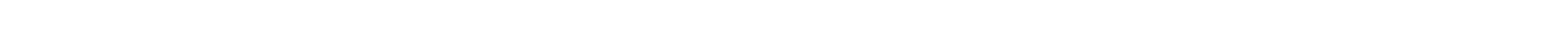

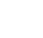

is

"น

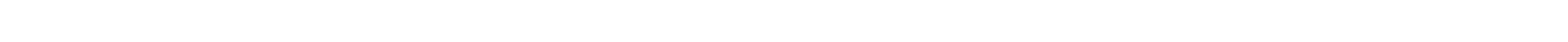

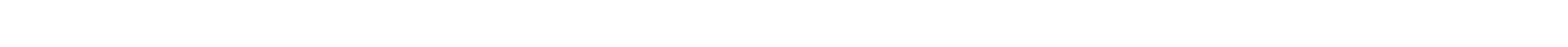

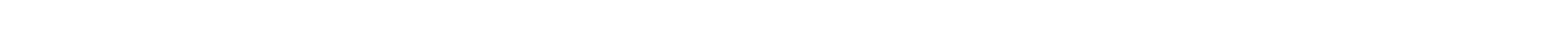

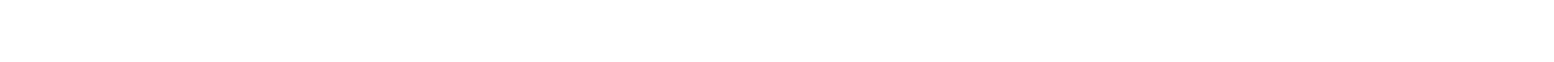

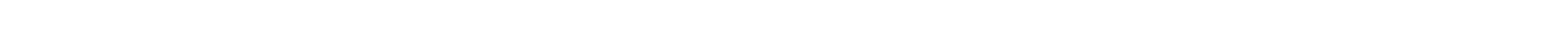
$x m m-\infty=0$ m

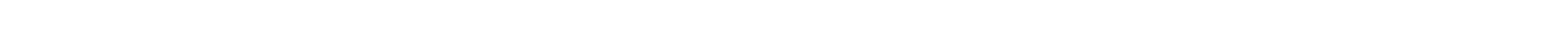

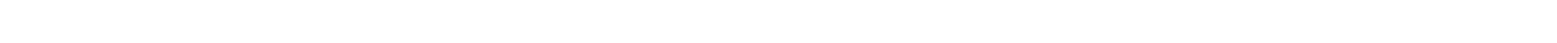

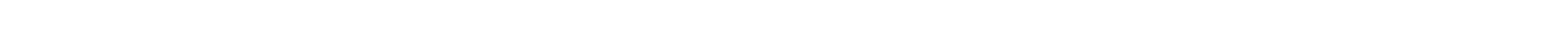
in

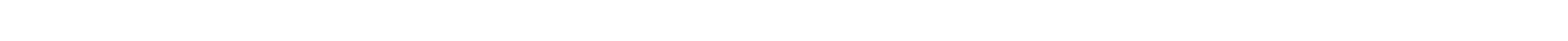
u ú

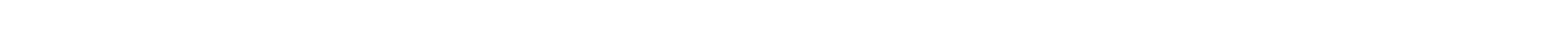

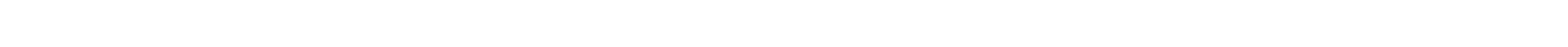

응 200000000

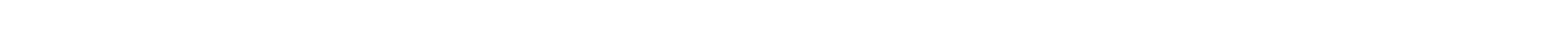

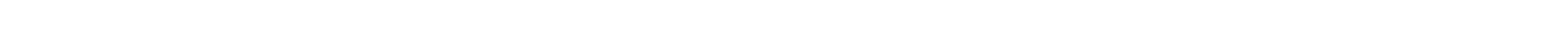

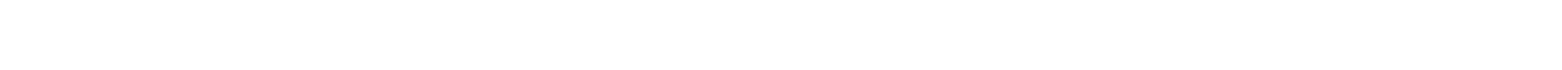



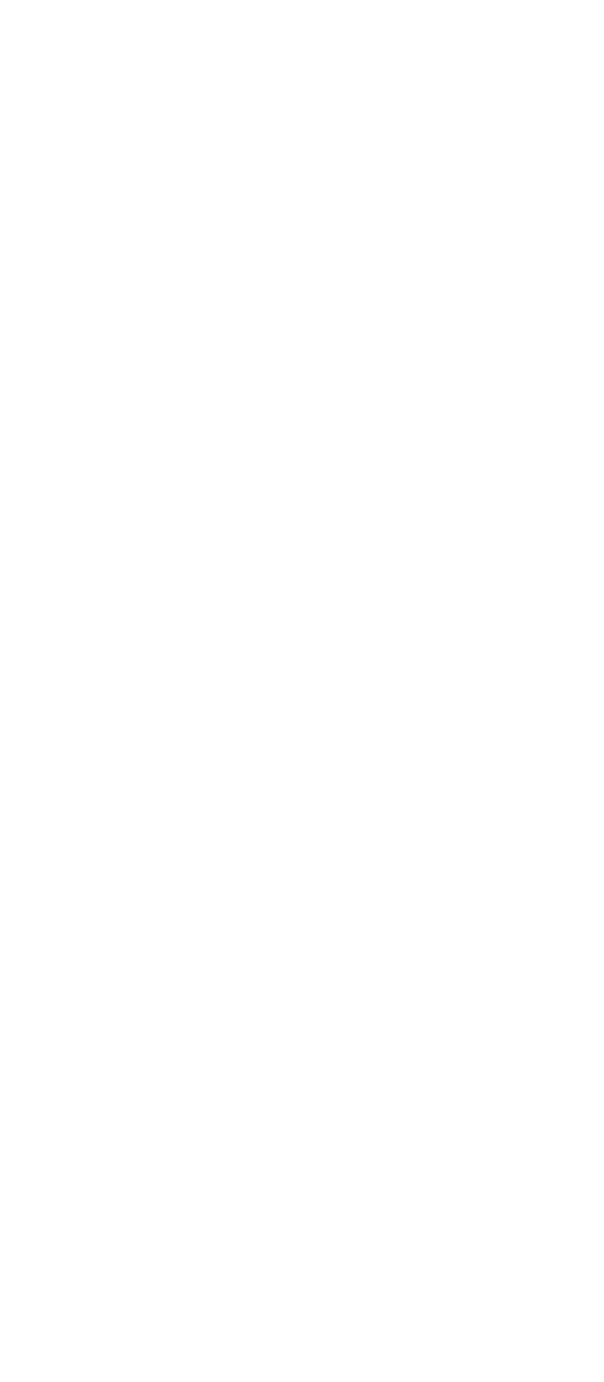

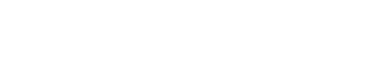

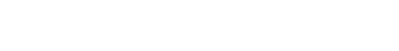
\&a mo

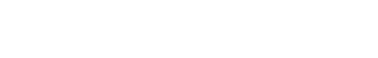

㟧

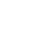

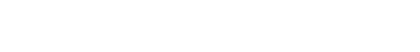
wrín்

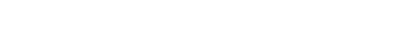

aaaaa웅

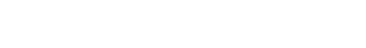
แニニニニニニラニニニララ

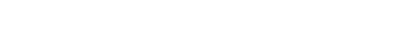

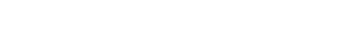



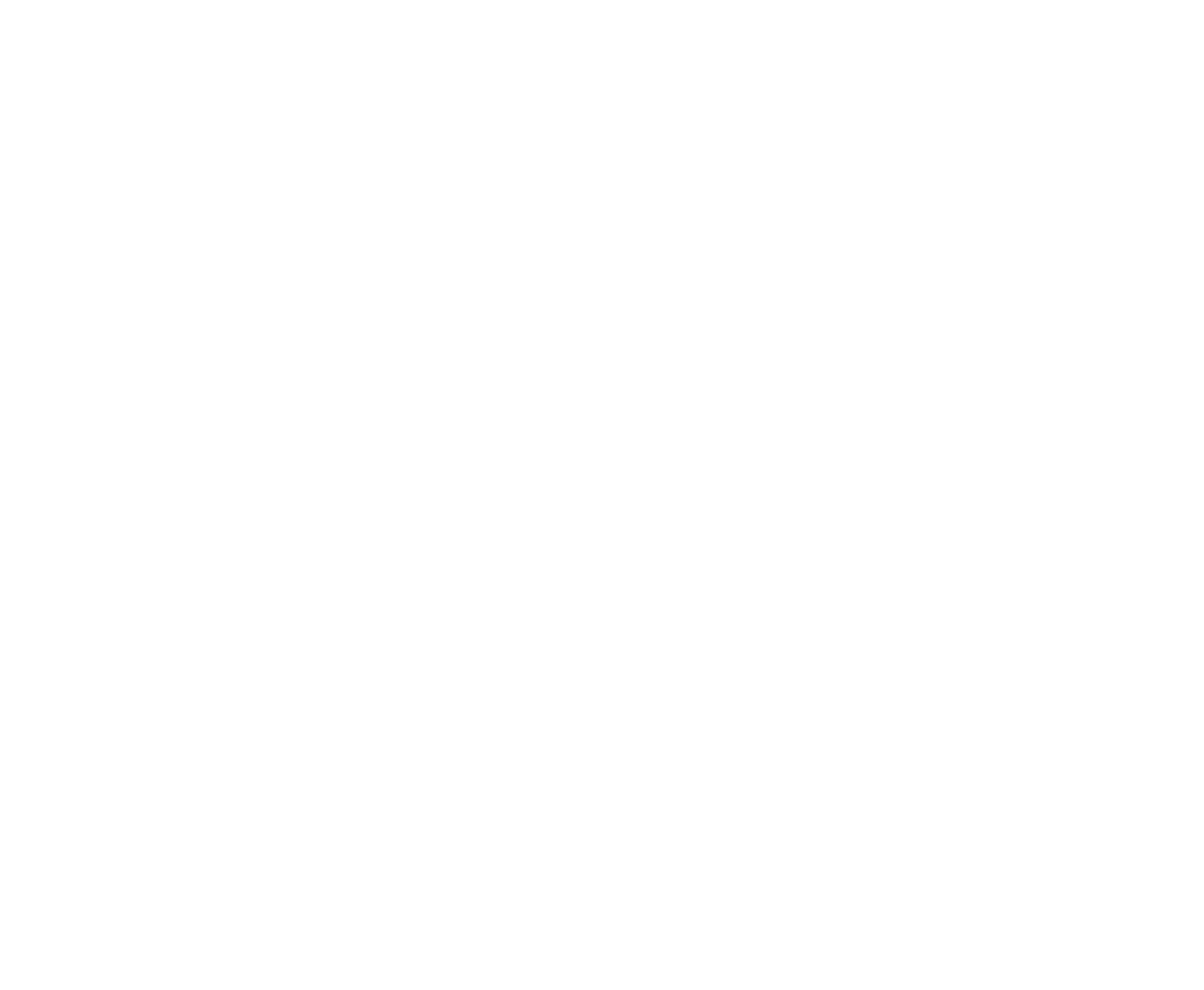

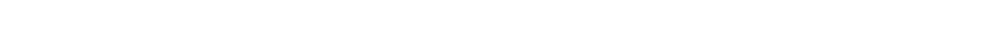

ANA

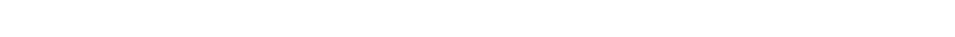

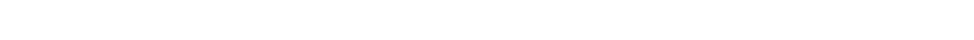

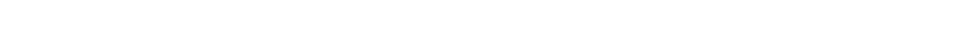

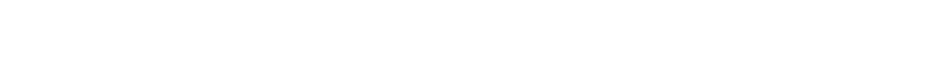

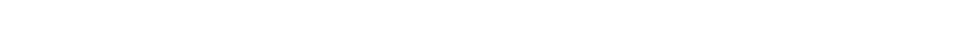

a

(n)

แ u w

- - उus

$\underset{u}{\ddot{u}} \stackrel{\ddot{u}}{a}$ ä $\overrightarrow{0}$ $\therefore \therefore \div$ $\sum_{11}$ $\stackrel{s}{a}$

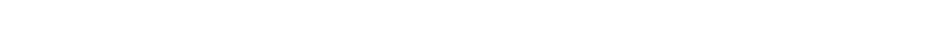

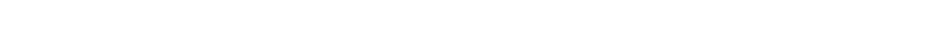

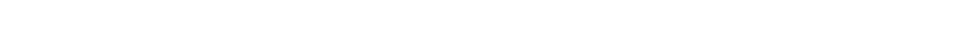

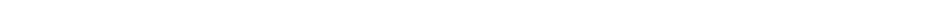

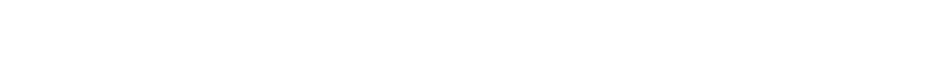

$-z \mathfrak{z}$ $0 \div 01$ $=0=-$

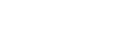
으응

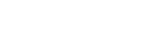

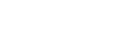

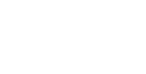
致峲 $\stackrel{a}{4}$

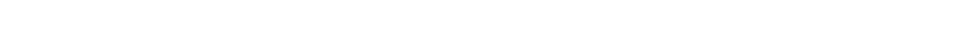
in

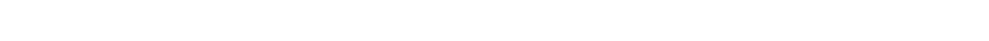

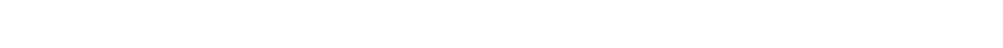

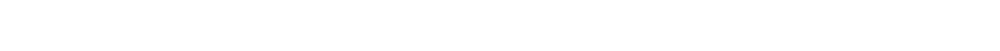

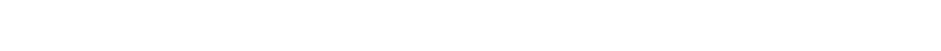

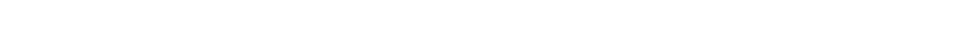

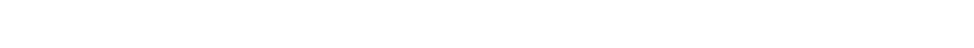

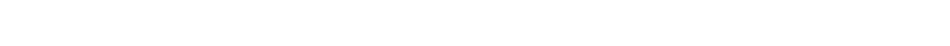

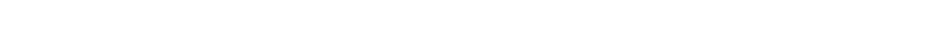




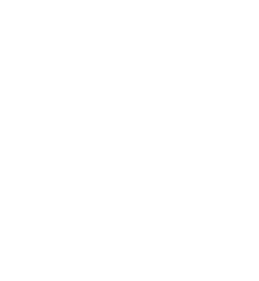

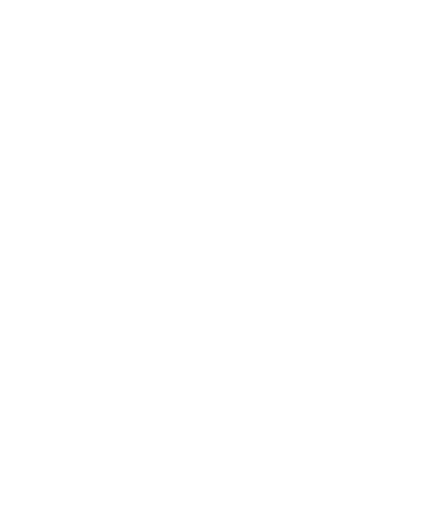

$\geqq$

空

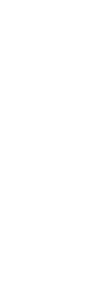

要

nonao

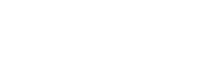

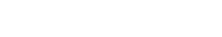

$0 \sim \approx \sim ⿻ N N$

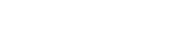

titesensenes

in

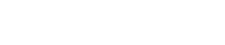

ü

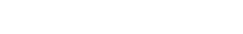

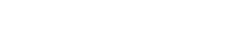

J aornms

- $\Rightarrow$ moñan

少

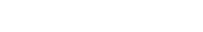

至合台合合

岕

$=$

ivenesesen

$\approx \quad 5 \sin 0 a n$

a $\quad \alpha+\infty \pi n+\infty \infty$

$\stackrel{1}{\longrightarrow} \dot{0} \dot{0} \dot{\infty} \dot{\infty}$

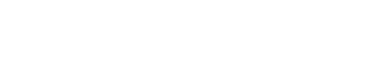

ณํํำ

a응

"ேニニニニ5

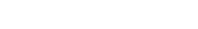

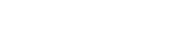




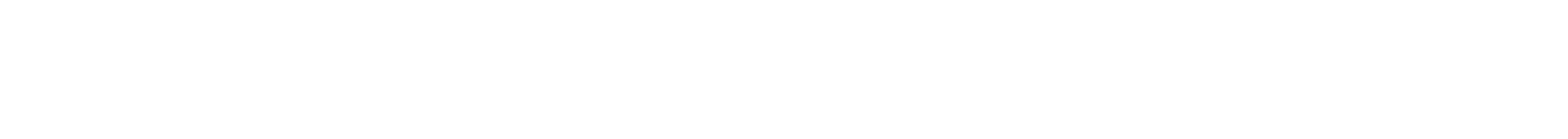

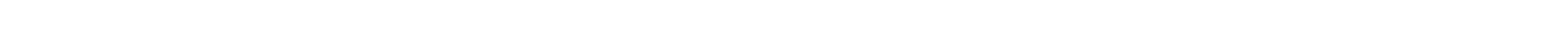

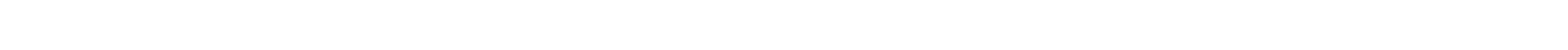
土 йm

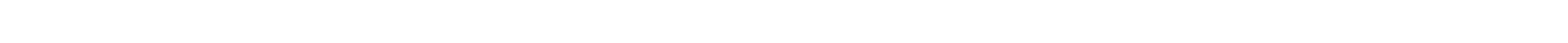

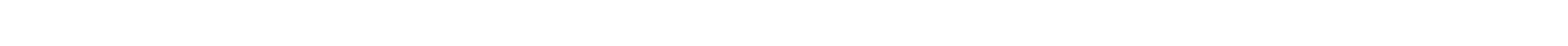

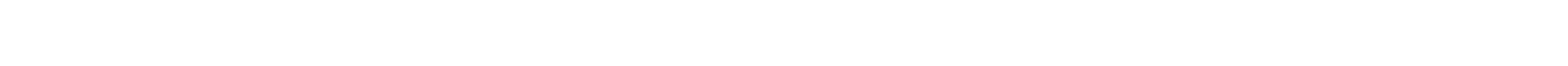

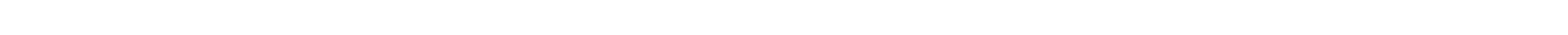

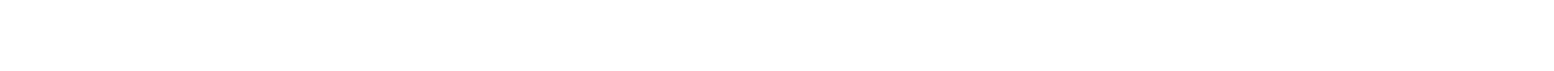

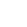

$\stackrel{\varpi}{\Sigma}$

in

๔

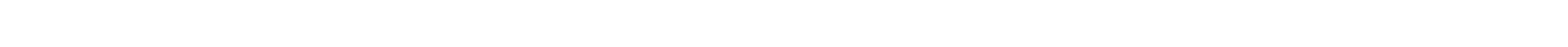

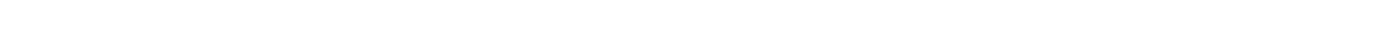

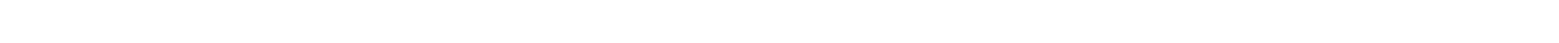

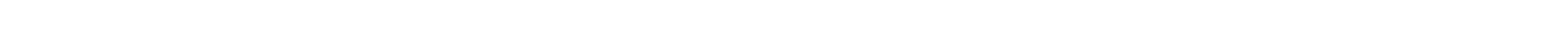

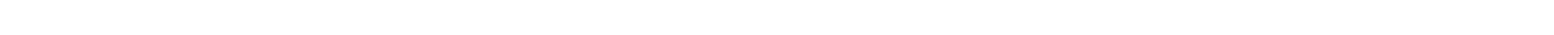

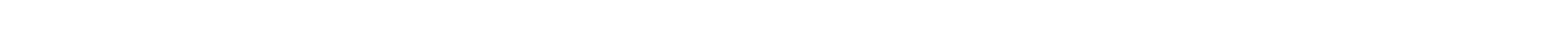

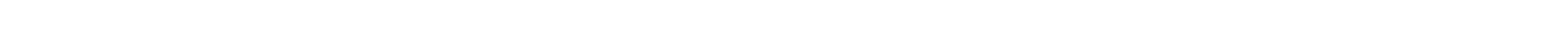
的

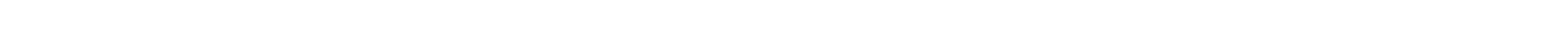
$\sum_{\Sigma}$ \&
n
n
n
is
is
하

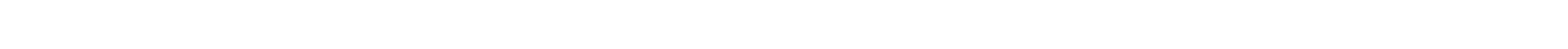

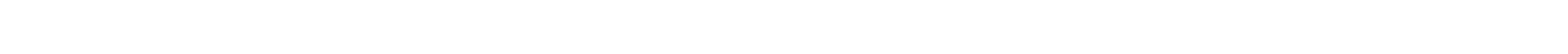

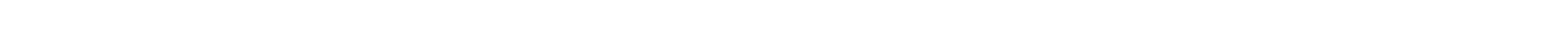

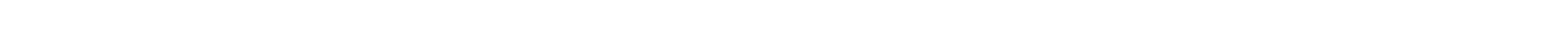

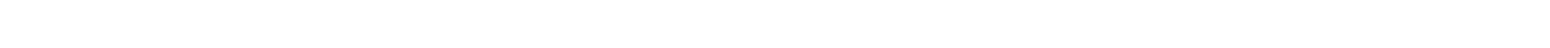
แ แ

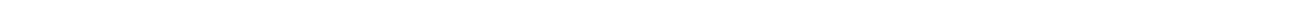

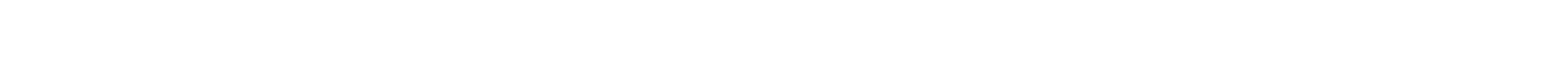
$\stackrel{w}{\Sigma} \sim$
in
n
n
in
n
n
$\stackrel{2}{n}$

n

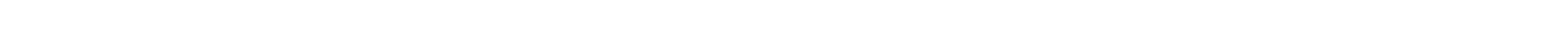

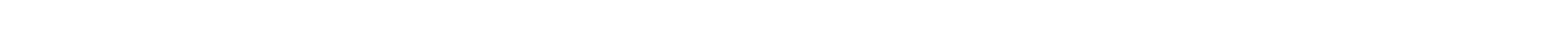

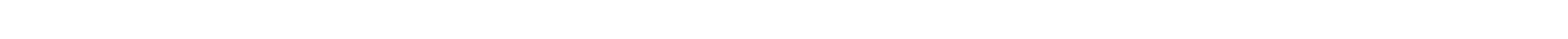

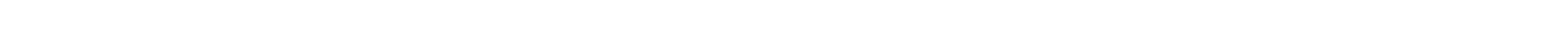

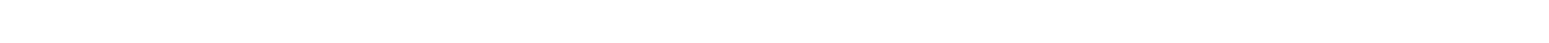

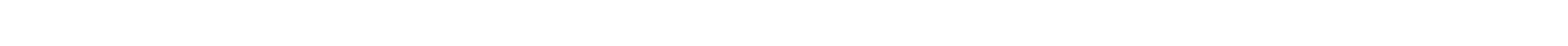

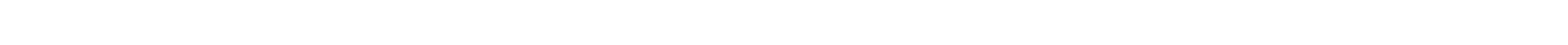

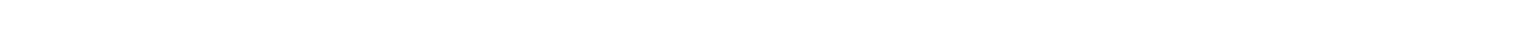

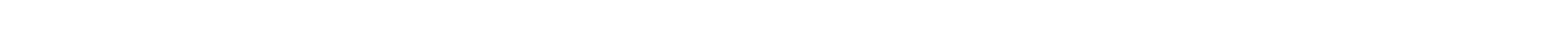




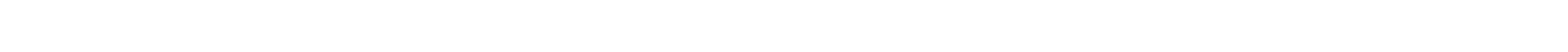

$i$

- 00 mk

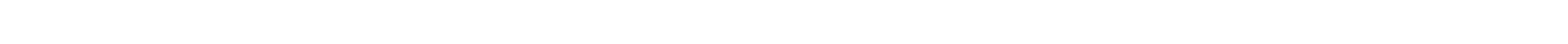

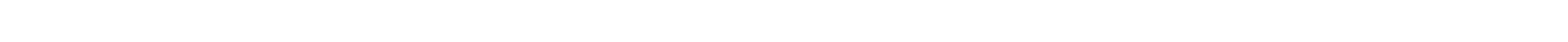

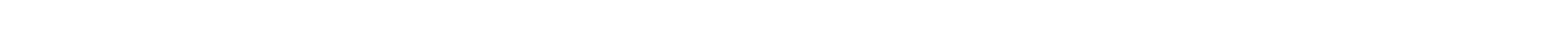

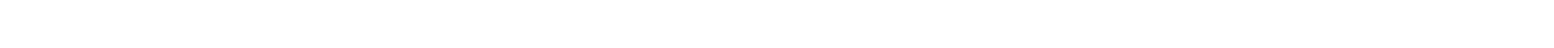
wE INニÑ

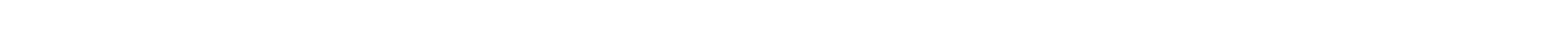
$\alpha$

is

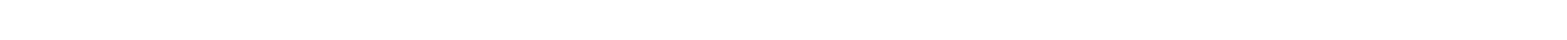
a s um - UNRR

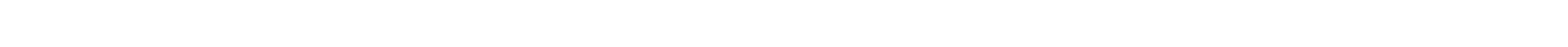

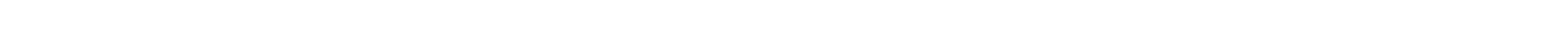

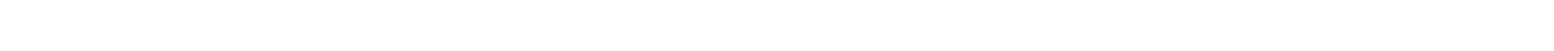
แ

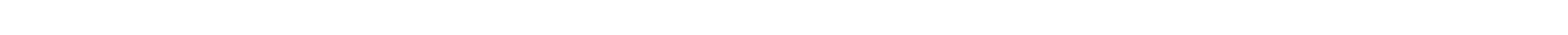

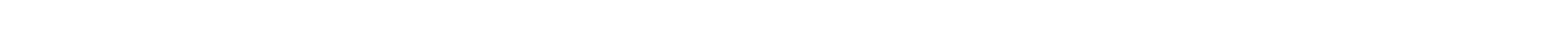

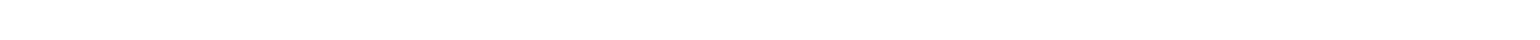

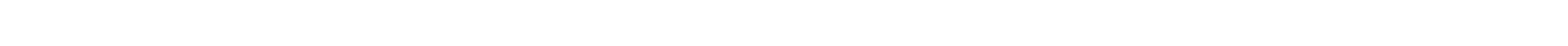
in

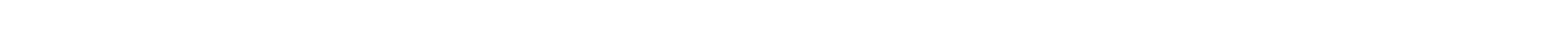
"w

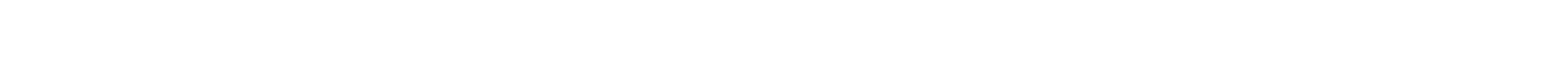

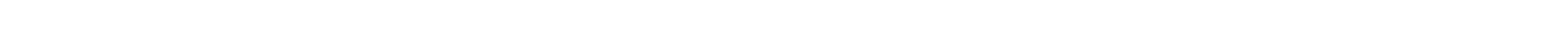

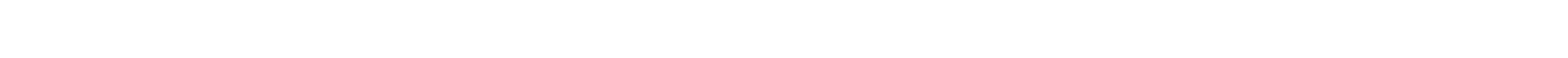

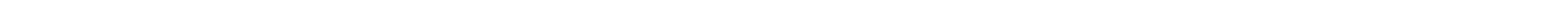

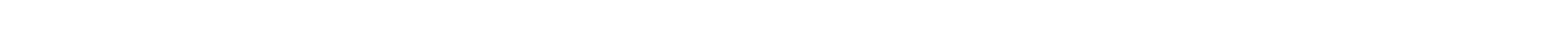
जó

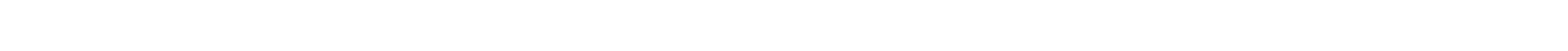
$\stackrel{a}{5}$

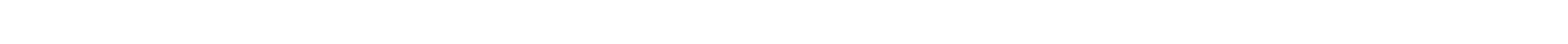
$w$ -

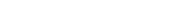

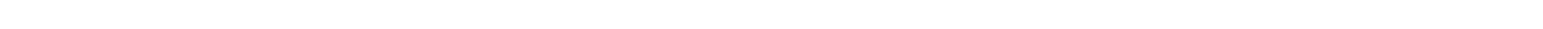

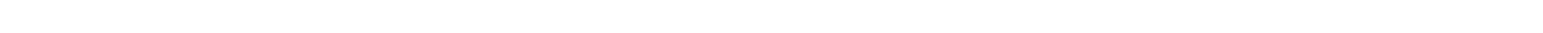
ñ w Lin

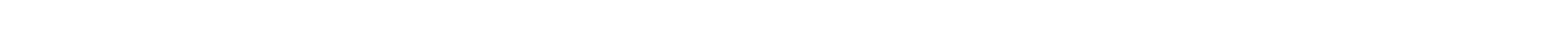

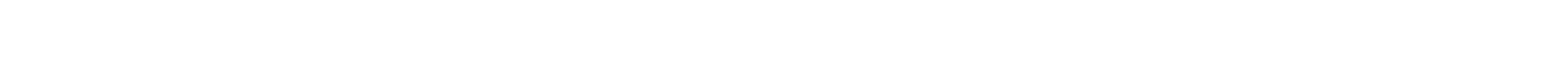




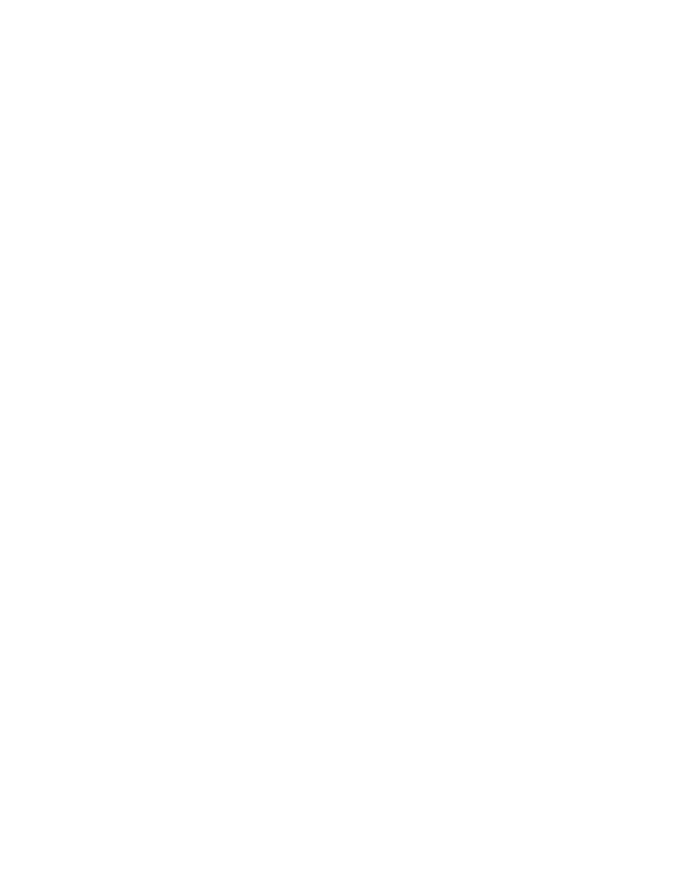

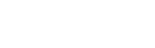

is

17 $\underset{\alpha}{\infty} \geq$

0 4 2

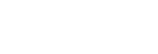

avm

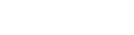

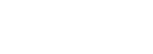

我背。

$->0$

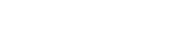

Zuc a m

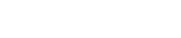

vaー上?

논이용

ง n

.

z.0

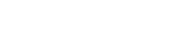

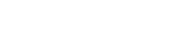

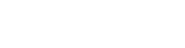

in 2 ?

굿요요

in

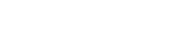

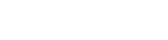

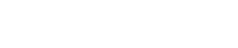

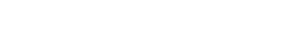
岁出 㟧 㟧

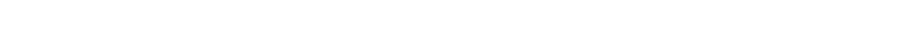

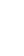

뉴:

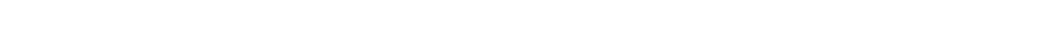
बwnR

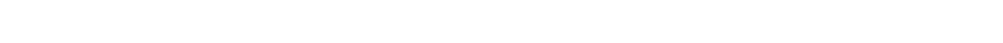

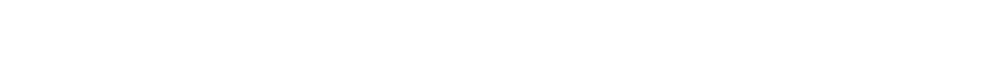

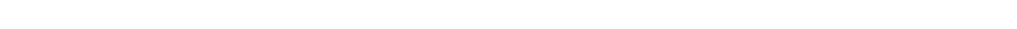

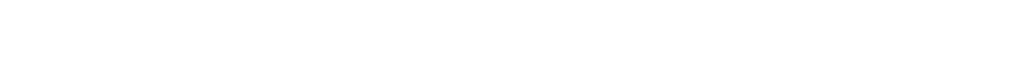

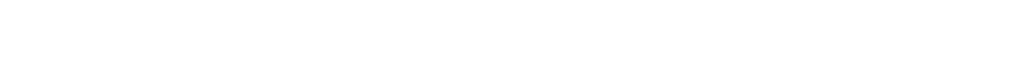

in

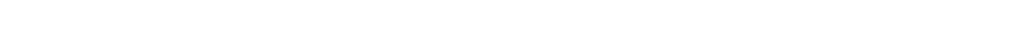

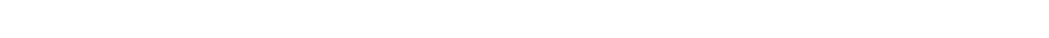

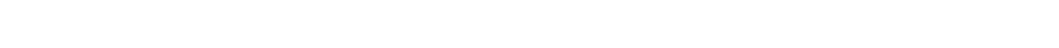

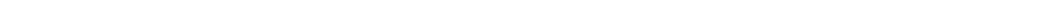

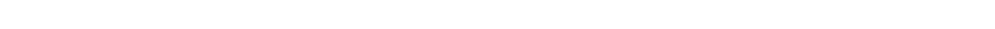

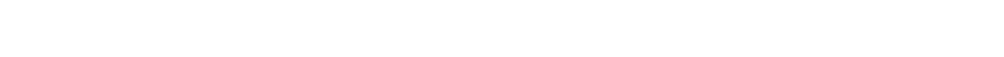

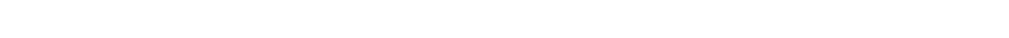

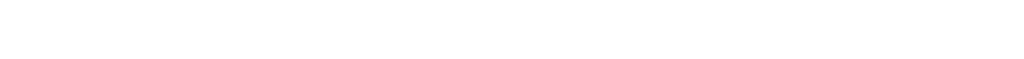

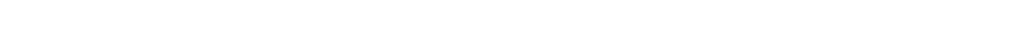

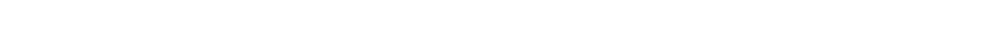

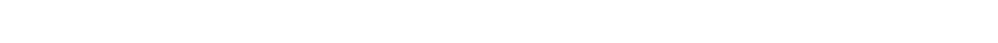

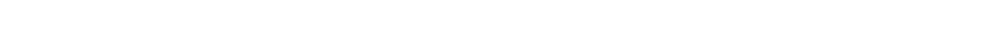

is

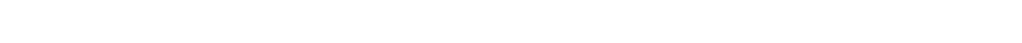
u.

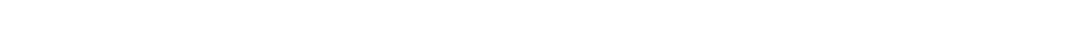

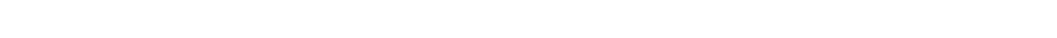

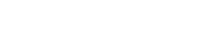
Tom

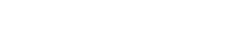

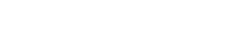

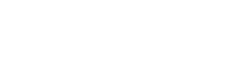

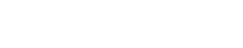
$\sum_{w} \sum_{w} \sum_{w} \sum_{w} \sum_{w}$ $\begin{array}{lllll}z & a & 1 & 1 & \vdots\end{array}$

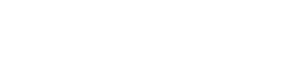

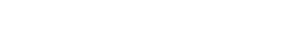

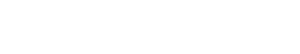

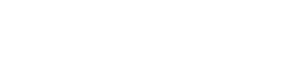

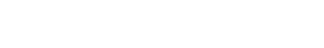

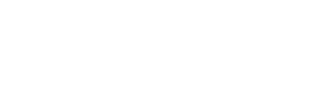
$a z z z=\frac{a}{z} z \frac{a}{z} z$

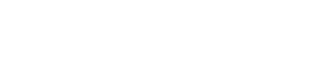
岕

000000000000000000000000000000000

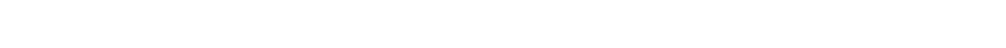

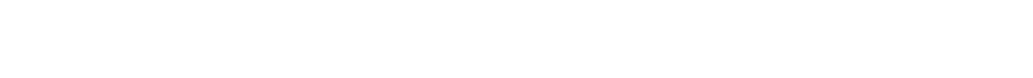

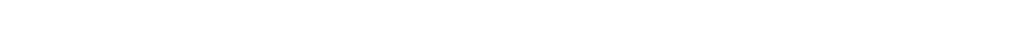

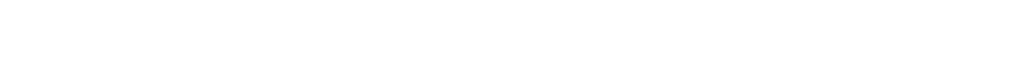

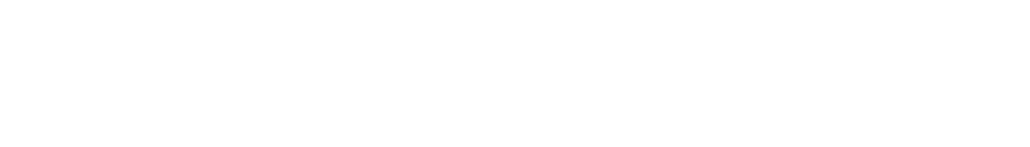

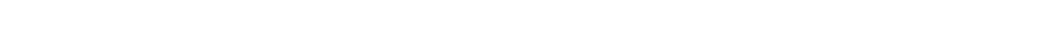

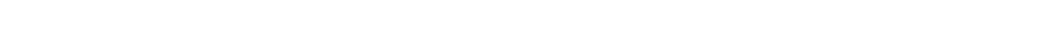

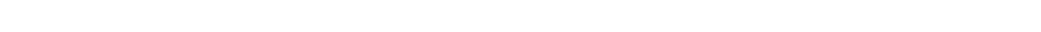

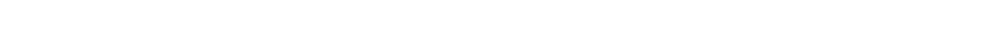

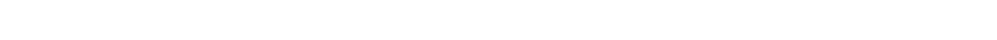
in

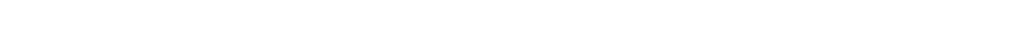

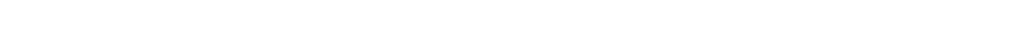

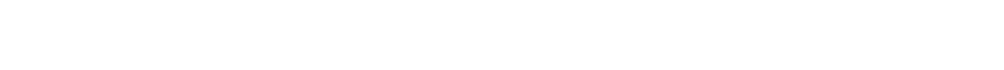




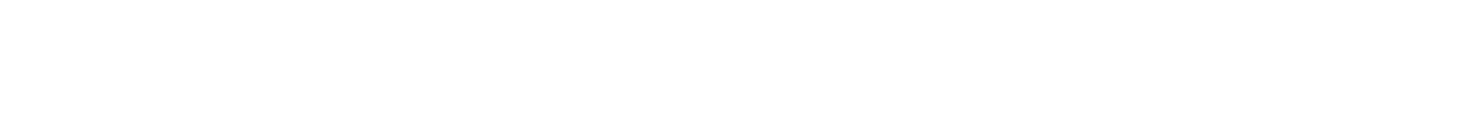

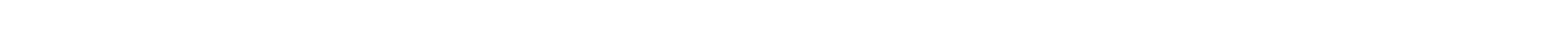

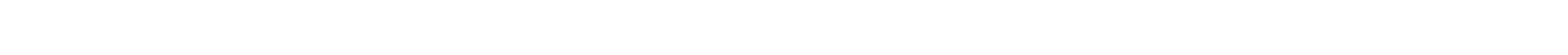

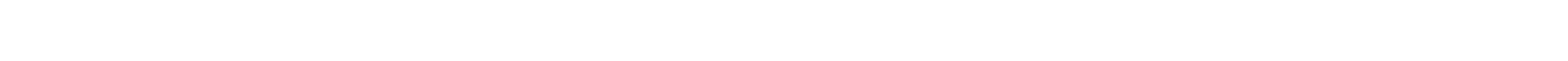

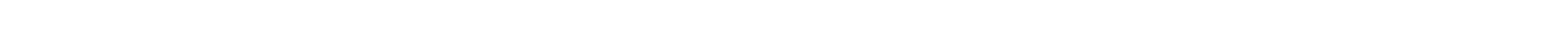

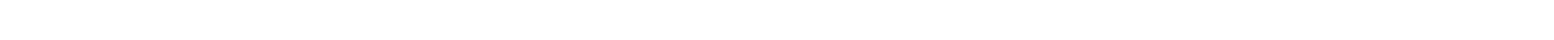

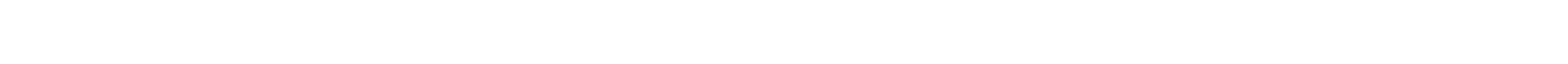

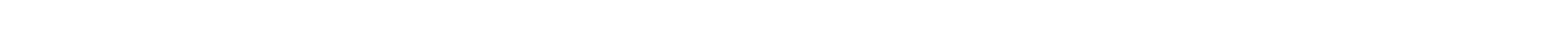

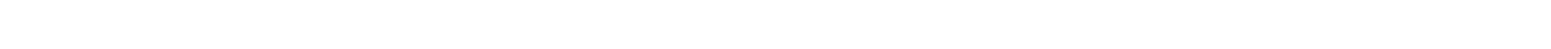

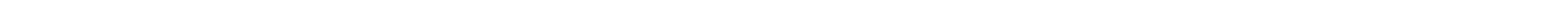
$\underset{\Sigma}{w}$ in
n
n
is
n
ns
in
n
in in

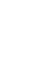

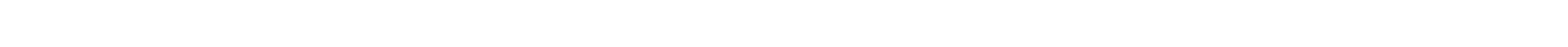

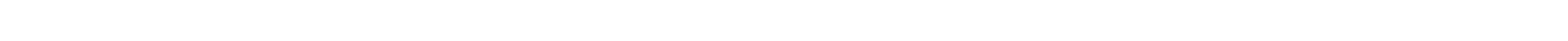

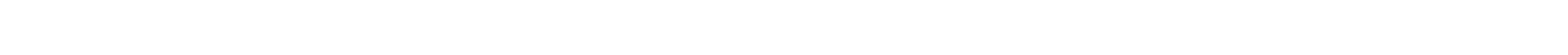
>

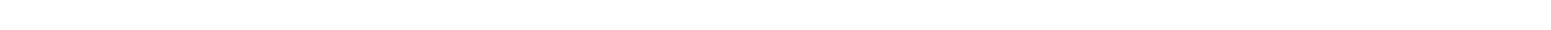

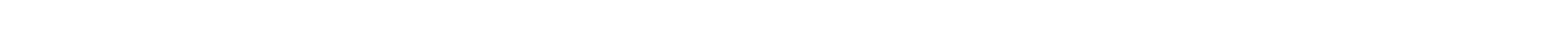

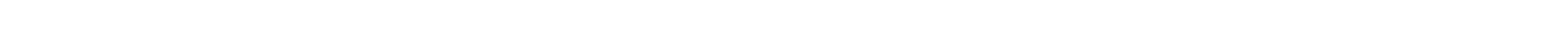

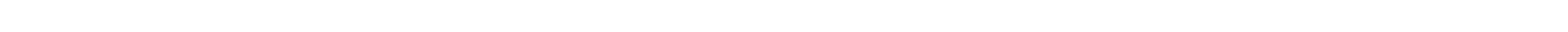

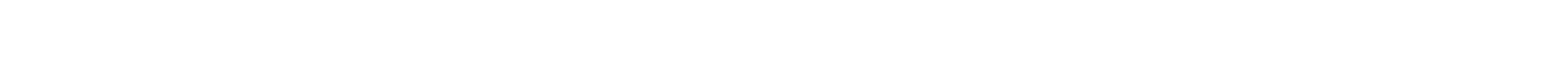

t. n

is

is

is

is

$\sin \sin$

is in es

in m

$\sim$

¿

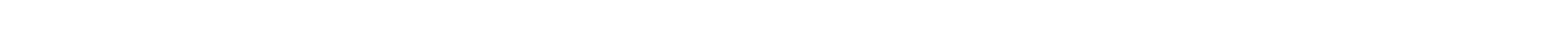
ü

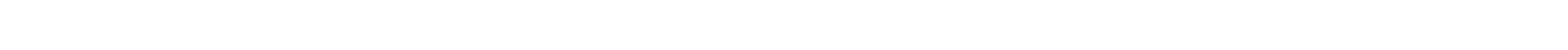

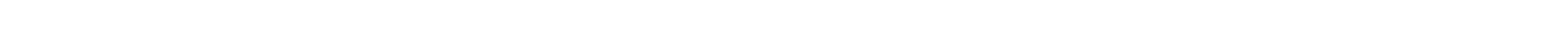

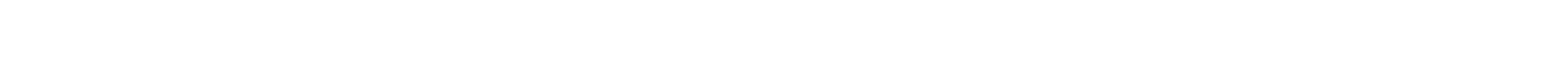

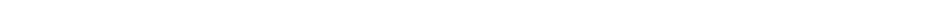

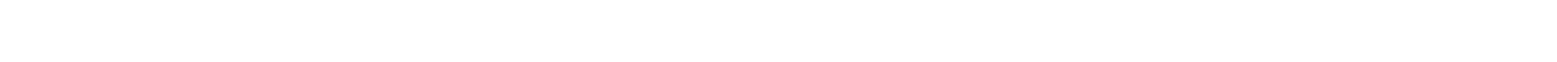

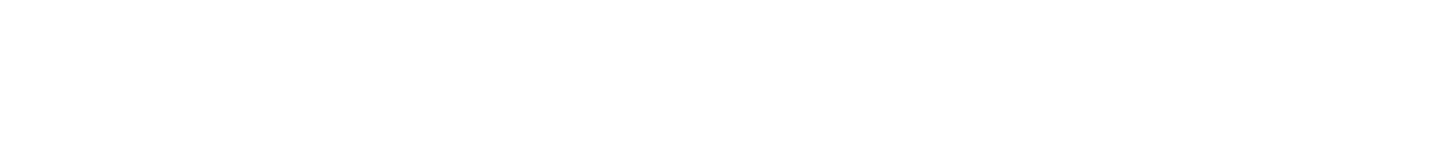

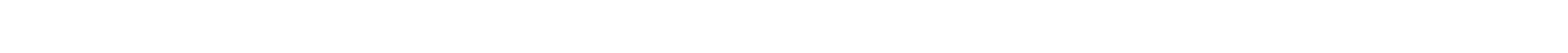

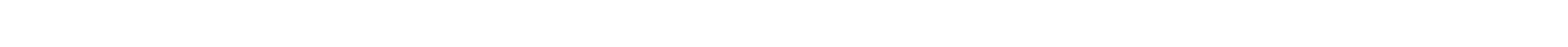

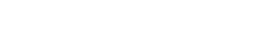

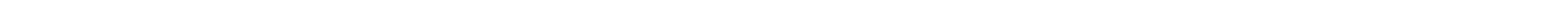

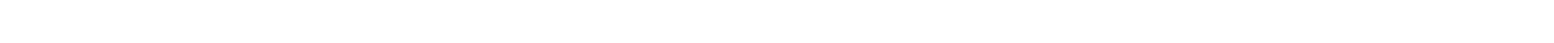

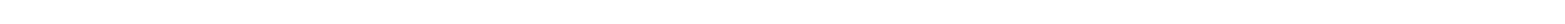

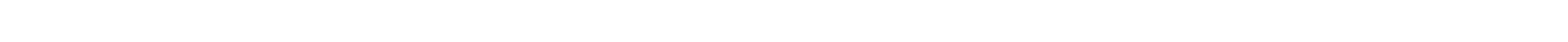

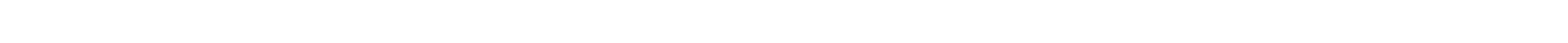

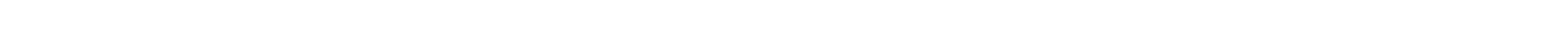




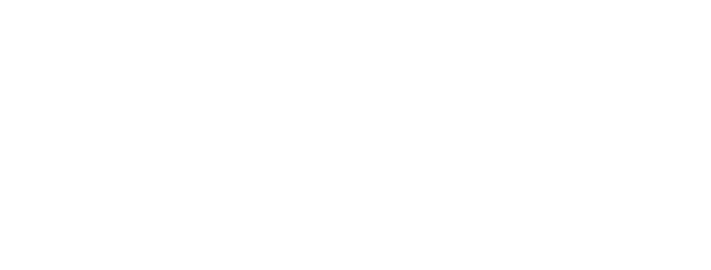

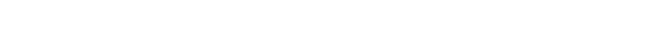

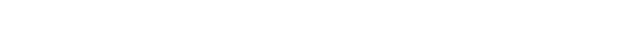
แ

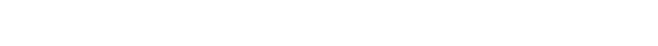

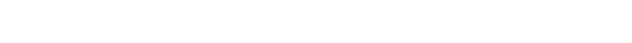

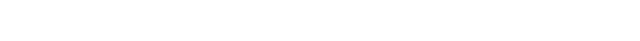

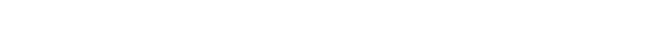
in

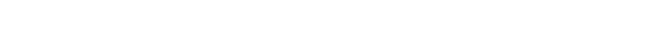
×

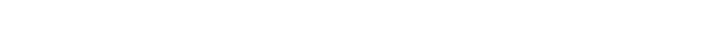

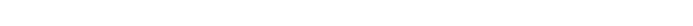

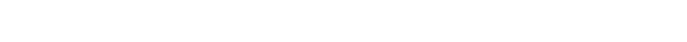

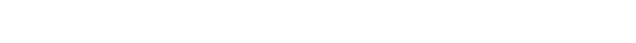

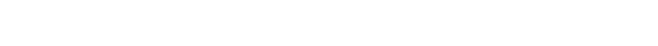

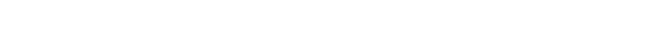

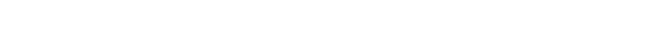

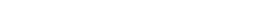

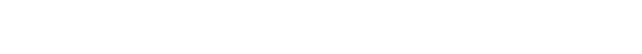

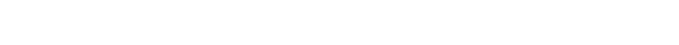

\&

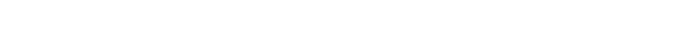
w w

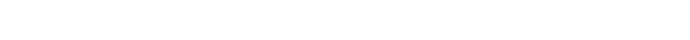

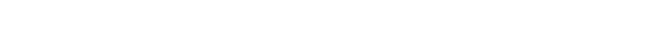

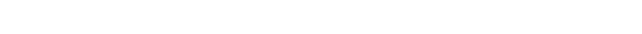

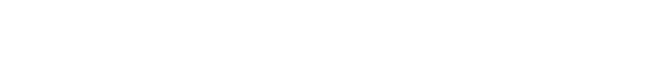
ONNNNNEN-ENNNNNMNーNENE

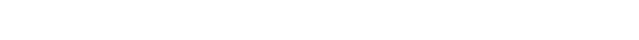

$$
\text { two }
$$

¿

naomamunm-nmónagommn a

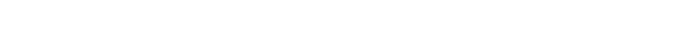

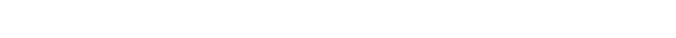

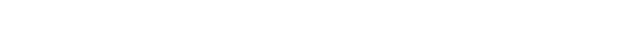

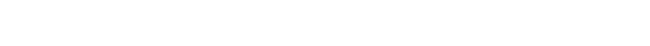

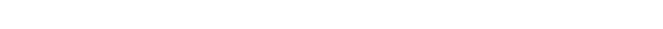

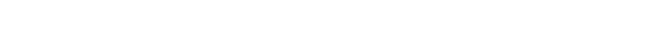

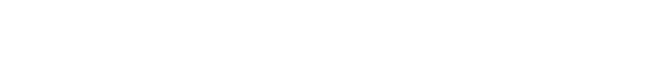



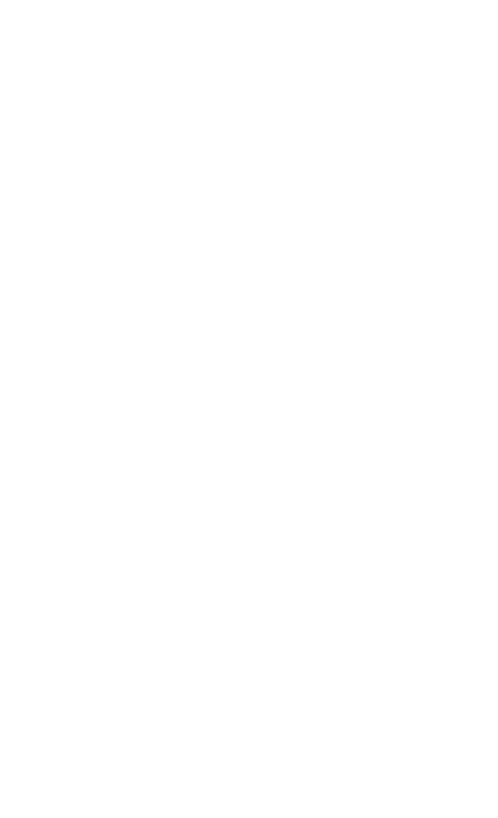

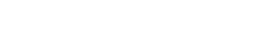

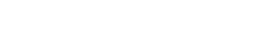
แ

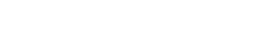

垈

tyencosonesoses in

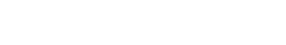

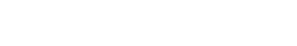
$\stackrel{\Delta}{\rightarrow} 000-000$

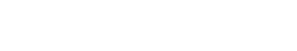

Un $\mathrm{Na}$ armo

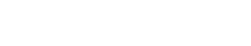
แニニニニニニニニ ommoñ

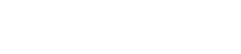

tosesencoseses $\frac{\omega}{\Sigma}$

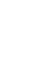

"

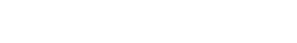
×

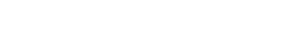

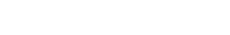
ํํำำㅇำ

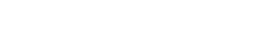

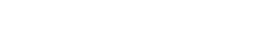
ふえニか人ニ的 

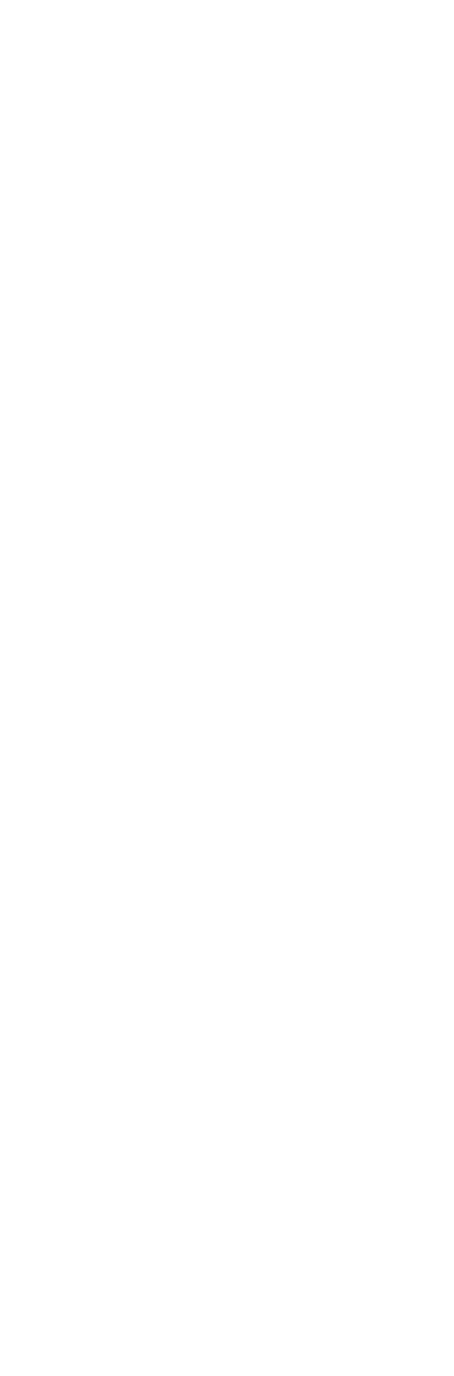

I nammoro

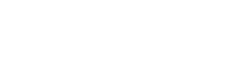

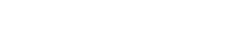

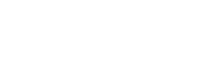

is

$z \quad \stackrel{5}{5} \cos e \operatorname{ses} c o s$

$\stackrel{a}{n}$

इ $\quad$ a

ü

- >

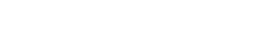

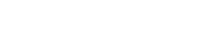

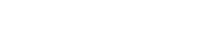

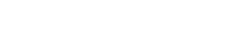

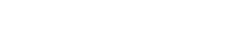

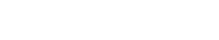



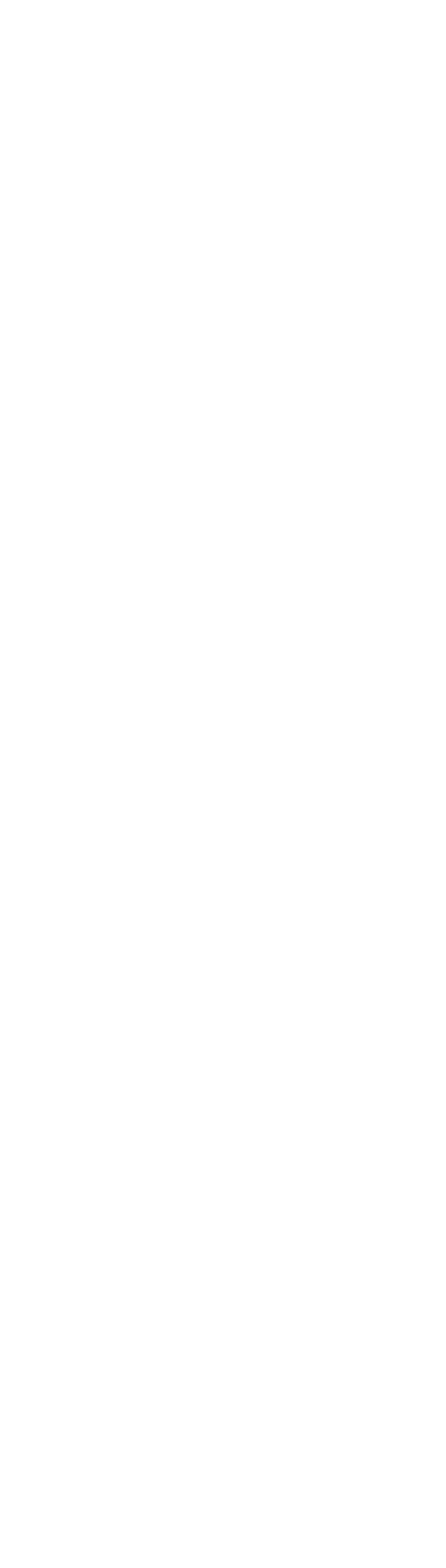

titanesenes $\leftleftarrows$

N n ำㅇำ แミニミミミミミ

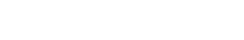

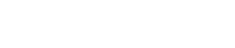




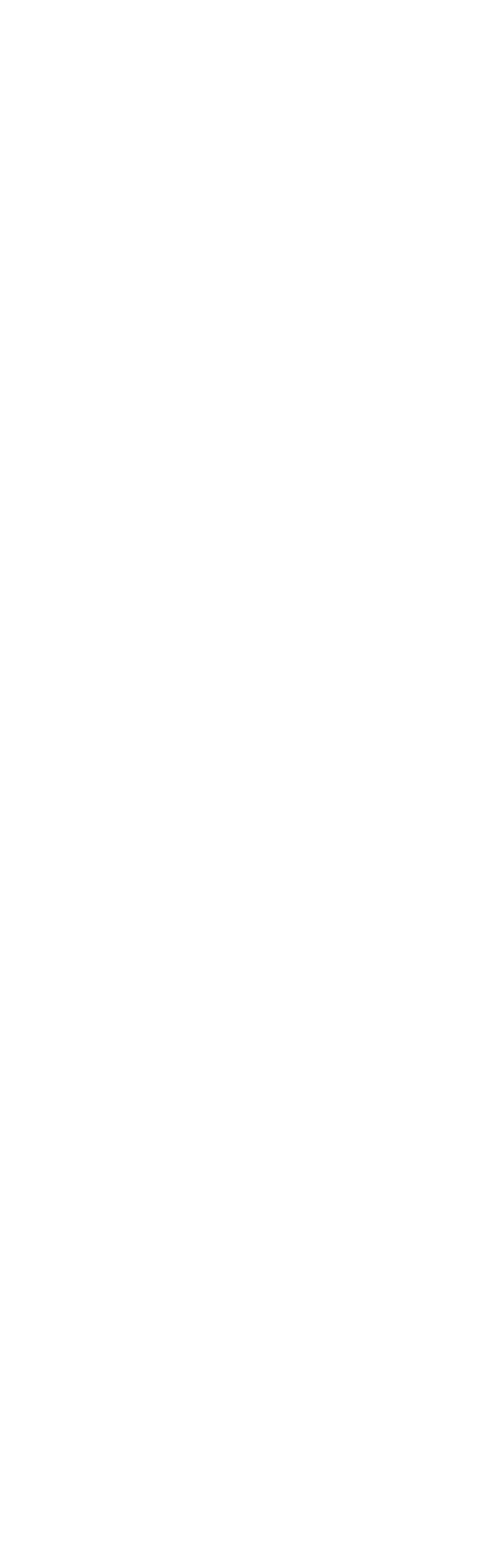

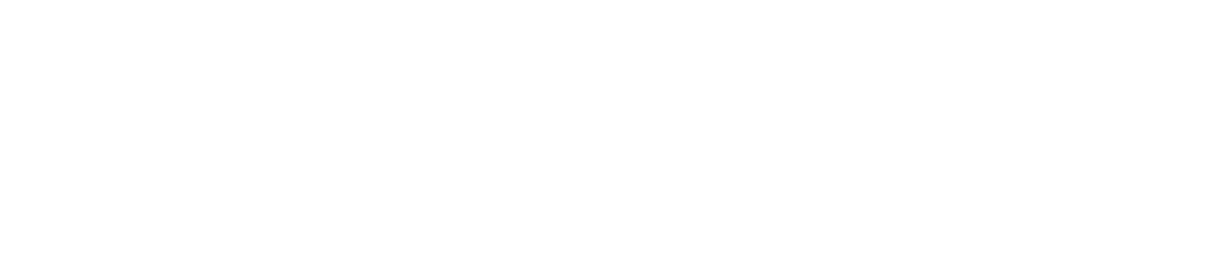

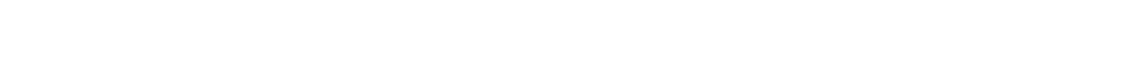
世上

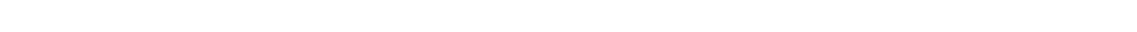

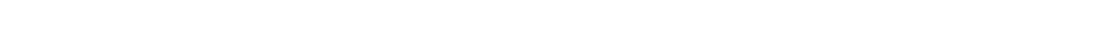

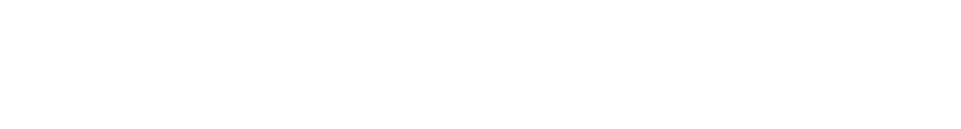

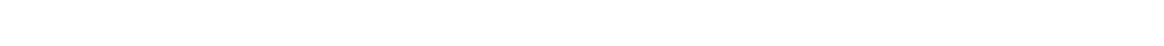

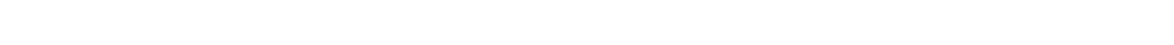

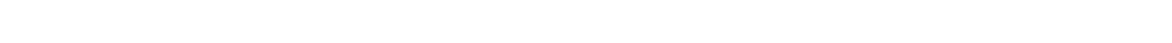

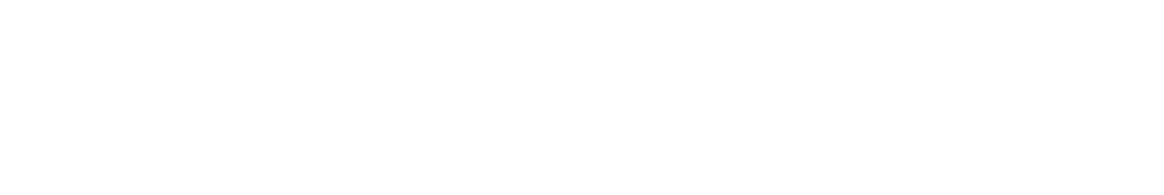

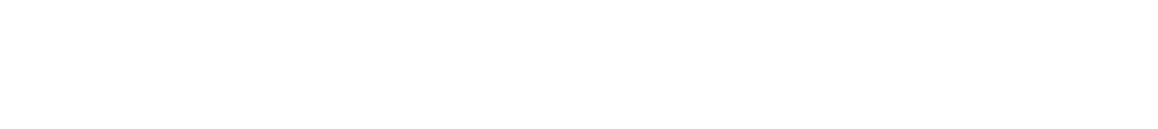
a \&

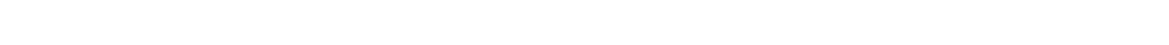

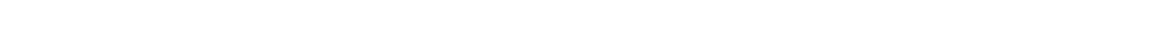

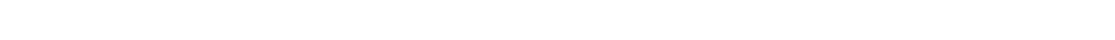

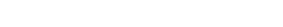

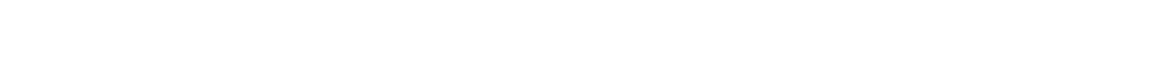

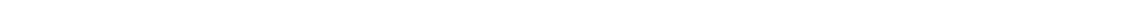

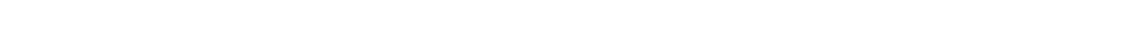

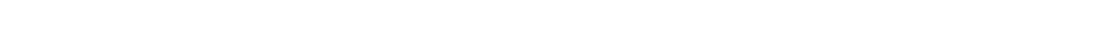

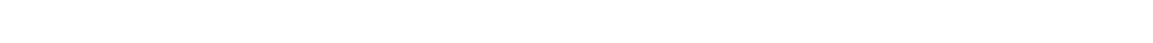

\&

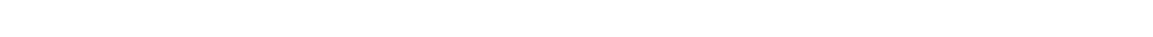
ш w व

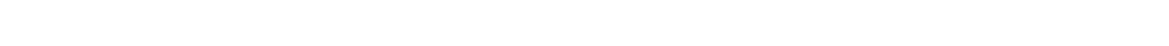

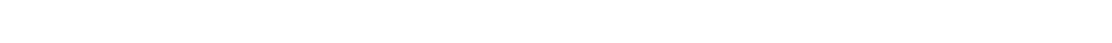

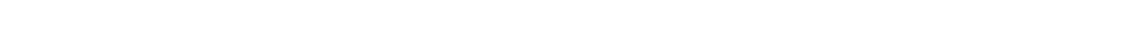

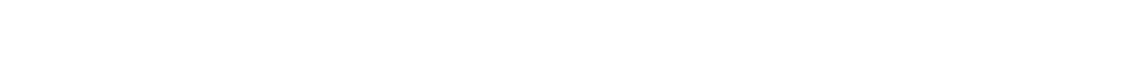

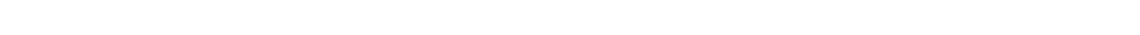

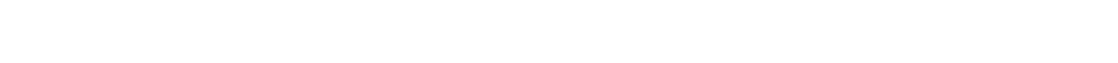




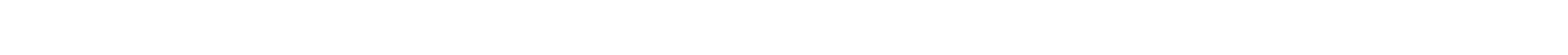

is

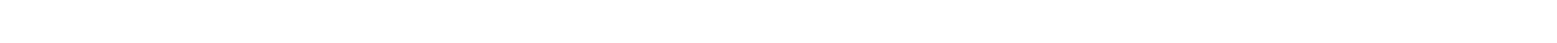
a

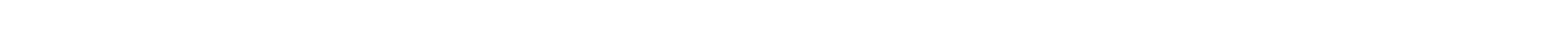

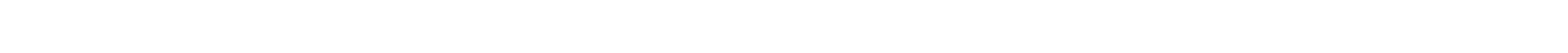

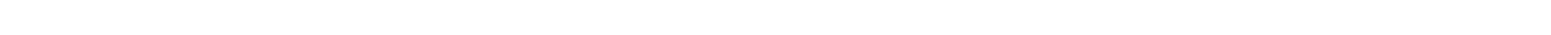

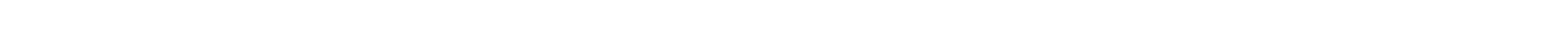

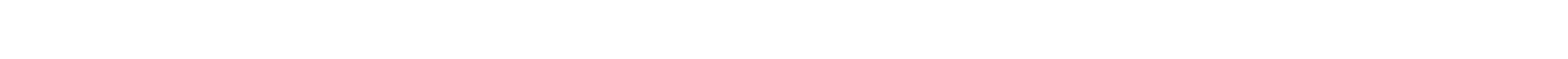

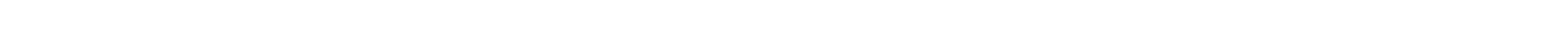

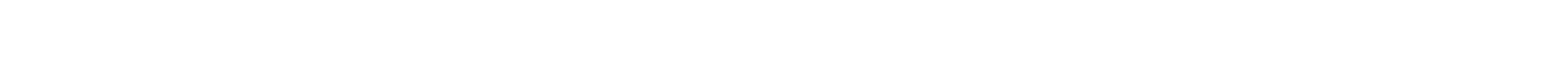

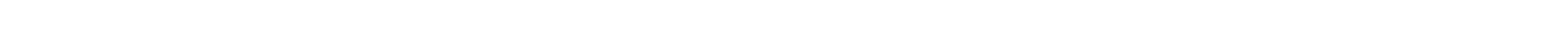
in

"

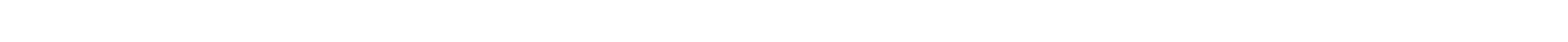

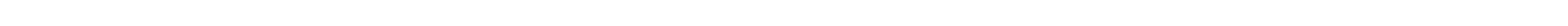

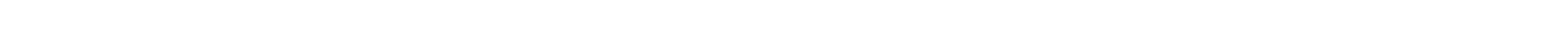

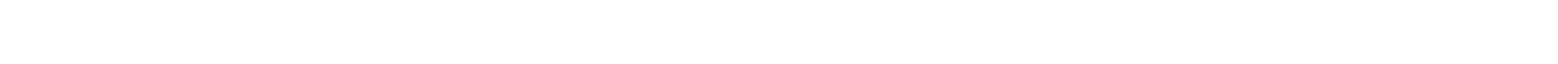

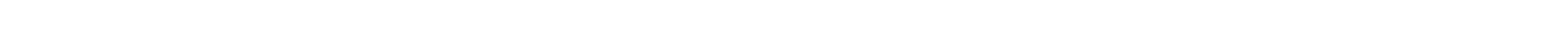

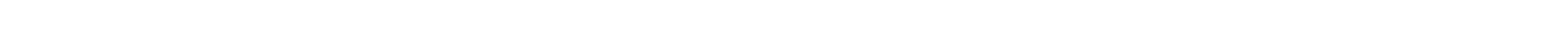

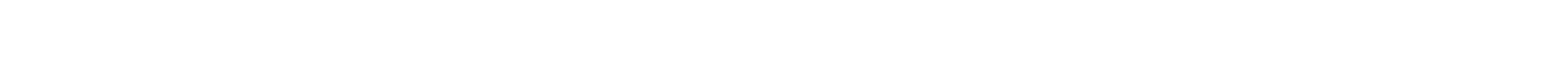
twencosenesen as as
es
n
is
n
nosunos
a

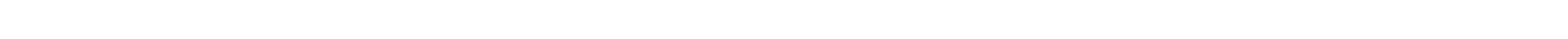

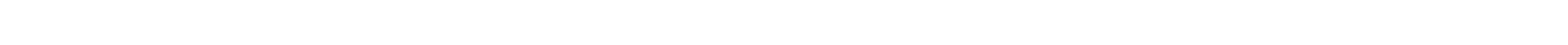

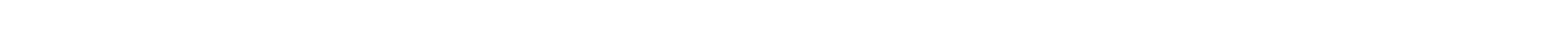

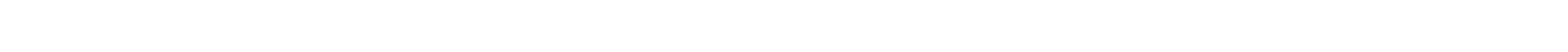

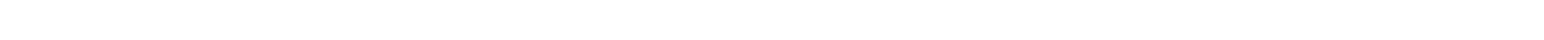

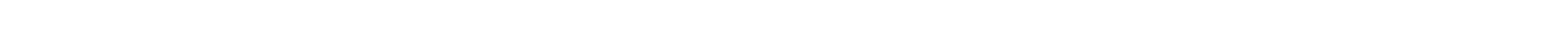

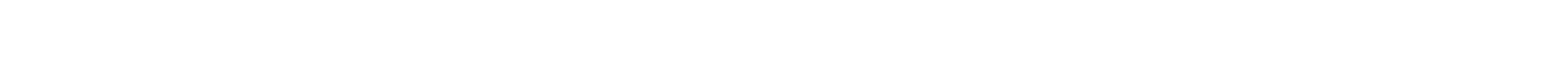

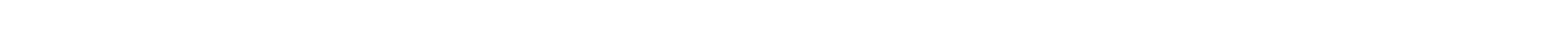

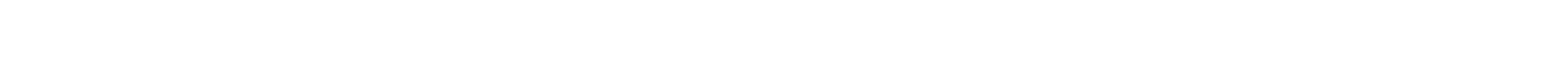

崖

in $n$

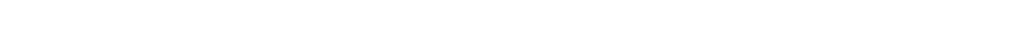

E

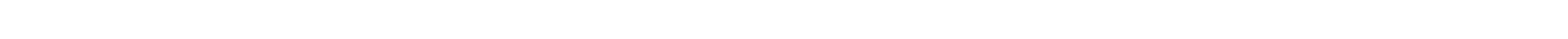
๔

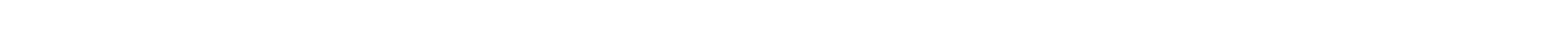

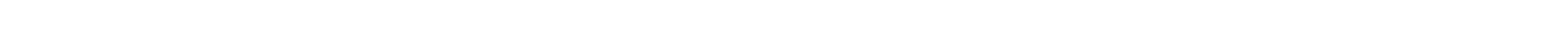

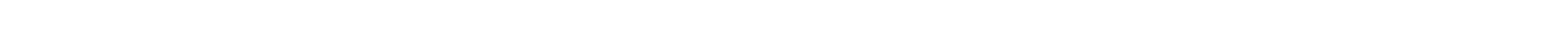

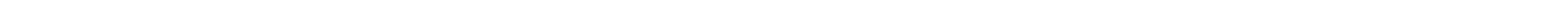

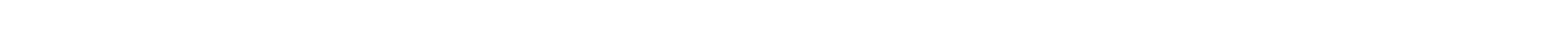

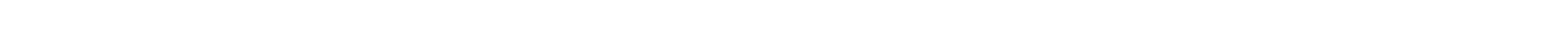

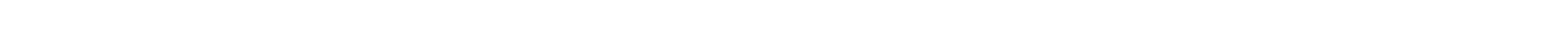

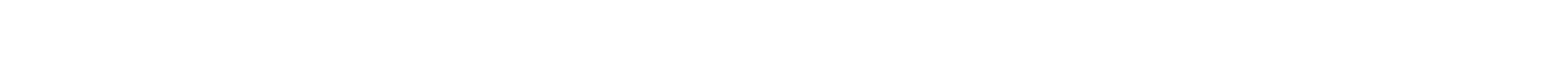



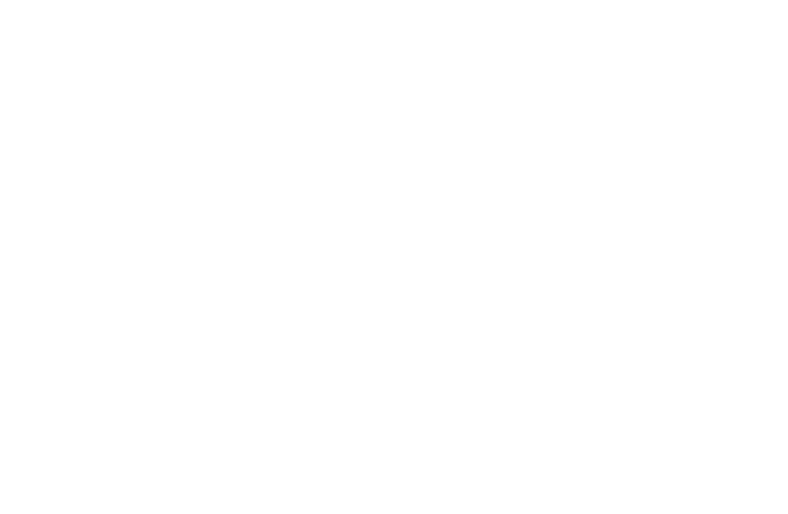

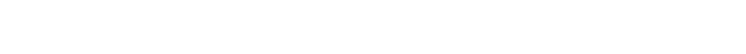

5

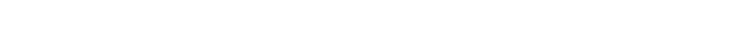

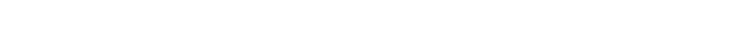

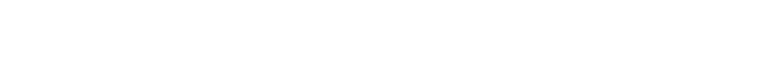

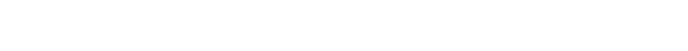

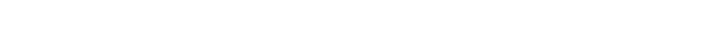

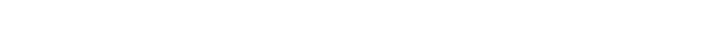

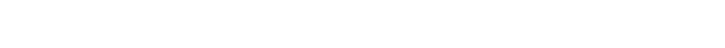

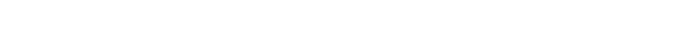

$m$

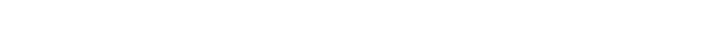

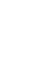

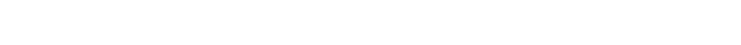
ж⿻儿口

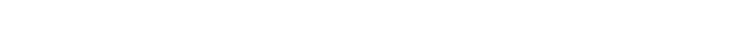

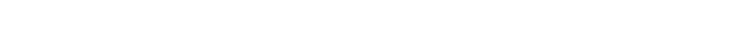

- n Nrr aล̃

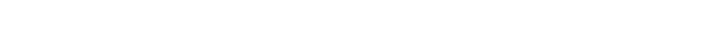

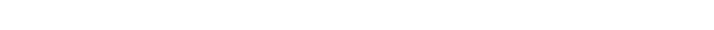

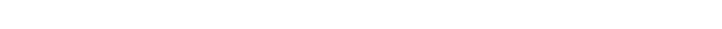

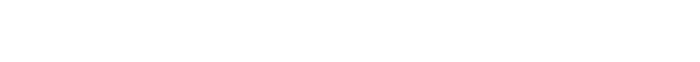

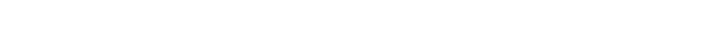
5

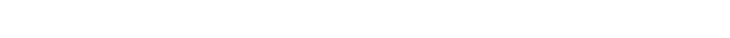
山س

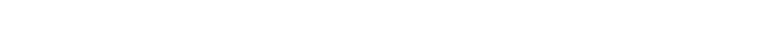

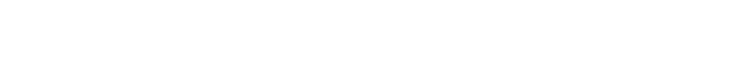

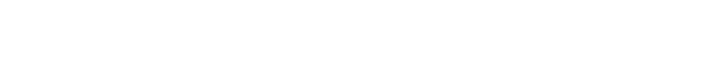

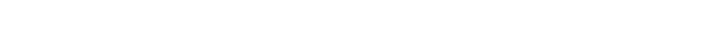

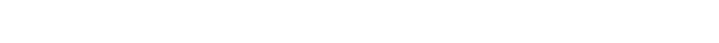

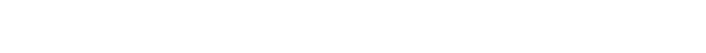




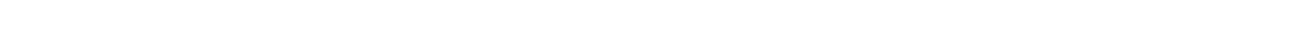

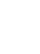

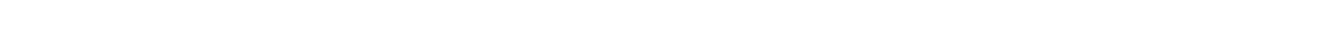

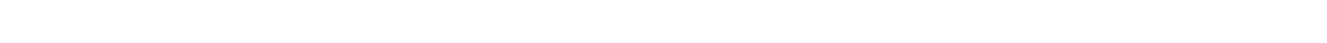

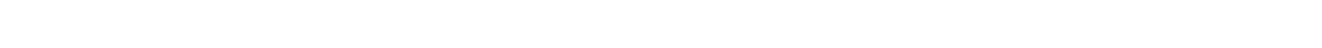

8

I文

t5: is

win Uே iz

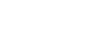
n $\infty$ 온ํำ

No 둥요 겅 z⿺⿻一𠃋十 $n \tilde{n}$ o는 tur a. $=$ a

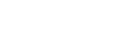
ong

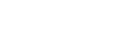
准 ant 드는 잉 조용

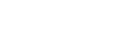

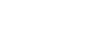
$\underset{4, z}{\alpha}=01$

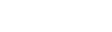

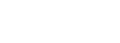
is $\alpha$

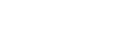
」几 ӑ 1. w0: 乎立足 铛 近员 $1 m-0$ a 네의 ㅁํำ ㄷํㅇ $20-0$ cin 진 $>m \sim$ i in 虫出的。 oษ 0 om 山䒕察少

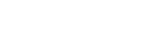
和包こ气

Nu

응 菏ㄴ․ 잉뭉 แ. 禹 汖
NNNNNmmmmmmmmmm+

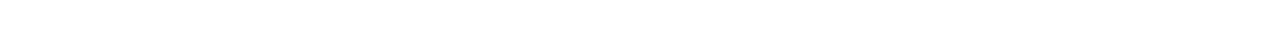

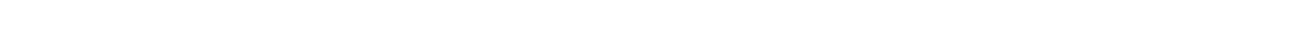

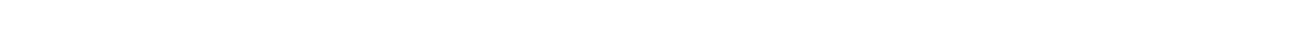

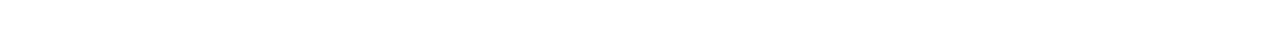

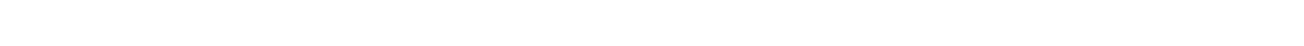
咅

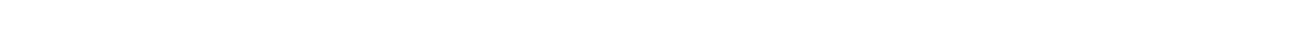
a dam

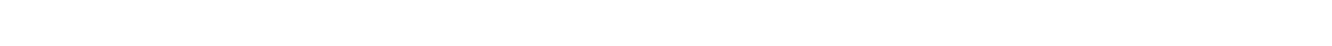
3
0

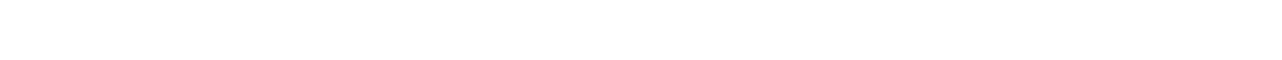

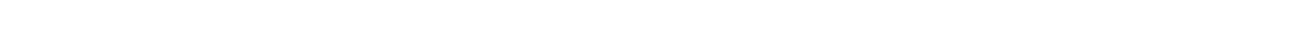
amvand

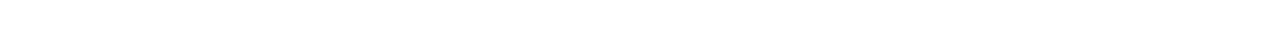

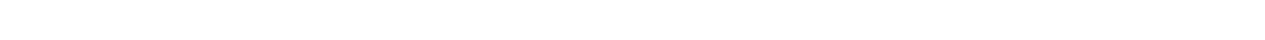

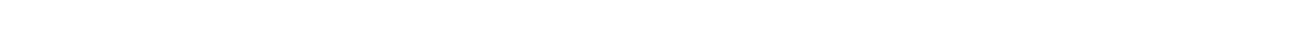
$\$$

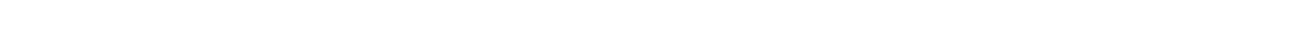
u w.

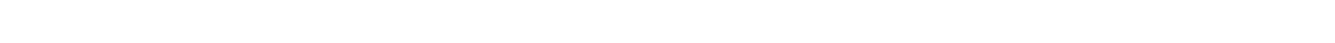

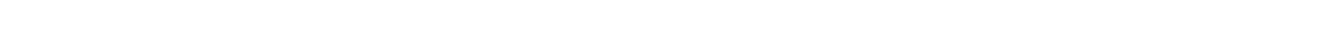

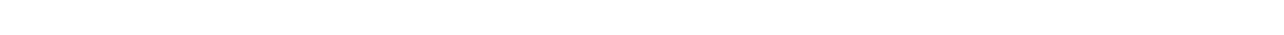

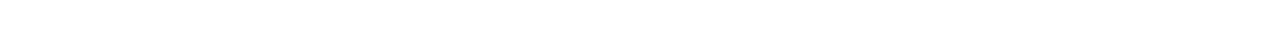
แ

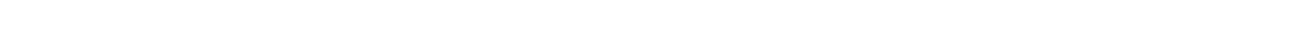

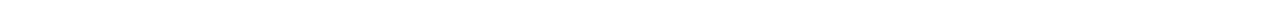

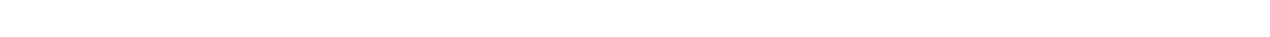

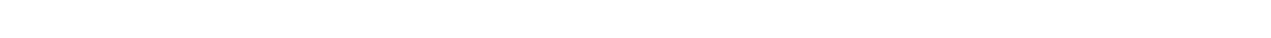

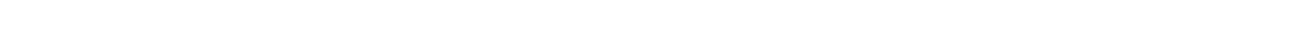
$\stackrel{\alpha}{\infty}$

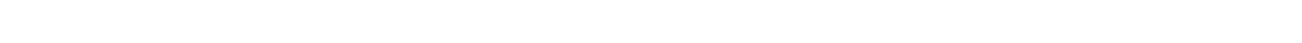

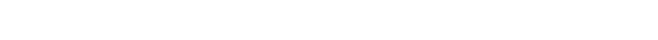

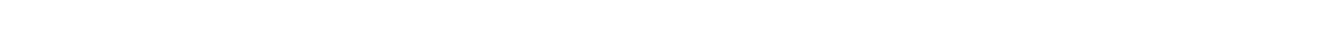

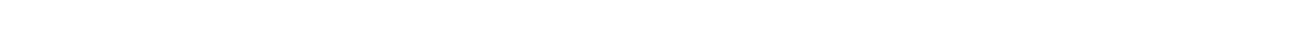

N N NMmMmmmmmmmmmmmmmmmmmmm to t

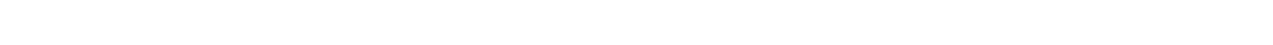
แ

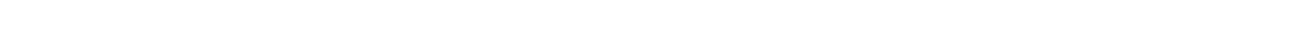

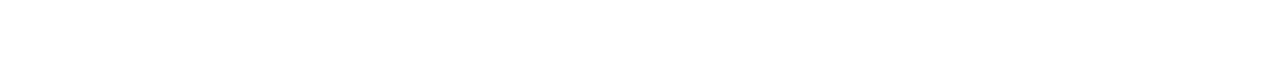




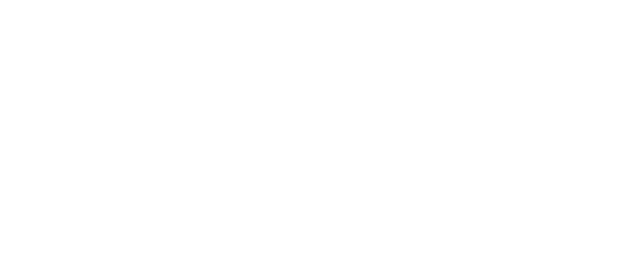

ก๊ ผี

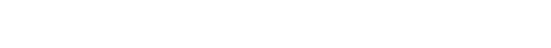

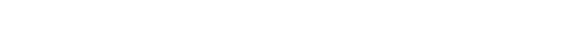

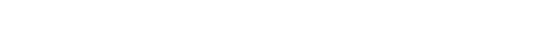

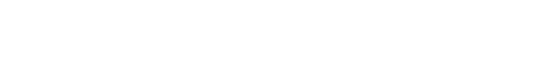

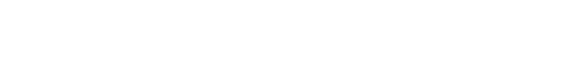

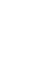

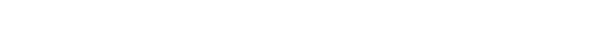
w山. - ưn a

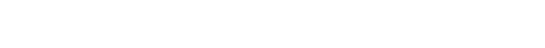

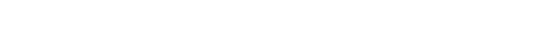

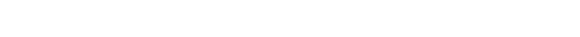

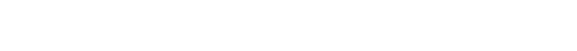

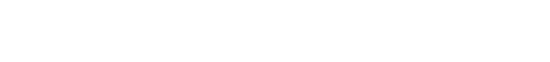

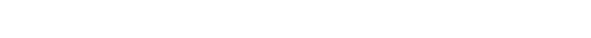
is

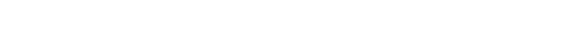

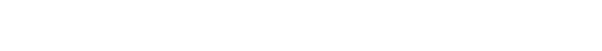

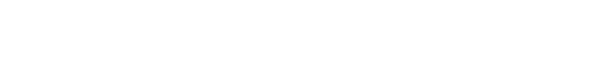

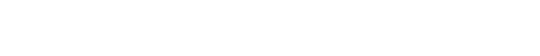

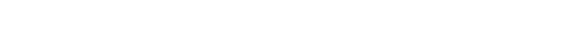

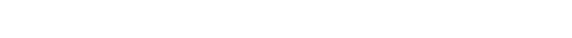

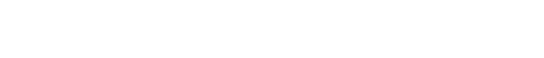

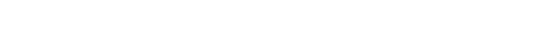

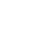

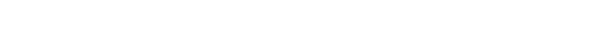
ü "

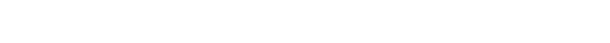

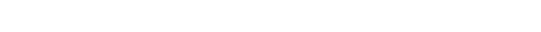

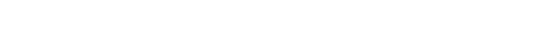

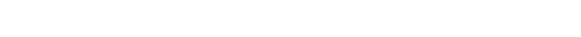

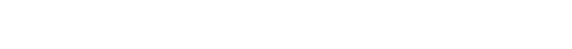

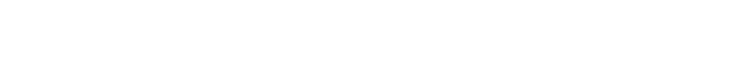




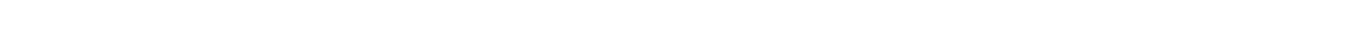

is

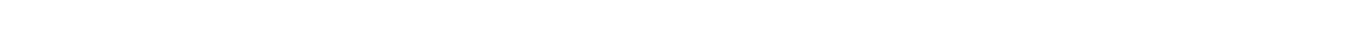

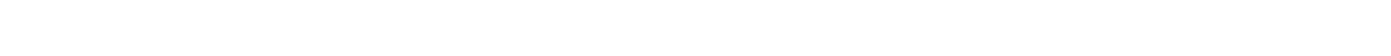

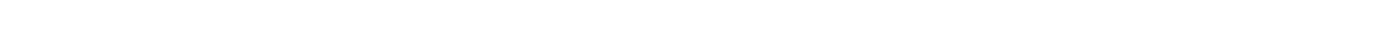
\begin{tabular}{lll}
7 \\
\hline
\end{tabular}

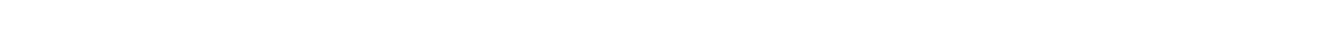

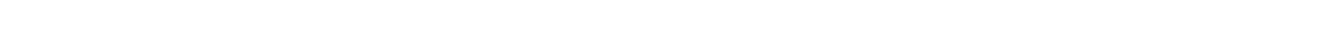

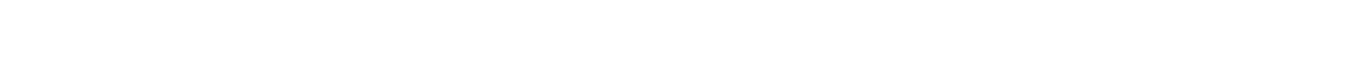

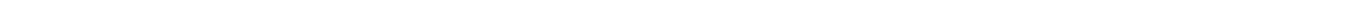

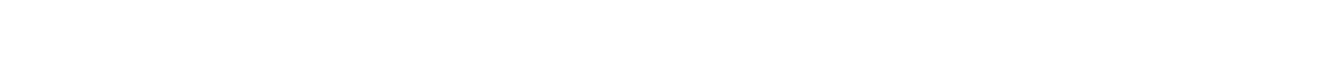

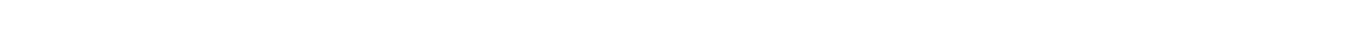

is

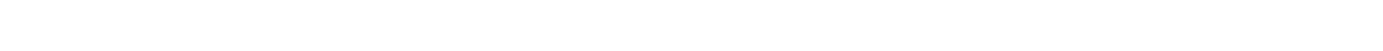
$\longrightarrow$ us

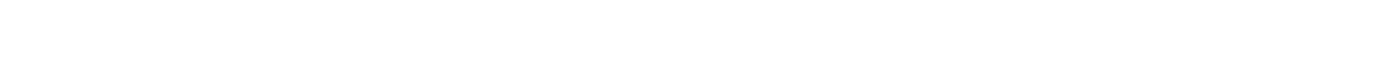

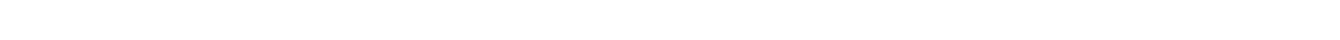

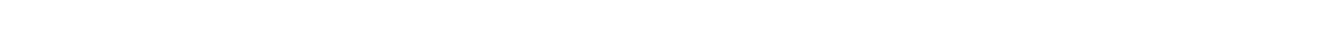

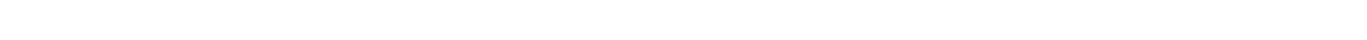

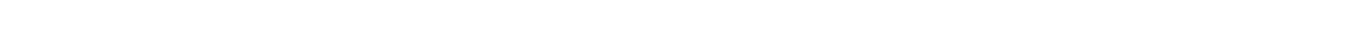

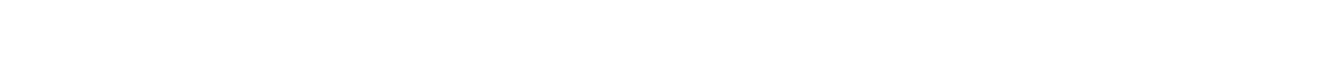

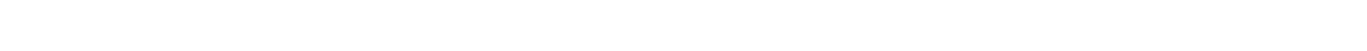
in

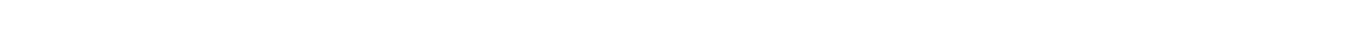

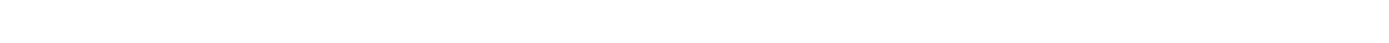

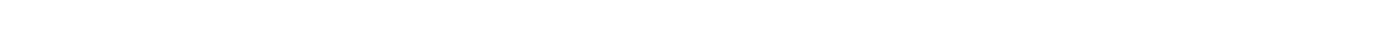

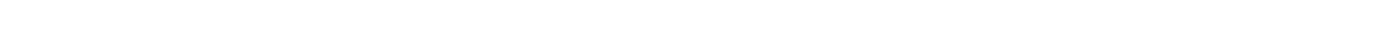

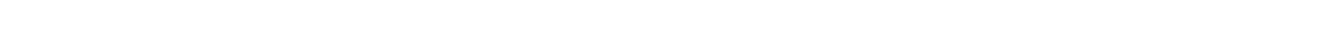

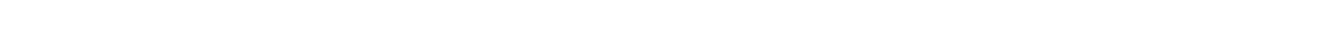

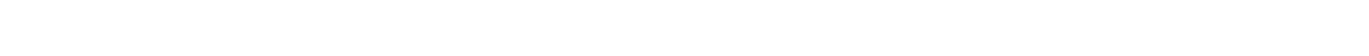

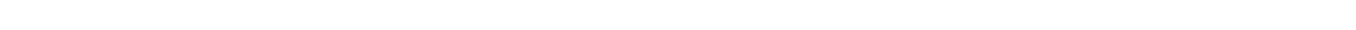

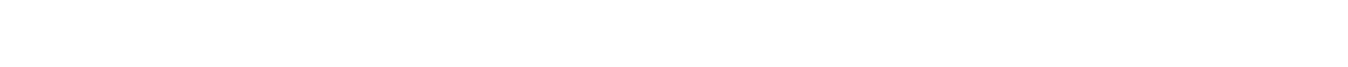

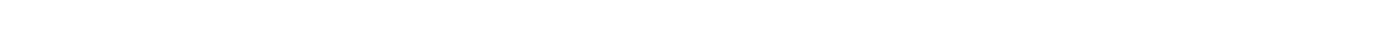
in

-nwarammana $\mu$ แ

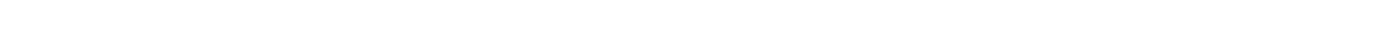

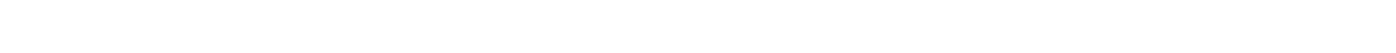

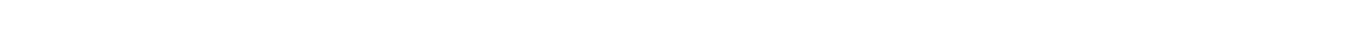

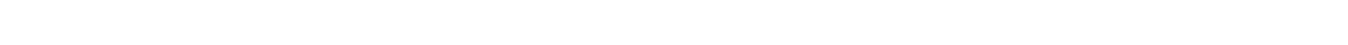

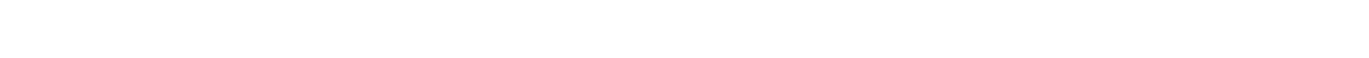

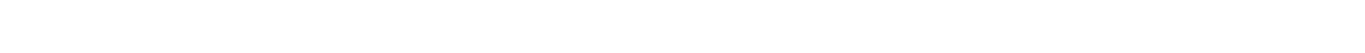

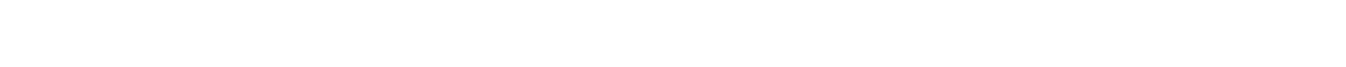




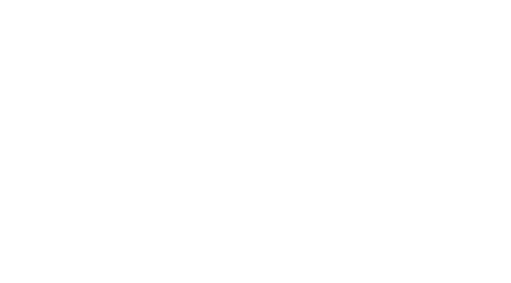

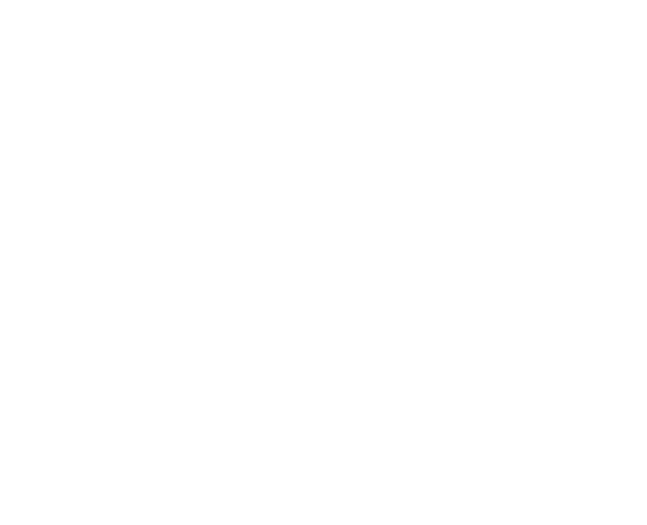
$z$

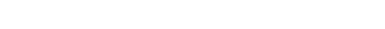

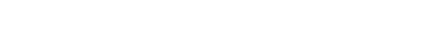

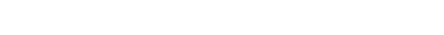

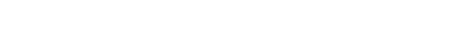

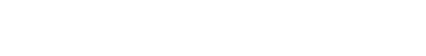

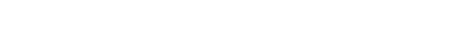

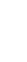

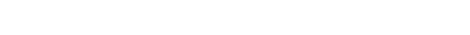

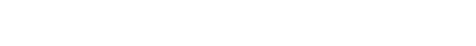

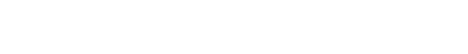

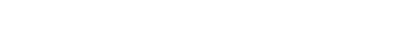

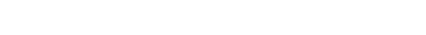

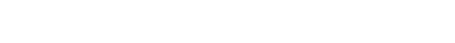

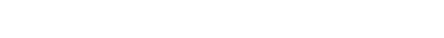

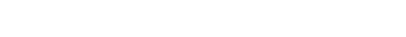

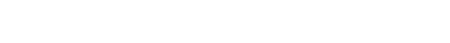
is $z$

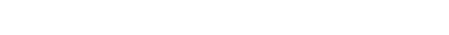

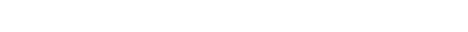

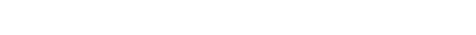

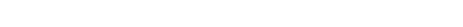

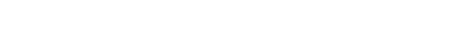

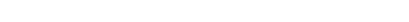

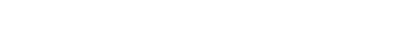
ய

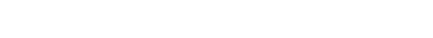

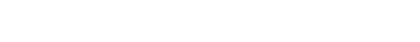

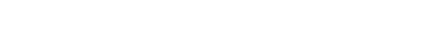


$2 \sum_{0}^{0}$ $2 \%$ , i के gm: 路 $\geq=$ 殀こ 就 舟出 응요 $z \alpha^{m}$ in 뜨의 J: 品

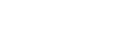
品 䏚. \& व

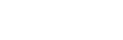
월

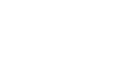
Es ${ }^{3}$ 可a 赵的品文 ज०品 wra a 0 z

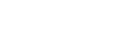

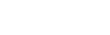
$\exists-0$ वे 级出 $\therefore \because n$ min 는른 $\sim \sim \alpha$ w 品: zn⿺ กIa 응응 $\rightarrow \therefore$ 든 แ少包: on $\vec{\alpha}=0$ 4 00 $\sum_{0}=$ $c 40$ $\because 0$ 落告 I

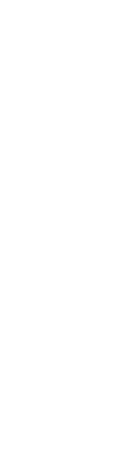
$\sim \geq n z$

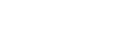
in ù 으의 .. ${ }^{\circ}$

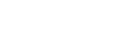
명용요

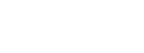
的出 $\underset{\Sigma}{w}$

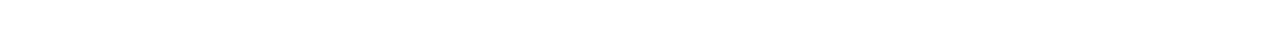
$\stackrel{2}{\infty}$

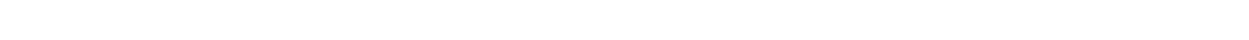
Hú

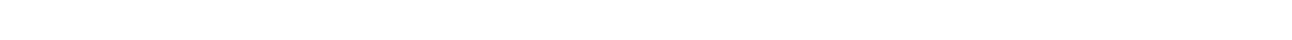

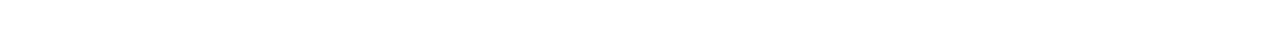

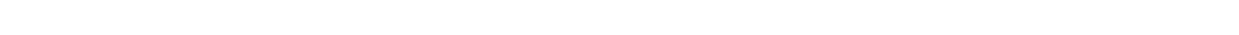

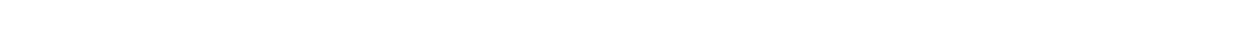

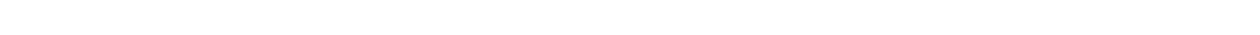
W5 5E5

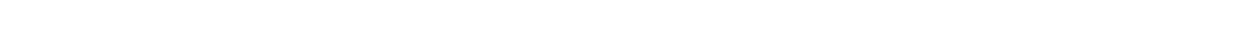

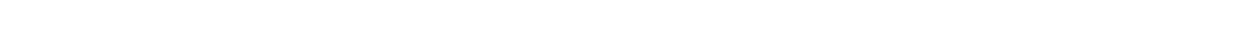

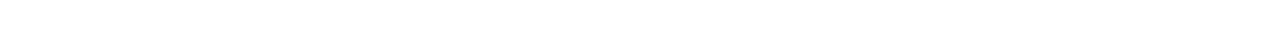

¿

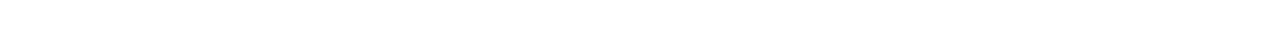
Wü

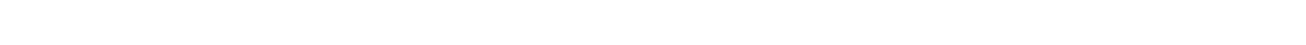

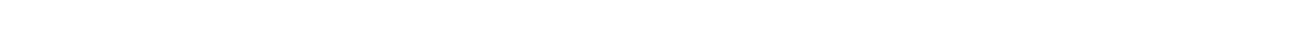

TRTE

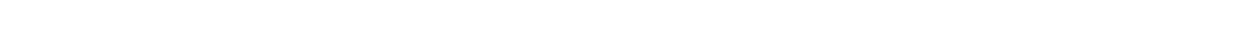

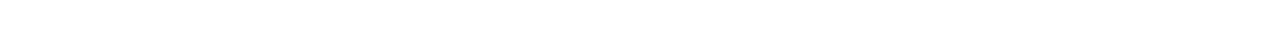

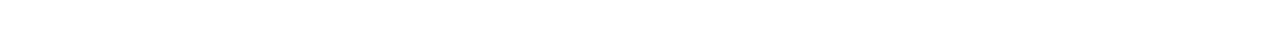

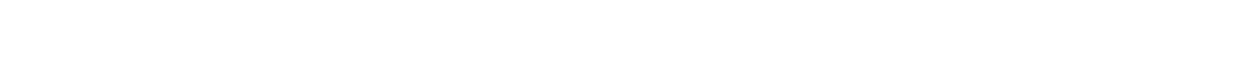

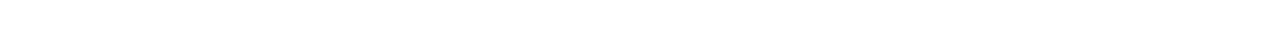
E

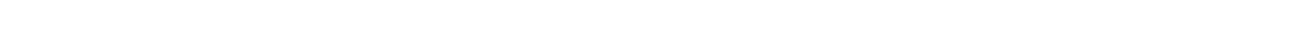
w

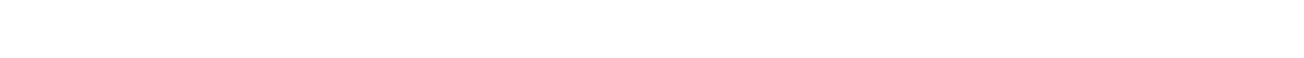

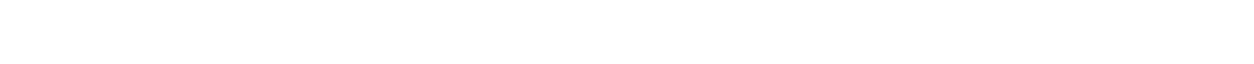
WE

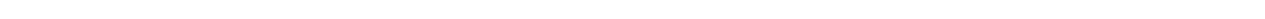

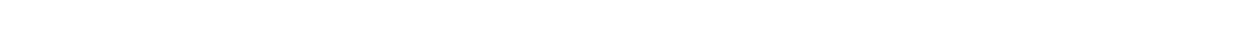

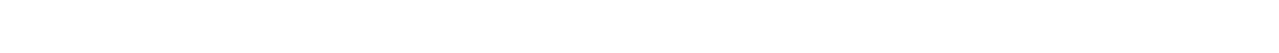
a

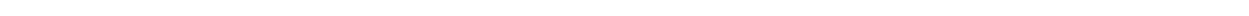
a म "ّ̈

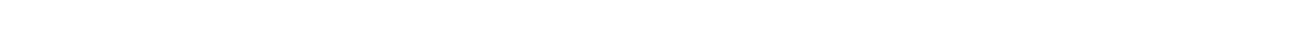

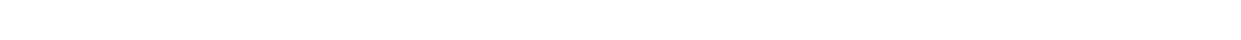

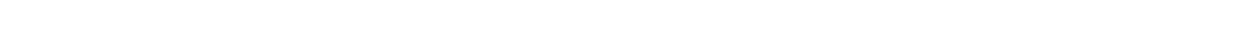

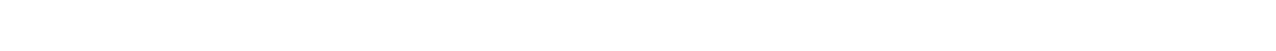

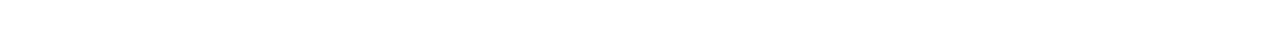

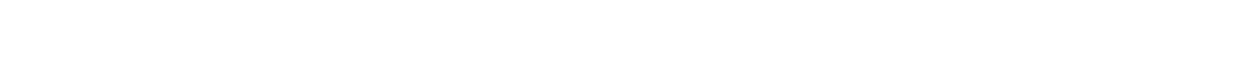




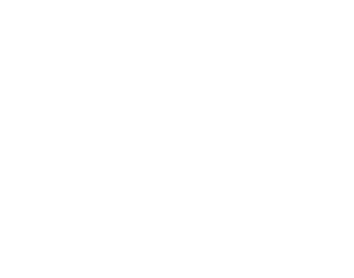

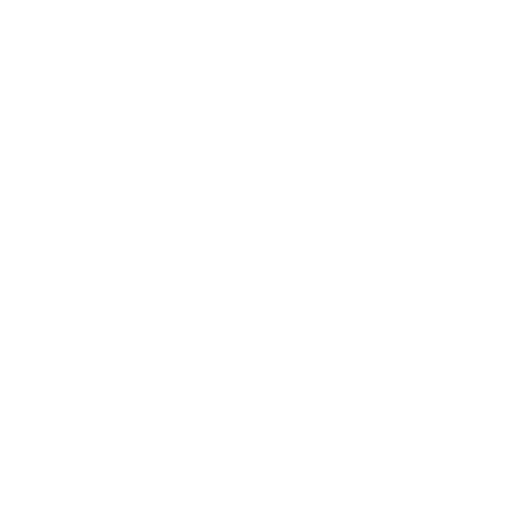

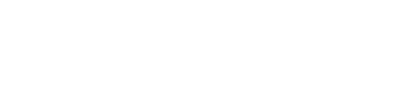

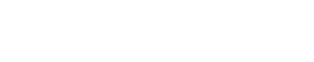

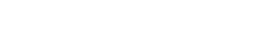

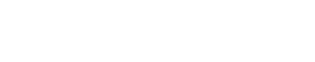
E

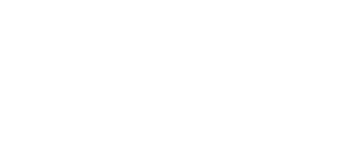

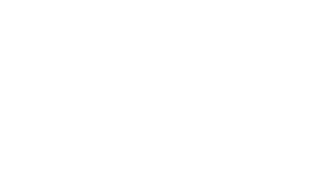

a.

Tusesencosenesenos $\Sigma$ is

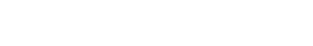
แ山 5 แ

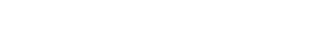

줏으무음

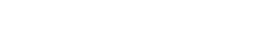
แேニニニニニラニニ ดั

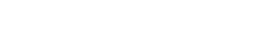




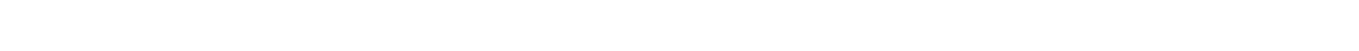

is

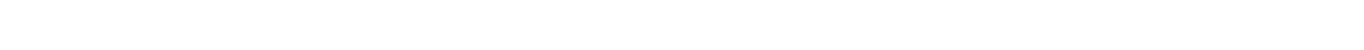

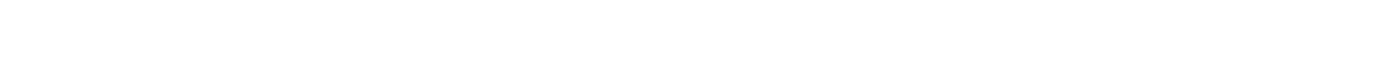

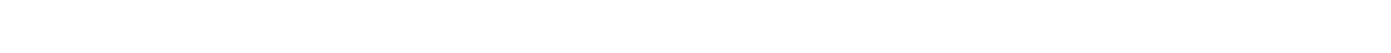

$\infty 0$

$\sum 0$

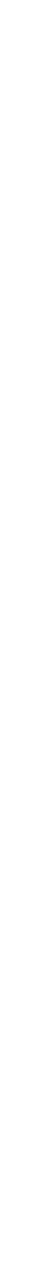

$\infty$

.

$\therefore$ $=\frac{1}{2}$

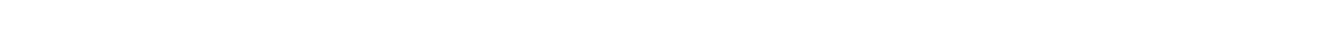

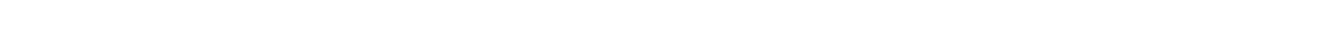

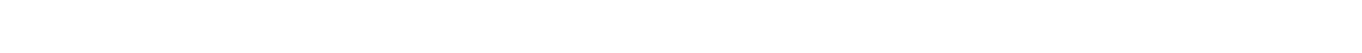

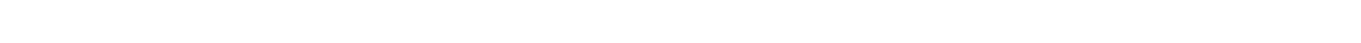

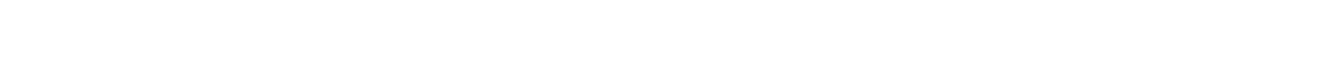

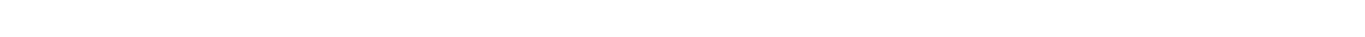
$\approx$

in

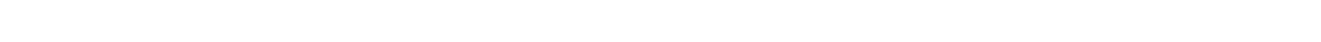
\&

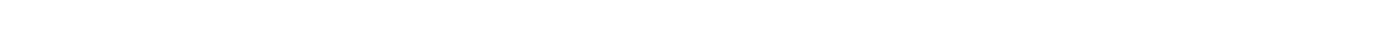

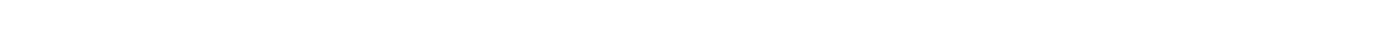

D00000000000-

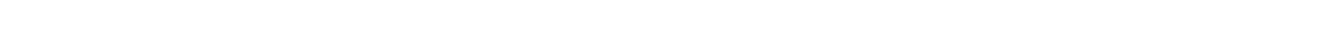

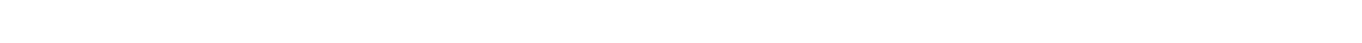

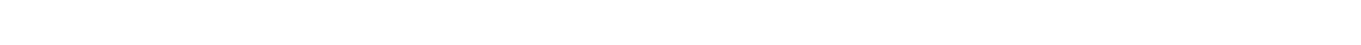

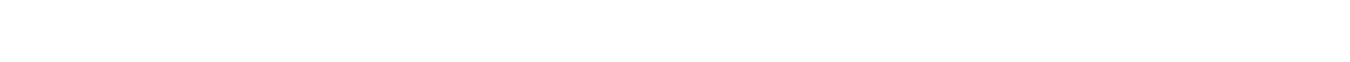

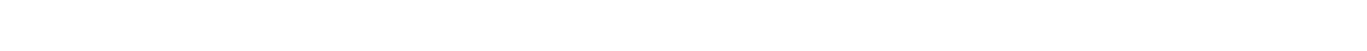

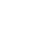

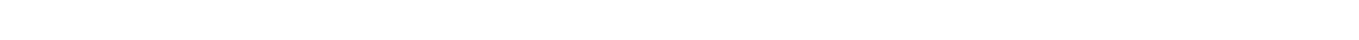

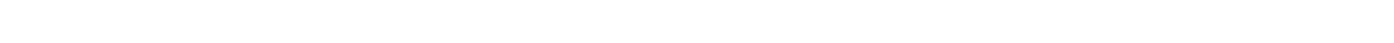

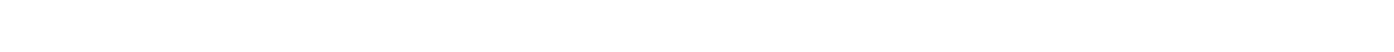

00 i

Iu

$\vec{\alpha} \cup \underline{0}$

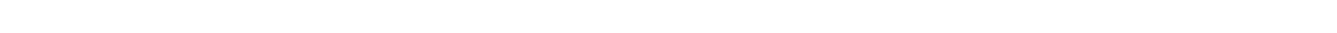

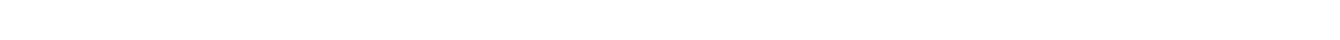

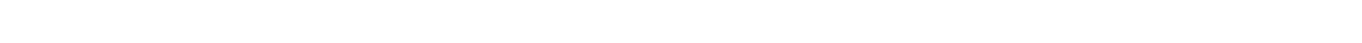

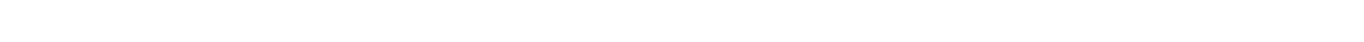

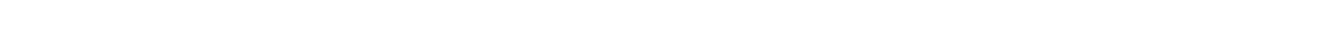

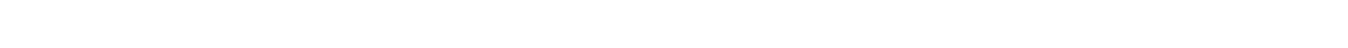

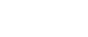
$m=z$

$\ddot{a r}$

$=n u$

$\stackrel{4}{u}$

$=\lim$

$\approx 2$

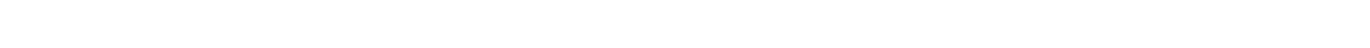
동 贸范

2 a 릉

퐁

Ju

แ⿻

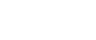

인준

응

is

๙ а д "“山 \begin{tabular}{ccc}
3 \\
\hline
\end{tabular}

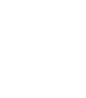
$\underset{\alpha}{2} \frac{2}{2}$

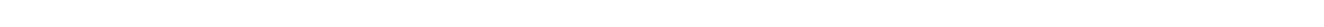

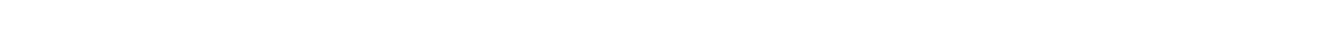

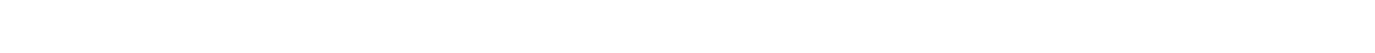

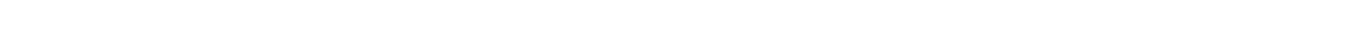

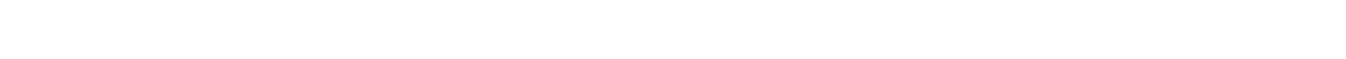



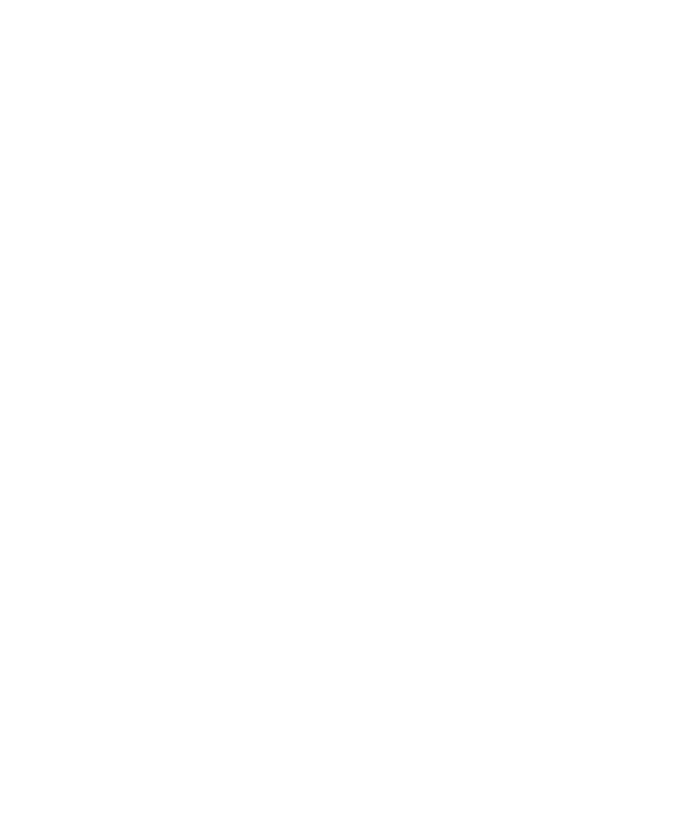

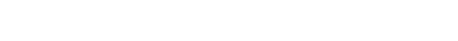

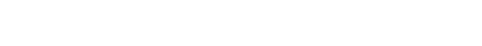

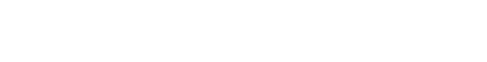

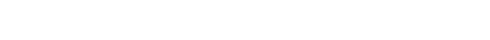

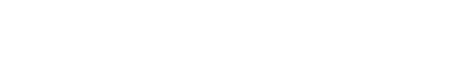

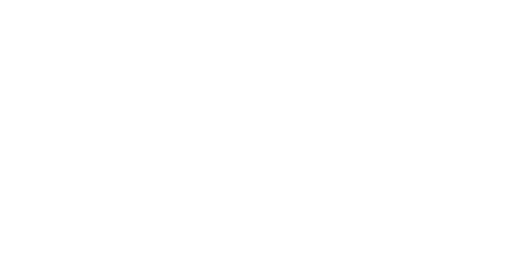

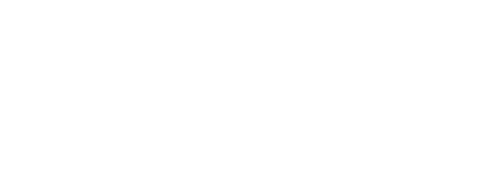

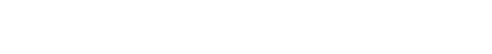
$\sum$

is amนnแก

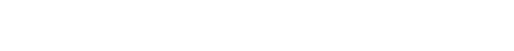
$\leftarrow$ anomomonomoñ

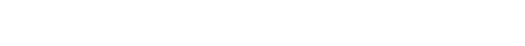

monomonataoga

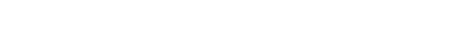

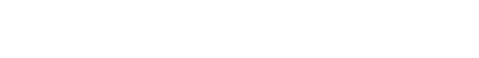

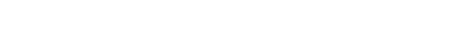

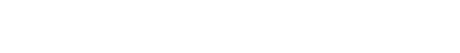




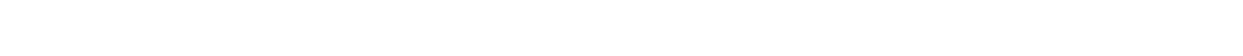

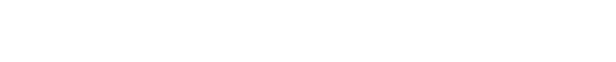

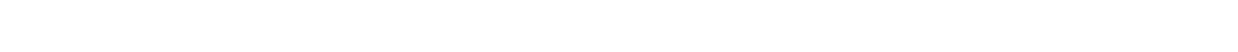

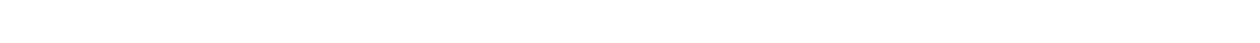
- >

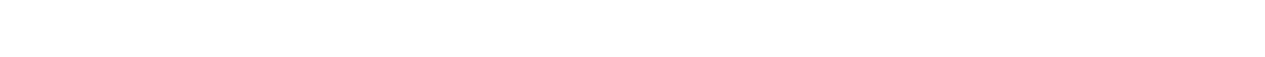

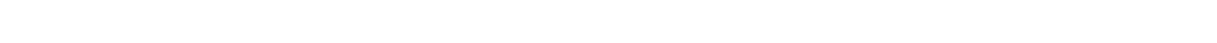

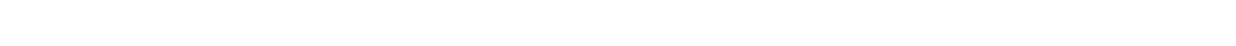

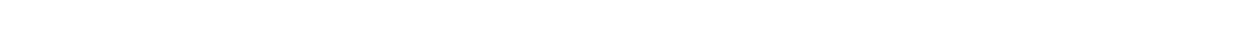

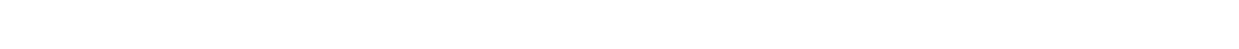

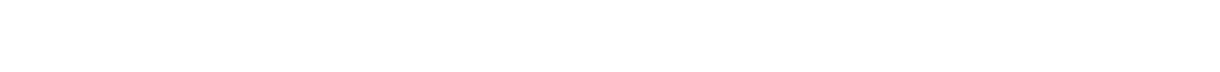

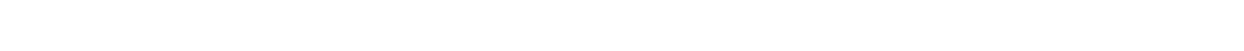
in

m

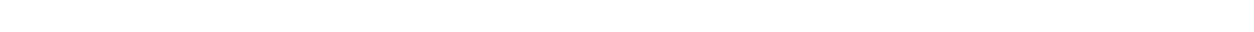

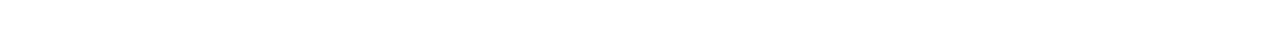
z

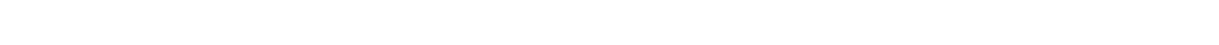

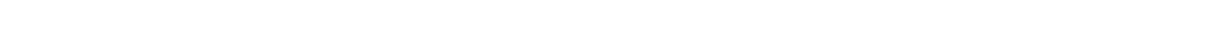
แ ดั

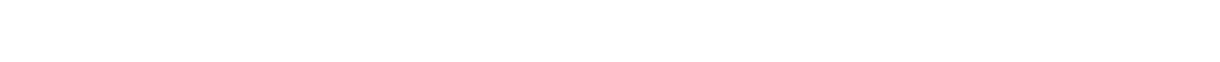

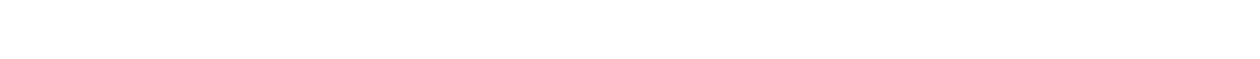

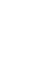

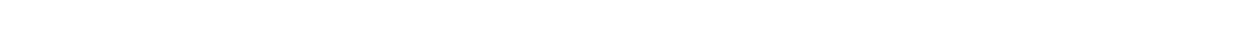

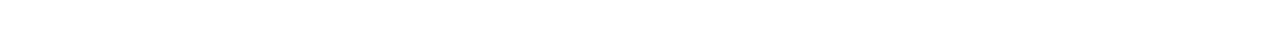
с й

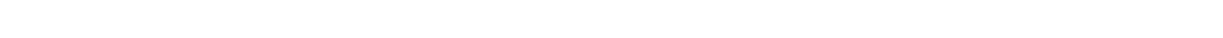

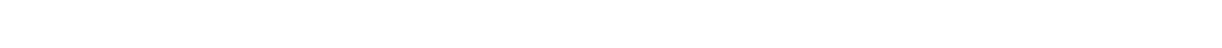

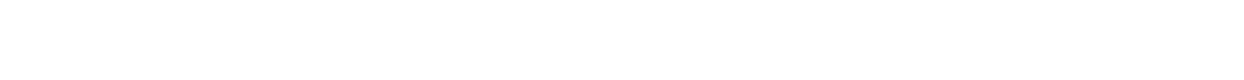

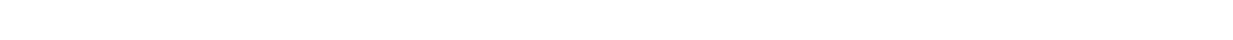

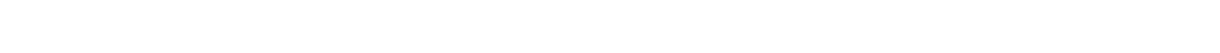

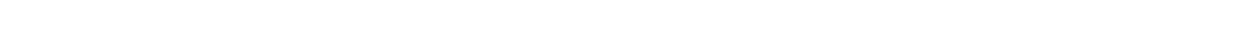
$\sum$

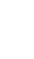

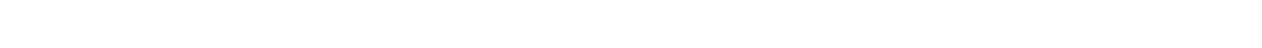
* แ แ

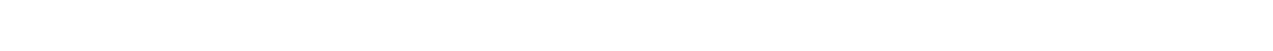

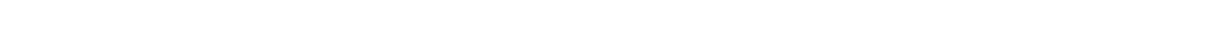

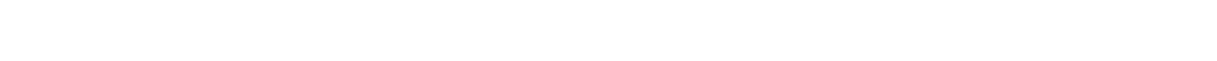

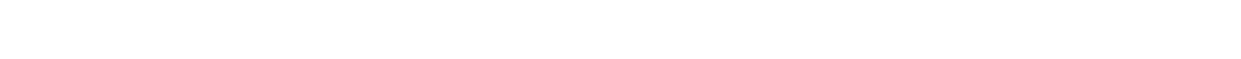

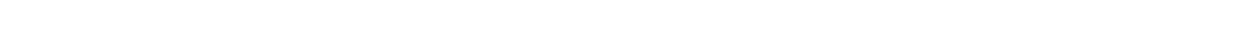

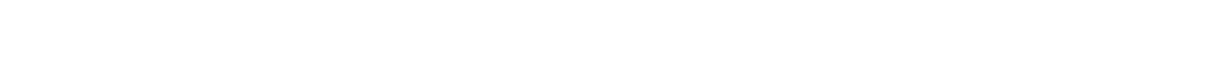



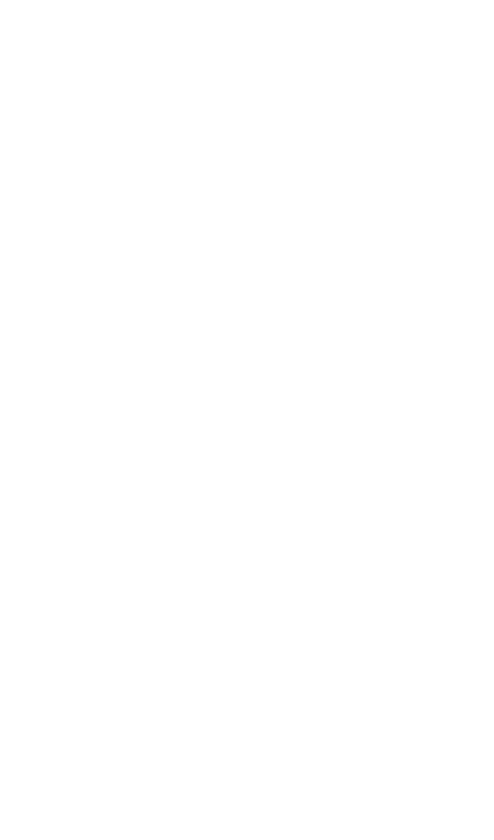

z

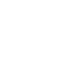

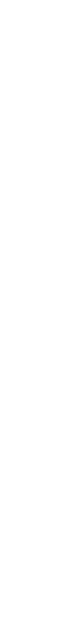

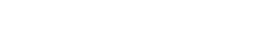

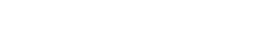
แニニ ニニニะ

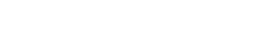

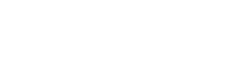

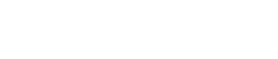
is 次 o

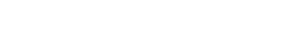

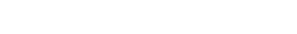

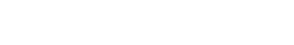

$-5-5-5-\infty$ aco u

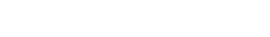
on $=n$ nก

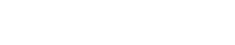

tur es cosesenenesos

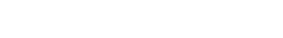
is

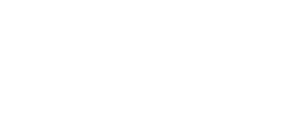

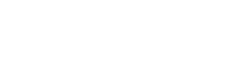
แேニラニニニニニ ธ์ํํำ

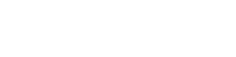



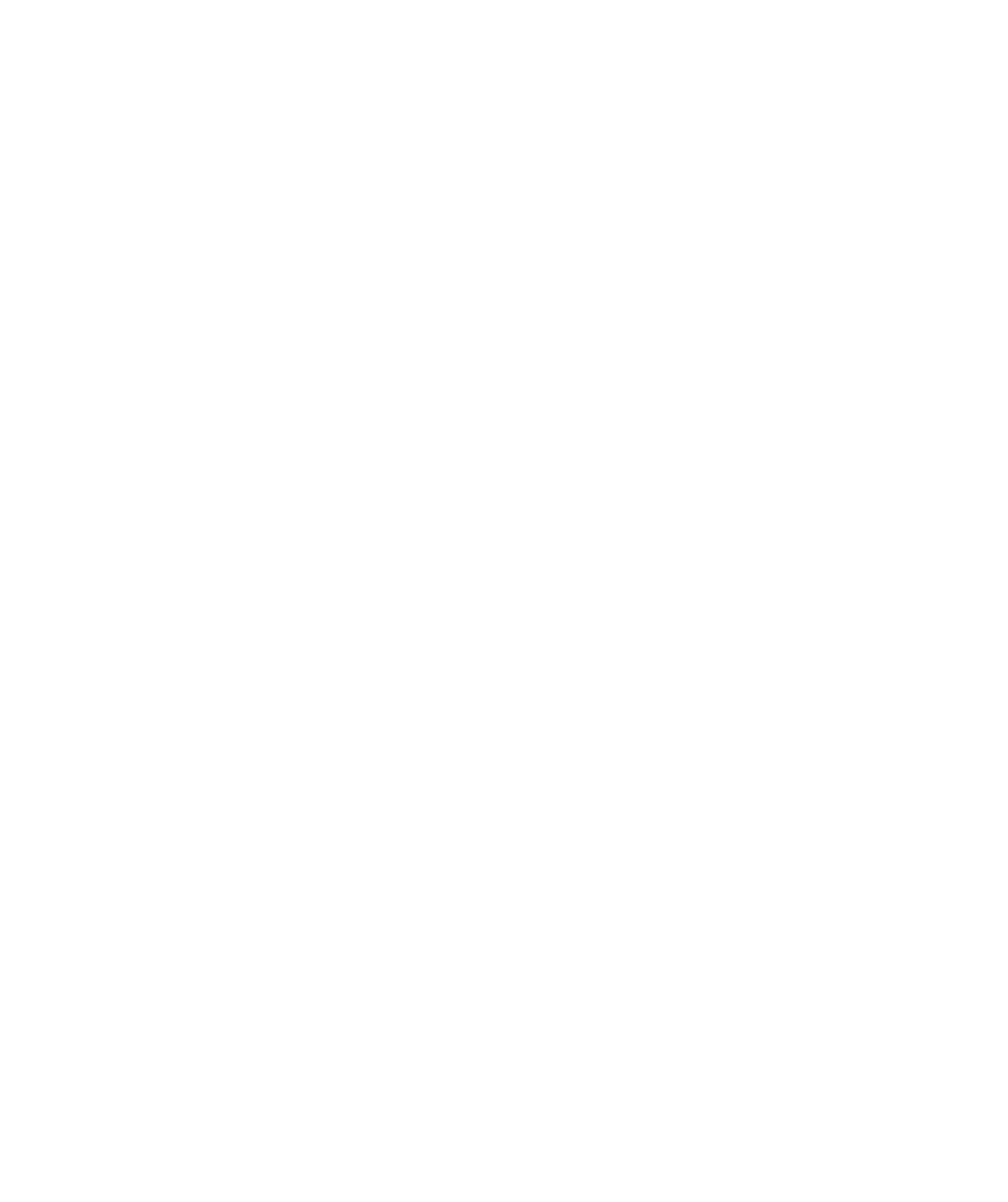

ผ

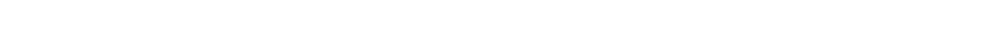

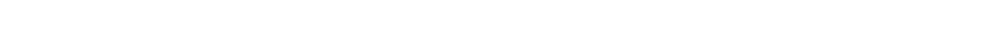

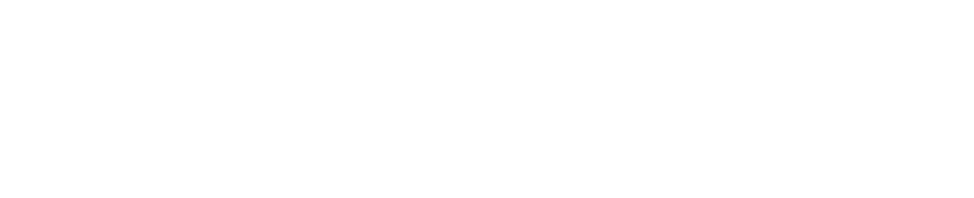

tom

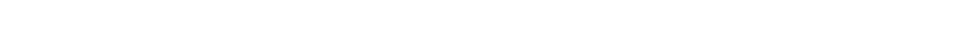

$\stackrel{a}{5}$

ڤ

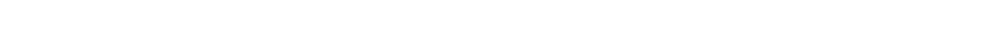

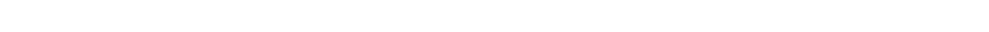

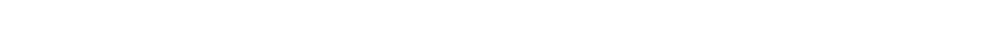

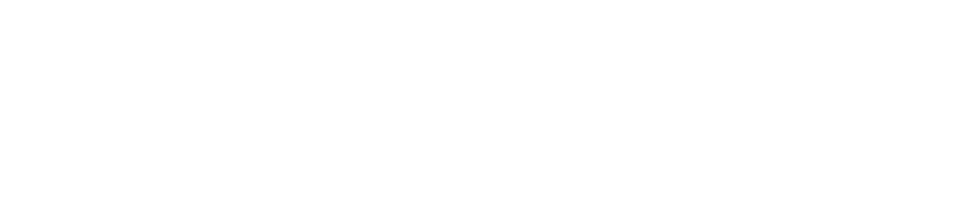

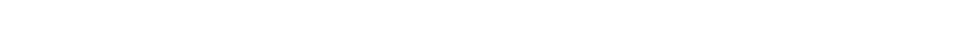
点

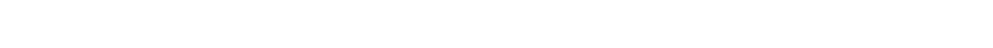
w w 出然m

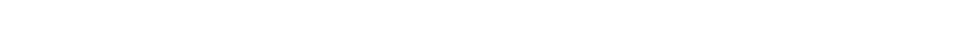

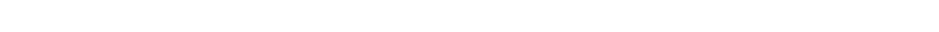

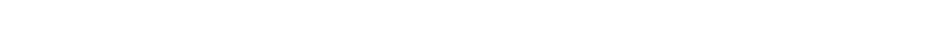

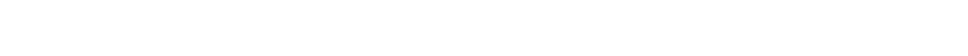

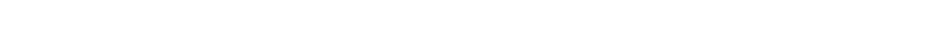
-

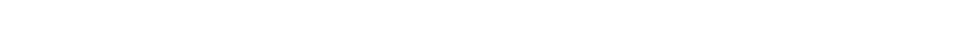

$\pm$

mก ü

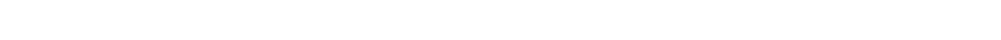

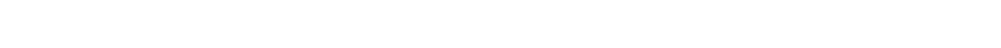

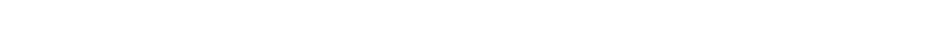

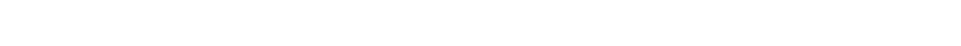

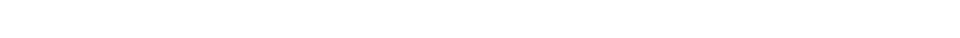

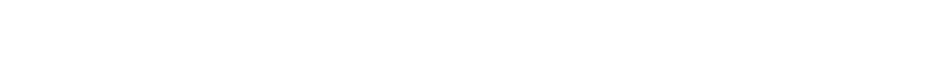



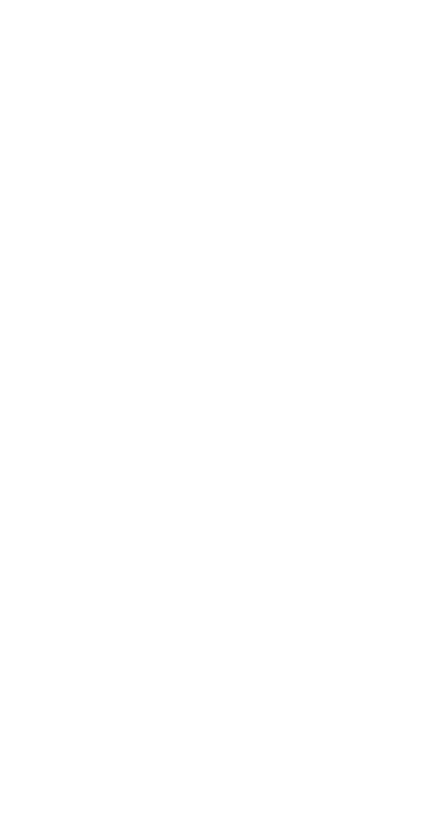

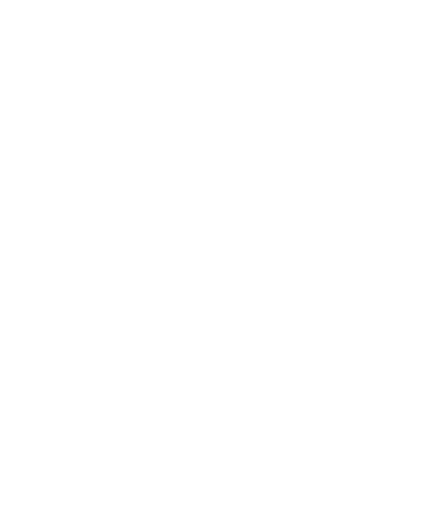

- annunan $\sin 2$

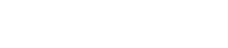
a

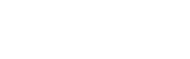

出

2

5

tosenosing $\Sigma$

is

$-0 \simeq-m n$

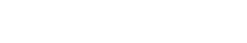

西药

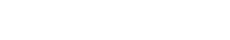

$\sim \sim N \sim N N$ क แேニニーニニ

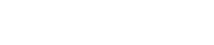

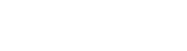




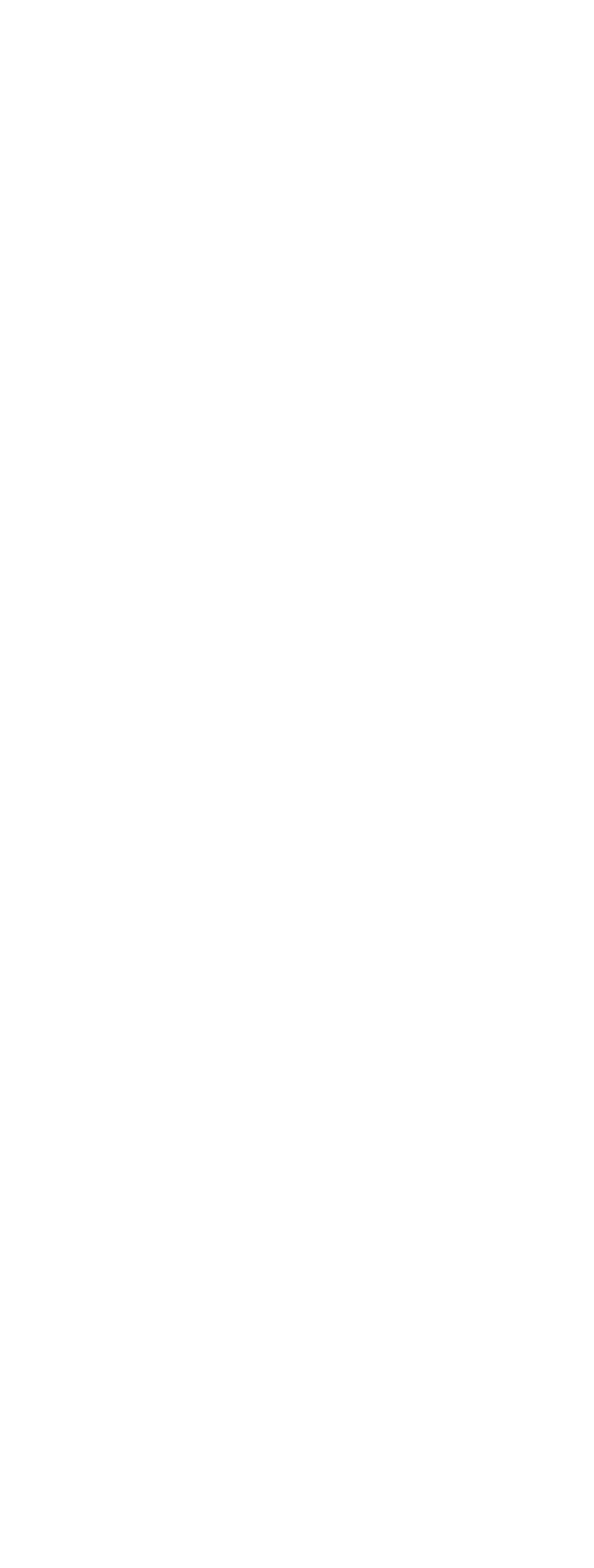

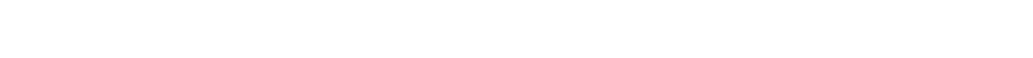

in

r v क

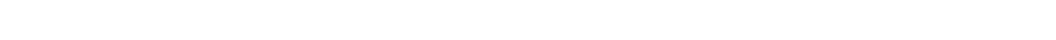

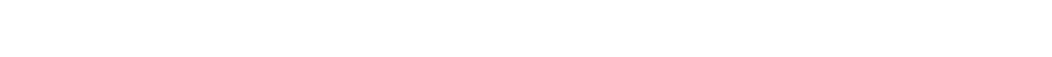

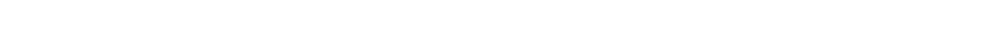
a a

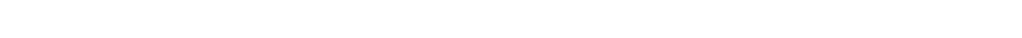

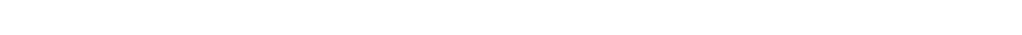

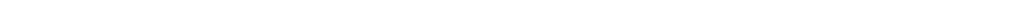

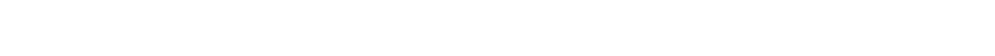

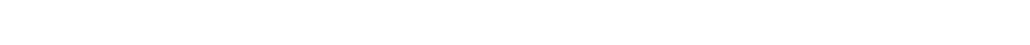

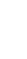

m. - тmmmññ

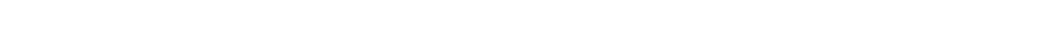

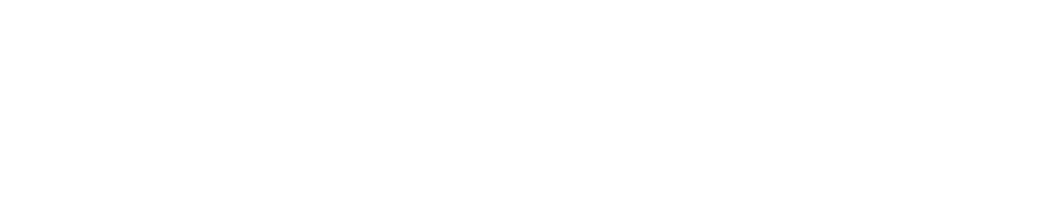

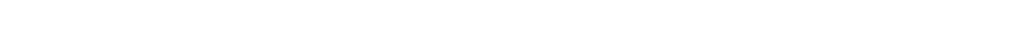
$\stackrel{a}{a}$

ม

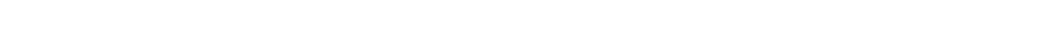
- דал

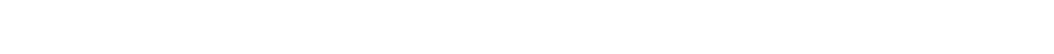

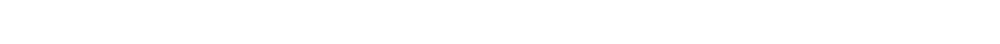

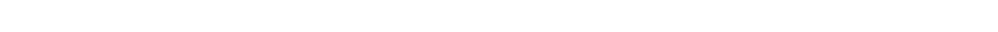

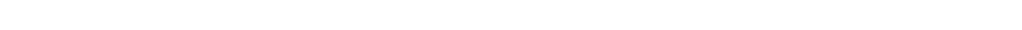

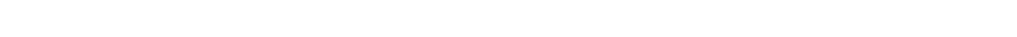

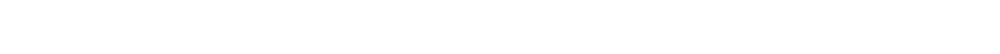

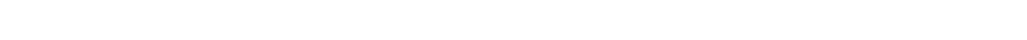
in

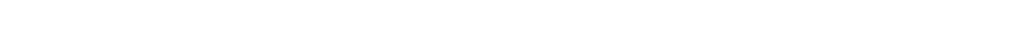
แ

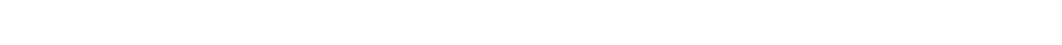

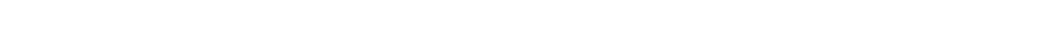

- - -

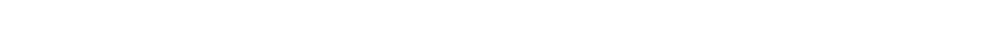

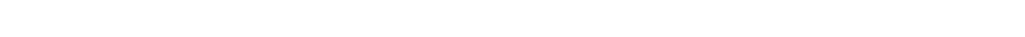

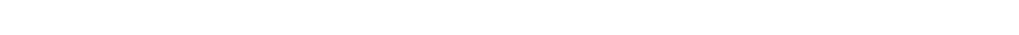

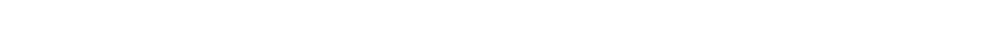

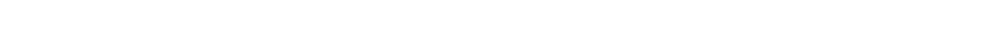



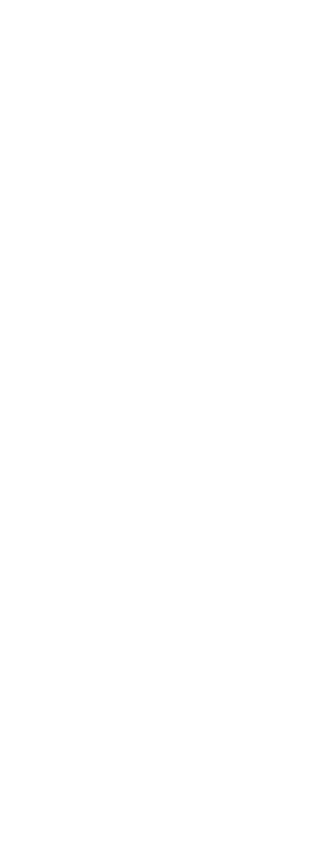

z

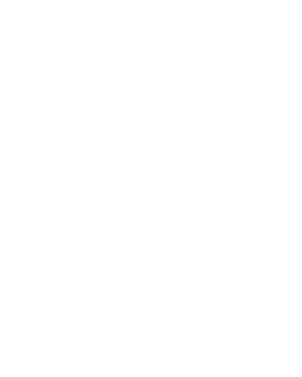

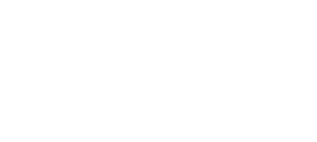

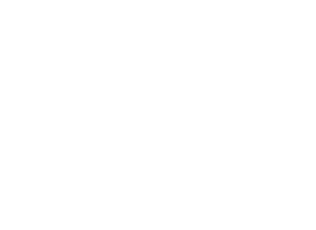

논

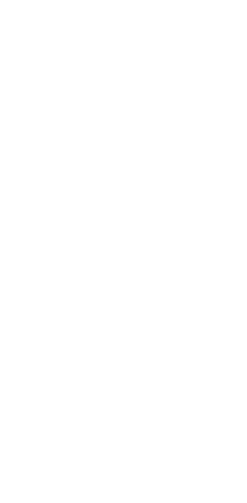



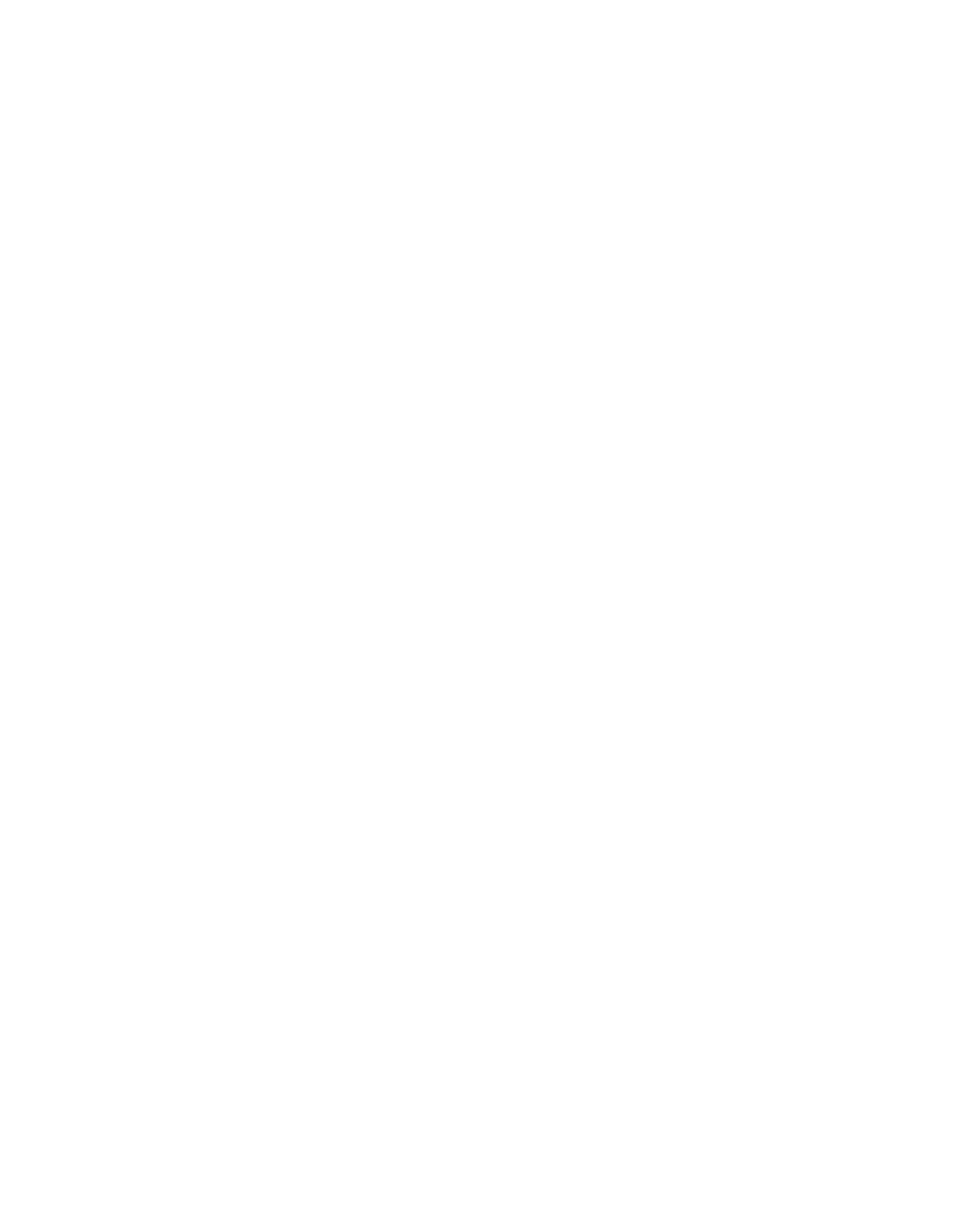

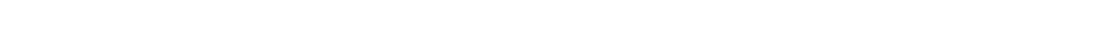

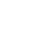

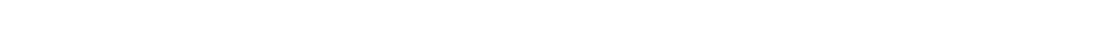

ü ü

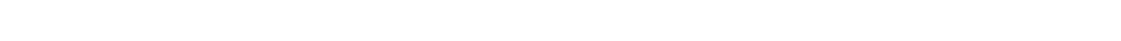

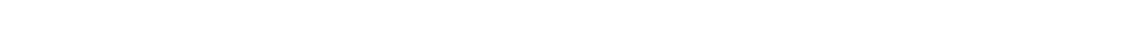

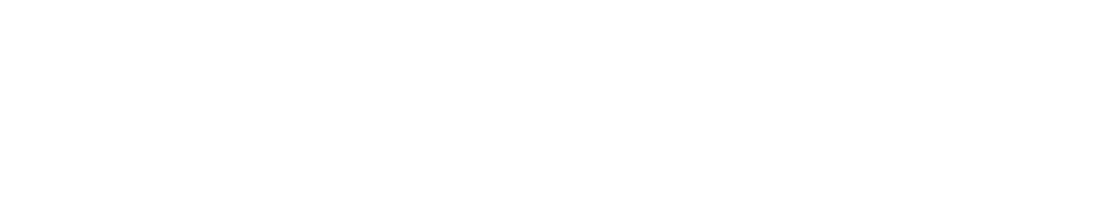

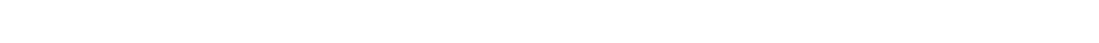
亲

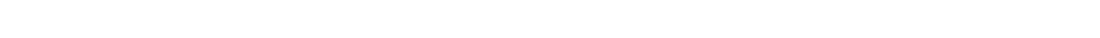
a

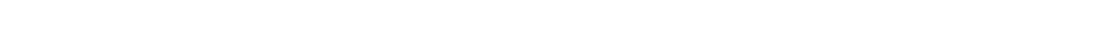

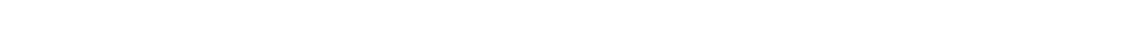

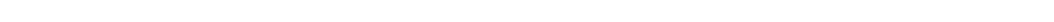

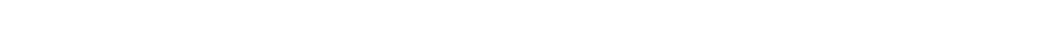
世ニニ a - Ñ

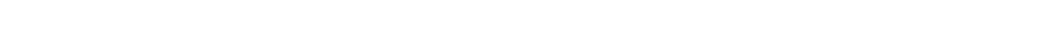

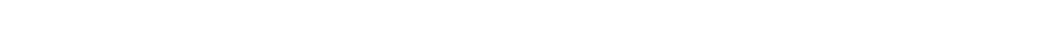

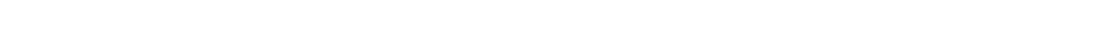
is

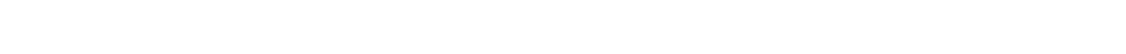
tu

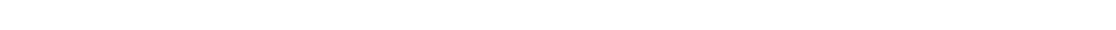

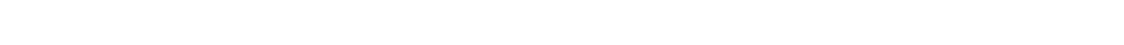

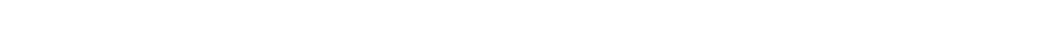

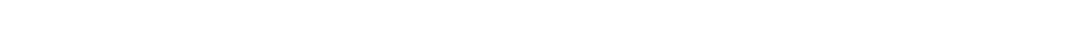
W ๔

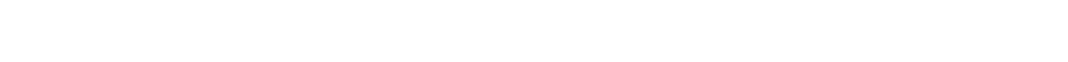


$\because \because a d a$

$-\dot{x}^{\infty}>$

ㅇํㅇํㅜ는

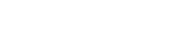

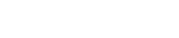

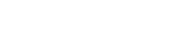

㳻舟a

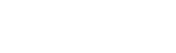
n.mo

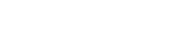
윤

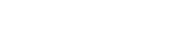
윤은

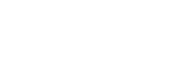
2us in mona. 우욤요

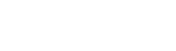
mE

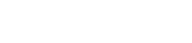

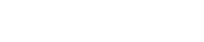
○。品告 $\because a$ : a

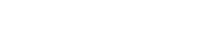
ơm:

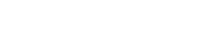
w屾

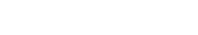
os $x$ UnO On

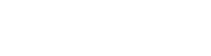
$\alpha \frac{\alpha}{2} \alpha$ w云交我

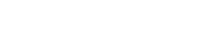

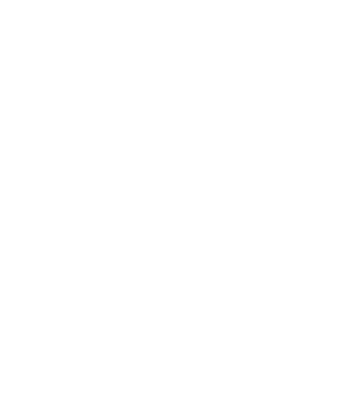

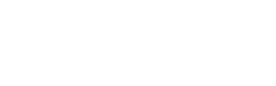

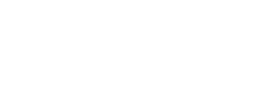

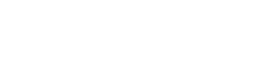
in a w w

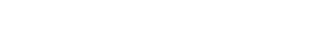

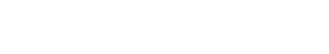

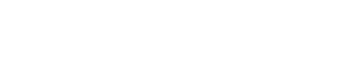

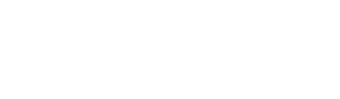
$-z \sim z \infty \omega \circ \omega-2 \pi$

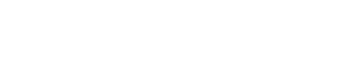

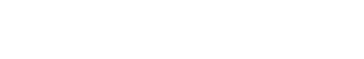

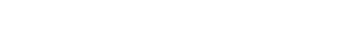

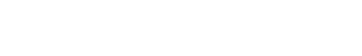
$\ddot{w}-\ddot{w} \sim \ddot{w} m \ddot{w} \rightarrow \ddot{w} \ddot{w}$ z七

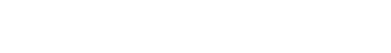
$z^{z} z^{z} z^{z} z^{z} z^{z} z^{2}$

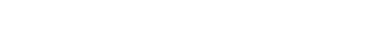
命岗 $\underset{w}{w}$

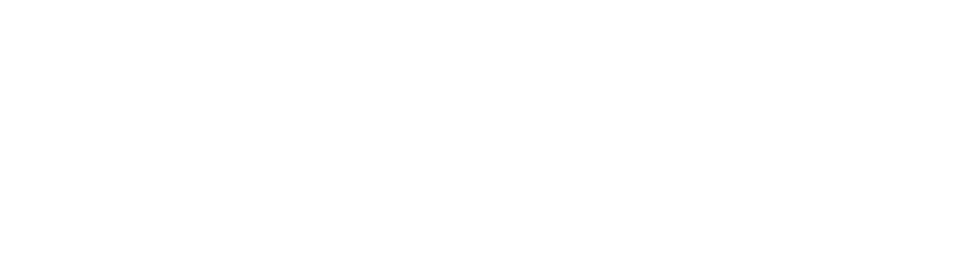

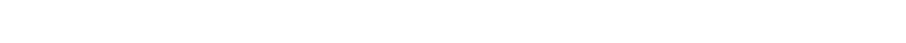

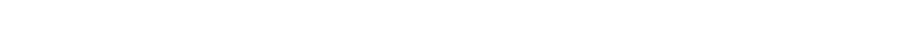

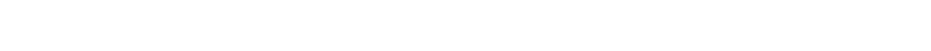

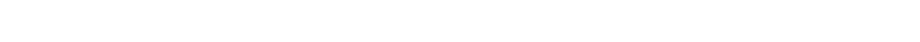

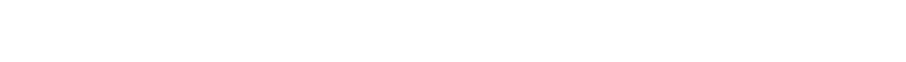

$$
\sum^{2}
$$

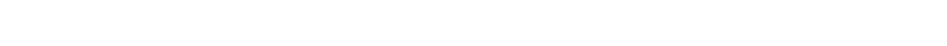
« स -

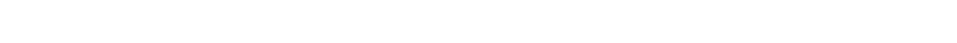

мmmmmmmmmmmm

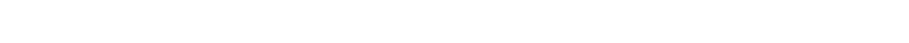
LIFE

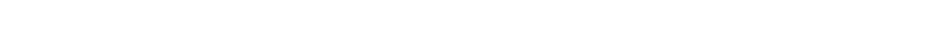

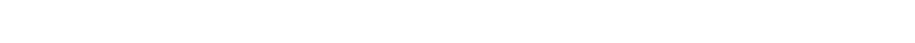

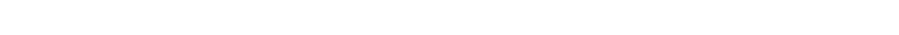

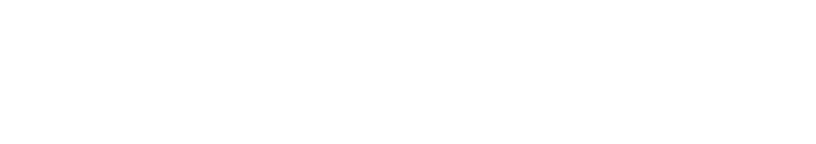

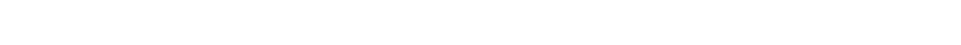
m a

mMmm

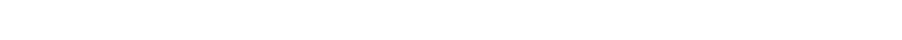

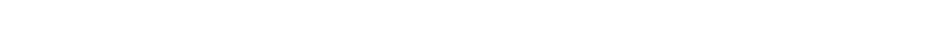
৯

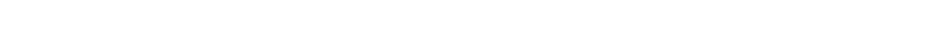

w

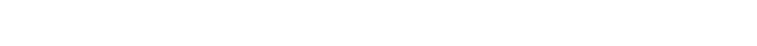

\&

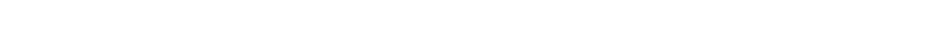
w w

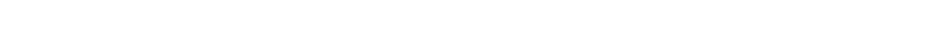

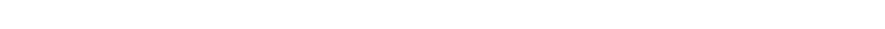

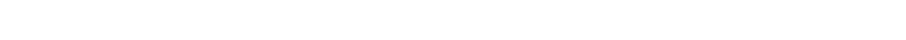

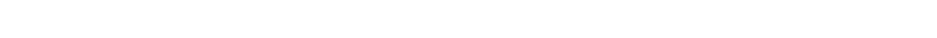
5ovo

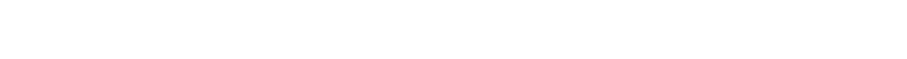




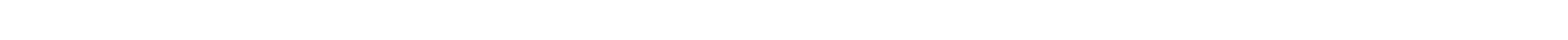
व

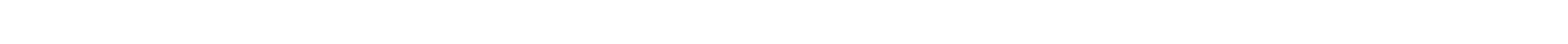

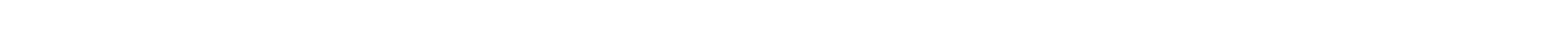

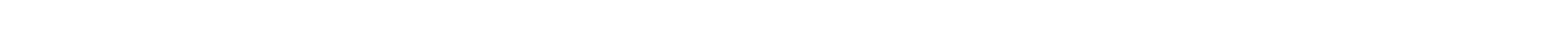

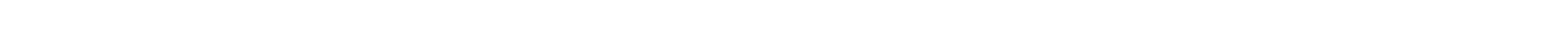

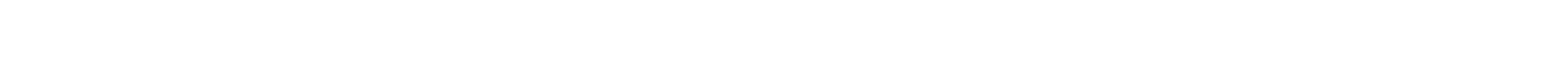

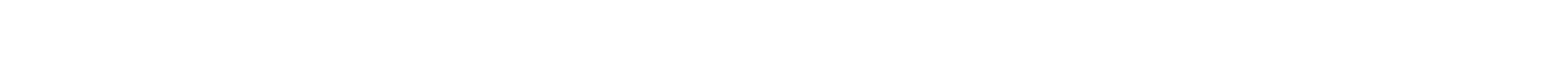

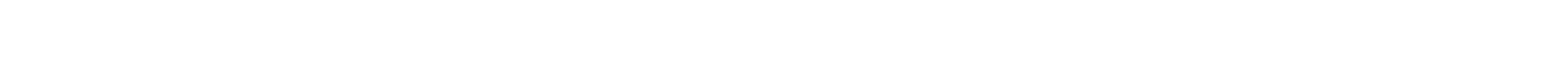

$\stackrel{-}{2}$

¿

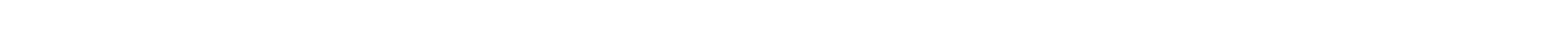
úp $->_{u}^{\infty}$ \begin{tabular}{ccc}
\hline \\
0
\end{tabular}

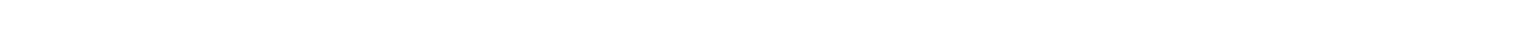

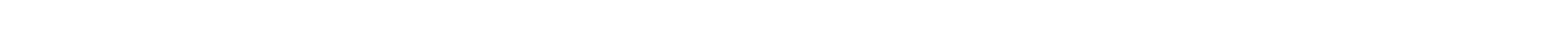

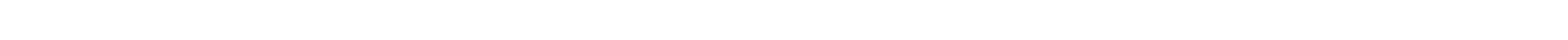
a

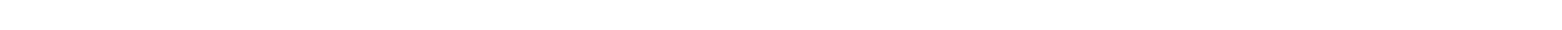

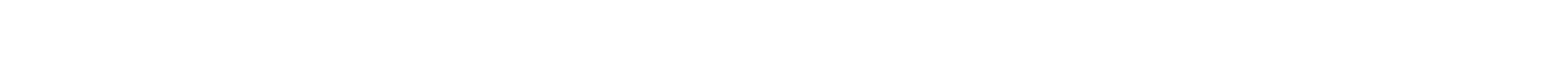

$\leftarrow$ m n

is

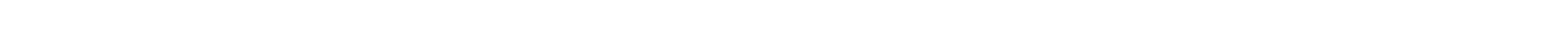
a d t tunn

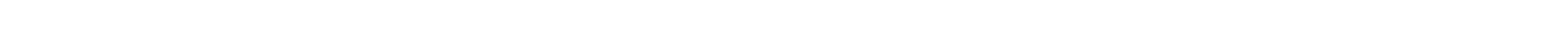

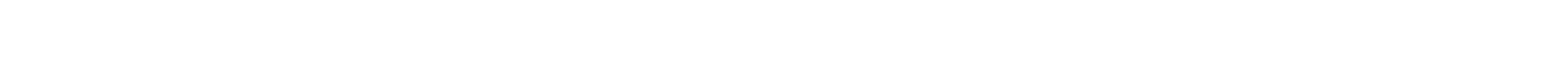

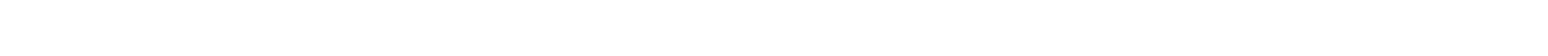

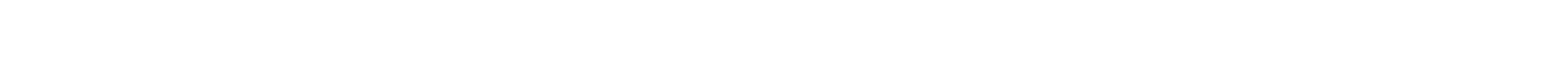
-

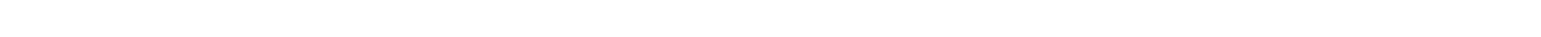

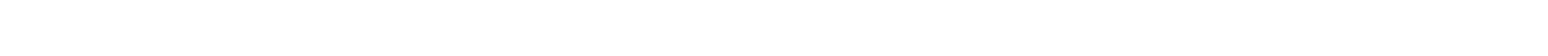

เ

2

" a "H

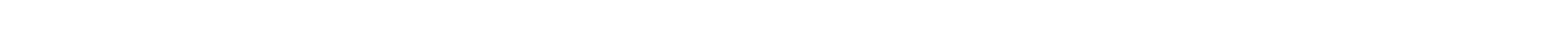

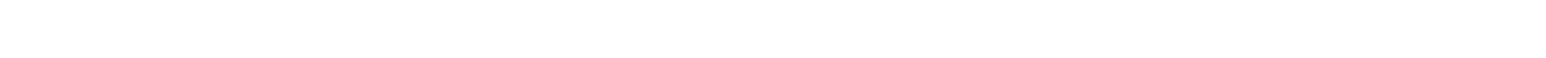

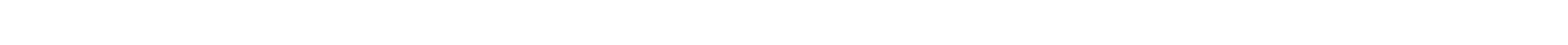

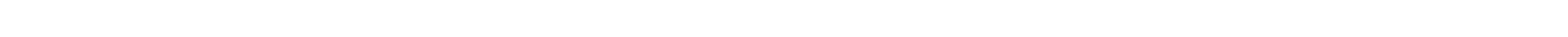

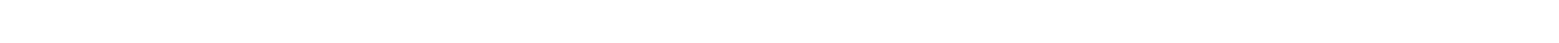

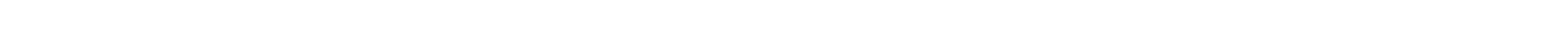




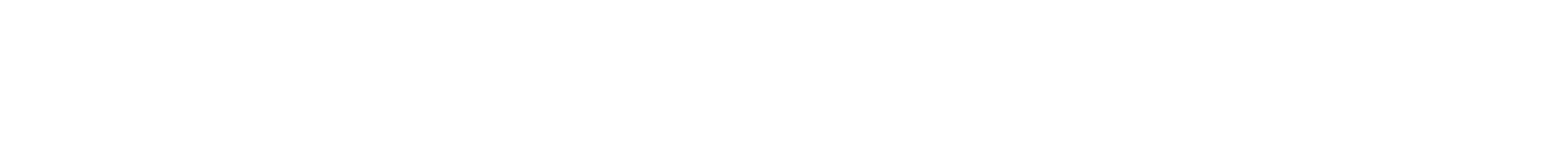

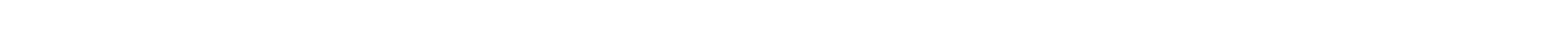

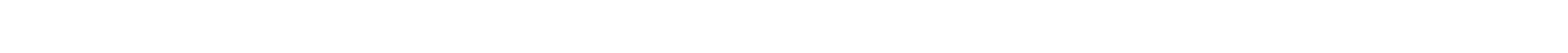

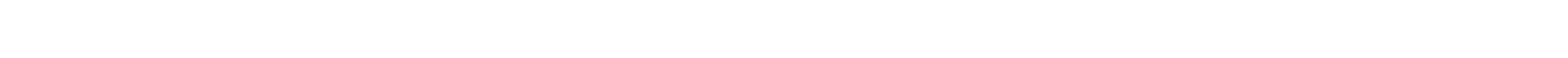

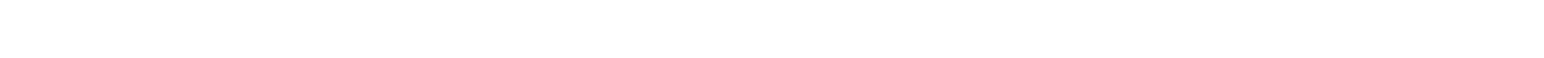

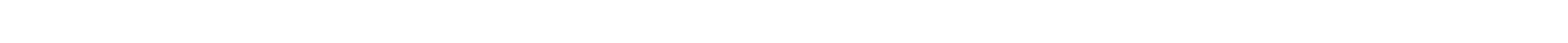

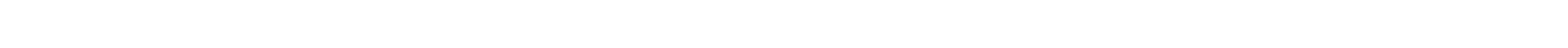

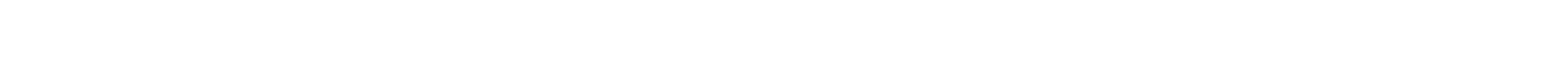
t

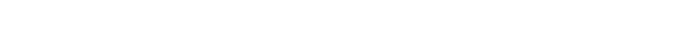
a

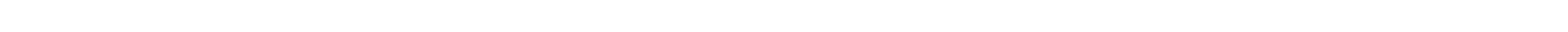

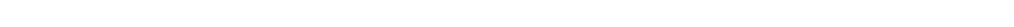

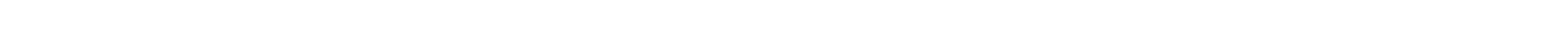

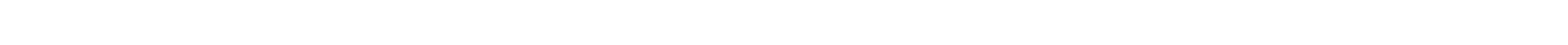

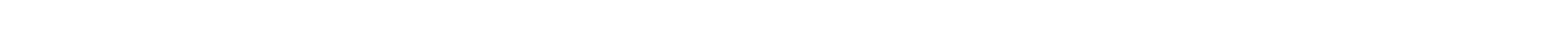

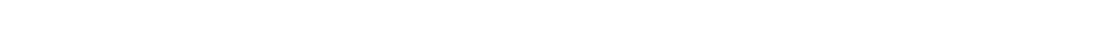

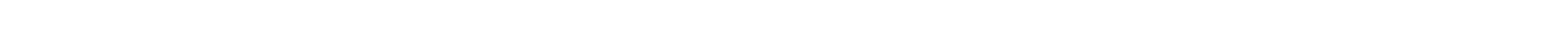

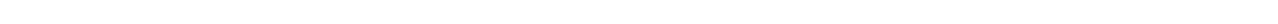

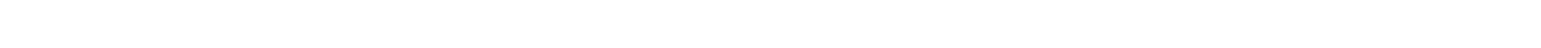

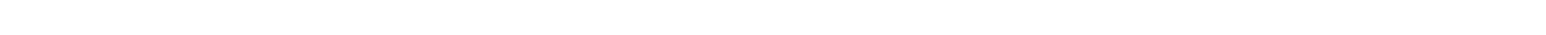

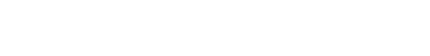
is
$\sim$
n
in

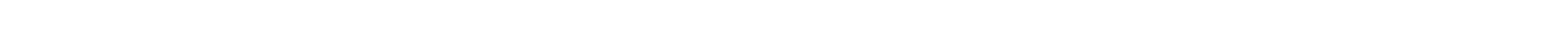

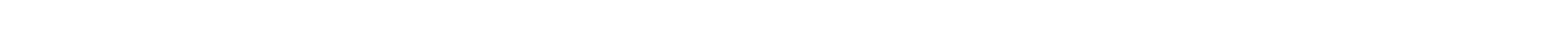

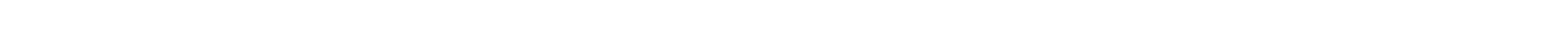

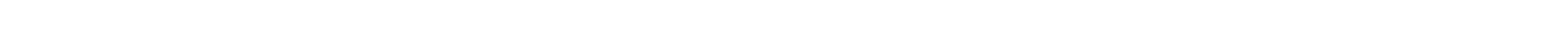

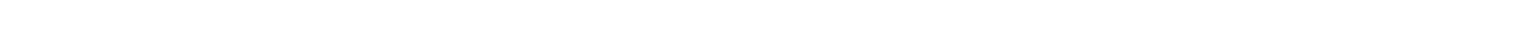

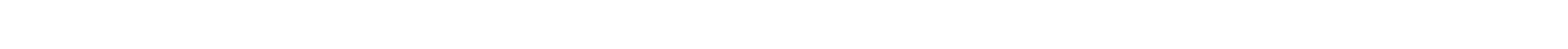

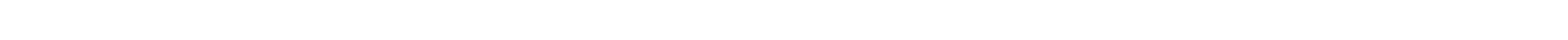

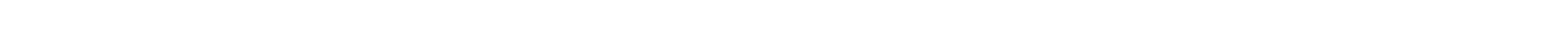

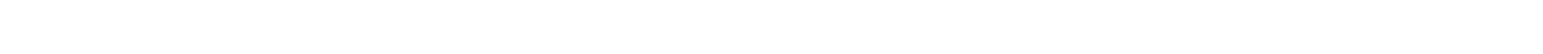

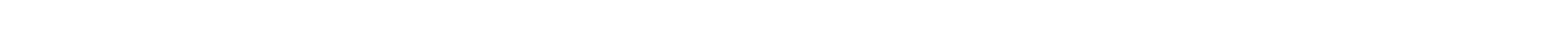

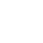

a mOO u.

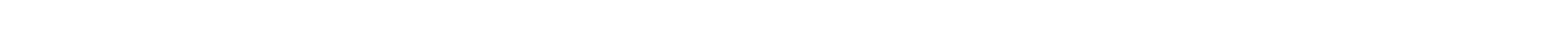

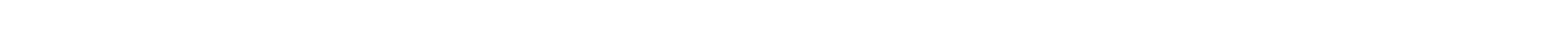

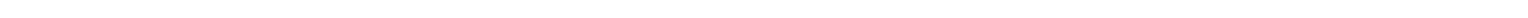

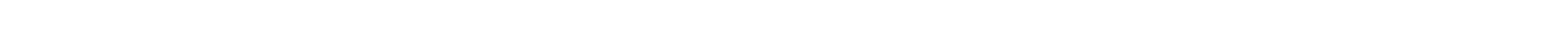

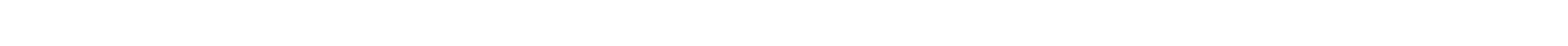

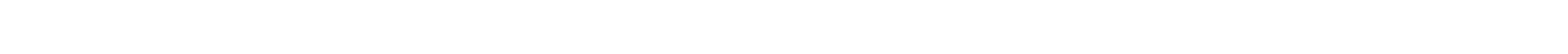

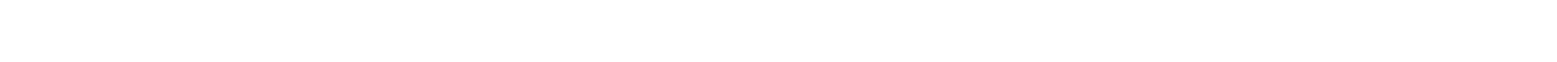




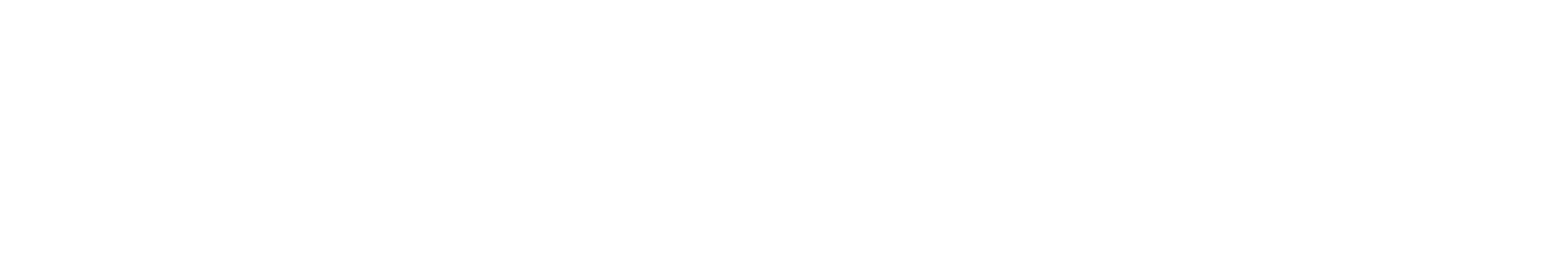

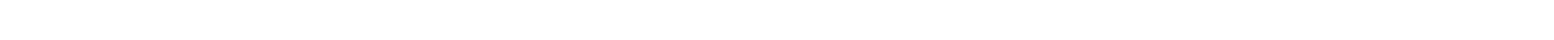

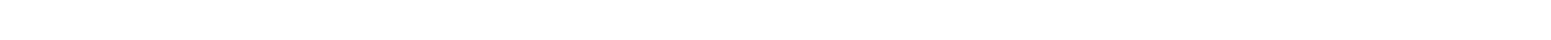

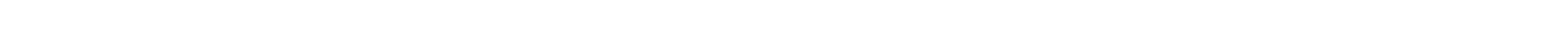

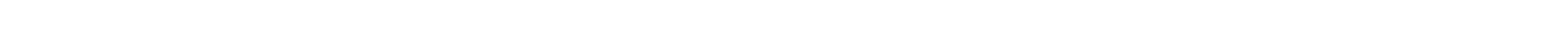

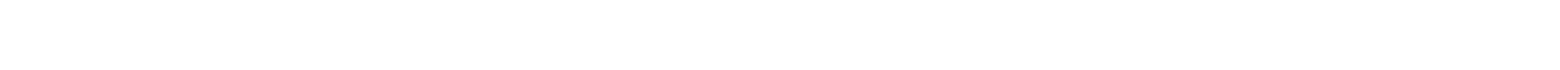

$\underline{n}$ a

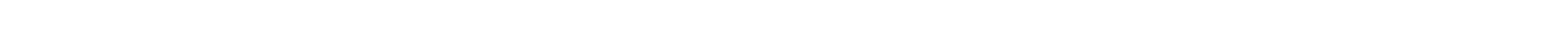

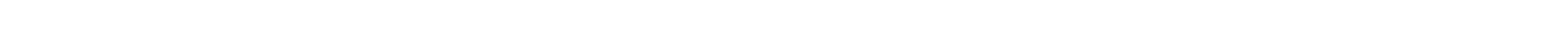

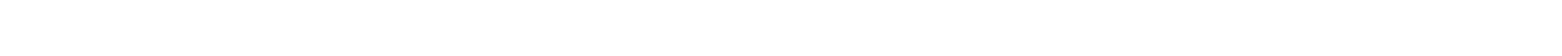

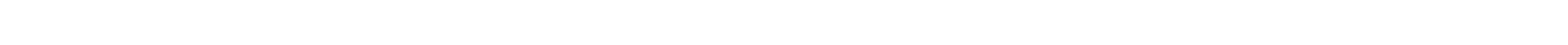

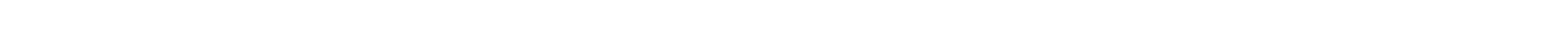

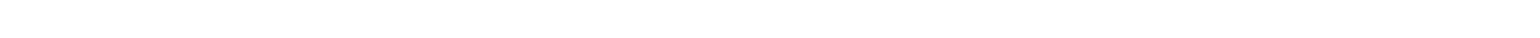

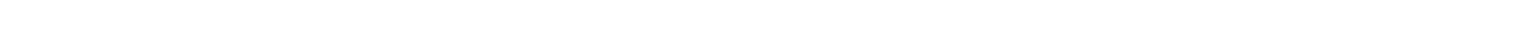

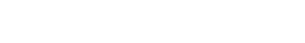
n
is
n
n
n in

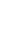

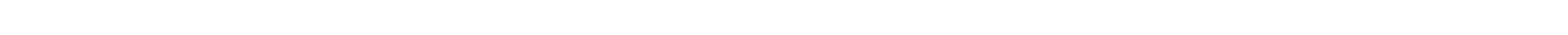
ü

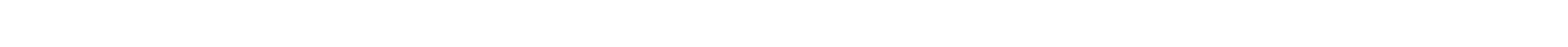

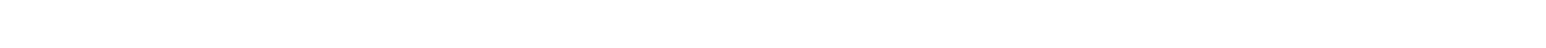

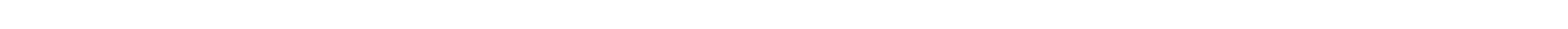

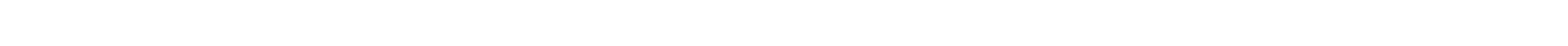

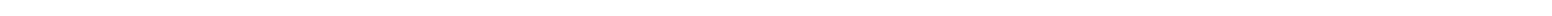

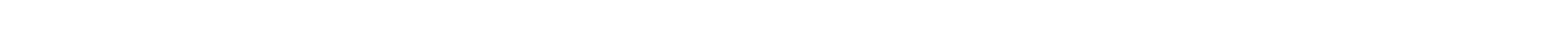

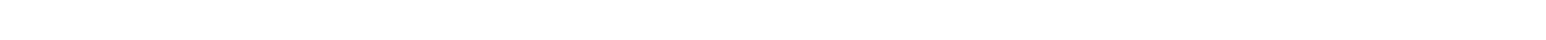
w or
in is

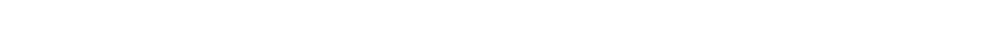
E

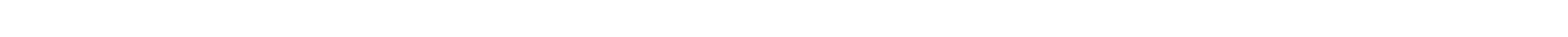
견

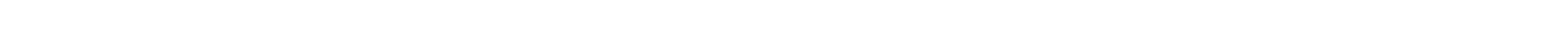
\begin{tabular}{lll}
\hline \\
\hline
\end{tabular}

का 2.

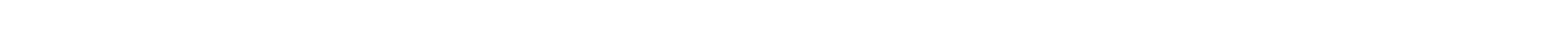

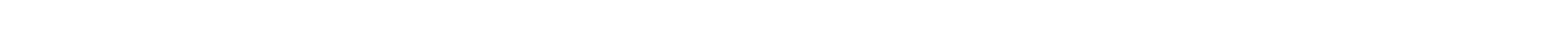

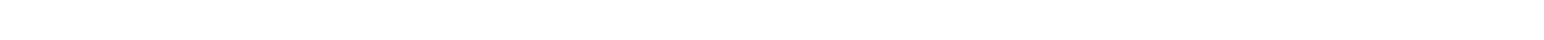

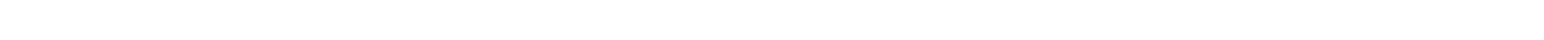




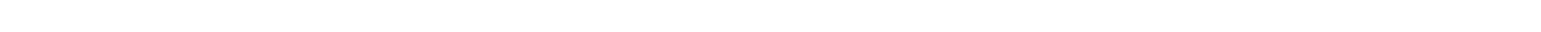

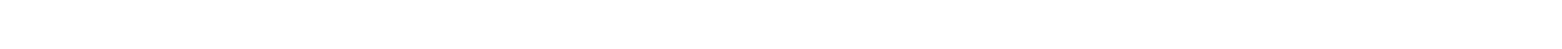

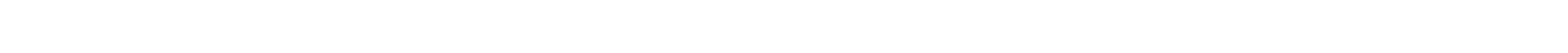

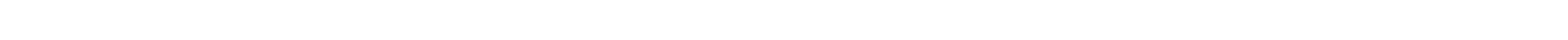

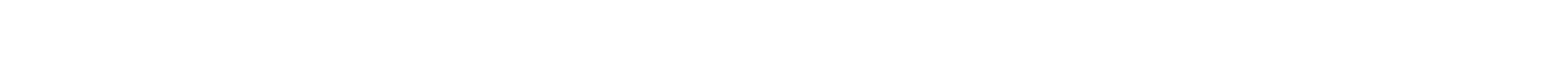
us

s:-

n

n

is

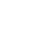

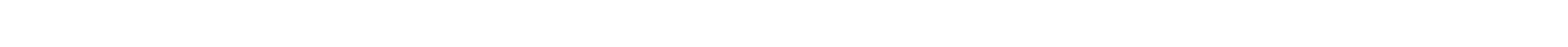
世

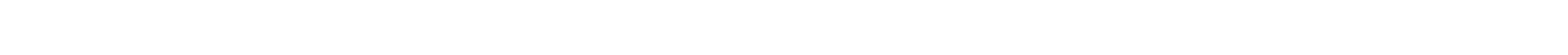

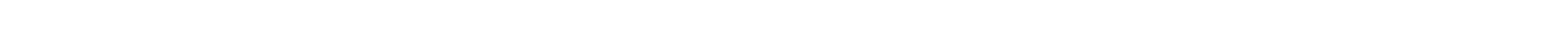

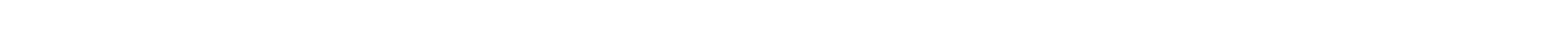
a

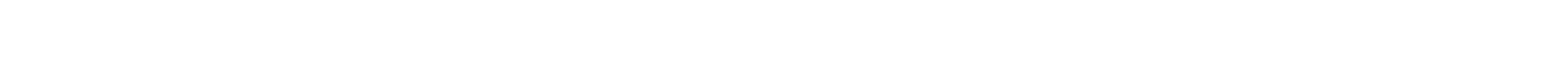
000000000000000000000000000000000000000000000000000

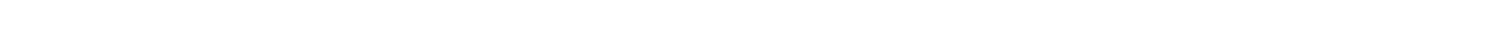

i

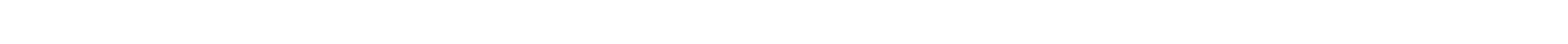

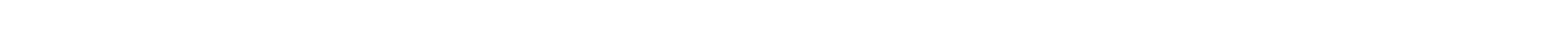

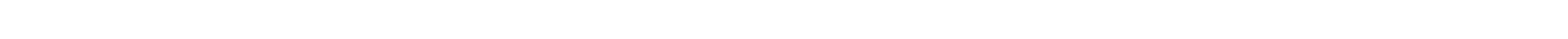

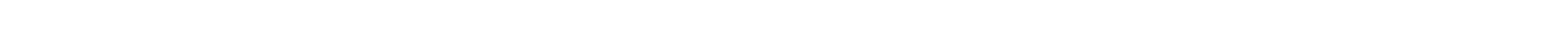

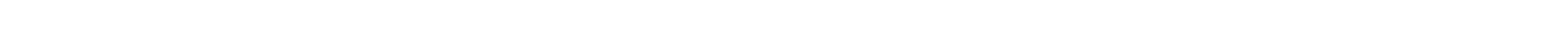

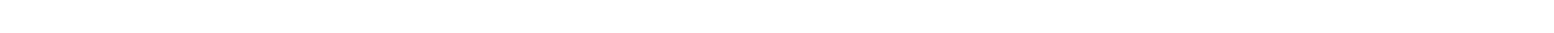

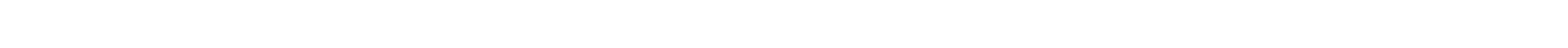
a

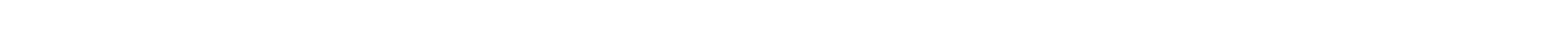

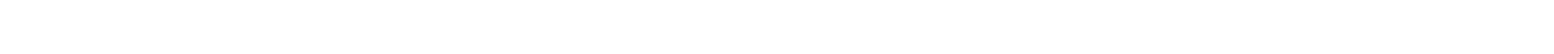

西

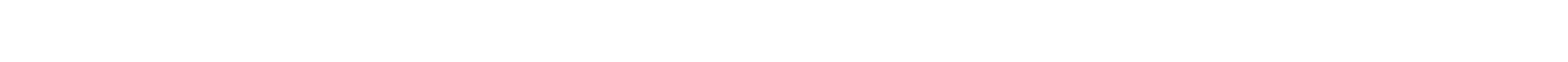
4. แ

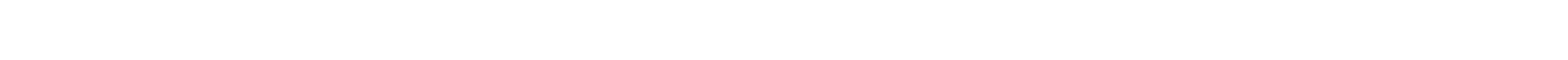

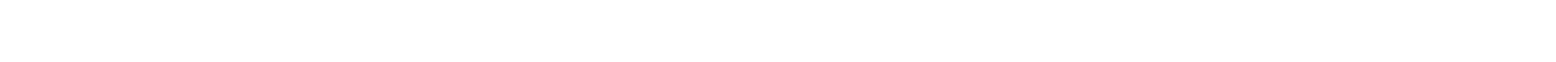

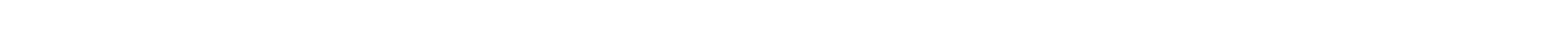

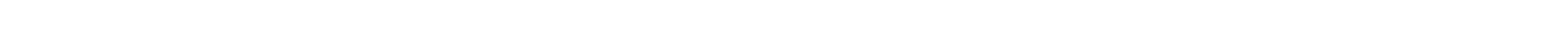

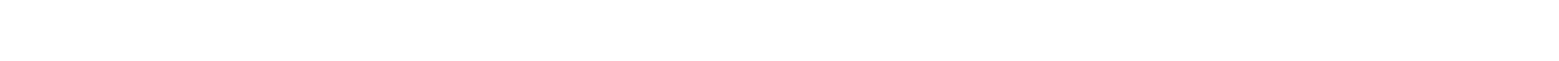


5

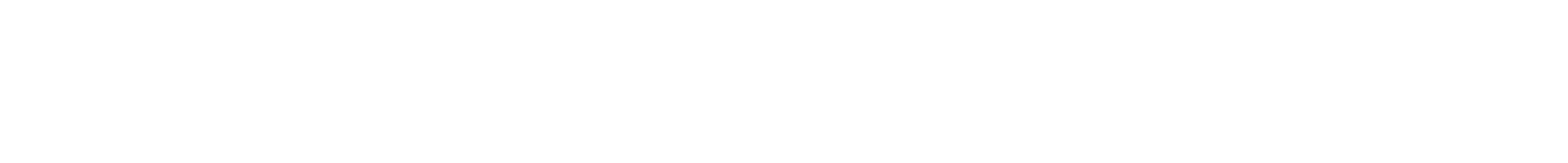

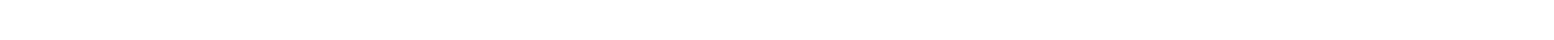

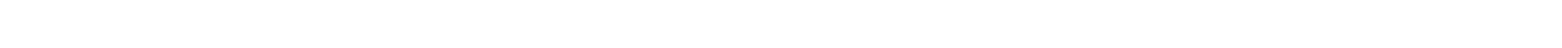

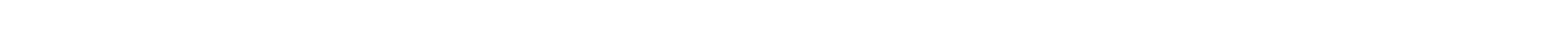

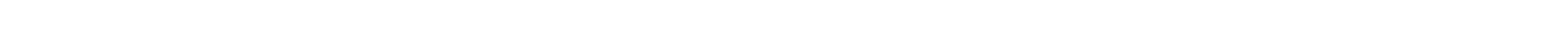

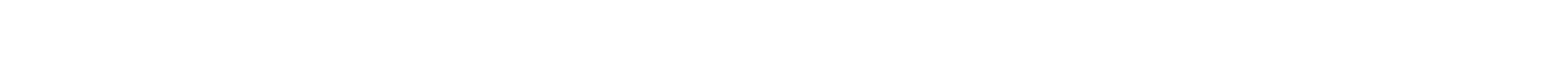

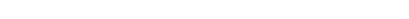

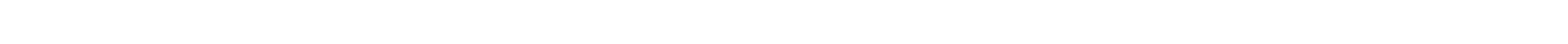

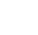

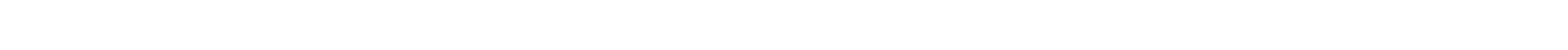

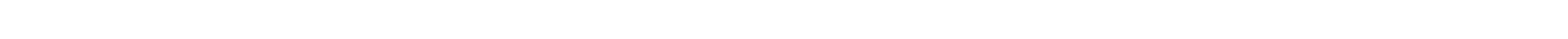

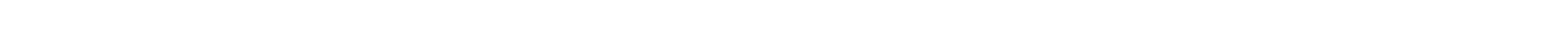
\begin{tabular}{ccc}
3 \\
\hline
\end{tabular}

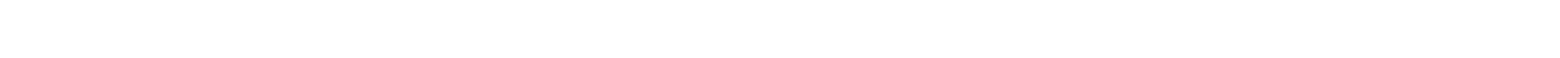

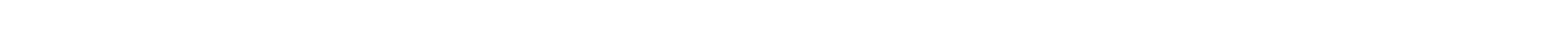

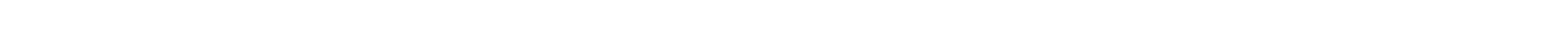

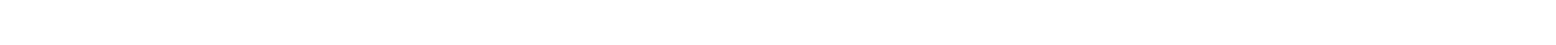

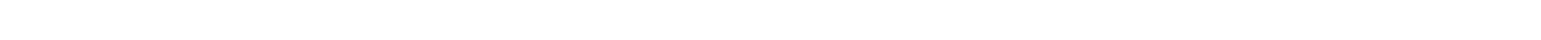
事
n
is
en
in

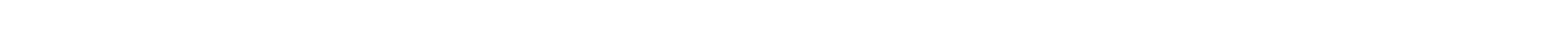

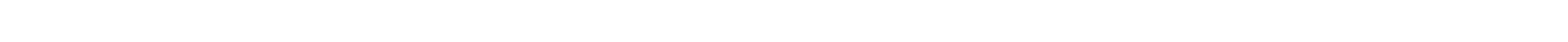

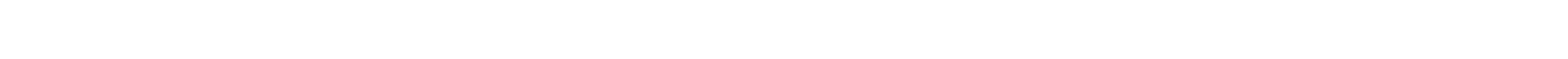

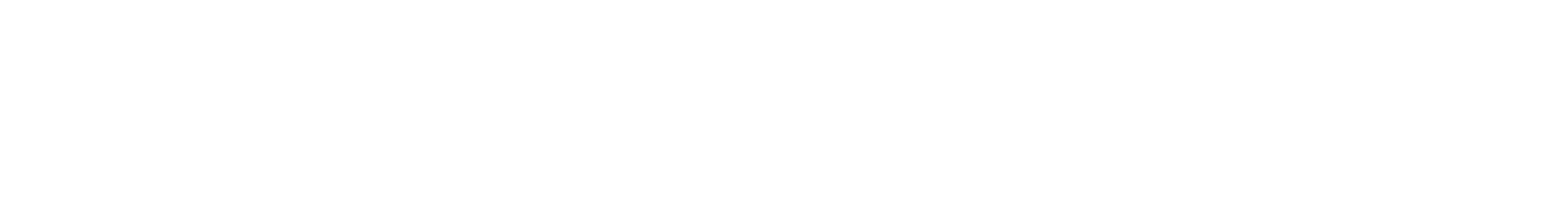

$\Sigma$

is

n

n

is

$\sim$

is

is

is

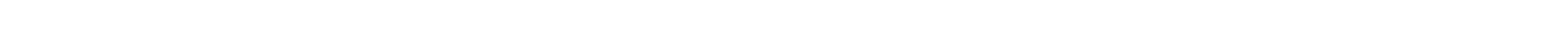

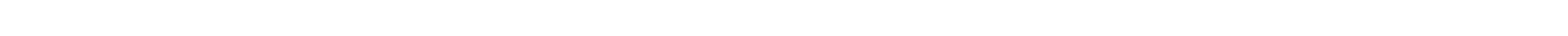

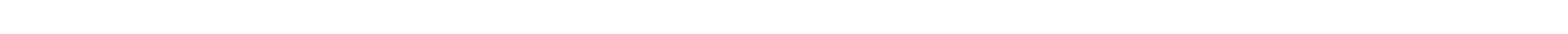
\begin{tabular}{ccc}
3 \\
\hline
\end{tabular}

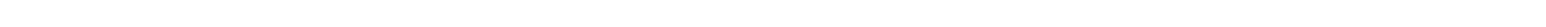

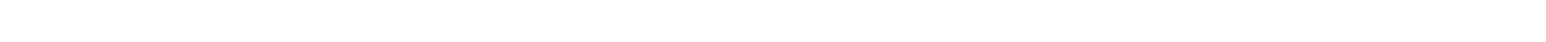

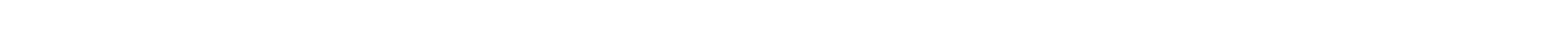

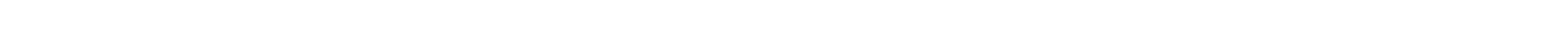

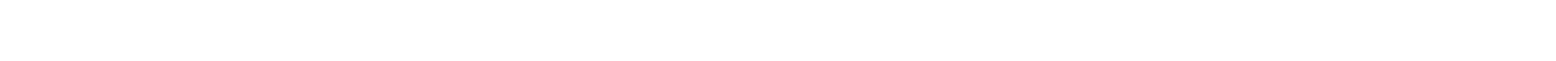




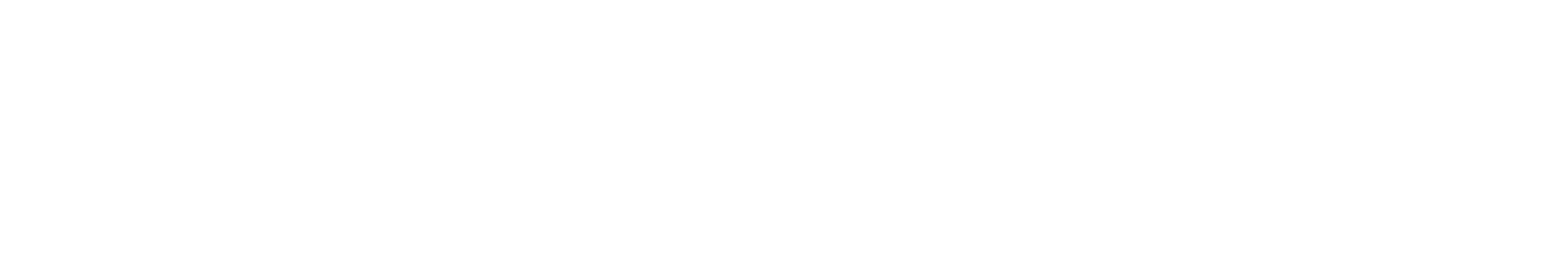

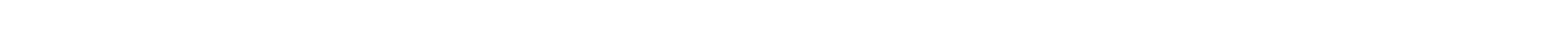

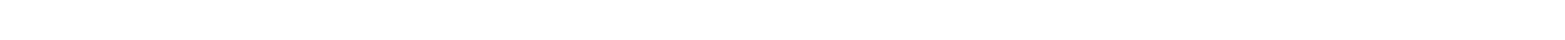

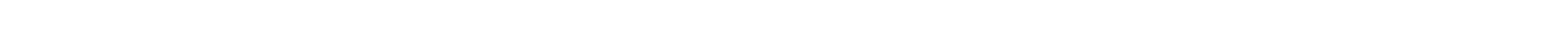

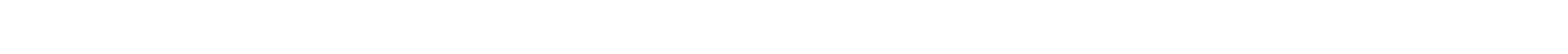

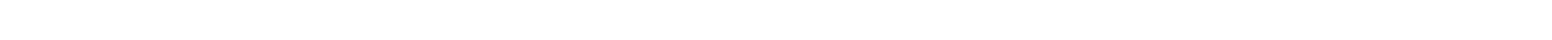

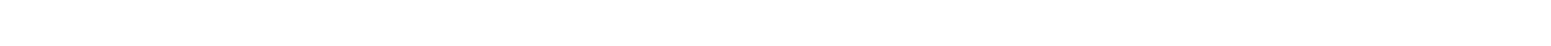

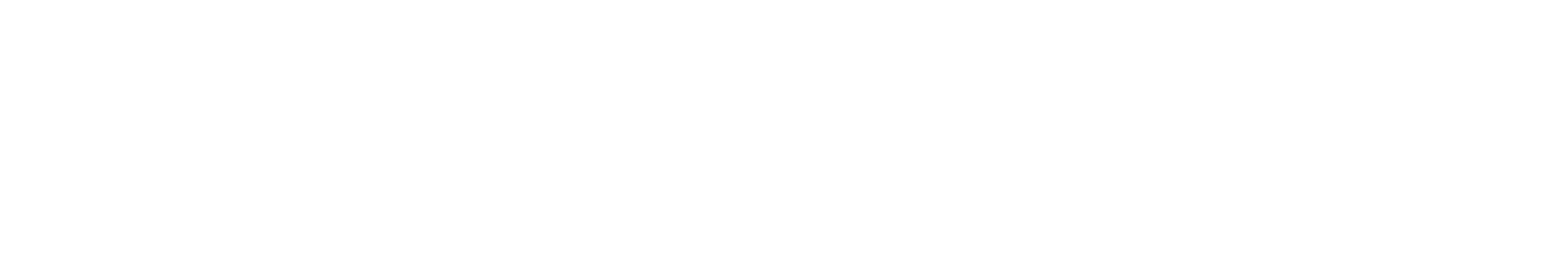

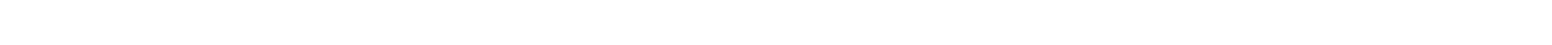
Кूаু แ

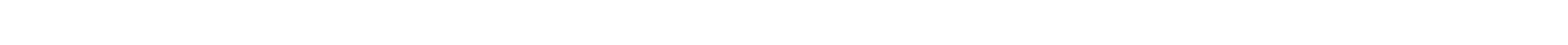

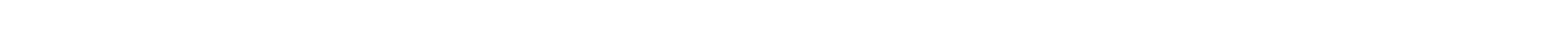

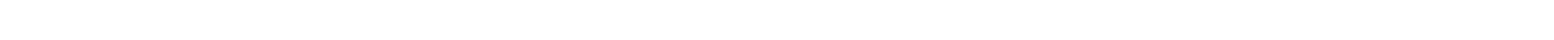

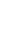

$\underline{m}$

n

n

$\sim$

m

$n$

n

$\stackrel{a}{\infty}$

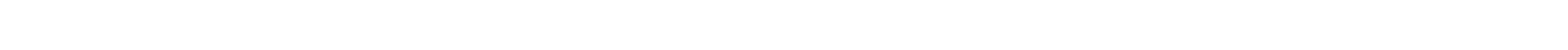
w

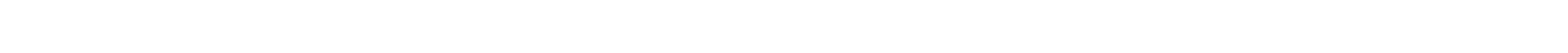

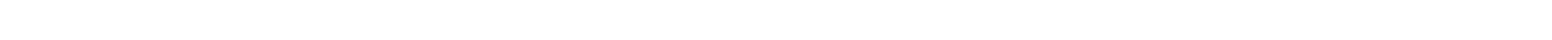

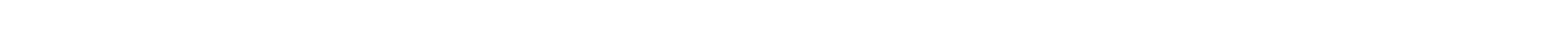

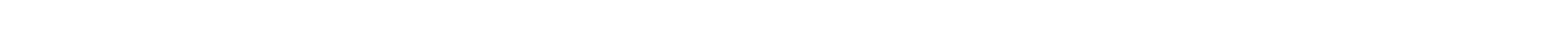

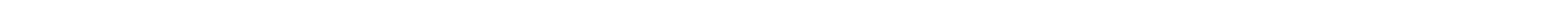

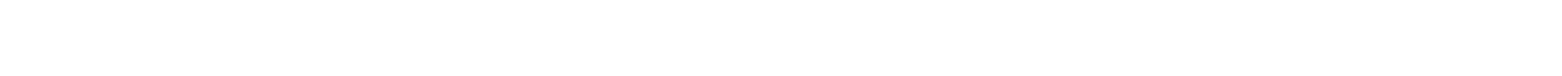
twen n
en
es
n
n
es
n

is

, $\mu$ แ

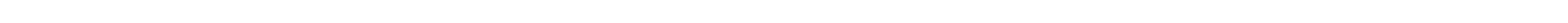

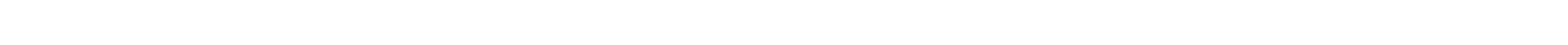

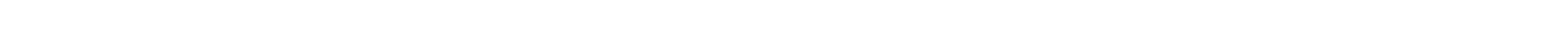

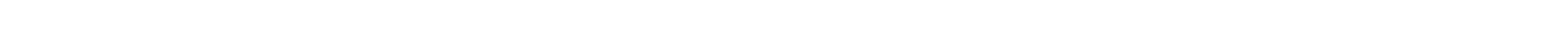
WE ๔

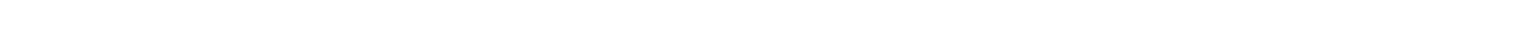

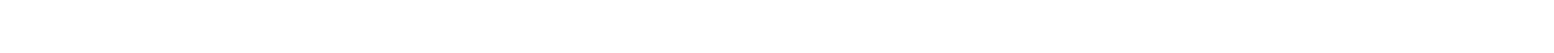



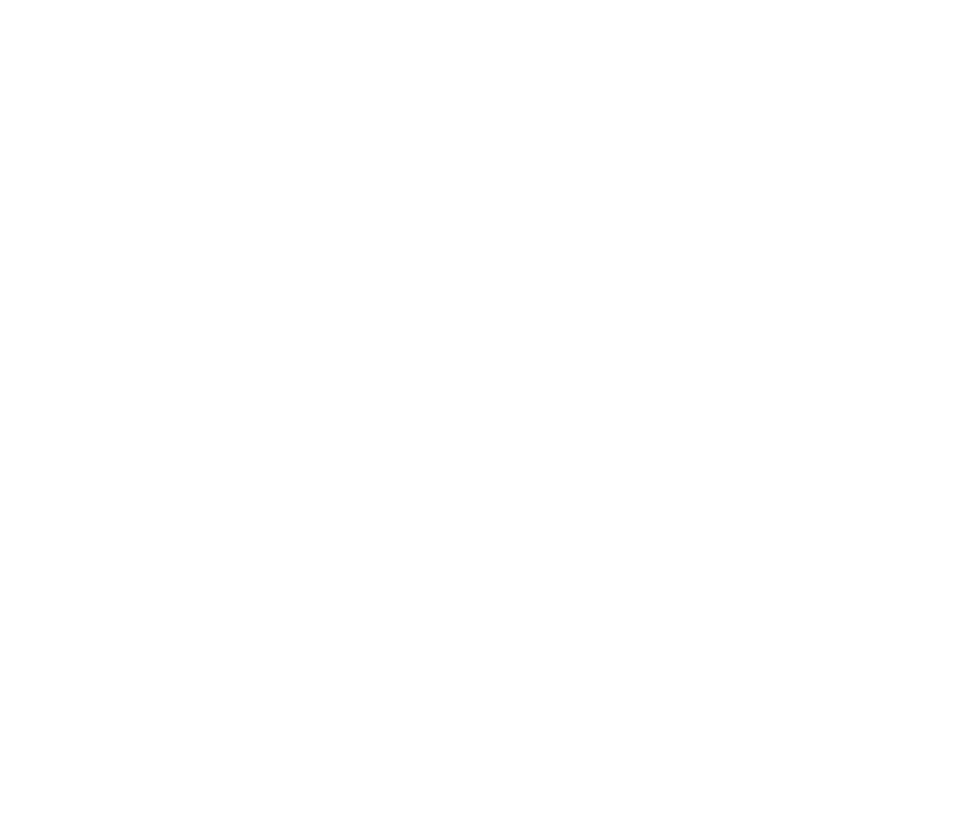

$z$

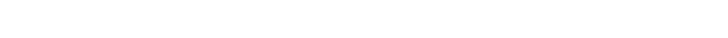
윰ำ

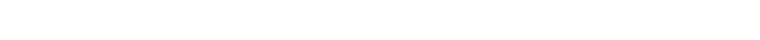

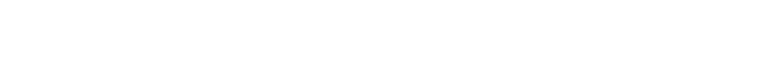

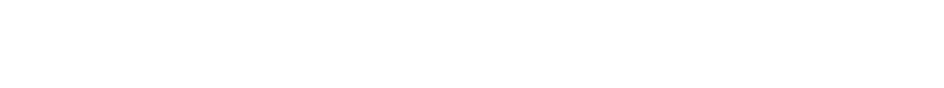

w

w on on

in

染

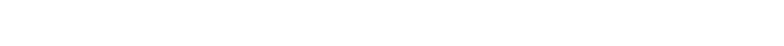

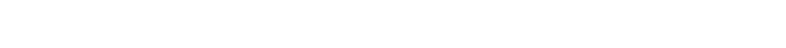

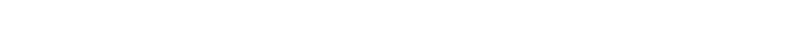

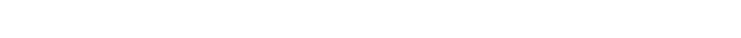

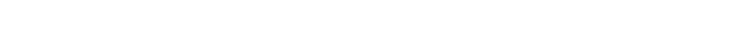

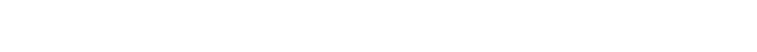

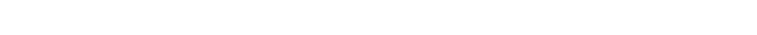

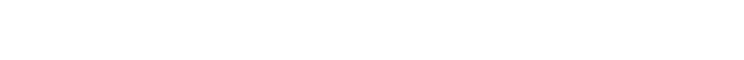

w

twis

is

in

is

$\underset{1}{a}$

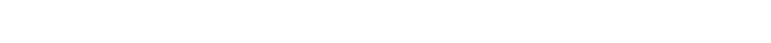

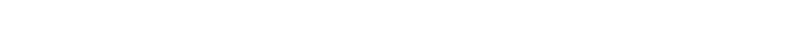

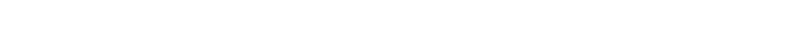

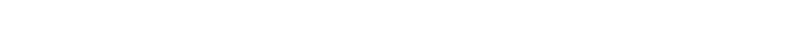

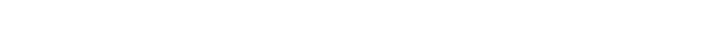

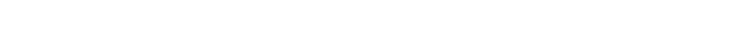

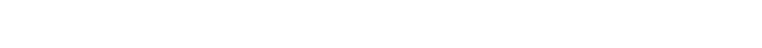

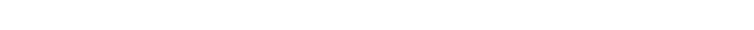

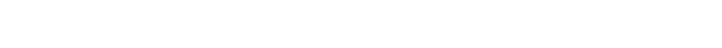




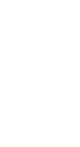

ำำ

西=-

$\cos$

$\alpha$ in

$m$

$\geq=u$

$\alpha \leftarrow z$

以的

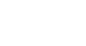

몽

름이

形各

w星

w

$\vec{a} \dot{0}$

$\therefore x=$

$+\infty$

แํㅇㅇ

든

ztm

똔.

잉

a

品东

mo

쏭

원욘

可

只放

-

$-\dot{0}$

(10)

느는

는

แa"

zัด

กัx운

.

웜

小을

wa

× 4

牙交

峛 $z$

은 $\stackrel{m}{\sim} \tilde{v}=$

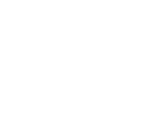

$-\stackrel{a}{a}-\frac{i}{z}$

- $\cong$

o

. in ..

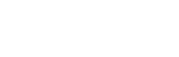

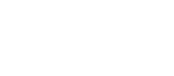

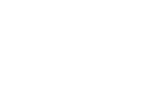

$n z+z a z$

ํํㅇำก

エNE-

는응

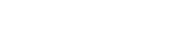

ํํㅇ응응응

- $\ddot{\omega}-\ddot{\omega} \sim \ddot{\omega}$

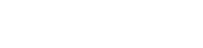

a

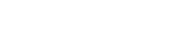

丞

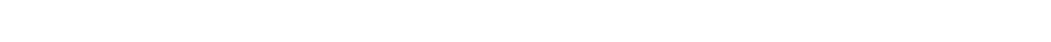

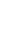

mombag-mmnOc.

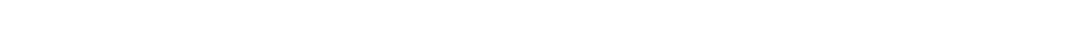

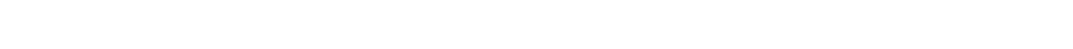

으ㅇㅠㅛ

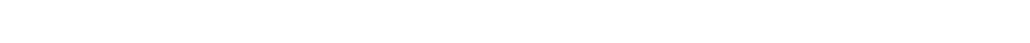

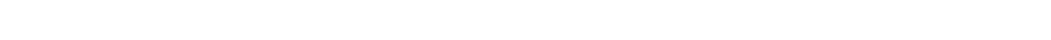

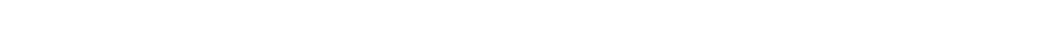

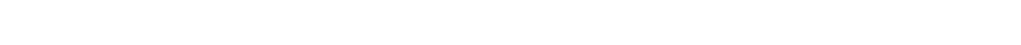

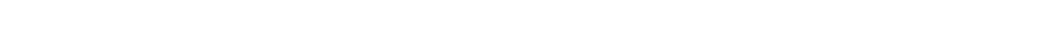
is

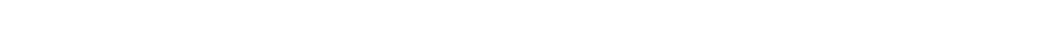

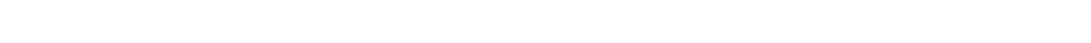
—

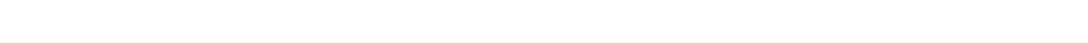

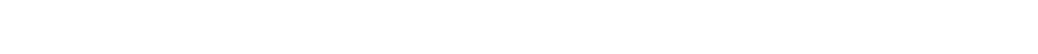

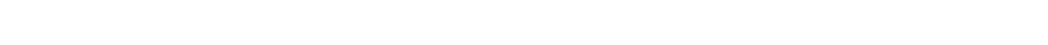
ニ

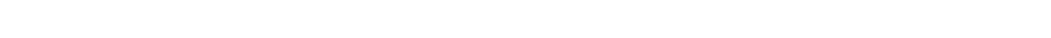

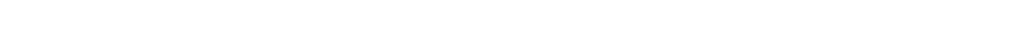

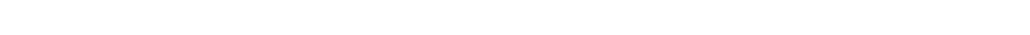

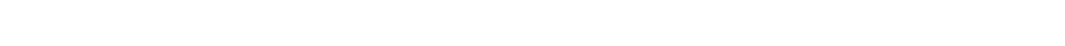

$\ln ^{2} \alpha$

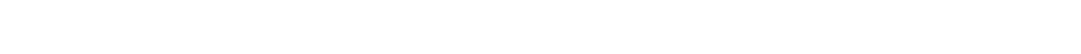
u

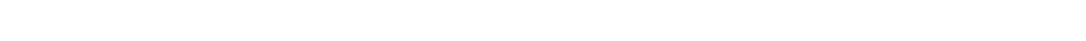

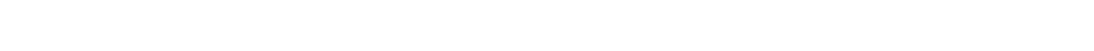

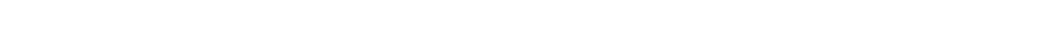

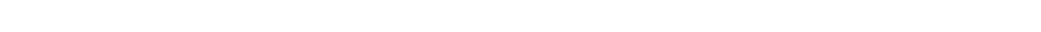

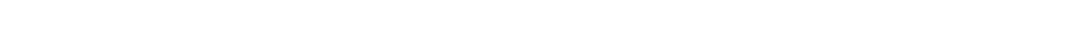
- -

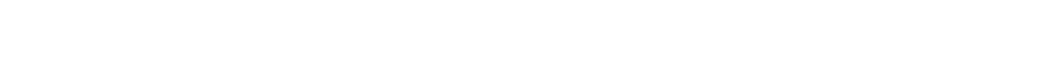

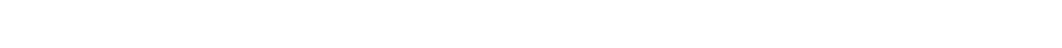
$\stackrel{\star}{5}$

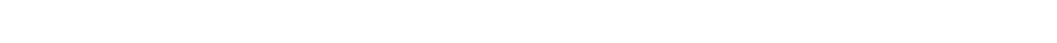
a แ

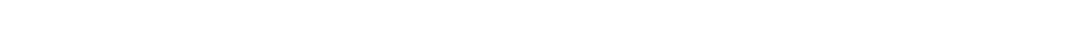

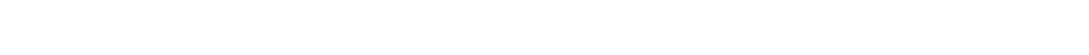

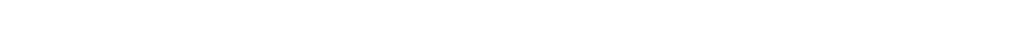

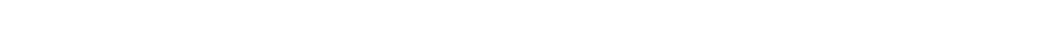

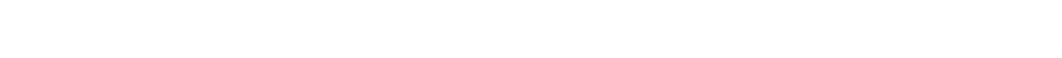

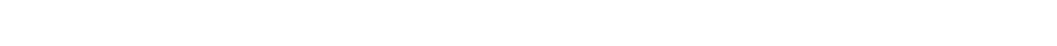

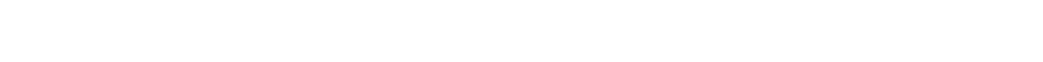




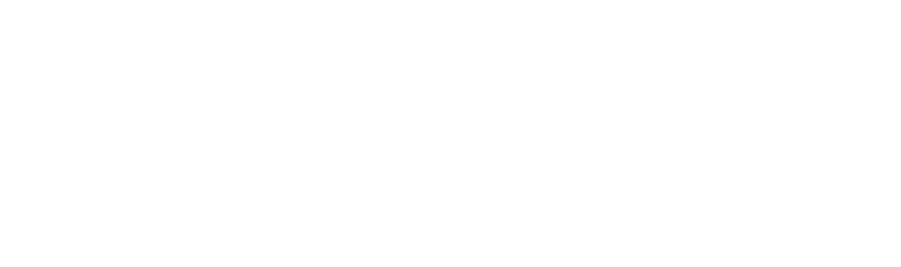

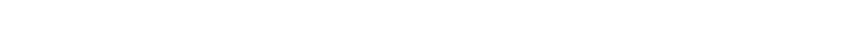

is

sonno-nanonnmora omonamumoonno a

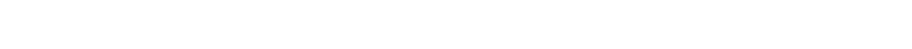
- यа̃

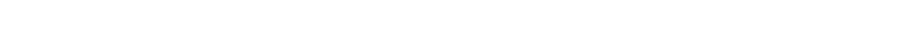

$$
\begin{aligned}
& \begin{array}{l}
\circ \\
\therefore \quad \\
-\quad 0
\end{array}
\end{aligned}
$$

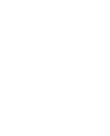

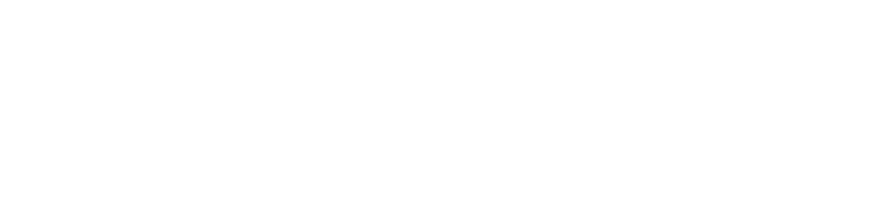

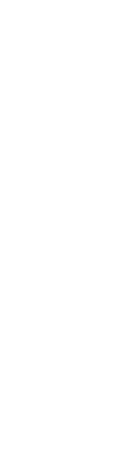

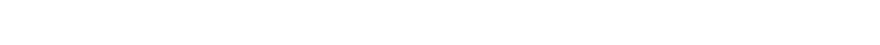

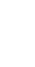

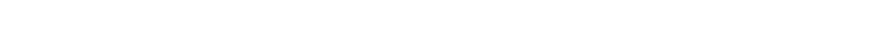

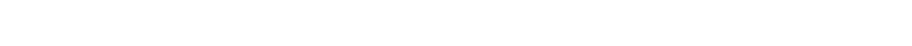

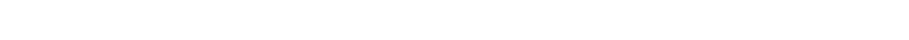

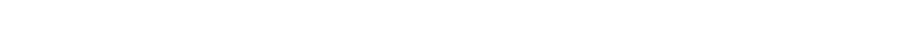

$m \equiv 0 \geq$ ㄴำ

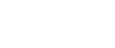
กับธั์

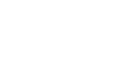
乞 $\sum_{0 \rightarrow 0}^{0}$

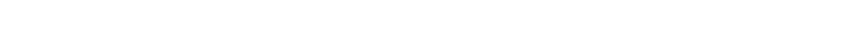

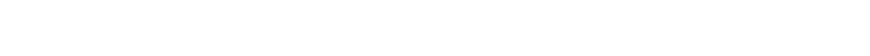

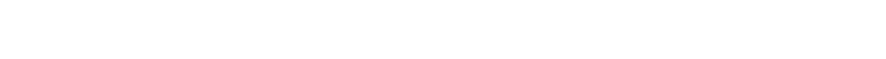

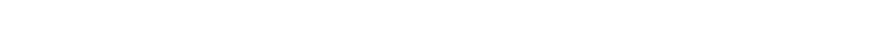

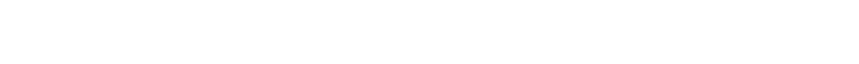

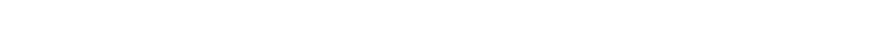

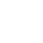
is

๔

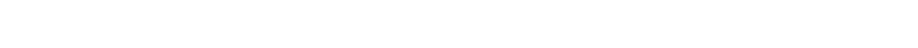

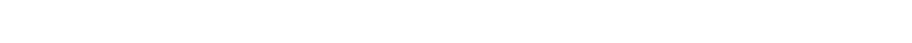

$z^{2} \geq$

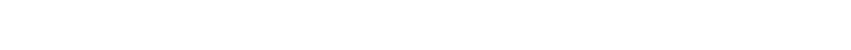

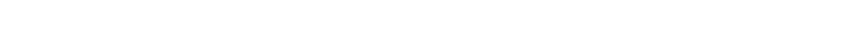

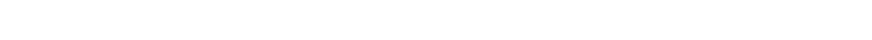

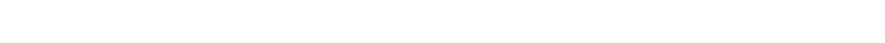

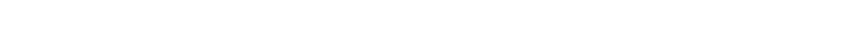



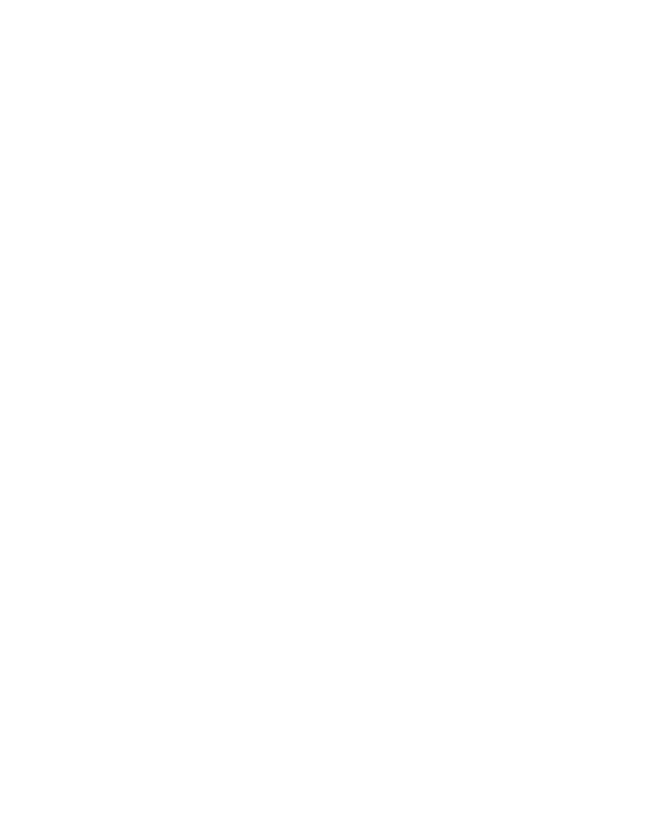

z

采 a

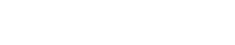

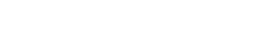

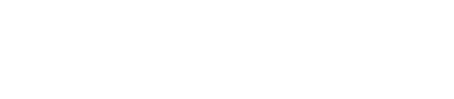

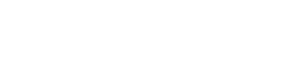

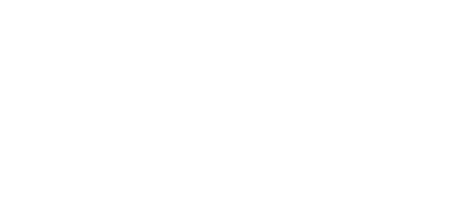

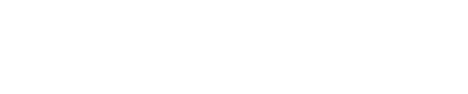

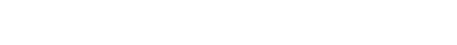

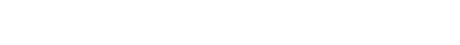

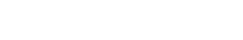

mzoz tónesosenosos 2⿺⿻一𠃋十 范芯皆 的望a 응 茈茫

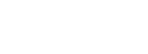

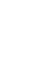
5

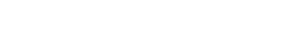
w

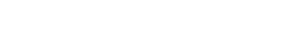

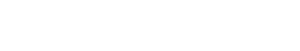
을을

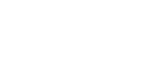
MMmMmmM⿻

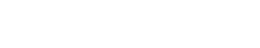

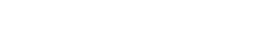

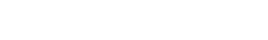

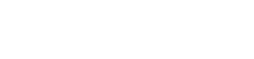




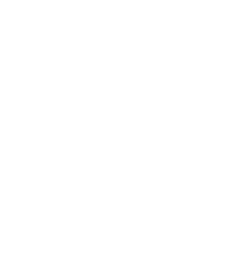

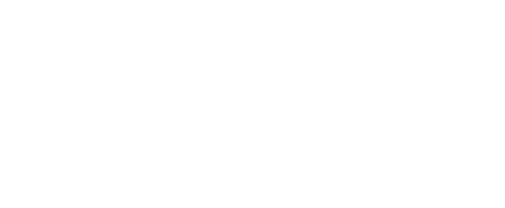

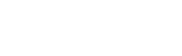
is

mmmom

嵒

$\rightarrow 0 n \dot{0} 0$

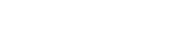

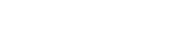

ü nrina

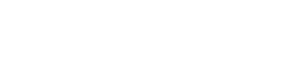

ㅁ añ்

यi

남ำ

$=n^{2}$

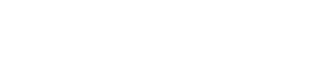

og a

-

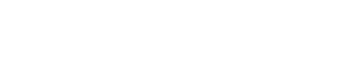

웅유.

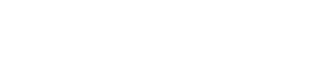

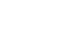

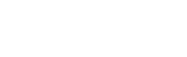

毃方立 

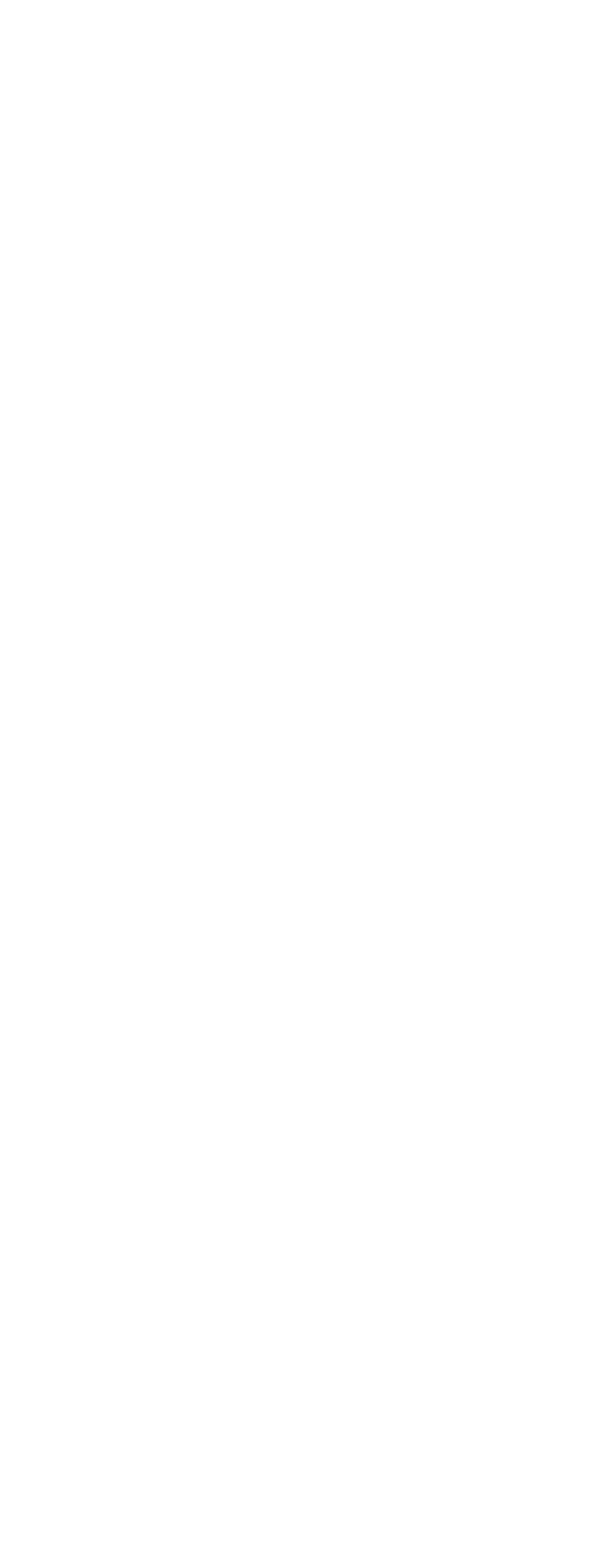

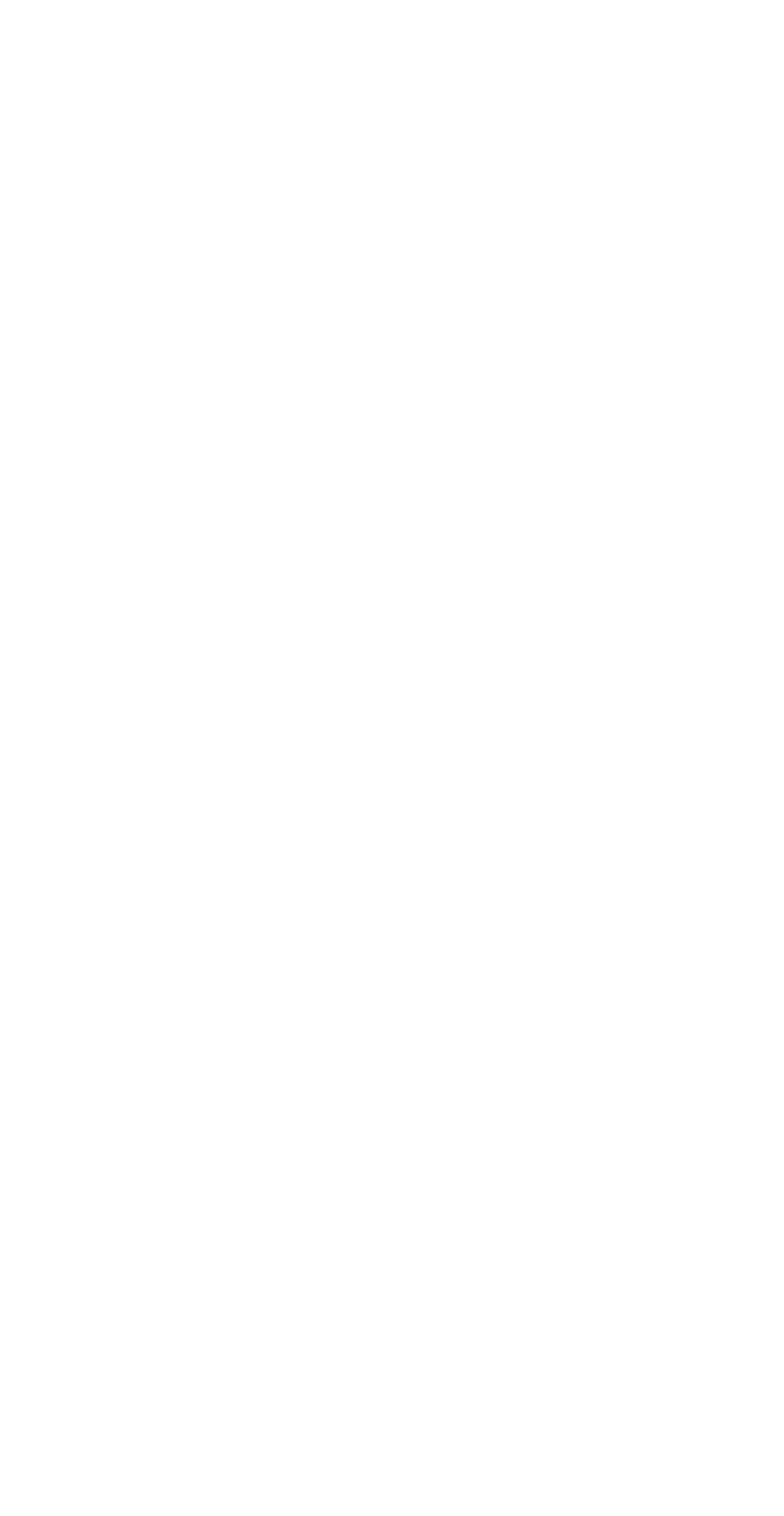

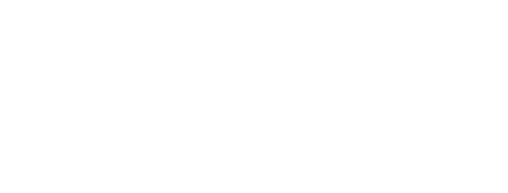

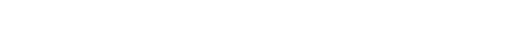

! i. 出 mmmmmmmmmmm

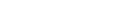

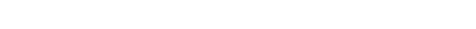

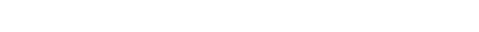
r $-0-n-n-0=-0-n=$

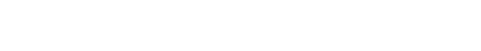

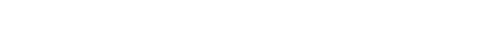
is

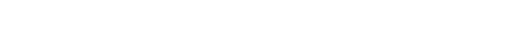
$-1-1-10-10$

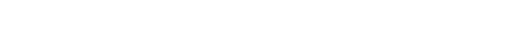

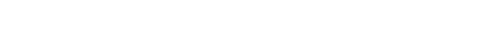

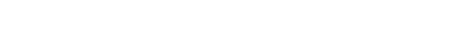

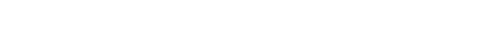

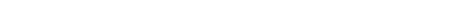

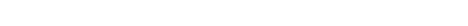
$\dddot{m}-\frac{\omega}{2}=E$

MUN ic 0 w id $=5$

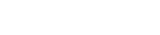

win $x=0$ 一⿻上丨 $>10$ aำ $\sum \Sigma z-r z$

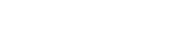

STEENRNNMMmMMmas

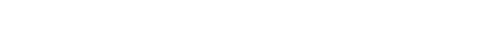

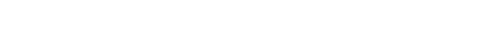

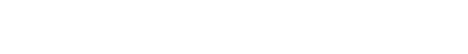

